\title{
DETECTION AND ANTIMICROBIAL ACTIVITY OF IMMOBILIZED QUATERNARY AMMONIUM ANTIMICROBIAL MONOLAYERS ON POROUS AND NON-POROUS SURFACES
}

by

\author{
Lukasz Porosa \\ Bachelor of Science in Applied Chemistry and Biology, \\ Ryerson University, Toronto, Canada, 2006 \\ Master of Science in Molecular Science, \\ Ryerson University, Toronto, Canada, 2008
}

\begin{abstract}
A dissertation presented to Ryerson University
in partial fulfillment of the requirements for the Doctorate of Philosophy

in the Program of Environmental Applied Science and Management
\end{abstract}

Toronto, Ontario, Canada, 2014

CLukasz Porosa 2014 


\section{AUTHOR'S DECLARATION}

I hereby declare that I am the sole author of this dissertation. This is a true copy of the dissertation, including any required final revisions, as accepted by my examiners.

I authorize Ryerson University to lend this dissertation to other institutions or individuals for the purpose of scholarly research

I further authorize Ryerson University to reproduce this dissertation by photocopying or by other means, in total or in part, at the request of other institutions or individuals for the purpose of scholarly research.

I understand that my dissertation may be made electronically available to the public. 


\title{
DETECTION AND ANTIMICROBIAL ACTIVITY OF IMMOBILIZED QUATERNARY AMMONIUM ANTIMICROBIAL MONOLAYERS ON POROUS AND NON-POROUS SURFACES
}

PhD, Lukasz Porosa, Environmental and Applied Science, Ryerson University, 2014

\begin{abstract}
This research describes the development of novel, environmentally-friendly, nonreleasing contact-active thin film coatings by immobilizing the quaternary ammonium (QA) antimicrobial group on a multitude of surfaces. Various chemical anchors based on organosilanes (i.e. textiles, silica, oxide surfaces), organosulfur comprising of thiol (noble metals), organophosphorus comprising of phosphonate and phosphonic acid (i.e. stainless steel (SS), titanium (Ti)), and catechol (Ti, SS) monolayers are employed to attach the QA antimicrobial onto metal surfaces, while benzophenone photoactive crosslinkers containing QA groups are used to coat plastic surfaces (C-H surfaces, i.e. polyethylene (PE), silicone (Si), polyvinylchloride (PVC)). Surfaces treated with covalently attached antimicrobial coatings function by killing microbes on contact, preventing surface attachment, colonization and contamination without releasing the chemical into the environment. The advantages of this method of delivery of the antimicrobial include a lower cost of application, decreased antimicrobial resistance, lower toxicity and increased environmental safety.
\end{abstract}

Samples prepared by an overnight immersion in an ethanolic solution of phosphorus containing quats followed by an overnight cure at $100^{\circ} \mathrm{C}$ showed the highest antimicrobial reduction versus electrospray application and no curing. Short chain phosphonic acid quats and the organosilane quat were inactive on titanium. Antimicrobial activity of long chain 
phosphonate quats prepared by dip coating and annealing on metal surfaces (Ti, SS, Al) was tested by growth enumeration in the dry state utilizing methods developed in the Wolfaardt lab. All samples showed a $100 \%$ reduction ( $10^{6}$ cells) of viable Salmonella, Arthrobacter, S.aureus and P.aeroguinosa after 2 hrs of contact time and maintained their activity over 24 hrs versus the uncoated controls. To demonstrate the phosphonate quats were truly immobilized, Ti samples from the first trial were washed in distilled $\mathrm{H}_{2} \mathrm{O}$, dried, and re-innoculated with $10^{6}$ Anthrobacter colonies. No visible colonies of Anthrobacter remained after 2 hrs of contact time with the Ti surfaces indicating a contact killing mechanism at play. 


\section{ACKNOWLEDGEMENTS}

First and foremost, I would like to thank my supervisors, Dr. Foucher and Dr. Gideon Wolfaardt for this research opportunity and providing me with what ever I asked for. Secondly, I would like to thank Joe Raich and Bioshield Technologies Canada Ltd. for teaming up with Ryerson University and providing the necessary funding to run this project successfully. Special recognition goes out to my committee/candidacy members Dr. Russ Viirre, Dr. Bryan Koivisto, Dr. Rob Gossage, and Dr. Donal Macartney for their insights, helpful advice and thesis corrections. I would also like to recognize Dr. Michal Bardecki for playing a key role in my acceptance to the EnSciMan program and for supporting Ryerson athletics over the years.

I would also like to thank all of the undergraduate students I had the pleasure to guide and work with over the past four years including Kamlesh Mistry, Shayan Hamzehi, Khaled Hamza, Amanda Mocella, Shayan Hamzehi and Hellen Deng. They were all instrumental in work and help on the Bioshield project over the years. Specifically, I would like to acknowledge the pioneer of the dansyl work, Kamlesh Mistry who contributed along with Grace Luk to collecting UV-VIS/fluorescence spectra. Thanks to Gabriel Wolfaardt who spent countless hours inoculating various bugs on metal and plastic surfaces treated with the QAC antimicrobials in the Wolfaardt and thanks to Alexander Caschera for his assistance with formatting the appendix. As well thanks to all of the graduate students (Aman, Sofija, Shane, Khrystyna, Michelle) who had to put up with me over the years. Most importantly, I would like to thank my patient, loving and supporting parents, Irena Porosa and Miroslaw Porosa who started everything and the reason you are reading this today. 


\section{DEDICATION}

To all my friends and family, especially my parents Mirek and Irena Porosa and my sister Natalia Porosa-Paoli for all the unconditional love and support over the years. 
TABLE OF CONTENTS

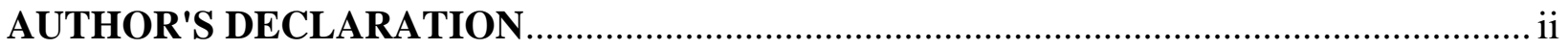

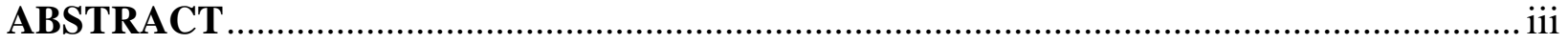

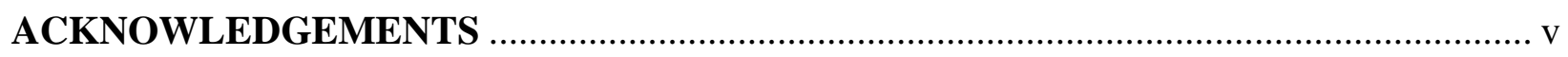

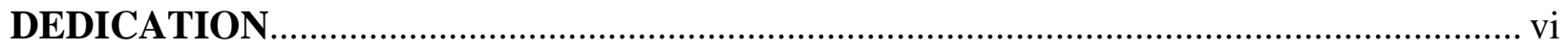

TABLE OF CONTENTS ............................................................................................... vii

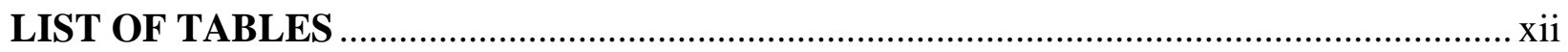

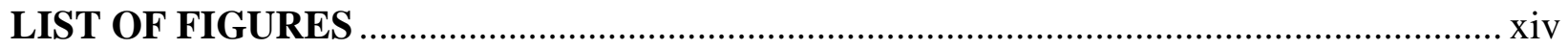

LIST OF ABBREVIATIONS ...........................................................................................

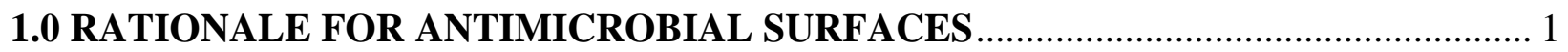

1.1 Examples and Preparation of Antimicrobial Surfaces.................................................... 5

$1.21^{\text {st }}$ Generation Antimicrobial Coatings: Literature Examples of Contact Active QA

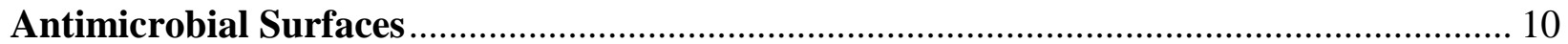

1.2.1 Polyhydroxylated Surfaces (Textiles, Silica, Glass) ......................................................... 10

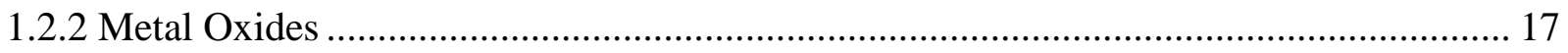

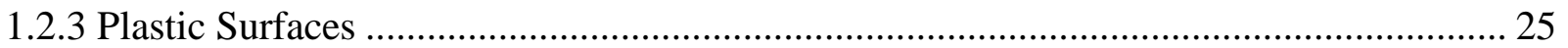

1.2.3.1 “QAC's as Additives” ........................................................................................... 28

1.2.3.2 Thermoset Antimicrobial Coatings: Cross Linked Networks ...................................... 29

1.2.3.3 “Grafting Onto” and “Grafting From” Plastic Surfaces ................................................ 31

$1.32^{\text {nd }}$ Generation Antimicrobial Coatings: Literature Examples of Dual Action

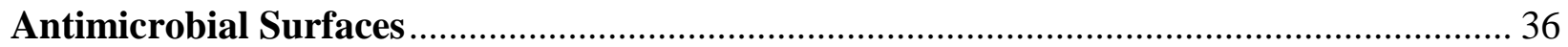

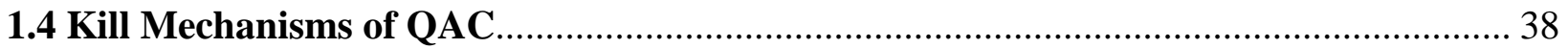

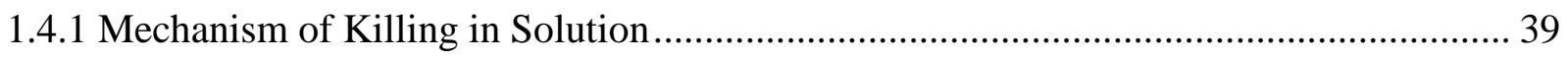

1.5. Mechanism of Immobilized Contact Active QAC.......................................................... 46

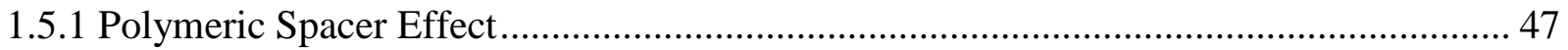

1.5.2 Phospholipid Sponge Effect ........................................................................................... 49

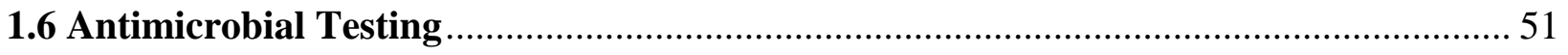

1.6.1 Growth Based Enumeration .............................................................................................. 52 


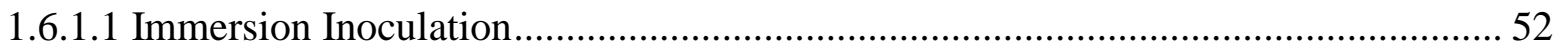

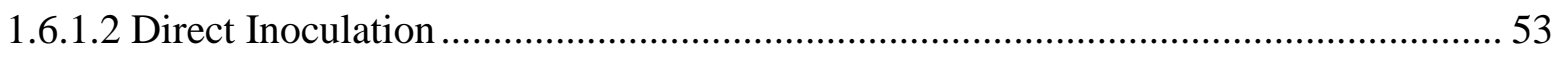

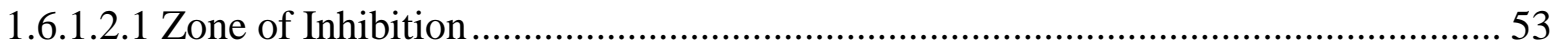

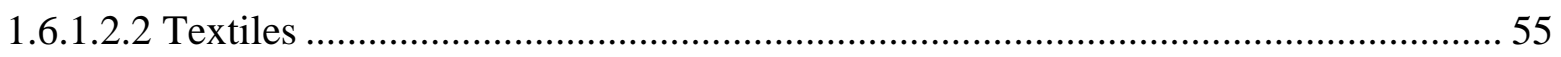

1.6.1.2.3 Hard Surfaces: Metals, Plastics, Glass ………………………………………........ 55

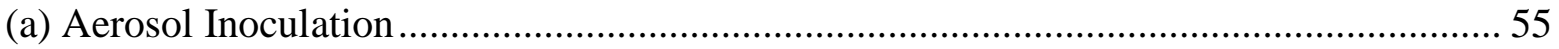

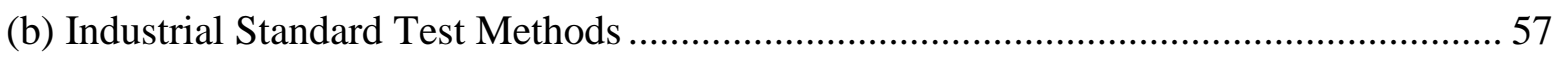

(c) Wolfaardt's Lab Modification of ISO 22196................................................................... 59

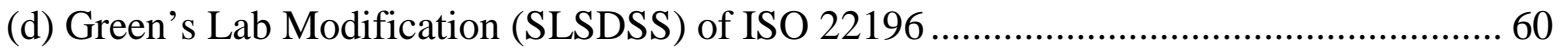

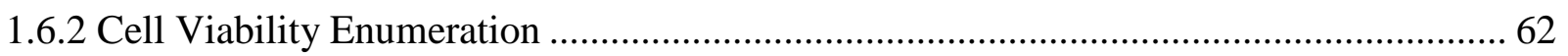

1.7 CHEMISTRY OF QUATERNARY AMMONIUM GROUPS AND

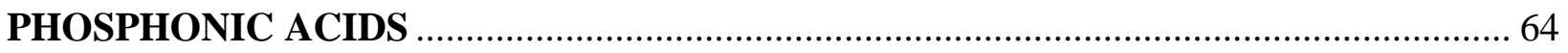

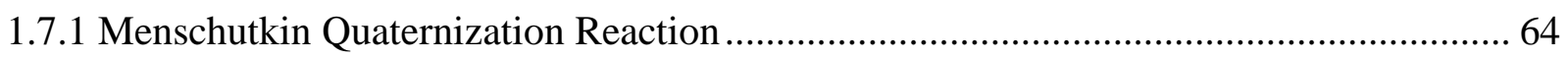

1.7.2 Precursors for the Menshutkin Reaction ............................................................................ 67

1.7.3 Stability of Quaternary Ammonium Groups ....................................................................... 71

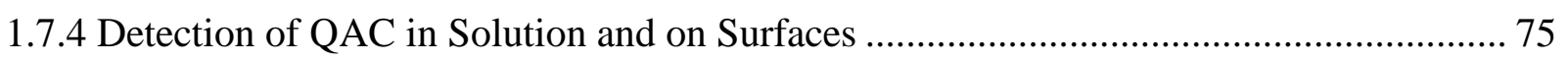

1.8 CHEMISTRY OF ORGANOPHOSPHORUS COMPOUNDS......................................... 79

1.8.1 Mono and Didealkylation of Phosphonate Esters .......................................................... 79

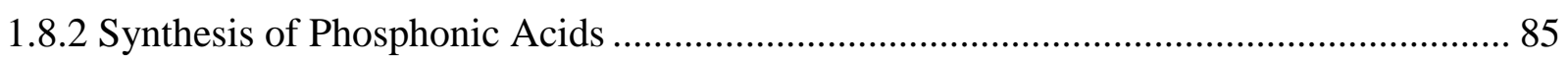

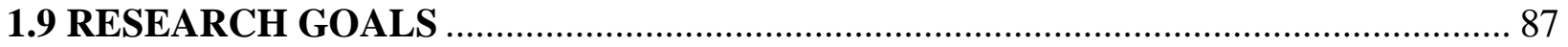

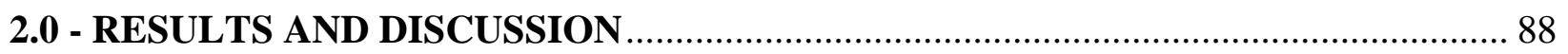

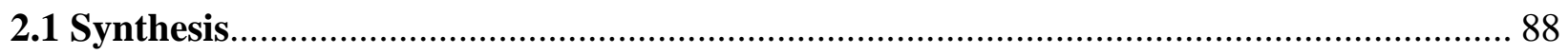

2.1.1 Alkoxysilane-Functional Quaternary Ammonium Cations (SiQAC) ........................... 88

2.1.2 Organophosphorus Functional Quaternary Ammonium Cations QAC Antimicrobials

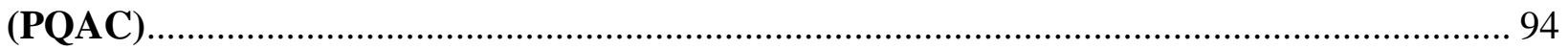

2.1.2.1 $\gamma$-Monophosphonic Acids QAC Antimicrobials ( $\gamma$-MPQAC) ........................................ 94

2.1.2.2 $\gamma$-Bisphosphonic Acids QAC Antimicrobials ( $\gamma$-BPQ) Synthesis .............................. 104

2.1.2.3 $\alpha$-CH Bisphosphonic Acids QAC Antimicrobials ( $\alpha$-CH-BPQA) Synthesis .............. 109

Method 1: Onepot Bis Addition of Dialkylphosphites to Aldehydes.................................. 110 
Method 2: Michael Addition to Diethylvinylphosphonate................................................... 116

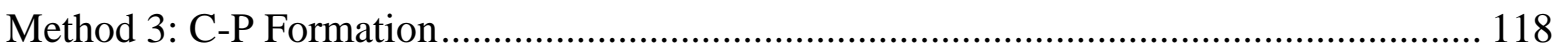

Method 4: Direct Alkylation....................................................................................... 120

2.1.2.4 Bisphosphonates via the 3-Component Reaction.................................................. 130

2.1.2.5 $\alpha$-Aminobisphosphonic acids QAC Antimicrobials ( $\alpha$-ABPQA) ............................... 131

2.1.2.6 $\beta$-Aminobisphosphonic Acids QAC Antimicrobials ( $\beta$-ABPQA) .............................. 143

2.1.2.7 Tridentate Phosphonic Acid QAC Antimicrobials (TPQA) ....................................... 154

2.1.2.8 Amine Scaffolds for Tetra Phosphonic Acid QAC Antimicrobials ............................ 159

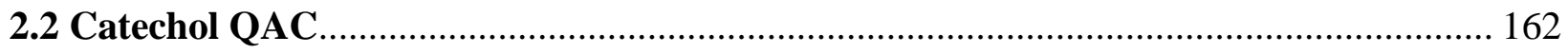

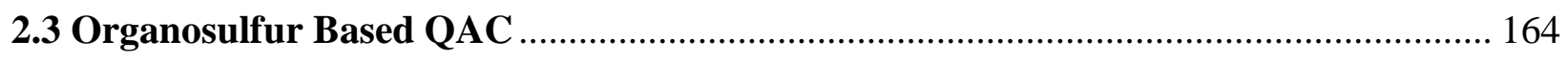

2.4 Benzophenone QAC (Plastic Coating) ……………………………………………..... 167

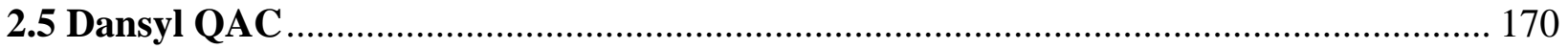

2.5.1 Fluorescent Antimicrobial Coating Detection............................................................. 170

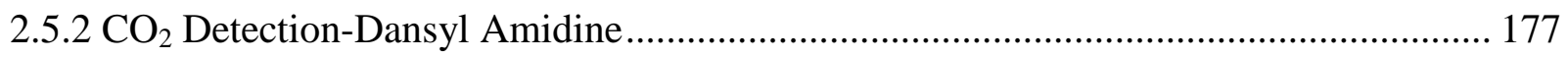

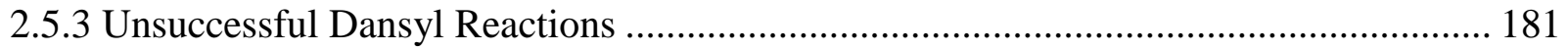

2.5.3 Fluorescence Properties of Dansyl QAC .................................................................... 185

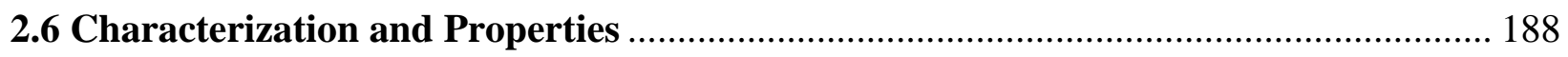

2.6.1 X-ray Crystallography .................................................................................................. 188

2.6.2 Antimicrobial Detection / Coating Procedures ................................................................ 195

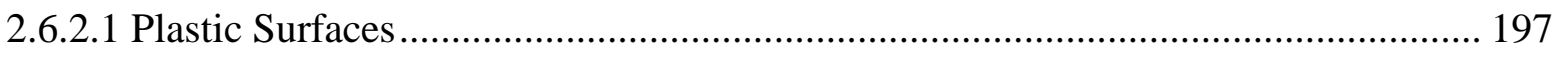

2.6.2.3 Metal Surfaces ………………………………….................................................... 200

2.6.3 Antimicrobial Activity ........................................................................................... 201

2.6.3.1 Solution Killing: Minimum Inhibitory Concentration’s (MIC’s).............................. 201

2.6.3.2 Contact Killing on Hard Surfaces............................................................................ 205

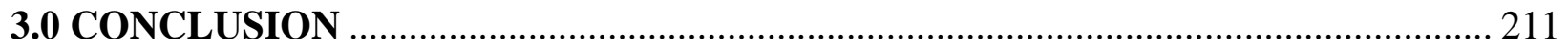

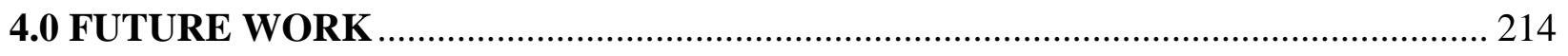

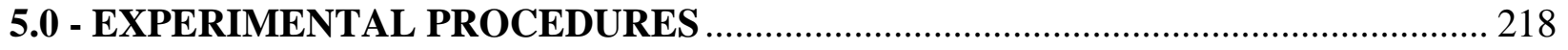

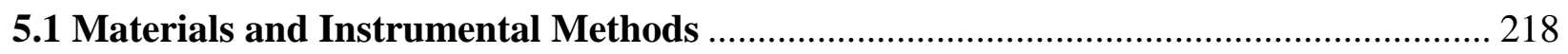




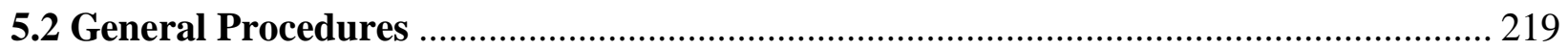

Method 5.2.1 Sealed Tube Reactions............................................................................... 219

Method 5.2.2 $\mu \mathrm{W}$ Reactions............................................................................................... 220

5.2.3 Purification/Preparation of Common Starting Materials ................................................ 220

5.2.4 Dow Antimicrobial Synthesis .................................................................................... 222

5.3 General Procedure for the Synthesis of ( $\gamma$-MPQA's) ..................................................... 222

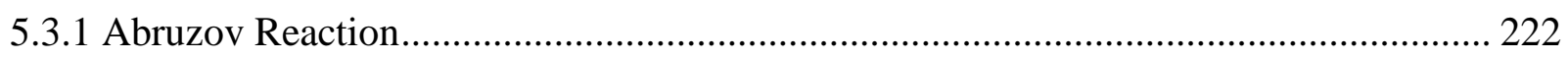

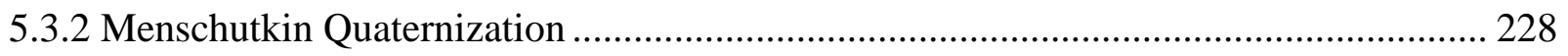

5.3.3 Didealkylation of Phosphonate diesters. .................................................................... 237

5.4 General Procedures for the Synthesis of $\alpha$-CH Bisphosphonic Acids QAC

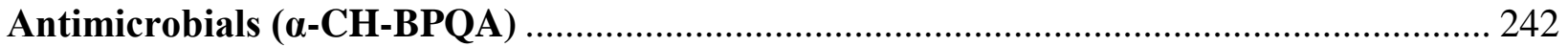

5.4.1 Method 1: Bis Addition of Dialkylphosphites to Aldehydes ......................................... 242

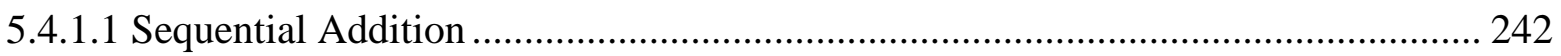

5.4.1.2 Synthesis of Aldehyde Precursors ....................................................................... 244

5.4.2 Method 2: Michael Addition to Diethylvinylphosphonate .............................................. 246

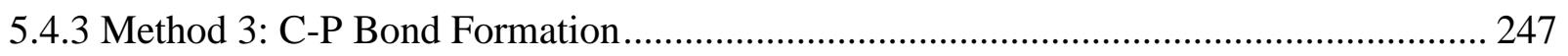

5.4.4 Method 4: Alkylation of Methylenebisphosphonate....................................................... 248

5.5.0 General Procedure for the 3-Component Reaction ....................................................... 257

5.6.0 General Procedure for the Bis Kabachnik Fields Reaction............................................. 258

5.7.0 General Procedure for the Bis Michael Addition of Amines onto Vinylphosphonates . 267

5.8.0 Preparation of Tris Phosphonic Acid Derivatives.......................................................... 276

5.9.0 Preparation of Bis Amines Scaffolds for Multidentate Phosphonic Acid Synthesis ..... 280

5.10 Preparation of Catechol QAC ................................................................................... 282

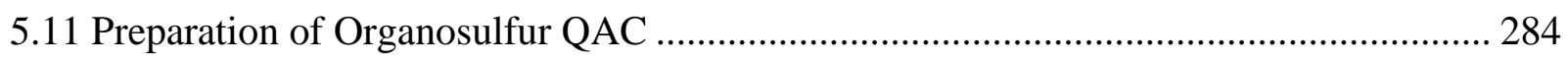

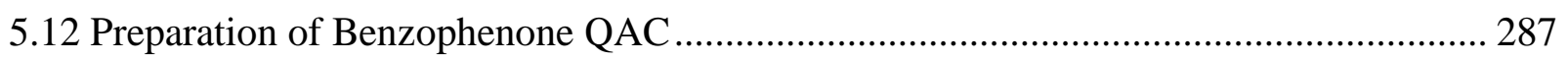

5.13 Preparation of Dansyl QAC ................................................................................... 290

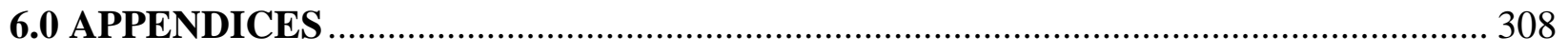

List of Appendix Tables................................................................................................. 308 


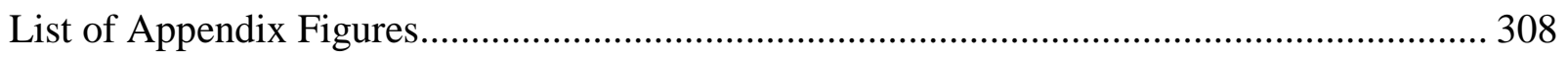

6.1 X-Ray Data Tables .................................................................................................. 320

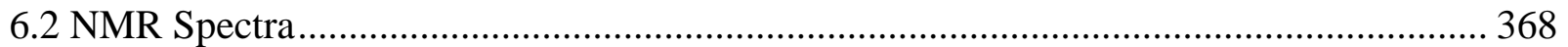

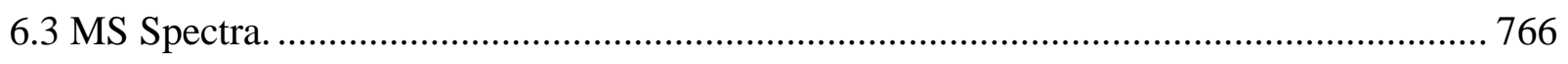

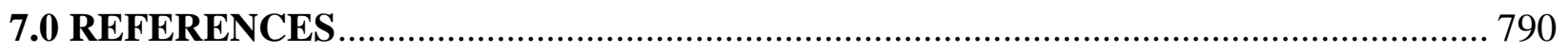




\section{LIST OF TABLES}

\section{SECTION 1.0}

Table 1.1: Measured persistence of different nosocomial pathogens on inanimate surfaces. ....... 2

Table 1.2: Measured bacterial loads $\mathrm{CFU} / \mathrm{cm}^{2}$ in the healthcare and food related surfaces........... 3

Table 1.3: Summary of different surfaces immobilized with antimicrobial organosilanes......... 16

Table 1.4: Common medical devices vulnerable to microbial contamination.............................. 26

Table 1.5: Polymers commonly used for food packaging materials........................................... 27

Table 1.6: Examples of the benzophenone group utilized to create antimicrobial surfaces........ 35

Table 1.7: MIC values for ADBAC and DDAC against increasing S. aureus concentrations after

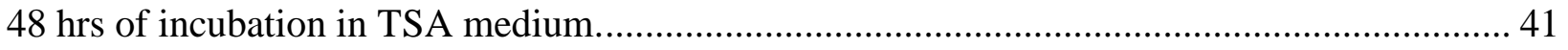

Table 1.8: MIC values of Commercial Disinfectant BAK 50 versus thiol QAC's...................... 44

Table 1.9: Antibacterial activity of compound 3 in solution. ..................................................... 53

Table 1.10: Relative rates of the Menschutkin quaternization in various solvents....................... 65

Table 1.11: Examples of literature Menschutkin reaction conditions. ………………................... 66

Table 1.12: Examples of the $S_{N} 2$ dequaternization reaction....................................................... 72

Table 1.13: Literature examples of the dealkylation of phosphonate esters with mineral acids. 80

Table 1.14: Literature examples of the dealkylation of phosphonate esters with $\mathrm{BBr}_{3} \ldots \ldots \ldots \ldots \ldots . . . . .84$ SECTION 2.0

Table 2.1: Literature/patent procedures describing the preparation of SiQAC 3. 89

Table 2.2: ${ }^{1} \mathrm{H}$ NMR $\left(\mathrm{CDCl}_{3}\right)$ monitoring of the formation of 3.................................................. 91

Table 2.3: Optimization of Step 1: Abruzov reaction between trialkyl phosphites (4, 5A-B) and dibromoalkanes $(\mathbf{6}, \mathbf{7}$ or $\mathbf{8})$ or bromoalkanes $(\mathbf{9}, \mathbf{1 0}$ or $\mathbf{1 1})$ 96 
Table 2.4: Optimization of Step 2: Menschutkin reaction between bromoalkylphosphonates 12-

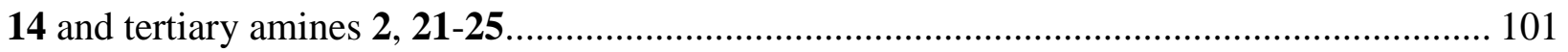

Table 2.5: Optimization of Step 4: Didealkylation of phosphonate diester quats. ................... 103

Table 2.6: Attempted Grignard addition reactions onto 68. ............................................. 117

Table 2.7: Preparation of bisphosphonates 49, 77-78 by Method 3. ................................... 119

Table 2.8: Synthesis of $\alpha$-C-H bisphosphonates by Method 4........................................... 122

Table 2.9: Optimization of the Kabachnik-Fields reaction................................................ 133

Table 2.10: Optimization of the Michael addition to $\beta$-aminobisphosphonates QAC 131-144.145

Table 2.11: Didealkylation of dansyl phosphonate diesters 211 and 212........................... 174

Table 2.12: MIC plate counts of samples using a $10^{-3}$ (about $200000 \mathrm{cfu} / \mathrm{ml}$ ) culture of S.aureus. 205

Table 2.13: Arthrobacter reduction by antimicrobial metal surfaces treated with 26 and 34A. 207 Table 2.14: $P$. aeroguinosa (PA01) reduction by antimicrobial metal surfaces treated with 26 and 34A. 208

Table 2.15: P. aeroguinosa (PA01) reduction by antimicrobial Ti surfaces treated with $34 \mathrm{~A}$ and retested. 209

Table 2.16: Determination of initial bacterial load dried on titanium surfaces from plate counts. 210

Table 2.17: Determination of initial bacterial load dried on SS surfaces from plate counts (raw data). 210 


\section{LIST OF FIGURES}

\section{SECTION 1.0}

Figure 1.1: Representation of bacterial adhesion and biofilm formation.

Figure 1.2: The role of surfaces and antimicrobial surface coatings in the epidemiology of

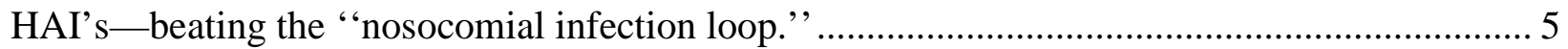

Figure 1.3: Example of various types of antimicrobial surfaces prepared in the literature .......... 6

Figure 1.4: Possible anchors to generate self assembled monolayers of small molecules and polymers on various substrates ....................................................................................... 7

Figure 1.5: Literature examples of different biocide immobilization strategies............................ 8

Figure 1.6: Initiators typically used for growing antimicrobial polymers from surfaces "grafting

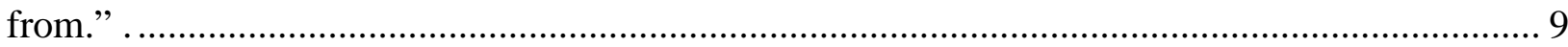

Figure 1.7: Examples of antimicrobial organosilanes described in the literature. ....................... 11

Figure 1.8: Typical procedure for immobilizing SiQAC on polyhydroxy surfaces. .................... 13

Figure 1.9: Anchoring of trialkoxysilyl compounds onto polyhydroxide surfaces..................... 14

Figure 1.10: Comparison of silanization to phosphonic acid monolayer formation on metal oxide surfaces. 18

Figure 1.11: Hydrolytic loss of dansyl-cysteine from a silane based SAM vs a phosphonate based SAM. 19

Figure 1.12: Mechanism of organophosphorus monolayer formation on metal oxide surfaces.. 20

Figure 1.13: IR spectrum of a deposited film of octadecylphosphonic acid.. 21

Figure 1.14: Example of literature QAC phosphonic acid antimicrobials.. ................................ 22

Figure 1.15: Bactericidal QAC prepared by plasma treated stainless steel via grafting to approach. 23 
Figure 1.16: Example of literature QAC catechol antimicrobials.

Figure 1.17: Example of a contact active plastic self-finishing coating................................. 29

Figure 1.18: Monomers and quats used to prepare polymer-QAC antimicrobial plastic coatings.

Figure 1.19: Bactericidal QAS polymers and small molecules prepared by plasma treated polypropylene. 32

Figure 1.20: Example of "grafting from" immobilization of biocidal polymers employing the benzophenone surface bound initiator. 33

Figure 1.21: Examples of benzophenone used to prepare antimicrobial surfaces. 34

Figure 1.22: Example of "grafting from” a plastic surface. Immobilization of biocidal polymers without employing a surface bound initiator. 35

Figure 1.23: Example of a releasing and repelling $2^{\text {nd }}$ generation dual action antimicrobial surface 37

Figure 1.24: Example of a dual action and reversible contact killing / hydrophobic antimicrobial surface 38

Figure 1.25: QAC disinfectants commercially employed. 40

Figure 1.26: Bacterial membrane destruction by QAC disinfectants in solution..... 40

Figure 1.27: Scanning electron micrographs of S. aureus strain ATCC 25923 indicating the effect didecyldimethylammonium chloride (DDAC) had on cell morphology. 42

Figure 1.28: The "Cut off Effect:” A hyperbolic trend observed in MIC values of surfactants as a function of the length of the hydrocarbon chain. 43

Figure 1.29: Effect of chain length on antimicrobial activity in solution.............................. 46

Figure 1.30: Example of the polymeric spacer effect with long chain QAC............................ 48 
Figure 1.31: Contact-killing via the polymeric spacer mechanism...................................... 48

Figure 1.32: Contact-killing via the phospholipid sponge mechanism.. ............................... 51

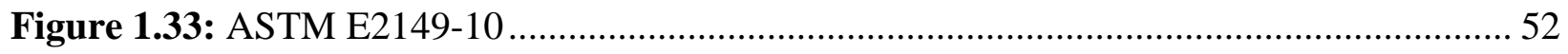

Figure 1.34: ZOI Agar diffusion method: Antimicrobial releasing plastic film surface on $A$. Niger. 54

Figure 1.35: Standard methods for testing antimicrobials on textiles. .................................. 55

Figure 1.36: Diagram of the aerosol inoculation method: (i) 5-50 × 10 6 CFU/mL (inoculum) .. 56

Figure 1.37: Results from the aerosol inoculum method with an immobilized QAS polymer onto a glass slide. 57

Figure 1.38: Industrial standard method for testing antimicrobials on hard surfaces by growth enumeration. 59

Figure 1.39: Modified method for testing antimicrobials on hard surfaces by growth enumeration in the dry state testing developed in the Wolfaardt lab. 60

Figure 1.40: A schematic diagram of the symmertric SSLDS method depicting three distinct antimicrobial agent states. 61

Figure 1.41: Direct innoculation SLDSS methods. (A) symmetric design, (B) asymmetric design.

Figure 1.42: Diagram of a high-thoughput biofilm retention assay that measures absorbance or fluorescence from a 96-well plate. 62

Figure 1.43: Polypropylene microscopy of control $~ 50 \mathrm{cfu} / \mathrm{mL}$ (A) vinyl terminated QAC sprayed (10\%) $0 \mathrm{cfu} / \mathrm{mL}(\mathrm{B})$. 63

Figure 1.44: SEM images of bacteria incubated with antimicrobial silica nanoparticles...... 64

Figure 1.45: Reactivity of the Menschutkin reaction. 65 
Figure 1.46: Preparation of Menschutkin precursors from primary amines by direct alkylation.68

Figure 1.47: Preparation of Menschutkin precursors from primary amines............................ 68

Figure 1.48: Mechanism of the Eschweiler-Clarke $N, N$-methylation. ................................... 69

Figure 1.49: Preparation of Menschutkin precursors from alcohols. .................................... 70

Figure 1.50: Side reactions favoured over the Menshutkin reaction. ................................... 74

Figure 1.51: Monophasic titration with bromophenol blue.............................................. 76

Figure 1.52: Monophasic titration with dichlorofluorescein. ........................................ 77

Figure 1.53: Biphasic ion pair titration based on bromophenol blue and SiQAC 3 ................. 77

Figure 1.54: Dansyl linkers previously synthesized in the Foucher lab. ............................... 78

Figure 1.55: Examples of reagents used to dealkylate phosphonate esters. ............................ 79

Figure 1.56: Mechanism of the didealkylation of phosphonate diesters in mineral acids.......... 81

Figure 1.57: Mechanism of the didealkylation of dialkylphosphonate esters via silylation and

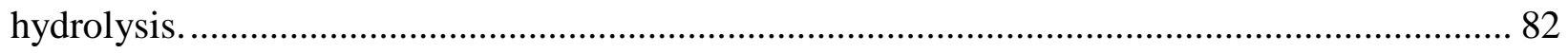

Figure 1.58: Possible products resulting from the hydrolysis of bistrimethylsilyl phosphonates.

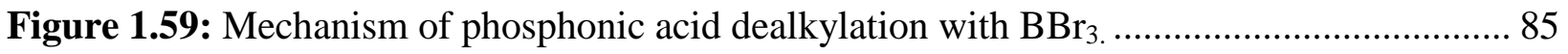

Figure 1.60: Literature routes describing the preparation of QA phosphonic acids.................. 86

\section{SECTION 2.0}

Figure 2.1: ${ }^{1} \mathrm{H}$ NMR $\left(\mathrm{CDCl}_{3}\right)$ spectrum of compound $\mathbf{1}+2$ in at $\mathrm{T}=0(\mathrm{~A})$, and the formation of

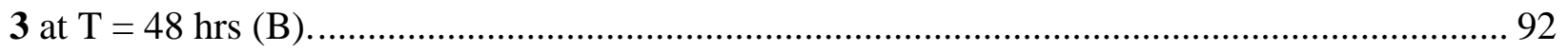

Figure 2.2: Comparison of trimethoxysilane pricing as of May 2013 (SA: Sigma Aldrich)..... 93

Figure 2.3: Cyclic oxaphospholane by-products formed during the Abruzov reaction.............. 99 
Figure 2.4: ${ }^{1} \mathrm{H}$ NMR $\left(\mathrm{CDCl}_{3}\right)$ spectra showing successful didealkylation of the ethyl phosphonate ester QAC $\mathbf{2 6}$ and isopropyl ester QAC 27 to the corresponding phosphonic acid QAC $34\left(\mathrm{D}_{2} \mathrm{O}\right)$. 104

Figure 2.5 : ${ }^{1} \mathrm{H}$ NMR $\left(\mathrm{D}_{2} \mathrm{O}\right)$ spectrum of $\gamma$-BPQAC 42. 107

Figure 2.6: ${ }^{31} \mathrm{P}$ NMR $\left(\mathrm{CDCl}_{3}\right)$ reaction monitoring of compound 45.................................. 109

Figure 2.7: Retrosynthesis of $\alpha-\mathrm{CH}$ bisphosphonic acid quats........................................ 109

Figure 2.8: Four retrosynthetic methods for the preparation of $\alpha-C H-B P Q A$ 's...................... 110

Figure 2.9: The Horner-Wadsworth-Emmons rearrangement of 49.................................. 113

Figure 2.10: ${ }^{1} \mathrm{H}$ NMR $\left(\mathrm{CDCl}_{3}\right)$ spectrum of QAC aldehyde precursor 66. ........................... 115

Figure 2.11: ${ }^{1} \mathrm{H}$ NMR (MeOD) spectrum of cyclic bisphosphonate by-product 97................ 125

Figure 2.12: ${ }^{31} \mathrm{P}$ NMR $\left(\mathrm{CDCl}_{3}\right)$ monitoring for compound 94 and 95 prepared by Method $4 . .125$

Figure 2.13: ${ }^{1} \mathrm{H}$ NMR $\left(\mathrm{CDCl}_{3}\right)$ spectrum of $\alpha$-CH-QAC 93 prepared by Method 4............... 127

Figure 2.14: ${ }^{1} \mathrm{H}$ NMR $\left(\mathrm{CDCl}_{3}\right)$ spectrum of precursor 102 ........................................... 129

Figure 2.15: ${ }^{1} \mathrm{H} \mathrm{NMR}\left(\mathrm{CDCl}_{3}\right)$ spectrum of the undesired product 124 isolated from the Kabachnik-Fields Kabachnik-Fields reaction............................................................. 135

Figure 2.16: Possible mechanistic routes involved in the Kabachnik-Fields reaction. ............ 136

Figure 2.17: Attempted quaternization of triazine 123 with bromoctadecane $\left({ }^{1} \mathrm{H} \mathrm{NMR}\left(\mathrm{CDCl}_{3}\right)\right)$. 139

Figure 2.18: ${ }^{1} \mathrm{H}$ NMR $\left(\mathrm{CDCl}_{3}\right)$ spectrum of $\alpha$-ABPQA 121 .............................................. 140

Figure 2.19: ${ }^{31} \mathrm{P}$ NMR $\left(\mathrm{CDCl}_{3}\right)$ reaction monitoring of 121 cleavage with $\mathrm{HBr}$ and TMSBr... 141

Figure 2.20: ${ }^{31} \mathrm{P}$ NMR $\left(\mathrm{CDCl}_{3}\right)$ reaction monitoring of tetraphosphonate 127..................... 143

Figure 2.21: Reaction monitoring of Michael addition reactions by ${ }^{31} \mathrm{P}$ NMR $\left(\mathrm{D}_{2} \mathrm{O}\right) \ldots \ldots \ldots \ldots . . . . .148$

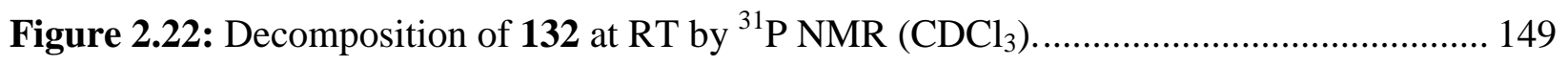


Figure 2.23: ${ }^{1} \mathrm{H}$ NMR $\left(\mathrm{CDCl}_{3}\right)$ spectrum of $\beta$-ABPQA 137. 150

Figure 2.24: Preparation of diethylvinylphosphonate 130 (crude mixture by ${ }^{31} \mathrm{P} \mathrm{NMR}\left(\mathrm{CDCl}_{3}\right)$ ). 151

Figure 2.25: ${ }^{1} \mathrm{H}$ NMR $\left(\mathrm{CDCl}_{3}\right)$ of $\mathbf{1 4 4}$ after $\mathrm{N}_{2} \mathrm{H}_{4}$ deprotection. 153

Figure 2.26: Mechanism of boiling $\mathrm{H}_{2} \mathrm{O}$ BOC deprotection. 158

Figure 2.27: ${ }^{1} \mathrm{H}$ NMR $\left(\mathrm{CDCl}_{3}\right)$ of catechol QAC 188 (upper) and 189 (lower)..................... 163

Figure 2.28: ${ }^{1} \mathrm{H}$ NMR $\left(\mathrm{CDCl}_{3}\right)$ spectra of organosulfur QAC 192 and 199.......................... 165

Figure 2.29: ${ }^{1} \mathrm{H},{ }^{13} \mathrm{C} \mathrm{NMR}\left(\mathrm{CDCl}_{3}\right)$ spectra of compound 206........................................ 169

Figure 2.30: ${ }^{1} \mathrm{H}$ NMR spectra of $208\left(\mathrm{CDCl}_{3}\right), 209\left(\mathrm{CDCl}_{3}\right)$, and $217\left(\mathrm{D}_{2} \mathrm{O}\right) \ldots \ldots \ldots \ldots \ldots \ldots \ldots . . . . . . . . . . . .175$

Figure 2.31: ${ }^{1} \mathrm{H},{ }^{13} \mathrm{C}$ NMR $\left(\mathrm{CDCl}_{3}\right)$ spectra of dansyl acrylamide 219............................... 177

Figure 2.32: ${ }^{1} \mathrm{H},{ }^{13} \mathrm{C}$ NMR $\left(\mathrm{D}_{2} \mathrm{O}\right)$ spectra of dansyl amine 221 (upper) and dansyl amidine 222 (lower) 180

Figure 2.33: Attempted deprotection of dansyl thioacetate 218 to dansyl thiol 212............... 181

Figure 2.34: ${ }^{1} \mathrm{H}$ NMR (DMSO-d $\mathrm{d}_{6}$ ) spectrum of compound 225....................................... 182

Figure 2.35: ESI-TOF MS spectrum of compound 225.............................................. 183

Figure 2.36: ${ }^{31} \mathrm{P}$ NMR $\left(\mathrm{D}_{2} \mathrm{O}\right)$ reaction monitoring of the formation of $\mathbf{2 2 6}$ after $3 \mathrm{~d}$. at RT.... 184

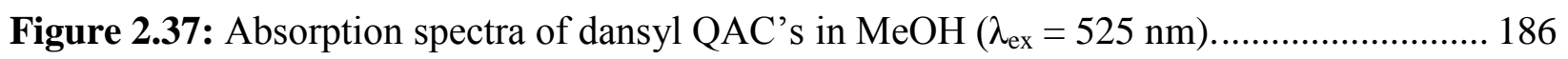

Figure 2.38: Absorption spectra of dansyl QAC 213 at different pH's $\left(\lambda_{\mathrm{ex}}=525 \mathrm{~nm}\right)$.......... 186

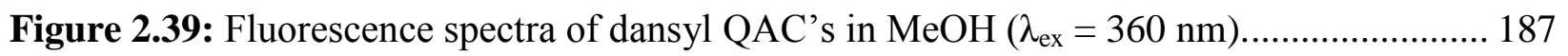

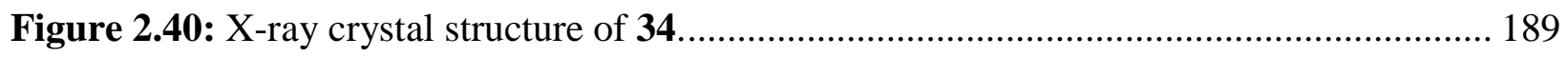

Figure 2.41: Crystal packing interactions of two molecules of 34 .................................... 190

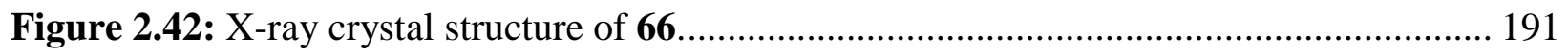

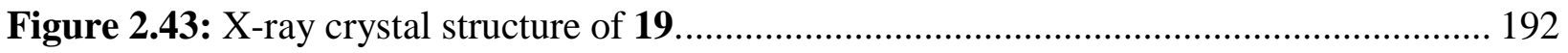


Figure 2.44: X-ray crystal structure of 220.

Figure 2.45: Coating procedure of QAC antimicrobials onto porous and nonporous surfaces. 196

Figure 2.46: Plastic coating experimental set-up....................................................... 198

Figure 2.47: Bromophenol blue (detection of QAC antimicrobials on plastic surfaces............ 198

Figure 2.48: Fluorescent detection of dansyl QAC's. .................................................... 199

Figure 2.49: Metal coating experimental set up using 20 mL glass screw cap vial open to air. 200

Figure 2.50: Experimentally determined MIC (CFU/mL) of SiQAC 3, 34A and 34B..... 204

Figure 2.51: Colony forming units per mL of Salmonella and S. aureus after 3 hrs of drying on Ti surfaces. 206

Figure 2.52: Agar growth method showing P. aeroguinosa (PA01) reduction by antimicrobial Ti surfaces treated with 34A.. 209 


\section{LIST OF SCHEMES}

\section{SECTION 2.0}

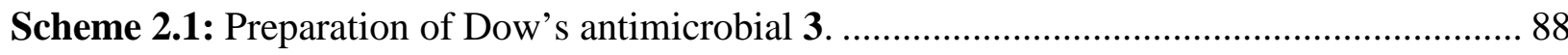

Scheme 2.2: Optimized conditions for the synthesis of $\gamma$-monophosphonic acid QAC's........... 95

Scheme 2.3: Preparation of $\gamma$-Bisphosphonic acid 41............................................................ 105

Scheme 2.4: Preparation of the potassium salt of 41............................................................... 106

Scheme 2.5: Attempted preparation of compound 47........................................................... 108

Scheme 2.6: Preparation of 50 by Method 1 ............................................................................. 111

Scheme 2.7: Attempted stepwise preparation of 49............................................................... 112

Scheme 2.8: Synthesis of bisphosphonates 57-59 from aldehydes............................................ 113

Scheme 2.9: Synthesis of aldehyde precursors 47, 54-55 used for Method 1............................ 114

Scheme 2.10: Possible synthetic routes leading to aldehyde QAC 56...................................... 115

Scheme 2.11: Preparation of tetraethyl ethene-1,1-diylbis(phosphonate) 73............................. 118

Scheme 2.12: Preparation of THP protected 3-bromopropanol 9............................................. 118

Scheme 2.13: Preparation of bisphosphonates by direct alkylation............................................ 120

Scheme 2.14: Intramolecular bisphosphonate alkylation........................................................... 124

Scheme 2.15: Preparation of bisphosphonate QAC 96 via alkylation. ....................................... 126

Scheme 2.16: Attempted preparation of trisphosphonic acid 102 via alkylation of $\mathbf{9 6}$............. 128

Scheme 2.17: Preparation of halo QAC 83 and 102 precursors for Method 4. ......................... 129

Scheme 2.18: Attempted preparation of bisphosphonate 106................................................... 130

Scheme 2.19: Synthetic route to $\alpha$-aminobisphosphonate QAC 121. Optimized conditions for

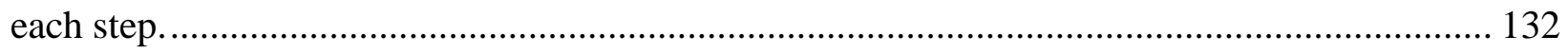

Scheme 2.20: Attempted stepwise preparation of 121............................................................. 139 
Scheme 2.21: Attempted didealkylation of $\alpha$-aminobisphosphonate QAC 121.

Scheme 2.22: Tetraphosphonate 127 by C-P alkylation of 113. 142

Scheme 2.23: Optimized synthetic route to $\beta$-aminobisphosphonate QAC 136. 144

Scheme 2.24: Attempted synthetic route to tetraphosphonate QAC 146 from $\beta$-amino bisphosphonate 141. 152

Scheme 2.25: Preparation of amine quat 144 153

Scheme 2.26: Attempted synthesis of tris- $\alpha$-hydroxyphosphonate QAC 161. 155

Scheme 2.27: Attempted synthesis of precursors 162 and 164 to tris- $\beta$-hydroxyphosphonate

QAC’s. 156

Scheme 2.28: Attempted synthesis of tris- $\gamma$-hydroxyphosphonate QAC 168.......................... 157

Scheme 2.29: Preparation of mesyl 158 and tosyl phosphonate 170 ................................. 158

Scheme 2.30: Preparation of tris-BOC 157 and deprotection in boiling $\mathrm{H}_{2} \mathrm{O}$ to tris 171......... 158

Scheme 2.31: Michael addition of $\mathrm{NMe}_{2}$ to tris acrylamide 173......................................... 159

Scheme 2.32: Attempted preparation of tetradentate $\beta$-amino QAC 179. .............................. 160

Scheme 2.33: Attempted preparation of tetradentate $\beta$-amino QAC 183............................. 161

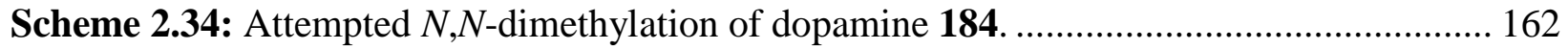

Scheme 2.35: Attempted preparation of catechol QAC 190................................................ 163

Scheme 2.36: Literature routes to target thiol QAC’s 194 and 199..................................... 166

Scheme 2.37: Preparation of the benzophenone QAC’s 205 and 206. ................................ 168

Scheme 2.38: Preparation of dansyl dimethyl 208 and halo precursors 209 and 210 for quaternization. 171

Scheme 2.39: Preparation of bifunctional dansyl anchors 211-216 for binding onto porous and non-porous surfaces 172 
Scheme 2.40: Preparation of dansyl acrylamide QAC 219............................................... 176

Scheme 2.41: Preparation of dansyl amindine switchable fluorophore 222 and 223. ............. 179

Scheme 2.42: Attempted preparation of dansyl DTC 225............................................... 182

Scheme 2.43: Attempted preparation of dansyl bis $\beta$-phosphonate 226. ............................. 184

\section{SECTION 4.0}

Scheme 4.1: Catalytic Finkelstein / Menschutkin reaction in the preparation of the Dow antimicrobial 3.

Scheme 4.2: Onepot preparation of $\alpha$-CH-bisphosphonates from the aldehyde quat precursor, $\mathbf{5 6 .}$

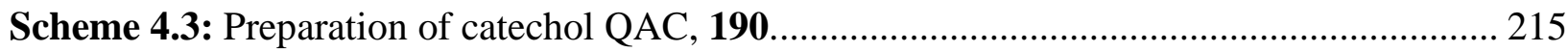

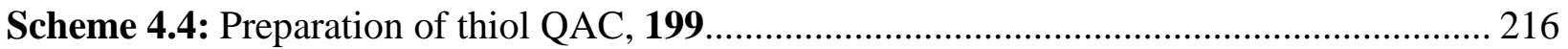

Scheme 4.5: Synthesis of multifunctional QAC antimicrobial polymer coating..................... 216

Scheme 4.6: Synthesis of multifunctional QAC antimicrobial polymer coating...................... 217 


\section{LIST OF ABBREVIATIONS}

Ac

$\mathrm{Ar}$

AMA

APA

ARI

Aq.

ATRP

BOC

$\mathrm{Bn}$

$t-\mathrm{Bu}$

Cat

Cbz

CDI

CFU

d.

DCM Dichloromethane

Diox. Dioxane

DIPEA Diisopropylethylamine

DMAEMA 2-(N,N-dimethylamino)ethyl Methacrylate

DMSO Dimethylsulfoxide

DTC Dithiocarbamate

EPA Environmental Protection Agency

Eq. Equation

Et

$\mathrm{Et}_{3} \mathrm{~N}$

Hrs

HAI

HMPA
Ethyl

Triethylamine

Hours

Hospital Acquired Infection

Hexamethylphosphoramide 


\begin{tabular}{|c|c|}
\hline HCWU & Health Care Workers Uniforms \\
\hline HRMS & High Resolution Mass Spectrometry \\
\hline IAI & Implant Associated Infections \\
\hline iAMA & Immobilized Antimicrobial Agent \\
\hline$i \mathrm{Pr}$ & Isopropanol \\
\hline $\mathrm{LAH}$ & Lithium Aluminum Hydride \\
\hline LG & Leaving Group \\
\hline Mon. & Months \\
\hline $\mathrm{Me}$ & Methyl \\
\hline MIC & Minimum Inhibitory Concentration \\
\hline MLC & Minimum Lethal Concentration \\
\hline $\mathrm{Mp}$ & Melting Point \\
\hline Mol & Moles \\
\hline MS & Mass Spectrometry \\
\hline NMR & Nuclear Magnetic Resonance \\
\hline $\mathrm{Nu}$ & Nucleophile \\
\hline Ms & Mesyl \\
\hline ON & Overnight \\
\hline PBS & Phosphate buffered saline \\
\hline PCC & Pyridinium Chlorochromate \\
\hline PMMA & Poly Methyl Methacrylate \\
\hline PMRA & Pest Management Regulatory Agency \\
\hline QA & Quaternary Ammonium \\
\hline QAC & Quaternary Ammonium Compound \\
\hline QAM & Quaternary Ammonium Monomer \\
\hline QUAT & Quaternary Ammonium Cation \\
\hline QAS & Quaternary Ammonium Salt \\
\hline $\mathrm{RBF}$ & Round Bottom Flask \\
\hline $\mathrm{RT}$ & Room Temperature \\
\hline
\end{tabular}




\begin{tabular}{ll} 
RXN & Reaction \\
SAM & Self-Assembled Monolayer \\
Si-QAC & 3-(trimethoxysilyl)propyldimethyl-Octadecyl Ammonium Chloride \\
SIP & Surface Initiated Polymerization \\
Solv. & Solvent \\
ST & Sealed Tube \\
THF & Tetrahydrofuran \\
TLC & Thin Layer Chromatrography \\
TOL & Toluene \\
PG & Protecting Group \\
PHT & Phthalimide \\
TMSCl & Trimethylsilylchloride \\
TMSBr & Trimethylsilylbromide \\
TMSI & Trimethylsilyliodide \\
TMTC & Too Many To Count \\
Tos & Tosyl \\
$\mu W$ & Microwave \\
UTI & Urinary Track Infection \\
Yr. & Year \\
W-VP & 4-Vinylpyridine \\
\hline
\end{tabular}




\section{CHAPTER 1 - INTRODUCTION}

\subsection{RATIONALE FOR ANTIMICROBIAL SURFACES}

Common surfaces that are frequently touched are inhabited by a variety of microorganisms such as bacteria, viruses and fungi which can persist on these "touch surfaces" anywhere from a couple of hours up to six months (Table 1.1). ${ }^{1}$ If pathogenic bacteria persist in healthcare and food preparation facilities, patients and workers can readily develop and spread nosocomial infections from touch surfaces such as door handles, pens, telephones, health care workers uniforms (HCWU's), stethoscopes, IV poles, faucets, food and food preparation surfaces (Table 1.2). ${ }^{2,3}$

The healthcare and food industry are facing an ever growing microbial contamination problem. Contamination of medical devices, healthcare products, $\mathrm{H}_{2} \mathrm{O}$ purification systems, food packaging and food storage are becoming a serious threat both in terms of cost and safety. ${ }^{4}$ To date, infection control counter measures which rely on personal hygiene, hand washing, masks and the use of disinfectants on hospital equipment to prevent the spread of infections have been largely unsuccessful. According to the World Health Organization (WHO), nosocomial or hospital-acquired infection (HAI's) are becoming a national economic burden resulting in prolonged hospitalization and can lead to serious complications and even death. ${ }^{5}$ For example, hospital-acquired infection (HAI's) from contact with pathogenic microorganisms affect approximately 2 million people and result in more than 100,000 deaths each year in the US. ${ }^{6}$ Such unintended infections require $10-20 \mathrm{~d}$. of additional patient hospitalization, costing the already strained US health-care system between $\sim 25,000-\$ 30,000$ per infection and billions of dollars per year. ${ }^{6}$ Another route for bacteria to infect patients is through hospital invasive support 
equipment such as intravascular lines and implanted medical devices such as artificial prosthetics, cardiovascular implants and urinary catheters. ${ }^{6,7}$ Implant associated infections (IAI's) occur in more than 1 million patients and cost an estimated \$3 billion in the US per year to treat. ${ }^{4}$ For example, approximately $10-50 \%$ of patients with implanted catheters run the risk of developing urinary tract infections (UTI’s) translating to an average of $\$ 200,000$ per infection in additional healthcare costs. The rise in the frequency and severity of (HAI's) and (IAI's) can be attributed to decreased antibiotic efficacy against drug-resistant strains of pathogens found in surface biofilms. $^{3}$

Table 1.1: Measured persistence of different nosocomial pathogens on inanimate surfaces. ${ }^{8}$

\begin{tabular}{|c|c|c|c|c|c|}
\hline $\begin{array}{c}\text { Type of } \\
\text { Bacterium }\end{array}$ & $\begin{array}{l}\text { Duration of } \\
\text { Persistence }\end{array}$ & $\begin{array}{c}\text { Type of } \\
\text { Virus }\end{array}$ & $\begin{array}{l}\text { Duration of } \\
\text { Persistence }\end{array}$ & $\begin{array}{l}\text { Type of } \\
\text { fungus }\end{array}$ & $\begin{array}{l}\text { Duration of } \\
\text { Persistence }\end{array}$ \\
\hline Acinetobacter spp. & 3 d. to 5 mon. & Adenovirus & 7 d. - 3 mon. & C.albicans & $1-120 \mathrm{~d}$ \\
\hline Bordetella pertussi, & $3-5 \mathrm{~d}$ & Astrovirus & 7 - $90 \mathrm{~d}$ & C.parapsilosis & $14 \mathrm{~d}$. \\
\hline C. difficile (spores) & 5 mon. & HIV & $7 \mathrm{~d}$. & $\begin{array}{l}\text { Torulopsis } \\
\text { glabrata }\end{array}$ & $102-150 \mathrm{~d}$. \\
\hline E. coli & $\begin{array}{l}1.5 \text { hrs to } 16 \\
\text { mon. }\end{array}$ & $\begin{array}{l}\text { Herpes } \\
\text { Simplex }\end{array}$ & $4.5 \mathrm{hrs}$ to $8 \mathrm{wks}$. & & \\
\hline H. influenza & $12 \mathrm{~d}$. & Influenza & $1-2 \mathrm{~d}$. & & \\
\hline M. tuberculosis & 1 d. to 4 mon. & Parvovirus & $1 \mathrm{yr}$. & & \\
\hline $\begin{array}{l}\text { S. aureus (including } \\
\text { MRSA) }\end{array}$ & 7 d. to mon. & Papiloma & $7 \mathrm{~d}$. & & \\
\hline S. pyogenes & 3 d. to 6.5 mon. & Rhino Virus & 2 hrs to $7 \mathrm{~d}$. & & \\
\hline
\end{tabular}


Table 1.2: Measured bacterial loads in the healthcare and food related surfaces (colony forming units, CFU/ $\left.\mathrm{cm}^{2}\right)^{9}$

\begin{tabular}{|c|c|c|}
\hline Site of Study & Site & Bacterial Load Found \\
\hline Food & Abbatoir surfaces & 8 to $1.3 \times 10^{4} \mathrm{cfu} / \mathrm{cm}^{2}$ \\
\hline Food & Food contact surfaces & 630 to $1.8 \times 10^{9} \mathrm{cfu} / \mathrm{cm}^{2}$ \\
\hline Food & Meat preparation surfaces & $10^{5} \mathrm{cfu} / \mathrm{cm}^{2}$ \\
\hline Food & Refrigerator surfaces & 813 to $6 \times 10^{8} \mathrm{cfu} / \mathrm{cm}^{2}$ \\
\hline Food & Vegetable preparation surfaces & $>10^{5} \mathrm{cfu} / \mathrm{cm}^{2}$ \\
\hline Healthcare & Nurse workstation & $<9 \mathrm{cfu} / \mathrm{cm}^{2}$ \\
\hline Healthcare & Hospital ward surfaces & 2.5 to $40 \mathrm{cfu} / \mathrm{cm}^{2}$, cleaning reduced to $<2.5 \mathrm{cfu} / \mathrm{cm}^{2}$ \\
\hline Healthcare & Hospital kitchen surfaces & $2-29 \mathrm{cfu} / \mathrm{cm}^{2}$ \\
\hline Healthcare & Hospital ward floors & $<5 \mathrm{cfu} / \mathrm{cm}^{2}$ \\
\hline Healthcare & Hospital underward bed & $<25 \mathrm{cfu} / \mathrm{cm}^{2}$ \\
\hline Healthcare & Stethoscope membrane & In $>54 \%$ of cases $>5 \mathrm{cfu} / \mathrm{cm}^{2}$, in $18 \%$ of cases $>29 \mathrm{cfu} / \mathrm{cm}^{2}$ \\
\hline
\end{tabular}

Surface biofilms are complex communities of bacteria that offer protection from environmental hazards (eg. biocides). Biofilm formation involves three phases beginning with the initial reversible adhesion of bacteria though polysaccarides and adhesion proteins on the bacterial membrane (phase I). After 2-3 hrs under appropriate conditions, bacteria irreversibly attach to a surface (phase II). Once formed (usually after 24 hrs) the bacterial biofilms secrete a protective peptidoglycan matrix (biofilm) capable of withstanding $1000 \times$ the antibiotic dose of non adherent bacteria (Figure 1.1). ${ }^{10}$ As a result, once the infection occurs, it becomes difficult to 
treat and strategies that prevent bacterial contamination or destroy adsorbed microorganisms that lead to biofilm formation are actively sought. ${ }^{10}$

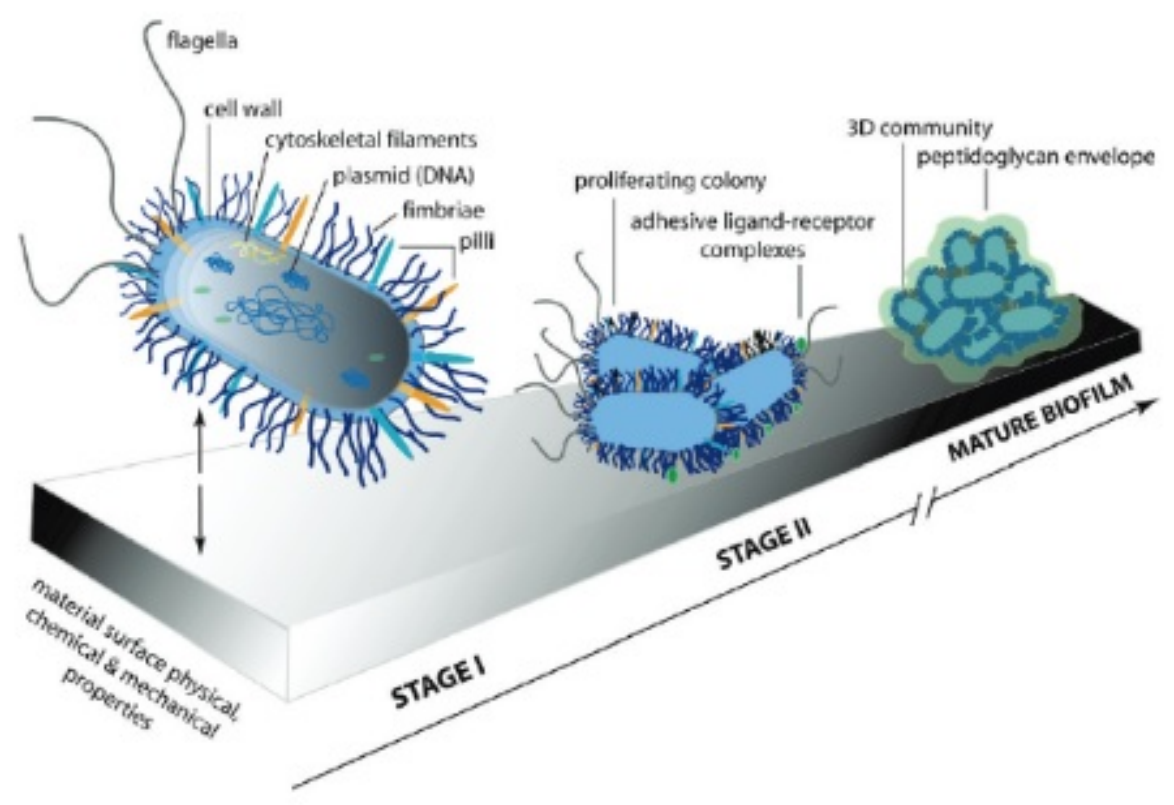

Figure 1.1: Representation of bacterial adhesion and biofilm formation (Used with permission from Ref. $^{10}$ ).

Consumer demand for odor and contamination free hygienic textile products has grown remarkably. Estimates of antimicrobial textile production worldwide were 100,000 tonnes in $2000 .{ }^{11}$ With an estimated growth of $15 \%$ per year in Western Europe, antimicrobial textiles are one of the fastest growing sectors in this industry. ${ }^{11}$ In addition, managers in the health care/food industries as well as medical device makers are actively seeking to introduce antimicrobial coatings as part of an infection control strategy combined with hygiene and disinfection protocols. In this regard, introducing antimicrobial surfaces that prevent biofilm formation could help reduce the spread of pathogenic bacterial infections from surfaces and implants to patients and healthcare workers, thereby closing the "nosocomial infection loop” (See Figure 1.2). ${ }^{8,9}$ 


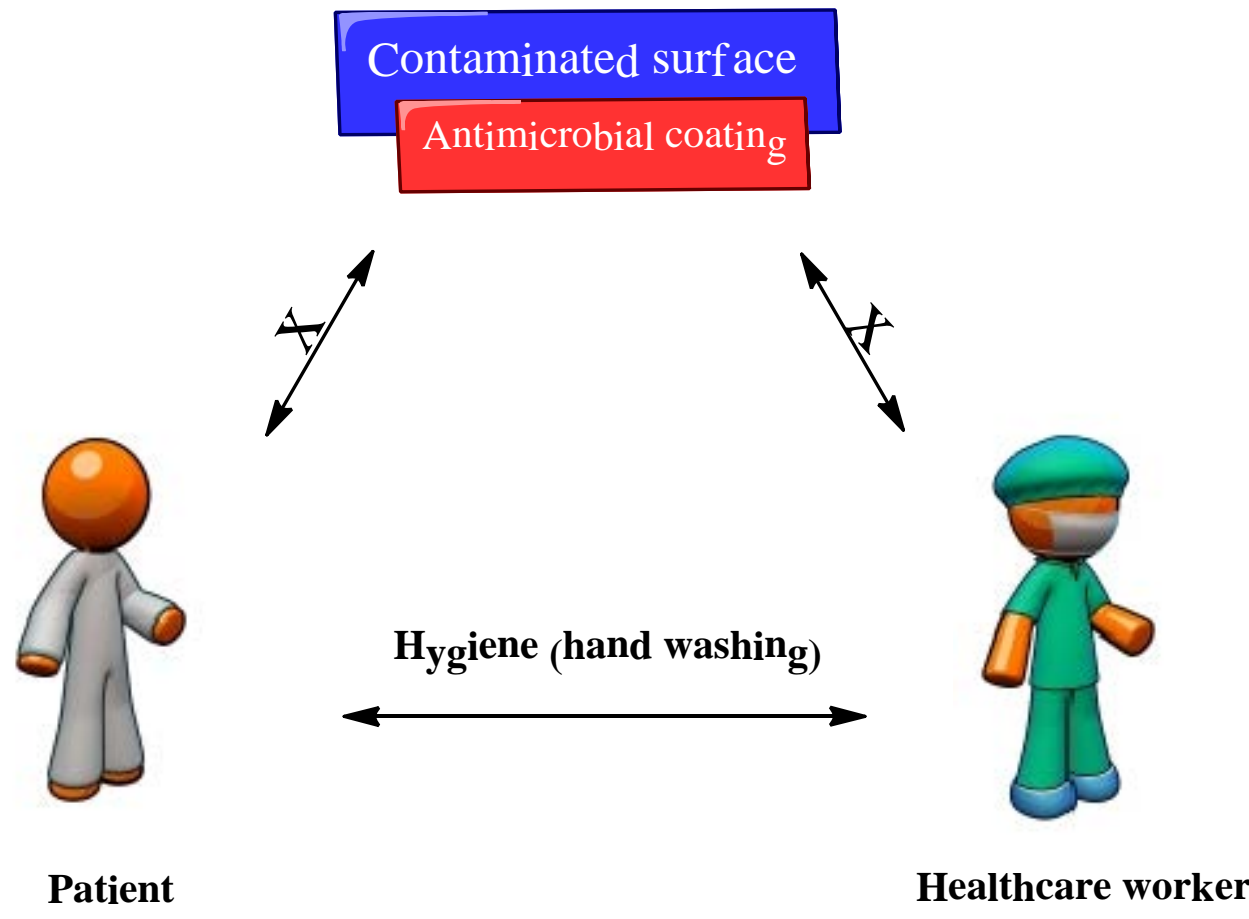

Figure 1.2: The role of surfaces and antimicrobial surface coatings in the epidemiology of HAI's-beating the "nosocomial infection loop." 8,9

\subsection{Examples and Preparation of Antimicrobial Surfaces}

In order to prevent the formation of biofilms, strategies utilizing antimicrobial surfaces have been employed to make surfaces inhospitable to bacteria. ${ }^{12,13}$ Small molecule monolayers or polymer thin films either "grafted to" or "grown from" a surface have been widely used to prepare antimicrobial surfaces and clothing. Figure 1.3 shows examples of the different approaches that have been used to prepare antimicrobial surfaces or objects. These prior art monolayers or polymer coatings include, for example, non-biofouling coatings which are passive strategies that rely on preventing bacterial adhesion with hydrophobic or zwitterionic thin films, but do not kill the approaching bacteria. A second class of antibacterial thin films kills microbes on contact either by releasing a biocidal agent or immobilizing a biocidal agent. A third class of 
antibacterial thin films utilize a combination strategy of including a non-biofouling and biocidal component into the coating (see Section 1.3). ${ }^{12,13}$

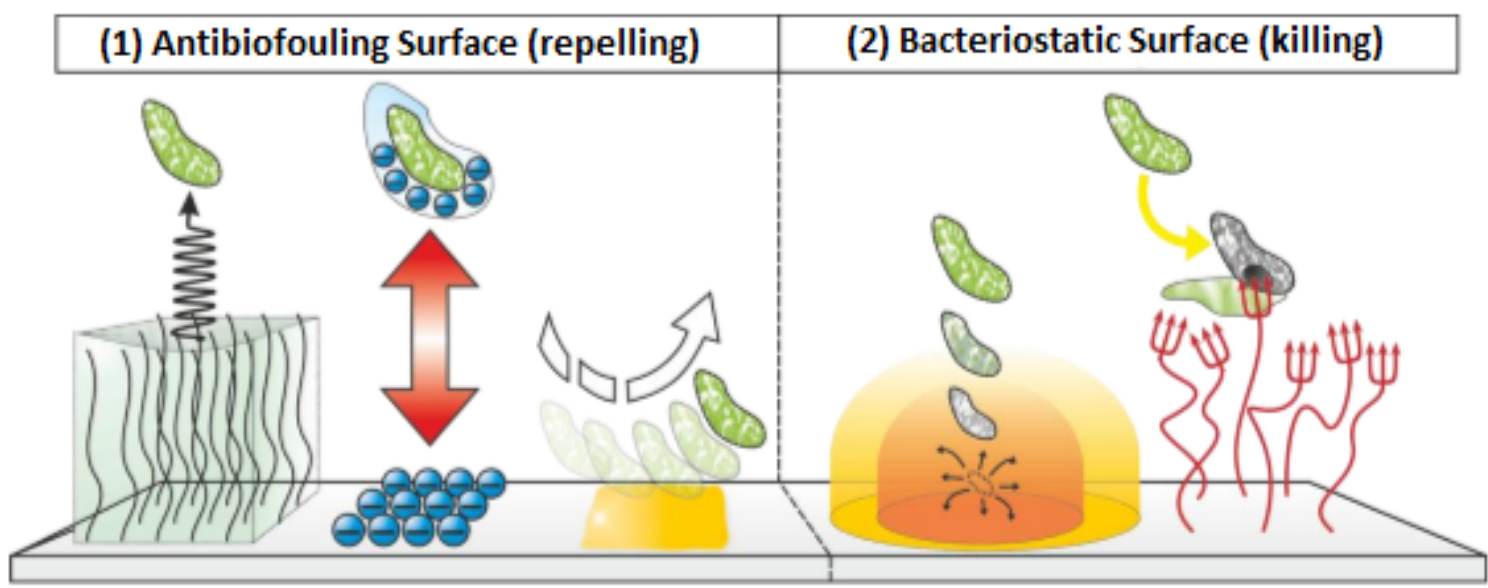

\section{(a) exclusion steric repulsion \\ (b) electrostatic (c) low surface repulsion energy \\ (d) biocide releasing \\ (e) contact active immobilized biocide}

Figure 1.3: Example of various types of antimicrobial surfaces prepared in the literature (Used with permission. ${ }^{12,13}$

Active releasing antimicrobial coatings based on the leaching of biocides are typically prepared by impregnating biocides in a polymer matrix coating. In this way, the leachable biocide is gradually released from the coating and kills adhered microorganisms via interaction with the cell depending on the biocide mode of action. ${ }^{14,15}$ The most popular leachable biocide is silver ion $\left(\mathrm{Ag}^{+}\right) .{ }^{16,17}$ Quaternary ammonium compounds, although used in leaching type systems, are largely immobilized onto surfaces as small molecules or polymers that provide contact killing without leaching. The advantages of the non-leaching immobilized approach include longer antimicrobial efficacy, less chance for the development of resistant bacteria and overall is more environmentally friendly. ${ }^{3,18,19}$ 
Quaternary ammonium biocides can be immobilized onto various surfaces either as small molecule monolayers with various anchors (Figure 1.4) or as polymers. ${ }^{20,21}$ Grafting of larger molecules onto surfaces such as polymers can be accomplished in one of three ways; (A) physisorption of a polymer to a surface (grafting to approach); (B) chemisorption via reaction of anchors in the polymer with complementary functional groups at the substrate surface (grafting to approach); (C) growth of polymer brushes via surface-initiated polymerization techniques such as Atom Transfer Radical Polymerization (ATRP) (grafting from approach) (Figure 1.5). Grafting from approaches use ATRP polymerization initiators such as 2-bromoisobutyryl bromide (BIBB) directly bound to a surface or immobilized through an anchor (Figure 1.6). ${ }^{22}$ Photochemical linkers have also been used to bind antimicrobials onto textiles and polymers. ${ }^{23-26}$

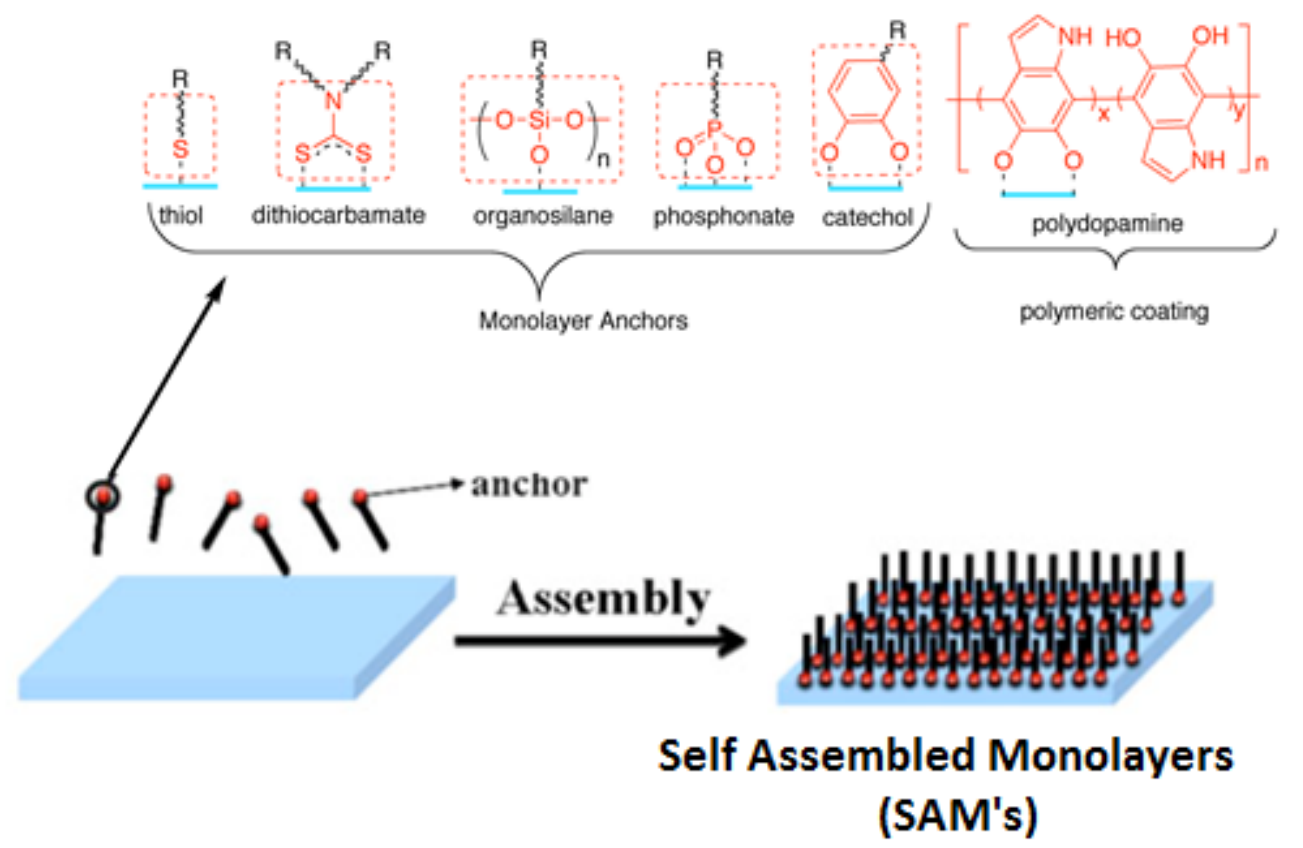

Figure 1.4: Possible anchors to generate self assembled monolayers of small molecules and polymers on various substrates (Adapted from Ref. ${ }^{27}$ ). 
(A) Adsorption
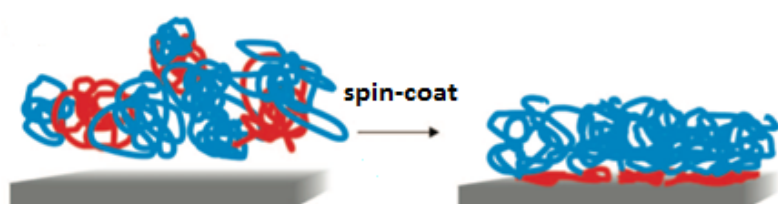

(C) "Graft From"

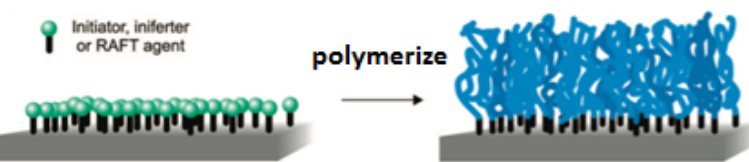

(B) "Graft To"

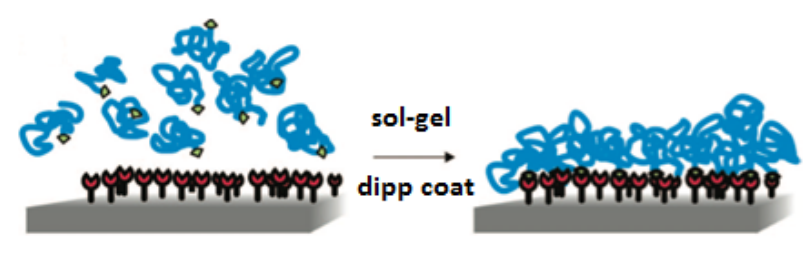

(D) Polymer Blends

Biocide added as additive of part of polymer

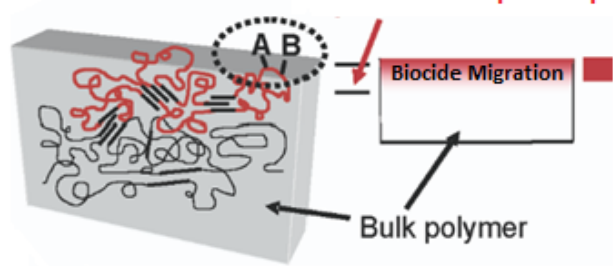

Figure 1.5: Literature examples of different biocide immobilization strategies. (A) Polymeric thin films coating are adsorbed or painted on compatible surfaces, (B) Self Assembling Polymers (SAP's) or Self Assembling Monolayers (SAM's) of small molecules with pending biocides have been employed, (C) biocidal polymers grown from a surface via a pre-immobilized Atom Transfer Radical Polymerization (ATRP) initiator, (D) self-finishing surface where the biocide is added during the polymerization process or is attached to the monomer prior to polymerization (Adapted from Ref. ${ }^{28}$ ). 


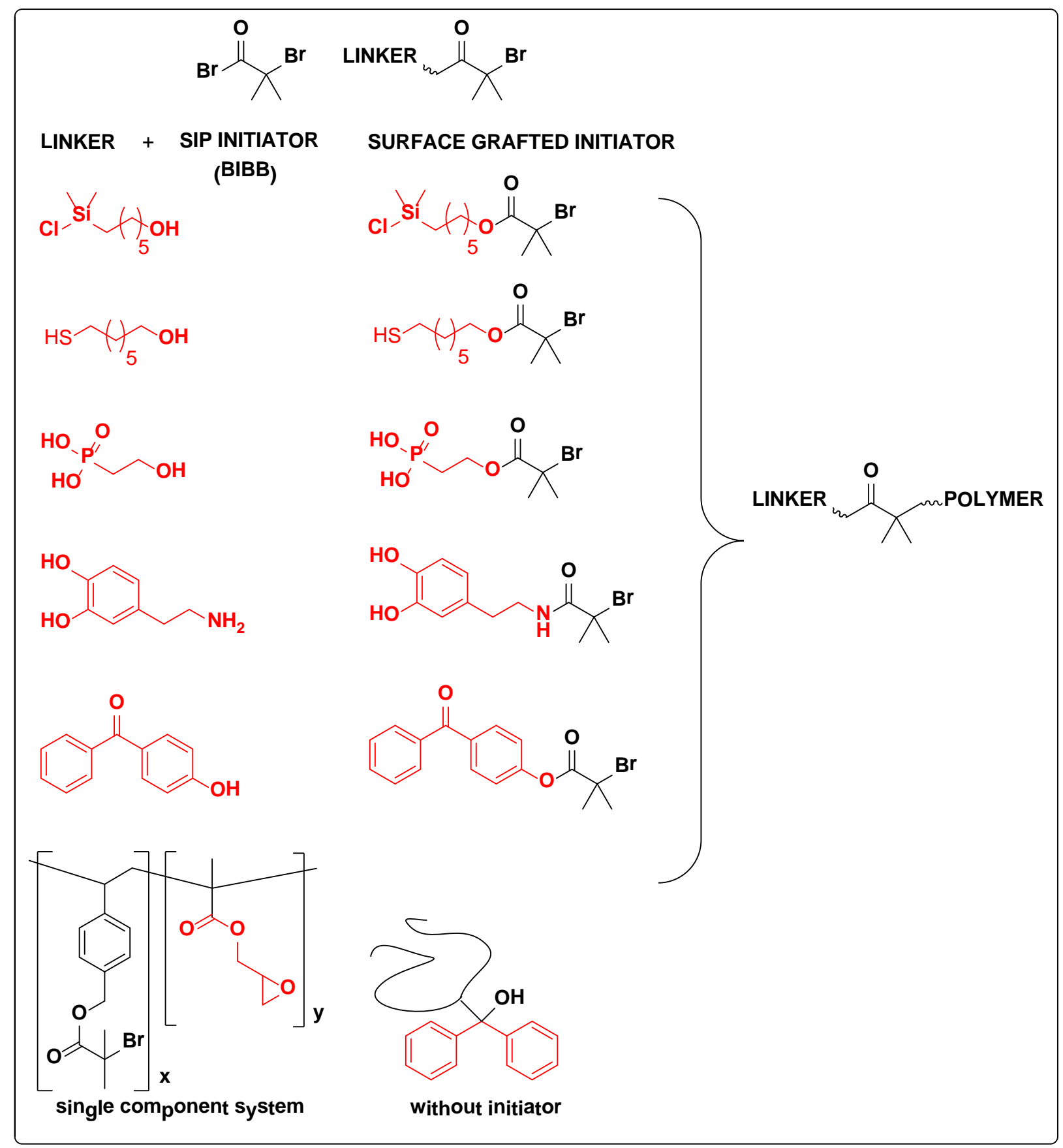

Figure 1.6: Initiators typically used for growing antimicrobial polymers from surfaces "grafting from." 29,30,31,32,33,34

The research proposed is based on developing novel, non-releasing, contact-active monolayer thin film coatings. The following sections will highlight literature examples of 
immobilized QA antimicrobials on porous (polyhydroxylated surfaces) and non-porous (metal and plastic) surfaces.

\section{$1.21^{\text {st }}$ Generation Antimicrobial Coatings: Literature Examples of Contact Active QA Antimicrobial Surfaces}

\subsubsection{Polyhydroxylated Surfaces (Textiles, Silica, Glass)}

Akin to the discovery of the antibiotic penicillin by Alexander Fleming, who noticed after a month long vacation that a petri dish contaminated by a fungus killed the growing bacteria, the first contact active antimicrobial surface coating was also discovered by accident. The first report describing a surface bound antimicrobial capable of killing microorganisms on contact was published in 1972 by Isquith et al., who prepared antimicrobial glass and cotton samples with octadecyldimethyl (3-trimethoxysilylpropyl)-ammonium chloride (ODDMAC or Si-QAC) (see Figure 1.7, Compound 3). ${ }^{35}$ The publication was based on Abbott's research at Dow Corning on silicone and silane based compounds for the control of algae. ${ }^{36}$ When measuring minimum inhibitory concentrations (MIC's) of silane quaternary ammonium compounds in solution, Abbott unexpectedly found that he was getting extremely low values (cfu $\approx 0$ ). ${ }^{36}$ He attributed his observations of false positives to the adsorption of the active compounds on to the wall of his

equipment. ${ }^{36}$ As a result, (3-trimethoxysilylpropyl)dimethylalkyl ammonium chlorides with alkyl chains from 6 to 22 carbons produced the highest algae reductions and were quickly patented by DOW Corning (Figure 1.7, Compounds 1-11). ${ }^{37}$ 


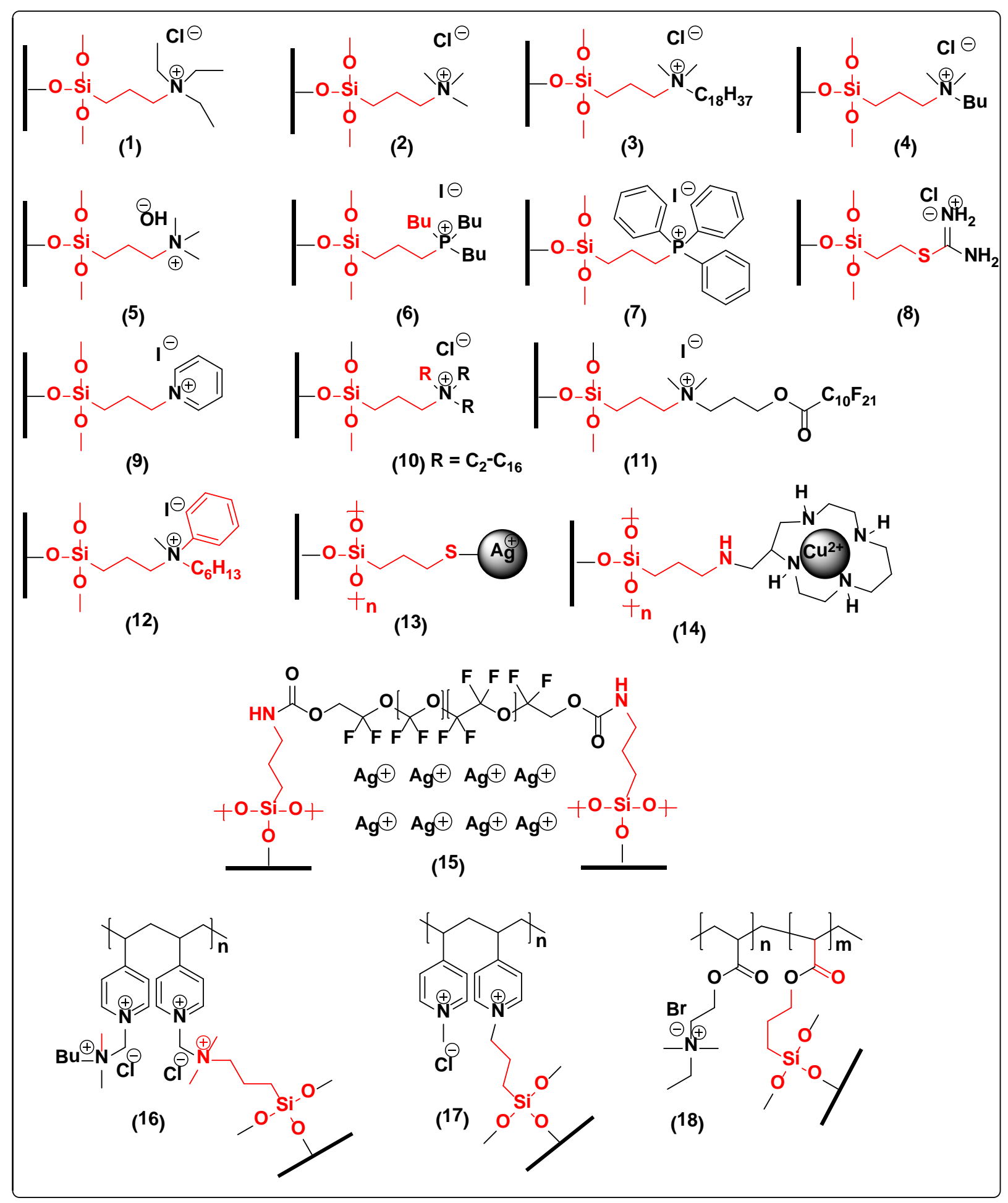

Figure 1.7: Examples of antimicrobial organosilanes described in the literature. $(\mathbf{1})^{38},(\mathbf{2 - 1 1})^{36}$, $(\mathbf{1 3})^{39},(\mathbf{1 4})^{40},(\mathbf{1 5})^{39,41},(\mathbf{1 6})^{42},(\mathbf{1 7})^{43},(\mathbf{1 8})^{44}$. 
After years of extensive toxicological testing, Dow Corning's antimicrobial quaternary ammonium agent 3, sold as an antimicrobial finish for textiles, was approved by the EPA in 1977 and received the Industrial Research and Development award as the best new commercialized product that year. ${ }^{3}$ SiQAC (Figure 1.7, Compound 3), is commercially prepared from 3chloropropyl trimethoxysilane (excess $\sim 1.2$ eq.) and $N, N$-dimethyloctadecylamine, and available as a $40-72 \%$ methanolic solution from the following companies: Aegis (AEM 5772), Piedmont (Ztrex72), Flexipel (Q-1000), and Dow Corning (Q9-6346), (Figure 1.7, Compound 3). ${ }^{45}$

In the original publication, Si-QAC (72\%) was typically applied as a $0.1 \mathrm{wt} \%$ solution in $\mathrm{H}_{2} \mathrm{O}$ on cleaned glass or cotton followed by annealing at $70^{\circ} \mathrm{C}$ for 30 min in order to form strong Si-O-Si bonds with free $-\mathrm{OH}$ groups on oxide surfaces, e.g glass, ceramics, cellulose, silica. However, for industrial applications the compound was limited by the ready polymerization of neighbouring silanols in $\mathrm{H}_{2} \mathrm{O}$ and precipitation of the product upon long term storage (Figure 1.8). In aqueous environments, alkoxy silanes are rapidly hydrolyzed under neutral conditions leading to condensation of neighbouring silanols, resulting in the undesired precipitation or gelling of the product (Figure 1.9). For large scale applications, methanol-based products are undesirable due to their toxicity, flammability and the highly regulated nature of methanol. ${ }^{46}$ 


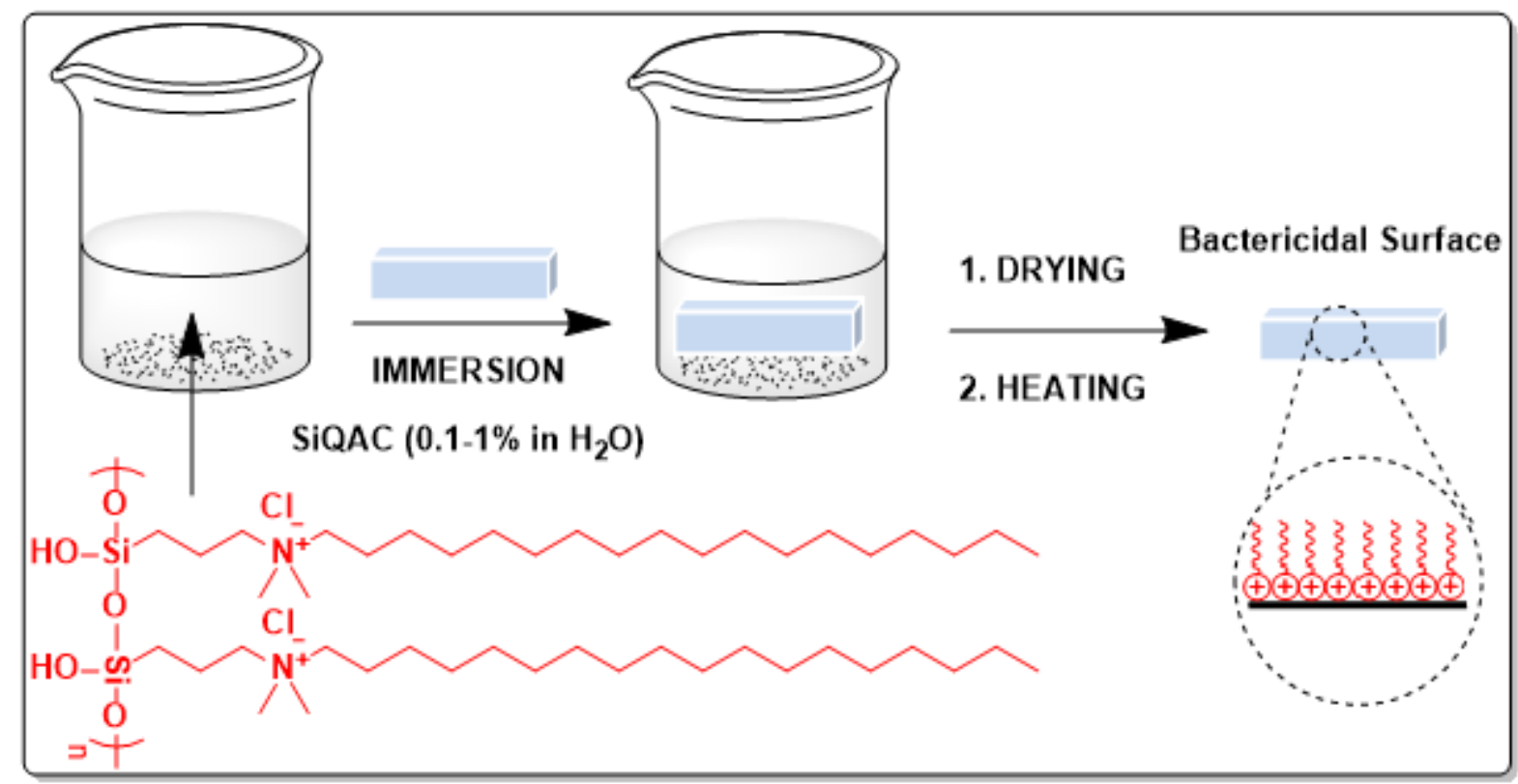

Figure 1.8: Typical procedure for immobilizing SiQAC on polyhydroxy surfaces. ${ }^{38}$ 


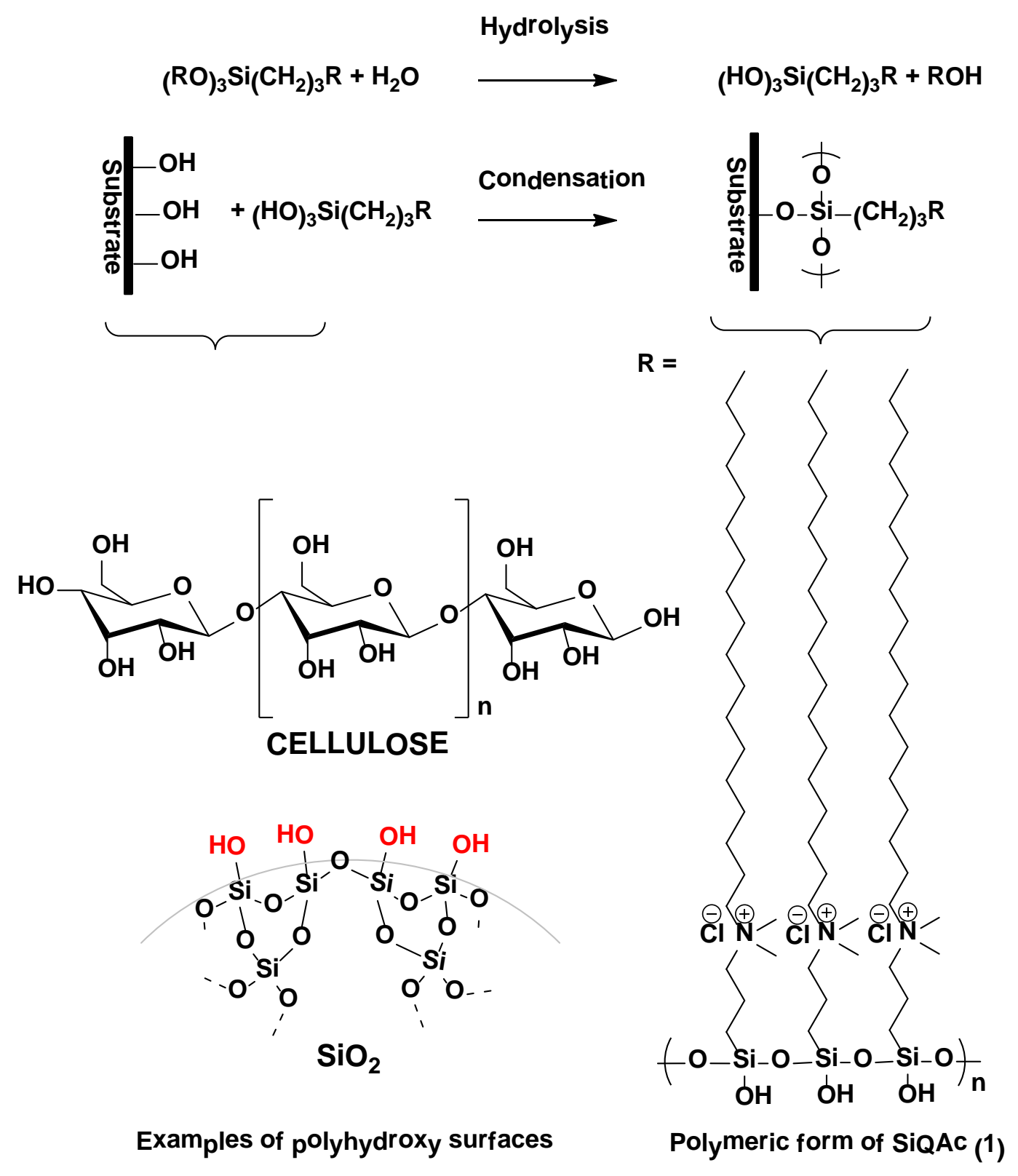

Figure 1.9: Anchoring of trialkoxysilyl compounds onto polyhydroxide surfaces.

It would take another 18 years to develop a safer and more environmentally friendly SiQAC. In 1995, Dr. Gary Allred and Dr. Lanny Liebeskind patented the Si-QAC as a 5\% active stabilized product in $\mathrm{H}_{2} \mathrm{O} .{ }^{47}$ The researchers from Emory University added a proprietary stabilizer that gave the organosilane an alternative to polymerization in $\mathrm{H}_{2} \mathrm{O}$ and resulted in a stabilized product. Currently the safer and more environmentally friendly Si-QAC is 
commercially available from Pureshield as a $\mathrm{H}_{2} \mathrm{O}$ soluble solution of the active quat at 5 wt \% and sold under different brand names such as AM 500, SiShield 500, BioProtect 500, or Microbe Guard in several countries. The product is currently used commercially as an antimicrobial finish on textiles such as cotton, nylon, polyester and wool. ${ }^{11,20}$

In the original publication, Isquith claimed that immobilized Si-QAC on different substrates such as siliceous surfaces, natural fibers, man-made fibers, metals and others had broad-spectrum antimicrobial activity. ${ }^{38}$ However, the publication only demonstrated antimicrobial activity of Si-QAC on glass against F. faecalis (gram-positive) and on cotton cloth against E. coli (gram-negative). The silane modified surfaces killed $>95 \%$ of the F. facealis visible colonies after 30 min measured with the Dow Corning Corporate Test Method 0923 (CTM-0923, See Section 1.6.1.1.1). ${ }^{38}$

The antibacterial treatment of Si-QAC and related organosilanes onto polyhydroxy surfaces such as textile fabrics, glass and silica nanoparticles (NP's) are well studied in the literature. Table 1.3 summarizes the historical account of various antimicrobial organosilane treated surfaces described in the literature starting from the original publication by Isquith in 1972 until 2013. Examples include different textiles surfaces: polyester fabrics (Entry ii), cotton polyester (Entry vi), microfibrillated cellulose (Entry xi), cotton gauze (Entry xiv), cotton textile (Entry xv) as well as glass (Entries i-ii, iv, xiii, xvii), titanium (Entry xii) and silica NP’s (Entry xvi). These treated materials were shown to be antimicrobicidal after treatment with the antimicrobial trialkoxysilane 1. Other surfaces normally inert to silanization such as silicone rubber and metal oxides were also coated with $\mathbf{1}$ but required prior surface pre-treatment: sanding (Ti, Entry ix) and plasma treatment (silicone, Entries vii and x) necessary to activate them towards silanization. Similarly, organosilane antimicrobials based on the release of $\mathrm{Ag}^{+}$ 
(Entries xiii and xv) and polymeric QAC's (Entries xv and xvi) were developed after the original silane QAC 3. However the application of the antimicrobial varies in each study as well as the type of bacteria tested and the testing methods.

Table 1.3: Summary of different surfaces immobilized with antimicrobial organosilanes.

\begin{tabular}{|c|c|c|c|}
\hline Entry & $\begin{array}{l}\text { Antimicrobial } \\
\text { silane }\end{array}$ & Immobilized Surface & Year \\
\hline $\mathbf{i}^{38,48}$ & 1 & $\begin{array}{l}\text { Siliceous surfaces, man-made fibres, natural fibres, metals, } \\
\text { leather, wood, rubber. }\end{array}$ & 1972 \\
\hline ii $^{48}$ & 1 & Glass, cotton, cellulose, polyester & 1973 \\
\hline iiii $^{36}$ & $2-16$ & Cellulose acetate, nylon 6,6, polyester, silica & 1982 \\
\hline $\mathbf{i v}^{49}$ & 1 & Glass & 1984 \\
\hline $\mathbf{v}^{50}$ & 1 & Polyurethane foam & 1985 \\
\hline $\mathbf{v i}^{51}$ & 1 & Cotton-polyester fabrics & 1988 \\
\hline vii $^{52}$ & 1 & Silicone rubber & 2002 \\
\hline viiii $^{53}$ & 1 & Polyethylene terephthalate (PET) & 2004 \\
\hline $\mathbf{i x}^{54}$ & 1 & Titanium dental implants & 2005 \\
\hline $\mathbf{x}^{55}$ & 1 & Silicone rubber stents & 2006 \\
\hline $\mathbf{x i}^{\mathbf{i}^{56}}$ & 1 & Microfibrillated cellulose & 2007 \\
\hline $\mathbf{x i i}^{42,57,58}$ & 16,17 & Glass, cotton, paper & 2008 \\
\hline xiiii $^{59}$ & 12 & Glass & 2009 \\
\hline $\mathbf{x i v}^{60}$ & 1 & Cotton gauze & 2010 \\
\hline $\mathbf{x v}^{61}$ & 1 & Cotton textile & 2011 \\
\hline $\mathbf{x v i} \mathbf{i}^{62}$ & 1 & $\mathrm{SiO}_{2}$ nanoparticles & 2011 \\
\hline xvii $^{63}$ & 1 & Glass & 2011 \\
\hline
\end{tabular}




\subsubsection{Metal Oxides}

Basic metal oxide surfaces (e.g. Ti, SS) typically contain far fewer surface functional groups $(-\mathrm{OH})$ than are necessary for grafting monolayers and often require surface pretreatment, also know as passivation, in order to increase surface - $\mathrm{OH}$ groups compared to the acidic metal oxide surface representative of silica. Passivation is typically achieved by either chemical means ${ }^{64,}{ }^{65}$ (dipping in Pirhana solution, sanding, or heating over $130^{\circ} \mathrm{C}$ in air) or physical methods (electrochemical grafting, plasma deposition). ${ }^{66}$

A simpler way to functionalize metal oxide surfaces without the use of expensive and surface altering plasma pre-treatment involves the direct formation of phosphonates and catechol monolayers on metal oxide surfaces. Phosphonate monolayers have been shown to be advantageous over self-assembled monolayers (SAMs) of thiols and silanes in terms of durability, long-term stability and surface coverage, especially on Ti and SS. ${ }^{67-69}$ Thiol-based SAM’s lack substrate specificity (mainly reserved for Au surfaces) and long-term stability needed for biomedical applications, (i.e. implants). ${ }^{70}$ Over time, thiols become oxidized to sulfonates, which lack affinity for Au and become displaced from the surface. ${ }^{71}$

In comparison to silane based SAM's on metal oxide surfaces, phosphonate based SAM's are advantageous because they resist hydrolysis under physiological conditions and higher surface coverage can be obtained without a harsh acid surface pretreatment (to increase $\mathrm{OH}$ content). ${ }^{72}$ The problem with silanization on the native oxide of titanium is the presence of only 15\% surface hydroxyl groups (XPS data). ${ }^{69}$ Silanization consumes surface OH sites and a lack of a neighbouring $\mathrm{OH}$ group can promote the cross-linking of nearby silanes to siloxanes. Siloxanes are known to be hydrolytically unstable and are easily hydrolyzed under physiological 
conditions. ${ }^{69}$ Once a phosphonic acid molecule coordinates to a metal, proton transfers between the coordinated phosphonate and the metal surface can take place which can create new $-\mathrm{OH}$ groups accessible for further monolayer formation and result in bidentate or tridentate coordination (Figure 1.10). ${ }^{69}$
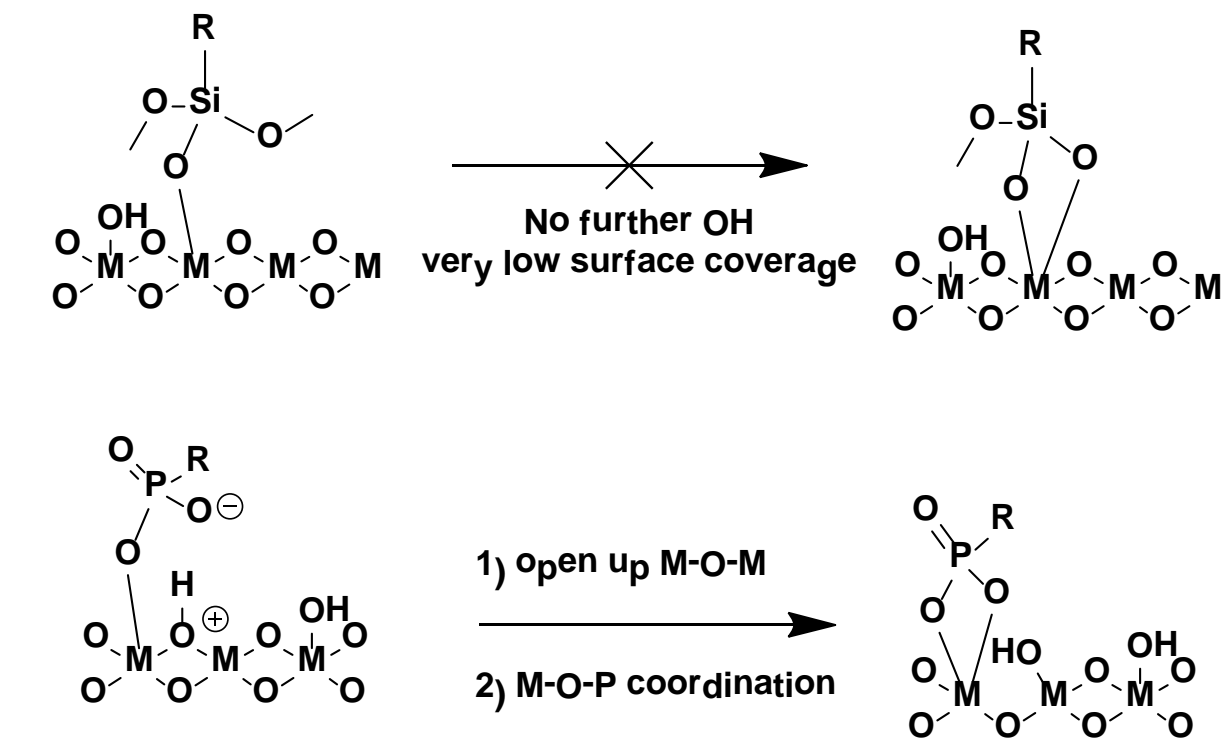

coordinated phosphonate can transfer $\mathrm{H}^{+}$to surface

Figure 1.10: Comparison of silanization to phosphonic acid monolayer formation on metal oxide surfaces. ${ }^{69}$

For example, the hydrolytic stability of silane to phosphonate based SAM's was directly compared with the incorporation of a fluorescent dansyl tag. ${ }^{73}$ The silane-dansyl molecule was assembled on Ti either by (i) attaching the silane linker aminopropyl(triethoxy)silane (APTES) on Ti followed by coupling with a maleimido reagent and capped with the fluorescent tag or (ii) attachment to $\mathrm{Ti}$ with preformed (3-triethoxysilylpropyl)-6- $N$-maleimidohexanamide followed by capping with the dansyl tag (Figure 1.11, (ii)). The phosphonic acid-dansyl reporter was prepared from Ti immobilized 11-hydroxyundecylphosphonic acid followed by coupling with the maleimido reagent and capped with the dansyl cysteine (Figure 1.11, (iii)). ${ }^{73}$ Both surface 
loadings and shear strengths of each monolayer were found to be durable enough for biomedical device surface coating. However, as expected, the Si-O-M bonds in the silane based film were completely hydrolyzed after $7 \mathrm{~d}$. in $\mathrm{pH} 7$ buffer, while the phosphonate film remained intact (Figure 1.11) ${ }^{73}$

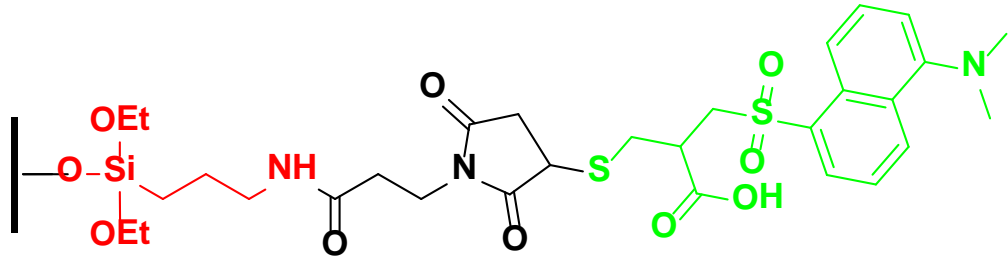

(i) prepared from aminopropyl(triethoxyssilane (APTES)

(ii) prepared from (APTES + maleimido coupling reagent)

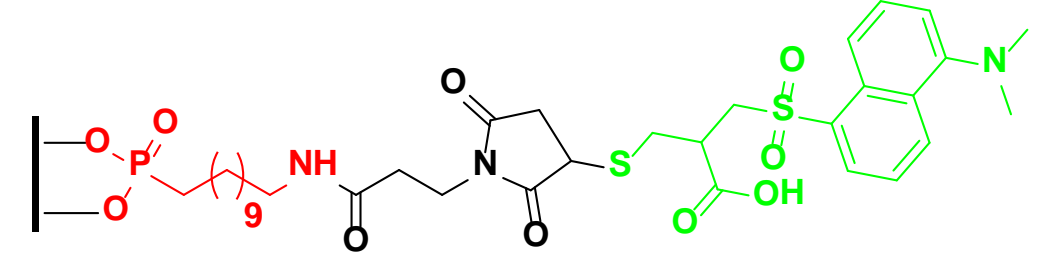

(iii) prepared from 11-Hydroxyundecylphosphonic acid

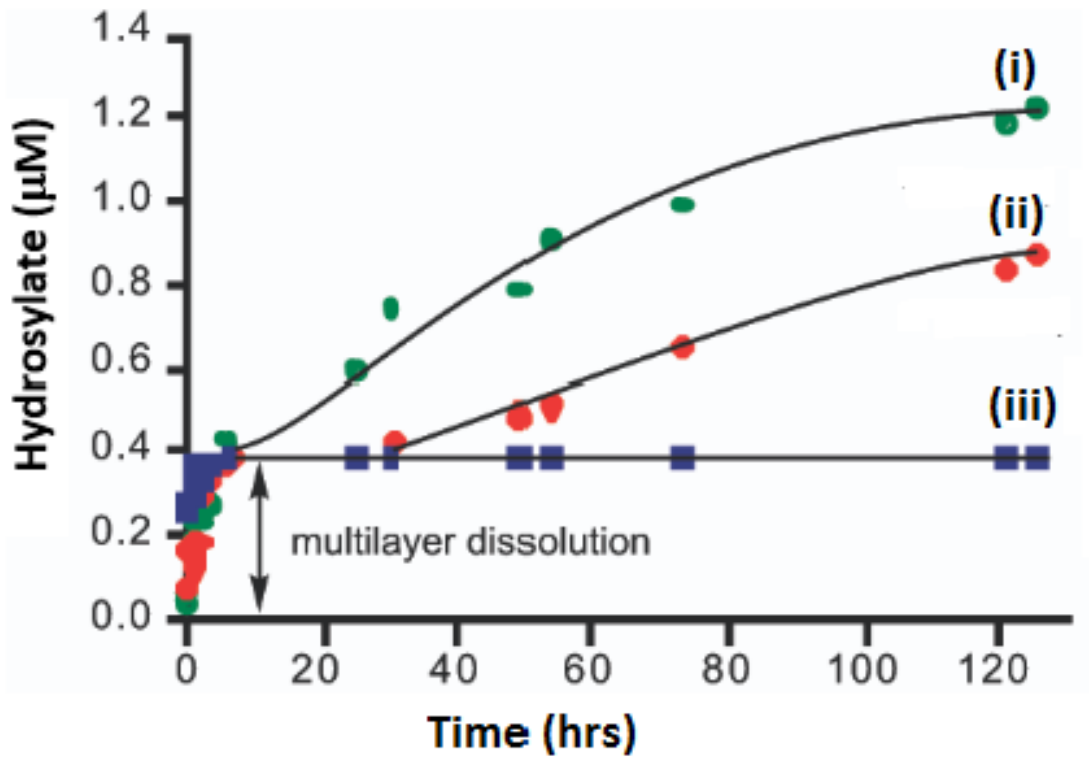

Figure 1.11: Hydrolytic loss of dansyl-cysteine from a silane based SAM vs a phosphonate based SAM (Used with permission from $\operatorname{Ref}^{73}$ ). 
Similar to the formation of silane monolayers on polyhydroxy surfaces, phosphonic acid monolayer formation on metal oxides requires a thermal curing step to drive off $\mathrm{H}_{2} \mathrm{O}$ resulting in a strong M-O-P bond. ${ }^{74}$ Both bidentate and tridentate phosphonate coordination modes to metal oxides are possible and depend on the metal surface and the temperature of the annealing step (Figure 1.12). For example, surface coverage of the phosphonate monolayer was enhanced 5-fold by depositing the monolayer with six cycles of spray/heat/rinse versus just one cycle (Figure 1.13 A vs. B). Six cycles of deposited phosphonate films were found to be very durable and resisted desorption by solvent rinsing or by a mechanical peeling test with tape (compared by IR peak intensities, Figure $1.13 \mathrm{~B}){ }^{72}$

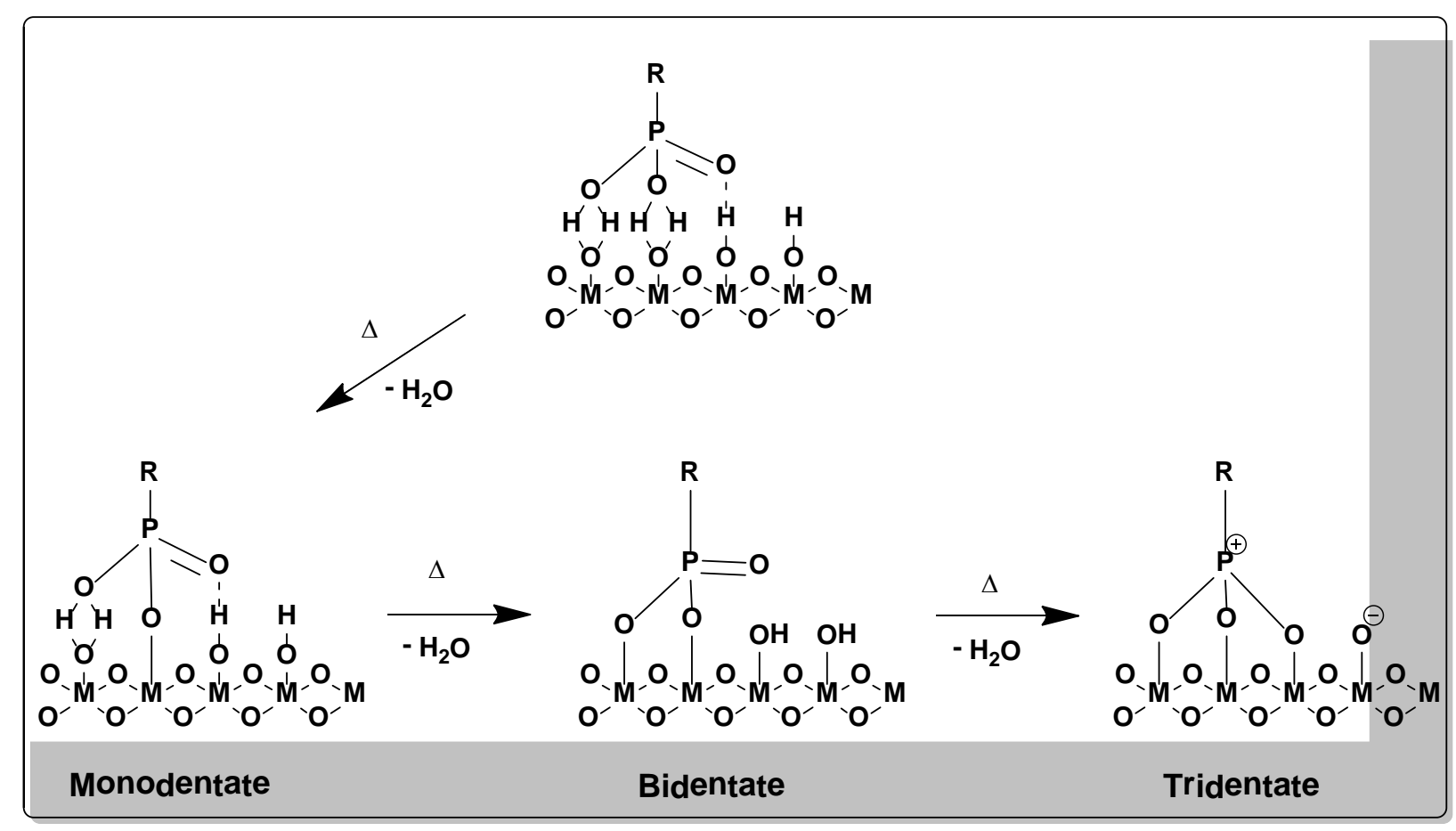

Figure 1.12: Mechanism of organophosphorus monolayer formation on metal oxide surfaces. ${ }^{75-}$ 


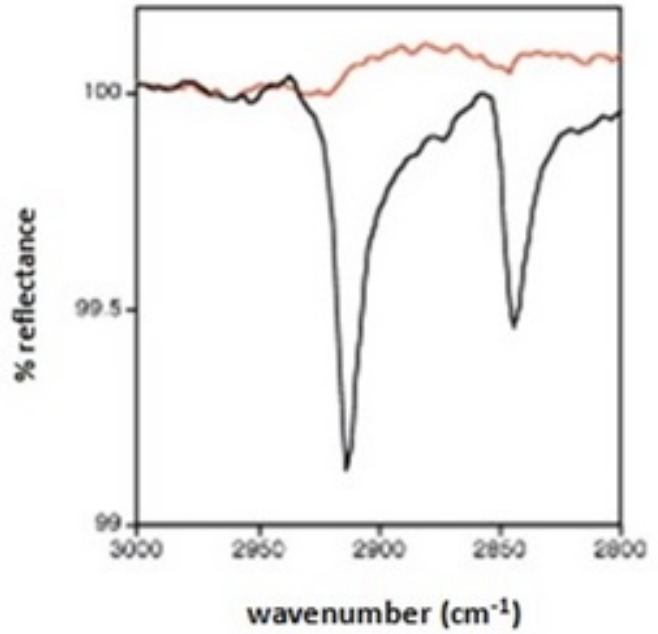

(A)

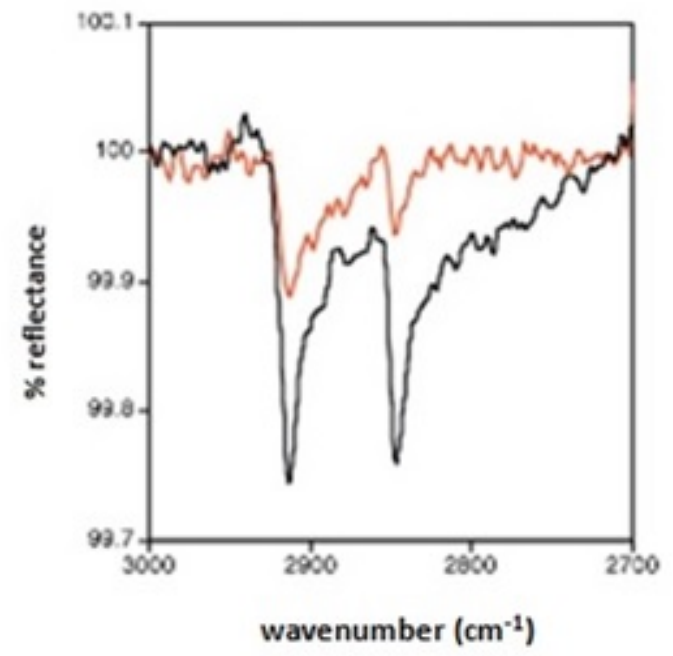

(B)

Figure 1.13: IR spectrum of a deposited film of octadecylphosphonic acid. (A) no heat, control, before (lower trace) and after solvent wash (upper trace), (B) monolayer after six cycles of deposition, with heat treatment (lower trace), compared with one cycle (upper trace), after both solvent wash and tape peel tests. ${ }^{72}$

Both active and passive strategies to prevent biofilm formation have been described with mono- or bisphosphonate monolayers and polymer thin films (Figure 1.14). Examples of active surfaces include contact killing monolayers employing immobilized quaternary ammonium salts (Figure 1.14, 19-21, 23, 24), and the antibiotics daptomycin and vanomycin (Figure 1.14, 25, 28). Active surface coatings releasing biocidal $\mathrm{NO}$ and $\mathrm{Ag}^{+}$ions have been patented (Figure 1.14, 20, 22). ${ }^{74}$ Passive strategies were described employing hydrophobic perfluorinated bisphosphonates on stainless steel, silicon, and titanium oxide surfaces for anticorrosion applications. $^{74}$ 


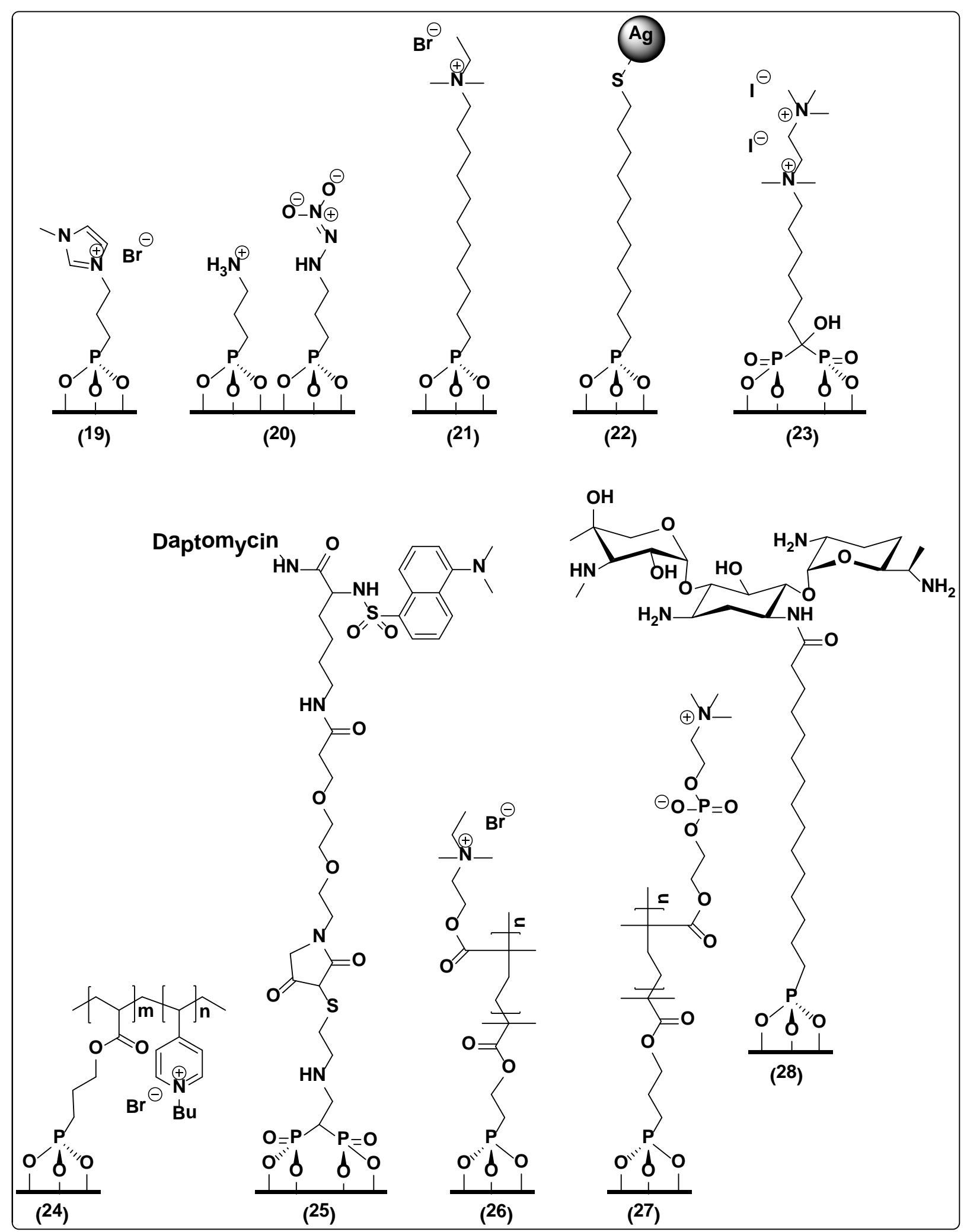

Figure 1.14: Example of literature QAC phosphonic acid antimicrobials. (19-21) ${ }^{78-80},(\mathbf{2 2})^{79,81}$, $(23)^{82,83},(24)^{84},(25)^{85},(26)^{86},(27)^{87}$. 
Another example describes an antimicrobial stainless steel (SS) surface with immobilized QAC's via plasma activation. To prepare the surface, an ethylenediamine low pressure plasma was used to functionalize the SS surface with primary amines. Quats were formed by reacting the plasma-deposited amines with hexyl bromide to generate secondary and tertiary $\mathrm{C}_{16}$ amines which were later quaternized with MeI (Figure 1.15). These QAC modified SS surfaces showed excellent bactericidal properties by killing more than $98 \%$ and $99.9 \%$ of K.pneumoniae and S.aureus respectively. ${ }^{66}$

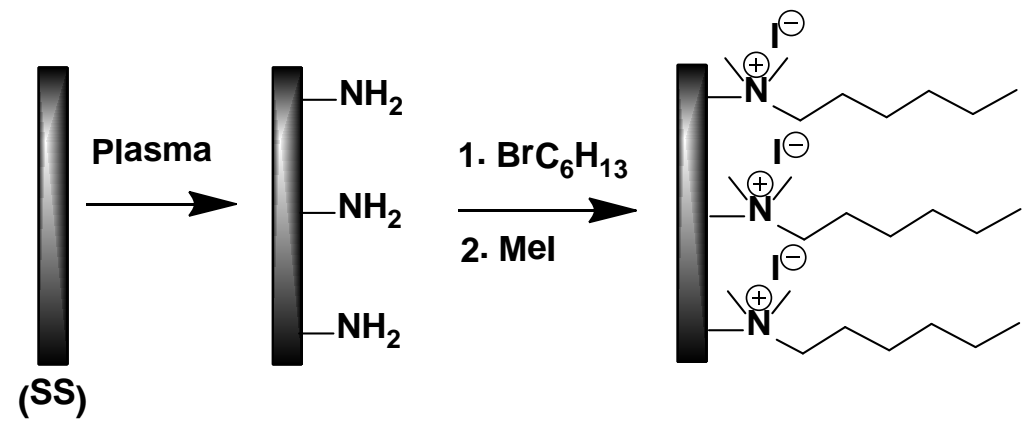

Figure 1.15: Bactericidal QAC prepared by plasma treated stainless steel via grafting to approach (Adapted from Ref. ${ }^{66}$ ).

Another linker strategy for immobilizing polymer thin films either by "graft to" or "graft from” methods onto a variety of metal oxide surfaces was introduced by the Messersmith group. Inspired by mussel adhesive proteins which contain the catechol group, the Messersmith group functionalized various polymers with this anchor and used it to create both passive and active antimicrobial surfaces for the purposes of biofilm prevention and control. ${ }^{88}$ Since the introduction of the catechol as a versatile linker, other groups created antibacterial metal surfaces by immobilizing polyethylene $(\mathrm{PE})$ based polymers with microbe repelling capabilities (compound 28), and various biocidal moieties such as antibiotics, antimicrobial peptides and quaternary QAS polymers via catechol groups (Figure 1.16, compounds 18-33). 


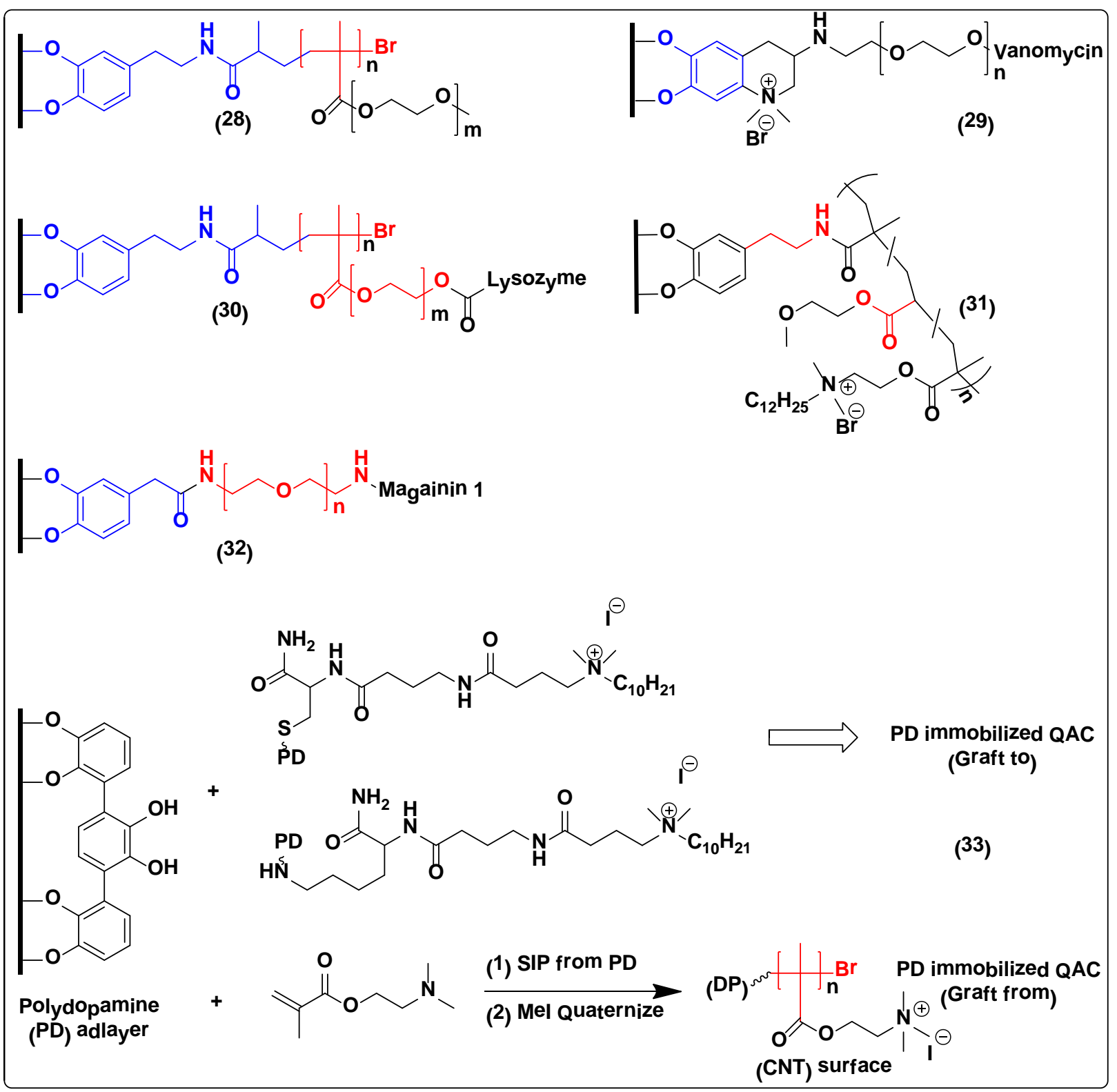

Figure 1.16: Example of literature QAC catechol antimicrobials. (28) $\left.{ }^{88,89},(\mathbf{2 9})^{90},(\mathbf{3 0})^{91}, \mathbf{( 3 1}\right)^{92}$, $(32)^{93},(33) \cdot{ }^{94}$ 


\subsubsection{Plastic Surfaces}

Common plastic or polymers surfaces widely used in the medical and food industries as medical devices and food packaging materials respectively (Tables $1.4,1.5$ ) are also very susceptible to biofilm colonization. ${ }^{95,96}$ Unlike polyhydroxy and metal oxide surfaces, plastic surfaces with functional C-H bonds such as polyethylene, polypropylene, and polystyrene or COR bonds in the case of polyesters are inert to direct silanization using the anchor chemistries previously described for -OH bearing surfaces / metal oxides, and thus require a different strategy to immobilize an antimicrobial group. The literature contains a limited number of examples of covalently immobilized antimicrobials as a finishing coating on plastics, however many examples are prevalent where the antimicrobial is introduced as an additive or polymerized into the plastic during the manufacturing process. Antimicrobials incorporated into plastics where the active species migrates to the surface during the polymerization process are termed “self-finishing” coatings. ${ }^{18}$ 
Table 1.4: Common medical devices vulnerable to microbial contamination. ${ }^{29}$

\begin{tabular}{ll}
\hline Medical Device & Polymeric Materials \\
\hline Breast implants & Silicones \\
Catheters & Silicones, PVC, urethanes, fluoropolymers \\
Contact lenses & Methacrylates, silicones \\
Dental implants & Silicones \\
Heart valves & Polyester, polyoxymethylene, \\
Hip and knee prostheses & UHMWPE, PMMA \\
Intraocular lens & Methacrylates, silicones \\
Left ventricular assist device & Urethanes, carbonates \\
Pacemakers & Urethane \\
Renal dialyzers & Polyacrylonitrile \\
\hline
\end{tabular}


Table 1.5: Polymers commonly used for food packaging materials. ${ }^{95,97}$

\begin{tabular}{|c|c|}
\hline Polymer & Formula \\
\hline \multicolumn{2}{|c|}{ Ethylene vin ${ }_{y} l$ acetate (EVA) } \\
\hline \multicolumn{2}{|c|}{ Ethylene methyl acetate (EMA) } \\
\hline \multicolumn{2}{|c|}{ 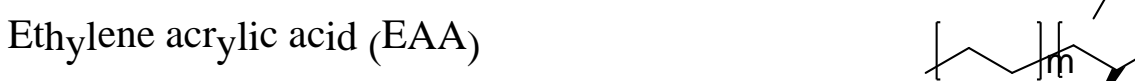 } \\
\hline \multicolumn{2}{|c|}{ Ethylene methacrylic acid (EMAA) } \\
\hline \multicolumn{2}{|l|}{ Ionomer } \\
\hline \multicolumn{2}{|l|}{$\mathrm{N}_{\mathrm{y}} \mathrm{lon}$} \\
\hline \multicolumn{2}{|c|}{$\begin{array}{l}\text { Polyvinylidene chloride (PVDC) } \\
\text { Polyvinyl chloride copolymer (PVC) }\end{array}$} \\
\hline \multicolumn{2}{|c|}{ Ethylene vinyl alcohol (EVOH) } \\
\hline \multicolumn{2}{|c|}{ Polyethylene ${ }^{\circ}{ }_{p}{ } l_{y}$ mer $(\mathrm{PE})$} \\
\hline Pol $_{y}$ st $_{y}$ rene (PS) & \\
\hline
\end{tabular}




\subsubsection{1 “QAC's as Additives”}

Wynne prepared polyurethane-QAC antimicrobial coatings by blending a long chain quat with oxyethylene groups into polyurethane. ${ }^{98}$ By increasing the length of the hydrophobic alkyl chain to octyl and decreasing the length of the oxyethylene groups from $n=4$ to $n=2$, allowed for increased migration of the QAC to the surface of the polyurethane resin (measured by X-ray photoelectron spectroscopy (XPS)) resulting in a 7-log reduction of S. aureus and E. coli (Figure 1.17). ${ }^{98}$ Furthermore, the antimicrobial plastics with the highest surface concentration $(\mathrm{n}=8, \mathrm{~m}$ = 2) maintained their activity after immersion in $\mathrm{H}_{2} \mathrm{O}$ for $7 \mathrm{~d}$., demonstrating they were truly surface bound. HPLC and antimicrobial analysis of the $\mathrm{H}_{2} \mathrm{O}$ phase further confirmed that the plastic sample was non-leaching and contact killing. This suggests that the QAC additives are initially mobile and able to surface-concentrate in the uncured polyurethane but become "locked" in place once the resin has cured. Thus, diffusion of the antimicrobial agents out of the film is prevented despite the inherent $\mathrm{H}_{2} \mathrm{O}$ solubility of the quat. ${ }^{98}$

The Foucher group successfully incorporated SiQAc into polypropylene (PP) during the injection molding process at 5wt \% and obtained significant reduction in biofilm growth, the treated surface stained blue with bromophenol blue indicating prescence of the antimicrobial at the surface. $^{99}$ 

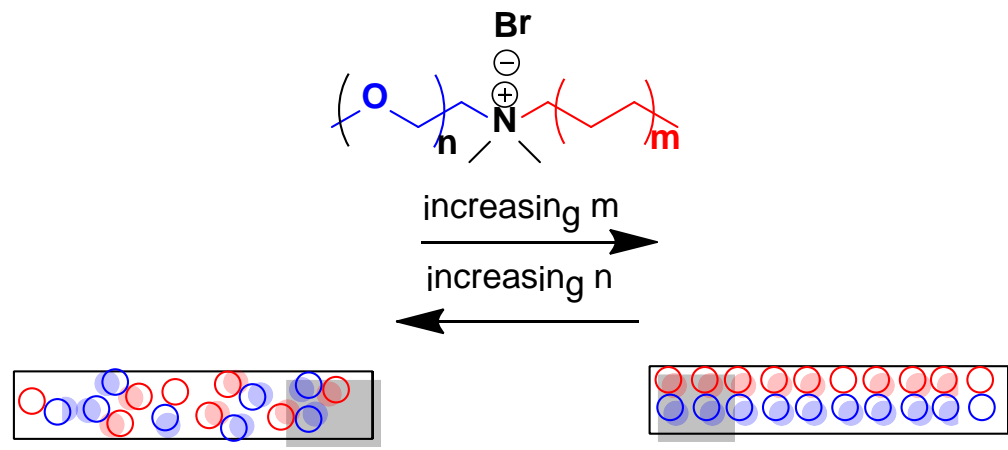

Figure 1.17: Example of a contact active plastic self-finishing coating. ${ }^{98}$

\subsubsection{Thermoset Antimicrobial Coatings: Cross Linked Networks}

Non-leaching antimicrobial-QAC thermoset plastic coatings are prepared by crosslinking the antimicrobial agent with polymerizable functional groups such as siloxanes, polyurethanes, epoxides and acrylates into polymerizable networks (Figure 1.18).

Clarkson first prepared SiQAC-(SE) plastics by incorporating the antimicrobial agent, SiQAC, into silicone elastomer (SE) polymers via crosslinking with the hydroxy groups present in the poly(dimethylsiloxane) polymer. The coating was toxic to the algae, Amphora coffeaeformis for up to 20 weeks, but lost activity after 23 weeks in sea $\mathrm{H}_{2} \mathrm{O}$. The toxicity of the SiQAC-SE surfaces was attributed to a slow leaching of residual uncrosslinked SiQAC from the coating. Once all excess uncrosslinked SiQAC was removed by boiling solvent Soxlet extraction, the polymer lost its anti-algal activity. ${ }^{100}$ Structures of crosslinked siloxanes, ${ }^{101}$ polyurethanes ${ }^{102}$, epoxides $^{103}$ and acrylates crosslinked by UV light ${ }^{104}$ with the antimicrobial QAC as part of the

polymer are shown in Figure 1.18. For example, the polyurethane QAC 35 prepared by a covalently bonded hydroxyl terminated QAC bearing a long perfluorinated tail with a 
polyisocyanate cross linker showed a $5 \log$ reduction of $S$. aureus and E. coli at low concentrations ( $0.5 \mathrm{wt} \%, 0.007 \mathrm{mmol} / \mathrm{g}$ ) with ISO 22196 and JIS Z 2801 protocols. ${ }^{102}$

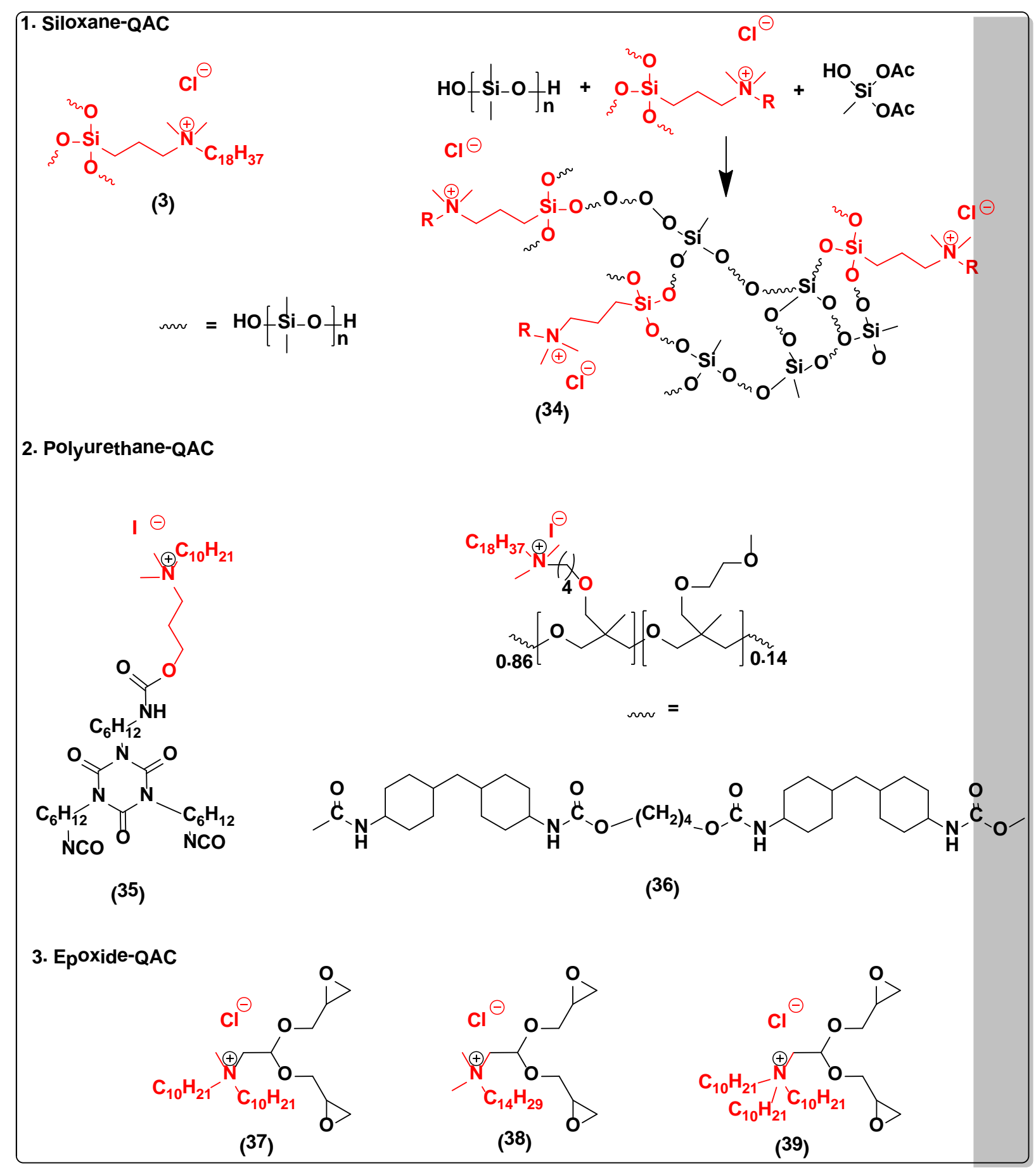




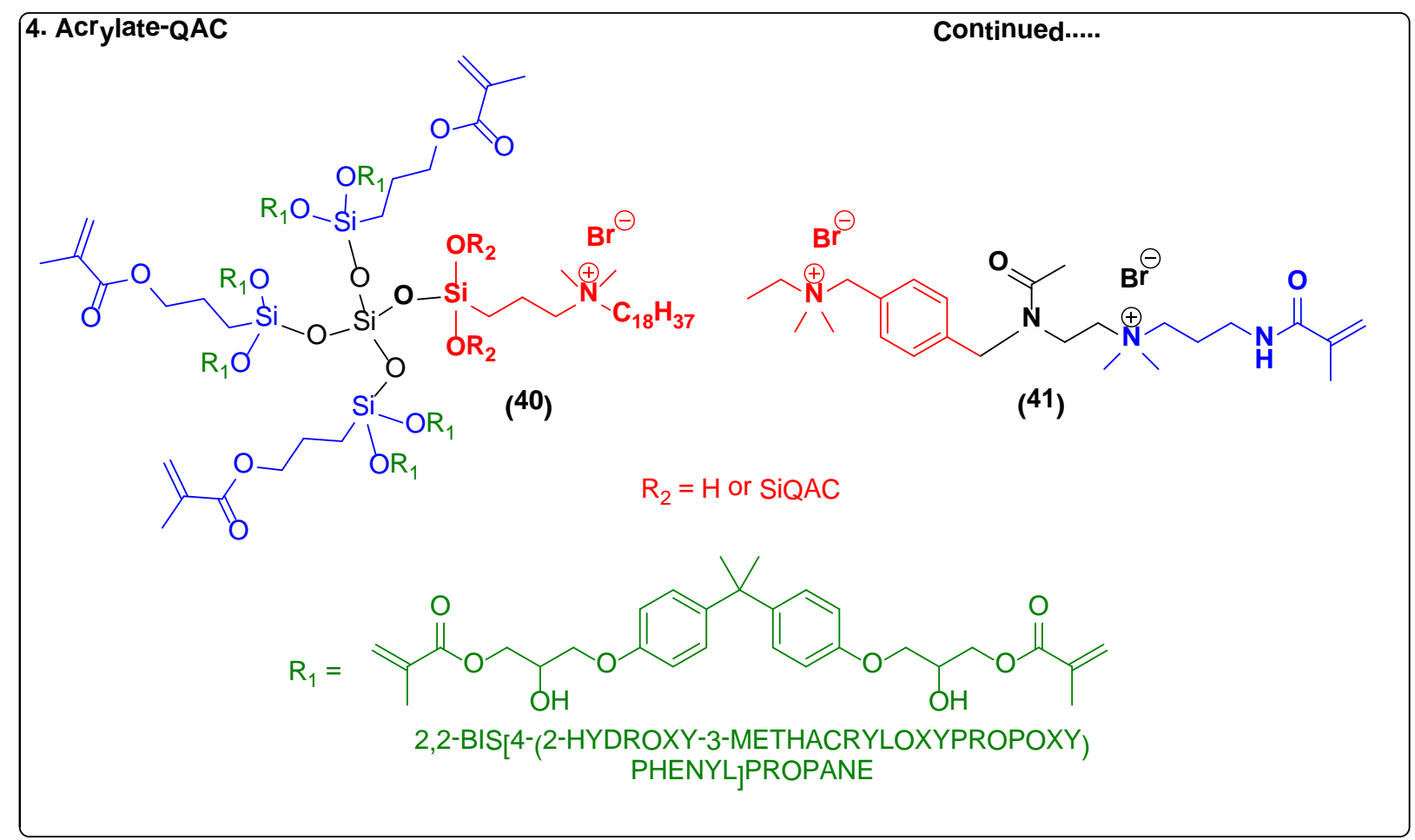

Figure 1.18: Monomers and quats used to prepare polymer-QAC antimicrobial plastic coatings. $(34)^{101},(35-36)^{102},(37-39)^{103},(40-41)^{104}$

\subsubsection{3 “Grafting Onto” and “Grafting From” Plastic Surfaces}

Covalently attached biocides such as QAS onto plastics can be achieved by involving either (i) plasma activation in order to functionalize the C-H surface with reactive groups such as $\mathrm{OH}$ or $\mathrm{NH}_{2}$ onto which biocidal polymers can be grafted to or from (ii) or UV light activation of functional groups known to directly react with inert $\mathrm{C}-\mathrm{H}$ groups present on the polymer backbone such as benzophenones, vinyls, propargyls. In 2002, Gotenboss described the $1^{\text {st }}$ antimicrobial QA plastic surfaces on an $\mathrm{OH}$ terminated silicone rubber after plasma treatement with SiQAC. In 2003, a patent described the plasma activation of polypropylene to either (A) 
generate radicals from which QAC monomers were "grafted from" or (B) introduce functional groups onto which QAC polymers were "grafted to” (Figure 1.19). ${ }^{52}$

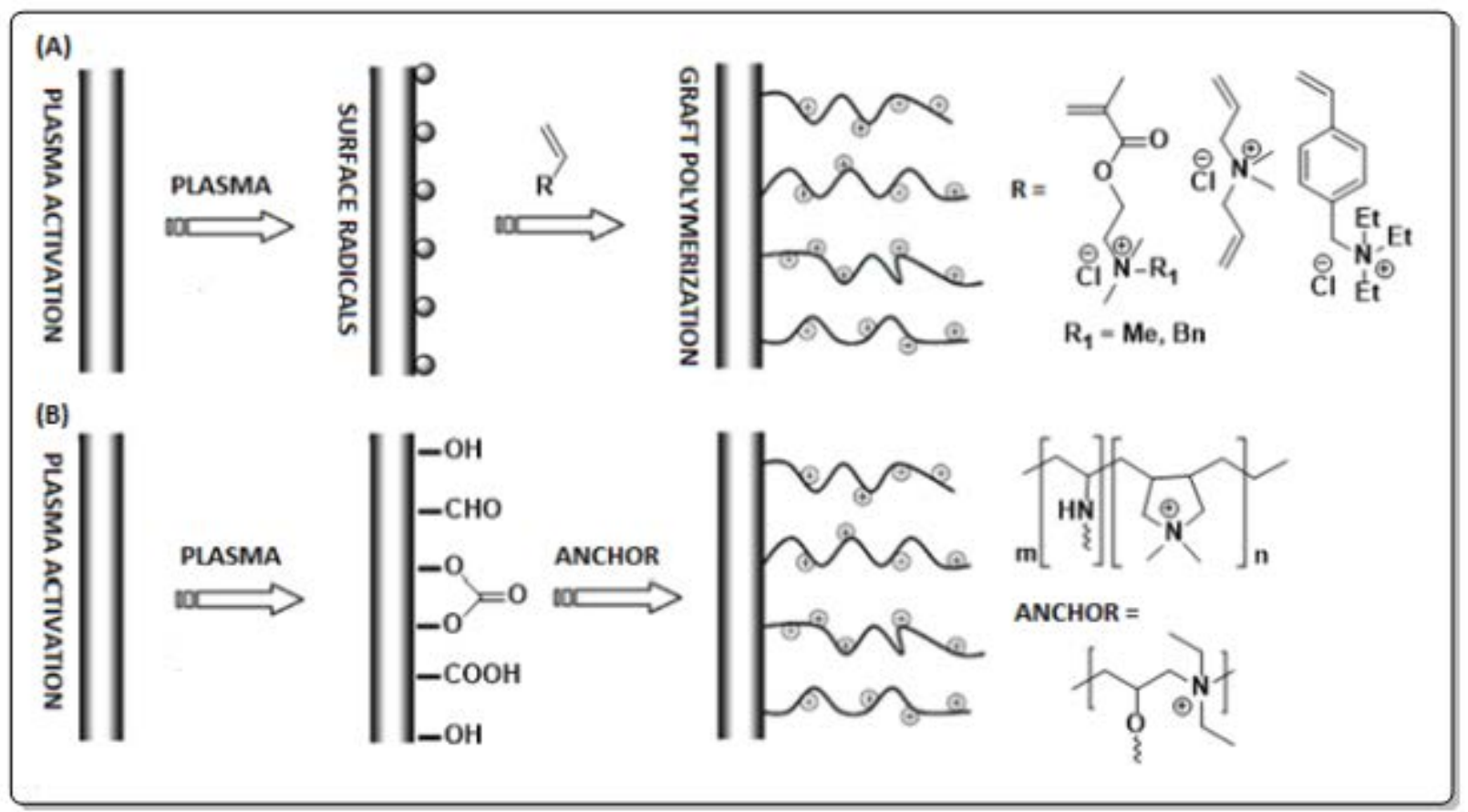

Figure 1.19: Bactericidal QAS polymers and small molecules prepared by plasma treated polypropylene (A) "grafting from” and (B) "grafting to" (Adapted from Ref. ${ }^{32}$ ).

An alternative way to effectively attach polymeric or monomeric antimicrobials to plastics surfaces is with photochemically active groups such as the benzophenone cross-linker. When irradiated with UV light (345-365 nm), the benzophenone group abstracts a hydrogen atom from a polymer (C-H) surface, forming a strong C-C bond (Figure 1.20). ${ }^{24}$ This linker has recently been used to attach thin polymer films to metal oxide and polyhydroxy surfaces via a phosphonic acid or silane group (Figure 1.20, 42, 45). Other examples include "grafting to" of antimicrobial QAC molecules and polymers with benzophenone groups to plastic surfaces with C-H groups under UV light (Figure 1.21, 43, 44, 46, 47). Dhende was first to covalently attach a quaternized polyethyleneimine (PEI) polymer onto various plastic surfaces ${ }^{25}$ (Figure 1.21, Table 
1.6, compound 43) while the Foucher group experienced success with a benzophenone terminated $\mathrm{C}_{18}$ quat on PVC and silicone grade medical tubing ${ }^{105}$ (Figure 1.21, Table 1.6, compound 44). The coating was visualized with bromophenol blue and found to be stable up to 3 rinse cycles after destaining with anionic detergents and restaining. Lastly, Yang et al., used benzophenone as a surface initiator and grew polymers from PP surfaces that were later either quaternized or first quaternized and crosslinked (Figures 1.21 and 1.22, Table 1.6, Compound 47). ${ }^{106}$ With the unquaternized surfaces, a surface charge density of $\sim 3.50 \mu \mathrm{mol} \mathrm{cm}{ }^{-2}$ was enough to kill both $E$. coli and S. aureus cells after 5 min. However, once the polymers were cross-linked, the surface lost its antimicrobial activity which was attributed to the loss of mobility of the immobilized polymeric cations. ${ }^{106}$<smiles>CCC(C)CC(C)CC(C)CC(C)CC(C)C</smiles>

Figure 1.20: Example of "grafting from" immobilization of biocidal polymers employing the benzophenone surface bound initiator. ${ }^{24}$ 


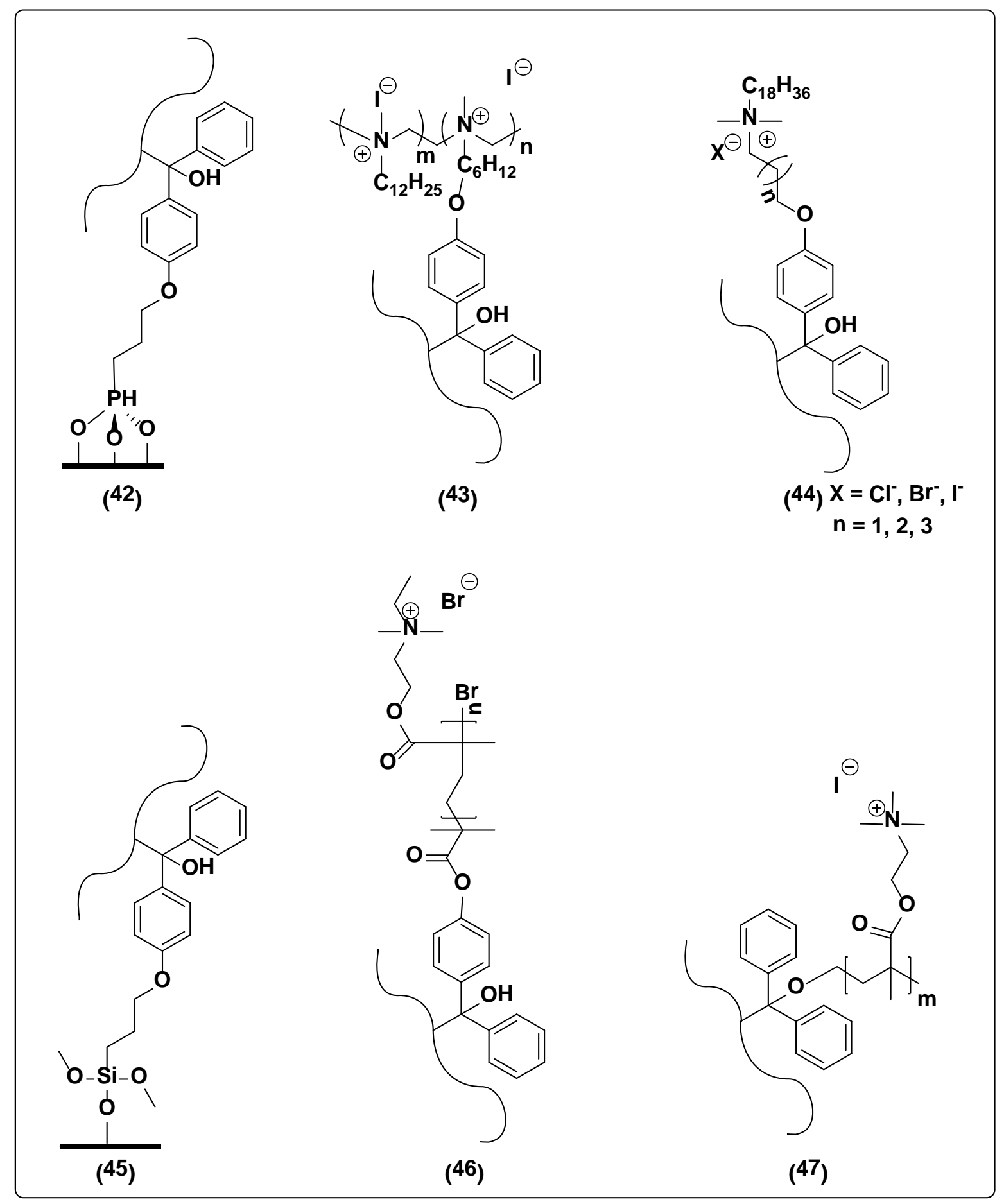

Figure 1.21: Examples of benzophenone used to prepare antimicrobial surfaces. $(\mathbf{4 2})^{23,107}$, (43) ${ }^{25},(\mathbf{4 4})^{105},(\mathbf{4 5})^{108},(\mathbf{4 6})^{32},(\mathbf{4 7})^{106}$. 
Table 1.6: Examples of the benzophenone group utilized to create antimicrobial surfaces.

\begin{tabular}{|c|c|c|c|}
\hline $\begin{array}{l}\text { Antimicrobial } \\
\text { Compound }\end{array}$ & $\begin{array}{l}\text { Surface } \\
\text { Tested }\end{array}$ & Immobilization type & $\begin{array}{l}\text { Contact } \\
\text { killing (\%) }\end{array}$ \\
\hline $42^{23,27}$ & $\mathrm{Ti}, \mathrm{Al}$ & $\begin{array}{l}\text { Bifunctional- } \mathrm{PO}_{3} \mathrm{H}_{2} \text { SAM on metal with } \\
\text { benzophenone polymer cap }\end{array}$ & N/A \\
\hline $43^{25}$ & $\begin{array}{l}\text { PP, PVC, } \\
\text { PE }\end{array}$ & $\begin{array}{l}\text { Benzophenone grafting onto ( } 1.5 \% \text { sprayed, } \\
\text { acetone), UV light ( } 365 \mathrm{~nm}, 180 \mathrm{~mW} / \mathrm{cm}^{2} \text { ) } \\
\text { for } 15 \mathrm{~min}\end{array}$ & $\begin{array}{l}99 \% \text { E. coli } \\
\text { and S.aureus } \\
\text { for }>35 \mathrm{~nm} \\
\text { thickness } \\
\text { coating }\end{array}$ \\
\hline $44^{105}$ & $\begin{array}{l}\text { PP, PVC, } \\
\text { Silicone }\end{array}$ & $\begin{array}{l}\text { Benzophenone grafting onto ( } 1-5 \% \text { sprayed, } \\
\text { EtOH), UV light }\left(365 \mathrm{~nm}, 180 \mathrm{~mW} / \mathrm{cm}^{2}\right) \text { for } \\
5 \mathrm{~min}\end{array}$ & $\begin{array}{l}99 \% \text { E. coli } \\
\text { reduction on } \\
\text { Silicone }\end{array}$ \\
\hline $45^{108}$ & Si Wafer & $\begin{array}{l}\text { Bifunctional-silane SAM on glass and } \\
\text { benzophenone to antimicrobial polymer cap }\end{array}$ & N/A \\
\hline $46^{32}$ & $\mathrm{PP}$ & $\begin{array}{l}\text { Benzophenone to BIBB SIP initiator ester } \\
\text { coupling followed by ATRP graft with } \\
\text { DMAEMA, quaternized with EtBr }\end{array}$ & 95\% S.aureus \\
\hline $47^{106}$ & PP & $\begin{array}{l}\text { Benzophenone adlayer to "grafting from" } \\
\text { with acrylate monomers then quaternizing }\end{array}$ & 95\% S.aureus \\
\hline
\end{tabular}

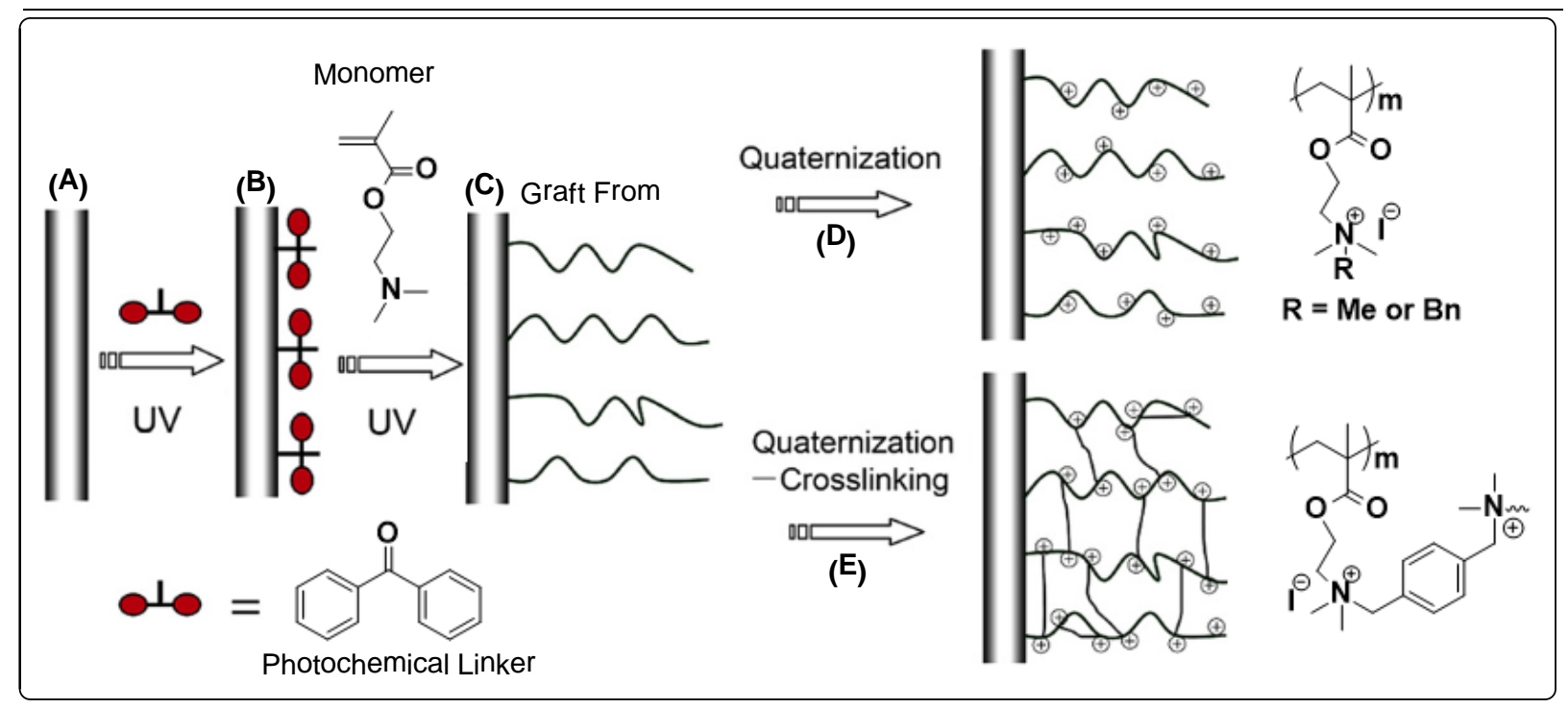

Figure 1.22: Example of "grafting from" a plastic surface. Immobilization of biocidal polymers without employing a surface bound initiator (Adapted from Ref. ${ }^{106}$ ). 


\section{$1.32^{\text {nd }}$ Generation Antimicrobial Coatings: Literature Examples of Dual Action Antimicrobial Surfaces}

Since the advent and popularity of contact active QAC antimicrobials, current research has shifted towards combining both passive and active antimicrobial coating strategies on a single surface, giving rise to $2^{\text {nd }}$ generation antimicrobials. ${ }^{109}$ These surfaces were designed to improve on the drawbacks of the $1^{\text {st }}$ generation coatings which provide only one level of protection, either a microbe killing or microbe repelling role. $2^{\text {nd }}$ generation coatings provide dual protection against invading bacteria by combining contact-killing with repelling capabilities so dead bacteria are swept or released from the surface after being killed. ${ }^{109}$ In theory, these ideal surface coatings are difficult and costly to prepare, with few literature examples (mainly on Au surfaces) known. One example of a dual killing surface with contact killing and biocide releasing (killing) capabilities was prepared by growing cationic chains from Au surfaces followed by impregnation with $\mathrm{Ag}^{+}$ions (Figure 1.23). ${ }^{109}$ One apparent problem with solely killing surfaces is the build up and attachment of dead cells or debris that can deactivate the surface if not reactivated by a cleaning step. One solution to this problem was realized with a polymeric coating on Au surfaces that can be hydrolyzed from the quaternary ammonium (killing) to the zwitterionic form (repelling) reversibly (Figure 1.24). ${ }^{110}$ However, this surface required a manual hydrolysis similar to a cleaning step, otherwise the surface won't automatically switch after a build up of cells is sensed (Figure 1.24). The Foucher group is also interested in biocidal and self-cleaning surface coatings. Synthesis of an azo-benzene QAC with inherent self-cleaning capabilities switching from the cis and trans configuration under UV light is currently under study. ${ }^{99}$ 
The $3^{\text {rd }}$ generation of antimicrobial coatings is foreseen to be capable of responding to stimuli so the surface can switch from a killing to a repelling one and vice-versa when necessary and target pathogenic bacteria exclusively. No literature reports of such stimuli responsive “switchable,” "smart,” futuristic surfaces are yet available.

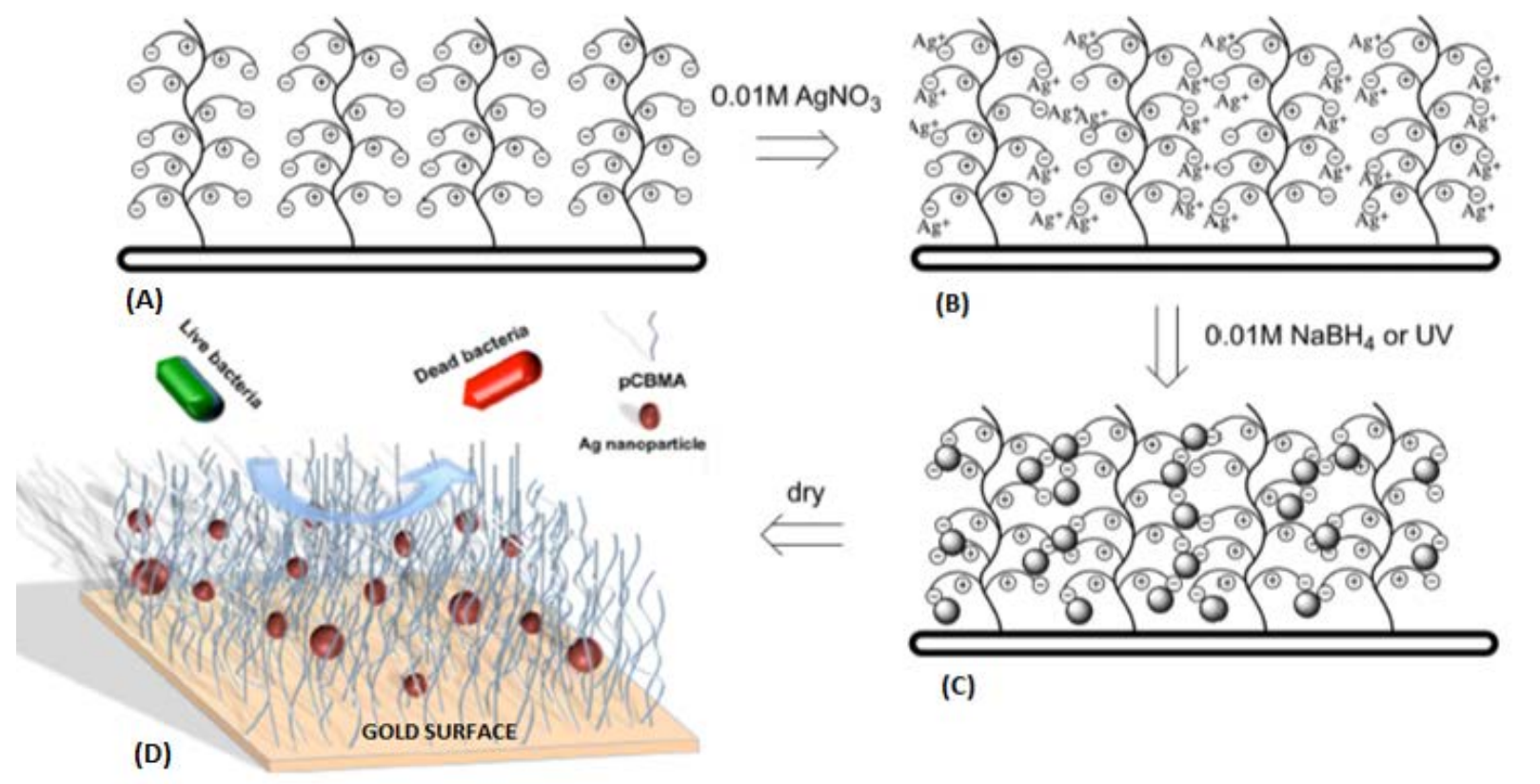

Figure 1.23: Example of a releasing and repelling $2^{\text {nd }}$ generation dual action antimicrobial surface. ${ }^{109}$ 

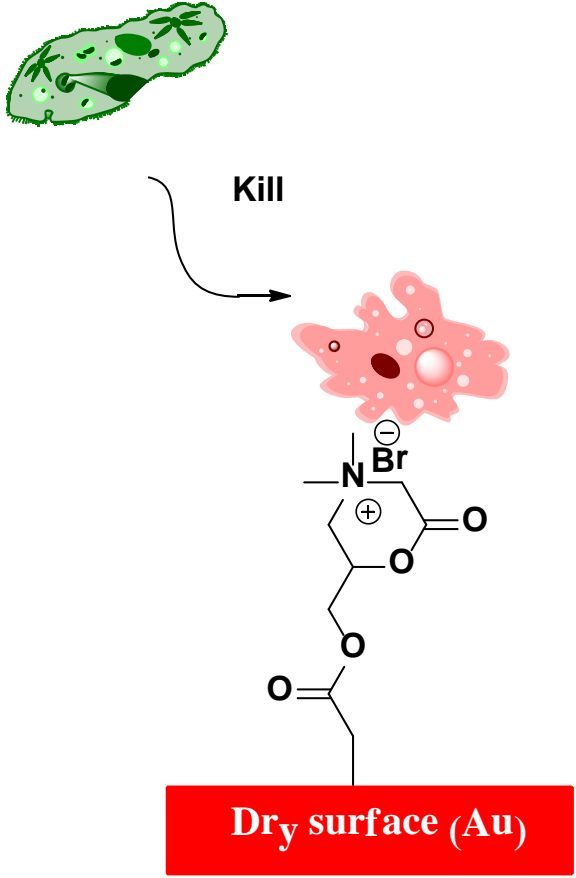
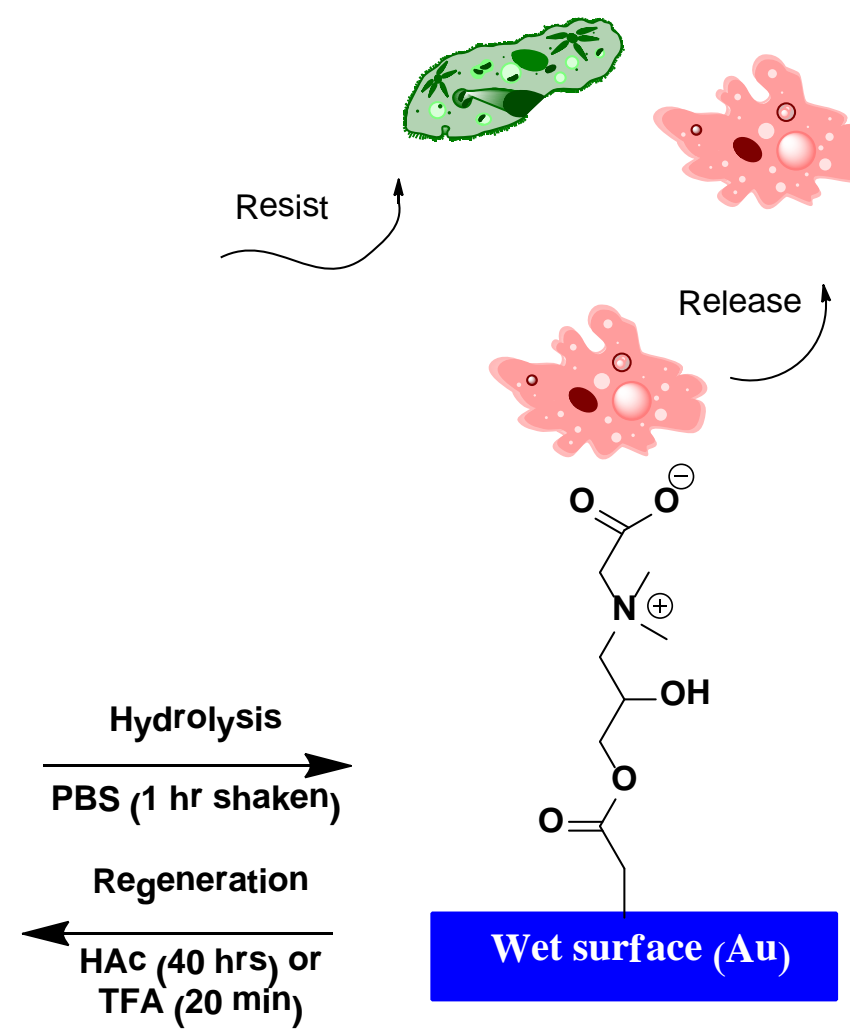

Figure 1.24: Example of a dual action and reversible contact killing / hydrophobic antimicrobial surface. ${ }^{110}$

\subsection{Kill Mechanisms of QAC}

The kill mechanism of QAC’s in solution or immobilized onto a surface is dependent on a host of factors that influence the compound's antimicrobial activity. Some of the factors include: (i) solution testing vs. surface testing, (ii) concentration/surface coverage, (iii) size (molecular weight), (iv) structural backbone, (v) type of counter ion, (vi) number of positive charges, and (vii) hydrophobicity of n-alkyl chains in the backbone of the QAC molecule or polymer. ${ }^{13,97,111,112}$ The bacterial cytoplasmic membrane is commonly targeted by different types of cationic QAC's that eventually result in cell death and is discussed below along with the 
structural factors influencing QAC's antimicrobial activity both in solution and on hard surfaces. $^{13}$

\subsubsection{Mechanism of Killing in Solution}

In solution, long chain monocationic QAC’s such as those employed commercially as disinfectants (Figure 1.25) destroy the bacterial cell wall and or cytoplasmic membranes by a two step process (Figure 1.26). ${ }^{113,114}$ First, the positively charged QAC’s are inititally attracted to the negatively charged bacterial cell after which the QAC's diffuse and firmly bind to the inner cytoplasmic membrane. QAC's form ion-pairs with the head groups of acidic-phospholipids namely phosphatidylethanolamine (70\% membrane composition) and in the process displace the $\mathrm{Mg}^{2+}$ and $\mathrm{Ca}^{2+}$ cations used to stabilize the lipid bilayer (Figure $1.26 \mathrm{~b}$ ). ${ }^{76}$ Membrane function is further disrupted by the hydrophobic portion of the molecule by denaturing structural membrane proteins and inserting itself into the cytoplasmic membrane (Figure $1.26 \mathrm{c}, \mathrm{d}$ ). With the QAC concentration approaching the minimum inhibitory concentration (MIC), the membrane begins to lose vital physiological functions such as osmoregularity and begins to leak $\mathrm{K}^{+}$ions and protons. ${ }^{76}$ Eventually, physiologically relevant membrane protein function is inhibited and the cell loses the ability to respire, transport solutes, and resynthesize the cell wall. ${ }^{113}$ At high concentrations, the disinfectants form micelluar aggregates that completely solubilize membrane phospholipids and proteins causing leakage of the intracellular material, cell lysis and eventually cell death (Figure 1.26 e, f). ${ }^{113,115}$ 


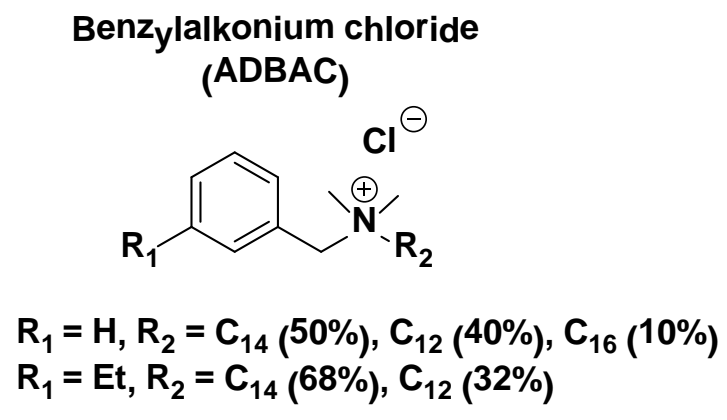

Dialkyldimethylammonium chloride

$$
\begin{aligned}
& \text { (DDAC) } \\
& \mathbf{R}_{\mathbf{1}}^{\prime}{\stackrel{\mathrm{N}}{\mathbf{C l}^{\prime}}}^{\ominus} \mathbf{R}_{\mathbf{2}} \\
& R_{1}=C_{8}, R_{2}=C_{10}(50 \%) \\
& \mathrm{R}_{1}=\mathrm{C}_{10}, \mathrm{R}_{\mathbf{2}}=\mathrm{C}_{10}(25 \%) \\
& R_{1}=C_{8}, R_{2}=C_{8}(25 \%)
\end{aligned}
$$

Figure 1.25: QAC disinfectants commercially employed. ${ }^{116}$

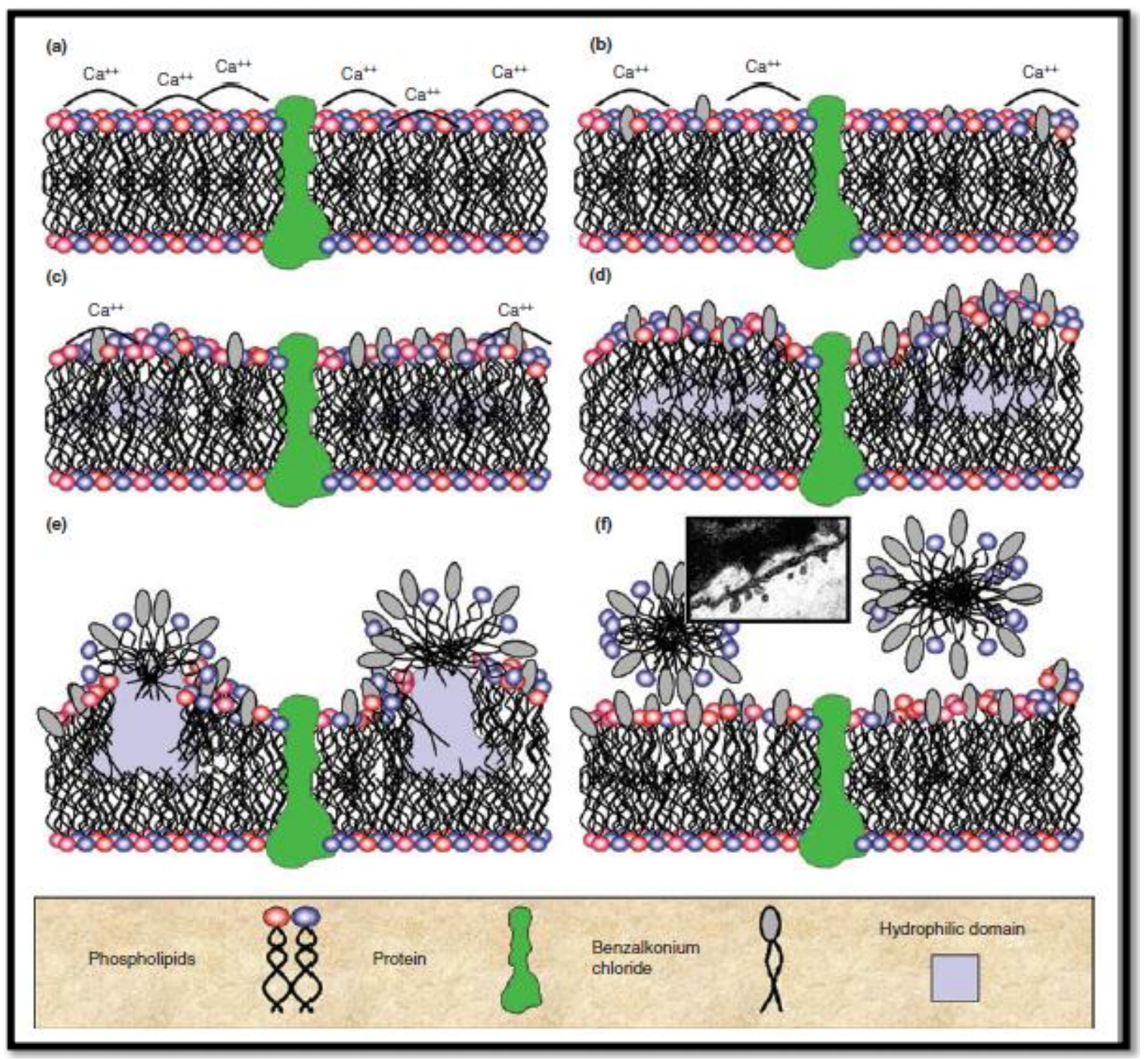

Figure 1.26: Bacterial membrane destruction by QAC disinfectants in solution. (Used with permission from Ref. ${ }^{113}$ ). 
For example, Iannou et al., found that the adherence of alkyldimethylbenzylammonium chloride (ADBAC, Figure 1.25) (a blend of $\mathrm{C}_{12}, \mathrm{C}_{14}$ and $\mathrm{C}_{16}$ alkyl homologues) and didecyldimethylammonium chloride (DDAC, Figure 1.25) to S. aureus cells occurred through slightly different mechanisms. ${ }^{117}$ ADBAC formed single monolayer coverage around the $S$. aureus outer membrane, while the DDAC formed a double layer. Both disinfectants eventually caused cell leakage and a total release of the intracellular $\mathrm{K}^{+}$and $260 \mathrm{~nm}$-absorbing proteins. Autolysis concentrations were similar regardless of the monolayers formed at $9 \mathrm{ug} / \mathrm{mL}$ (for both disinfectants), along with the depletion of approximately $30 \%$ of the internal $\mathrm{K}^{+}$pool. ${ }^{117}$ The authors attributed the lethality of ADBAC and DDAC to the autolysis of S. aureus, however, it was concluded that mechanical lysis also seemed to be involved because cell autolytic enzymes became inhibited at the disinfectant concentrations employed (Table 1.7). ${ }^{117}$

Table 1.7: MIC values for ADBAC and DDAC against increasing $S$. aureus concentrations after 48 hrs of incubation in TSA medium. ${ }^{117}$

\begin{tabular}{|c|c|c|c|c|c|}
\hline \multirow{2}{*}{ Biocide } & \multicolumn{5}{|c|}{ Biocide MIC $(\mu \mathrm{g} / \mathrm{mL})$ for inoculum test conc. (CFU/mL) } \\
\cline { 2 - 6 } & $10^{5}$ & $10^{6}$ & $10^{7}$ & $10^{8}$ & $10^{9}$ \\
\hline ADBAC & 0.6 & 0.6 & 0.7 & 1.0 & 1.8 \\
\hline DDAC & 0.4 & 0.4 & 0.4 & 0.6 & 1.6 \\
\hline
\end{tabular}

In another example, electron microscopy was used to study the interaction and effects of QAC's on the S. aureus membrane. The micrographs (Figure 1.27) clearly showed the different stages of insertion of the QAC into S. aureus strain ATCC 25923. On the right, the arrows indicate tiny holes showing nodule formation (ND), indicating the beginning of cellular material leaking out of the cell. Cell deformity and cell leakage (CL) was also evident. Interestingly, after performing elemental analysis on QAS treated and untreated cells, the authors were unable to 
detect the QAC chlorine counterion after it was taken up by the cells. It was theorized that the chlorine was pumped out of the cell on its own or together with the QAC from the cell prior to cell lysis. ${ }^{114}$ Another possibility could be that upon binding to the membrane, the chlorine counterion was diplaced from the QAC forming $\mathrm{MgCl}_{2}$ with structural cations within the microbial membrane.

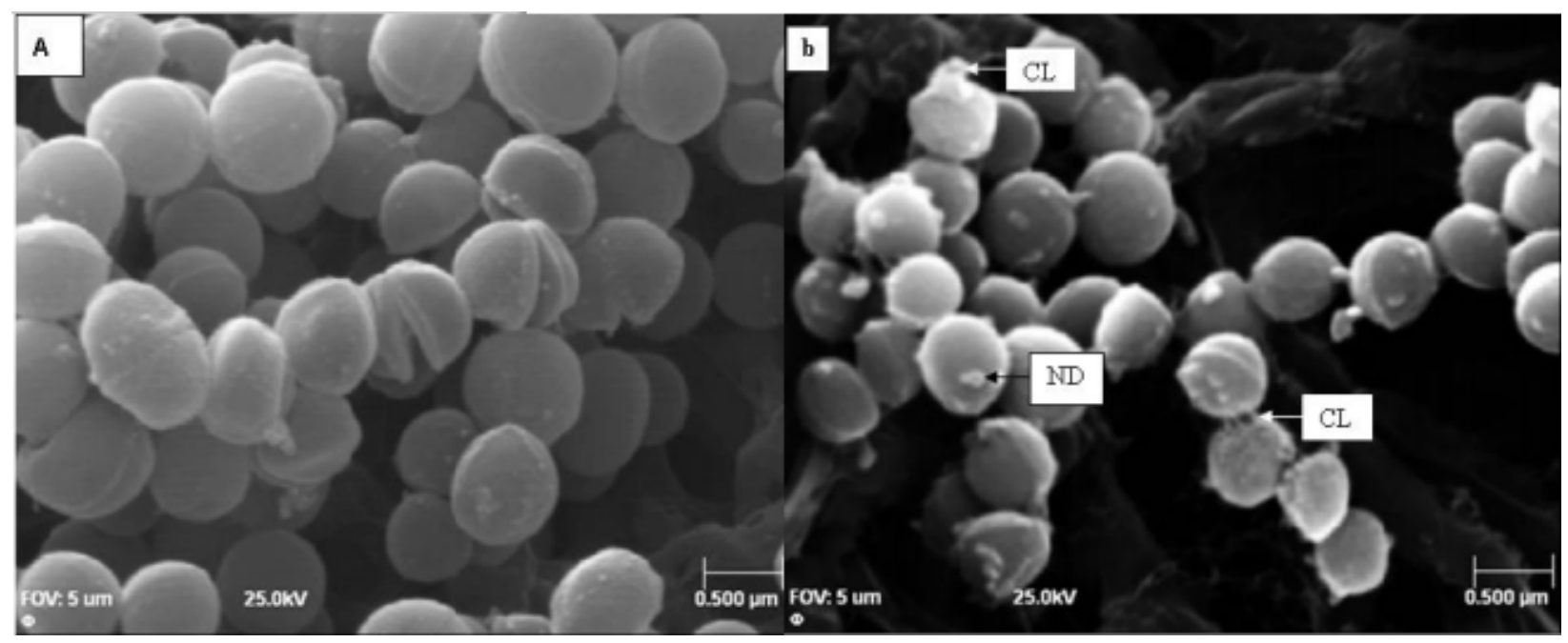

Figure 1.27: Scanning electron micrographs of S. aureus strain ATCC 25923 indicating the effect didecyldimethylammonium chloride (DDAC) had on cell morphology, (a) control $S$. aureus cells containing no DDAC displayed normal spherical shapes, (b) cells treated with 20 g/L DDAC for $10 \mathrm{~min}$. The arrows indicated where cell leakage (CL) and nodule formation (ND) was observed when the $S$. aureus cells were incubated with DDAC. (Used with permission from Ref $\left.{ }^{114}\right)$.

The antimicrobial activity of the disinfectants discussed thus far are dependent on the length of the hydrophobic chain which follows a parabolic trend of MIC values (Figure 1.28). This phenomenon is called the "cut-off effect" where QAC's with $\mathrm{C}_{12}-\mathrm{C}_{14}$ alkyl chain lengths show the highest activity against gram-positive bacteria and yeast, while those with longer alkyl chains $\left(\mathrm{C}_{14}-\mathrm{C}_{16}\right)$ show the highest activity against gram-negative bacteria. Compounds with short alkyl chains $(\mathrm{n}<4)$ or very long alkyl chains $(\mathrm{n}>18)$ showed the lowest activity. ${ }^{113}$ QAC's 
with the highest antimicrobial activity and lowest MIC values are observed when the total alkyl chain length is ( $\mathrm{n}>10)$. In solution, QAC's with the optimum alkyl chain length ( $\mathrm{n}>10)$, exist as dimers because the attractive forces of neighbouring hydrophobic tails overcome the repulsive positive charges of neighbouring cations and thus show the highest kill activity. Dimeric-QAC's interact and solubilize bacterial membranes more freely in comparison to the monomeric QAC's. As a result the most effective QAC disinfectants are sold as a mixture of different chain lengths in order to ensure broad spectrum activity against different types of bacteria. ${ }^{116}$
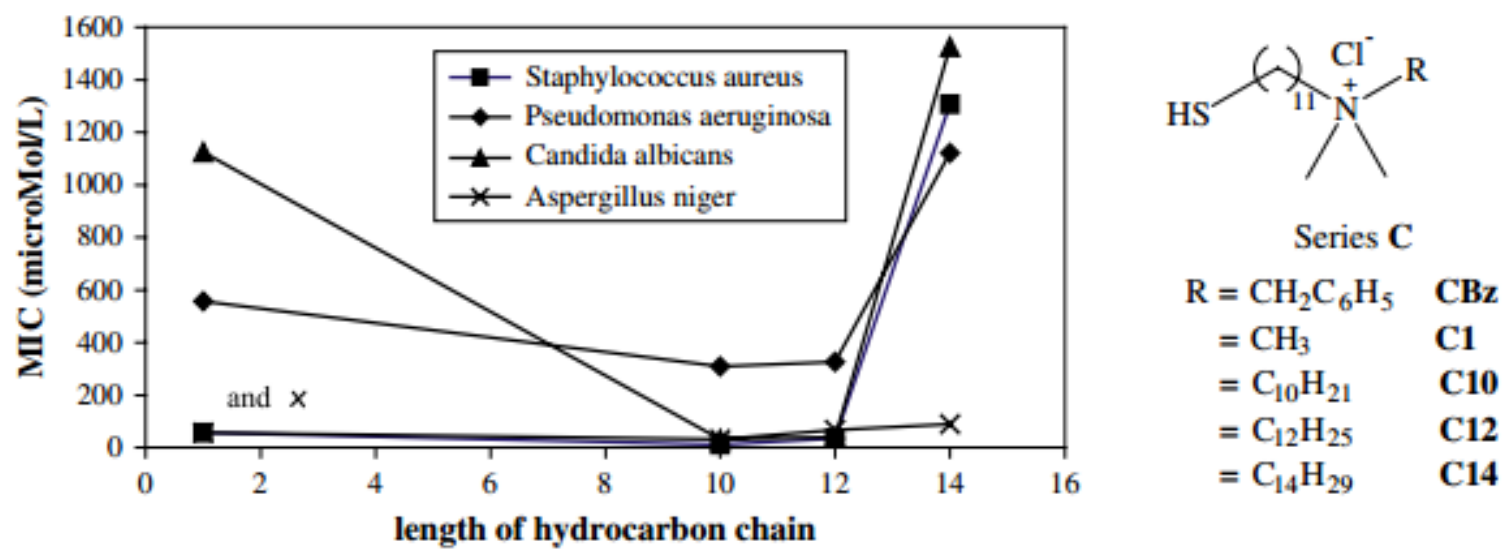

Figure 1.28: The "Cut off Effect:" A hyperbolic trend observed in MIC values of surfactants as a function of the length of the hydrocarbon chain. (Used with permission from Ref. ${ }^{118}$ ).

When QAC disinfectants are used at concentrations above bacterial MIC's, developing resistance is unlikely, however, when bacteria are exposed to sub-MIC concentrations various resistant genes are acquired. ${ }^{119,120}$ In fact, gram-negative bacteria such as $E$. coli ${ }^{121}$ and $P$. aeruginosa $^{122}$ have acquired QAC efflux pumps capable of removing quats out of the cell. P.aeroguinosa also contain enzymes capable of metabolizing QAC's as a source of carbon and nitrogen, therefore, the MIC’s are much higher (upto $10 \times$ ) for this bacterium (Table 1.8). Further adaptive resistance to QAC's exists in bacteria capable of forming a biofilm, where the biofilm secreted lipopolysacharide layer can protect the bacteria from coming into contact with the 
biocide. Single strain bacterial biofilms resistant to QAC's studied include those of P.aeroguinosa and Salmonella Enterica Serovar Enteritidis ATCC $4931 .^{123}$ Lastly, grampositive bacterial membranes are less resistant to QAC's due to the loosely packed peptidoglycan outer layer allowing easier access to their inner cytoplasmic membrane and typically show lower MIC values (Table 1.8, S. aureus). Meanwhile, gram-negative bacterial membranes are inherently more resistant because a QAC must first cross the outer membrane with a bilayer phospholipid structure before it can gain access to the inner cytoplasmic membrane.

Table 1.8: MIC values of Commercial Disinfectant BAK 50 versus thiol QAC's. ${ }^{118}$

\begin{tabular}{|c|c|c|c|c|}
\hline Compound & \multicolumn{4}{|c|}{ MIC $(\mu \mathrm{mol} / \mathrm{L})$} \\
\hline $\begin{array}{c}\mathbf{C l}^{\ominus} \\
\mathrm{CS}_{\mathbf{1 0}}^{\oplus} \mathbf{N}^{\mathbf{R}}\end{array}$ & S.aureus & P.aeruginosa & C.albicans & A.niger \\
\hline BAK 50 & 3.2 & 93 & 8.36 & 4.74 \\
\hline $\mathrm{R}=\mathrm{CH}_{2} \mathrm{C}_{6} \mathrm{H}_{5}$ & 56.6 & 556.9 & 1124.8 & 56.6 \\
\hline $\mathrm{R}=\mathrm{CH}_{3}$ & 681.6 & 1055.9 & 471.6 & 351.0 \\
\hline $\mathrm{R}=\mathrm{C}_{10} \mathrm{H}_{21}$ & 11.6 & 308.9 & 31.6 & 31.6 \\
\hline $\mathrm{R}=\mathrm{C}_{12} \mathrm{H}_{25}$ & 34.7 & 326.2 & 39.5 & 67.2 \\
\hline $\mathrm{R}=\mathrm{C}_{14} \mathrm{H}_{29}$ & 1306.3 & 1121.5 & 1524.6 & 90.0 \\
\hline
\end{tabular}

When dealing with polymeric QAC's in solution, factors such as polymer size, type of counter ion, the length of the hydrophobic backbone and location of the positive charge along the backbone all have an affect on the antimicrobial activity. Typically, polymeric biocides are designed with the QAC's spread throughout the backbone as repeat units and such polymer 
systems show a dependence on molecular weight where a $1.4-9.4 \times 10^{4}$ Da range shows maximum activity. Polymers exceeding the cut-off range are inactive because they are too large to adhere to or enter the bacterial cytoplasm. ${ }^{97}$

QAC-polymers with loosely bound counter anions foster increased antimicrobial activity because the weaker ion pair readily dissociates from the quat, which leads to ion-pair formation with the negatively charged bacterial membrane. On the other hand, strong ion-pair counterion associations with the polymer show decreased antimicrobial activity because the tightly held counter ion will prevent the polymeric quats from associating with the bacteria. ${ }^{124}$

The spacer length or alkyl chain length refers to the length of the carbon chain that composes the polymer backbone. The length of this chain has been investigated to see if it affects the antimicrobial activity of the polymer. Results have generally shown that longer alkyl chains result in higher antimicrobial activity. There are two primary explanations for this effect. Firstly, longer chains have more active sites available for adsorption with the bacteria cell wall and cytoplasmic membrane. Secondly, longer chains aggregate differently than shorter chains, which again may provide a better means for adsorption. ${ }^{124}$

Lastly, with polymeric QAC's, changing the hydrophobic portions can have a drastic effect on the efficacy of the antimicrobial in solution. Tiller's group synthesized a $C_{12}$ mono quaternary oxazoline polymer with a terminal hydrophobic end group with either 10 or 16 hydrocarbons in length. ${ }^{125}$ Only the hexadecyl polymer demonstrated antimicrobial activity, while the shorter decyl chain prevented the polymer from refolding into the right orientation necessary to lyse the bacterial membrane (Figure 1.29). ${ }^{125}$ 


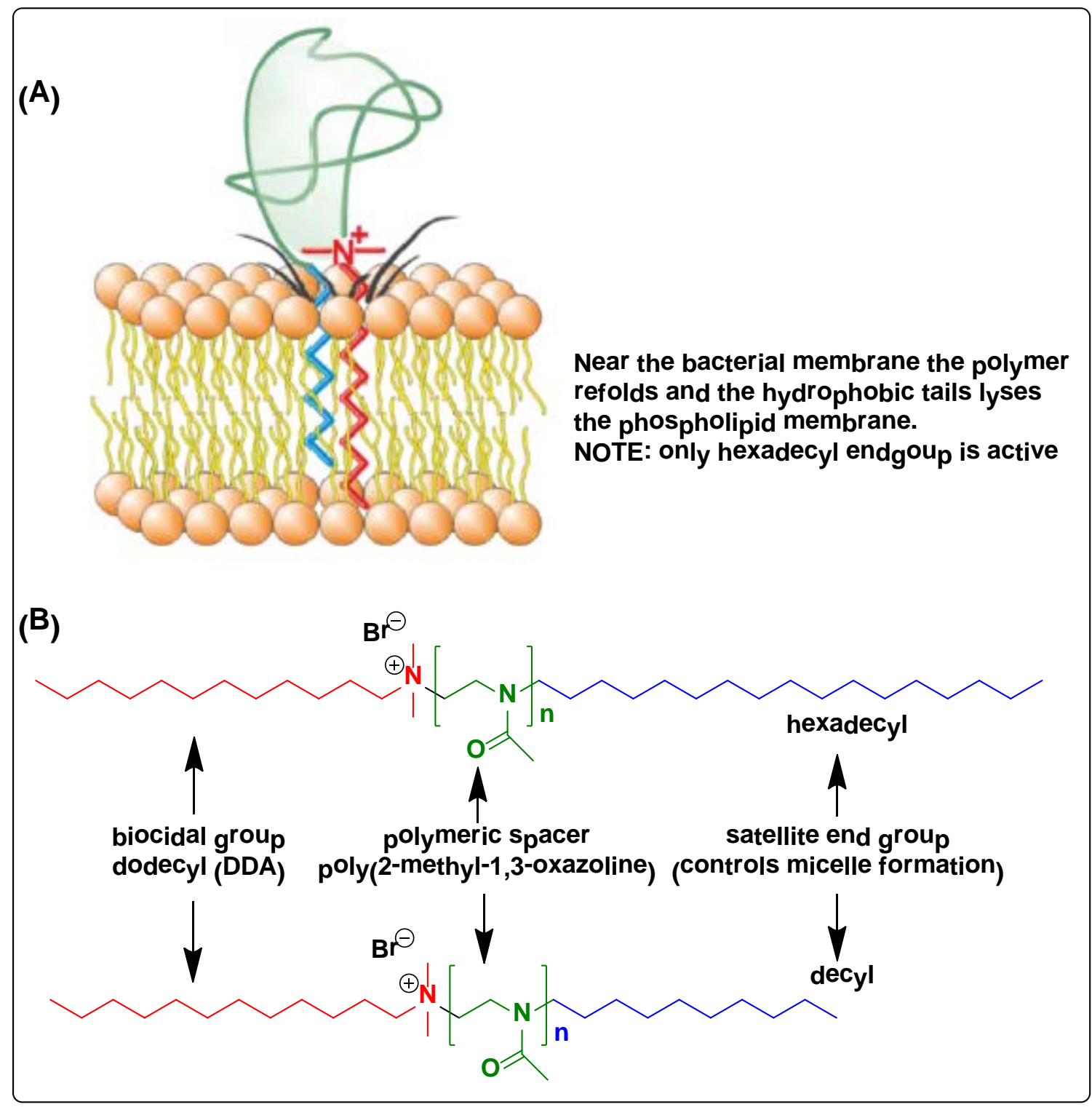

Figure 1.29: Effect of chain length on antimicrobial activity in solution (Adapted from Ref. ${ }^{125}$ ).

\subsection{Mechanism of Immobilized Contact Active QAC}

Some authors suggest that surfaces immobilized with QAC compounds kill microbes on contact by disruption of the microbial membranes based on observations with structurally different polycationic polymers. Two contact killing hypotheses were proposed and are based on 
disruption of the microbial membranes either by (i) physical means, e.g. membrane penetration/disruption or (ii) electrostatic/ionic interactions with oppositely charged bacterial phospholipids and the polycationic molecules/polymers. Both mechanisms are described below.

\subsubsection{Polymeric Spacer Effect}

The first contact killing hypothesis was proposed in 2001 by Klibanov et al., after showing that long chain cationic polymers of poly(4-vinyl- $N$-hexyl)pyridinium bromide “grafted-from” or $N$-alkyl PEI adsorbed onto glass slides were highly antimicrobial (Figure 1.30). ${ }^{126-128}$ Glass surfaces modified with large cationic polymers ranging from $25 \mathrm{kD}$ to $750 \mathrm{kD}$ resulted in instant killing, meanwhile the shorter chain polymers (2 kD) were inactive towards airborne S. aureus cells sprayed onto these surfaces (Section 1.6.1.2.3 (a)). Similarly, glass slides immobilized with these antimicrobial polymers were capable of reducing both gram-positive and gram-negative bacteria by a factor of $10^{9}$ cells after immersing these surfaces into bacterial solutions according to live/dead staining. Since bacterial cell walls typically range from 16 to 80 $\mathrm{nm}$, the surface grafted cationic polymer coatings needed to be long and flexible enough to be able to penetrate the cell wall and disrupt the negatively charged inner bacterial membranes, e.g. a polymeric spacer of $50 \mathrm{~nm}$ would be required to destroy the E. coli cytoplasmic membrane which are $~ 46 \mathrm{~nm}$ thick (Figure 1.31). ${ }^{126}$ 


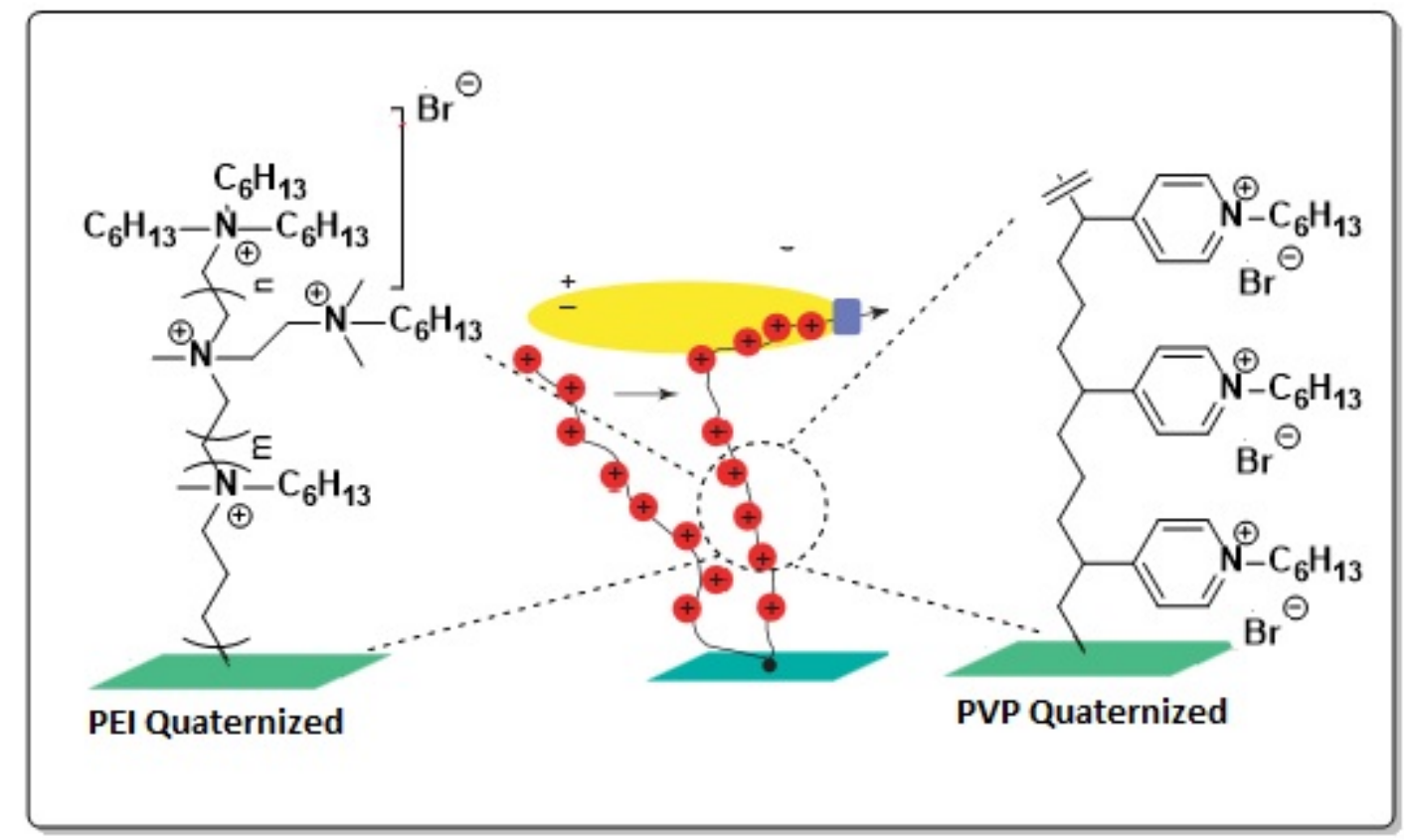

Figure 1.30: Example of the polymeric spacer effect with long chain QAC. PEI: polyethyleneimine, PVP: polyvinylpyridine. (Adapted from Ref. ${ }^{129}$ ).

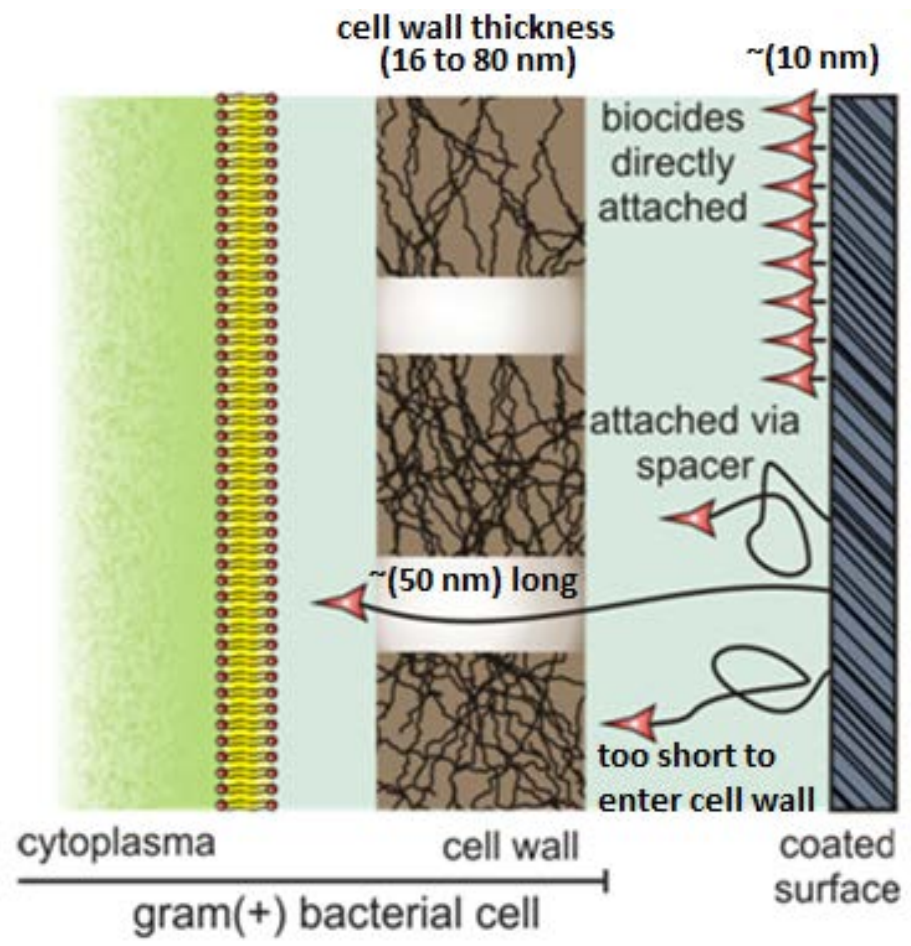

Figure 1.31: Contact-killing via the polymeric spacer mechanism. (Used with permission from Ref. $^{12,13}$ ). 


\subsubsection{Phospholipid Sponge Effect}

A second membrane disrupting, contact-killing mechanism was proposed after observations that the short chain Si-QAC 3 and other shorter polymer brushes grown from surfaces (less than $10 \mathrm{~nm}$ ) were also highly antimicrobial. Instead of being long enough to penetrate the cell wall, these surface immobilized shorter chain polycationic antimicrobials were hypothesized to disrupt membranes via ion-exchange between the polycationic biocides and structurally important mobile cations $\left(\mathrm{Ca}^{2+}\right.$ and $\left.\mathrm{Mg}^{2+}\right)$ within the bacterial cytoplasmic membrane (Figure 1.32). ${ }^{130}$ Based on observations with short chain polycationic polymers 'grafted onto' glass slides via silane linkers, surfaces with a charge density of QA cations $\left(10^{12}\right.$ $10^{16} \mathrm{~N}^{+}$positive charges per $\mathrm{cm}^{2}$ ) were required for killing after 10 min contact time depending on the type of bacterium. Approximately $\sim 1 \times 10^{10}$ QA or 0.015 pmol $\mathrm{N}^{+}$was calculated to be necessary to kill one bacterium, thus a surface with $3.2 \times 10^{14} \mathrm{~N}^{+} / \mathrm{cm}^{2}$ would roughly kill $\sim 0.3 \times$ $10^{5}$ bacteria $/ \mathrm{cm}^{2}$. In all cases, surfaces grafted with more charges $/ \mathrm{cm}^{2}$ of short chain QAC's caused faster release of counterions and resulted in higher and faster killing. ${ }^{42,131}$

In addition, Tiller proposed that highly charged surfaces without polymeric spacers need a certain degree of hydrophobicity around the quat in order to effectively kill microbes on contact. Based on observations with quaternized $N$-butyl or $N$-dodecyl- $N, N$ dimethyldeoxyammonium cellulose polymer surfaces prepared from tosyl cellulose, polymers with only 50\% of the tosyl groups (hydrophobic residues) substituted with the non-biocidal short chain ( $N$-butyl-quat) were found to be biocidal whereas polymers with the same amount of $\mathrm{N}^{+}$ charges but with a low degree of substitution of the biocidal longer chain ( $N$-dodecyl-quat) were 
not biocidal. However, tosyl celluloses fully quaternized with the $\mathrm{C}_{12}$ tail were highly biocidal while the same polymers substituted with the $\mathrm{C}_{4}$ chain had no antimicrobial activity. ${ }^{132,133}$

To further prove that a contact active mechanism was involved, the $\mathrm{C}_{12}$ fully substituted cellulose coatings were deactivated with sodium dodecyl sulfate (SDS, 1g/L, $1 \mathrm{~min}$ ) and an oppositely charged phospholipid (liposomes with 10\% phosphatidylglycerol). As expected, antimicrobial activity against $S$. aureus was lost as these anionic molecules completely blocked the cationic sites on the antimicrobial surface, preventing the removal of bacterial phospholipids. In contrast, the same coating but with a polyethyloxazoline spacer ( 100 polymeric units) between the $\mathrm{C}_{12}$ quat and the cellulose backbone was unaffected by the same oppositely charged deactivating treatment and killed all $S$. aureus cell in support of the polymeric spacer mechanism. The authors proposed that in the phospholipid sponge mechanism, the $\mathrm{H}_{2} \mathrm{O}$ insoluble bacterial phospholipids are removed from the bacterial membrane and transverse through small holes in the bacterial cell-wall as micelles in order to reach the oppositely charged antimicrobial coating (Figure 1.32). A hydrophobic portion surrounding the positive charge may be required to further stabilize the newly formed ion-pair, utilizing van der Waals interactions with the hydrophobic tail of the removed lipids. 


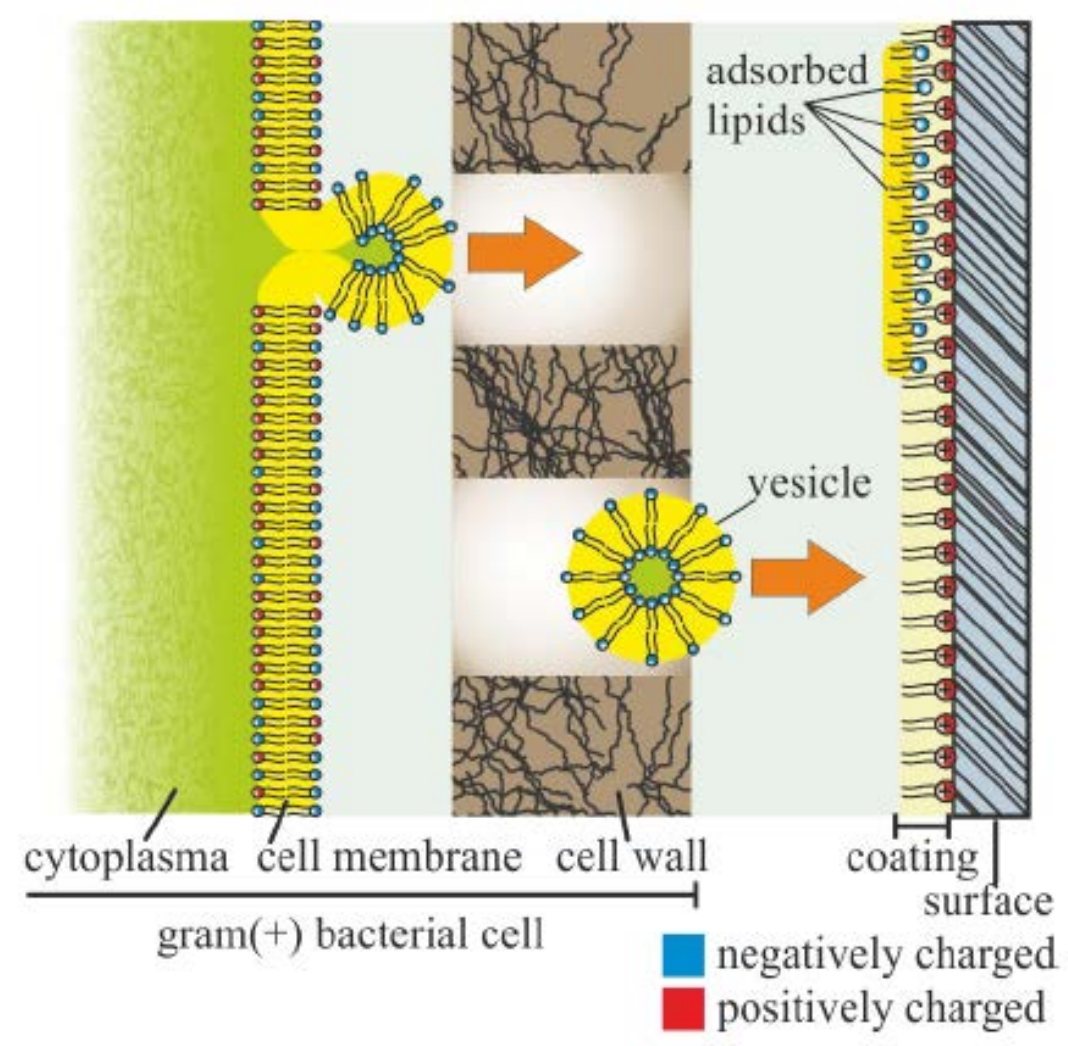

Figure 1.32: Contact-killing via the phospholipid sponge mechanism. (Used with permission from Ref. ${ }^{13,133}$ ).

\subsection{Antimicrobial Testing}

A variety of methods are available for testing non-leaching (immobilized) antimicrobial products or surfaces based on either (i) growth enumeration of recovered bacterial colonies or (ii) cell viability enumeration after coming into contact with the active surface. With growth based methods, each method was tailored to a specific type of surface (textiles, metals, plastics) and differ in the way the bacterial inoculum is applied. Some methods require immersion of a test substrate with innoculum, while others apply the innoclum directly via (i) aerosol spraying and/ or (ii) placing a drop of inoculum and allowing it to dry on the surface prior to enumeration. Cell viability enumeration is typically based on fluorescence staining combined with real time 
microscopy or assayed with various molecular biology techniques. Both growth and cell viability enumeration methods are discussed below along with their drawbacks and limitations.

\subsubsection{Growth Based Enumeration}

\subsubsection{Immersion Inoculation}

\subsection{ASTM E2149-10}

Originally developed by Isquith et al., the Dow Corning Corporate Test Method 0923 $(\mathrm{CTM}-0923)^{35}$ became known as the ASTM E2149-01, ${ }^{18}$ the standard test method for determining the antimicrobial activity of immobilized antimicrobial agents under dynamic contact and was later superseded by ASTM E2149-10. ${ }^{134}$ Active surfaces are tested by immersion with inoculum following shaking and removal of samples at different times. Cells are serially diluted and colonies are enumerated on agar plates (Figure 1.33).

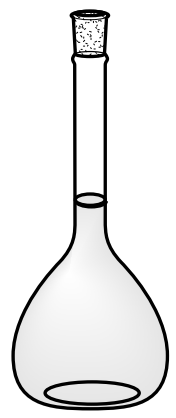

i
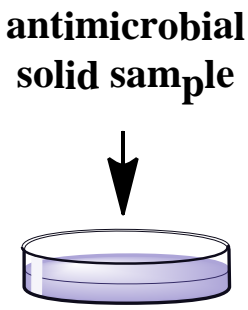

ii

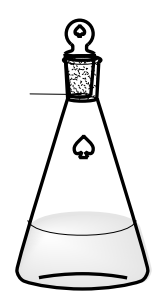

iii
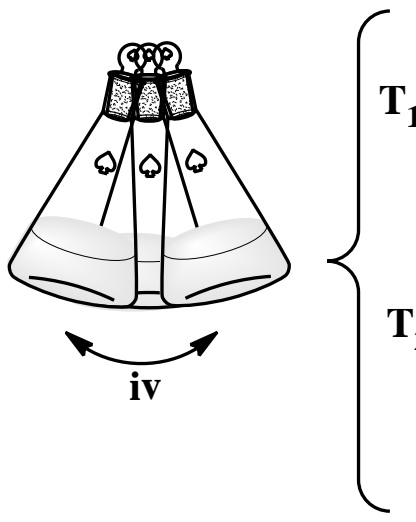

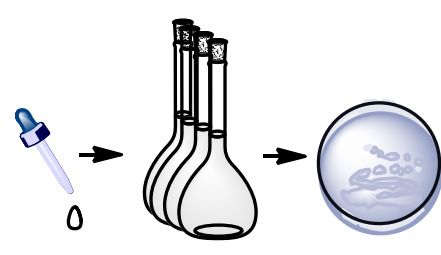

$\mathbf{T}_{2}$

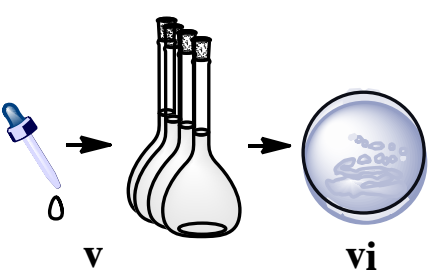

Figure 1.33: ASTM E2149-10: (i) 1.5-3.0 × 105 CFU/mL (inoculum), (ii) treated surface cut into smaller pieces, (iii) treated surface mixed with $50 \mathrm{~mL}$ inoculum, (iv) place on mechanical shaker, (v) periodically remove $1.0 \mathrm{~mL}$ samples $\left(\mathrm{T}_{1}-\mathrm{T}_{4}\right)$, (vi) dilute serially + growth on agar, (vii) compare to controls and calculate percent or log 10 reduction of viable cells. ${ }^{18}$

Even though the ASTM E2149 method has become the industrial benchmark for testing non-leaching (immobilized) antimicrobial products or surfaces, it has been debated whether it 
could clearly distinguish between a contact killing surface and a biocide releasing one. ${ }^{63}$ The growth based enumeration test method assumes that an overall reduction of living cells (CFU's, colony forming units) is due to contact killing without taking into account that some of the killing could be due to slow leaching of the immobilized antimicrobial over time. ${ }^{63}$ Since the minimum inhibitory concentration (MIC) and minimum lethal concentrations (MLC) of Si-QAC in solution are very low, it is possible to observe bacterial kill from surface leaching of the active over time. For example, White et al., reported a very low MIC for gram-positive bacteria (MIC = $10 \mathrm{ug} / \mathrm{mL}$, S. aureus) and a much higher concentration for gram-negative bacteria (MIC = 100 $\mu \mathrm{g} / \mathrm{mL}$, E. coli $)$ and $(\mathrm{MIC}=1000 \mu \mathrm{g} / \mathrm{mL}$, A. niger $) .{ }^{27}$ Le Song also reported a similar result (MLC $=84 \mu \mathrm{g} / \mathrm{mL}$, E. coli). ${ }^{24}$ However, in saline solution, the activity of compound 3 was negligible (see Table 1.9). ${ }^{24}$

Table 1.9: Antibacterial activity of compound 3 in solution. ${ }^{24}$

\begin{tabular}{|c|c|c|}
\hline Test medium & Compound & MLC $(\mu \mathrm{g} / \mathrm{mL})$ \\
\hline Deionized $\mathrm{H}_{2} \mathrm{O}$ & $\mathbf{3}$ & $<84$ \\
\hline Artificial sea $\mathrm{H}_{2} \mathrm{O}$ & $\mathbf{3}$ & 8000 \\
\hline
\end{tabular}

\subsubsection{Direct Inoculation}

\subsection{Zone of Inhibition}

In the agar diffusion test, or the Kirby-Bauer disk-diffusion method, the zone of inhibition is often used to determine if an antimicrobial surface releases biocides or if it kills on contact. ${ }^{13}$ An agar plate with a visible zone of dead bacteria around the antimicrobial surface is attributed to the release of the active biocide that exceeds the MIC, meanwhile an uncolonized surface with no such zone is typically considered to kill on contact (Figure 1.34). The size of the 
zone depends on factors such as the diffusion kinetics through the nutrient media and the original concentration of the active chemical present. With this test, however, it is difficult to prove if the immobilized surface is truly contact killing solely by the absence of a zone of inhibition. For example, monolayers of immobilized biocides typically contain a very small amount of the active molecule and thus the absence of a zone of inhibition could be mistaken for a nonreleasing surface when in fact the concentration of the antimicrobial agent is above the MIC. ${ }^{13}$ On the other hand, interferences such as charged proteins and other growth media nutrients that can deactivate cationic immobilized antimicrobials on the test surface would raise the MIC and result in a smaller or no zone in an eluting surface. ${ }^{13}$

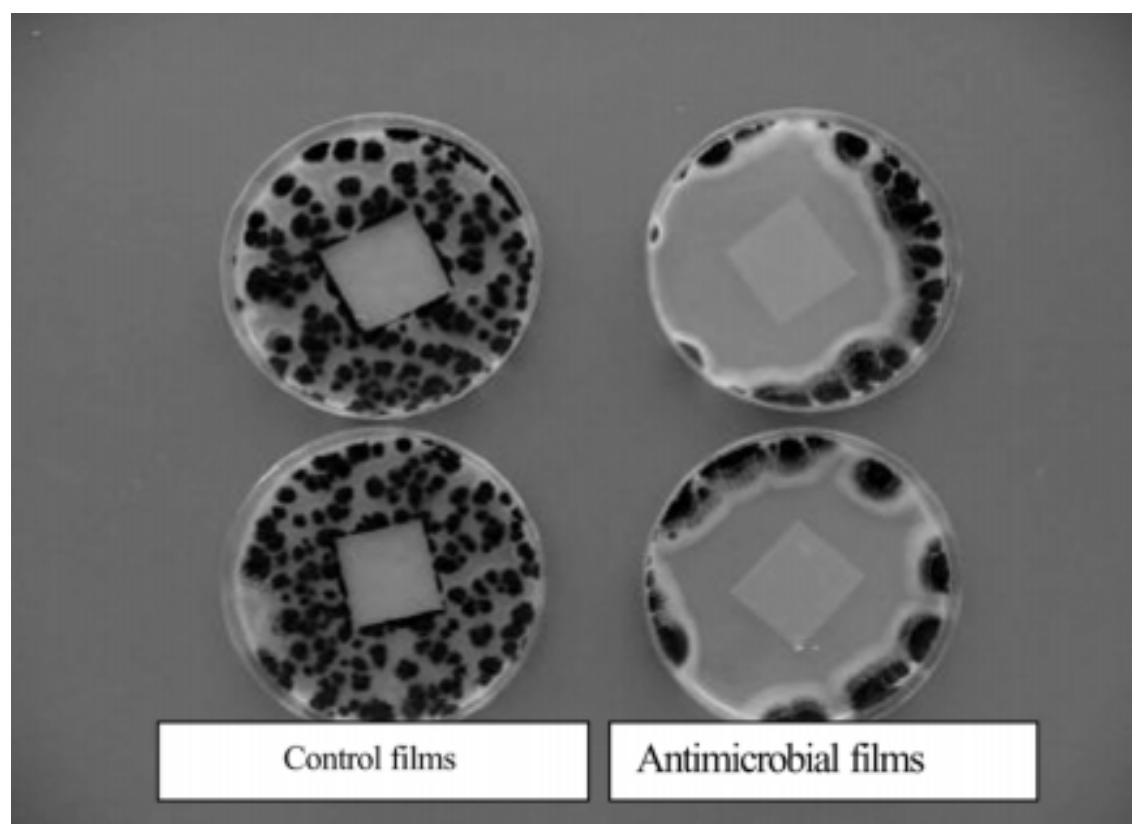

Figure 1.34: ZOI Agar diffusion method: Antimicrobial releasing plastic film surface on $A$. niger. (Used with permission from Ref. ${ }^{95}$ ) 


\subsection{Textiles}

The AATCC 100-2004 test method or the "padding test" is very similar to the Dow suspension test except only textile samples are tested and no shaking takes place. Circular texile samples are inoculated, incubated and colonies are counted after serial dilutions (Figure 1.35). ${ }^{18}$

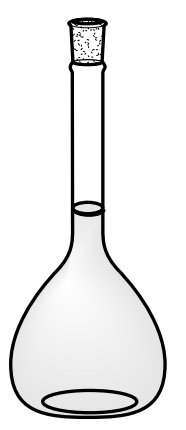

i

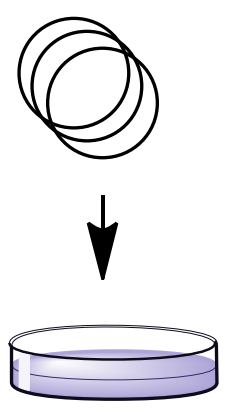

ii

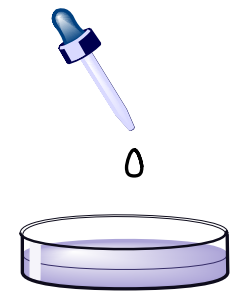

iii

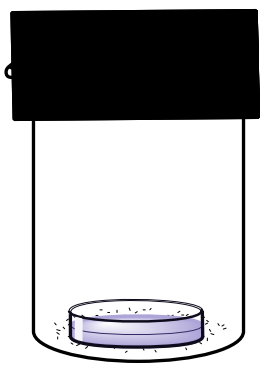

iv
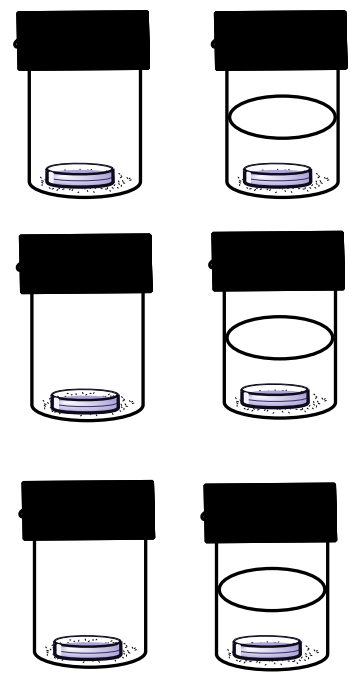

$\mathbf{v}$

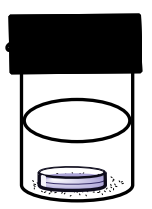

vi

Figure 1.35: Standard methods for testing antimicrobials on textiles. (i) $1-2 \times 10^{5} \mathrm{CFU} / \mathrm{mL}$ (inoculum), (ii) place textile in petri dish, (iii) $1 \mathrm{~mL}$ inncolum is added (iv) transfer to jar to prevent evaporation, (v) incubate, (vi) add neutralizer solution, (vii) serial dilutions, incubate and agar plating, (viii) compare to controls and calculate percent of log 10 reduction. $^{18}$

\subsection{Hard Surfaces: Metals, Plastics, Glass}

\section{(a) Aerosol Inoculation}

This method, designed to mimic airborne deposition of microorganisms on surfaces, utilizes a readily available TLC sprayer to deposit a bacterial inoculum as a mist on hard surfaces followed by air drying, agar incubation and viable colony counts (Figure 1.36). Simple in design, the drawback of this method is reproducibility in the delivery of the inoculum which makes it difficult to compare to non-aerosol methods. ${ }^{18}$ Also the aerosol method does not distinguish 
between contact killing or release killing from the surface. ${ }^{63}$ A literature example described by Klibanov shows the results of the aerosol method of a glass slide with covalently attached cationic antimicrobial PVP polymer (Figure 1.37). ${ }^{127}$

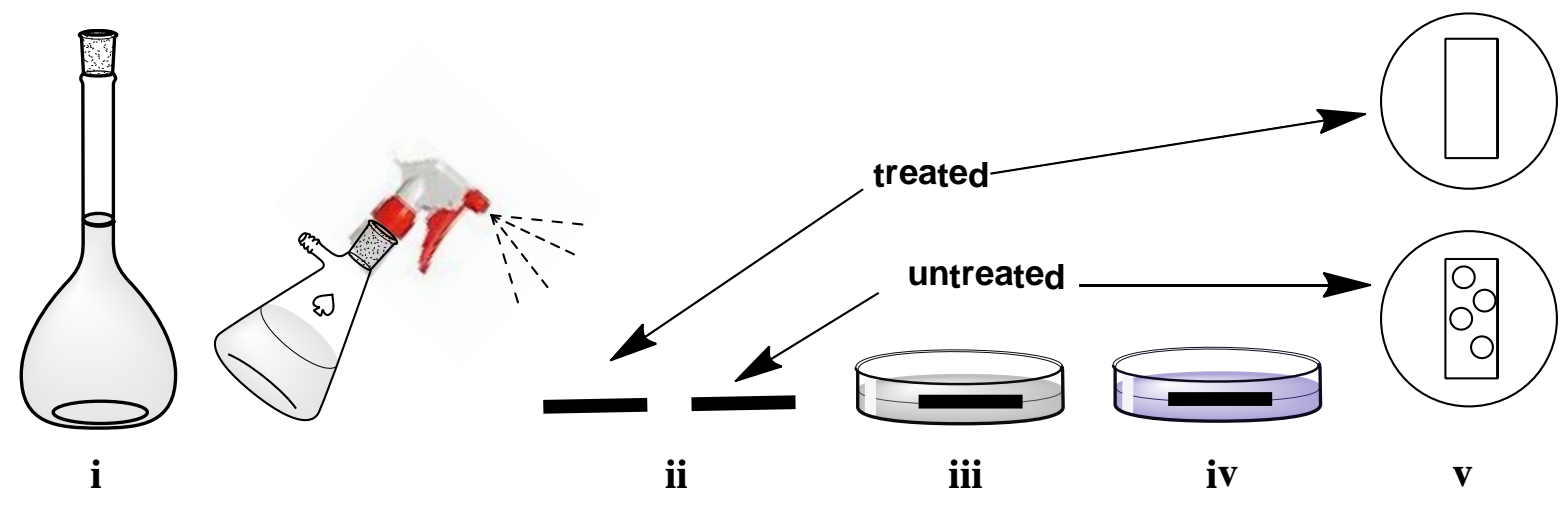

Figure 1.36: Diagram of the aerosol inoculation method: (i) 5-50 × $10^{6} \mathrm{CFU} / \mathrm{mL}$ (inoculum), (ii) inoculum sprayed onto treated and control surfaces and allowed to air dry for 2 min, (iii) cover with agar and incubate $37^{\circ} \mathrm{C}$ ON, (iv) compare to controls and calculate percent or log 10 reduction. ${ }^{18}$ 

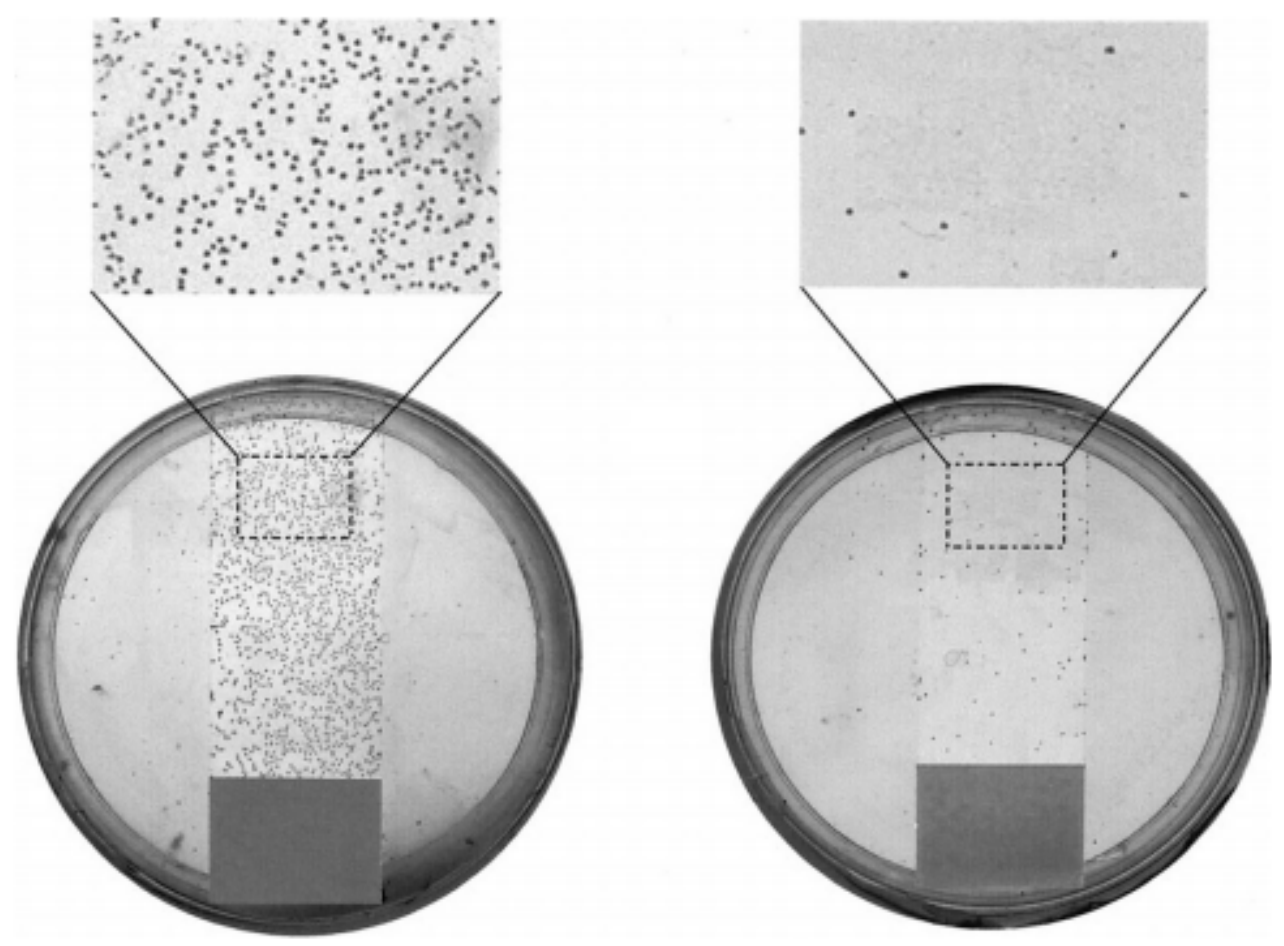

Figure 1.37: Results from the aerosol inoculum method with an immobilized QAS polymer onto a glass slide. Left (control), right (polymer modified slide). (Used with permission from Ref. ${ }^{127}$ )

\section{(b) Industrial Standard Test Methods}

A variety of standard test methods designed to quantitatively test microbial inhibition via direct innoculation on solid surfaces include the (i) JIS Z 2801, the Japanese standard, developed in 2006 and superseded by (ii) ISO 22196, the international standard method. Other similar standard methods for hard surfaces include (iii) EN ISO 846, plastics-evaluation of the action of microorganisms, (iv) ASTM G21-90, standard practice for determining resistance of synthetic polymeric materials to fungi, and (v) ASTM G22-76, standard practice for determining resistance of synthetic polymeric materials to bacteria. ${ }^{18,95}$

As with the immersion inoculation method, the test microorganism is cultured, usually by growth in a liquid culture medium. The suspension of test microorganism is standardized by 
dilution in a nutritive broth (this affords microorganisms the potential to grow during the test). ${ }^{95}$ Control and test surfaces are inoculated with microorganisms in triplicate, and then the microbial inoculum is covered with a thin, sterile film. Covering the inoculum spreads it, prevents it from evaporating, and ensures close contact with the antimicrobial surface. Microbial concentrations are determined at "time zero" by elution followed by dilution and plating. A control is run to verify that the neutralization/elution method effectively neutralizes the antimicrobial agent in the antimicrobial surface being tested. ${ }^{95}$ Inoculated covered control and antimicrobial test surfaces are allowed to incubate undisturbed in a humid environment for 24 hrs. ${ }^{95}$ After incubation, microbial concentrations on surfaces are determined. The reduction of microorganisms relative to initial concentrations and the control surface is then calculated (Figure 1.38).

The drawback of the ISO 22196 method is that it is not representative of the actual antimicrobial surface contamination scenario. Typically upon landing, microbial contaminants dry very quickly on surfaces, whereas with this method, the use of a cover slip keeps the contact between bacteria and the surface in a wet state typically overnight. In reality, bacteria rarely spend that long of an interaction with the surface in the wet state. ${ }^{135}$ As well, cells adhered to the cover slip are not typically taken into account, inflating log reduction values (LRV's). ${ }^{136}$ This method has recently been modified by the Wolfaardt lab, by forgoing the glass cover slip and allowing the inoculum to dry on the surface prior to analysis and by the Green lab, by using a polypropylene spacer in between the substrate surface and the coverslip in order to distinguish release killing vs. contact killing (discussed below). ${ }^{63}$ 


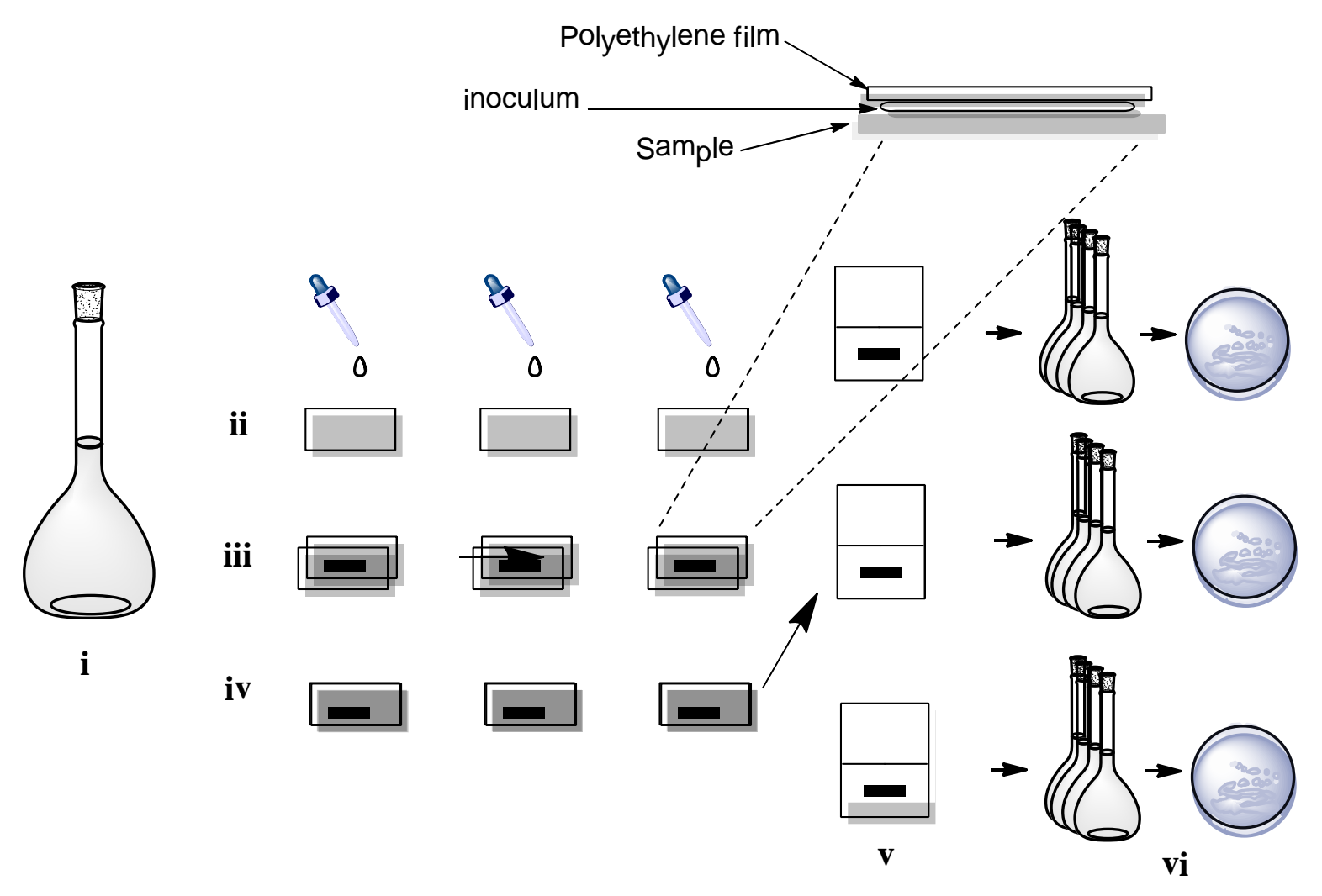

Figure 1.38: Industrial standard method for testing antimicrobials on hard surfaces by growth enumeration, (i) $1 \times 10^{6} \mathrm{CFU} / \mathrm{mL}$ (inoculum), (ii) $400 \mu \mathrm{L}$ inoculum added to each $50 \mathrm{~mm} \times 50$ mm sample, (iii) cover incoluum with $40 \mathrm{~mm} \times 40 \mathrm{~mm}$ sterile polypropylene coverslip, (iv) incubate for $24 \mathrm{hrs}$, (v) transfer to individual containers with $10 \mathrm{~mL}$ neutralizer (vi) serial dilutions, incubate and agar plating, (vii) compare to controls and calculate percent of log 10 reduction. $^{18}$

\section{(c) Wolfaardt's Lab Modification of ISO 22196}

The Wofaardt method differs from the ISO 22196 method by foregoing the coverslip altogether and allowing a droplet of inoculum to dry in direct contact on the treated surface. Once dried, the surface is shaken with saline to remove unbound cells followed by serial dilution and enumeration on agar as with the other methods (Figure 1.39). ${ }^{137}$ 


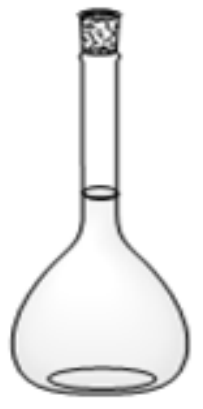

i

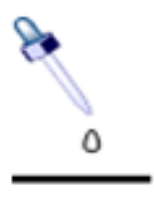

ii

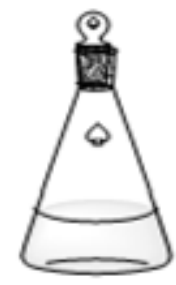

iii

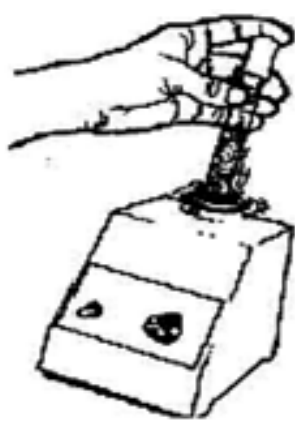

iv

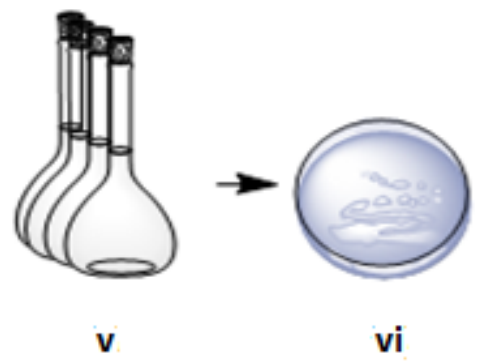

Figure 1.39: Modified method for testing antimicrobials on hard surfaces by growth enumeration in the dry state testing developed in the Wolfaardt lab. (i) $1 \times 10^{10} \mathrm{CFU} / \mathrm{mL}$ (inoculum), (ii) $1 \mathrm{~mL}$ inoculum added to $1 \mathrm{~cm} \times 1 \mathrm{~cm}$ solid sample and left to dry for appropriate time 2-24 hrs, (iii) sample is added to $0.9 \%$ saline solution, (iv) vortex saline solution to remove attached cells, (v) serial dilutions and agar plating, (vi) compare to controls and calculate percent of $\log 10$ reduction.

\section{(d) Green's Lab Modification (SLSDSS) of ISO 22196}

Recently Green et al., reported an improved microbiological test method that differentiates between contact killing and release killing from immobilized antimicrobial surfaces. The new method, known as the semi-quantitative surface-separated live-dead staining (SSLDS) technique, is a modification of the traditional live-dead staining technique based on fluorescence microscopy. ${ }^{63}$ By utilizing $50 \mathrm{~mm}$ polystyrene spacers between the treated surface and a control surface, the method can distinguish three outcomes: (A) live or dead cells on the control surface, (B) live or dead cells on the treated surface, (C) live or dead cells leaching (Figure 1.40). Thus in the first outcome (A) the immobilized agent is ineffective and no bacteria are killed (cells stained green), (B) the treated surface agent if firmly immobilized and only kills bacteria at the treated surface (cells are stained red) while cells on the coverslip survive (stain green) (C) the antimicrobial leaches from the treated surface via the spacer and kill cells throughout, all cells are stained red or dead (Figure 1.40). ${ }^{63}$ 

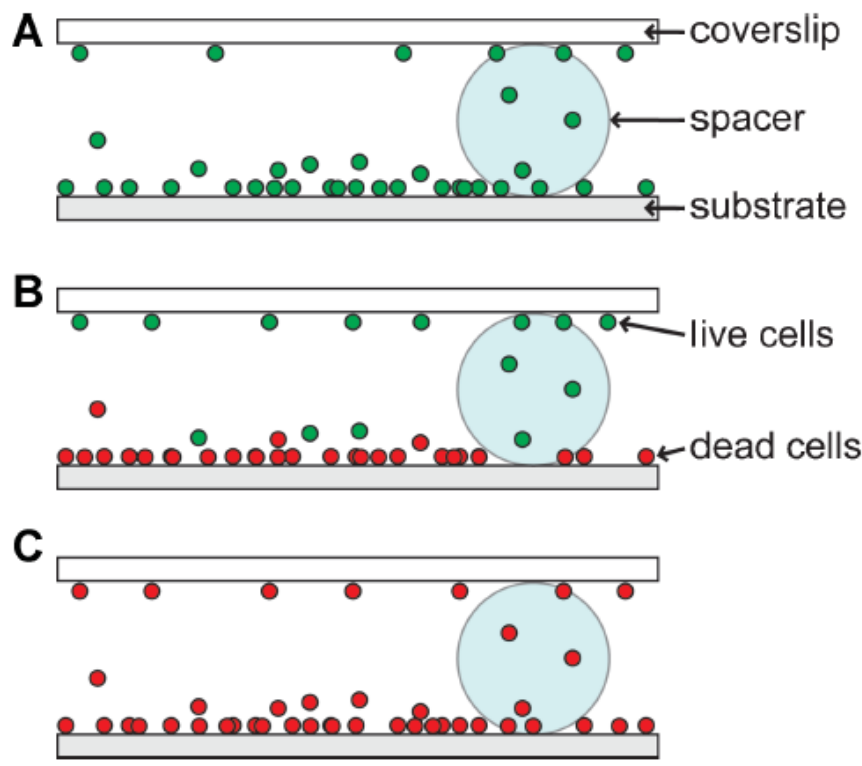

Figure 1.40: A schematic diagram of the symmertric SSLDS method depicting three distinct antimicrobial agent states: (A) no kill, all cells are stained live (green), (B) on cell that come into contact with immobilized antimicrobial are killed (bottom), (C) all cells are killed because of antimicrobial leaching. ${ }^{63}$

The Green lab modified their SSLDS method further by incorporating an asymmetric direct inoculation design in comparison to their standard symmetric design. The difference between the two designs being in the asymmetric design with both treated active glass surfaces are separated by a spacer, meanwhile the symmetric design uses an untreated control glass (coverslip) on top and an active treated slide with the immobilized antimicrobial agent on the bottom (Figure 1.41). In this way, false positives resulting from non-adherent cells or interaction of the fluorescent dyes with the antimicrobial substrate found in the symmetric SSLDS are minimized. For example, if the bacterial strains fail to adhere to the active surface and after being killed detach to the coverslip, then false conclusions can be drawn that the surface was eluting. ${ }^{136}$ 

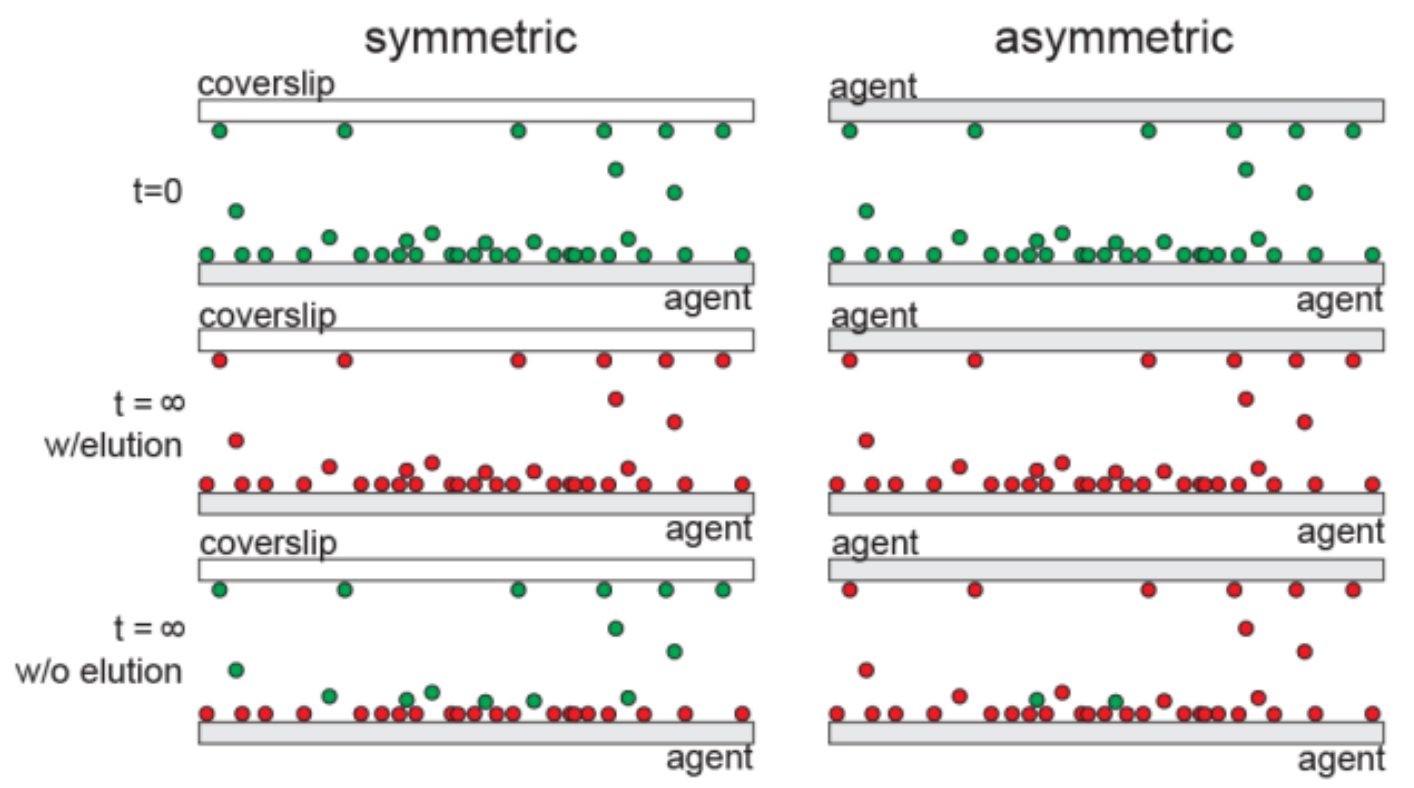

Figure 1.41: Direct innoculation SLDSS methods. (A) symmetric design, (B) asymmetric design. ${ }^{136}$

\subsubsection{Cell Viability Enumeration}

Cell viability on the surface can be quantified with fluorescence based on live dead staining combined with real time microscopy or assayed with various molecular biology techniques that measure (i) cellular ATP with bioluminescence, or (ii) $\mathrm{CO}_{2}$ respiration with flow cells or with (iii) redox active indicator dye (2,3-bis-(2-methoxy-4-nitro-5-sulfophenyl)-2Htetrazolium-5-carboxanilide) (XTT) measured spectrophotometrically (Figure 1.42).

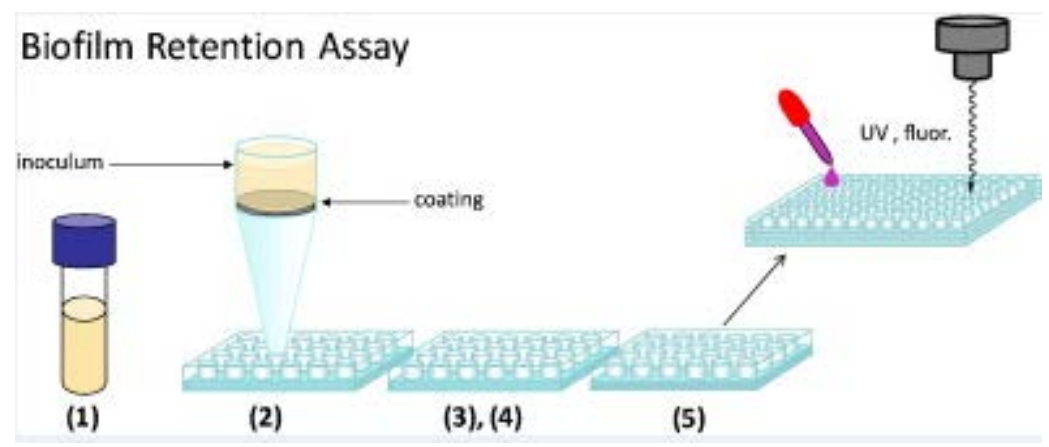

Figure 1.42: Diagram of a high-thoughput biofilm retention assay that measures absorbance or fluorescence from a 96-well plate. ${ }^{18}$ 
Microscopy techniques can be employed as tools to test antimicrobial activity on a surface. The Foucher group in collaboration with the Wolfaardt group tested QAC immobilized PP surfaces for bacterial growth. SEM microscopy showed considerable bacterial colonization on the control (left) and virtually no growth on the treated surface indicative of a contact killing surface (Figure 1.43). ${ }^{138}$

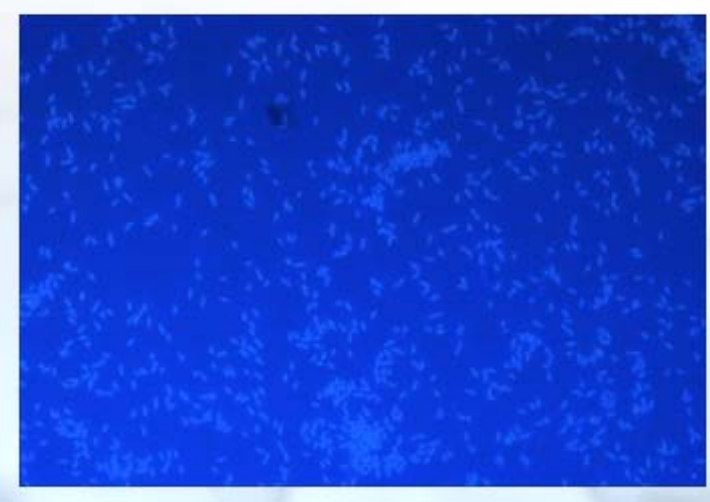

(A)

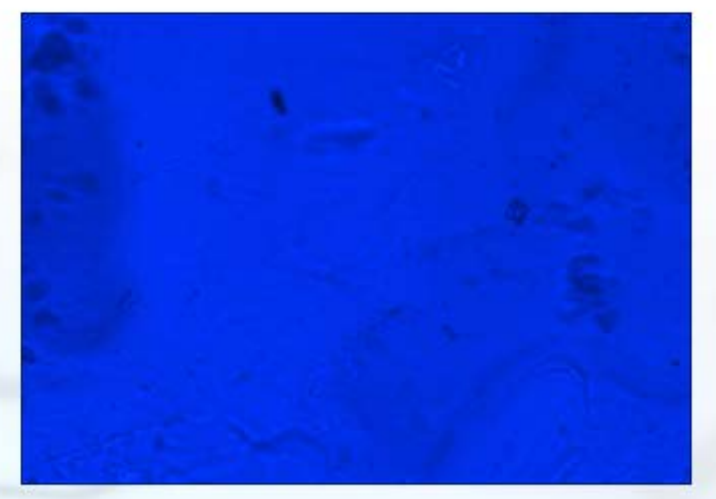

(B)

Figure 1.43: Polypropylene microscopy of control $\sim 50 \mathrm{cfu} / \mathrm{mL}$ (A) vinyl terminated QAC sprayed (10\%) 0 cfu/mL (B). ${ }^{138}$

Other groups captured the disruption of gram-positive and gram-negative bacterial membranes with SEM images after incubation with QAC-silica nanoparticles. Both the untreated and non quat treated nanoparticles (NP) showed normal cell morphology, meanwhile the QAS treated NP’s showed cell lysis and cell death. (Figure 1.44). 


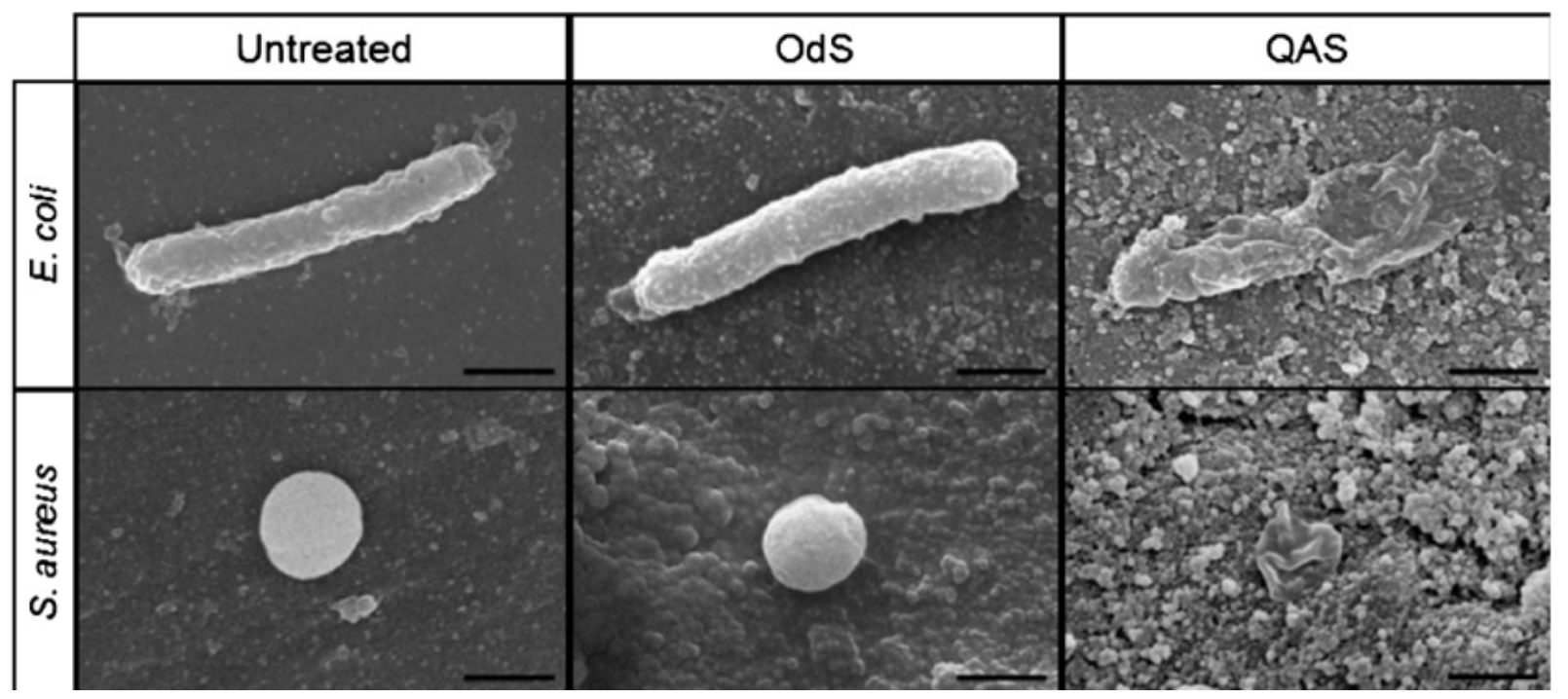

Figure 1.44: SEM images of bacteria incubated with antimicrobial silica nanoparticles. OdS = silica with $\mathrm{C}_{18}$ chain lacking a quat, QAS = SiQAC 3. (Used with permission from Ref. ${ }^{62}$ ).

\subsection{CHEMISTRY OF QUATERNARY AMMONIUM GROUPS AND PHOSPHONIC ACIDS}

\subsubsection{Menschutkin Quaternization Reaction}

Discovered in 1890 by Nicoli Menschutkin, the quaternization or quat formation reaction is widely used industrially for the synthesis of antimicrobials, phase transfer catalysis, pesticides, ionic liquids and natural products. ${ }^{139}$ A typical quaternization reaction involves the reaction between two neutral molecules, a tertiary amine with an alkylhalide or sulfonate. Upon nucleophilic attack, the amine substitutes the alkylhalide or sulfonate leaving group in an $\mathrm{S}_{\mathrm{N}} 2$ fashion producing a positively charged quaternary amine with four bonds and a negatively charged counter ion, thus forming an ion pair. In accordance with an $\mathrm{S}_{\mathrm{N}} 2$ mechanism, the reaction is accelerated by more nucleophilic amines, increased leaving group ability, increased pressure and elevated temperature (Figure 1.45). ${ }^{140}$ Polar solvents also speed up the reaction by stabilizing the charged transition state (Table 1.10). ${ }^{141}$ With alkyltosyl or mesylates the reaction 
is favored at lower temperatures and polar protic solvents in order to avoid the competing elimination reaction. ${ }^{142}$

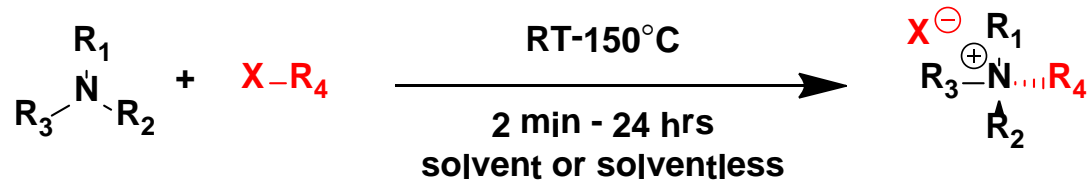

$\mathrm{X}=\mathrm{OMS}>\mathrm{I}>\mathrm{Br}>\mathrm{Cl}$
$\begin{aligned} & \text { Order of } \\ & \text { reactivity }\end{aligned}$
$\mathrm{H}_{\mathbf{2}} \mathrm{O} \sim \mathrm{DMF}>\mathrm{ACN}>\mathrm{ROH}>\mathrm{TOL}$
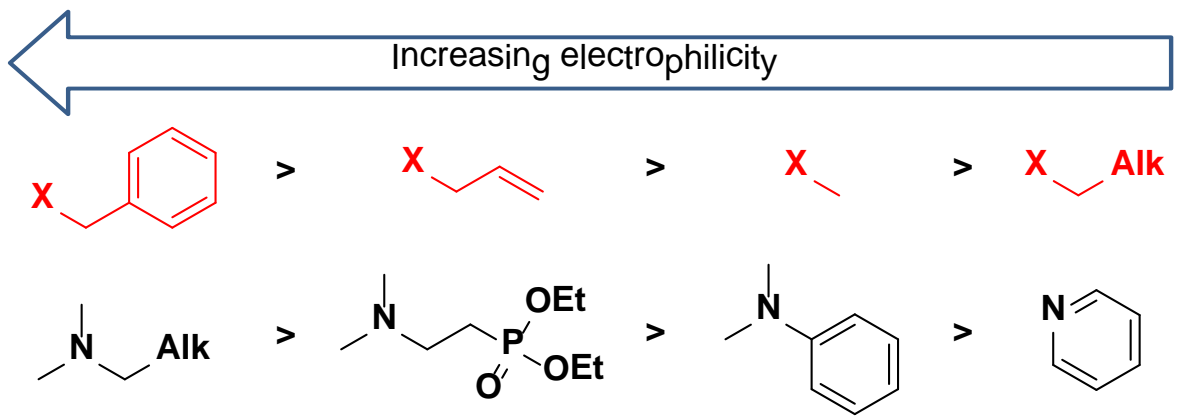
Increasing nucleophilicity

Figure 1.45: Reactivity of the Menschutkin reaction.

Table 1.10: Relative rates of the Menschutkin quaternization in various solvents (Adapted from Ref. $^{143}$ ).

\begin{tabular}{|c|c|c|c|}
\hline Solvent & Relative Rate $\left.\mathbf{( s}^{\mathbf{- 1}}\right)$ & Solvent & Relative Rate $\left.\mathbf{( s}^{-\mathbf{1}}\right)$ \\
\hline $\mathrm{CH}_{3}\left(\mathrm{CH}_{2}\right)_{4} \mathrm{CH}_{3}$ & 1 & $\mathrm{EtOH}$ & 200 \\
\hline $\mathrm{Et}_{2} \mathrm{O}$ & 4 & $\mathrm{MeOH}$ & 285 \\
\hline $\mathrm{C}_{6} \mathrm{H}_{6}$ & 38 & $\left(\mathrm{CH}_{3}\right)_{2} \mathrm{CO}$ & 340 \\
\hline$n-\mathrm{BuOH}$ & 70 & $\mathrm{ACN}$ & 375 \\
\hline $\mathrm{CHCl}$ & 100 & $\mathrm{CH}_{3} \mathrm{NO}_{2}$ & 500 \\
\hline $\mathrm{EtOAC}$ & 125 & $\mathrm{DMF}$ & 900 \\
\hline
\end{tabular}


In accordance with the aims of this thesis, the synthesis of quaternary ammonium antimicrobials for surface immobilization will be conducted through the Menshutkin quaternization reaction. Typically, the reaction goes to completion by employing refluxing temperatures in polar solvents from which QAS products crystallize or can be precipitated with the addition of $\mathrm{Et}_{2} \mathrm{O}$ or hexanes. Selected literature quaternization examples showing reaction conditions used to prepare surface bound antimicrobials are presented in Table 1.11.

Table 1.11: Examples of literature Menschutkin reaction conditions.

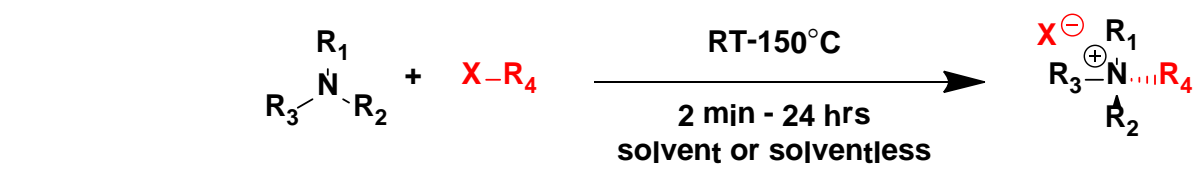

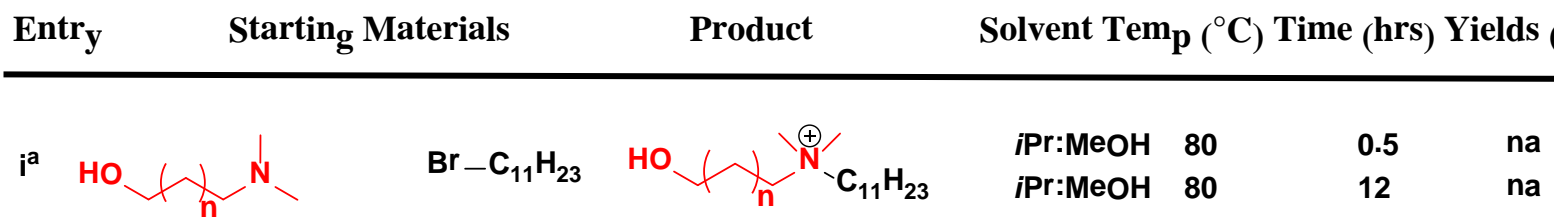<smiles>CO[Si](CCCCl)(OC)OC</smiles>
$\mathrm{iii}^{\mathrm{C}} \underset{1}{1}$
MeI<smiles>CO[Si](O)(O)CCC[N+](C)(C)c1ccccc1</smiles>
THF
RT
24
76
ivc EtO $_{\text {Eto' }}^{\text {P' }} \sim_{\text {Br }}$<smiles>c1ccncc1</smiles><smiles>CCOP(=O)(CC[n+]1ccccc1)OCC</smiles>
$\mathrm{H}_{2} \mathrm{O}$
82
48
na

Continued.... 


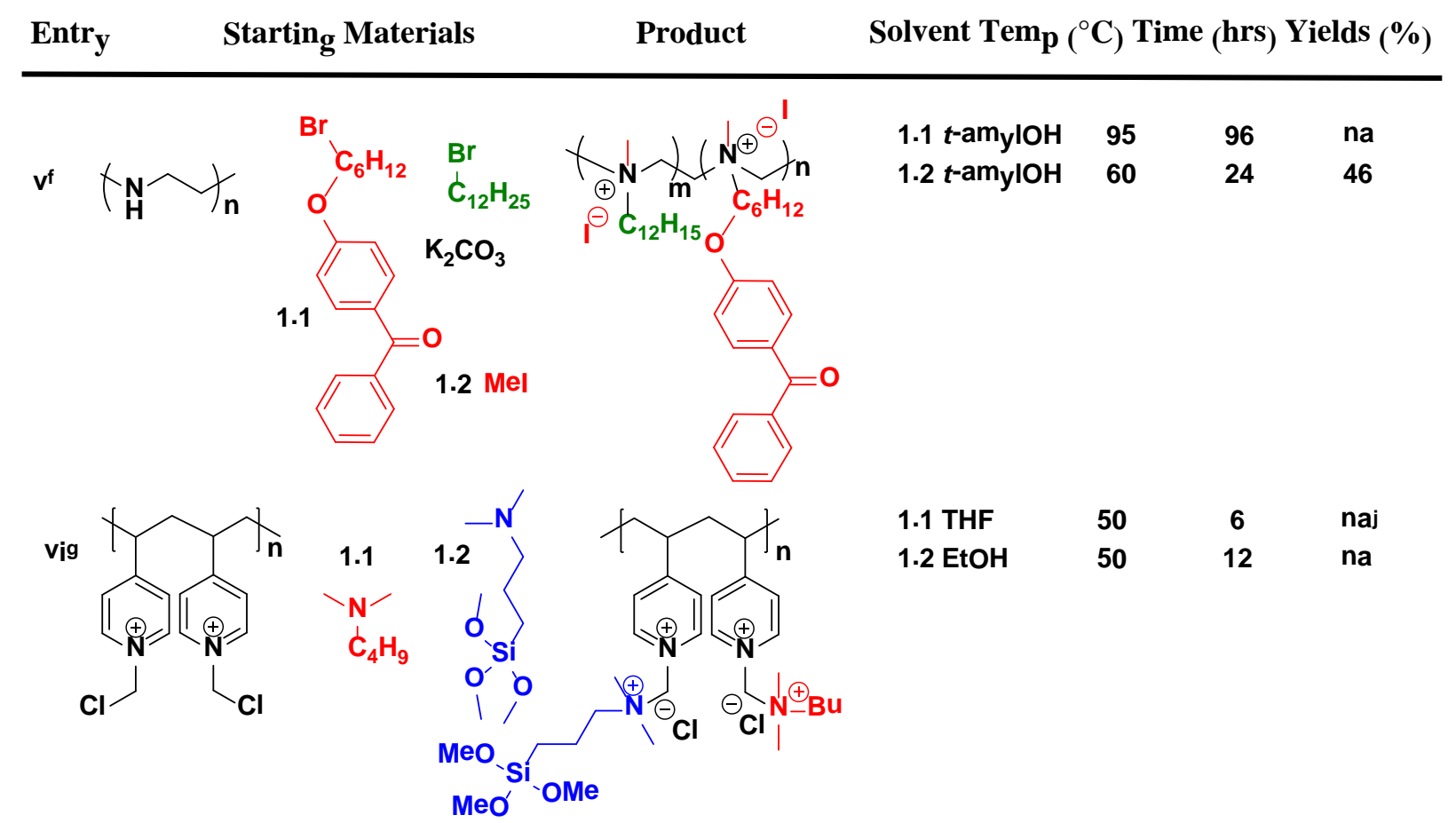

(i), ${ }^{102}$ (ii), ${ }^{38}$ (iii), ${ }^{59}$ (iv) ${ }^{144},(\mathrm{v}),{ }^{25}$ (vi), ${ }^{42}$; b (diluted directly to $50 \mathrm{wt} \%$ in MeOH) c (used directly following hydrolysis with $\mathrm{HCl}$, not shown), f-g (used directly in step 1.2, final yield not reported).

\subsubsection{Precursors for the Menshutkin Reaction}

Unless commercially available, the precursor amine and alkylhalide or sulfonate often require synthesis. The most direct method for the preparation of $N$-alkyl secondary or tertiary amines is alkylation of a primary amine with an alkylhalide (or sulfonate) known as the "Hoffman alkylation." ${ }^{145}$ Although the method seems simple at first glance, it lacks control due to the possibility of overalkylation (Figure 1.46). ${ }^{145,146}$ When starting with unactivated primary amines the higher substituted $N$-alkyl amine products formed can react with the alkyl halide giving a mixture of primary, secondary, tertiary and QAS that are impossible to separate. In practice, completion only to the secondary amine stage can be achieved by using a large excess $(10-100 \times)$ of the primary amine (Figure 1.46). However the drawbacks are the price of the starting amine used in excess and the extra purification steps required (normally distillation) to 
remove the excess amine. The cost of the starting amine limit the scope of the reaction because only inexpensive simple primary amines such as methyl and benzyl amines could realistically be used. ${ }^{145}$

Recent developments showed that by adding a small amount of an additive (silica ${ }^{147}$, $\mathrm{CsOH}^{148}$ ) one can selectively stop the $N$-alkylation reaction at the secondary or tertiary amine without QAS formation. Stopping at the tertiary amine is also possible with microwave $(\mu \mathrm{W})$ radiation under aqueous conditions $\left(\mathrm{NaOH} / \mathrm{H}_{2} \mathrm{O}^{149,150}\right.$ or $\left.\mathrm{NaHCO}_{3} / \mathrm{H}_{2} \mathrm{O}^{151}\right)$. Additionally, with activated substrates such as allylhalide or $\mathrm{CH}_{3} \mathrm{I}$, a primary amine can be driven to the quaternary amine as the sole product with the addition of a base (Figure 1.46, further discussion below).

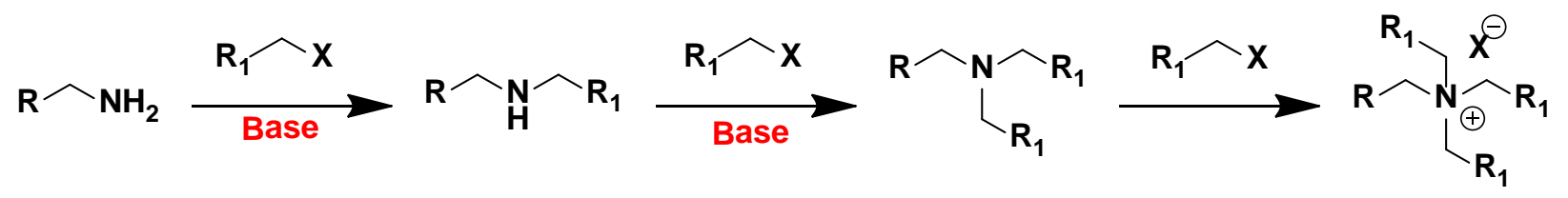

Figure 1.46: Preparation of Menschutkin precursors from primary amines by direct alkylation.

If more control is needed, two alternative reactions can be used to give $N, N$-methylated tertiary amines as the sole products (Figure 1.47).

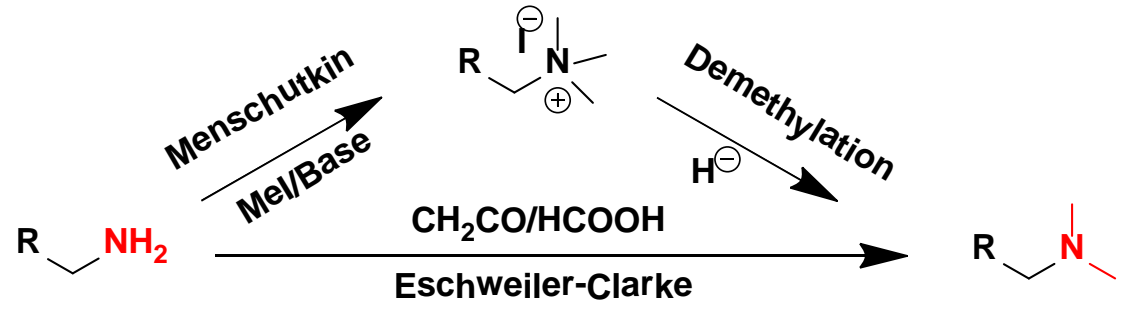

Figure 1.47: Preparation of Menschutkin precursors from primary amines.

$N, N$-dimethyltertiaryamines are most commonly synthesized by the classic Eschweiler Clarke (EC) reaction from primary or secondary amines using formaldehyde as the source of the methyl groups and formic acid as the reducing agent generating $\mathrm{H}_{2} \mathrm{O}$ and $\mathrm{CO}_{2}$ in the mechanism 
(Figure 1.48). ${ }^{152}$ Alternative reducing agents include the borohydrides, $\mathrm{NaBH}_{4},{ }^{153}$ $\mathrm{NaBH}(\mathrm{OAc})_{3},{ }^{154,155} \mathrm{NaCNBH}_{4},{ }^{156}$ and zinc reagents, $\mathrm{ZnCl}_{2} / \mathrm{NaBH}_{4} / \mathrm{DCM}^{157}$ and $\mathrm{Zn} / \mathrm{AcOH} / \mathrm{H}_{2} \mathrm{O} .{ }^{158}$ The advantage of the EC reductive methylation over amine methylations with MeI is that the reaction halts at the tertiary amine without producing quaternary ammonium salt because tertiary amines are inert to further imine formation with formaldehyde. However, the intermediate imine formed in the reaction can sometimes isomerize, and upon hydrolysis, yield the carbonyl derivative of the starting amine plus methylamine. ${ }^{152,159}$ As a result of by-product formation, the EC reaction can give poor yields and can be difficult to purify if mixtures of mono- and dimethylated products are formed. An alternative synthetic strategy to $\mathrm{N}, \mathrm{N}$-dimethyl tertiary amines can be considered.

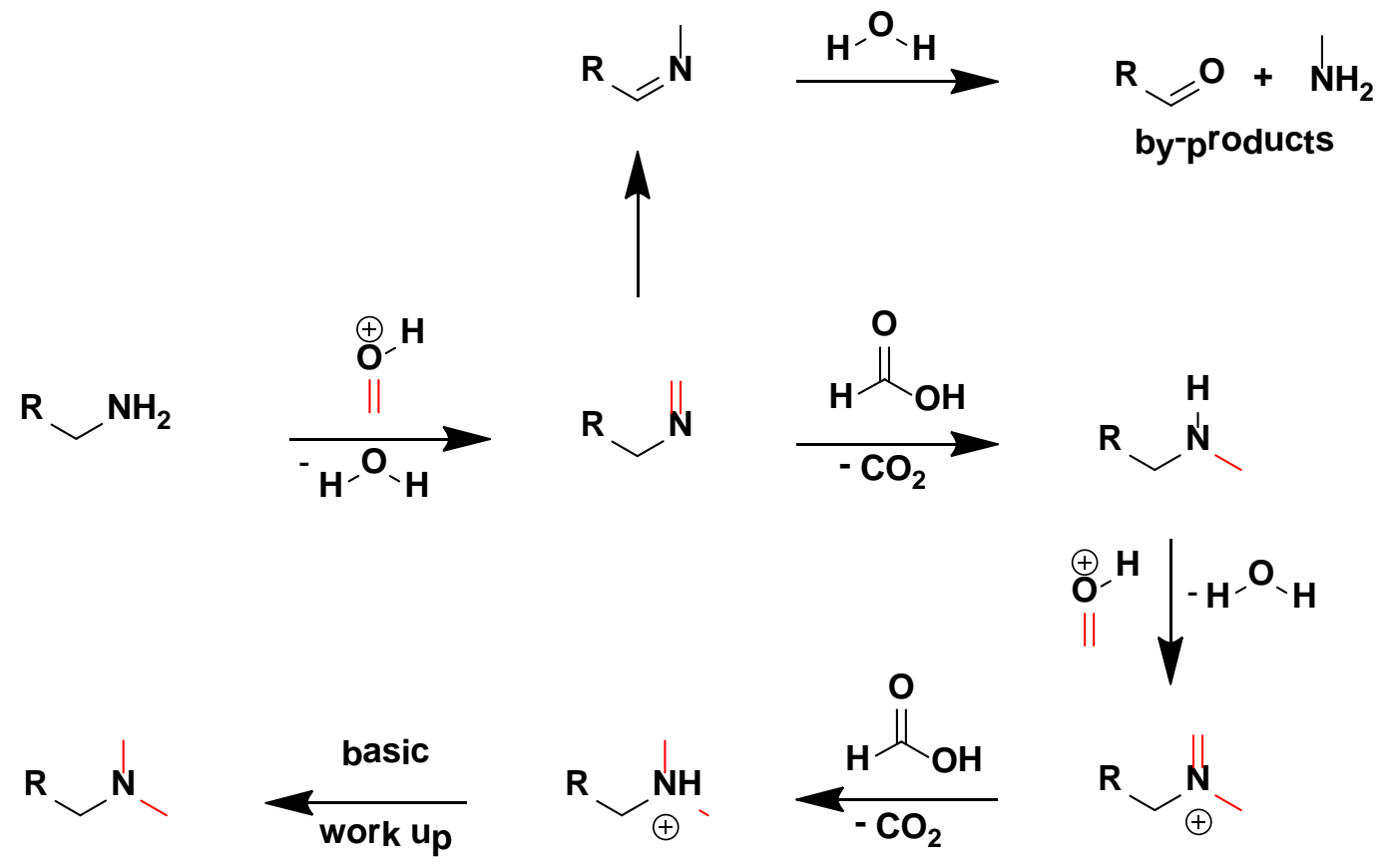

Figure 1.48: Mechanism of the Eschweiler-Clarke $N, N$-methylation.

Combination of the Menshutkin quaternization with demethylation is another route towards $N, N$-dimethyl tertiary amines which avoids epimerization like the EC reaction. For 
example the reaction has to be performed sequentially when the demethylating nucleophilc hydride is LAH. ${ }^{159}$ The first step performed in $\mathrm{MeOH} / \mathrm{bicarbonate}$ gives $N, N, N$-trimethylalkyl amines which are isolated and demethylated in THF/LAH (Figure 1.47). A one-pot synthesis of $\mathrm{N}, \mathrm{N}$-dimethylalkyl amines is achieved by tandem quaternization/demethylation when $\mathrm{NaBH}_{4}$ was used as the nucleophile. ${ }^{160} \mathrm{~A}$ onepot procedure involved quaternization of either primary, secondary or tertiary amines with MeI in a polar aprotic solvent (DMF, DMSO, ACN) utilizing a sterically hindered organic base ${ }^{143}$ of greater basicity than the starting amine such as 2,6 lutidine (pKa 6.77), tri-n-butylamine (pKa 10.89), 1,2,2,6,6-pentamethylpiperidine or PMP (pKa 11.2), to mop up the HI formed in the reaction. This sequence is followed by demethylation with the addition of $\mathrm{NaBH}_{4}$ to the reaction without isolation of the trimethylalkylquaternary ammonium salt. ${ }^{160}$ Alternatively, alcohols can be used as starting materials to prepare halo and $N, N$-dimethylamine functional groups either directly with the Appel reaction $\left(\mathrm{CBr}_{4} / \mathrm{PPh}_{3}\right)^{161}$ or indirectly by substitution of an activated alcohol either by mesylation $\left(\mathrm{MsCl} / \mathrm{NMe}_{3} \cdot \mathrm{HCl}\right.$, $\mathrm{LiBr}^{162}$, or silylation (TMSCl/LiBr) ${ }^{163}$ (Figure 1.49).

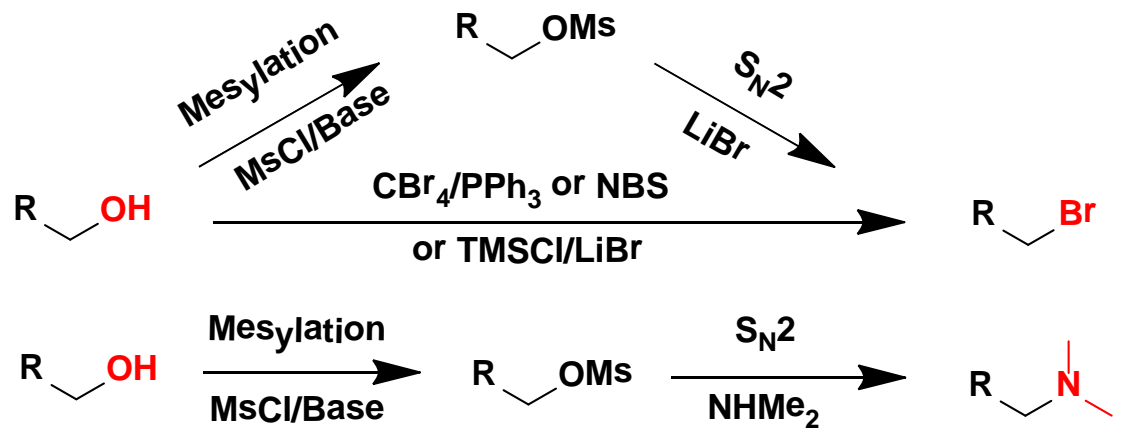

Figure 1.49: Preparation of Menschutkin precursors from alcohols. 


\subsubsection{Stability of Quaternary Ammonium Groups}

Quaternary ammonium salts undergo dequaternization or dealkylation (debenzylation, deallylation, demethylation, dealkylation) by various nucleophiles in an $\mathrm{S}_{\mathrm{N}} 2$ fashion producing tertiary amines and transalkylated by-products bound to the nucleophile. By varying the nucleophile and the alkyl leaving groups the substitution can be selective, giving the desired tertiary amine or can occur randomly, providing a mixture of products (Table 1.12). ${ }^{164}$ Selective dequaternization of QAS is an effective method used to prepare tertiary amines having different alkyl substituents. ${ }^{146,165}$ Selective dequaternization is only possible when one of the alkyl groups is a better leaving group compared to the other three groups on the quaternary nitrogen. Two examples demonstrate the utility of this discriminating method for the preferential dealkylation. In the first approach, heating a QAC bearing a 2-hydroxyethyl group with hydroxide ion allows for its preferential and clean cleavage. This is because upon heating, only the 2-hydroxyethyl group leaves as ethylene oxide whereas other alkyl groups remain untouched by hydroxide (Table 1.12, Entry i). ${ }^{146}$ In the second approach, a QAC attached through a benzyl group to a solid support resin (Merrifield resin) and bearing three different substituents can be preferentially removed from the resin by selective debenzylation over demethylation. This is possible because the alkyl leaving group ability from QAS increases with resonance stabilization and the loss of a benzyl group and allyl group is preferred over the loss of a methyl group which itself is favored over the loss of longer alkyl groups (Table 1.12, Entry iii). ${ }^{165}$ Other nucleophiles capable of selective QAC demethylation to the desired tertiary amines include the hydrides; $\mathrm{LAH}^{159}$ $\mathrm{NaBH}_{4}$ (HMPA, DMSO, sulfone $\left.)^{160}, \mathrm{LiBH}_{(\mathrm{Et}}\right)_{3},{ }^{159} \mathrm{LiBH}(\mathrm{S}-\mathrm{Bu})_{3},{ }^{159}$ and also $\mathrm{Na} / \mathrm{NH}_{3},{ }^{166}$ $\mathrm{OH}\left(\mathrm{CH}_{2}\right)_{2} \mathrm{NH}_{2},{ }^{167} \mathrm{LiI}^{167}{ }^{167} \mathrm{PhS}^{-} \mathrm{Na}^{+},{ }^{168} \mathrm{CH}_{3}\left(\mathrm{CH}_{2}\right)_{2} \mathrm{~S}^{-} \mathrm{Li}^{+},{ }^{169}$ and piperidine ${ }^{170}$ (Table 1.12, Entry iii). 
Table 1.12: Examples of the $S_{N} 2$ dequaternization reaction.

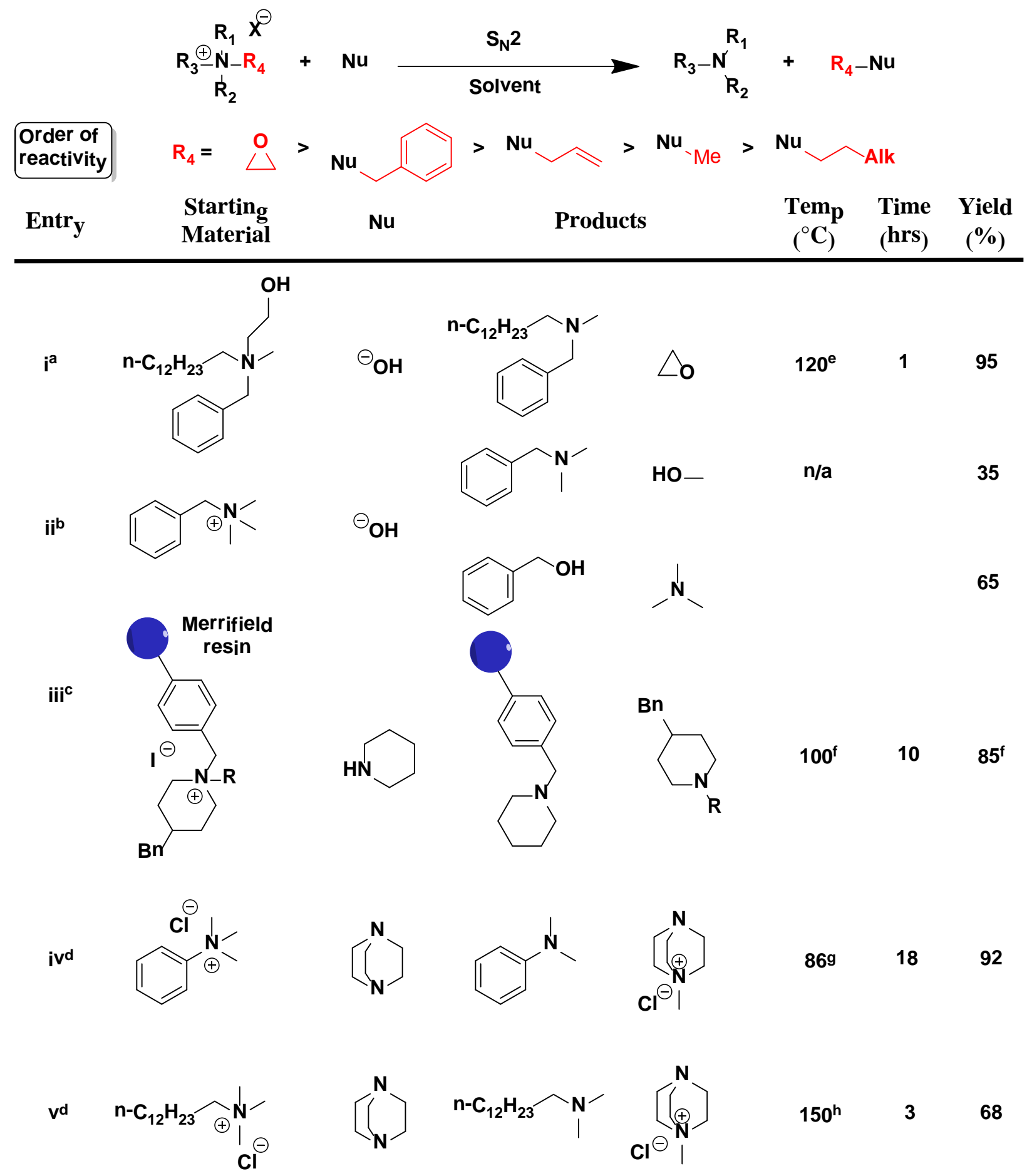

$\mathrm{a}^{146}, \mathrm{~b}^{171}, \mathrm{c}^{165}, \mathrm{~d}^{170}$,e (no solvent), $\mathrm{f}\left(\mathrm{R}=n\right.$-Bu (85\%), Et (83\%)), Me (83\%) ${ }^{165}$, (no solvent), $\mathrm{g}$ $(\mathrm{EtOH}), \mathrm{h}(\mathrm{EtOH}=$ no reaction, $\mathrm{DMF})$. 
Otherwise, when a non-selective nucleophile such as $\mathrm{OH}^{-}$is reacted with a QAC possesing benzyl and methyl substitutents, a mixture of products is possible (Table 1.12, Entry ii). For example, heating benzyltrimethylammonium hydroxide gives a mixture of benzyldimethyl amine and $\mathrm{MeOH}$ (35\%) resulting from an $\mathrm{S}_{\mathrm{N}} 2$ attack on one of the methyl groups, while attack on the benzyl carbon results in benzyl alcohol and $\mathrm{Et}_{3} \mathrm{~N}$ (65\%). Elimination products (alkenes and tertiary amines) are not possible in this case because this substrate lacks $\beta$ hydrogens necessary for elimination to take place. Of course E2 elimination, favoured by sterically hindered bases can occur only on QAC substrates with $\beta$-hydrogens and the leaving group in the trans configuration. ${ }^{172}$ On the otherhand, the E1 mechanism is possible only on QAC substrates with sterically hindered $\alpha$ and $\beta$-carbons atoms. ${ }^{172}$

Lastly, depending on the nature of the alkyl halide and the basicity of the amine, competing side reactions may take place in favor of the Menschutkin reaction. For example when an electron withdrawing group (eg. ethyl ester) is located two carbons away from the halide atom, the elimination reaction dominates and the thermodynamically favoured ethyl acrylate is formed as the sole product over the quat (Figure $1.50 \mathrm{a}$ ). Similarly the prescence of acidic functional groups with a lower pKa than the amine result in protonation of the nucleophilic amine instead of the $\mathrm{S}_{\mathrm{N}} 2$ quaternization (Figure $1.50 \mathrm{~b}$ ). In order to prepare such carboxylic acid quats, the tertiary amine are quaternized with propriolactone followed by acidic work up (Figure 1.50 c). ${ }^{173}$ 


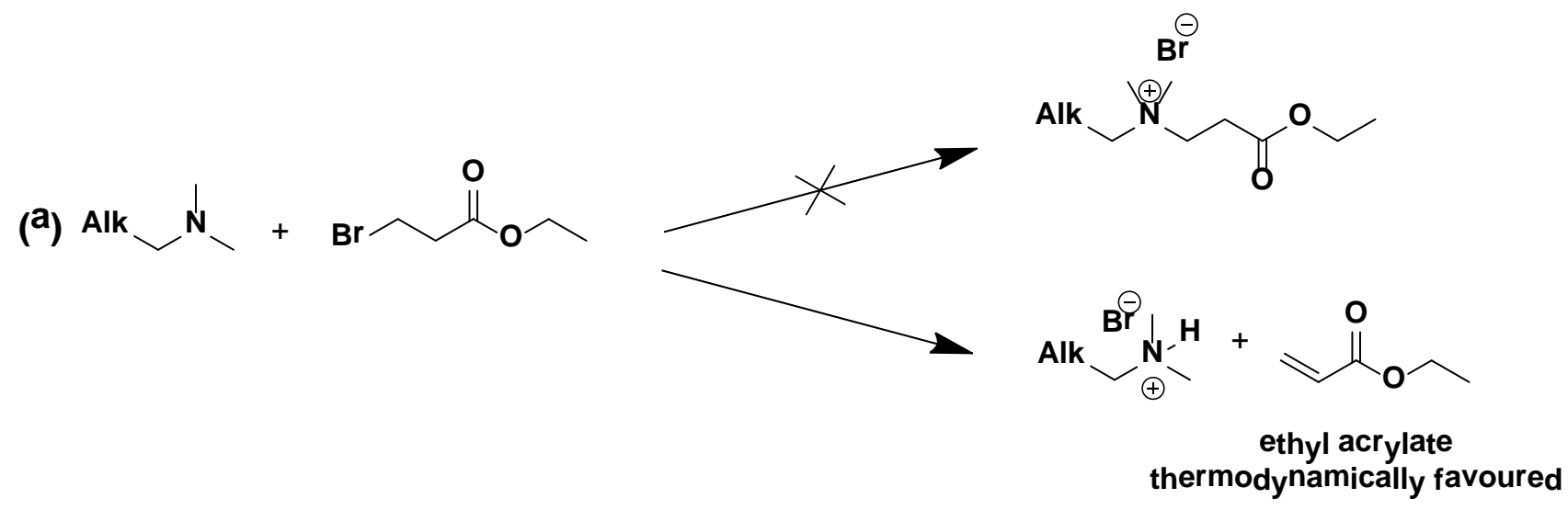

(b) Alk $^{\mathrm{N}} \sim^{\mathrm{N}} \sim \mathrm{H}_{\mathrm{OH}}^{\mathrm{O}}$
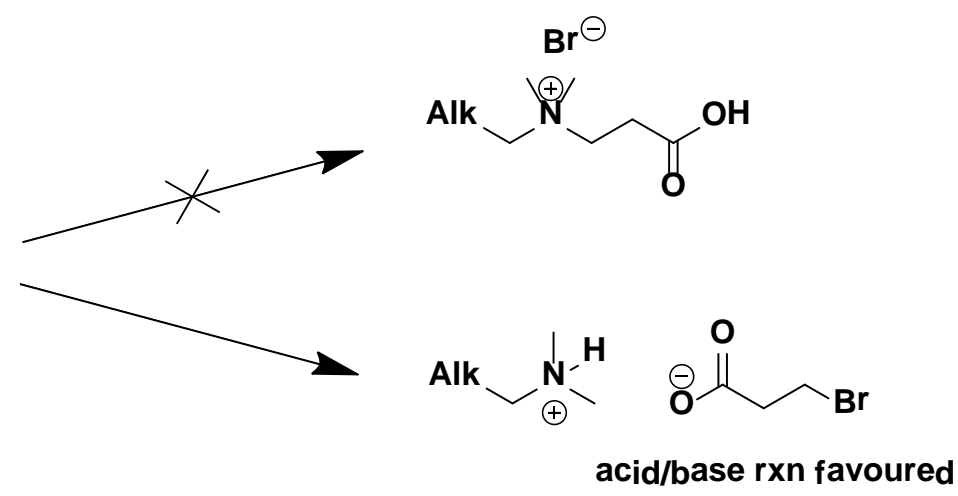

(c) $\mathrm{Alk}^{\mathrm{N}}++$
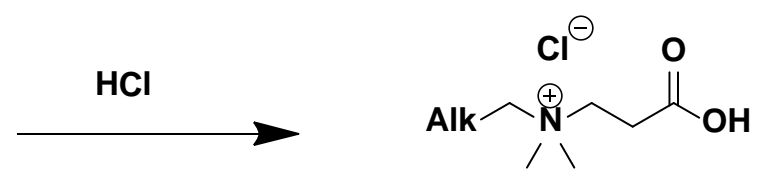

Figure 1.50: Side reactions favoured over the Menshutkin reaction. 


\subsubsection{Detection of QAC in Solution and on Surfaces}

A variety of titration methods employing organic dyes were developed to quantify the amount of quaternary ammonium salts widely employed as antimicrobials in consumer products

and pesticides in waste $\mathrm{H}_{2} \mathrm{O} .{ }^{174,175}$ The most popular methods employ bromophenol blue and dichlorofluorescein as dyes capable of binding to the quaternary ammonium through an ion pair interaction. Biphasic titrations employed bromphenol blue (Figure 1.51, 1.53) whereas dichlorofluorescein was used in a monophasic titration to determine QAS directly (Figure 1.52). ${ }^{176}$ The biphasic titration ${ }^{176}$ involves an ion pair complex formation between the active ingredient quaternary ammonium cation and the indicator bromophenol blue anion which is readily soluble in DCM (blue bottom organic phase, Figure 1.53 b). Upon addition of $\left[\mathrm{BPh}_{4}\right]^{-} \mathrm{Na}^{+}$ the bromophenol blue anion was displaced from the complex and migrated up into the top aqueous layer turning the organic bottom layer clear at the end point of the titration (Figure 1.53, c). 
(A) Start of biphasic titration with bromophenol blue

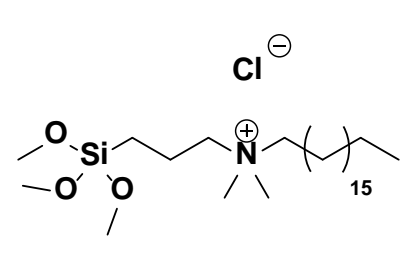

SiQAC (3)

(B) End point<smiles>O=C(O)c1cc(C2(c3cc(Br)c(O)c(Br)c3)OS(=O)(=O)c3ccccc32)cc(Br)c1Br</smiles><smiles>CCC(C)(C)CC[N+](C)(C)CCC[Si](OC)(OC)OC</smiles>

Bromophenol blue ion pair $[\mathrm{BPB}]\left[\mathrm{R}_{4} \mathrm{~N}\right]^{+}$formation with SIQAC (3)

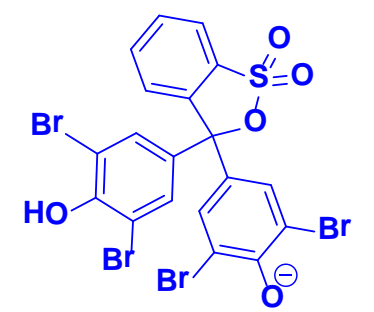

Bromophenol blue dye $\left.{ }^{\mathrm{BPB}}\right]^{-}$

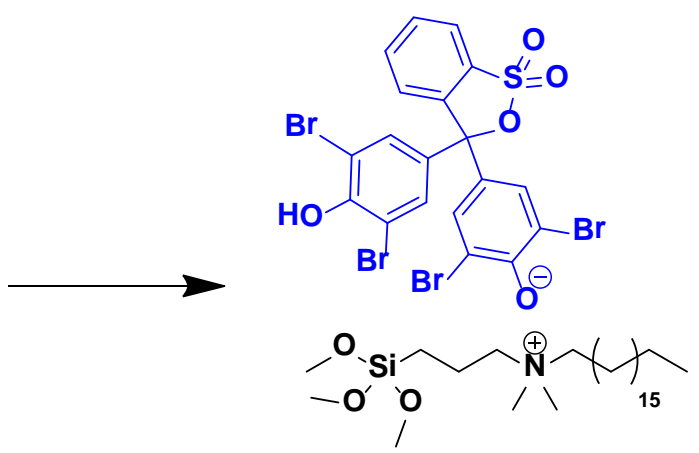

Bromophenol blue ion pair $[B P B]^{-}\left[\mathrm{R}_{4} \mathrm{~N}^{+}\right.$formation with SiQAC (3) (blue complex, organic layer)

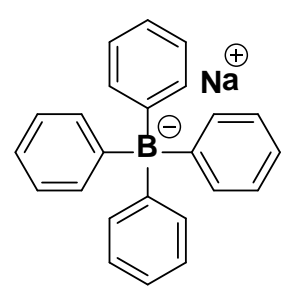

Sodium tetraphenylborate $\left[\mathrm{BPh}_{4}\right]^{-\mathrm{Na}^{+}}$titrant

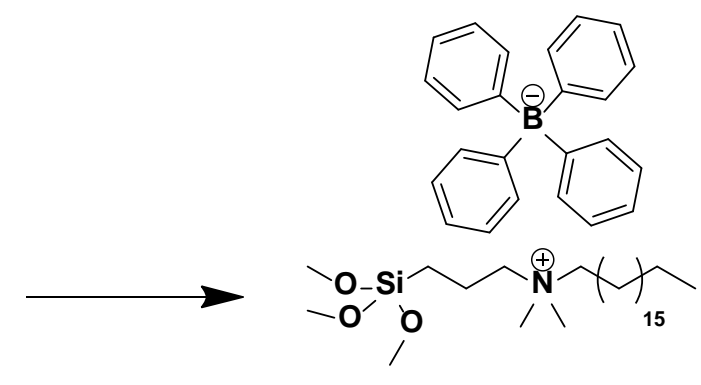

Titrant ion pair $\left[\mathrm{R}_{4} \mathrm{~N}^{+}{ }^{+} \mathrm{BPh}_{4}\right]^{-}$ with SiQAC (3)

(organic layer, clear)

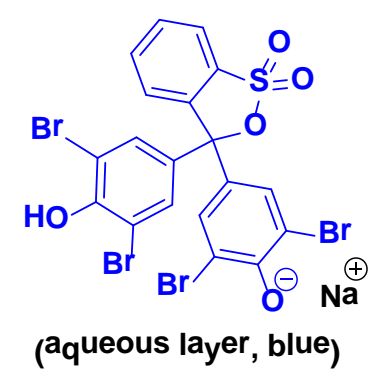

Figure 1.51: Monophasic titration with bromophenol blue. 


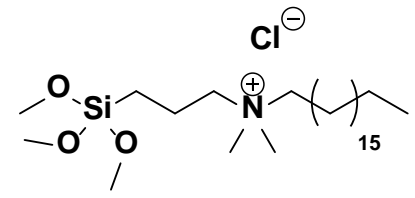

SiQAC (3)

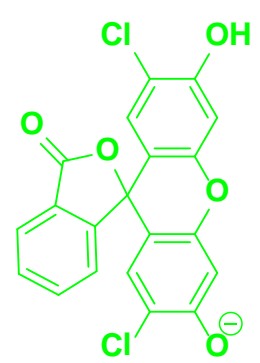

Dichlorofluorescein ion pair [DCF]

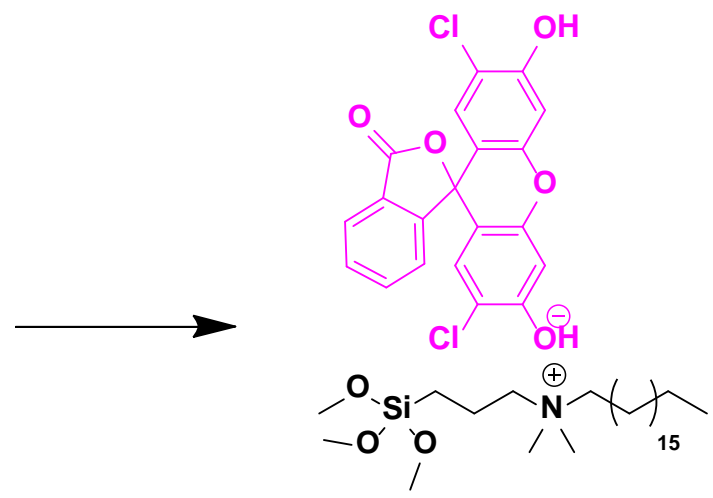

Dichlorofluorescein ion pair $[\mathrm{DCF}]^{-}\left[\mathrm{R}_{4} \mathrm{~N}^{+}{ }^{+}\right.$formation with SiQAC (3) (pink complex)

Figure 1.52: Monophasic titration with dichlorofluorescein.

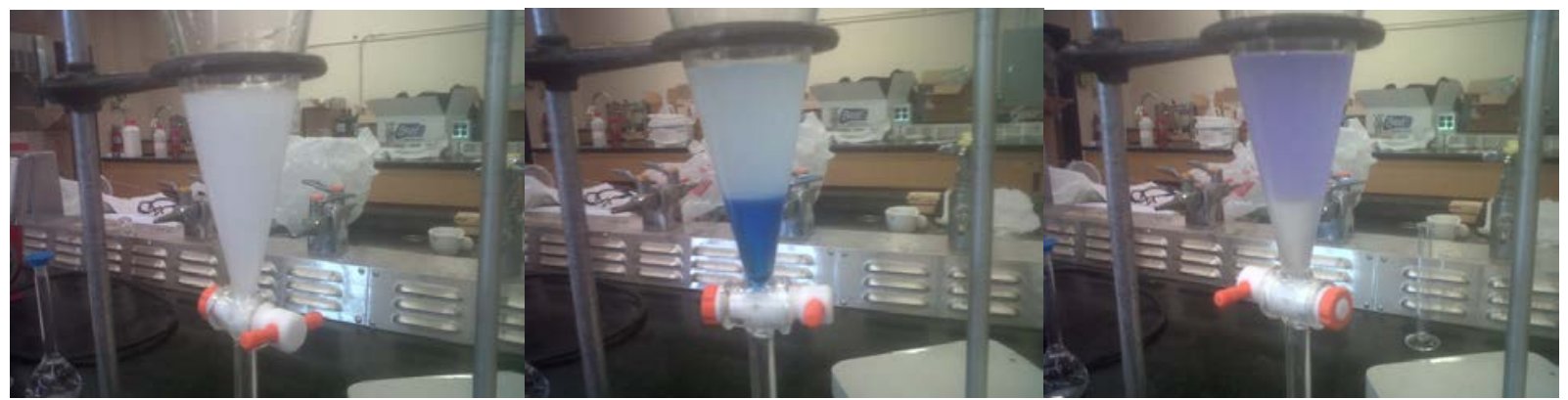

(a)

(b)

(c)

Figure 1.53: Biphasic ion pair titration based on bromophenol blue and SiQAC 3, (a) prior to addition of indicator (b) start of titration, (c) past end point.

The amount of QAS in the test sample employing the titration methods mentioned above is calculated using the following equation: \% QUAT $=\left(\mathrm{V}_{\text {tit }} \times \mathrm{N}_{\text {tit }} \times \mathrm{M}_{\mathrm{QAS}}\right) / \mathrm{Wt}_{\mathrm{QAS}}$. where $\mathrm{V}_{\text {tit }}$ is the volume of titrant, STPB added to the test solution to reach the end-point (in $\mathrm{mL}$ ), $\mathrm{N}_{\mathrm{tit}}$ is the normality of titrant (in mol/L), $\mathrm{M}_{\mathrm{QAS}}$ is the equivalent molecular weight of the $\mathrm{QAS}, \mathrm{Wt}_{\mathrm{QAS}}$ is the gross weight of the QAS (in grams).

The bromophenol blue dye has also been used to directly visualize quaternary ammonium compounds coated on fabric surfaces (porous surfaces). This $\mathrm{H}_{2} \mathrm{O}$ soluble anionic dye complexes 
surface bound quaternary ammonium compounds forming an ion pair which stains the surface a blue colour. ${ }^{2}$ However, the bromophenol blue dye once complexed to the fabric stains the surface, potentially damaging the test material for future use. The same dyes may be utilized to indirectly detect the prescence of surface bound quats on non-porous surfaces such as glass, and plastic.

A simple alternative to bromophenol blue for the detection of QAC surface coverage and coating uniformity is fluorescent detection. Thus, treated surfaces would glow fluorescent under exposure to a low power UV lamp, identifying areas of poor adhesion as well as missed areas during application. Additionally, it can also act as a unique product identifier and security feature for treated surfaces when added in trace amounts to functional antimicrobial solutions. Previously in the Foucher group, dansyl tags with organosilane, ${ }^{177}$ phosphonate, ${ }^{177}$ benzophenone ${ }^{105}$ and vinyl ${ }^{138}$ linkers were synthesized (Figure 1.54).

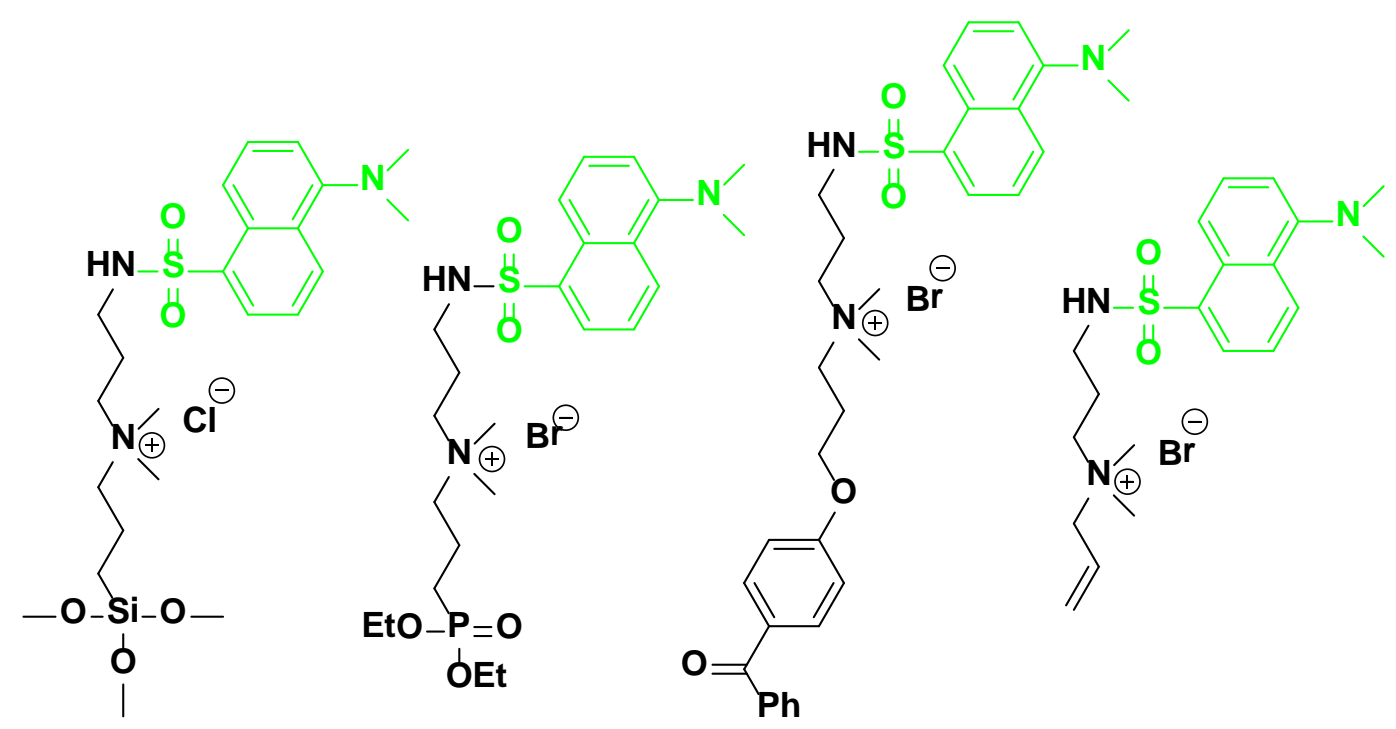

Figure 1.54: Dansyl linkers previously synthesized in the Foucher lab. ${ }^{105,138,177}$ 


\subsection{CHEMISTRY OF ORGANOPHOSPHORUS COMPOUNDS}

\subsubsection{Mono and Didealkylation of Phosphonate Esters}

Dialkyl phosphonates undergo selective dealkylation with heteroatom nucleophiles such as $\mathrm{KOH}, \mathrm{NH}_{4} \mathrm{OH}$, LiI or can be directly didealkylated with mineral acids, silicone or boron reagents (Figure 1.55)

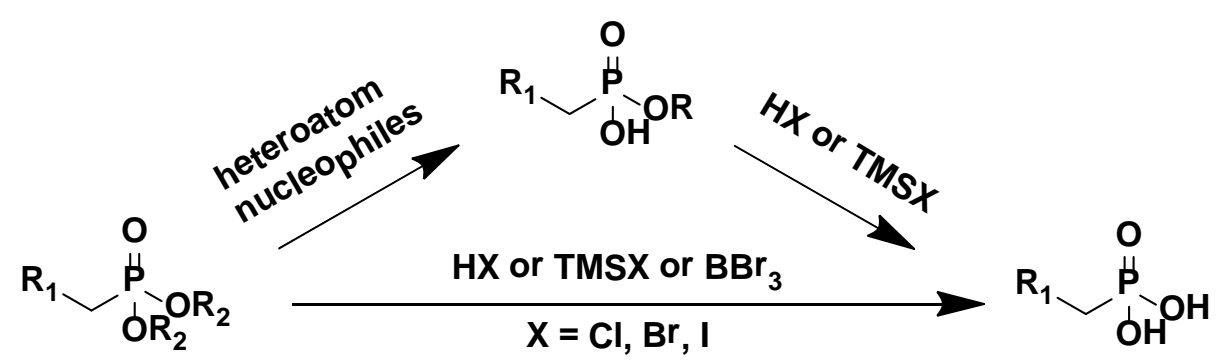

Figure 1.55: Examples of reagents used to dealkylate phosphonate esters.

Mineral acids used to completely dealkylate phosphonate esters include both $\mathrm{HCl}$ and $\mathrm{HBr}$, which are added in excess (10-100 × ) and typically require long refluxing conditions (12-24 hrs) especially with the less reactive $\mathrm{HCl}$. Under $\mu \mathrm{W}$ radiation the reaction times are shortened to 30 min with a stoichiometric amount of $\mathrm{HCl}^{178,179}$ Selected literature examples of the dealkylation of phosphonate esters with mineral acids is shown in Table 1.13 and the mechanism of this transformation is shown in Figure 1.56. Since the formation of the carbocation intermediate is the rate determining step, the more stable $i \operatorname{Pr}$ carbocations and more reactive $\mathrm{Br}^{-}$ ions generally give shorter reaction times (Table 1.13). 
Table 1.13: Literature examples of the dealkylation of phosphonate esters with mineral acids.

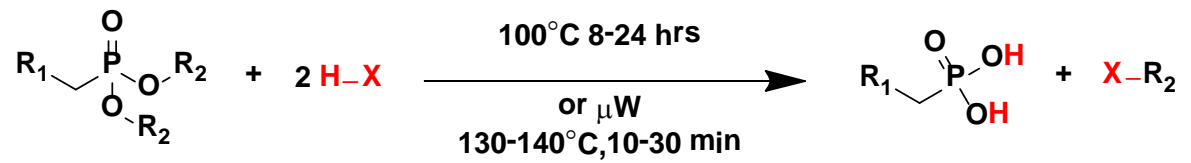

$$
\begin{aligned}
& \begin{array}{l}
\begin{array}{l}
\text { Order of } \\
\text { reactivity }
\end{array} \\
\mathrm{R}_{2}=i \mathrm{Pr}>n \mathrm{BB}>\mathrm{Bt}<\mathrm{Me}
\end{array}
\end{aligned}
$$

Entry Starting Material $\quad$ Products $\quad H X\left(\# e_{q \cdot)} \quad \operatorname{Tem}_{p}\left({ }^{\circ} \mathrm{C}\right) \quad\right.$ Time (hrs) $\quad$ Yields $(\%)$

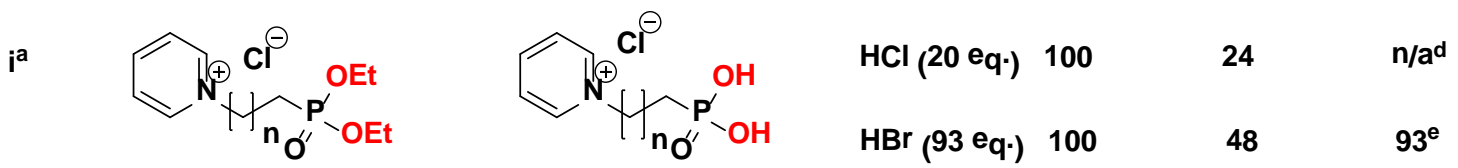

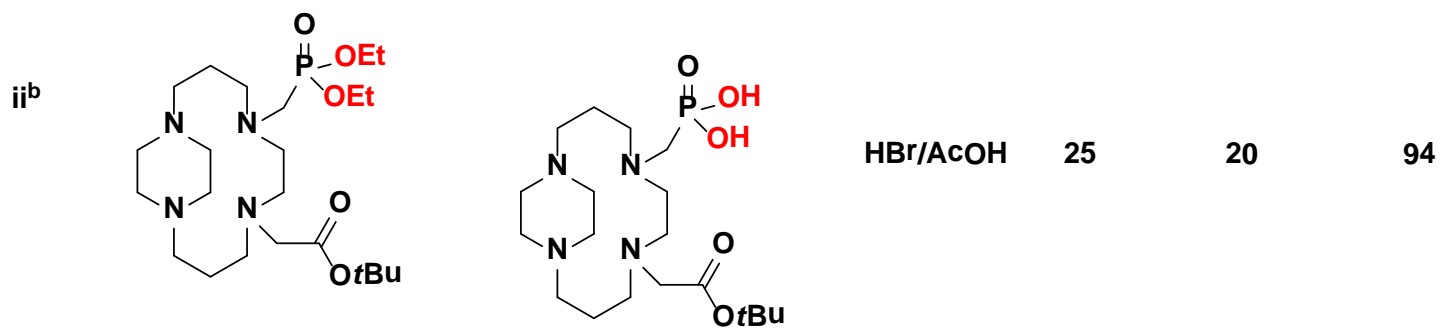<smiles>CCOP(=O)(CCCl)OCC</smiles><smiles></smiles><smiles>CCCP(=O)(CCCl)OC(C)C</smiles>

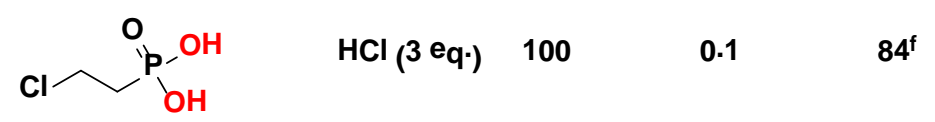<smiles>CCOP(=O)(COCCn1cnc2c(N)ncnc21)OCC</smiles><smiles>Nc1ncnc2c1ncn2CCOCP(=O)(O)O</smiles><smiles>CCCOP(=O)(COCCn1cnc2c(N)ncnc21)OC(C)C</smiles><smiles>Nc1ncnc2c1ncn2CCOCP(=O)(O)O</smiles>
$\mathrm{HCl}(2 \mathbf{e q} \cdot) \quad 130$ 0.1 $78^{f}$ $a,{ }^{178,}{ }^{179} \mathrm{~b},{ }^{180} \mathrm{c},{ }^{181} \mathrm{~d},(\mathrm{n}=1)$, (no yield reported), e, $(\mathrm{n}=5,9,13), \mathrm{f},(\mu \mathrm{W}$ reactions) 


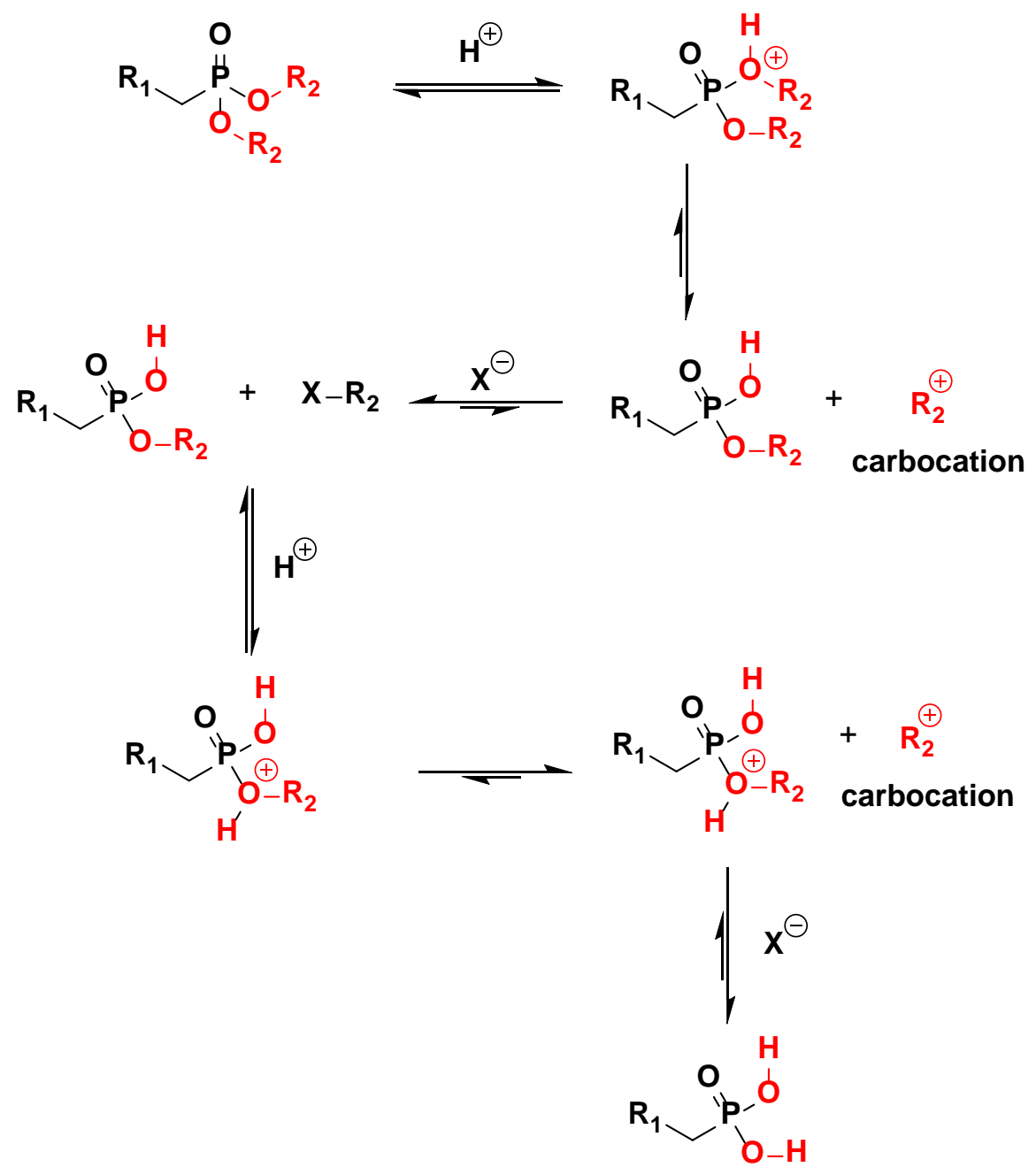

Figure 1.56: Mechanism of the didealkylation of phosphonate diesters in mineral acids.

On the other hand, dealkylation of phosphonate esters with TMSX $\left(\mathrm{X}=\mathrm{Cl}^{-}, \mathrm{Br}^{-}, \mathrm{I}^{-}\right)$ occurs via a different mechanism whereby the halide ion acts as both the leaving group and the nucleophile in the reaction (Figure 1.57) ${ }^{182-184}$ In the first step of the mechanism the acidic phosphoryl $\mathrm{sp}^{3}$ oxygen reversibly attacks the electrophilic silicon of the silylhalide forming a charged phosphonium intermediate while displacing the halide. Irreversible substitution with the nucleophilic halide only occurs with the attack on the phosphonate alkyl group producing one equivalent of an alkyl halide and a mixed trimethylsilylated ester. With TMSI bearing the more nucleophilic iodide ion, the reaction proceeds at a faster rate than $\mathrm{TMSBr}$ and occurs predictably 
the slowest with TMSCl due to the weakly nucleophilic chloride (typically d. at refluxing temperatures). Another cycle of the mechanism produces dealkylated bis(trimethylsilyl) phosphonate which at this point can be distilled or hydrolysed with $\mathrm{H}_{2} \mathrm{O}$ or lower alcohols to the free phosphonic acid. ${ }^{184}$ Aqueous hydrolysis always leads to the free phosphonic acids while the use of higher chain alcohols (decanol and above) can result in a mixture of products depending if the nucleophilic attack site is either silicon or phosphorus (Figure 1.58).
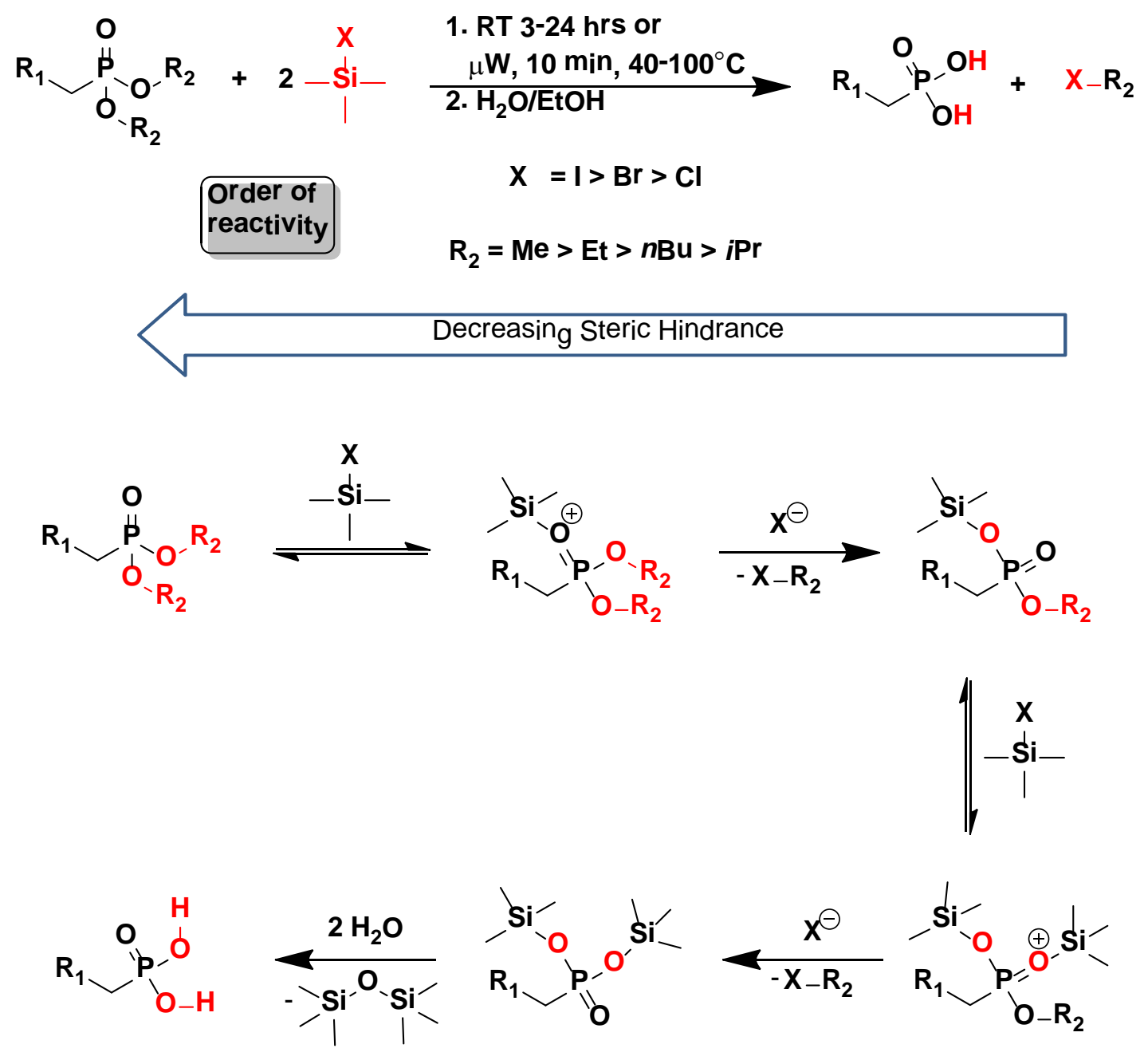

Figure 1.57: Mechanism of the didealkylation of dialkylphosphonate esters via silylation and hydrolysis. 

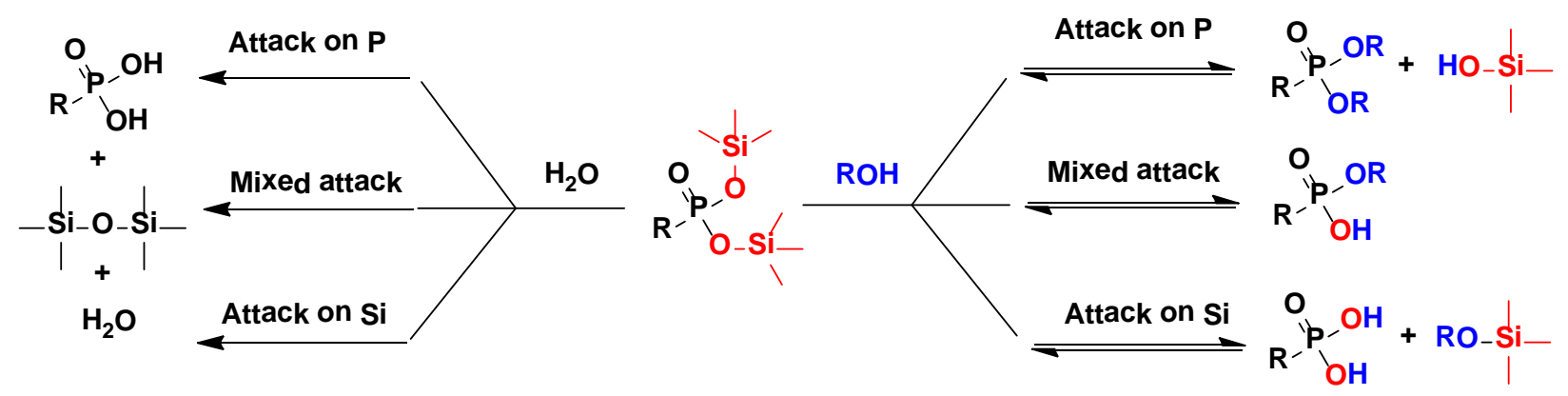

Figure 1.58: Possible products resulting from the hydrolysis of bistrimethylsilyl phosphonates.

Similarly, nucleophilic displacement of alkyl groups from phosphonate esters goes to completion with lithium trialkylborohydrides via a $\mathrm{S}_{\mathrm{N}} 2$ meachanism (Table 1.14, Figure 1.59). ${ }^{185}$ The advantages of using silicone and boron reagents include milder reaction conditions (RT) and greater function group sensitivity. Additionally, these reagents are compatible with aryl phosphonate esters, carboxylic ester, ethers, halo alkyl, alkyne and alkene functional groups. ${ }^{186,}$ 187 
Table 1.14: Literature examples of the dealkylation of phosphonate esters with $\mathrm{BBr}_{3}$.

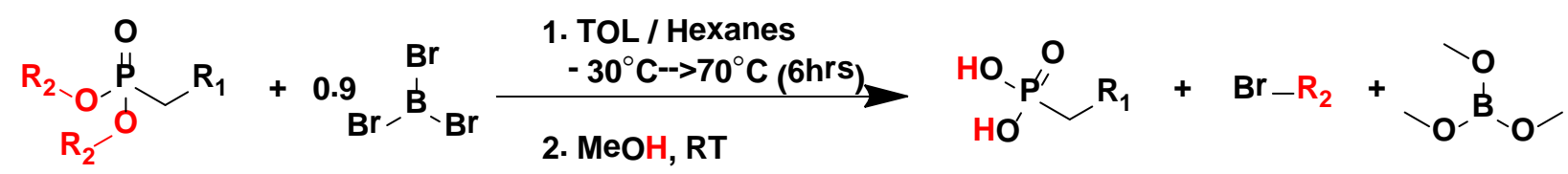

\begin{tabular}{cccc} 
Entry & Starting Material & Products & Yield (\%) \\
\hline i & $\mathrm{RO}_{-\mathrm{P}^{\prime}} \mathrm{O}^{\prime}$ & $\mathrm{HO} \mathrm{O}_{-\mathrm{P}^{\prime}}^{\mathrm{O}}$ & \\
& $\mathrm{RO}^{\prime}$ & $\mathrm{HO}^{\prime}$ & $90^{\mathrm{a}}, 95^{\mathrm{b}}, 89^{\mathrm{c}}, 84^{\mathrm{d}}$
\end{tabular}

ii<smiles>CCCCCCP(=O)(CC)OCC</smiles>

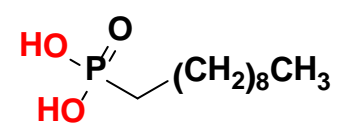

ii<smiles>C=CCP(=O)(OC)OC</smiles><smiles>C=CCP(=O)(O)O</smiles>

iv<smiles>CCOP(=O)(Cc1ccccc1)OCC</smiles>

v<smiles>CCOP(=O)(CCBr)CCBr</smiles>

vi<smiles>CCOP(=O)(CC#N)OCC</smiles>

vii

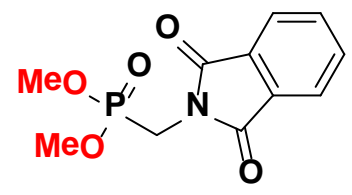<smiles>O=P(O)(O)Cc1ccccc1</smiles><smiles>O=P(O)(O)CCBr</smiles><smiles>N#CCP(=O)(O)O</smiles><smiles>O=C1c2ccccc2C(=O)N1CP(=O)(O)O</smiles>

80

80

89

$\left.\underline{\text { (Redrawn from Ref. }}{ }^{186}\right)$ a, $(\mathrm{R}=\mathrm{Me}), \mathrm{b},(\mathrm{R}=\mathrm{Et}), \mathrm{c},(\mathrm{R}=i \mathrm{Pr}), \mathrm{d},(\mathrm{R}=t-\mathrm{Bu})$ 

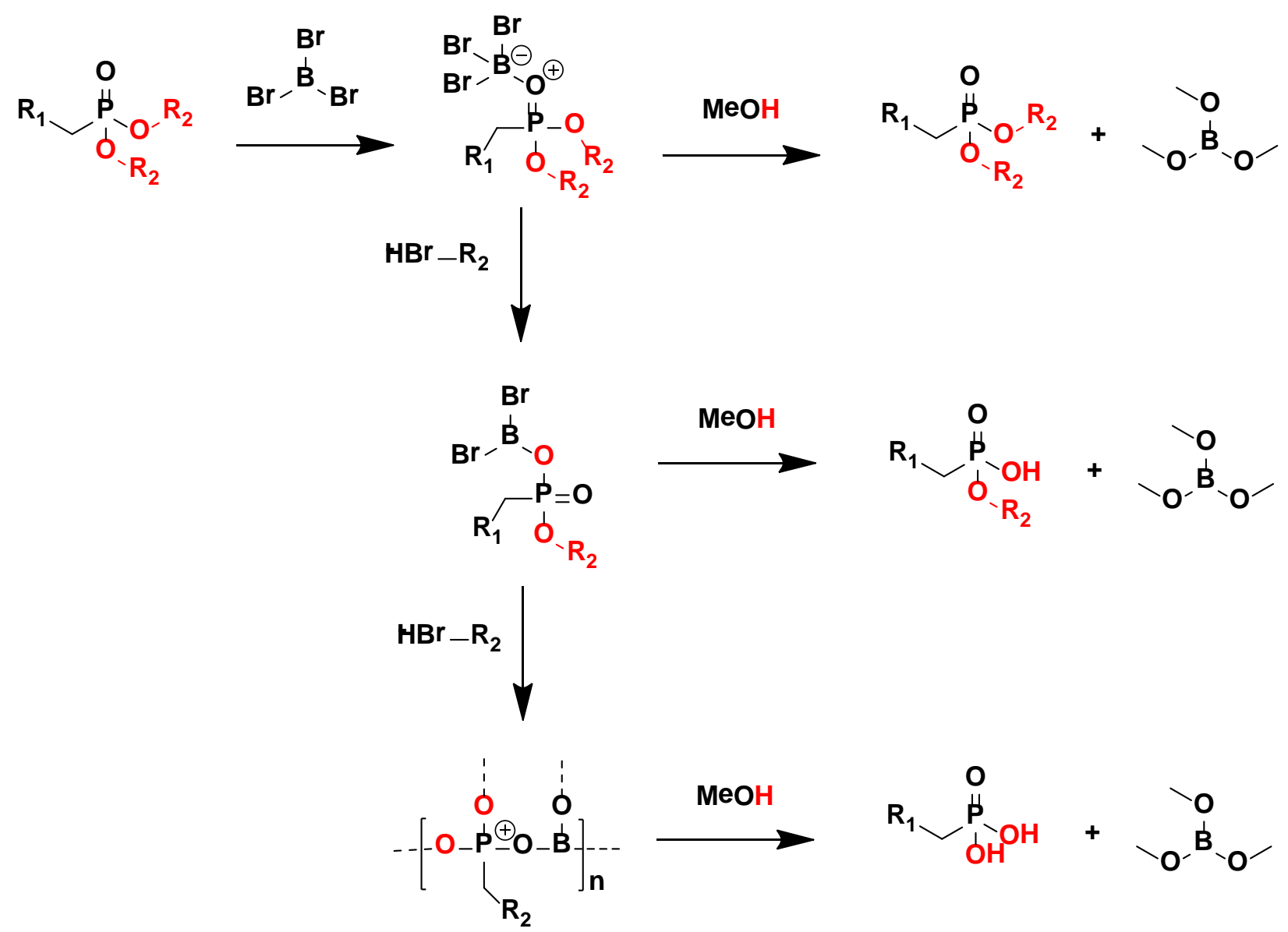

Figure 1.59: Mechanism of phosphonic acid dealkylation with $\mathrm{BBr}_{3}{ }^{188,189}$

\subsubsection{Synthesis of Phosphonic Acids}

The synthesis of $\gamma$-monophosphonic acid QAC’s with hydrophobic tails typically from 12 to 18 carbons long are known in the literature. Two syntheses describe the preparation of a $\mathrm{C}_{18} \gamma$ monophosphonic acid QAC (Figure 1.60), one starting with the bromophosphonate (Route a) while the other with the bromoQAC (Route b), and both are obtained from an Abruzov reaction. Quaternary ammonium phosphonate compounds prepared by Route b were patented in 1955 for use as synthetic detergents. ${ }^{190,191}$ In the reported synthesis, the final product could only be isolated as Na salt of the phosphobetaine after hydrolysis of the phosphonate ester with $\mathrm{HCl}$ followed by treatment with $\mathrm{NaHCO}_{3}$ (Figure 1.60, a). Route b was used by Germanaud who 
isolated the quats as betaines after purification on an anion exchange resin (Figure 1.60,b). ${ }^{192}$ The products synthesized by this method were not spectroscopically characterized in the patents and were used as is, while Germanaud's purification is costly and doesn't isolate the product as a phosphonic acid. ${ }^{192}$ The free phosphonic acid with a $\mathrm{C}_{18}$ was never reported (Figure 1.60, d), however the preparation of the $\mathrm{C}_{12}$ analog by method c starting from 3-bromophosphonic acid is known. ${ }^{193}$ Purification called for extraction of the $\mathrm{H}_{2} \mathrm{O}$ soluble $\mathrm{C}_{12}$ betaine into $\mathrm{CHCl}_{3}$, and no mention of emulsions was reported. ${ }^{193}$

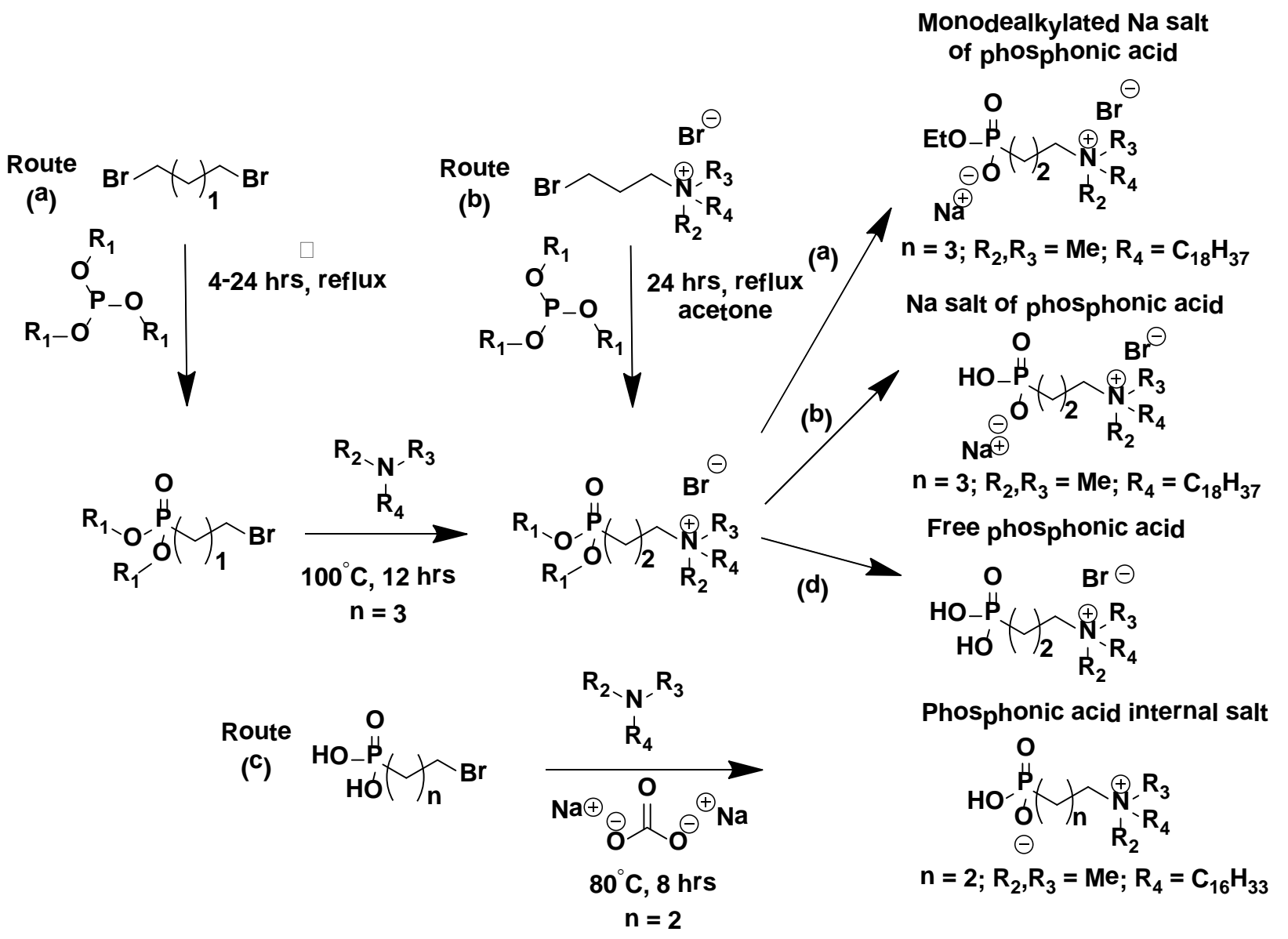

Figure 1.60: Literature routes describing the preparation of QA phosphonic acids. ${ }^{189-192}$ 


\subsection{RESEARCH GOALS}

The initial aims of this research were to prepare the EPA approved Dow antimicrobial (3(trimethoxysilyl)-propyldimethyloctadecyl ammonium chloride) ${ }^{37}$ or (SiQAC, 3) and to improve on the original synthesis by altering the reaction parameters and by employing $\mu \mathrm{W}$ heating.

The primary research goals were to synthesize cost-effective and commercialy viable QAC antimicrobials capable of adhering to surfaces other than porous ones (i.e. Ti, SS, plastics) for use in the health care and food handling facilities to help curb the spread of nonsomical infection spread by direct contact with "touch surfaces" colonized by pathogenic bacteria. These new antimicrobials are expected to (i) possess broad spectrum antimicrobial activity, (ii) maintain activity / stability on the treated surface over extended periods of time (months) and (iii) kill microbes on contact without leaching of the chemical into the environment (prevent development of bacterial resistance).

It was hypothesized that the replacement of the trimethoxysilane $\left(\mathrm{SiOMe}_{3}\right)$ anchor present in Dow's commercial antimicrobial with a phosphonate $\left(\mathrm{PO}_{3} \mathrm{R}_{2}\right)$, phosphonic acid $\left(\mathrm{PO}_{3} \mathrm{H}_{2}\right)$, catechol or thiol end group would dramatically improve adhesion to non-porous surfaces, specifically metals, while retaining the broad spectrum antimicrobial activity of the quaternary ammonium compound. Therefore, a variety of novel and synthetically unknown compounds were targeted comprising of either a mono- (34-37), bis-(42, 47, 96, 126, 141), tris-

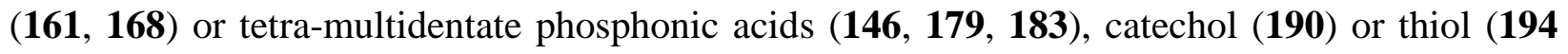
and 199) end group connected to a long chain quaternary ammonium group. Additionally, the synthesis of a new photoactive benzophenone-silane QAC 206 for coating onto non-porous surfaces, specifically plastic surfaces (C-H surfaces, i.e. polyethylene, silicones) is described. 
Following synthesis, antimicrobial properties in solution and of monophosphonates 26 and $34 \mathbf{A}$ on metal surfaces ( $\mathrm{SS}, \mathrm{Ti}, \mathrm{Al}$ ) as well as their detection with dansyl QAC fluorophores and or bromophenol blue was investigated.

\section{0 - RESULTS AND DISCUSSION}

\subsection{Synthesis}

\subsubsection{Alkoxysilane-Functional Quaternary Ammonium Cations (SiQAC)}

The Dow antimicrobial (SiQAC, 3), synthesized according to published procedures ${ }^{37}$ suffers from poor purity and a lower than anticipated level of the active antimicrobial in the concentrate formula (< $72 \mathrm{wt} \%)$. Commercially, compound 3 is prepared from 3-chloropropyl trimethoxysilane 1 (excess $\sim 1.2$ eq.) and $N, N$-dimethyloctadecylamine 2 (Scheme 2.1) as a concentrate in methanol by the following companies: Aegis (AEM 5772); Piedmont (Ztrex72); Flexipel (Q-1000), and Dow Corning (Q9-6346). ${ }^{45}$ Industrially, the final concentrate is used "as is” without a purification step to remove any unreacted starting materials, and as a result, a varying amount of active quat is often produced (Table 2.1, Entry i). The composition of the concentrate on average ranges from $\sim 72 \mathrm{wt} \%$ for the the active quat $3, \sim 15 \mathrm{wt} \%$ of unreacted 1, 1-5 wt \% of 2, and 13 wt \% MeOH (Table 2.1, Entry i).

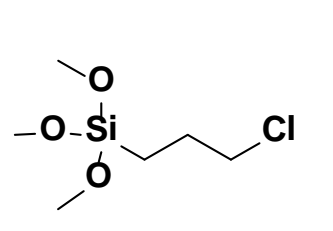

$(\mathbf{1})$

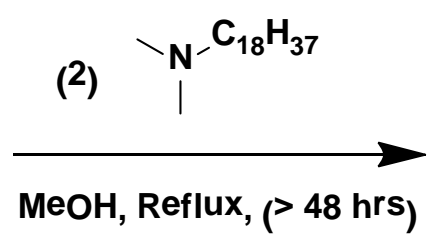

MeOH, Reflux, (> $48 \mathrm{hrs}$ )

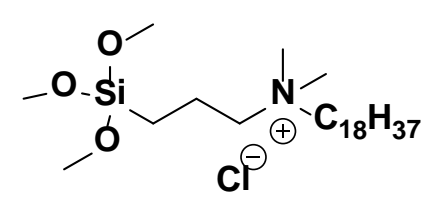

(3)

Scheme 2.1: Preparation of Dow's antimicrobial 3.

Literature (Table 2.2, Entries ii, iii, and v) and patent procedures (Table 2.2, Entries i and iv) describe the preparation of 3 with typically long reaction times ( $>24 \mathrm{hrs)} \mathrm{but} \mathrm{fail} \mathrm{to} \mathrm{provide}$ 
any spectral (NMR) characterization data for the product. Klaus observed complete conversion of starting materials to 3 only after 3 to $4 \mathrm{~d}$. in $\mathrm{MeOH}$ without reporting any spectral data (Table 2, Entry ii) ${ }^{194}$ Similarly, Chisholm performed the reaction under solventless conditions at $110^{\circ} \mathrm{C}$ for $48 \mathrm{hrs}$ in a sealed tube and directly prepared a $50 \mathrm{wt} \% \mathrm{MeOH}$ solution without initial characterization (Table 2.1, Entry iii). ${ }^{195}$ In a recent patent, Ludwig reacted $\mathbf{1}$ with a variety of commercially available products containing alkyl amine mixtures with varying carbon chain lengths $\left(\mathrm{C}_{10}, \mathrm{C}_{12}, \mathrm{C}_{14}, \mathrm{C}_{16}, \mathrm{C}_{18}\right.$ in different ratios) under neat conditions and reported a $100 \%$ conversion at $90^{\circ} \mathrm{C}$ after 48 hrs with a commercial starting material consisting mostly of $\mathbf{2}$ (Table 2.1, Entry iv). ${ }^{45}$ Instead of NMR, completion of the reaction was determined by diluting aliquots of the reaction mixture first in propylene glycol (1:1, $2 \mathrm{~g}$ total) followed by $\mathrm{H}_{2} \mathrm{O}(1000 \mathrm{~mL}$ of $\mathrm{pH}$ 3, $500 \mathrm{ppm}$ final dilution) at different time intervals and titrating for the $\mathrm{Cl}^{-}$ion content (CHEMetrics, Inc., Calverton, Va). ${ }^{45}$ Lastly, Huang reported the synthesis of $\mathbf{3}$ using $\mu \mathrm{W}$ radiation in a $89 \%$ yield at $150^{\circ} \mathrm{C}$ for $1.5 \mathrm{hrs}$ in $\mathrm{MeOH}$ (Table 2.1, Entry v). ${ }^{196}$

Table 2.1: Literature/patent procedures describing the preparation of SiQAC 3.

\begin{tabular}{|c|c|c|c|c|c|c|c|c|}
\hline \multicolumn{4}{|c|}{} & \multicolumn{3}{c|}{$\begin{array}{c}\text { Composition of reaction (rxn) } \\
\text { mixture (\%) }\end{array}$} \\
\hline Entry & Solvent & $\begin{array}{c}\text { Time } \\
\text { (hrs) }\end{array}$ & $\begin{array}{c}\text { Temp } \\
\left.\mathbf{(}^{\mathbf{0}} \mathbf{C}\right)\end{array}$ & $\begin{array}{c}\text { Ratio } \\
\mathbf{( 1 ) : ( 2 )}\end{array}$ & $\mathbf{1}$ & $\mathbf{2}$ & $\mathbf{3}$ & MeOH \\
\hline $\mathrm{i}^{37}$ & $\mathrm{MeOH}$ & $\mathrm{n} / \mathrm{a}$ & $\mathrm{n} / \mathrm{a}$ & $1.2: 1$ & 15 & $1-5$ & 72 & 13 \\
\hline $\mathrm{ii}^{197}$ & $\mathrm{MeOH}$ & $72-96$ & 110 & $1: 1$ & $\mathrm{n} / \mathrm{a}$ & $\mathrm{n} / \mathrm{a}$ & $\mathrm{n} / \mathrm{a}$ & $\mathrm{n} / \mathrm{a}$ \\
\hline $\mathrm{iii}^{195}$ & Neat & 48 & 110 & $1: 0.95$ & $\mathrm{n} / \mathrm{a}$ & $\mathrm{n} / \mathrm{a}$ & $\mathrm{n} / \mathrm{a}$ & 50 \\
\hline $\mathrm{iv}^{45}$ & Neat & 48 & 90 & $1: 1$ & 0 & 0 & 100 & Neat \\
\hline $\mathrm{v}^{196}$ & $\mathrm{MeOH}$ & 1.5 & 150 & - & - & - & 89 & - \\
\hline
\end{tabular}
5


Due to the added costs and difficulties associated with distillation ${ }^{197}$ and column chromagraphy necessary to purifying the final product, driving the reaction to completion employing solventless conditions and or $\mu \mathrm{W}$ heating would (i) save on energy costs, (ii) avoid the shipment of the concentrate with flammable organic solvents and (iii) reduce the environmental impact of the toxic unreacted impurities $(\mathbf{1}, \mathbf{2})$ in the concentrate and (iv) potentially require less stabilizer.

Microwave $(\mu \mathrm{W})$ heating was investigated in the synthesis of $\mathbf{3}$ according to Scheme 2.1 in order to improve the yield ( $72 \%$, commercially) by altering the reaction time, temperature and solvent choice. Results from both sealed tube (ST) and $\mu \mathrm{W}$ reactions employing solvent and neat conditions are summarized in Table 2.2. Complete conversion to 3 employing a 1:1 or a 1:0.95 ratio of 1:2 was never observed even after 48 hrs reflux in $\mathrm{MeOH}$ (Table 2.2, Entry v) or 72 hrs at $110^{\circ} \mathrm{C}$ under neat conditions (Table 2.2, Entry iii). These results clearly contrast those reported by Ludwig (Table 2.1, Entry ii), where complete conversion was observed at $90^{\circ} \mathrm{C}$ after $3 \mathrm{~d}$. Monitoring the reaction by ${ }^{1} \mathrm{H}$ NMR is advantageous over the titration method for free $\mathrm{Cl}^{-}$ content used by Ludwig and clearly shows that the reaction never achieves completion. At best, a 76-80\% yield is obtained only after prolongued heating times (> $48 \mathrm{hrs}$ ).

Additional $\mu \mathrm{W}$ reactions (4 mmol scale) were attempted in hopes of obtaining a further conversion to quat $\mathbf{3}$ by employing higher temperatures and shorter reaction times. Performing the reaction neat in the $\mu \mathrm{W}$ (Table 2.2, Entry vi) resulted in only a 10\% conversion after 45 min at $150^{\circ} \mathrm{C}$ whereas under similar reaction conditions, but with $\mathrm{MeOH}$, the reaction reached $58 \%$ conversion (Table 2.2, Entry vii). These results support those obtained by Huang who observed an $89 \%$ conversion after $90 \mathrm{~min}$ at $150^{\circ} \mathrm{C}$ in $\mathrm{MeOH}$ under $\mu \mathrm{W}$ heating (Table 2.1, Entry v). ${ }^{196}$ Another $\mu \mathrm{W}$ experiment employing the higher boiling $i \mathrm{PrOH}$ instead of $\mathrm{MeOH}$, at $165^{\circ} \mathrm{C}$ for 60 
min (Table 2.2, Entry xiii), also failed to drive the quaternization to completion with product conversion reaching only $64 \%$.

Table 2.2: ${ }^{1} \mathrm{H}$ NMR $\left(\mathrm{CDCl}_{3}\right)$ monitoring of the formation of 3 .

(\%) Conversion (limiting reagent) by ${ }^{1} \mathrm{H}$ NMR $\left(\mathrm{CDCl}_{3}\right)$

\begin{tabular}{|c|c|c|c|c|c|}
\hline Entry & Solvent & Time (hrs) & Temp ( $\left.{ }^{\mathbf{0}} \mathbf{C}\right)$ & Ratio (1):(2) & PRD. (3 \%) \\
\hline i & Neat & 24 & 110 & $1: 0.95$ & $\sim 30$ \\
\hline ii & Neat & 48 & 110 & $1: 0.95$ & $\sim 53$ \\
\hline iii & Neat & 72 & 110 & $1: 0.95$ & $\sim 80$ \\
\hline iv & MeOH & 24 & Reflux & $1: 1$ & $\sim 45$ \\
\hline v & MeOH & 48 & Reflux & $1: 1$ & $\sim 76$ \\
\hline vi & Neat & 0.45 & $150^{\mu \mathrm{w}}$ & $1: 1$ & $\sim 10$ \\
\hline vii & MeOH & 0.45 & $150^{\mu \mathrm{w}}$ & $1: 1$ & $\sim 58$ \\
\hline viii & iPrOH & 0.6 & $165^{\mu \mathrm{w}}$ & $1: 0.95$ & 64 \\
\hline
\end{tabular}

n/a : not attempted.

Progress of the formation of 3 was monitored with ${ }^{1} \mathrm{H}$ NMR $\left(\mathrm{CDCl}_{3}\right)$ by observing the disappearance of the overlapping dimethylamino and amino $-\mathrm{CH}_{2}$ protons $\left[-\underline{\mathrm{CH}}_{2}-\mathrm{N}\left(\underline{\mathrm{CH}}_{3}\right)_{2}\right]$ at $2.2 \mathrm{ppm}$ from the limiting starting amine $\mathbf{2}$ and the appearance of two new upfield resonance at $3.5 \mathrm{ppm}\left[-\underline{-\mathrm{CH}_{2}} \underline{\mathrm{N}}^{+}\left(\mathrm{CH}_{3}\right)_{2}\right]$ and $~ 3.3 \mathrm{ppm}\left[\left(-\mathrm{N}^{+}-\left(\underline{\mathrm{CH}_{3}}\right)_{2}\right]\right.$ resulting from the quaternized product (Figure 2.1). ${ }^{101}$ Percent conversion was calculated from ${ }^{1} \mathrm{H}$ NMR $\left(\mathrm{CDCl}_{3}\right)$ integrations of the $N, N$-dimethyl peaks from the limiting starting amine 2 and the quat dimethyl peak in the product $\mathbf{3}$ according to the following formula:

$$
\% \text { Conversion }=(\mathrm{x} / 6) /((\mathrm{x} / 6)+(\mathrm{y} / 8))
$$


where $\mathrm{y}=$ the disappearance of the $\left(-\mathrm{N}\left(\mathrm{CH}_{3}\right)_{2}\right) \mathrm{SM} .2$ peak $\sim 2.3 \mathrm{ppm}$, and $\mathrm{x}=$ the formation of [(-N $\left.{ }^{+}-\left(\underline{\mathrm{CH}}_{3}\right)_{2}\right]$ PRD. 3 peak at 3.3 ppm (See, Figure 2.1 A and B, Appendix 1.2, Figure A18, A19). For example, \% conversion $(2.48 / 6) /[(2.48 / 6)+(2.97 / 8)]=53 \%$ (Table 2.2, Entry ii).

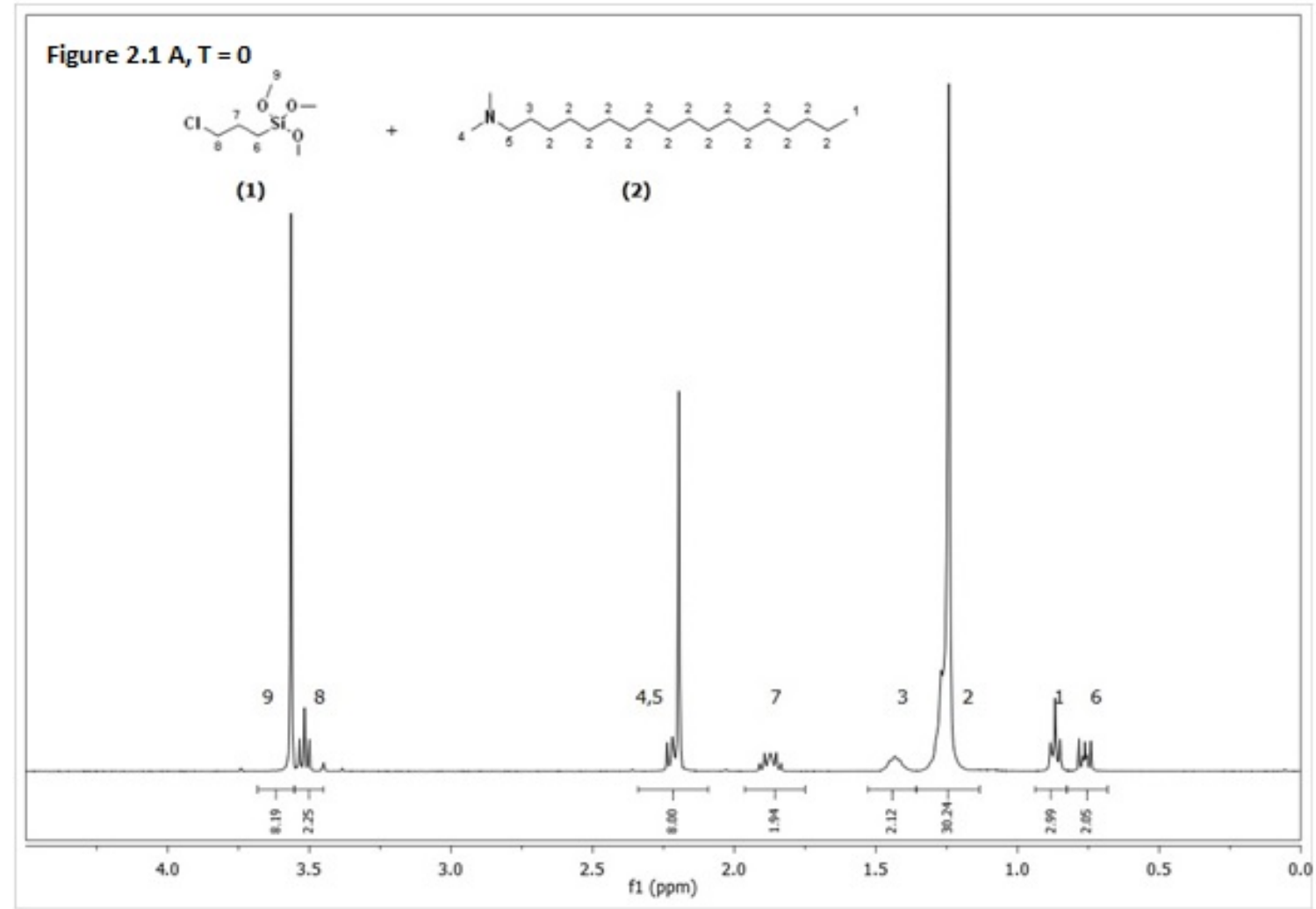




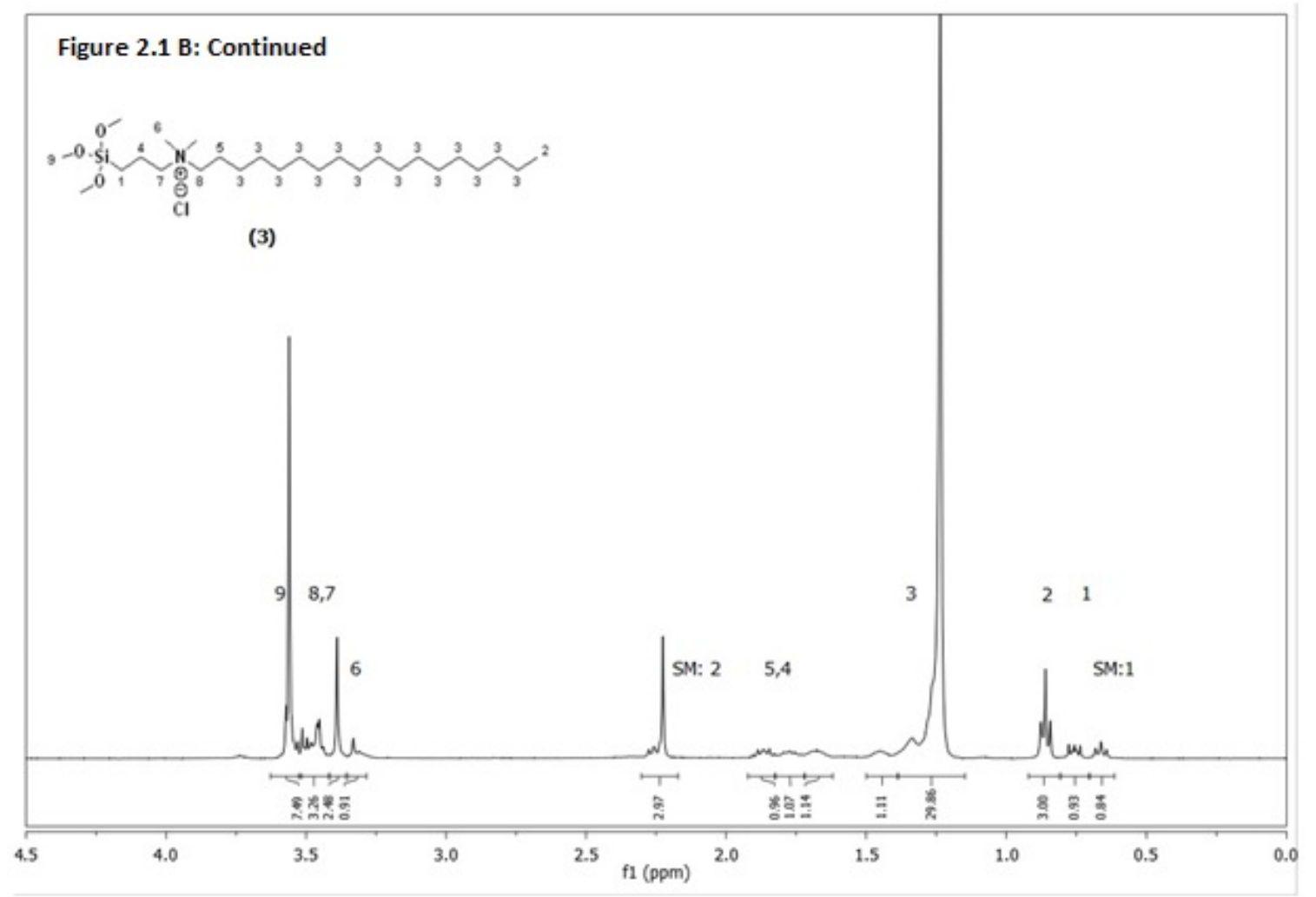

Figure 2.1: ${ }^{1} \mathrm{H}$ NMR $\left(\mathrm{CDCl}_{3}\right)$ spectra of compound $1+2$ in at $\mathrm{T}=0$ (A), and the formation of 3 at $\mathrm{T}=48 \mathrm{hrs}(\mathrm{B})$.

Another option to save on energy during manufacturing is replacing the cheap but unreactive 1 with more reactive silanes (Figure 2.2). Employing other trimethoxysilanes like the bromo or iodo derivative would significantly speed up the Menshutkin reaction, however due to their high costs (> $100 \times$ more expensive) versus the chloro derivative, this option is commercially unrealistic (Figure 2.2).

\begin{tabular}{|c|c|c|c|}
\hline $\mathrm{O}_{\mathrm{O}_{-}}^{-\mathrm{Si}} \widehat{\mathrm{Cl}}_{\mathrm{O}}$ & ${ }^{-}{ }^{-} \mathrm{Si}_{\mathrm{O}_{-}} \sim_{\mathrm{Br}}$ & ${ }_{0}^{-0} \mathrm{Si}_{\mathrm{O}}$ & $\mathrm{O}_{\mathrm{O}}^{-\mathrm{Si}}$ \\
\hline $\begin{array}{l}\$ 0.25 \text { I } 1 \mathrm{~mL} \text { SA } \\
\$ 0.05 \text { / } 1 \mathrm{~mL} \text { Gelest }\end{array}$ & $\begin{array}{l}\$ 61.4 \text { I 1 mL SA } \\
\$ 5.7 \text { I 1 mL Gelest }\end{array}$ & $\begin{array}{l}\$ 5.38 \text { / } 1 \mathrm{~mL} \text { SA } \\
\$ 2.16 / 1 \mathrm{~mL} \text { Gelest }\end{array}$ & $\begin{array}{l}\$ 6.86 \text { / } 1 \mathrm{~mL} \mathrm{SA} \\
\$ 3.58 \text { / } 1 \mathrm{~mL} \text { Gelest }\end{array}$ \\
\hline
\end{tabular}

Figure 2.2: Comparison of trimethoxysilane pricing as of May 2013 (SA: Sigma Aldrich). 


\subsubsection{Organophosphorus Functional Quaternary Ammonium Cations QAC Antimicrobials (PQAC)}

\subsubsection{1 $\gamma$-Monophosphonic Acids QAC Antimicrobials ( $\gamma$-MPQAC)}

A series of $\gamma$-monophosphonic acid QAC’s ( $\gamma$-MPQAC) derivatives 34-37 for binding onto $\mathrm{Ti}$, SS and $\mathrm{Al}$ were prepared in three steps (Scheme 2.2). First, the Abruzov reaction between trialkyl phosphites (4, 5A-B) and dibromoalkanes 6-16 or haloalkanes 18-19 produced the phosphonate esters 12-19 in 50-93 \% yield (Tables 2.3, Entries i-xix). Compounds 12-14 were quaternized with various tertiary amines $\mathbf{2 - 2 4}$ by the Menshutkin reaction to produced QAC phosphonate esters 26-32, while compounds 12-13 were alkylated with $\mathrm{HNMe}_{2}$ and $\mathrm{NaN}_{3}$ to give precursors 32 and 33 for use in Section 2.1.3.2, Method 3. Didealkylation of 26-30 with either $\mathrm{HX}(\mathrm{X}=\mathrm{Cl}$ or $\mathrm{Br})$ or $\mathrm{TMSBr}$ afforded the desired antimicrobial phosphonic acid derivatives 3437. All three steps were optimized with $\mu \mathrm{W}$ heating which resulted in shorter reaction times at higher temperatures and improved yields (Tables 2.3 - 2.5, Scheme 2.2). 
Step 1 - Abruzov Reaction

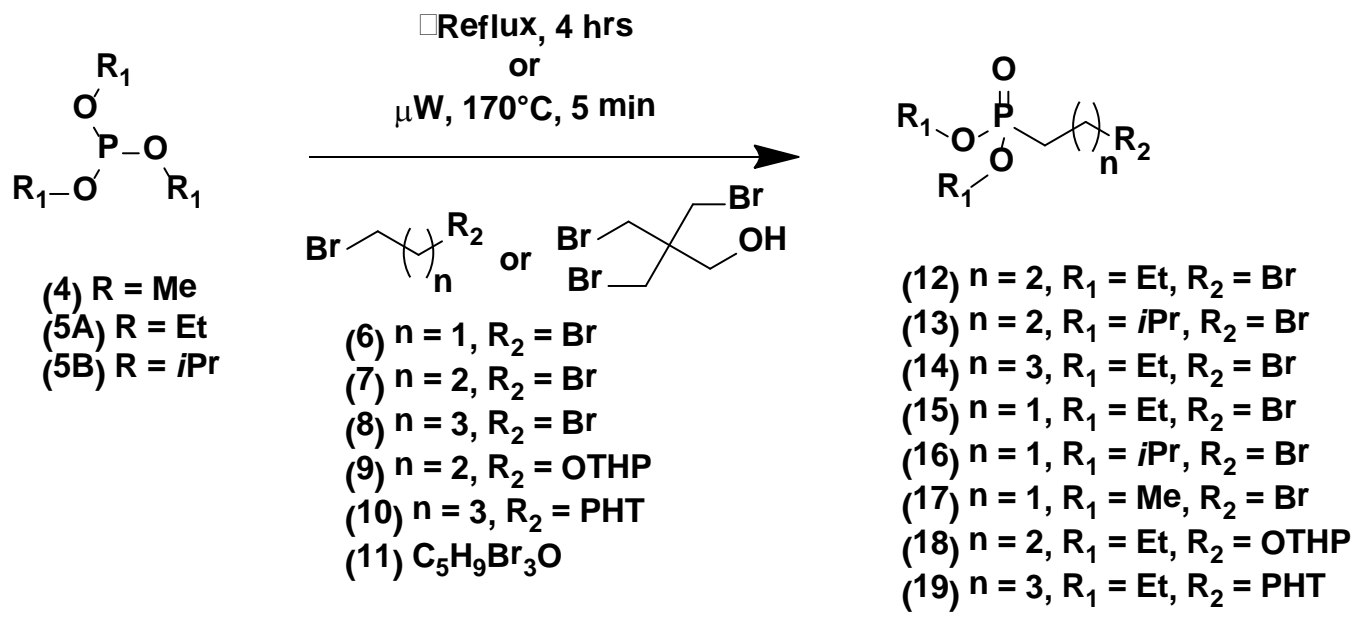

Step 2 - Alkylation, Quaternary Amine Formation (Menschutkin Reaction)

Neat, $100^{\circ} \mathrm{C}, 35 \mathrm{~min}$ or

$\mathrm{ACN}, \mu \mathrm{W}, 150^{\circ} \mathrm{C}, 2 \mathrm{~min}$

$(12-14)$

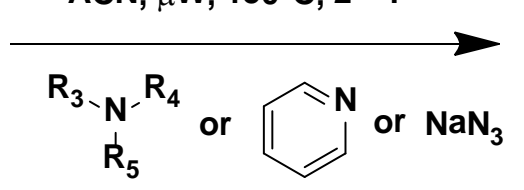

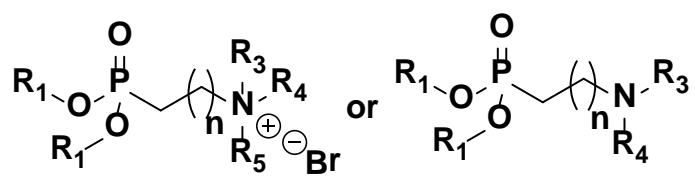
(2) $\mathrm{R}_{3}, \mathrm{R}_{4}=\mathrm{Me}, \mathrm{R}_{5}=\mathrm{C}_{18} \mathrm{H}_{37}$
(20) $R_{3}, R_{4}, R_{5}=M e$
(21) Pyridine
(22) $R_{3}, R_{4}=M e, R_{5}=C_{12} H_{25}$
(23) $\mathrm{R}_{3}=\mathrm{Me}, \mathrm{R}_{4}=\mathrm{H}, \mathrm{R}_{5}=\mathrm{C}_{18} \mathrm{H}_{37}$
(24) $R_{3}, R_{4}=M e, R_{5}=H$
(25) $\mathrm{NaN}_{3}$

(26) $n=2, R_{1}=E t, R_{3}, R_{4}=M e, R_{5}=C_{18} H_{37}$

(27) $\mathrm{n}=2, \mathrm{R}_{1}=i \mathrm{Pr}, \mathrm{R}_{3}, \mathrm{R}_{4}=\mathrm{Me}, \mathrm{R}_{5}=\mathrm{C}_{18} \mathrm{H}_{37}$

(28) $n=2, R_{1}=i P r, R_{3}, R_{4}, R_{5}=M e$

(29) $n=2, R_{1}=T M S, R_{3}, R_{4}, R_{5}=$ pyridinium

(30) $n=2, R_{1}=E t, R_{3}, R_{4}=M e, R_{5}=C_{12} H_{25}$

(31) $n=2, R_{1}=E t, R_{3}, R_{4}=M e, R_{5}=C_{18} H_{37}$

(32) $n=3, R_{3}, R_{4}=M e$

(33) $n=2, R_{3}, R_{4}, R_{5}=N_{3}$

Step 3 - Phosphonate Ester Didealkylation

A) TMSBr, DCM, RT, ON or $\mathrm{ACN}, \mu \mathrm{W}, 60^{\circ} \mathrm{C}, 10 \mathrm{~min}$

$(26-30)$
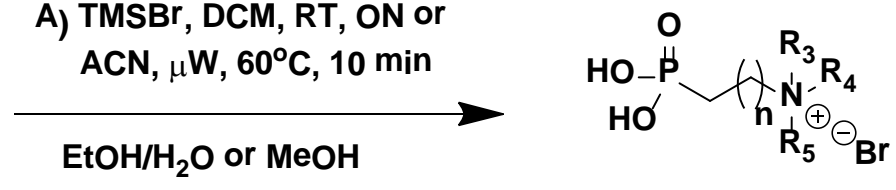

B) 1-6 M HX, $100^{\circ} \mathrm{C}, 1-8 \mathrm{hrs}$
or $\mu \mathrm{W}, 140^{\circ} \mathrm{C}, 4-10 \mathrm{~min}$

$$
\begin{aligned}
& (34 A) n=2, R_{3}, R_{4}=M e, R_{5}=C_{18} H_{37} \\
& (34 B) n=2, R_{3}, R_{4}=M e, R_{5}=C_{18} H_{37}\left(N^{+} \text {salt }\right) \\
& (35) n=2, R_{3}, R_{4}, R_{5}=M e \\
& (36) n=2, R_{3}, R_{4}, R_{5}=\text { pyridinium } \\
& (37) n=2, R_{3}, R_{4}=M e, R_{5}=C_{12} H_{25}
\end{aligned}
$$

Scheme 2.2: Optimized conditions for the synthesis of $\gamma$-monophosphonic acid QAC's. 
Short chain $n$-bromoalkylphosphonates are commercially available although rather expensive ( \$101.50/5 mL, for compound 12 (S-A, 95\%)). As mentioned above, their preparation is achieved through the classic Abruzov reaction and involves heating the two reactants (4, 5A-B) with 6,7 or 8) neat under conventional heating (at reflux) or under $\mu \mathrm{W}$ radiation followed by distillation to isolate the target products 12-17. Table 2.3 summarizes the optimization experiments to obtain products 12-17 and details the major by-products that codistilled together with attempted purification.
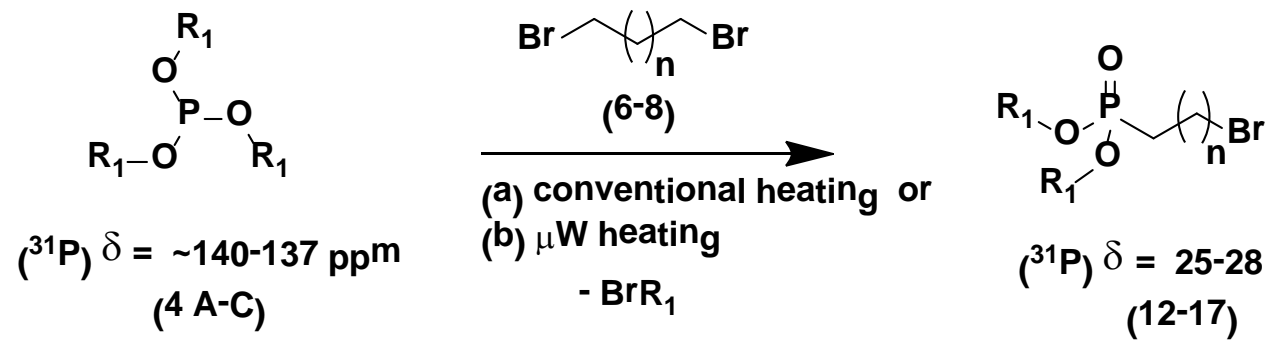

(b) $\mu$ Weatin

$$
\left({ }^{31} \mathrm{P}\right) \delta=25-28 \mathrm{ppm}
$$

$(12-17)$

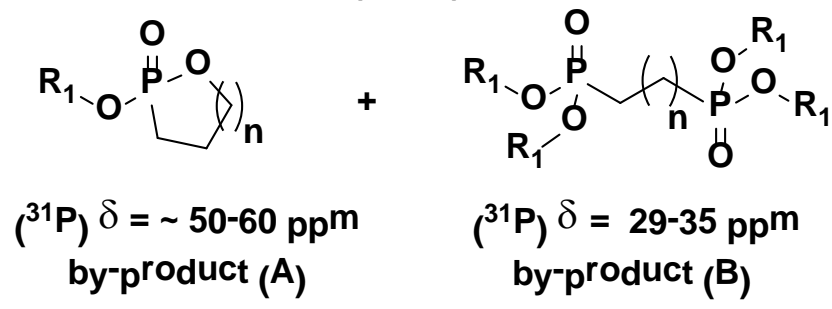

Table 2.3: Optimization of Step 1: Abruzov reaction between trialkyl phosphites $(\mathbf{4}, \mathbf{5 A - B})$ and dibromoalkanes $(\mathbf{6}, \mathbf{7}$ or $\mathbf{8})$ or bromoalkanes $(\mathbf{9}, \mathbf{1 0}$ or $\mathbf{1 1})$.

\begin{tabular}{|c|c|c|c|c|c|c|c|}
\hline \multicolumn{8}{|c|}{ Rxn. Comp. (\%) by $\left({ }^{1} \mathrm{H},{ }^{31} \mathrm{P}, \mathrm{CDCl}_{3}\right) \mathrm{NMR}$} \\
\hline Entry & Compound & Time & $\begin{array}{c}\text { Temp. } \\
\left({ }^{\circ} \mathrm{C}\right)\end{array}$ & $\begin{array}{l}\text { Ratio of } \\
\text { reactants }\end{array}$ & PRD. & $\begin{array}{l}\text { BY- } \\
\text { PRD. A } \\
\%\end{array}$ & $\begin{array}{c}\text { BY- } \\
\text { PRD. B } \\
\%\end{array}$ \\
\hline $\mathrm{i}$ & 0 & $20 \mathrm{hrs}$ & Reflux & $(5 A+7) / \underline{1}: \underline{5}$ & $52^{\mathrm{D}}$ & $4^{\mathrm{D}}$ & 0 \\
\hline ii & \multirow{2}{*}{ (12) } & $20 \mathrm{hrs}$ & Reflux & $(5 \mathrm{~A}+7) / \underline{1}: \underline{4}$ & $89.9^{\mathrm{D}}$ & $7^{\mathrm{D}}$ & $0.1^{\mathrm{D}}$ \\
\hline iii & & $6 \mathrm{hrs}$ & Reflux & $(5 \mathrm{~A}+7) / \underline{1}: \underline{4}$ & $79^{\mathrm{D}}$ & $\mathrm{n} / \mathrm{d}$ & $\mathrm{n} / \mathrm{d}$ \\
\hline
\end{tabular}




\begin{tabular}{|c|c|c|c|c|c|c|c|}
\hline Entry & Compound & Time & $\begin{array}{c}\text { Temp. } \\
\left({ }^{\circ} \mathrm{C}\right)\end{array}$ & $\begin{array}{l}\text { Ratio of } \\
\text { reactants }\end{array}$ & PRD. & $\begin{array}{c}\text { BY- } \\
\text { PRD. A } \\
\quad \%\end{array}$ & $\begin{array}{c}\text { BY- } \\
\text { PRD. B } \\
\quad \%\end{array}$ \\
\hline iv & & $3 \mathrm{hrs}$ & Reflux & $(5 B+7) / \underline{1}: \underline{4}$ & 79 & 21 & 0 \\
\hline $\mathrm{V}$ & & $3.4 \mathrm{hrs}$ & Reflux & $(5 B+7) / \underline{1}: \underline{4}$ & 80 & 14 & 0 \\
\hline vi & (13) & $2 \min$ & 170 & $\begin{array}{c}(5 \mathrm{~B}+7) / \\
1: \underline{1.2}\end{array}$ & 48 & 17 & 0 \\
\hline vii & & $5 \mathrm{~min}$ & 170 & $\begin{array}{c}(5 \mathrm{~B}+7) / \\
1: 1.2\end{array}$ & $74^{\mathrm{D}}$ & $10^{\mathrm{D}}$ & 0 \\
\hline viii & & 20 hrs & Reflux & $(5 \mathrm{~A}+8) / \underline{1}: \underline{4}$ & 50 & 15 & 35 \\
\hline $\mathrm{xi}$ & & $5 \min$ & 190 & $(5 \mathrm{~A}+8) / \underline{1}: \underline{2}$ & $72^{\mathrm{D}}$ & $10^{\mathrm{D}}$ & 0 \\
\hline $\mathrm{X}$ & (14) & $5 \min$ & 170 & $\begin{array}{c}(5 \mathrm{~A}+8) / \\
1: \underline{1.2}\end{array}$ & 70.4 & 19 & 0 \\
\hline $\mathrm{xi}$ & & $5 \mathrm{~min}$ & 170 & $(5 \mathrm{~A}+8) / \underline{1}: \underline{2}$ & 74 & 2 & 10 \\
\hline xii & 0 & $2.2 \mathrm{hrs}$ & Reflux & $(5 \mathrm{~A}+6) / \underline{1}: \underline{4}$ & 67 & 15.4 & 0 \\
\hline xiii & & $4.3 \mathrm{hrs}$ & Reflux & $(5 \mathrm{~A}+6) / \underline{1}: \underline{4}$ & 71.6 & 15.4 & 0 \\
\hline xiv & (15) & 36 hrs & $110^{\mathrm{TOL}}$ & $(5 A+6) / \underline{1}: \underline{4}$ & 54 & 14 & 0 \\
\hline $\mathrm{XV}$ & $\underbrace{\text { OP }}_{\text {OiPI }}$ & $12 \mathrm{hrs}$ & Reflux & $(5 B+6) / \underline{1}: \underline{4}$ & 81 & 10 & 0 \\
\hline xvi & $\begin{array}{c}\text { O } \\
\text { OM-O } \\
\text { OMe } \\
\text { (17) }\end{array}$ & $12 \mathrm{hrs}$ & Reflux & $(4+6) / \underline{1}: \underline{2}$ & 65 & 0 & 0 \\
\hline xvii & (18) & $6 \mathrm{hrs}$ & Reflux & $(5 B+9) / \underline{3}: \underline{1}$ & $90^{\mathrm{D}}$ & $\mathrm{n} / \mathrm{a}$ & $\mathrm{n} / \mathrm{a}$ \\
\hline
\end{tabular}




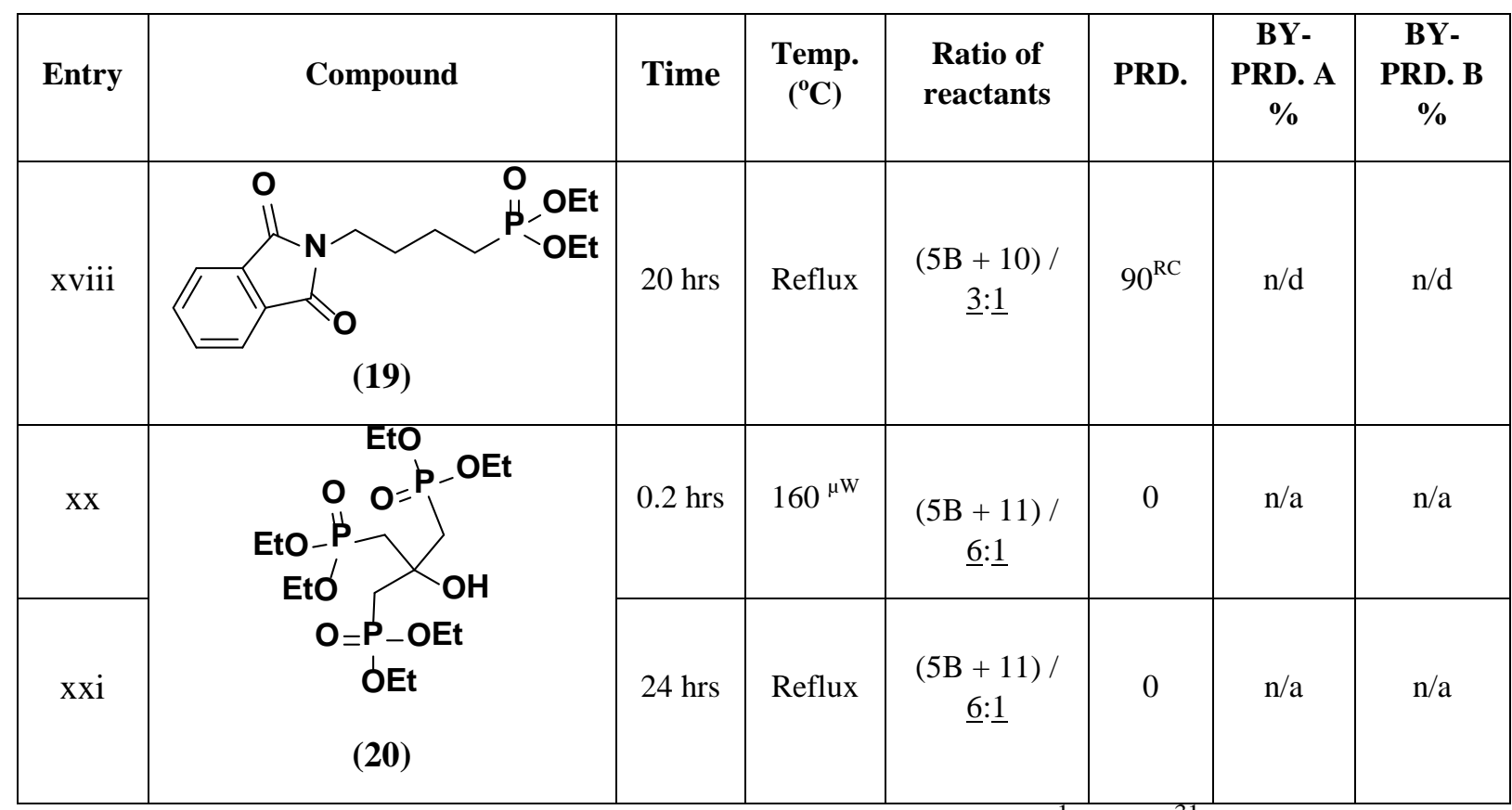

Unless indicated, the crude reaction composition was checked by ${ }^{1} \mathrm{H}$ and ${ }^{31} \mathrm{P}$ NMR $\left(\mathrm{CDCl}_{3}\right)$ after the time indicated. $\mathrm{D}=$ distilled product, $\mathrm{RC}$ = recrystallized product. By-product (A) is a result of dialkylation, by-product (B) is a result of intramolecular alkylation.

Compounds 12-16 were formed in $\sim 70 \%$ yield on average, with the rest of the mass corresponding to phosphorus by-products identified by ${ }^{1} \mathrm{H}$ and ${ }^{31} \mathrm{P}$ NMR $\left(\mathrm{CDCl}_{3}\right)$ including the higher boiling 5- and 6-membered cyclized oxaphospholanes (Table 2.3, by-product A) as well as the corresponding bisphosphonates (Table 2.3, by-product B). The thermodynamically favourable cyclic 5- and 6-membered by-products (Table 2.3, Entries i-x) were a likely result of intramolecular attack by oxygen of the phosphoryl group of the electrophilic $\mathrm{C}_{3}$ and $\mathrm{C}_{4}$ carbon atoms, respectively. The driving force is likely to be the energy gain in the formation of 5- and 6membered rings (Figure 2.3). ${ }^{198}$ 


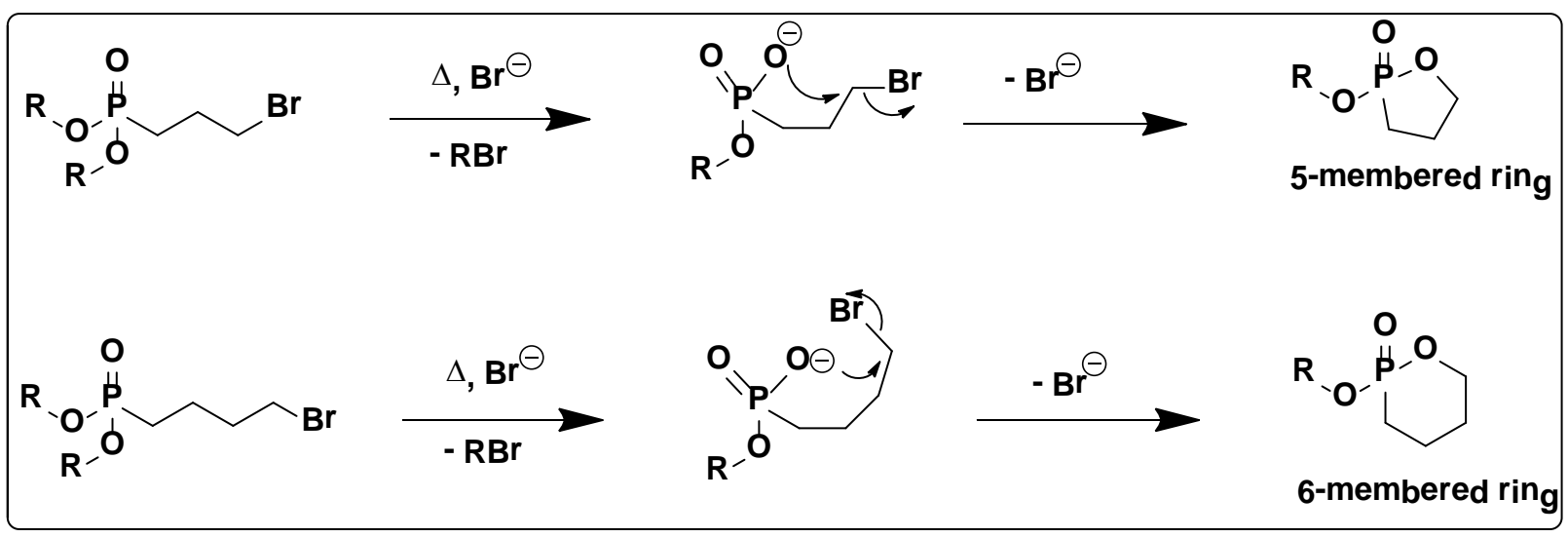

Figure 2.3: Cyclic oxaphospholane by-products formed during the Abruzov reaction.

Most of the lower boiling by-products which include ethyl or isopropyl bromides, and triethyl or triisopropyl phosphates were removed by distillation from the mixture while traces of the corresponding bisphosphonates (by-product B) co-distilled with the product (Table 2.3, Entries i, ii, vi, viii). With compound 14, a new ${ }^{31} \mathrm{P}$ NMR $\left(\mathrm{CDCl}_{3}\right)$ resonance was observed at 24 ppm and was attributed to the cyclic by-product, A, due to intramolecular cyclization. Compound 14 was also difficult to separate with distillation as it codistilled with the by-product upon purification. Both by-products (A and B) were readily removed in the subsequent Menschutkin reaction either by an aqueous extraction of the short chain phosphonate QAC's 28-29 or after precipitation of the longer chain phosphonates QAC’s 26-27.

Compounds 15-17 were prepared as precursors for dialkyl vinylphosphonates (Section 2.1.2.6). Compound 15 was used directly after distillation without characterization by NMR. Compounds 18 and 19 (Table 2.3, Entries xvi, xvii, xviii) were prepared in high yield employing an excess of $\mathrm{P}(\mathrm{OEt})_{3}$ (3 eq.), (Section 2.1.2.3, Method 3). A crystal structure of compound 19 was obtained after distillation to remove excess $\mathrm{P}(\mathrm{OEt})_{3}$ and re-crystallization from EtOAc (Section 2.6.1). 
Preparation of compound $\mathbf{2 0}$ was attempted under $\mu \mathrm{W}$ heating followed by distillation which resulted in thermal decomposition of the reactants and products that led to the formation of multiple peaks observed by ${ }^{31} \mathrm{P}$ NMR. Another attempt to prepare compound $\mathbf{2 0}$ was performed under reflux conditions with purification on column chromatography. After 24 hrs of reflux, the ${ }^{31} \mathrm{P}$ NMR spectrum $\left(\mathrm{CDCl}_{3}\right)$ of the crude reaction mixture revealed mainly unreacted $\mathrm{P}(\mathrm{OEt})_{3}$, likely as a result of steric hindrance. Chromatography on a short pad of silica eluted mostly $\mathrm{P}(\mathrm{OEt})_{3}$ which was subsequently converted to $\mathrm{HP}(\mathrm{OEt})_{2}$ on the column (Table 2.3, Entries xx and xxi).

The second step involving the Meshchutkin reaction was optimized (Table 2.4, Compounds 12-14). With the bromo leaving group, the Menshutkin reaction was more rapid compared to the chloro derivative (48-72 hrs, reflux, compound 3, Figure 1.45). Employing a sealed screw cap glass vial in refluxing ACN, the reaction was complete in 3-4 hrs (Table 2.4, Entries ii, iv, ix) while under $\mu \mathrm{W}$ heating at $150^{\circ} \mathrm{C}$ the reaction was complete in 2-10 min (Table 2.4, Entries iii, v, vi, vii, viii). Compound 28 was prepared from $\mathrm{NMe}_{3} \cdot \mathrm{HCl}$ by neutralizing the amine in ethanol $/ \mathrm{NaOH}$ followed by the Abruzov reaction with $\mathbf{1 3}$ directly in the $\mu \mathrm{W}$ without filtering off $\mathrm{NaCl}$. Compound $\mathbf{2 9}$ was reacted for 10 min to ensure complete conversion of the weaker nucleophilic pyridine and then dealkylated directly in the same vial with either TMSBr/ACN or $\mathrm{HBr} / \mathrm{H}_{2} \mathrm{O}$ (Table 2.4). ACN was the preferred solvent for the quaternization by enhancing the rate and in most cases the products solidified directly from the reaction mixture or with the addition of $\mathrm{Et}_{2} \mathrm{O}$. Compounds $\mathbf{3 2}$ and $\mathbf{3 8}$ were prepared by nucleophilic substitution of 14 by $\mathrm{NHMe}_{2}$ and 12 by $\mathrm{NaN}_{3}$ for use in Section 2.1.3.2, Method 3. 
Neat, $100^{\circ} \mathrm{C}, 35 \mathrm{~min}$ or

$\mathrm{ACN}, \mu \mathrm{W}, 150^{\circ} \mathrm{C}, 2 \mathrm{~min}$

$(12-14)$

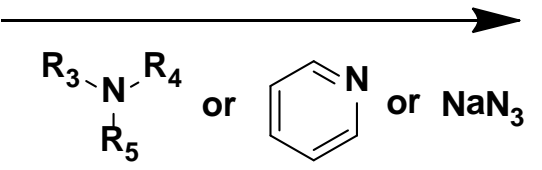

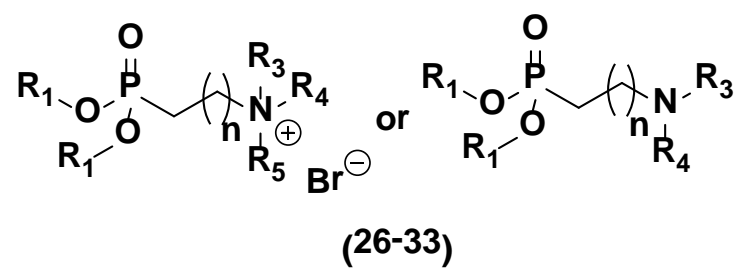

Table 2.4: Optimization of Step 2: Menschutkin reaction between bromoalkylphosphonates 1214 and tertiary amines 2, 21-25.

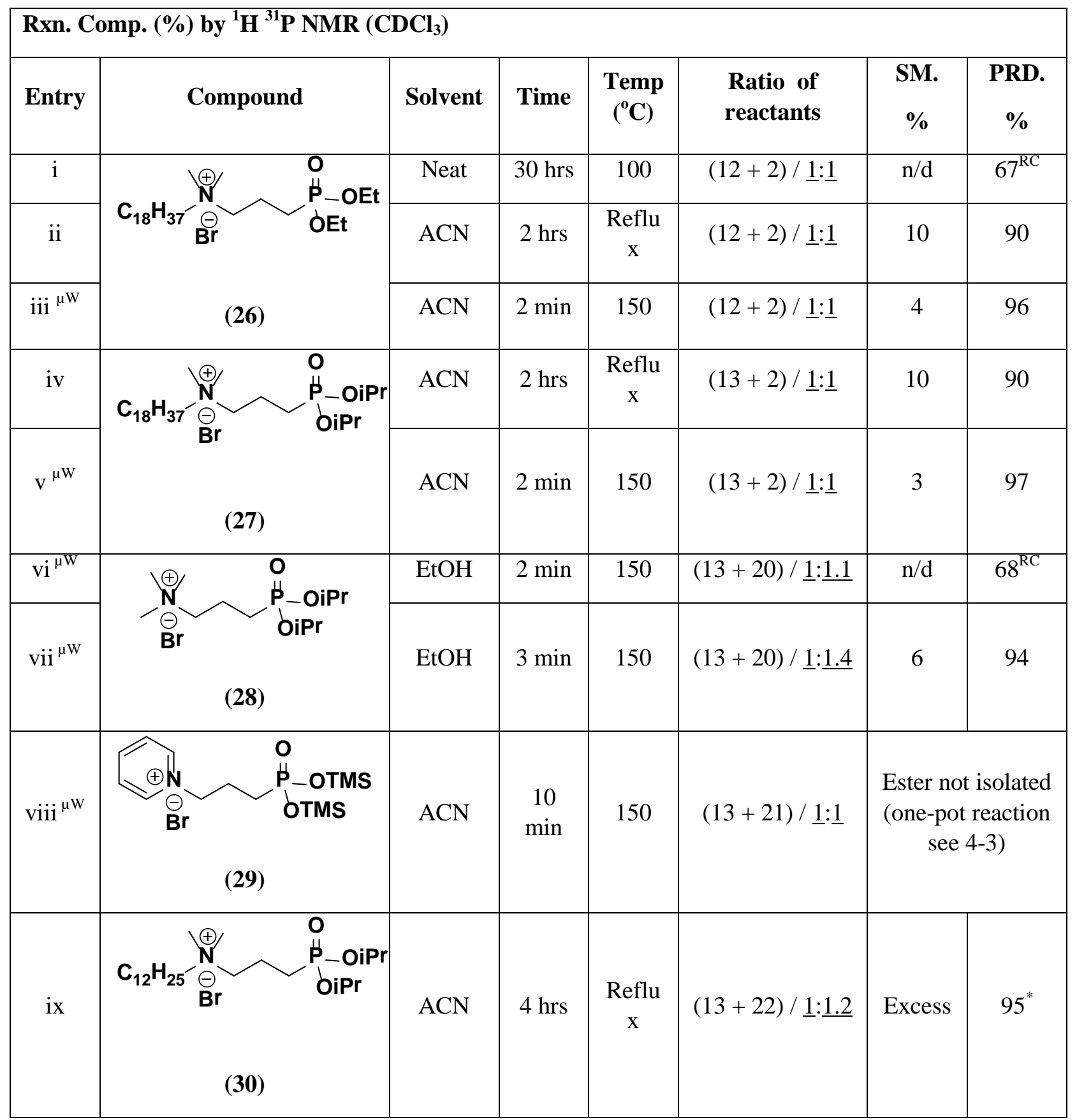




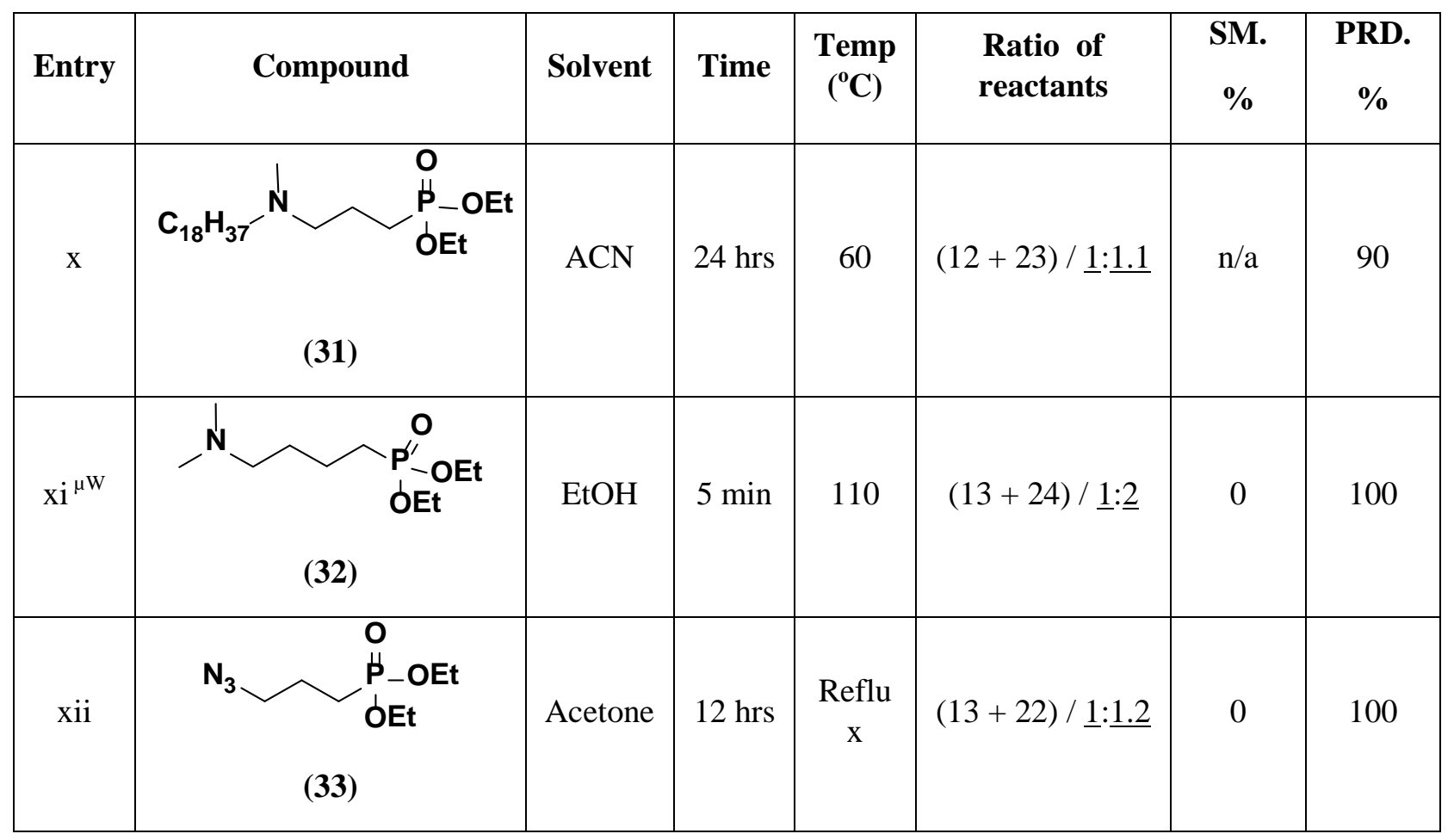

Didealkylation of compound 26-29 was first explored with TMSBr and then optimized with $\mathrm{HCl}$ and $\mathrm{HBr}$ (Table 2.5, Entries i-xi). With anhydrous TMSBr the reaction was performed in DCM overnight and complete conversion was observed, however in situ generation of the reagent from $\mathrm{TMSCl} / \mathrm{NaI}$ in $\mathrm{ACN}$ failed to give the dealkylated product (Table 2.5, Entry ii). This can be attributed to poor solubility of quat $\mathbf{2 6}$ in ACN vs DCM. Next, the phosphonate quats were successfully dealkylated in $\mathrm{H}_{2} \mathrm{O}$ with a two fold excess of $\mathrm{HCl}$ or $\mathrm{HBr}$. Since the formation of the carbocation intermediate is the rate determining step in the mineral acid didealkylation of phosphonate esters (see Figure 1.53), the more stable iPr carbocations and the more reactive $\mathrm{Br}^{-}$ions proceeded faster compared to Et esters (Table 2.5, Entry iv vs Entry v and Entry vi vs Entry vii) and $\mathrm{HBr}$ was faster than $\mathrm{HCl}$ (Table 2.5, Entry iv vs Entry vi and Entry v vs Entry vii). $\mu \mathrm{W}$ heating was also optimized with compounds 27 and 28 with complete conversion observed within 6-10 min at $140-150^{\circ} \mathrm{C}$ (Table 2.5, Entries ix and xi). 

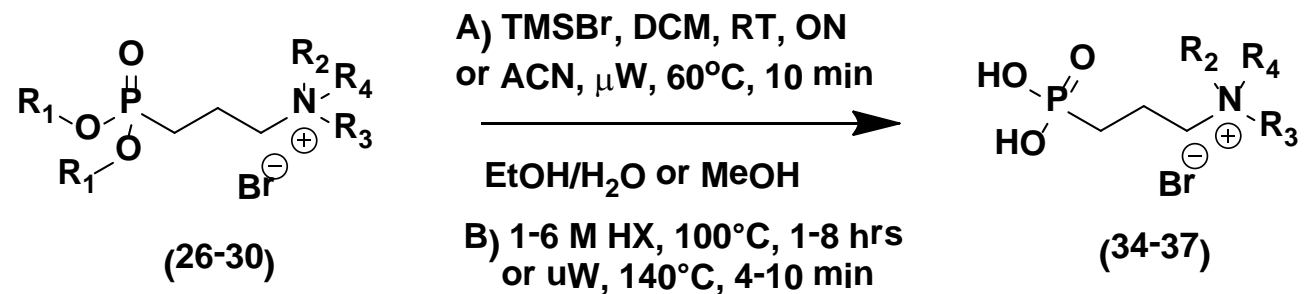

B) 1-6 $\mathrm{M} \mathrm{HX}, 100^{\circ} \mathrm{C}, 1-8 \mathrm{hrs}$ or $\mathrm{uW}, 140^{\circ} \mathrm{C}, 4-10 \mathrm{~min}$

(34-37)

Table 2.5: Optimization of Step 4: Didealkylation of phosphonate diester quats.

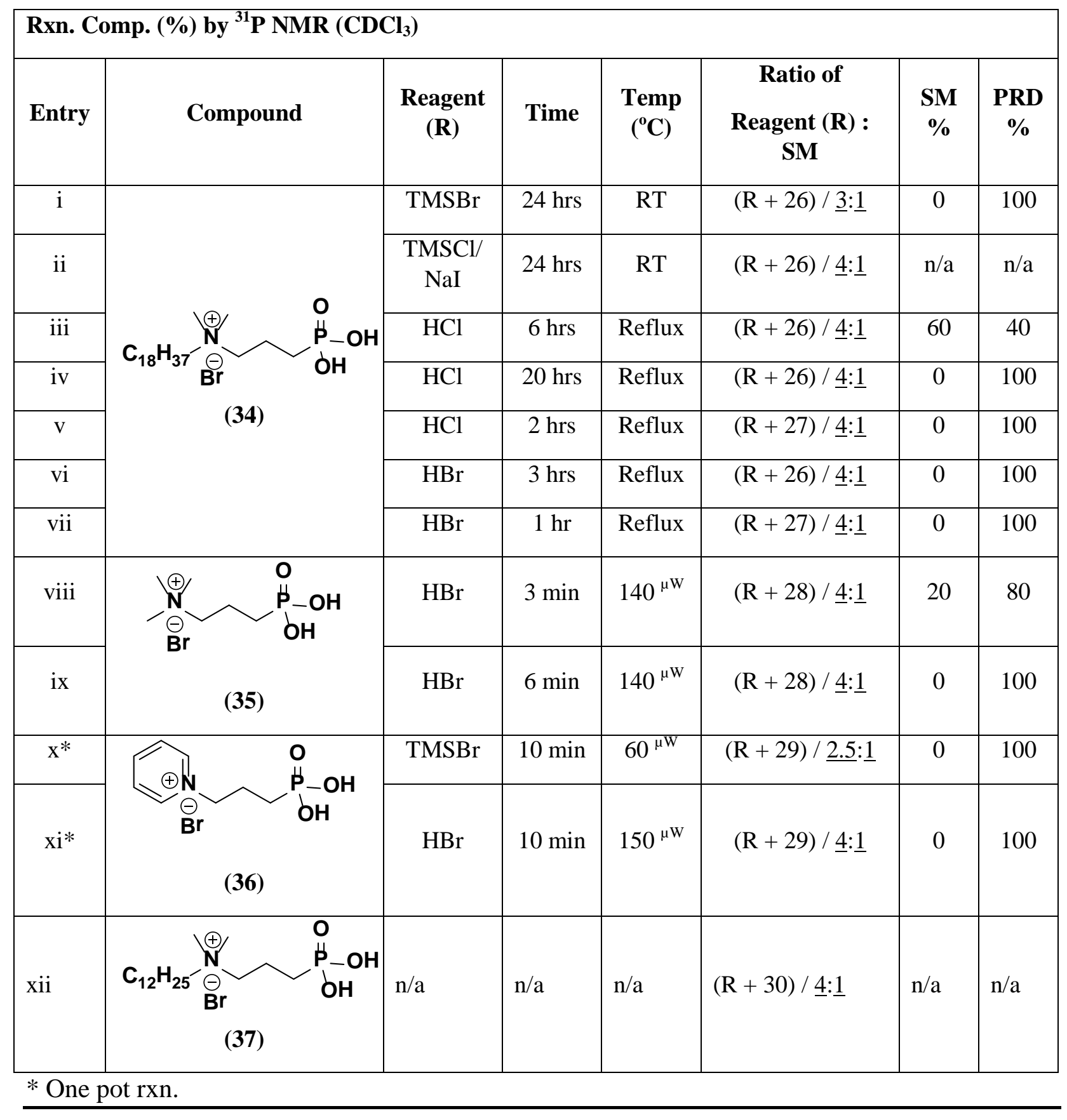


The long chain $\mathrm{C}_{18}$ phosphonic acid quat, 34, previously only isolated as the Na salt of the phosphobetaine or as an internal salt after purification on an expensive anion exchange resin (see Figure 1.57) was successfully recrystallized from EtOAc:EtOH and X-ray quality crystals were obtained (Figure 2.4, and Figure 2.40).

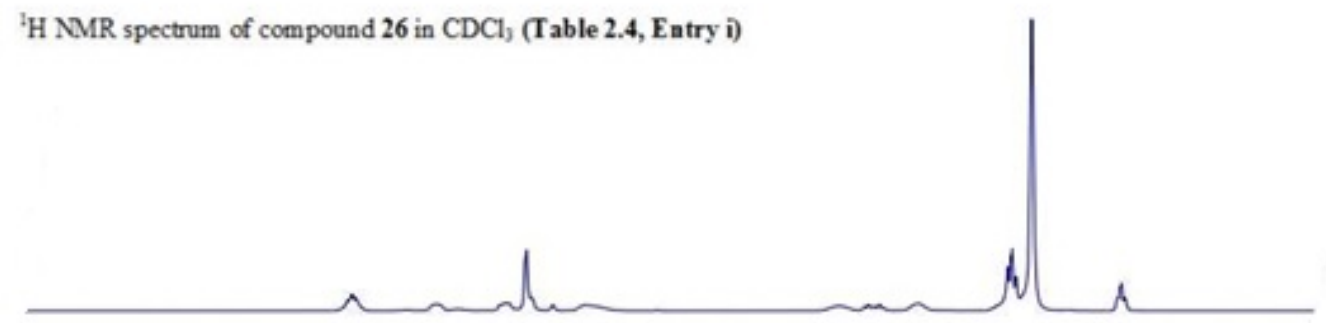

'H NMR spectrum of compound 27 in $\mathrm{CDCl}_{3}$ ( $\mathrm{T}$ able 2.4, Entry iv)

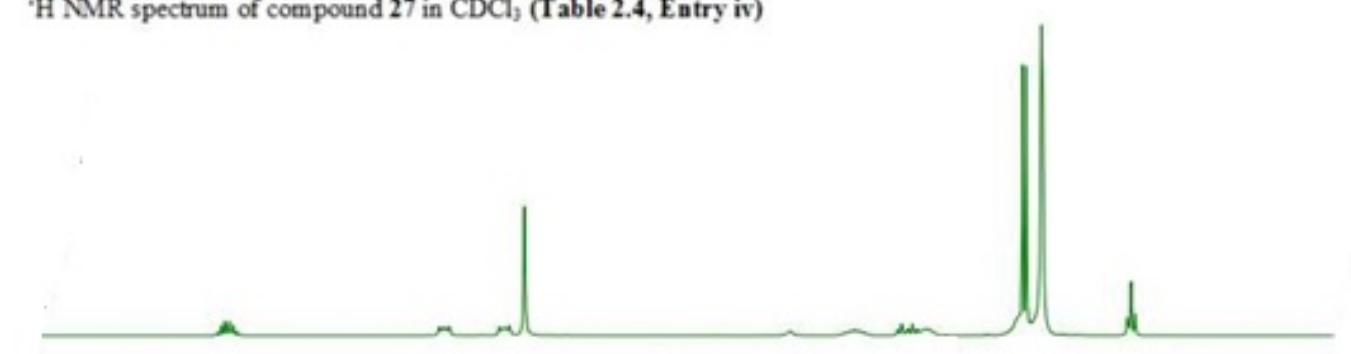

'H NMR spectrum of compound 34 in $\mathrm{D}_{2} \mathrm{O}$ (Table 2.5, Entry i)

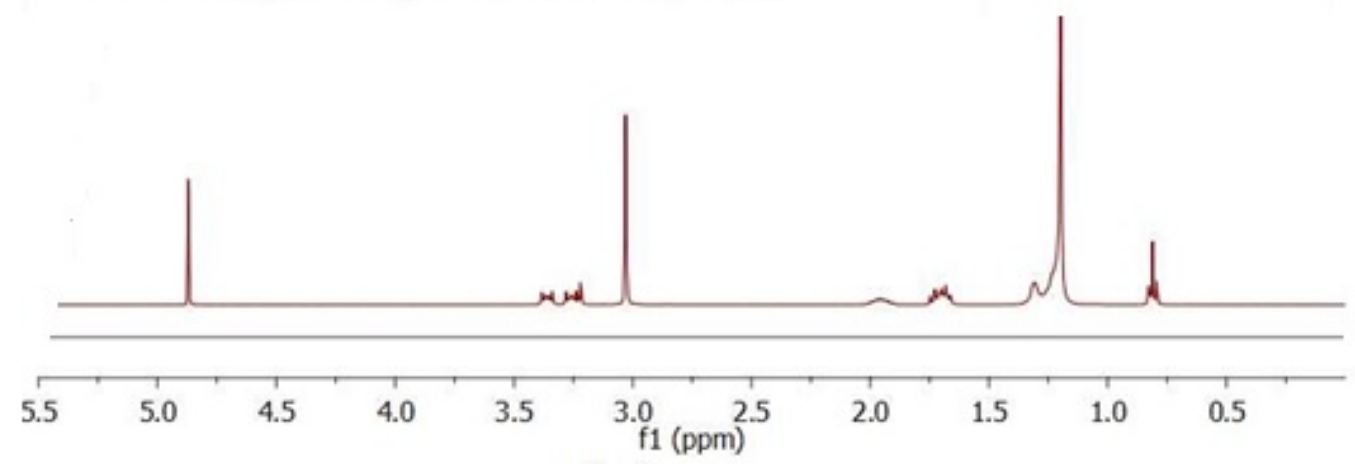

Figure 2.4: ${ }^{1} \mathrm{H}$ NMR $\left(\mathrm{CDCl}_{3}\right)$ spectra showing successful didealkylation of the ethyl phosphonate ester QAC $\mathbf{2 6}$ and isopropyl ester QAC $\mathbf{2 7}$ to the corresponding phosphonic acid QAC $34\left(\mathrm{D}_{2} \mathrm{O}\right)$.

\subsubsection{2 $\gamma$-Bisphosphonic Acids QAC Antimicrobials ( $\gamma$-BPQ) Synthesis}

Bisalkylation of the commercially available octadecylamine 38 with 13 employing Hunig's base afforded the desired iPr-bisphosphonate 39 in good yield with conventional 
(solventless) or $\mu \mathrm{W}$ heating (ACN). Trialkylbromophosphonates (where alkyl = Me, Et, $i \mathrm{Pr}$ ) may be used interchangeably in this reaction, however $\mathbf{1 3}$ was used instead of $\mathbf{1 2}$ due to easier cleavage with $\mathrm{HBr}$ during the last step of the synthesis. Any trace amounts of unreacted starting materials in the crude sample of $\mathbf{3 9}$ had no effect on the quaternization reaction with MeI (1.2 eq.), resulting in quantitative conversion to $\mathbf{4 0}$. These by-products may be purified at any stage in the preparation of $\mathbf{4 1}$ (Scheme 2.3). The last dealkylation step to obtain $\mathbf{4 1}$ proved to be a challenge due to insolubility of the bisphosphonic acid product.

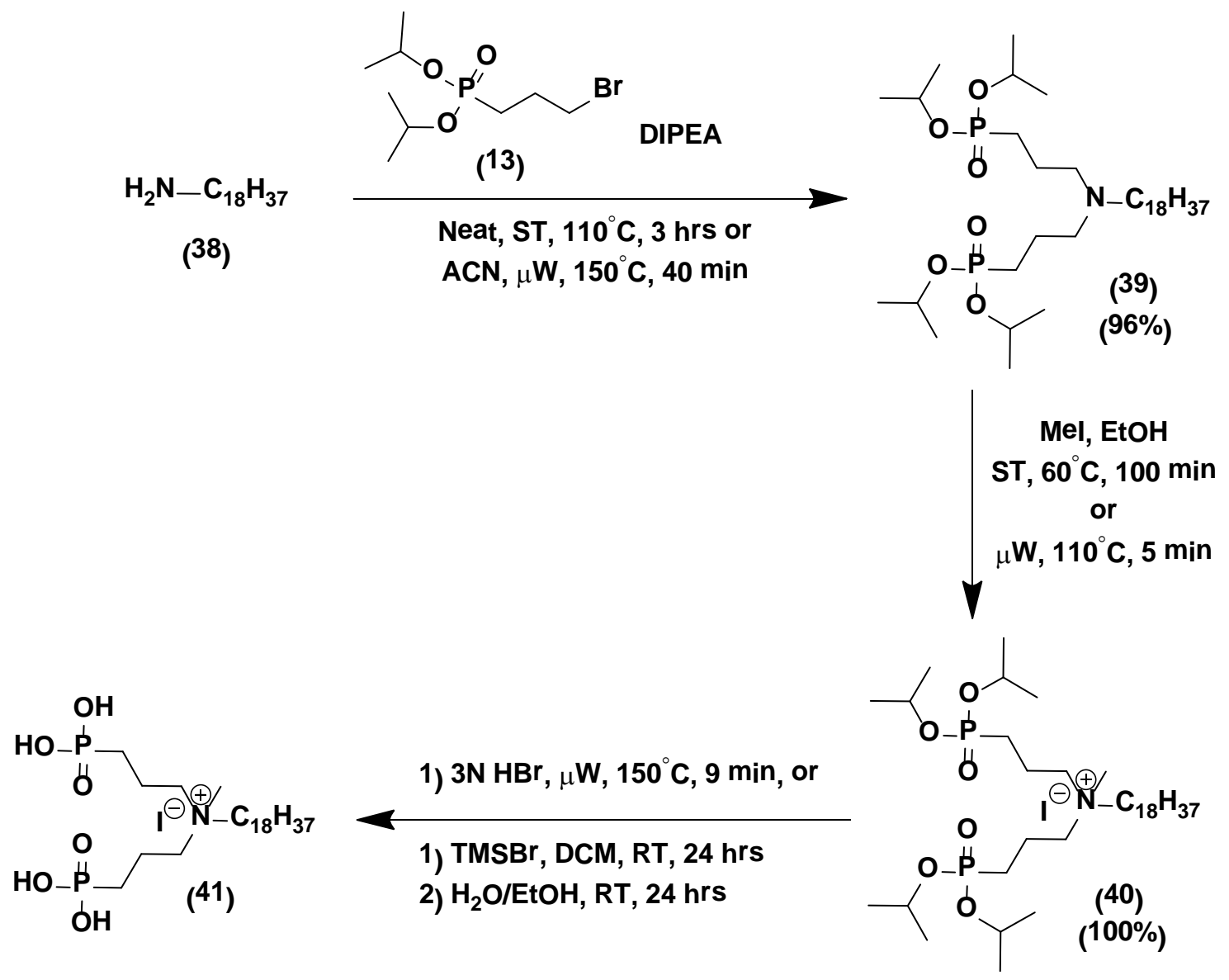

Scheme 2.3: Preparation of $\gamma$-Bisphosphonic acid 41.

Initially the reaction was attempted with $\mu \mathrm{W}$ heating, however it was difficult to monitor due to solidification of the product in the NMR tube $\left(\mathrm{D}_{2} \mathrm{O}\right)$. Other deuturated solvents such as 105 
$\mathrm{CDCl}_{3}$ or DMSO also failed to show any ${ }^{31} \mathrm{P}$ signals of the crude reaction mixture. After evaporation of the crude product to an orange paste, the residue was treated with DCM/TMSBr at RT overnight in an attempt to isolate a crystalline compound after TMS ester hydrolysis. Once again, after extensive solubility analysis, the residue remained practically insoluble in every solvent including the NMR solvents mentioned above.

Compound $\mathbf{4 1}$ is insoluble in $\mathrm{H}_{2} \mathrm{O}$ due the presence of two phosphonic acid groups and the hydrophobic $\mathrm{C}_{18}$ tail and the quat contributes to its insolubility in most polar solvents. Only after treatment with 2 eq. $\mathrm{KOH}$ to make the potassium salt of the bisphosphonic acid, 42, was it possible to solubilize the compound in $\mathrm{D}_{2} \mathrm{O}$ and obtain a clean NMR (Scheme 2.4, Figure 2.5).
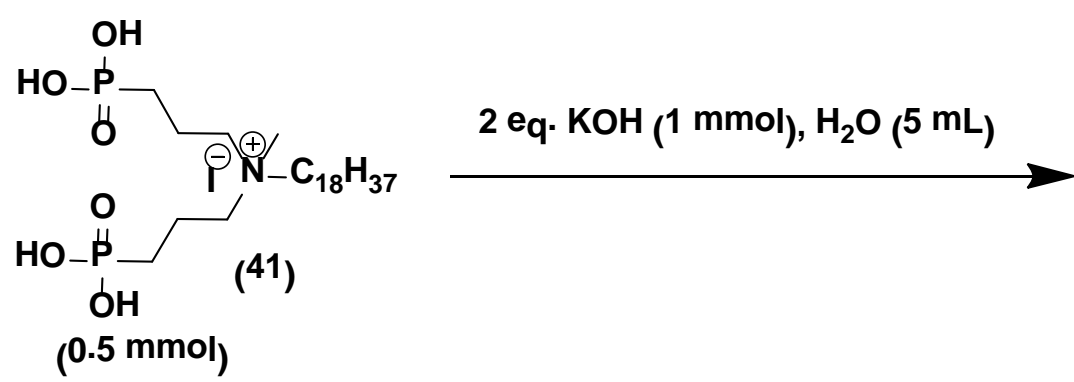

Scheme 2.4: Preparation of the potassium salt of 41. 


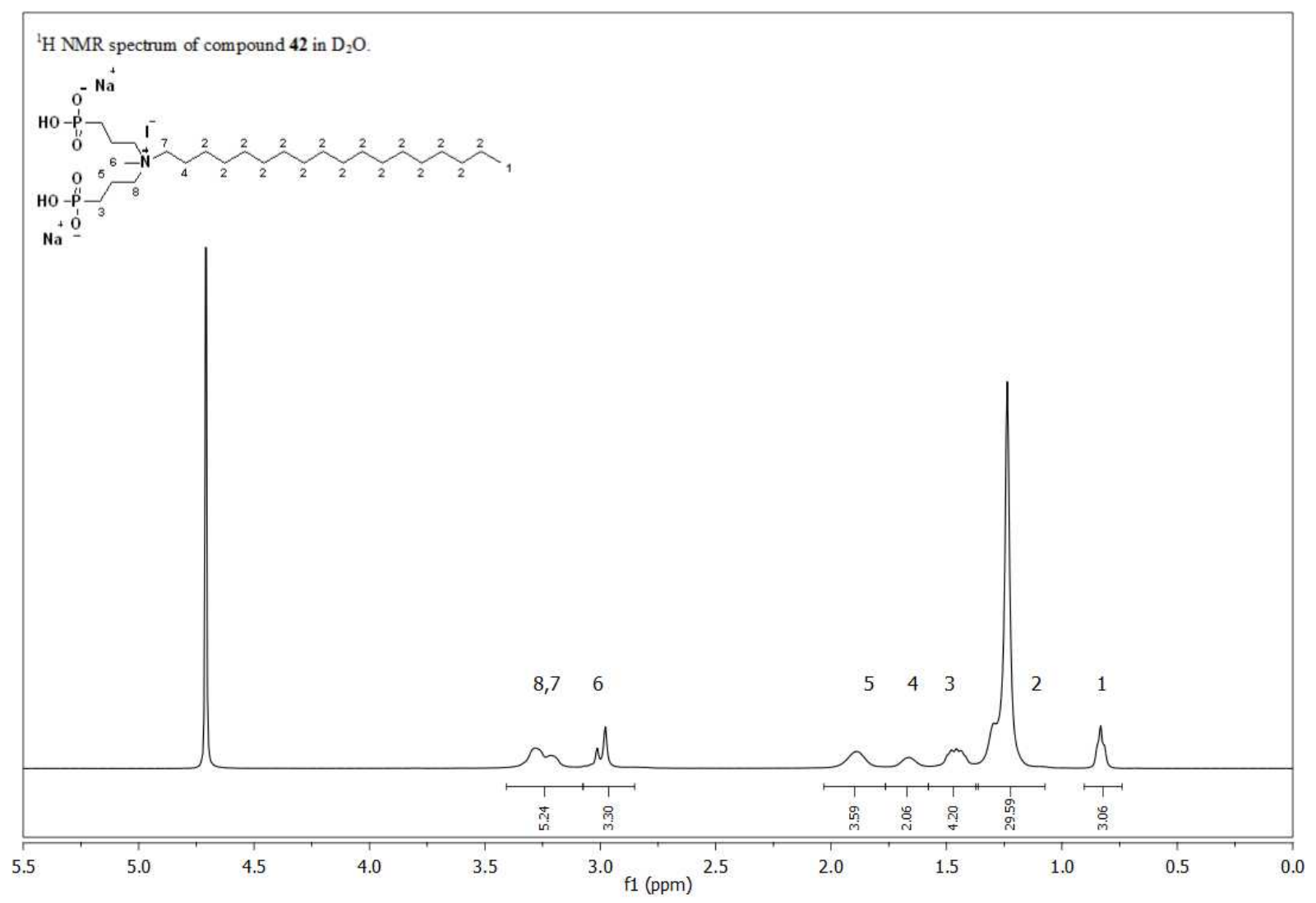

Figure 2.5 : ${ }^{1} \mathrm{H}$ NMR $\left(\mathrm{D}_{2} \mathrm{O}\right)$ spectrum of $\gamma$-BPQAC 42.

An alternative ( $\gamma$-BPQ) synthesis was attempted starting with $N^{1}, N^{1}$-bis(2aminoethyl)ethane-1,2-diamine scaffold based on Yoshimura's work with a tridodecyl quaternized star-shaped precursor $\mathbf{4 4}$ (Scheme 2.5). ${ }^{199}$ Precursor $\mathbf{4 4}$ was prepared by a modified procedure starting with paraformaldehyde instead of formalin and heated in ACN, employing anhydrous $\mathrm{HCl}$ and $\mathrm{NaOEt}$ instead of $\mathrm{HCl}(\mathrm{aq})$ and $\mathrm{NaOH}$ to obtain the free base amine (see Experimental, Section 5.3.3). Attempted synthesis of $\mathbf{4 5}$ by quaternizing with 0.33 eq. bromooctadecane followed by 0.66 eq. of $\mathbf{1 3}$ or vice-versa failed to give the desired quaternized product peak $\left({ }^{31} \mathrm{P}\right.$ NMR $\delta \sim 29 \mathrm{ppm}$, Scheme 2.5, Figure 2.6). Instead, an unidentified upfield peak from the starting material 13 was observed $\left({ }^{31} \mathrm{P}\right.$ NMR $\delta \sim 30 \mathrm{ppm}$, Figure 2.6), in a 1:1 ratio with unreacted 13 (Figure 2.6). Since the reaction was performed without filtering off $\mathrm{NaCl}$ after 
44 was freebased with NaOEt prior to adding 13, any excess base could have reacted with 13 . No further experimentation was attempted with this scaffold.<smiles>NCCN(CCN)CCN</smiles>
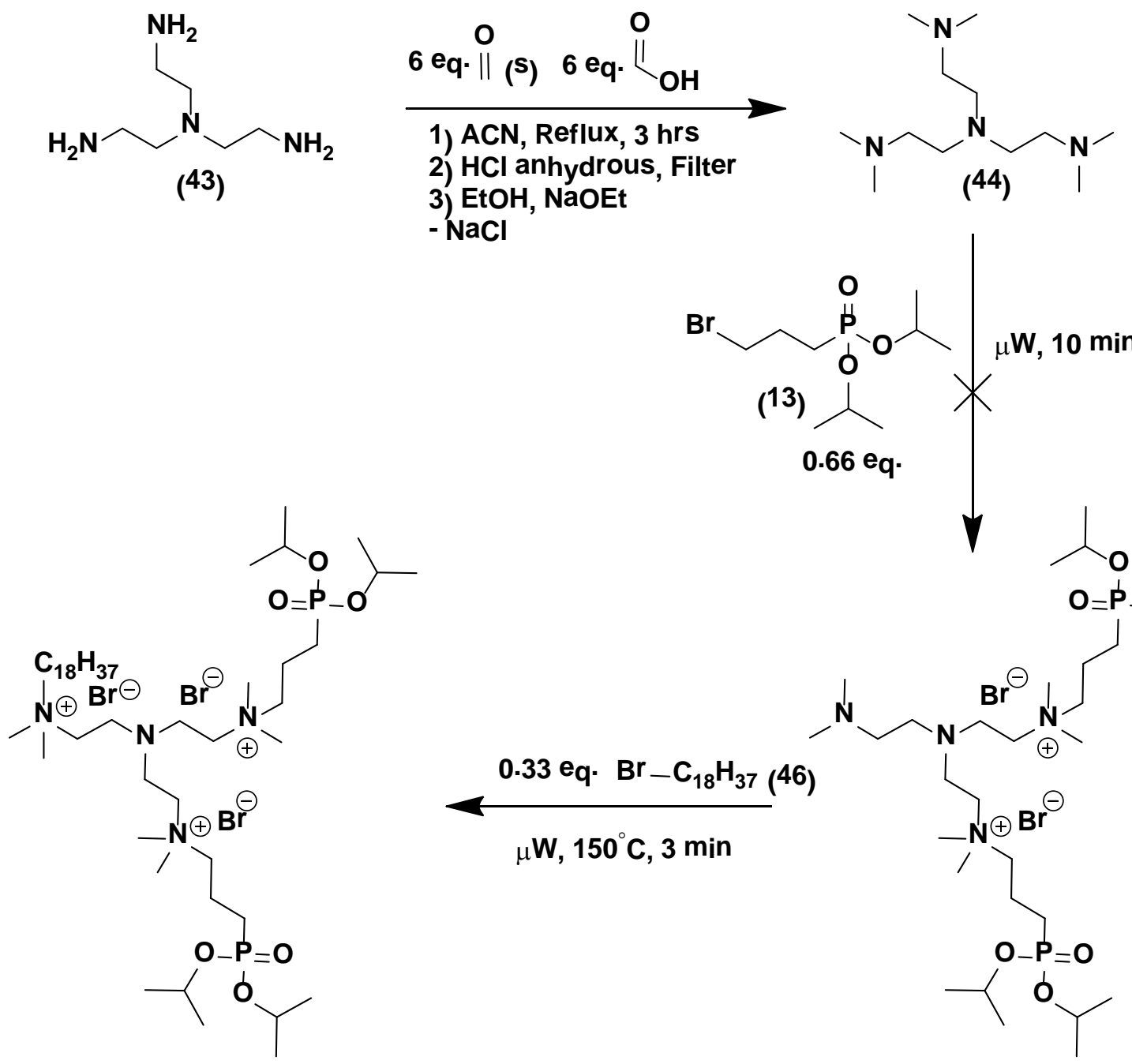

(47)

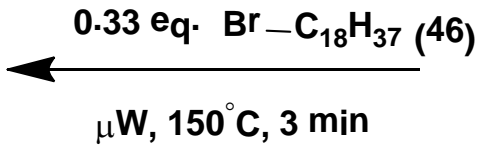

Scheme 2.5: Attempted preparation of compound 47. 


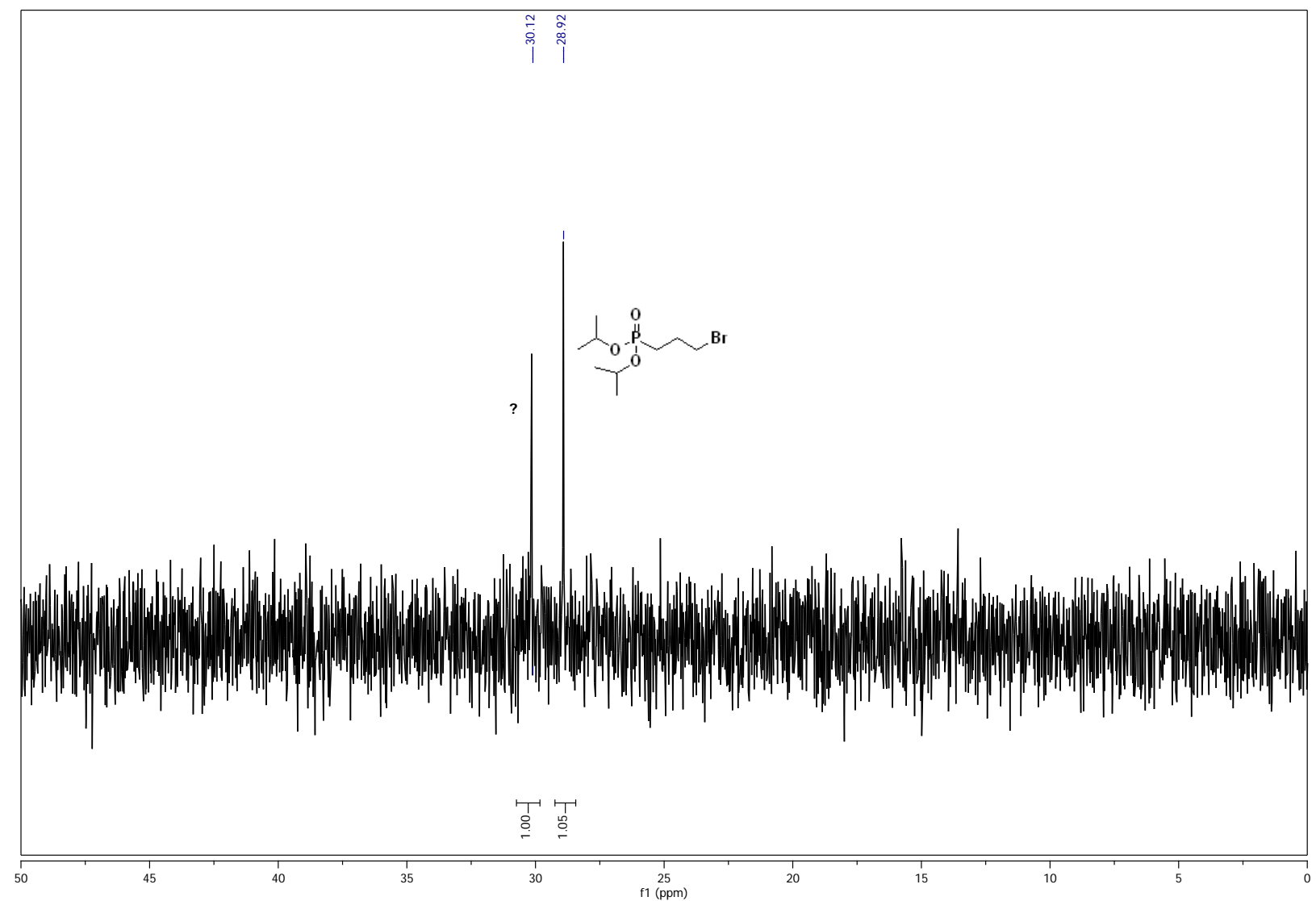

Figure 2.6: ${ }^{31} \mathrm{P} \mathrm{NMR}\left(\mathrm{CDCl}_{3}\right)$ reaction monitoring of compound $\mathbf{4 5}$.

\subsubsection{3 $\alpha$-CH Bisphosphonic Acids QAC Antimicrobials ( $\alpha-C H-B P Q A)$ Synthesis}

$\alpha-\mathrm{CH}$ Bisphosphonic acid quats envisioned coming from alkylated methylenebis (phosphonate) (Figure 2.7) were also patentable synthetic targets of various small molecule multidentate phosphonic acid antimicrobials.

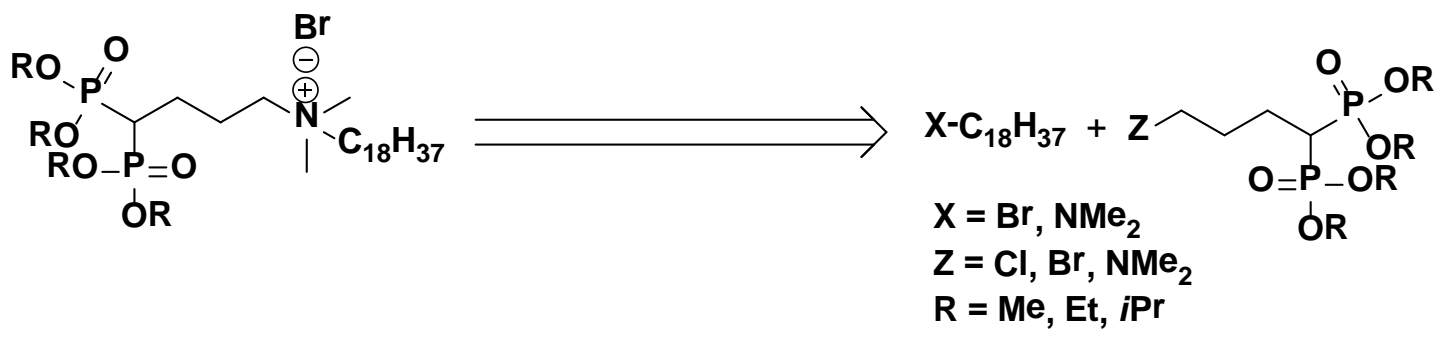

Figure 2.7: Retrosynthesis of $\alpha-\mathrm{CH}$ bisphosphonic acid quats. 
Four methods were identified in the literature for the preparation of halo or $N, N$-dimethyl substituted methylene bisphosphonate precursors for the Menshutkin reaction. Surprisingly, none of these have been used to make QAC's (Figure 2.8). The following starting materials can be used to prepared alkylated methylene bisphosphonates: (i) aldehydes, (ii) monophosphonates, (iii) dialkylvinylphosphonate (iv) methylene bisphosphonates. Each of the four methods are individually discussed in the following sections.

Method 1

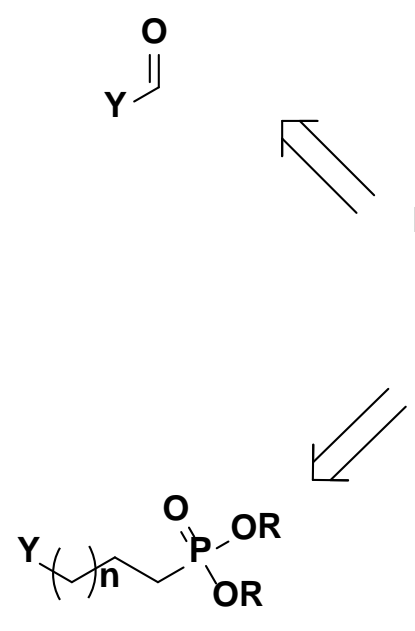

Method 3
Method 2

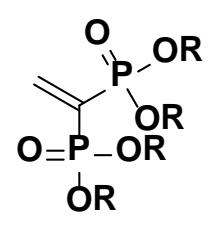

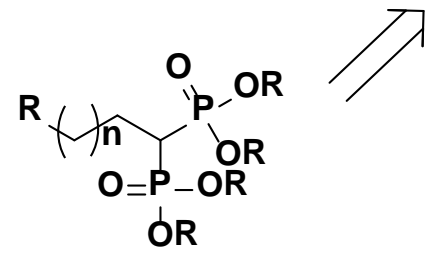
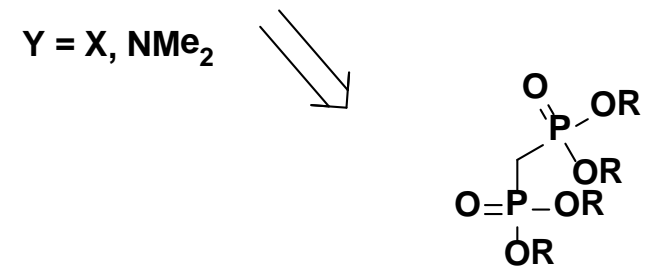

Method 4

$$
\begin{aligned}
& \mathrm{R}=\mathrm{Et} \\
& \mathrm{R}=i \mathrm{Pr}
\end{aligned}
$$

Figure 2.8: Four retrosynthetic methods for the preparation of $\alpha$-CH-BPQA's.

\section{Method 1: Onepot Bis Addition of Dialkylphosphites to Aldehydes}

The most cost effective reaction to prepare the $\alpha$-CH bisphosphonate scaffold involves the one pot addition reaction of 1 eq. of $\mathrm{HP}(\mathrm{OEt})_{2}$ to an aldehyde followed by mesylation of the in situ formed $\alpha$-hydroxyl phosphonate and subsequent substitution by another equivalent of 
nucleophilic diethylphosphite (Scheme 2.6). The only drawback here is the extra step required to prepare the commercially unavailable aldehydes.

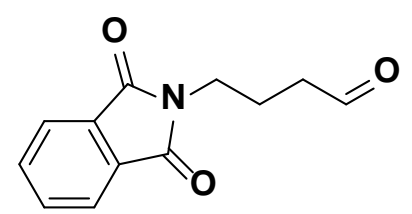

(48)

(49)

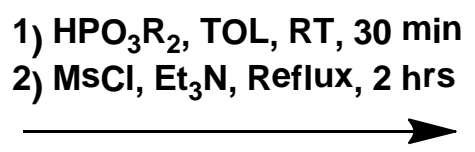

1) $\mathrm{H}_{2} \mathrm{~N}-\mathrm{NH}_{2}$, EtOH, Reflux, $2 \mathrm{hrs}$ 2) $\mathrm{CHO}, \mathrm{CH}_{3} \mathrm{CHO}, \mathrm{Zn}$

3) $\mathrm{C}_{18}-\mathrm{Br}, 100^{\circ} \mathrm{C}, 1 \mathrm{hr}$

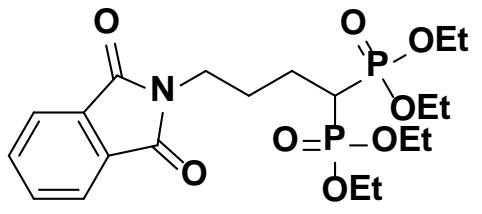

$(49)$

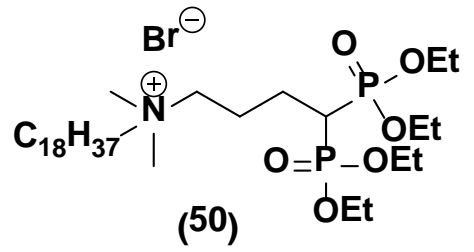

Scheme 2.6: Preparation of $\mathbf{5 0}$ by Method 1.

Using Method 1, bisphosphonic quat $\mathbf{5 0}$ was targeted from the phthalimide protected aldehyde $\mathbf{4 8}$ after hydrazine deprotection of $\mathbf{4 9}$ followed by $N, N$-methylation and quaternization (Scheme 2.6). However, the first reaction in the sequence failed to give the desired product $\left({ }^{31} \mathrm{P}\right.$ NMR $24 \mathrm{ppm})$ by the one-pot method. Instead, the one pot reaction was performed sequentially isolating each intermediate according to Scheme 2.7. 


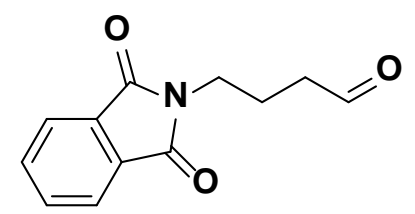

(48)

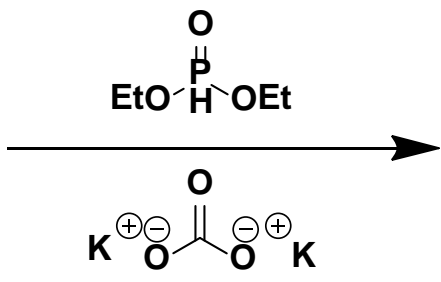

$A C N, 60^{\circ} \mathrm{C}, 10 \mathrm{~min}$

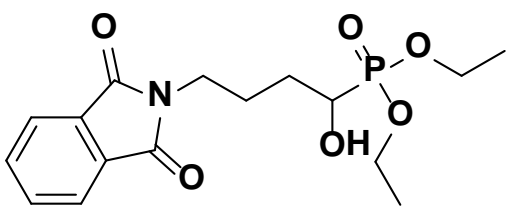

(51)

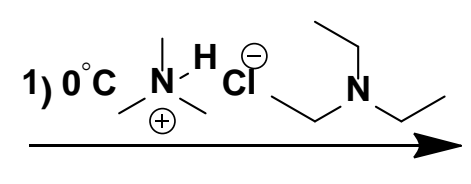

2) Dropwise, $10 \mathrm{~min} R T$

0

- 10<smiles>CCOP(=O)(OCC)C(CCCN1C(=O)c2ccccc2C1=O)OS(C)(=O)=O</smiles><smiles>CCOP(=O)(OCC)C(CCCN1C(=O)c2ccccc2C1=O)OS(C)(=O)=O</smiles>

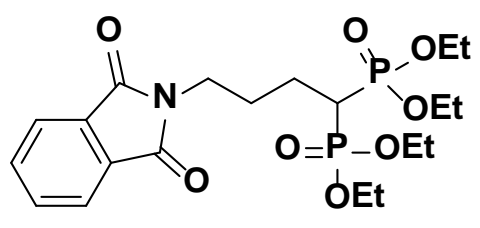

(49)

Scheme 2.7: Attempted stepwise preparation of $\mathbf{4 9}$.

Synthesis of the bisphosphonate 49 from 52 was unsuccessful, leading to a mixture of products $\left({ }^{31} \mathrm{P} \quad \mathrm{NMR}, \mathrm{CDCl}_{3}\right)$. All base/solvent combinations including $\mathrm{K}_{2} \mathrm{CO}_{3} / \mathrm{EtOH}$, $\mathrm{Cs}_{2} \mathrm{CO}_{3} / \mathrm{ACN}, \mathrm{Cs}_{2} \mathrm{CO}_{3} / \mathrm{ACN}$, pyridine/TOL also failed to give the desired ${ }^{31} \mathrm{P}$ resonance at $\delta \sim$ 23-24 ppm, with unidentified peaks at $17.7 \mathrm{ppm}$ and $0.7 \mathrm{ppm}$ always observed.

A closer investigation of the literature revealed that the phthalimide protecting group is incompatible with Method 1, leading instead to heterocyclic products. ${ }^{200}$ Deprotonation of the newly formed bisphosphonate by the one pot reaction creates a nucleophilic carbanion that undergoes an intramolecular Horner-Wadsworth-Emmons reaction between the formed imide and the $\beta$-functionalized phosphonate (Figure 2.9). 


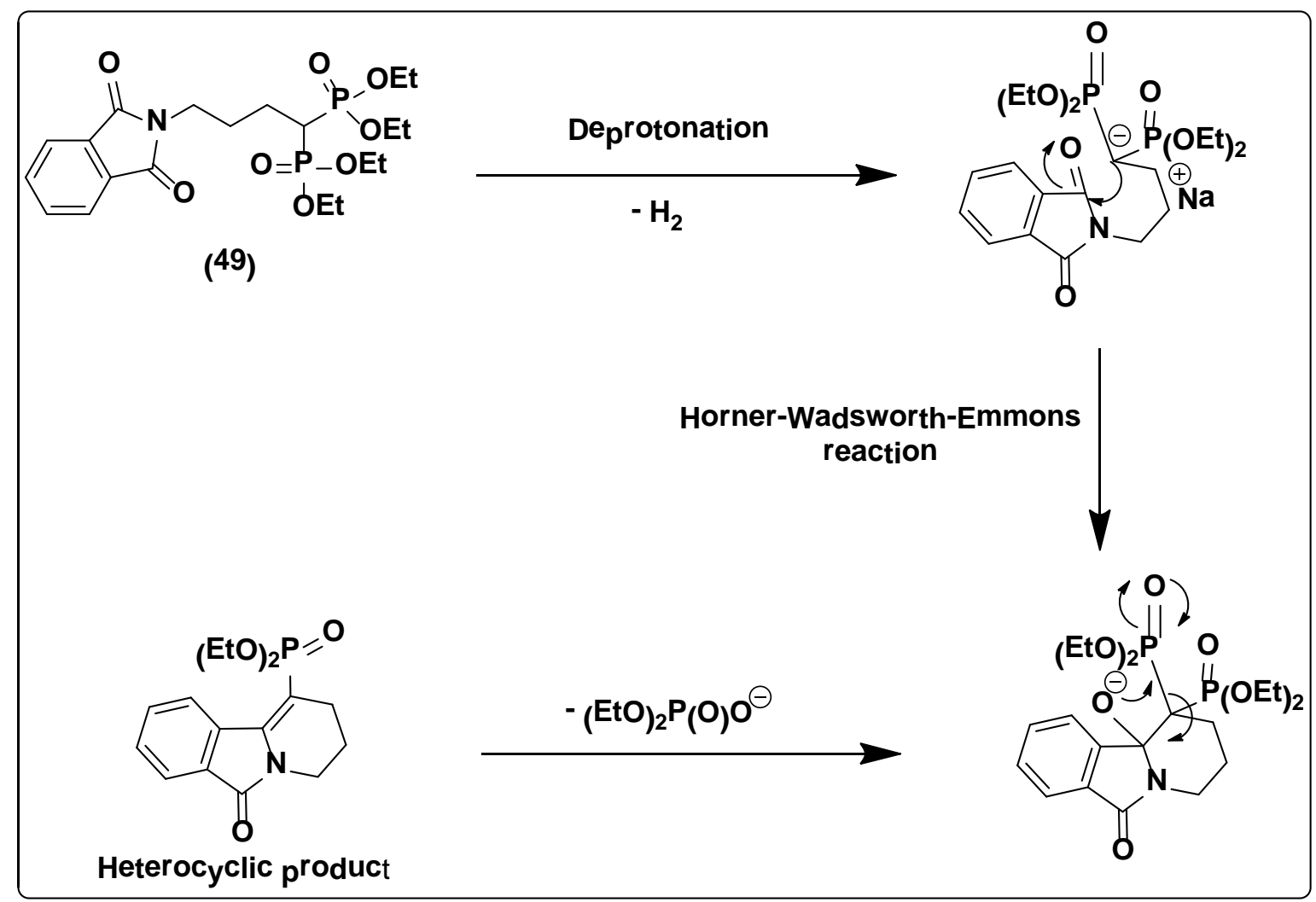

Figure 2.9: The Horner-Wadsworth-Emmons rearrangement of $\mathbf{4 9} .^{200}$

Due to the rearrangement observed with precursor 49, it was hypothesized that switching to a different aldehyde would allow the one pot reaction to proceed without heterocycle formation. Synthesis of various halo substituted 54 and 55 and the quat aldehyde 56 was attempted, but were problematic to isolate by recrystallization (Scheme 2.8).
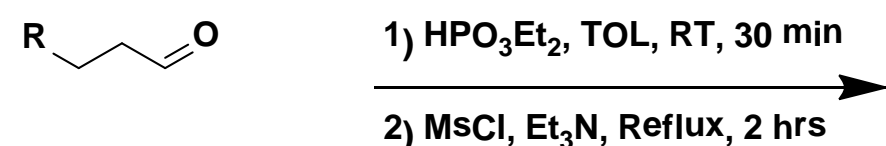

$$
\begin{aligned}
& \text { (54) } R=C l \\
& \text { (55) } R=B r \\
& \text { (56) } R=B^{-} N^{+} M_{2} C_{18} H_{37}
\end{aligned}
$$

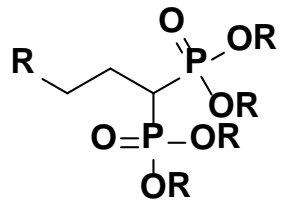

(57) $\mathrm{R}=\mathrm{Cl}$

(58) $\mathrm{R}=\mathrm{Br}$

(59) $\mathrm{R}=\mathrm{Br}^{-} \mathrm{N}^{+} \mathrm{Me}_{2} \mathrm{C}_{18} \mathrm{H}_{37}$

Scheme 2.8: Synthesis of bisphosphonates 57-59 from aldehydes. 
Synthesis of QAC aldehyde $\mathbf{5 6}$ was first attempted by quaternizing the commercially available acetal 65 with 2 . The desired product was obtained along with an unidentified inseparable impurity by ${ }^{1} \mathrm{H}$ NMR $\left(\mathrm{CDCl}_{3}\right)$ after numerous attempts at recrystallization. No further reaction with this compound was pursued. The second way to obtain $\mathbf{5 6}$ is the oxidation of the quat alcohols 66 or $\mathbf{6 7}$ prepared in high yield (98\%) and purity (Figure 2.10, Section 2.6.1, see crystal structure). Compounds $\mathbf{6 6}$ and $\mathbf{6 7}$ remain to be oxidized with pyridinium chlorochromate (PCC) as shown in Scheme 2.10. Preparation of the chloro and bromo aldehydes precursors 54 and 55 was attempted by employing refluxing $\mathrm{HCl}$ or TFA/DCM with loss of the volatile product aldehydes upon distillation (Scheme 2.10). Additionally, a potential problem with Method 1 is the possibility of the unwanted substitution of the halo group with nucleophilic $\mathrm{HP}(\mathrm{OEt})_{2}$. Instead, Methods 2-4 were explored in order to prepare $\alpha$-CH-BPQA's.

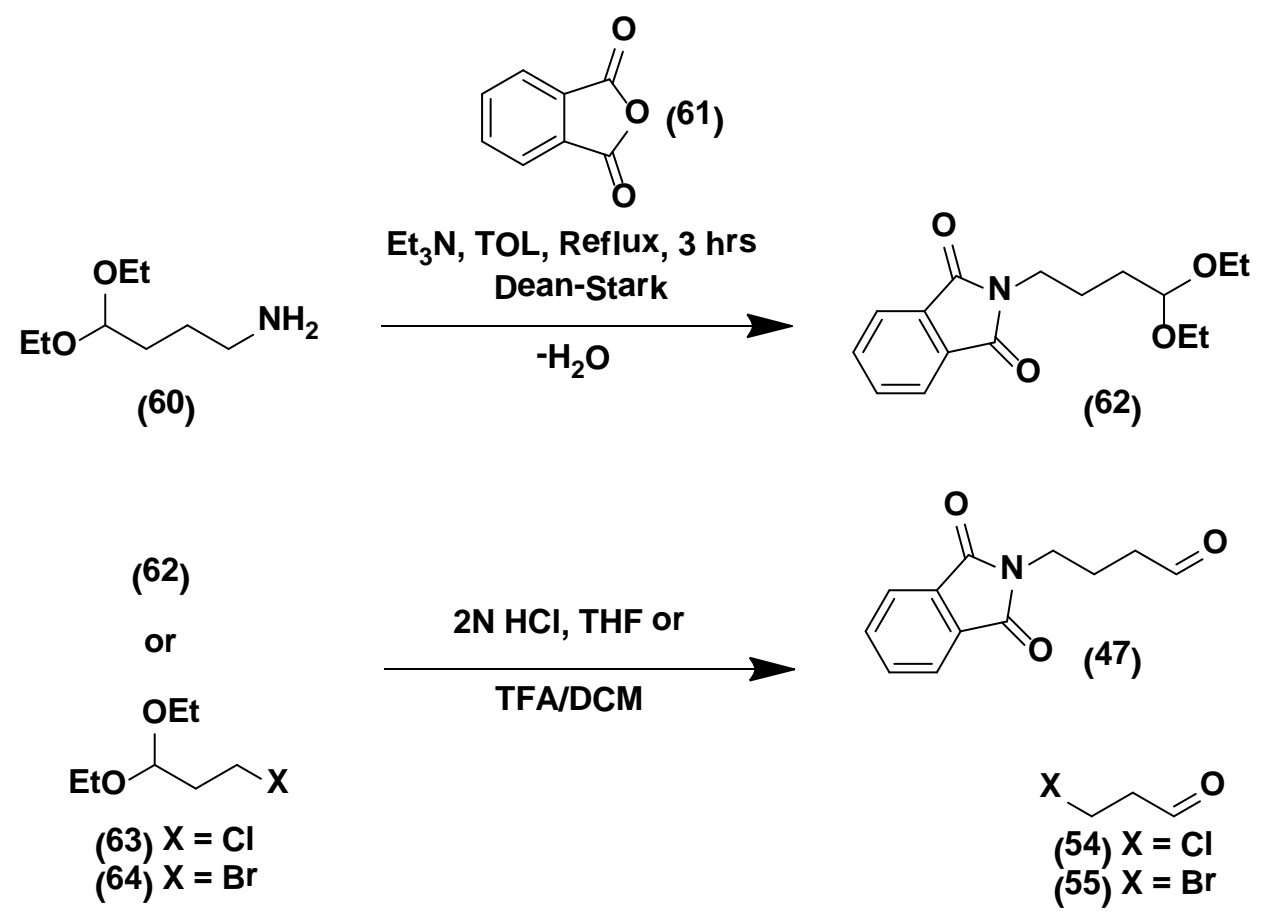

Scheme 2.9: Synthesis of aldehyde precursors 47, 54-55 used for Method 1. 


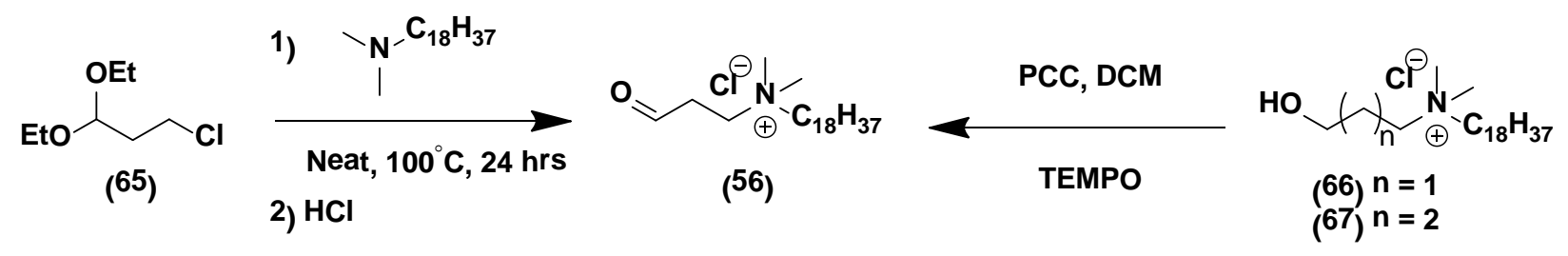

Scheme 2.10: Possible synthetic routes leading to aldehyde QAC 56.

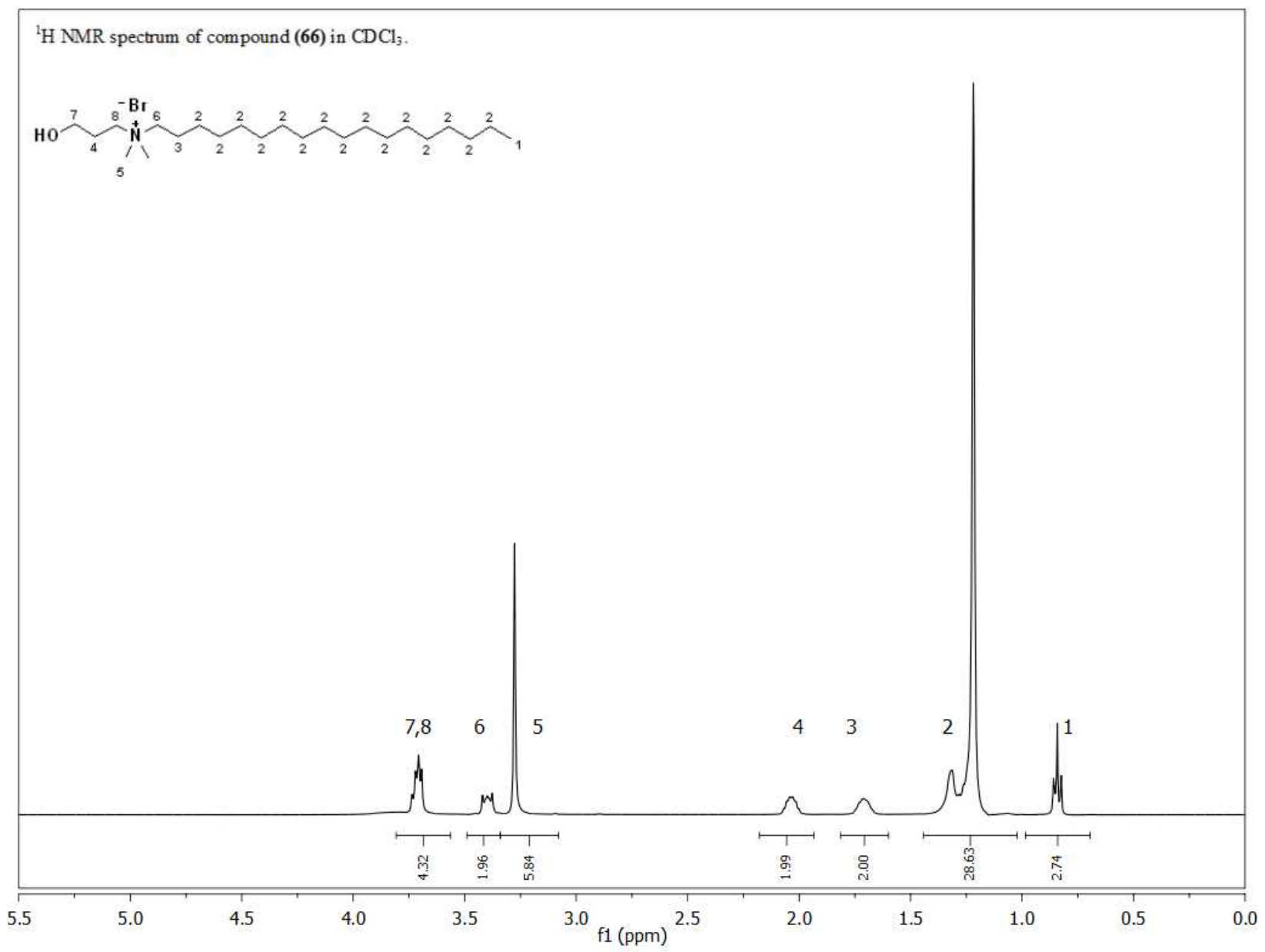

Figure 2.10: ${ }^{1} \mathrm{H}$ NMR $\left(\mathrm{CDCl}_{3}\right)$ spectrum of QAC aldehyde precursor 66. 


\section{Method 2: Michael Addition to Diethylvinylphosphonate}

THP protected bisphosphonate intermediate $\mathbf{7 1}$ and $\mathbf{7 2}$ are known literature compounds prepared by Method 2 (see Experimental, Section 5.4.2). However numerous attempts to obtain these compounds via the Michael addition of the Grignard to $\mathbf{6 8}$ failed to give the desired product according to Table 2.6. In all cases, the reaction did not proceed (Table 2.6, Entries i and ii) or resulted in a mixture of products by ${ }^{31} \mathrm{P} \mathrm{NMR}\left(\mathrm{CDCl}_{3}\right)$ after EtOAc extraction (Table 2.6, Entry iii). The failure of Method 2 can be attributed to an incomplete conversion of the alkyl halide to the Grignard nucleophile or its decomposition after it was prepared. A variety of ways to prepare the Grignard reagent were investigated. First, overnight sonication of $\mathrm{Mg}$ metal in $\mathrm{Et}_{2} \mathrm{O}$ was tested, however, the next day incomplete consumption of the metal remained (Table 2.6, Entry i). Activation of Mg by refluxing for 2 hrs followed by stirring at RT ON resulted in consumption of $\mathrm{Mg}$ and a cloudy solution was obtained (Table 2.6, Entry ii). In another trial, Mg was stirred at RT ON before adding it to 68 (Table 2.6, Entry iii). In all cases, no Michael addition products were detected by ${ }^{31} \mathrm{P}$ NMR $\left(\mathrm{CDCl}_{3}\right)$. 


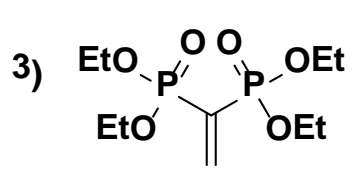

(68)

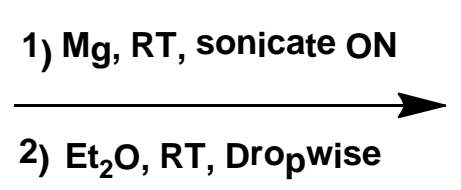

$\widehat{R}_{(69-70)}^{B r}$

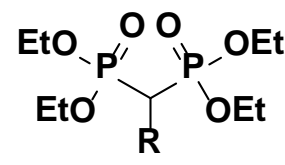

(71) $\mathrm{R}=\left(\mathrm{CH}_{2}\right)_{3} \mathrm{Cl}$

(72) $\mathrm{R}=\left(\mathrm{CH}_{2}\right)_{3} \mathrm{OTHP}$

Table 2.6: Attempted Grignard addition reactions onto 68.

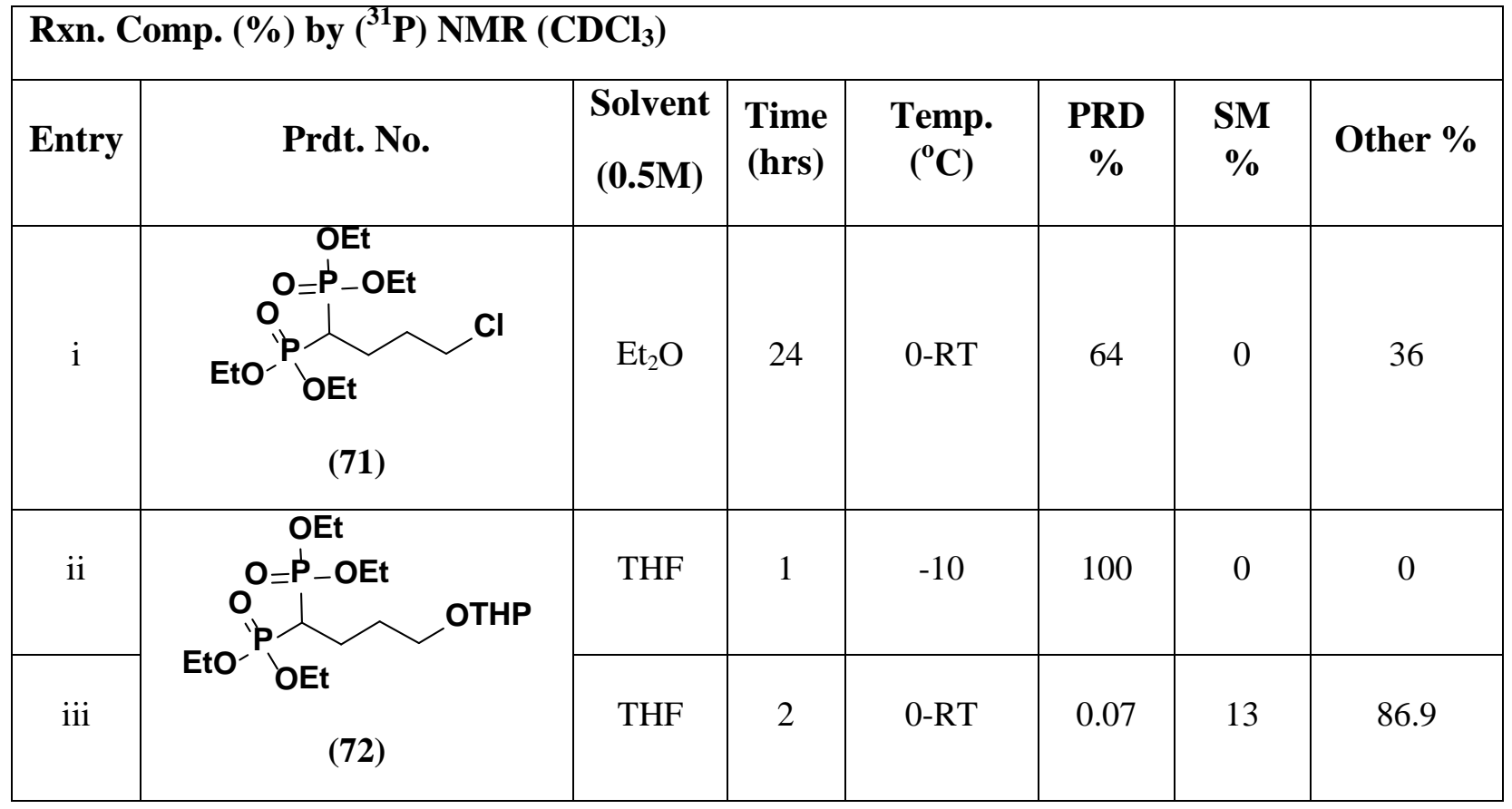

Precursors for the Michael addition were prepared by literature procedures (see Experimental, Section 5.4.2). Compound $\mathbf{6 8}$ was prepared from the commercially available tetraethyl methyl bisphosphonate $\mathbf{7 3}$ and purified by distillation according to Scheme 2.11, while 74 was protected with tetrahydropyran and purified by column chromatography (Scheme 2.12). After performing the THP protection numerous times it was found that the reaction was complete after $1 \mathrm{hr}$ without added catalyst. 


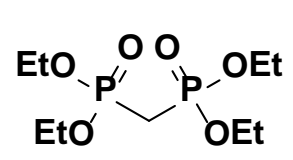

(73)

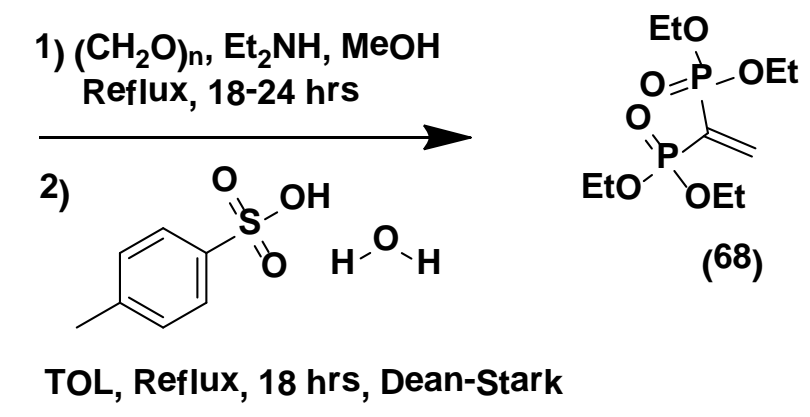

Scheme 2.11: Preparation of tetraethyl ethene-1,1-diylbisphosphonate 73.

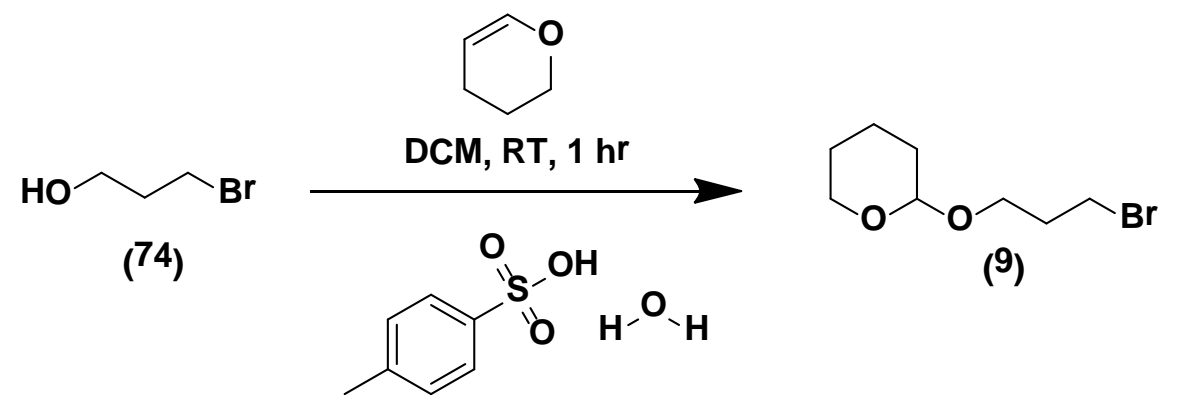

Scheme 2.12: Preparation of THP protected 3-bromopropanol 9.

\section{Method 3: C-P Formation}

Method 3 was attempted in order to prepare the amine protected $\mathbf{4 9}$ and $\mathbf{7 7 - 7 8}$ according to Table 2.7. Compound $\mathbf{7 7}$ was only successfully prepared on a small scale ( $<1 \mathrm{~g}$ ) when LDA was made in situ from $n$-BuLi/diisopropylamine (Table 2.7, Entry iii) in $\sim 60 \%$ yield and purified by column chromatography. Unfortunately LDA failed to deprotonate the phthalimide protected monophosphonate $\mathbf{1 9}$ at $-78{ }^{\circ} \mathrm{C}$ due to solubility issues (Table 2.7, Entry iv). When the same reaction was performed at $-10^{\circ} \mathrm{C}$, a mixture of two unknown products by ${ }^{31} \mathrm{P} \mathrm{NMR}\left(\mathrm{CDCl}_{3}\right)$ were observed at $\delta=31.43 \mathrm{ppm}(30 \%)$ and $\delta=-13.13 \mathrm{ppm}$ (70\%) respectively, but not the desired bisphosphonate 49 ( $\delta_{\text {expected }} \sim 24 \mathrm{ppm}$ ) (Table 2.7, Entry v). Lastly the $N, N$-dimethyl phosphonate 32 also could not be deprotonated due to insolubility in THF (Table 2.7, Entry vi). For large scale preparation of 77, Method 4 was investigated and is discussed below. 
<smiles>[R]CCCP(=O)(OCC)OCC</smiles>

(18) $R=$ OTHP

(19) $\mathrm{R}=\mathrm{PHT}$

(32) $\mathrm{R}=\mathrm{CH}_{2} \mathrm{NMe}_{2}$

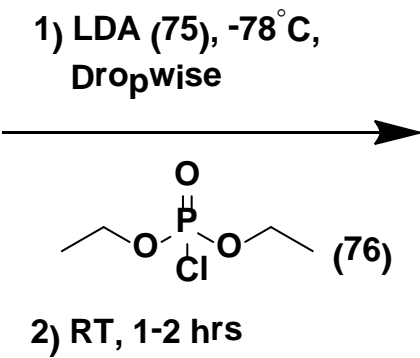

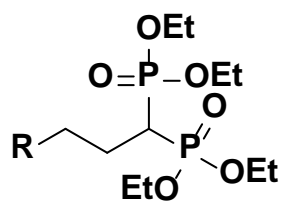

(49) $\mathrm{R}=\mathrm{PTH}$

(77) $R=$ OTHP

(78) $\mathrm{R}=\mathrm{NMe}_{2}$

Table 2.7: Preparation of bisphosphonates 49, 77-78 by Method 3.

\begin{tabular}{|c|c|c|c|c|c|c|}
\hline \multicolumn{7}{|c|}{ Rxn. Comp. (\%) by ${ }^{1} \mathrm{H},{ }^{31} \mathrm{P}$ NMR $\left(\mathrm{CDCl}_{3}\right)$} \\
\hline Entry & Reactant & Base & $\begin{array}{l}\text { Time } \\
\text { (hrs) }\end{array}$ & $\begin{array}{c}\text { Temp. } \\
\left({ }^{\circ} \mathrm{C}\right)\end{array}$ & $\begin{array}{c}\text { PRD. } \\
\%\end{array}$ & $\begin{array}{c}{ }^{31} \mathbf{P} \\
\text { NMR } \\
\text { (ppm) }\end{array}$ \\
\hline $\mathrm{i}$ & & NaHMDS & 2 & -78 & 0 & $\mathrm{n} / \mathrm{a}$ \\
\hline ii & & LDA & 2 & -78 & 10 & 24.14 \\
\hline iii & (77) & $\begin{array}{c}n \text {-BuLi / } \\
\text { DIA }\end{array}$ & 2 & -78 & 60 & 23.78 \\
\hline iv & & LDA & 2 & -78 & 0 & $\mathrm{n} / \mathrm{a}$ \\
\hline $\mathrm{v}$ & (49) & LDA & 2 & -10 & $0^{*}$ & $\mathrm{n} / \mathrm{a}$ \\
\hline vi & (78) & & n/a (SM ( & insolubl & n THF) & \\
\hline
\end{tabular}

Precursors 18 and 19 were prepared by the Abruzov reaction in one step while $\mathbf{3 2}$ was prepared in two steps utilizing the Abruzov reaction followed by alkylation with dimethylamine (see Table 2.3, Section 2.1.2.1). A pure sample of 32, a new compound, was isolated by column chromatography (note: compound turns dark on storage at RT). 


\section{Method 4: Direct Alkylation}

In contrast to Methods 1-3, all of the starting materials used in Method 4 are commercially available and were employed to prepare the bisphosphonate QAC 93 on a large scale after a lengthy optimization of the first alkylation step according to Scheme 2.13.

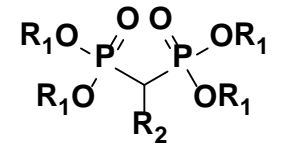

(72) $R_{1}=E t, R_{2}=H$

(79) $\mathrm{R}_{\mathbf{1}}=i \mathrm{Pr}, \mathrm{R}_{\mathbf{2}}=\mathrm{H}$
1) $\mathrm{NaH}, \mathrm{DMSO}, 0^{\circ} \mathrm{C}, \mathrm{RT}, 30 \mathrm{~min}$

2)

$$
\mathbf{R}_{3} \widehat{\gamma / n} \mathrm{R}_{4}
$$

(7-10)

(80) $n=4, R_{3}, R_{4}=B r$

(81) $n=1, R_{3}=C l, R_{4}=B r$

(82) $n=1, R_{3}=C l, R_{4}=I$

(83) $n=1, R_{3}=B r, R_{4}=B^{-} N^{+} M e_{2} C_{18} H_{37}$

(84) $n=1, R_{3}=B r, R_{4}=N_{2}$<smiles>[R3]C=C([R12])CC(P([R20])(=O)O[R20])P(=O)(O[R20])O[R2]</smiles>

(85) $\mathrm{n}=1, \mathrm{R}_{1}=\mathrm{Et}, \mathrm{R}_{3}=\mathrm{Br}$

(86) $\mathrm{n}=1, \mathrm{R}_{1}=i \mathrm{Pr}, \mathrm{R}_{3}=\mathrm{Br}$

(87) $\mathrm{n}=2, \mathrm{R}_{1}=i \mathrm{Pr}, \mathrm{R}_{3}=\mathrm{Br}$

(88) $n=2, R_{1}=E t, R_{3}=B r$

(89) $\mathrm{n}=4, \mathrm{R}_{1}=i \mathrm{Pr}, \mathrm{R}_{3}=\mathrm{Br}$

(90) $\mathrm{n}=4, \mathrm{R}_{1}=i \mathrm{Pr}, \mathrm{R}_{3}=\mathrm{Br}$

(91) $\mathrm{n}=1, \mathrm{R}_{1}=i \mathrm{Pr}, \mathrm{R}_{3}=\mathrm{Cl}$

(92) $\mathrm{n}=1, \mathrm{R}_{1}=i \mathrm{Pr}, \mathrm{R}_{3}=\left(\mathrm{CH}_{2}\right)_{3} \mathrm{PHT}$

(93) $\mathrm{n}=1, \mathrm{R}_{1}=i \mathrm{Pr}, \mathrm{R}_{3}=\mathrm{Br}^{-} \mathrm{N}^{+} \mathrm{Me}_{2} \mathrm{C}_{18} \mathrm{H}_{37}$

(94) $n=1, R_{1}=E t, R_{3}=\left(C_{2}\right)_{3}$ OTHP

(95) $\mathrm{n}=1, \mathrm{R}_{1}=i \mathrm{Pr}, \mathrm{R}_{3}=\left(\mathrm{CH}_{2}\right)_{3} \mathrm{OTHP}$

(96) $n=1, R_{3}=\mathrm{NMe}_{2}$

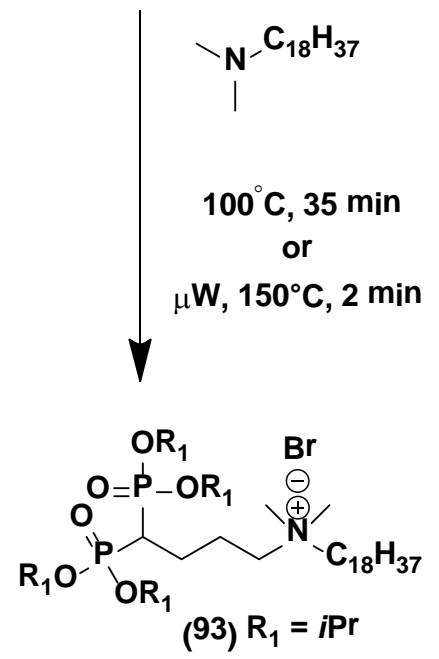

Scheme 2.13: Preparation of bisphosphonates by direct alkylation.

The direct alkylation of $\mathbf{7 2}$ or $\mathbf{7 9}$ to $\mathbf{8 5 - 9 6}$ was explored with different alkyl halides employing different solvents and bases (Table 2.8). Reaction progress was monitored by ${ }^{31} \mathrm{P}$ 
$\operatorname{NMR}\left(\mathrm{CDCl}_{3}\right)$ by observing the consumption of $72 \mathrm{OEt}(\delta=19.27 \mathrm{ppm})$, and $79 \mathrm{OiPr}(\delta=18.06$ ppm, Table 2.8). In all cases, the alkylation was incomplete and resulted in a mixture containing the desired product along with unreacted starting material, often requiring a difficult column chromatography purification (Table 2.8, Entries xiv, xv, xvi).

The choice of alkylhalide proved to be the most critical step in the reaction. Alkylation with the bromopropyl QAC (Table 2.8, Entry xviii) that could directly lead to the desired product in one step, failed to react $\left({ }^{31} \mathrm{P}\right.$ NMR) while the $N, N$-dimethyl 84 intermediate, also failed to alkylate. Next, dihalo precursors of various chain lengths $\mathrm{C}_{3}-\mathrm{C}_{6}$ were employed (Table 2.8, 7 $\left.\left(\mathrm{C}_{3}\right), \mathbf{8}\left(\mathrm{C}_{4}\right), \mathbf{8 0}\left(\mathrm{C}_{6}\right)\right)$ resulting in low yields. When NaH/DMSO (Table 2.8, Entries iii, xviii, xix, xxii) was employed with the dihalo compounds, higher yields were obtained versus THF while the $n$-BuLi/THF reaction resulted in a complicated mixture of products (Table 2.8, Entry iv). When an excess of NaH in DMSO was used, compound $\mathbf{8 8}$ was unexpectedly dialkylated due to an intramolecular reaction (Scheme 2.14, Table 2.8, Entry xvii, Figure 2.11).

As a result, a protective group strategy employing compound $\mathbf{1 0}$ was used as the alkyl halide to prepare 93 on a large scale (Figure 2.12). Compound 95, a known compound, was previously synthesized in the literature by Method 4 with NaH/THF in low yield (30\%). As a result both $\mathrm{NaH} / \mathrm{THF}$ and $\mathrm{NaH} / \mathrm{DMSO}$ combinations were tested in an attempt to improve the yield. Even though the NaH/DMSO conditions were higher yielding (Table 2.8, Entry xxvi), it was difficult to remove the solvent fully during extraction or distillation. Instead, when THF was dried ( $4^{\circ} \mathrm{A}$ MS) ON (Table 2.8, Entry xxiii) a $60 \%$ yield was obtained and the unreacted starting material $\mathbf{7 9}$ was carried throughout a lengthy deprotection/mesylation/substitution procedure to convert the alcohol to either a bromo 87 or $N, N$-dimethyl group 96 (Scheme 2.16). 


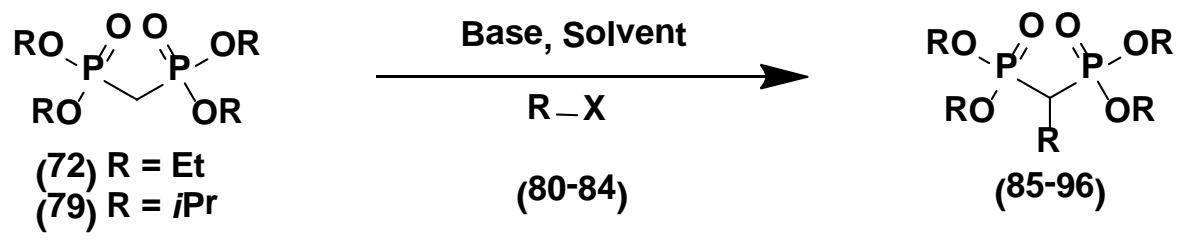

Table 2.8: Synthesis of $\alpha-\mathrm{C}-\mathrm{H}$ bisphosphonates by Method 4 .

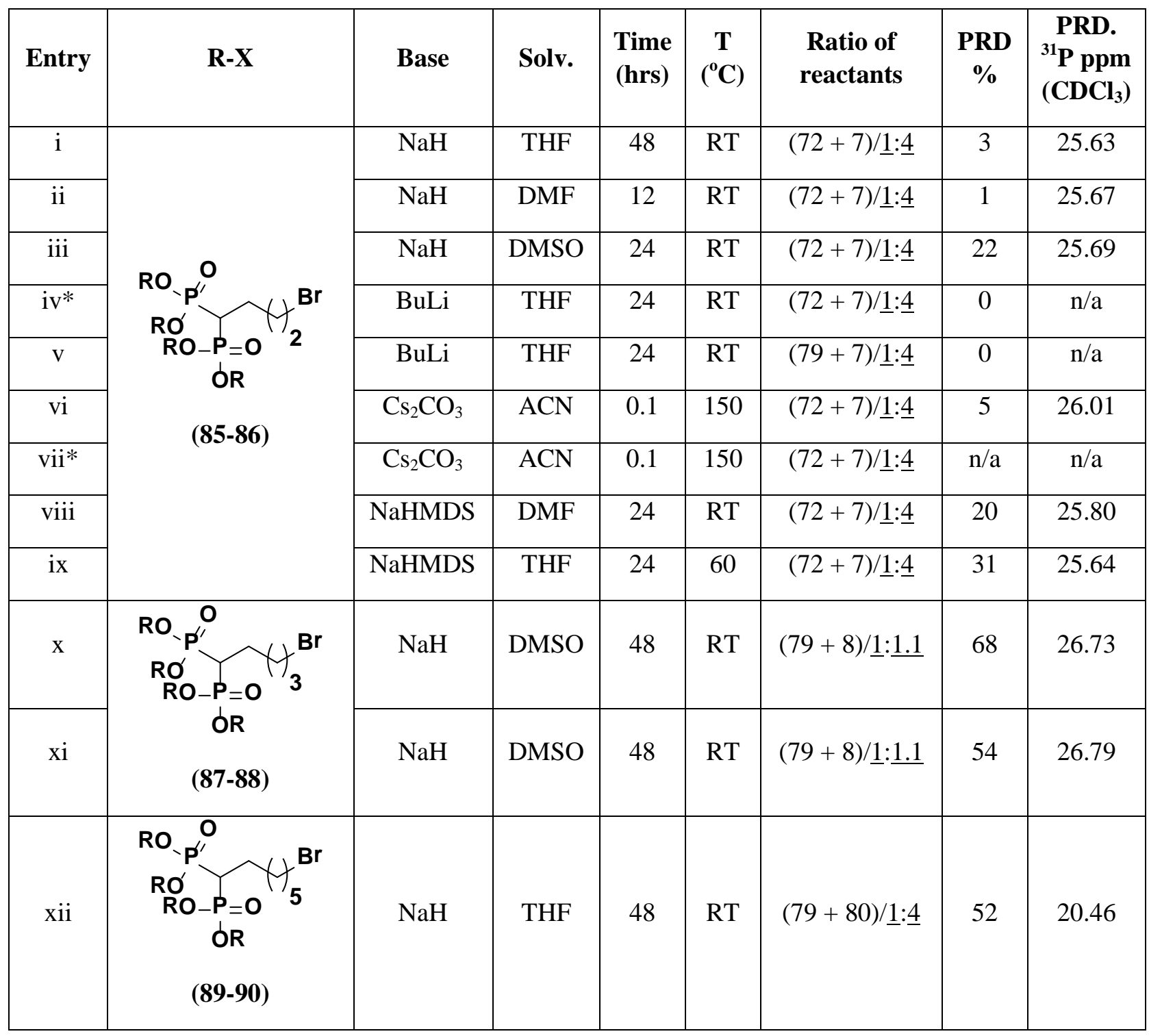




\begin{tabular}{|c|c|c|c|c|c|c|c|c|}
\hline Entry & $\mathbf{R}-\mathbf{X}$ & Base & Solv. & $\begin{array}{l}\text { Time } \\
\text { (hrs) }\end{array}$ & $\begin{array}{c}\mathrm{T} \\
\left({ }^{\circ} \mathrm{C}\right)\end{array}$ & $\begin{array}{l}\text { Ratio of } \\
\text { reactants }\end{array}$ & $\begin{array}{c}\text { PRD } \\
\%\end{array}$ & $\begin{array}{c}\text { PRD. } \\
{ }^{31} \mathrm{P} \mathrm{ppm} \\
\left(\mathrm{CDCl}_{3}\right)\end{array}$ \\
\hline xiii & & $\mathrm{NaH}$ & THF & 24 & 60 & $(79+81) / \underline{1}: \underline{4}$ & 0 & $\mathrm{n} / \mathrm{a}$ \\
\hline xiv & OR & $\mathrm{NaH}$ & Diox. & 24 & 60 & $(79+81) / \underline{1}: \underline{4}$ & 0 & $\mathrm{n} / \mathrm{a}$ \\
\hline $\mathrm{xV}^{*}$ & & $\mathrm{NaH}$ & THF & 24 & 70 & $(79+82) / \underline{1}: \underline{1.1}$ & $\mathrm{n} / \mathrm{a}$ & $\mathrm{n} / \mathrm{a}$ \\
\hline xvi & RO_ $\mathbf{p}^{\prime \prime}$ & $\mathrm{NaH}$ & DMSO & 48 & RT & $(79+10) / \underline{1}: \underline{1.1}$ & 0 & $\mathrm{n} / \mathrm{a}$ \\
\hline xvii & $\begin{array}{l}\text { OR } \\
\text { (92) }\end{array}$ & $\mathrm{NaH}$ & Diox. & 24 & 80 & $(79+10) / \underline{1}: \underline{1.1}$ & 8 & 24.98 \\
\hline xviii & 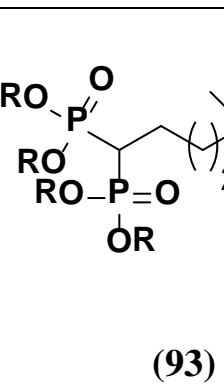 & $\mathrm{NaH}$ & DMSO & 24 & 80 & $(79+83) / \underline{1}: \underline{1.1}$ & 24 & 21.07 \\
\hline xix & ${ }^{\text {Eto }}{ }^{\mathrm{O}} \mathrm{P}$ & $\mathrm{NaH}$ & DMSO & 24 & RT & $(72+7) / \underline{1}: \underline{1.5}$ & 99 & 28.50 \\
\hline $\mathrm{XX}$ & 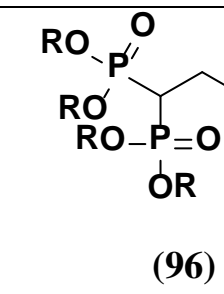 & $\mathrm{NaH}$ & DMSO & 24 & 70 & $(72+84) / \underline{1}: \underline{1}$ & 0 & $\mathrm{n} / \mathrm{a}$ \\
\hline
\end{tabular}

* multiple peaks ,** PCT (phase transfer catalyst). 


\begin{tabular}{|c|c|c|c|c|c|c|c|c|}
\hline \multicolumn{9}{|c|}{ Rxn. Comp. (\%) by ${ }^{31}$ P NMR $\left(\mathrm{CDCl}_{3}\right)$} \\
\hline Entry & $\mathbf{R}-\mathbf{X}$ & Base & Solv. & $\begin{array}{l}\text { Time } \\
\text { (hrs) }\end{array}$ & $\begin{array}{c}\mathrm{T} \\
\left({ }^{\circ} \mathrm{C}\right)\end{array}$ & $\begin{array}{l}\text { Ratio of } \\
\text { reactants }\end{array}$ & $\begin{array}{c}\text { PRD } \\
\%\end{array}$ & $\begin{array}{c}\text { PRD. } \\
{ }^{31} \text { P ppm } \\
\left(\mathrm{CDCl}_{3}\right)\end{array}$ \\
\hline xxi & \multirow{7}{*}{ 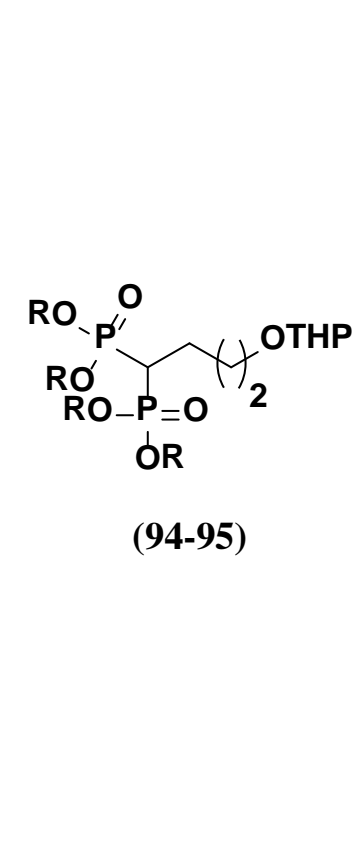 } & $\mathrm{NaH}$ & THF & 48 & 60 & $(79+9) / \underline{1}: \underline{1}$ & 47 & 23.94 \\
\hline xxii & & $\mathrm{NaH}$ & THF & 72 & RT & $(72+9) / \underline{1}: \underline{1.1}$ & $\mathrm{n} / \mathrm{a}$ & $\mathrm{n} / \mathrm{a}$ \\
\hline xxiii & & $\mathrm{NaH}$ & THF & 48 & 70 & $(79+9) / \underline{1}: \underline{1}$ & 60 & $\mathrm{n} / \mathrm{a}$ \\
\hline xxiv * & & $\mathrm{Cs}_{2} \mathrm{CO}_{3}$ & $\mathrm{ACN}$ & 48 & 90 & $(79+9) / \underline{1}: \underline{2}$ & 23 & 23.78 \\
\hline $\mathrm{xxv}$ & & $\mathrm{Cs}_{2} \mathrm{CO}_{3}$ & $\mathrm{ACN}$ & 72 & 90 & $(79+9) / \underline{1}: \underline{1}$ & 44 & 23.75 \\
\hline xxvi & & $\mathrm{NaH}$ & $\begin{array}{c}\text { DMS } \\
\text { O }\end{array}$ & RT & 24 & $(79+9) / \underline{1}: \underline{5}$ & 62 & 23.71 \\
\hline xxvii & & $\mathrm{NaH}^{\mathrm{eq}}$ & $\begin{array}{c}\text { DMS } \\
\text { O }\end{array}$ & RT & 96 & $(79+9) / \underline{1}: \underline{1}$ & 92 & 23.66 \\
\hline
\end{tabular}

* multiple peaks , ** PCT (phase transfer catalyst).

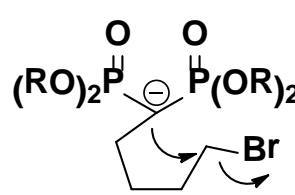

(88b) $\mathrm{R}=i \mathrm{Pr}$

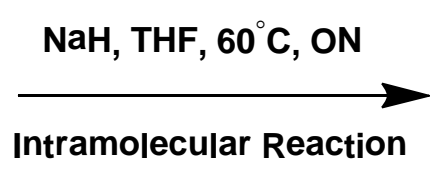

(97) $\mathrm{R}=i \mathrm{Pr}$

Isolated Product

Scheme 2.14: Intramolecular bisphosphonate alkylation. 


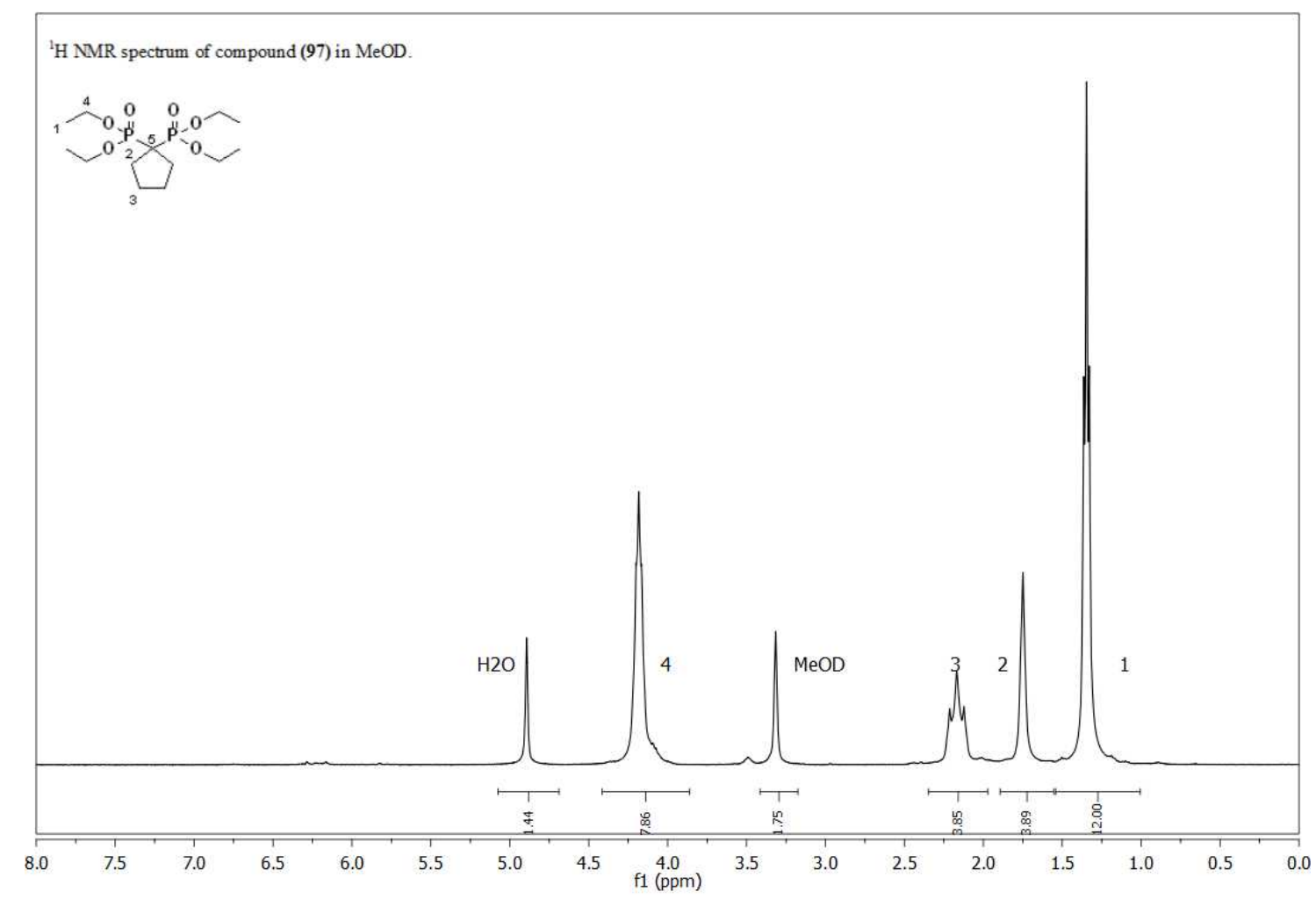

Figure 2.11: ${ }^{1} \mathrm{H}$ NMR (MeOD) spectrum of cyclic bisphosphonate by-product 97.
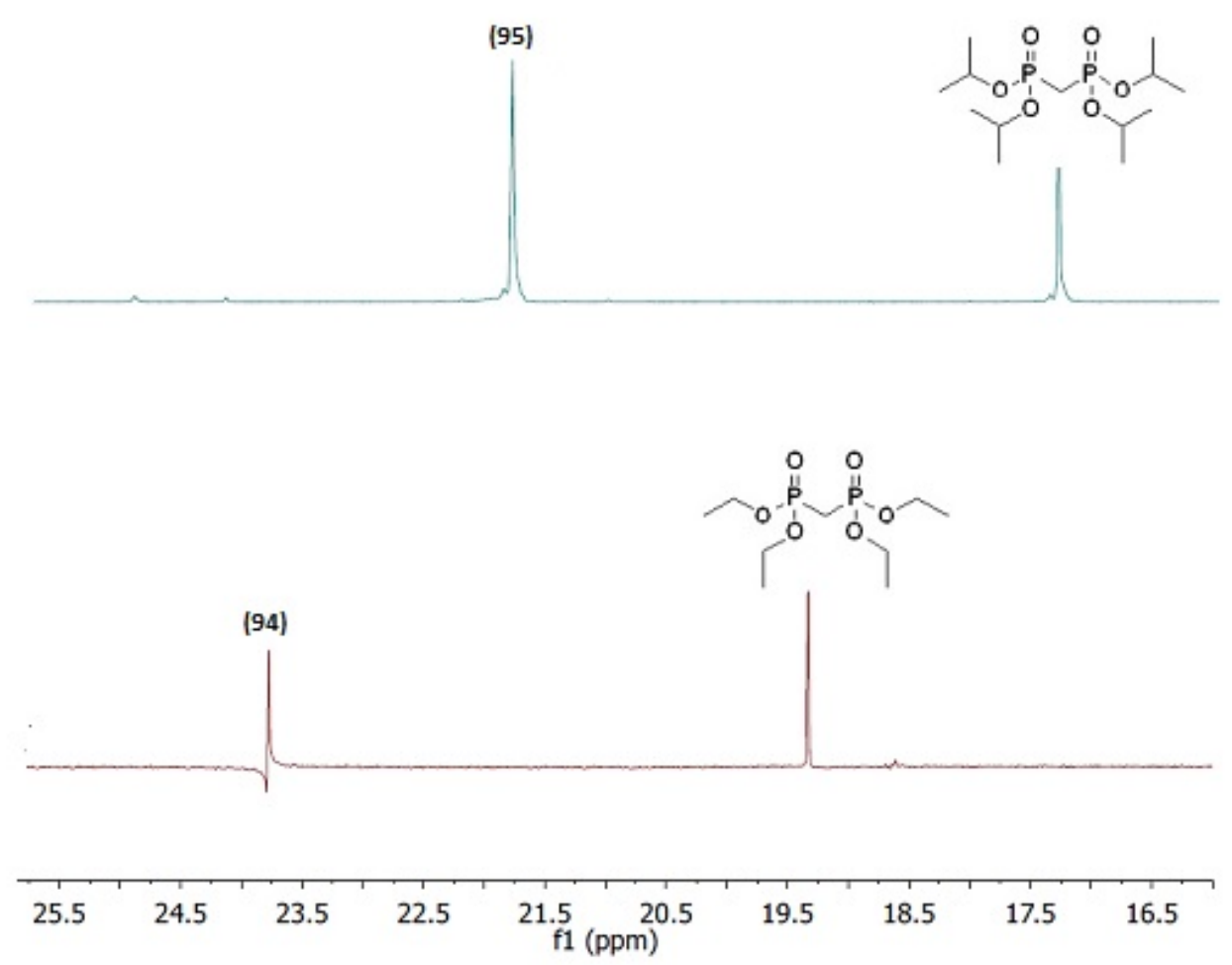

Figure 2.12: ${ }^{31} \mathrm{P}$ NMR $\left(\mathrm{CDCl}_{3}\right)$ monitoring for compound 94 and 95 prepared by Method 4. 125 


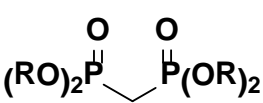

(79) $\mathrm{R}=i \mathrm{Pr}$

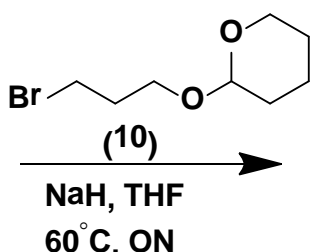

$60^{\circ} \mathrm{C}$, ON

(93)

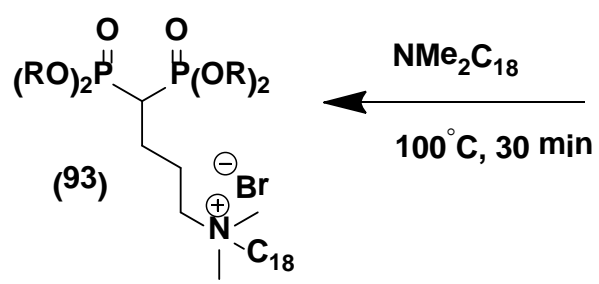

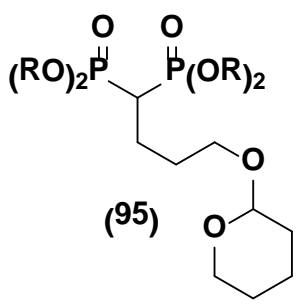

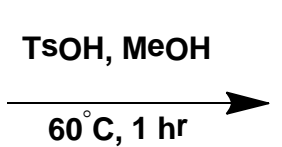

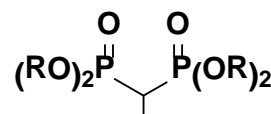

(98)

$\mathrm{MSCl}, \mathrm{Et}_{3} \mathrm{~N}$
$\mathrm{RT}, 1 \mathrm{hr}$

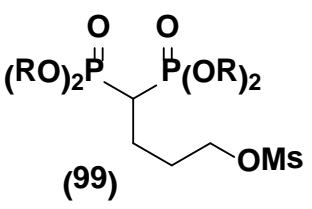

Scheme 2.15: Preparation of bisphosphonate QAC 96 via alkylation.

Quaternization of 87 (Scheme 2.15) gave the desired bisphosphonate quat 93 (Figure 2.13), however, dealkylation with $\mathrm{HBr}(\mu \mathrm{W}, 8 \mathrm{~min})$ could not be monitored due to precipitation of the product 97 in the NMR tube $\left(\mathrm{D}_{2} \mathrm{O}\right)$ as well as several other deuturated solvents $\left(\mathrm{CDCl}_{3}\right.$, DMSO), failing to show any ${ }^{31} \mathrm{P}$ NMR signals of the crude reaction mixture. 


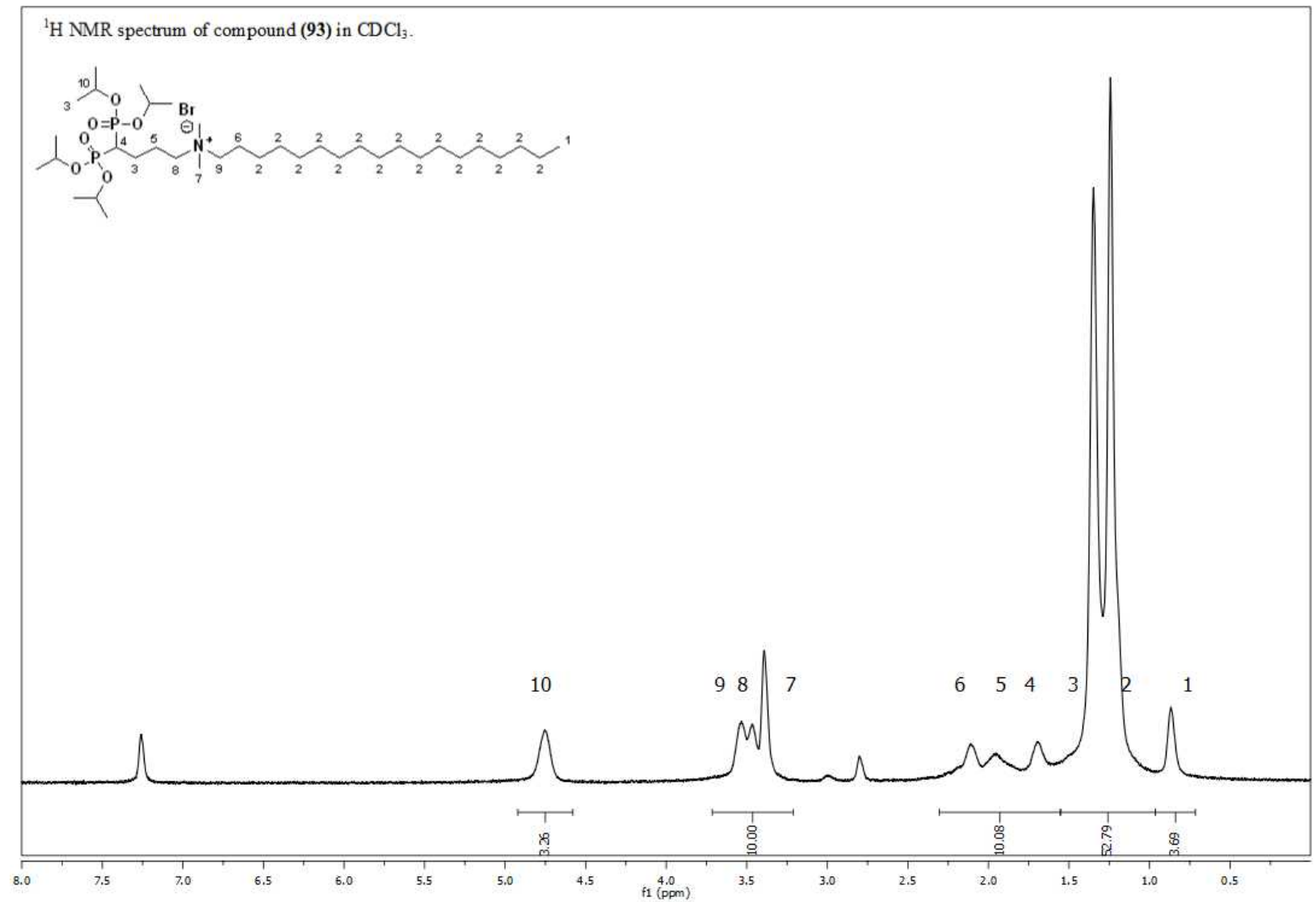

Figure 2.13: ${ }^{1} \mathrm{H}$ NMR $\left(\mathrm{CDCl}_{3}\right)$ spectrum of $\alpha$-CH-QAC 93 prepared by Method 4.

The rest of compound $\mathbf{8 7}$ was used to make the dimethyl derivative $\mathbf{9 6}$ in order to prepare trisphosphonate 101 via alkylation with NaHMDS/DECP (Scheme 2.16). 


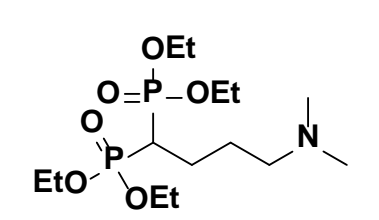

(96)

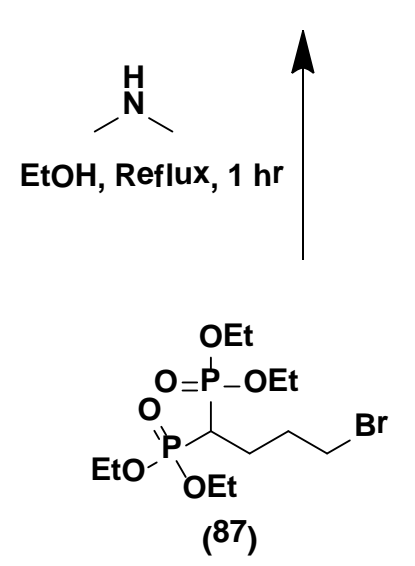

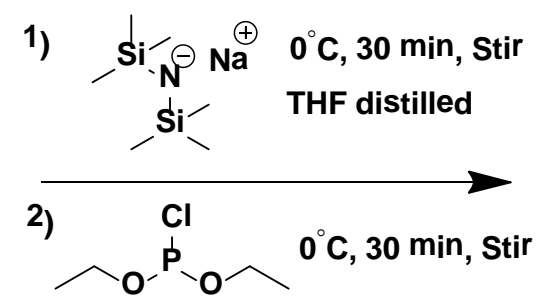

3) $\mathrm{H}_{2} \mathrm{O}_{2}$ Dropwise, $0^{\circ} \mathrm{C}, 30 \mathrm{~min}$, Stir
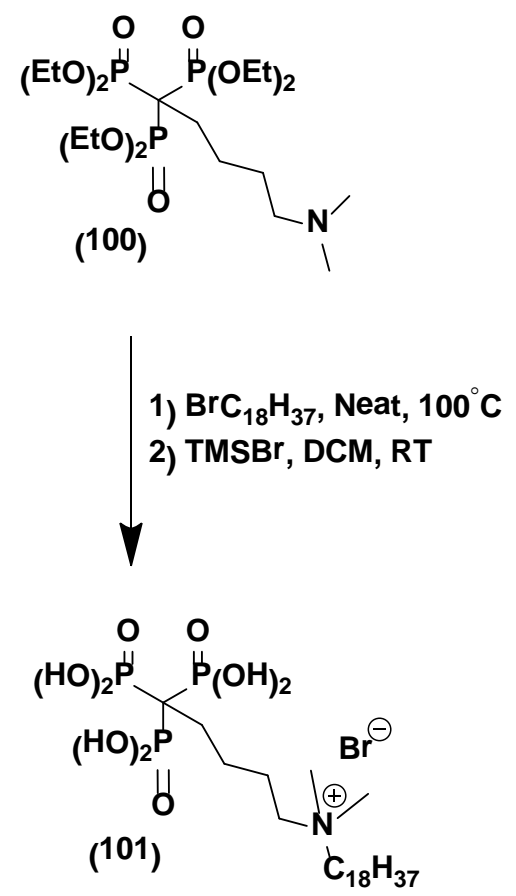

Scheme 2.16: Attempted preparation of trisphosphonic acid 101 via alkylation of $\mathbf{9 6} .^{201}$

Halo QAC’s 83 (bromo) and 102 (chloro) were synthesized by the Menschutkin reaction as precursors for alkylation of $\mathbf{7 9}$ in order to directly obtain the target bisphosphonate QAC $\mathbf{9 3}$ in one step with Method 4 (Scheme 2.17 and Scheme 2.13). Alkylation with bromo precursor 83 resulted in only a $24 \%$ conversion by NMR spectroscopy $\left({ }^{1} \mathrm{H},{ }^{31} \mathrm{P}, \mathrm{CDCl}_{3}\right)$ (Table 2.8, Entry xviii). Purifying the product from the quat mixture was never attempted. The less reactive chloro precursor was made and characterized by NMR spectroscopy $\left({ }^{1} \mathrm{H},{ }^{31} \mathrm{P}, \mathrm{CDCl}_{3}\right.$, Figure 2.14$)$ but never employed in the alkylation reaction with $\mathbf{7 9}$ due the low product conversion with the bromo precursor. 


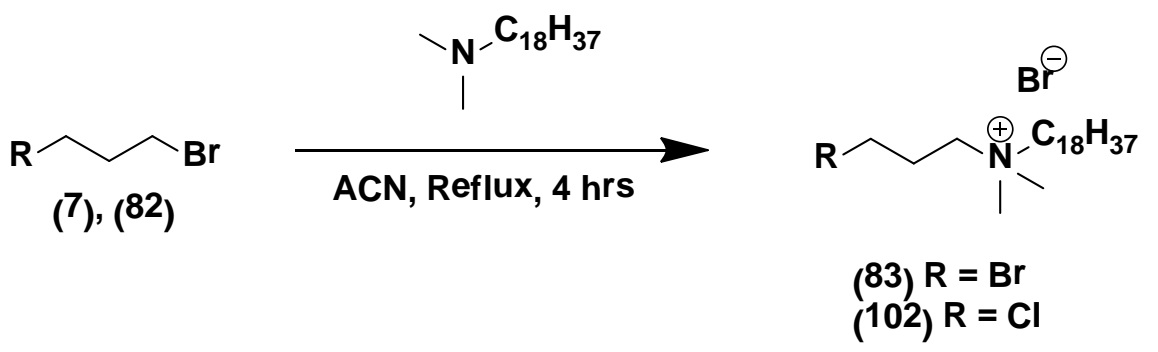

Scheme 2.17: Preparation of halo QAC 83 and 102 precursors for Method 4.

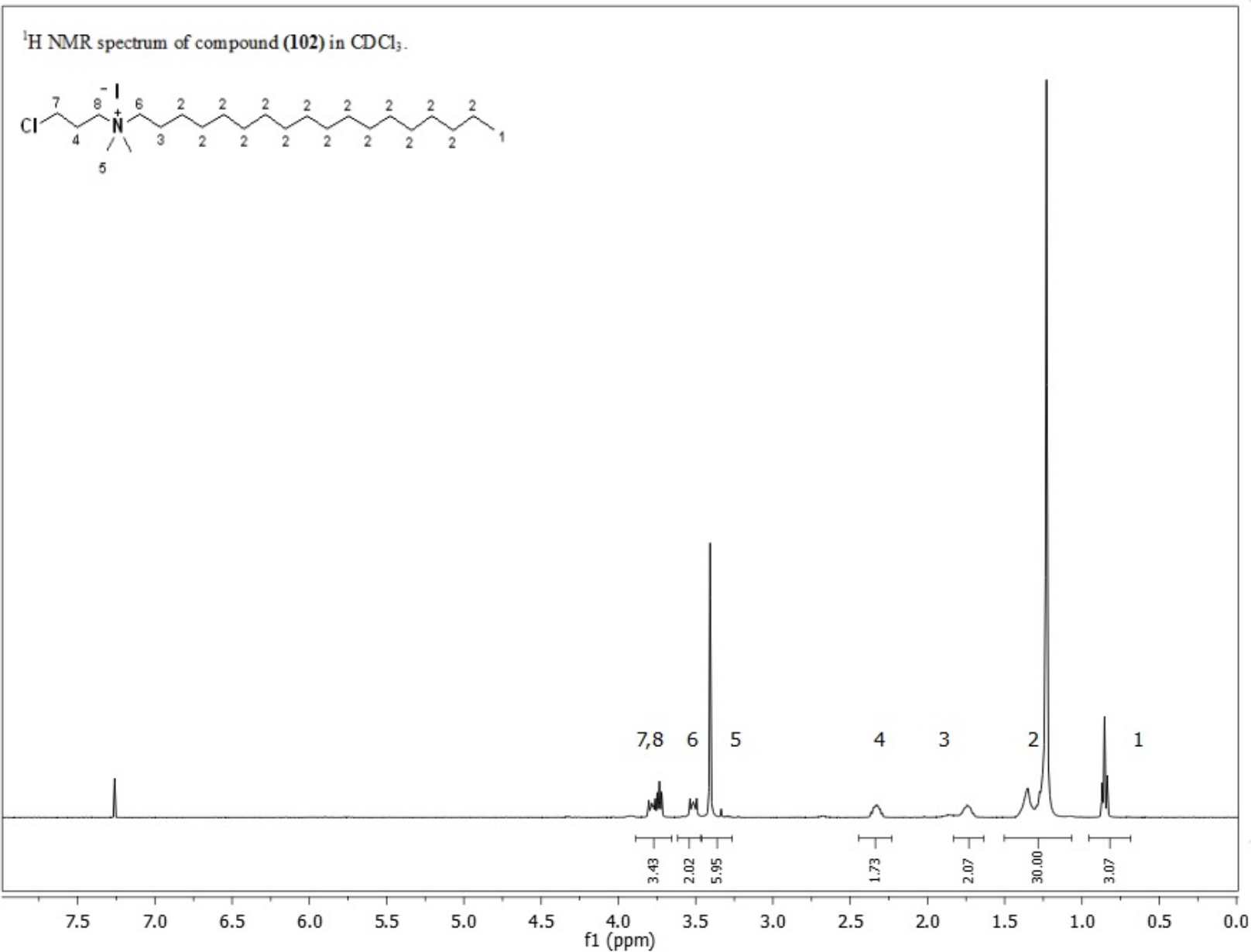

Figure 2.14: ${ }^{1} \mathrm{H}$ NMR $\left(\mathrm{CDCl}_{3}\right)$ spectrum of precursor 102. 


\subsubsection{Bisphosphonates via the 3-Component Reaction}

A 3-component reaction was used to prepare precursor 105 in 75\% yield (Experimental, Section 5.5.0) according to known procedures and quaternized with bromooctadecane (Scheme 2.18). ${ }^{202}$ No useful NMR data was obtained for compound 106 due to difficulties with purification. It remains doubtful whether 105, a weak and hindered nucleophile due to the presence of two electron withdrawing phosphonate ester groups could be quaternized.

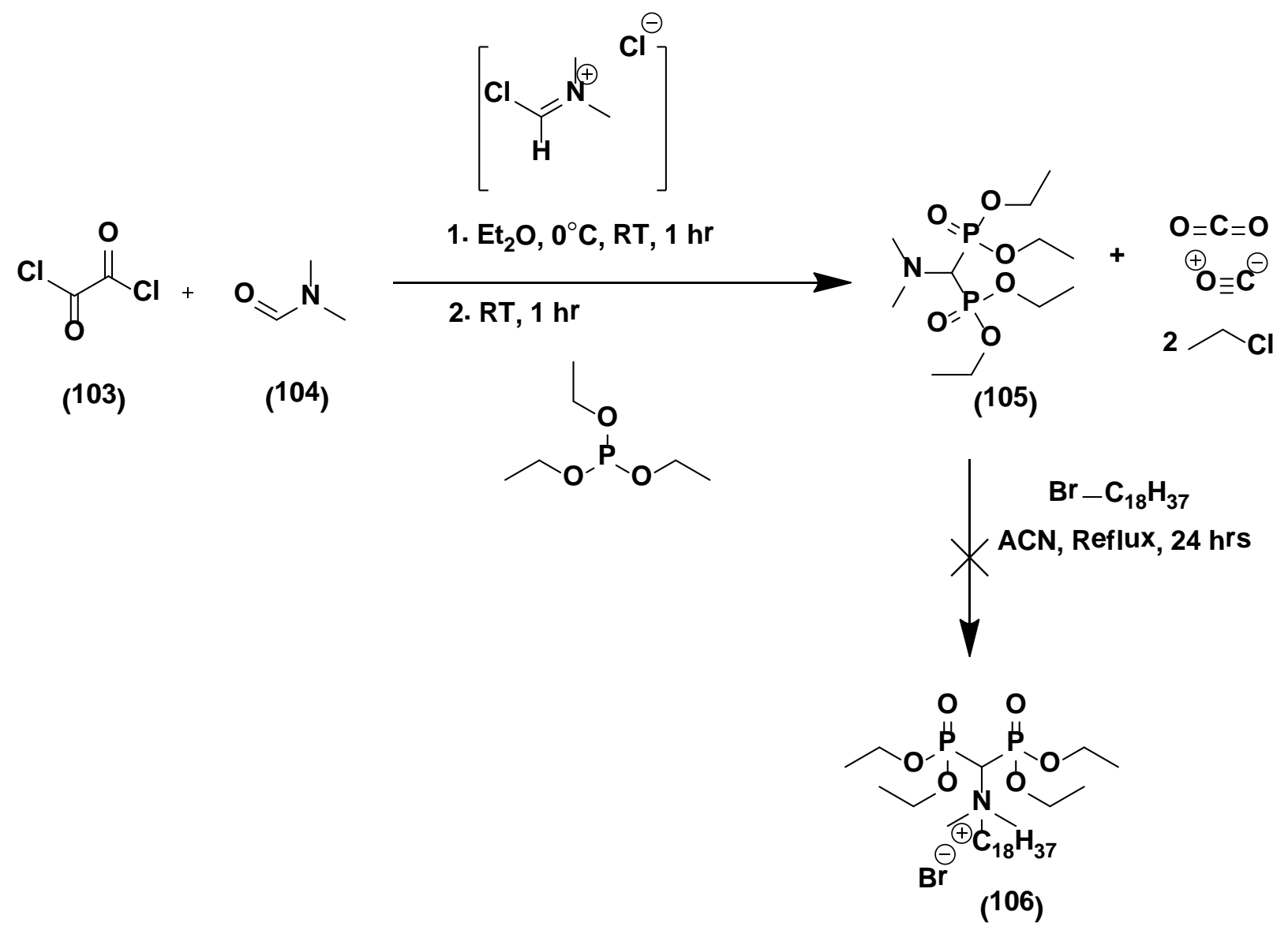

Scheme 2.18: Attempted preparation of bisphosphonate 106. 


\subsubsection{5 $\alpha$-Aminobisphosphonic acids QAC Antimicrobials ( $\alpha$-ABPQA)}

$\alpha$-Aminobisphosphonic acid QAC’s were synthesized either directly via the three component Kabachnik-Fields reactions between formaldehyde, a primary amine and dialkyl phosphite (Scheme 2.19) or sequentially by isolating the imine intermediate (Scheme 2.20). The synthesis required a three step process starting with the Kabachnik-Fields reaction to generate novel bifunctional molecules with the $\alpha$-bisphosphonates anchored at one end and either a $N, N$ dimethylamino 113 or halo 114-117, 120 end group required for the quaternization reaction. The third and final step required didealkylation of the QAC bisphosphonate $\mathbf{1 2 1}$ to the free phosphonic acid 126 (Scheme 2.21). Initially $N^{1}, N^{1}$-dimethylpropane-1,3-diamine 108 seemed like the most logical choice of starting material for the Kabachnik-Fields reaction in order to obtain compound 113. Compound $\mathbf{1 0 8}$ is an inexpensive commercially available liquid and required no additional steps to free the primary amine as was the case with the halo precursors 109, 110 and sold as the $\mathrm{HCl}$ and $\mathrm{HBr}$ salts, respectively. 


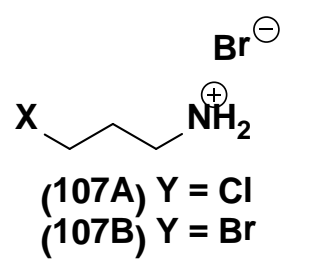<smiles>[X]CCCN</smiles>

$$
\begin{aligned}
& (108) X=\mathrm{NMe}_{2} \\
& (109) X=\mathrm{Cl} \\
& (110) X=\mathrm{Br} \\
& (111) X=\mathrm{OH}
\end{aligned}
$$

Step 1: KF reaction

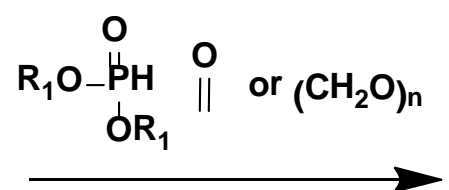
1) $\mathrm{RT}, 30 \mathrm{~min}, 2) 100^{\circ} \mathrm{C}, 1 \mathrm{hr}$
or $\mu \mathrm{W}, 130^{\circ} \mathrm{C}, 5 \mathrm{~min}$

$$
\begin{aligned}
& (112 A) R_{1}=O M e \\
& \text { (112B) } R_{1}=O E t
\end{aligned}
$$

(113) $X=\mathrm{NMe}_{2}, \mathrm{R}_{1}=\mathrm{Me}$

(114) $X=C l, R_{1}=E t$

(115) $X=B r R_{1}=M e$

(116) $X=B r, R_{1}=E t$

(117) $X=O H, R_{1}=E t$

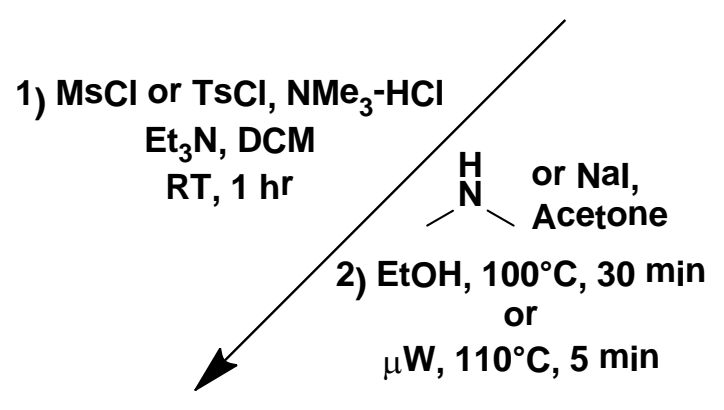

Step 2: Quaternization
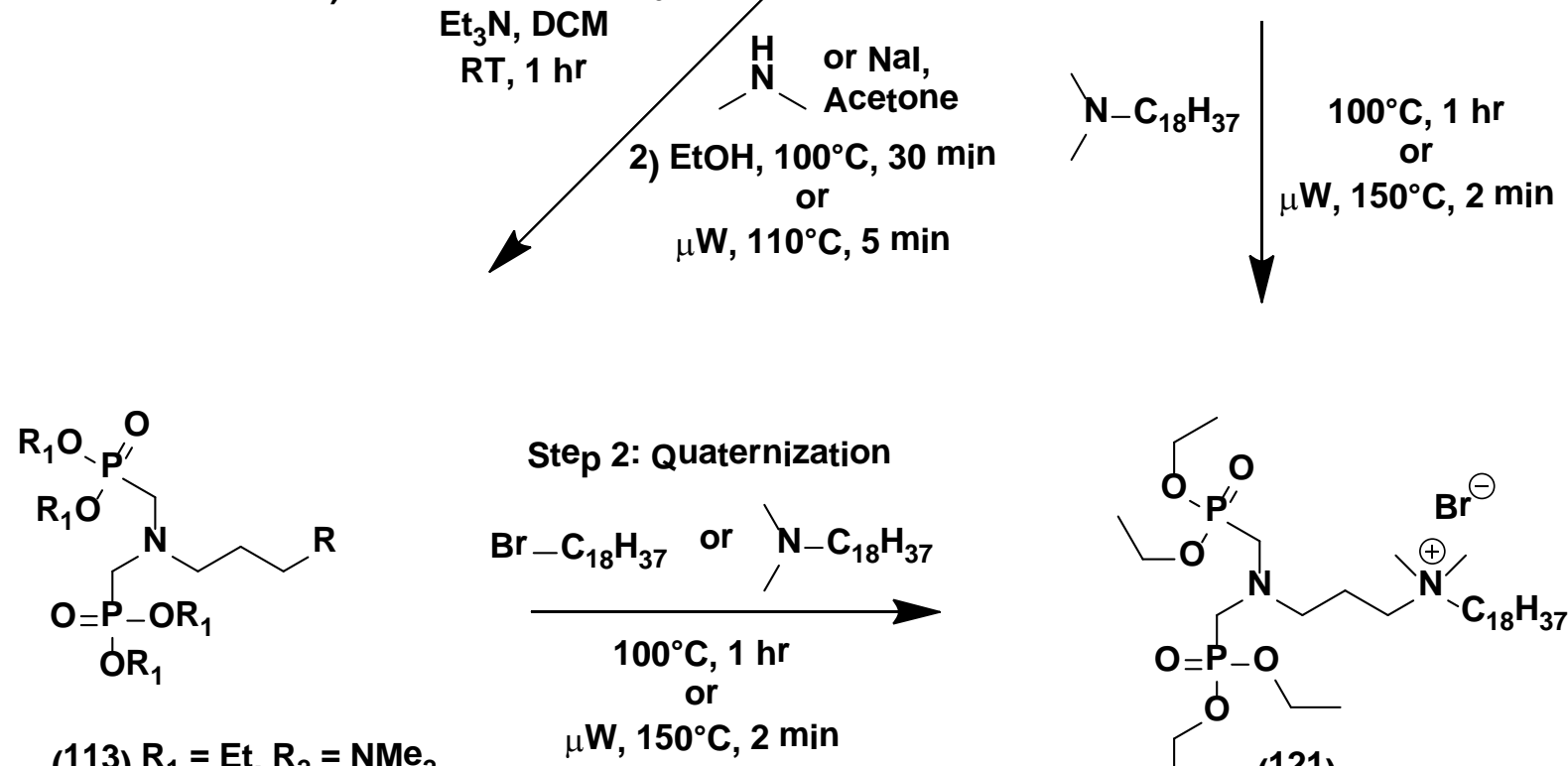

(113) $R_{1}=\mathrm{Et}, \mathrm{R}_{2}=\mathrm{NMe}_{2}$

(118) $R_{1}=E t, R_{2}=O M s$

(119) $R_{1}=E t, R_{2}=$ OTOS

(120) $R_{1}=E t, R_{2}=1$

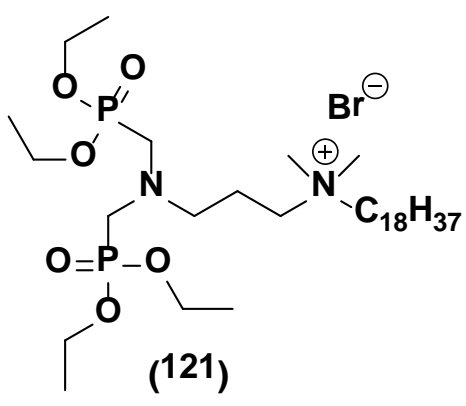

Scheme 2.19: Synthetic route to $\alpha$-aminobisphosphonate QAC 121. Optimized conditions for each step.

As a result, optimization of the Kabachnik-Fields reaction was first explored with $\mathbf{1 0 8}$ (Table 2.10, Entries i-iv) as well as with the triazine intermediate 123 (Table 2.10, Entry v). In 
all cases, the expected $\alpha$-bisphosphonate 113 product peak at $\delta \sim 26-24 \mathrm{ppm}\left({ }^{31} \mathrm{P}\right.$ NMR, $\left.\mathrm{CDCl}_{3}\right)$ was observed while monitoring the reaction, however it was incorrectly assigned as compound 113. After minimal mass was recovered in the organic layer after extraction, the reaction was directly purified by column chromatography and the peak at $\sim 26 \mathrm{ppm}$ was attributed to compound 124 after a further ${ }^{1} \mathrm{H} \mathrm{NMR}\left(\mathrm{CDCl}_{3}\right)$ analysis (Figure 2.15).

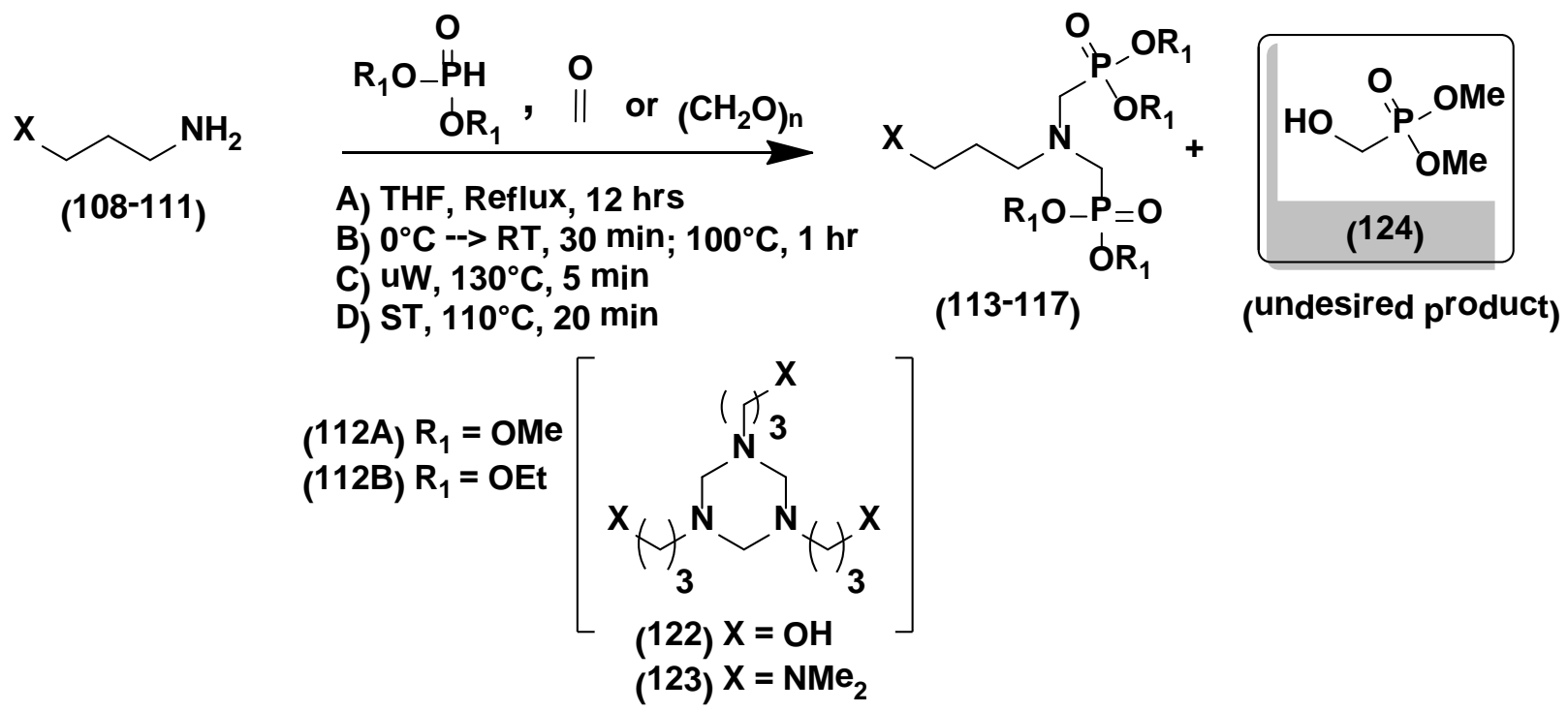

Table 2.9: Optimization of the Kabachnik-Fields reaction.

\begin{tabular}{|c|c|c|c|c|c|c|c|c|}
\hline \multicolumn{9}{|c|}{ Rxn. Comp. $(\%)$ by $\left({ }^{31} \mathrm{P}\right)$ ppm NMR $\left(\mathrm{CDCl}_{3}\right)$} \\
\hline Entry & Reactant & SM & Base & $\begin{array}{l}\text { Temp. } \\
\left({ }^{\circ} \mathrm{C}\right)\end{array}$ & Time & Solv. & $\begin{array}{c}\text { PRD } \\
\%\end{array}$ & $\begin{array}{c}\text { PRD } \\
{ }^{31} \mathbf{P} \mathbf{~ p p m}\end{array}$ \\
\hline $\mathrm{i}$ & \multirow{5}{*}{ 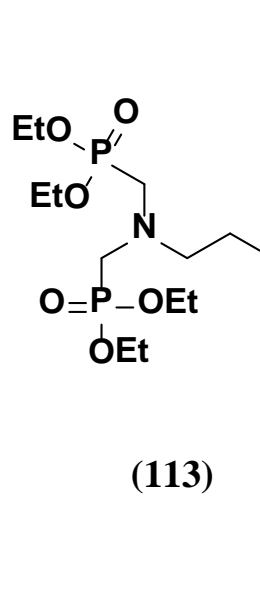 } & $\begin{array}{c}112 \mathrm{~A}+ \\
108\end{array}$ & $\mathrm{n} / \mathrm{a}$ & RT & 24 hrs & $\mathrm{H}_{2} \mathrm{O}$ & 70 & 26.85 \\
\hline ii & & $\begin{array}{c}112 \mathrm{~A}+ \\
108\end{array}$ & $\mathrm{n} / \mathrm{a}$ & RT & $5 \mathrm{~min}$ & THF & 36 & 26.42 \\
\hline iii & & $\begin{array}{c}112 \mathrm{~A}+ \\
108\end{array}$ & $\mathrm{n} / \mathrm{a}$ & 80 & 4 hrs & THF & 66 & 26.61 \\
\hline iv & & $\begin{array}{c}112 \mathrm{~A}+ \\
108\end{array}$ & $\mathrm{n} / \mathrm{a}$ & 80 & 20 hrs & THF & 76 & 26.61 \\
\hline $\mathrm{v}$ & & $\begin{array}{c}112 \mathrm{~A}+ \\
108\end{array}$ & $\mathrm{n} / \mathrm{a}$ & Reflux & $3 \mathrm{hrs}$ & ACN & 50 & 25.01 \\
\hline
\end{tabular}




\begin{tabular}{|c|c|c|c|c|c|c|c|c|}
\hline \multicolumn{5}{|c|}{ Table 2.9 continued } & \multicolumn{4}{|c|}{$\begin{array}{c}\text { Rxn. Comp. }(\%) \text { by }\left({ }^{31} \mathrm{P}\right) \mathrm{ppm} \\
\text { NMR }\left(\mathrm{CDCl}_{3}\right)\end{array}$} \\
\hline Entry & Reactant & SM & Base & $\begin{array}{l}\text { Temp. } \\
\left({ }^{\circ} \mathrm{C}\right)\end{array}$ & Time & Solv. & $\begin{array}{l}\text { PRD } \\
. \%\end{array}$ & $\begin{array}{c}\text { PRD } \\
{ }^{31} \mathbf{P} \\
\text { ppm }\end{array}$ \\
\hline vi & $\begin{array}{c}\text { Eto-P } \mathbf{P}^{\prime \prime} \\
\text { Eto' }\end{array}$ & $112 B+108$ & $\mathrm{NMe}_{2}$ & Reflux & $30 \mathrm{~min}$ & $\mathrm{EtOH}$ & 62 & 24.89 \\
\hline vii & $\begin{array}{l}\text { OEt } \\
\text { (113) }\end{array}$ & $112 B+108$ & $\mathrm{NMe}_{2}$ & 110 & $5 \min$ & $\mathrm{EtOH}$ & 65 & 24.52 \\
\hline viii & Eto & $\begin{array}{c}112 \mathrm{~A}+ \\
110\end{array}$ & $\mathrm{Et}_{3} \mathrm{~N}$ & 70 & 20 hrs & THF & 94 & 8.32 \\
\hline ix & & $112 B+110$ & $\mathrm{NaOH}$ & 100 & $1 \mathrm{hr}$ & $\mathrm{H}_{2} \mathrm{O}$ & 1 & $\mathrm{n} / \mathrm{a}$ \\
\hline $\mathrm{X}$ & $O=F$ & $112 B+110$ & $\mathrm{NaOH}$ & 100 & $1 \mathrm{hr}$ & $\mathrm{H}_{2} \mathrm{O}$ & 21 & 24.69 \\
\hline$x i$ & & $112 B+110$ & $\mathrm{NaOH}$ & 100 & $1 \mathrm{hr}$ & $\mathrm{H}_{2} \mathrm{O}$ & 53 & 24.69 \\
\hline $\mathrm{xii}^{\mu \mathrm{W}}$ & 5-116) & $112 \mathrm{~B}+110$ & $\mathrm{NaOH}$ & 130 & $5 \min$ & Neat & 40 & 24.68 \\
\hline xiii & $\begin{array}{l}\text { Eto-P } \\
\text { Eto' }\end{array}$ & $112 B+109$ & $\mathrm{NaOH}$ & 100 & $1 \mathrm{hr}$ & $\mathrm{H}_{2} \mathrm{O}$ & 46 & 24.65 \\
\hline xiv & $\begin{array}{l}\text { OEt } \\
(\mathbf{1 1 4})\end{array}$ & $112 B+109$ & $\mathrm{KOH}$ & 100 & $1 \mathrm{hr}$ & $\mathrm{H}_{2} \mathrm{O}$ & 48 & 24.40 \\
\hline $\mathrm{XV}$ & $\begin{array}{c}\text { Eto_ } \mathbf{P}^{\prime \prime} \\
\text { EtO' }\end{array}$ & $112 B+111$ & $\mathrm{n} / \mathrm{a}$ & 80 & $4 \mathrm{hrs}$ & THF & 63 & 27.42 \\
\hline xvi & (117) & $112 B+111$ & $\mathrm{n} / \mathrm{a}$ & 80 & 20 hrs & THF & 85 & 26.60 \\
\hline
\end{tabular}




\begin{tabular}{|c|c|c|c|c|c|c|c|c|}
\hline \multicolumn{5}{|c|}{ Table 2.9 continued } & \multicolumn{4}{|c|}{$\begin{array}{c}\text { Rxn. Comp. }(\%) \text { by }\left({ }^{31} \mathrm{P}\right) \mathrm{ppm} \\
\text { NMR }\left(\mathrm{CDCl}_{3}\right)\end{array}$} \\
\hline Entry & Reactant & SM & Base & $\begin{array}{c}\text { Temp. } \\
\left({ }^{\circ} \mathrm{C}\right)\end{array}$ & Time & Solv. & $\begin{array}{c}\text { PRD } \\
\%\end{array}$ & $\begin{array}{l}\text { PRD } \\
{ }^{31} \mathbf{P} \\
\text { ppm }\end{array}$ \\
\hline xvii* & & $112 B+111$ & $\mathrm{n} / \mathrm{a}$ & Reflux & $20 \mathrm{hrs}$ & ACN & 80 & 25.00 \\
\hline$\underset{\mu \mathrm{W}}{\mathrm{xvii}}$ & & $112 B+111$ & $\mathrm{n} / \mathrm{a}$ & 130 & $5 \min$ & Neat & 73 & 24.83 \\
\hline 19 & & $112 B+111$ & n/a & 110 & $0.5 \mathrm{hr}$ & Neat & 85 & 24.84 \\
\hline
\end{tabular}

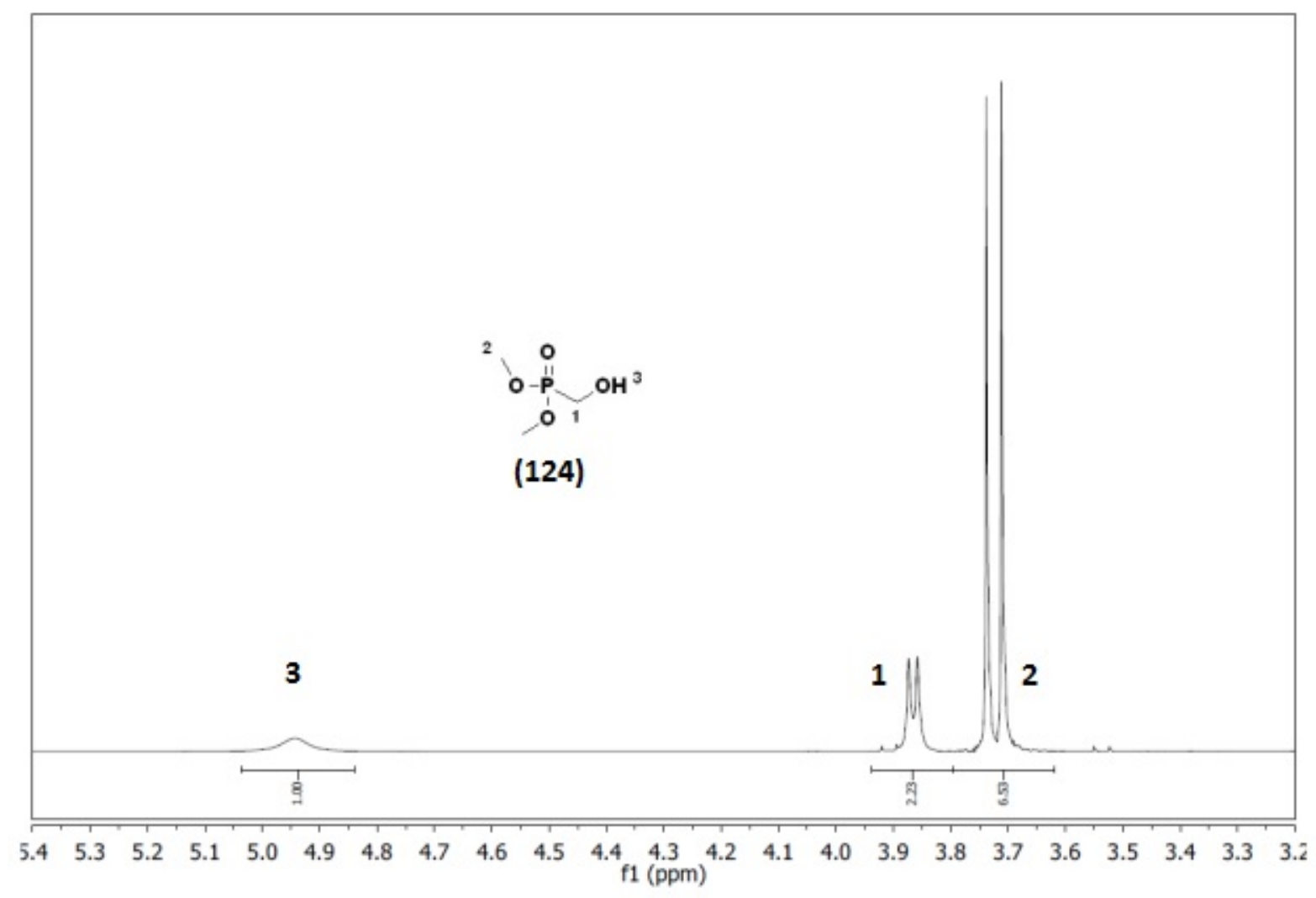

Figure 2.15: ${ }^{1} \mathrm{H}$ NMR $\left(\mathrm{CDCl}_{3}\right)$ spectrum of the undesired product 124 isolated from the Kabachnik-Fields reaction. 
Based on the Kabachnik-Fields mechanism (Figure 2.16), the basic $N, N$-dimethylamine appears to play a catalytic role in driving the Abramov addition of dialkylphosphite to $\mathrm{C}=\mathrm{O}$ bond forming $\alpha$-hydroxy phosphonate (Route B) over the Pudovik addition of dialkylphosphite to the imine (Figure 2.16, Route A). In fact, the literature preparation of $\mathbf{1 2 4}$ requires a catalytic amount of base (see Section 2.1.2.7). In contrast, when starting materials without a basic group such as the halo or $\mathrm{OH}$ derivatives were used, the Kabachnik-Fields reaction mechanism is favoured (Figure 2.16, Route A) and the bisphosphonates, 114-117 were obtained when 2 eq. of the dialkylphosphite was employed.

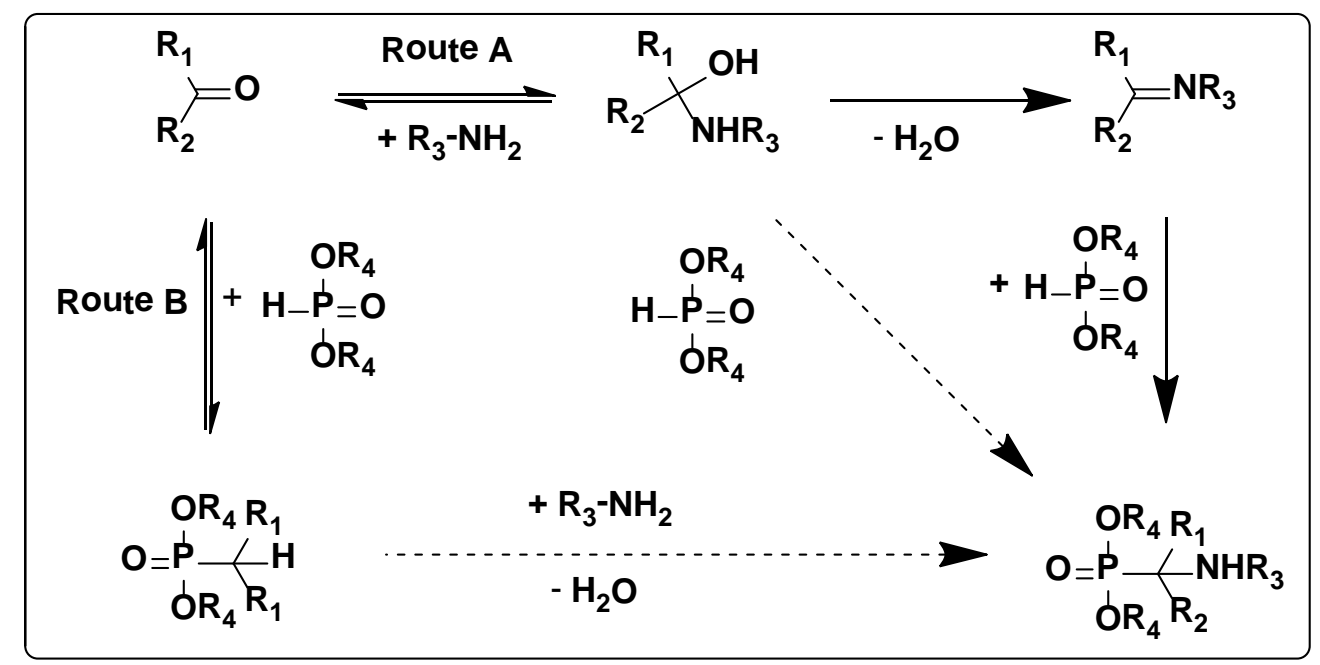

Figure 2.16: Possible mechanistic routes involved in the Kabachnik-Fields reaction. ${ }^{203}$

Next, the halo starting materials 107A and 107B (Scheme 2.19) were investigated in the Kabachnik-Fields reaction. As mentioned earlier, both the bromo 107A and chloro 107B sold as the $\mathrm{HCl}$ and $\mathrm{HBr}$ salts respectively, required an extra step to free base the amine salt prior to addition of formaldehyde and dialkylphosphonate. When $\mathrm{Et}_{3} \mathrm{~N}$ or $\mathrm{NaOH}$ (1 eq.) were stirred with 107A for 20 min prior to the addition of formaldehyde and dialkylphosphonate in the same pot, the desired bisphosphonate was never observed (Table 2.9, Entries viii and ix). Instead of the desired bisphosphonate product with a ${ }^{31} \mathrm{P}$ NMR $\left(\mathrm{D}_{2} \mathrm{O}\right)$ peak at $\sim 24 \mathrm{ppm}$, a major downfield ${ }^{31} \mathrm{P}$ 
NMR peak at $\delta \sim 8 \mathrm{ppm}$ was observed with complete consumption of $\mathrm{HPO}(\mathrm{OMe})_{2}\left({ }^{31} \mathrm{P}, \delta=14.7\right.$ ppm). In order for the reaction to proceed, $\mathbf{1 0 9}$ and $\mathbf{1 1 0}$ required a tedious extraction from $6 \mathrm{~N}$ $\mathrm{NaOH}$ or $\mathrm{KOH}$ with isolation of the separated free bases as yellow oils that had to be used directly otherwise self-polymerization took place (Table 2.9, Entries x-xiv). No solvent was added during the extraction because when $\mathrm{DCM}, \mathrm{CHCl}_{3}$, or EtOAc were employed for the free based halo derivatives, which prefered the aqueous phase over the organic phase ( 5\% extraction efficiency by weight). As a result, the oily extracts of the free based compounds were contaminated with $\sim 40 \% \mathrm{H}_{2} \mathrm{O}\left({ }^{1} \mathrm{H}\right.$ NMR), resulting in only $\sim 50 \%$ overall extraction yield starting from the amine salts (Table 2.9, Entries xx-xiv, note low product yields, 21-53\%). Lastly, $\mathrm{Et}_{3} \mathrm{~N} / \mathrm{ACN}$ was employed in order to deprotonate the starting materials in situ without the need to extract and thus improve the yield, however the resulting triethylamine salt failed to precipitate from the solution and could not be removed prior to the Kabachnik-Fields reaction.

Due to problems with starting materials 108-110 in the Kabachnik-Fields reaction described above, the reaction was screened with the amino alcohol $\mathbf{1 1 1}$ used in the literature to make 117 in refluxing THF (Experimental, Section 5.6.0). Under reflux conditions, the aminoalcohol 111 (Table 2.9, Entries xv and xvi, THF) or the triazine 122 (Table 2.9, Entry xv, ACN) worked well in the Kabachnik-Fields reaction with solid paraformaldehyde, resulting in yields over $70 \%$ by ${ }^{31} \mathrm{P}$ NMR $\left(\mathrm{CDCl}_{3}\right)$. Similarly, $\mu \mathrm{W}$ heating under neat conditions gave a $74 \%$ yield when reaction parameters were optimized to $5 \mathrm{~min}$ at $130^{\circ} \mathrm{C}$ (Table 2.9, Entry xxviii). This is the first $\mu \mathrm{W}$ synthesis of $\mathbf{1 1 7}$, while synthesis of $\alpha$-bisphosphonates with only alkyl chains (starting with 3-aminopropanol), has been previously reported with the Kabachnik-Fields reaction under $\mu \mathrm{W}$ heating $\left(60 \mathrm{~min}, 100^{\circ} \mathrm{C}\right) .{ }^{204,205}$ After success under solventless conditions in the $\mu \mathrm{W}$, a sealed tube experiment was thus perfomed with the alcohol starting material under 
neat conditions for $30 \mathrm{~min}$ at $110^{\circ} \mathrm{C}$ which resulted in a $85 \%$ yield after column chromatography (Table 2.9, Entry xix). With the successful preparation of 117, the conversion to either a bromo, iodo (Experimental, Section 5.6.0) or $\mathrm{NMe}_{2}$ (Table 2.9, Entries vi and vii) required two extra steps, activation via mesyl or $\mathrm{TsCl}$ followed by the $\mathrm{S}_{\mathrm{N}} 2$ reaction. All sulfonyl chloride reactions resulted in higher yields and faster conversion times when the catalyst $\mathrm{NMe}_{3} \cdot \mathrm{HCl}^{162}$ was employed (30 min vs 24 hrs for non-catalyzed reaction).

When the amine starting materials $\mathbf{1 0 8}$ or $\mathbf{1 1 1}$ were reacted solely with formaldehyde without addition of $\mathrm{HP}(\mathrm{OEt})_{2}$, the corresponding imines were formed respectively. However at RT, the imine compounds were in equilibrium with the trimer compounds $\mathbf{1 2 2}$ and $\mathbf{1 2 3}$ as observed by ${ }^{1} \mathrm{H}$ NMR $\left(\mathrm{CDCl}_{3}\right.$, Scheme 2.20). ${ }^{206}$ Trimer 123 was purified by an aqueous wash and subjected to quaternization with bromooctadecane. However, purification of the final product failed to give clean samples for characterization by ${ }^{1} \mathrm{H}$ NMR spectroscopy (Figure 2.17). Instead, compound 121 was prepared from 115 and quaternized with bromooctadecane. Compound 115 was prepared via a longer route by activating the alcohol 111 via the mesyl 118 or tosyl 119 group refluxing in excess $\mathrm{HNMe}_{2}$ (Scheme 2.19, Step 1-2). Pure 121 was finally obtained after column chromatography eluting with $\mathrm{EtOH}\left(\mathrm{NaBr}_{\text {sat }}\right)$ : $\mathrm{ACN}(1: 3)$ (Figure 2.18). 


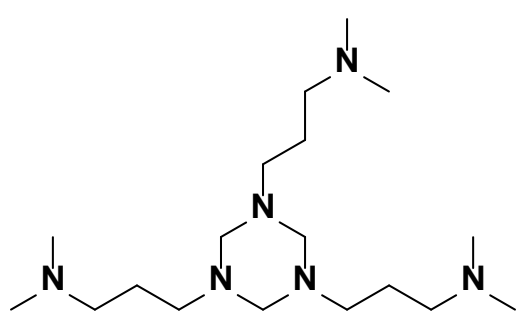

(123)
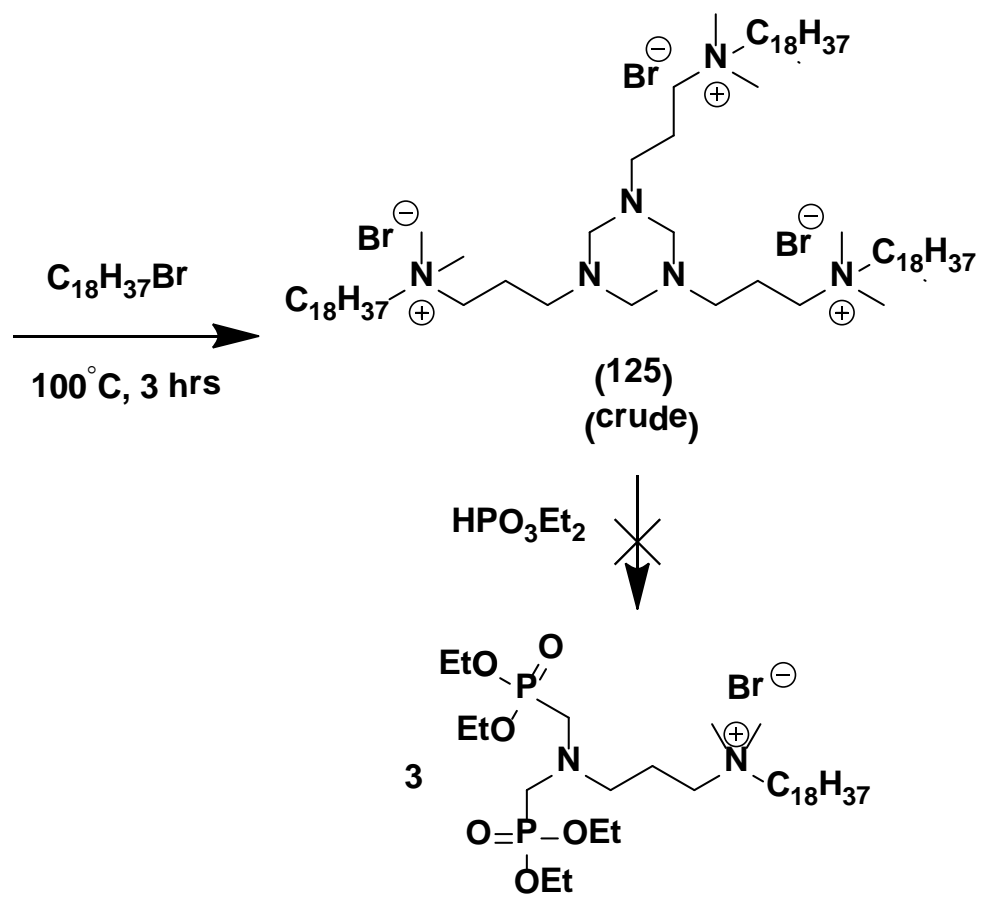

(121)

Scheme 2.20: Attempted stepwise preparation of 121.

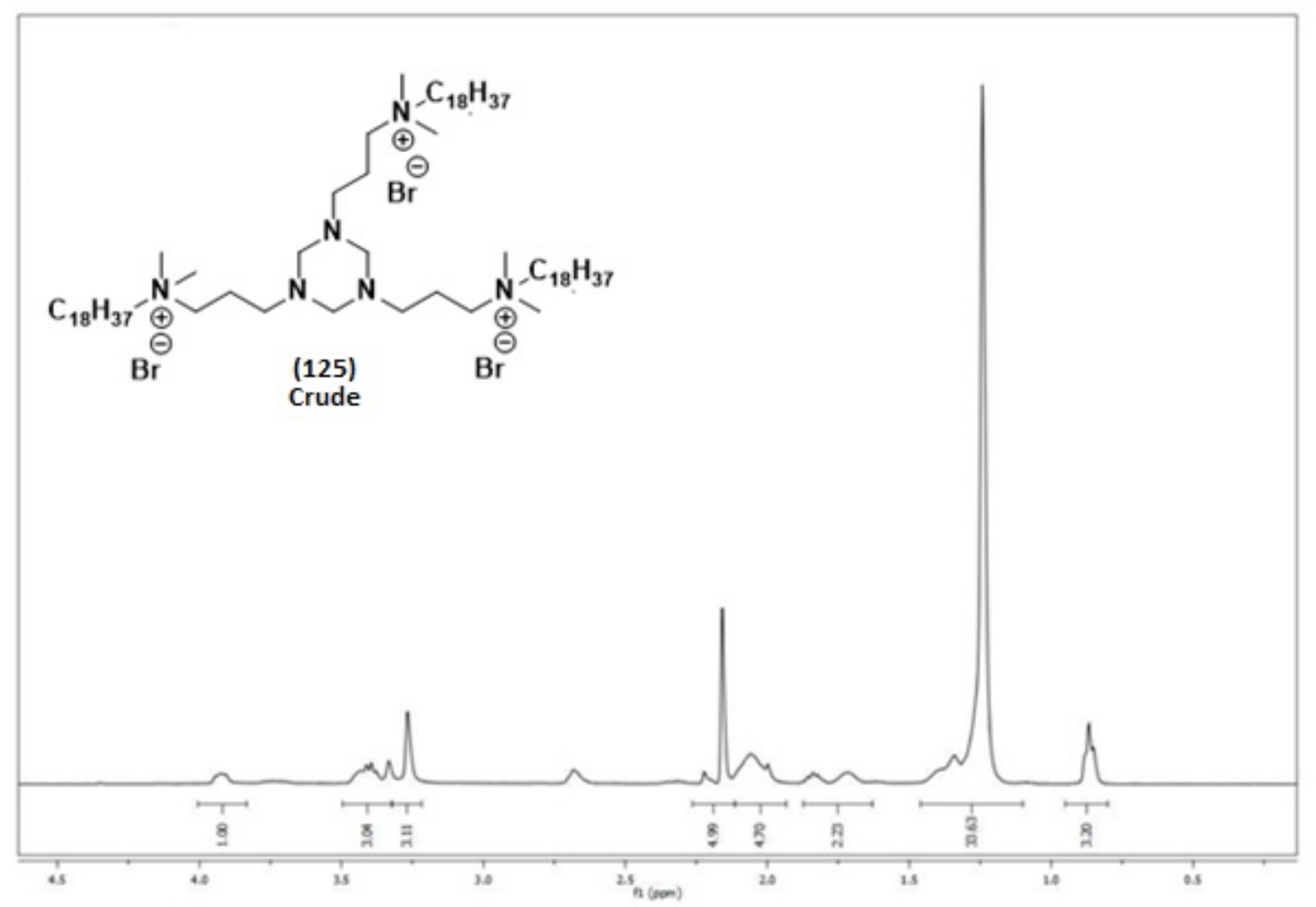

Figure 2.17: Attempted quaternization of triazine 123 with bromoctadecane $\left({ }^{1} \mathrm{H} \mathrm{NMR}\left(\mathrm{CDCl}_{3}\right)\right)$. 


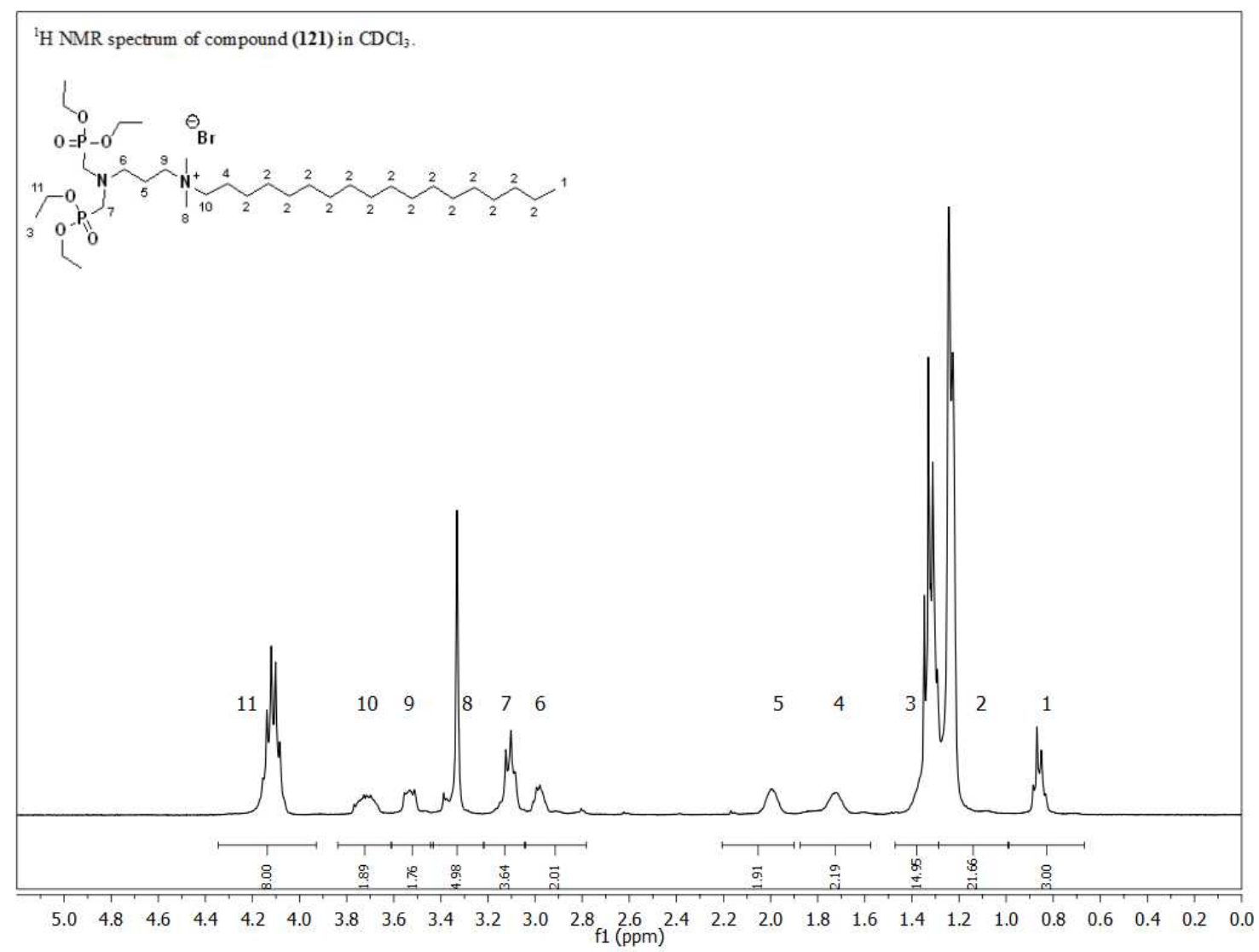

Figure 2.18: ${ }^{1} \mathrm{H}$ NMR $\left(\mathrm{CDCl}_{3}\right)$ spectrum of $\alpha$-ABPQA 121.

Dealkylation of ester $\mathbf{1 2 1}$ to the desired $\alpha$-bisphosphonic acid 126 was unsuccessful with both $\mathrm{HBr}$ and TMSBr (Scheme 2.21, Figure 2.19, a and b) and resulted in a mixture of products by ${ }^{31} \mathrm{P}$ NMR $\left(\mathrm{D}_{2} \mathrm{O}\right)$. The TMSBr reaction seemed promising at first by ${ }^{31} \mathrm{P}$ NMR with a major peak at $~ 10$ ppm attributed to the TMS ester (Figure 2.19, b). However, after hydrolysis with $\mathrm{H}_{2} \mathrm{O}$ and evaporation of volatiles, the product was insoluble in both $\mathrm{D}_{2} \mathrm{O}$ and $\mathrm{CDCl}_{3}$. After treatment of $\mathbf{1 2 6}$ with 1 eq. of $\mathrm{NaOH}$ to make the sodium salt, decomposition was observed with three unidentified ${ }^{31} \mathrm{P}$ NMR $\left(\mathrm{D}_{2} \mathrm{O}\right)$ signals at $\delta \sim 21,16$ and $6.5 \mathrm{ppm}$ resulting in an inseparable mixture of products (Figure 2.19, c). According to Guerrero, instead of the phosphonic acid, phosphonate esters may also bind to metal oxide surfaces with the loss of an ethyl group. ${ }^{207}$ 


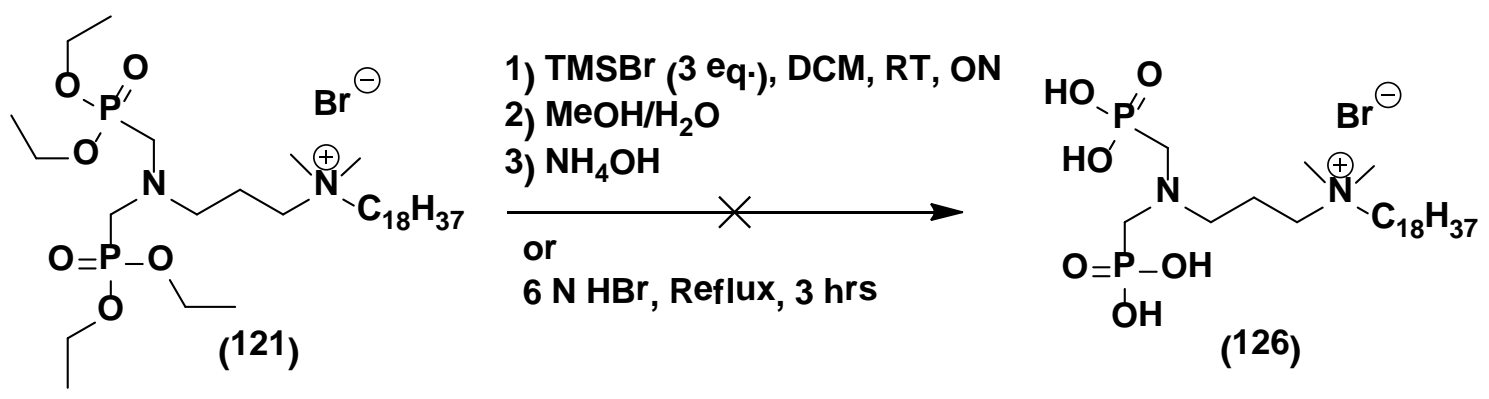

Scheme 2.21: Attempted didealkylation of $\alpha$-aminobisphosphonate QAC 121.

a) $\mathrm{HBr}, \mathrm{uW}, 10 \mathrm{~min}, 150^{\circ} \mathrm{C}$

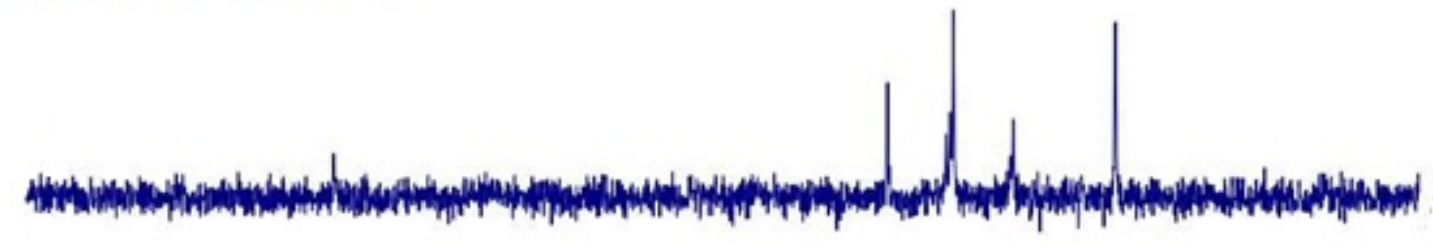

b) TMSBr, ON , RT , 24 hrs

c) TMSBr , ON , RT , 24 hrs, $\mathrm{H}_{2} \mathrm{O}: \mathrm{EtOH}, 1 \mathrm{hr}$ deprotect, Na Salt

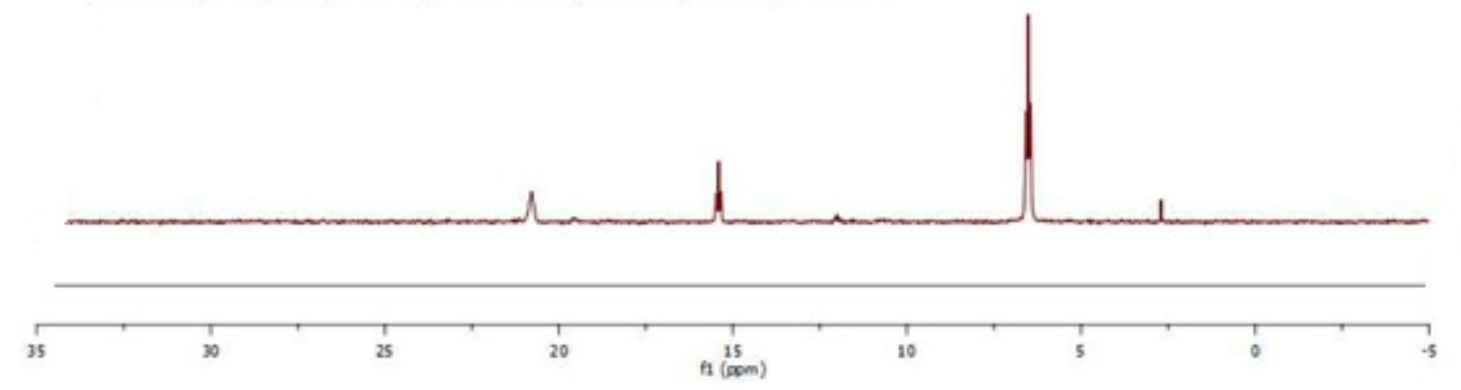

Figure 2.19: ${ }^{31} \mathrm{P} \mathrm{NMR}\left(\mathrm{CDCl}_{3}\right)$ reaction monitoring of 121 cleavage with $\mathrm{HBr}$ and TMSBr. 
Synthesis of tetraphosphonate 127 was attempted from $\alpha$-bisphosphonate 113 after deprotonation with a strong base (LDA or $\mathrm{NaH}$ ) and substitution with $\mathrm{ClP}(\mathrm{OEt})_{2}$ (Scheme 2.22). Monitoring the reaction with LDA as base at $-10^{\circ} \mathrm{C}$ after 10 min by ${ }^{31} \mathrm{P}$ NMR spectroscopy $\left(\mathrm{CDCl}_{3}\right)$ showed no product $\mathbf{1 2 7}$ formation (Figure 2.20 a). Employing the same conditions as above but allowing the reaction to stir for $60 \mathrm{~min}$ at $\mathrm{RT}$ and extracting into EtOAc recovered mainly unreacted SM 113. Switching to $\mathrm{NaH}$ made no difference and no reaction took place (Figure $2.20 \mathrm{c}$ ). Allowing the deprotonation to occur at $-78^{\circ} \mathrm{C}$ with LDA prior to the addition of $\mathrm{ClP}(\mathrm{OEt})_{2}$ seemed promising with consumption of $\mathbf{1 1 3}$ by ${ }^{31} \mathrm{P}$ NMR spectroscopy (Figure 2.20 e), however after extraction into EtOAc and column chromatography of the reaction mixture no product corresponding to $\mathbf{1 2 7}$ was eluted.

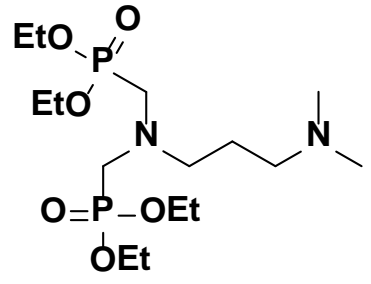

(113)

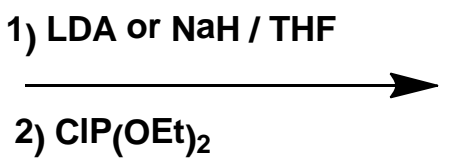

2) $\mathrm{ClP}(\mathrm{OEt})_{2}$

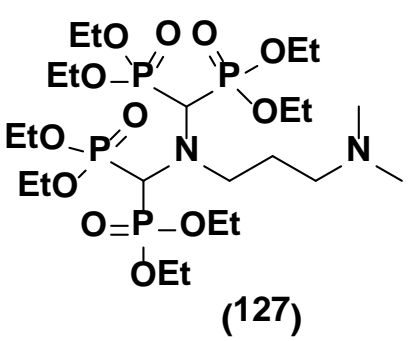

Scheme 2.22: Tetraphosphonate 127 by C-P alkylation of 113. 
a) LDA (4.4 eq), phosphonate (2.2 eq), $-10^{\circ} \mathrm{C}, 10 \mathrm{~min} \mathrm{H}_{2} \mathrm{O}$ quench and evap (113) PCIO(OEt) 2

b) LDA (4.4 eq), phosphonate $(2.2 \mathrm{eq}),-10^{\circ} \mathrm{C}, 60 \mathrm{~min}$, EtOAc extract

(113)

c) $\mathrm{NaH}(4.4 \mathrm{eq})$, phosphonate $(2.2 \mathrm{eq}), 0^{\circ} \mathrm{C}, 60 \mathrm{~min}$, crd

PCIO(OEt) 2

d) LDA (4.4 eq), phosphonate (3.2 eq), $-78^{\circ} \mathrm{C}, 60 \mathrm{~min}$, crd

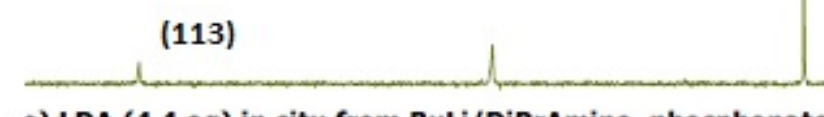

e) LDA (4.4 eq) in situ from BuLi/DiPrAmine, phosphonate ( $2.2 \mathrm{eq}),-78^{\circ} \mathrm{C}, 60 \mathrm{~min}$, crd

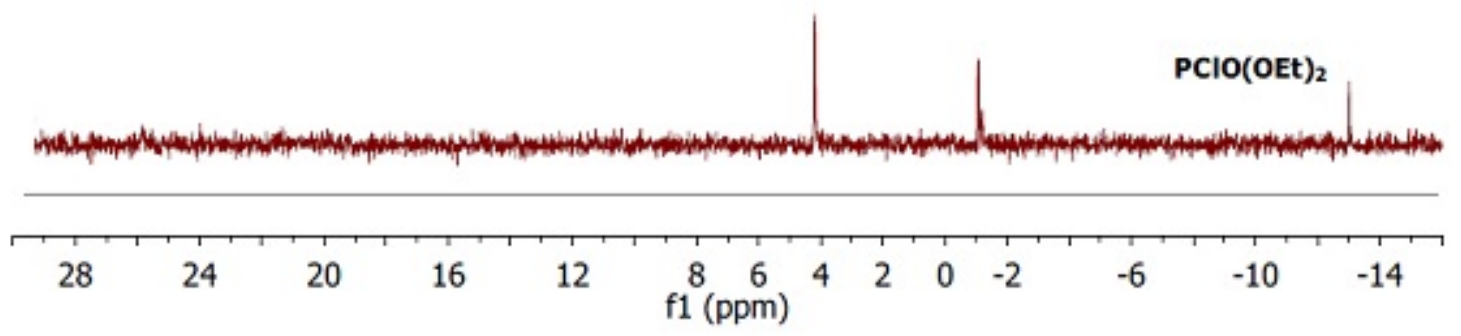

Figure 2.20: ${ }^{31} \mathrm{P}$ NMR $\left(\mathrm{CDCl}_{3}\right)$ reaction monitoring of tetraphosphonate 127.

\subsubsection{6 $\beta$-Aminobisphosphonic Acids QAC Antimicrobials ( $\beta$-ABPQA)}

Inspired by Matveeva's work ${ }^{208}$ on the $\mathrm{H}_{2} \mathrm{O}$ based synthesis of $\beta$-aminobisphosphonates, the reaction was explored with various bi-functional amines in order to prepare precursor halo or dimethylamino substituted $\beta$-aminobisphosphonates necessary for quaternization (Scheme 2.23, Table 2.10). Similarly to $\alpha$-aminobisphosphonates (Section 2.1.2.5), the most direct way to prepare $N, N$-dimethyl or halo $\beta$-aminobisphosphonates is the addition of vinyl phosphonate to amines 108-110. The reaction progress was monitored by NMR $\left({ }^{31} \mathrm{P}, \mathrm{D}_{2} \mathrm{O}\right)$ and reaction 
completeness was ascertained with the consumption of dialkylphosphite $\mathbf{1 2 9}(\delta=19.97$ to 23.71 ppm) and $130(\delta=18.10$ ppm to 20.85 ppm, Table 2.10).

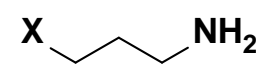

(108) $X=\mathrm{NMe}_{2}$

(109) $X=\mathrm{Cl}$

(110) $X=B r$

(111) $X=\mathrm{OH}$

(128) $\mathrm{X}=\mathrm{Br}^{-} \mathrm{N}^{+} \mathrm{Me}_{2} \mathrm{C}_{18} \mathrm{H}_{37}$

(135), (136)

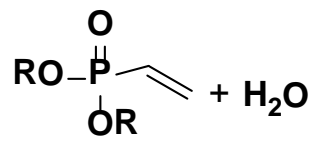

RT, 24 hrs

(129) $\mathrm{R}=\mathrm{Me}$

(130) $R=E t$

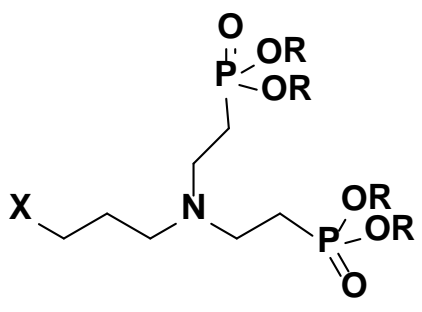

(131) $X=\mathrm{NMe}_{2}, \mathrm{R}=\mathrm{OEt}$

(132) $X=\mathrm{Cl}, \mathrm{R}=\mathrm{OEt}$

(133) $X=B r, R=O M e$

(134) $X=B r, R=O E t$

(135) $X=O H, R=O M e$

(136) $X=O H, R=O E t$

(137) $X=$ Br-N $^{+} \mathrm{Me}_{2} \mathrm{C}_{18} \mathrm{H}_{37}, \mathrm{R}=\mathrm{OMe}$
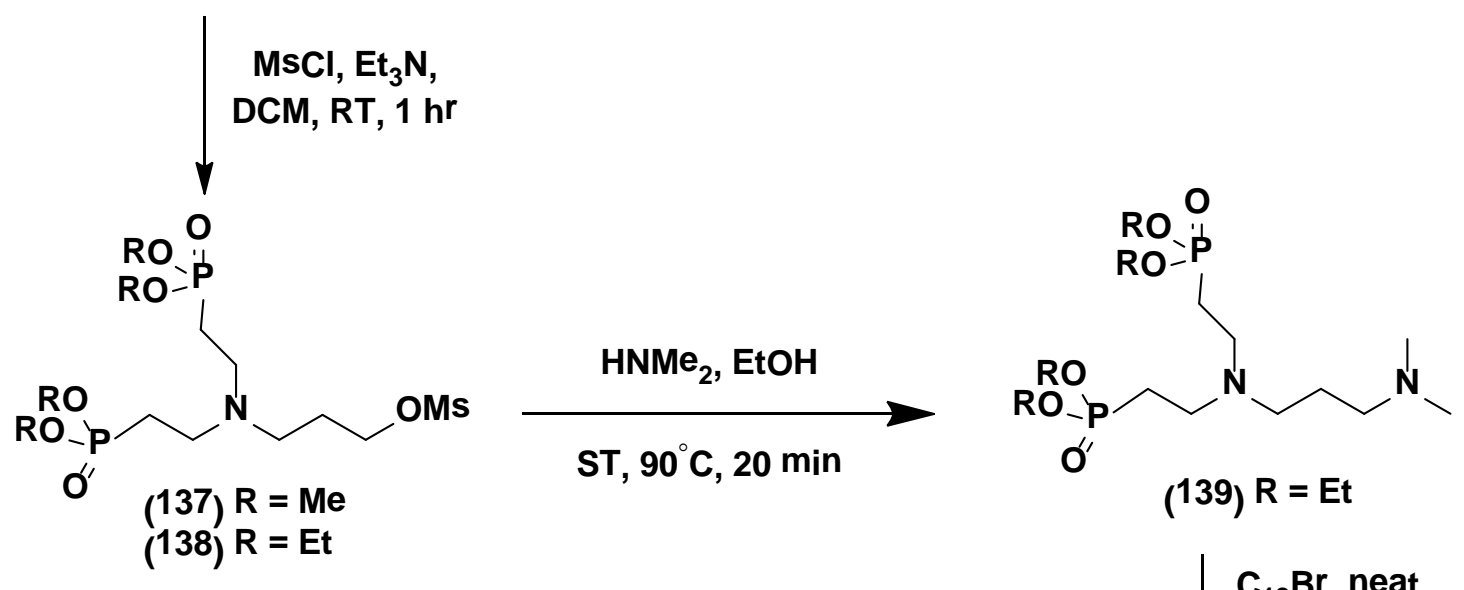

$\mid \begin{gathered}\mathrm{C}_{18} \mathrm{Br}, \text { neat } \\ 100^{\circ} \mathrm{C}, 1-4 \mathrm{hrs}\end{gathered}$<smiles>CN(CCCN(CCP(=O)(O)O)CCP(=O)(O)O)C(C)(C)C</smiles>

1) TMSBr/ DCM, RT, ON

2) $\mathrm{H}_{2} \mathrm{O} / \mathrm{EtOH}$

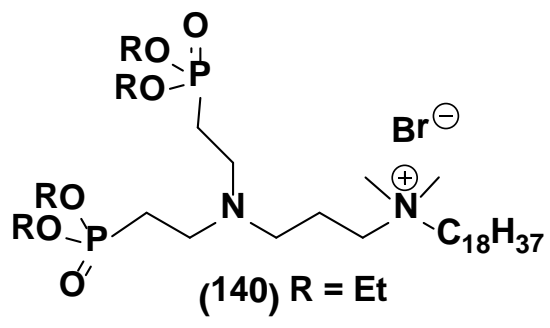

Scheme 2.23: Optimized synthetic route to $\beta$-aminobisphosphonate QAC 141. 
<smiles>[X]CCCN</smiles>

(108) $X=\mathrm{NMe}_{2}$

(109) $X=\mathrm{Cl}$

(110) $X=B r$

(111) $X=O H$

(128) $\mathrm{X}=\mathrm{Br}^{-} \mathrm{N}^{+} \mathrm{Me}_{2} \mathrm{C}_{18} \mathrm{H}_{37}$

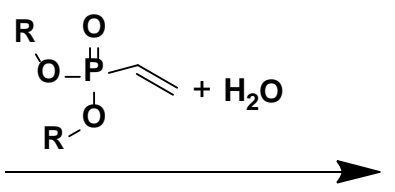

RT, 24 hrs

(129) $R=M e$ (130) $R=E t$<smiles>[X]CCCNCCP(=O)(O[R7])O[R7](=O)OP([R7])(=O)CCN(CCC[X])CC[PH]([R])([R])[O-]</smiles>

(131) $X=\mathrm{NMe}_{2}, \mathrm{R}=\mathrm{OEt}$

(132) $X=C l, R=O E t$

(133) $X=B r, R=O M e$

(134) $X=B r, R=O E t$

(135) $X=O H, R=O M e$

(136) $X=O H, R=O E t$

(137) $\mathrm{X}=\mathrm{Br}^{-} \mathrm{N}^{+} \mathrm{Me}_{2} \mathrm{C}_{18} \mathrm{H}_{37}, \mathrm{R}=\mathrm{OMe}$

Table 2.10: Optimization of the Michael addition to $\beta$-aminobisphosphonates QAC 131-144.

\begin{tabular}{|c|c|c|c|c|c|c|c|}
\hline \multicolumn{8}{|c|}{ Rxn. Comp. (\%) by $\left({ }^{31} \mathrm{P}\right.$ NMR, $\left.\mathrm{D}_{2} \mathrm{O}\right)$} \\
\hline Entry & Reactant & SM & Base & $\begin{array}{l}\text { Time } \\
\text { (hrs) }\end{array}$ & $\begin{array}{l}\text { Temp } \\
\cdot\left({ }^{\circ} \mathrm{C}\right)\end{array}$ & $\begin{array}{c}\text { SM } \\
(\% / p p m)\end{array}$ & $\begin{array}{c}\text { PRD. } \\
\text { (\%/ppm) }\end{array}$ \\
\hline $\mathrm{i}$ & \multirow{3}{*}{$\underset{\mathrm{O}^{\prime \prime}}{\mathrm{RO}_{-}^{\mathrm{RO}}} \mathrm{(131)}^{\mathrm{N}}$} & $129+108$ & $\mathrm{n} / \mathrm{a}$ & 3 & 100 & 0 & $\mathrm{n} / \mathrm{a}$ \\
\hline ii & & $129+108$ & $\mathrm{n} / \mathrm{a}$ & 24 & RT & 36/23.52 & 55/36.18 \\
\hline iii & & $129+108$ & $\mathrm{n} / \mathrm{a}$ & 48 & RT & $30 / 23.47$ & $59 / 36.14$ \\
\hline iv & \multirow{3}{*}{$\underset{(133-134)}{\mathrm{RO}_{-\mathrm{P}}^{\mathrm{RO}}} \mathrm{O}^{\prime \prime}$} & $129+110$ & $\mathrm{NaOH}$ & 3 & RT & $40 / 23.56$ & 5/36.16 \\
\hline V & & $129+110$ & $\mathrm{NaOH}$ & 24 & $\mathrm{RT}$ & $20 * / 23.51$ & 10/36.16 \\
\hline $\mathrm{vi}^{*}$ & & $130+110$ & $\mathrm{NaOH}$ & 24 & $\mathrm{RT}$ & 23.51 & $\mathrm{n} / \mathrm{a}$ \\
\hline vii & \multirow{2}{*}{$\underbrace{\mathrm{RO}_{-P}}_{\mathrm{O}^{\prime}} \mathrm{(132)}^{\mathrm{R}}$} & $130+109$ & DIPEA & 64 & RT & $21 * / 20.85$ & 31/33.36 \\
\hline viii & & $130+109$ & $\mathrm{NaOMe}$ & 48 & $\mathrm{RT}$ & $17 / 20.85$ & $50 / 33.40$ \\
\hline
\end{tabular}




\begin{tabular}{|c|c|c|c|c|c|c|c|}
\hline \multicolumn{5}{|c|}{ Table 2.10 continued } & \multicolumn{3}{|c|}{$\begin{array}{c}\text { Rxn. Comp. }(\%) \text { by }\left({ }^{31} \mathrm{P}\right. \\
\left.\text { NMR, } \mathrm{D}_{2} \mathrm{O}\right)\end{array}$} \\
\hline Entry & Reactant & SM & Base & $\begin{array}{l}\text { Time } \\
\text { (hrs) }\end{array}$ & $\begin{array}{l}\text { Temp } \\
\cdot\left({ }^{\circ} \mathrm{C}\right)\end{array}$ & $\begin{array}{c}\text { SM } \\
(\% / p p m)\end{array}$ & $\begin{array}{c}\text { PRD. } \\
\text { (\%/ppm) }\end{array}$ \\
\hline ix & (140) & $130+128$ & $\mathrm{n} / \mathrm{a}$ & 48 & RT & $51 / 23.68$ & 49/35.41 \\
\hline $\mathrm{x}^{* *}$ & $\begin{array}{l}\text { RO } \\
\text { RO }\end{array}$ & $129+111$ & $\mathrm{n} / \mathrm{a}$ & 24 & RT & $1 / 23.71$ & $100 / 36.39$ \\
\hline $\mathrm{xi}^{* *}$ & $\begin{array}{c}\text { RO } \\
\text { RO-P }_{-}\end{array}$ & $129+111$ & $\mathrm{n} / \mathrm{a}$ & 24 & RT & $1 / 24.54$ & $100 / 36.50$ \\
\hline xii** & $(135-136)$ & $130+111$ & $\mathrm{n} / \mathrm{a}$ & 24 & RT & $10 / 20.82$ & $90 / 30.00$ \\
\hline
\end{tabular}

* excess $\mathrm{NaOH}$ used (1.2 eq.), ** excess 129-130 was used. n/a: multiple peaks were observed and none of which corresponded to the product.

In most cases the addition to amines $\mathbf{1 0 8 - 1 1 0}$ and the quat amine 128 resulted in a mixture containing the product with unreacted starting material (Table 2.10, Entries i-ix) or in the case of the halo amines with a mixture of mono- and bis- $\beta$-aminophosphonates identified by ${ }^{31} \mathrm{P}$ NMR (Figure 2.21 and Table 2.10, Entries iv, v, vii, viii). The added base used (Table 2.10, Entries iv-viii) to freebase the amine salts 107A, 107B hindered the bis Michael addition to vinylphosphonate. Even when the based/amine salt was added in a 0.9/1 ratio, the reaction still resulted in an inseparable mixture of mono and bisphosphonates along with unreacted SM in the following ratios: (Figure 2.21 and Table 2.10, Entry v (1.0:0.12:0.32), Entry vii (1.0:0.65:0.43), Entry viii (0.66:1.0:0.34). Surprisingly, the quat amine 128 having no basic functionalities gave a low yield of product 140 with considerable unreacted starting material ( 1:1 ratio) and was left unpurified (Figure 2.21 and Table 2.10, Entry ix). As a result bis $\beta$-aminobisphosphonates were 
successfully prepared by employing a longer indirect method that involving 3-amino-1-propanol 111. When the reaction was performed with $\mathbf{1 1 1}$ and either dimethyl- or diethylvinylphosphonate (Table 2.10, Entries x-xii) a clean reaction was observed with complete consumption of the starting material. Excess vinylphosphonate was removed by column chromatography to give pure 135 or 136 (Figure 2.21 and Table 2.10, Entries x-xii).

Further functional group transformations were necessary to activate the alcohol $\mathbf{1 1 1}$ into a better leaving group through mesylation followed by substitution with excess dimethylamine and quaternization to give 140 (Scheme 2.23). The mesyl chloride 137 intermediate decomposed over time at RT and was reacted directly (Figure 2.22). Finally, quaternization with bromooctadecane gave quat $\mathbf{1 4 0}$ that was isolated after column chromatography along with $10 \%$ of impurities (27.6 and 26.8 ppm by ${ }^{31} \mathrm{P}$ NMR, $\mathrm{CDCl}_{3}$ ), (Figure 2.23, Experimental, Section 5.7.0). No dealkylation of $\mathbf{1 4 0}$ to the phosphonic acid $\mathbf{1 4 1}$ was attempted at this time. 
(Table 2.10, Entry ii)

\begin{tabular}{|c|c|c|c|}
\hline & \multicolumn{2}{|c|}{131} & 129 \\
\hline \multicolumn{4}{|l|}{ (Table 2.10, Entry v) } \\
\hline 1 & 133 & 144 & 129 \\
\hline
\end{tabular}

(Table 2.10, Entry vii)

\begin{tabular}{l|l|l|l|} 
(Table 2.10, Entry vii) & 142 & 130 \\
& 132 & 142
\end{tabular}

(Table 2.10, Entry viii)

\begin{tabular}{l|l|l|l}
132 & 142 & 130 \\
\hline
\end{tabular}

(Table 2.10, Entry ix)

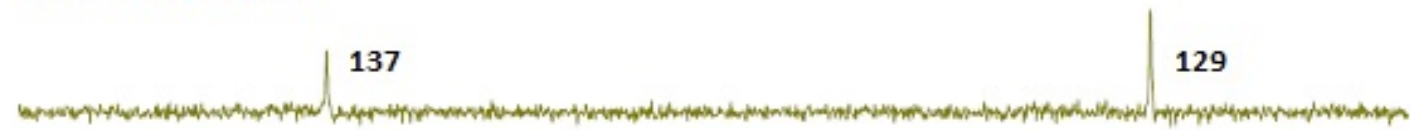

(Table 2.10, Entry x)

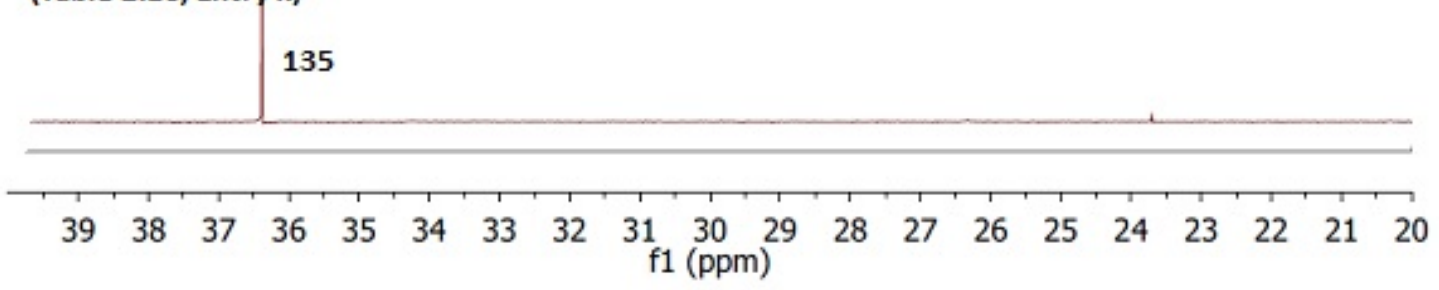

Figure 2.21: Reaction monitoring of Michael addition reactions by ${ }^{31} \mathrm{P} N \mathrm{NM}\left(\mathrm{D}_{2} \mathrm{O}\right)$. 


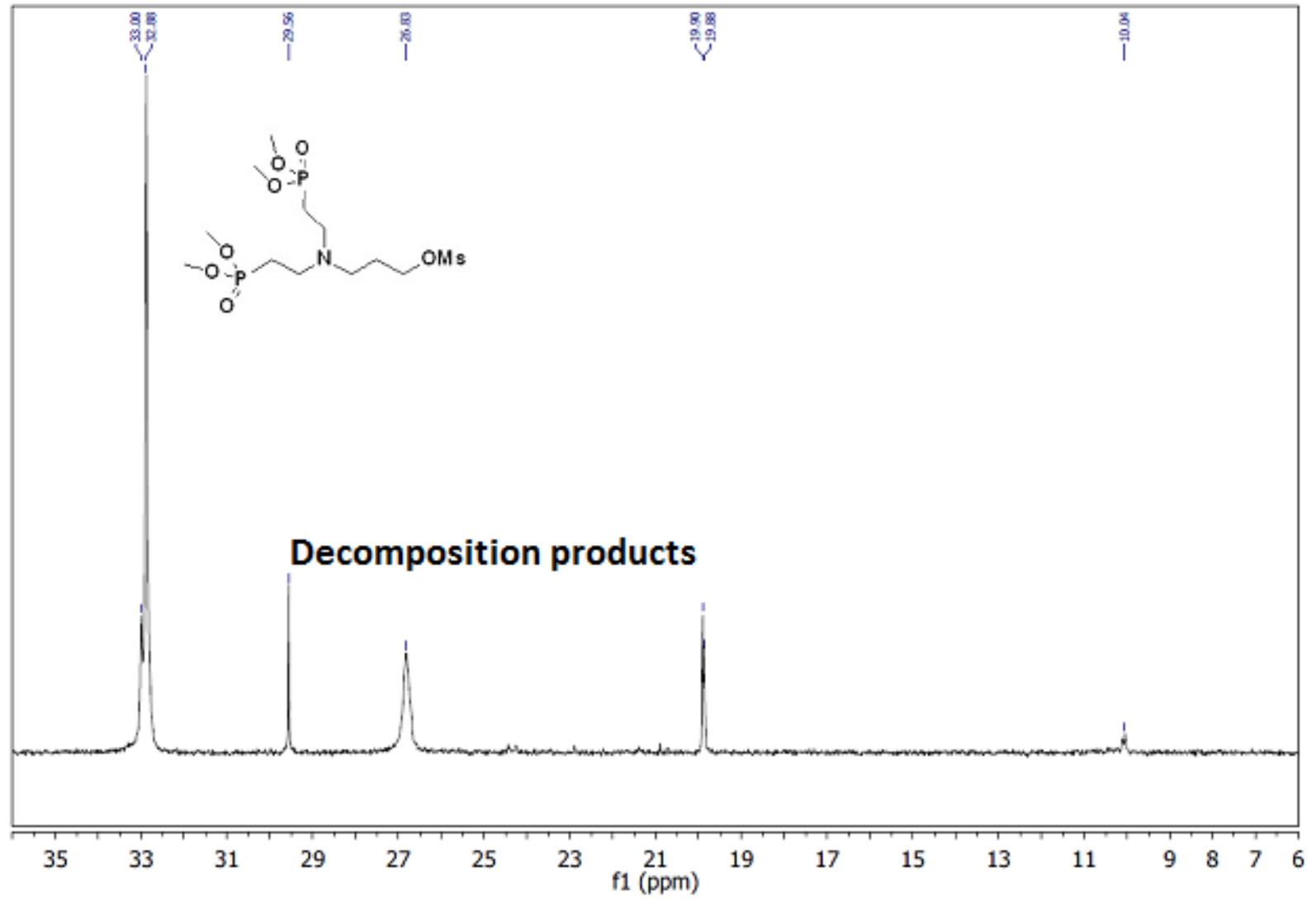

Figure 2.22: Decomposition of $\mathbf{1 3 2}$ at $\mathrm{RT}$ by ${ }^{31} \mathrm{P} \mathrm{NMR}\left(\mathrm{CDCl}_{3}\right)$. 


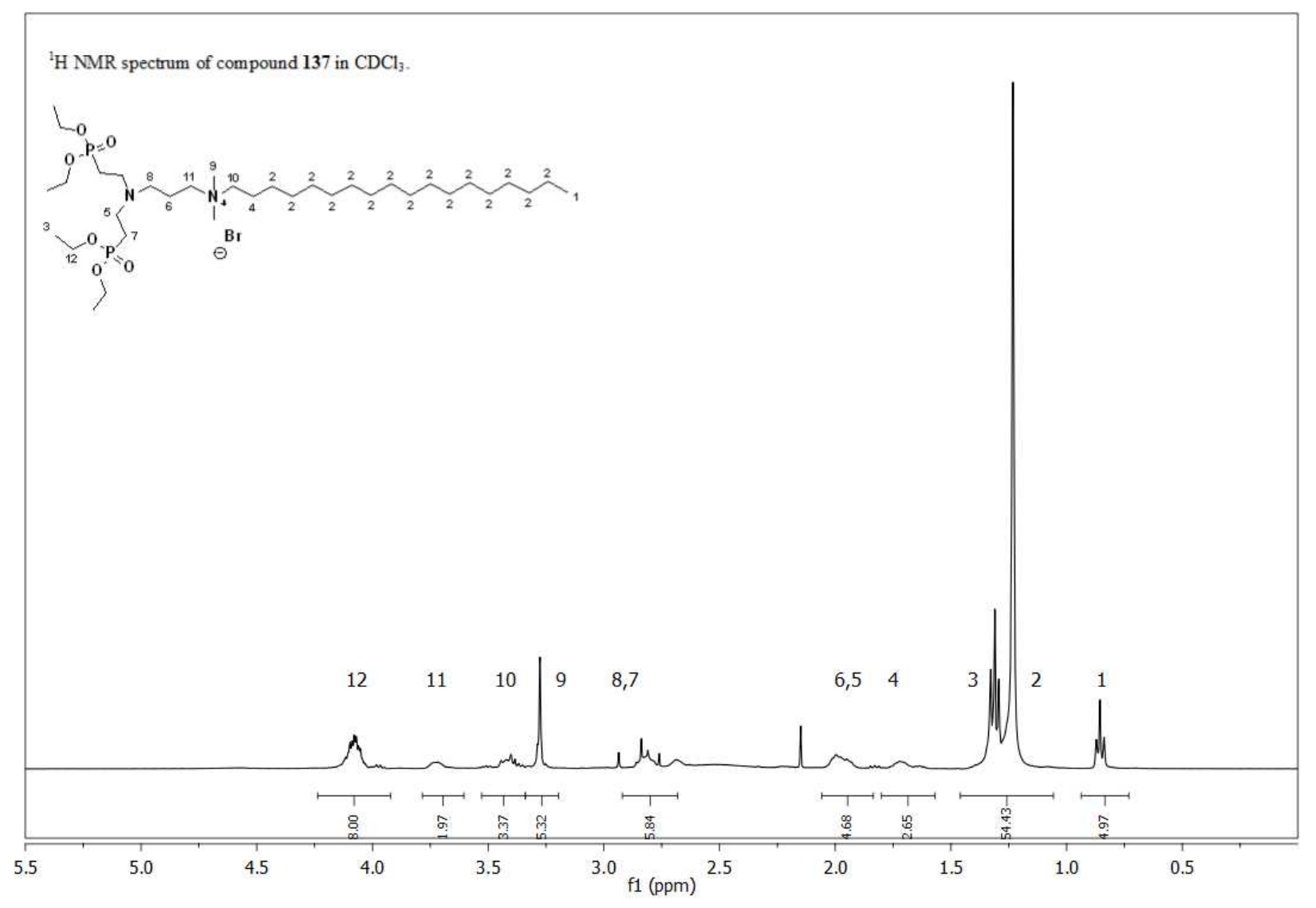

Figure 2.23: ${ }^{1} \mathrm{H}$ NMR $\left(\mathrm{CDCl}_{3}\right)$ spectrum of $\beta$-ABPQA 140.

The commercially available starting material vinyl phosphonates 129-130 were quite expensive to purchase ( $\sim 213 / 5 \mathrm{~mL}, 95 \%$ for $\mathrm{R}_{1}=\mathrm{Et}, \mathrm{S}-\mathrm{A}$ ) and as a result, all of the optimization reactions were performed on a very small scale $(\sim 1 \mathrm{mmol})$. Preparation of 130 was attempted from 15 and $\mathrm{KOH}$ in $\mathrm{EtOH}$ following literature procedures (Section 2.1.2.1). ${ }^{209}$ However, the major product was difficult to purify by distillation from unreacted $\mathbf{1 5}$ and the byproduct, diethyl (2-hydroxyethyl)phosphonate, resulting from the competing elimination reaction (Figure 2.24). 


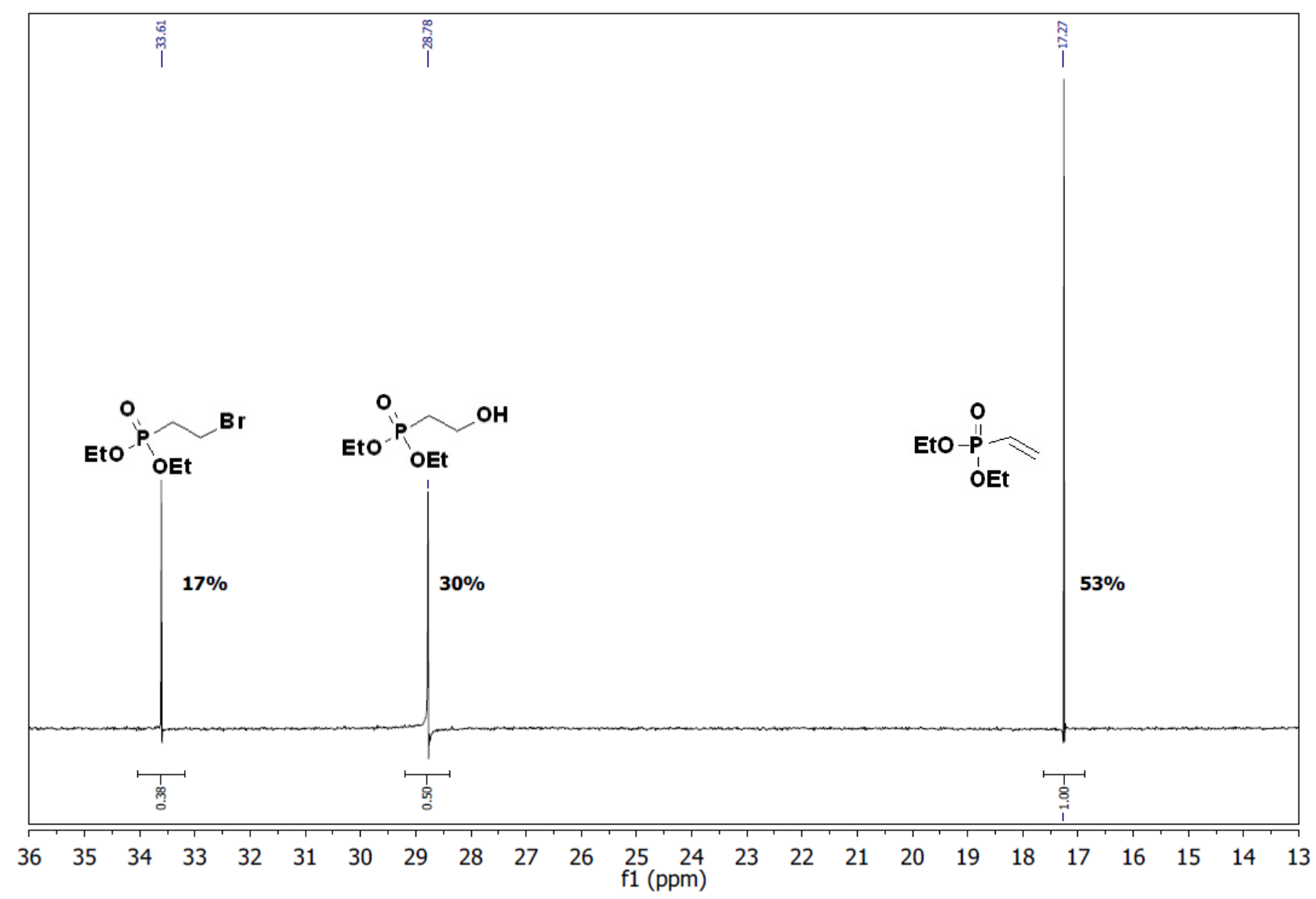

Figure 2.24: Preparation of diethylvinylphosphonate 130 (crude mixture by ${ }^{31} \mathrm{P} \mathrm{NMR}\left(\mathrm{CDCl}_{3}\right)$ ).

Preparation of tetraphosphonates starting from $\beta$-aminobisphosphonate $\mathbf{1 4 1}$ according to (Scheme 2.24) was also targeted but abandoned. It was difficult to isolate the THP protected 145 intermediate even after employing an excess of $p$-TosOH catalyst. As a result no further reactions leading to 146 were possible. 
<smiles>NCCCO</smiles>

(111)

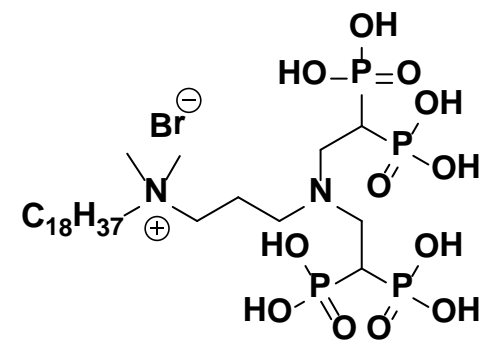

(146)

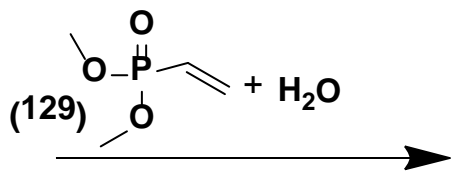

RT, 24 hrs

1) LDA, CIP(OEt)2

2) Amberslyt H-15/MeOH, $45^{\circ} \mathrm{C}, 1 \mathrm{hr}$

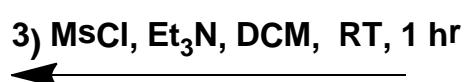

4) $\mathrm{C}_{18} \mathrm{Br}$, Neat, $100^{\circ} \mathrm{C}$

5) TMSBr/ DCM, RT
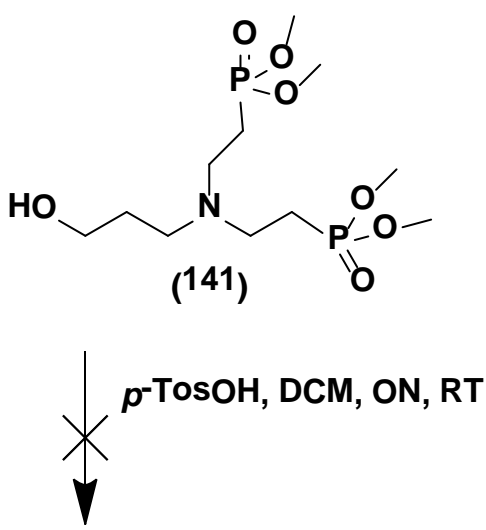

Scheme 2.24: Attempted synthetic route to tetraphosphonate QAC 146 from $\beta$-amino bisphosphonate 141.

The amine QAC precusor 144 utilized in the Michael addition was prepared via the Menshutkin reaction from amine protected alkylhalides 147-151 and $N, N$-dimethylocteadecyl amine in good yields. However, only the CBz 156 and PHT 152 protecting groups were successfully deprotected to the free quat amine 144 with $\mathrm{H}_{2} / \mathrm{PdC}$ and excess hydrazine hydrate respectively (Scheme 2.25). There is only one literature report describing the preparation of 144 from the amide 153 after basic hydrolysis. However in our hands, extraction of 144 into $\mathrm{CHCl}_{3}$ upon purification was problematic and led to to inseparable emulsions and low product recovery. Figure 2.25 shows the ${ }^{1} \mathrm{H}$ NMR spectrum $\left(\mathrm{D}_{2} \mathrm{O}\right)$ of 144 prepared from hydrazine cleavage of 152. 

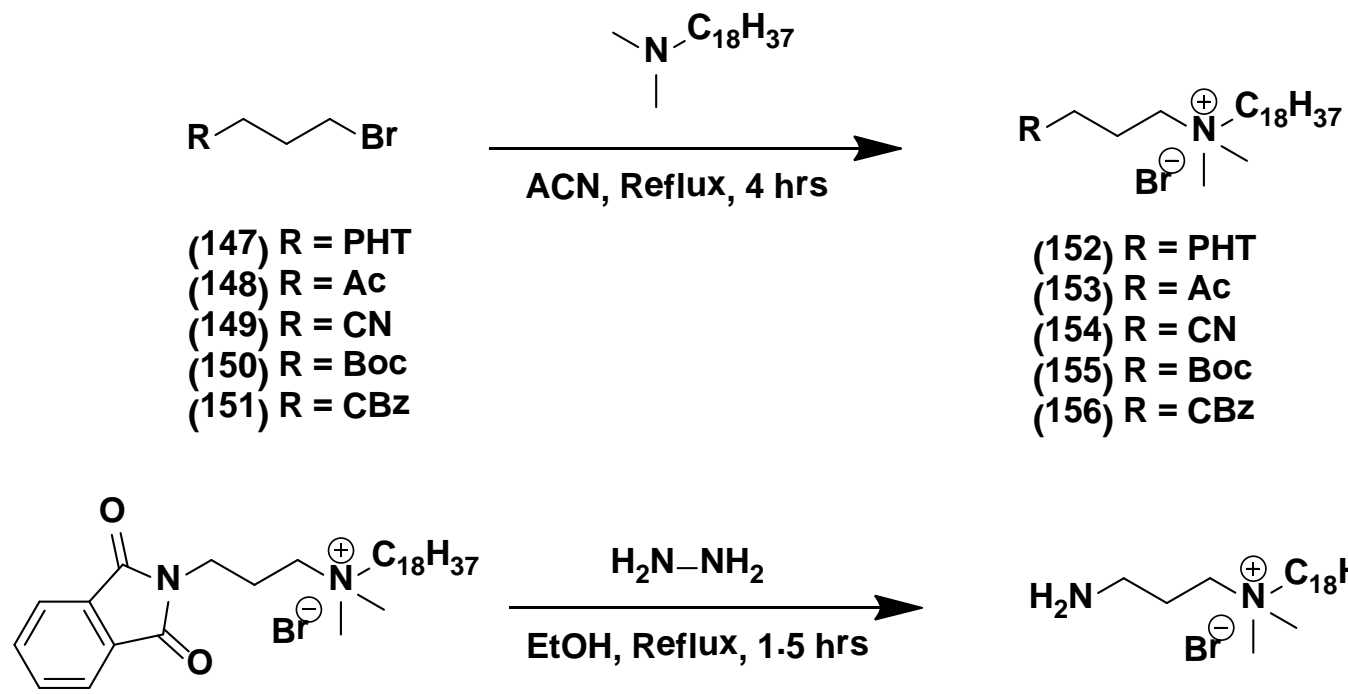

(152)

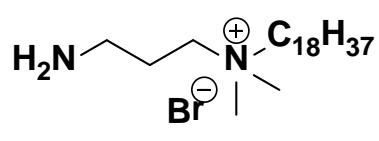

(144)

Scheme 2.25: Preparation of amine quat 144.

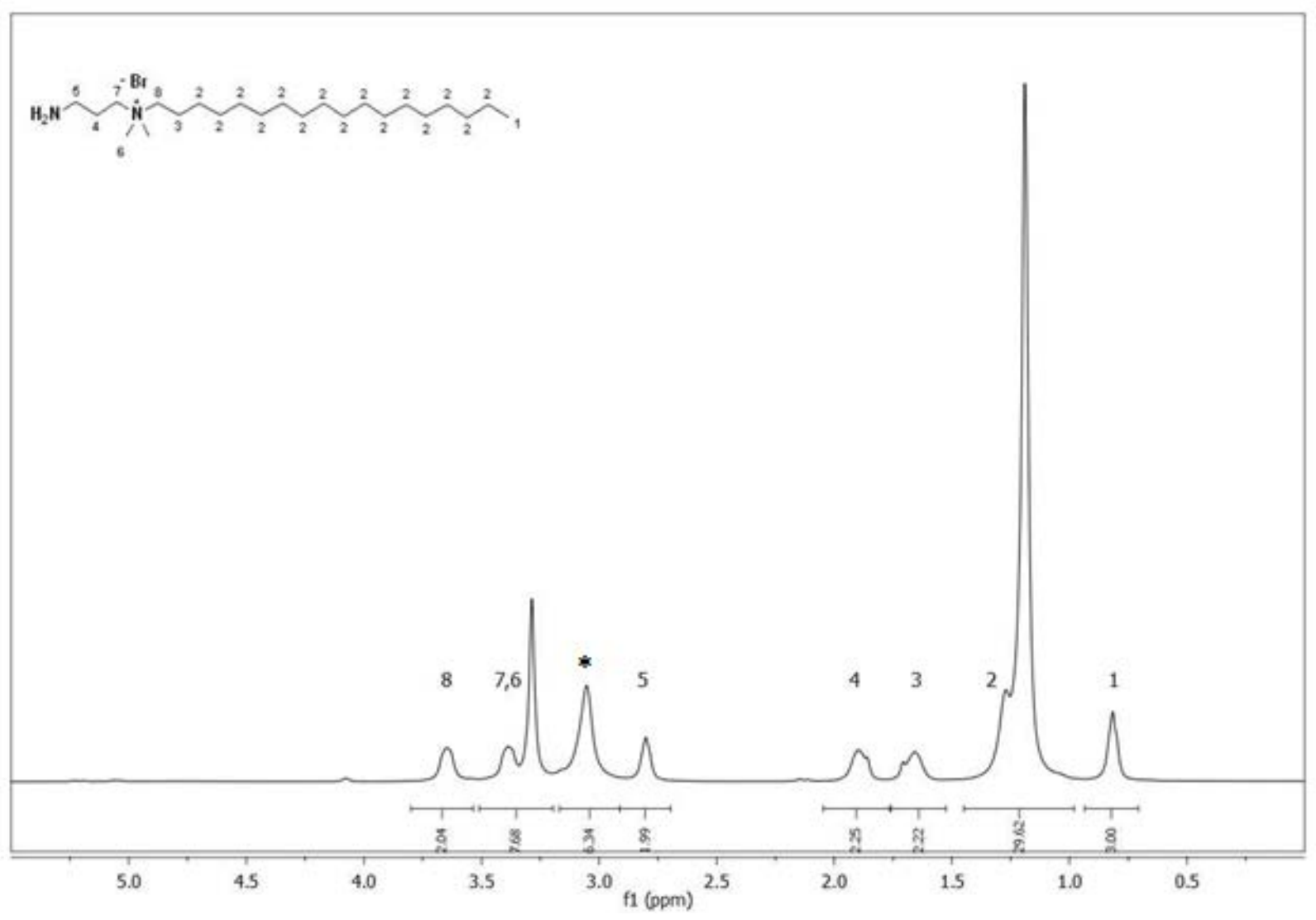

Figure 2.25: ${ }^{1} \mathrm{H}$ NMR $\left(\mathrm{CDCl}_{3}\right)$ spectrum of 144 after $\mathrm{N}_{2} \mathrm{H}_{4}$ deprotection (* unidentified impurity). 


\subsubsection{Tridentate Phosphonic Acid QAC Antimicrobials (TPQA)}

Synthesis of novel multidentate tris- $\alpha$-hydroxy phosphonic acid, tris- $\beta$-hydroxy phosphonic acid and or tris- $\gamma$-hydroxy phosphonic acid QAC was explored with the inexpensive and commercially available polyhydroxy 2-amino-2-hydroxymethyl-propane-1,3-diol (tris) scaffold. Prior to installing the phosphonate group from the hydroxyl groups, the primary amine from tris was BOC protected as $\mathbf{1 5 7}$ and used to prepare tris- $\alpha$-hydroxy phosphonic acid (Scheme 2.26), tris- $\beta$-hydroxy phosphonic acid (Scheme 2.27 a) and tris- $\gamma$-hydroxy phosphonic acid QAC (Scheme 2.28). Meanwhile, the tris- $N, N$-dimethylacrylamide 163 was also tried in an attempt to prepare tris- $\beta$-hydroxy phosphonic acid but was unsuccessful (Scheme $2.27 \mathrm{~b}$ ).

First, in the preparation of tris- $\alpha$-hydroxy phosphonic acid 161, the hydroxyl groups from tris-BOC were subjected to nucleophilic substitution with mesylphosphonate $\mathbf{1 5 8}$ according to the first step in Scheme 2.26. Unfortunately, the first step failed and none of the three nucleophilic OH's substituted the mesylphosphonate 158 with the following solvent/base combinations: $\mathrm{NaH} / \mathrm{DMF}, \mathrm{KOt}-\mathrm{Bu} / \mathrm{DMF}, \mathrm{KOt}-\mathrm{Bu} / \mathrm{t}-\mathrm{BuOH}$. It is possible that steric hindrance along with the prescence of multiple nucleophiles and insolubility of tris-BOC in $t$-BuOH played a negative role on the reaction outcome. 

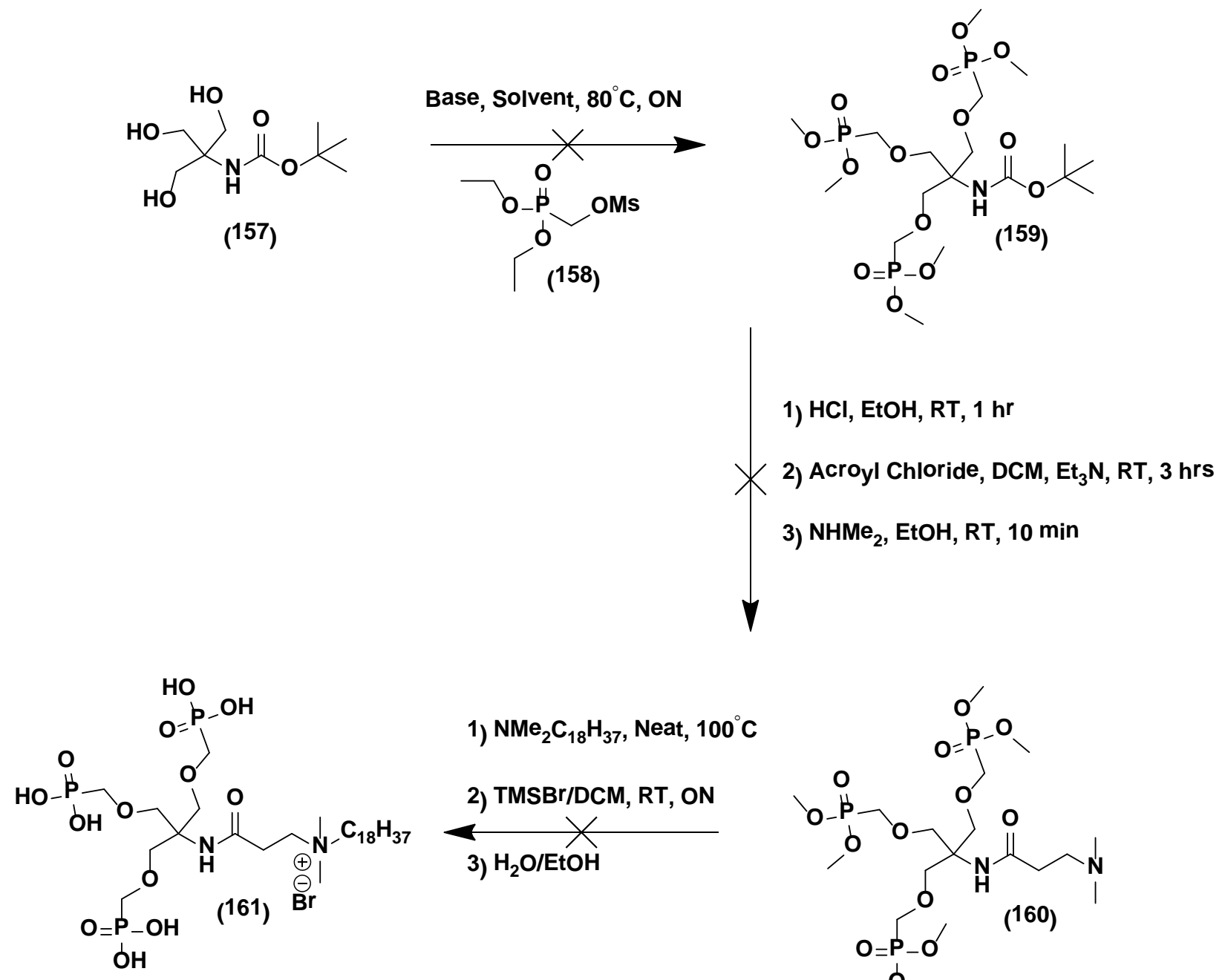

1) $\mathrm{NMe}_{2} \mathrm{C}_{18} \mathrm{H}_{37}$, Neat, $100^{\circ} \mathrm{C}$
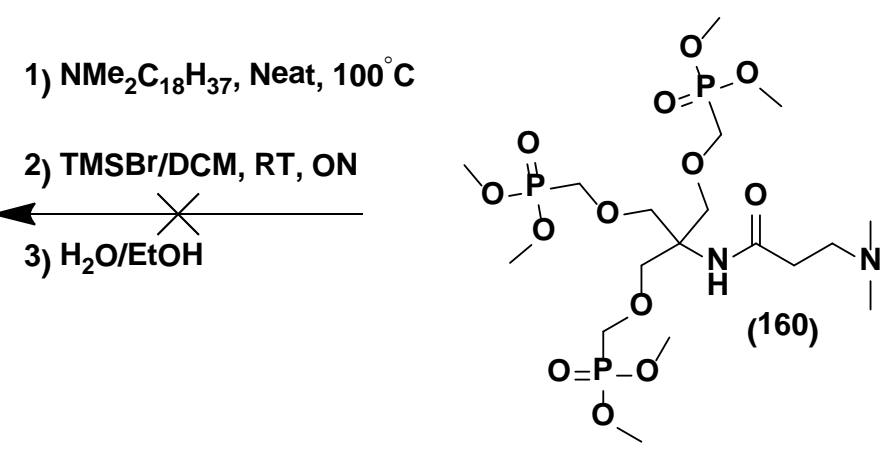

Scheme 2.26: Proposed synthesis of tris- $\alpha$-hydroxyphosphonate QAC 161.

Next, tris- $\beta$-hydroxyphosphonate QAC’s 162 and 164 were targeted via the oxa-Michael addition of the three hydroxy groups from both tris-BOC 157 and tris $N, N$-dimethylacrylamide 163 onto dimethylvinyl phosphonate according to Scheme 2.27. As was the case with tri- $\alpha-$ hydroxyphosphonate QAC, both starting materials were inert in this reaction and no addition products were observed by TLC and NMR $\left({ }^{31} \mathrm{P},{ }^{1} \mathrm{H}, \mathrm{D}_{2} \mathrm{O}\right)$. Once again insolubility and steric factors were likely the result of the failed addition reaction. In order to increase solubility and decrease the steric strain of the three hydroxyl groups in close proximity to each other the 155 
synthesis of tri- $\gamma$-hydroxyphosphonate QAC was explored in DMF and a longer carbon spacer $\left(\mathrm{C}_{3}\right)$ was used between the phosphonate and oxygen atoms.<smiles>CC(C)(C)OC(=O)NC(CO)(CO)CO</smiles>
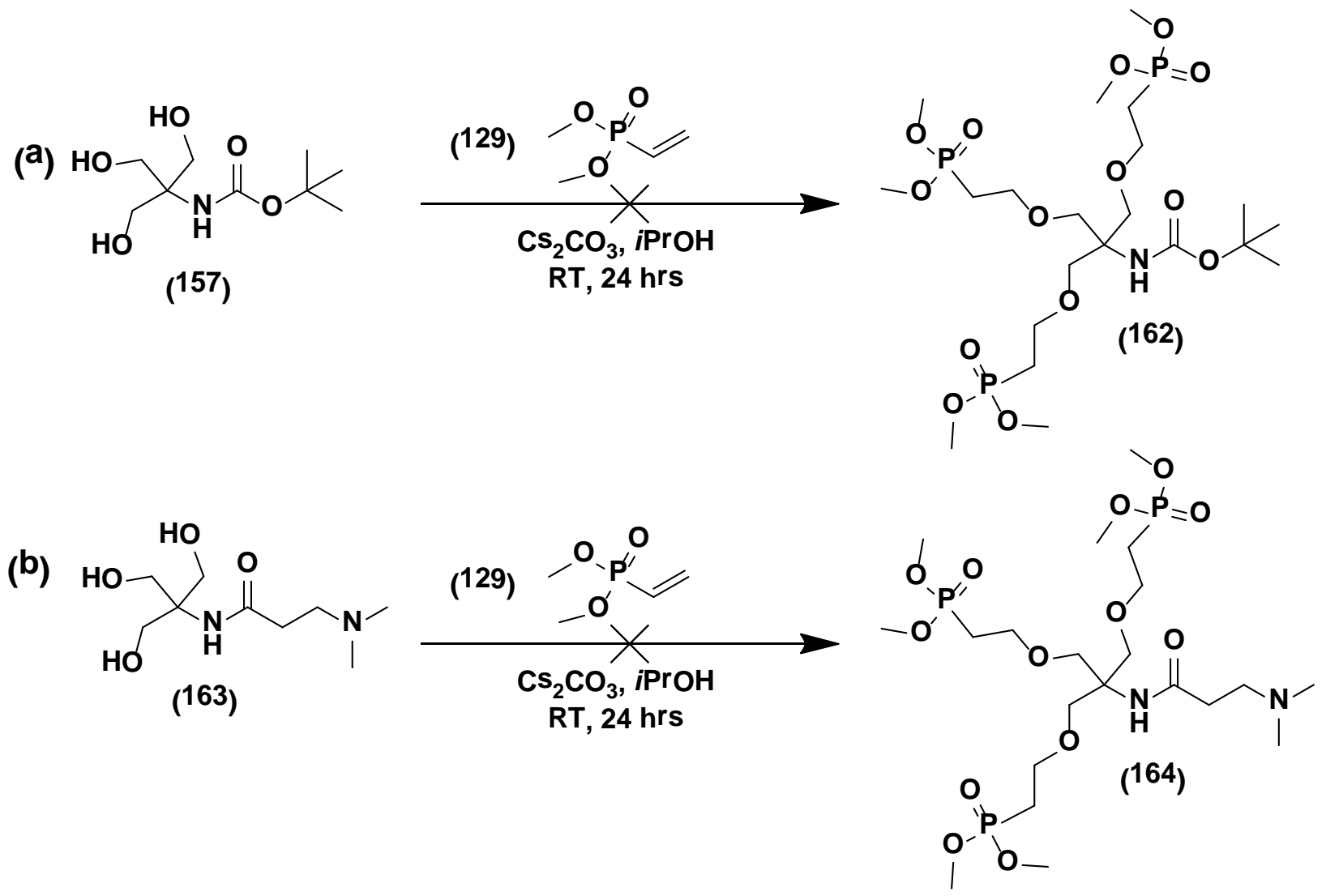

Scheme 2.27: Attempted synthesis of precursors 162 and $\mathbf{1 6 4}$ to tris- $\beta$-hydroxyphosphonate QAC's.

The conversion of BOC protected trivinyl derivative 165 and tris-BOC 157 to tri- $\gamma$ hydroxyphosphonate was attempted according to Scheme 2.28. However, like the case with the preparation of tri- $\alpha$-hydroxyphosphonate and tri- $\beta$-hydroxyphosphonate QAC's, the first step of the reaction also failed to add the desired phosphonate groups onto these starting materials. As a result, no further reactions with these starting materials was pursued. 


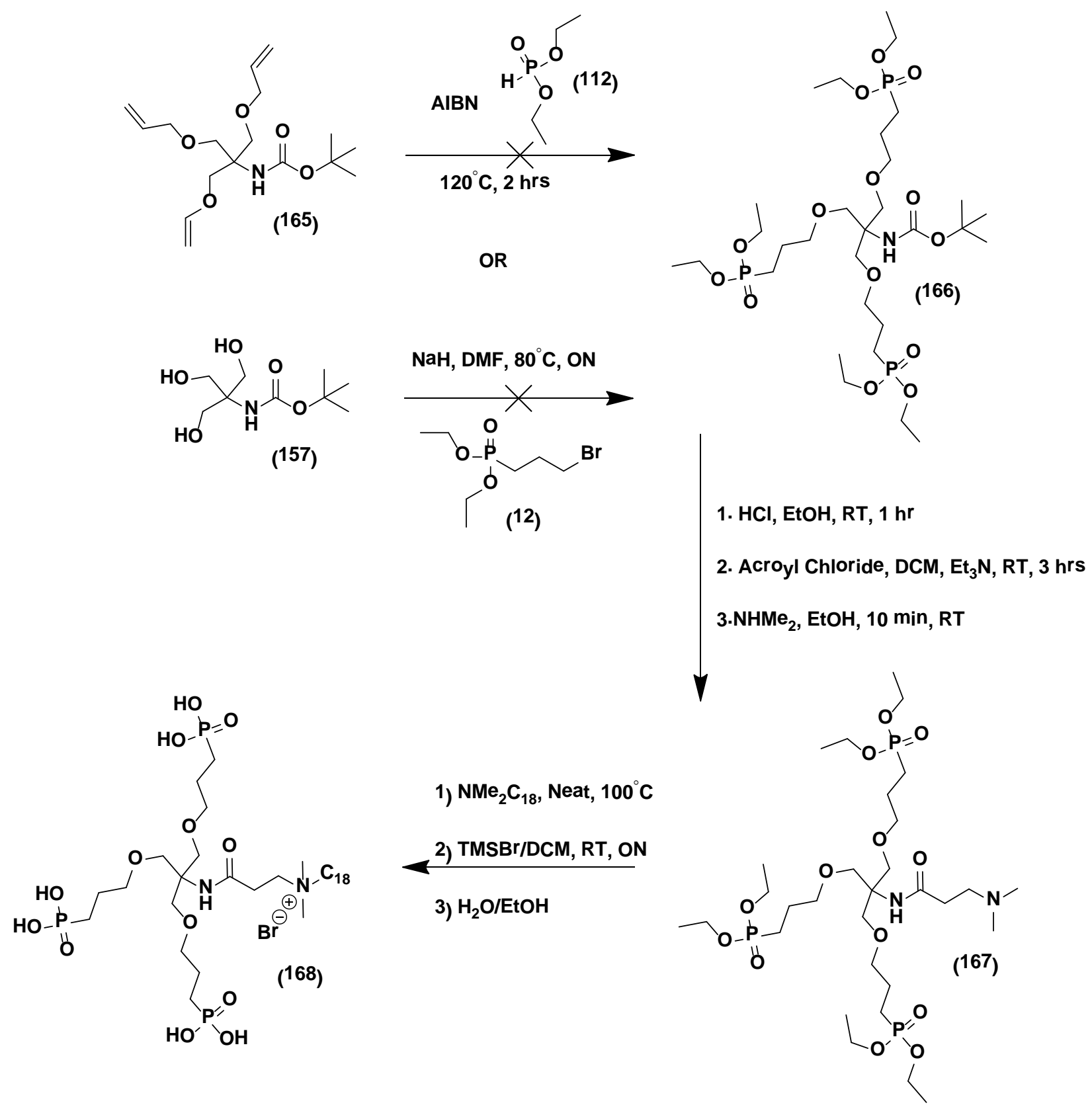

Scheme 2.28: Attempted synthesis of tris- $\gamma$-hydroxyphosphonate QAC 168.

Commercially unavailable mesyl phosphonate 158 utilized in the proposed synthesis of tris- $\alpha$-hydroxyphosphonate QAC’s 161 and 168 (Scheme 2.26) was prepared in > 90\% yield in two steps via the addition of dialkylphosphite to paraformaldehyde forming $\alpha$-hydroxy phosphonate 158 followed by sulfonation with catalytic $\mathrm{Me}_{3} \mathrm{~N} \cdot \mathrm{HCl}$ (Scheme 2.29). The tosyl 
phosphonate $\mathbf{1 7 0}$ was also prepared in an analogous fashion, but never utilized in any of the reactions involving the tris scaffold.

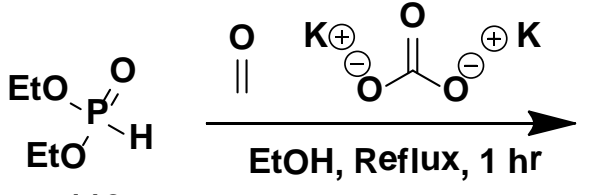

(112)

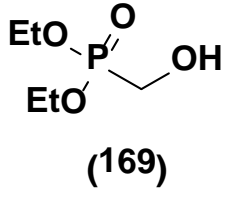

1. $0^{\circ} \mathrm{C}, \mathrm{DCM}$

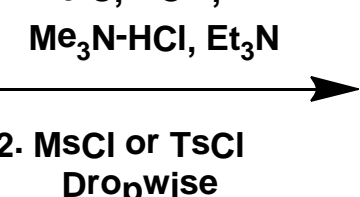

Scheme 2.29: Preparation of mesyl 158 and tosyl phosphonate 170.

BOC protection of the commercially available tris $\mathbf{1 7 1}$ followed by recrystallization from EtOAc gave precursor 157 in good yield (Scheme 2.30). A neutral BOC deprotection procedure that could be applied to any of the envisioned tris-BOC phosphonate intermediates 159, 162, 166 was identified using boiling $\mathrm{H}_{2} \mathrm{O}$ that was successfully used in deprotecting $\mathbf{1 5 7}$ to the free amine (Scheme 2.30). According to Wang et al., at elevated temperatures $\mathrm{H}_{2} \mathrm{O}$ plays the role of a dual acid/base catalyst resulting in the free neutral amine whereas the BOC group breaks down to the easily removable by products, $t$ - $\mathrm{BuOH}$ and $\mathrm{CO}_{2}$ (Figure 2.26). ${ }^{210}$

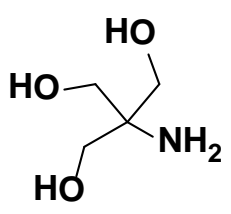

(171)
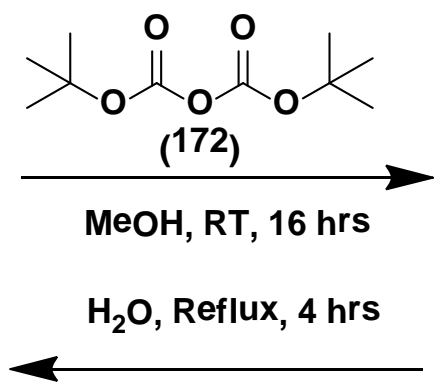

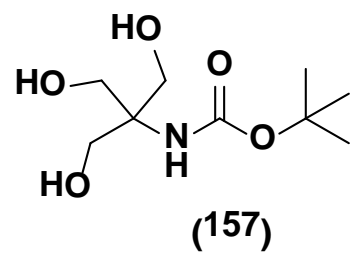

Scheme 2.30: Preparation of tris-BOC 157 and deprotection in boiling $\mathrm{H}_{2} \mathrm{O}$ to tris $\mathbf{1 7 1}$.

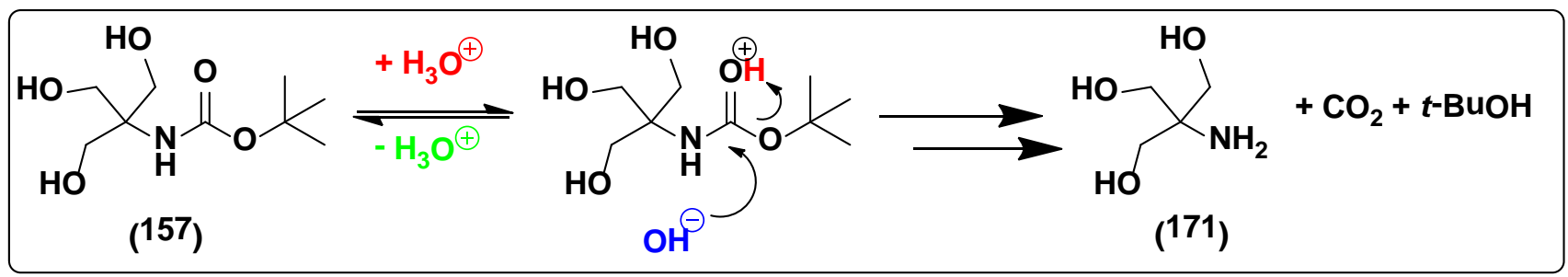

Figure 2.26: Mechanism of boiling $\mathrm{H}_{2} \mathrm{O}$ BOC deprotection. ${ }^{210}$ 
Lastly, precursor 163, a new compound was prepared from the commercially available tris acrylamine 173, and was synthesized via the rapid Michael addition with dimethyl amine (5 min by TLC) according to Scheme 2.31. Quaternization of $\mathbf{1 6 3}$ was attempted with $\mathrm{BrC}_{18} \mathrm{H}_{37}$ in DMF, but no clean NMR could be obtained at this time.
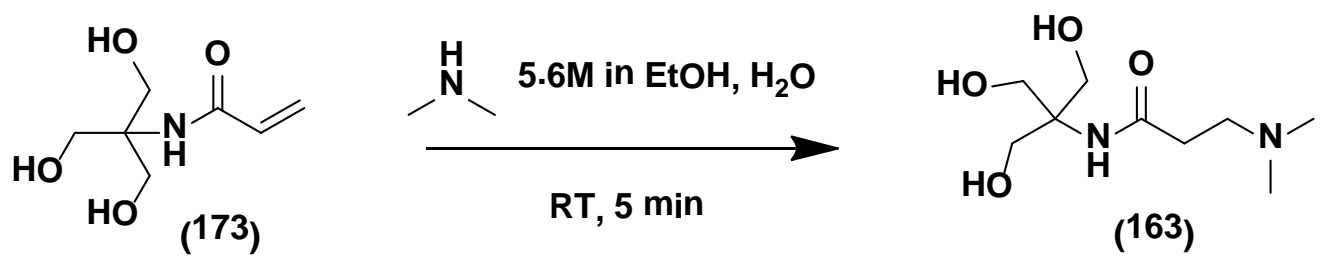

Scheme 2.31: Michael addition of $\mathrm{HNMe}_{2}$ to tris acrylamide $173 .^{211}$

\subsubsection{Amine Scaffolds for Tetra Phosphonic Acid QAC Antimicrobials}

Synthesis of tetradentate $\beta$-amino phosphonate QAC 179 and 183 were targeted via the Michael addition of two free primary amines onto 129 after selective BOC protection of commercially available triamine building blocks 174 and 180 according to Schemes 2.32 and 2.33. Compound $\mathbf{1 7 7}$ decomposed upon BOC deprotection, meanwhile compound $\mathbf{1 8 1}$ was insoluble in $\mathrm{H}_{2} \mathrm{O}$. Since the Phospha-Micheal reaction requires $\mathrm{H}_{2} \mathrm{O}$ to work, the addition of $50 \%$ EtOH to dissolve the starting material hampered the reaction and only SM was recovered after 72 hrs at RT by ${ }^{31}$ P NMR. No further reactions were carried out with these polyamine scaffolds. 
<smiles>NCCNCCN</smiles><smiles>COPOCCOPOC</smiles><smiles>COP(C)CCNCCCO</smiles><smiles>COP(=O)(CCN(CCN(CCCO)CCCO)CCP(=O)(OC)OC)OC</smiles>

1) $2 \mathrm{M} \mathrm{HCl}$ (IPA,EtOAC), RT, $1 \mathrm{hr}$ 2) NaOMe/MeOH neutralize 3)<smiles>COP(C=[14CH2+]O)(=C[14CH3])OC</smiles>
RT, 24 hrs

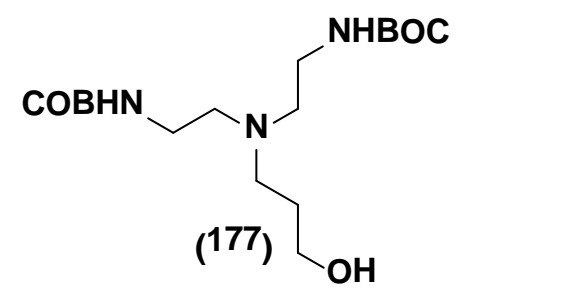

HO $\widehat{B r}^{(74)}$ DIPEA, ACN, Reflux, ON

1) $\mathrm{MsCl}, \mathrm{Et}_{3} \mathrm{~N}, \mathrm{DCM}, \mathrm{RT}, 1 \mathrm{hr}$

2) $\mathrm{NMe}_{2} \mathrm{C}_{18} \mathrm{H}_{37}$, Neat, $100^{\circ} \mathrm{C}$

3) TMSBr/DCM, RT, ON

4) $\mathrm{H}_{2} \mathrm{O} / \mathrm{EtOH}$

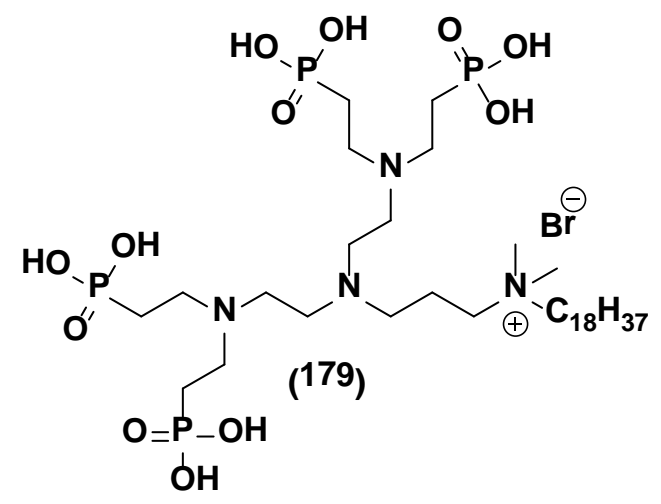

Scheme 2.32: Attempted preparation of tetradentate $\beta$-amino QAC 179. 

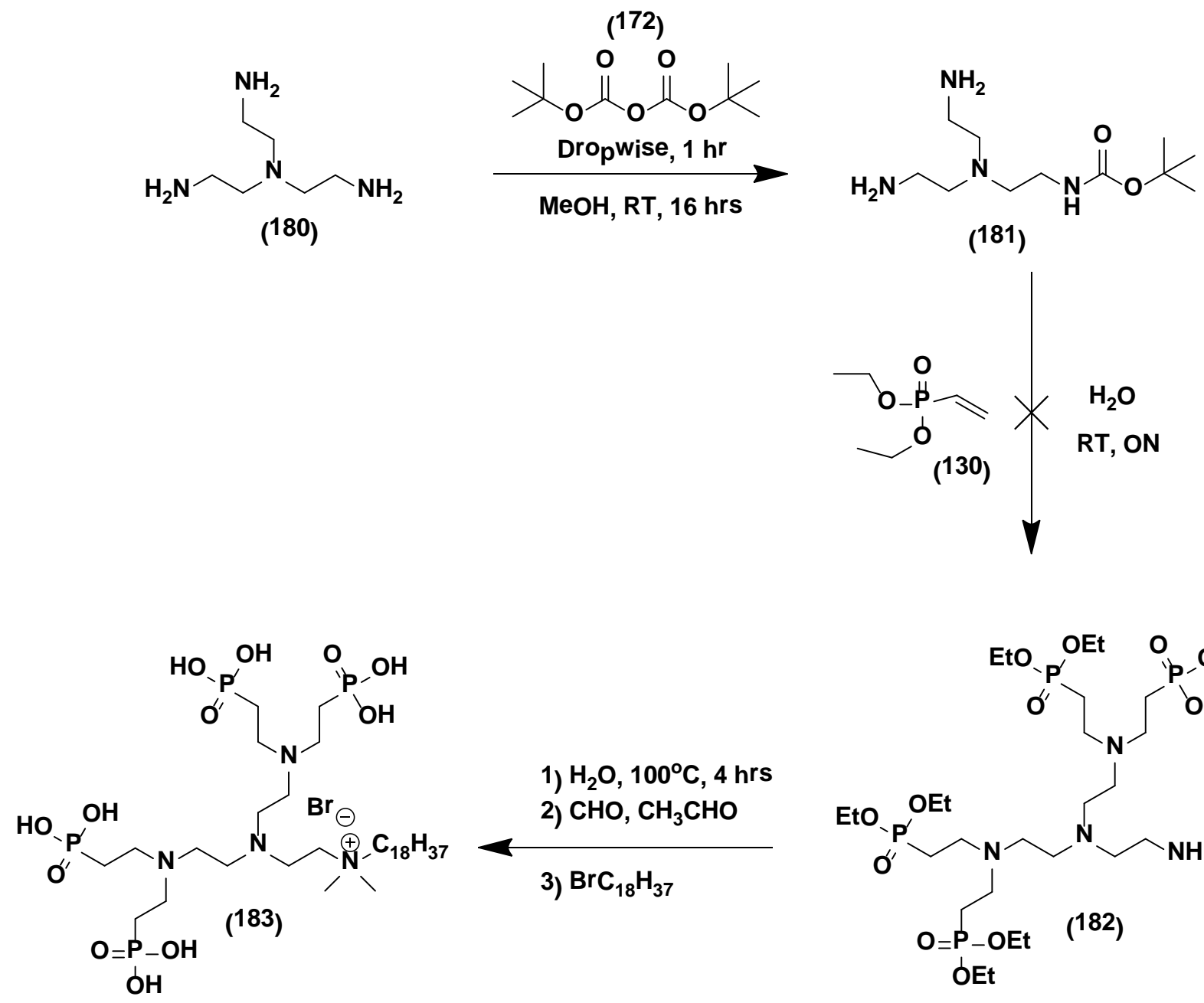

Scheme 2.33: Attempted preparation of tetradentate $\beta$-amino QAC 183. 


\subsection{Catechol QAC}

Research efforts have also focused on trying to design new types of quats from amino acids (AA) with functional linkers for attachment onto metal oxide surfaces. Since AA's are readily available in bulk at low cost and very biodegradable, QAC's based on AA's and their derivatives were envisioned as cheaper and more environmentally friendly source of antimicrobials in addition to the phosphonate QAC's. The target AA-QAC was anticipated from the dopamine amino acid analogue (L-DOPA) for binding to Ti and SS according to Scheme 2.34. Dopamine derivative $\mathbf{1 8 4}$ was the target starting material, but after problems with $N, N$ dimethylation of the primary amine resulting in only the tetrahydroisoquinolinone product 185 due to intramolecular cyclization (Scheme 2.34) ${ }^{211}$, it was decided to start from the commercially available $\mathrm{OH}$ derivative $\mathbf{1 8 7}$ and convert it to an alkyl halide $\mathbf{1 8 8}$ via the Appel reaction (Scheme 2.35). Thus, the bromo derivative $\mathbf{1 8 8}$ was quaternized with bromooctadecane but failed to

crystallize as pure $\mathbf{1 8 9}$ and was analyzed as a mixture by NMR spectroscopy $\left({ }^{1} \mathrm{H},{ }^{31} \mathrm{P}\right.$ NMR $\left(\mathrm{CDCl}_{3}\right)$, Figure 2.27). No attempt at this time was made at removing the benzyl ethers groups to the target surface active catechol QAC $\mathbf{1 9 0 .}$

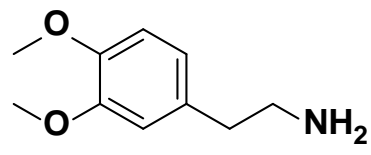

(184)

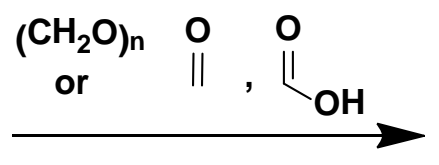

Reflux, 3-24 hrs or $\mu \mathrm{W}, 110^{\circ} \mathrm{C}, 5 \mathrm{~min}$
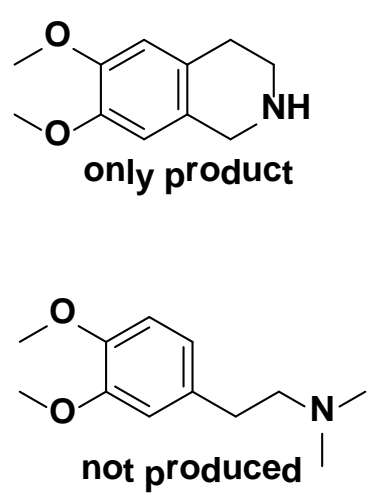

(185)

Scheme 2.34: Attempted $N, N$-dimethylation of dopamine $184 .^{212}$ 
<smiles>COc1ccc(CCO)cc1OC</smiles>

(188)

(189)
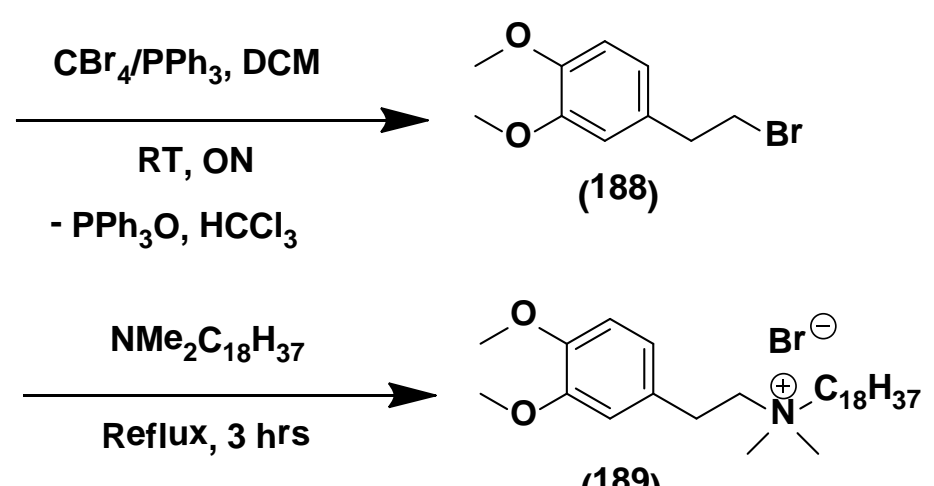

(189)

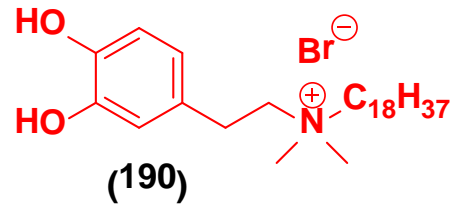

Scheme 2.35: Attempted preparation of catechol QAC 190.

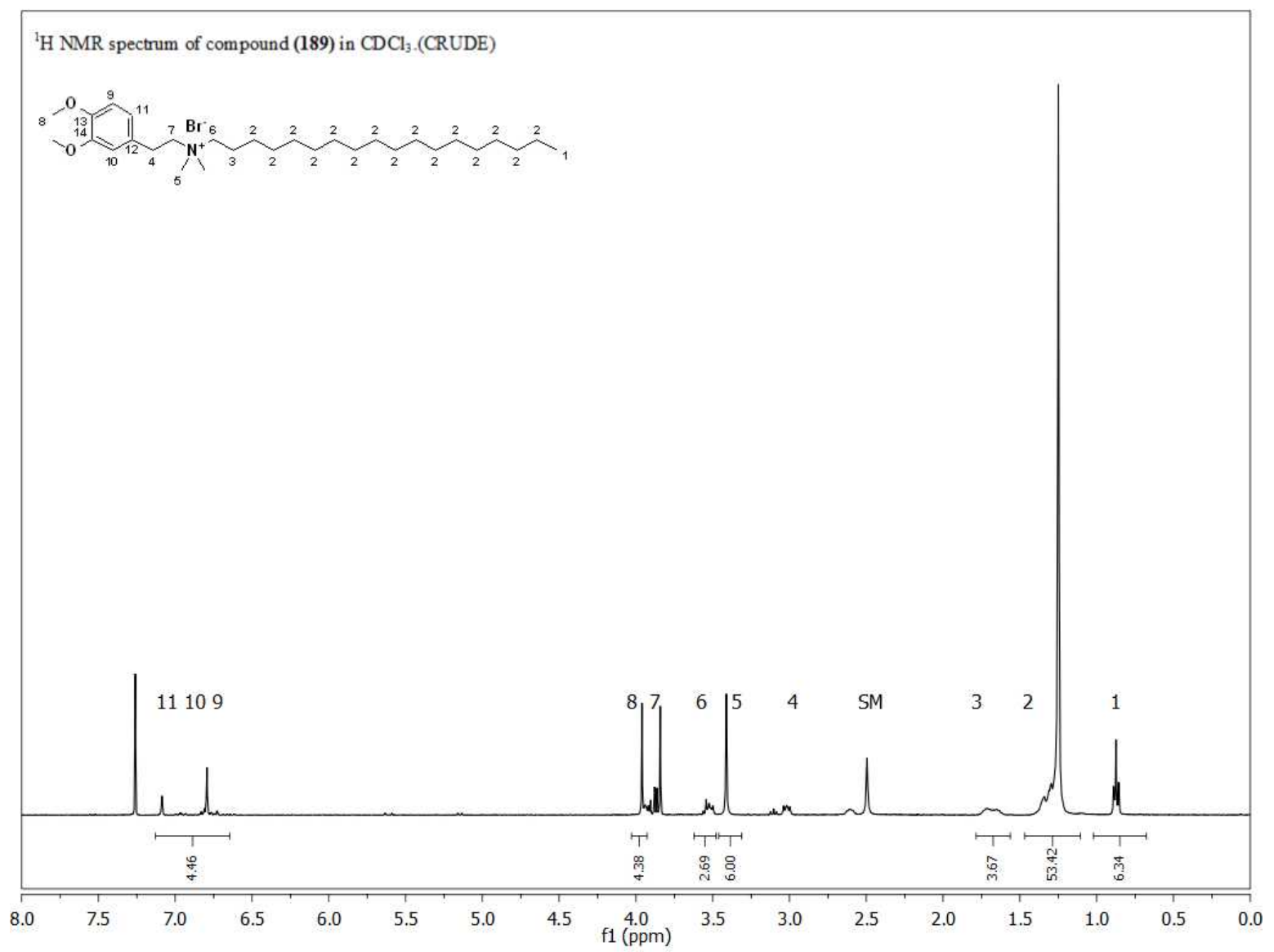

Figure 2.27: ${ }^{1} \mathrm{H}$ NMR $\left(\mathrm{CDCl}_{3}\right)$ spectrum of catechol QAC 189. 


\subsection{Organosulfur Based QAC}

The thiol group represents another functional group suitable for anchoring antimicrobials onto metals. Replacement of the methoxysilane $\left(\mathrm{SiOMe}_{3}\right)$ of the commercially available Dow silane antimicrobial with a thiol (S-H) group anchors this QAC to select metal surfaces. The thiol quat forms self-assembled monolayers specifically on $\mathrm{Au}, \mathrm{Ag}, \mathrm{Cu}$ and other suitable metal surfaces while retaining the antimicrobial activity of the quaternary ammonium compound.

The possible synthetic routes identified in the literature for the preparation of the thiolQAC largely involve commercially unavailable starting precursors (Scheme 2.36, Route iii) or require multiple preparative steps (Scheme 2.36, Route ia, ii). All of the routes feature a common nucleophilic substitution reaction between an alkylhalide (R-X) and a nitrogen nucleophile.

Due to commercial availability of 3-chloropropyl thioacetate 191 and the disulfide 197 routes ib and iv were chosen to prepare the target thiol QAC’s 194 and 199. In the thioacetate route, 3-chloropropylthioacetate was quaternized with 2 in high yield (90\%) followed by cleavage with $\mathrm{KOH} / \mathrm{MeOH}$ to give 194 in a 83\% yield (Scheme 2.36, Route ia, Figure 2.28). Numerous attempts at obtaining a crystal structure of 194 were unsuccessful. 


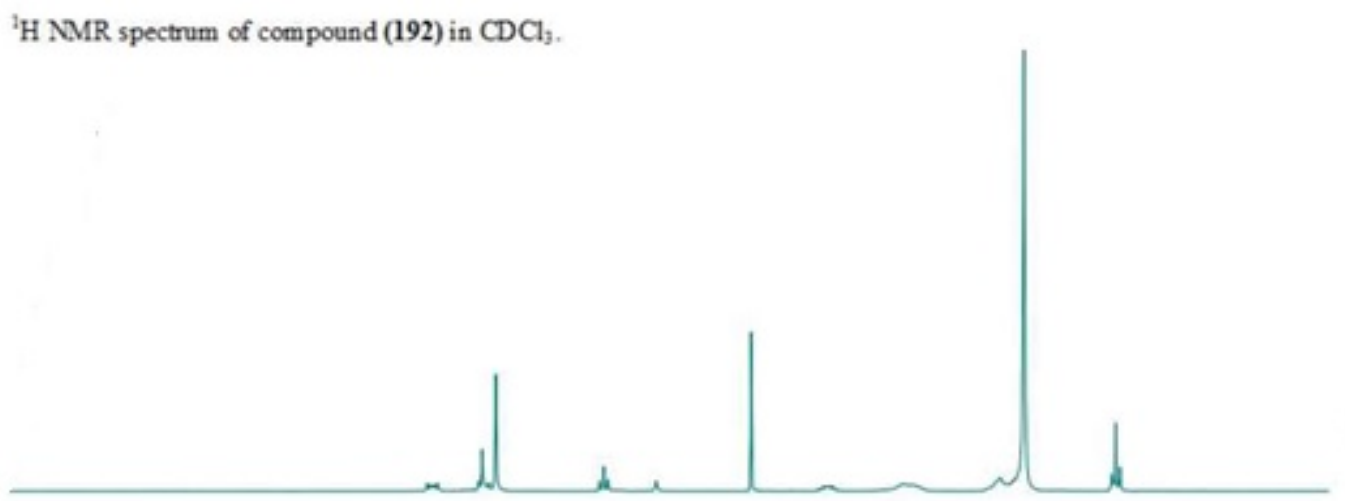

${ }^{2} \mathrm{H}$ NMR spectrum of compound (199) in $\mathrm{CDCl}_{3}$.

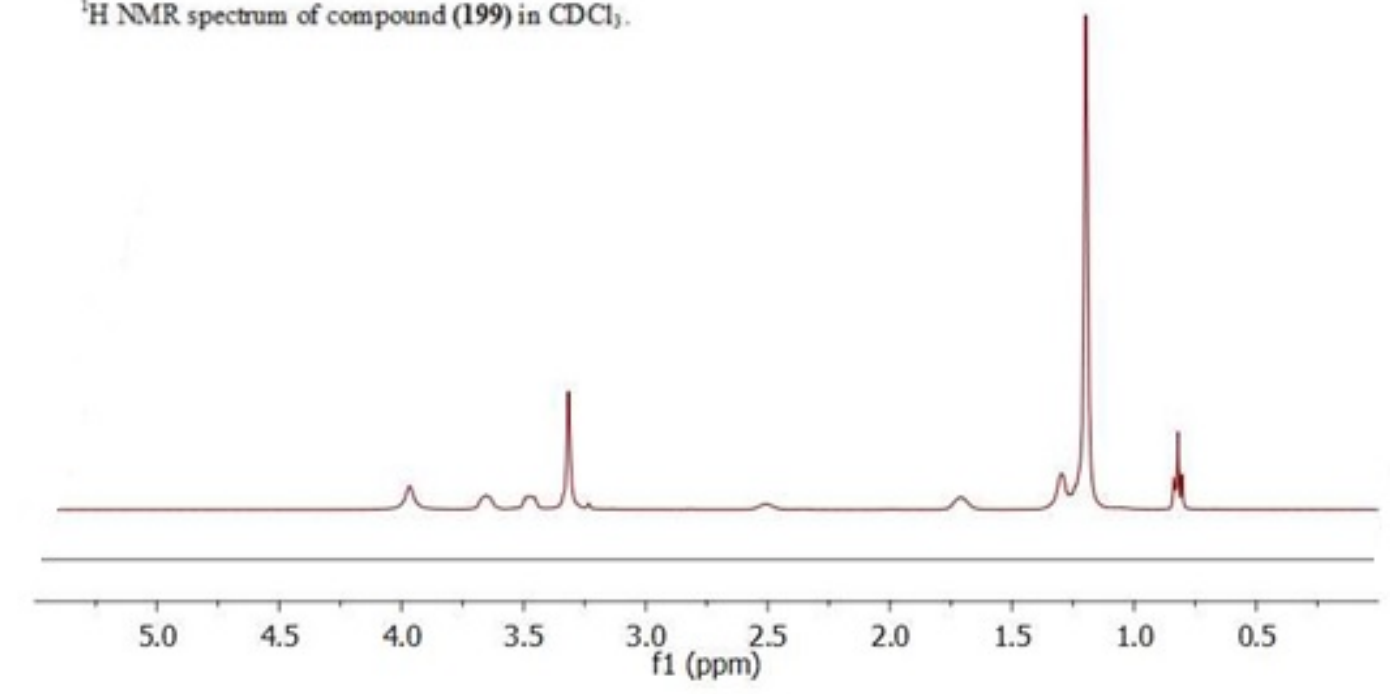

Figure 2.28: ${ }^{1} \mathrm{H}$ NMR $\left(\mathrm{CDCl}_{3}\right)$ spectra of organosulfur QAC 192 (upper) and 199 (lower).

Alternatively, the $\mathrm{C}_{2}$ thiol quat $\mathbf{1 9 9}$ was prepared from the commercially available disulfide. Deprotonation of $\mathbf{1 9 7}$ with $\mathrm{NaOMe}$ in $\mathrm{EtOH}$ and the subsequent filtering of $\mathrm{NaCl}$ yields the free base which was then quaternized in situ with 2 eq. bromooctadecane to 198 in a $80 \%$ yield. Cleavage of the disulfide bond was attempted with $\mathrm{HCl} / \mathrm{Zn}$ and $\mathrm{NaBH}_{4} / \mathrm{EtOH}$, but the final product could not be spectroscopically characterized due to difficulties with purification and reformation of the disulfide in open air. The use of triphenyl phosphine to cleave the disulfide 198 was planned, but not pursued at this time (Scheme 2.36, Route iv). 
(ia)

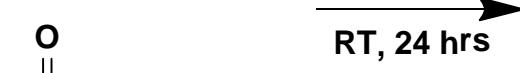

(ib)$$
\text { (191) }
$$$$
\begin{aligned}
& \text { 3-Chloropropyl } \\
& \text { thiolacetate } \\
& \$ 4.3 / g
\end{aligned}
$$

(ii)

$$
\begin{aligned}
& B r \chi_{B r} \\
& (7)^{n=3}
\end{aligned}
$$

(iiia) $\chi_{n} \chi_{B r}+\chi_{H}^{N} \chi_{n} S H$

(46)

(195)

(iiib)

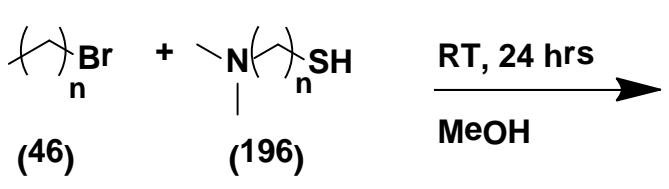

1) NaOMe/iPr

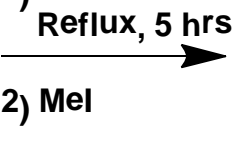

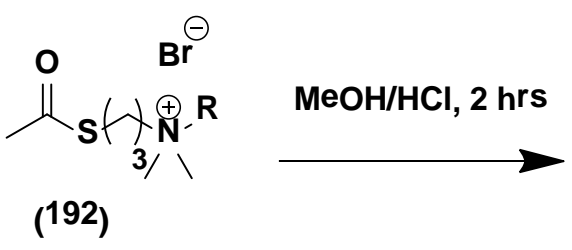
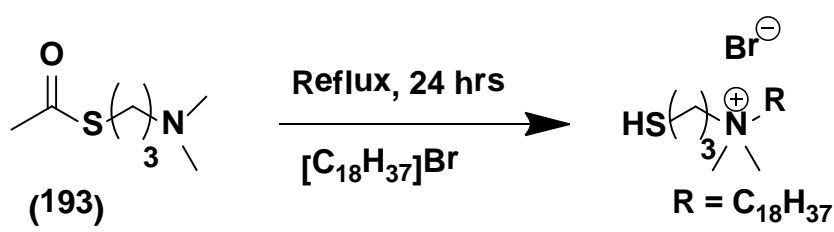

(194)

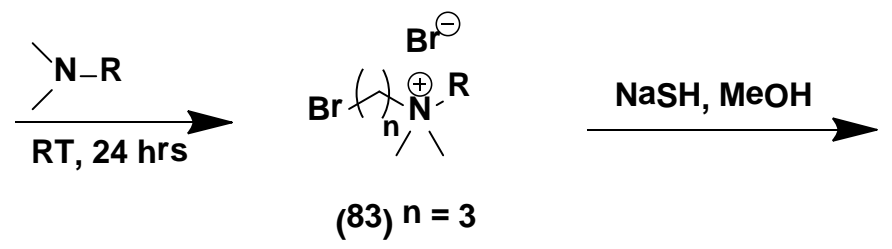

$$
\begin{aligned}
& \text { (194) } \mathrm{R}=\mathrm{C}_{18} \mathrm{H}_{37} \\
& N_{3}^{\mathrm{B} r^{\ominus}}
\end{aligned}
$$

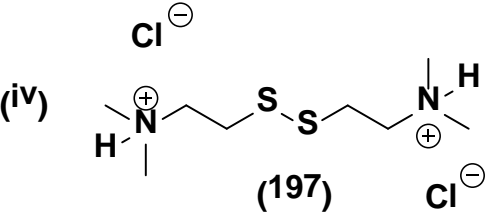

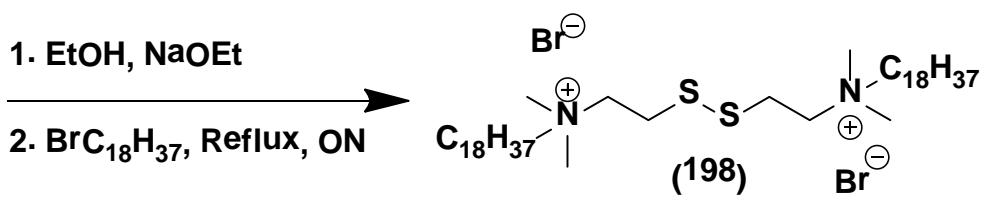

(198)

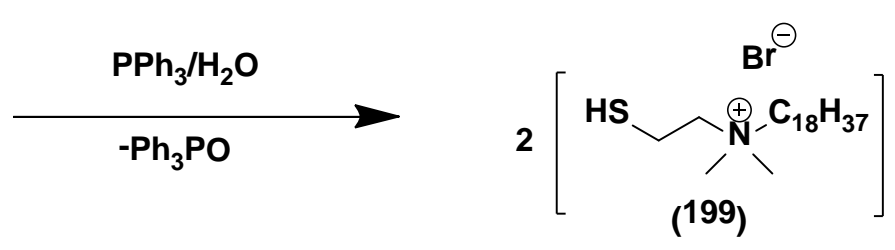

Scheme 2.36: Literature routes to target thiol QAC's $194^{213}$ and $\mathbf{1 9 8}^{214}$. 
Previous literature describing the first thiol QAC's SAM failed to prepare a stable coating. An ethanolic solution of the thiol was coated onto Au. However, no surface antimicrobial data was reported, only MIC’s in solution were reported. ${ }^{118,215}$ Further work with thiol SAM's was discontinued with greater effort focused on the preparation of organophosphonate QAC’s (Section 2.1.2.1-2.1.2.6).

\subsection{Benzophenone QAC (Plastic Coating)}

Based on previous work in our lab on benzophenone QAC $\mathbf{2 0 5 ^ { 1 0 5 }}$, the reaction conditions were optimized under $\mu \mathrm{W}$ heating by shortening the reaction time to $2 \mathrm{~min}$ at $150^{\circ} \mathrm{C}$ compared to 24 hrs reflux (Scheme 2.37). Dansyl benzophenone 214 was also synthesized for fluorescence testing onto plastics.

In addition a novel benzophenone QAC with a silane linker 206 was synthesized and tested on polypropylene and nylon (Section 2.6.2.1). Post quaternization, compound 206 showed the characteristic upfield ${ }^{1} \mathrm{H}$ resonances $\left(\delta=3.47 \mathrm{ppm}, \mathrm{CDCl}_{3}\right)$ due to the two methyl groups on the quaternary amine and the characteristic silane resonance $(\delta=3.59 \mathrm{ppm})$ due to the silane methyl groups $-\mathrm{SiOMe}_{3}$ (Figure 2.29). ${ }^{13} \mathrm{C}$ NMR spectrum reveled that compound 206 was contaminated with a trace of unreacted SM that was difficult to separate from the mixture. 


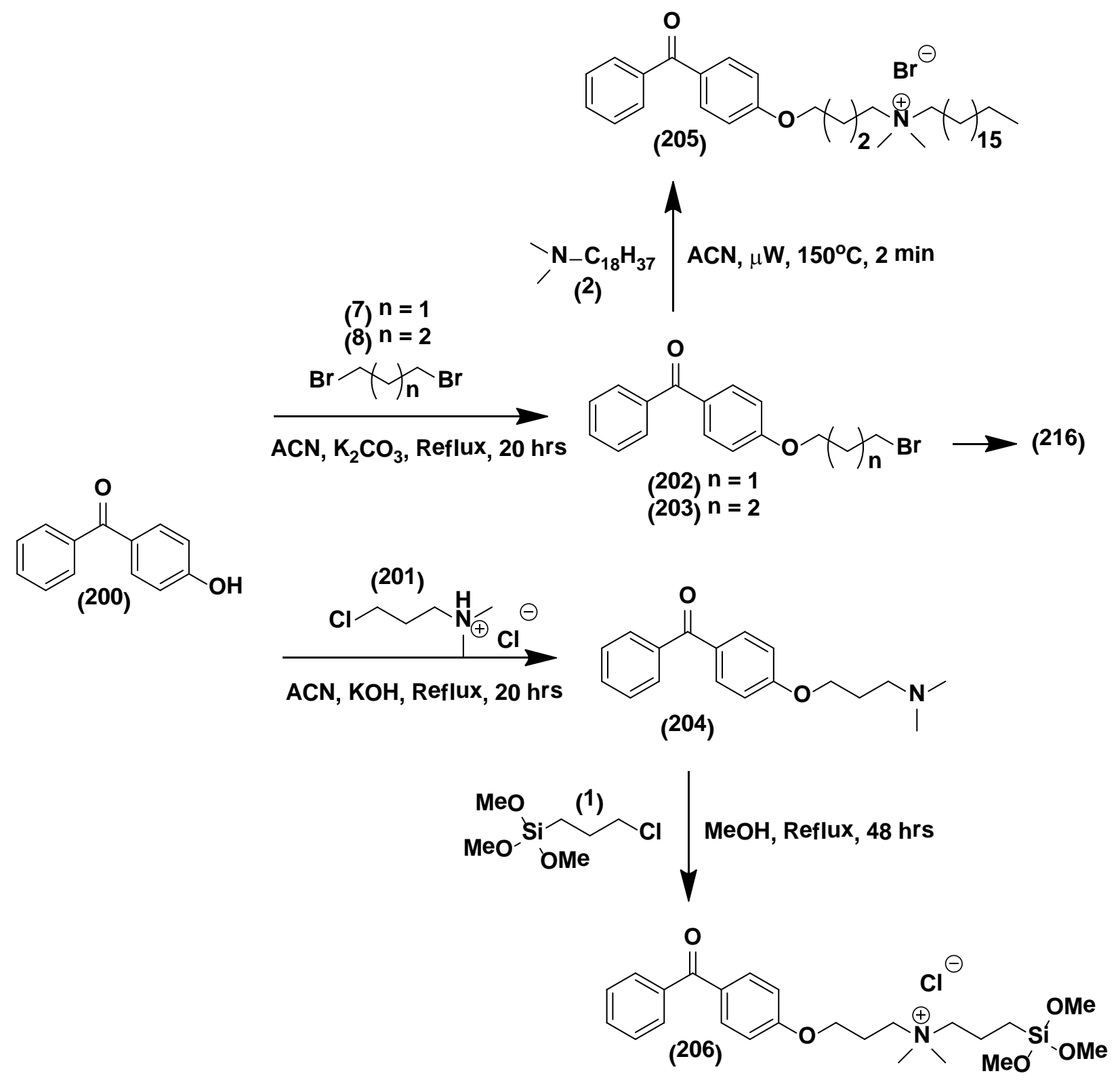

Scheme 2.37: Preparation of the benzophenone QAC’s 205 and 206. 

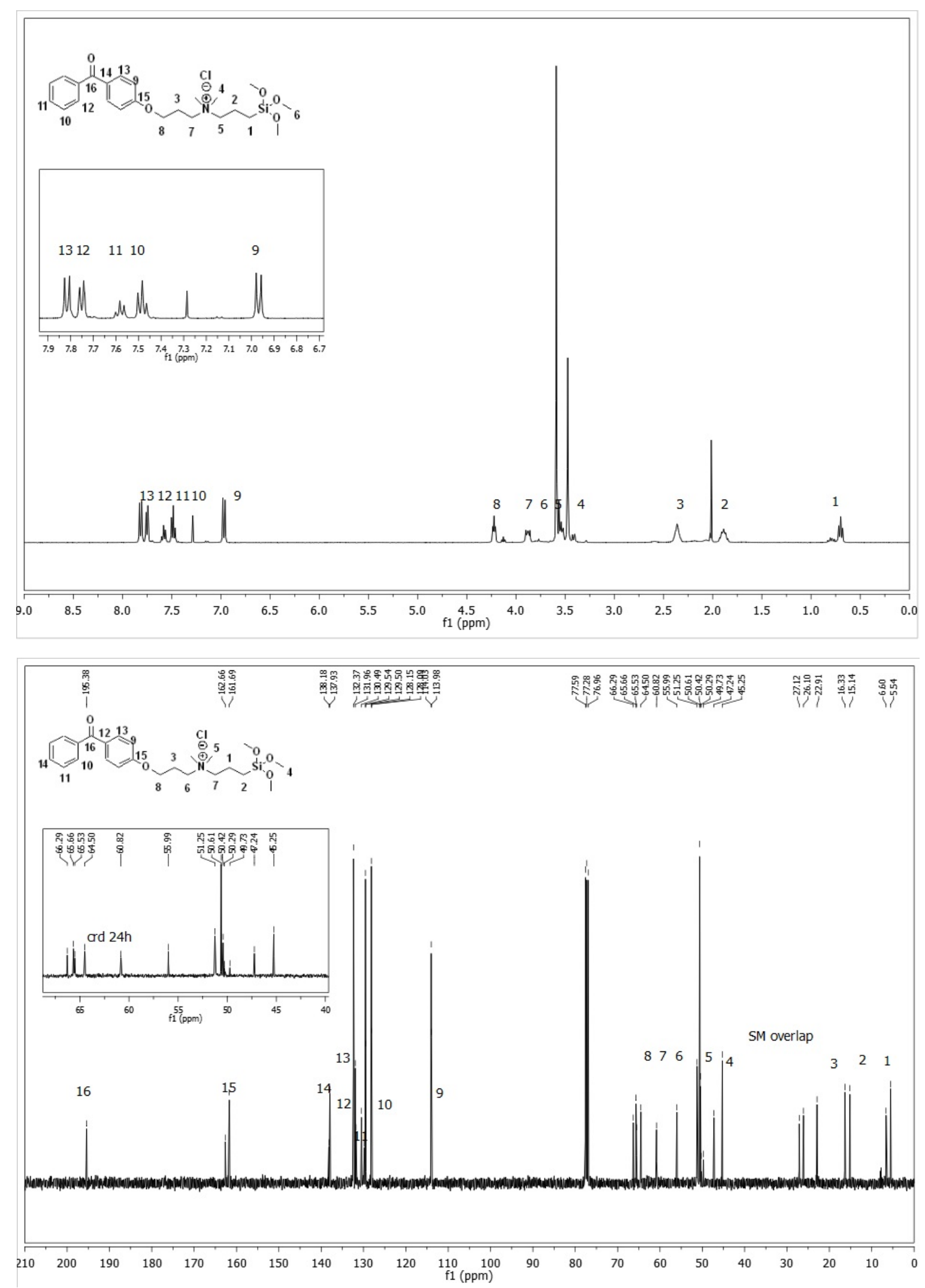

Figure 2.29: ${ }^{1} \mathrm{H}$ (upper), ${ }^{13} \mathrm{C}$ (lower) $\mathrm{NMR}\left(\mathrm{CDCl}_{3}\right)$ spectra of compound 206. 


\subsection{Dansyl QAC}

\subsubsection{Fluorescent Antimicrobial Coating Detection}

Fluorescence detection of antimicrobial QAC is advantageous over traditional dye based methods (i.e. bromophenol blue) because it allows for easy detection of the Si-QAC once applied by simply irradiating the treated surface with a UV lamp without destructive or permanent binding type testing. Accordingly, three fluorescent dansyl QAC derivatives were synthesized from previously developed procedures in our lab and used in product testing onto different

surfaces (see Section 2.7.2). ${ }^{105,177}$ The compounds, dansylphosphonate 211-212 (metals), dansylsilane 213 (non-porous surfaces), dansyl benzophenone 216 (plastic surfaces) previously synthesized in our lab were prepared on a larger scale via the Menschutkin reaction between dansyl dimethyl obtained from dansyl chloride (Scheme 2.38) and the appropriate alkyl halide (Scheme 2.39). New dansyl derivatives reported herein were prepared in the same fashion and include dansyl isopropyl phosphonate 212, dansyl thioacetate 214 and dansyl $\alpha$-bisphosphonate 215 (Scheme 2.39). All dansyl compounds 211-216, 217 and 219 comprising of a dansyl group and a quaternary amine salt, represent a novel class of $\mathrm{H}_{2} \mathrm{O}$ soluble fluorescent reporters. 

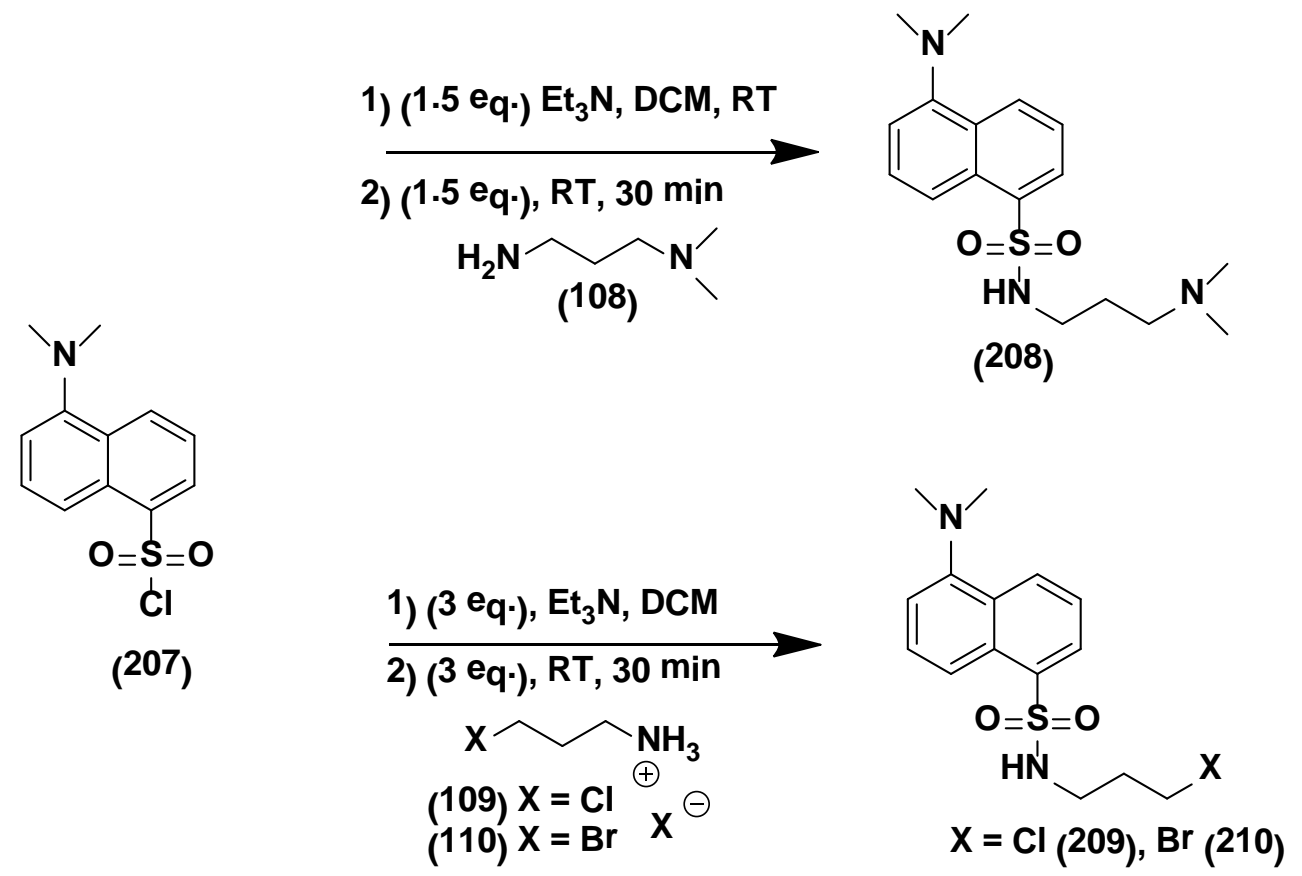

Scheme 2.38: Preparation of dansyl dimethyl 208 and halo precursors 209 and 210 for quaternization. 


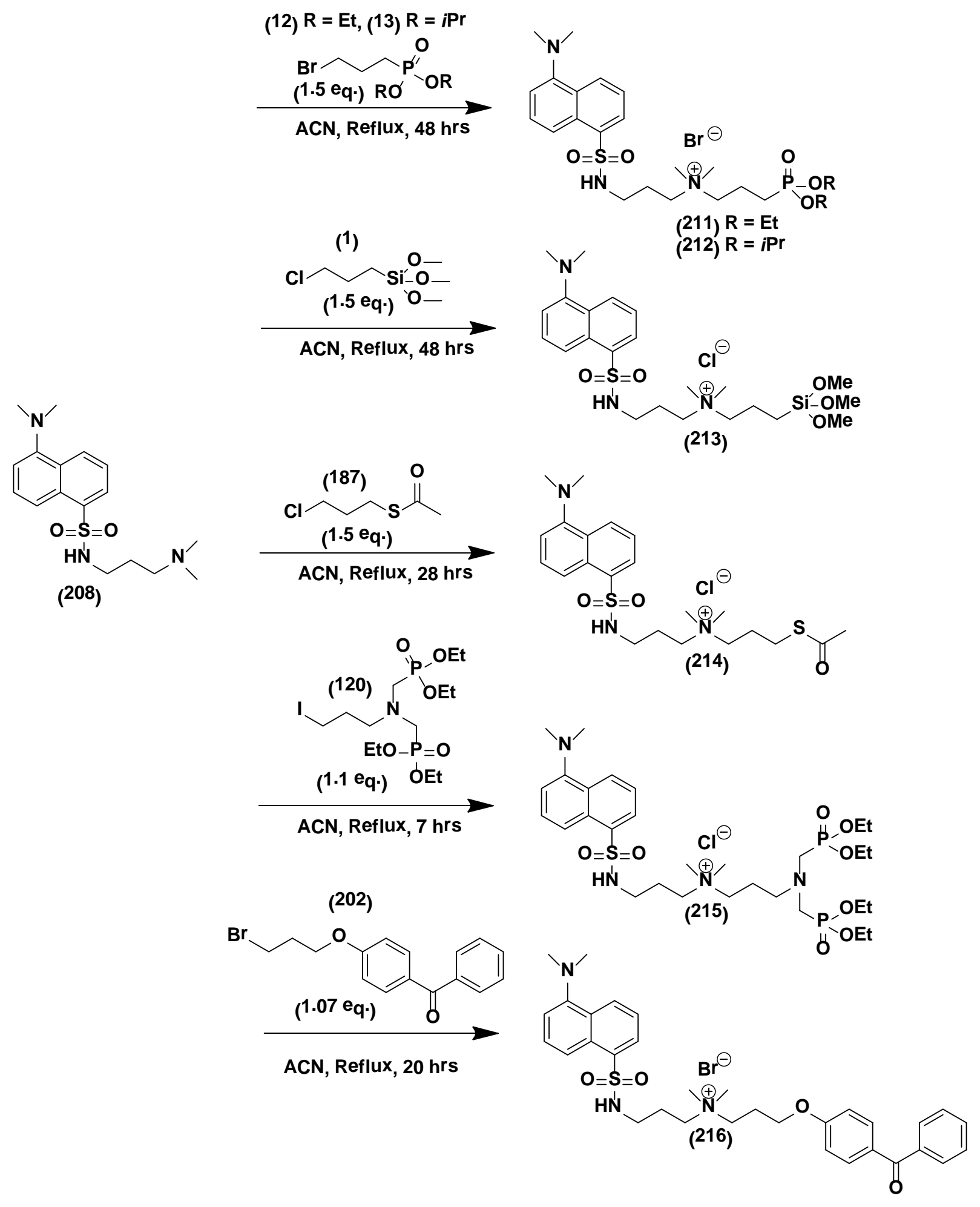

Scheme 2.39: Preparation of bifunctional dansyl anchors 211-216 for binding onto porous and non-porous surfaces. 
Didealkylation of dansyl QAC diethyl and diisopropyl phosphonate esters 211 and 212 respectively was accomplished in the absence of previous literature describing the dealkylation of such compounds. Herein are presented optimized dealkylation conditions with the appropriate reagents according to Table 2.11. Both TMSBr in DCM (Table 2.11, Entry i) and HX (aq) reagents (Table 2.11, Entries iv-viii) were successful at generating the free phosphonic acid in quantitative yield while leaving the quat group intact. However, in situ generation of TMSBr from $\mathrm{TMSCl} / \mathrm{LiBr}$ was incompatible with these compounds due to poor solubility of the quat group in ACN (Table 2.11, Entry ii).

Of the mineral acids employed under both (ST) (Table 2.11, Entries iv-v and vii-viii) and $\mu \mathrm{W}$ heating (Table 2.11, Entry vi), it was necessary to use aqueous $\mathrm{HBr}$ as anhydrous $\mathrm{HBr}(20 \%$ in EtOH) tested intitially with these compounds was significantly slower at cleaving the phosphonate ethyl ester and never reached completion even after prolonged heating (Table 2.11, Entry iii). In aqueous $\mathrm{HBr}$ (Table 2.11, Entry iv), the reaction was complete after 2 hrs whereas after 24 hrs of heating in ethanolic $\mathrm{HBr}$ (Table 2.11, Entry iii) the reaction was only 71\% completed.

$\mathrm{HBr}$ (aq) (Table 2.11, Entries iv and vii) as expected resulted in faster dealkylation compared to $\mathrm{HCl}$ (Table 2.11, Entries vi-vii) and the $i$ Pr leaving group (Table 2.11, Entries vii and viii) also resulted in faster dealkylation versus the Et group (Table 2.11, Entries iv and v). Both the $\mathrm{HBr}$ and the $i \mathrm{Pr}$ leaving groups produced more stable intermediates during the course of the reaction and these are supported by the observations in Table 2.11. Compound 217 is the first example of a fluorescent dansyl QA phosphonic acid compound connected through a quaternary ammonium group with excellent solubility in aqueous solution (Figure 2.30). 

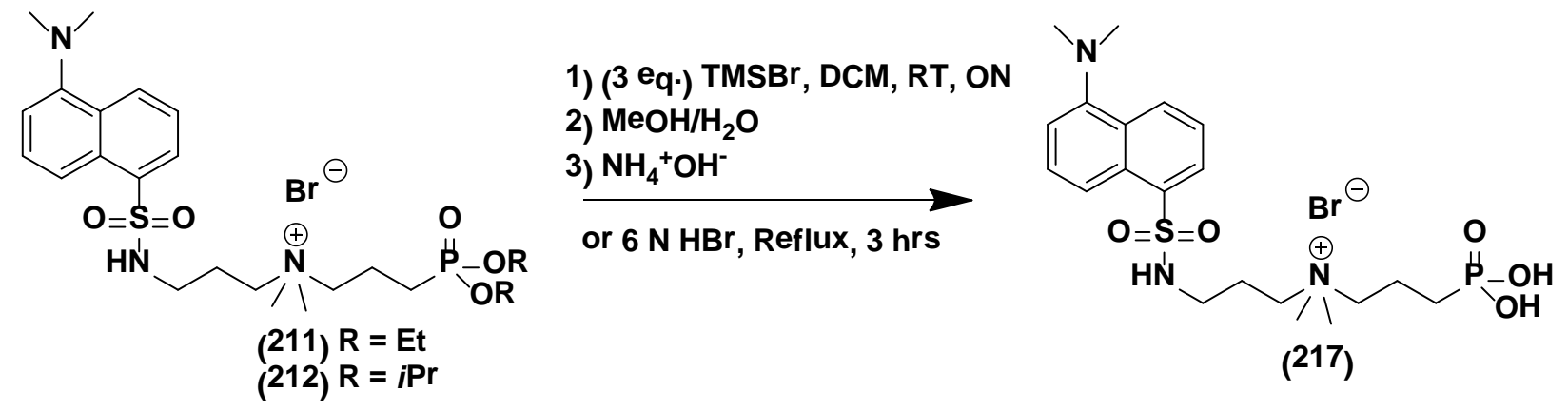

Table 2.11: Didealkylation of dansyl phosphonate diesters 211 and 212.

\begin{tabular}{|c|c|c|c|c|c|c|c|}
\hline \multirow[b]{2}{*}{ Entry } & \multirow[b]{2}{*}{$\mathbf{R}$} & \multirow[b]{2}{*}{ Reagent } & \multirow[b]{2}{*}{ Solvent } & \multirow[b]{2}{*}{ Time (hrs) } & \multirow[b]{2}{*}{$\begin{array}{c}\text { Temp. } \\
\left({ }^{\circ} \mathrm{C}\right)\end{array}$} & \multicolumn{2}{|c|}{$\begin{array}{l}\text { Rxn. Comp. (\%) by }{ }^{31} P \\
\text { NMR }\left(D_{2} O\right)\end{array}$} \\
\hline & & & & & & SM & PRD. \\
\hline $\mathrm{i}$ & Et & $\mathrm{TMSBr}$ & DCM & 24 & RT & 0 & 100 \\
\hline ii & Et & TMSCl/LiBr & $\mathrm{ACN}$ & 24 & RT & $\mathrm{n} / \mathrm{a}$ & $\mathrm{n} / \mathrm{a}$ \\
\hline iii & Et & $20 \% \mathrm{HBr}$ & EtOH & 24 & Reflux & 29 & 71 \\
\hline iv* & Et & $\mathrm{HBr}$ & $\mathrm{H}_{2} \mathrm{O}$ & 2 & Reflux & 0 & 100 \\
\hline $\mathrm{v}$ & Et & $\mathrm{HCl}$ & $\mathrm{H}_{2} \mathrm{O}$ & 12 & Reflux & 0 & 100 \\
\hline vi & Et & $\mathrm{HCl}$ & $\mathrm{H}_{2} \mathrm{O}$ & 0.3 & 150 & 0 & 100 \\
\hline vii* & $i \mathrm{Pr}$ & $\mathrm{HBr}$ & $\mathrm{H}_{2} \mathrm{O}$ & 1 & Reflux & 0 & 100 \\
\hline viii & $i \mathrm{Pr}$ & $\mathrm{HCl}$ & $\mathrm{H}_{2} \mathrm{O}$ & 2 & Reflux & 0 & 100 \\
\hline
\end{tabular}



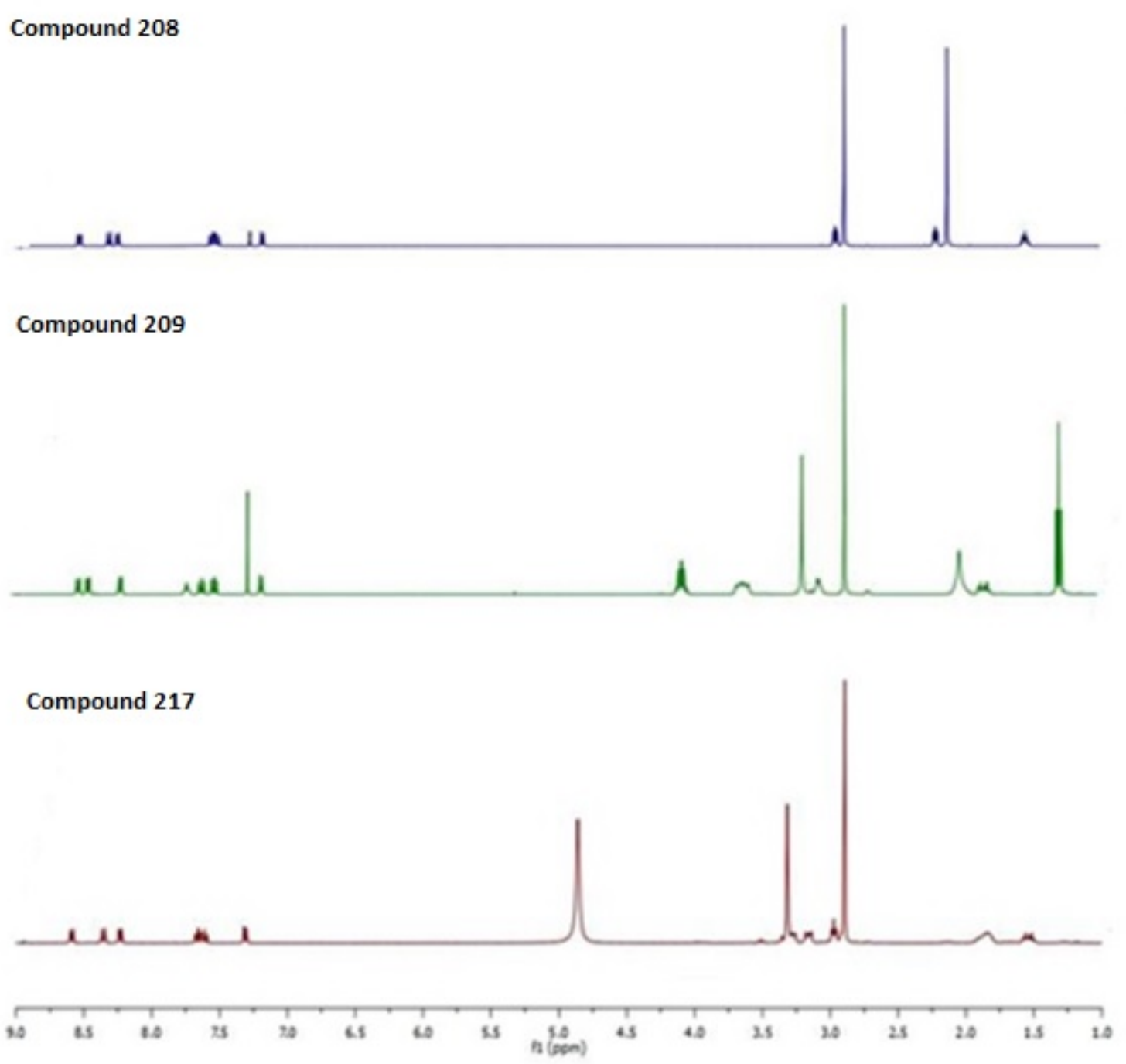

Figure 2.30: ${ }^{1} \mathrm{H}$ NMR spectra of $208\left(\mathrm{CDCl}_{3}\right), 209\left(\mathrm{CDCl}_{3}\right)$, and $217\left(\mathrm{D}_{2} \mathrm{O}\right)$.

The thioacetate protected dansyl derivative $\mathbf{2 1 4}$ for binding onto noble metals was also successfully prepared from 208 and the commercially available 3-chloropropylthioacetate. NMR analysis $\left({ }^{1} \mathrm{H},{ }^{13} \mathrm{C}, \mathrm{CDCl}_{3}\right)$ spectrum for 214 revealed characteristic hydrogen $\left({ }^{1} \mathrm{H}, \delta=2.28 \mathrm{ppm}\right)$ and carbon resonances $\left({ }^{13} \mathrm{C}, \delta=195.80\right.$ and $\left.\delta=30.77 \mathrm{ppm}\right)$ typical for the thioacetate group.

Dansyl acrylamide, 219, was prepared in an analogous fashion to compounds 209-214 by the Menshutkin reaction except by employing the dansyl bromo precusor $\mathbf{2 1 0}$ instead of dansyl 
dimethyl 208 and quaternized with the commercially available $N, N$-dimethylacrylamide derivative 218 (Scheme 2.40). Compound 219 was characterized by $\left({ }^{1} \mathrm{H},{ }^{13} \mathrm{C}\right)$ NMR spectroscopy and showed typical proton resonances corresponding to the acrylamide protons at $\delta \approx 6.38$ and $6.20 \mathrm{ppm}$. The ${ }^{13} \mathrm{C}$ NMR spectrum revealed the expected ketone carbon resonance at $\delta \approx 166.5$ ppm and two new resonances $\delta \approx 130.3$ and $126.1 \mathrm{ppm}$ due to the vinyl carbons (Figure 2.31). Compound 219 may find use as a solid fluorescent marker additive during the injection molding of plastics.

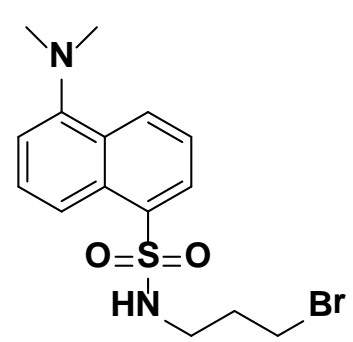

(210)

Br

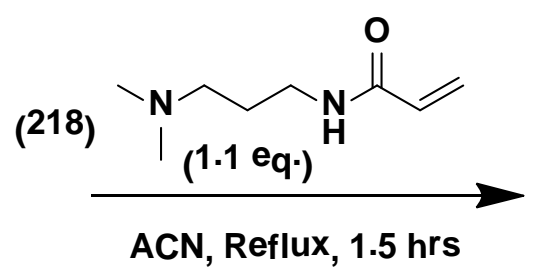

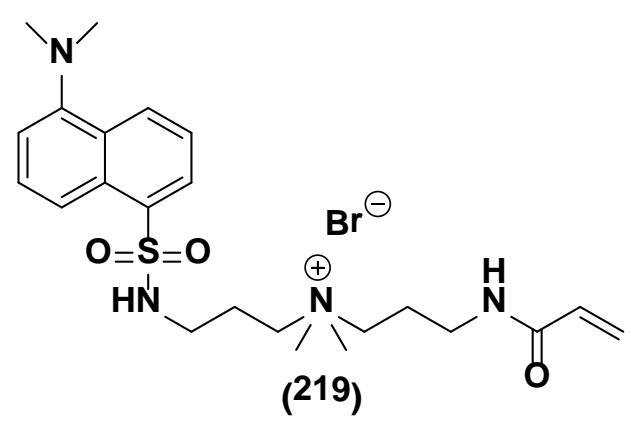

Scheme 2.40: Preparation of dansyl acrylamide QAC 219.

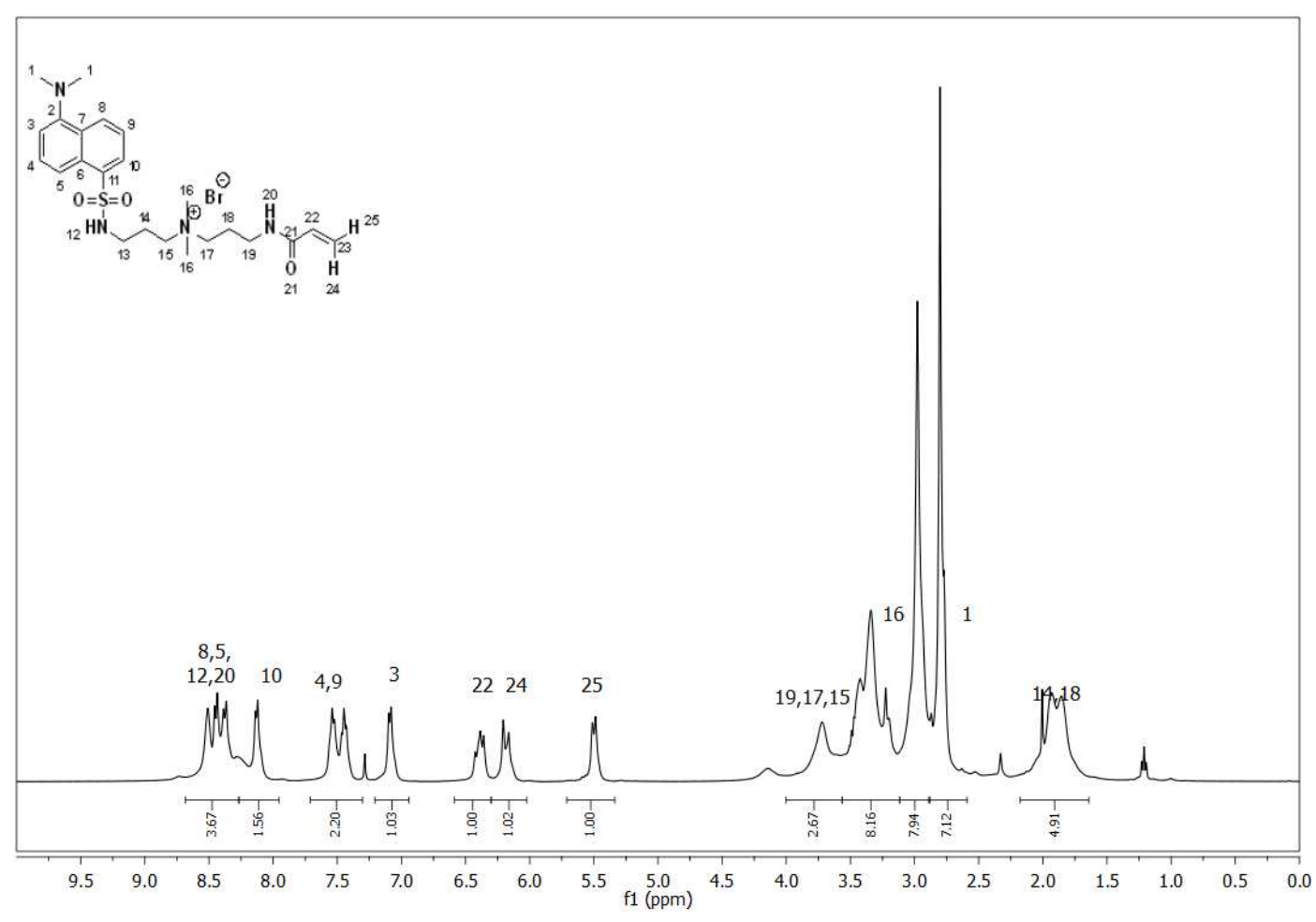


Scheme 2.24 Continued.....

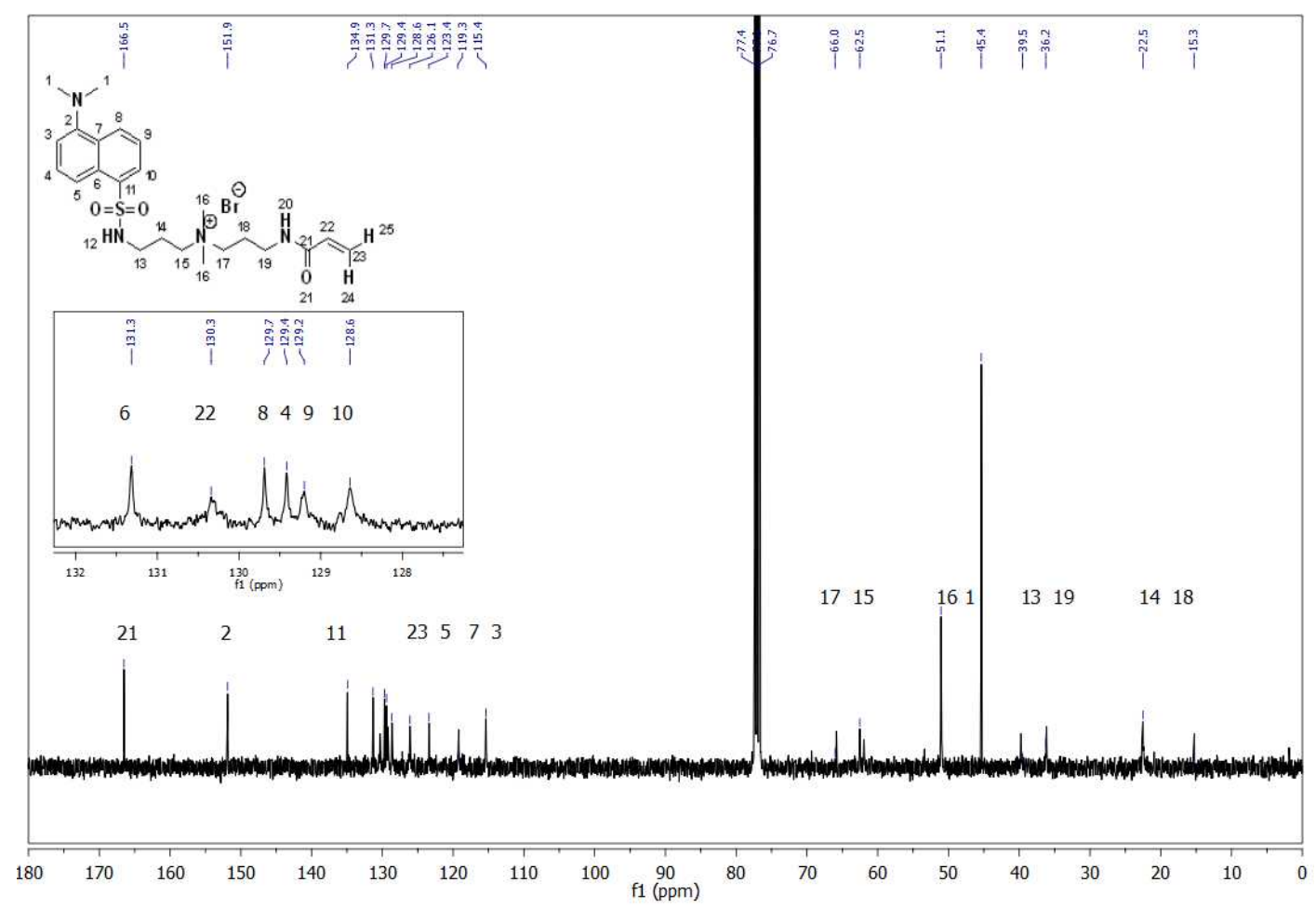

Figure 2.31: ${ }^{1} \mathrm{H}$ (upper), ${ }^{13} \mathrm{C}$ (lower) NMR $\left(\mathrm{CDCl}_{3}\right)$ spectra of dansyl acrylamide 219.

\subsection{2 $\mathrm{CO}_{2}$ Detection-Dansyl Amidine}

Synthesis of amidine containing dansyl fluorophore 222 was achieved from dansyl amine 221 which was synthesized via the Menschutkin reaction similarly to dansyl QAC's 209-214 described above (Scheme 2.41). The amidine group in 222 was attached according to a procedure from Jessup et al., by employing EtOH instead of THF. ${ }^{216}$ In a typical amidine synthesis, the condensation of a primary amine with $\mathrm{N}, \mathrm{N}$-dimethylacetamide dimethyl acetal yields a mixture of acetamidine and imidate ester depending on the temperature, solvent, and structure of the primary amine. It was possible to suppress the formation of the imidate ester by performing the reaction in the presence of excess dimethyl amine, yielding acetamidine as the exclusive product. The synthesized primary amine 221 (obtained from hydrazine deprotection of 220) was used to 
prepare amidine 222 in good yield after high vacuum purification. NMR analysis $\left({ }^{1} \mathrm{H},{ }^{13} \mathrm{C}\right.$, $\left.\mathrm{CDCl}_{3}\right)$ spectrum for 222 revealed characteristic amidine hydrogen $\left({ }^{1} \mathrm{H}, \delta=2.47, \delta=1.83 \mathrm{ppm}\right)$ and carbon resonances $\left({ }^{13} \mathrm{C}, \delta=174.11, \delta=50.29\right.$ and $\delta=22.05 \mathrm{ppm}$, Figure 2.32).

In the prescence of $\mathrm{CO}_{2}$, fluorophore $\mathbf{2 2 2}$ underwent a reversible phase change to $\mathbf{2 2 3}$ that could act as a fluorescence reporter for bacterial respiration. It was thought the reversible phase change to $\mathbf{2 2 3}$ would cause fluorescence quenching that would be directly proportional to the rate of $\mathrm{CO}_{2}$ produced by bacterial biofilms. However, no such change was observed with UV-light after 60 min of $\mathrm{CO}_{2}$ bubbling at RT. A $\mathrm{H}_{2} \mathrm{O}$ soluble fluorophore capable of reporting biofilm $\mathrm{CO}_{2}$ production remains a challenge at this point. 
<smiles>CN(C)CCCNS(=O)(=O)c1cccc2c(N(C)C)cccc12</smiles>

(208)

(220)

(221)

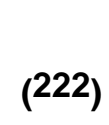

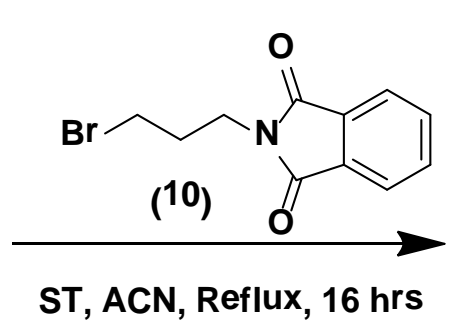
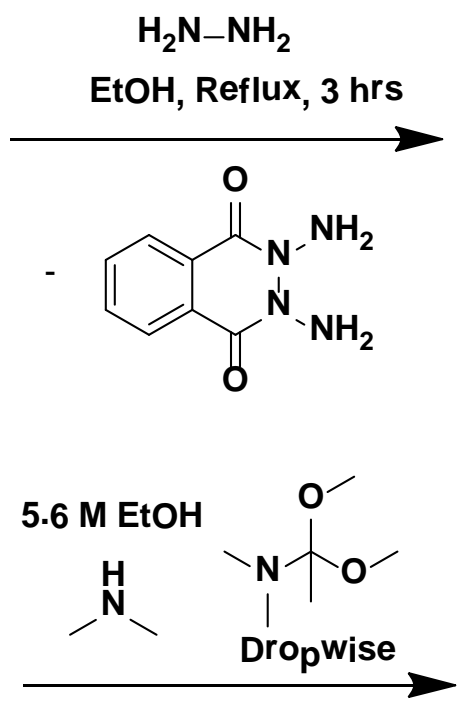

EtOH, RT, Dark, $18 \mathrm{hrs}$

High vac, $80^{\circ} \mathrm{C}, 8 \mathrm{hrs}$

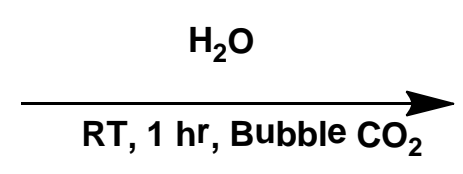

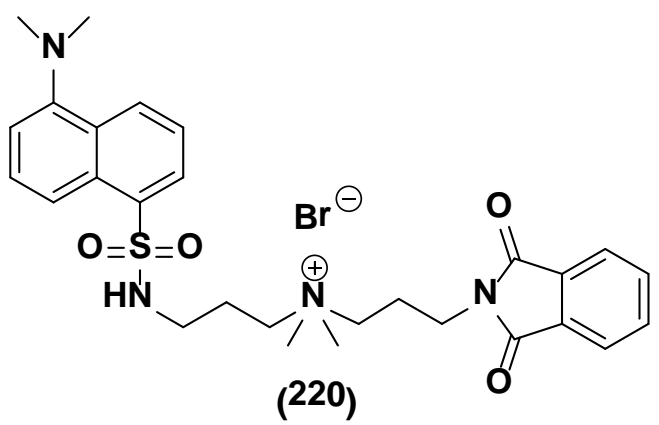

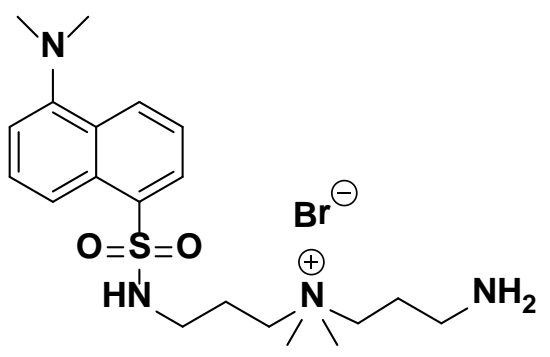

(221)

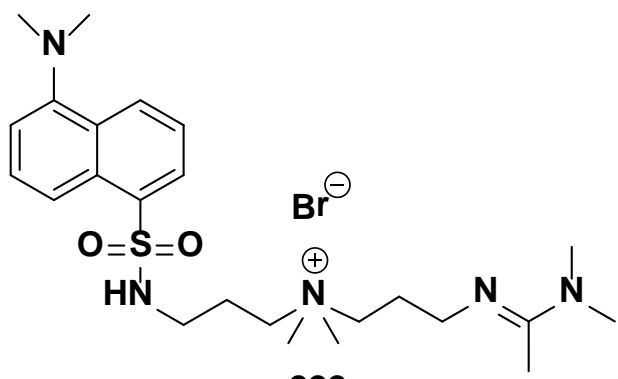

(222)

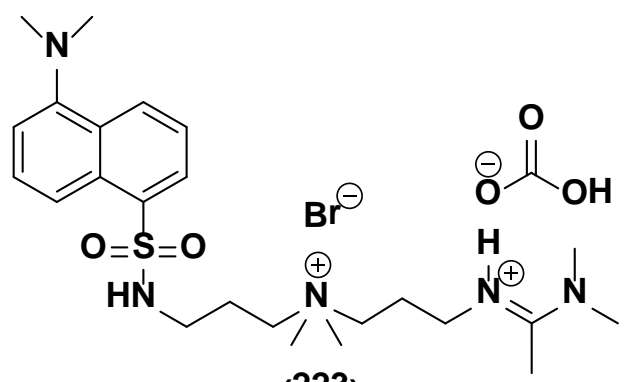

(223)

Scheme 2.41: Preparation of dansyl amindine switchable fluorophore 222 and 223. 

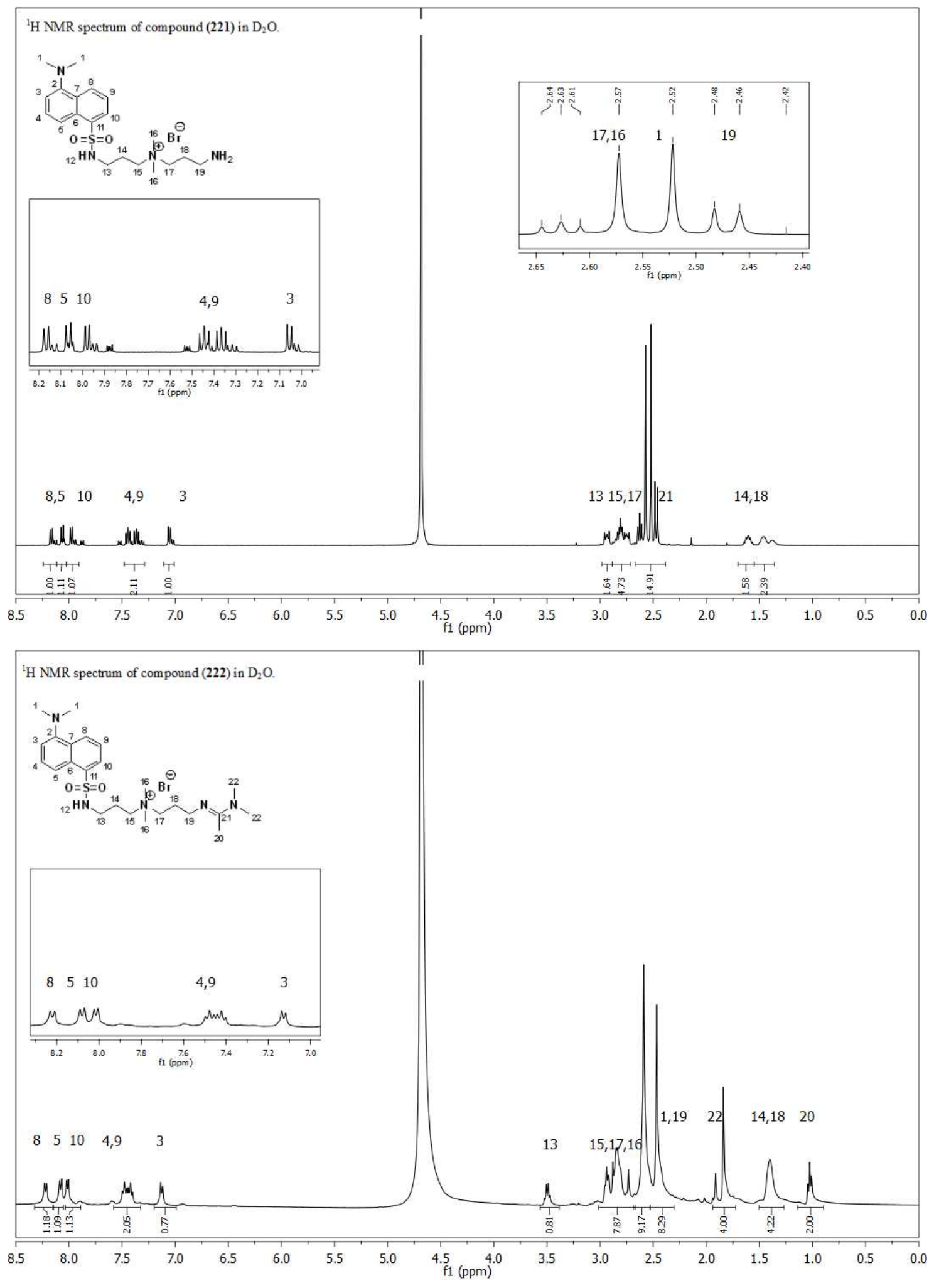

Figure 2.32: ${ }^{1} \mathrm{H}$ NMR $\left(\mathrm{D}_{2} \mathrm{O}\right)$ spectra of dansyl amine 221 (upper) and dansyl amidine 222 (lower). 


\subsubsection{Unsuccessful Dansyl Reactions}

Attempted deprotection of dansyl thiolacetate 214 to the free thiol 224 with $\mathrm{KOH}$ was unsuccessful and the reaction turned from a light yellow to green and finally to a deep purple colour after stirring at RT for 60 min (Figure 2.33). ${ }^{1} \mathrm{H}$ NMR $\left(\mathrm{D}_{2} \mathrm{O}\right)$ analysis of the crude mixture showed a complicated spectrum due to decomposition of the free thiol and disulfide formation. No further attempts to make the free thiol derivative were carried out.

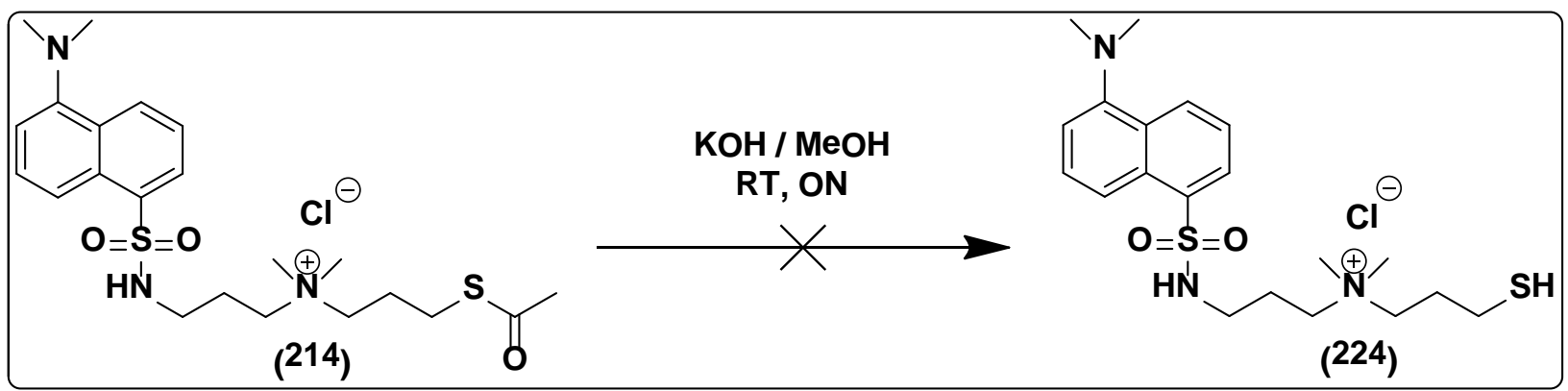

Figure 2.33: Attempted deprotection of dansyl thioacetate 218 to dansyl thiol 212.

In addition to thiol fluorophore QAC 224, a dithiocarbamate (DTC) anchored QAC was also targeted as an improved anchor for binding onto metals such as Au, Ag and Cu. The DTC $\mathrm{N}-\mathrm{C}-\mathrm{S}_{2}$ group forms a resonance structure that allows bidentate coordination to noble metals versus only monodentate coordination for thiol groups. Thus the DTC binding group forms a stronger and more stable monolayer on Au versus the thiol anchor. To the best of my knowledge, no QAC’s dithiocarbamate exist in the literature.

The synthesis of fluorophore 225 was attempted via the dropwise addition of $\mathrm{CS}_{2}$ to a stirred solution of $\mathbf{2 2 1}$ in methanolic $\mathrm{KOH}$. A precipitate was observed after a few minutes and spectroscopically characterized (Experimental, Section 5.13). From the NMR evidence it was inconclusive whether 225 exists as the bis salt or the internal salt (Figure 2.34, Scheme 2.42). 
Additionally characterization of $\mathbf{2 2 5}$ by HRMS spectrometry (ESI-TOF) accounted for a mass of (m/z: 469.1760, $\mathrm{C}_{21} \mathrm{H}_{33} \mathrm{~N}_{4} \mathrm{O}_{2} \mathrm{~S}_{3}$ ) indicative of the internal salt (Figure 2.35).

(221)
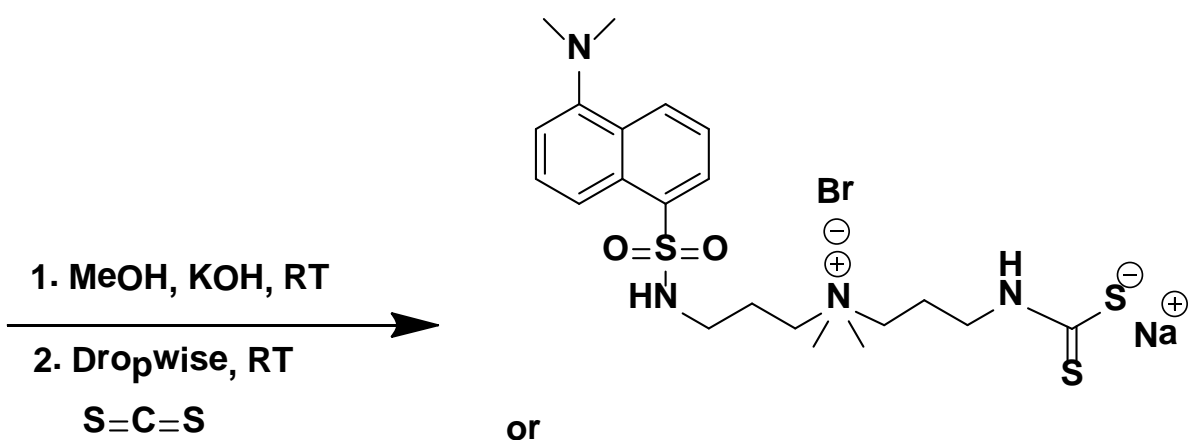

or

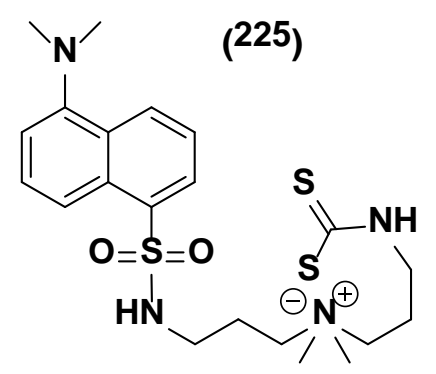

Scheme 2.42: Attempted preparation of dansyl DTC 225.

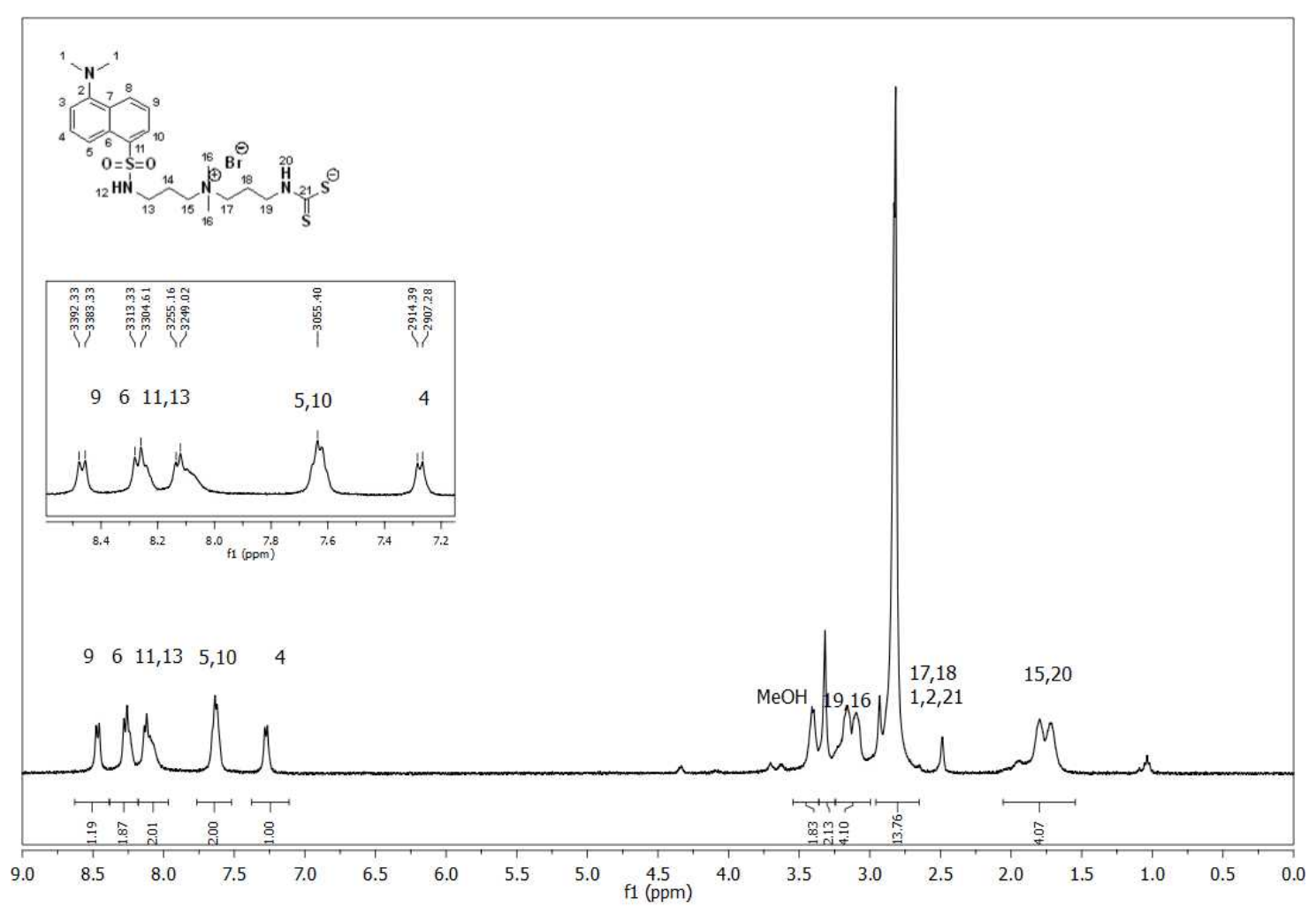

Figure 2.34: ${ }^{1} \mathrm{H}$ NMR (DMSO- $\mathrm{d}_{6}$ ) spectrum of compound 225. 


\begin{tabular}{|c|l|l|l|l|l|}
\hline \multicolumn{1}{|c|}{ Formula } & \multicolumn{1}{|c|}{ Calculated m/z (amu) } & \multicolumn{1}{|c|}{ mDa Error } & PPM Error & DBE \\
\hline 1 & C28 H25 N2 05 & 469.1757 & 0.0 & -0.2 & 17.5 \\
\hline 2 & C21 H33 N4 O2 S3 & 469.1760 & -0.3 & -0.6 & 7.5 \\
\hline 3 & C20 H29 N4 07 S & 469.1751 & 0.5 & 1.1 & 8.5 \\
\hline 4 & C21 H25 N8 03 S & 469.1764 & -0.7 & -1.6 & 13.5 \\
\hline 5 & C29 H29 N2 S2 & 469.1766 & -0.9 & -2.0 & 16.5 \\
\hline
\end{tabular}

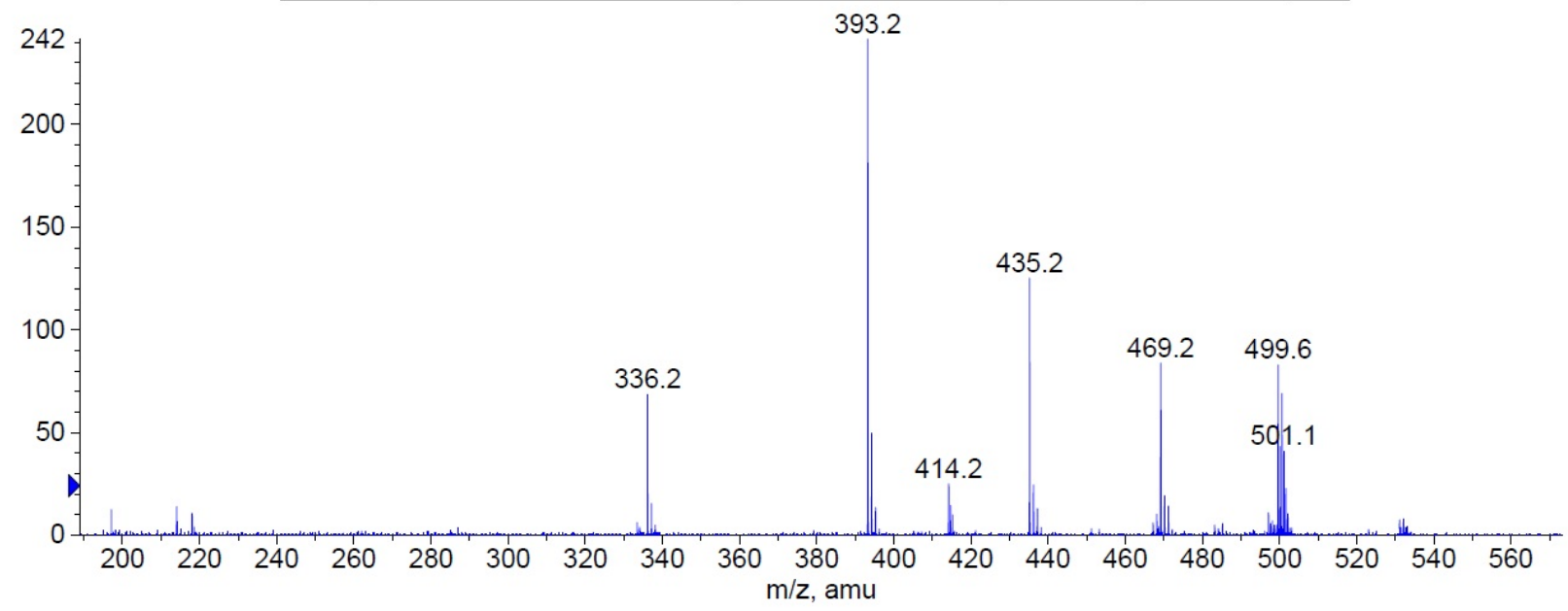

Figure 2.35: ESI-TOF MS spectrum of compound 225.

Lastly, the synthesis of bis $\beta$-amino phosphonate fluorophore $\mathbf{2 2 6}$ was undertaken from the amine quat 221 (Scheme 2.43), however the reaction resulted in a mixture of unreacted starting material, the monophosphonate, and the desired product in a $(0.51: 0.71: 1.0)$ ratio by ${ }^{31} \mathrm{P}$ NMR spectroscopy $\left(\mathrm{CDCl}_{3}\right)$ after $3 \mathrm{~d}$. Purification of the crude reaction mixture of quats was unsuccessful by column chromatography (Figure 2.36). 
(221)

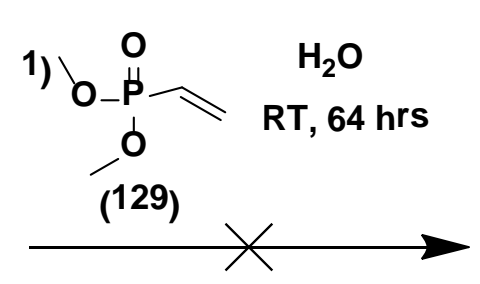

2) TMSBr, DCM, RT, ON

3) $\mathrm{H}_{2} \mathrm{O} / \mathrm{EtOH}$

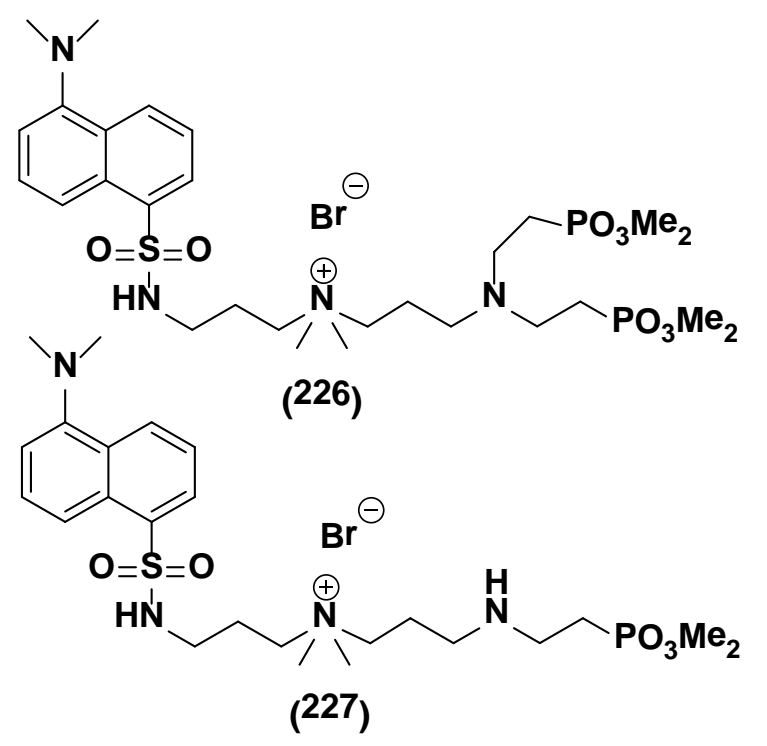

Scheme 2.43: Attempted preparation of dansyl bis $\beta$-phosphonate 226.

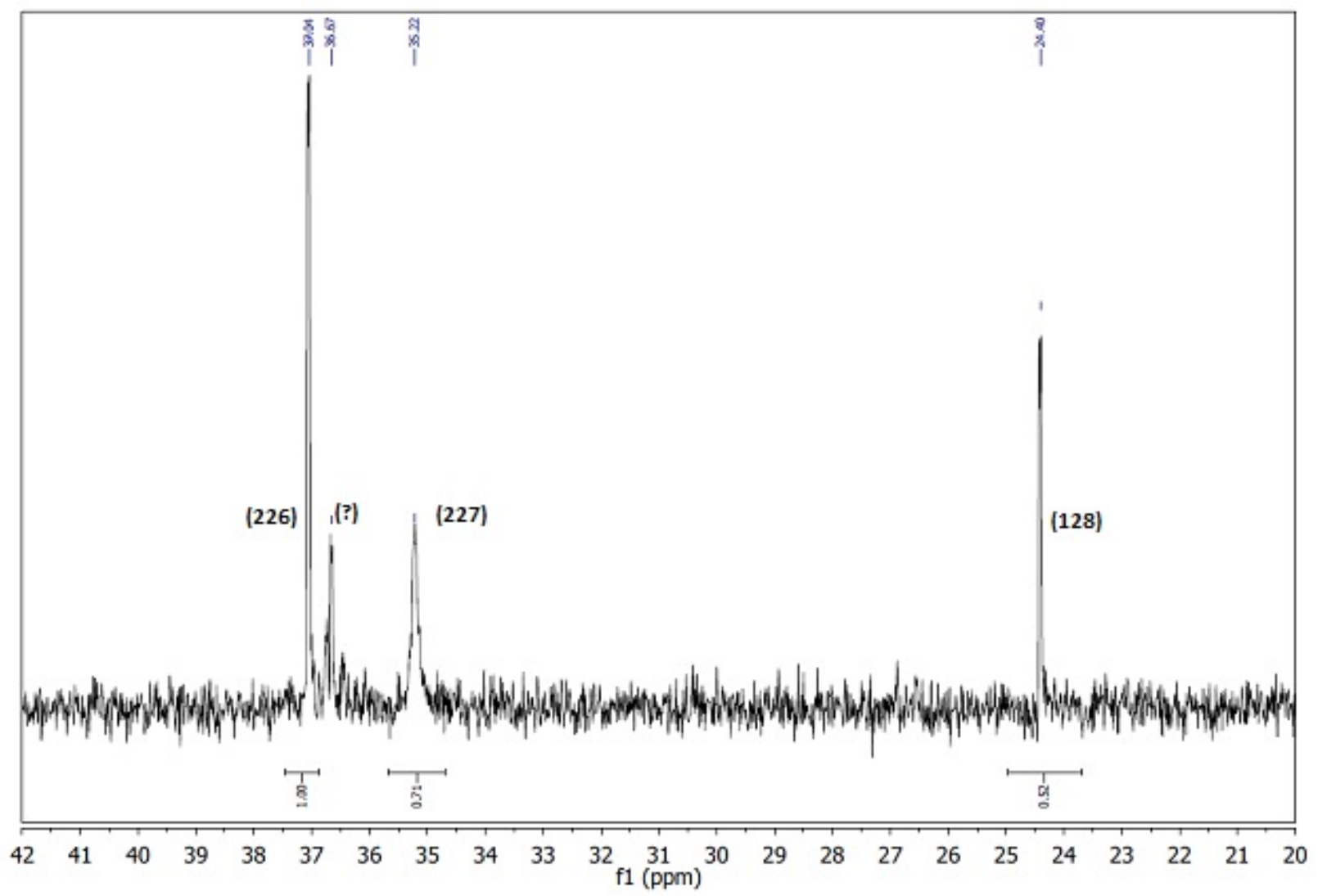

Figure 2.36: ${ }^{31} \mathrm{P}$ NMR $\left(\mathrm{D}_{2} \mathrm{O}\right)$ reaction monitoring of the formation of $\mathbf{2 2 6}$ after $3 \mathrm{~d}$. at $\mathrm{RT}$. 


\subsubsection{Fluorescence Properties of Dansyl QAC}

As expected, all dansyl QAC derivatives analyzed in $\mathrm{MeOH}$ exhibited similar fluorescence emission $\left(\lambda_{\text {emission }}=525 \mathrm{~nm}\right)$ and absorption spectra $\left(\lambda_{\text {absorption }}=340 \mathrm{~nm}\right)$ characteristic of the green-yellow dansyl tag $\left(\lambda_{\text {emission }}=525 \mathrm{~nm}, \lambda_{\text {absorption }}=360 \mathrm{~nm}\right)$. Only compound 216 exhibited a different major absorption wavelength at $\sim 300 \mathrm{~nm}$ due to the benzophenone group (Figure 2.37). However, in aqeous environments at low $\mathrm{pH}(0.1 \mathrm{~N} \mathrm{HCl})$ fluorescence quenching of the dansyl fluorophore $\mathbf{2 1 3}$ was observed due to protonation of the aromatic $N, N$-dimethylamine (Figure 2.38). At $\mathrm{pH} 7$ (phosphate buffer) no deviation from the characteristic dansyl fluorescence spectra occured, only as slight decrease in intensity was observed at the same concentration versus analysis in $\mathrm{MeOH}$. At high $\mathrm{pH}(0.1 \mathrm{~N} \mathrm{NaOH})$ absorption was shifted to $310 \mathrm{~nm}$ which produced a lower intensity emission at $325 \mathrm{~nm}$ when

excited at $340 \mathrm{~nm}$ (Figure 2.39). Noticable fluorescence quenching was also observed with derivative 220 likely due to $\pi$ stacking interactions of the phthalimide group and the aromatic amine from the dansyl group (Figure 2.39). 


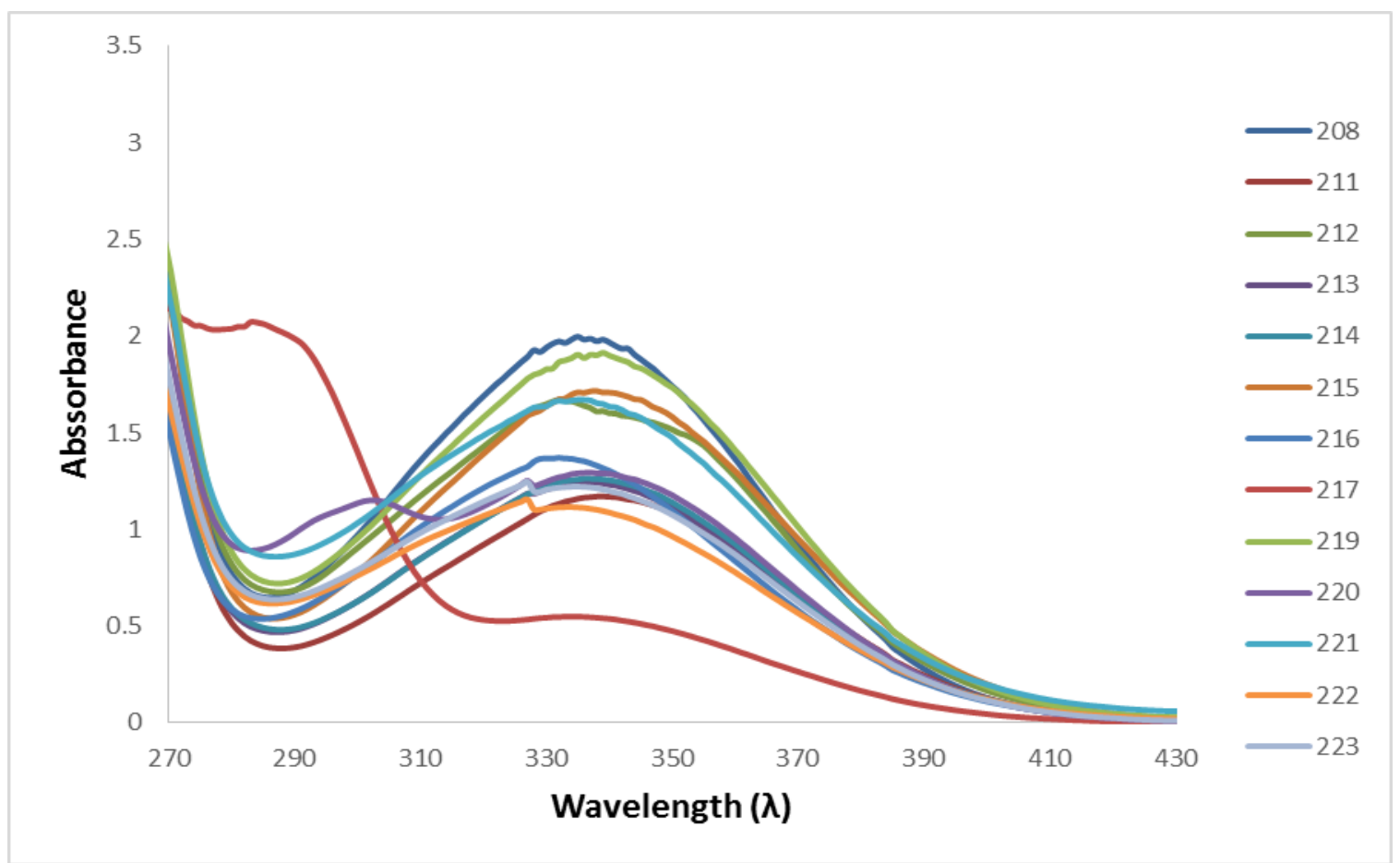

Figure 2.37: Absorption spectra of dansyl QAC's $\left(\sim 1 \times 10^{-3} \mathrm{M}\right)$ in $\mathrm{MeOH}\left(\lambda_{\mathrm{ex}}=330 \mathrm{~nm}\right)$.

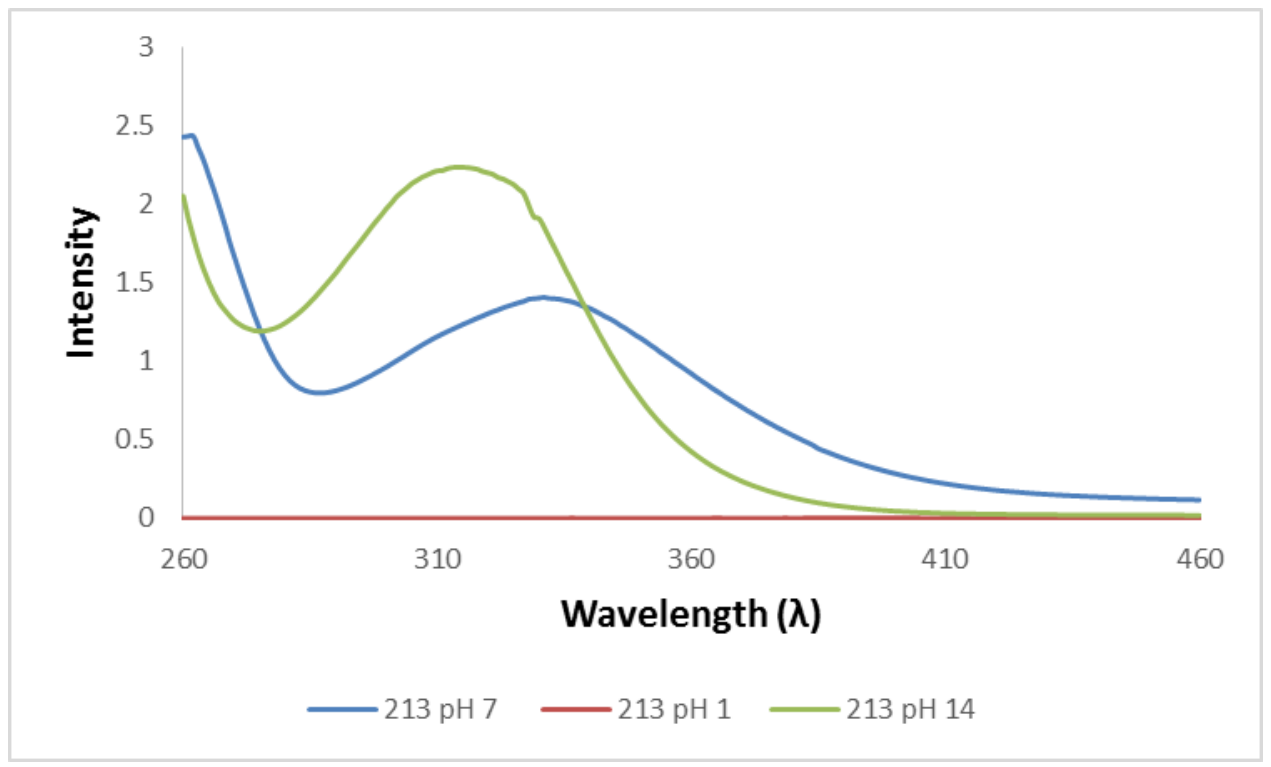

Figure 2.38: Absorption spectra of dansyl QAC $213\left(\sim 1 \times 10^{-3} \mathrm{M}\right)$ at different $\mathrm{pH}$ 's $\left(\lambda_{\mathrm{ex}}=330\right.$ $\mathrm{nm}$ ). 


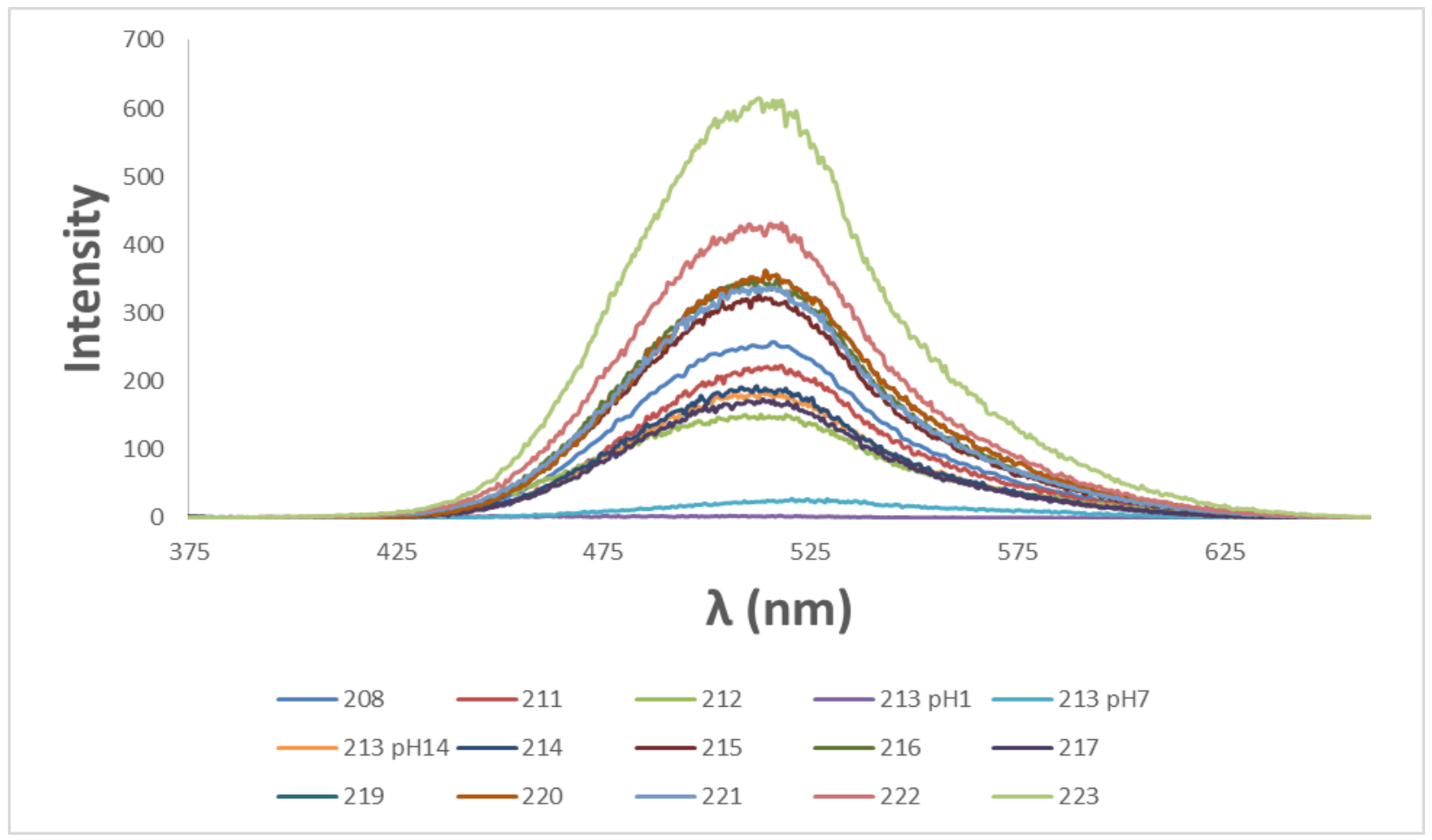

Figure 2.39: Fluorescence spectra of dansyl QAC's $\left(\sim 1 \times 10^{-3} \mathrm{M}\right)$ in $\mathrm{MeOH}\left(\lambda_{\mathrm{ex}}=525 \mathrm{~nm}\right)$. 


\subsection{Characterization and Properties}

\subsubsection{X-ray Crystallography}

Four novel crystal structures of 34, 19, 66 and 220 were obtained during the course of this research and are depicted in Figures 2.24, 2.25, 2.26, 2.27. The phosphonic acid quat 34 was re-crystallized using a $80 \% \mathrm{EtOH} / \mathrm{EtOAc}$ mixture, resulting in the isolation of long crystals growing vertically from the solution, representative of the long $\mathrm{C}_{18}$ chain (Figure 2.40). A hydrogen donor and acceptor group found at the end of both 34 and $\mathbf{6 6}$ was necessary in the formation of X-ray quality crystals of these long chain molecules (Figure 2.41). Structures with $\mathrm{C}_{18}$ tails are difficult to crystallize and are rare in the literature.

In addition to 34, a crystal structure of the $\mathrm{C}_{18}$ QAC intermediate $\mathbf{6 6}$ was obtained with a hydroxyl terminal end group instead of a phosphonic acid (Figure 2.42). This compound was recrystallized from ACN by slow evaporation of the solvent at RT, resulting in the isolation of fine, white, prismatic crystals. Compound $\mathbf{6 6}$ had been previously reported in the literature, but a crystal structure of this long chain QAC was unknown.

Crystals of both 34 and $\mathbf{6 6}$ exhibited average carbon-nitrogen bond lengths typical for an ammonium salt (1.499 $\AA$ ). ${ }^{217}$ From the data obtained (see supplementary information) the bond lengths for $\mathrm{N}\left({ }_{1}\right)-\mathrm{C}\left({ }_{23}\right), \mathrm{N}\left({ }_{1}\right)-\mathrm{C}\left({ }_{22}\right), \mathrm{N}\left({ }_{1}\right)-\mathrm{C}\left({ }_{4}\right)$, and $\mathrm{N}\left({ }_{1}\right)-\mathrm{C}\left({ }_{3}\right)$ were $1.486\left({ }_{8}\right) \AA, 1.505(7) \AA, 1.522\left({ }_{8}\right)$ $\AA$, and $1.523\left({ }_{8}\right) \AA$ respectively. These bond lengths agree with the above mentioned $\mathrm{C}-\mathrm{N}_{\mathrm{QAC}}$ average bond lengths and along with other characterization data (Section 6.0, Appendix 1.1) support the identity of $\mathbf{3 4}$ and $\mathbf{6 6 .}$ 


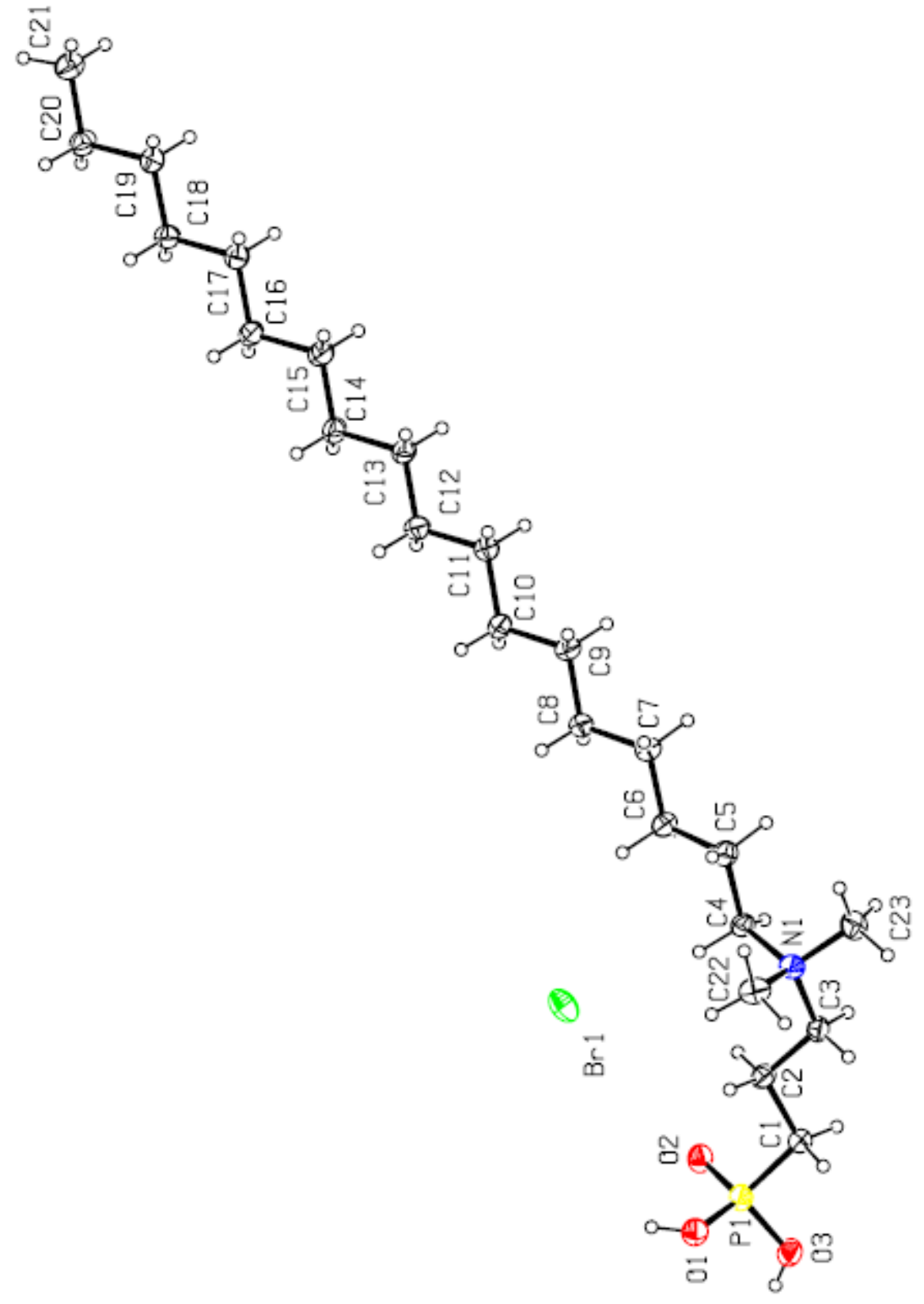

Figure 2.40: X-ray crystal structure of 34 . 


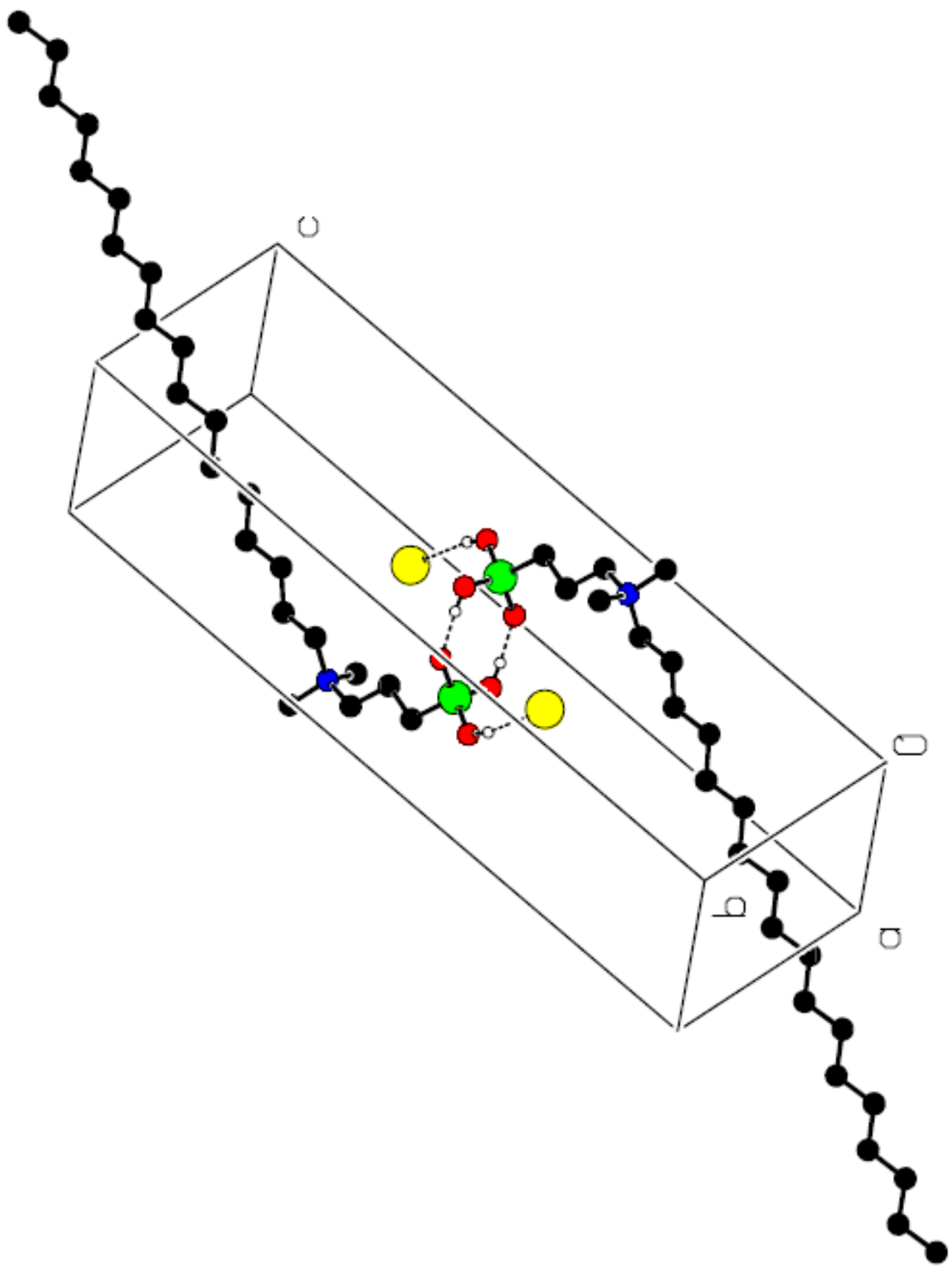

Figure 2.41: Crystal packing interactions of two molecules of 34 . 


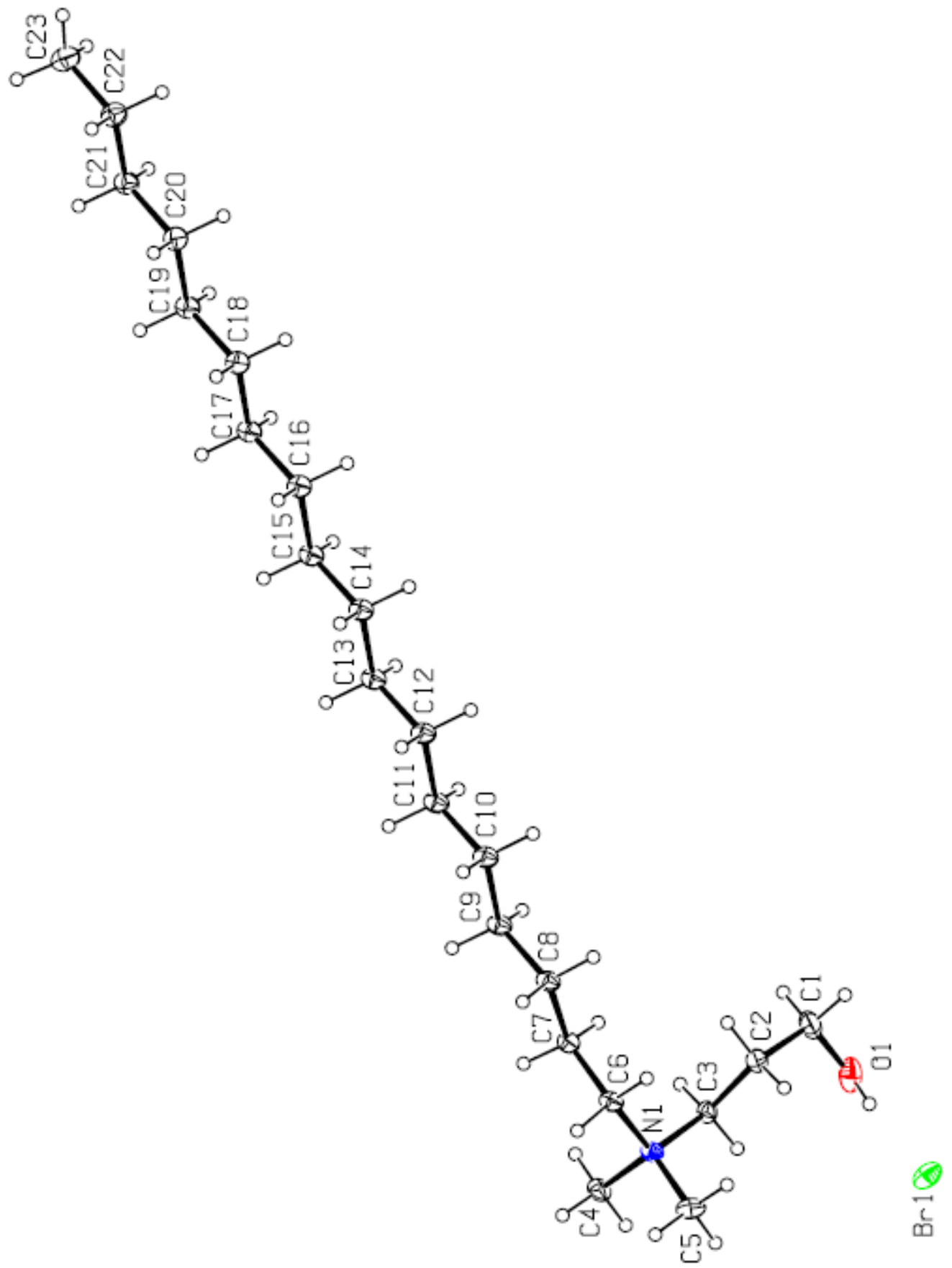

Figure 2.42: X-ray crystal structure of 66. 


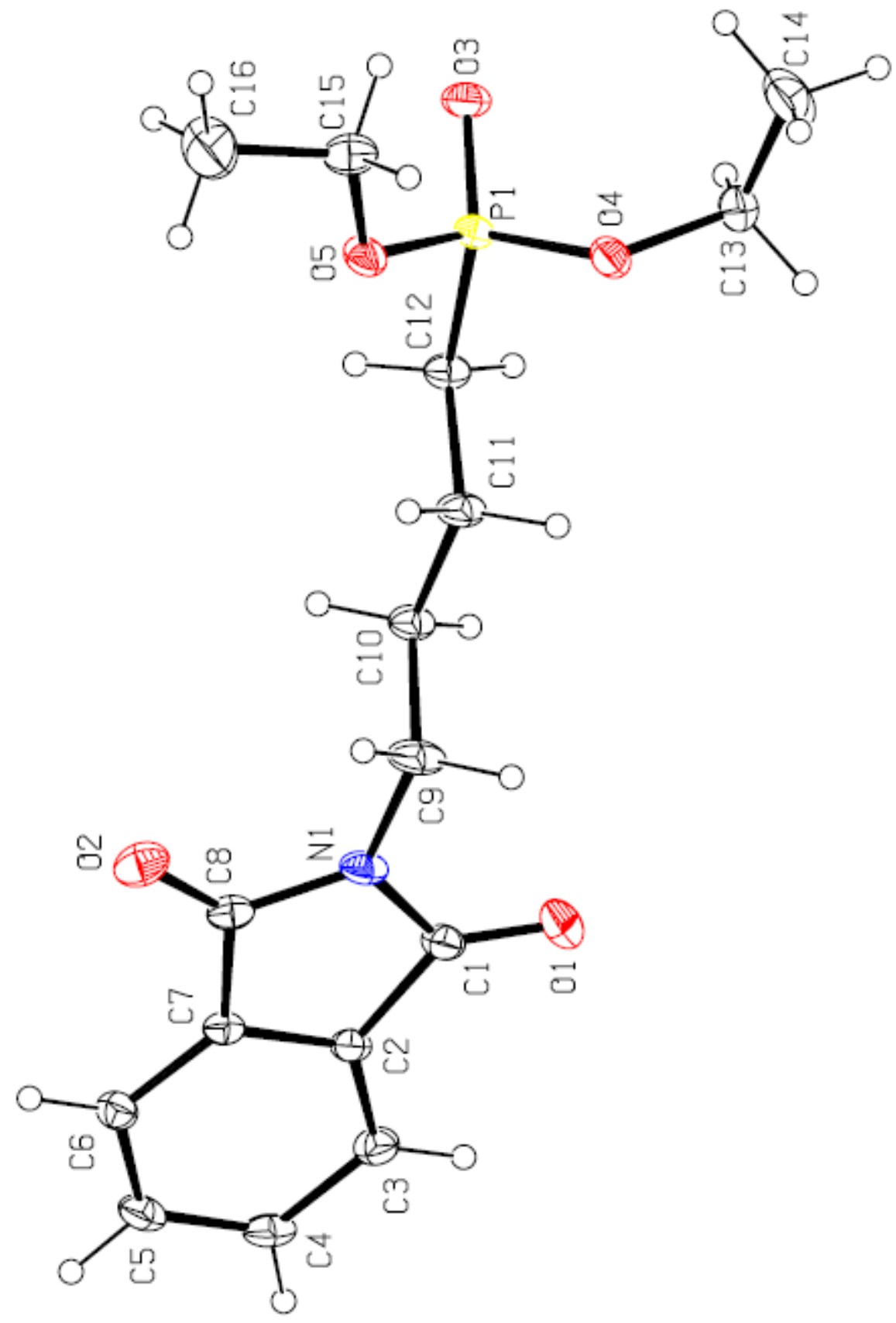

Figure 2.43: X-ray crystal structure of 19.

Two intermediate compounds 19 and 220 containing phthalimide protecting groups were crystallized and X-ray structures were obtained. The diethylphosphonate intermediate, 19, was 
crystallized from EtOAc at $-20^{\circ} \mathrm{C}$, forming square, clear crystals (Figure 2.43). The dansyl phthalimide QAC intermediate $\mathbf{2 2 0}$ was crystallized inside an NMR tube from $\mathrm{D}_{2} \mathrm{O}$ at RT, forming star shaped, clear crystals. From the crystal structure, a close interaction of the phthalimide group near the dansyl group can be observed (Figure 2.44). This interaction supports the fluorescence quenching data for this compound due to $\pi$ - $\pi$ stacking of the two aromatic rings. The bond lengths and bond angles for all of these compounds are provided (Section 6.0, Appendix 1.1). 


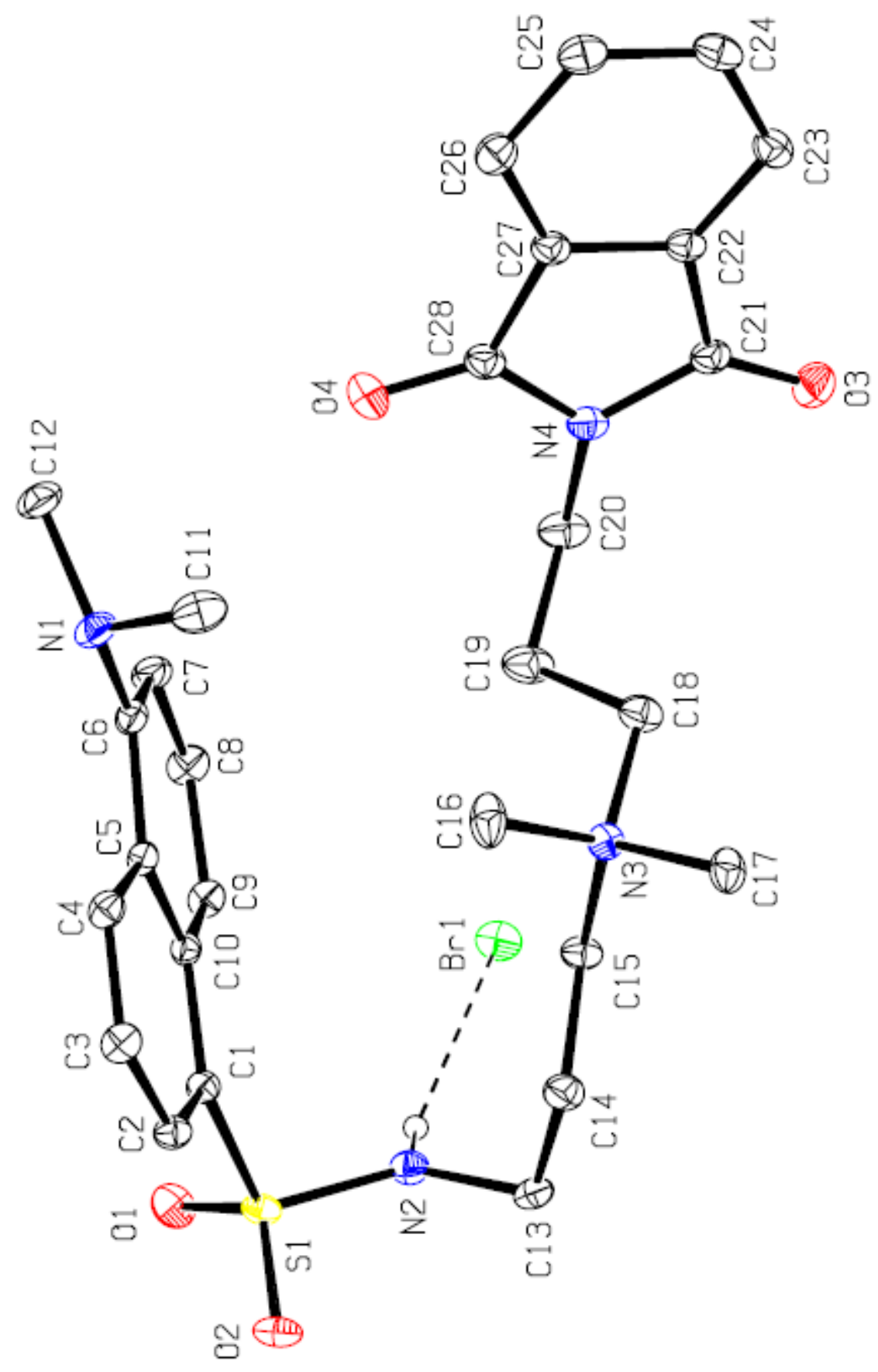

Figure 2.44: X-ray crystal structure of 220. 


\subsubsection{Antimicrobial Detection / Coating Procedures}

Both non-fluorescent and fluorescent dansyl quats were attached to porous (cotton, $\mathrm{SiO}_{2}$ ) and non-porous (metals, plastics) by dip coating a given surface in the appropriate compound according to Figure 2.45. The dansyl QAC compounds were visualized under UV light while

non-fluorescent QAC’s were visualized with bromophenol blue. Coating procedures with examples on plastics and metals are described below. 


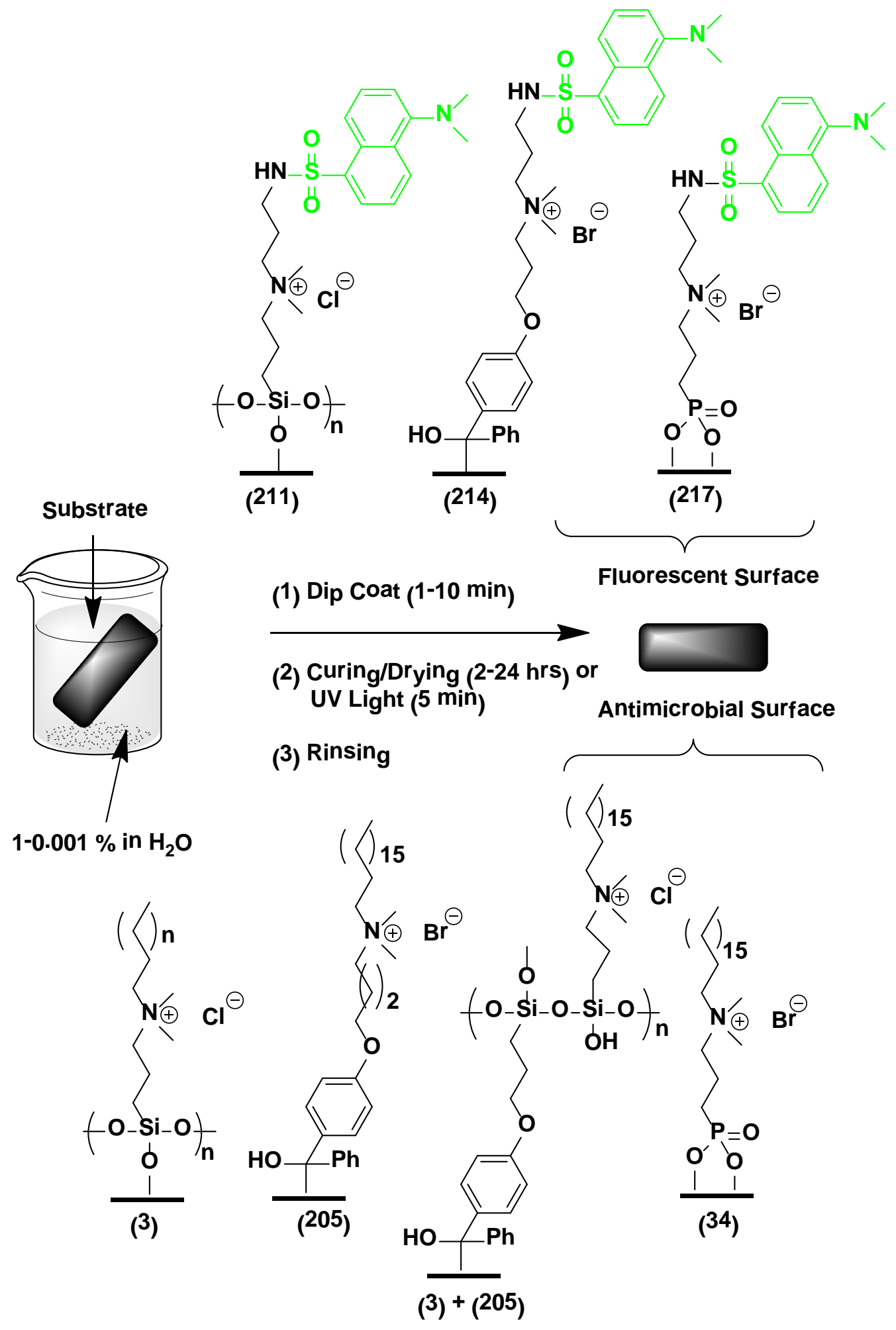

Figure 2.45: Coating procedure of QAC antimicrobials onto porous and nonporous surfaces. 


\subsubsection{Plastic Surfaces}

Grafting of non-fluorescent benzophenone compounds 205 and $3+205$ onto polypropylene (PP) and silicone was performed as follows: plastic samples were cut into rectangles $(3.5 \times 2.5 \mathrm{~cm})$, rinsed with $\mathrm{H}_{2} \mathrm{O}$ and $\mathrm{MeOH}$ and air dried prior to their use. The clean substrates were dipped into a $0.01 \%$ (w/v) solution of 214 and irradiated with UV (5 min) and any unbound material was rinsed from the substrates using $\mathrm{H}_{2} \mathrm{O}$ prior to visualization with bromophenol blue (Figure 2.46). Both PP and silicone surfaces stained a deep blue colour indicating the presence of quats on these plastic surfaces, while the controls remained unstained (Figure 2.47).

Dansyl fluorescent compounds were successfully visualized without the bromophenol blue dye. Medical grade silicone tubing coated with 214, silica NP's treated with 211, PP coated with 214, and SS coated with 217 all fluoresced green, indicating their prescence on these surfaces when imaged under UV light (Figure 2.48). 


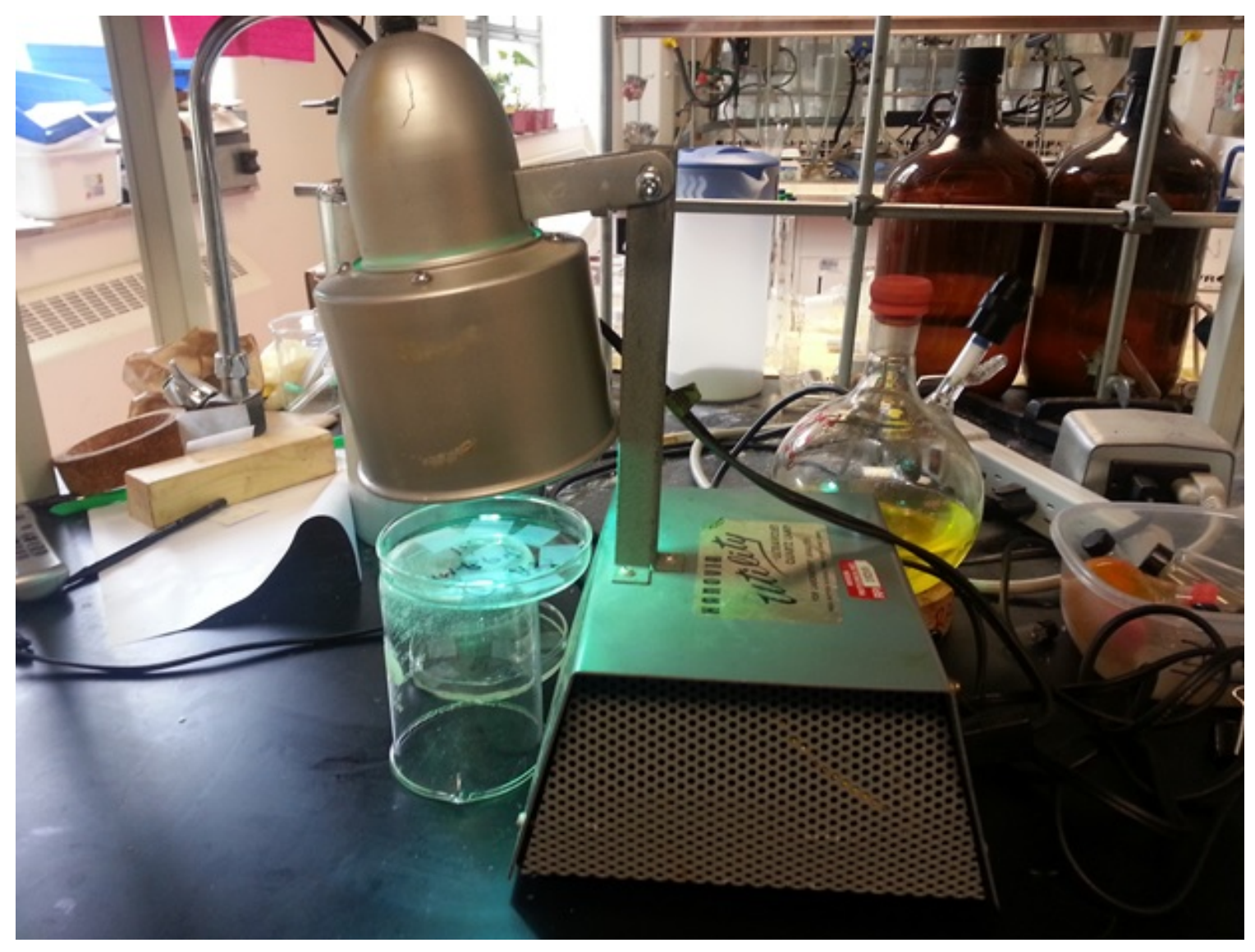

Figure 2.46: Plastic coating experimental set-up.

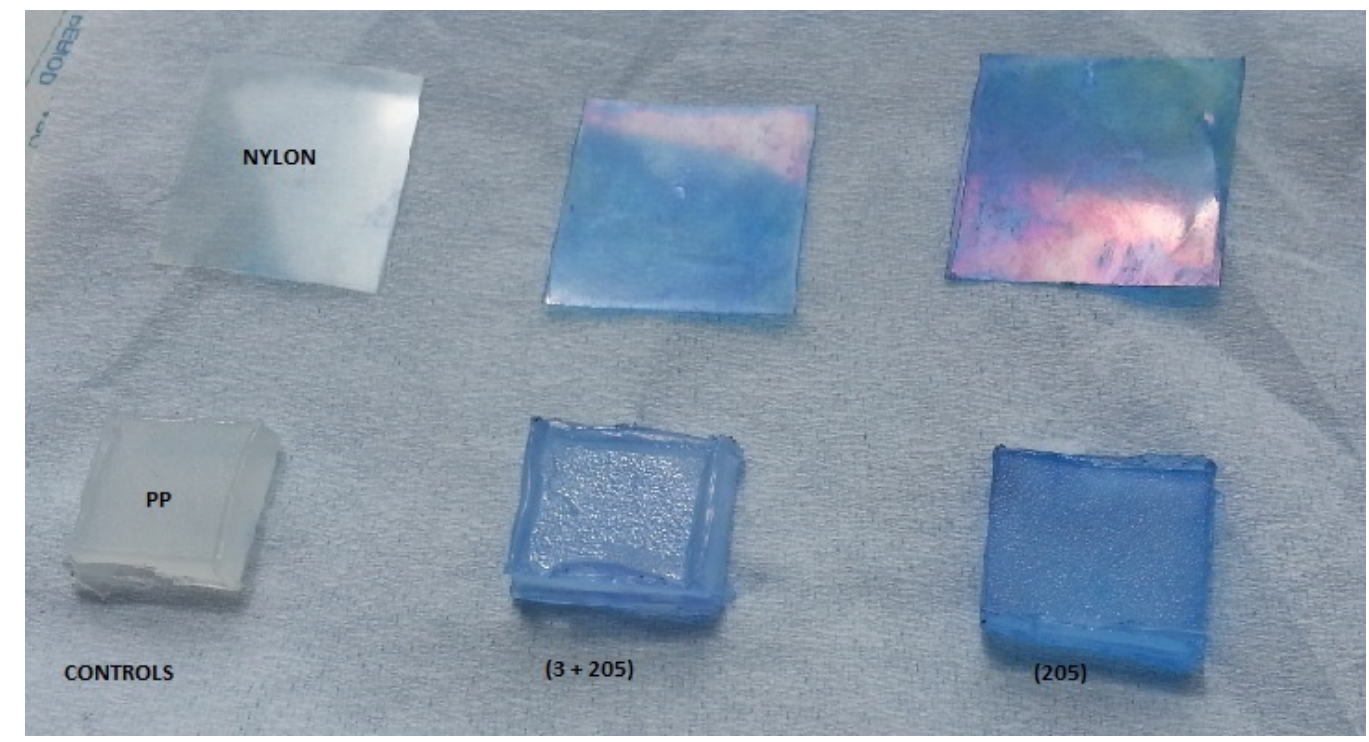

Figure 2.47: Bromophenol blue (0.01 M) detection of QAC antimicrobials on plastic surfaces. 

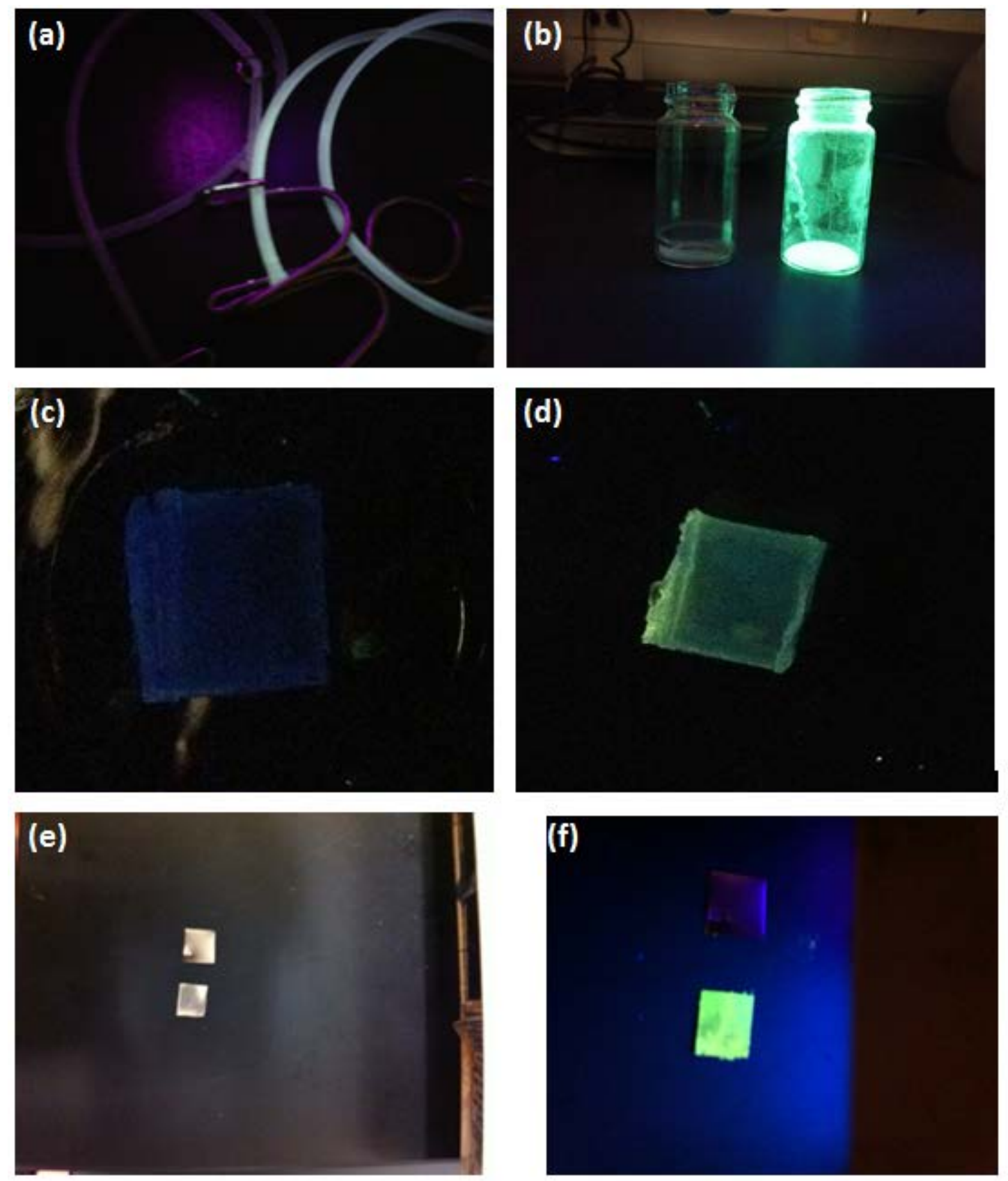

Figure 2.48: Fluorescent detection of dansyl QAC's. (a) Medical grade silicone tubing coated with control (left) and with 214 (right), imaged under UV light, (b) silica NP's treated with 211, PP plastic coated (c) control and (d) 214, (e) SS coated with control (upper) and 217 (lower) imaged under ambient light, (f) same image as (e) but imaged in the dark under UV light (TLC lamp). 


\subsubsection{Metal Surfaces}

Preparation of self-assembled monolayers of compound 211 on cotton and silica NP's were prepared by dip coating the samples in $0.01 \%(w / v) \mathrm{H}_{2} \mathrm{O}$ or $\mathrm{MeOH}$ solution of $211 \mathrm{ON}$ or until the solvent evaporated, followed by heating (100 $\left.{ }^{\circ} \mathrm{C}, 24 \mathrm{hrs}\right)$ and drying (Figure 2.49). Note a faster procedure was developed by placing the vials into an oven $\left(100{ }^{\circ} \mathrm{C}\right)$ and letting the solvent evaporate ( 30-60 min) followed by a rinsing step (MeOH) and a short oven cure (100 $\left.{ }^{\circ} \mathrm{C}, 1 \mathrm{hr}\right)$. Samples prepared in both ways were found to possess the same antimicrobial activity while uncured samples were inactive.

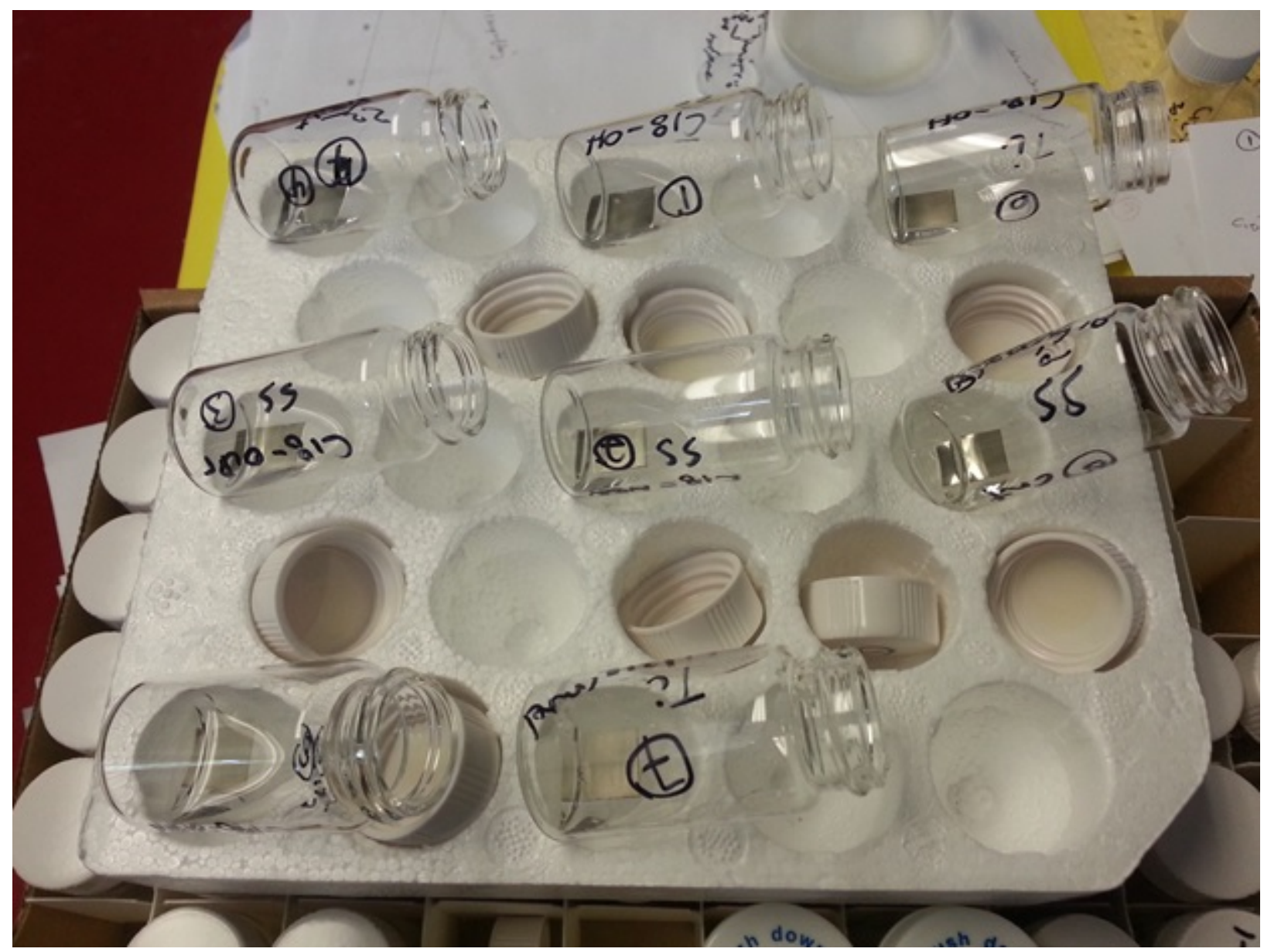

Figure 2.49: Metal coating experimental set up using $20 \mathrm{~mL}$ glass screw cap vial open to air. 


\subsubsection{Antimicrobial Activity}

\subsubsection{Solution Killing: Minimum Inhibitory Concentration's (MIC’s)}

Minimum inhibitory concentration (MIC) experiments were performed according to standard procedures ${ }^{218}$ with both gram-positive and gram-negative bacterial strains: S. aureus, Listeria, Salmonella, and E. coli. Solutions of long chain QAC antimicrobials $(0.01 \mathrm{~g} / \mathrm{mL}$ or $1 \%)$ were dissolved in $\mathrm{H}_{2} \mathrm{O}$, inoculated with the test organism and serially diluted from $10^{-1}-10^{-3}$, representing a dilution range from $(0.01 \mathrm{~g} / \mathrm{mL}$ to $0.00001 \mathrm{~g} / \mathrm{mL})$, followed by plate counts to determine the MIC values (Figure 2.50).

Samples 1-3 were prepared with (1\%) solutions of the $\mathrm{C}_{18}$ phosphonic acid QAC 34A obtained from hydrolysis of the phosphonate ester $\mathbf{2 6}$ or $\mathbf{2 7}$. Sample 1 is compound 34A obtained by TMSBr dealkylation of the ethyl ester (Et, TMSBr), sample 2 is the sodium salt 34B (Na salt) which is freely soluble in $\mathrm{H}_{2} \mathrm{O}$ and sample 3 is compound $34 \mathrm{~A}$ obtained by $\mathrm{HCl}$ dealkylation of the $i \mathrm{Pr}$ ester 27 (i-Pr, $\mathrm{HCl}$ ). Sample 4 which was initially a (1\%) solution of 34A used for ON dip coating experiments with Ti metal, is the residual liquid (unknown concentration) after the metal coupon was removed for antimicrobial analysis.

Samples 1-3 prepared with 1\% starting solutions of 34A exhibited similar MIC values but demonstrated $100 \times$ higher efficacy with Listeria and Salmonella and $10 \times$ higher efficacy against E. coli compared to the silane QAC 3 (sample 5 and 6) (Figure 2.50). All compounds were inhibitory towards the gram-positive S.aureus bacterium, susceptible to QAC compounds in solution (Figure 2.34). The phosphonate ester 26 (1\% in $\left.\mathrm{H}_{2} \mathrm{O}\right)$ was also tested for inhibition 
against S. aureus and was found to be highly inhibitory even at $100 \mu \mathrm{g} / \mathrm{mL}$ concentrations (Table 2.12).

As expected, sample 4, the retested aqueous phase containing residual 34A performed poorly with Listeria, Salmonella, and E. coli and no inhibition was observed at the starting concentration. This can be explained by the fact the phosphonic acid molecules in solution formed monolayers on the surface to Ti metal thereby decreasing the concentration 10-100 fold. So in fact the starting concentration was closer to a $0.001-0.0001 \mathrm{~g} / \mathrm{mL}$ which after further dilution was no longer inhibitory (Figure 2.50).

A stock solution (1\%) of the silane quat 3 prepared from the $5 \%$ stabilized solution in $\mathrm{H}_{2} \mathrm{O}$ (SiQAC 3 (stabilized)) or prepared from the $72 \%$ concentrate in $\mathrm{MeOH}$ and diluted to $1 \%$ in $\mathrm{H}_{2} \mathrm{O}$ (SiQAC 3 (no stabilizer)) worked extremely well (Figure 2.50). All bacterial strains tested with these samples were inhibited at concentrations up $100 \mu \mathrm{g} / \mathrm{mL}$ which is in close agreement agreement with literature MIC values $(84 \mu \mathrm{g} / \mathrm{mL}){ }^{61}$ 


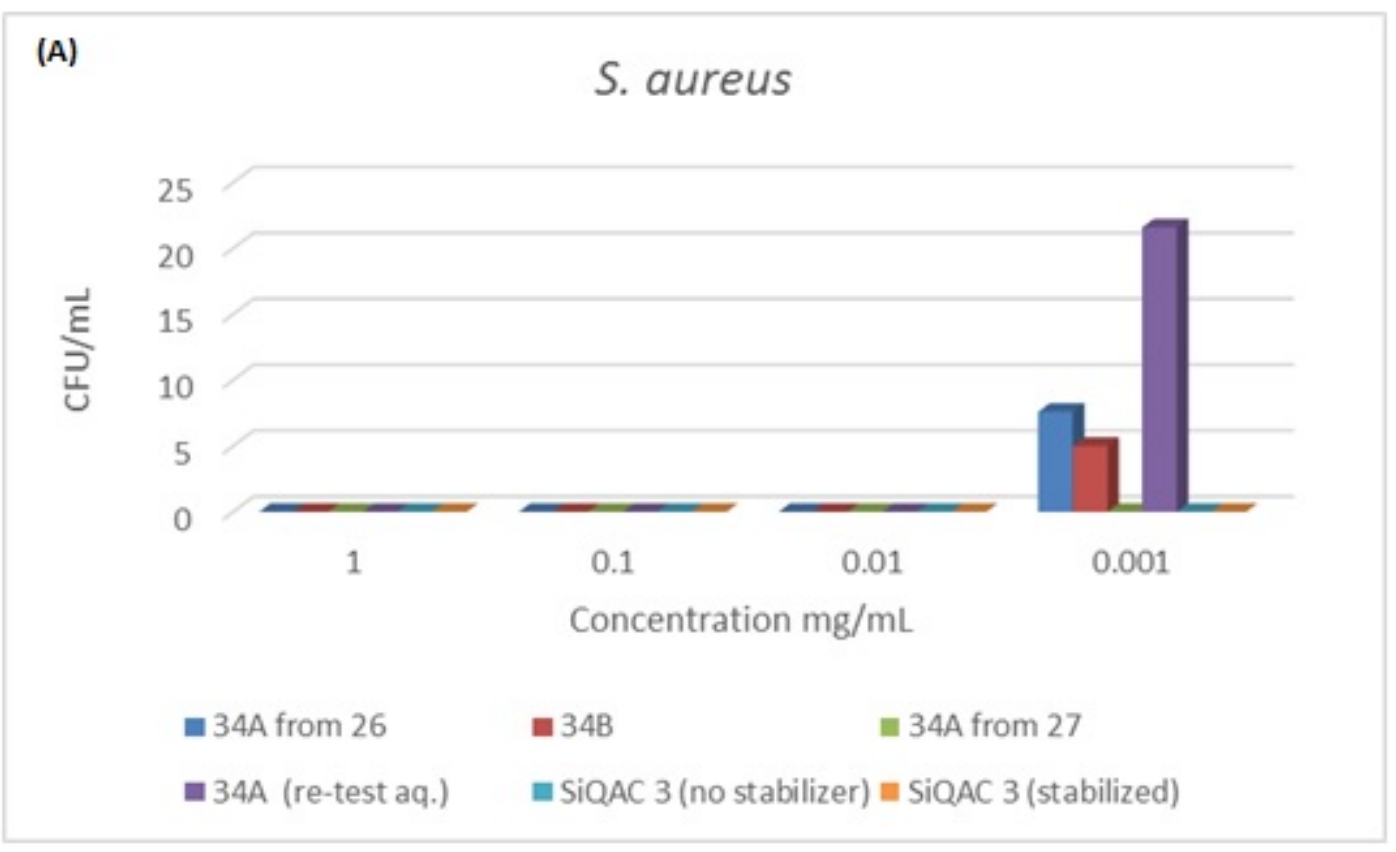

(B)

\section{Listeria}

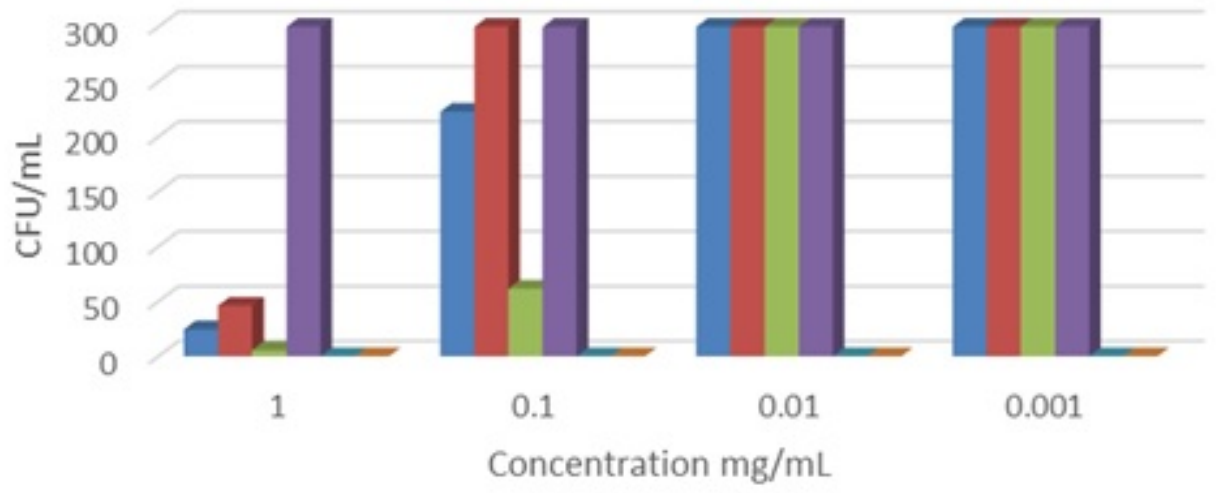

- 34A from 26

- $34 \mathrm{~B}$

- 34A from 27

- 34A (re-test aq.)

- SiQAC 3 (no stabilizer) $=$ SiQAC 3 (stabilized) 
(C)

\section{Salmonella}

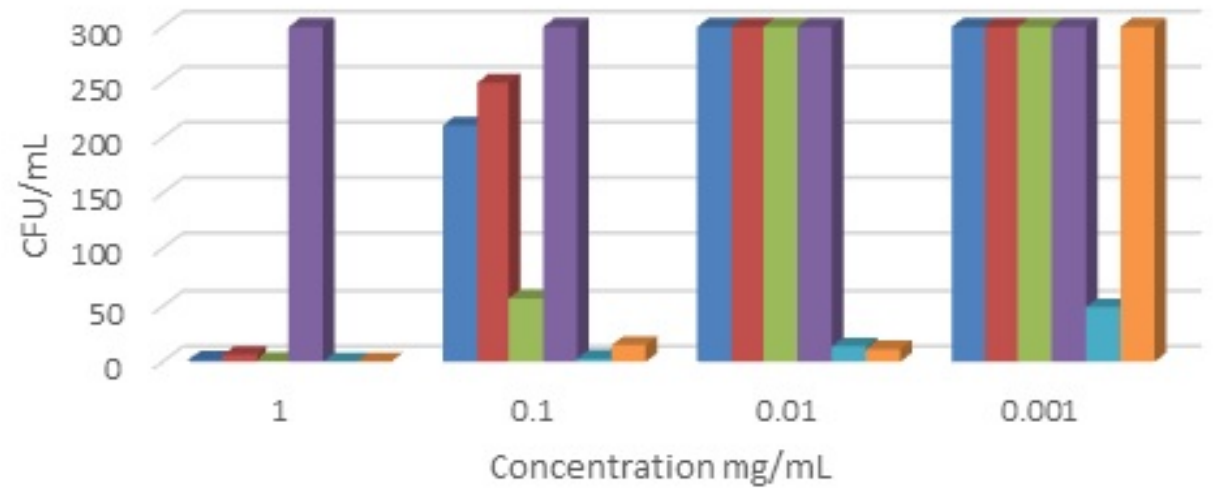
口 $34 \mathrm{~A}$ from 26
- 348
34A from 27
- 34A (re-test Aq.)
- SIQAC 3 (no stabilizer) I SIQAC 3 (stabilized)

(D)
E. coli

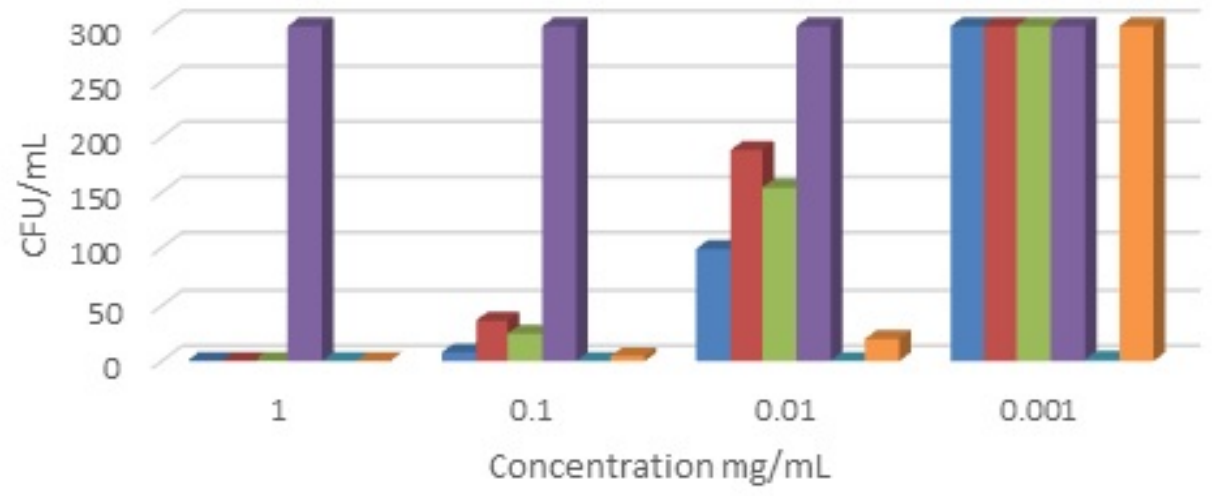

$$
\begin{aligned}
& \text { - } 34 \mathrm{~A} \text { from } 26 \\
& \text { ש } 34 \mathrm{~B} \\
& \text { a } 34 \mathrm{~A} \text { from } 27 \\
& \text { 口 34A (re-test Aq.) }
\end{aligned}
$$

Figure 2.50: Experimentally determined MIC (CFU/mL) of SiQAC 3, 34A and 34B. MIC plate counts of samples were perfomed using a $10^{-3}$ (about $200000 \mathrm{cfu} / \mathrm{ml}$ ) culture of $S$. aureus (A), Listeria (B), Salmonella (C), E. coli (D). Solutions of QAC samples were prepared in $\mathrm{H}_{2} \mathrm{O}$ starting at $1 \mathrm{mg} / \mathrm{mL}$ or $1 \%$ and diluted up $10^{3}$. 
Table 2.12: MIC plate counts of samples using a $10^{-3}$ (about $200000 \mathrm{cfu} / \mathrm{mL}$ ) culture of S.aureus.

\begin{tabular}{|l|l|l|l|l|}
\hline QAC & $0.01 \mathrm{~g} / \mathrm{mL}$ & $0.001 \mathrm{~g} / \mathrm{mL}$ & $0.0001 \mathrm{~g} / \mathrm{mL}$ & $0.00001 \mathrm{~g} / \mathrm{mL}$ \\
\hline (26) & No Growth & No Growth & No Growth & No Growth \\
\hline
\end{tabular}

\subsubsection{Contact Killing on Hard Surfaces}

Antimicrobial activity of phosphonate QAC coatings and the silane QAC 3 for comparison purposes were prepared on metal surfaces ( $\mathrm{Ti}, \mathrm{SS}, \mathrm{Al})$ and tested by growth enumeration in the dry state testing method developed in the Wolfaardt lab against various strains of bacteria. Intially, $1 \%$ solutions of compound 34A were electrosprayed in triplicate onto (Ti, SS, Al) and cured overnight at $120^{\circ} \mathrm{C}$. However, no significant bacterial reduction was observed and the electrosprayed metal coupons were indistinguishable to the unsprayed controls (results not shown). Ti samples prepared by an overnight immersion in an aqueous or ethanolic solution of compound 34A followed by an overnight cure at $100^{\circ} \mathrm{C}$ showed promising results versus uncured samples. Ti samples were coated with compound 34A obtained by either TMSBr dealkylation of the ethyl ester (Et, TMSBr) or of the $i \mathrm{Pr}$ ester by the $\mathrm{HCl}$ method (iPr, $\mathrm{HCl}$ ). In both cases the ${ }^{31} \mathrm{P}$ NMR spectrum were identical, however, 34A prepared by the TMSBr method was only slightly soluble in $\mathrm{H}_{2} \mathrm{O}$ and was tested in ethanol, while 34A prepared by the $\mathrm{HCl}$ method was tested in $\mathrm{H}_{2} \mathrm{O}$ at $10 \mathrm{mg} / \mathrm{mL}$. S. aureus and Salmonella inoculated antimicrobial Ti surfaces were sampled after 3 hrs of drying and showed 99-100\% reduction in viable bacteria versus the controls and uncured surfaces (Figure 2.51). 


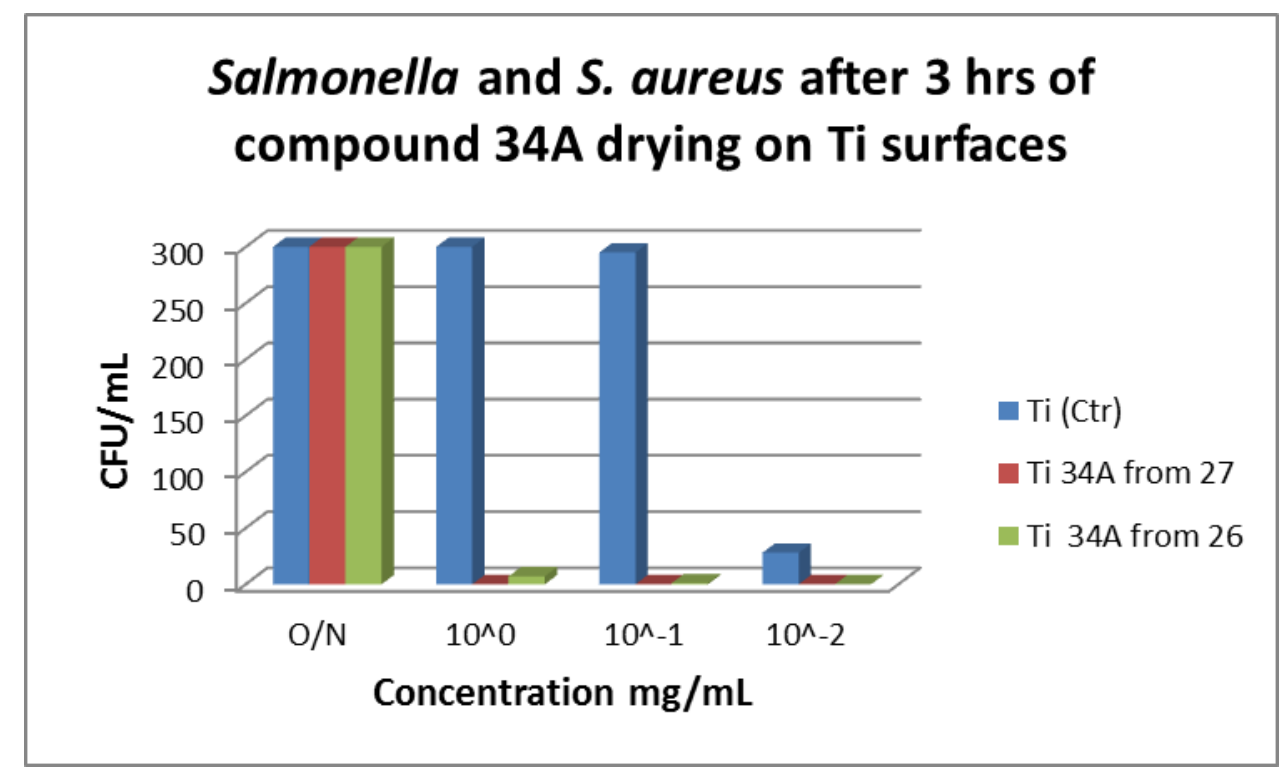

Figure 2.51: Colony forming units per $\mathrm{mL}$ of Salmonella and S. aureus after 3 hrs of drying on Ti surfaces. $\mathrm{O} / \mathrm{N}$ is the initial concentration $\left(10^{5}\right.$ cells $\left./ \mathrm{ml}\right)$. 300 is the maximum cfu/mL. Blue rectangles $=$ control untreated $\mathrm{Ti}$, Red rectangles $=$ compound $34 \mathrm{~A}$ coated $\mathrm{Ti}$ ( obtained from dealkylation of the $i \mathrm{Pr}$ ester with $\mathrm{HCl}$ ), Green = compound 34A coated Ti (obtained from dealkylation of the ethyl ester with TMSBr)

After success with compound 34A on Ti, other organophosphorus QAC's including the ester 26, trimethylammnoium 35, pyridinium 36 as well as the silane quat SiQAC 3 on Ti were evaluated against a strain of P.aeroguinosa (PAO1) known for its robust survival on dry surfaces. Only compounds 26 and 34A showed a significant reduction of bacterial colonies after 4 hrs of sampling while the shorter chain phosphonic acid QAC’s 35, 36 and the silane QAC 3 were indistinguishable from control samples (results not shown). As a result, no further sampling was performed on these surfaces. Instead the active compounds 26 and $\mathbf{3 4 A}$ were used to coat other metals SS, Al along with $\mathrm{Ti}$ and these surfaces were tested with other bacterial strains.

Contact killing was observed immediately with compounds 26 and 34A on all surfaces tested (Ti, SS, Al). After 2 hrs these antimicrobial coatings had killed all of the cells initially dried on each surface $\left(10^{6}\right.$ bacterial cells, Table 2.13 for Arthrobacter, Table 2.14 for PAO1). 
Control samples without such coatings showed some bacterial reduction but only after extended periods of time. Typically, 24 hrs were required to reduce the bacterial surface population from $10^{6}$ cells to $10^{5}-10^{3}$ depending on the surface of the controls (Table 2.13). Aluminum control surfaces showed the largest reduction of PAO1 by a factor of $10^{3}$ after 24 hrs while their survival was highest on SS with $10^{5}$ cells remaining after 24 hrs. Such reductions are typical over time on surfaces with limited nutrients necessary for bacterial survival and reproduction. From these observations, the phospholipid sponge mechanism is believed to be responsible for the rapid contact killing with the longer chain QAC's. These monolayer forming organophosphorus antimicrobials required both a quat and a long hydrophobic tail to effectively remove bacterial phospholipids when in close proximity to the cells whereas QAC's without a long hydrophobic chain such as $\mathbf{3 5}$ and $\mathbf{3 6}$ were completely inactive even though they contained the same number of ammonium $\left(\mathrm{N}^{+}\right)$charges on the surface.

Table 2.13: Arthrobacter reduction by antimicrobial metal surfaces treated with 26 and 34A.

\begin{tabular}{|l|l|l|l|l|l|}
\hline \multicolumn{7}{|l|}{ Arthrobacter (CFU/mL) } \\
\hline Entry & Sample & $\mathbf{0 ~ h r s}$ & $\mathbf{2 ~ h r s ~}$ & $\mathbf{4}$ hrs & 24 hrs \\
\hline I & Ti (Ctr) & $10^{6}$ & $10^{4}$ & $10^{2}$ & $10^{2}$ \\
\hline Ii & Ti (26) & $10^{6}$ & 0 & 0 & 0 \\
\hline Iii & Ti (34A) & $10^{6}$ & 0 & 0 & 0 \\
\hline Iv & SS (Ctr) & $10^{6}$ & $10^{4}$ & $10^{2}$ & $10^{2}$ \\
\hline V & SS (26) & $10^{6}$ & 0 & 0 & 0 \\
\hline Vi & SS (34A) & $10^{6}$ & 0 & 0 & 0 \\
\hline
\end{tabular}


Table 2.14: $P$. aeroguinosa (PA01) reduction by antimicrobial metal surfaces treated with 26 and 34A.

\begin{tabular}{|c|c|c|c|c|c|}
\hline \multicolumn{6}{|c|}{ P.aeroguinosa PA01 (CFU/mL) } \\
\hline Entry & Sample & 0 hrs & 2 hrs & $4 \mathrm{hrs}$ & 24 hrs \\
\hline I & Ti (Ctr) & $10^{6}$ & $10^{6}$ & $10^{5}$ & $10^{4}$ \\
\hline Ii & $\mathrm{Ti}(\mathbf{2 6})$ & $10^{6}$ & 0 & 0 & 0 \\
\hline Iii & $\operatorname{Ti}(\mathbf{3 4 A})$ & $10^{6}$ & 0 & 0 & 0 \\
\hline Iv & $\operatorname{Ti}(34 \mathrm{~A})$ & $10^{6}$ & 0 & 0 & 0 \\
\hline $\mathrm{V}$ & SS (Ctr) & $10^{6}$ & $10^{6}$ & $10^{5}$ & $10^{5}$ \\
\hline $\mathrm{Vi}$ & SS (26) & $10^{6}$ & 0 & 0 & 0 \\
\hline Vii & SS (34A) & $10^{6}$ & 0 & 0 & 0 \\
\hline Viii & $\mathrm{Al}$ (Ctr) & $10^{6}$ & $10^{6}$ & $10^{4}$ & $10^{3}$ \\
\hline Ix & $\mathrm{Al} \mathrm{(26)}$ & $10^{6}$ & 0 & 0 & 0 \\
\hline $\mathrm{X}$ & $\mathrm{Al}(34 \mathrm{~A})$ & $10^{6}$ & 0 & 0 & 0 \\
\hline
\end{tabular}

Surfaces treated with compound 34A (Table 2.13, Entries i-iii) were further tested to determine if antimicrobial activity was retained. Samples stored in saline for 24 hrs from the first antimicrobial trial were removed, dried, washed in distilled $\mathrm{H}_{2} \mathrm{O}$, dried, re-innoculated and then retested. All samples from the second trial showed similar Anthrobacter colony reductions $\left(10^{6}\right.$ to 0 ) indicating the molecule was truly immobilized (Table 2.15). The effectiveness of the antimicrobial Ti treated with 34A to withstand Anthrobacter colonization was also demonsrated on agar. The control samples were fully colonized by the bacterium, while the antimicrobial treated Ti sample, effectively killed Anthrobacter on contact and no colonies were observed around the metal coupons (Figure 2.52). 
Table 2.15: P. aeroguinosa (PA01) reduction by antimicrobial Ti surfaces treated with 34A and retested.

\begin{tabular}{|l|l|l|l|l|}
\hline \multicolumn{6}{|l|}{ P. aeroguinosa (PA01) (CFU/mL) } \\
\hline Entry & Sample & 0 hrs & 2 hrs & 4 hrs \\
\hline i & $\mathrm{Ti}(\mathrm{Ctr})$ & $10^{6}$ & $10^{4}$ & $10^{2}$ \\
\hline ii & $\mathrm{Ti}(34 \mathrm{~A})$ & $10^{6}$ & 0 & 0 \\
\hline iii & $\mathrm{Ti}$ (34A) & $10^{6}$ & 0 & 0 \\
\hline iv & $\mathrm{Ti}(34 \mathrm{~A})$ & $10^{6}$ & 0 & 0 \\
\hline
\end{tabular}

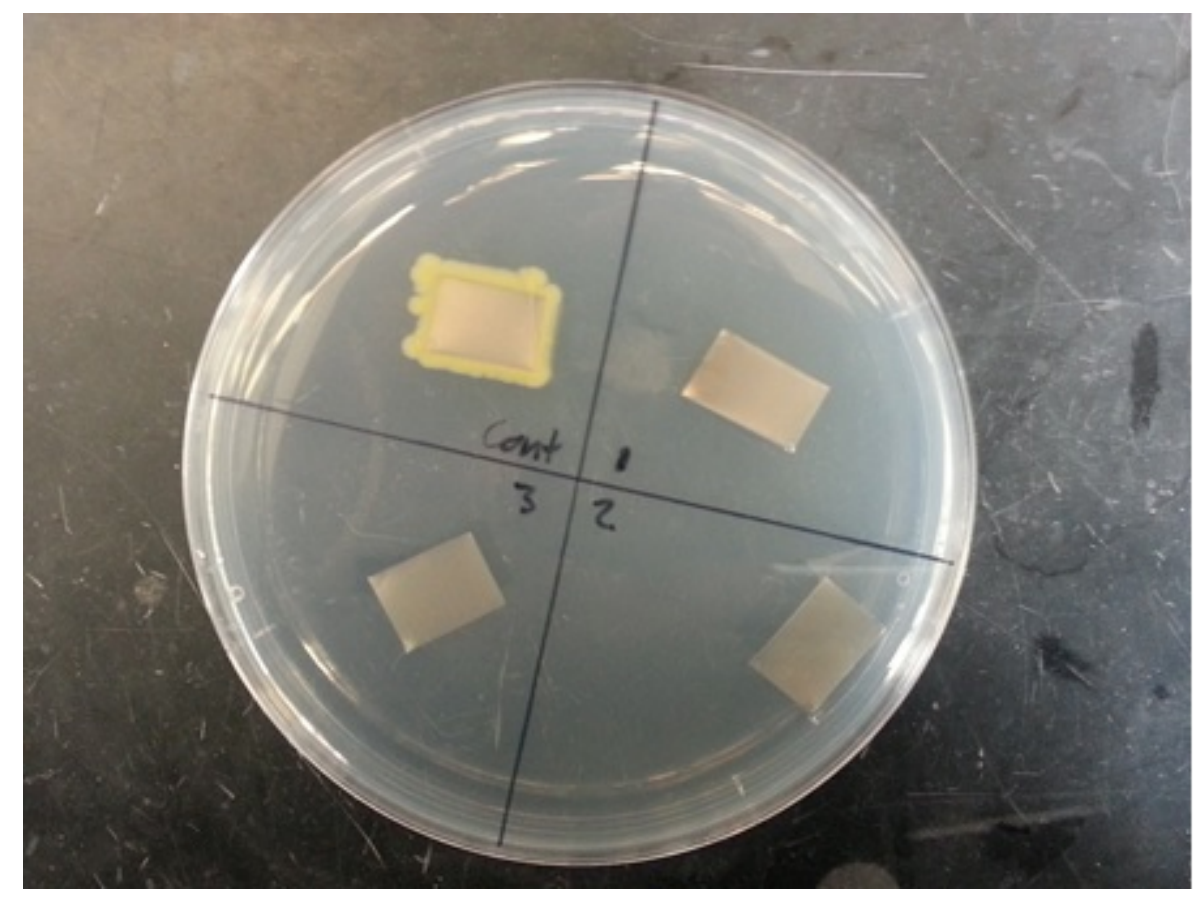

Figure 2.52: Agar growth method showing P. aeroguinosa (PA01) reduction by antimicrobial Ti surfaces treated with 34A. $($ Cont $=$ control, 1, 2, $3=$ compound 34A).

The amount of bacteria intitially dried on the metal surfaces tested was determined by serial dilutions and agar plate counts until a countable amount of colonies was observed. For example after six dilutions of the original stock of bacteria applied to $\mathrm{Ti}$ and SS, 58 colonies were counted at the beginning of sampling $(\mathrm{T}=0)$ which corresponds to a concentration of $\sim 10^{6}$ cells (Table 2.16, 2.17). 
Table 2.16: Determination of initial bacterial load dried on titanium surfaces from plate counts (raw data). Tmtc = too many to count.

\begin{tabular}{|l|llllllll|}
\hline Arthrobacter (Titanium) (CFU/mL) & & & & & \\
\hline Control & $\mathbf{1 0}^{\mathbf{0}}$ & $\mathbf{1 0}^{-\mathbf{1}}$ & $\mathbf{1 0}^{-\mathbf{2}}$ & $\mathbf{1 0}^{-\mathbf{3}}$ & $\mathbf{1 0}^{-4}$ & $\mathbf{1 0}^{-5}$ & $\mathbf{1 0}^{-\mathbf{6}}$ & $\mathbf{1 0}^{-\mathbf{7}}$ \\
\hline $0 \mathrm{hrs}$ & tmtc & tmtc & tmtc & tmtc & tmtc & tmtc & 58 & 5 \\
$4 \mathrm{hrs}$ & tmtc & 285 & 24 & 0 & 0 & 0 & 0 & 0 \\
$24 \mathrm{hrs}$ & tmtc & tmtc & 35 & 3 & 0 & 0 & 0 & 0 \\
\hline
\end{tabular}

Table 2.17: Determination of initial bacterial load dried on SS surfaces from plate counts (raw data).

\begin{tabular}{|l|llllllll|}
\hline Arthrobacter (Stainless Steel) & (CFU/mL) & & & & \\
\hline Control & $\mathbf{1 0}^{\mathbf{0}}$ & $\mathbf{1 0}^{-1}$ & $\mathbf{1 0}^{-2}$ & $\mathbf{1 0}^{-3}$ & $\mathbf{1 0}^{-4}$ & $\mathbf{1 0}^{-5}$ & $\mathbf{1 0}^{-\mathbf{6}}$ & $\mathbf{1 0}^{-7}$ \\
\hline $0 \mathrm{hrs}$ & Tmtc & tmtc & tmtc & tmtc & tmtc & tmtc & 40 & 2 \\
$4 \mathrm{hrs}$ & Tmtc & tmtc & 32 & 5 & 0 & 0 & 0 & 0 \\
$24 \mathrm{hrs}$ & Tmtc & tmtc & 34 & 4 & 0 & 0 & 0 & 0 \\
\hline
\end{tabular}




\subsection{CONCLUSION}

Water soluble quats with anchors specific for forming monolayers onto porous $\left(\left(\left[\mathrm{C}_{18} \mathrm{H}_{37^{-}}\right.\right.\right.$ $\left.\mathrm{NH}-\left(\mathrm{CH}_{2}\right)_{3}-\mathrm{NMe}_{2}-\mathrm{R}^{+}\right]\left[\mathrm{Cl}^{-}\right]$(3; anchor $\left.=-\mathrm{Si}(\mathrm{OMe})_{3}\right)$ and non-porous metal oxide (Ti, SS, Al) $\left(\left(\left[\mathrm{C}_{18} \mathrm{H}_{37}-\mathrm{NH}-\left(\mathrm{CH}_{2}\right)_{3}-\mathrm{NMe}_{2}-\mathrm{R}^{+}\right]\left[\mathrm{Cl}^{-}\right] \quad \text { (26; anchor = -PO(OEt }\right)_{2}\right.$, 34; $\left.-\mathrm{PO}(\mathrm{OH})_{2}\right)$ and plastic surfaces (silicones, PP) $\left(\left(\left[\mathrm{C}_{18} \mathrm{H}_{37}-\mathrm{NH}-\left(\mathrm{CH}_{2}\right)_{4}-\mathrm{NMe}_{2}-\mathrm{R}^{+}\right]\left[\mathrm{Br}^{-}\right]\right.\right.$(205; anchor = -benzophenone), ([benzophenone-NH- $\left.\left(\mathrm{CH}_{2}\right)_{3}-\mathrm{NMe}_{2}-\mathrm{R}^{+}\right]\left[\mathrm{X}^{-}\right]\left(\right.$206; $\left.\mathrm{R}=-\mathrm{Si}(\mathrm{OMe})_{3}, \mathrm{X}^{-}=\mathrm{Cl}^{-}\right)$were synthesized by the Menschutkin reaction and used to prepare antimicrobial coating on these surfaces. New compounds were successfully characterized by NMR spectroscopy $\left({ }^{1} \mathrm{H},{ }^{13} \mathrm{C},{ }^{29} \mathrm{Si}\right.$, and ${ }^{31} \mathrm{P}$ where required), HRMS spectroscopy and in a few instances by X-ray crystallography (34, 66, 19, 220).

Improvements in the synthesis of the organosilane QA antimicrobial dimethyloctadecyl[3-(trimethoxysilyl)propyl]ammonium chloride 3 , approved by the Environmental Protection Agency (EPA) and the Pest Management Regulatory Agency (PMRA) was performed both under solventless $\left(72 \mathrm{hrs}, 100^{\circ} \mathrm{C}, 80 \%\right)$ and solvent (MeOH, $\left.48 \mathrm{hrs}, 76 \%\right)$ conditions in a sealed tube (ST), as well as with $\mu \mathrm{W}$ radiation in $\mathrm{MeOH}\left(45 \mathrm{~min}, 150^{\circ} \mathrm{C}, 58 \%\right)$. In all cases, the reaction never reached full conversion even after prolonged heating.

Various monodentate organophosphorus phosphonates and phosphonic acid QAC's 3437 with short and long chains were successfully synthesized and used to prepare antimicrobial surfaces on Ti, SS and Al. The didealkylation of phosphoate QAC's was explored for the first time under $\mu \mathrm{W}$ heating and proved to be extremely effective and rapid with the following reagents; $\mathrm{TMSBr}\left(\mathrm{ACN}, 10 \mathrm{~min}, 60^{\circ} \mathrm{C}\right), \mathrm{HCl}\left(3 \mathrm{M}, 30 \mathrm{~min}, 150^{\circ} \mathrm{C}\right)$ or $\mathrm{HBr}(3 \mathrm{~N}, 10 \mathrm{~min}$, $150^{\circ} \mathrm{C}$ ). Three bisphosphonate QAC’s 93, 121 and $\mathbf{1 4 0}$ were also prepared but their dealkylation to the corresponding phosphonic acids 96, 126, 141 was unsuccessful using either $\mathrm{HBr}$ or 211 
TMSBr due to insolubility of the formed products in $\mathrm{H}_{2} \mathrm{O}$ and organic solvents. Other multidentate phosphonic acid quats were targeted $(\mathbf{1 6 1}, \mathbf{1 6 8}, \mathbf{1 7 9}, \mathbf{1 8 3})$ but their synthesis was unsuccessful based on the synthetic schemes attempted. Novel thiol and catechol QAC’s 194 and 189 were synthesized, both of which require further testing on metal surfaces.

Various new quaternary ammonium dansyl containing compounds with both alkoxysilane 213, phosphonate 211-212, 217, thiolacetate $\mathbf{2 1 4}$, acrylamide $\mathbf{2 1 9}$, were similarly prepared and evaluated as potential fluorescent probes in antimicrobial coatings. Physical attachment of dansyl silane 213 to $\mathrm{SiO}_{2}$ nanoparticles (NP's) and cotton surfaces after immersion in solutions containing the fluorescent dye was verified by exposure to UV light and by complexation with bromophenol blue that rendered the surfaces visibly blue in colour. Plastic surfaces (PP, silicon medical tubing) were UV cured with dansyl benzophenone 216 and resulted in the physical attachment of the dye visualized by UV light. Dansyl phosphonic acid, 217, was attached to a stainless steel surface by exposure to an aqueous solution containing this dye, followed by a thermal cure $\left(100^{\circ} \mathrm{C}, \mathrm{ON}\right)$ resulting in the formation of a self-assembled fluorescent monolayer. Synthesis of a $\mathrm{C}_{18}$ benzophenone QAC 205 was improved with $\mu \mathrm{W}$ heating by lowering the reaction time to 2 min vs. an ON reflux in ACN. A new benzophenone QAC with a silane linker 206 was made and successfully visualized with bromophenol blue on PP and silicone surfaces with bromophenol blue. Antimicrobial activity of $\mathbf{2 0 5}$ and $\mathbf{2 0 6}$ on plastic surfaces remains to be tested. A dansyl amidine probe $\mathbf{2 2 2}$ prepared from dansyl amine $\mathbf{2 2 1}$ was expected to find use in the detection of microbial respiration $\mathrm{CO}_{2}$, however, no change in fluorescence was observed after exposure to $\mathrm{CO}_{2}$.

Presence of non-fluorescent antimicrobial QAC coatings on surfaces was successfully detected using the bromophenol blue test. Antimicrobial activity was tested in solution by MIC 
determinations and in the solid state (metal surfaces only, Ti, SS, Al) by growth enumeration in the dry state. SiQAC 3 was found to be the most biocidal in solution with an MIC of $~ 100 \mu \mathrm{g} / \mathrm{g}$ which is in close agreement to the literature value of $80 \mu \mathrm{g} / \mathrm{g}$. Compounds $\mathbf{2 6}$ and $\mathbf{3 4}$ on the other hand were more biocidal on metal oxide surfaces and were capable of reducing initial concentrations of Salmonella, Arthrobacter, S. aureus and P. aeroguinosa by a factor of $10^{6}$ (100\%) after a contact time of 2 hrs and maintained their activity after 24 hrs. Re-testing the same active antimicrobial $\mathrm{Ti}$ surfaces after 3 weeks with another challenge of bacteria was performed and was also successful. Identical reductions in Arthrobacter colonies after a contact time of 2 hrs were observed $10^{6}$ cells $\rightarrow 0$ cells (100\%). These antimicrobial surfaces are believed to kill bacteria on contact because their MIC's are higher in solution compared to compound $\mathbf{3}$ and the monolayer concentrations of $\mathbf{2 6}$ and $\mathbf{3 4}$ are too small to effectively kill $10^{6}$ cells if these surfaces released the antimicrobial upon testing. 


\subsection{FUTURE WORK}

Another possibility to improving the commercial synthesis of the Dow antimicrobial (SiQAC, 3) which utilizes the unreactive chlorosilane 1 (see Section 2.1.1), would entail the addition of a catalytic or stoichiometric amount of $\mathrm{NaI}$ or $\mathrm{LiBr}$ for a onepot Finkelstein / Menschutkin reaction. This would lead the the in situ formation of the more reactive iodo or bromosilane and would allow for shorter reaction times and complete conversion to the quat, previously impossible with the chlorosilane (Scheme 4.1 A). Alternatively the Finkelstein/Menschutkin reaction could be performed sequentially with isolation of the iodosilane and the quaternization with dimethyloctadecylamine (Scheme 4.1 B).

(A) One Pot

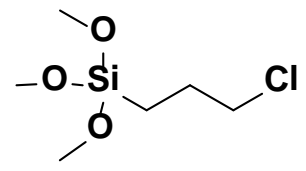

(1)

(B) Sequential

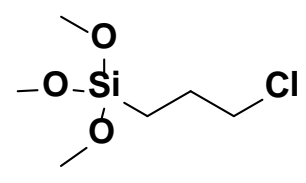

(1)

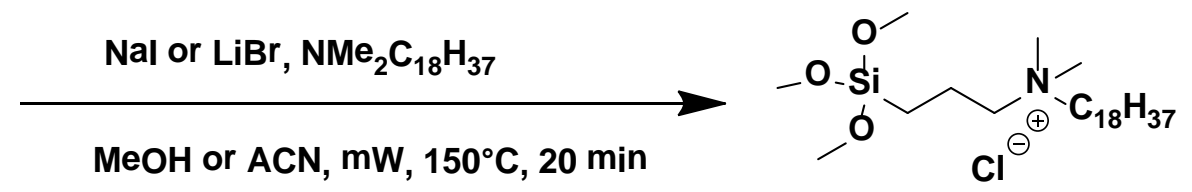

(3)

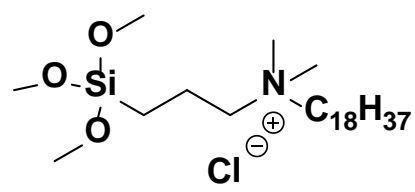

(3)

Scheme 4.1: Catalytic Finkelstein / Menschutkin reaction in the preparation of the Dow antimicrobial 3.

The onepot bis addition of dialkylphosphites to aldehydes (see Section 2.1.2.3) needs to be further explored with the aldehyde quat precursor, 56, obtained by oxidation of the quat 
alcohol with PCC/DCM (see Section 2.1.2.3). Precursor 56 would be an ideal starting material in preparing the target bisphosphonate, 59, in high yielding single pot reaction (Scheme 4.2).
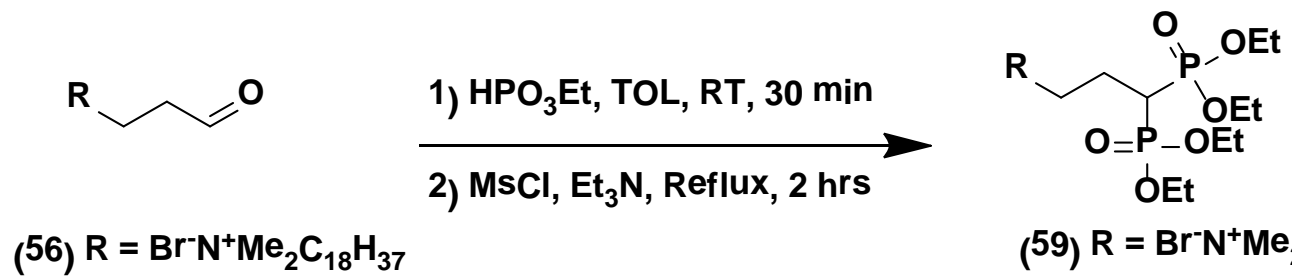

(59) $\mathrm{R}=\mathrm{Br}^{-} \mathrm{N}^{+} \mathrm{Me}_{2} \mathrm{C}_{18} \mathrm{H}_{37}$

Scheme 4.2 .Onepot preparation of $\alpha$-CH-bisphosphonates from the aldehyde quat precursor, 56.

The syntheses of catechol and disulfide QAC’s 190 and 199 respectively remains to be finished (see Section 2.3 and 2.4). The final deprotection of catechol QAC 189 remains to be attempted with either $\mathrm{BBr}_{3} / \mathrm{DMC}$ or $\mathrm{HBr}$ under microwave irradiation (Scheme 4.3). Likewise the final cleavage of the disulfide QAC with $\mathrm{PPh}_{3} / \mathrm{H}_{2} \mathrm{O}$ remains to be performed to obtain the target thiol QAC 199 (Scheme 4.4).<smiles>COc1ccc(CCO)cc1OC</smiles>

(187)

(188)

(189)
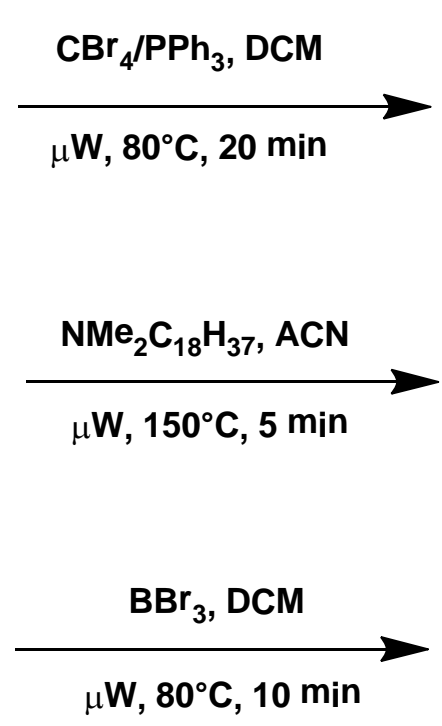
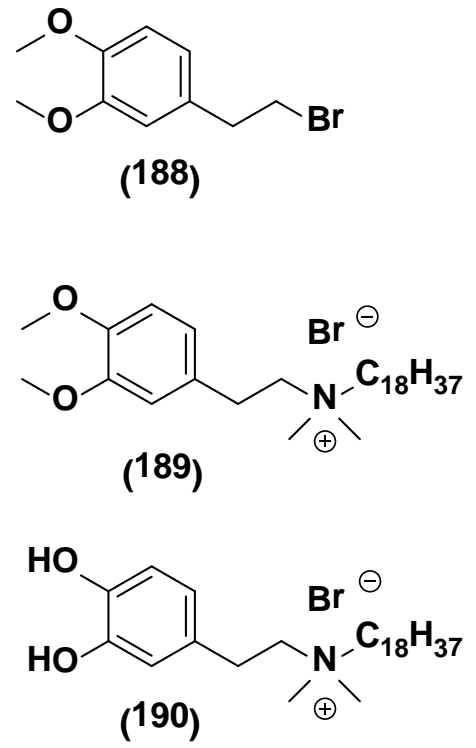

Scheme 4.3: Preparation of catechol QAC, 190. 


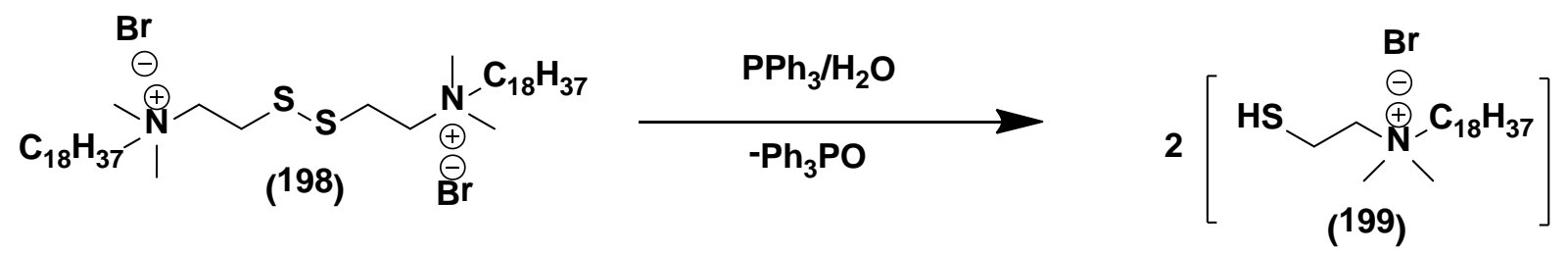

Scheme 4.4: Preparation of thiol QAC, 199.

The next generation of QA antimicrobials would be capable of binding to every possible surface (eg. both porous and non-porous: metal and plastic surfaces). Synthesis of a universal surface antimicrobial with multiple functional anchors is attractive with the commercially available polymer (Scheme 4.5). Different anchor groups including: silane, phosphonate, catechol and benzophenone groups could potentially be quaternized onto the polymer backbone. Dual cleavage of the catechol, and phosphonate groups may be achieved with $\mathrm{BBr}_{3}$ if desired. Alternatively, a series of diallyl monomers with different anchors can be co-polymerized to create multifunctional polymer coatings (Scheme 4.6).

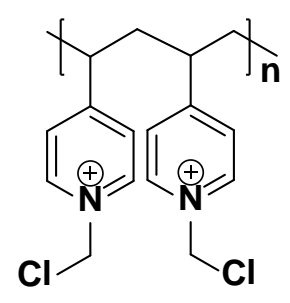

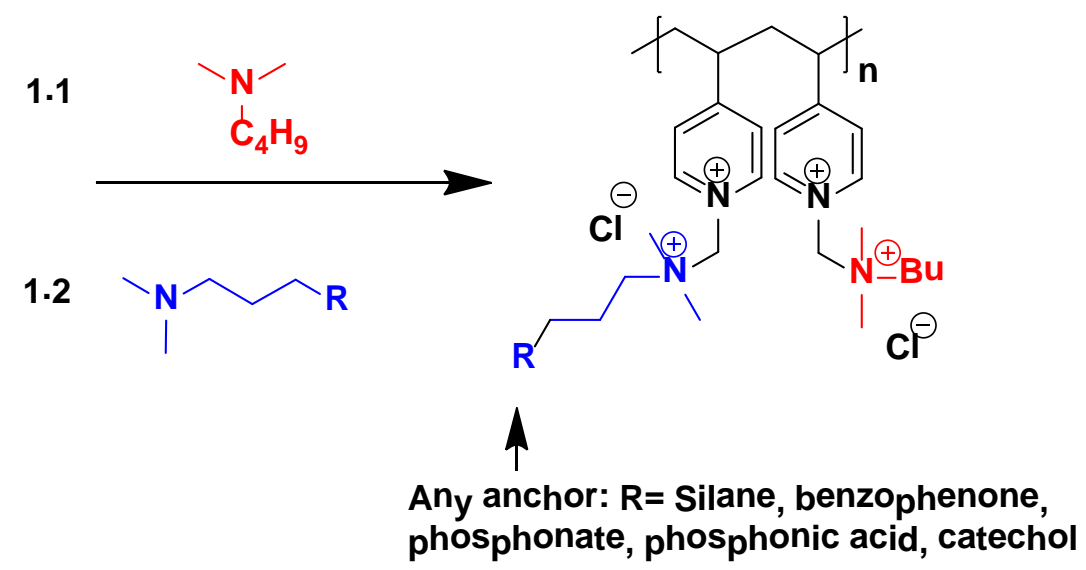

Scheme 4.5: Synthesis of multifunctional QAC antimicrobial polymer coating. 


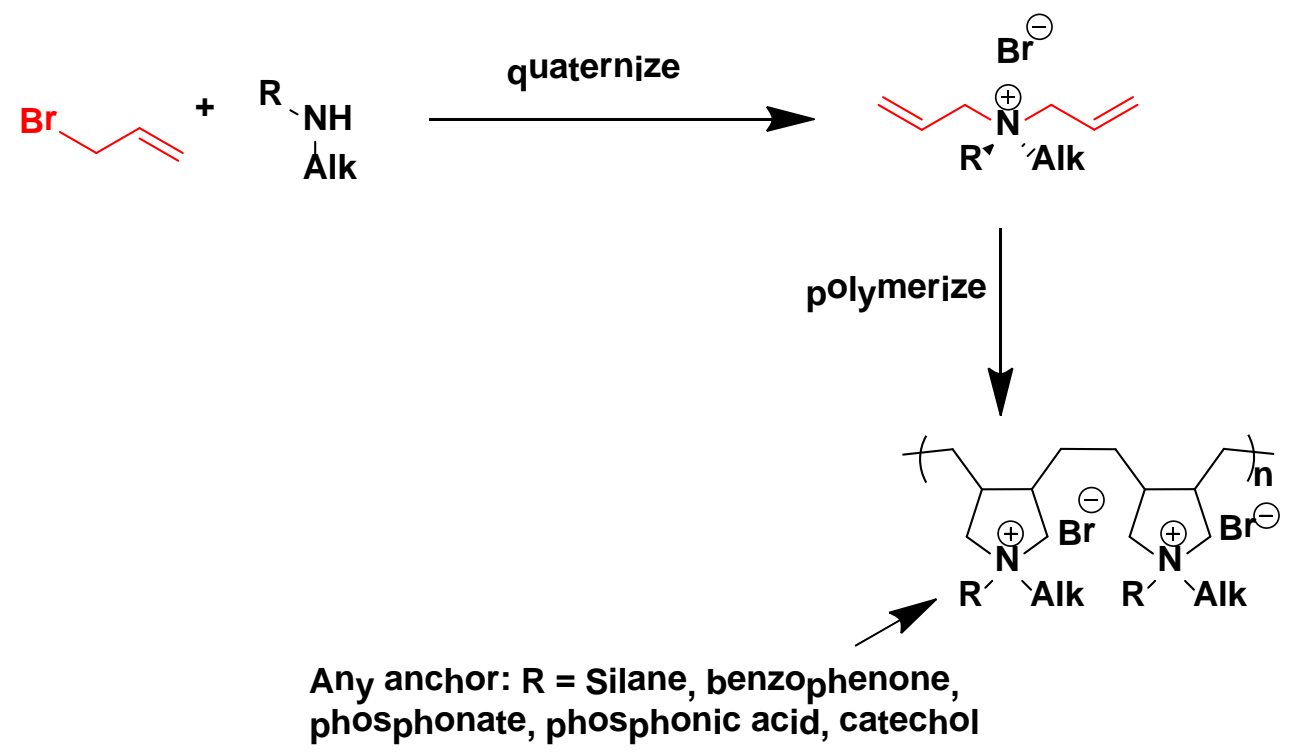

Scheme 4.6: Synthesis of multifunctional QAC antimicrobial polymer coating. 


\section{0 - EXPERIMENTAL PROCEDURES}

\subsection{Materials and Instrumental Methods}

The majority of reagents and solvents were obtained from Sigma-Aldrich (S-A) or Alfa Aesar (A-A) and used as received. Reagents that were purified by vaccum distillation prior to use and include $N, N$-dimethyloctadecylamine (89\%), octadecylamine, triethyl and triisopropyl phosphites. Bromooctadecane was purified by column chromatorgarphy eluting with 10\% acetone/hexanes.

The following anhydrous solvents, ACN (99.8\%), 1,4-dioxane, DMF, DMSO, TOL and non-anhydrous solvents, $\mathrm{MeOH}$, EtOH (95\%), $\mathrm{iPrOH}$, EtOAc and hexanes were purchased from Aldrich and used as received. Anhydrous DCM and THF were obtained from a mBraun solvent purification system by passage of the wet solvent through a bed of activated molecular sieves under an atmosphere of dry nitrogen.

Nuclear magnetic resonance (NMR) experiments were recorded on a $400 \mathrm{MHz}$ Bruker Avance II Spectrometer (Ryerson University) using deuterated chloroform $\left(\mathrm{CDCl}_{3}\right)$ as the solvent unless otherwise indicated. ${ }^{1} \mathrm{H}$ and ${ }^{13} \mathrm{C}$ NMR spectra were referenced to the residual $\mathrm{CDCl}_{3}(7.26 \mathrm{ppm}$ and $77.0 \mathrm{ppm})$ solvent signal while ${ }^{31} \mathrm{P}$ spectrum were referenced externally to $\mathrm{H}_{3} \mathrm{PO}_{4}(0 \mathrm{ppm})$. Proton chemical shift assignments are given in $\delta(\mathrm{ppm})$ and were interpreted with the aid of 2-D COSY spectra (Section 6, Appendix 1.2), while carbon chemical shift assignments are given in $\delta(\mathrm{ppm})$ and were interpreted with the aid of 2-D HSQC spectra (Section 6, Appendix 1.2).

$\mu \mathrm{W}$ reactions were performed in sealed glass reaction tubes utilizing the Biotage ${ }^{\circledR}$ Initiator $\mu \mathrm{W}$ Synthesizer (2.45 GHz) at the Ryerson University Analytical Centre (RUAC). UV- 
VIS and fluorescence measurements were recorded on a Perkin Elmer Spectrophotometer (Lambda 20) at the Ryerson University Analytical Centre (RUAC).

High resolution mass spectra (HRMS) were recorded by direct analysis in real time by DART-TOF or by electrospray time-of-flight (ESI-TOF) at the Advanced Instrumentation for Molecular Structure (AIMS) laboratory at the University of Toronto. X-ray crystal structure analysis was performed with a Bruker-Nonius Kappa-CCD diffractometer at the University of Toronto X-ray facilities.

Thin Layer Chromatography (TLC) was carried out on Silica gel 60 aluminum backed plates and visualized with a UV lamp or staining with $\left(\mathrm{KMnO}_{4}\right.$ or ninhydrin). Melting points were measured using a Fischer Scientific melting point apparatus. All prepared intermediates and compounds were stored in glass vials at RT while the dansyl derivatives were stored in the dark at RT.

\subsection{General Procedures}

\section{Method 5.2.1 Sealed Tube Reactions}

The appropriate reactants were placed, along with a magnetic stirring bar, into a $20 \mathrm{~mL}$ glass scintillation vial and sealed with a screw cap. The reaction mixture was heated using an oil or sand bath at the indicated time and temperature. The reaction was transferred to a round bottom flask, and volatiles removed on a rotary evaporator and the crude material purified either

by distillation, Dry Column Vacuum Chromatography (DCVC) on silica gel or crystallization as indicated. In the case of dansyl derivatives, $\mathrm{Et}_{2} \mathrm{O}$ was added directly to the reaction mixture followed by decanting (repeat $\mathrm{Et}_{2} \mathrm{O}$ wash $\times 2$ ) and drying under high vaccum. 


\section{Method 5.2.2 $\mu \mathrm{W}$ Reactions}

The appropriate reactants were placed, along with a magnetic stirring bar, into the appropriate size glass reaction tube (5 mL or $20 \mathrm{~mL}$ supplied from Biotage) and sealed with a septum/aluminum cap. The reaction mixture was heated in the Biotage ${ }^{\circledR}$ Initiator $\mu \mathrm{W}$ Synthesizer at the indicated time and temperature. Reaction mixtures were transferred to a round bottom flask and removed on a rotary evaporator and the crude material purified either by distillation, crystallization or Dry Column Vacuum Chromatography (DCVC) on silica gel as indicated.

\subsubsection{Purification/Preparation of Common Starting Materials}
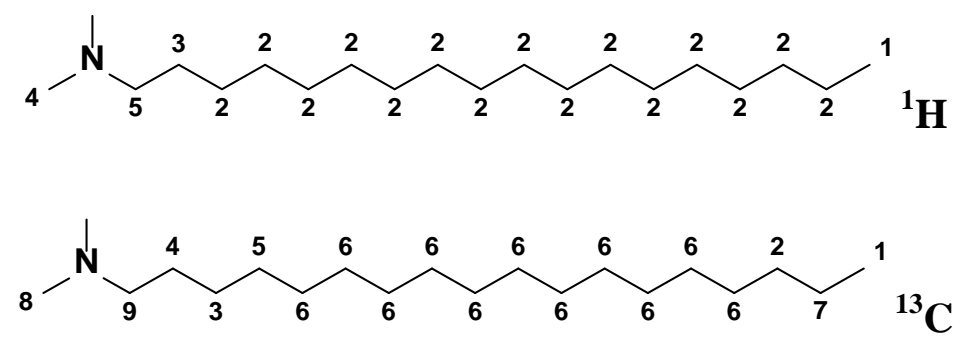

\section{$N, N$-dimethyloctadecan-1-amine (2):}

This amine was purified by vacuum distillation $\left(150^{\circ} \mathrm{C}, 0.5 \mathrm{mmHg}\right)$ using a shortpath distillation head attached to a Schlenk line utilizing a silicon oil bath $\left(200^{\circ} \mathrm{C}\right)$ and isolated as a clear, colourless liquid that solidified at RT. ${ }^{1} \mathbf{H}$ NMR (400 MHz, $\left.\mathrm{CDCl}_{3}, \delta\right): 2.24-2.14$ (m, 8H, (H4, H5 overlap)), 1.45-1.36 (m, 2H, H3) 1.22 (brs, 30H, H2 overlap), 0.84 (t, $J=7.1 \mathrm{~Hz}, 3 \mathrm{H}, \mathrm{H} 1$ ) ppm; ${ }^{13}$ C NMR (100 MHz, $\mathrm{CDCl}_{3}$, ): 60.0 (C9), 45.5 (C8), 31.9 (C7), 29.7-29.6 (C6 overlap), 29.3 (C5), 27.8 (C4), 27.5 (C3), 22.7 (C2), 14.1 (C1) ppm.

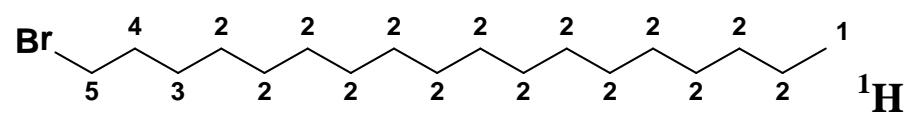




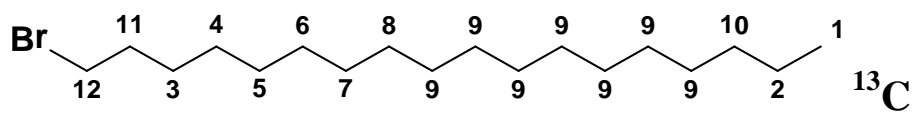

\section{1-Bromooctadecane (46):}

This starting material from Aldrich (brown solid, 97\%) was packed onto silica (20g / ( $20 \mathrm{~mL}$ SM $)$ and purified by dry column chromatography $(4.5 \mathrm{~cm} \times 5.0 \mathrm{~cm}$ frit, 50 g silica $)$ eluting with 10\% acetone/hexanes $(150 \mathrm{~mL})$ to afford $\sim 9 \mathrm{~g}$ of a clear oil that solidified at RT. (Note: the brown coloured impurities remained on column under these conditions but will elute in 50\% acetone/hexanes). ${ }^{1} \mathbf{H}$ NMR (400 MHz, $\mathrm{CDCl}_{3}, \delta$ ): 2.21-2.14 (m, 2H, H5), 1.88-1.81 (m, 2H, H4), 1.46-1.38 (m, 2H, H3), 1.24 (brs, 28H, H2), 0.86 (t, $J=7.0 \mathrm{~Hz}, 3 \mathrm{H}, \mathrm{H} 1) \mathrm{ppm} ;{ }^{13} \mathbf{C}$ NMR (100 MHz, $\mathrm{CDCl}_{3}, \delta$ ): 33.9 (C12), 32.8 (C11), 31.9 (C10), 29.7-29.6 (C8, C9 overlap), 29.5 (C7), 29.4 (C6), 29.3 (C5), 28.7 (C4), 28.2 (C3), 22.7 (C2), 14.1 (C1) ppm.

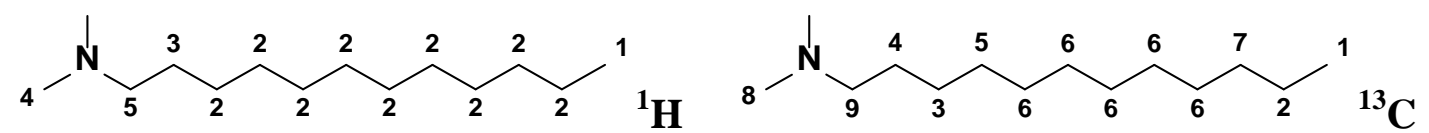

\section{$N, N$-dimethyldodecan-1-amine (22): ${ }^{193}$}

This compound was prepared by Method 5.2.2 with the EC reaction. ${ }^{1} \mathbf{H}$ NMR (400 MHz, $\mathrm{CDCl}_{3}, \delta$ ): 2.21-2.14 (m, 8H, (H4 + H5 overlap)), 1.44-1.36 (m, 2H, H3) 1.21 (brs, 30H, H2 overlap), 0.84 (t, $J=7.0 \mathrm{~Hz}, 3 \mathrm{H}, \mathrm{H1}$ ) ppm; ${ }^{13} \mathrm{C}$ NMR (100 MHz, $\mathrm{CDCl}_{3}, \delta$ ): 60.0 (C9), 45.5 (C8), 31.9 (C7), 29.7-29.6 (C6 overlap), 29.3 (C5), 27.8 (C4), 27.5 (C3), 22.7 (C2), 14.1 (C1) ppm. (Agrees well with literature NMR values). ${ }^{193}$ 


\subsubsection{Dow Antimicrobial Synthesis}
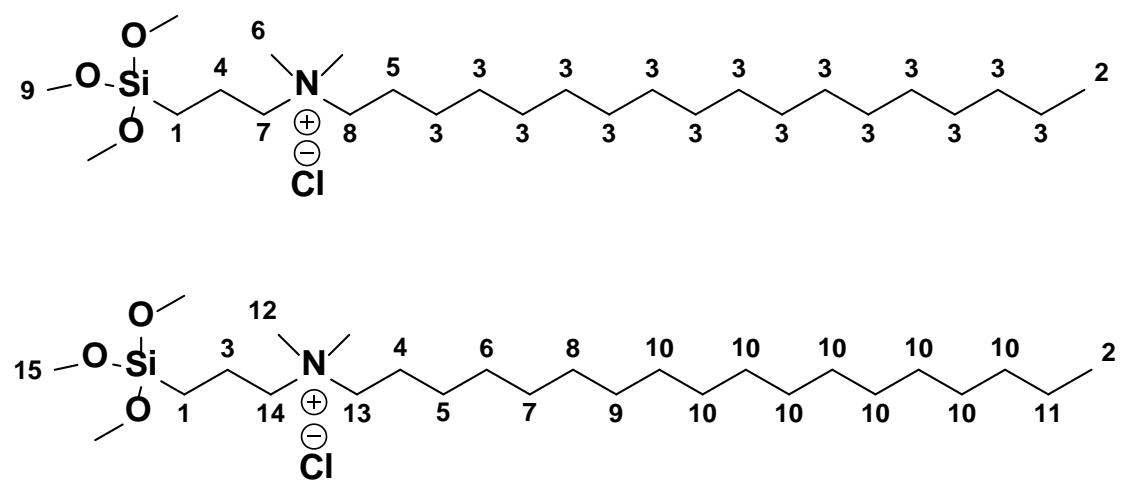

$N, N$-dimethyl- $N$-(3-(trimethoxysilyl)propyl)nonadecan-1-ammonium chloride (3): ${ }^{194,}, 219$

See (Table 2.2, Entry iii). This compound was synthesized according to Method 5.2.1 by heating 3-chloropropyltrimethoxysilane 1 (0.814 g, 4.1 mmol, 1.05 eq.) and $N, N$-dimethyloctadecyl-1amine (DMOA) 2 (1.190 g, 4 mmol, 1.0 eq.) neat for 72 hrs at $110^{\circ} \mathrm{C}$. Crude yield: $~ 80 \%$ by ${ }^{1} \mathrm{H}$ NMR spectroscopy (Section 6, Appendix 1.2, Figure A19). ${ }^{\mathbf{1}} \mathbf{H} \mathbf{N M R}$ (400 MHz, $\mathrm{CDCl}_{3}, \delta$ ): 3.33 (s, 9H, H9), 3.32-3.08 (m, 10H, (H8 + H7 + H6 overlap)), 1.67-1.41 (m, 4H, (H5 + H4 overlap)),

1.01 (s, 30H, H3), 0.63 (t, $J=7.0 \mathrm{~Hz}, 2 \mathrm{H}, \mathrm{H} 2), 0.44$ (t, $J=7.4 \mathrm{~Hz}, 2 \mathrm{H}, \mathrm{H} 1) \mathrm{ppm}$; ${ }^{13} \mathbf{C}$ NMR (100 MHz, $\mathrm{CDCl}_{3}, \delta$ ): 65.56 (C15), 64.14 (C14), 50.61 (C13), 31.69 (C11), 29.5-29.35 (C10 overlap), 29.26 (C9), 29.18 (C8), 29.12 (C7), 29.03 (C6), 22.44 (C5), 16.26 (C4), 15.02 (C3), 13.85 (C2), 5.40 (C1) ppm. (No NMR values reported in the literature).

\subsection{General Procedure for the Synthesis of ( $\gamma$-MPQA's)}

\subsubsection{Abruzov Reaction}

According to general procedures ${ }^{220,221}$ for the Abruzov reaction, to a flame dried round bottom flask equipped with a reflux condenser connected to an inert atmosphere manifold, were added the appropriate reagents according to (Table 2.3). The flask was evacuated ( $2 \mathrm{~min}$ ), backfilled 
with $\mathrm{N}_{2}$ (g) and the reaction mixture refluxed for the appropriate time according to (Table 2.3) using an oil bath $\left(175^{\circ} \mathrm{C}\right)$. The solution was then cooled to RT and excess dibromoalkane, trialkylphosphite and lower boiling by-products were removed by vacuum distillation $\left(100^{\circ} \mathrm{C}\right.$, $1 \times 10^{-2} \mathrm{~mm} \mathrm{Hg}$ ) using a shortpath distillation head attached to a Schlenk line. Compounds (2-12-7) were vacuum distilled as clear, colourless liquids, while compound 2-8 was recrystallized from EtOAc as a brittle crystalline solid. This reaction was also performed under $\mu \mathrm{W}$ radiation according to Method 5.2.2 and Table 2.3.

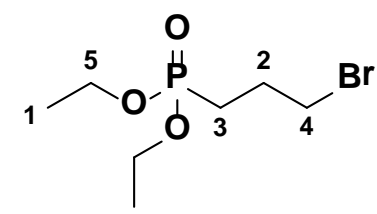

\section{Diethyl (3-bromopropyl)phosphonate (12): 220}

See (Table 2.3, Entry iii). This compound was synthesized according to Method 5.2.1 by refluxing 1,3-dibromopropane (40 mL, 394 mmol, 4.0 eq.) and $\mathrm{P}(\mathrm{OEt})_{3}(13 \mathrm{~mL}, 75.8 \mathrm{mmol}, 1.0$ eq.) for 6 hrs. Yield: 79\% (15.54 g). TLC (10\% MeOH:EtOAc, $\mathrm{KMnO}_{4}$ stain), $\mathrm{R}_{\mathrm{f}}=0.65 ;{ }^{1} \mathbf{H}$ NMR (400 MHz, $\mathrm{CDCl}_{3}, \delta$ ): 4.12-4.00 (m, 4H, H5), 3.43 (t, 2H, $\left.J=4.3 \mathrm{~Hz}, \mathrm{H} 4\right), 2.16-2.05$ (m, 2H, H3), 1.90-1.81 (m, 2H, H2), 1.28 (t, $J=7.0 \mathrm{~Hz}, 6 \mathrm{H}, \mathrm{H1}) \mathrm{ppm} ;{ }^{13} \mathbf{C}$ NMR (100 MHz, CDCl 3 ,

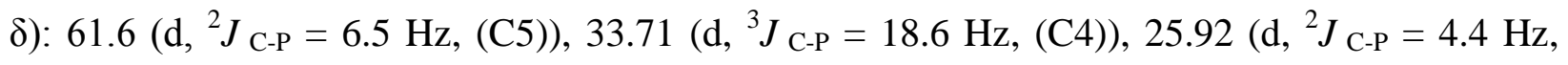
(C2)), 23.64 (d, $\left.{ }^{1} J_{\mathrm{C}-\mathrm{P}}=142.5 \mathrm{~Hz},(\mathrm{C} 3)\right), 16.4$ (d, $\left.{ }^{2} J_{\mathrm{C}-\mathrm{P}}=6.2 \mathrm{~Hz},(\mathrm{C} 1)\right) \mathrm{ppm} ;{ }^{31} \mathbf{P}$ NMR (121.45 $\mathrm{MHz}, \mathrm{CDCl}_{3}, \delta$ ): $30.2 \mathrm{ppm}$. (Agrees well with literature NMR values). ${ }^{220}$

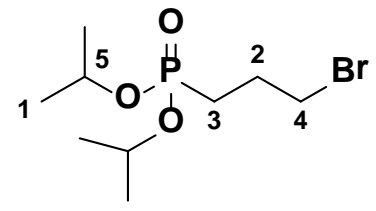




\section{Diisopropyl (3-bromopropyl)phosphonate (13): ${ }^{222}$}

See (Table 2.3, Entry vii). This compound was synthesized according to Method 5.2.2 by $\mu \mathrm{W}$ heating 1,3-dibromopropane (5.48 mL, 53.9 mmol, 1.1 eq.) and $\mathrm{P}(\mathrm{OiPr})_{3}(11.16 \mathrm{~mL}, 49 \mathrm{mmol}$, 1.0 eq.) for $5 \mathrm{~min}$ at $170^{\circ} \mathrm{C}$. The title compound $\mathbf{1 3}$ was isolated as a mixture after distillation with tetraisopropyl propane-1,3-diylbis(phosphonate) by-product A $\quad$ and 1isopropoxyphospholane 1-oxide by-product $\mathrm{B}$ in a $0.05: 0.05: 1$ mass ratio determined by ${ }^{31} \mathrm{P}$ NMR spectroscopy. Of the $10.45 \mathrm{~g}$ isolated, approximately (5\%, $0.522 \mathrm{~g}$ ) was the bisphosphonate and the other (5\%, 0.522 g) was the cyclic 5-membered ring oxaphospholane. Yield: 64\% (9.45 g). TLC (10\% MeOH, EtOAc, $\mathrm{KMnO}_{4}$ stain), $\mathrm{R}_{\mathrm{f}}=0.7 ;{ }^{1} \mathbf{H} \mathbf{N M R}(400 \mathrm{MHz}$, $\left.\mathrm{CDCl}_{3}, \delta\right): 4.70-4.61$ (m, 2H, H5), 3.42 (t, 2H, $J=6.5$ Hz, H4), 2.15-2.04 (m, 2H, H3), 1.85-1.75 (m, 2H, H2), 1.27 (d, $J=7.21 \mathrm{~Hz}, 12 \mathrm{H}, \mathrm{H1}) \mathrm{ppm} ;{ }^{13} \mathrm{C}$ NMR (100 MHz, $\left.\mathrm{CDCl}_{3}, \delta\right): 70.12\left(\mathrm{~d},{ }^{2} J\right.$ C-P $=6.51 \mathrm{~Hz},(\mathrm{C} 5)), 33.70\left(\mathrm{~d},{ }^{3} J_{\mathrm{C}-\mathrm{P}}=20.38 \mathrm{~Hz},(\mathrm{C} 4)\right), 26.19\left(\mathrm{~d},{ }^{1} J_{\mathrm{C}-\mathrm{P}}=4.32 \mathrm{~Hz},(\mathrm{C} 2)\right), 25.76(\mathrm{~d}$, $\left.{ }^{2} J_{\mathrm{C}-\mathrm{P}}=25.76 \mathrm{~Hz},(\mathrm{C} 3)\right), 24.01\left(\mathrm{t},{ }^{2} J_{\mathrm{C}-\mathrm{P}}=3.44 \mathrm{~Hz},(\mathrm{C} 1)\right) \mathrm{ppm} ;{ }^{31} \mathbf{P}$ NMR (121.45 MHz, $\mathrm{CDCl}_{3}$, $\delta): 28.34$ ppm. (Agrees well with literature NMR values). ${ }^{222}$

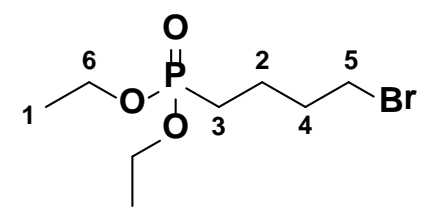

\section{Diethyl (4-bromobutyl)phosphonate (14): ${ }^{223}$}

See (Table 2.3, Entry ix). This compound was synthesized according to Method 5.2.2 by $\mu \mathrm{W}$ heating 1,4-dibromobutane (0.88 mL, 8.69 mmol, 1.1 eq.) and $\mathrm{P}(\mathrm{OEt})_{3}(1.32 \mathrm{~mL}, 7.9 \mathrm{mmol}, 1.0$ eq.) for $5 \mathrm{~min}$ at $190^{\circ} \mathrm{C}$. Isolated as a mixture with the bisphosphonate tetraethyl butane-1,4diylbis(phosphonate) and $\mathrm{P}(\mathrm{OEt})_{3}$ in a 0.1:0.29:1 mass ratio determined by ${ }^{31} \mathrm{P}$ NMR. Of the 1.32 
g isolated, approximately ( 10\%, 0.132 g) was the bisphosphonate by-product B and ( 29\%, 0.27 g) was by-product A. Yield: 63\% (0.95 g). TLC (10\% MeOH:EtOAc, $\mathrm{KMnO}_{4}$ stain), $\mathrm{R}_{\mathrm{f}}=$ 0.65; ${ }^{1} \mathbf{H}$ NMR (400 MHz, $\left.\mathrm{CDCl}_{3}, \delta\right): 4.10-3.93$ (m, 4H, H6), 3.38-3.29 (m, 2H, H5), 1.91-1.80 (m, 2H, H4), 1.75-1.60 (m, 4H, (H2, H3)), 1.30-1.20 (m, 6H, H1) ppm; ${ }^{31} \mathbf{P}$ NMR (121.45 MHz, $\mathrm{CDCl}_{3}, \delta$ ): $31.23 \mathrm{ppm}$. (Agrees well with literature NMR values). ${ }^{223}$

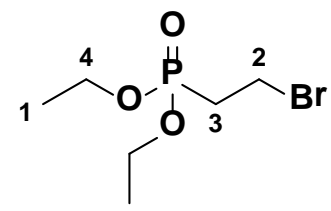

\section{Diethyl (2-bromoethyl)phosphonate (15): ${ }^{221}$}

See (Table 2.3, Entry xiii). This compound was synthesized according to Method 5.2.1 by refluxing 1,2-dibromoethane (40 mL, $394 \mathrm{mmol}, 4.0$ eq.) and $\mathrm{P}(\mathrm{OEt})_{3}(13 \mathrm{~mL}, 75.8 \mathrm{mmol}, 1.0$ eq.) for 4.3 hrs. The title compound $\mathbf{1 5}$ was purified by distillation and used directly without ${ }^{1} \mathrm{H}$ and ${ }^{13} \mathrm{C}$ NMR characterization in the synthesis of diethyl vinyl phosphonate $\mathbf{1 3 0}$.

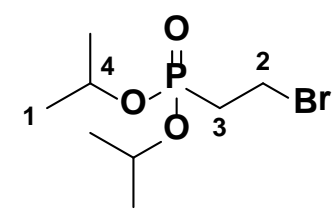

\section{Diisopropyl (2-bromoethyl)phosphonate (16): ${ }^{224}$}

See (Table 2.3, Entry xv). This compound was synthesized according to Method 5.2.1 by refluxing 1,2-dibromoethane (49.6 mL, 576 mmol, 4.0 eq.) and $\mathrm{P}(\mathrm{OiPr})_{3}(32.8 \mathrm{~mL}, 144 \mathrm{mmol}$, 1.0 eq.) for 12 hrs. The title compound was isolated as a mixture after distillation with tetraisopropyl ethane-1,2-diylbis(phosphonate) by-product B and by-product A in a 0.09:0.1:1 mass ratio determined by ${ }^{31} \mathrm{P}$ NMR spectroscopy. Of the $24.82 \mathrm{~g}$ isolated, approximately ( $\sim 10 \%$, 
$2.36 \mathrm{~g})$ was identified as bisphosphonate and the other ( 10\%, $2.36 \mathrm{~g})$ was the by-product A. Yield: 64\% (20.10 g). TLC (10\% MeOH, EtOAc, $\mathrm{KMnO}_{4}$ stain), $\mathrm{R}_{\mathrm{f}}=0.7 ;{ }^{1} \mathbf{H}$ NMR (400 MHz, $\mathrm{CDCl}_{3}, \delta$ ): 4.68-4.58 (m, 2H, H4), 3.43 (q, $J=8.8 \mathrm{~Hz}, 2 \mathrm{H}, \mathrm{H} 3$ ), 3.31-2.20 (m, 2H, H2), 1.24 (d, $J=6.2 \mathrm{~Hz}, 12 \mathrm{H}, \mathrm{H} 1) \mathrm{ppm} ;{ }^{13} \mathbf{C}$ NMR $\left(100 \mathrm{MHz}, \mathrm{CDCl}_{3}, \delta\right): 70.61\left(\mathrm{~d},{ }^{2} J_{\mathrm{C}-\mathrm{P}}=6.70 \mathrm{~Hz},(\mathrm{C} 4)\right)$, $32.05\left(\mathrm{~d},{ }^{2} J_{\mathrm{C}-\mathrm{P}}=134.80 \mathrm{~Hz},(\mathrm{C} 2)\right), 24.10$ (s, C3), 23.90 (t, $\left.{ }^{2} J_{\mathrm{C}-\mathrm{P}}=3.0 \mathrm{~Hz},(\mathrm{C} 1)\right) \mathrm{ppm} ;{ }^{31} \mathbf{P}$ NMR (121.45 MHz, $\mathrm{CDCl}_{3}, \delta$ ): 28.34 ppm. (Agrees well with literature NMR values). ${ }^{224}$

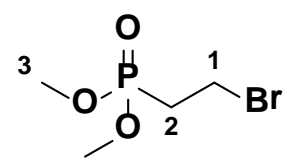

Dimethyl (2-bromoethyl)phosphonate (17): ${ }^{225}$

See (Table 2.3, Entry xvi). This compound was synthesized according to Method 5.2.1 by refluxing 1,2-dibromoethane (24.12 mL, $280 \mathrm{mmol}, 4.0$ eq.) and $\mathrm{P}(\mathrm{OMe})_{3}(11.05 \mathrm{~mL}, 70 \mathrm{mmol}$, 1.0 eq.) for 12 hrs. Yield: 65\% (10 g). TLC (10\% MeOH, EtOAc, $\mathrm{KMnO}_{4}$ stain), $\mathrm{R}_{\mathrm{f}}=0.7 ;{ }^{1} \mathbf{H}$ NMR (400 MHz, $\left.\mathrm{CDCl}_{3}, \delta\right): 3.60-3.46$ (m, 6H, H3), 1.30-1.26 (m, 2H, H2), 1.25-1.21 (m, 2H, H1), ppm; ${ }^{13} \mathrm{C}$ NMR (100 MHz, $\left.\mathrm{CDCl}_{3}, \delta\right): 51.9\left(\mathrm{~d},{ }^{2} J_{\mathrm{C}-\mathrm{P}}=6.6 \mathrm{~Hz},(\mathrm{C} 3)\right), 10.2$ (C1), 8.8 (C2) ppm; ${ }^{31} \mathbf{P}$ NMR (121.45 MHz, $\mathrm{CDCl}_{3}, \delta$ ): $33.10 \mathrm{ppm}$. (Agrees well with literature NMR values). ${ }^{225}$

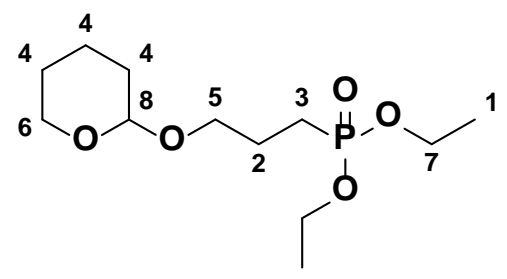

Diethyl (3-((tetrahydro-2H-pyran-2-yl)oxy)propyl)phosphonate (18): ${ }^{226}$ 
To a $20 \mathrm{~mL}$ conical round bottom flask was added the THP protected bromopropylalcohol 10 (4.55 g, $20 \mathrm{mmol})$ followed by excess $\mathrm{P}(\mathrm{OEt})_{3}(10.0 \mathrm{~mL}, 60.0 \mathrm{mmol}, 3.0$ eq.). The reaction was heated at reflux $\left(175^{\circ} \mathrm{C}\right)$ overnight. Excess $\mathrm{P}(\mathrm{OEt})_{3}$ was vacuum distilled at reduced pressure providing the pure product as a clear, viscous oil. Yield 89\% (5.0 g). ${ }^{1} \mathbf{H}$ NMR (400 MHz, $\mathrm{CDCl}_{3}, \delta$ ): 4.57 (t, $\left.J=3.54 \mathrm{~Hz}, 1 \mathrm{H}, \mathrm{H8}\right), 4.17-4.04$ (m, 4H, H7), 3.86-3.72 (m, 2H, H6), 3.523.40 (m, 2H, H5), 1.93-1.77 (m, 4H, H3 + H4), 1.73-1.67 (m, 4H, H2), 1.31 (t, $J=7.04$ Hz, 6H, H1) ppm; ${ }^{13}$ C NMR (100 MHz, $\mathrm{CDCl}_{3}, \delta$ ): 98.90 (C8), 67.50 (d, ${ }^{3} J_{\mathrm{C}-\mathrm{P}}=6.5 \mathrm{~Hz},(\mathrm{C} 5)$ ), 62.3 (C6), 61.45 (d, ${ }^{2} J_{\mathrm{C}-\mathrm{P}}=3.7 \mathrm{~Hz},(\mathrm{C} 7)$ ), 32.90 (C4), 30.65 (d, ${ }^{3} J_{\mathrm{C}-\mathrm{P}}=9.9 \mathrm{~Hz},(\mathrm{C} 5)$ ), 25.43 (C4), $23.15\left(\mathrm{~d},{ }^{2} J_{\mathrm{C}-\mathrm{P}}=3.7 \mathrm{~Hz},(\mathrm{C} 3)\right), 19.45\left(\mathrm{~d},{ }^{2} J_{\mathrm{C}-\mathrm{P}}=2.3 \mathrm{~Hz},(\mathrm{C} 2)\right) 16.45\left(\mathrm{~d},{ }^{3} J_{\mathrm{C}-\mathrm{P}}=5.9 \mathrm{~Hz},(\mathrm{C} 1)\right)$ ppm; ${ }^{31} \mathbf{P}$ NMR (121.45 MHz, $\mathrm{CDCl}_{3}, \delta$ ): $32.32 \mathrm{ppm}$. (Agrees well with literature NMR values). ${ }^{226}$

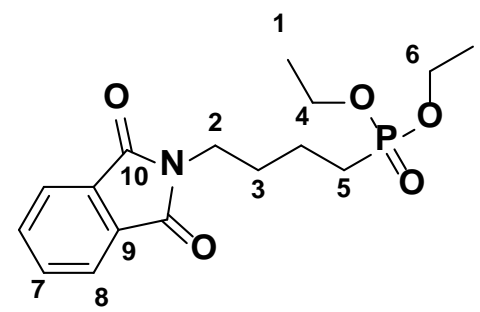

\section{Diethyl (4-(1,3-dioxoisoindolin-2-yl)butyl) phosphonate (19): ${ }^{27}$}

To a flame dried $50 \mathrm{~mL}$ round bottom flask equipped with a reflux condenser was added $\mathrm{N}$-(4bromobutyl)-phthalimide (5g, $17.7 \mathrm{mmol}, 1.0$ eq.) followed by $\mathrm{P}(\mathrm{OEt})_{3}(18.24 \mathrm{~mL}, 106.3 \mathrm{mmol}$, 6 eq.) and the mixture was refluxed overnight $\left(175^{\circ} \mathrm{C}\right)$ using a sand bath. The reaction was then cooled to RT and excess $\mathrm{P}(\mathrm{OEt})_{3}$ was vacuum distilled using a shortpath distillation head attached to a Schlenk line. Once all of the excess $\mathrm{P}(\mathrm{OEt})_{3}$ was removed, the title compound was placed under high vacuum ( $30 \mathrm{~min})$ until it solidified. Further recrystallization from EtOAc (5 
$\mathrm{mL}$ ) at $-20^{\circ} \mathrm{C}$ provided pure product. Colourless crystals. Yield: $90 \%$ (5.43 g). TLC (5\% MeOH: EtOAc), $\mathrm{R}_{\mathrm{f}}=0.90, \mathrm{Mp}=80-81^{\circ} \mathrm{C} ;{ }^{1} \mathbf{H}$ NMR $\left(400 \mathrm{MHz}, \mathrm{CDCl}_{3}, \delta\right): 7.82-7.77(\mathrm{~m}, 2 \mathrm{H}$, H8), 7.70-7.66 (m, 2H, H7), 4.11-3.98 (m, 4H, H6), 3.66 (m, $J=7.0 \mathrm{~Hz}, 2 \mathrm{H}, \mathrm{H} 5), 1.81-1.71$ (m, 4H, (H4, H3)), 1.67-1.56 (m, 2H, H2), 1.27 (t, $J=7.1 \mathrm{~Hz}, \mathrm{H} 1)$ ppm; ${ }^{13} \mathbf{C}$ NMR (100 MHz, $\mathrm{CDCl}_{3}, \delta$ ): 168.29 (C10), 133.91 (C9), 132.06 (C7), 123.18 (C8), 61.48 (d, ${ }^{2} J_{\mathrm{C}-\mathrm{P}}=6.5 \mathrm{~Hz},(\mathrm{C} 6)$ ), $37.23\left(\mathrm{~d},{ }^{1} J_{\mathrm{C}-\mathrm{P}}=1.33 \mathrm{~Hz},(\mathrm{C} 5)\right), 29.25\left(\mathrm{~d},{ }^{2} J_{\mathrm{C}-\mathrm{P}}=16.77 \mathrm{~Hz}(\mathrm{C} 4)\right), 24.44$ (C2), 19.81 (d, ${ }^{3} J_{\mathrm{C}-\mathrm{P}}=$ $5.01 \mathrm{~Hz},(\mathrm{C} 3)), 16.42\left(\mathrm{~d},{ }^{3} \mathrm{~J}_{\mathrm{C}-\mathrm{P}}=6.01 \mathrm{~Hz},(\mathrm{C} 1)\right) \mathrm{ppm} ;{ }^{31} \mathbf{P}$ NMR (121.45 MHz, CDCl 3 , $\left.\delta\right): 31.48$ ppm. HRMS-DART (m/z): $\left[\mathrm{M}^{+}\right]$calculated for $\mathrm{C}_{16} \mathrm{H}_{23} \mathrm{NO}_{5} \mathrm{P}$, 340.1308; found, 340.1317 . (Agrees well with literature NMR values). ${ }^{227}$

\subsubsection{Menschutkin Quaternization}

General Procedure for the Menschutkin Quaternization. The appropriate tertiary amine and alkyl halide (1-2 eq.) were mixed with ACN or EtOH (0.5 M) employing Method 5.2.1 or Method 5.2.2 and heated for the appropriate length of time (Table 2.4) until ${ }^{31} \mathrm{P}$ NMR spectroscopy showed the consumption of the starting phosphonates. The vial was allowed to cool to RT and the crude product was purified either by extraction (aq. phase isolated after washing with $\mathrm{Et}_{2} \mathrm{O}$ for $\mathrm{H}_{2} \mathrm{O}$ soluble compound only), centrifugation with a non-polar solvent, recrystallization, or dry packed onto silica and purified via dry column chromatography $(4.5 \mathrm{~cm}$ $\times 5.0 \mathrm{~cm}$ frit, $40 \mathrm{~g}$ silica), eluting with 6\% $\mathrm{NaBr}$ in $\mathrm{MeOH} / \mathrm{ACN}$ (20:80). This method was also successfully used to make compounds 26-33, $\mathbf{4 0}$ (Scheme 2.2). 

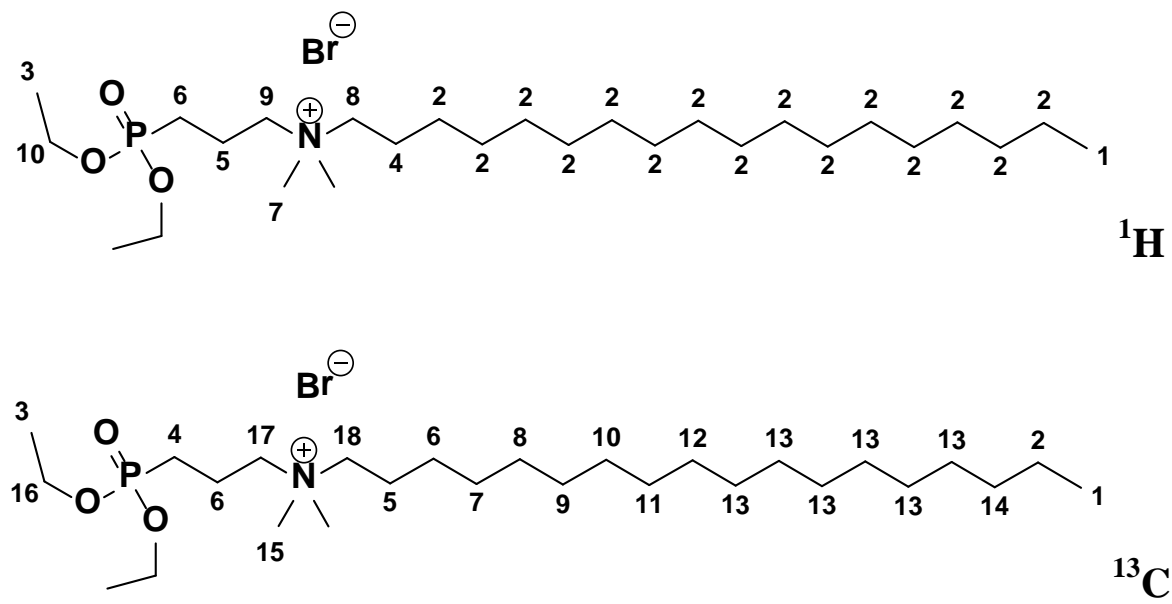

$N$-(3-diethoxyphosphorylpropyl)- $N, N$-dimethyloctadecan-1-ammonium bromide (26): ${ }^{192,228}$

This compound was prepared by the Menshutkin reaction using Method 5.2.1, dimethyl (3bromopropyl)phosphonate 12 (1.264g, $4.88 \mathrm{mmol})$ and $N, N$-dimethyloctadecylamine (DMOA) (1.71 g, $5.1 \mathrm{mmol}, 1.1$ eq.) were reacted neat for $35 \mathrm{~min}$ at $100^{\circ} \mathrm{C}$ until the mixture solidified. The mixture was then cooled to RT, centrifuged from hexanes (15 mL), and recrystallized from $20 \mathrm{~mL}$ EtOAc / hexanes (1:5) to afford 26. Yield: 67\% (1.82 g). Mp = 54-55 ${ }^{\circ} \mathrm{C} ;{ }^{\mathbf{1}} \mathbf{H}$ NMR (400 MHz, $\mathrm{CDCl}_{3}, \delta$ ): 4.09-4.01 (m, 2H, H10), 3.66-3.22 (m, 2H, H9) 3.43-3.38 (m, 2H, H8), 3.31 (s, 6H, H7), 2.03 (brs, 2H, H6), 1.84-1.80 (m, 2H, H5), 1.67 (brs, 2H, H4), 1.33-1.25 (m, 6H, H3), 1.19 (brs, 30H, H2), 0.83-0.79 (m, 3H, H1) ppm; ${ }^{13} \mathrm{C}$ NMR (100 MHz, $\mathrm{CDCl}_{3}, \delta$ ): 64.25 (C18), $63.09\left(\mathrm{~d},{ }^{3} J_{C-P}=6.54 \mathrm{~Hz},(\mathrm{C} 17)\right), 62.14\left(\mathrm{~d},{ }^{2} J_{C-P}=6.54 \mathrm{~Hz},(\mathrm{C} 16)\right), 51.25(\mathrm{C} 15), 31.86(\mathrm{C} 14)$, 29.66-29.58 (C13 overlap), 29.55 (C12), 29.44 (C11), 29.38 (C10), 29.29 (C9), 29.19 (C8), 26.25 (C7), 22.69 (C6), 22.62 (C5), 16.49 (C4), 16.45 (C3), 16.39 (C2), 14.05 (C1) ppm; ${ }^{31} \mathbf{P}$ NMR (121.45 MHz, $\mathrm{CDCl}_{3}, \delta$ ): 29.54 ppm. HRMS-DART (m/z): [M+] - $\mathrm{Br}^{-}$calculated for $\mathrm{C}_{27} \mathrm{H}_{59} \mathrm{NO}_{3} \mathrm{P}$, 476.4227; found, 476.4240. (Agrees well with literature NMR values). ${ }^{192,228}$ 


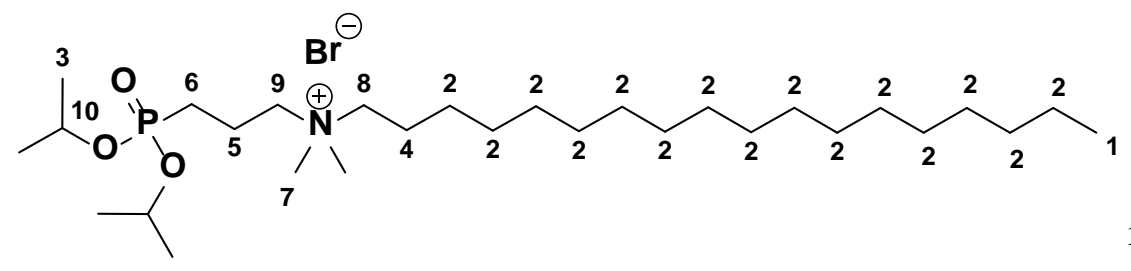

${ }^{1} \mathbf{H}$

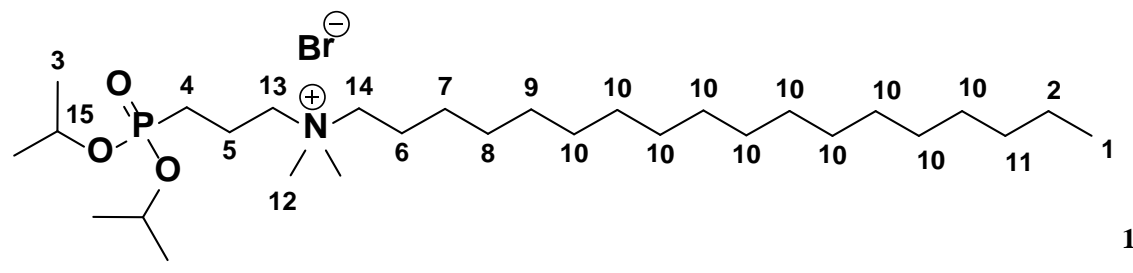

${ }^{13} \mathrm{C}$

\section{$N$-(3-(diisopropoxyphosphoryl)propyl)- $N, N$-dimethyloctadecan-1-ammonium bromide}

(27):

See (Table 2.4, entry iv). This compound was prepared by the Menshutkin reaction using Method 5.2.1. Diisopropylyl (3-bromopropyl)phosphonate 13 (0.964g, $3.36 \mathrm{mmol}$ ) and DMOA (1g, 3.36 mmol, 1.0 eq.) were refluxed in ACN for 3 hrs. The mixture was then cooled to RT, poured into $20 \mathrm{~mL}$ of $\mathrm{Et}_{2} \mathrm{O}$, and placed into a freezer $\left(-20^{\circ} \mathrm{C}\right)$ for $60 \mathrm{~min}$ to precipitate 27 as a white waxy solid. Yield: 89\% (2.53 g). $\mathrm{Mp}=54-55^{\circ} \mathrm{C} ;{ }^{1} \mathbf{H}$ NMR (400 MHz, $\left.\mathrm{CDCl}_{3}, \delta\right): 4.68-$ 4.60 (m, 2H, H10), 3.78-3.67 (m, 2H, H9) 3.50-3.42 (m, 2H, H8), 3.39 (s, 6H, H7), 2.05-1.92 (m, 2H, H5), 1.83-1.62 (m, 4H, H4 + H6), 1.28 (d, $\left.{ }^{2} J=6.20 \mathrm{~Hz}, 12 \mathrm{H}, \mathrm{H} 3\right) 1.21$ (brs, 30H, H2), 0.84 (t, $J=7.03 \mathrm{~Hz}, 3 \mathrm{H}, \mathrm{H1}) \mathrm{ppm} ;{ }^{13} \mathrm{C}$ NMR (100 MHz, $\left.\mathrm{CDCl}_{3}, \delta\right): 70.65\left(\mathrm{~d},{ }^{2} J_{\mathrm{C}-P}=6.54 \mathrm{~Hz}\right.$, (C15)), 64.09 (C14), 62.85 (d, ${ }^{3} J_{C-P}=6.54 \mathrm{~Hz},(\mathrm{C} 13)$ ), 51.55 (C12), 31.84 (C11), 29.66-29.55 (C10 overlap), 29.40 (C9), 29.29 (C8), 29.18 (C7), 22.41 (d, ${ }^{2} J_{C-P}=\mathrm{Hz},(\mathrm{C} 6)$ ), 23.95 (d, ${ }^{1} J_{C-P}=$ Hz, (C5)), 22.61 (C4), 22.42 (C3), 16.70 (C2), 14.06 (C1) ppm; ${ }^{31}$ P NMR (121.45 MHz, CDCl

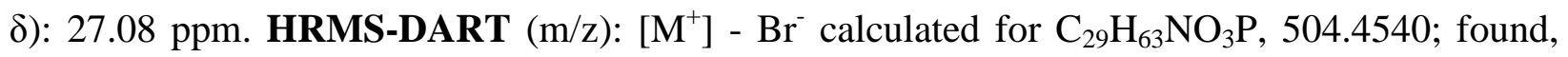
504.4546. 


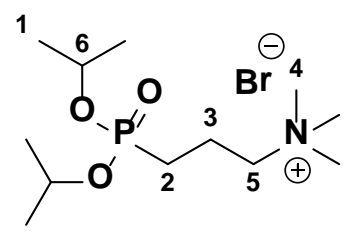

\section{3-(diisopropoxyphosphoryl)- $N, N, N$-trimethylpropan-1-ammonium bromide (28):}

See (Table 2.4, Entry vi). $\mathrm{NMe}_{3} \cdot \mathrm{HCl}(0.5 \mathrm{~g}, 1.5$ eq., $5.2 \mathrm{mmol})$ and $\mathrm{NaOH}(0.196$ g, 1.4 eq., 4.9 mmol) were placed, with a magnetic stirring bar, into a $20 \mathrm{~mL}$ glass reaction tube and sealed. EtOH (18 mL) was injected via a syringe and the mixture was stirred for $2 \mathrm{~min}$ at RT to free base the $\mathrm{NMe}_{3} \cdot \mathrm{HCl}$. Next diisopropyl (3-bromopropyl)phosphonate $13(1.0 \mathrm{~g}, 3.48 \mathrm{mmol})$ was introduced via syringe and the reaction mixture was heated in the $\mu \mathrm{W}$ at $150{ }^{\circ} \mathrm{C}(3 \mathrm{~min})$. Volatiles were removed on a rotary evaporator and the crude material purified by dissolving the mixture in $\mathrm{CHCl}_{3}(30 \mathrm{~mL})$, filtering off salts through a short pad of Celite and extracting the title compound into $30 \mathrm{~mL}$ of $\mathrm{H}_{2} \mathrm{O} . \mathrm{H}_{2} \mathrm{O}$ was co-evaporated from ACN (100 mL), and the final product was further dried under high vaccum ( $1 \mathrm{hr}$ ). Compound 28 was isolated as a clear, colourless oil. Yield: $80 \%$ (0.96 g). ${ }^{1} \mathbf{H}$ NMR (400 MHz, $\mathrm{CDCl}_{3}, \delta$ ): 4.69-4.53 (m, 2H, H6), 3.83-3.70 (m, 2H, H5) 3.51-3.36 (brs, 9H, H4), 2.10-1.93 (m, 2H, H3), 1.83-1.67 (m, 2H, H2), 1.36-1.20 (m,12H, H1) ppm; ${ }^{31} \mathbf{P}$ NMR (121.45 MHz, $\left.\mathrm{CDCl}_{3}, \delta\right): 26.77$ ppm.

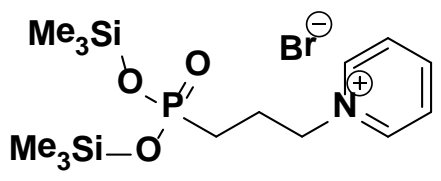

Bis(trimethylsilyl)-3-propylphosphonatepyridin-1-ium bromide (29):

See (Table 2.4, Entry viii). This compound was prepared in a one pot reaction on a $1.74 \mathrm{mmol}$ scale by reacting pyridine (0.137g, $1.74 \mathrm{mmol}$ ) with diisopropyl (3-bromopropyl)phosphonate 13 
(0.5 g, $1.74 \mathrm{mmol})$ in ACN $(1.5 \mathrm{~mL})$ under $\mu \mathrm{W}$ heating $\left(150^{\circ} \mathrm{C}, 10 \mathrm{~min}\right)$. Next TMSBr $(0.6 \mathrm{~mL}$, $\sim 2.5$ eq.) was syringed into the same vial and heated in the $\mu \mathrm{W}\left(60{ }^{\circ} \mathrm{C}, 10 \mathrm{~min}\right)$ to obtain a complete conversion of the TMS ester. ${ }^{31} \mathbf{P}$ NMR $\left(121.45 \mathrm{MHz}, \mathrm{CDCl}_{3}, \delta\right): 10.60 \mathrm{ppm}$. This reactive intermediate was hydrolyzed directly with $\mathrm{H}_{2} \mathrm{O}$ to compound 36 (Table 2.5, Entry ix).
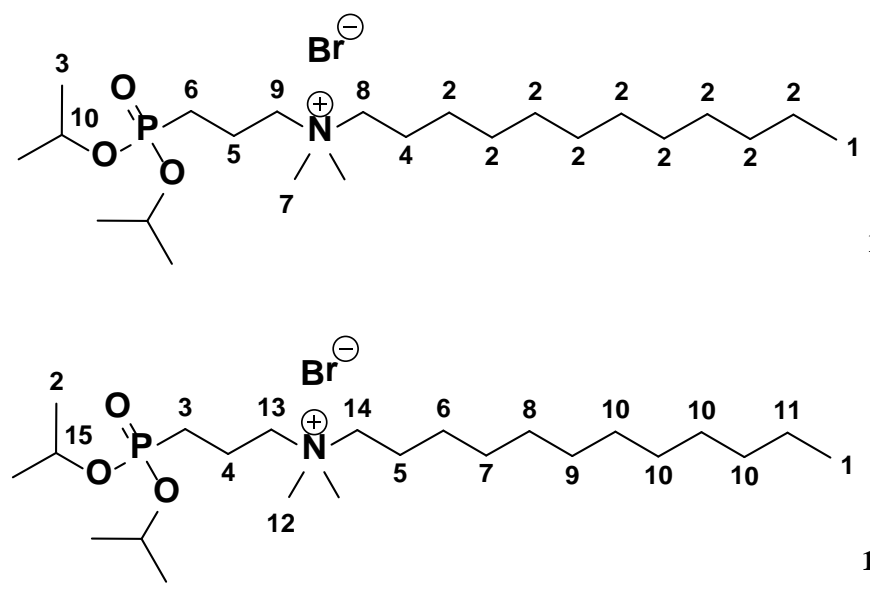

${ }^{1} \mathbf{H}$

${ }^{13} \mathrm{C}$

\section{$N$-(3-(diisopropoxyphosphoryl)propyl)- $N, N$-dimethyldodecan-1-ammonium bromide (30):}

See (Table 2.4, Entry ix). This compound was prepared by the Menshutkin reaction using Method 5.2.1: diisopropylyl (3-bromopropyl)phosphonate 13 (1.13 g, 4.11 mmol, 1.15 eq.) and dimethyldodecylamine DMDA 22 (0.665 g, 3.58 mmol, 1.0 eq.) were refluxed in ACN for 4 hrs. The mixture was then cooled to RT, poured into $20 \mathrm{~mL}$ of $\mathrm{Et}_{2} \mathrm{O}$, and placed into a freezer $\left(-20^{\circ} \mathrm{C}\right)$ for 24 hrs. No precipitation of the title compound was observed so volatiles were evaporated and the crude mixture containing 28\% excess diisopropylyl (3-bromopropyl)phosphonate 13 ( $\delta=$ $28.31 \mathrm{ppm},{ }^{31} \mathrm{P}$ NMR) was partitioned between $30 \mathrm{~mL}$ of $\mathrm{H}_{2} \mathrm{O}$ and $30 \mathrm{~mL}$ DCM and left to settle overnight. In the absence of a clear separation, the volatiles were evaporated and the crude material was chromatographed eluting with acetone / $\mathrm{MeOH}$ (9:1) resulting in a mixed fraction and as a result $\mathbf{3 0}$ was analyzed impure with left over starting material $\mathbf{1 3}$. Purification by further column chromatography was unsuccessful. Crude yield: 95\% (1.70 g). ${ }^{1} \mathbf{H}$ NMR (400 MHz, 
$\mathrm{CDCl}_{3}, \delta$ ): 4.72-4.61 (m, 2H, H10), 3.79-3.73 (m, 2H, H9) 3.52-3.36 (m, 2H, H8), 3.40 (s, 6H, H7), 2.17-1.94 (m, 2H, H6), 1.88-1.67 (m, 4H, H4 + H5), 1.36-1.17 (m, 30H, (H2 + H3 overlap)), 0.86 (t, $J=7.03 \mathrm{~Hz}, 3 \mathrm{H}, \mathrm{H1}) \mathrm{ppm} ;{ }^{13} \mathbf{C} \mathbf{N M R}\left(100 \mathrm{MHz}, \mathrm{CDCl}_{3}, \delta\right): 70.77$ (d, ${ }^{2} J_{C-P}=$ 6.70 Hz, (C15)), 70.19 (C14), 64.33 (C13), 51.27 (C12), 31.87 (C11), 29.60-29.50 (C10), 29.42 (C9), 29.38 (C8), 29.29 (C7), 29.20 (C6), 26.28 (C5), 24.01 (d, ${ }^{2} J_{C-P}=4.29 \mathrm{~Hz},(\mathrm{C} 4)$ ), 22.69 (d, $\left.{ }^{1} J_{C-P}=8.99 \mathrm{~Hz},(\mathrm{C} 3)\right), 16.79(\mathrm{~d}, J=4.78 \mathrm{~Hz},(\mathrm{C} 2)), 14.08$ (C1) ppm; ${ }^{31} \mathbf{P}$ NMR (121.45 MHz, $\left.\mathrm{CDCl}_{3}, \delta\right): 27.04$ ppm.
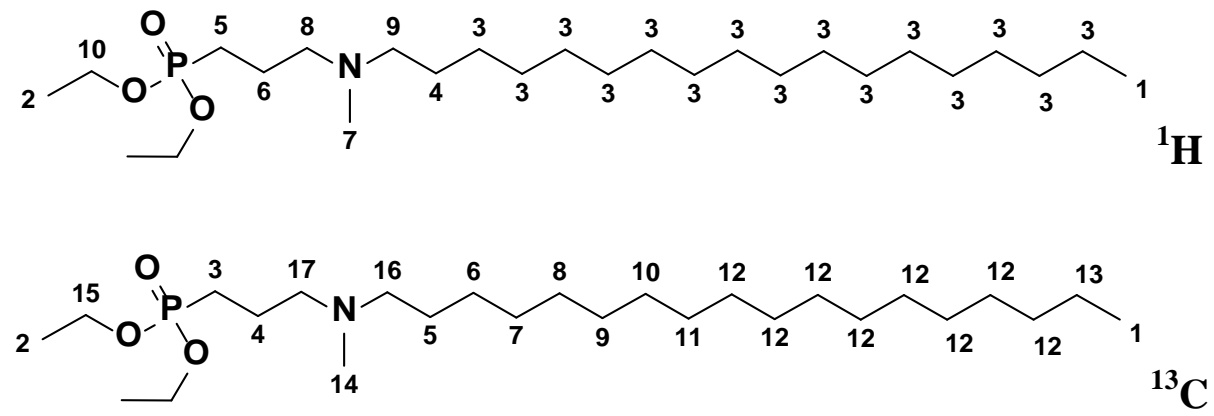

\section{Diethyl (3-(methyl(octadecyl)amino)propyl)phosphonate (31):}

A solution of $N$-methyloctadecylamine $(0.284 \mathrm{~g}, 1 \mathrm{mmol}), N, N$-diisopropylethylamine $(0.26 \mathrm{~mL}$, 1.5 mmol, 1.5 eq.), diethyl (3-bromopropyl)phosphonate 12 (0.2 mL, 1.1mol, 1.1eq.) in $10 \mathrm{~mL}$ ACN were heated (24 hrs). After completion of the reaction (monitored by TLC) the reaction mixture was evaporated to dryness under reduced pressure. The residue was dissolved in DCM (40 mL) and washed with $\mathrm{H}_{2} \mathrm{O}(5 \mathrm{~mL})$. The organic layer was dry packed onto Celite and purified by (DVCC) pre-eluting with acetone $(150 \mathrm{~mL})$ and eluting with 30\% IPA/acetone (300 $\mathrm{mL}$ ). The solvent was removed under reduced pressure to yield $\mathbf{3 1}$ as a white waxy solid. Yield: 90\% (0.363 g). $\mathrm{Mp}=36{ }^{\circ} \mathrm{C} ;{ }^{1} \mathbf{H}$ NMR (400 MHz, $\mathrm{CDCl}_{3}, \delta$ ): 3.50-3.40 (m, 4H, H10), 2.09 (t, $J=$ $6.2 \mathrm{~Hz}, 2 \mathrm{H}, \mathrm{H} 9), 2.00$ (t, $J=7.8 \mathrm{~Hz}, 2 \mathrm{H}, \mathrm{H} 8), 1.81(\mathrm{~s}, 3 \mathrm{H}, \mathrm{H} 7), 1.25-1.14$ (m, 4H, (H5 + H6 
overlap)), 0.97-0.87 (m, 2H, H4), 0.71-0.60 (m, 34H, (H2 + H3 overlap)), 0.24 (t, $J=6.1 \mathrm{~Hz}$, 3H, H1) ppm; ${ }^{13}$ C NMR (100 MHz, $\left.\mathrm{CDCl}_{3}, \delta\right): 61.92\left(\mathrm{~d},{ }^{3} \mathrm{~J}_{\mathrm{C}-\mathrm{P}}=43.40 \mathrm{~Hz},(\mathrm{C} 17)\right), 56.86$ (C16), 56.60 (d, $\left.{ }^{2} J_{C-P}=17.22 \mathrm{~Hz},(\mathrm{C} 15)\right), 40.21$ (C14), 31.69 (C13), 29.44-29.36 (C12 overlap), 29.31 (C11), 29.27 (C10), 29.11 (C9), 26.84 (C8), 25.46 (C7), 23.87 (C6), 22.36 (C4), 21.91 (d $^{2} J_{C-P}=$ $21.91 \mathrm{~Hz},(\mathrm{C} 5)), 18.64$ (d, $\left.{ }^{1} J_{C-P}=4.41 \mathrm{~Hz},(\mathrm{C} 3)\right), 15.35$ (d, $\left.{ }^{3} J_{C-P}=6.04 \mathrm{~Hz},(\mathrm{C} 2)\right), 13.09$ (C1) ppm; ${ }^{31}$ P NMR (121.45 MHz, $\left.\mathrm{CDCl}_{3}, \delta\right): 32.34$ ppm.

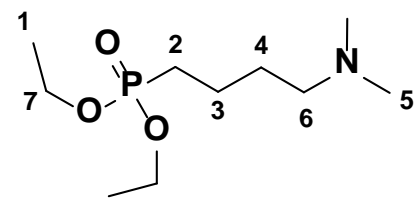

\section{Diethyl (4-(dimethylamino)butyl)phosphonate (32): ${ }^{153}$}

A mixture of diethyl (4-bromobutyl)phosphonate 14 (5.0 g, $18.3 \mathrm{mmol}$ ) with $\mathrm{NHMe}_{2}$ (5.6 $\mathrm{M}$ in EtOH, $10 \mathrm{~mL}$, excess) was placed, with a magnetic stirring bar, into a $20 \mathrm{~mL}$ glass reaction tube and sealed. The reaction mixture was placed in the $\mu \mathrm{W}$ at $110{ }^{\circ} \mathrm{C}(5 \mathrm{~min})$. Volatiles were removed on a rotary evaporator and the crude material purified by DCVC (50 g silica, $3.5 \mathrm{~cm} \times$ $5.5 \mathrm{~cm})$ eluting first with $150 \mathrm{~mL}(10 \% \mathrm{MeOH} /$ acetone) collecting $250 \mathrm{~mL}(10 \% \mathrm{MeOH} / 10 \%$ $\mathrm{NH}_{4}{ }^{+} \mathrm{OH}^{-} / 80 \%$ acetone) and evaporated to a yellow oil. Yield: 81\% (3.55 g); TLC (20\% $\mathrm{NH}_{4}{ }^{+} \mathrm{OH}^{-}$/acetone), $\mathrm{R}_{\mathrm{f}}=0.50 ;{ }^{1} \mathrm{H}$ NMR (400 MHz, $\mathrm{CDCl}_{3}, \delta$ ): 4.06-3.92 (m, 4H, H7), 2.85 (t, 2H, $J=7.96 \mathrm{~Hz}, \mathrm{H6}$ ), 2.62 (s, 6H, H5), 1.83-1.53 (m, 6H, (H4 + H3 + H2 overlap)), 1.22 (t, 6H, $J=7.04 \mathrm{~Hz}, \mathrm{H} 1) \mathrm{ppm} ;{ }^{13} \mathrm{C}$ NMR $\left(100 \mathrm{MHz}, \mathrm{CDCl}_{3}, \delta\right): 61.71\left(\mathrm{~d},{ }^{2} J_{\mathrm{C}-\mathrm{P}}=6.60 \mathrm{~Hz},(\mathrm{C} 7)\right), 57.68$ (C6), 43.58 (C5), 25.68 (t, $\left.{ }^{1} J_{C-P}=14.07 \mathrm{~Hz},(\mathrm{C} 2)\right), 24.13$ (C4), $19.90\left(\mathrm{~d},{ }^{2} J_{C-P}=4.60 \mathrm{~Hz}\right.$, (C3)), $16.41\left(\mathrm{~d},{ }^{3} J_{C-P}=6.22 \mathrm{~Hz},(\mathrm{C} 1)\right) \mathrm{ppm} ;{ }^{31} \mathbf{P}$ NMR $\left(121.45 \mathrm{MHz}, \mathrm{CDCl}_{3}, \delta\right): 30.94 \mathrm{ppm}$. (Agrees well with literature NMR values). ${ }^{153}$ 


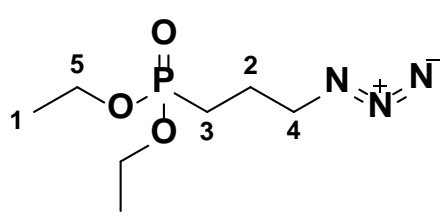

\section{Diethyl (3-azidopropyl)phosphonate (33): ${ }^{229}$}

To a stirred solution of diethyl (3-bromopropyl)phosphonate 12 (1 mL, $5.2 \mathrm{mmol}$ ) in acetone (20 $\mathrm{mL}$ ) was added $\mathrm{NaN}_{3}(0.68,10.4 \mathrm{mmol}, 2$ eq.) and the mixture was refluxed for 12 hrs. After cooling to RT, the mixture was filtered through Celite, washing with acetone and evaporated in vacuo to give the title compound as a yellow oil. Yield: 98\% (1.13 g); TLC (60\% acetone/hexanes), $\mathrm{R}_{\mathrm{f}}=0.50 ;{ }^{1} \mathbf{H}$ NMR (400 MHz, $\left.\mathrm{CDCl}_{3}, \delta\right): 4.16-4.02$ (m, 4H, H5), 3.37 (t, 2H, $J=6.52 \mathrm{~Hz}, \mathrm{H} 4), 1.92-1.74$ (m, 4H, (H3 + H2 overlap)), 1.31 (t, 2H, $J=7.06 \mathrm{~Hz}, \mathrm{H1}) \mathrm{ppm} ;{ }^{13} \mathrm{C}$ NMR (100 MHz, CDCl $3, \delta): 61.64\left(\mathrm{~d},{ }^{2} J_{C-P}=6.59 \mathrm{~Hz},(\mathrm{C} 5)\right), 51.45\left(\mathrm{~d},{ }^{3} J_{C-P}=16.29 \mathrm{~Hz}(\mathrm{C} 4)\right)$, $22.86\left(\mathrm{~d},{ }^{1} J_{C-P}=4.98 \mathrm{~Hz},(\mathrm{C} 3)\right), 22.40\left(\mathrm{~d},{ }^{2} J_{C-P}=143.06 \mathrm{~Hz},(\mathrm{C} 2)\right), 16.43\left(\mathrm{~d},{ }^{2} J_{C-P}=5.91 \mathrm{~Hz}\right.$, (C1)) ppm; ${ }^{31} \mathbf{P}$ NMR $\left(121.45 \mathrm{MHz}, \mathrm{CDCl}_{3}, \delta\right): 30.75 \mathrm{ppm}$. (Agrees well with literature NMR values). ${ }^{229}$

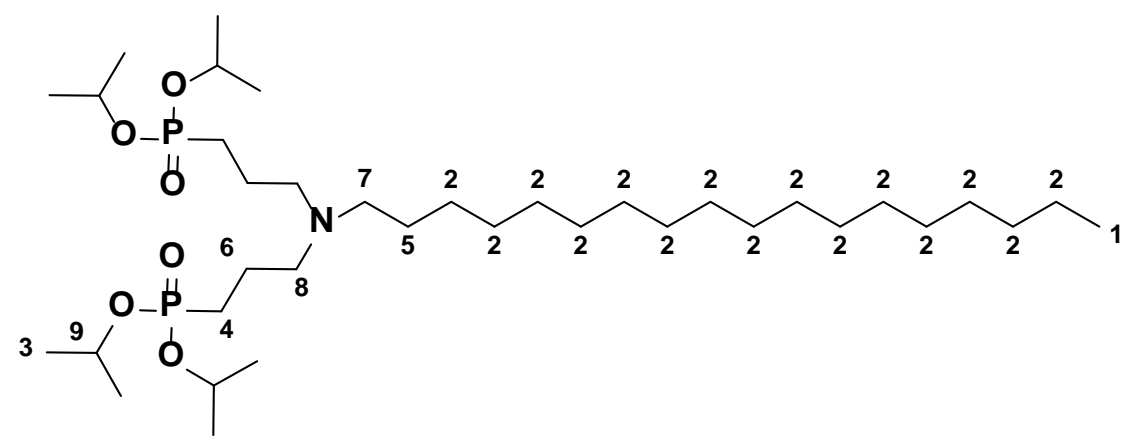

Tetraisopropyl ((octadecylazanediyl)bis(propane-3,1-diyl))bis(phosphonate) (39):

A solution of octadecylamine (1.0 g, $3.7 \mathrm{mmol}), N, N$-diisopropylethylamine $(0.26 \mathrm{~mL}, 10 \mathrm{mmol}$, 2.7 eq.), diethyl (3-bromopropyl)phosphonate 12 (2.34, $8.16 \mathrm{~mol}$, 2.2 eq.) were placed in a 20 
$\mathrm{mL}$ vial, sealed and heated at $110^{\circ} \mathrm{C}(3 \mathrm{hrs})$. After completion of the reaction (monitored by ${ }^{31} \mathrm{P}$ NMR) the reaction was cooled to RT and the orange residue dissolved in DCM (60 mL) and washed with $\mathrm{H}_{2} \mathrm{O}(60 \mathrm{~mL})$. The organic layer was dry packed onto Celite and purified by (DVCC) pre-eluting with 30\% acetone/hexanes (150 mL) and eluting with 10\% $\mathrm{MeOH} /$ acetone (160 mL). The solvent was removed under reduced pressure to yield the title compound as a yellow oil. Note: this compound may be used without further purification in the next reaction. Yield: 96\% (2.43 g). ${ }^{1} \mathbf{H}$ NMR (400 MHz, $\mathrm{CDCl}_{3}, \delta$ ): 4.70-4.59 (m, 4H, H9), 2.44-2.28 (m, 6H, (H8 + H7 overlap)), 1.71-1.59 (m, 10H, (H6 + H5 + H4 overlap)), 1.42-1.14 (m, 54H, (H3 + H2 overlap)), 0.83(t, $J=5.4 \mathrm{~Hz}, 3 \mathrm{H}, \mathrm{H1}) \mathrm{ppm} ;{ }^{31} \mathbf{P}$ NMR (121.45 MHz, $\left.\mathrm{CDCl}_{3}, \delta\right): 30.09$ ppm.

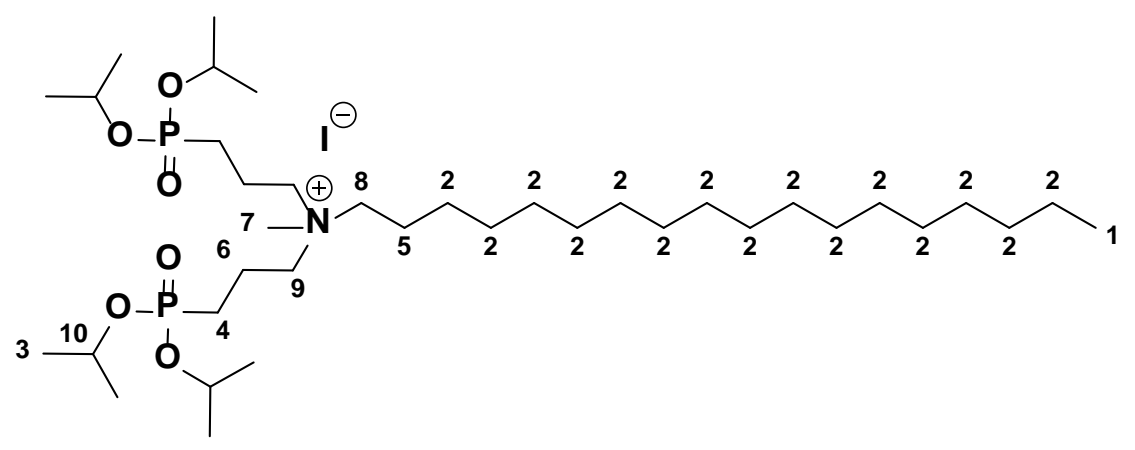

\section{$N, N$-bis(3-(diisopropoxyphosphoryl)propyl)- $N$-methyloctadecan-1-ammonium iodide (40):}

To a solution of 39 ( $4.0 \mathrm{~g}, 5.8 \mathrm{mmol})$ in IPA ( $3 \mathrm{~mL}$ ) was added MeI (0.45 mL, $7 \mathrm{mmol}, 1.2$ eq.), in a $20 \mathrm{~mL}$ glass reaction tube and sealed. The reaction mixture was heated in the $\mu \mathrm{W}$ at $110^{\circ} \mathrm{C}$ (5 min). Volatiles were removed on a rotary evaporator and the crude material purified by DCVC (100 g silica, $4.5 \mathrm{~cm} \times 5.5 \mathrm{~cm})$ pre-eluting first with $200 \mathrm{~mL}(10 \% \mathrm{MeOH} /$ acetone $)$ and collecting $300 \mathrm{~mL}$ (50\% MeOH/ acetone). Yield: 74\% (3.55 g); TLC (50\% MeOH/ acetone), $\mathrm{R}_{\mathrm{f}}$ $=0.30 ;{ }^{1} \mathbf{H}$ NMR (400 MHz, $\mathrm{CDCl}_{3}, \delta$ ): 4.72-4.59 (m, 4H, H10), 3.80-3.62 (m, 4H, H9), 3.403.32 (m, 4H, H8), 3.28 (s, 3H, H7), 2.11-1.96 (m, 4H, H6), 1.87-1.68 (m, 6H, (H4 + H5 
overlap)), 1.40-1.15 (m, 54H, (H3 + H2 overlap)), 0.84 (t, $J=6.9$ Hz, 3H, H1) ppm; ${ }^{31} \mathbf{P}$ NMR (121.45 MHz, $\left.\mathrm{CDCl}_{3}, \delta\right): 27.20 \mathrm{ppm}$.

\subsubsection{Didealkylation of Phosphonate diesters.}
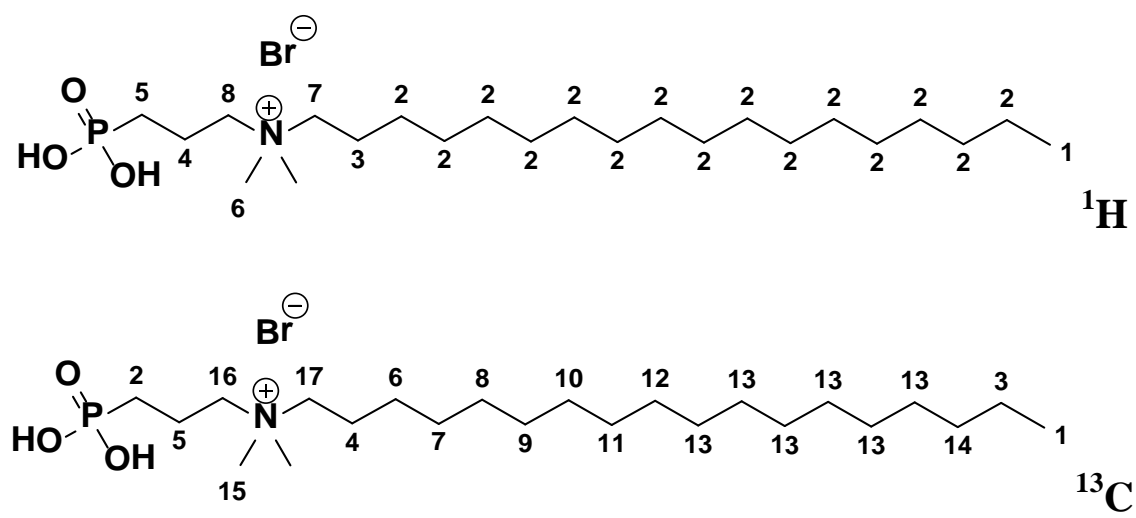

\section{$\boldsymbol{N}, \boldsymbol{N}$-dimethyl- $N$-(3-phosphonopropyl)octadecan-1-ammonium bromide (34): ${ }^{230,231}$}

Inside a flame dried and evacuated $20 \mathrm{~mL}$ screw cap vial $N$-(3-(diethoxyphosphoryl)propyl)$N, N$-dimethyloctadecan-1-ammonium bromide $26(0.27 \mathrm{~g}, 0.46 \mathrm{mmol})$ was dissolved in anhydrous DCM (5 mL). To the clear stirred solution was added TMSBr $(0.25 \mathrm{~mL}, 1.9 \mathrm{mmol}$, 4.0 eq.) through a rubber septum via syringe and the reaction was stirred at RT overnight. Completion of the reaction was followed by ${ }^{31} \mathrm{P}$ NMR spectroscopy after which the reaction was quenched with EtOH (10 mL) and stirred for $1 \mathrm{hr}$ followed by addition of $\mathrm{H}_{2} \mathrm{O}(1 \mathrm{~mL})$. Volatiles were removed with a rotary evaporator connected to a high vacuum Schlenk line and the crude product was centrifuged with $\mathrm{Et}_{2} \mathrm{O}(2 \times 10 \mathrm{~mL})$ to remove brown coloured impurities isolating 41 as a white solid. Yield: $94 \%$ (0.942 g). A small portion of the title compound was recrystallized as clear, long needles from EtOAc/IPA for MS and X-ray analysis. Mp $=118-120{ }^{\circ} \mathrm{C} ;{ }^{1} \mathbf{H}$ NMR (400 MHz, MeOD, $\delta$ ): 3.38-3.33 (m, 2H, H8), 3.28-3.23 (m, 2H, H7), 3.02 (s, 6H, H6), 2.021.90 (m, 2H, H5), 1.75-1.65 (m, 4H, H4, H3), 1.20 (brs, 30H, H3), 0.82 (t, $J=6.9$ Hz, 3H, H1), 
ppm; ${ }^{13} \mathrm{C}$ NMR $\left(\mathrm{CDCl}_{3}, 100 \mathrm{MHz}, \delta\right): 64.24$ (C17), 63.54 (d, $\left.{ }^{1} J_{\mathrm{C}-\mathrm{P}}=16.5 \mathrm{~Hz}, \mathrm{C} 16\right), 49.94$ (C15), 31.68 (C14), 29.45-29.37 (C13 overlap), 29.37 (C12), 29.35 (C11), 29.27 (C10), 29.20 (C9), 29.08 (C8), 29.86 (C7), 26.02 (C6), 23.15 (d, $\left.{ }^{2} J_{C-P}=141.29 \mathrm{~Hz}, \mathrm{C} 5\right), 22.34$ (C4), 22.17 (C3), 16.47 (d, $\left.{ }^{1} J_{C-P}=4.07 \mathrm{~Hz}, \mathrm{C} 2\right), 13.08$ (C1) ppm; ${ }^{31} \mathbf{P}$ NMR (121.45 MHz, $\mathrm{CDCl}_{3}, \delta$ ): 26.92 ppm; HRMS-DART (m/z): [M+] - $\mathrm{Br}^{-}$calculated for $\mathrm{C}_{23} \mathrm{H}_{51} \mathrm{NO}_{3} \mathrm{P}$, 420.3601; found, 420.3608 . (No NMR values reported in the literature). ${ }^{230,231}$

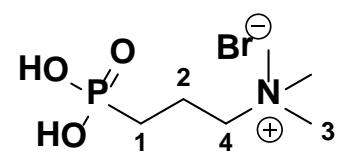

\section{$N, N, N$-trimethyl-3-phosphonopropan-1-ammonium bromide (35):}

Table 2.4 (Entry xiiii). A mixture of diisopropylyl (3-bromopropyl)phosphonate 28 (0.96g, 2.78 mmol) and $\mathrm{HBr}$ (6 M, $1.85 \mathrm{~mL}$, 4 eq.) was placed, with a magnetic stirring bar, into a $5 \mathrm{~mL}$ glass reaction tube and sealed. The reaction mixture was heated in the $\mu \mathrm{W}$ at $140{ }^{\circ} \mathrm{C}(10 \mathrm{~min})$. Volatiles were removed on a rotary evaporator and the crude material was purified by recrystallization from $\mathrm{MeOH} / \mathrm{IPA}(1: 1,20 \mathrm{~mL})$ by slow evaporation of solvent $\mathrm{ON}$ and recovered as white crystals. Yield 80\% (0.582 g); ${ }^{1} \mathbf{H}$ NMR (400 MHz, $\left.\mathrm{CDCl}_{3}, \delta\right)$ : 3.35-3.29 (m, 2H, H4) 3.04 (s, 9H, H3), 2.03-1.91 (m, 2H, H2), 1.77-1.66 (m, 2H, H1) ppm; ${ }^{13} \mathbf{C}$ NMR (100 MHz, $\left.\mathrm{CDCl}_{3}, \delta\right): 66.24\left(\mathrm{dt},{ }^{3} J_{C-P}=2.92 \mathrm{~Hz}, \mathrm{C} 4\right), 52.92\left(\mathrm{t},{ }^{4} J_{C-P}=3.93 \mathrm{~Hz}, \mathrm{C}\right), 22.6\left(\mathrm{~d},{ }^{1} J_{C-P}=\right.$ $137.50 \mathrm{~Hz}, \mathrm{C} 1), 16.69$ (d, $\left.{ }^{2} J_{\mathrm{C}-\mathrm{P}}=3.55 \mathrm{~Hz}, \mathrm{C} 2\right) \mathrm{ppm} ;{ }^{31} \mathbf{P} \mathbf{N M R}\left(121.45 \mathrm{MHz}, \mathrm{CDCl}_{3}, \delta\right): 26.77$ ppm.

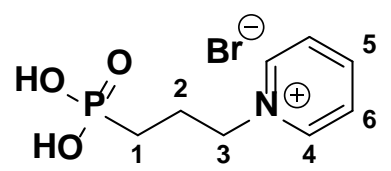




\section{3-phosphonopropan-1-pyridin-1-ium bromide (36):}

This compound was made by a one pot reaction in either ACN/TMSBr or $\mathrm{H}_{2} \mathrm{O} / \mathrm{HBr}$. (See $\mathbf{1 3}$ for procedure, Table 2.4 (Entry xi)). A mixture of $13(0.5 \mathrm{~g}, 1.74 \mathrm{mmol})$ and $\mathrm{HBr}(2 \mathrm{M}, 3.1 \mathrm{~mL}, 4$ eq.) was placed, with a magnetic stirring bar, into a $5 \mathrm{~mL}$ glass reaction tube and sealed. The reaction mixture was heated in the $\mu \mathrm{W}$ at $\left(150^{\circ} \mathrm{C}, 10 \mathrm{~min}\right)$. The aqueous phase was neutralized with $\mathrm{NH}_{4}{ }^{+} \mathrm{OH}^{-}(2 \mathrm{~mL})$ in $20 \mathrm{~mL} \mathrm{H} \mathrm{H}_{2} \mathrm{O}$ and washed with $\mathrm{CHCl}_{3}(10 \mathrm{~mL})$ followed by evaporation of the organic phase (100 mL ACN co-evap) to a solid which was was purified by recrystallization from $\mathrm{MeOH} / \mathrm{IPA}(2: 1,30 \mathrm{~mL})$ by slow evaporation of solvent $\mathrm{ON}$ and recovered as white crystals. Yield 90\% (0.441 g); ${ }^{1} \mathbf{H}$ NMR (400 MHz, $\left.\mathrm{CDCl}_{3}, \delta\right): 8.78$ (t, $J=$ $5.52 \mathrm{~Hz}, 2 \mathrm{H}, \mathrm{H6}$ ), 8.48 (t, $J=7.0 \mathrm{~Hz}, 1 \mathrm{H}, \mathrm{H} 5), 7.99$ (t, $J=5.60 \mathrm{~Hz}, 2 \mathrm{H}, \mathrm{H} 4), 4.80-4.60$ (m, 2H, H3 overlap w $\mathrm{D}_{2} \mathrm{O}$ ), 2.27-2.14 (m, 2H, H2), 1.77-1.66 (m, 2H, H1) ppm; ${ }^{13}$ C NMR (100 MHz, $\left.\mathrm{CDCl}_{3}, \delta\right){ }^{13} \mathrm{C}$ NMR (100 MHz, $\mathrm{CDCl}_{3}, \delta$ ): 147.20 (C5), 144.78 (C6), 128.33 (C4), 61.35 (d, ${ }^{3} J_{C-}$ $\left.{ }_{P}=18.35 \mathrm{~Hz},(\mathrm{C} 3)\right), 25.04\left(\mathrm{~d},{ }^{2} J_{\mathrm{C}-\mathrm{P}}=4.38 \mathrm{~Hz},(\mathrm{C} 2)\right), 23.21\left(\mathrm{~d},{ }^{1} J_{\mathrm{C}-\mathrm{P}}=139.29 \mathrm{~Hz},(\mathrm{C} 1)\right) \mathrm{ppm} ;{ }^{31} \mathbf{P}$ NMR (121.45 MHz, $\left.\mathrm{CDCl}_{3}, \delta\right): 26.99 \mathrm{ppm}$. HRMS-DART (m/z): $\left[\mathrm{M}^{+}\right]-\mathrm{Br}^{-}$calculated for $\mathrm{C}_{8} \mathrm{H}_{13} \mathrm{NO}_{3} \mathrm{P}$, 202.0628; found, 202.0624. 

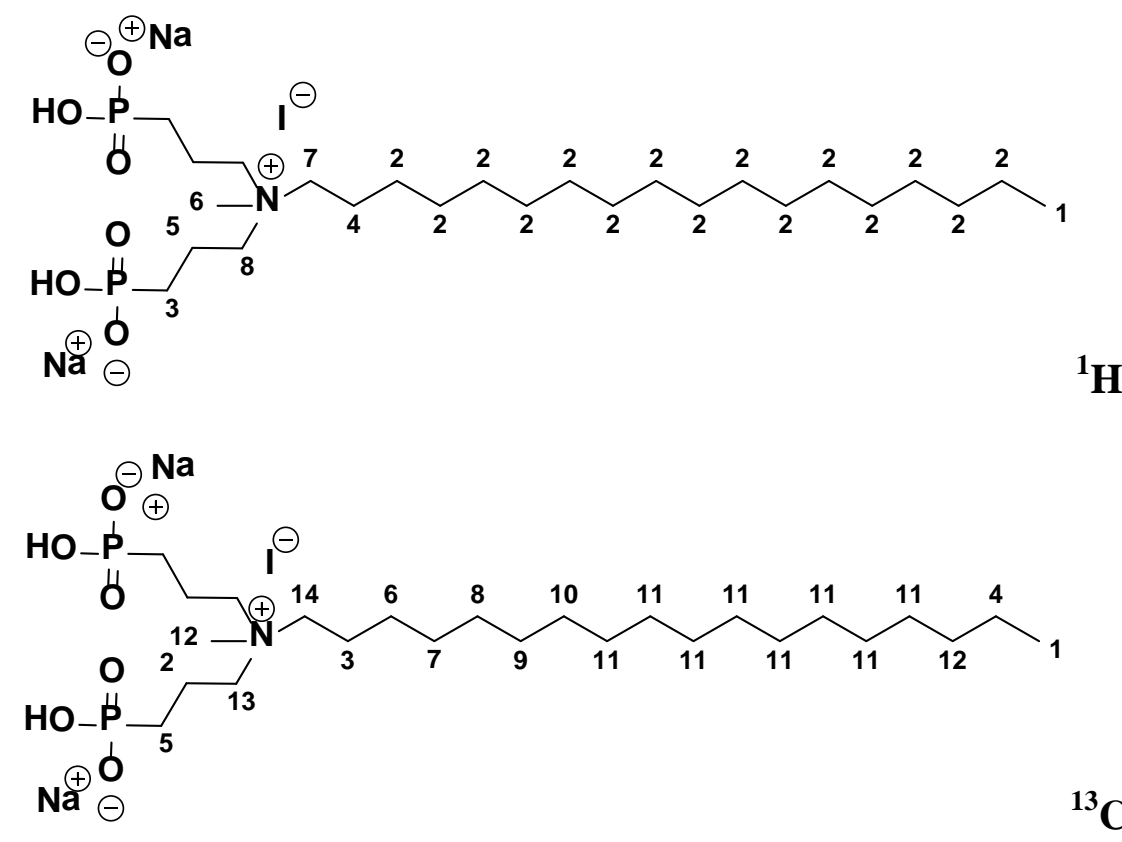

Sodium ((methyl(octadecyl)ammonio)bis(propane-3,1-diyl))bis(hydrogenphosphonate) iodide (42):

A mixture of 41 (3.288 g, $4.82 \mathrm{mmol})$ and $\operatorname{HBr}(2 \mathrm{M}, 8.4 \mathrm{~mL}, 3.5$ eq.) were placed, with a magnetic stirring bar, into a $10 \mathrm{~mL}$ glass reaction tube and sealed. The reaction mixture was heated in the $\mu \mathrm{W}$ at $150{ }^{\circ} \mathrm{C}(10 \mathrm{~min})$. Volatiles were removed on a rotary evaporator and the crude material was purified by adding $\mathrm{NaOH}$ (2eq. in $10 \mathrm{~mL} \mathrm{H}_{2} 0$ ) to make the monosodium salt of the bisphosphonic acid. Evaporation of $\mathrm{H}_{2} \mathrm{O}$ gave the title compound as a white solid. Yield: 90\% (3.15 g); ${ }^{1}$ H NMR (400 MHz, $\mathrm{D}_{2} \mathrm{O}, \delta$ ): 3.34-3.16 (m, 4H, (H8 + H7 overlap)), 2.97 (s, 3H, H6), 1.97-1.83 (m, 2H, H5), 1.71-1.61 (m, 2H, H4), 1.50-1.40 (C3),1.23 (brs, 30H, H2), 0.83 (t, $J=5.6 \mathrm{~Hz}, 3 \mathrm{H}, \mathrm{H} 1), \mathrm{ppm} ;{ }^{13} \mathrm{C}$ NMR $\left(\mathrm{D}_{2} \mathrm{O}, 100 \mathrm{MHz}, \delta\right): 62.52$ (C15), $61.85\left(\mathrm{~d},{ }^{3} J_{\mathrm{C}-\mathrm{P}}=19.94\right.$ Hz, C14), 47.96 (C13), 32.00 (C12), 30.20-29.85 (C11 overlap), 29.81 (C10), 29.65 (C9), 29.49 (C8), 29.21 (C7), 26.26 (C6), 25.02 (d, $\left.{ }^{1} J_{C-P}=132.27 \mathrm{~Hz}, \mathrm{C} 5\right), 22.68$ (C4), 22.01 (C3), 17.24 (C2), 13.96 (C1) ppm; ${ }^{31}$ P NMR (121.45 MHz, $\mathrm{D}_{2} \mathrm{O}, \delta$ ): 21.11 ppm. 


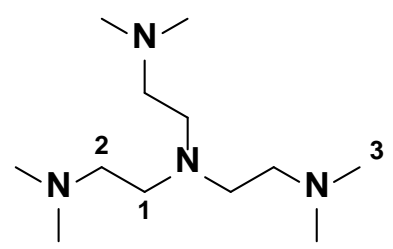

$N^{1}, N^{1}$-bis(2-(dimethylamino)ethyl)- $N^{2}, N^{2}$-dimethylethane-1,2-diamine (44): ${ }^{199}$

This compound was made by a modification of the literature procedure ${ }^{199}$ by employing paraformaldehyde and anydrous $\mathrm{HCl}$. ACN (200 mL) was added to a $500 \mathrm{~mL}$ RBF containing tris(2-aminoethyl)amine (7.3115 g, $50 \mathrm{mmol})$ and paraformaldehyde (10 g, $333 \mathrm{mmol}, 6.66$ eq.) followed by formic acid (17 mL, $295 \mathrm{mmol}, 5.9$ eq.) at RT, and the mixture was refluxed for 3 hrs. The mixture was then cooled to RT, placed on ice and anydrous HCl (2 N in EtOH/IPA, 100 $\mathrm{mL}$ ) was added, and a white precipitate was isolated. The residual solid was diluted with $\mathrm{MeOH}$ (200 mL), filtered through a Buchner funnel and washed twice with $\mathrm{MeOH}(2 \times 100 \mathrm{~mL})$ to give tris ( $N, N$-dimethyl-2-aminoethyl)amine hydrochloride as a yellow/white solid (12 g, 70\%). Next, tris ( $N, N$-dimethyl-2-aminoethyl)- amine hydrochloride (2.0 g, $6.89 \mathrm{mmol})$ was added slowly to $50 \mathrm{~mL}$ of an ethanolic solution containing NaOEt (1.4g, $20.67 \mathrm{mmol})$, and stirred at RT for $1 \mathrm{hr}$. The mixture was filtered through Celite, evaporated in vacuo, $\mathrm{CHCl}_{3}$ was added to the residue, and the solution was filtered to remove more of the inorganic precipitate $(\mathrm{NaCl})$. The evaporation of the solvent on a Schlenk line gave the title compound as a brown liquid. Yield: 65\% (7.49 g); ${ }^{1} \mathbf{H}$ NMR (400 MHz, $\mathrm{CDCl}_{3}, \delta$ ): 3.28-3.19 (m, 6H, H3), 2.98-2.85 (m, 6H, H2), 2.81 (s, 18H, H1) ppm. ${ }^{13} \mathrm{C}$ NMR (100 MHz, $\mathrm{CDCl}_{3}, \delta$ ): 53.89 (C3), 47.24 (C2), 43.16 (C1) ppm. (Agrees well with literature NMR values). ${ }^{199}$ 


\subsection{General Procedures for the Synthesis of a-CH Bisphosphonic Acids QAC Antimicrobials ( $\alpha$-CH-BPQA)}

\subsubsection{Method 1: Bis Addition of Dialkylphosphites to Aldehydes}

\subsubsection{Sequential Addition}

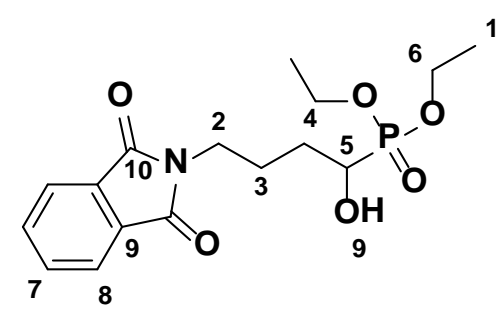

Diethyl (4-(1,3-dioxoisoindolin-2-yl)-1-hydroxybutyl) phosphonate (51):

A $25 \mathrm{~mL}$ round bottom flask, equipped with a magnetic stir bar and a condenser was charged with the aldehyde 48 (2.28 g, $10.5 \mathrm{mmol})$, diethylphosphonate (1.52 g, $11.0 \mathrm{mmol}, 1.05$ eq.), $\mathrm{K}_{2} \mathrm{CO}_{3}(0.073 \mathrm{~g}, 0.53 \mathrm{mmol}, 0.05$ eq.) and ACN (5 mL). The heterogeneous solution was stirred at $60^{\circ} \mathrm{C}$ for $15 \mathrm{~min}$ at which point TLC showed disappearance of the starting aldehyde $(60 \%$ EtOAc in hexanes, $10 \mathrm{~mL}$ ). The reaction was cooled to $0^{\circ} \mathrm{C}$, filtered and evaporated. The resulting yellow oil solidified under high vacuum $(10 \mathrm{~min})$ and was recrystallized from hot EtOAc (5 mL) after cooling for $20 \mathrm{~min}$ at $0^{\circ} \mathrm{C}$. Yield 69.1\% (2.578 g); TLC (60\% EtOAc in hexanes), $\mathrm{R}_{\mathrm{f}}=0.2 ; \mathrm{Mp}=91-94^{\circ} \mathrm{C} ;{ }^{1} \mathbf{H}$ NMR $\left(400 \mathrm{MHz}, \mathrm{CDCl}_{3}, \delta\right): 7.83-7.79$ (m, 4H, H8), 7.717.64 (m, 4H, H7), 4.18-4.07 (m, 4H, H6), 3.89 (quintet, $J=4.59$ Hz, 1H, H5), 3.77-3.66 (m, 2H, H4), 2.05-1.95 (m, 2H, H3), 1.87-1.68 (m, 2H, H2), 1.29 (t, $J=7.08 \mathrm{~Hz}, 6 \mathrm{H}, \mathrm{H1}) \mathrm{ppm} ;{ }^{13} \mathrm{C}$ NMR (100 MHz, $\mathrm{CDCl}_{3}, \delta$ ): 168.37 (C10), 133.90 (C9), 132.10 (C7), 123.18 (C8), 68.12 (C5), $62.65\left(\mathrm{q}^{2}, J_{\mathrm{C}-\mathrm{P}}=7.3 \mathrm{~Hz}, \mathrm{C} 6\right), 37.52$ (C2), $28.43\left(\mathrm{~d},{ }^{1} J_{\mathrm{C}-\mathrm{P}}=1.45 \mathrm{~Hz}, \mathrm{C} 5\right), 25.02$ (C3), 24.96 (C4), 
$16.46\left(\mathrm{~d},{ }^{3} \mathrm{~J}_{\mathrm{C}-\mathrm{P}}=5.20 \mathrm{~Hz}, \mathrm{C1}\right) \mathrm{ppm} ;{ }^{31} \mathbf{P}$ NMR $\left(121.45 \mathrm{MHz}, \mathrm{CDCl}_{3}, \delta\right): 24.64 \mathrm{ppm}$. ESI-TOF (m/z): $\left[\mathrm{M}^{+}\right]$calculated for $\mathrm{C}_{16} \mathrm{H}_{22} \mathrm{NO}_{6} \mathrm{P}$, found, 434.1.

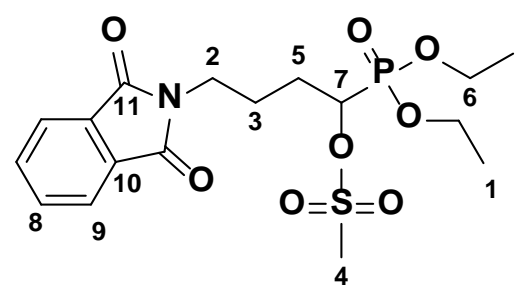

Example 14 - 1-(diethoxyphosphoryl)-4-(1,3-dioxoisoindolin-2-yl) butyl methanesulfonate (52):

To a flame dried and evacuated $50 \mathrm{~mL}$ round bottom flask, equipped with a magnetic stir bar was added sequentially $\mathrm{NMe}_{3} \cdot \mathrm{HCl}\left(0.062 \mathrm{~g}, 0.62 \mathrm{mmol}, 0.20\right.$ eq.), $\mathrm{DCM}(2 \mathrm{~mL}), \mathrm{Et}_{3} \mathrm{~N}(0.65 \mathrm{~mL}, 4.63$ mmol, 1.5 eq.) and $51(1.097 \mathrm{~g}, 3.09 \mathrm{mmol})$ and the solution was cooled to $0^{\circ} \mathrm{C}$ in an ice bath. To the chilled stirred solution was added, dropwise, mesyl chloride $(0.25 \mathrm{~mL}, 3.70 \mathrm{mmol}, 1.2$ eq.) in anhydrous DCM (2 mL) and the cloudy yellow mixture was stirred for $20 \mathrm{~min}$ at RT at which point TLC showed disappearance of the starting amine (10\% MeOH in EtOAc, $10 \mathrm{~mL})$. The reaction was diluted with $\mathrm{H}_{2} \mathrm{O}(1 \times 10 \mathrm{~mL})$ and extracted with DCM $(2 \times 5 \mathrm{~mL}$ total $)$, the combined organic layers were dried over $\mathrm{MgSO}_{4}$, filtered and evaporated to give a yellow oil. The crude product (1.409 g) containing traces of DCM and excess mesyl chloride by ${ }^{1} \mathrm{H}$ NMR, was placed under high vacuum at $60^{\circ} \mathrm{C}$ for $1 \mathrm{hr}$. Yield 93\% $(1.201 \mathrm{~g})$; TLC $(10 \% \mathrm{MeOH}$ in EtOAc), $\mathrm{R}_{\mathrm{f}}=0.5 ;{ }^{1} \mathbf{H}$ NMR (400 MHz, $\left.\mathrm{CDCl}_{3}, \delta\right): 7.85-7.81(\mathrm{~m}, 2 \mathrm{H}, \mathrm{H} 9), \quad 7.73-7.70(\mathrm{~m}, 2 \mathrm{H}$, H8), 4.94-4.88 (m, 1H, H7), 4.20-4.15 (m, 4H, H6), 3.77-3.70 (m, 2H, H5), 3.15 (s, 3H, H4), 1.95-1.82 (m, 4H, H2, H3), 1.41-1.25 (m, 6H, H1) ppm; ${ }^{13} \mathrm{C}$ NMR (100 MHz, CDCl $\left.3, \delta\right)$ : 168.26 (C11), 133.98 (C10), 132.08 (C9), 123.22 (C8), 74.79 (C7), 63.31 (q, ${ }^{2} J_{\text {C-P }}=7.3$ Hz, C6), 
52.56 (C2) , 39.11 (C4), 27.58 (C3), $24.45\left(\mathrm{~d},{ }^{2} J_{\mathrm{C}-\mathrm{P}}=11.67 \mathrm{~Hz}\right.$ C5), $16.45\left({ }^{2} J_{\mathrm{C}-\mathrm{P}}=5.20 \mathrm{~Hz}, \mathrm{C} 1\right)$ ppm; ${ }^{31}$ P NMR (121.45 MHz, $\left.\mathrm{CDCl}_{3}, \delta\right): 17.63$ ppm.

\subsubsection{Synthesis of Aldehyde Precursors}
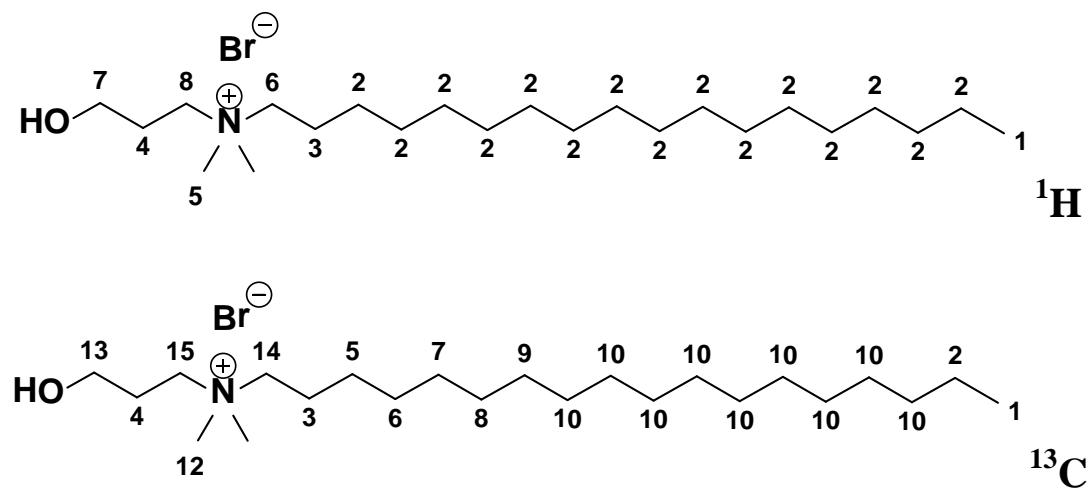

\section{$N$-(3-hydroxypropyl)- $N, N$-dimethyloctadecan-1-ammonium (66): ${ }^{232}$}

This compound was prepared by the Menshutkin reaction using Method 5.2.1: 3(dimethylamino)propan-1-ol (0.515 g, 5 mmol) and 1-bromooctadecane (1.667 g, 5 mmol, 1.0 eq.) were reacted at $100^{\circ} \mathrm{C}$ neat for $1 \mathrm{hr}$ until the mixture solidified. The mixture was then dissolved in $\mathrm{MeOH}(40 \mathrm{~mL})$ and hot filtered from charcoal. Volatiles were removed on a rotary evaporator and the crude was recrystallized from $\mathrm{MeOH} / \mathrm{ACN}$ (1:3, $40 \mathrm{~mL}$ ) by slow evaporation over a few d. as a brittle crystalline solid. A small sample was used to obtain a crystal structure. Yield: $90 \%$ (1.962 g). $\mathrm{Mp}=93-95^{\circ} \mathrm{C} ;{ }^{1} \mathbf{H}$ NMR $\left(400 \mathrm{MHz}, \mathrm{CDCl}_{3}, \delta\right): 3.74-3.68$ (m, 4H, (H8 + H7 overlap)), 3.43-3.37 (m, 2H, H6), 3.27 (s, 6H, H5), 2.07-1.99 (m, 2H, H4), 1.75-1.67 (m, 2H, H3), 1.21 (brs, 30H, H2), 0.84 (t, $J=7.0 \mathrm{~Hz}, 3 \mathrm{H}, \mathrm{H1}) \mathrm{ppm} ;{ }^{13} \mathbf{C}$ NMR (100 MHz, $\left.\mathrm{CDCl}_{3}, \delta\right)$ : 64.59 (C15), 62.65 (C14), 58.20 (C13), 51.24 (C12), 31.89 (C11), 29.73-29.60 (C10 overlap), 29.51 (C9), 29.45 (C8), 29.33 (C7), 29.24 (C6), 26.33 (C5), 25.98 (C4), 22.82 (C3), 22.66 (C2), 
14.09 (C1) ppm. HRMS-DART (m/z): $\left[\mathrm{M}^{+}\right]$- Br calculated for $\mathrm{C}_{23} \mathrm{H}_{50} \mathrm{NO}$, 356.3886; found, 356.3891. (Agrees well with literature NMR values). ${ }^{232}$
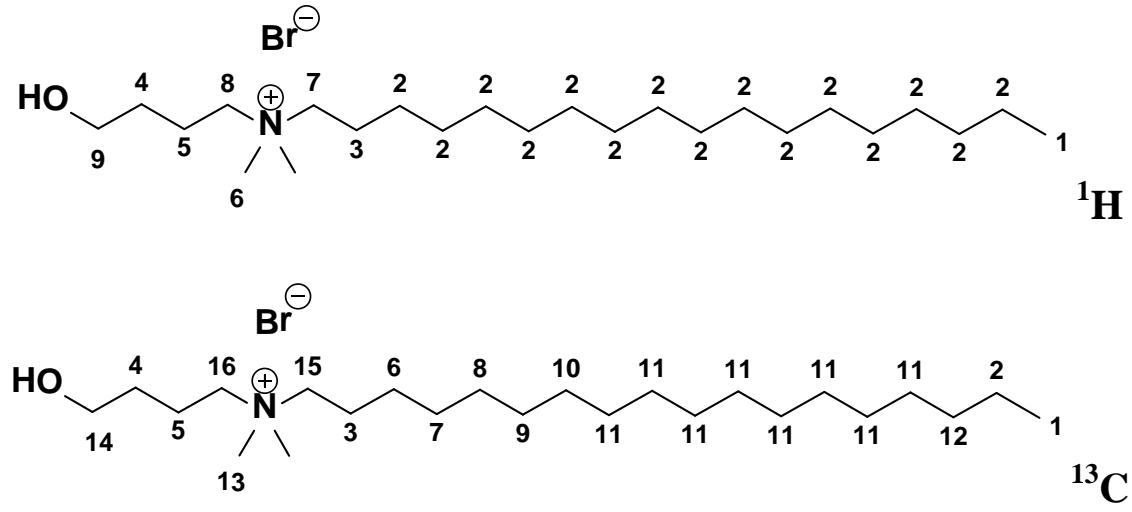

\section{$N$-(4-hydroxybutyl)- $N, N$-dimethyloctadecan-1-ammonium bromide (67):}

This compound was prepared by the Menshutkin reaction using method 5.2.1, 4(dimethylamino)butan-1-ol (0.586 g, 9.8 mmol) and 1-bromooctadecane (1.667 g, 9.8 mmol, 1.0 eq.) were reacted at $110^{\circ} \mathrm{C}$ neat for 20 min until the mixture solidified The mixture was then dissolved in EtOH (20 mL) and hot filtered from charcoal. Volatiles were removed on a rotary evaporator and the crude was recrystallized from acetone (20 mL) by slow evaporation after 20 min as fine white crystalline needles. Yield: 63\% (2.8 g). $\mathrm{Mp}=94^{\circ} \mathrm{C} ;{ }^{1} \mathbf{H} \mathbf{~ N M R}(400 \mathrm{MHz}$, MeOD, $\delta$ ): 3.64 (t, $J=6.13 \mathrm{~Hz}, 2 \mathrm{H}, \mathrm{H9}), 3.39-3.31$ (m, 4H, (H7 + H8 overlap)), 3.11 (s, 6H, H6), 1.89-1.80 (m, 4H, (H5 + H4 overlap)), 1.64-1.57 (m, 2H, H3), 1.30 (brs, 30H, H2), 0.91 (t, $J=7.0 \mathrm{~Hz}, 3 \mathrm{H}, \mathrm{H1}$ ) ppm; ${ }^{13} \mathrm{C}$ NMR (100 MHz, MeOD, $\delta$ ): 63.99 (C16), 63.70 (C15), 60.43 (C14), 49.86 (C13), 31.69 (C12), 29.42-29.35 (C11 overlap), 29.28 (C10), 29.20 (C9), 29.10 (C8), 28.87 (C7), 28.68 (C6), 26.03 (C5), 22.36 (C4), 22.18 (C3),18.98 (C2), 13.10 (C1) ppm. 


\subsubsection{Method 2: Michael Addition to Diethylvinylphosphonate}

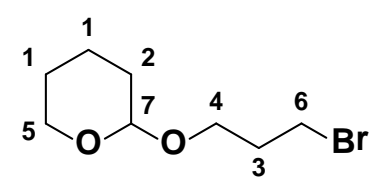

\section{2-(3-bromopropoxy)tetrahydro-2H-pyran (9): ${ }^{233}$}

To a stirred solution inside a $125 \mathrm{~mL}$ round bottom flask containing 3-bromo-1-propanol (6.95 g, 50 mmol, 1 eq.) in DCM (25 mL) was added 3,4-dihydropyran (5.93 mL, 65 mmol, 1.3 eq.). The mixture was stirred overnight at RT at which point TLC showed disappearance of 3-bromo-1propanol (20\% EtOAc in hexanes, $10 \mathrm{~mL}, \mathrm{KMnO}_{4}$ ). The reaction was evaporated and the crude material was purified by flash chromatography on silica gel $(20 \mathrm{~g}$ silica, $1.5 \mathrm{~cm}$ i.d) eluting with 10\% EtOAc: hexanes $(100 \mathrm{~mL})$ to obtain the title compound as a clear oil. Yield: $86.4 \%$ (9.637 g); TLC (20\% EtOAc in hexanes), $\mathrm{R}_{\mathrm{f}}=0.85 ;{ }^{1} \mathbf{H}$ NMR (400 MHz, $\left.\mathrm{CDCl}_{3}, \delta\right): 4.59$ (t, $1 \mathrm{H}, J=$ 3.52 Hz, H7), 3.90-3.81 (m, 2H, H6), 3.55-3.47 (m, 4H, (H4, H5)), 2.16-2.08 (m, 2H, H3), 1.90-

1.64 (m, 2H, H2), 1.57-1.50 (m, 4H, H1) ppm; ${ }^{13} \mathrm{C}$ NMR (100 MHz, $\mathrm{CDCl}_{3}, \delta$ ): 98.90 (C7), 64.88 (C6), 62.26 (C5), 32.90 (C3), 30.59 (d, ${ }^{2} J=6.04$ Hz, C4), 25.41 (C2), 19.48 (C1) ppm. (Agrees well with literature NMR values). ${ }^{233}$

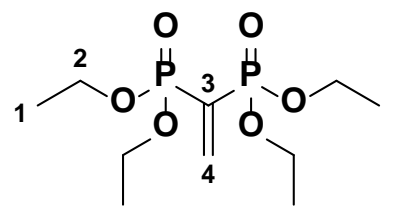

\section{Tetraethyl ethene-1,1-diylbis(phosphonate) (68): ${ }^{234}$}

A $50 \mathrm{~mL}$ round bottom flask was charged with paraformaldehyde (6.3 g, $200 \mathrm{mmol}, 4.0$ eq.) and $\mathrm{HNEt}_{2}$ (5.2 mL, $50 \mathrm{mmol}, 1$ eq.) in $\mathrm{MeOH}(125 \mathrm{~mL})$ and the mixture was stirred under reflux until a clear solution was obtained ( 5 min). Tetraethylmethylene bisphosphonate was added via 
syringe (12.4 mL, $50 \mathrm{mmol}, 1.0$ eq.) and the solution was refluxed overnight ( $24 \mathrm{hrs})$. The clear solution was concentrated in vacuo and then re-evaporated from TOL $(2 \times 10 \mathrm{~mL})$ completely removing residual $\mathrm{MeOH}$ to give the intermediate methyl ether as a clear oil. The residue was dissolved in TOL (100 mL), treated with p-toluenesulphonic acid (38 mg, $0.02 \mathrm{mmol}$ ), and refluxed through a Dean-Stark trap overnight. The orange solution was concentrated in vacuo, dissolved in $\mathrm{CHCL}_{3}(50 \mathrm{~mL})$, washed with $\mathrm{H}_{2} \mathrm{O}(2 \times 10 \mathrm{~mL})$, dried over $\mathrm{MgSO}_{4}$, and concentrated in vacuo. A portion of the orange oil (6 g) was further distilled under high vacuum. Yield: 90\% (5.40 g); TLC (EtOAc), $\mathrm{R}_{\mathrm{f}}=0.2 ;{ }^{1} \mathbf{H}$ NMR (400 MHz, $\left.\mathrm{CDCl}_{3}, \delta\right)$ : 7.02-6.86 (m, H3, 2H), 4.10-4.05 (m, H2, 8H), 1.34-1.21 (m, H1, 12H) ppm; ${ }^{13}$ C NMR (100 MHz, $\mathrm{CDCl}_{3}, \delta$ ): 149.04 (m, C4), 133.77-129.71 (m, C3), $62.54\left(\mathrm{t},{ }^{2} J_{\mathrm{C}-\mathrm{P}}=2.88 \mathrm{~Hz}, \mathrm{C} 2\right), 16.17\left(\mathrm{t},{ }^{3} J_{\mathrm{C}-\mathrm{P}}=3.15 \mathrm{~Hz}\right.$, C1) ppm; ${ }^{31} \mathbf{P}$ NMR (121.45 MHz, $\mathrm{CDCl}_{3}, \delta$ ): $21.0 \mathrm{ppm}$. (Agrees well with literature NMR values). ${ }^{234}$

\subsubsection{Method 3: C-P Bond Formation}

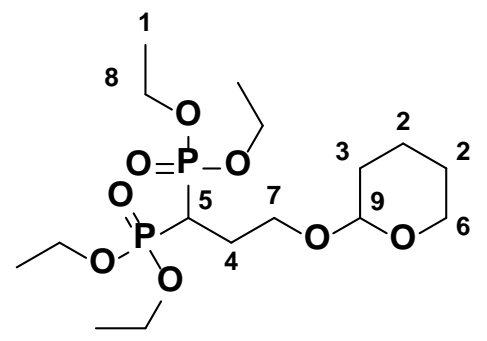

Tetraisopropyl (3-((tetrahydro-2H-pyran-2-yl)oxy)propane-1,1-diyl)bis(phosphonate) (77 ): $: 35$

To a solution of freshly prepared LDA ( $n$-BuLi (1.6 M in hexane, $4.93 \mathrm{~mL}, 7.88 \mathrm{mmol}$ ) and $\mathrm{HN}\left(i \mathrm{Pr}_{2}\right)(1.12 \mathrm{~mL}, 7.97 \mathrm{mmol})$ in anhydrous THF $\left.(10 \mathrm{~mL})-78{ }^{\circ} \mathrm{C}\right)$ was added $18(1.10 \mathrm{~g}, 3.94$ mmol) in THF ( $5 \mathrm{~mL})$. After $0.5 \mathrm{~h}$, diethyl chlorophosphate $(0.84 \mathrm{~mL}, 4.73 \mathrm{mmol})$ was added 
dropwise via syringe, and the mixture stirred for $1 \mathrm{hr}$ at $-78{ }^{\circ} \mathrm{C}$ and $30 \mathrm{~min}$ at $\mathrm{RT}$. The reaction was quenched with $\mathrm{NH}_{4} \mathrm{Cl}_{\text {sat }}(10 \mathrm{~mL})$ and extracted with EtOAc $(1 \times 10 \mathrm{~mL})$. The organic layer separated, dried over $\mathrm{MgSO}_{4}$, evaporated in vacuo and the crude product (in a 0.8:0.2 ratio with excess unreacted diethyl chlorophosphate) was loaded onto silica (10 g, EtOAc, $15 \mathrm{~mL}$ ) and purified by column chromatography on silica gel $(50 \mathrm{~g})$ pre-eluting with EtOAc $(1 \times 50 \mathrm{~mL})$ to remove unextracted diethyl chlorophosphate. The column was eluted with MeOH:EtOAc (20\%, $60 \mathrm{~mL})$ to afford the title compound as a light yellow oil. Yield: 74\% (0.741 g); TLC (10\% MeOH:EtOAc), Rf = 0.30; ${ }^{1} \mathbf{H}$ NMR (400 $\left.\mathrm{MHz} \mathrm{CDCl}_{3}, \delta\right): 4.59-4.55$ (m, 1H, H9), 4.22-4.06 (m, 2H, H8), 3.90-3.77 (m, 2H, H7), 3.62-3.42 (m, 2H, H6), 2.66-2.48 (m, 1H, H5), 2.26-2.09 (m, 2H, H4), 1.82-1.61 (m, 2H, H3), 1.58-1.42 (m, 4H, H2), 1.33 (t, $J=7.03 \mathrm{~Hz}, 12 \mathrm{H}, \mathrm{H} 1) ;{ }^{13} \mathrm{C}$ NMR (100 MHz, $\mathrm{CDCl}_{3}, \delta$ ): 98.57 (C9), 65.13 (C7), 62.63-63.39 (m, C8), 62.19 (C6), 32.81 (t, ${ }^{1} J_{C-P}=134.7 \mathrm{~Hz}$, (C6)), 30.59 (C3), 25.40 (C4), 19.46 (C2), 16.34 (t, $\left.{ }^{3} J_{C-P}=2.81 \mathrm{~Hz},(\mathrm{C} 2)\right) \mathrm{ppm}$; ${ }^{31} \mathbf{P}$ NMR (121.45 MHz, $\mathrm{CDCl}_{3}, \delta$ ): 23.77 ppm. (Agrees well with literature NMR values). ${ }^{235}$

\subsubsection{Method 4: Alkylation of Methylenebisphosphonate}

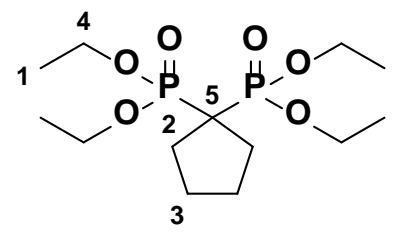

\section{Tetraethyl cyclopentane-1,1-diylbis(phosphonate) (97): ${ }^{236}$}

To a stirred solution (250 mL Schlenk flask, under argon) of tetraethyl methylidene bisphosphonate (7.45 mL, $30 \mathrm{mmol}, 1.0$ eq.) in DMSO $(60 \mathrm{~mL})$ at $0^{\circ} \mathrm{C}$ was added excess solid $\mathrm{NaH}$ (60\% dispersion in mineral oil, $2.4 \mathrm{~g}, 60 \mathrm{mmol}, 2.0$ eq.) portionwise. The reaction was brought to RT and stirred (30 min), washed with more DMSO (60 mL) and evacuated and 
backfilled with $\operatorname{Ar}$ (2 cycles).When hydrogen gas evolution ceased and the mixture cleared ( $\sim 0$ min later), 1,4-dibromobutane (5.37 mL, $45.0 \mathrm{mmol}, 1.5$ eq.) was added and the resulting yellow orange mixture was stirred overnight at RT. The solution was then cooled to RT, neutralized with $\mathrm{NH}_{4}{ }^{+} \mathrm{Cl}^{-}$sat: $\mathrm{H}_{2} \mathrm{O}(1: 3,400 \mathrm{~mL})$ and extracted with EtOAc $(1 \times 400 \mathrm{~mL}, 1 \times 300 \mathrm{~mL})$. The organic phases were washed with brine $(1 \times 50 \mathrm{~mL})$, dried over $\mathrm{MgSO}_{4}$, filtered and concentrated in vacuo. The crude product (only one peak by ${ }^{31} \mathrm{P}$ NMR at $28.50 \mathrm{ppm}$ ) was loaded onto silica (40 g, $30 \mathrm{~mL}$ EtOAc) and purified by column chromatography (100 g) pre-eluting with EtOAc $(2 \times 60 \mathrm{~mL})$ to remove any unextracted DMSO. The column was eluted with $10 \%$ $\mathrm{MeOH} /$ acetone $(200 \mathrm{~mL})$ to afford the title compound as a light yellow oil. Yield: 78\% (10.0 g); TLC (20\% MeOH in acetone), $\mathrm{R}_{\mathrm{f}}=0.60 ;{ }^{1} \mathbf{H}$ NMR $\left(400 \mathrm{MHz}, \mathrm{CDCl}_{3}, \delta\right): 4.18(\mathrm{t}, J=6.61 \mathrm{~Hz}$, 8H, H4), 2.16 (t, $J=18.17 \mathrm{~Hz}, 4 \mathrm{H}, \mathrm{H} 3), 1.80-1.70$ (m, 4H, H2), 1.34 (t, 12H, $J=6.97 \mathrm{~Hz}, \mathrm{H} 1)$ ppm; ${ }^{13} \mathrm{C}$ NMR (100 MHz, $\left.\mathrm{CDCl}_{3}, \delta\right): 63.00$ (t, ${ }^{2} J=2.81 \mathrm{~Hz}$, (C5, C4 overlap)), 30.96 (t, ${ }^{3} \mathrm{~J}=$ $4.14 \mathrm{~Hz}, \mathrm{C} 3), 26.48$ (t, $\left.{ }^{2} J=4.28 \mathrm{~Hz}, \mathrm{C} 2\right), 15.37\left(\mathrm{t},{ }^{3} \mathrm{~J}=2.67 \mathrm{~Hz}, \mathrm{C} 1\right) \mathrm{ppm} ;{ }^{31} \mathbf{P}$ NMR (121.45 $\mathrm{MHz}, \mathrm{CDCl}_{3}, \delta$ ): 28.40. (Agrees well with literature NMR values). ${ }^{236}$

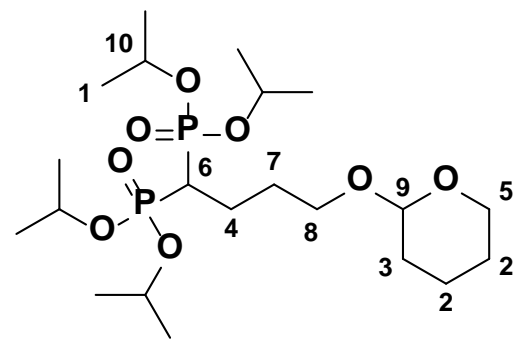

Tetraisopropyl (4-((tetrahydro-2H-pyran-2-yl)oxy)butane-1,1-diyl) bis (phosphonate) (95): ${ }^{237}$

To a stirred suspension (250 mL Schlenk flask, under Ar) of $\mathrm{NaH}$ (60\% dispersion in mineral oil, $1.76 \mathrm{~g}, 60 \mathrm{mmol}, 2.0$ eq.) in anhydrous THF (100 mL, pre-dried ON over MS) was added 
dropwise (canula) tetraisopropyl methylidene bisphosphonate ( $14 \mathrm{~mL}, 44 \mathrm{mmol}, 1.0$ eq.) in THF $(20 \mathrm{~mL})$ at $0^{\circ} \mathrm{C}$. The reaction was brought to RT and stirred (30 min), and evacuated and backfilled with Ar (2 cycles). When hydrogen gas evolution ceased and the mixture cleared, 2(3-bromopropoxy)tetrahydro-2H-pyran (5.976 g, $43 \mathrm{mmol}, 0.98 \mathrm{eq.}$ ) in THF (20 mL) was added dropwise by canula and the resulting clear mixture was refluxed (72 hrs). The solution was then cooled to RT, neutralized with $\mathrm{NH}_{4}{ }^{+} \mathrm{Cl}^{-}$sat: $\mathrm{H}_{2} \mathrm{O}(1: 1,100 \mathrm{~mL})$ and extracted with EtOAc $(1 \times 150$ $\mathrm{mL})$. The organic phases were washed with brine $(1 \times 50 \mathrm{~mL})$, dried over $\mathrm{MgSO}_{4}$, filtered and concentrated in vacuo. The crude product (in a 0.62:0.38 ratio with unreacted tetraisopropyl methylidenebisphosphonate) was loaded onto silica (40 g, 10\% acetone/hexanes, $30 \mathrm{~mL}$ ) and purified by column chromatography on silica gel (100 g) pre-eluting with 10\% acetone/hexanes $(2 \times 100 \mathrm{~mL})$ to remove unextracted (3-bromopropoxy)tetrahydro-2H-pyran. The column was eluted with acetone $(200 \mathrm{~mL})$ to afford the title compound in a 0.62:0.38 ratio with unreacted tetraisopropyl methylidenebisphosphonate by $\left({ }^{31} \mathrm{P}\right.$ NMR) as a light yellow oil (14.78 g). Yield 49.5\% (9.163 g); TLC (acetone), $\mathrm{R}_{\mathrm{f}}=0.50 ;{ }^{1} \mathbf{H}$ NMR (400 MHz, $\left.\mathrm{CDCl}_{3}, \delta\right): 4.85-4.65$ (m, 4H, H10), 4.59-4.50 (m, 1H, H9), 3.86-3.60 (m, 2H, H8), 3.50-3.20 (m, 2H, H7), 2.27-2.06 (m, 1H, H6), 2.08-1.71 (m, 4H, (H5, H4)), 1.70-1.40 (m, 6H, (H3 + H2)), 1.30 (s, 24H, H1); ${ }^{13}$ C NMR (100 MHz, $\mathrm{CDCl}_{3}, \delta$ ): 98.51 (C9), 70.96 (C10), 66.57 (C8), 62.09 (C5), 38.21 (C6), 30.62 (C7), 29.01 (C3), 25.45 (C4), 23.85 (C1), 19.22 (C2) ppm; ${ }^{31}$ P NMR (121.45 MHz, CDCl,, ): 21.83 ppm. (Agrees well with literature NMR values). ${ }^{237}$

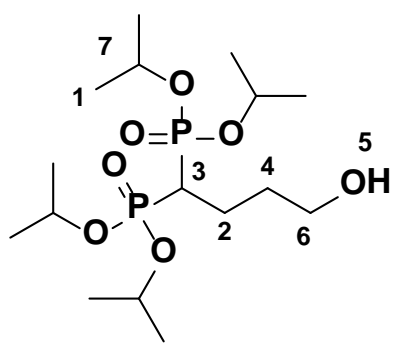


Tetraisopropyl (3-hydroxbutane-1,1-diyl)bis(phosphonate) (98): ${ }^{237,238}$

To a stirred solution of the crude product 95 (10 g, $14.4 \mathrm{mmol})$ in $\mathrm{MeOH}$ (50 mL) was added Amberlite IR-120 (0.5 g). The reaction mixture was heated to $50^{\circ} \mathrm{C} \mathrm{ON}$, filtered (Celite), and concentrated in vacuo. The crude product containing the unreated tetraispropyl methylenebisphosphonate (38\% by weight, 3.8g, ${ }^{31} \mathrm{P}$ NMR, $\delta=17.36 \mathrm{ppm}$ ) was used directly in the next reaction without purification. Yield: 100\% (5.791 g); TLC (15\% MeOH/EtOAc), $\mathrm{R}_{\mathrm{f}}=$ 0.35; ${ }^{1} \mathbf{H}$ NMR (400 MHz, $\left.\mathrm{CDCl}_{3}, \delta\right):$ 4.80-4.65 (m, 4H, H7), 3.60 (m, 2H, H6), 3.11 (s, 1H, H5), 2.25-1.70 (m, 5H, (H4 + H3 + H2 overlap)), 1.29 (s, 32H, H1); ${ }^{13}$ C NMR (100 MHz, $\mathrm{CDCl}_{3}, \delta$ ): 71.13 (C7), 61.19 (C6), 37.25 (t, $\left.{ }^{1} J=138.0 \mathrm{~Hz}, \mathrm{C} 3\right), 31.98$ (t, $\left.{ }^{2} J=5.64 \mathrm{~Hz}, \mathrm{C} 2\right)$, 23.88 (d, $\left.{ }^{3} J=2.52 \mathrm{~Hz}, \mathrm{C} 1\right), 21.63$ (t, $\left.{ }^{3} J=5.33 \mathrm{~Hz}, \mathrm{C} 4\right)$ ppm; ${ }^{31} \mathbf{P}$ NMR $\left(121.45 \mathrm{MHz}, \mathrm{CDCl}_{3}, \delta\right)$ : 22.33 ppm. (Agrees well with literature NMR values). ${ }^{237,238}$

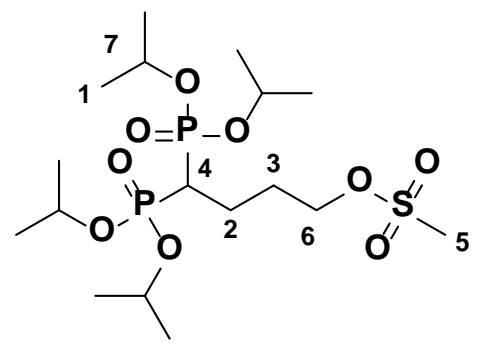

\section{4,4-bis(diisopropoxyphosphoryl)butyl methanesulfonate (99):}

To a flame dried and evacuated $125 \mathrm{~mL}$ round bottom flask, equipped with a magnetic stir bar was added sequentially $\mathrm{NMe}_{3} \cdot \mathrm{HCl}\left(1.5 \mathrm{~g}, 1.44 \mathrm{mmol}, 0.1\right.$ eq.), DCM (20 mL), $\mathrm{Et}_{3} \mathrm{~N}(17.43 \mathrm{~mL}$, $21.6 \mathrm{mmol}, 1.5$ eq.) the crude alcohol 98 (5.791 g, $14.4 \mathrm{mmol}, 1.0$ eq.) and the solution cooled to $0^{\circ} \mathrm{C}$ (ice bath). To the chilled, stirred solution was added, dropwise, $\mathrm{MsCl}$ (1.22 mL, $15.8 \mathrm{mmol}$, 1.1 eq.) followed by rinsing the addition funnel with anhydrous DCM (5 mL) and the cloudy yellow mixture was stirred for $30 \mathrm{~min}$ at RT at which point TLC showed disappearance of the 
starting alcohol (15\% MeOH in EtOAc, $10 \mathrm{~mL})$. The reaction was diluted with $\mathrm{H}_{2} \mathrm{O}(1 \times 100$ $\mathrm{mL})$ and extracted with DCM (100 mL total), the organic layer was re-washed with brine $(1 \times 50$ $\mathrm{mL}$ ), dried over $\mathrm{MgSO}_{4}$, filtered and evaporated to give a light yellow oil. The crude product (10.72 g) containing the unreated tetraispropyl methylenebisphosphonate (38\% by weight, 3.80 g, ${ }^{31} \mathrm{P}$ NMR, $\left.\delta=17.36 \mathrm{ppm}\right)$ was used directly in the next reaction without purification. Yield $100 \%$ (6.919 g); TLC (15\% MeOH/EtOAc), $\mathrm{R}_{\mathrm{f}}=0.45 ;{ }^{1} \mathbf{H}$ NMR (400 MHz, $\left.\mathrm{CDCl}_{3}, \delta\right): 4.83-$ 4.60 (m, 4H, H7), 4.24-4.17 (m, 2H, H6), 2.98 (m, 3H, H5), 2.40-2.25 (m, 1H, H4), 2.11-1.86 (m, 4H, (H3 + H2)), 1.46-1.14 (m, 24H, H1) ppm; ${ }^{13} \mathrm{C}$ NMR (100 MHz, $\left.\mathrm{CDCl}_{3}, \delta\right): 71.09$ (C7), 69.50 (C6), 37.42 (d, $\left.{ }^{3} J=2.81 \mathrm{~Hz}, \mathrm{C} 4\right), 36.46$ (C5), 28.36 (C3), 24.06 (C1), 22.29 (C2) ppm; ${ }^{31} \mathbf{P}$ NMR (121.45 MHz, $\left.\mathrm{CDCl}_{3}, \delta\right): 20.95$ ppm.

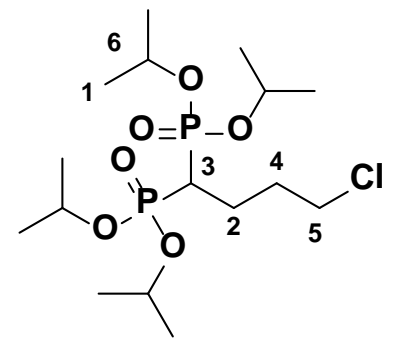

\section{Tetraisopropyl (4-chlorobutane-1,1-diyl)bis(phosphonate) (91):}

To a stirred solution (250 mL Schlenk flask, under argon) of tetraisopropyl methylidenebisphosphonate (9.0 mL, $25 \mathrm{mmol}, 1$ eq.) in DMSO $(40 \mathrm{~mL})$ at $0^{\circ} \mathrm{C}$ was added solid $\mathrm{NaH}$ (60\% dispersion in mineral oil, 1.05 g, $26.4 \mathrm{mmol}, 1.05$ eq.) portionwise. The reaction was brought to RT and then stirred at $60^{\circ} \mathrm{C}$ (10 min), washed with additional DMSO (50 $\left.\mathrm{mL}\right)$, evacuated and backfilled with Ar (2 cycles). When hydrogen gas evolution ceased and the mixture cleared (30 min later), 3-bromo-1-chloropropane (2.47 mL, $25.0 \mathrm{mmol}, 1$ eq.) was added and the resulting yellow mixture was stirred overnight at RT. The solution was then cooled to RT, neutralized with $\mathrm{NH}_{4}{ }^{+} \mathrm{Cl}^{-}(300 \mathrm{~mL}$ ) and extracted with ethyl acetate (300 mL). The organic 
phase was washed with brine $(1 \times 300 \mathrm{~mL})$, dried over $\mathrm{MgSO}_{4}$, filtered and concentrated in vacuo. The crude product ( 7.712 g, after high vac, 3 hrs) was loaded onto silica (40 g, $30 \mathrm{~mL}$ hexanes) and purified by column chromatography on silica gel (100 g) pre-eluting with hexanes (200 mL), followed by 30\% EtOAc/hexanes (100 mL) to remove any unextracted DMSO. The column was eluted with $50 \%$ acetone/hexanes ( $50 \mathrm{~mL}$ ) to afford the title compound, a colourless oil (1.0 g) while the rest of the crude product was recovered by eluting with acetone (200 $\mathrm{mL})$ as a yellow oil (5.305 g) that was comprised of a mixture of the title compound with unreacted tetraisopropyl methylidenebisphosphonate in a 0.38:0.62 ratio by ${ }^{31} \mathrm{P}$ NMR spectroscopy. Yield: 25.1\% (3.02 g); TLC (70\% acetone in hexanes), $\mathrm{R}_{\mathrm{f}}=0.45 ;{ }^{1} \mathbf{H}$ NMR (400 MHz, $\left.\mathrm{CDCl}_{3}, \delta\right): 4.56-$ 4.40 (m, 4H,H6), 3.10-3.00 (m, 2H, H5), 2.38-2.20 (m, 4H, (H4 + H3 overlap)), 1.99-1.85 (m, 2H, H2), 1.11 (s, 24H, H1) ppm; ${ }^{13}$ C NMR (100 MHz, $\mathrm{CDCl}_{3}, \delta$ ): 78.07 (C5), 71.72 (t, ${ }^{2} \mathrm{~J}=3.46$ Hz, C6), 24.56 (C3), 22.98 (d, $\left.{ }^{3} J=22.38 \mathrm{~Hz}, \mathrm{C} 1\right), 16.77$ (C4) ppm; ${ }^{31}$ P NMR (121.45 MHz, $\left.\mathrm{CDCl}_{3}, \delta\right): 24.34 \mathrm{ppm}$.

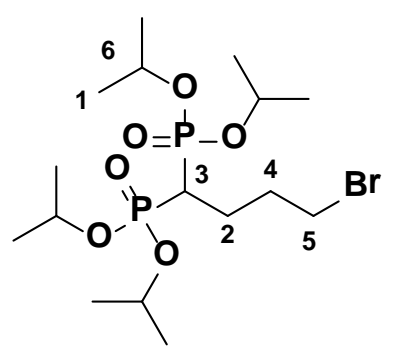

Tetraisopropyl (4-bromobutane-1,1-diyl)bis(phosphonate) (87):

To a stirred solution of the crude product 99 (3.459 g, $7.2 \mathrm{mmol})$ in ACN (25 mL) was added $\mathrm{LiBr}$ (1.25 g, $14.4 \mathrm{mmol}, 2.0$ eq.) and the reaction was refluxed (1.5 hrs). The solution was then cooled to RT, quenched with $\mathrm{H}_{2} \mathrm{O}(50 \mathrm{~mL})$, extracted with ethyl acetate $(100 \mathrm{~mL})$, dried over $\mathrm{MgSO}_{4}$, filtered and concentrated in vacuo. The crude product containing the unreated 
tetraispropyl methylenebisphosphonate (38\% by weight, $1.9 \mathrm{~g},{ }^{31} \mathrm{P}$ NMR $\left.\left(\mathrm{CDCl}_{3}\right)=17.36 \mathrm{ppm}\right)$ was used directly in the next reaction without purification and recovered as an orange oil.Yield: $100 \%$ (3.35 g); TLC (15\% MeOH/EtOAc), $\mathrm{R}_{\mathrm{f}}=0.45 ;{ }^{1} \mathbf{H}$ NMR (400 MHz, $\left.\mathrm{CDCl}_{3}, \delta\right): 4.82-4.62$ (m, 4H,H6), 3.41-3.28 (m, 2H, H5), 2.31 (t, $J=\mathrm{Hz}, 3 \mathrm{H}, \mathrm{H} 5), 2.19-1.86$ (m, 4H, (H3 + H2 overlap)), 1.29 (s, 24H, H1) ppm; ${ }^{31}$ P NMR (121.45 MHz, $\left.\mathrm{CDCl}_{3}, \delta\right): 21.36$ ppm.

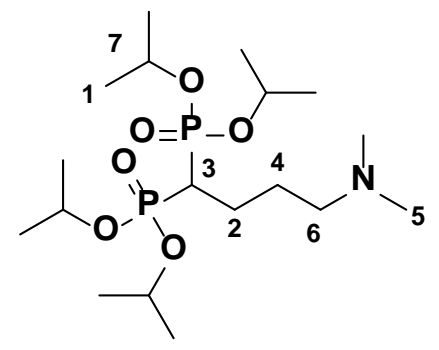

\section{Tetraisopropyl (4-(dimethylamino)butane-1,1-diyl)bis(phosphonate) (96):}

To a $20 \mathrm{~mL}$ glass screw cap vial equipped with a magnetic stir bar was added the crude product 87 (3.459 g, $7.2 \mathrm{mmol}$ ), $\mathrm{NHMe}_{2}$ (5.6 M in EtOH, $3.8 \mathrm{~mL}$, excess) and stirred at reflux sealed for $1.5 \mathrm{hrs}$, at which point the TLC plate showed disappearance of the starting material (1\% $\mathrm{NH}_{4}{ }^{+} \mathrm{OH}^{-}$in acetone, $10 \mathrm{~mL}, \mathrm{R}_{\mathrm{f}}=0.95$ ). The reaction was cooled to RT, evaporated, filtered (Celite), washed with EtOAc and loaded onto silica (40 g, $30 \mathrm{~mL}$ EtOAc). The title compound was purified by column chromatography on silica gel (100 g) pre-eluting with $10 \%$ $\mathrm{MeOH} / \mathrm{EtOAc}(50 \mathrm{~mL})$, and eluted with $\left(10 \% \mathrm{NH}_{4} \mathrm{OH} / \mathrm{ACN}, 100 \mathrm{~mL}\right)$. The eluted fraction was separated from $\mathrm{H}_{2} \mathrm{O}$ by coevaporation from $\mathrm{ACN}$, stirring in $\mathrm{CHCl}_{3}(10 \mathrm{~mL})$ for $5 \mathrm{~min}$, filtering (Celite) and drying under high vaccum (60 min) and recovered as a yellow oil. Yield: 64\% (2.0

g); TLC (1\% $\mathrm{NH}_{4}{ }^{+} \mathrm{OH}^{-}$in Acetone, $\left.10 \mathrm{~mL}\right)$ or $\left(20 \% \mathrm{MeOH}(6 \% \mathrm{NaBr}): \mathrm{ACN}, \mathrm{R}_{\mathrm{f}}=0.40 ;{ }^{1} \mathbf{H}\right.$ NMR (400 MHz, $\mathrm{CDCl}_{3}, \delta$ ): 4.82-4.65 (m, 4H,H7), 2.29-2.07 (m, 2H, H6), 2.17 (s, 6H, H5), 1.91-1.62 (m, 6H, (H4 + H3 + H2 overlap)), 1.29 (s, 24H, H1) ppm; ${ }^{13}$ C NMR (100 MHz, 
$\mathrm{CDCl}_{3}, \delta$ ): 70.87 (q, $\left.{ }^{2} \mathrm{~J}=\mathrm{Hz}, \mathrm{C} 7\right), 59.50$ (C6), 45.34 (C5), 38.36 (d, $\left.{ }^{1} \mathrm{~J}=\mathrm{Hz}, \mathrm{C} 3\right), 27.06\left(\mathrm{t},{ }^{2} \mathrm{~J}=\right.$ Hz,C2), 24.19 (C4), 23.90 (t, $\left.{ }^{3} J=\mathrm{Hz}, \mathrm{C} 1\right) \mathrm{ppm}{ }^{31} \mathbf{P}$ NMR (121.45 MHz, $\mathrm{CDCl}_{3}, \delta$ ): 24.34 ppm.
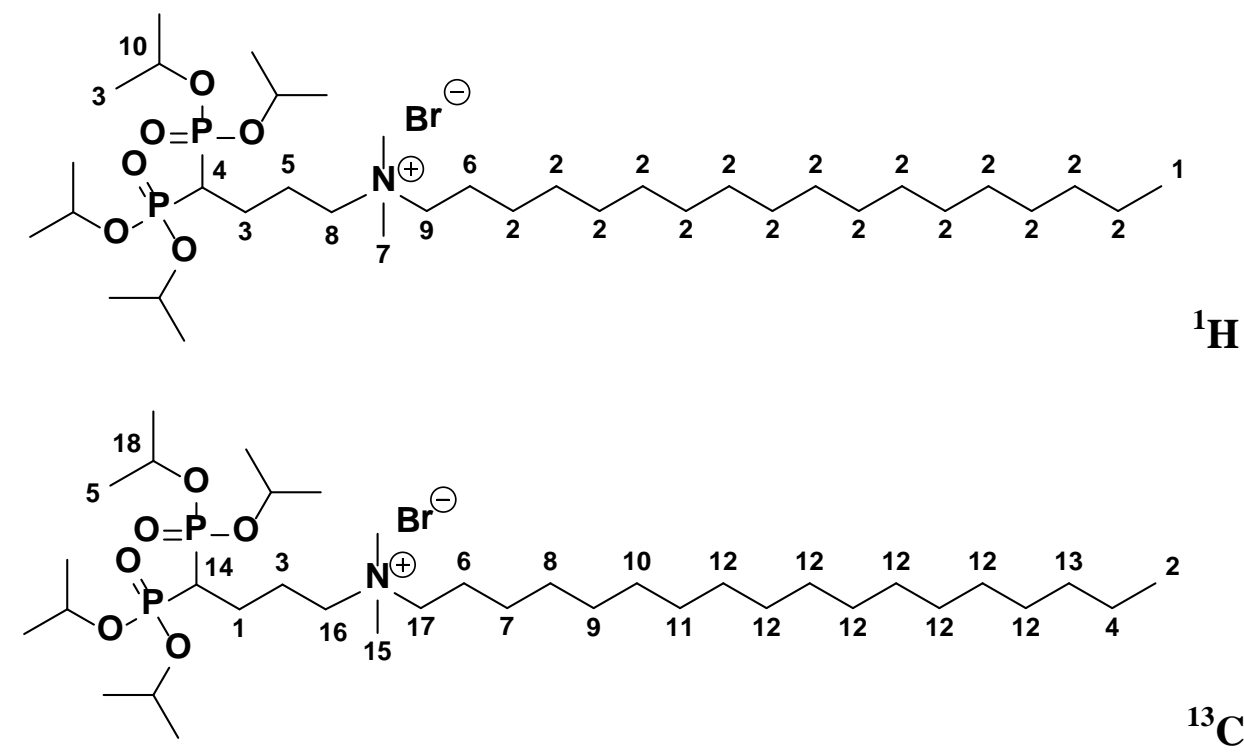

\section{$N$-(4,4-bis(diisopropoxyphosphoryl)butyl)- $N, N$-dimethyloctadecan-1-ammonium (93):}

This compound was prepared by the Menshutkin reaction using Method 5.2.1: 87 (2.28 g, 5.57 mmol) and DMOA (1.65 g, $5.57 \mathrm{mmol}, 1.0$ eq.) in ACN (10 mL) were refluxed for 4 hrs. The mixture was then cooled to RT, poured into $20 \mathrm{~mL}$ of $\mathrm{Et}_{2} \mathrm{O}$, and placed into a freezer $\left(-20^{\circ} \mathrm{C}\right)$ ON. The title compound did not solidify so the crude product was loaded onto silica (40 g, 30 $\mathrm{mL}$ acetone) and purified by column chromatography on silica gel (100 g) pre-eluting with acetone $(100 \mathrm{~mL})$, and eluted with $20 \%(6 \% \mathrm{NaBr}$ in $\mathrm{MeOH})$ : $\mathrm{ACN}(150 \mathrm{~mL})$ to give the title compound as a yellow oil. Yield: 50\% (2.12 g); ${ }^{1} \mathbf{H}$ NMR (400 MHz, $\left.\mathrm{CDCl}_{3}, \delta\right): 3.82-3.71$ (m, 4H, (H8 + H7 overlap)), 3.56-3.49 (m, 2H, H6), 3.40 (s, 6H, H5), 2.36-2.28 (m, 2H, H4), 1.801.69 (m, 2H, H3), 1.23 (brs, 30H, H2), 0.85 (t, $J=7.0 \mathrm{~Hz}, 3 \mathrm{H}, \mathrm{H1}$ ) ppm; ${ }^{13} \mathbf{C}$ NMR (100 MHz, $\mathrm{CDCl}_{3}, \delta$ ): 65.88 (C15), 61.55 (C14), 52.14 (C13), 41.14 (C12), 31.90 (C11), 29.71-29.62 (C10 
overlap), 29.46 (C9), 29.34 (C8), 29.18 (C7), 26.15 (C6), 26.14 (C5), 22.75 (C4), 22.66 (C3), 22.66 (C2), 14.10 (C1) ppm.
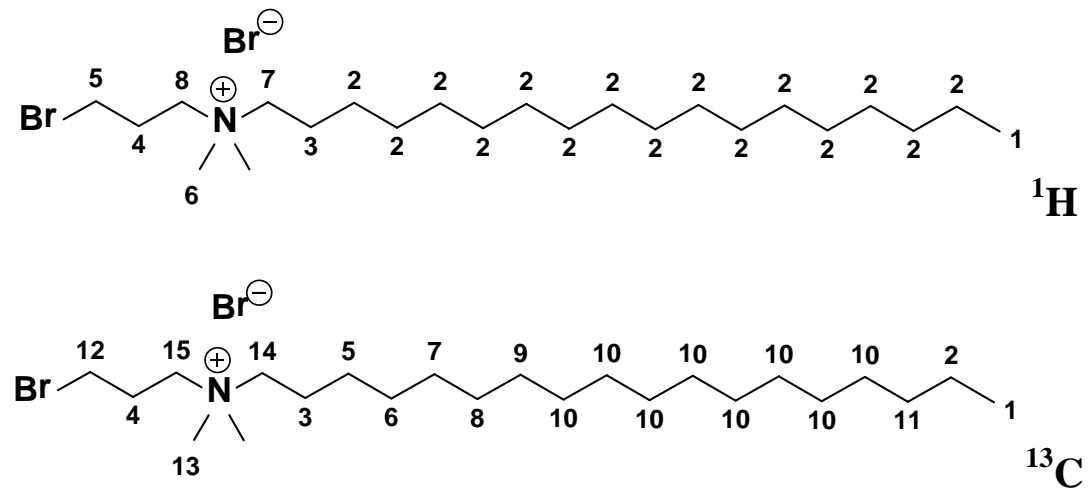

\section{N-(4-hydroxybutyl)-N,N-dimethyloctadecan-1-ammonium bromide (83): $:^{239}$}

This compound was prepared by the Menshutkin reaction using Method 5.2.1: 1,3dibromopropane (4.079 mL, $44 \mathrm{mmol}, 4$ eq.) and DMOA (2.9 g, $9.8 \mathrm{mmol}, 1.0$ eq.) in ACN:EtOH (6:1, $70 \mathrm{~mL})$ were refluxed for 3 hrs. The mixture was then cooled to RT, poured into $30 \mathrm{~mL}$ of $\mathrm{Et}_{2} \mathrm{O}$, and placed into a freezer $\left(-20^{\circ} \mathrm{C}\right)$ for $10 \mathrm{~min}$ to precipitate the title compound as a white solid. Yield: $50 \%(2.95 \mathrm{~g}) . \mathrm{Mp}=93-95^{\circ} \mathrm{C} ;{ }^{1} \mathbf{H}$ NMR $\left(400 \mathrm{MHz}, \mathrm{CDCl}_{3}, \delta\right)$ : 3.84-3.77 (m, 4H, H8), 3.53-3.44 (m, 2H, H7), 3.36 (s, 6H, H6), 2.33-2.19 (m, 4H, (H5 + H4

overlap)), 1.80-1.71 (m, 2H, H3), 1.23 (brs, 30H, H2), 0.86 (t, $J=7.0 \mathrm{~Hz}, 3 \mathrm{H}, \mathrm{H1}$ ) ppm; ${ }^{13} \mathrm{C}$ NMR (100 MHz, $\mathrm{CDCl}_{3}$, \&): 66.47 (C15), 60.90 (C14), 51.29 (C13), 34.54 (C12), 31.92 (C11), 29.95-29.62 (C10 overlap), 29.55 (C9), 29.48 (C8), 29.36 (C7), 29.29 (C6), 26.32 (C5), 22.95 (C4), 22.68 (C3), 20.88 (C2), 14.12 (C1) ppm. (Agrees well with literature NMR values). ${ }^{239}$ 

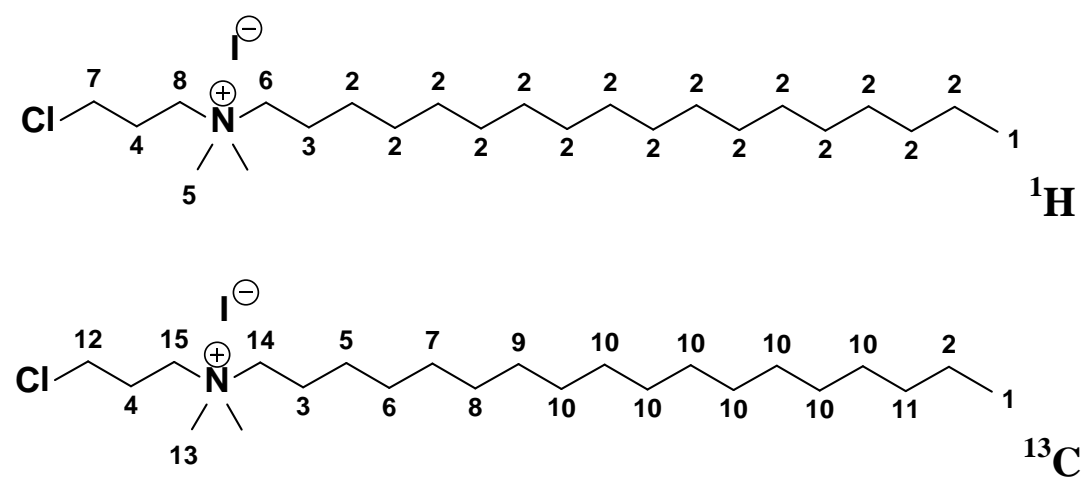

\section{$N$-(3-chloropropyl)- $N, N$-dimethyloctadecan-1-ammonium iodide (102): ${ }^{240}$}

This compound was prepared by the Menshutkin reaction using Method 5.2.1: 3-chloro-1-iodo propane (2.019 g, $9.8 \mathrm{mmol})$ and DMOA (2.9 g, $9.8 \mathrm{mmol}, 1.0$ eq.) in ACN (10 mL) were refluxed for 1.5 hrs. The mixture was then cooled to RT, poured into $20 \mathrm{~mL}$ of $\mathrm{Et}_{2} \mathrm{O}$, and placed into a freezer $\left(-20^{\circ} \mathrm{C}\right)$ for $10 \mathrm{~min}$ to precipitate the title compound as a white solid. Yield: $69 \%$ (3.1 g). $\mathrm{Mp}=91^{\circ} \mathrm{C} ;{ }^{1} \mathbf{H}$ NMR (400 $\left.\mathrm{MHz} \mathrm{CDCl}_{3}, \delta\right): 3.82-3.71$ (m, 4H, (H8 + $\mathrm{H} 7$ overlap)), 3.56-3.49 (m, 2H, H6), 3.40 (s, 6H, H5), 2.36-2.28 (m, 2H, H4), 1.80-1.69 (m, 2H, H3), 1.23 (brs, 30H, H2), 0.85 (t, $J=7.0 \mathrm{~Hz}, 3 \mathrm{H}, \mathrm{H1}) \mathrm{ppm} ;{ }^{13} \mathrm{C}$ NMR (100 MHz, $\mathrm{CDCl}_{3}, \delta$ ): 65.88 (C15), 61.55 (C14), 52.14 (C13), 41.14 (C12), 31.90 (C11), 29.71-29.62 (C10 overlap), 29.46 (C9), 29.34 (C8), 29.18 (C7), 26.15 (C6), 26.14 (C5), 22.75 (C4), 22.66 (C3), 22.66 (C2), 14.10 (C1) ppm. (Agrees well with literature NMR values). ${ }^{240}$

\subsubsection{General Procedure for the 3-Component Reaction}

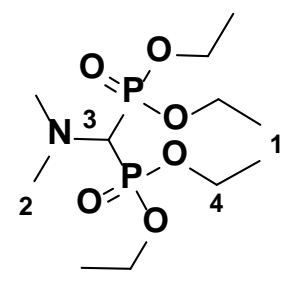

Tetraethyl dimethylaminomethylenediphosphonate (105): ${ }^{202}$ 
To a chilled solution of DMF (3.87 mL, $50 \mathrm{mmol})$ in DCM $(75 \mathrm{~mL}$ ) was added dropwise with stirring a solution of oxalyl chloride ( $25 \mathrm{~mL}, 2 \mathrm{M}$ in DCM, $50 \mathrm{mmol}$ ). Following addition, the mixture was allowed to warm to RT and stirred for $1 \mathrm{hr}$. P(OEt) $)_{3}(18.77 \mathrm{~mL}, 109.5 \mathrm{mmol}, 2.19$ eq.) was then added dropwise with stirring. After $1 \mathrm{hr}$ the mixture was concentrated under reduced pressure to a yellow oil. Yield: $75.5 \%$ (12.42 g). ${ }^{1} \mathbf{H}$ NMR (400 MHz, $\mathrm{CDCl}_{3}, \delta$ ): 4.214.14 (m, 8H, H4), 3.22 (dt, 1H, ${ }^{1} \mathrm{~J}=24.98 \mathrm{~Hz},{ }^{2} \mathrm{~J}=24.98 \mathrm{~Hz}, \mathrm{H} 3$ ), 2.58 (s, 6H, H2), 3.18 (dt, $\left.12 \mathrm{H},{ }^{1} J=7.07 \mathrm{~Hz},{ }^{2} J=7.06 \mathrm{~Hz}, 1 \mathrm{H}\right) \mathrm{ppm} ;{ }^{13} \mathbf{C} \mathbf{~ N M R}\left(100 \mathrm{MHz}, \mathrm{CDCl}_{3}, \delta\right): 62.70\left(\mathrm{t},{ }^{1} J_{\mathrm{C}-\mathrm{P}}=3.05\right.$ $\mathrm{Hz}, \mathrm{C} 3), 62.40\left(\mathrm{t},{ }^{2} J_{\mathrm{C}-\mathrm{P}}=3.61 \mathrm{~Hz}, \mathrm{C} 4\right), 44.11\left(\mathrm{t},{ }^{3} J_{\mathrm{C}-\mathrm{P}}=4.71 \mathrm{~Hz}, \mathrm{C} 2\right), 16.39\left(\mathrm{q},{ }^{3} J_{\mathrm{C}-\mathrm{P}}=3.01 \mathrm{~Hz}\right.$, C1) ppm; ${ }^{31} \mathbf{P}$ NMR (121.45 MHz, $\mathrm{CDCl}_{3}, \delta$ ): $19.15 \mathrm{ppm}$. (Agrees well with literature NMR values). ${ }^{202}$

\subsubsection{General Procedure for the Bis Kabachnik Fields Reaction}

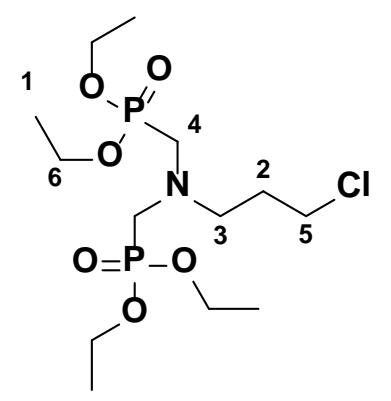

\section{Tetraethyl (((3-chloropropyl)azanediyl)bis(methylene))bis(phosphonate) (114):}

To a $20 \mathrm{~mL}$ glass screw cap vial, equipped with a magnetic stir bar was added diethylphosphite ( $2.86 \mathrm{~g}, 20.74 \mathrm{mmol}, 2.0$ eq.). The vial was placed on ice to cool. In a separate beaker, 3aminopropyl-1-chloride hydrochloride (2.0 g, 11.4 mmol) was treated with $\mathrm{NaOH}(\sim 12 \mathrm{~N}, 2.0 \mathrm{~g}$ in $5 \mathrm{~mL}$ ) and stirred at $0^{\circ} \mathrm{C}$ until a yellow oil appeared $(\sim 5 \mathrm{~min})$. The mixture was then extracted without solvent, adding the upper yellow layer of the free base 3-aminopropyl-1- 
chloride to the vial containing diethylphosphite cooled to $0-5{ }^{\circ} \mathrm{C}$ (ice bath). To the chilled solution was added formalin, dropwise, via syringe (37\%, $2.15 \mathrm{~mL}, 25.79$ mmol, 2.5 eq.) over 10 min maintaining the reaction temp under $10^{\circ} \mathrm{C}$. The mixture was then warmed, with stirring, to RT for $10 \mathrm{~min}$, then heated to $100^{\circ} \mathrm{C}$ for 30 min. Excess formaldehyde and $\mathrm{H}_{2} \mathrm{O}$ were removed via rotary evaporator and the crude material purified by DCVC (20 g silica, $3.5 \mathrm{~cm} \times 4.5 \mathrm{~cm})$ eluting with $80 \mathrm{~mL}$ EtOAc and collecting $50 \mathrm{~mL}$ (20\% MeOH/EtOAc). Yield: 50\% (2.03 g); TLC (10\% MeOH in EtOAc), $\mathrm{R}_{\mathrm{f}}=0.70 ;{ }^{1} \mathbf{H}$ NMR (400 MHz, $\left.\mathrm{CDCl}_{3}, \delta\right): 4.18-4.09$ (m, 8H, H6), 3.62 (t, 2H, $J=6.6 \mathrm{~Hz}, \mathrm{H} 5), 3.17$ (d, 4H, $J=8.6 \mathrm{~Hz}, \mathrm{H} 4), 2.97$ (t, 2H, $J=6.6 \mathrm{~Hz}, \mathrm{H} 3), 2.00$ (m, 2H, H2), 1.33 (t, 12H, $J=7.1 \mathrm{~Hz}, \mathrm{H1}) ;{ }^{13} \mathbf{C}$ NMR (100 MHz, $\left.\mathrm{CDCl}_{3}, \delta\right): 61.84$ (m, C6), 53.93 (t, $\left.{ }^{3} J_{C-P}=7.44 \mathrm{~Hz},(\mathrm{C} 5)\right), 50.18\left(\mathrm{dd},{ }^{1} J_{C-P}=6.08 \mathrm{~Hz},{ }^{1} J_{C-P}=6.00 \mathrm{~Hz},(\mathrm{C} 4)\right), 42.47$ (C3), 30.77 (C2), $16.45\left(\mathrm{t},{ }^{3} J_{\mathrm{C}-\mathrm{P}}=2.94 \mathrm{~Hz},(\mathrm{C} 1)\right) ;{ }^{31} \mathbf{P}$ NMR $\left(121.45 \mathrm{MHz}, \mathrm{CDCl}_{3}, \delta\right): 24.40 \mathrm{ppm}$.

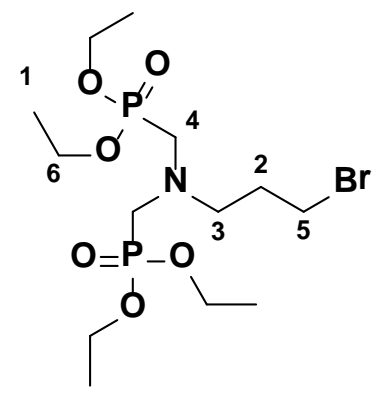

\section{Tetraethyl (((3-bromopropyl)azanediyl)bis(methylene))bis(phosphonate) (116):}

To a $20 \mathrm{~mL}$ glass screw cap vial, equipped with a magnetic stir bar was added diethylphosphite (2.77 mL, $21.56 \mathrm{mmol}, 2.2$ eq.) and the vial was placed on ice meanwhile 3-aminopropyl-1bromide hydrobromide ( $\sim 2.5 \mathrm{~g}, \sim 11 \mathrm{mmol}$ ) was treated with $\mathrm{KOH}(6 \mathrm{~N}, 6 \mathrm{~g}$ in $20 \mathrm{~mL}$ ) and stirred at $0^{\circ} \mathrm{C}$ until a yellow oil appeared $(\sim 5 \mathrm{~min})$. The mixture was then extracted without solvent, collecting the upper yellow layer of the free base aminopropyl-1-bromide (incompletely 
dry by NMR, $50 \% \mathrm{H}_{2} \mathrm{O}$ present). The amine $(1.350 \mathrm{~g}, 9.78 \mathrm{mmol})$ was added to the vial containing diethylphosphite and cooled at $0-5{ }^{\circ} \mathrm{C}$ (ice bath). To the chilled, stirred solution was added formalin, dropwise (37\%, $2.12 \mathrm{~mL}, 25.43 \mathrm{mmol}, 2.6$ eq.) over 10 min while maintaining the reaction temp under $10^{\circ} \mathrm{C}$, then warming the mixture to RT for $30 \mathrm{~min}$, and finally to $100{ }^{\circ} \mathrm{C}$ for $1 \mathrm{hr}$. The reaction was diluted with $0.2 \mathrm{~N} \mathrm{NaOH}(\sim 300 \mathrm{mg}$ in $40 \mathrm{~mL})$ and extracted with $\mathrm{CHCl}_{3}(1 \times 30 \mathrm{~mL}, 1 \times 10 \mathrm{~mL})$, the organic layer was separated, washed with brine $(1 \times 20 \mathrm{~mL})$ and dried over anhydrous $\mathrm{MgSO}_{4}$ filtered and concentrated to afford a yellow oil. The title compound was in poor yield, however analysis by ${ }^{1} \mathrm{H}$ NMR spectroscopy revealed $>98 \%$ purity and required no further purification. Yield: $20.9 \%$ (0.76 g); TLC (5\% MeOH in EtOAc), $\mathrm{R}_{\mathrm{f}}=$ 0.48; ${ }^{1} \mathbf{H}$ NMR (400 MHz, $\mathrm{CDCl}_{3}, \delta$ ): 4.17-4.07 (m, 8H, H6), 3.47 (t, 2H, $J=6.7 \mathrm{~Hz}, \mathrm{H} 5$ ), 3.14 (d, 4H, $J=8.5 \mathrm{~Hz}, \mathrm{H} 4), 2.93$ (t, 2H, $J=6.6 \mathrm{~Hz}, \mathrm{H} 3), 2.00$ (q, 2H, $J=6.58 \mathrm{~Hz}, \mathrm{H} 2), 1.31$ (t, 12H, $J=7.1 \mathrm{~Hz}, \mathrm{H1}) ;{ }^{13} \mathrm{C}$ NMR $\left(100 \mathrm{MHz}, \mathrm{CDCl}_{3}, \delta\right): 61.9\left(\mathrm{t},{ }^{2} J_{\mathrm{C}-\mathrm{P}}=3.36 \mathrm{~Hz}, \mathrm{C} 6\right), 55.08$ ( C3), 49.43 (C4), 31.09 (C5), 30.96 (C2), 16.49 (t, $\left.{ }^{3} J_{C-P}=2.94 \mathrm{~Hz}, \mathrm{C} 1\right) ;{ }^{31} \mathbf{P}$ NMR (121.45 MHz, $\left.\mathrm{CDCl}_{3}, \delta\right): 24.60 \mathrm{ppm}$.

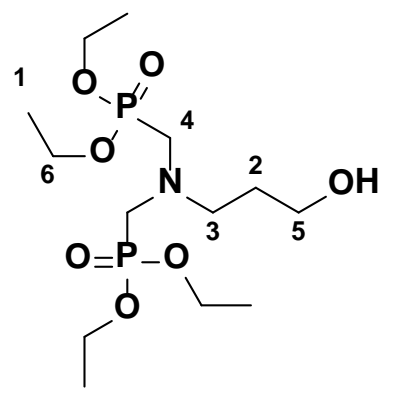

\section{Tetraethyl (((3-hydroxypropyl)azanediyl)bis(methylene))bis(phosphonate) (117): ${ }^{241-243}$}

To a $20 \mathrm{~mL}$ glass screw cap vial, equipped with a magnetic stir bar was added diethylphosphite (2.86 g, $20.74 \mathrm{mmol}, 2.0$ eq.) and 3-amino-1-propanol (0.768 g, $10.24 \mathrm{mmol}$,) and the mixture cooled to $0-5{ }^{\circ} \mathrm{C}$ (ice bath). To the chilled solution was added formalin, dropwise, via syringe 
(37\%, $2.15 \mathrm{~mL}, 25.79 \mathrm{mmol}, 2.5$ eq.) over $10 \mathrm{~min}$ maintaining the reaction temp under $10^{\circ} \mathrm{C}$. The mixture was warmed, with stirring, to RT for $30 \mathrm{~min}$, then heated to $100^{\circ} \mathrm{C}$ for $60 \mathrm{~min}$. Excess formaldehyde and $\mathrm{H}_{2} \mathrm{O}$ were removed via rotary evaporator and the crude material purified by DCVC $(20 \mathrm{~g}$ silica, $3.5 \mathrm{~cm} \times 4.5 \mathrm{~cm})$ eluting with $100 \mathrm{~mL}$ EtOAc $(20 \%$ $\mathrm{MeOH} / \mathrm{EtOAc}$ ) to give a light yellow oil after evaporation of solvent. Yield: 50\% (2.03 g); TLC (10\% MeOH in EtOAc), $\mathrm{R}_{\mathrm{f}}=0.50 ;{ }^{1} \mathbf{H}$ NMR (400 MHz, $\left.\mathrm{CDCl}_{3}, \delta\right): 4.18-4.09$ (m, 8H, H6), 3.62 (t, 2H, $J=6.6 \mathrm{~Hz}, \mathrm{H}$ ), 3.17 (d, 4H, $J=8.6 \mathrm{~Hz}, \mathrm{H} 4), 2.97$ (t, 2H, $J=6.6 \mathrm{~Hz}, \mathrm{H3}), 1.61$ (m, 2H, H2), 1.32 (t, $12 \mathrm{H}, J=7.1 \mathrm{~Hz}, \mathrm{H1}) \mathrm{ppm} ;{ }^{13} \mathrm{C}$ NMR $\left(100 \mathrm{MHz}, \mathrm{CDCl}_{3}, \delta\right): 62.06\left(\mathrm{t},{ }^{2} J_{\mathrm{C}-\mathrm{P}}=3.61\right.$ Hz, C6), 59.04 (C5), 53.44 (t, $\left.{ }^{3} J_{C-P}=7.46 \mathrm{~Hz}, \mathrm{C} 3\right), 50.66$ (dd, $\left.{ }^{1} J_{C-P}=9.14 \mathrm{~Hz}, \mathrm{C} 4\right), 29.78$ (C2), $16.43\left(\mathrm{t},{ }^{3} \mathrm{~J}_{\mathrm{C}-\mathrm{P}}=2.93 \mathrm{~Hz}, \mathrm{C} 1\right) \mathrm{ppm} ;{ }^{31} \mathbf{P} \mathbf{N M R}\left(121.45 \mathrm{MHz}, \mathrm{CDCl}_{3}, \delta\right): 25.0 \mathrm{ppm}$. (Agrees well with literature NMR values). ${ }^{241-243}$

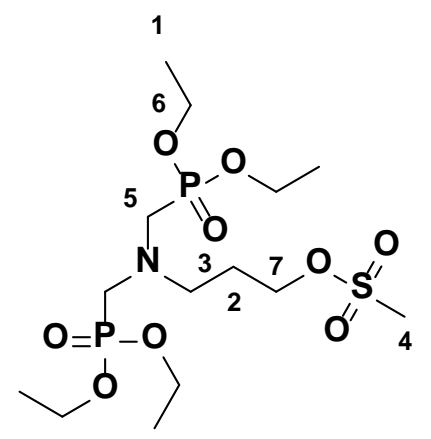

\section{3-(bis((diethoxyphosphoryl)methyl)amino)propyl methanesulfonate (118):}

To a flame dried and evacuated $125 \mathrm{~mL}$ round bottom flask, equipped with a magnetic stir bar was added sequentially $\mathrm{NMe}_{3} \cdot \mathrm{HCl}$ (1.50 g, 5 mmol, 0.1 eq.), DCM (50 mL), Et ${ }_{3} \mathrm{~N}$ (17.43 mL, 75 mmol, 1.5 eq.), 117 (18.76 g, $50 \mathrm{mmol}, 1.0$ eq.) and the solution cooled to $0^{\circ} \mathrm{C}$ in an ice bath. To the chilled, stirred solution was added, dropwise, mesyl chloride ( $4.5 \mathrm{~mL}, 58.1 \mathrm{mmol}, 1.16 \mathrm{eq}$.) followed by rinsing the addition funnel with anhydrous DCM $(15 \mathrm{~mL})$ and the cloudy yellow 
mixture was stirred for $30 \mathrm{~min}$ at RT at which point TLC showed disappearance of the starting amine $(5 \% \mathrm{MeOH}$ in EtOAc, $10 \mathrm{~mL})$. The reaction was diluted with $\mathrm{H}_{2} \mathrm{O}(1 \times 75 \mathrm{~mL})$ and extracted with DCM (100 mL total), the organic layer was re-washed with $\mathrm{H}_{2} \mathrm{O}(2 \times 75 \mathrm{~mL})$, dried over $\mathrm{MgSO}_{4}$, filtered and evaporated to give a light orange oil. The crude material was used without further purification. Yield: 74\% (13.39 g); TLC (30\% MeOH in EtOAc), $\mathrm{R}_{\mathrm{f}}=0.45$; ${ }^{1}$ H NMR (400 MHz, CDCl, 8$): 4.33$ (t, 12H, $J=6.44$ Hz, 2H, H7), 4.16-4.06 (m, 8H, H6), 3.12 (d, 4H, $J=8.57 \mathrm{~Hz}, \mathrm{H}$ ), 3.01 (s, 3H, H4), 2.97 (t, 2H, $J=6.57 \mathrm{~Hz}, \mathrm{H} 3$ ), 1.94-1.85 (m, 2H, H2), 1.31 (t, $12 \mathrm{H}, J=7.05 \mathrm{~Hz}, \mathrm{H} 1) ;{ }^{13} \mathrm{C}$ NMR $\left(100 \mathrm{MHz}, \mathrm{CDCl}_{3}, \delta\right): 67.90$ (C7), 61.84 (t, ${ }^{2} J_{\mathrm{C}-\mathrm{P}}=$ $3.50 \mathrm{~Hz}, \mathrm{C}$ ), $52.50\left(\mathrm{t},{ }^{3} \mathrm{~J}_{\mathrm{C}-\mathrm{P}}=7.61 \mathrm{~Hz}, \mathrm{C} 3\right) 52.54,49.30$ (dd, $\left.{ }^{1} J_{\mathrm{C}-P}=6.57 \mathrm{~Hz}, \mathrm{C} 5\right), 37.07$ (C4), 27.21 (C2), 16.39 (t, $\left.{ }^{3} J_{C-P}=2.81 \mathrm{~Hz}, \mathrm{C} 1\right) \mathrm{ppm} ;{ }^{31} \mathbf{P}$ NMR (121.45 MHz, $\left.\mathrm{CDCl}_{3}, \delta\right): 24.50 \mathrm{ppm}$.

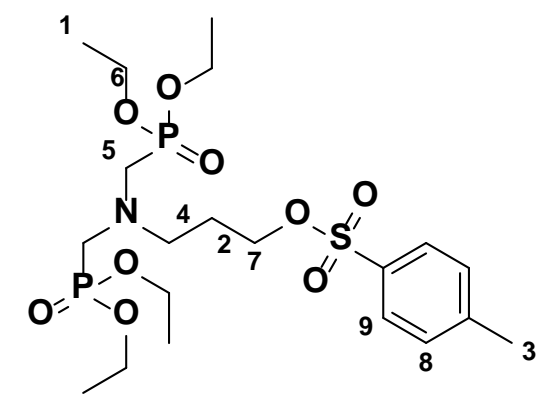

\section{3-(bis((diethoxyphosphoryl)methyl)amino)propyl 4-methylbenzenesulfonate (119):}

To a flame dried and evacuated $25 \mathrm{~mL}$ round bottom flask, equipped with a magnetic stir bar was added sequentially $\mathrm{NMe}_{3} \cdot \mathrm{HCl}\left(0.045 \mathrm{~g}, 0.24 \mathrm{mmol}, 0.24\right.$ eq.), DCM (1 mL), $\mathrm{Et}_{3} \mathrm{~N}(0.58 \mathrm{~mL}, 2.5$ mmol, 2.5 eq.), $117(0.375 \mathrm{~g}, 1 \mathrm{mmol})$ and the solution cooled to $0^{\circ} \mathrm{C}$ in an ice bath. To the chilled, stirred solution was added, dropwise, TsCl (0.286 mg, $1.5 \mathrm{mmol}$, 1.5 eq.) in anhydrous DCM (2 mL) and the cloudy yellow mixture was stirred for $1 \mathrm{hr}$ at RT at which point TLC showed disappearance of the starting amine (5\% MeOH in EtOAc, $10 \mathrm{~mL})$. The reaction was diluted with $\mathrm{H}_{2} \mathrm{O}(1 \times 15 \mathrm{~mL})$ and extracted with DCM $(10 \mathrm{~mL}$ total $)$, the aqueous layer was re- 
extracted with EtOAC (15 mL) and the combined organic layers were dried over $\mathrm{MgSO}_{4}$, filtered and evaporated to give a yellow oil. The crude material was purified by flash chromatography on silica gel (20 g silica, $1.5 \mathrm{~cm}$ i.d) with gradient elution: 100\% EtOAc (35 mL) then 5\% MeOH:EtOAc (90 mL) to obtain the title compound as a yellow oil. Yield: 56.7\% (0.300 g); TLC (5\% MeOH in EtOAc), $\mathrm{R}_{\mathrm{f}}=0.42 ;{ }^{1} \mathbf{H}$ NMR $\left(400 \mathrm{MHz}, \mathrm{CDCl}_{3}, \delta\right): 7.76(\mathrm{~d}, 2 \mathrm{H}, J=8.24 \mathrm{~Hz}$, H9), 7.32 (d, 2H, $J=8.04 \mathrm{~Hz}, \mathrm{H8}$ ), 4.13-4.05 (m, 10H, (H7, H6)), 3.08 (d, 4H, $J=8.40 \mathrm{~Hz}, \mathrm{H}$ ), $2.83(\mathrm{t}, 2 \mathrm{H}, J=6.70 \mathrm{~Hz}, \mathrm{H} 4), 2.42$ (s, 3H, H3), 1.81 (t, 2H, $J=6.65 \mathrm{~Hz}, \mathrm{H} 2), 1.30$ (t, 12H, $J=$ $7.08 \mathrm{~Hz}, \mathrm{H1}) ;{ }^{13} \mathrm{C}$ NMR (100 MHz, $\mathrm{CDCl}_{3}, \delta$ ): 44.67 (C11), 133.19 (C10), 129.81 (C8), 127.83 (C9), 68.50 (C7), 61.86 (t, $\left.{ }^{2} J_{C-P}=3.19 \mathrm{~Hz}, \mathrm{C} 6\right), 52.67$ (dd, $\left.{ }^{1} J_{C-P}=6.02 \mathrm{~Hz}, \mathrm{C} 5\right), 52.67$ (C4), 27.22 (C2), 21.57 (C3), $16.46\left(\mathrm{t},{ }^{3} J_{\mathrm{C}-\mathrm{P}}=2.78 \mathrm{~Hz}, \mathrm{C} 1\right) \mathrm{ppm} ;{ }^{31} \mathbf{P}$ NMR (121.45 MHz, $\mathrm{CDCl}_{3}, \delta$ ): $24.56 \mathrm{ppm}$.

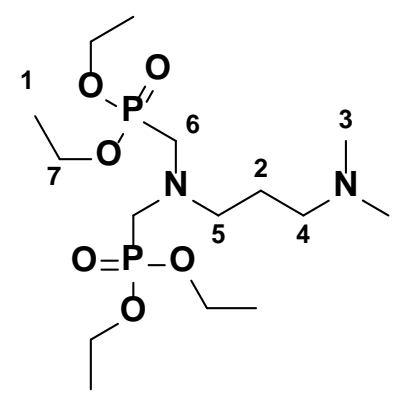

\section{Tetraethyl (((3-(dimethylamino)propyl)azanediyl)bis(methylene)) bis(phosphonate) (113):}

To a $20 \mathrm{~mL}$ glass screw cap vial equipped with a magnetic stir bar containing the bromo amino bisphosphonate (0.954 g, $1.8 \mathrm{mmol}$ ) was added $\mathrm{NHMe}_{2}$ (5.6 M in EtOH, $2.5 \mathrm{~mL}$, excess) followed by $\mathrm{H}_{2} \mathrm{O}(0.5 \mathrm{~mL})$ and the clear mixture was stirred at reflux sealed for $1.5 \mathrm{hr}$, at which point TLC showed disappearance of the starting material $\left(1 \% \mathrm{NH}_{4}{ }^{+} \mathrm{OH}^{-}\right.$in Acetone, $10 \mathrm{~mL}, \mathrm{R}_{\mathrm{f}}$ $=0.95)$. The cooled yellow reaction diluted with $\mathrm{H}_{2} \mathrm{O}(1 \times 20 \mathrm{~mL}, \mathrm{pH}$ was 11$)$ and extracted with $\mathrm{CHCl}_{3}(2 \times 30 \mathrm{~mL})$, dried over $\mathrm{MgSO}_{4}$, filtered and evaporated to give an orange oil. The title 
compound was isolated $>98 \%$ purity $\left({ }^{1} \mathrm{H}\right.$ and ${ }^{31} \mathrm{P}$ NMR $)$ and required no further purification. Yield $62 \%(0.446 \mathrm{~g})$; TLC $\left(1 \% \mathrm{NH}_{4}{ }^{+} \mathrm{OH}^{-}\right.$in Acetone, $\left.10 \mathrm{~mL}\right)$ or $(20 \% \mathrm{MeOH}(6 \% \mathrm{NaBr}))$ : ACN, $\mathrm{R}_{\mathrm{f}}=$ 0.47; ${ }^{1} \mathbf{H}$ NMR (400 MHz, $\mathrm{CDCl}_{3}, \delta$ ): 4.15-4.06 (m, 8H, H7), 3.11 (d, 4H, J = 8.9 Hz, H6), 2.80 (t, 2H, J = $6.8 \mathrm{~Hz}, \mathrm{H5}$ ), 2.27 (t, 2H, J = $7.5 \mathrm{~Hz}, \mathrm{H} 4), 2.18$ (s, 6H, H3), 1.61 (p, 2H, J $=7.15 \mathrm{~Hz}, \mathrm{H} 2), 1.28(\mathrm{t}, 12 \mathrm{H}, \mathrm{J}=7.0 \mathrm{~Hz}, \mathrm{H} 1) \mathrm{ppm} ;{ }^{13} \mathbf{C} \mathbf{N M R}\left(100 \mathrm{MHz}, \mathrm{CDCl}_{3}, \delta\right): 61.8\left(\mathrm{t},{ }^{2} \mathrm{~J}_{\mathrm{C}}\right.$ $\mathrm{P}=3.3 \mathrm{~Hz}, \mathrm{C} 7), 57.24$ (C4), 55.03 (C5), $50.92\left(\mathrm{dd},{ }^{1} \mathrm{~J}_{\mathrm{C}-\mathrm{P}}=7.1 \mathrm{~Hz}, \mathrm{C} 6\right), 49.36\left(\mathrm{dd},{ }^{1} \mathrm{~J}_{\mathrm{C}-\mathrm{P}}=6.7 \mathrm{~Hz}\right.$, C6), 45.48 (C3), 25.65 (C2), 16.48 (t, $\left.{ }^{3} \mathrm{~J}_{\mathrm{C}-\mathrm{P}}=2.8 \mathrm{~Hz}, \mathrm{C} 1\right) \mathrm{ppm} ;{ }^{31} \mathbf{P}$ NMR (121.45 MHz, $\mathrm{CDCl}_{3}$,

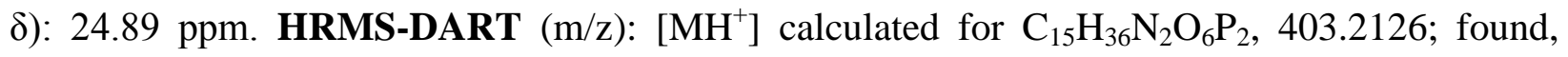
403.2135 .

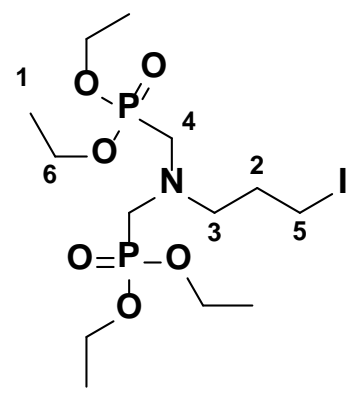

\section{Tetraethyl (((3-iodopropyl)azanediyl)bis(methylene))bis(phosphonate) (120):}

A mixture of diethyl (4-bromobutyl)phosphonate (1.0 g, 2.21 mmol, 1.0 eq.) and NaI (0.69 g, 4.6 mmol, 2.08 eq.) in acetone ( $3 \mathrm{~mL}$ ) were placed, with a magnetic stirring bar, into a $5 \mathrm{~mL} \mu \mathrm{W}$ glass reaction tube and sealed. The reaction mixture was heated in the $\mu \mathrm{W}$ at $100{ }^{\circ} \mathrm{C}(5 \mathrm{~min})$. The yellow solid was transferred to a $100 \mathrm{~mL}$ RBF washing with acetone $(50 \mathrm{~mL})$. Volatiles were removed on a rotary evaporator and the crude material was diluted with brine $(10 \mathrm{~mL})$ and extracted with $\mathrm{CHCl}_{3}(1 \times 10 \mathrm{~mL})$. The organic layer was separated, dried over anhydrous $\mathrm{MgSO}_{4}$ filtered, concentrated and recovered as a yellow oil without further purification. Yield: $75 \%$ (0.80 g); TLC (10\% $\mathrm{NH}_{4}{ }^{+} \mathrm{OH}^{-} /$acetone), $\mathrm{R}_{\mathrm{f}}=0.85 ;{ }^{1} \mathbf{H}$ NMR (400 MHz, $\left.\mathrm{CDCl}_{3}, \delta\right): 4.20-$ 
4.09 (m, 8H, H6), 3.24 (t, 2H, $J=7.01 \mathrm{~Hz}, \mathrm{H} 5), 3.15$ (d, 4H, $J=8.75 \mathrm{~Hz}, \mathrm{H} 4), 2.89$ (t, 2H, $J=$ 6.6 Hz, H3), 2.03-1.94 (m, 2H, H2), 1.33 (t, 12H, $J=7.03 \mathrm{~Hz}, \mathrm{H} 1) ;{ }^{31} \mathbf{P}$ NMR (121.45 MHz, $\left.\mathrm{CDCl}_{3}, \delta\right): 24.79 \mathrm{ppm}$.
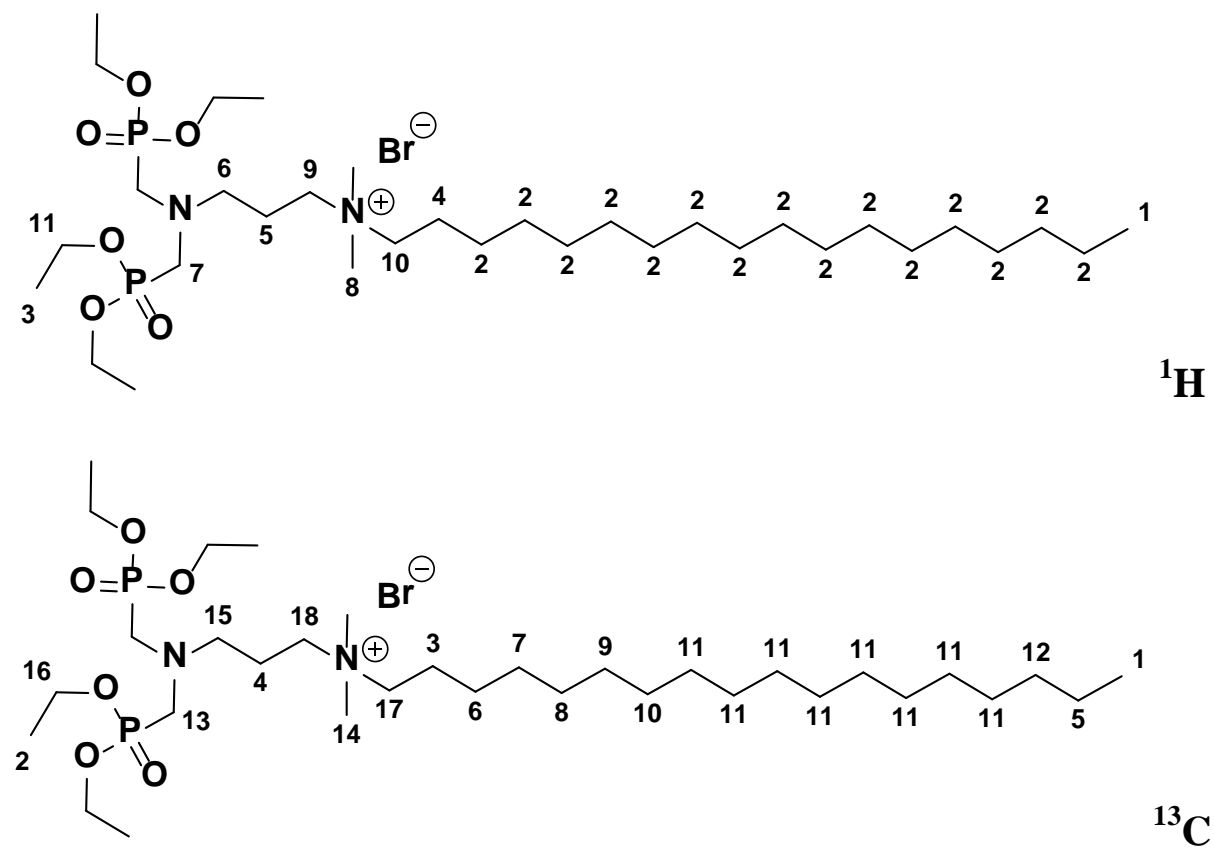

\section{$N$-(3-(bis((diethoxyphosphoryl)methyl)amino)propyl)- $N, N$-dimethyloctadecan-1- ammonium (121):}

To a flame dried and evacuated $20 \mathrm{~mL}$ screw cap vial, equipped with a magnetic stir bar was added a mixture of bromoaminobisphosphonate $(0.20 \mathrm{~g}, 0.51 \mathrm{mmol})$ and DMOA $(0.143 \mathrm{~g}, 0.6$ mmol, 1.19 eq.) was which was sealed and heated to $100^{\circ} \mathrm{C}$ on a sand bath. After $1 \mathrm{hr}$, TLC showed the disappearance of the starting amine (5\% MeOH in EtOAc, $10 \mathrm{~mL}$ ). The mixture was partitioned between hexanes $(\sim 7 \mathrm{~mL})$ and $\mathrm{MeOH} / \mathrm{H}_{2} \mathrm{O}(4: 1,5 \mathrm{~mL})$, the bottom yellow methanolic layer was separated and concentrated $\left(2 \times 5 \mathrm{~mL}\right.$ ACN to azeotrope excess $\left.\mathrm{H}_{2} \mathrm{O}\right)$ to afford a yellow oily solid (0.308 g). The crude material was purified by DCVC (20 g silica, 3.5 $\mathrm{cm} \times 4.5 \mathrm{~cm})$ pre-washed with $60 \mathrm{~mL} \mathrm{20 \%}(\mathrm{NaBr} 6 \%$ in $\mathrm{MeOH})$ : ACN then eluting with the 
same eluent ( $1^{\text {st }} 40 \mathrm{~mL}$ removed upper $\mathrm{R}_{\mathrm{f}}$ impurity, compound $\mathbf{1 2 1}$ was obtained in the next 7 fractions totaling $(95 \mathrm{~mL})$ ) as a yellow oil after filtering off $\mathrm{NaBr}$ (Celite), washing with $\mathrm{CHCl}_{3}$. Yield: 46.1\% (0.162 g). TLC (20\% MeOH (NaBr 6\%): ACN), $\mathrm{R}_{\mathrm{f}}=0.5 ;{ }^{1} \mathbf{H}$ NMR (400 MHz, $\mathrm{CDCl}_{3}, \delta$ ) 4.15-4.08 (m, 8H, H11), 3.72-3.69 (m, 2H, H10), 3.55-3.51 (m, 2H, H9), 3.33 (s, 6H, H8), 3.12-3.08 (m, 4H, H7), 2.99-2.97 (m, 2H, H6), 2.0-1.98 (m, 2H, H5), 1.74-1.71 (m, 2H, H4), 1.24-1.20 (br m, 42H, (H2 + H3 overlap)), 0.88-0.83 (m, 3H, H1) ppm; ${ }^{13}$ C NMR (100 MHz, $\mathrm{CDCl}_{3}, \delta$ ): 65.19-61.96 (C19-C16 overlap, from HSQC: 65.19 (C18), 62.52 (C17), 61.96 (C16)), 53.64 (C15), 50.86 (C14), 50.5 (d, C13), 31.90 (C12), 29.68-29.60 (C11 overlap), 29.57 (C10), 29.47 (C9), 29.39 (C8), 29.34 (C7), 29.26 (C6) 26.12 (C5), 22.83 (C4), 22.66 (C3), 16.58-16.50 (m, ${ }^{3} J_{C-P}=$ unresolved, C2), 14.10 (C1) ppm; ${ }^{31} \mathbf{P}$ NMR (121.45 MHz, $\left.\mathrm{CDCl}_{3}, \delta\right)$ 24.40 ppm. HRMS-DART (m/z): $\left[\mathrm{M}^{+}\right]-\mathrm{Br}^{-}$calculated for $\mathrm{C}_{33} \mathrm{H}_{73} \mathrm{~N}_{2} \mathrm{O}_{6} \mathrm{P}_{2}$, 655.4937; found, 655.4938.

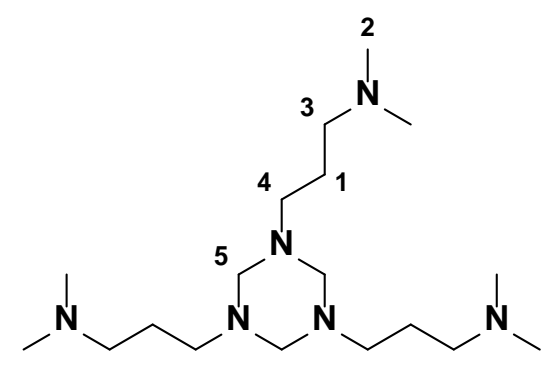

3,3',3"-(1,3,5-triazinane-1,3,5-triyl)tris(N,N-dimethylpropan-1-amine) (123): ${ }^{244-245}$

To a $125 \mathrm{~mL}$ round bottom flask, paraformaldehyde (1.652 g, $55 \mathrm{mmol}, 1.1$ eq.) was added to a solution of $N, N$-dimethylpropane-1,3-diamine $(6.29 \mathrm{~mL}, 50 \mathrm{mmol})$ in TOL $(15 \mathrm{~mL})$. The reaction was refluxed using a Dean-Stark trap for 1.5 hrs. TOL was evaporated and a portion of the residue (1.96 g) was partitioned between $\mathrm{CHCl}_{3}(15 \mathrm{~mL})$ and $\mathrm{H}_{2} \mathrm{O}(5 \mathrm{~mL})$. The organic layer was separated, dried with $\mathrm{MgSO}_{4}$ and concentrated to give a clear oil. Yield: 66\% (1.307 g). 
TLC (20\% MeOH in EtOAc, $10 \mathrm{~mL}), \mathrm{R}_{\mathrm{f}}=0.05 ;{ }^{1} \mathbf{H}$ NMR (400 MHz, $\left.\mathrm{CDCl}_{3}, \delta\right): 3.29$ (brs, $6 \mathrm{H}$, H5), 2.40 (t, 6H, $J=7.5 \mathrm{~Hz}, \mathrm{H} 4), 2.25$ (t, 6H, $J=7.5 \mathrm{~Hz}, \mathrm{H} 3$ ), 2.18 (s, 18H, H2), 1.59 (p, 6H, $J$ $=7.5 \mathrm{~Hz}, \mathrm{H1}$ ) ppm; ${ }^{13} \mathrm{C}$ NMR (100 MHz, $\mathrm{CDCl}_{3}, \delta$ ): 74.65 (C5), 57.83 (C3), 50.78 (C4), 45.54 (C2), 25.88 (C1) ppm. (Agrees well with literature NMR values). ${ }^{244-245}$

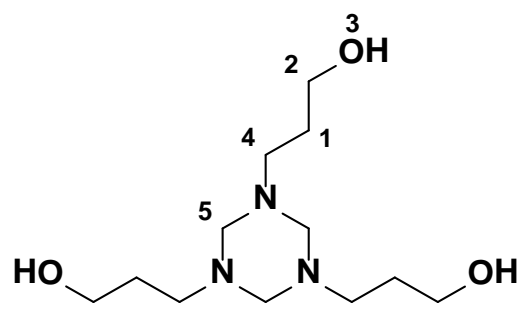

\section{3,3',3"'-(1,3,5-triazinane-1,3,5-triyl)tris(propan-1-ol) (122): ${ }^{40,246}$}

To a $125 \mathrm{~mL}$ round bottom flask, formalin $(0.813 \mathrm{~mL}, 10 \mathrm{mmol})$ was added to a solution of 3 amino-1-propanol (0.751 g, $10 \mathrm{mmol})$ in ACN $(10 \mathrm{~mL})$. The reaction was stirred at RT overnight. Evaporation of volatiles followed by DCVC (20 g silica, $3.5 \mathrm{~cm} \times 4.5 \mathrm{~cm})$ eluting with $5 \% \mathrm{NH}_{4}{ }^{+} \mathrm{OH}^{-}$in acetone $(50 \mathrm{~mL})$ then collecting $(150 \mathrm{~mL})$, provided pure product as a clear oil. Yield: $92 \%$ (0.8 g). TLC ( $5 \% \mathrm{NH}_{4}{ }^{+} \mathrm{OH}^{-}$in acetone, $10 \mathrm{~mL}$ ), $\mathrm{R}_{\mathrm{f}}=0.3 ;{ }^{1} \mathbf{H}$ NMR (400 MHz, $\mathrm{CDCl}_{3}, \delta$ ): 4.37 (s, 6H, H5), 3.84 (t, 6H, $\left.J=7.0 \mathrm{~Hz}, \mathrm{H} 4\right), 3.71$ (s, 3H, H3), 2.97 (t, 6H, $J=5.6$ Hz, H2), 1.60 (q, 6H, $J=5.40 \mathrm{~Hz}, \mathrm{H1}) \mathrm{ppm} ;{ }^{13} \mathrm{C}$ NMR (100 MHz, $\mathrm{CDCl}_{3}, \delta$ ): 83.06 (C4), 68.12 (C3), 47.78 (C2), 22.45 (C1) ppm. (Agrees well with literature NMR values). ${ }^{40,246}$

\subsubsection{General Procedure for the Bis Michael Addition of Amines onto Vinylphosphonates}

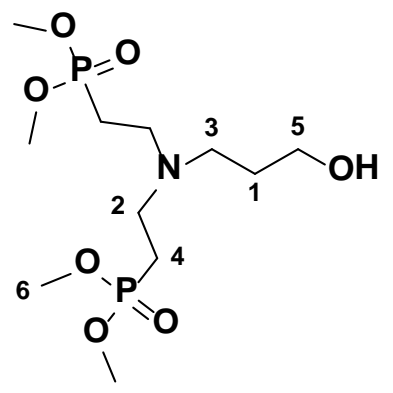




\section{Tetramethyl (((3-hydroxypropyl)azanediyl)bis(ethane-2,1-diyl))bis(phosphonate) (135):}

To a $25 \mathrm{~mL}$ round bottom flask equipped with a magnetic stir bar, was added a stirred solution of the primary amine $(0.448 \mathrm{~g}, 5.9 \mathrm{mmol})$ in distilled $\mathrm{H}_{2} \mathrm{O}(5 \mathrm{~mL})$ at RT. Two equivalents of diethyl vinylphosphonate (1.637 g, $12.03 \mathrm{mmol}, 2.01$ eq.) was then added and the reaction stirred at RT ON. The reaction was transferred to a $125 \mathrm{~mL}$ round bottom flask along with $30 \mathrm{~mL}$ ACN and evaporated to a clear oil (2.14 g, containing 7\% starting material by ${ }^{31} \mathrm{P}$ NMR). The crude material was purified by DCVC (20 g silica, $3.5 \mathrm{~cm} \times 4.5 \mathrm{~cm}$ ) eluting with $30 \% \mathrm{MeOH}$ :EtOAc (30 mL fractions, $240 \mathrm{~mL}$ ). Fractions (2-7) containing were filtered (Celite) and evaporated to obtain the title compound as a yellow oil. Yield: $95 \%$ (1.968 g); TLC (30\% MeOH:EtOAc), $\mathrm{R}_{\mathrm{f}}=$ 0.33; ${ }^{1} \mathbf{H}$ NMR (400 MHz, $\mathrm{CDCl}_{3}, \delta$ ): 3.73 (d, $\left.J=11.0 \mathrm{~Hz}, 12 \mathrm{H}, \mathrm{H} 6\right), 3.74-3.70$ (m, 2H, H5), 2.82-2.74 (m, 4H, H4), 2.60 (t, 2H, $J=6.0 \mathrm{~Hz}, \mathrm{H} 3), 1.98-1.88$ (m, 4H, H2), 1.71-1.64 (m, $2 \mathrm{H}$, Hz, H1) ppm; ${ }^{13}$ C NMR (100 MHz, $\mathrm{CDCl}_{3}, \delta$ ): 61.54 (C5), 52.65 (d, $\left.{ }^{2} J_{C-P}=6.61 \mathrm{~Hz}, \mathrm{C} 6\right), 50.69$ (C3), 46.26 (C4), 28.69 (C1), 21.84 (d, $\left.{ }^{2} J_{C-P}=138.47 \mathrm{~Hz}, \mathrm{C} 1\right)$ ppm. ${ }^{31} \mathbf{P}$ NMR (121.45 MHz, $\left.\mathrm{CDCl}_{3}, \delta\right): 32.66 \mathrm{ppm}$.

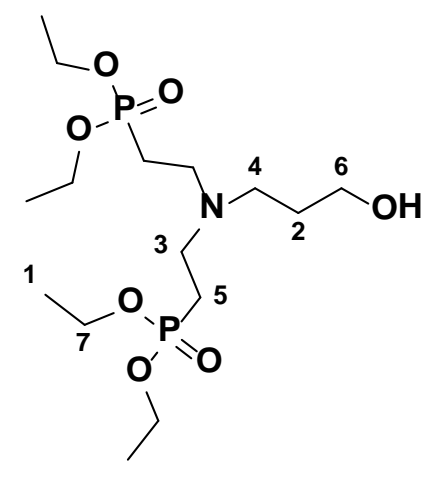

\section{Tetraethyl (((3-hydroxypropyl)azanediyl)bis(ethane-2,1-diyl))bis(phosphonate) (136): ${ }^{247}$}

To a $125 \mathrm{~mL}$ round bottom flask equipped with a magnetic stir bar, was added a stirred solution of the primary amine $(1.016 \mathrm{~g}, 13.5 \mathrm{mmol})$ in distilled $\mathrm{H}_{2} \mathrm{O}(15 \mathrm{~mL})$ at RT. Two equivalents of 
diethyl vinylphosphonate (4.44 g, $27.0 \mathrm{mmol}, 2.00$ eq.) was then added and the reaction stirred at RT ON. $\mathrm{H}_{2} \mathrm{O}$ was co-evaporated from ACN (80 mL) and evaporated to a yellow oil (containing 14\% starting material by ${ }^{31} \mathrm{P}$ NMR). The crude material was purified by DCVC (50 g silica, 3.5 $\mathrm{cm} \times 4.5 \mathrm{~cm}$ ) pre-eluting with acetone $(50 \mathrm{~mL})$ and then eluting with 35\% EtOH:Acetone (150 $\mathrm{mL}$ ) to obtain the title compound as a yellow oil. Yield: 50\% (2.722 g); TLC (30\% EtOH:Acetone), $\mathrm{R}_{\mathrm{f}}=0.40 ;{ }^{1} \mathbf{H}$ NMR (400 MHz, $\left.\mathrm{CDCl}_{3}, \delta\right): 4.16-4.03$ (m, 8H, H7), 3.75-3.67 (m, 3H, H6), 2.83-2.75 (m, 4H, H5), 2.64-2.59 (m, 2H, H4), 1.97-1.86 (m, 4H, H3), 1.68 (q, 2H, $J=5.58 \mathrm{~Hz}, \mathrm{H} 2), 1.31$ (t, $12 \mathrm{H}, J=7.06 \mathrm{~Hz}, \mathrm{H} 1) \mathrm{ppm} ;{ }^{31} \mathbf{P}$ NMR (121.45 MHz, CDCl $\left.3, \delta\right): 30.00$ ppm. $^{247}$

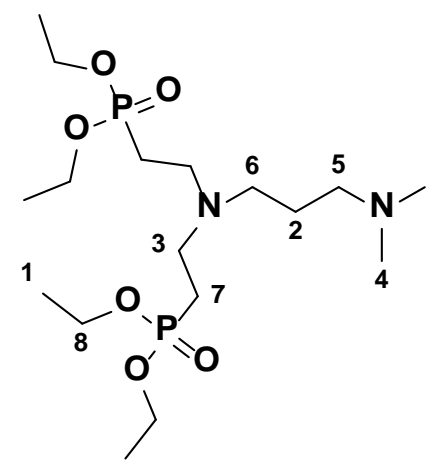

Tetraethyl (((3-(dimethylamino)propyl)azanediyl)bis(ethane-2,1-diyl)) bis (phosphonate) (139):

Synthesized from alcohol via mesylate and dimethylamine, see Tetraethyl (((3(dimethylamino)propyl)azanediyl)bis(methylene))bis(phosphonate) $\mathbf{1 1 3}$ procedure; ${ }^{\mathbf{1}} \mathbf{H} \mathbf{N M R}$ (400 MHz, $\mathrm{CDCl}_{3}, \delta$ ): 4.11-3.99 (m, 8 H, H8), 2.76-2.69 (m, 4H, H7), 2.40 (t, 2H, J = 7.12 Hz, H6), 2.22 (t, H5, $J=7.14$ Hz, H5), 2.16 (s, 6H, H4), 1.91-1.81 (m, 4H, H3), 1.60-1.53 (m, H2, 2H), $1.28(\mathrm{t}, \mathrm{H} 1, J=7.04 \mathrm{~Hz}) \mathrm{ppm} ;{ }^{31} \mathbf{P} \mathbf{N M R}\left(121.45 \mathrm{MHz}, \mathrm{CDCl}_{3}, \delta\right): 30.57 \mathrm{ppm}$. 

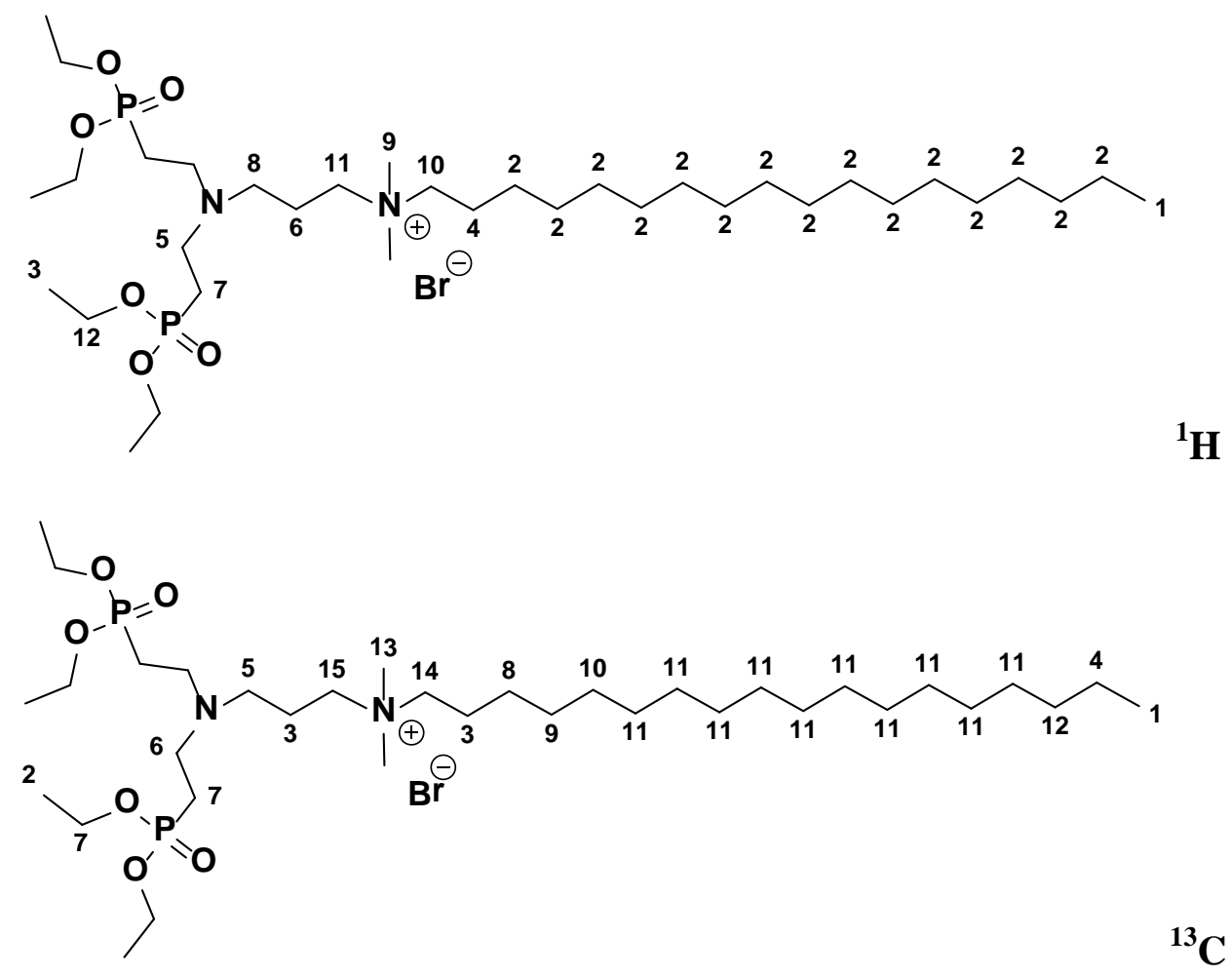

\section{$N$-(3-(bis(2-(diethoxyphosphoryl)ethyl)amino)propyl)- $N, N$-dimethyloctadecan-1- ammonium bromide (140):}

To a flame dried and evacuated $20 \mathrm{~mL}$ screw cap vial, equipped with a magnetic stir bar was added a mixture of 139 (0.181 g, $0.42 \mathrm{mmol})$ and bromooctadecane (0.140 g, $0.42 \mathrm{mmol}, 1.0$ eq.) was sealed and heated to $100^{\circ} \mathrm{C}$ on a sand batch. After $1 \mathrm{hr}$, TLC showed the disappearance of the starting amine (5\% MeOH in EtOAc, $10 \mathrm{~mL}$ ). The mixture was drypacked onto silica and the crude material was purified by DCVC (20 g silica, $3.5 \mathrm{~cm} \times 4.5 \mathrm{~cm})$ pre-washed with $2 \times 40 \mathrm{~mL}$ 20\% $\mathrm{MeOH}(\mathrm{NaBr} 6 \%)$ : ACN then eluting with the same eluent (50 mL), evaporating, redissolving with $\mathrm{CHCl}_{3}$ and filtering off $\mathrm{NaBr}$ through a pad of Celite, washing with $\mathrm{CHCl}_{3}$ to provide a yellow wax. Yield: 44\% (0.15 g). TLC (20\% MeOH (NaBr 6\%): ACN), $\mathrm{R}_{\mathrm{f}}=0.3 ;{ }^{1} \mathbf{H}$ NMR (400 MHz, $\left.\mathrm{CDCl}_{3}, \delta\right)$ 4.14-4.04 (m, 8H, H12), 3.72-3.67 (m, 2H, H11), 3.47-3.36 (m, 2H, H10), 3.27 (s, 6H, H9), 2.85-2.76 (m, 4H, H8), 2.05-1.09 (m, 4H, (H6 + H5)), 1.75-1.65 (m, 2H, 
H4), 1.31 (t, $J=7.03 \mathrm{~Hz}, 6 \mathrm{H}, \mathrm{H} 3$ ), 1.23 (brs, $30 \mathrm{H}, \mathrm{H} 2$ ), 0.86 (t, $J=6.94 \mathrm{~Hz}, 3 \mathrm{H}, \mathrm{H} 1$ ) ppm; ${ }^{13} \mathrm{C}$ NMR (100 MHz, $\mathrm{CDCl}_{3}, \delta$ ): 67.49 (C15), 61.93 (C14), 61.78 (C13), 31.89 (C12), 29.68 (C11 overlap), 29.63 (C10), 29.49 (C9), 29.42 (C8), 29.33 (C7), 29.26 (C6), 26.29 (C5), 25.60 (C4), 22.66 (C3), $16.48\left(\mathrm{~d},{ }^{3} J_{C-P}=6.03 \mathrm{~Hz}, \mathrm{C} 2\right), 14.08$ (C1) ppm; ${ }^{31} \mathbf{P}$ NMR (121.45 MHz, $\left.\mathrm{CDCl}_{3}, \delta\right)$ $24.40 \mathrm{ppm}$.
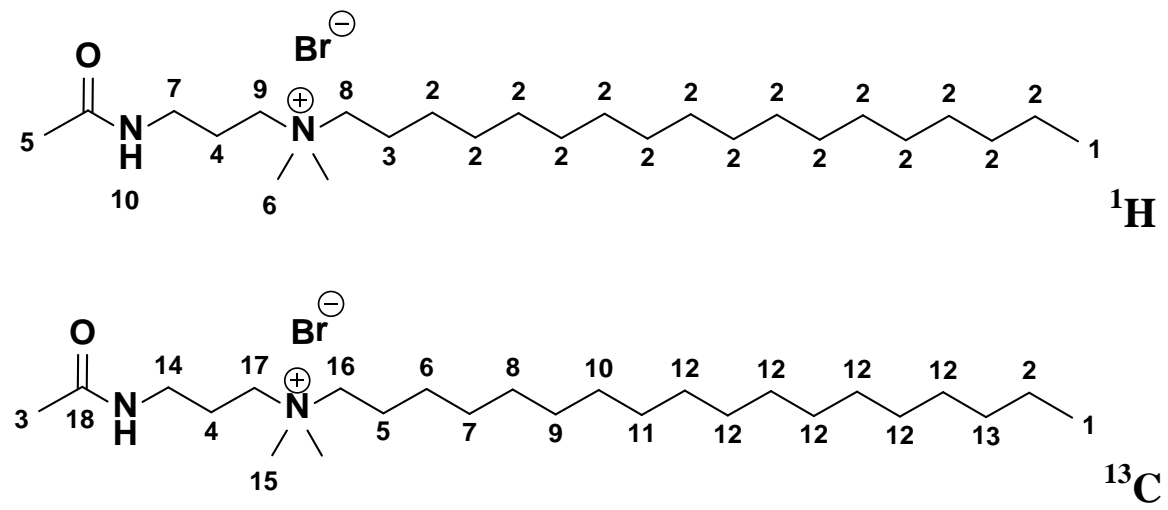

\section{$N$-(3-acetamidopropyl)- $N, N$-dimethyloctadecan-1-ammonium (153): ${ }^{248}$}

This compound was prepared by the Menshutkin reaction using Method 5.2.1: $N$-(3(dimethylamino)propyl)acetamide 148 (0.285 g, $1.97 \mathrm{mmol})$ and 1-bromooctadecane (0.664 g, $1.99 \mathrm{mmol}, 1.01$ eq.) were reacted at $100^{\circ} \mathrm{C}$ neat for 4 hrs until the mixture solidified. The mixture was then dissolved in $\mathrm{MeOH}(10 \mathrm{~mL})$ and hot filtered from charcoal. Volatiles were removed on a rotary evaporator and the crude was recrystallized from acetone $(15 \mathrm{~mL})$ by placing the solution on ice (15 min) as a white solid. Yield: $74 \%\left(0.720\right.$ g). $\mathrm{Mp}=93-95^{\circ} \mathrm{C} ;{ }^{1} \mathbf{H}$ NMR (400 MHz, $\left.\mathrm{CDCl}_{3}, \delta\right): 7.98$ (t, $\left.J=7.98 \mathrm{~Hz}, 1 \mathrm{H}, \mathrm{H} 10\right), 3.84-3.78$ (m, 2H, H9), 3.39-3.32 (m, 4H, (H7 + H8 overlap)), 3.26 (s, 6H, H5), 2.10-2.00 (m, 2H, H5), 2.04 (s, 3H, H4), 1.75-1.65 (m, 2H, H3), 1.22 (brs, 30H, H2), 0.84 (t, $J=6.79 \mathrm{~Hz}, 3 \mathrm{H}, \mathrm{H1}) \mathrm{ppm} ;{ }^{13} \mathrm{C}$ NMR (100 MHz, $\mathrm{CDCl}_{3}, \delta$ ): 171.60 (C18), 64.97 (C17), 63.00 (C16), 51.13 (C15), 36.22 (C14), 32.00 (C13), 
29.80-29.74 (C12 overlap), 29.73 (C11), 29.66 (C10), 29.53 (C9), 29.46 (C8), 29.44 (C7), 29.25

(C6), 26.38 (C5), 23.36 (C4), 22.85 (C3), 22.76 (C2), 14.20 (C1) ppm. (Agrees well with literature NMR values). ${ }^{248}$

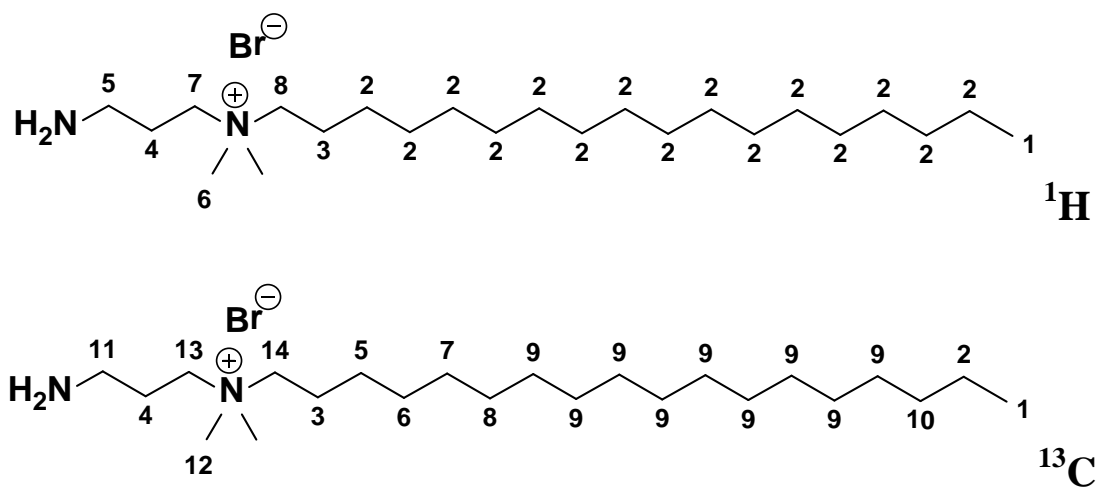

\section{$N$-(3-aminopropyl)- $N, N$-dimethyloctadecan-1-ammonium bromide (144): ${ }^{248}$}

This compound was prepared by dissolving $152(10.80 \mathrm{~g}, 14.16 \mathrm{mmol})$ in EtOH $(80 \mathrm{~mL})$ and deprotecting with hydrazine hydrate ( $5.5 \mathrm{~mL}, 5$ eq.) under reflux $1.5 \mathrm{hrs}$. The mixture was then cooled to RT, diluted with $\mathrm{CHCl}_{3} / \mathrm{ACN}(1: 1,100 \mathrm{~mL})$ and filtered through Celite. Volatiles were removed in vacuo and the crude product (8.647 g, yellow gum) was again diluted with $\mathrm{CHCl}_{3} / \mathrm{ACN}(1: 1,100 \mathrm{~mL})$ and placed in the freezer $\left(-20^{\circ} \mathrm{C}, 30 \mathrm{~min}\right)$ to further precipitate the pthalylhydrazide impurity (white solid) and filtered through Celite. Volatiles were evaporated and the sample was placed under high vaccum ( $1 \mathrm{hr})$ to obtain the title compound as a yellow/white waxy solid. Yield: 87\% (5.361 g); ${ }^{1} \mathbf{H}$ NMR (400 MHz, $\mathrm{CDCl}_{3}, \delta$ ): 3.75-3.66 (m, 2H, H8), 3.48-3.39 (m, 2H, H7), 3.33 (s, 6H, H6), 2.29-2.82 (m, 2H, H5), 2.01-1.87 (m, 2H, H4), 1.79-1.67 (m, 2H, H3), 1.24 (brs, 30H, H2), 0.86 (t, $J=7.06$ Hz, 3H, H1) ppm; ${ }^{13}$ C NMR (100 MHz, $\mathrm{CDCl}_{3}, \delta$ ): 64.15 (C11), 62.27 (C10), 51.02 (C9), 38.28 (C8), 31.55 (C7), 29.40-27.31 (C6 overlpa), 26.32 (C5), 25.84 (C4), 22.54 (C3 ), 22.44(C2), 13.86 (C1) ppm. ${ }^{248}$ 


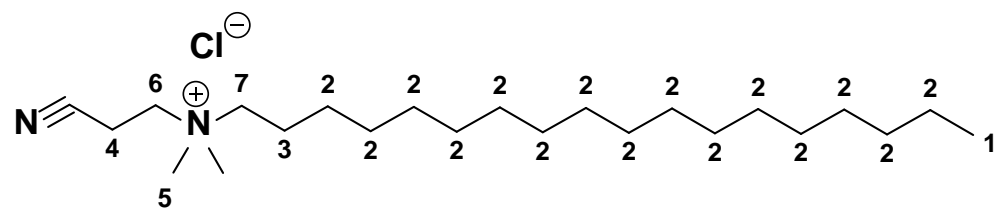

\section{$\mathrm{N}$-(2-cyanoethyl)- $N, N$-dimethyloctadecan-1-ammonium (154):}

This compound was prepared by the Menshutkin reaction using Method 5.2.1: 3chloropropanenitrile 149 (1.429 g, 18.9 mmol) and DMOA (6.258 g, $18.7 \mathrm{mmol}$, 1.0 eq.) were reacted at $100^{\circ} \mathrm{C}$ neat for 5 min until the mixture solidified. To the solid mixture was added $\mathrm{MeOH}(10 \mathrm{~mL})$ and heating was continued for another $30 \mathrm{~min}$. Volatiles were removed on a rotary evaporator and the crude product was recrystallized from acetone $(200 \mathrm{~mL})$ by placing the solution on ice (15 min) recovered a waxy white solid. Yield: $88 \%\left(6.0\right.$ g). $\mathrm{Mp}=54-55^{\circ} \mathrm{C} ;{ }^{1} \mathbf{H}$ NMR (400 MHz, $\left.\mathrm{CDCl}_{3}, \delta\right): 3.72-3.67$ (m, 2H, H7), 3.56 (s, 6H, H6), 2.09-1.98 (m, 2H, H5), 1.86-1.76 (m, 2H, H4), 1.42-1.32 (s, 2H, H2), 1.24 (brs, 30H, H2), 0.87 (t, $J=6.68$ Hz, 3H, H1) ppm.
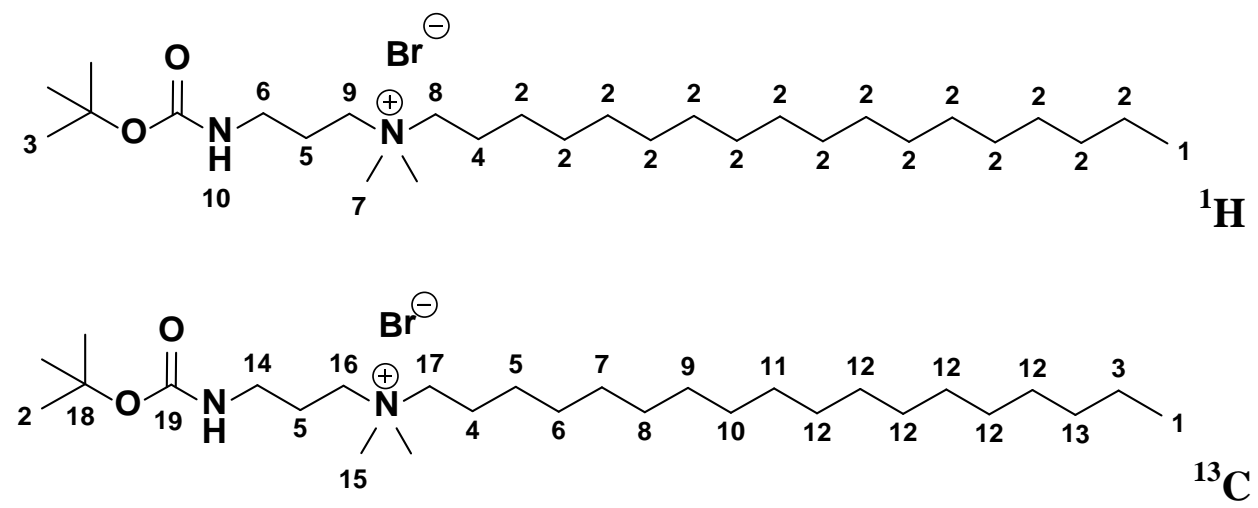

$N$-(3-((tert-butoxycarbonyl)amino)propyl)- $N, N$-dimethyloctadecan-1-ammonium (155): ${ }^{249}$ 
This compound was prepared by the Menshutkin reaction using Method 5.2.1: tert-butyl (3bromopropyl)carbamate 150 (1.421 g, $5.96 \mathrm{mmol}$ ) and DMOA (2.4649 g, 7.37 mmol, 1.23 eq.) were reacted at $100^{\circ} \mathrm{C}$ neat for 35 min until the mixture solidified. The crude reaction was recrystallized from acetone $(20 \mathrm{~mL})$ by placing the solution on ice (10 min) and recovered as an off white waxy solid. The final product was isolated as a mixture with the starting material DMOA in a 0.63:0.37 ratio by ${ }^{1} \mathrm{H}$ NMR and used without further purification. Yield: $70 \%$ (2.243 g). $\mathrm{Mp}=98-100^{\circ} \mathrm{C} ;{ }^{1} \mathbf{H}$ NMR (400 $\mathrm{MHz} \mathrm{CDCl}_{3}, \delta$ ): 5.8 (s, 1H, H10), 3.65-3.56 (m, 2H, H9), 3.44-3.33 (m, 2H, H8), 3.28 (s, 6H, H7), 3.25-3.17 (m, 2H, H6), 2.07-1.95 (m, 2H, H5), 1.741.60 (m, 2H, H4), 1.38 (s, 12H, H3), 1.23 (brs, 30H, H2), 0.83 (t, J = $7.02 \mathrm{~Hz}, 3 \mathrm{H}, \mathrm{H} 1$ ) ppm; ${ }^{13} \mathbf{C}$ NMR (100 MHz, $\left.\mathrm{CDCl}_{3}, \delta\right): 156.92$ (C23), 136.77 (C22), 128.49 (C21), 128.07 (C20), 128.0 (C19), 66.52 (C18), 64.50 (C17), 62.33 (C16), 51.07 (C15), 37.88 (C14), 31.90 (C13), 29.8029.74 (C12 overlap), 29.65 (C11), 29.59 (C10), 29.47 (C9), 29.40 (C8), 29.34 (C7), 29.19 (C6), 26.25 (C5), 23.19 (C4), 22.73 (C3), 22.67 (C2), 14.11 (C1) ppm. (Agrees well with literature NMR values). ${ }^{249}$
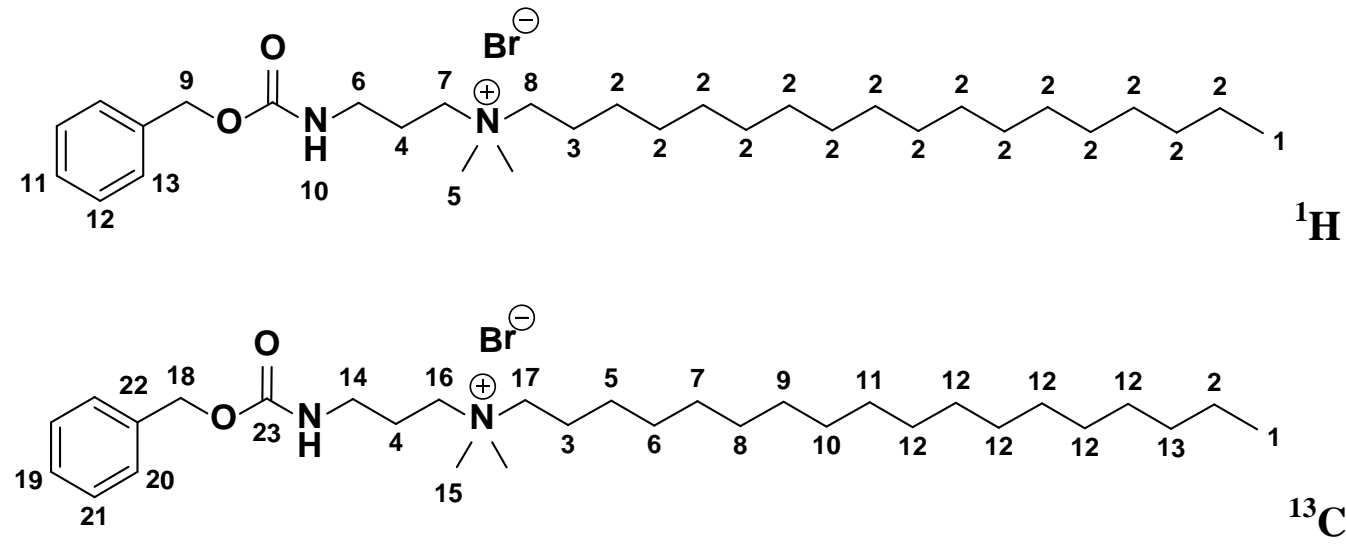

$N$-(3-(((benzyloxy)carbonyl)amino)propyl)- $N, N$-dimethyloctadecan-1-ammonium (156): 
This compound was prepared by the Menshutkin reaction using Method 5.2.1: benzyl (3bromopropyl)carbamate 151 (0.991 g, $3.64 \mathrm{mmol})$ and DMOA (1.21 g, $3.62 \mathrm{mmol}$, 1.0 eq.) were reacted at $100^{\circ} \mathrm{C}$ neat for 35 min until the mixture solidified. The mixture was then cooled to $\mathrm{RT}$, poured into $20 \mathrm{~mL}$ of $\mathrm{Et}_{2} \mathrm{O}$, and placed into a freezer $\left(-20^{\circ} \mathrm{C}\right)$ for 60 min to precipitate the title compound as a white waxy solid. Yield: $70 \%$ (1.52 g). $\mathrm{Mp}=98-100{ }^{\circ} \mathrm{C} ;{ }^{1} \mathbf{H} \mathbf{~ N M R}(400$ MHz, $\left.\mathrm{CDCl}_{3}, \delta\right):$ 7.32-7.27 (m, 3H, H11-13), 6.51 (t, $J=6.51$ Hz, H10), 5.06 (s, 2H, H9), 3.653.56 (m, 2H, H8), 3.35-3.25 (m, 4H, (H6, H7)), 3.18 (s, 6H, H5), 2.05-1.95 (m, 2H, H4), 1.651.50 (m, 2H, H3), 1.23 (brs, 30H, H2), 0.86 (t, $J=6.69 \mathrm{~Hz}, 3 \mathrm{H}, \mathrm{H} 1) \mathrm{ppm} ;{ }^{13} \mathrm{C}$ NMR (100 MHz, $\mathrm{CDCl}_{3}, \delta$ ): 156.92 (C23), 136.77 (C22), 128.49 (C21), 128.07 (C20), 128.0 (C19), 66.52 (C18),64.50 (C17), 62.33 (C16), 51.07 (C15), 37.88 (C14), 31.90 (C13), 29.80-29.74 (C12 overlap),29.65 (C11), 29.59 (C10), 29.47 (C9), 29.40 (C8), 29.34 (C7), 29.19 (C6), 26.25 (C5), 23.19 (C4), 22.73 (C3), 22.67 (C2), 14.11 (C1) ppm.
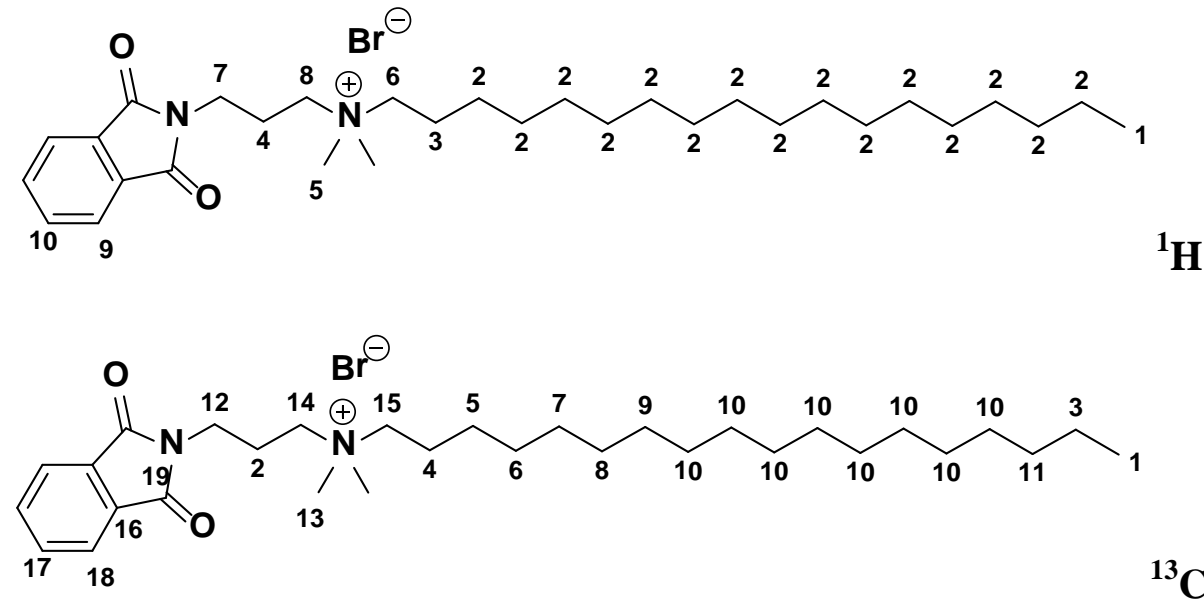

$N$-(3-(1,3-dioxoisoindolin-2-yl)propyl)- $N, N$-dimethyloctadecan-1-ammonium bromide (152): 
This compound was prepared by the Menshutkin reaction using Method 5.2.2: 3bromopropylphthalimide 10 (8.00 g, 29.38 mmol, 1.0 eq.) and DMOA (8.80 g, 29.38 mmol, 1.0 eq.) in ACN ( $80 \mathrm{~mL}$ ) were refluxed for $4.2 \mathrm{hrs}$. The mixture was then cooled to RT, poured into $100 \mathrm{~mL}$ of $\mathrm{Et}_{2} \mathrm{O}$, and left at RT for 30 min to precipitate the title compound as a white solid. Yield: 84\% (14.172 g). $\mathrm{Mp}=100-105^{\circ} \mathrm{C} ;{ }^{1} \mathbf{H}$ NMR (400 MHz, $\left.\mathrm{CDCl}_{3}, \delta\right): 7.82-7.76$ (m, 2H, H10), 7.72-7.64 (m, 2H, H9), 3.81 (t, $J=6.62 \mathrm{~Hz}, 2 \mathrm{H}, \mathrm{H8}$ ), 3.76-3.64 (m, 2H, H7), 3.53-3.46 (m, 2H, H6), 3.41 (s, 6H, H5), 2.24-2.13 (m, 2H, H4), 1.75-1.58 (m, 2H, H3), 1.22 (brs, 30H, H2), 0.84 (m, $J=6.8 \mathrm{~Hz}, 3 \mathrm{H}, \mathrm{H1}$ ) ppm; ${ }^{13} \mathbf{C}$ NMR (100 MHz, $\left.\mathrm{CDCl}_{3}, \delta\right): 168.23$ (C19), 134.29 (C18),131.78 (C17), 123.50 (C16), 64.32 (C15), 61.32 (C14), 51.41 (C13), 34.92 (C12), 31.90 (C11), 29.75-29.65 (C10 overlap), 29.58 (C9), 29.47 (C8), 29.34 (C7), 29.21 (C6), 26.22 (C5), 23.80 (C4), 22.66 (C3), 22.48 (C2), 14.10 (C1) ppm.

\subsubsection{Preparation of Tris Phosphonic Acid Derivatives}

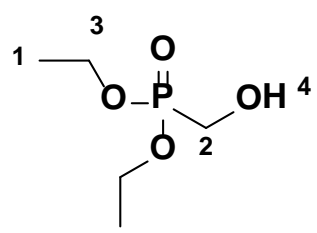

\section{Diethyl (hydroxymethyl)phosphonate (169): ${ }^{250}$}

Into a Schlenk flask (250 mL flask), equipped with a condenser and a magnetic stirrer, was introduced diethylhydrogenphosphonate $\mathrm{HP}(\mathrm{O})(\mathrm{OEt})_{2}(20 \mathrm{~g}, 144.8 \mathrm{mmol})$, paraformaldehyde (5.2 g, 1.2 eq.), EtOH (30 mL) and powdered $\mathrm{K}_{2} \mathrm{CO}_{3}(1 \mathrm{~g}, 0.05$ eq.) and the mixture was refluxed for $60 \mathrm{~min}$. At the end of the reaction, the solution was evaporated and filtered through a short pad of Celite from acetone (50 mL). The solvent was removed under vacuum to obtain the title compound as a clear liquid which may be distilled (bp $=95^{\circ} \mathrm{C}, \sim 5 \times 10^{-2} \mathrm{mbar}$ ) but was used 
without any further purification. Yield: 94\% (22.84 g). ${ }^{1} \mathbf{H}$ NMR (400 MHz, $\mathrm{CDCl}_{3}, \delta$ ): 4.87 (s, 1H, H4); 4.14 (t, $\left.{ }^{2} J=5.8 \mathrm{~Hz}, 4 \mathrm{H}, \mathrm{H} 3\right) ; 3.57\left(\mathrm{~d},{ }^{2} J=5.9 \mathrm{~Hz}, 2 \mathrm{H}, \mathrm{H} 2\right) ; 1.29\left(\mathrm{t},{ }^{3} J=7.0 \mathrm{~Hz}, 6 \mathrm{H}\right.$, H1) ppm; ${ }^{13}$ C NMR (100 MHz, $\left.\mathrm{CDCl}_{3}, \delta\right): 62.45\left(\mathrm{~d},{ }^{1} J=6.7 \mathrm{~Hz}, \mathrm{C} 3\right), 56.90\left(\mathrm{~d},{ }^{1} J=162.6 \mathrm{~Hz}\right.$, C2), 16.35 (d, $\left.{ }^{1} J=5.6 \mathrm{~Hz}, \mathrm{C} 1\right)$ ppm; ${ }^{31} \mathbf{P}$ NMR (121.45 MHz, $\mathrm{CDCl}_{3}, \delta$ ): $24.72 \mathrm{ppm}$. (Agrees well with literature NMR values). ${ }^{250}$

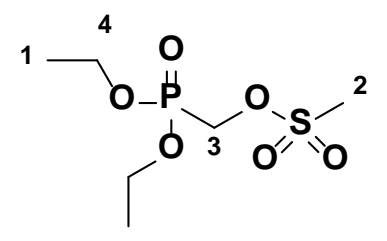

\section{(Diethoxyphosphoryl)methyl methanesulfonate (158): ${ }^{250}$}

To a flame dried and evacuated $125 \mathrm{~mL}$ round bottom flask, equipped with a magnetic stir bar was added sequentially $\mathrm{NMe}_{3} \cdot \mathrm{HCl}\left(2.41 \mathrm{~g}, 12.68 \mathrm{mmol}, 0.20\right.$ eq.), DCM (100 mL), $\mathrm{Et}_{3} \mathrm{~N}(13.26$ mL, 95.1 mmol, 1.5 eq.) and the alcohol 169 (10.60 g, 63.4 mmol, 1.0 eq.) and the solution was cooled to $0^{\circ} \mathrm{C}$ in an ice bath. To the chilled stirred solution was added, dropwise, mesyl chloride ( $~ 8.5 \mathrm{~mL}, 69.74 \mathrm{mmol}, 1.1$ eq.) in anhydrous DCM $(2 \mathrm{~mL})$ and the cloudy yellow mixture was stirred for $30 \mathrm{~min}$ at RT at which point TLC showed disappearance of the starting alcohol (10\% $\mathrm{MeOH}$ in EtOAc, $10 \mathrm{~mL})$. The reaction was diluted with $\mathrm{H}_{2} \mathrm{O}(1 \times 80 \mathrm{~mL})$ and extracted. The organic layer was washed with brine (60 mL) and dried over $\mathrm{MgSO}_{4}$, filtered and evaporated to give a yellow oil. The crude product was packed onto silica and purified by dry column chromatography $(4.5 \mathrm{~cm} \times 5.0 \mathrm{~cm}$ frit, 40 g silica) pre-eluting with EtOAC/Hexanes (60\%, 150 $\mathrm{mL}$ ) then eluting with EtOAC/MeOH (25\%,200 mL) to afford the title compound as a yellow oil.Yield: 99\% (15.50g). ${ }^{1} \mathbf{H}$ NMR (400 MHz, $\mathrm{CDCl}_{3}, \delta$ ): 4.36 (d, $\left.{ }^{2} J=7.9 \mathrm{~Hz} 4 \mathrm{H}, \mathrm{H} 4\right)$; 4.204.11 (m, 2H, H3); 3.07 (s, 3H, H2); 1.31 (t, $\left.{ }^{3} \mathrm{~J}=7.1 \mathrm{~Hz}, 6 \mathrm{H}, \mathrm{H} 1\right)$ ppm; ${ }^{13} \mathbf{C}$ NMR (100 MHz, 
$\left.\mathrm{CDCl}_{3}, \delta\right): 63.45\left(\mathrm{~d},{ }^{1} \mathrm{~J}=6.4 \mathrm{~Hz}, \mathrm{C} 3\right), 61.0\left(\mathrm{~d},{ }^{2} J=169.5 \mathrm{~Hz}, \mathrm{C} 4\right), 37.80(\mathrm{C} 2), 16.35\left(\mathrm{~d},{ }^{1} J=5.7\right.$ Hz, C1) ppm; ${ }^{31} \mathbf{P}$ NMR (121.45 MHz, $\mathrm{CDCl}_{3}, \delta$ ): 15.65 ppm. (Agrees well with literature NMR values). ${ }^{250}$

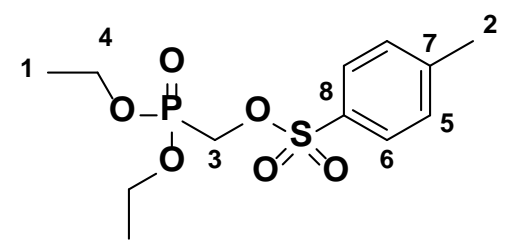

\section{(Diethoxyphosphoryl)methyl 4-methylbenzenesulfonate (170): ${ }^{251}$}

To a chilled and stirred solution of the alcohol $169(0.168 \mathrm{~g}, 1 \mathrm{mmol}), \mathrm{NMe}_{3} \cdot \mathrm{HCl}(0.040 \mathrm{~g}, 0.21$ mmol, 0.21 eq.), $\mathrm{Et}_{3} \mathrm{~N}$ (0.21 mL, $1.5 \mathrm{mmol}, 1.5$ eq.) in $\mathrm{ACN}$ (1 mL) inside a flame dried and evacuated $25 \mathrm{~mL}$ round bottom flask was added TsCl (0.210 g, $1.1 \mathrm{mmol}, 1.1 \mathrm{eq}$.) in ACN (1 $\mathrm{mL}$ ) at $0{ }^{\circ} \mathrm{C}$. The clear and cloudy mixture was stirred for $1 \mathrm{hr}$ at $\mathrm{RT}$ at which point TLC showed disappearance of the starting amine (5\% $\mathrm{MeOH}$ in EtOAc , $10 \mathrm{~mL}$ ). The reaction was diluted with $\mathrm{H}_{2} \mathrm{O}(2 \mathrm{~mL})$ and extracted with EtOAc $(2 \times 5 \mathrm{~mL})$, the organic layer was washed with brine (2 mL), dried over $\mathrm{MgSO}_{4}$, filtered and evaporated to give a clear oil. The crude material was purified by flash chromatography on silica gel (20 g silica, $1.5 \mathrm{~cm}$ i.d) with gradient elution: 100\% EtOAc (35 mL) then 5\% MeOH:EtOAc $(90 \mathrm{~mL})$ to obtain the title compound as a yellow oil. Yield 93\% (0.30 g); TLC (5\% MeOH in EtOAc), $\mathrm{R}_{\mathrm{f}}=0.42 ;{ }^{1} \mathbf{H}$ NMR (400 MHz, $\left.\mathrm{CDCl}_{3}, \delta\right): 7.73(\mathrm{t}, J=8.0 \mathrm{~Hz}, 4 \mathrm{H}, \mathrm{H} 6) ; 7.29(\mathrm{t}, J=3.8 \mathrm{~Hz}, 4 \mathrm{H}, \mathrm{H} 5) ; 4.14-4.02$ (m, 6H, (H3 + H4 overlap)); 3.39 (s, 3H, H2); 1.23 (t, $J=7.1 \mathrm{~Hz}, 6 \mathrm{H}, \mathrm{H} 1) \mathrm{ppm} ;{ }^{13} \mathbf{C} \mathbf{N M R}\left(100 \mathrm{MHz}, \mathrm{CDCl}_{3}, \delta\right)$ : 145.52 (C8), 131.64 (C7), 129.99 (C6), 129.99 (C5), 128.13 (C6), 63.31 (d, ${ }^{1} J=36.53 \mathrm{~Hz}$, C3),61.28 (d, $\left.{ }^{2} J=168.91 \mathrm{~Hz}, \mathrm{C} 4\right), 21.60$ (C2),16.25 (d, $\left.{ }^{1} J=5.81 \mathrm{~Hz}, \mathrm{C} 1\right) \mathrm{ppm} ;{ }^{31} \mathbf{P}$ NMR (121.45 MHz, $\left.\mathrm{CDCl}_{3}, \delta\right): 15.12 \mathrm{ppm}$. (Agrees well with literature NMR values). ${ }^{251}$ 


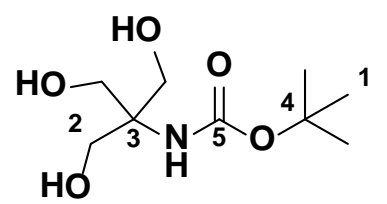

Tert-butyl (1,3-dihydroxy-2-(hydroxymethyl)propan-2-yl)carbamate (157): ${ }^{252}$

To a solution of 2-amino-2-(hydroxymethyl)propane-1,3-diol (tris) 171 (6.057 g; 50 mmol) dissolved in $\mathrm{MeOH}(180 \mathrm{~mL})$ was added a solution of $\mathrm{Boc}_{2} \mathrm{O}$ (11.350 g; $\left.52 \mathrm{mmol}\right)$ in $\mathrm{MeOH}$ (40 $\mathrm{mL}$ over 30 min and the reaction mixture was stirred at RT overnight. Volatiles were evaporated to dryness, and the title compound recrystallized from EtOAc $(100 \mathrm{~mL})$ as white cotton like needles. Yield 94\% (10.00 g); ${ }^{1} \mathbf{H}$ NMR (400 MHz, D $\left.{ }_{2} \mathrm{O}, \delta\right): 3.65$ (s, 9H, H2); 1.38 (s, 6H, H1) ppm; ${ }^{13}$ C NMR (100 MHz, DMSO, $\delta$ ): 155.25 (C5), 78.10 (C4), 60.54 (C2), 60.43 (C3), 28.39 (C1) ppm. (Agrees well with literature NMR values). ${ }^{252}$

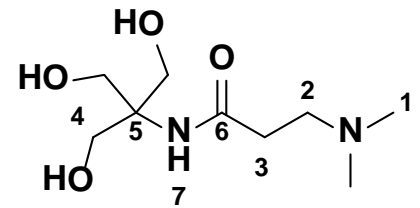

\section{N-(1,3-dihydroxy-2-(hydroxymethyl)propan-2-yl)-3-(dimethylamino)propanamide (163):}

To a solution of $N$-[tri(hydroxy- methyl)methyl]acrylamide $\mathbf{1 7 3}$ (1.051g, 6 mmol, 1.0 eq.) in $\mathrm{H}_{2} \mathrm{O}$ (3 mL) was added $\mathrm{HNMe}_{2}$ (2 mL, 5.6 M in EtOH, 2 eq.) at RT and the reaction was stirred for 5 min at which point TLC (EtOAc:MeOH: 1:1, $20 \mathrm{~mL}$ ) showed consumption of the starting amine. The reaction was separated from $\mathrm{H}_{2} \mathrm{O}$ by coevaporation from $\mathrm{ACN}$, stirring in $\mathrm{CHCl}_{3}(10$ $\mathrm{mL}$ ) for 5 min with decanting followed by drying under high vaccum (60 min) and recovered as a white solid. Yield 100\% (1.321 g); TLC (50\% MeOH in EtOAc), Rf = 0.05; ${ }^{\mathbf{H}}$ NMR (400 MHz, $\mathrm{D}_{2} \mathrm{O}, \delta$ ): 3.76 (s, 6H, H4); 2.67 (t, $J=7.03$ Hz, 2H, H3); 2.48 (t, $J=3.46$ Hz, 2H, H2); 2.23 
(s, 6H, H1) ppm; ${ }^{13} \mathrm{C}$ NMR (100 MHz, $\mathrm{CDCl}_{3}, \delta$ ): 175.0 (C6), 62.95 (C5), 60.27 (C4), 54.12

(C3), 43.59 (C1), 33.38 (C2) ppm. HRMS-DART (m/z): [M+] calculated for $\mathrm{C}_{9} \mathrm{H}_{20} \mathrm{~N}_{2} \mathrm{O}_{4}$, 221.15013; found, 221.14964.

\subsubsection{Preparation of Bis Amines Scaffolds for Multidentate Phosphonic Acid Synthesis}

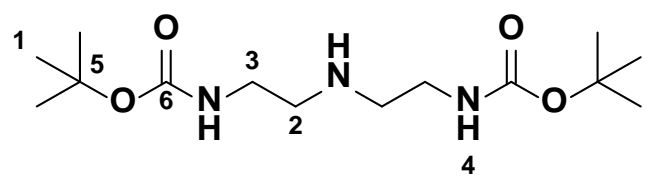

\section{Di-tert-butyl (azanediylbis(ethane-2,1-diyl))dicarbamate (176): ${ }^{253}$}

To a stirred solution of 1,1'-carbonyldiimidazole (CDI) (12.97 g. 80 mmol, 2 eq.), KOH (0.112 g, $2 \mathrm{mmol}$ ) and $t$-BuOH (7.65 mL, $80 \mathrm{mmol}, 2$ eq.) in anhydrous TOL (300 mL) preheated to $60^{\circ} \mathrm{C}$ for 3 hrs was added $N$-(2-aminoethyl)ethane-1,2-diamine dropwise (4.34 mL, 40 mmol, 1.0 eq.) via a syringe. The reaction was further stirred at $60^{\circ} \mathrm{C}$ for another $3 \mathrm{hrs}$ and then cooled to RT and rotovaped to remove volatiles. The residue was dissolved in DCM (100 mL) and washed with $\mathrm{H}_{2} \mathrm{O}(3 \times 50 \mathrm{~mL})$, dried with $\mathrm{MgSO}_{4}$, evaporated and placed under high vaccum for 4 hrs to give a thick light yellow oil. Yield: 62\% (7.51 g). ${ }^{\mathbf{1}} \mathbf{H}$ NMR (400 $\left.\mathrm{MHz}^{\mathrm{CDCl}} \mathrm{CDC}_{3}, \delta\right)$ : 4.10-3.93 (m, 4H, H6), 3.38-3.29 (m, 2H, H5), 1.91-1.80 (m, 2H, H4), 1.75-1.60 (m, 4H, (H2 + H3)), 1.301.20 (m, 6H, H1) ppm. (Agrees well with literature NMR values). ${ }^{253}$

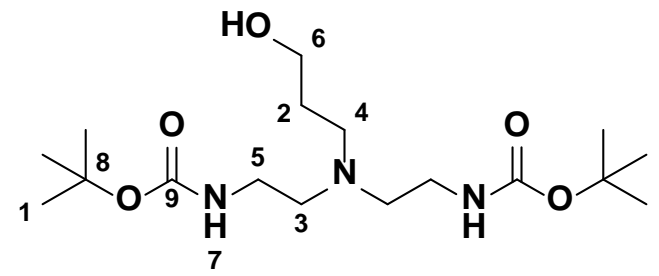

Di-tert-butyl (((3-hydroxypropyl)azanediyl)bis(ethane-2,1-diyl))dicarbamate (177): 
A solution of the secondary amine 176 (7.511 g, $24.7 \mathrm{~mol}), N, N$-diisopropylethylamine (5.17 mL, 37.13 mmol, 1.2 eq.), 3-bromo-1-propanol 74 (2.46 mL, 27.23 mmol, 1.1eq.) in $100 \mathrm{~mL}$ ACN were refluxed (24 hrs). After completion of the reaction (monitored by TLC) the reaction mixture was evaporated to dryness under reduced pressure. The residue was dissolved in DCM (200 mL) and washed with $\mathrm{H}_{2} \mathrm{O}(200 \mathrm{~mL})$ followed by another extraction with DCM (200 mL) and a brine wash $(100 \mathrm{~mL})$ of the organic phases. Volatiles were removed under reduced pressure and the yellow liquid was placed under hight vaccum (15 min) to give a yellow thick oil that solidified in the freezer $\left(-20^{\circ} \mathrm{C}\right)$. The collected organic fractions were dried over $\mathrm{MgSO}_{4}$ and the solvent was removed under reduced pressure to yield the crude product as a yellow oil. Yield: 81\% (6.0 g). ${ }^{1} \mathbf{H}$ NMR (400 MHz, $\mathrm{CDCl}_{3}, \delta$ ): 5.06 (s, 2H, H7), 3.70 (t, $J=5.57 \mathrm{~Hz}, 2 \mathrm{H}$, H6), 3.22-3.15 (m, 4H, H5), 2.61 (t, $J=6.14 \mathrm{~Hz}, 2 \mathrm{H}, \mathrm{H} 4), 2.52$ (t, $J=6.02 \mathrm{~Hz}, 4 \mathrm{H}, \mathrm{H3}), 1.61$ (m, 2H, H2), 1.42 (s, 18H, H1) ppm; ${ }^{13}$ C NMR (100 MHz, $\mathrm{CDCl}_{3}$, d): 156.301 (C8), 79.15 (C7), 61.97 (C6), 53.94 (C3), 52.70C (4), 38.20 (C5), 30.45 (C2), 28.38 (C1) ppm.

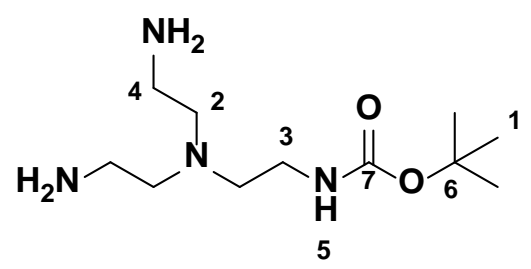

\section{Tert-butyl (2-(bis(2-aminoethyl)amino)ethyl)carbamate (181):}

To a stirred solution of tris(2-aminoethyl)amine (36.5 g, $249 \mathrm{mmol})$ in dioxane (200 mL) was added $\mathrm{Boc}_{2} \mathrm{O}$ dropwise $(6.91 \mathrm{~mL}, 30 \mathrm{mmol}, 0.12$ eq. ) over $15 \mathrm{~min}$, followed by rinsing the addition funnel with dioxane $(10 \mathrm{~mL})$. The reaction was stirred at RT overnight ( 20 hrs) and then dioxane was removed under reduced pressure. The residue was dissolved in $\mathrm{H}_{2} \mathrm{O}(80 \mathrm{~mL})$ and repeatedly extracted with DCM $(5 \times 100 \mathrm{~mL})$ followed by a brine wash $(80 \mathrm{~mL})$. Volatiles 
were removed under reduced pressure and the yellow liquid was placed under high vaccum (15 min) to give a yellow thick oil that solidified in the freezer $\left(-20^{\circ} \mathrm{C}\right)$. Note the NMR spectrum recorded the final product was contamined with dioxane even after high vaccum. Yield: 81\% (6.0g). ${ }^{1} \mathbf{H}$ NMR (400 MHz, $\mathrm{CDCl}_{3}, \delta$ ): 5.21 (s, 1H, H6), 3.16-3.07 (m, 4H, H4), 2.71 (t, $J=6.15$ Hz, 2H, H3), 2.53-2.46 (m, 6H, H2), 1.40 (s, 18H, H1) ppm; ${ }^{13}$ C NMR (100 MHz, $\mathrm{CDCl}_{3}, \delta$ ): 156.30 (C7), 79.13 (C6), 57.31 (C2), 54.07 C(3), 54.07 (C3), 39.15 (C4), 28.40 (C1) ppm. (Agrees well with literature NMR values). ${ }^{254}$

\subsection{Preparation of Catechol QAC}

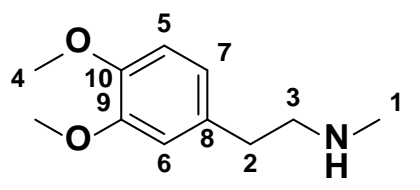

\section{2-(3,4-dimethoxyphenyl)-N-methylethanamine (185): ${ }^{255}$}

This compound was prepared by the Echweiler clarke reaction using Method 5.2.1: To a refluxing solution of 2-(3,4-dimethoxyphenyl)ethanamine (3.0 g, $16.5 \mathrm{mmol}, 1.0$ eq.) in $\mathrm{MeOH}$ (5 mL) was added by syringe formalin ( $4.5 \mathrm{~mL}, 44.8 \mathrm{mmol}, 2.7$ eq.), formic acid (5.5 mL, 105.05 mmol, 6.3 eq.) and the mixture was left to reflux overnight. $\mathrm{MeOH}$ was evaporated in vacuo and the reaction $\mathrm{pH}$ was brought to $\mathrm{pH}=14$ with $\mathrm{KOH}\left(5.7 \mathrm{~g}\right.$ in $50 \mathrm{~mL} \mathrm{H}_{2} \mathrm{O}$ ) at which point a white solid precipitated out on ice and was filtered to afford the crude monomethylated amine as a yellow white solid. This compound was contaminated with the starting material dopamine in $~ 8 \%$ by ${ }^{1} \mathrm{H}$ NMR spectroscopy. Yield: 66\% (2.30 g); ${ }^{1} \mathbf{H}$ NMR (400 MHz, $\mathrm{CDCl}_{3}$, ঠ): 6.73-6.65 (m, 1H, H5), 6.52 (s, 1H, H6), 6.43 (s, 1H, H7), 3.75 (d, J = 6.5 Hz, 9H, H4), 2.75 (t, $J=5.8 \mathrm{~Hz}, 2 \mathrm{H}, \mathrm{H} 3), 2.57$ (t, $J=6.0 \mathrm{~Hz}, 2 \mathrm{H}, \mathrm{H} 2), 2.35$ (s, 3H, H1) ppm. ${ }^{13} \mathbf{C}$ NMR (100 MHz, $\mathrm{CDCl}_{3}, \delta$ ): 147.48 (C9), 147.17 (C10), 126.52 (C8), 125.67 (C7), 111.40 (C6), 109.35 
(C5), 55.81 (d, $J=4.15 \mathrm{~Hz}, \mathrm{C} 4), 52.88$ (C1), 45.94 (C2), 28.72 (C3) ppm. (Agrees well with literature NMR values). ${ }^{255}$

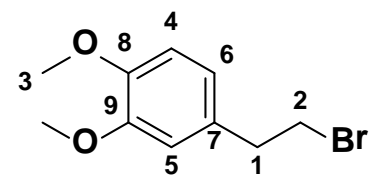

\section{4-(2-bromoethyl)-1,2-dimethoxybenzene (188): ${ }^{256}$}

To a solution of 2-(3,4-dimethoxyphenyl)ethanol (0.51 g, $2.8 \mathrm{mmol})$ and $\mathrm{CBr}_{4}$ (1.083, $3.2 \mathrm{mmol}$, 1.14 eq.) dissolved in DCM (10 mL) was added a solution of $\mathrm{PPh}_{3}(0.907 \mathrm{~g}, 3.45 \mathrm{mmol}, 1.23$ eq.) in DCM (10 mL ) over a 30 min period. The reaction was stirred at RT overnight. The crude product was packed onto silica and purified by dry column chromatography $(4.5 \mathrm{~cm} \times 5.0 \mathrm{~cm}$ frit, $40 \mathrm{~g}$ silica) pre-eluting with hexanes $(150 \mathrm{~mL})$ then with 5\% EtOAC/Hexanes $(100 \mathrm{~mL})$ to remove excess of $\mathrm{CBr}_{4}$ and then eluting with $10 \%$ EtOAC/Hexanes (150 mL) to yield the product as a viscous oil, which solidified under high vaccum as a white/yellow solid. TLC (10\% EtOAc/hexanes), $\mathrm{R}_{\mathrm{f}}=$ 0.3; Yield: 56\% (0.64 g). Mp. $57{ }^{\circ} \mathrm{C} ;{ }^{1} \mathbf{H}$ NMR (400 MHz, $\left.\mathrm{CDCl}_{3}, \delta\right)$ : 6.85-6.70 (m, 3H, (H4 + H5 + H6), 3.87 (d, $J=6.4$ Hz, 6H, H3), 3.53 (t, $J=7.6$ Hz, 2H, H2), 3.09 (t, $J=7.7 \mathrm{~Hz}, 2 \mathrm{H}, \mathrm{H} 1)$ ppm. ${ }^{13} \mathrm{C}$ NMR (100 MHz, $\left.\mathrm{CDCl}_{3}, \delta\right): 148.96$ (C9), 147.98 (C8), 131.51 (C7), 120.68 (C6), 111.89 (C5), 111.28 (C4), 55.90 (C3), 39.07 (C2), 33.25 (C1) ppm. (Agrees well with literature NMR values). ${ }^{256}$

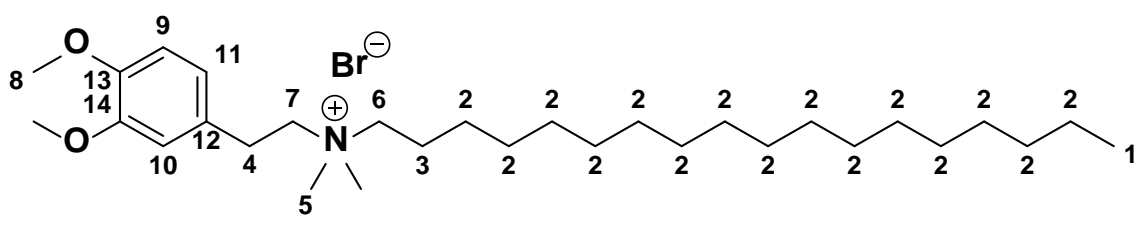

$N$-(3,4-dimethoxyphenethyl)- $N, N$-dimethyloctadecan-1-ammonium bromide (189): 
To a stirred solution of 188 (0.540 g, $2.2 \mathrm{mmol})$, in ACN (5 mL) was added DMOA (0.6640 g, 2.43 mmol, 1.1 eq.), and the mixture was refluxed ( 3 hrs). The reaction was cooled to RT and placed in the fridge (60 min) until a white precipitate appeared. The solid was filtered and dried to give the title compound as an impure mixture with $N, N$-dimethyloctadecylamine (white/yellow solid) and was not purified any further. TLC $\left(10 \% \mathrm{NH}_{4}{ }^{+} \mathrm{OH}^{-} /\right.$acetone), $\mathrm{R}_{\mathrm{f}}=0.4$; Yield: 56\% (0.82 g); ${ }^{1} \mathbf{H}$ NMR (400 MHz, $\mathrm{CDCl}_{3}, \delta$ ): 7.12-7.05 (m, 1 H, H11), 6.86-6.77 (m, 2H, (H10, H9)), 3.96 (s, 6H, H9), 3.93-3.86 (m, 2H, H7), 3.57-3.47 (m, 2H, H6), 3.41 (s, 6H, H5), 3.05-2.98 (m, 2H, H4), 1.76-1.59 (m, 2H, H3), 1.24 (brs, 30H, H2), 0.87 (t, $J=7.0$ Hz, 3H, H1) ppm.

\subsection{Preparation of Organosulfur QAC}
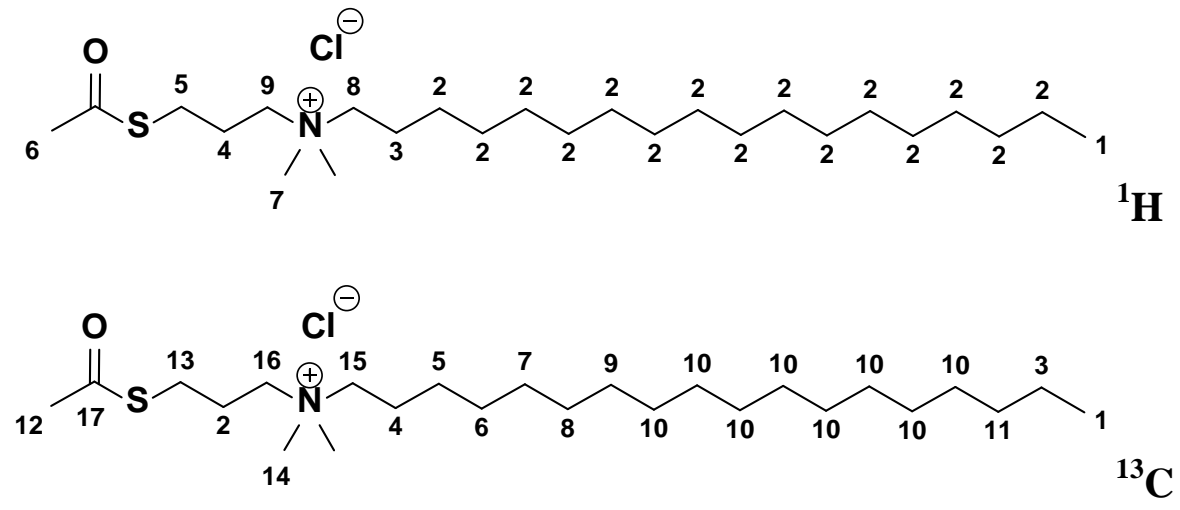

\section{N-(3-(acetylthio)propyl)-N,N-dimethyloctadecan-1-ammonium bromide (192):}

This compound was prepared by the Menshutkin reaction using Method 5.2.1: 3chloropropylthioacetate (7 mL, $53 \mathrm{mmol}, 90 \%$ from SA) and DMOA ( 17 g, $51 \mathrm{mmol}, 89 \%$ from FLUKA 1.1 eq.) were reacted neat for $24 \mathrm{hrs}$ at $120^{\circ} \mathrm{C}$. The hot gummy mixture was dissolved in acetone: $\mathrm{Et}_{2} \mathrm{O}(1: 1,70 \mathrm{~mL})$ and after $5 \mathrm{~min}$ at $\mathrm{RT}$ the white solid was filtered. A small portion was further centrifuged from $\mathrm{Et}_{2} \mathrm{O}(15 \mathrm{~mL})$, and recrystallized from $6 \mathrm{~mL}$ acetone 
(5 min on ice) to afford the title compound as a white solid. Yield: $70 \%$ (16.27 g). Mp $=54-$ 55두 ${ }^{1} \mathbf{H}$ NMR (400 MHz, $\mathrm{CDCl}_{3}, \delta$ ): 3.69-3.64 (m, 2H, H9), 3.49-3.44 (m, 2H, H8), 3.40 (s, 6H, H7), 2.36 (s, 3H, H6), 2.09-2.01 (m, 2H, H5), 1.78-1.64 (m, 4H, (H3, H4)), 1.24 (brs, 30H, H2), 0.87 (t, $J=6.9 \mathrm{~Hz}, 3 \mathrm{H}, \mathrm{H1}$ ) ppm; ${ }^{13} \mathbf{C}$ NMR (100 MHz, $\left.\mathrm{CDCl}_{3}, \delta\right): 195.71$ (C17), 71.17 (C16), 69.07 (C15), 55.36 (C14), 45.78 (C13), 30.71 (C12), 29.69 (C11), 29.71-29.68 (C10), 29.56 (C9), 29.35 (C8), 29.32 (C7), 29.19 (C6), 26.21 (C6), 25.72 (C4), 23.42 (C3), 22.68 (C2), $14.11(\mathrm{C} 1) \mathrm{ppm}$.
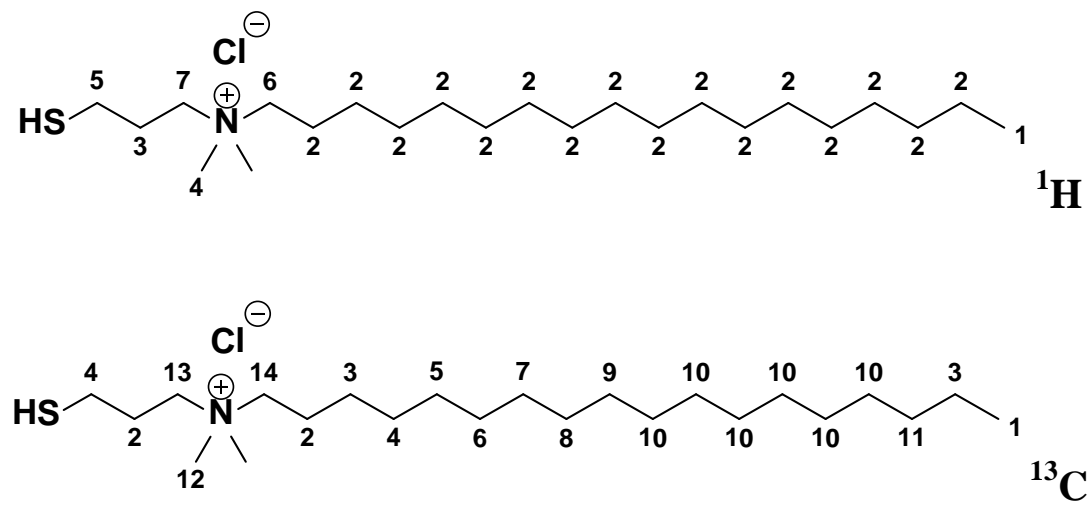

\section{$N$-(3-mercaptopropyl)- $N, N$-dimethyloctadecan-1-ammonium chloride (194): ${ }^{213}$}

To a flame dried and evacuated $20 \mathrm{~mL}$ screw cap vial was added $N$-(3-(acetylthio)propyl)- $N, N$ dimethyloctadecan-1-ammonium chloride (0.490g, $1 \mathrm{mmol}$ ) followed by $\mathrm{HBr}$ by pasteur pipette $(1 \mathrm{~mL}, 8.88 \mathrm{M})$ and $5 \mathrm{~mL} \mathrm{MeOH}$. The mixture was purged with nitrogen and placed in a $100{ }^{\circ} \mathrm{C}$ sand batch for 12 hrs. The mixture was then cooled to RT and concentrated to remove $\mathrm{H}_{2} \mathrm{O}$ and methanol, re-dissolved in $5 \mathrm{~mL} \mathrm{MeOH}$ and stirred with activated charcoal for 3 hrs at RT. The mixture was filtered through Celite (washed with $2 \times 10 \mathrm{~mL} \mathrm{MeOH}$ ), concentrated and recrystallized from $\mathrm{CHCl}_{3} /$ pentanes to afford a white solid. Yield $83 \%(0.339 \mathrm{~g}) . \mathrm{Mp}=58-60^{\circ} \mathrm{C}$; ${ }^{1} \mathbf{H}$ NMR (400 MHz, $\left.\mathrm{CDCl}_{3}, \delta\right): 3.85-3.41$ (m, 2H, H7), 3.75-3.54 (m, 2H, H6), 3.52-3.40 (m, 2H, H5), 3.31 (s, 6H, H4), 1.82-1.60 (m, 2H, H3), 1.19 (brs, 30H, H2), 0.82 (t, $J=6.9 \mathrm{~Hz}, 3 \mathrm{H}$, 
H1) ppm; ${ }^{13}$ C NMR (100 MHz, $\mathrm{CDCl}_{3}, \delta$ ): 65.79 (C14), 60.58 (C13), 51.35 (C12), 31.89 (C11), 29.80-29.85 (C10), 29.71 (C9), 29.65 (C8), 29.61 (C7), 29.39 (C6), 29.34 (C5), 26.38 (C4), 22.95 (C3), 22.64 (C2), 14.06 (C1) ppm. HRMS-DART (m/z): $\left[\mathrm{M}^{+}\right]-\mathrm{Cl}^{-}$calculated for $\mathrm{C}_{24} \mathrm{H}_{52} \mathrm{NS}$, 386.3814; found, 386.3821. (Agrees well with literature NMR values). ${ }^{213}$

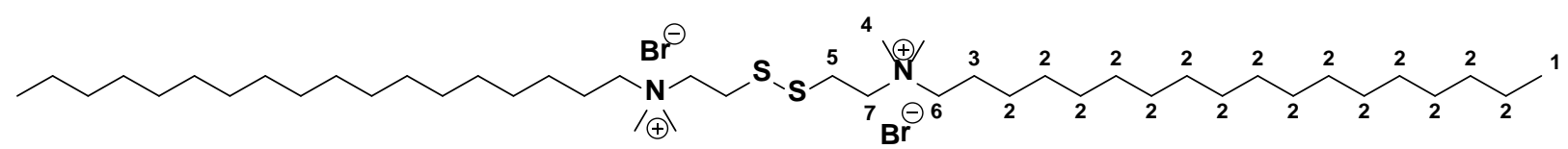

${ }^{1} \mathbf{H}$

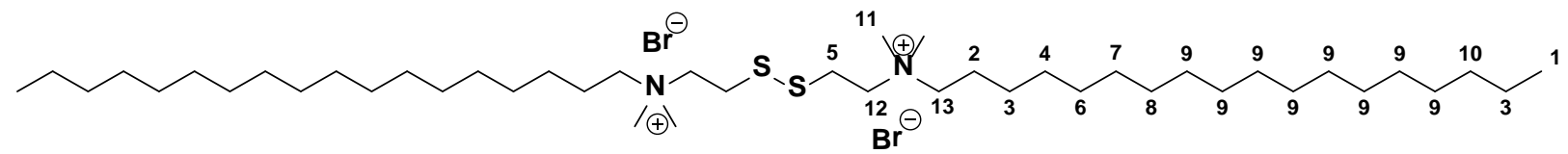

${ }^{13} \mathrm{C}$

2,2'-disulfanediylbis $N, N$-dimethyloctadecan-1-ammonium bromide (198): ${ }^{214}$

This compound was prepared by first stirring 2,2'-disulfanediylbis( $N, N$-dimethylethanaminium) $\mathrm{HCl}(0.896 \mathrm{~g}, 3.18 \mathrm{mmol})$ with NaOEt $(0.433 \mathrm{~g}, 6.37 \mathrm{mmol}, 2$ eq.) in EtOH (10 mL) for $20 \mathrm{~min}$ at RT to free base the bisamine. Next the mixture was filtered through Celite and bromooctadecane was added ( $2.30 \mathrm{~g}, 6.9 \mathrm{mmol}, 2.2$ eq.) followed by reflux overnight. The mixture was hot filtered from charcoal. Volatiles were removed on a rotary evaporator and the crude was recrystallized from acetone $(60 \mathrm{~mL})$ by slow evaporation overnight at RT as a white solid. Yield: $70 \%$ (1.11 g). $\mathrm{Mp}=98-100{ }^{\circ} \mathrm{C} ;{ }^{1} \mathbf{H}$ NMR (400 MHz, $\left.\mathrm{CDCl}_{3}, \delta\right): 3.90-3.77$ (m, 2H, H7), 3.64-3.44 (m, 4H, (H6, H5)), 3.37 (s, 6H, H4), 1.82-1.62 (m, 2H, H3), 1.23 (brs, 30H, H2), 0.85 (t, $J=6.9 \mathrm{~Hz}, 3 \mathrm{H}, \mathrm{H1}) \mathrm{ppm} ;{ }^{13} \mathrm{C}$ NMR (100 MHz, $\left.\mathrm{CDCl}_{3}, \delta\right): 64.64$ (C13), 62.13 (C12), 51.59 (C11), 31.92 (C10), 29.80-29.70 (C9), 29.67 (C8), 29.58 (C7), 29.42 (C6), 29.36 (C5), 26.35 (C5), 22.96 (C3), 22.67 (C2), 14.10 (C1) ppm. (Agrees well with literature NMR values). ${ }^{214}$ 


\subsection{Preparation of Benzophenone QAC}

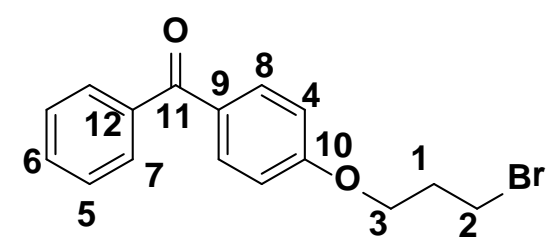

\section{4-O-(4-bromobutyl)benzophenone (202): ${ }^{105}$}

A $50 \mathrm{~mL}$ round bottom flask was charged with 1,3-dibromopropane ( $4 \mathrm{~mL}, 40 \mathrm{mmol}, 4$ eq.), $\mathrm{K}_{2} \mathrm{CO}_{3}$ (2.76 g, $20 \mathrm{mmol}, 2$ eq.) and ACN (10 mL). A solution of 4-hydroxybenzophenone (1.989 g, $10 \mathrm{mmol}$ ) in ACN (4 mL) was prepared and added dropwise to the previous mixture under reflux. The resultant yellow mixture was heated at reflux until a colourless solution was obtained or until TLC showed the disappearance of starting material 4-hydroxybenzophenone ( 20 hrs). The excess $\mathrm{KBr}$ salt was filtered through Celite and washed with acetone (10 mL). The solution was evaporated under reduced pressure to give the crude product. The crude product was packed onto silica and purified by dry column chromatography $(4.5 \mathrm{~cm} \times 5.0 \mathrm{~cm}$ frit, 40 g silica) pre-eluting with 5\% EtOAC/Hexanes (150 mL) then eluting with 100\% acetone (200 mL) to afford $2.7 \mathrm{~g}$ of the desired product contaminated with trace of starting material. The resulting yellow oil was recrystallized from hexanes/EtOAc (8:2) to yield clear, colourless crystals. Yield: $90 \%$ (2.3 g). TLC (50\% acetone/hexanes, $\mathrm{KMnO}_{4}$ stain), $\mathrm{R}_{\mathrm{f}}=0.8, \mathrm{Mp}=52-$ $53^{\circ} \mathrm{C}^{1}{ }^{1} \mathbf{H}$ NMR (400 MHz, $\left.\mathrm{CDCl}_{3}, \delta\right): 7.83$ (d, 2H, $\left.J=8.7 \mathrm{~Hz}, \mathrm{H} 1\right), 7.77$ (d, 2H, $\left.J=8.3 \mathrm{~Hz}, \mathrm{H} 7\right)$, 7.58 (t, 1H, $J=7.4 \mathrm{~Hz}, \mathrm{H3}), 7.48$ (t, 2H, $J=7.7 \mathrm{~Hz}, \mathrm{H} 2), 6.98$ (d, 2H, $J=8.7 \mathrm{~Hz}, \mathrm{H} 8), 4.20$ (t, $2 \mathrm{H}, \quad J=5.8 \mathrm{~Hz}, \mathrm{H} 10), 3.63(\mathrm{t}, 2 \mathrm{H}, J=6.3 \mathrm{~Hz}, \mathrm{H} 12), 2.37$ (q, 2H, $J=6.0 \mathrm{~Hz}, \mathrm{H} 11) \mathrm{ppm} ;{ }^{13} \mathrm{C}$ NMR (100 MHz, $\mathrm{CDCl}_{3}, \delta$ ): 195.51 (C5), 162.30 (C9), 138.23 (C4), 132.58 (C3), 131.94 (C7), 130.36 (C6), 129.74 (C1), 128.21 (C2), 114.04 (C8), 65.53 (C10), 32.13 (C12), 29.74 (C11) 
ppm. HRMS-DART (m/z): [ $\left.\mathrm{MH}^{+}\right]$calculated for $\mathrm{C}_{16} \mathrm{H}_{15} \mathrm{BrO}_{2}, 319.0334$; found, 319.0329. (Agrees well with literature NMR values). ${ }^{105}$

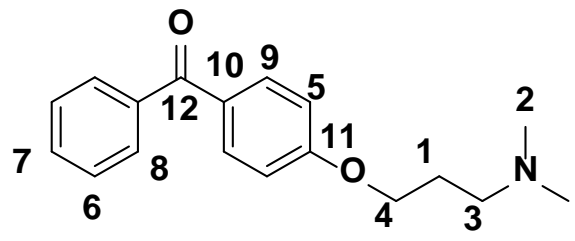

\section{(4-(3-(dimethylamino)propoxy)phenyl)(phenyl)methanone (204): ${ }^{257}$}

In a $50 \mathrm{~mL}$ round bottom flask, powdered $\mathrm{KOH}$ was added ( $0.8 \mathrm{~g}, 20 \mathrm{mmol}, 2 \mathrm{eq}$.) to a stirred solution of 4-hydroxybenzophenone (1.98 g, $10 \mathrm{mmol})$ in ACN (4 mL) and the mixture was brought to reflux for $15 \mathrm{~min}$. Dimethylaminopropionylchloride $\mathrm{HCl}$ (1.58 g, $10 \mathrm{mmol})$ was added to the hot mixture in one portion turning the reaction initially from a yellow colour to a clear colour and after a few seconds, back to yellow. The mixture was refluxed ON. The mixture was cooled to RT, evaporated under reduced pressure, packed onto silica and purified by dry column chromatography $(4.5 \mathrm{~cm} \times 5.0 \mathrm{~cm}$ frit, 40 g silica) pre-eluting with $100 \%$ acetone (100 $\mathrm{mL}$ ) then eluting with $10 \% \mathrm{Et}_{3} \mathrm{~N} /$ acetone $(150 \mathrm{~mL})$ to afford of the desired product as a yellow oil. Yield: 50\% (1.426 g). TLC (30\% MeOH/acetone), $\mathrm{R}_{\mathrm{f}}=0.3 ;{ }^{1} \mathbf{H}$ NMR (400 MHz, $\mathrm{CDCl}_{3}, \delta$ ): 7.77 (d, $J=8.8,2 \mathrm{H}, \mathrm{H} 9), 7.72$ (d, $J=7.0,2 \mathrm{H}, \mathrm{H} 8$ ), 7.52 (t, $J=7.4,1 \mathrm{H}, \mathrm{H} 7), 7.43$ (t, $J=7.6,2 \mathrm{H}$, H6), 6.93 (d, $J=8.8,2 \mathrm{H}, \mathrm{H} 5), 4.07$ (t, $J=6.4 \mathrm{~Hz}, 2 \mathrm{H}, \mathrm{H} 4), 2.43$ (t, $J=7.0 \mathrm{~Hz}, 2 \mathrm{H}, \mathrm{H} 3), 2.23$ (s, 6H, H2) 1.97 (m, 2H, H1) ppm; ${ }^{13} \mathrm{C}$ NMR (100 MHz, $\mathrm{CDCl}_{3}, \delta$ ): 195.4 (C12), 162.7 (C11), 138.3 (C7), 132.5 (C9), 131.8 (C10), 129.7 (C6), 128.1 (C8), 114.0 (C5), 66.4 (C4), 56.2 (C3), 45.5 (C2), 27.4 (C1) ppm. (Agrees well with literature NMR values). ${ }^{257}$ 

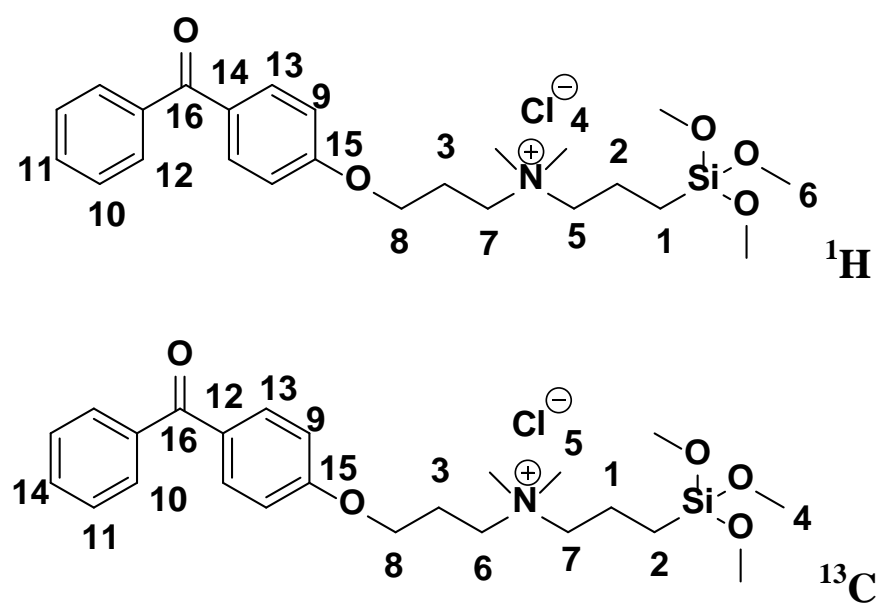

\section{3-(4-benzoylphenoxy)- $N, N$-dimethyl- $N$-(3-(trimethoxysilyl)propyl)propan-1-ammonium} chloride (206).

This compound was prepared according to Method 5.2.1 employing compound 204 (1.426 g, 5 mmol) and 3-chloropropyltrimethoxysilane 1 (1.7075g, 5.1 mmol, 1.1 eq.) in $\mathrm{MeOH}$ (2.5 mL) for 48 hrs. To purify, $\mathrm{Et}_{2} \mathrm{O}(2 \times 15 \mathrm{~mL})$ was added directly to the reaction mixture and the mixture was triturated followed by drying under high vaccum to give a yellow oil. Yield: $67 \%$ (1.76 g); ${ }^{1} \mathbf{H}$ NMR (400 MHz, $\mathrm{CDCl}_{3}, \delta$ ): 7.81 (d, $\left.J=8.74,2 \mathrm{H}, \mathrm{H} 13\right), 7.74$ (d, $J=7.37,2 \mathrm{H}$, H12), 7.58 (t, $J=7.37,1 \mathrm{H}, \mathrm{H} 11), 7.48$ (t, $J=7.77,2 \mathrm{H}, \mathrm{H} 10), 6.87$ (d, $J=8.79,2 \mathrm{H}, \mathrm{H} 9), 4.22$ (t, $J=5.33 \mathrm{~Hz}, 2 \mathrm{H}, \mathrm{H} 8), 3.93-3.82$ (m, 2H, H7), 3.59 (s, 9H, H6), 3.53-3.57 (m, 2H, H5), 3.47 (m, 6H, H4), 2.43-2.28 (m, 2H, H3), 1.95-1.82 (m, 2H, H2), 0.69 (t, J = 7.0 Hz, 2H, H1) ppm; ${ }^{13} \mathrm{C}$ NMR (100 MHz, $\mathrm{CDCl}_{3}, \delta$ ): 195.38 (C16), 161.69 (C15), 137.92 (C14), 132.37 (C13), 130.48 (C12), 129.53 (C11), 128.15 (C10), 114.12 (C9), 64.28 (C8), 60.71 (C7), 55.99 (C6), 50.42 (C5), 45.25 (C4), 22.91 (C3), 15.14 (C2), 5.54 (C1) ppm.

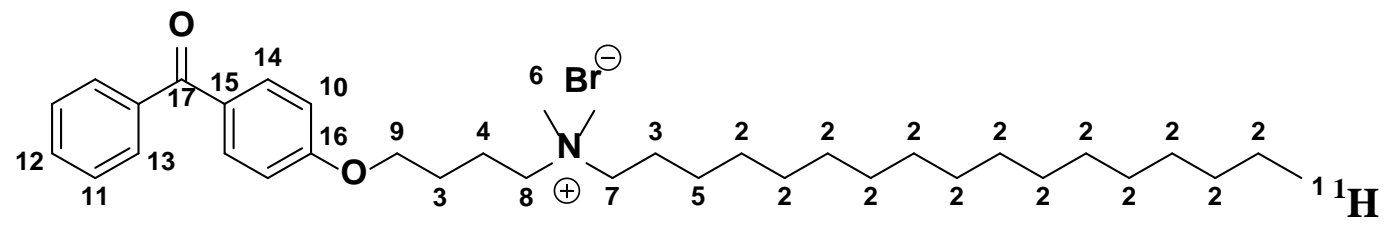




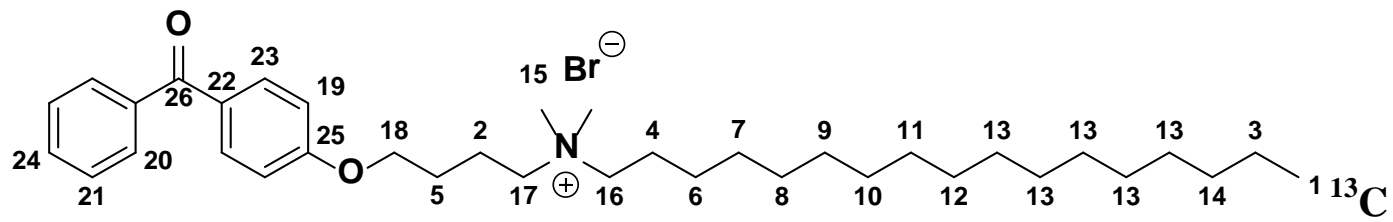

$N$-(4-(4-benzoylphenoxy)butyl)- $N, N$-dimethylheptadecan-1-ammonium bromide (205): ${ }^{258}$

This compound was prepared by the Menshutkin reaction using Method 5.2.2: 4-O-(4bromobutyl)benzophenone (0.333 g, $1 \mathrm{mmol})$ and DMOA (0.238 g,1.0 mmol, 1.0 eq.) in ACN (0.5 mL) were heated at $150{ }^{\circ} \mathrm{C}(2 \mathrm{~min})$. The title compound was placed on ice for $5 \mathrm{~min}$ and precipitated from the reaction vial upon cooling as a white solid. Yield: 65\% (0.40 g). Mp = 84$8^{\circ} \mathrm{C} ;{ }^{1} \mathbf{H}$ NMR (400 MHz, $\mathrm{CDCl}_{3}, \delta$ ): 7.77 (d, $\left.J=3.28,2 \mathrm{H}, \mathrm{H} 14\right), 7.71$ (d, $J=6.94,2 \mathrm{H}, \mathrm{H} 13$ ), 7.55 (t, $J=7.25,1 \mathrm{H}, \mathrm{H} 12), 7.44$ (t, $J=7.7,2 \mathrm{H}, \mathrm{H} 11), 6.93$ (t, $J=8.82,2 \mathrm{H}, \mathrm{H} 10), 4.15-4.08$ (m, 2H, H9), 3.78-3.69 (m, 2H, H8), 3.51-3.43 (m, 2H, H7), 3.38 (s, 6H, H6), 1.99-1.87 (m, 4H, (H6,H5)), 1.78-1.58 (m, 2H, H3), 1.21 (brs, 30H, H2), 0.84 (t, $J=7.07 \mathrm{~Hz}, 3 \mathrm{H}, \mathrm{H} 1)$ ppm; ${ }^{13} \mathrm{C}$ NMR (100 MHz, $\left.\mathrm{CDCl}_{3}, \delta\right): 195.47$ (C26), 162.15 (C15), 138.07 (C24), 132.55 (C23), 131.99 (C22), 129.68 (C21), 128.18 (C20), 114.08 (C19), 67.10 (C18), 67.11 (C17), 66.97 (C16), 51.19 (C15), 31.88 (C14), 29.67 (C13), 29.62 (C12), 29.57 (C11), 29.45 (C10), 29.38 (C9), 29.32 (C8), 29.20 (C7), 27.73 (C6), 26.27 (C5), 25.81 (C4), 22.65 (C3), 19.75 (C2), 14.09 (C1) ppm. (Agrees well with literature NMR values). ${ }^{258}$

\subsection{Preparation of Dansyl QAC}

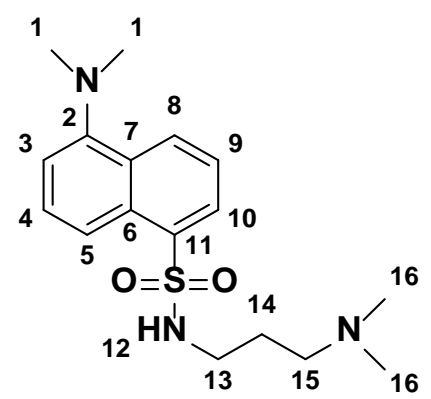


5-(dimethylamino)-N-(3-(dimethylamino)propyl)naphthalene-1-sulfonamide (208): ${ }^{177,259}$

To a flame dried $500 \mathrm{~mL}$ round bottom flask with a reflux condenser connected to an inert atmosphere manifold anhydrous DCM (300 mL) was added followed by dansyl chloride (10.0 g, $37.07 \mathrm{mmol}), \mathrm{Et}_{3} \mathrm{~N}(\sim 8 \mathrm{~mL}, 55.61 \mathrm{mmol})$. While the solution was stirring at RT, 3-(dimethylamino)propylamine $(7.0 \mathrm{ml}, 55.61 \mathrm{mmol})$ was added drop wise via syringe resulting in a colour change from orange to lime-green. After stirring for $1 \mathrm{hr}$, $\mathrm{HCl}$ (g) was bubbled through the solution until pH 2 was reached. The resulting mixture was evaporated to dryness, then re-dissolved in saturated brine $\mathrm{H}_{2} \mathrm{O}(100 \mathrm{~mL})$ and basified to $\mathrm{pH} 11$ with $6 \mathrm{~N} \mathrm{NaOH}(15 \mathrm{~mL})$ at $0{ }^{\circ} \mathrm{C}$ until white-yellow precipitate was observed. The mixture was refrigerated overnight enhancing further precipitation of product. The precipitate was filtered washing with $\mathrm{H}_{2} \mathrm{O}$ and the filtrate was extracted with DCM (500 mL) and evaporated to dryness to afford a white solid. Yield: 97\% (12.1 g). (Recrystallized using $\left.80 \% \mathrm{EtOH} / \mathrm{H}_{2} \mathrm{O}\right) . \mathrm{Mp}=122-124^{\circ} \mathrm{C}$; TLC $\left(5 \% \mathrm{NH}_{4}{ }^{+} \mathrm{OH}^{-}\right.$:Acetone), UV-Vis $\left(\mathrm{MeOH}, 1 \times 10^{-3} \mathrm{M}\right), \lambda_{\mathrm{Abs} \max }=516 \mathrm{~nm}, \varepsilon=447 \mathrm{M}^{-1} \mathrm{~cm}^{-1} .{ }^{1} \mathbf{H} \mathbf{N M R}(400 \mathrm{MHz}$, $\left.\mathrm{CDCl}_{3}, \delta\right): 8.52(\mathrm{~d}, 1 \mathrm{H}, J=8.5 \mathrm{~Hz}, \mathrm{H} 8), 8.31(\mathrm{~d}, 1 \mathrm{H}, J=8.6 \mathrm{~Hz}, \mathrm{H} 5), 8.23$ (dd, $1 \mathrm{H},{ }^{1} J=$ $\left.1.2 \mathrm{~Hz},{ }^{2} J=7.3 \mathrm{~Hz}, \mathrm{H} 10\right), 7.58-7.50$ (m, 2H, H4, H9), 7.17 (d, 1H, $J=7.5: \mathrm{H} 3$ ), 2.94 (t, 2H, $J=5.5 \mathrm{~Hz}, \mathrm{H} 13$ ), 2.88 (s, 6H, H1), 2.21 (t, 2H, $J=5.5$ Hz, H15), 2.12 (s, 6H, H16), 1.58-1.52 (m, 2H, H14) ppm; ${ }^{13} \mathbf{C}$ NMR (100 MHz, $\left.\mathrm{CDCl}_{3}, \delta\right): 151.90$ (C2), 134.77 (C11), 129.98 (C6), 128.89 (C8), 129.71 (C4), 129.65 (C9), 128.07 (C10), 123.17 (C5), 119.03 (C7), 115.00 (C3), 59.58 (C15), 45.42 (C1, C16), 44.54 (C13), 24.61 (C14) ppm. HRMS-DART (m/z): [ $\left.\mathrm{M}^{+}\right]$calculated for $\mathrm{C}_{17} \mathrm{H}_{26} \mathrm{~N}_{3} \mathrm{O}_{2} \mathrm{~S}_{1}$, 336.1736; found, 336.1745 .

(This compound has also been prepared on a $25 \mathrm{~g}, 92.6 \mathrm{mmol}$ scale). (Agrees well with literature NMR values). ${ }^{177,259}$ 


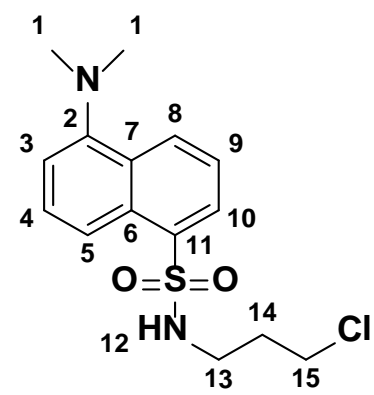

\section{$N$-(3-chloropropyl)-5-(dimethylamino)naphthalene-1-sulfonamide (209): ${ }^{260}$}

A flame dried $100 \mathrm{~mL}$ round bottom flask was charged with DCM $(30 \mathrm{~mL}), \mathrm{Et}_{3} \mathrm{~N}(\sim 1.6 \mathrm{~mL}$, $11.1 \mathrm{mmol}, 3.0$ eq.) and 3-chloropropan-1-aminium chloride (1.0 g, $5.56 \mathrm{mmol}, 1.5$ eq.). The reaction was stirried at RT until the amine salt dissolved, afterwhich the dansyl chloride (1.0 g, $3.7 \mathrm{mmol}$ ) was added in one portion resulting in a colour change from dark yellow to lime-green. After stirring for 30 min the reaction was quenched with $\mathrm{H}_{2} \mathrm{O}(50 \mathrm{~mL})$ and the organic layer was extracted, washed with brine (30 mL), dried with $\mathrm{MgSO}_{4}$ and evaporated to dryness to afford a brown orange solid that was used without any further purification. Yield: 95\% (1.15 g). TLC (5\% $\mathrm{NH}_{4}{ }^{+} \mathrm{OH}^{-}$:Acetone), $\mathrm{R}_{\mathrm{f}}=0.85:{ }^{1} \mathbf{H}$ NMR (400 MHz, MeOD, $\delta$ ): 8.66-8.58 (m, 1H, H8), 8.33 (d, $J=7.9 \mathrm{~Hz}, 1 \mathrm{H}, \mathrm{H} 5), 8.26$ (d, $J=6.4 \mathrm{~Hz}, 1 \mathrm{H}, \mathrm{H} 10), 7.39$ (q, 2H, $J=7.57 \mathrm{~Hz}, 2 \mathrm{H},(\mathrm{H} 4+\mathrm{H} 9)$ ), 7.29-7.21 (m, 1H, H3), 3.34-3.28 (m, 2H, H13), 3.11-3.03 (m, 2H, H15), 2.94 (s, 6H, H1), 1.95 (t, $J=6.1 \mathrm{~Hz}, 2 \mathrm{H}, \mathrm{H} 14) \mathrm{ppm} ;{ }^{13} \mathrm{C}$ NMR (100 MHz, MeOD, $\left.\delta\right): 151.9$ (C3), 135.5 (C12), 129.8 127.7 (m, (C5, C7, C9, C10, C11 overlap)), 122.9 (C6), 119.0 (C8), 115.0 (C4), 44.4 (C1, C2), 44.1 (C16), 39.5 (C14), 32.3 (C15) ppm. (Agrees well with literature NMR values). ${ }^{260}$ 


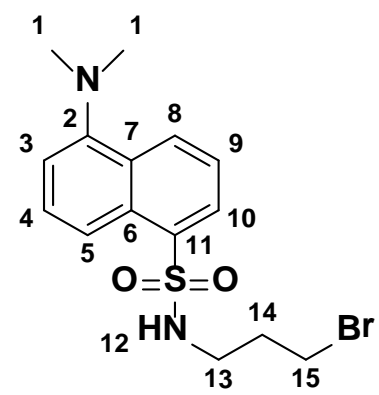

\section{$N$-(3-bromopropyl)-5-(dimethylamino)naphthalene-1-sulfonamide (210): ${ }^{261}$}

A flame dried $250 \mathrm{~mL}$ round bottom flask was charged with DCM (50 mL), Et ${ }_{3} \mathrm{~N}$ ( $1.6 \mathrm{~mL}$, 11.1 mmol, 3.0 eq.) and 3-bromopropan-1-aminium bromide (0.9 g, $4.1 \mathrm{mmol}, 1.1$ eq.). The reaction was stirred at RT to dissolve the amine salt, afterwhich the dansyl chloride (1.0 g, 3.7 mmol) was added in one portion resulting in a colour change from orange to lime-green. After stirring for 40 min the reaction was quenched with $\mathrm{H}_{2} \mathrm{O}(50 \mathrm{~mL})$ and the organic layer was extracted, washed with brine (30 mL), dried with $\mathrm{MgSO}_{4}$ and evaporated to dryness to afford a brown orange solid that was used without any further purification. Yield: 95\% (1.30 g). TLC (10\% IPA:Acetone), $\mathrm{R}_{\mathrm{f}}=0.80:{ }^{1} \mathbf{H}$ NMR (400 MHz, MeOD, $\left.\delta\right): 8.61$ (d, $J=8.2 \mathrm{~Hz}, 1 \mathrm{H}, \mathrm{H} 8$ ), 8.32 (d, $J=7.9 \mathrm{~Hz}, 1 \mathrm{H}, \mathrm{H} 5), 8.26$ (d, $J=6.4 \mathrm{~Hz}, 1 \mathrm{H}, \mathrm{H} 10), 7.57$ (q, 2H, $J=8.4 \mathrm{~Hz}, 2 \mathrm{H},(\mathrm{H} 4+$ H9)), 7.30-7.21 (m, 1H, H3), 3.30 (t, $J=3.3 \mathrm{~Hz}, 2 \mathrm{H}, \mathrm{H} 13), 3.13-3.02$ (m, 2H, H15), 2.90 (s, 6H, H1), 1.94 (t, $J=6.2 \mathrm{~Hz}, 2 \mathrm{H}, \mathrm{H} 14$ ) ppm. (Agrees well with literature NMR values). ${ }^{261}$

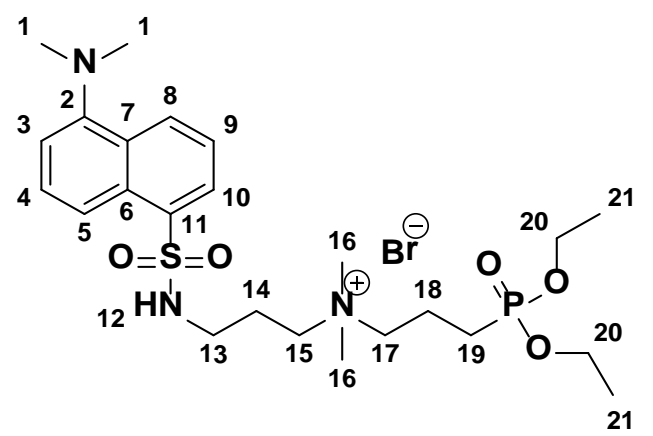


3-(diethoxyphosphoryl)- $N$-(3-(5-(dimethylamino)naphthalene-1-sulfonamido)propyl)- $N, N$ dimethylpropan-1-ammonium bromide (211): ${ }^{177}$

This compound was prepared according to Method 5.2.1: To a stirred solution of compound 208 (2.057 g, 6.13 mmol, 1.0 eq.) in refluxing ACN (15 mL) was added diethyl(3bromopropyl)phosphonate 12 ( 2.4 g, 9.2 mmol, 1.5 eq.) via syringe, and the vial was capped and refluxed for 20 hrs. To purify, $\mathrm{Et}_{2} \mathrm{O}(2 \times 40 \mathrm{~mL})$ was added directly to the reaction mixture and the mixture was triturated with decanting to remove any unreacted starting material followed by drying under high vaccum and recovered as a yellow solid. Yield: 90\% (3.64 g). UV-Vis $\left(\mathrm{MeOH}, 1 \times 10^{-3} \mathrm{M}\right), \lambda_{\text {Abs max }}=334 \mathrm{~nm}, \varepsilon=505 \mathrm{M}^{-1} \mathrm{~cm}^{-1} .{ }^{1} \mathbf{H} \mathbf{N M R}\left(400 \mathrm{MHz}, \mathrm{CDCl}_{3}, \delta\right): 8.51$ (d, $1 \mathrm{H}, J=8.3 \mathrm{~Hz}, \mathrm{H} 8), 8.43(\mathrm{~d}, 1 \mathrm{H}, J=8.6 \mathrm{~Hz}, \mathrm{H} 5), 8.20\left(\mathrm{dd}, 1 \mathrm{H},{ }^{1} J=1.0 \mathrm{~Hz},{ }^{2} J=7.3 \mathrm{~Hz}\right.$, H10), 7.71 (s, 1H, H12), 7.59 (t, 1H, $J=8.4$ Hz, H9), 7.50 (t, 1H, $J=7.3$ Hz, H4), 7.16 (d, 1H, $J$ = 7.4 Hz, H3), 4.11-4.02 (m, 4H, H20), 3.67-3.56 (m, 4H, H15, H17), 3.17 (s, 6H, H16), 3.102.99 (m, 2H, H13), 2.86 (s, 6H, H1), 2.10-1.95 (m, 4H, H14, H18), 1.89-1.77 (m, 2H, H19), 1.28 (t, 6H, $J=7.1 \mathrm{~Hz}, \mathrm{H} 21) \mathrm{ppm} ;{ }^{13} \mathbf{C}$ NMR (100 MHz, $\mathrm{CDCl}_{3}, \delta$ ): 151.79 (C2), 134.62 (C11), 130.29 (C4), 129.79 (C6), 129.47 (C8), 129.34 (C9), 128.60 (C10), 123.28 (C5), 119.28 (C7), 115.30 (C3), 62.23-62.16 (overlap, C15, C17, C20), 51.31 (C16), 45.43 (C1), 39.73 (C13), 24.75-22.83 (C14, C18, C19), 16.45 (d, $\left.{ }^{2} \mathrm{~J}=6.0 \mathrm{~Hz}, \mathrm{C} 21\right) \mathrm{ppm} ;{ }^{31} \mathbf{P}$ NMR (121.45 MHz, $\mathrm{CDCl}_{3}$,

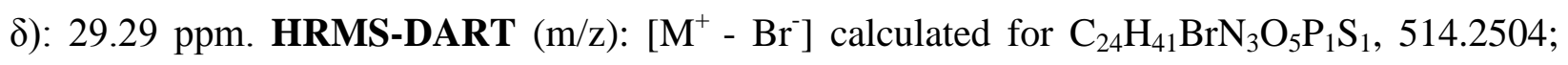
found, 514.2519. (Agrees well with literature NMR values). ${ }^{177}$ 


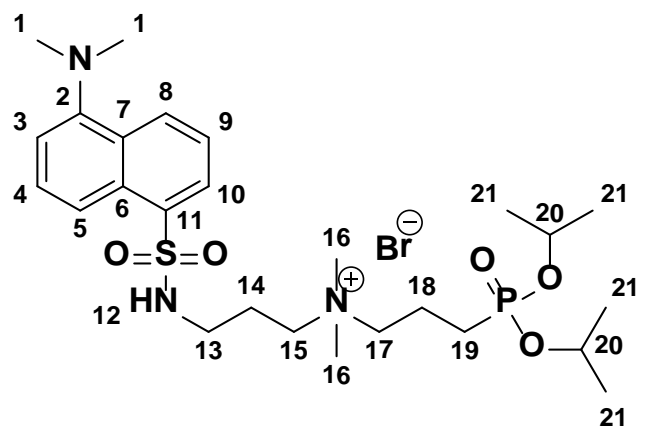

3-(diisopropoxyphosphoryl)- $N$-(3-(5-(dimethylamino)naphthalene-1-sulfonamido)propyl)$N, N$-dimethylpropan-1-ammonium (212):

This compound was prepared according to Method 5.2.1: To a stirred solution of compound 208 (0.5 g, $1.5 \mathrm{mmol})$ in refluxing ACN (3 mL) was added diisopropyl(3bromopropyl)phosphonate $13(0.46 \mathrm{~g}, 1.6 \mathrm{mmol})$ via syringe, and the vial was capped and refluxed for 7 hrs. To purify, $\mathrm{Et}_{2} \mathrm{O}(2 \times 15 \mathrm{~mL})$ was added directly to the reaction mixture and the mixture was triturated with decanting to remove any unreacted starting material followed by drying under high vaccum and recovered as a yellow solid. Yield: 70\% (0.653 g). UV-Vis $\left(\mathrm{MeOH}, 1 \times 10^{-3} \mathrm{M}\right), \lambda_{\text {Abs max }}=340 \mathrm{~nm}, \varepsilon=519 \mathrm{M}^{-1} \mathrm{~cm}^{-1} .{ }^{\mathbf{1}} \mathbf{H} \mathbf{~ N M R}$ (400 MHz, $\left.\mathrm{CDCl}_{3}, \delta\right): 8.50(\mathrm{~d}, 1 \mathrm{H}, J=8.3 \mathrm{~Hz}, \mathrm{H} 8), 8.43$ (d, 1H, $\left.J=8.6 \mathrm{~Hz}, \mathrm{H} 5\right), 8.22$ (dd, $\left.1 \mathrm{H},{ }^{1} J=1.0 \mathrm{~Hz},{ }^{2} J=7.3 \mathrm{~Hz}, \mathrm{H} 10\right), 7.75$ (s, 1H, H12), 7.59 (t, 1H, $J=8.4 \mathrm{~Hz}, \mathrm{H9}$ ), 7.49 (t, 1H, $J=7.3 \mathrm{~Hz}, \mathrm{H} 4), 7.15$ (d, 1H, $J=7.4$ Hz, H3), 4.68-4.56 (m, 2H, H20), 3.663.49 (m, 4H, H15, H17), 3.16 (s, 6H, H16), 3.09-2.97 (m, 2H, H13), 2.85 (s, 6H, H1), 2.05-1.90 (m, 4H, H14, H18), 1.80-1.69 (m, 2H, H19), 1.26 (t, 12H, J = 7.1 Hz, H21) ppm; ${ }^{13}$ C NMR (100 MHz, $\left.\mathrm{CDCl}_{3}, \delta\right): 151.79$ (C2), 134.62 (C11), 130.31 (C4), 129.87 (C6), 129.49 (C8), 129.31 (C9), 128.64 (C10), 123.28 (C5), 119.28 (C7), 115.30 (C3), 70.69 (C20), 62.23-62.16 (overlap, C15, C17), 51.19 (C16), 45.42 (C1), 41.80 (C13), 39.60 (C21), 33.76 (C19), 24.08 (C14, C18) ppm; ${ }^{31} \mathbf{P}$ NMR (121.45 MHz, $\mathrm{CDCl}_{3}, \delta$ ): 295 
27.32 ppm. HRMS-ESI-TOF (m/z): $\left[\mathrm{M}^{+}-\mathrm{Br}^{-}\right]$calculated for $\mathrm{C}_{26} \mathrm{H}_{45} \mathrm{BrN}_{3} \mathrm{O}_{5} \mathrm{P}_{1} \mathrm{~S}_{1}$, 542.2813; found, 542.2815.

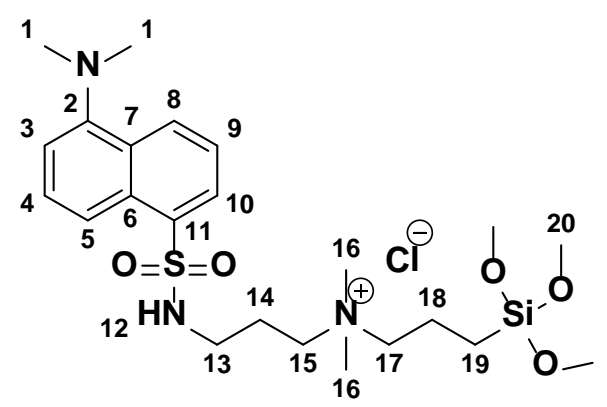

\section{3-(5-(dimethylamino)naphthalene-1-sulfonamido)- $N, N$-dimethyl- $N$-(3-(trimethoxysilyl) propyl)propan-1-ammonium chloride (213): ${ }^{177}$}

To a flame dried $25 \mathrm{~mL}$ round bottom flask with a reflux condenser connected to an inert atmosphere manifold, ACN (20 mL) was added followed by 5-(dimethylamino)-N-(3(dimethylamino)propyl)naphthalene-1-sulfonamide 208 (3.35 g, 10 mmol). While stirring, (3chloropropyl)trimethoxysilane $\mathbf{1}$ ( $5 \mathrm{~mL}, 25 \mathrm{mmol}$ ) was added via an inert syringe. The solution was stirred for $48 \mathrm{hrs}$ at $110^{\circ} \mathrm{C}$, and the solution turned to yellow-brownish oil. The oil precipitated in cold (1:1) DCM : $\mathrm{Et}_{2} \mathrm{O}$ mixture, forming two layers; a gummy layer and a white liquid layer. The white liquid layer was separated inside a $10 \mathrm{~mL}$ syringe and collected in a second $25 \mathrm{~mL}$ round bottom flask; the gummy layer left behind was dissolved using DCM and collected in a $25 \mathrm{~mL}$ round bottom flask. Excess DCM was evaporated using rotary evaporator resulting in a light yellow powder. Yield: $72.0 \%$ (3.849 g). $\mathrm{Mp}=85-87^{\circ} \mathrm{C} . \mathrm{UV}-\mathrm{Vis}(\mathrm{MeOH}, 1 \times$ $\left.10^{-3} \mathrm{M}\right), \lambda_{\mathrm{Abs} \max }=340 \mathrm{~nm}, \varepsilon=413 \mathrm{M}^{-1} \mathrm{~cm}^{-1} .{ }^{1} \mathbf{H} \mathbf{N M R}\left(400 \mathrm{MHz}, \mathrm{CDCl}_{3}, \delta\right): 8.48(\mathrm{~d}, 2 \mathrm{H}, J=$ $8.6 \mathrm{~Hz}, \mathrm{H} 8, \mathrm{H} 5), 8.37$ (s, 1H, H12), 8.19 (d, 1H, $J=7.3 \mathrm{~Hz}, \mathrm{H} 10), 7.59$ (t, 1H, $J=7.9 \mathrm{~Hz}, \mathrm{H} 4$ ), 7.49 (t, 1H, $J=8.2 \mathrm{~Hz}, \mathrm{H9}$ ), 7.13 (d, 1H, $J=7.6 \mathrm{~Hz}, \mathrm{H} 3$ ), 3.57-3.52 (m, 2H, H17), 3.49 (s, 9H, H20), 3.27-3.20 (m, 2H, H15), 3.10 (s, 6H, H16), 3.08-3.03 (m, 2H, H13), 2.84 (s, 6H, H1), 
2.01-1.90 (m, 2H, H14), 1.71-1.60 (m, 2H, H18), 0.54 (t, 2H, $J=7.8 \mathrm{~Hz}, \mathrm{H} 19) \mathrm{ppm} ;{ }^{13} \mathbf{C}$ NMR (100 MHz, $\left.\mathrm{CDCl}_{3}, \delta\right): 151.70$ (C2), 135.06 (C11), 130.18 (C4), 129.75 (C6), 129.58 (C8), 128.93 (C9), 128.53 (C10), 123.35 (C5), 119.45 (C7), 115.14 (C3), 65.62 (C15), 62.27 (C17), 50.79 (C16), 50.68 (C20), 45.40 (C1), 39.81 (C13), 26.00 (C14), 16.16 (C18), 5.35 (C19) ppm; ${ }^{29} \mathrm{Si}$ NMR $\left(79.4 \mathrm{MHz}, \mathrm{CD}_{3} \mathrm{OD}, \delta\right):-68.86 \mathrm{ppm}$. HRMS-DART (m/z): $\left[\mathrm{M}^{+}-\mathrm{Cl}^{-},-\mathrm{CH}_{3}\right]$, calculated for $\mathrm{C}_{23} \mathrm{H}_{40} \mathrm{ClN}_{3} \mathrm{O}_{5} \mathrm{SSi}$, 484.2314; found 484.2315. (Agrees well with literature NMR values). ${ }^{177}$

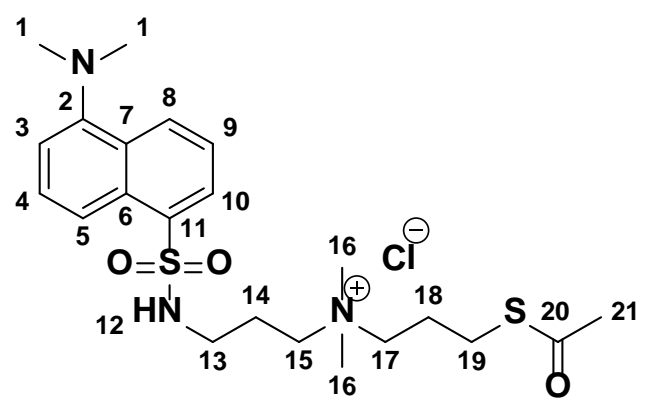

\section{3-(acetylthio)- $N$-(3-(5-(dimethylamino)naphthalene-1-sulfonamido)propyl)- $N, N$ -} dimethylpropan-1-ammonium chloride (214):

This compound was prepared according to Method 5.2.1: To a stirred solution of compound 208 (1.0 g, $2.98 \mathrm{mmol})$ in refluxing EtOH (4 mL) was added 3-chloropropylthioacetate (90\%) (0.65 $\mathrm{mL}, 4.9 \mathrm{mmol}, 1.5$ eq.) via syringe, and the vial was capped and refluxed for 24 hrs. To purify, $\mathrm{Et}_{2} \mathrm{O}(2 \mathrm{x} 15 \mathrm{~mL})$ was added directly to the reaction mixture and the mixture was triturated with decanting to remove any unreacted starting material followed by drying under high vaccum recovered a yellow powder. Yield: $63 \%(1.10 \mathrm{~g}) . \mathrm{Mp}=35-40^{\circ} \mathrm{C} ; \boldsymbol{\varepsilon}=439 \mathrm{M}^{-1} \mathrm{~cm}^{-1}$. UV-VIS $\left(\mathrm{MeOH}, 1 \times 10^{-3} \mathrm{M}\right), \lambda_{\text {Abs } \max }=334 \mathrm{~nm}, \varepsilon=439 \mathrm{M}^{-1} \mathrm{~cm}^{-1} .{ }^{1} \mathbf{H}$ NMR $\left(400 \mathrm{MHz}, \mathrm{CDCl}_{3}, \delta\right): 8.49$ (d, $1 \mathrm{H}, J=8.4 \mathrm{~Hz}, \mathrm{H} 8$ ), 8.43 (d, 1H, $J=8.5 \mathrm{~Hz}, \mathrm{H} 5$ ), 8.18 (d, 1H, $J=7.3 \mathrm{~Hz}, \mathrm{H} 10$ ), 7.57 (t, 1H, $J=8.0 \mathrm{~Hz}, \mathrm{H} 4), 7.49$ (t, 1H, $J=8.2 \mathrm{~Hz}, \mathrm{H} 9), 7.15$ (d, 1H, $J=7.5 \mathrm{~Hz}, \mathrm{H} 3), 3.57-3.48$ (m, 2H, 
H15), 3.40-3.32 (m, 2H, H17), 3.10 (s, 6H, H16), 3.05-2.98 (m, 2H, H13), 2.86 (s, 6H, H1), 2.83-2.77 (m, 2H, H19), 2.28 (s, 3H, H21), 2.03-1.85 (m, 4H, H14, H18) ppm; ${ }^{13}$ C NMR (100 MHz, $\mathrm{CDCl}_{3}, \delta$ ): 195.80 (C21), 149.79 (C2), 134.73 (C11), 130.34 (C4), 129.67 (C6), 129.43 (C8), 128.32 (C9), 128.71 (C10),124.64 (C5), 123.52 (C7), 115.45 (C3), 62.92 (C15), 62.73 (C17), 55.14 (C16), 45.32 (C1), 42.90 (C13), 30.77 (C21), 25.57 (C19), 23.16 (C18), 23.00 (C14) ppm. HRMS-ESI-TOF (m/z): [ $\mathrm{M}^{+}$- $\left.\mathrm{Cl}^{-}\right]$calculated for $\mathrm{C}_{22} \mathrm{H}_{34} \mathrm{ClN}_{3} \mathrm{O}_{3} \mathrm{~S}_{2}, 452.2036$; found, 452.2037.

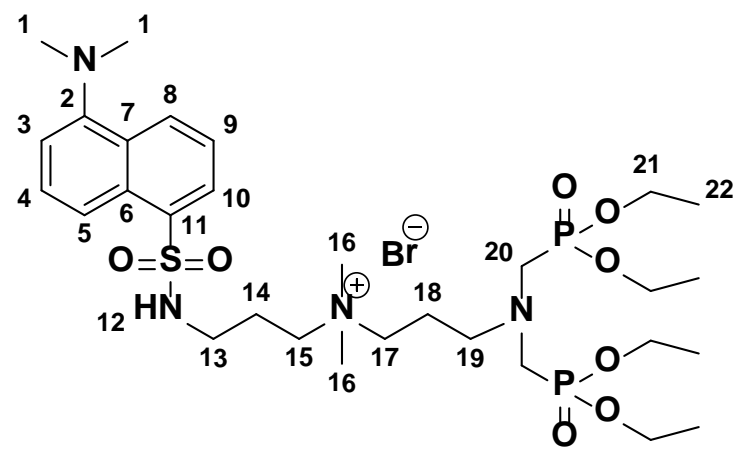

\section{3-(bis((diethoxyphosphoryl)methyl)amino)- $N$-(3-(5-(dimethylamino)naphthalene-1-} sulfonamido)propyl)- $N, N$-dimethylpropan-1-ammonium bromide (215):

This compound was prepared according to Method 5.2.1: To a stirred solution of compound 208 (0.658 g, $1.35 \mathrm{mmol})$ in refluxing ACN (3 mL) was added compound 120 (0.658 g, $1.35 \mathrm{mmol})$, and the vial was capped and refluxed for $7 \mathrm{hrs}$. To purify, $\mathrm{Et}_{2} \mathrm{O}(2 \times 15 \mathrm{~mL})$ was added directly to the reaction mixture and the mixture was triturated with decanting to remove any unreacted starting material followed by drying under high vaccum to give a yellow powder. Yield: $60 \%$ (0.626 g). $\mathrm{Mp}=35-36^{\circ} \mathrm{C}$, UV-Vis $\left(\mathrm{MeOH}, 1 \times 10^{-3} \mathrm{M}\right), \lambda_{\mathrm{Abs} \max }=340 \mathrm{~nm}, \varepsilon=526 \mathrm{M}^{-1} \mathrm{~cm}^{-1} .{ }^{1} \mathbf{H}$ NMR (400 MHz, $\mathrm{CDCl}_{3}, \delta$ ): 8.48-8.38 (m, 2H, H8), 8.37-8.29 (m, 1H, H5), 8.19-8.12 (m, 1H, H10), 7.72 (s, 1H, H12), 7.56-7.38 (m, 2H, H4, H9), 7.15 (m, 1H, H2), 4.16-3.97 (m, 8H, H21), 
3.62-3.25 (m, 4H, H15, H17), 3.05-2.85 (s, 6H, H16), 3.10-2.91 (m, 8H, H19, H20, H13), 2.81 (s, 6H, H1), 1.99-1.75 (m, 4H, H14, H18), 1.25 (t, 12H, $J=7.0$ Hz, H22) ppm; ${ }^{13}$ C NMR (100 MHz, $\mathrm{CDCl}_{3}, \delta$ ): 151.85 (C2), 135.16 (C11), 130.18 (C6), 129.76 (C8), 129.47 (C4), 129.12 (C9), 128.38 (C10), 123.34 (C5), 119.34 (C7), 115.19 (C3), 62.74 (C15), 62.24 (t, ${ }^{2} J_{C-P}=3.5 \mathrm{~Hz}$, C21), 61.84 (C17), 53.78 (C19), 51.65 (C16), 50.49 (C20), 45.38 (C1), 39.61 (C13), 22.79 (C14), 21.22 (C18), 16.51 (d, $\left.{ }^{2} J_{C-P}=2.5 \mathrm{~Hz}, \mathrm{C} 22\right)$ ppm; ${ }^{31} \mathbf{P}$ NMR (121.45 MHz, $\left.\mathrm{CDCl}_{3}, \delta\right)$ : $24.42 \mathrm{ppm}$. HRMS-ESI-TOF (m/z): $\left[\mathrm{MH}^{+}-\mathrm{I}^{-}\right]$calculated for $\mathrm{C}_{30} \mathrm{H}_{55} \mathrm{IN}_{4} \mathrm{O}_{8} \mathrm{P}_{2} \mathrm{~S}_{1}, 693.3210$; found, 693.3213.

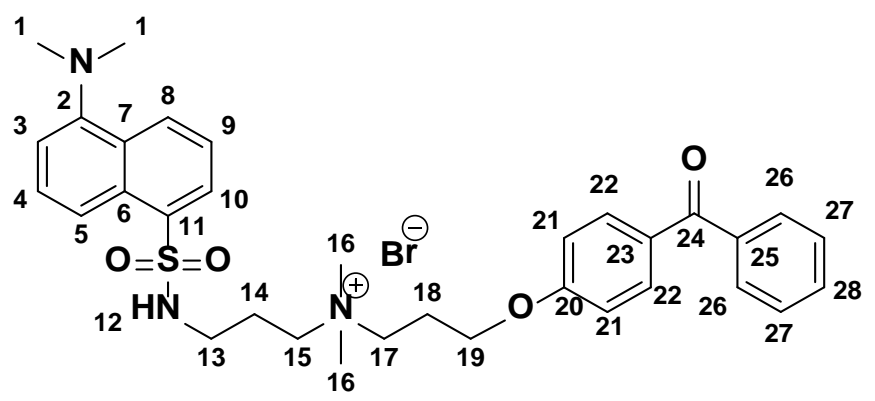

\section{3-(4-benzoylphenoxy)- $N$-(3-(5-(dimethylamino)naphthalene-1-sulfonamido)propyl)- $N, N$ - dimethylpropan-1-ammonium bromide (216): ${ }^{105}$}

This compound was prepared according to Method 5.2.1: To a stirred solution of compound 208 (0.5 g, $1.5 \mathrm{mmol})$ in refluxing ACN (4 mL) was added (4-(3bromopropoxy)phenyl)(phenyl)methanone (0.72 g, 2.25 mmol, 1.5 eq.) via syringe, and the vial was capped and refluxed for 24 hrs. To purify, $\mathrm{Et}_{2} \mathrm{O}(2 \times 15 \mathrm{~mL})$ was added directly to the reaction mixture and the mixture was triturated with decanting to remove any unreacted starting material followed by drying under high vaccum to a yellow powder. Yield: $93 \%$ (0.91 g). $\mathrm{Mp}=75-76^{\circ} \mathrm{C}$; UV-VIS $\left(\mathrm{MeOH}, 1 \times 10^{-3} \mathrm{M}\right), \lambda_{\mathrm{Abs} \max }=$ $335 \mathrm{~nm}, \varepsilon_{1}=524 \mathrm{M}^{-1} \mathrm{~cm}^{-1}, \lambda_{\text {Abs max }}=288 \mathrm{~nm}, \varepsilon_{2}=327 \mathrm{M}^{-1} \mathrm{~cm}^{-1} .{ }^{1} \mathbf{H} \mathbf{N M R}(400 \mathrm{MHz}$, 
$\mathrm{CDCl}_{3}, \delta$ ): 8.49-8.43 (m, 2H, H5, H10), 8.18 (d, 1H, $\left.J=7.3 \mathrm{~Hz}, \mathrm{H} 8\right), 7.76-7.68$ (m, 5H, H28, H22, H12, H9), 7.59-7.52 (m, 2H, H27), 7.47-7.42 (m, 3H, H26, H4), 7.07 (d, 1H, J = 7.6 Hz, H3), 6.82 (d, 2H, $J=8.8 \mathrm{~Hz}, \mathrm{H} 21), 4.00(\mathrm{t}, 2 \mathrm{H}, J=5.4 \mathrm{~Hz}, \mathrm{H} 19), 3.73-3.69$ (m, 2H, H15), 3.62-3.59 (m, 2H, H17), 3.22 (s, 6H, H16), 3.11-3.03 (m, 2H, H13), 2.81 (s, 6H, H1), 2.29-2.09 (m, 2H, H14), 2.05-1.95 (m, 2H, H18) ppm; ${ }^{13}$ C NMR (100 MHz, $\mathrm{CDCl}_{3}, \delta$ ): 195.47 (C24), 161.69 (C20), 151.86 (C2), 137.99 (C11), 134.86 (C25), 132.42 (C6), 132.06 (C28), 130.51 (C23), 130.37 (C8), 129.71 (C22), 129.44 (C26), 129.23 (C4), 129.20 (C9), 128.71 (C10), 128.25 (C27), 123.36 (C5), 119.39 (C7), 115.30 (C3), 114.15 (C21), 65.82 (C17), 64.50 (C15), 62.50 (C19), 51.37 (C16), 45.34 (C1), 39.87 (C13), 22.90 (C18), 15.26 (C14) ppm. HRMS-ESI-TOF (m/z): $\left[\mathrm{M}^{+}-\mathrm{Br}^{-}\right]$calculated for $\mathrm{C}_{33} \mathrm{H}_{40} \mathrm{BrN}_{3} \mathrm{O}_{4} \mathrm{~S}$, 574.2749; found, 574.2734.

(Agrees well with literature NMR values). ${ }^{105}$

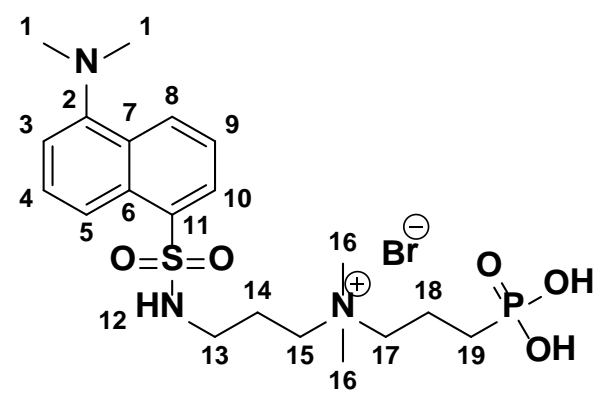

3-(5-(dimethylamino)naphthalene-1-sulfonamido)- $N, N$-dimethyl- $N$-(3phosphonopropyl)propan-1-ammonium bromide (217):

Inside a flame dried and evacuated $20 \mathrm{~mL}$ screw cap vial $N$-(3-(diethoxyphosphoryl)propyl)$N, N$-dimethyloctadecan-1-ammonium bromide $(0.35 \mathrm{~g}, 0.58 \mathrm{mmol})$ was dissolved in anhydrous DCM (5 mL). To the clear stirred solution was added TMSBr (0.23 mL, $1.76 \mathrm{mmol}, 3.0$ eq.) through a rubber septum via syringe and the reaction was stirred at RT overnight. Completion of 300 
the reaction was followed by ${ }^{31} \mathrm{P}$ NMR spectroscopy after which the reaction was quenched with EtOH $(10 \mathrm{~mL})$ and stirred for $1 \mathrm{hr}$ followed by addition of $\mathrm{H}_{2} \mathrm{O}(1 \mathrm{~mL})$. Volatiles were removed with a rotary evaporator connected to a high vacuum Schlenk line and the crude product was triturated with $\mathrm{Et}_{2} \mathrm{O}(2 \times 10 \mathrm{~mL})$ to remove brown colored impurities. Further purification entailed extraction with $\mathrm{NH}_{4}{ }^{+} \mathrm{OH}^{-}: \mathrm{H}_{2} \mathrm{O}(1: 10,10 \mathrm{~mL})$ and washing with $\mathrm{Et}_{2} \mathrm{O}(1 \times 5 \mathrm{~mL})$. The aqueous fluorescent layer was evaporated from ACN $(1 \times 50 \mathrm{~mL})$ to give the pure product as beige solid. Yield: $79 \%(0.25 \mathrm{~g}) . \mathrm{Mp}=165-168^{\circ} \mathrm{C} ; \varepsilon=449 \mathrm{M}^{-1} \mathrm{~cm}^{-1}$. UV-Vis $\left(\mathrm{MeOH}, 1 \times 10^{-3}\right.$ M), $\lambda_{\text {Abs max }}=342 \mathrm{~nm}, \varepsilon=449 \mathrm{M}^{-1} \mathrm{~cm}^{-1} .{ }^{1} \mathbf{H}$ NMR $\left(400 \mathrm{MHz}, \mathrm{D}_{2} \mathrm{O}, \delta\right): 8.58(\mathrm{~d}, 1 \mathrm{H}, J=8.5 \mathrm{~Hz}$, H8), 8.35 (d, 1H, $J=8.5$ Hz, H5), 8.23 (d, 1H, $J=7.0 \mathrm{~Hz}, \mathrm{H} 10$ ), 7.70-7.58 (m, 2H, H4, H9), 7.31 (d, 1H, $J$ = 7.5 Hz, H3), 3.32 (s, 6H, H16), 3.30-3.22 (m, 2H, H17 overlap), 3.19-3.11 (m, 2H, H15), 2.97 (t, 2H, J = 6.0 Hz, H13), 2.89 (s, 6H, H1), 1.93-1.86 (m, 4H, H14, H18), 1.571.49 (m, 2H, H19) ppm; ${ }^{13}$ C NMR (100 MHz, $\mathrm{D}_{2} \mathrm{O}, \delta$ ): 133.80 (C2), 131.15 (C11), 130.15 (C6), 129.76 (C8), 128.99 (C4), 128.61 (C9), 128.40 (C10), 124.54 (C5), 119.81 (C7), 116.59, (C3), $64.32\left(\mathrm{~d},{ }^{3} J_{C-P}=18.8 \mathrm{~Hz}, \mathrm{C} 17\right), 61.33$ (C15), 53.99 (C16), 45.08 (C1), 39.04 (C13), 24.77 (d, $\left.{ }^{1} J_{C-P}=136.1 \mathrm{~Hz}, \mathrm{C} 19\right), 21.96$ (C14), $16.85\left(\mathrm{~d},{ }^{2} J_{C-P}=3.2\right.$, C18) ppm; ${ }^{31} \mathrm{P}$ NMR (121.45 MHz, $\left.\mathrm{D}_{2} \mathrm{O}, \delta\right): 20.99$ ppm. HRMS-ESI-TOF (m/z): $\left[\mathrm{M}^{+}-\mathrm{Br}^{-}\right]$calculated for $\mathrm{C}_{20} \mathrm{H}_{33} \mathrm{BrN}_{3} \mathrm{O}_{5} \mathrm{P}_{1} \mathrm{~S}_{1}$, 458.1873; found, 458.1868.

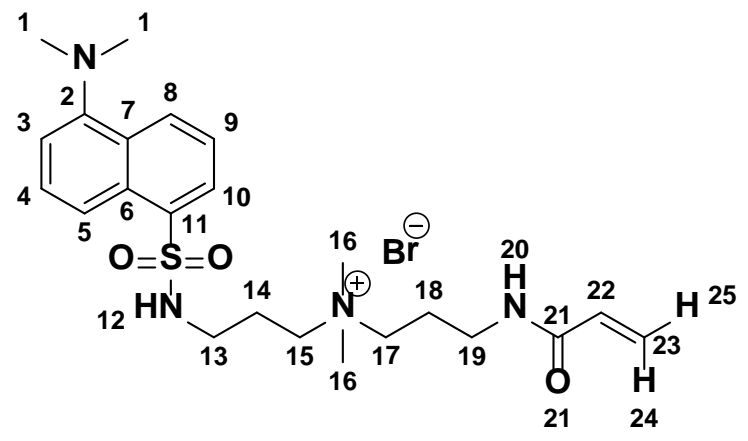


3-acrylamido- $N$-(3-(5-(dimethylamino)naphthalene-1-sulfonamido)propyl)- $N, N$ dimethylpropan-1-ammonium bromide (219):

This compound was prepared according to Method 5.2.1: To a stirred solution of compound 210 (1 g, 2.9 mmol, 1.0 eq.) in refluxing ACN (4 mL) was added $N$-(3-(dimethylamino) propyl)acrylamide (0.5 g, $3.2 \mathrm{mmol}, 2.14$ eq.) and the vial was capped and refluxed for 24 hrs. To purify, $\mathrm{Et}_{2} \mathrm{O}(2 \times 15 \mathrm{~mL})$ was added directly to the reaction mixture and the mixture was triturated with decanting to remove any unreacted starting material followed by drying under high vaccum and recovered as a yellow solid. Yield: $90 \%\left(1.16\right.$ g). $\mathrm{Mp}=34-36{ }^{\circ} \mathrm{C} ;{ }^{1} \mathbf{H} \mathbf{~ N M R}$ (400 MHz, $\left.\mathrm{CDCl}_{3}, \delta\right): 8.61-8.26$ (m, 2H, 4 H, (H8, H5, H12, H20)), 8.31 (d, J = 8.13 Hz, 1H, H10), 7.54 (t, $J=7.3 \mathrm{~Hz}, 1 \mathrm{H}, \mathrm{H} 4), 7.45$ (t, $J=7.3 \mathrm{~Hz}, 1 \mathrm{H}, \mathrm{H} 5), 7.09$ (d, $J=7.0 \mathrm{~Hz}, 1 \mathrm{H}, \mathrm{H} 3$ ), 6.38 (t, $J=10.3 \mathrm{~Hz}, 1 \mathrm{H}, \mathrm{H} 22), 6.18$ (d, $J=16.6 \mathrm{~Hz}, 1 \mathrm{H}, \mathrm{H} 24), 5.50$ (d, $J=9.9 \mathrm{~Hz}, 1 \mathrm{H}, \mathrm{H} 25)$, 3.60-3.12 (m, 6H, (H19, H17, H15)), 2.97 (s, 6H, H16), 2.80 (s, 6H, H1), 2.12-1.69 (m, 4H, (H14, H18)) ppm; ${ }^{13}$ C NMR (100 MHz, $\mathrm{CDCl}_{3}, \delta$ ): 166.5 (C21), 151.9 (C2), 134.9 (C11),131.3 (C6), 130.2 (C22), 129.7 (C8), 129.4 (C4), 129.2 (C9), 128.6 (C10), 126.1 (C23), 123.4 (C5), 119.2 (C7), 115.2 (C3), 66.0 (C17), 62.5 (C15), 51.1 (C16), 45.4 (C1), 39.5 (C13), 36.2 (C19), 22.5 (C14), 15.3 (C18) ppm.

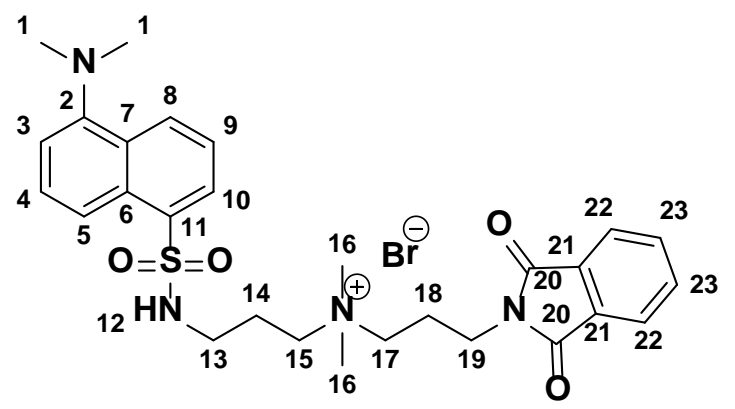

3-(5-(dimethylamino)naphthalene-1-sulfonamido)- $N$-(3-(1,3-dioxoisoindolin-2-yl)propyl)$N, N$-dimethylpropan-1-ammonium bromide (220): 
This compound was prepared according to Method 5.2.1 (500 mL glass bottle): A stirred solution of compound 208 (9.02 g, 26.88 mmol, 1.0 eq.) and 3-bromopropylphthalimide 10 (8.716 g, 32.5 mmol, 1.2 eq.) in $\mathrm{EtOH}(50 \mathrm{~mL})$ was sealed and refluxed for 16 hrs. To purify, $\mathrm{Et}_{2} \mathrm{O}(1 \times 300$ $\mathrm{mL}$ ) was added directly to the reaction mixture and the mixture was triturated with decanting to remove any unreacted starting material followed by another rinse with $\mathrm{Et}_{2} \mathrm{O}$ :acetone (3:1, 150 $\mathrm{mL}$ ). The bottle was placed under high vaccum $(60 \mathrm{~min})$ to give a light yellow powder. X-ray quality crystals were obtained by recrystalizaiton from boiling EtOH: $\mathrm{H}_{2} \mathrm{O}(4: 1,100 \mathrm{~mL})$, cooled to RT and placed at $-20^{\circ} \mathrm{C}$ (2 hrs) or formed $\mathrm{ON}$ at RT in an NMR tube $\left(\mathrm{D}_{2} \mathrm{O}\right)$. Star shaped, colourless crystals were recovered. Yield: $43 \%$ (7.0 g). $\mathrm{Mp}=123-125^{\circ} \mathrm{C} ;{ }^{1} \mathbf{H}$ NMR (400 MHz, $\mathrm{CDCl}_{3}, \delta$ ): 8.44 (d, $\left.J=8.3 \mathrm{~Hz}, 1 \mathrm{H}, \mathrm{H8}\right), 8.26$ (d, $\left.J=8.6 \mathrm{~Hz}, 1 \mathrm{H}, \mathrm{H} 5\right), 8.10$ (d, $J=6.9 \mathrm{~Hz}, 1 \mathrm{H}$, H10), 7.86 (t, $J=8.4$ Hz, 4H, (H22 + H23)), 7.66-7.56 (m, 2H, (H9, H4)), 7.24-7.20 (d, $J=7.2$ Hz, 1H, H3), 3.69-3.51 (m, 2H, H19), 3.37-3.24 (m, 2H, H17), 3.23-3.09 (m, 2H, H15), 2.87 (s, 6H, H16), 2.79 (s, 6H, (H1, (H2 + H14 overlap)), 2.02-1.85 (m, 2H, H14), 1.81-1.66 (m, 2H, H18) ppm; ${ }^{13}$ C NMR (100 MHz, $\mathrm{CDCl}_{3}, \delta$ ): 168.4 (C20), 151.89 (C2), 134.88 (C23),130.07 (C6), 129.47 (C8), 129.38 (C4), 128.98 (C9), 128.49 (C10), 124.16 (C5), 123.54 (C8), 119.48 (C7), 115.23 (C3), 61.76 (C17), 61.59 (C15), 50.26 (C16), 45.50 (C1), 39.50 (C13), 35.14 (C19), 23.01 (C18), 22.12 (C14) ppm.

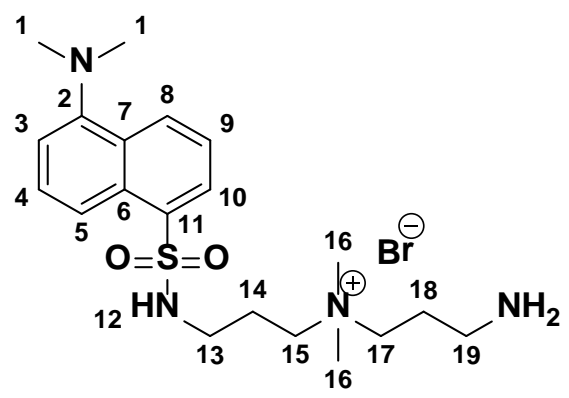


3-amino- $N$-(3-(5-(dimethylamino)naphthalene-1-sulfonamido)propyl)- $N, N$-dimethylpropan -1-ammonium bromide (221):

This compound was prepared by dissolving 220 (6.655 g, $11 \mathrm{mmol})$ in EtOH $(50 \mathrm{~mL})$ and deprotecting with hydrazine hydrate $(5.0 \mathrm{~mL}, 44 \mathrm{mmol}, 4$ eq.) under reflux 2 hrs. The mixture was then cooled to RT, diluted with $\mathrm{Et}_{2} \mathrm{O}(100 \mathrm{~mL})$ and filtered throught Celite. Volatiles were removed in vacuo and the crude product (8.647 g, yellow gum) was again diluted with $\mathrm{Et}_{2} \mathrm{O}$ (100 $\mathrm{mL}$ ) and triturated followed by decanting and placed under high vaccum (1 hr) to obtain the title compound as a yellow/white puffy solid. Yield: 99\% (5.2 g); Mp $=35-40{ }^{\circ} \mathrm{C} ;{ }^{1} \mathbf{H}$ NMR (400 MHz, $\mathrm{D}_{2} \mathrm{O}, \delta$ ): 8.17 (d, $\left.J=8.61 \mathrm{~Hz}, 1 \mathrm{H}, \mathrm{H} 8\right), 8.07$ (d, $\left.J=8.68 \mathrm{~Hz}, 1 \mathrm{H}, \mathrm{H} 5\right), 8.04$ (dd, ${ }^{1} J=0.95$ $\left.\mathrm{Hz},{ }^{2} J=7.35 \mathrm{~Hz}, 1 \mathrm{H}, \mathrm{H} 10\right), 7.45$ (t, $\left.J=7.79 \mathrm{~Hz}, 1 \mathrm{H}, \mathrm{H} 4\right), 7.36$ (t, $\left.J=7.49 \mathrm{~Hz}, 2 \mathrm{H}, \mathrm{H} 9\right), 7.05$ (d, $J=7.05 \mathrm{~Hz}, 1 \mathrm{H}, \mathrm{H} 3), 2.97-2.86$ (m, 2H, H13), 2.83-2.57 (m, 2H, H15), 2.63 (t, $J=2.63 \mathrm{~Hz}, 2 \mathrm{H}$, H17), 2.57 (s, 6H, H16), 2.52 (s, 6H, H1), 2.47 (d, J = 2.47 Hz, 2H, H19), 1.66-1.54 (m, 2H, H14), 1.49-1.40 (m, 2H, H18) ppm; ${ }^{13}$ C NMR (100 MHz, D $\left.2 \mathrm{O}, \delta\right): 150.90$ (C2), 138.82 (C11), 130.12 (C6), 129.70 (C8), 129.89 (C4), 128.75 (C9), 128.65 (C10), 124.02 (C5), 118.80 (C7), 115.85 (C3), 61.53-61.24 (C15, C17), 50.53 (C16), 44.76 (C1), 39.09 (C13), 33.85 (C19), 22.75 (C18), 22.13 (C14) ppm. DART (m/z): [ $\left.\mathrm{M}^{+}-\mathrm{Br}\right]$ calculated for $\mathrm{C}_{20} \mathrm{H}_{33} \mathrm{~N}_{4} \mathrm{O}_{2} \mathrm{~S}$, found, 407.3.

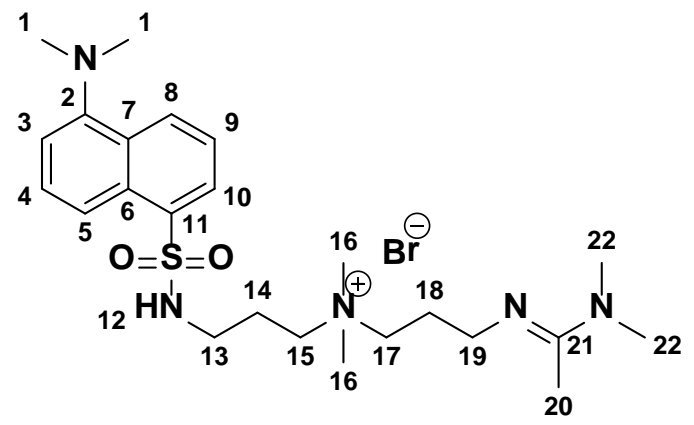




\section{(E)-3-((1-(dimethylamino)ethylidene)amino)- $N$-(3-(5-(dimethylamino)naphthalene-1-}

\section{sulfonamido)propyl)- $N$, $N$-dimethylpropan-1-ammonium bromide (222):}

To a stirred solution (20 ml ST) of dansylamine $221(0.5 \mathrm{~g}, 1.06 \mathrm{mmol})$ and $\mathrm{HNMe}_{2}(1.0 \mathrm{~mL}$ of $5.6 \mathrm{M}$ in EtOH, $5.6 \mathrm{mmol})$ in EtOH $(1 \mathrm{~mL})$ was added $N, N$-dimethylacetamide dimethyl acetal (0.2 g, $1.5 \mathrm{mmol})$ dropwise via a syringe. The reaction mixture was sealed, wrapped in aluminum foil stirred $\mathrm{ON}$ at RT in the dark. Volatile $\mathrm{EtOH}$ and $\mathrm{Me}_{2} \mathrm{NH}$ were evaporated in vacuo followed by placing the vial under high vaccum $\left(50^{\circ} \mathrm{C}, 8 \mathrm{hrs}\right)$ and recovered as a light orange solid. Yield: 99\% (0.57 g). ${ }^{1}$ H NMR (400 MHz, $\mathrm{D}_{2} \mathrm{O}, \delta$ ): 8.22 (d, $J=8.2 \mathrm{~Hz}, 1 \mathrm{H}, \mathrm{H} 8$ ), 8.08 (d, $J=8.5 \mathrm{~Hz}$, 1H, H5), 8.01 (d, $J=7.1 \mathrm{~Hz}, 1 \mathrm{H}, \mathrm{H} 10), 7.52-7.38$ (m, 2H, (H4 + H9)), 7.12 (d, $J=7.36 \mathrm{~Hz}, 1 \mathrm{H}$, H3), 2.97-2.70 (m, 6H, (H15 + H17 + H13)), 2.58 (s, 4H, H16), 2.47 (s, 8H, (H1 + H22)), 1.83 (s, 3H, H20), 1.50-1.27 (m, 4H, (H14 + H19)), 1.03 (t, $J=6.97$ Hz, 2H, H19) ppm; ${ }^{13}$ C NMR (100 MHz, $\mathrm{D}_{2} \mathrm{O}, \delta$ ): 174.11 (C21), 150.85 (C2), 134.05 (C11), 130.07 (C7), 129.72 (C9), 128.87 (C10), 128.73 (C5), 128.61 (C3), 124.10 (C6), 118.88 (C8), 115.91 (C4), 61.73 (C18), 61.10 (C15), 57.34 (C16), 50.29 (C22), 44.77 (C1), 39.11 (C13), 35.84 (C20), 22.01 (C18), 21.80 (C14), 16.70 (C19) ppm. ESI-TOF (m/z): [ $\left.\mathrm{M}^{+}\right]$calculated for $\mathrm{C}_{24} \mathrm{H}_{40} \mathrm{~N}_{5} \mathrm{O}_{2} \mathrm{P}$, found, 435.2

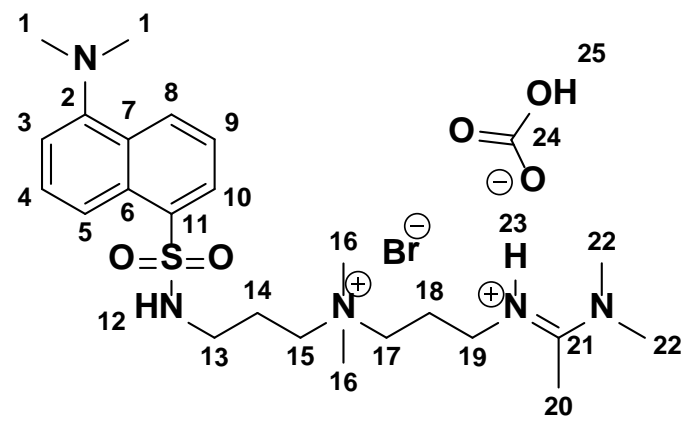


(E)- $N^{1}$-(1-(dimethylamino)ethylidene)- $N^{3}$-(3-(5-(dimethylamino)naphthalene-1-

sulfonamido)propyl)- $N^{3}, N^{3}$-dimethylpropane-1,3-diammonium bromide

hydrogencarbonate (223):

This compound was prepared by dissolving 222 in $\mathrm{D}_{2} \mathrm{O}$ and bubbling $\mathrm{CO}_{2}$ directly into the solution for 60 min. ${ }^{1} \mathbf{H}$ NMR (400 MHz, $\left.\mathrm{D}_{2} \mathrm{O}, \delta\right): 8.26(\mathrm{~d}, J=8.56 \mathrm{~Hz}, 1 \mathrm{H}, \mathrm{H} 8), 8.12$ (d, $J=$ 8.64 Hz, 1H, H5), 8.01 (d, $J=7.28 \mathrm{~Hz}, 1 \mathrm{H}, \mathrm{H} 10), 7.52$ (t, $J=8.24 \mathrm{~Hz}, 1 \mathrm{H}, \mathrm{H} 4), 7.46$ (t, $J=8.02$ Hz, 1H, H9), 7.15(d, $J=7.68 \mathrm{~Hz}, 1 \mathrm{H}, \mathrm{H3}), 3.00$ (t, $J=6.80 \mathrm{~Hz}, 2 \mathrm{H}, \mathrm{H} 19), 2.93-2.89$ (m, 6H, (H15 + H17)), 2.71-2.70 (m, 2H, H13), 2.62 (s, 6H, H1), 2.54 (s, 6H, H22), 1.90 (s, 3H, H20),

1.51-1.44 (m, 4H, (H14 + H18)) ppm; ${ }^{13} \mathrm{C}$ NMR (100 MHz, $\left.\mathrm{D}_{2} \mathrm{O}, \delta\right): 174.17$ (C21), 151.00 (C2), 133.98 (C11), 130.23 (C6), 129.81 (C8), 128.98 (C4), 128.81 (C9), 128.26 (C10), 124.13 (C5), 118.42 (C7), 115.95 (C3), 61.62 (C17), 61.10 (C16), 50.38 (C16, C22), 44.83 (C1), 38.96 (C13), 35.92 (C19), 22.05 (C20), 21.90 (C14), 21.86 (C18) ppm.

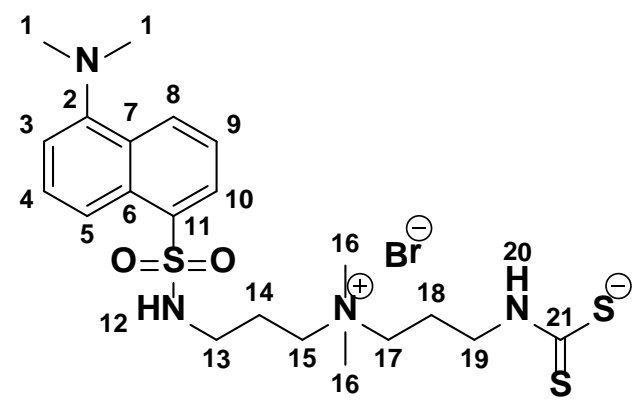

(3-((3-(5-(dimethylamino)naphthalene-1-sulfonamido)propyl) dimethylammonio) propyl) carbamodithioate (225):

To a stirred solution of flourophore $221(0.5 \mathrm{~g}, 1.05 \mathrm{mmol})$ in methanolic $\mathrm{KOH}(5 \mathrm{~mL}, 65 \mathrm{mg}$, 1.15 mmol, 1.1 eq.) was added $\mathrm{CS}_{2}(0.1 \mathrm{~mL}, 1.65 \mathrm{mmol}, 1.57$ eq.) dropwise via a syringe. A precipitate was observed after a few min and the reaction was further stirred for an additional 5 
min until an oily precipitate formed around the stir bar and stopped stirring. The mixture was then diluted with EtOH $(10 \mathrm{~mL})$ and decanted. The yellow sticky solid left behind was again diluted with $\mathrm{Et}_{2} \mathrm{O}(10 \mathrm{~mL})$, triturated followed by decanting and placed under high vaccum (1 hr) to obtain the title compound as a yellow/white powder. This compound was insoluble in $\mathrm{CDCl}_{3}$, $\mathrm{D}_{2} \mathrm{O}$ and MeOD. Yield: 81\% (0.4 g); $\mathrm{Mp}=220^{\circ} \mathrm{C} ;{ }^{1} \mathbf{H}$ NMR (400 MHz, DMSO, $\delta$ ): 8.46 (d, J = $8.46 \mathrm{~Hz}, 1 \mathrm{H}, \mathrm{H8}), 8.27$ (d, $J=8.72 \mathrm{~Hz}, 2 \mathrm{H}, \mathrm{H} 5), 8.16-8.03$ (m, 2H, (H10 + H12)), 7.69-7.57 (m, 2H, (H4 + H9)), 7.27 (d, J = 7.11 Hz, 1H, H3), 3.26-3.03 (m, 2H, (H15 + H17)), 2.81 (brs, 14H, (H1 + H16 + H20)), 1.85-1.65 (m, 4H, (H14 + H19)), ppm; ${ }^{13}$ C NMR (100 MHz, DMSO, $\left.\delta\right):$ 216.09 (C21), 152.10 (C2), 136.07 (C11), 130.30 (C6), 129.66 (C8), 129.56 (C4), 129.20 (C9), 128.78 (C10), 124.36 (C5), 119.47 (C7), 115.92 (C3), 62.23 (C17), 61.24 (C15), 50.67 (C16), 45.71 (C1), 22.97 (C19), 22.37 (C14), 19.20 (C18) ppm. ESI-TOF (m/z): [M+1 calculated for $\mathrm{C}_{21} \mathrm{H}_{32} \mathrm{~N}_{4} \mathrm{O}_{2} \mathrm{~S}_{3}$, found, 393.2. 


\subsection{Appendix}

\section{List of Appendix Tables}

Table A 1. Crystal data and structure refinement for 34........................................................ 319

Table A 2. Atomic coordinates and equivalent isotropic displacement parameters for 34 ........ 320

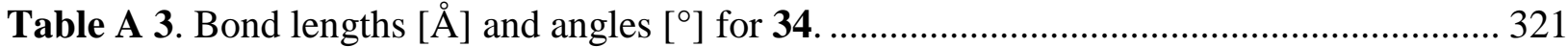

Table A 4. Anisotropic displacement parameters for 34............................................................. 328

Table A 5. Hydrogen coordinates and isotropic displacement parameters ................................. 329

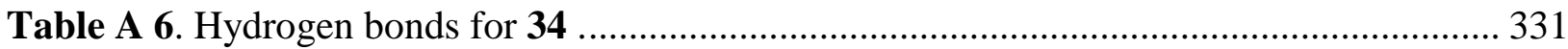

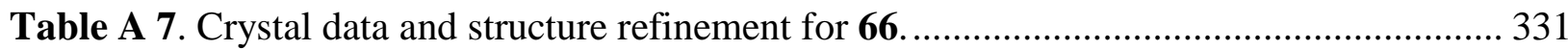

Table A 8. Atomic coordinates and equivalent isotropic displacement parameters for $\mathbf{6 6}$...... 332

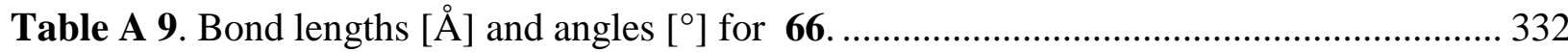

Table A 10. Anisotropic displacement parameters for 66........................................................ 340

Table A 11. Hydrogen coordinates and isotropic displacement parameters for 66. .................. 341

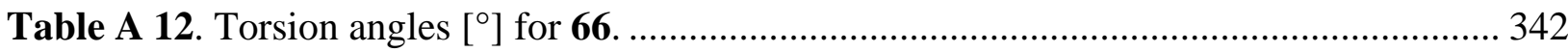

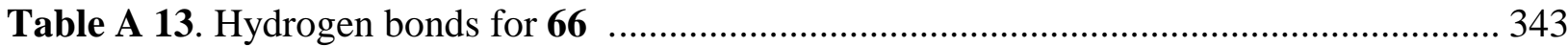

Table A 14. Crystal data and structure refinement for 19........................................................ 343

Table A 15. Atomic coordinates and equivalent isotropic displacement parameters for $19 \ldots 344$

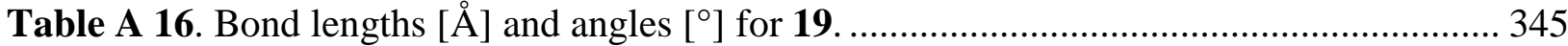

Table A 17. Anisotropic displacement parameters for 19.......................................................... 349

Table A 18. Hydrogen coordinates and isotropic displacement parameters for 19. ................... 350

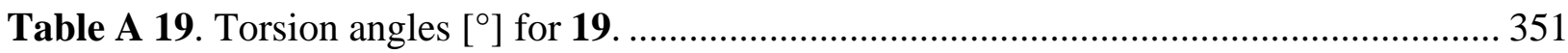

Table A 20. Crystal data and structure refinement for 220 ................................................... 353

Table A 21. Atomic coordinates and equivalent isotropic displacement parameters for 220.... 354

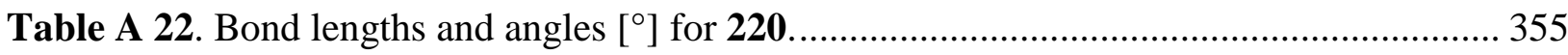

Table A 23. Anisotropic displacement parameters for 222...................................................... 361

Table A 24. Hydrogen coordinates and isotropic displacement parameters for 220 ................. 363

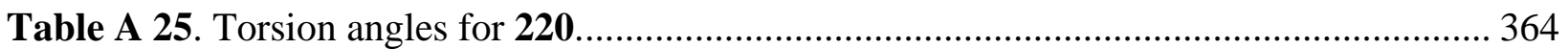

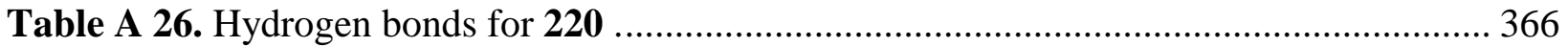

\section{List of Appendix Figures}

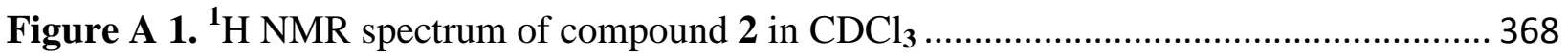

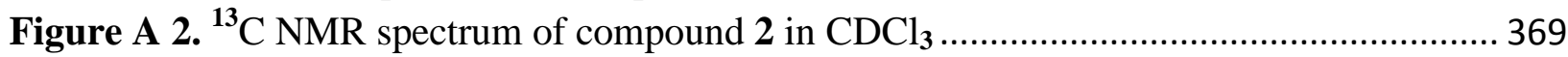

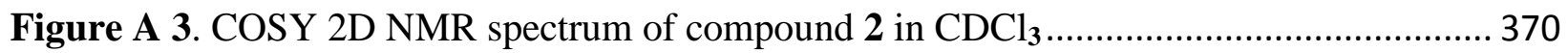

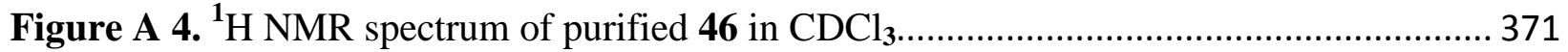

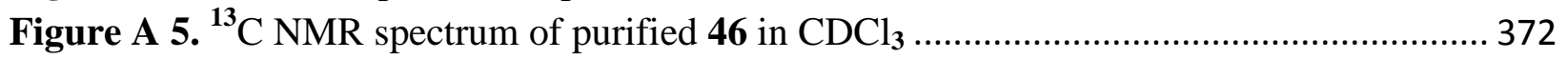

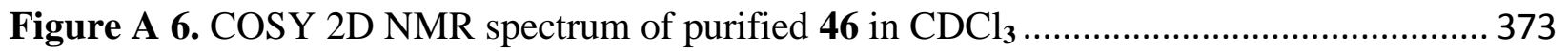

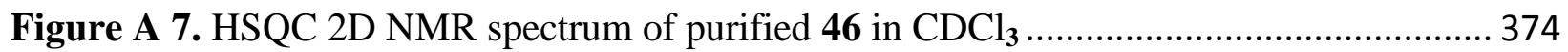

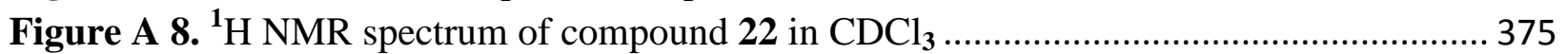

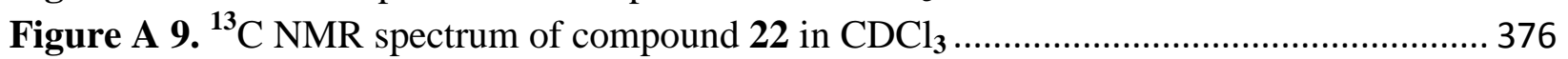




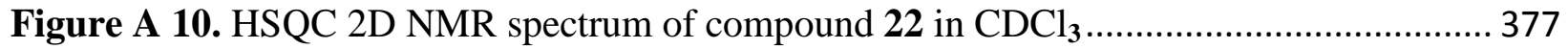

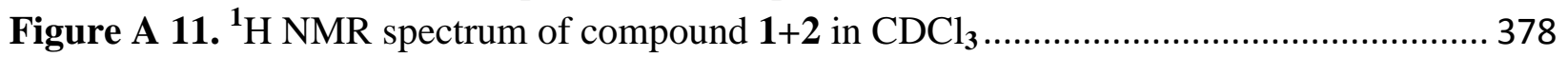

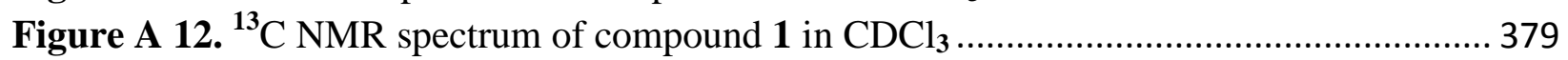

Figure A 13. ${ }^{1} \mathrm{H}$ NMR spectrum of compound 3 in $\mathrm{CDCl}_{3}$ (Table 2.1, entry i).................... 380

Figure A 14. ${ }^{13} \mathrm{C}$ NMR spectrum of compound 3 in $\mathrm{CDCl}_{3}$ (Table 2.1, entry i).................... 381

Figure A 15. COSY 2D NMR spectrum of compound 3 in $\mathrm{CDCl}_{3}$ (Table 2.1, entry i) ......... 382

Figure A 16. HSQC 2D NMR spectrum of compound 3 in $\mathrm{CDCl}_{3}$ (Table 2.1, entry i) ......... 383

Figure A 17. ${ }^{1} \mathrm{H}$ stacked NMR spectra of compound 3 in $\mathrm{CDCl}_{3}$ (Table 2.1, entry i-iii) ........ 384

Figure A 18. ${ }^{1} \mathrm{H}$ stacked NMR spectra of compound 3 in $\mathrm{CDCl}_{3}$ (Table 2.1, entry iv-v, viii) 385

Figure A 19. ${ }^{1} \mathrm{H}$ NMR spectrum of compound 3 in $\mathrm{CDCl}_{3}$ (Table 2.2, entry iii) .................. 386

Figure A 20. ${ }^{13} \mathrm{C}$ NMR spectrum of compound 3 in $\mathrm{CDCl}_{3}$ (Table 2.2, entry iii) .................. 387

Figure A 21. ${ }^{1} \mathrm{H}$ NMR spectrum of compound 12 in $\mathrm{CDCl}_{3}$ (Table 2.3, entry iii) ................ 388

Figure A 22. ${ }^{13} \mathrm{C}$ NMR spectrum of compound 12 in $\mathrm{CDCl}_{3}$ (Table 2.3, entry iii) ................ 389

Figure A 23. COSY 2D NMR spectrum of compound 12 in $\mathrm{CDCl}_{3}$ (Table 2.3, entry iii) ...... 390

Figure A 24. HSQC 2D NMR spectrum of compound 12 in $\mathrm{CDCl}_{3}$ (Table 2.3, entry iii) ...... 391

Figure A 25. ${ }^{31} \mathrm{P}$ NMR spectrum of compound 12 in $\mathrm{CDCl}_{3}$ (Table 2.3, entry iii)................. 392

Figure A 26. ${ }^{1} \mathrm{H}$ NMR spectrum of compound 13 in $\mathrm{CDCl}_{3}$ (Table 2.3, entry vii)................ 393

Figure A 27. ${ }^{13} \mathrm{C}$ NMR spectrum of compound 13 in $\mathrm{CDCl}_{3}$ (Table 2.3, entry vii) ............... 394

Figure A 28. COSY 2D NMR spectrum of compound 13 in $\mathrm{CDCl}_{3}$ (Table 2.3, entry vii) ..... 395

Figure A 29. HSQC 2D NMR spectrum of compound 13 in $\mathrm{CDCl}_{3}$ (Table 2.3, entry vii) ..... 396

Figure A 30. ${ }^{31} \mathrm{P}$ NMR spectrum of compound 13 in $\mathrm{CDCl}_{3}$ (Table 2.3, entry vii)................ 397

Figure A 31. ${ }^{1} \mathrm{H}$ NMR spectrum of compound 14 in $\mathrm{CDCl}_{3}$ (Table 2.3, entry ix)................ 398

Figure A 32. ${ }^{13} \mathrm{C}$ NMR spectrum of compound 14 in $\mathrm{CDCl}_{3}$ (Table 2.3, entry ix)................ 399

Figure A 33. COSY 2D NMR spectrum of compound 14 in $\mathrm{CDCl}_{3}$ (Table 2.3, entry ix) ...... 400

Figure A 34. HSQC 2D NMR spectrum of compound 14 in $\mathrm{CDCl}_{3}$ (Table 2.3, entry ix) ...... 401

Figure A 35. ${ }^{31} \mathrm{P}$ NMR spectrum of compound 14 in $\mathrm{CDCl}_{3}$ (Table 2.3, entry ix) ................ 402

Figure A 36. ${ }^{1} \mathrm{H}$ NMR spectrum of compound 16 in $\mathrm{CDCl}_{3}$ (Table 2.3, entry xv)............... 403

Figure A 37. ${ }^{13} \mathrm{C}$ NMR spectrum of compound 16 in $\mathrm{CDCl}_{3}$ (Table 2.3, entry xv)............... 404

Figure A 38. COSY 2D NMR spectrum of compound 16 in $\mathrm{CDCl}_{3}$ (Table 2.3, entry xv) ..... 405

Figure A 39. HSQC 2D NMR spectrum of compound 16 in $\mathrm{CDCl}_{3}$ (Table 2.3, entry xv) ..... 406

Figure A 40. ${ }^{31} \mathrm{P}$ NMR spectrum of compound 16 in $\mathrm{CDCl}_{3}$ (Table 4.3, entry xv) ............... 407

Figure A 41. ${ }^{1} \mathrm{H}$ NMR spectrum of compound 17 in $\mathrm{CDCl}_{3}$ (Table 2.3, entry xvi)............... 408

Figure A 42. ${ }^{13} \mathrm{C}$ NMR spectrum of compound 17 in $\mathrm{CDCl}_{3}$ (Table 2.3, entry xvi)............... 409

Figure A 43. COSY 2D NMR spectrum of compound 17 in $\mathrm{CDCl}_{3}$ (Table 2.3, entry xvi) .... 410

Figure A 44. HSQC 2D NMR spectrum of compound 17 in $\mathrm{CDCl}_{3}$ (Table 2.3, entry xvi) .... 411

Figure A 45. ${ }^{31} \mathrm{P}$ NMR spectrum of compound 17 in $\mathrm{CDCl}_{3}$ (Table 2.3, entry xvi) .............. 412

Figure A 46. ${ }^{1} \mathrm{H}$ NMR spectrum of compound 18 in $\mathrm{CDCl}_{3}$ (Table 2.3, entry xvii).............. 413

Figure A 47. ${ }^{13} \mathrm{C}$ NMR spectrum of compound 18 in $\mathrm{CDCl}_{3}$ (Table 2.3, entry xvii) ............. 414

Figure A 48. COSY 2D NMR spectrum of compound 18 in $\mathrm{CDCl}_{3}$ (Table 2.3, entry xvii) ... 415

Figure A 49. ${ }^{31} \mathrm{P}$ NMR spectrum of compound 18 in $\mathrm{CDCl}_{3}$ (Table 4.3, entry xvii)............. 416 
Figure A 50. ${ }^{1} \mathrm{H}$ NMR spectrum of compound 19 in $\mathrm{CDCl}_{3}$ (Table 2.3, entry xviii) ............. 417

Figure A 51. ${ }^{13} \mathrm{C}$ NMR spectrum of compound 19 in $\mathrm{CDCl}_{3}$ (Table 2.3, entry xviii) ........... 418

Figure A 52. COSY 2D NMR spectrum of compound 19 in $\mathrm{CDCl}_{3}$ (Table 2.3, entry xviii) .. 419

Figure A 53. HSQC 2D NMR spectrum of compound 19 in $\mathrm{CDCl}_{3}$ (Table 2.3, entry xviii) .. 420

Figure A 54. ${ }^{31} \mathrm{P}$ NMR spectrum of compound 19 in $\mathrm{CDCl}_{3}$ (Table 2.3, entry xviii)............. 421

Figure A 55. ${ }^{1} \mathrm{H}$ NMR spectrum of compound 26 in $\mathrm{CDCl}_{3}$ (Table 2.4, entry i)................... 422

Figure A 56. ${ }^{13} \mathrm{C}$ NMR spectrum of compound 26 in $\mathrm{CDCl}_{3}$ (Table 2.4, entry i).................. 423

Figure A 57. COSY 2D NMR spectrum of compound 26 in $\mathrm{CDCl}_{3}$ (Table 2.4, entry i) ....... 424

Figure A 58. HSQC 2D NMR spectrum of compound 26 in $\mathrm{CDCl}_{3}$ (Table 2.4, entry i) ....... 425

Figure A 59. ${ }^{31} \mathrm{P}$ NMR spectrum of compound 26 in $\mathrm{CDCl}_{3}$ (Table 2.4, entry i) .................. 426

Figure A 60. ${ }^{1} \mathrm{H}$ NMR spectrum of compound 27 in $\mathrm{CDCl}_{3}$ (Table 4.4, entry iv)................. 427

Figure A 61. ${ }^{13} \mathrm{C}$ NMR spectrum of compound 27 in $\mathrm{CDCl}_{3}$ (Table 2.4, entry iv)............... 428

Figure A 62. COSY 2D NMR spectrum of compound 27 in $\mathrm{CDCl}_{3}$ (Table 2.4, entry iv) ...... 429

Figure A 63. ${ }^{31} \mathrm{P}$ NMR spectrum of compound 27 in $\mathrm{CDCl}_{3}$ (Table 2.4, entry iv) ................ 430

Figure A 64. ${ }^{1} \mathrm{H}$ NMR spectrum of compound 28 in $\mathrm{CDCl}_{3}$ (Table 2.4, entry vii)................ 431

Figure A 65. ${ }^{13} \mathrm{C}$ NMR spectrum of compound 28 in $\mathrm{CDCl}_{3}$ (Table 2.4, entry vii) ............... 432

Figure A 66. COSY 2D NMR spectrum of compound 28 in $\mathrm{CDCl}_{3}$ (Table 2.4, entry vii) ..... 433

Figure A 67. ${ }^{31} \mathrm{P}$ NMR spectrum of compound 28 in $\mathrm{CDCl}_{3}$ (Table 2.4, entry vii) ................ 434

Figure A 68. ${ }^{31} \mathrm{P}$ NMR spectrum of compound 29 in $\mathrm{CDCl}_{3}$ (Table 2.4, entry viii).............. 435

Figure A 69. ${ }^{1} \mathrm{H}$ NMR spectrum of compound 30 in $\mathrm{CDCl}_{3}$ (Table 2.4, entry ix)................. 436

Figure A 70. ${ }^{13} \mathrm{C}$ NMR spectrum of compound 30 in $\mathrm{CDCl}_{3}$ (Table 2.4, entry ix)................ 437

Figure A 71. COSY 2D NMR spectrum of compound 30 in $\mathrm{CDCl}_{3}$ (Table 4.4, entry ix) ...... 438

Figure A 72. HSQC 2D NMR spectrum of compound 30 in $\mathrm{CDCl}_{3}$ (Table 2.4, entry ix) ...... 439

Figure A 73. ${ }^{31} \mathrm{P}$ NMR spectrum of compound 30 in $\mathrm{CDCl}_{3}$ (Table 2.4, entry ix) ................ 440

Figure A 74. ${ }^{1} \mathrm{H}$ NMR spectrum of compound 31 in $\mathrm{CDCL}_{3}$ (Table 2.4, entry $\mathbf{x}$ )................. 441

Figure A 75. ${ }^{13} \mathrm{C}$ NMR spectrum of compound 31 in $\mathrm{CDCL}_{3}$ (Table 2.4, entry $\mathbf{x}$ )................ 442

Figure A 76. COSY 2D NMR spectrum of compound 31 in $\mathrm{CDCL}_{3}$ (Table 2.4, entry x) ...... 443

Figure A 77. HSQC 2D NMR spectrum of compound 31 in $\mathrm{CDCL}_{3}$ (Table 4.4, entry x) ...... 444

Figure A 78. ${ }^{31} \mathrm{P}$ NMR spectrum of compound 31 in $\mathrm{CDCL}_{3}$ (Table 2.4, entry $\mathbf{x}$ ) ................. 445

Figure A 79. ${ }^{1} \mathrm{H}$ NMR spectrum of compound 32 in $\mathrm{CDCl}_{3}$ (Table 2.4, entry xi) ................. 446

Figure A 80. ${ }^{13} \mathrm{C}$ NMR spectrum of compound 32 in $\mathrm{CDCl}_{3}$ (Table 2.4, entry xi)................ 447

Figure A 81. HSQC 2D NMR spectrum of compound 32 in $\mathrm{CDCl}_{3}$ (Table 2.4, entry xi)...... 448

Figure A 82. ${ }^{31} \mathrm{P}$ NMR spectrum of compound 32 in $\mathrm{CDCl}_{3}$ (Table 2.4, entry xi) ................ 449

Figure A 83. ${ }^{1} \mathrm{H}$ NMR spectrum of compound 33 in $\mathrm{CDCL}_{3}$ (Table 2.4, entry xii)................ 450

Figure A 84. ${ }^{13} \mathrm{C}$ NMR spectrum of compound 33 in $\mathrm{CDCL}_{3}$ (Table 2.4, entry xii) .............. 451

Figure A 85. COSY 2D NMR spectrum of compound 33 in $\mathrm{CDCl}_{3}$ (Table 2.4, entry xii) ..... 452

Figure A 86. HSQC 2D NMR spectrum of compound 33 in $\mathrm{CDCl}_{3}$ (Table 2.4, entry xii) ..... 453

Figure A 87. ${ }^{31} \mathrm{P}$ NMR spectrum of compound 33 in $\mathrm{CDCL}_{3}$ (Table 2.4, entry xii)............... 454

Figure A 88. ${ }^{1} \mathrm{H}$ NMR spectrum of compound 39 in $\mathrm{CDCl}_{3}$............................................ 455

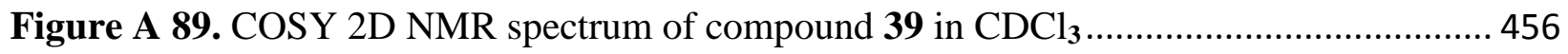




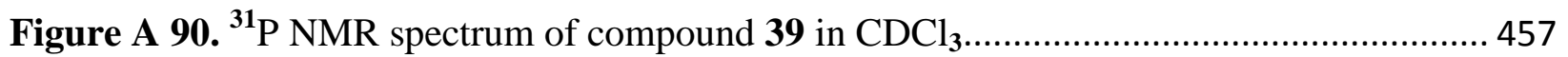

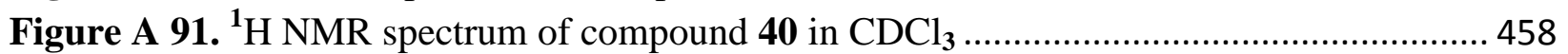

Figure A 92. ${ }^{31} \mathrm{P}$ NMR spectrum of compound 40 in $\mathrm{CDCl}_{3}$ (BEFORE COLUMN) .............. 459

Figure A 93. ${ }^{31} \mathrm{P}$ NMR spectrum of compound 40 in $\mathrm{CDCl}_{3}$ (AFTER COLUMN) ................ 460

Figure A 94. ${ }^{1} \mathrm{H}$ NMR spectrum of compound 34 in MeOD (Table 2.5, entry i) .................. 461

Figure A 95. ${ }^{13} \mathrm{C}$ NMR spectrum of compound 34 in MeOD (Table 2.5, entry i) .................. 462

Figure A 96. ${ }^{1} \mathrm{H}$ NMR spectrum of compound 34 in MeOD (Table 2.5, entry i) ................... 463

Figure A 97. ${ }^{1} \mathrm{H}$ NMR spectrum of compound 34 in MeOD (Table 2.5, entry i) .................. 464

Figure A 98. ${ }^{31} \mathrm{P}$ NMR spectrum of compound 34 in MeOD (Table 2.5, entry i).................. 465

Figure A 99. ${ }^{1} \mathrm{H}$ NMR spectrum of compound 35 in $\mathrm{D}_{2} \mathrm{O}$ (Table 4.5, entry ix) .................... 466

Figure A 100. ${ }^{13} \mathrm{C}$ NMR spectrum of compound 35 in $\mathrm{D}_{2} \mathrm{O}$ (Table 2.5, entry ix) ................. 467

Figure A 101. HSQC spectrum of compound 35 in $\mathrm{D}_{2} \mathrm{O}$ (Table 2.5, entry xi) .................... 468

Figure A 102. COSY spectrum of compound 35 in $\mathrm{D}_{2} \mathrm{O}$ (Table 2.5, entry ix) ...................... 469

Figure A 103. ${ }^{31} \mathrm{P}$ spectrum of compound 35 in $\mathrm{D}_{2} \mathrm{O}$ (Table 2.5, entry ix)........................... 470

Figure A 104. ${ }^{1} \mathrm{H}$ NMR spectrum of compound 35 in $\mathrm{D}_{2} \mathrm{O}$ (Table 2.5, entry ix) .................. 471

Figure A 105. ${ }^{13} \mathrm{C}$ NMR spectrum of compound 36 in $\mathrm{D}_{2} \mathrm{O}$ (Table 2.5, entry $\mathbf{x}$ ) ................. 472

Figure A 106. ${ }^{1} \mathrm{H}$ NMR spectrum of compound 36 in $\mathrm{D}_{2} \mathrm{O}$ (Table 2.5, entry x) ................... 473

Figure A 107. HSQC NMR spectrum of compound 36 in $\mathrm{D}_{2} \mathrm{O}$ (Table 2.5, entry x)............. 474

Figure A 108. ${ }^{31} \mathrm{P}$ NMR spectrum of compound 36 in $\mathrm{D}_{2} \mathrm{O}$ (Table 2.5, entry $\mathbf{x}$ ).................. 475

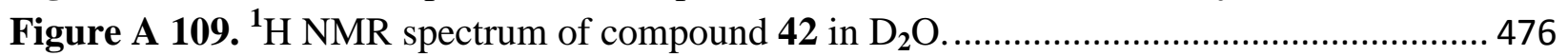

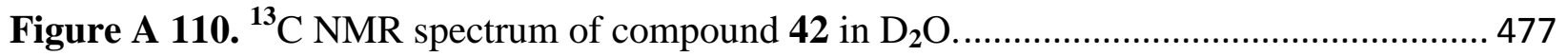

Figure A 111. COSY 2D NMR spectrum of compound 42 in $\mathrm{D}_{2} \mathrm{O}$..................................... 478

Figure A 112. HSQC 2D NMR spectrum of compound 42 in $\mathrm{D}_{2} \mathrm{O}$. .................................. 479

Figure A 113. ${ }^{31} \mathrm{P}$ NMR spectrum of compound 42 in $\mathrm{D}_{2} \mathrm{O}$............................................ 480

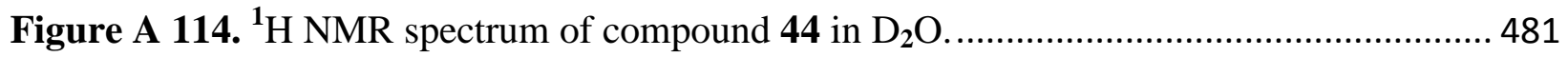

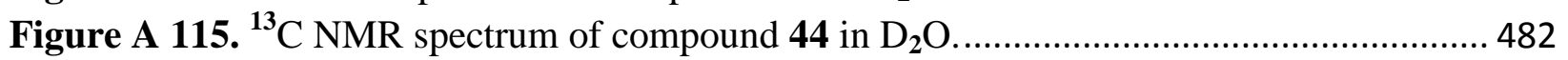

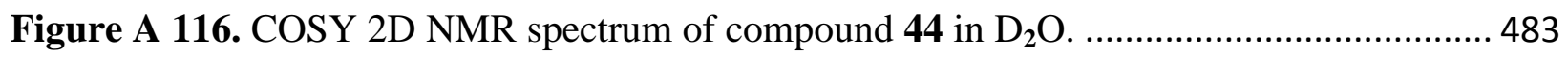

Figure A 117. HSQC 2D NMR spectrum of compound 44 in $\mathrm{D}_{2} \mathrm{O}$. ...................................... 484

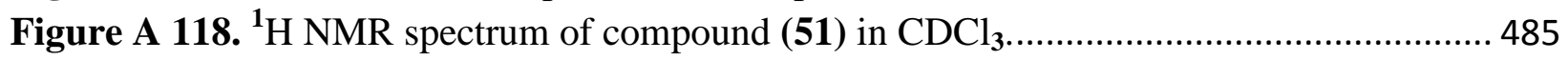

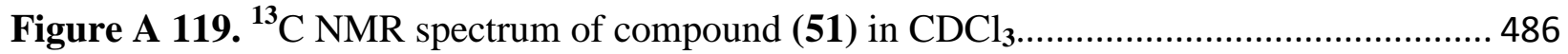

Figure A 120. COSY 2D NMR spectrum of compound (51) in $\mathrm{CDCl}_{3}$.............................. 487

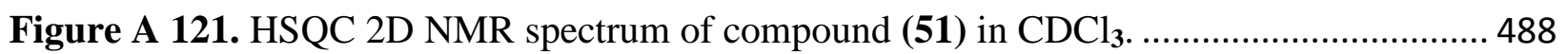

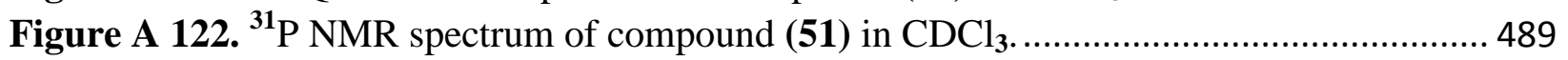

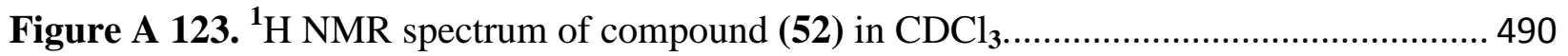

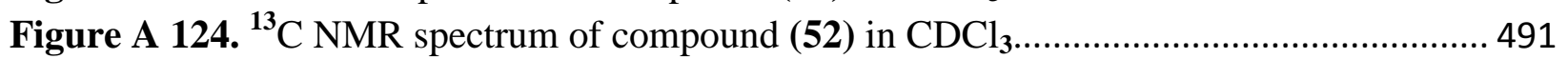

Figure A 125. COSY 2D NMR spectrum of compound (52) in $\mathrm{CDCl}_{3}$.............................. 492

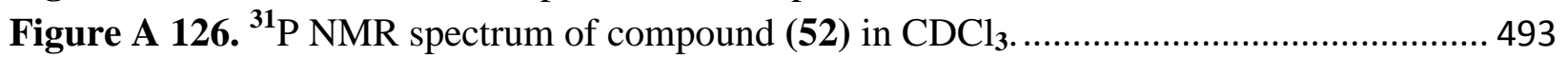

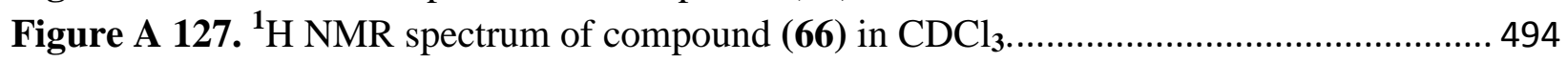

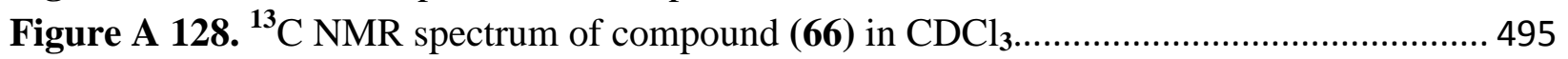

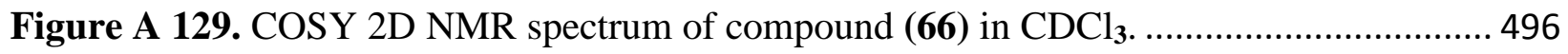




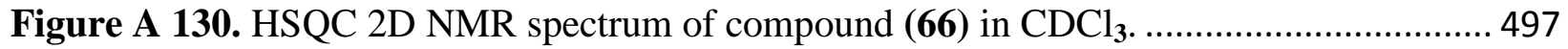

Figure A 131. ${ }^{1} \mathrm{H}$ NMR spectrum of compound (67) in MeOD............................................ 498

Figure A 132. ${ }^{13} \mathrm{C}$ NMR spectrum of compound (67) in MeOD.......................................... 499

Figure A 133. COSY 2D NMR spectrum of compound (67) in MeOD................................ 500

Figure A 134. HSQC 2D NMR spectrum of compound (67) in MeOD.................................. 501

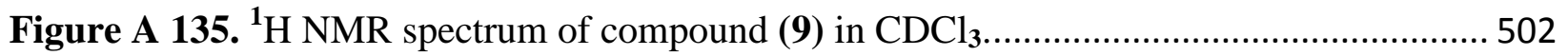

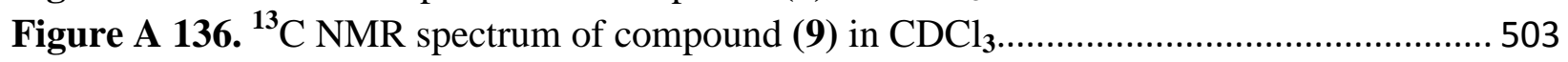

Figure A 137. COSY 2D NMR spectrum of compound (9) in $\mathrm{CDCl}_{3}$................................ 504

Figure A 138. HSQC 2D NMR spectrum of compound (9) in $\mathrm{CDCl}_{3}$............................... 505

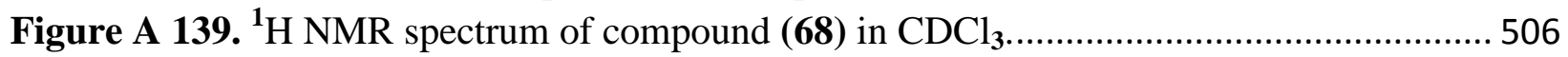

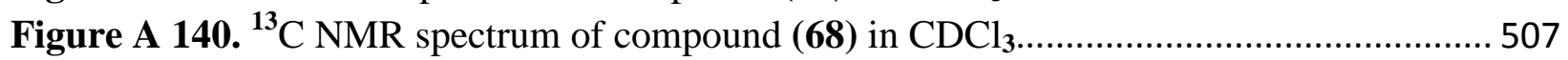

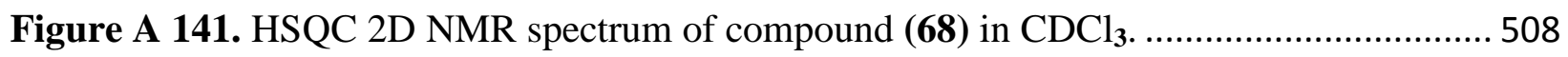

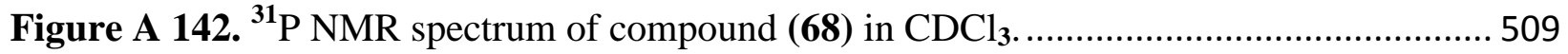

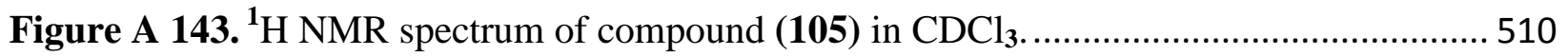

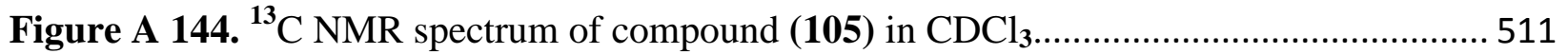

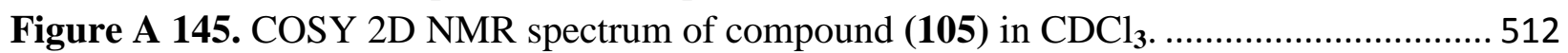

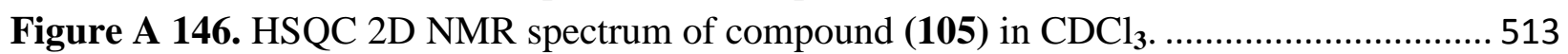

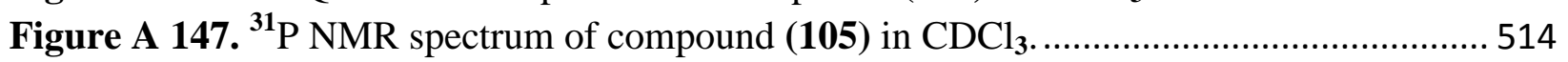

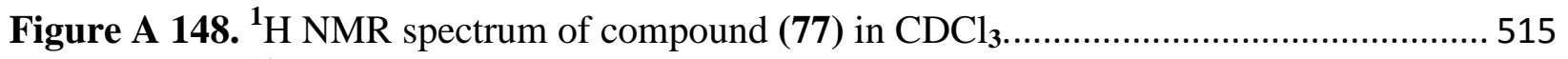

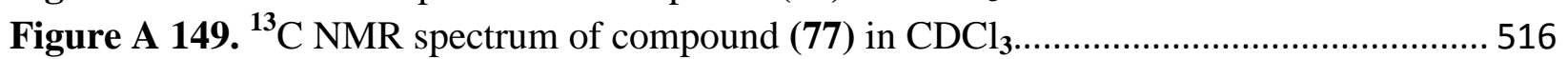

Figure A 150. HSQC 2D NMR spectrum of compound (77) in $\mathrm{CDCl}_{3}$............................... 517

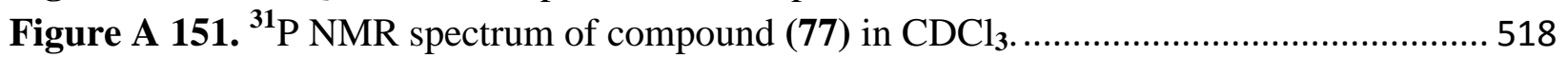

Figure A 152. ${ }^{1} \mathrm{H}$ NMR spectrum of compound (97) in MeOD. .............................................. 519

Figure A 153. ${ }^{13} \mathrm{C}$ NMR spectrum of compound (97) in MeOD.......................................... 520

Figure A 154. COSY 2D NMR spectrum of compound (97) in MeOD................................ 521

Figure A 155. HSQC 2D NMR spectrum of compound (97) in MeOD............................... 522

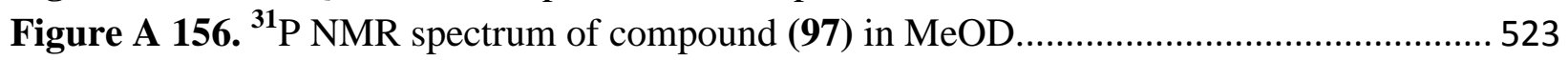

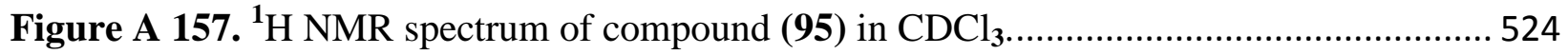

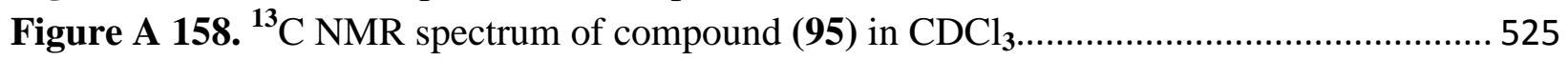

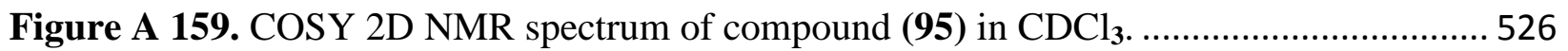

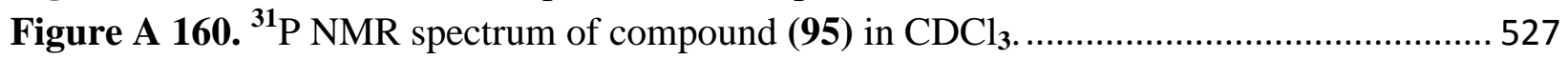

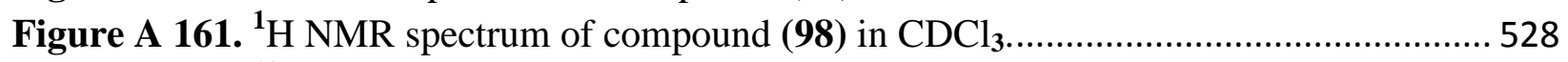

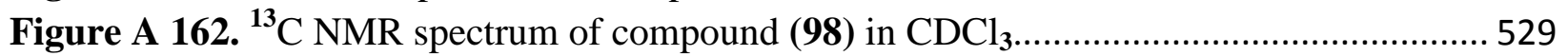

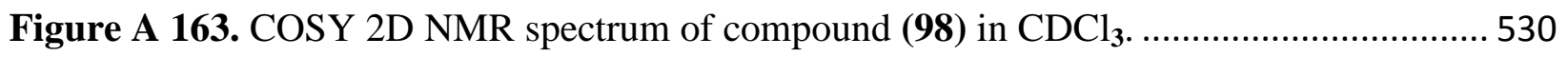

Figure A 164. HSQC 2D NMR spectrum of compound (98) in $\mathrm{CDCl}_{3}$............................... 531

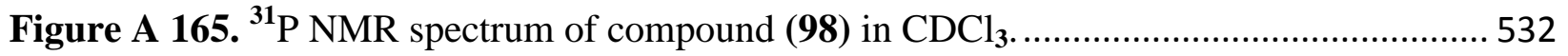

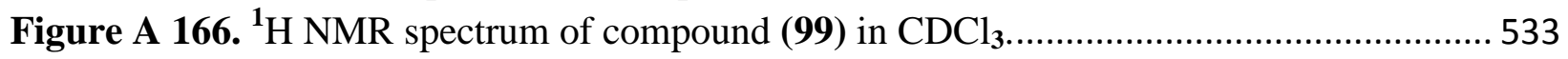

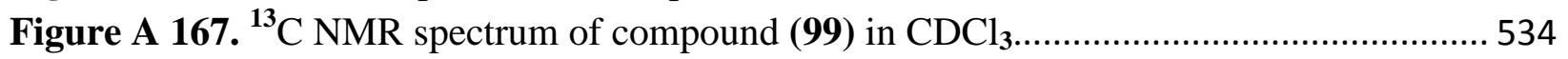

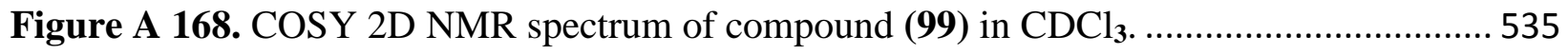

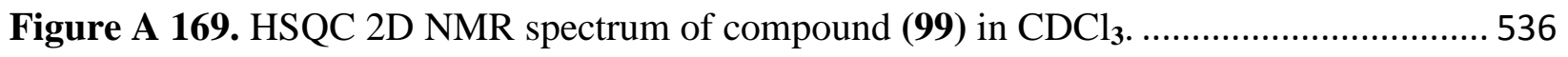




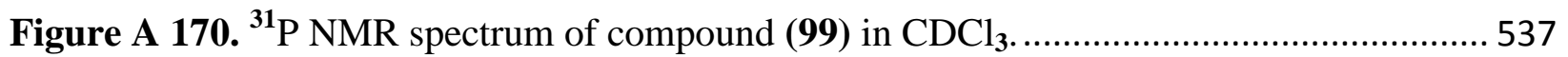

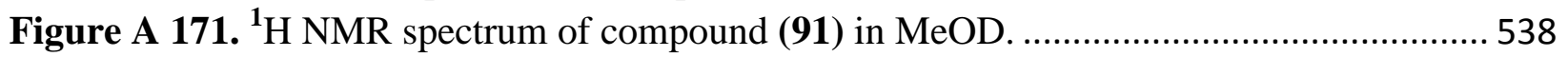

Figure A 172. ${ }^{13} \mathrm{C}$ NMR spectrum of compound (91) in MeOD............................................ 539

Figure A 173. COSY 2D NMR spectrum of compound (91) in MeOD................................. 540

Figure A 174. HSQC 2D NMR spectrum of compound (91) in MeOD................................. 541

Figure A 175. ${ }^{31} \mathrm{P}$ NMR spectrum of compound (91) in MeOD. (CRUDE) ………................. 542

Figure A 176. ${ }^{31} \mathrm{P}$ NMR spectrum of compound (91) in MeOD. (COLUMNED FRACTION) 543

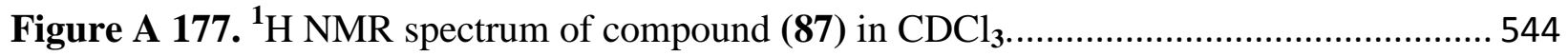

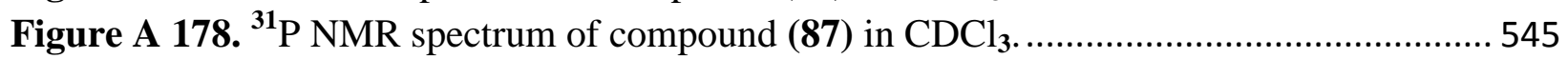

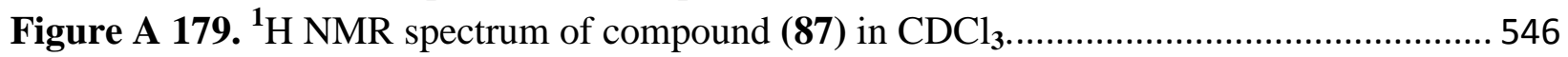

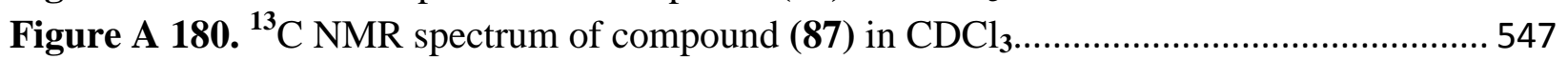

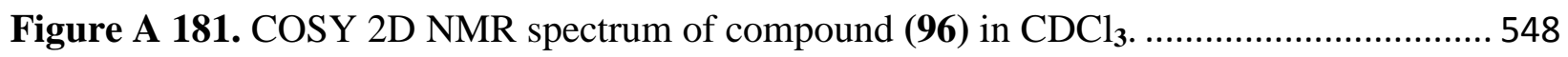

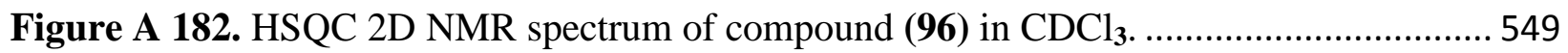

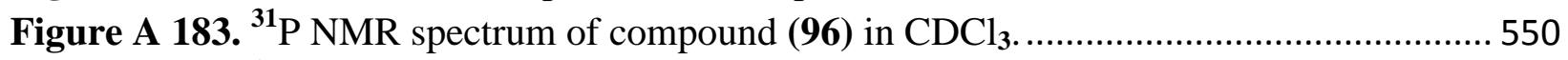

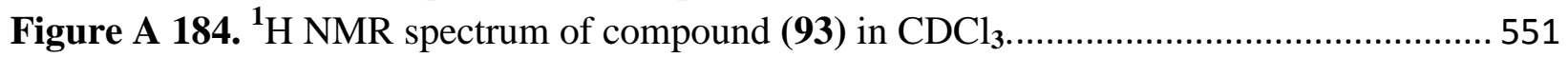

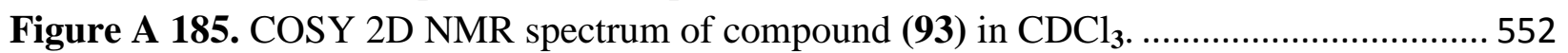

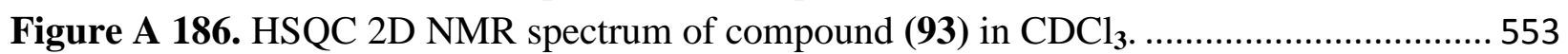

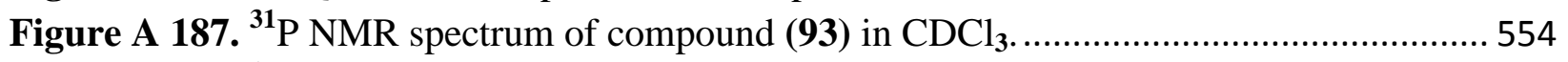

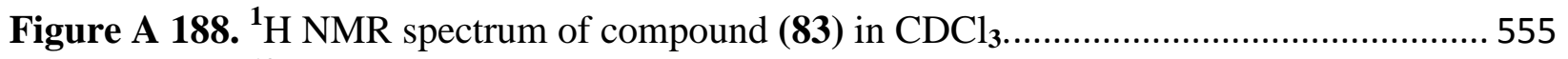

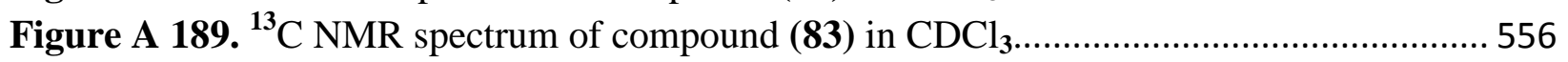

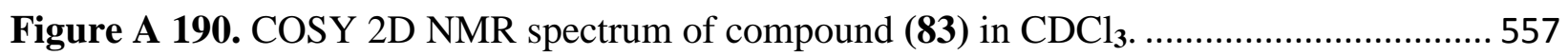

Figure A 191. HSQC 2D NMR spectrum of compound (83) in $\mathrm{CDCl}_{3} \ldots \ldots \ldots \ldots \ldots \ldots \ldots \ldots \ldots \ldots . . . . . . . . . . . . . . . .558$

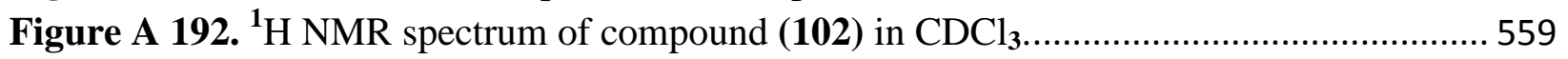

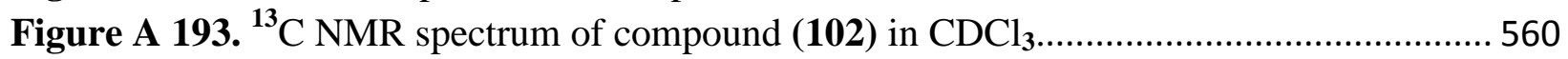

Figure A 194. COSY 2D NMR spectrum of compound (102) in $\mathrm{CDCl}_{3} \ldots \ldots \ldots \ldots \ldots \ldots \ldots \ldots \ldots \ldots . . . . . . . . . . . . . . . . .561$

Figure A 195. HSQC 2D NMR spectrum of compound (102) in $\mathrm{CDCl}_{3} \ldots \ldots \ldots \ldots \ldots \ldots \ldots \ldots \ldots \ldots . . . . . . . . . . . . . . .562$

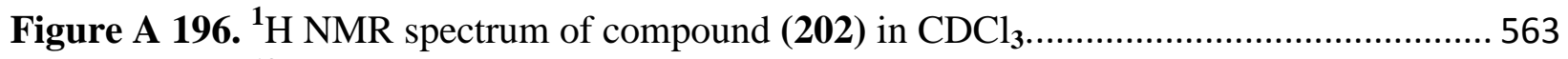

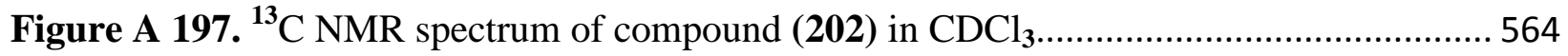

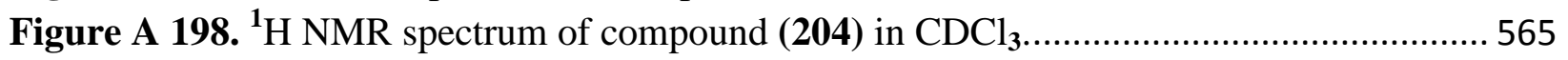

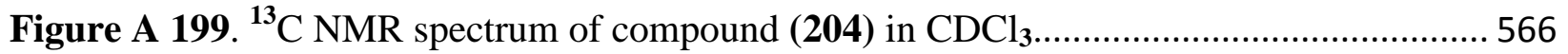

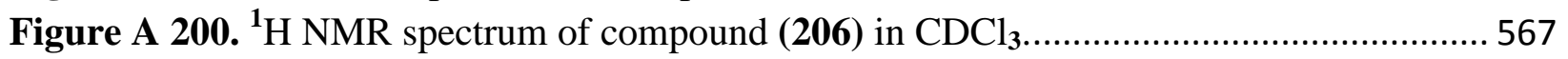

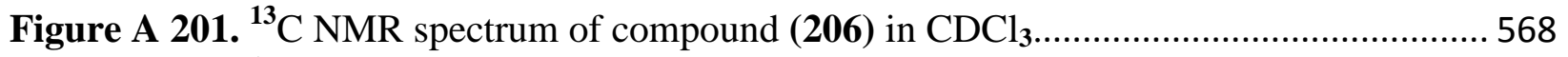

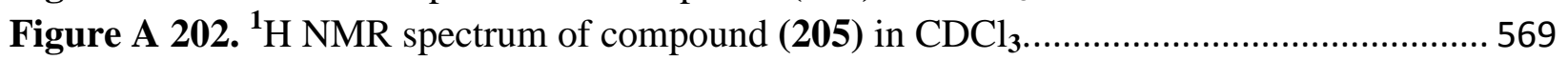

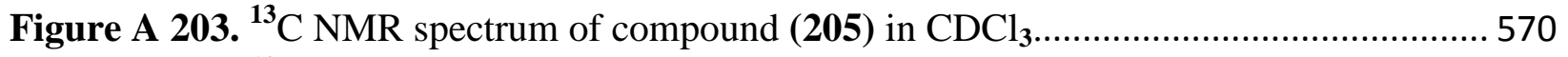

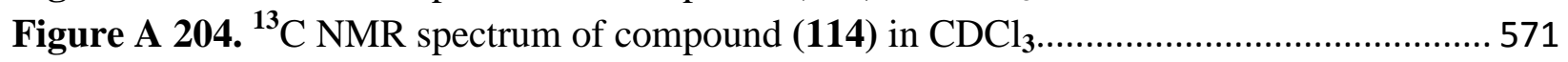

Figure A 205. COSY 2D NMR spectrum of compound (114) in $\mathrm{CDCl}_{3} \ldots \ldots \ldots \ldots \ldots \ldots \ldots \ldots \ldots \ldots . . . . . . . . . . . . . . . .572$

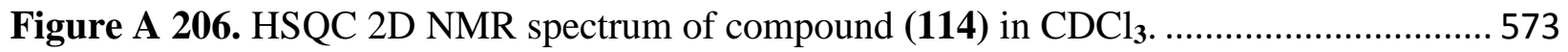

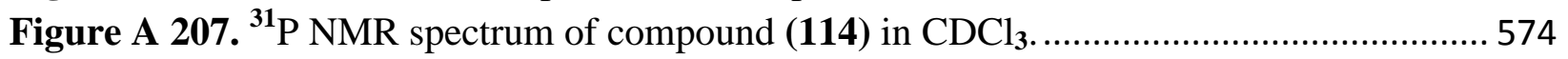

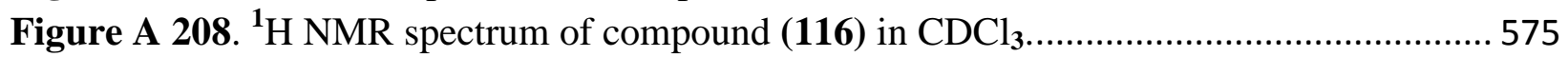

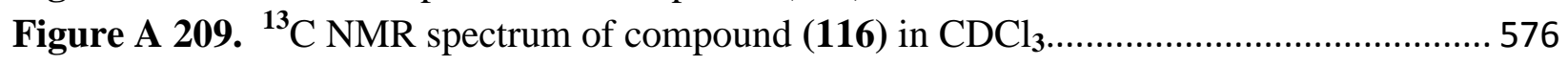




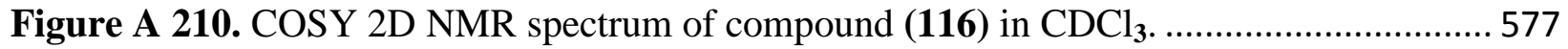

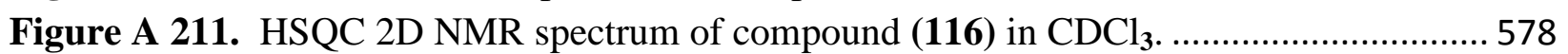

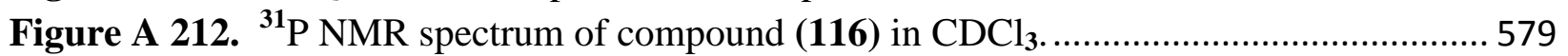

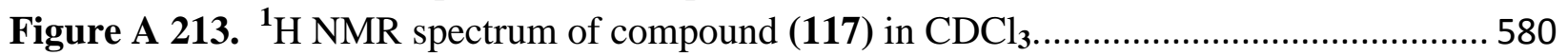

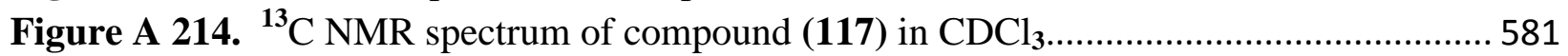

Figure A 215. COSY 2D NMR spectrum of compound (117) in $\mathrm{CDCl}_{3}$............................. 582

Figure A 216. HSQC 2D NMR spectrum of compound (117) in $\mathrm{CDCl}_{3}$........................... 583

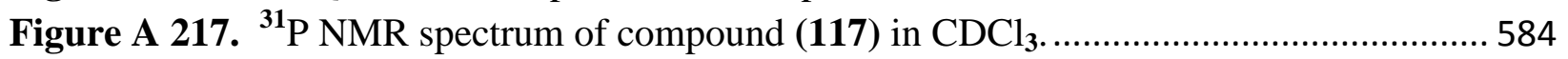

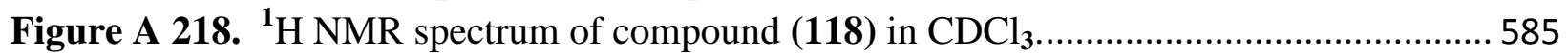

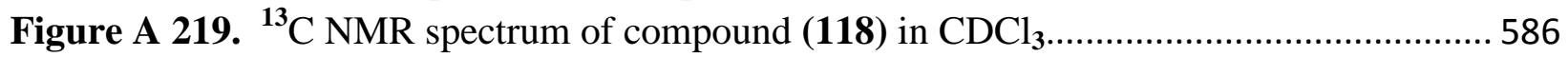

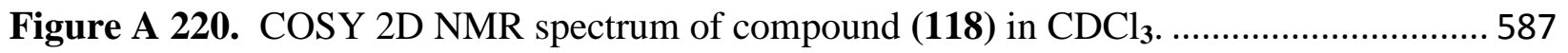

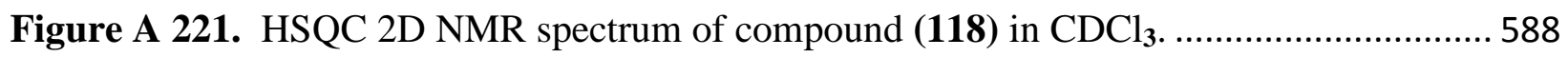

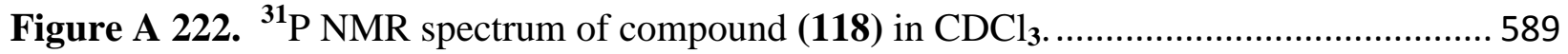

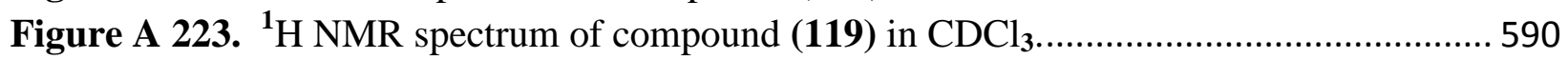

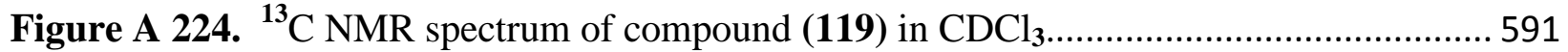

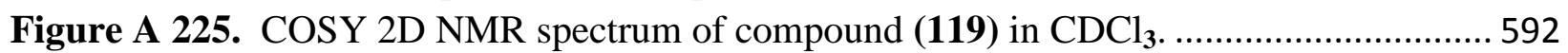

Figure A 226. HSQC 2D NMR spectrum of compound (119) in $\mathrm{CDCl}_{3}$............................ 593

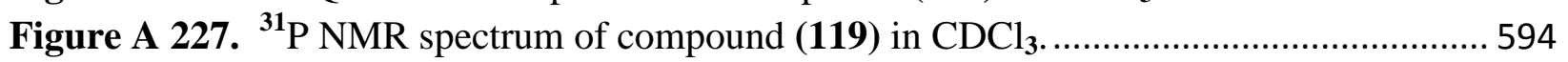

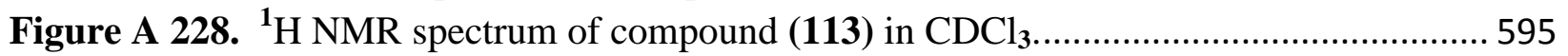

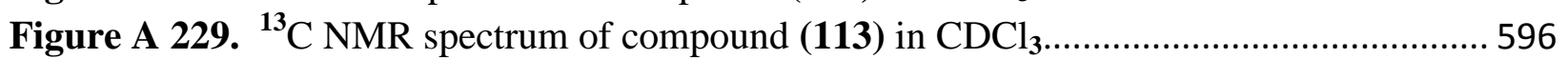

Figure A 230. COSY 2D NMR spectrum of compound (113) in $\mathrm{CDCl}_{3}$............................ 597

Figure A 231. HSQC 2D NMR spectrum of compound (113) in $\mathrm{CDCl}_{3}$............................. 598

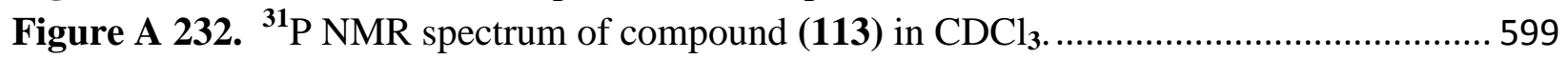

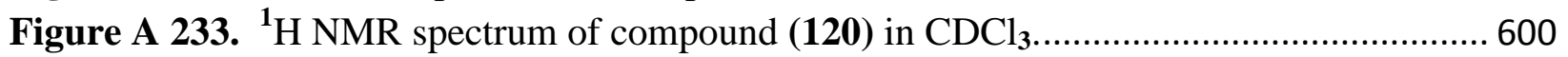

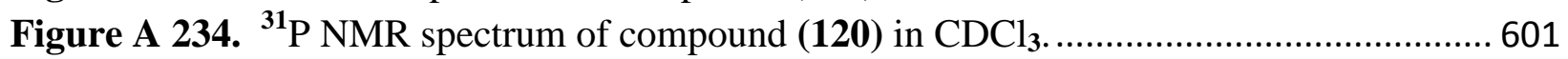

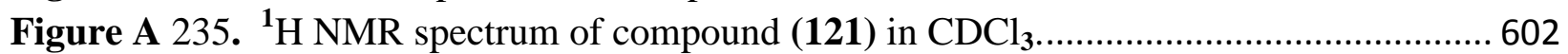

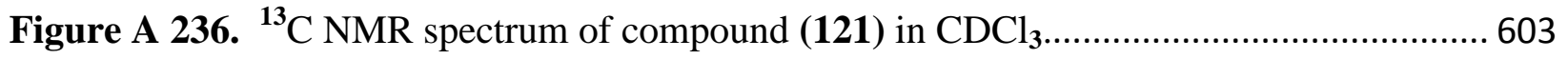

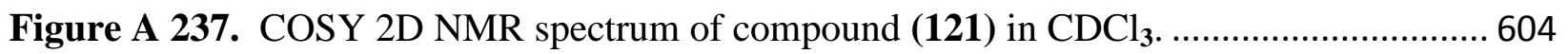

Figure A 238. HSQC 2D NMR spectrum of compound (121) in $\mathrm{CDCl}_{3}$.............................605

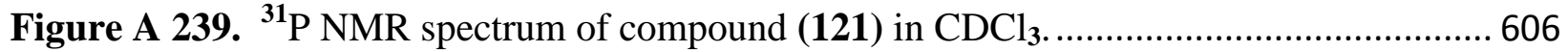

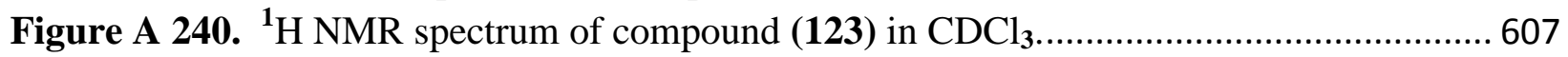

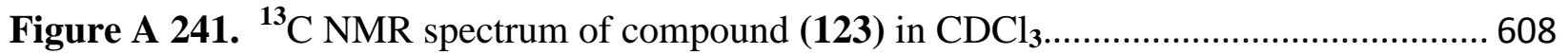

Figure A 242. HSQC 2D NMR spectrum of compound (123) in $\mathrm{CDCl}_{3}$........................... 609

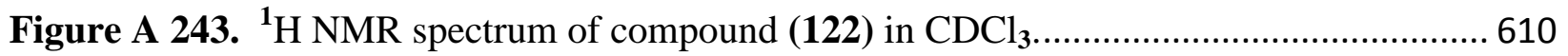

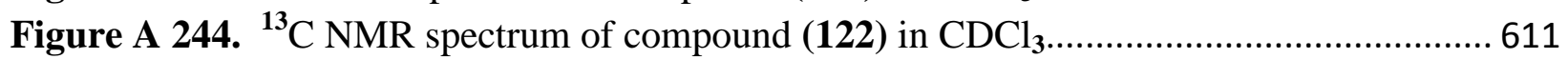

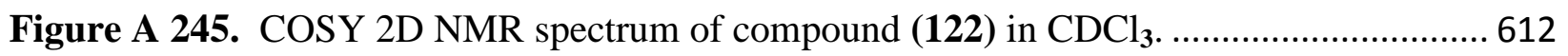

Figure A 246. HSQC 2D NMR spectrum of compound (122) in $\mathrm{CDCl}_{3}$........................... 613

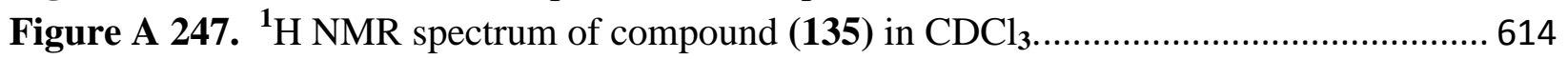

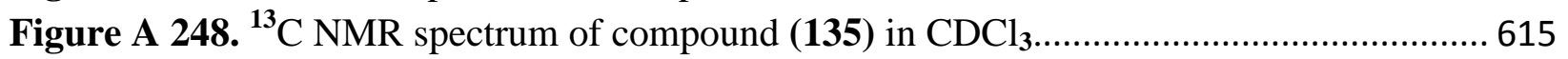

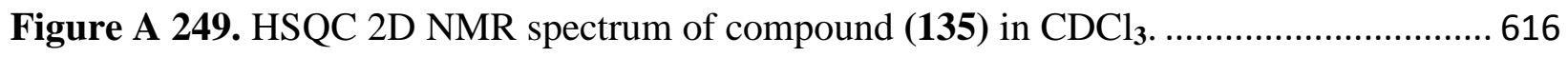




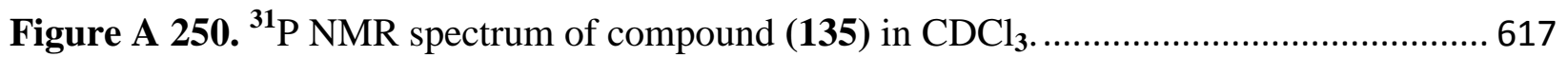

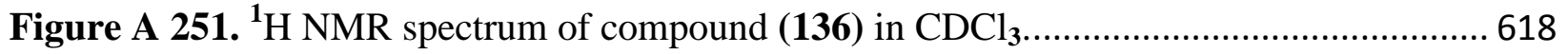

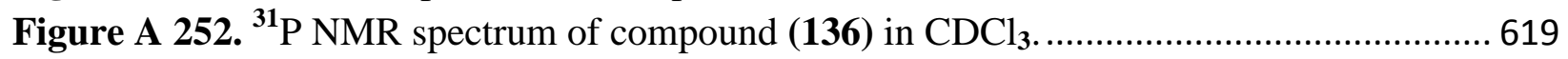

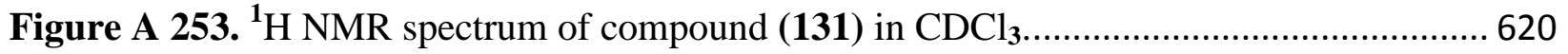

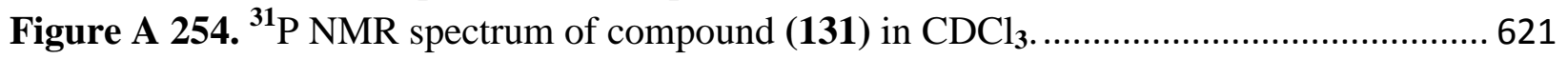

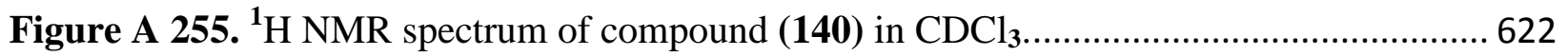

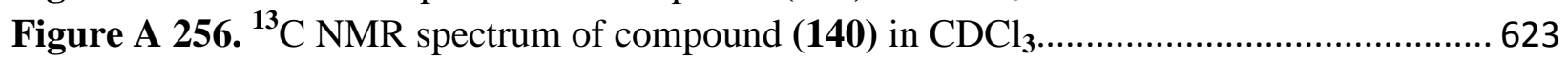

Figure A 257. COSY 2D NMR spectrum of compound (140) in $\mathrm{CDCl}_{3}$............................ 624

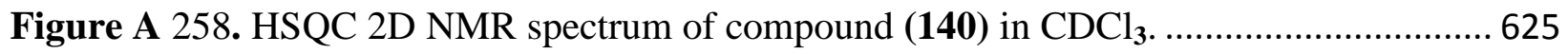

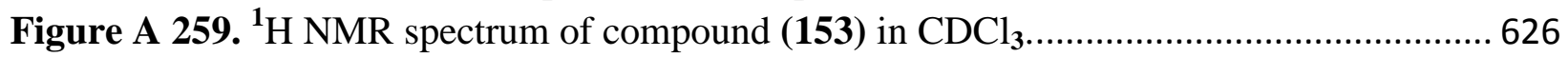

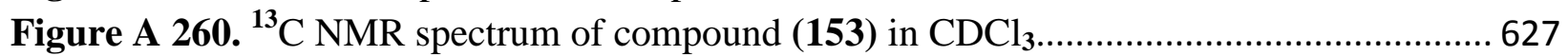

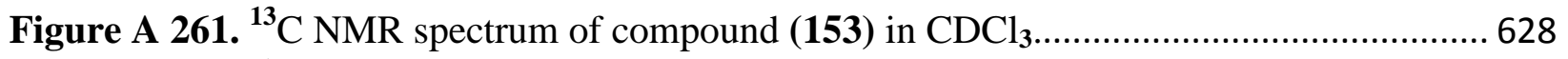

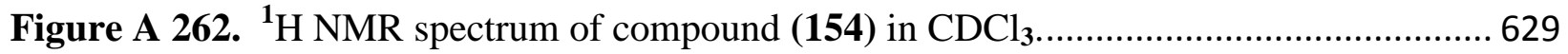

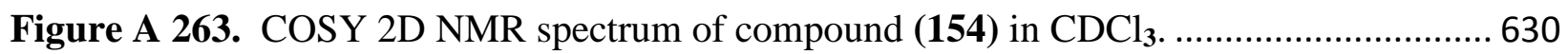

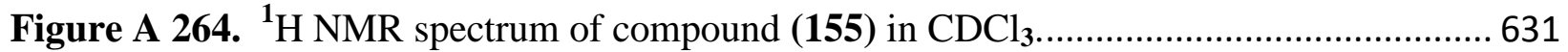

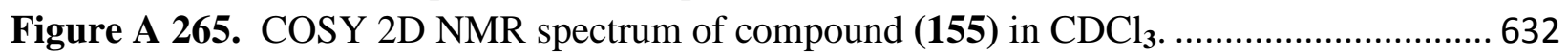

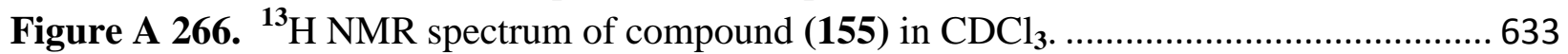

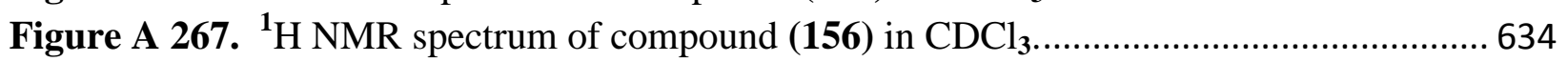

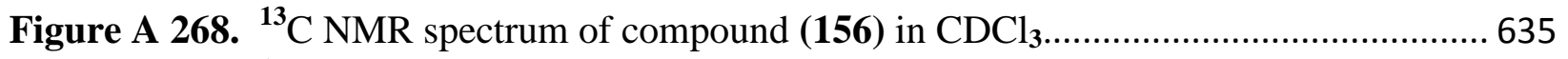

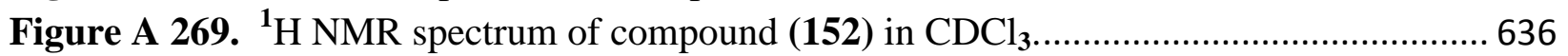

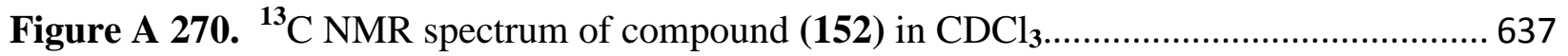

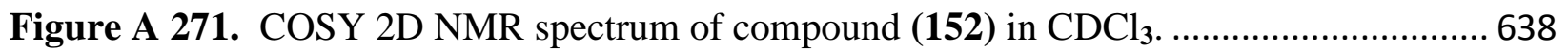

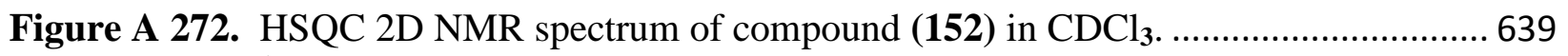

Figure A 273. ${ }^{1} \mathrm{H}$ NMR spectrum of compound (144) from (152) in $\mathrm{CDCl}_{3} \ldots \ldots \ldots \ldots \ldots \ldots . . . . . . . . . . .640$

Figure A 274. ${ }^{1} \mathrm{H}$ NMR spectrum of compound (144) from (156) in $\mathrm{CDCl}_{3}$...................... 641

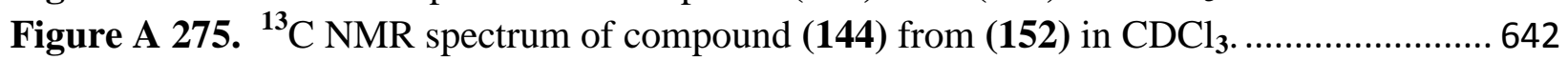

Figure A 276. COSY 2D NMR spectrum of compound (144) from (152) in $\mathrm{CDCl}_{3} \ldots \ldots \ldots \ldots . . . .643$

Figure A 277. HSQC 2D spectrum of compound (144) from (152) in $\mathrm{CDCl}_{3} \ldots \ldots \ldots \ldots \ldots \ldots \ldots . . . . . . . . . .644$

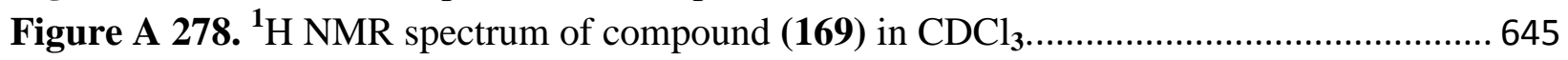

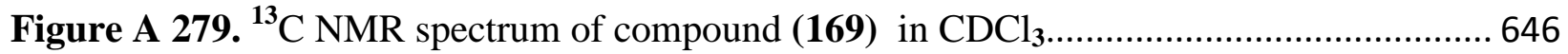

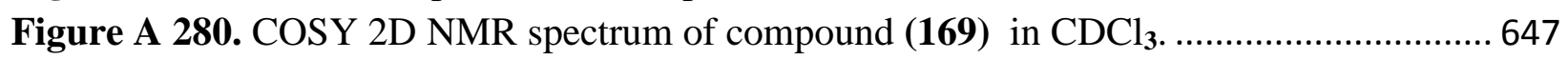

Figure A 281. HSQC 2D NMR spectrum of compound (169) in $\mathrm{CDCl}_{3} \ldots \ldots \ldots \ldots \ldots \ldots \ldots \ldots . . . . . . . . . . . . . . .648$

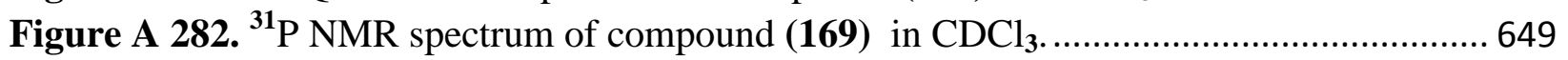

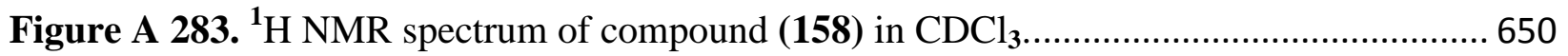

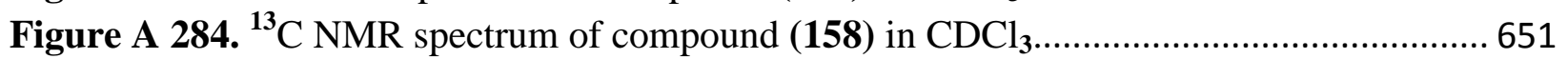

Figure A 285. COSY 2D NMR spectrum of compound (158) in $\mathrm{CDCl}_{3}$.............................. 652

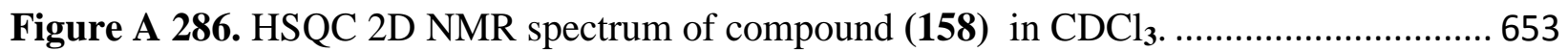

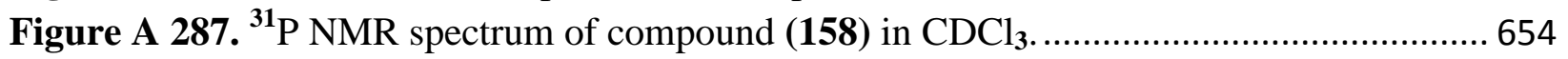

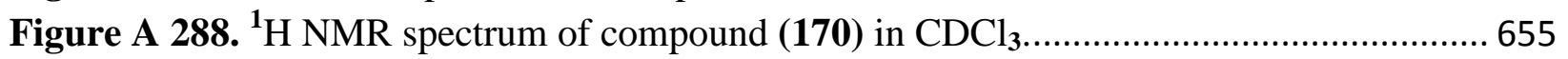

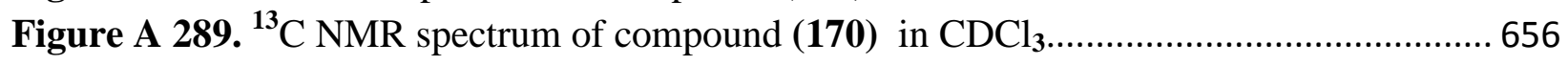


Figure A 290. COSY 2D NMR spectrum of compound (170) in $\mathrm{CDCl}_{3} \ldots \ldots \ldots \ldots \ldots \ldots \ldots \ldots \ldots . . . . . . . . . . . . . . . . .657$

Figure A 291. HSQC 2D NMR spectrum of compound (170) in $\mathrm{CDCl}_{3} \ldots \ldots \ldots \ldots \ldots \ldots \ldots \ldots \ldots . . . . . . . . . . . . . . .658$

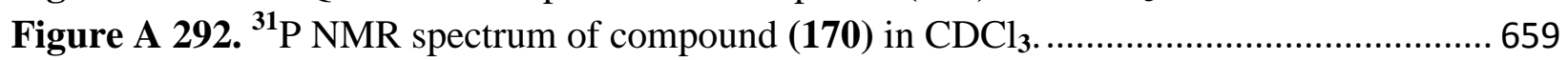

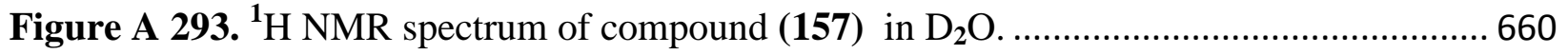

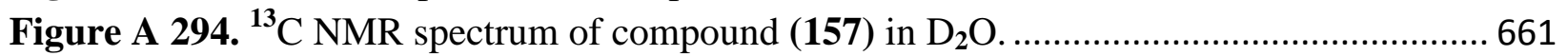

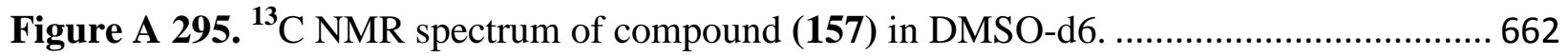

Figure A 296. HSQC 2D NMR spectrum of compound (157) in $\mathrm{CDCl}_{3} \ldots \ldots \ldots \ldots \ldots \ldots \ldots \ldots \ldots \ldots . . . . . . . . . . . . . . .663$

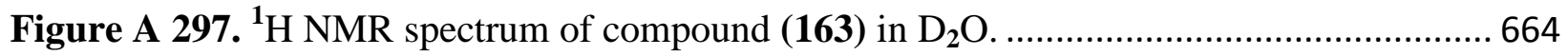

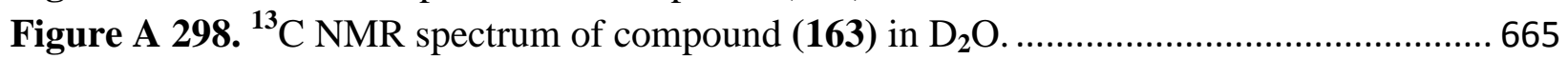

Figure A 299. COSY 2D NMR spectrum of compound (163) in $\mathrm{CDCl}_{3} \ldots \ldots \ldots \ldots \ldots \ldots \ldots \ldots \ldots . . . . . . . . . . . . . . . .666$

Figure A 300. HSQC 2D NMR spectrum of compound (163) in $\mathrm{CDCl}_{3} \ldots \ldots \ldots \ldots \ldots \ldots \ldots \ldots \ldots . . . . . . . . . . . . . . .667$

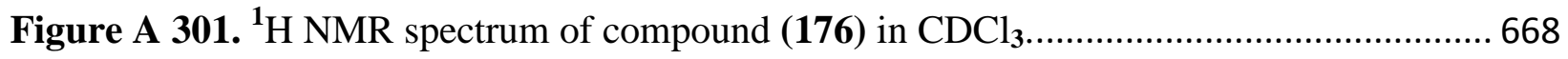

Figure A 302. COSY 2D NMR spectrum of compound (176) in $\mathrm{CDCl}_{3} \ldots \ldots \ldots \ldots \ldots \ldots \ldots \ldots \ldots \ldots . . . . . . . . . . . . . . . .669$

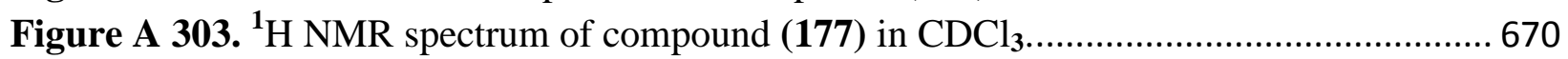

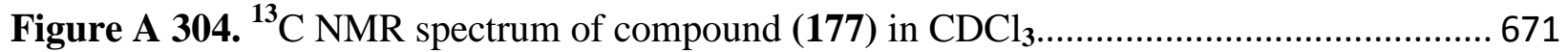

Figure A 305. COSY 2D NMR spectrum of compound (177) in $\mathrm{CDCl}_{3} \ldots \ldots \ldots \ldots \ldots \ldots \ldots \ldots \ldots \ldots . . . . . . . . . . . . . . . . .672$

Figure A 306. HSQC 2D NMR spectrum of compound (177) in $\mathrm{CDCl}_{3} \ldots \ldots \ldots \ldots \ldots \ldots \ldots \ldots \ldots . . . . . . . . . . . . . . .673$

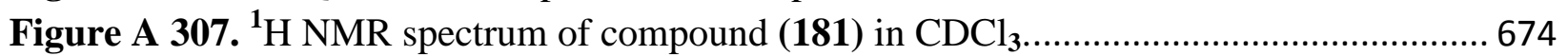

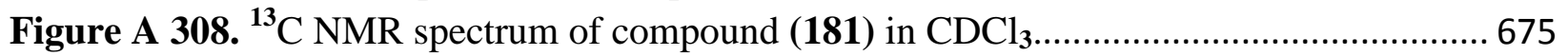

Figure A 309. COSY 2D NMR spectrum of compound (181) in $\mathrm{CDCl}_{3} \ldots \ldots \ldots \ldots \ldots \ldots \ldots \ldots \ldots \ldots . . . . . . . . . . . . . . .676$

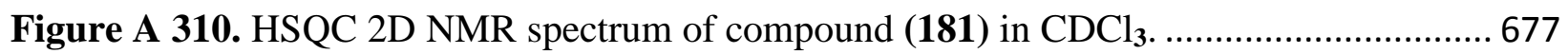

Figure A 311. ${ }^{1} \mathrm{H}$ NMR spectrum of compound (185) in $\mathrm{CDCl}_{3}$. (CRUDE) ............................ 678

Figure A 312. ${ }^{13} \mathrm{C}$ NMR spectrum of compound (185) in $\mathrm{CDCl}_{3}$.(CRUDE) .......................... 679

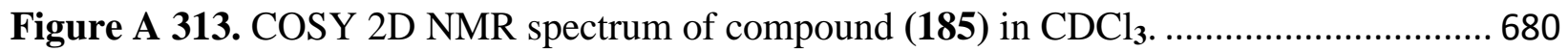

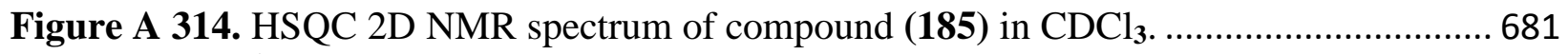

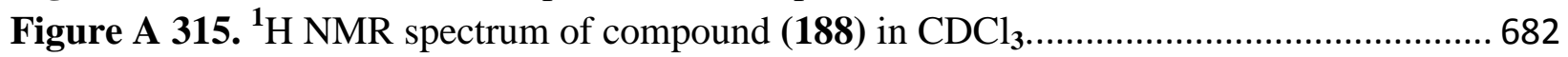

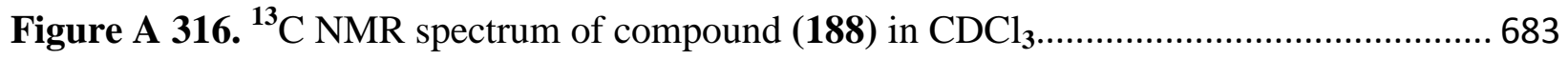

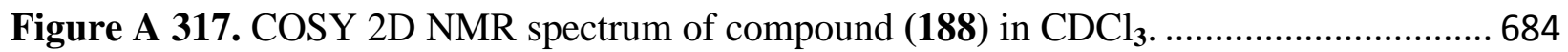

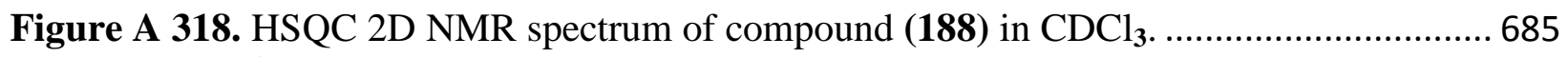

Figure A 319. ${ }^{1} \mathrm{H}$ NMR spectrum of compound (189) in $\mathrm{CDCL}_{3}$.(CRUDE) ............................. 686

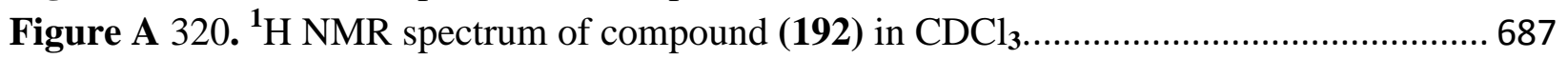

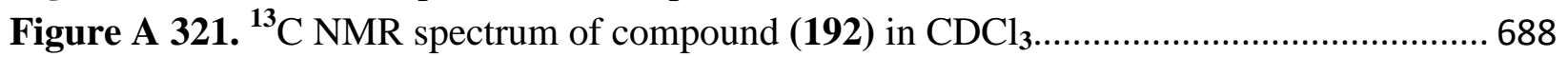

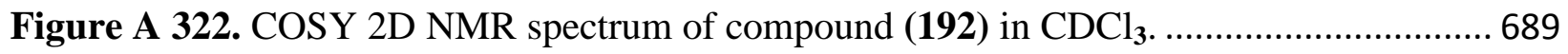

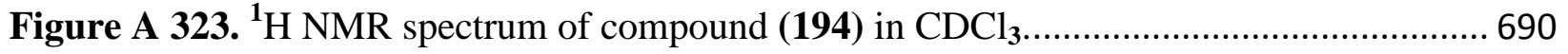

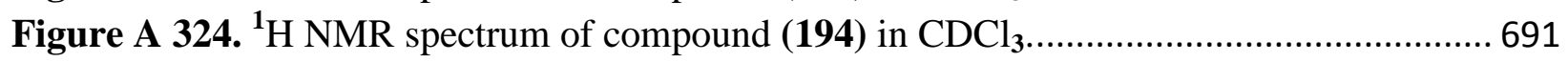

Figure A 325. COSY 2D NMR spectrum of compound (194) in $\mathrm{CDCl}_{3} \ldots \ldots \ldots \ldots \ldots \ldots \ldots \ldots \ldots \ldots . . . . . . . . . . . . . . . . . .692$

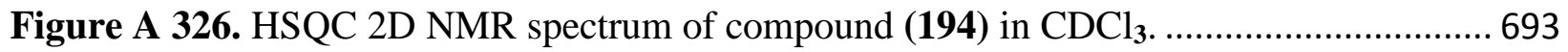

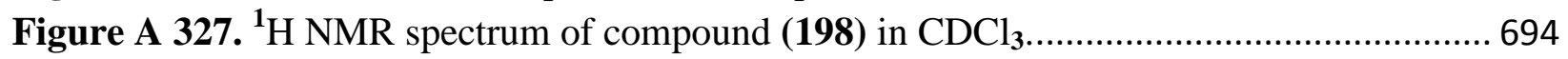

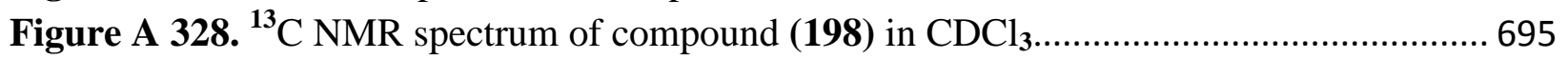

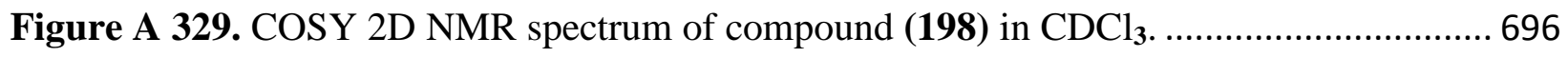




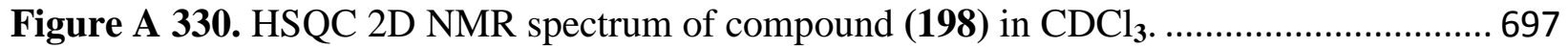

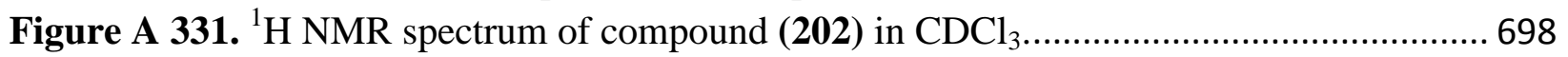

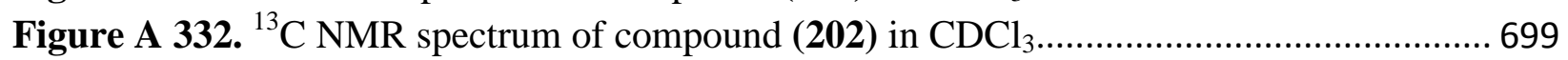

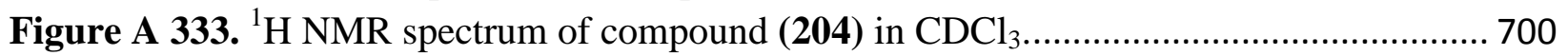

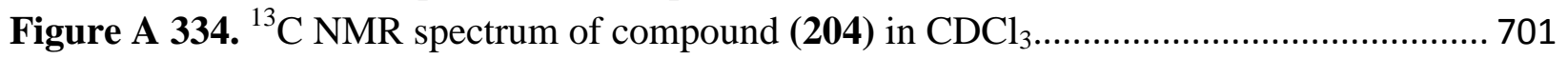

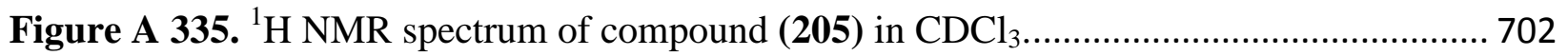

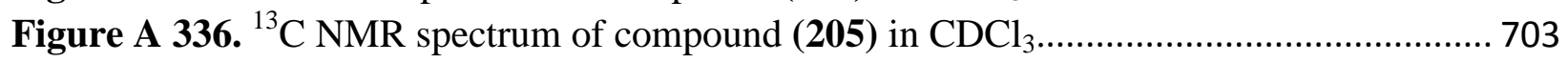

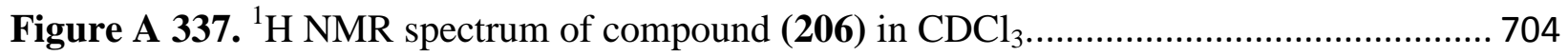

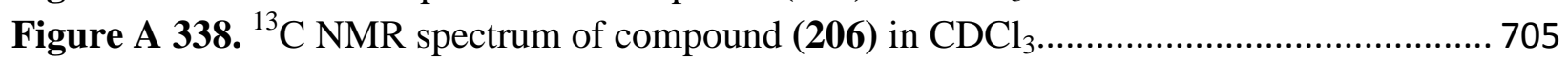

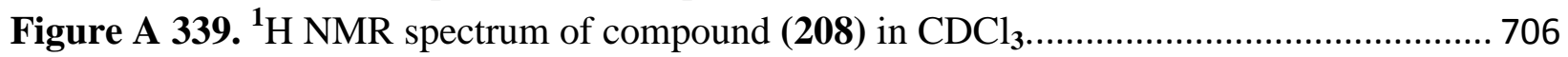

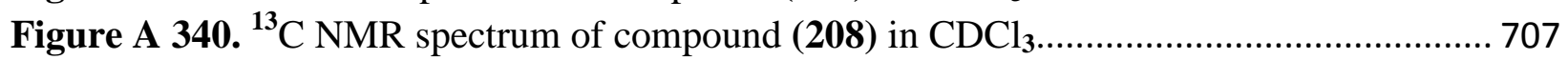

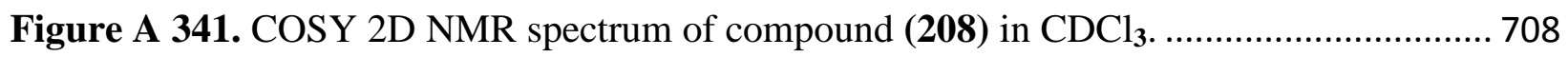

Figure A 342. HSQC 2D NMR spectrum of compound (208) in $\mathrm{CDCl}_{3}$............................ 709

Figure A 343. ${ }^{1} \mathrm{H}$ NMR spectrum of compound (209) in MeOD. .......................................... 710

Figure A 344. ${ }^{13} \mathrm{C}$ NMR spectrum of compound (209) in MeOD......................................... 711

Figure A 345. COSY 2D NMR spectrum of compound (209) in MeOD.............................. 712

Figure A 346. HSQC 2D NMR spectrum of compound (209) in MeOD.............................. 713

Figure A 347. ${ }^{1} \mathrm{H}$ NMR spectrum of compound (210) in MeOD .......................................... 714

Figure A 348. COSY 2D NMR spectrum of compound (210) in MeOD............................. 715

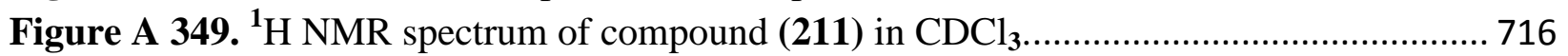

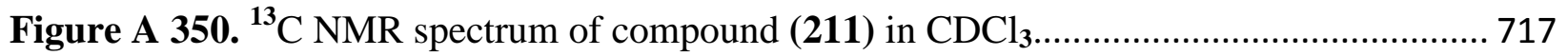

Figure A 351. COSY 2D NMR spectrum of compound (211) in $\mathrm{CDCl}_{3}$.............................. 718

Figure A 352. HSQC 2D NMR spectrum of compound (211) in $\mathrm{CDCl}_{3}$............................ 719

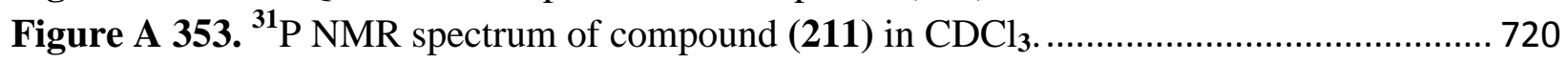

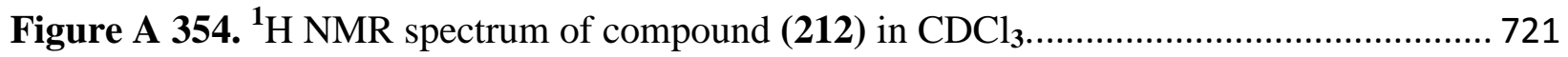

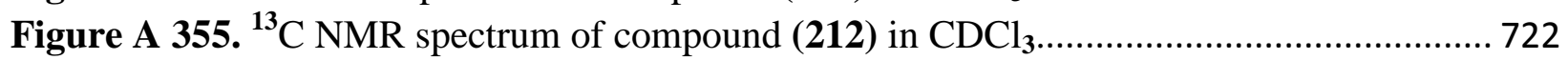

Figure A 356. HSQC 2D NMR spectrum of compound (212) in $\mathrm{CDCl}_{3}$.......................... 723

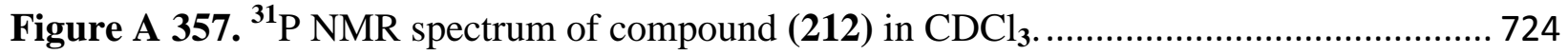

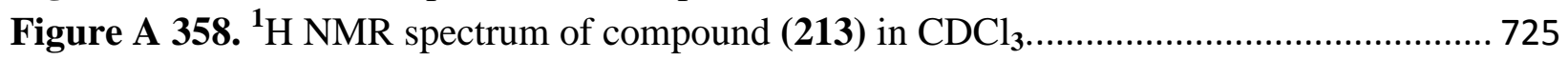

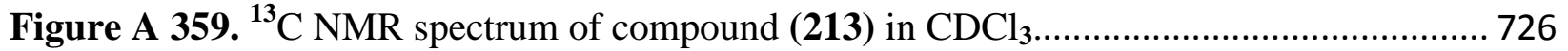

Figure A 360. COSY 2D NMR spectrum of compound (213) in $\mathrm{CDCl}_{3}$............................ 727

Figure A 361. HSQC 2D NMR spectrum of compound (213) in $\mathrm{CDCl}_{3}$............................ 728

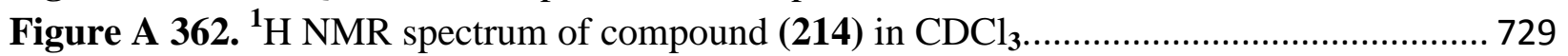

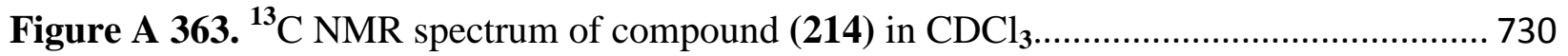

Figure A 364. COSY 2D NMR spectrum of compound (214) in $\mathrm{CDCl}_{3}$............................ 731

Figure A 365. HSQC 2D NMR spectrum of compound (214) in $\mathrm{CDCl}_{3}$.............................. 732

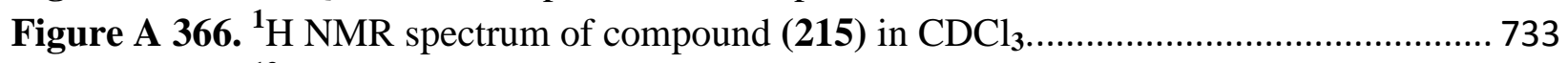

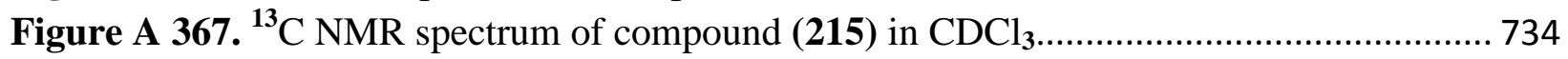

Figure A 368. COSY 2D NMR spectrum of compound (215) in $\mathrm{CDCl}_{3}$........................... 735

Figure A 369. HSQC 2D NMR spectrum of compound (215) in $\mathrm{CDCl}_{3}$........................... 736 


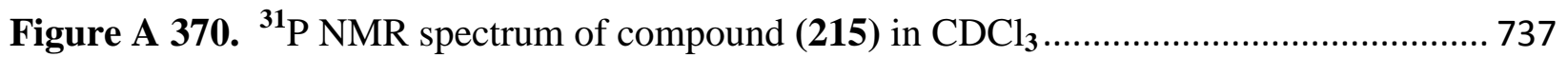

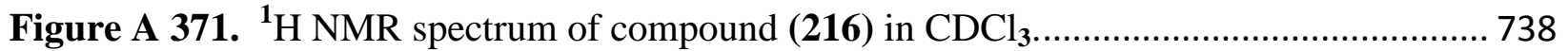

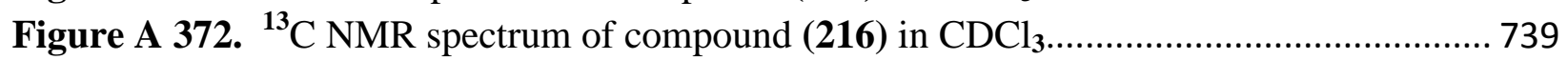

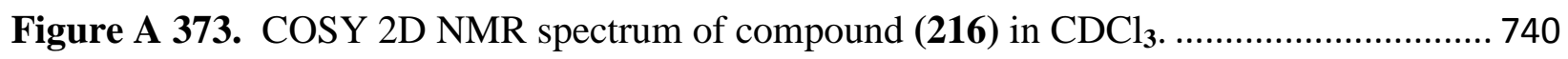

Figure A 374. HSQC 2D NMR spectrum of compound (216) in $\mathrm{CDCl}_{3}$............................. 741

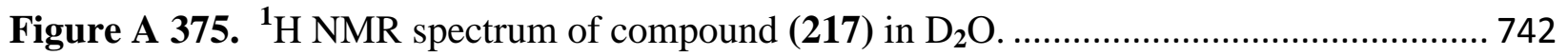

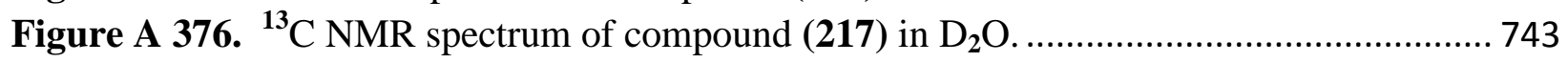

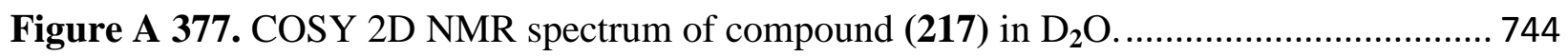

Figure A 378. HSQC 2D NMR spectrum of compound (217) in $\mathrm{D}_{2} \mathrm{O}$................................ 745

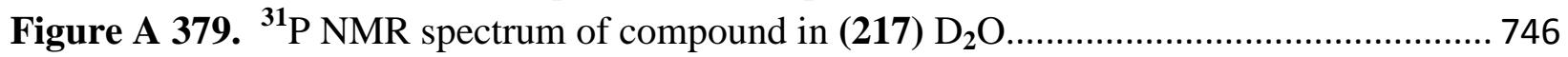

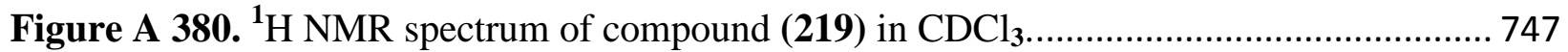

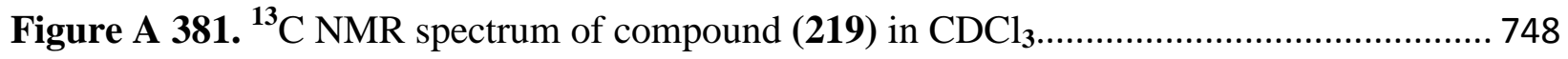

Figure A 382. COSY 2D NMR spectrum of compound (219) in $\mathrm{CDCl}_{3}$............................ 749

Figure A 383. ${ }^{1} \mathrm{H}$ NMR spectrum of compound (220) in DMSO-d6. ..................................... 750

Figure A 384. ${ }^{13} \mathrm{C}$ NMR spectrum of compound (220) in DMSO-d6................................... 751

Figure A 385. HSQC 2D NMR spectrum of compound (220) in DMSO-d6........................ 752

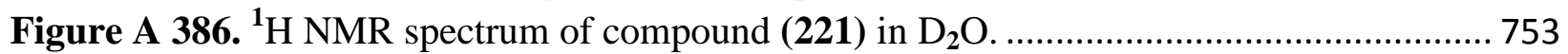

Figure A 387. ${ }^{13} \mathrm{C}$ NMR spectrum of compound (221) in $\mathrm{D}_{2} \mathrm{O}$............................................ 754

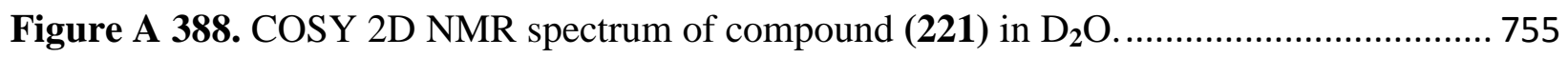

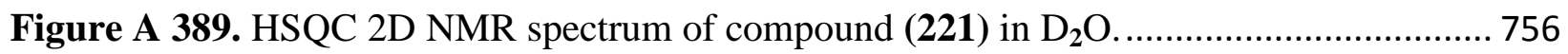

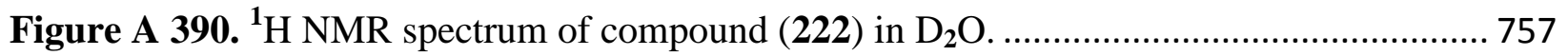

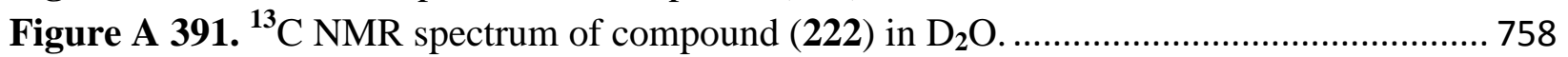

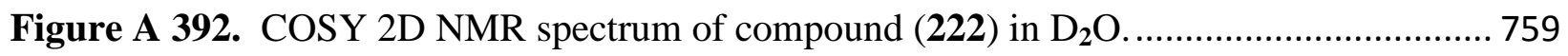

Figure A 393. HSQC 2D NMR spectrum of compound (222) in $\mathrm{D}_{2} \mathrm{O}$............................... 760

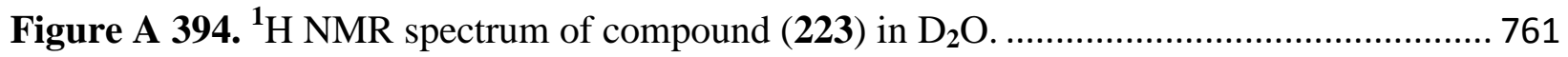

Figure A 395. ${ }^{13} \mathrm{C}$ NMR spectrum of compound (223) in $\mathrm{D}_{2} \mathrm{O}$.......................................... 762

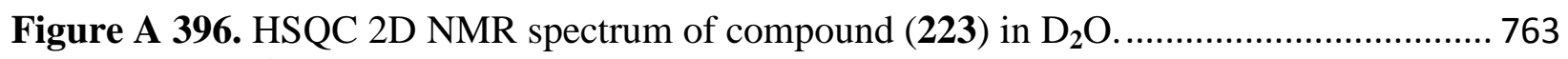

Figure A 397. ${ }^{1} \mathrm{H}$ NMR spectrum of compound (225) in DMSO-d6. ................................... 764

Figure A 398. ${ }^{13} \mathrm{C}$ NMR spectrum of compound (225) in DMSO-d6................................... 765

Figure A 399. ESI-TOF spectrum of compound 19.......................................................... 766

Figure A 400. ESI-TOF spectrum of compound 26....................................................... 767

Figure A 401. HRMS ESI-TOF spectrum of compound 27. ........................................... 768

Figure A 402. ESI-TOF spectrum of compound 34........................................................... 769

Figure A 403. HRMS ESI-TOF spectrum of compound 36.......................................... 770

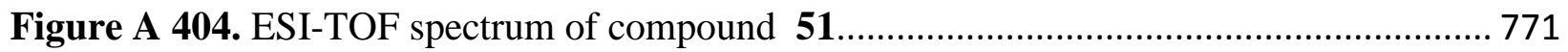

Figure A 405. ESI-TOF spectrum of compound 66.......................................................... 772

Figure A 406 .DART-HRMS spectrum of compound 113............................................. 773

Figure A 407. ESI-TOF spectrum of compound 121.................................................. 774

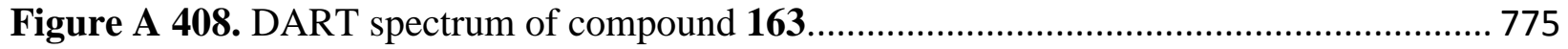

Figure A 409. HRMS-DART spectrum of compound 163.......................................... 776 
Figure A 410. ESI-TOF spectrum of compound 194.................................................... 777

Figure A 411. ESI-TOF spectrum of compound 205................................................. 778

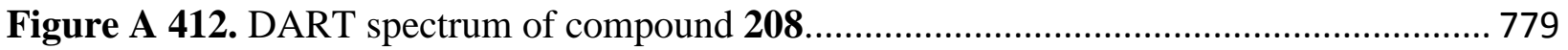

Figure A 413. HRMS-DART spectrum of compound 208........................................... 780

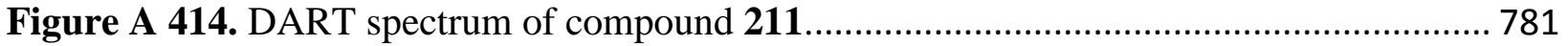

Figure A 415. HRMS-DART spectrum of compound 211 ............................................... 782

Figure A 416. ESI-TOF spectrum of compound 212 ...................................................... 783

Figure A 417. HRMS-DART spectrum of compound 213................................................ 784

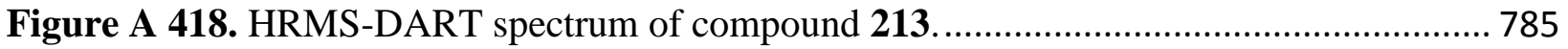

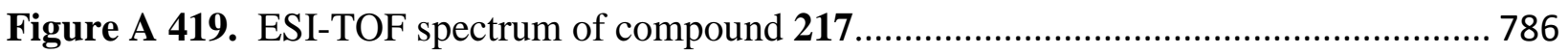

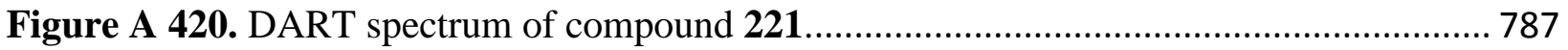

Figure A 421. ESI-TOF spectrum of compound 222 ................................................... 788

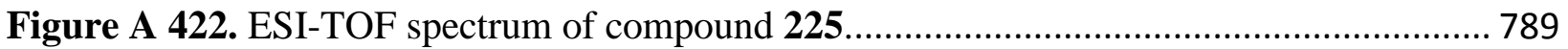




\subsection{Appendix 1.1 - X-Ray Data}

Table A 1. Crystal data and structure refinement for 34.

Identification code

Empirical formula

Formula weight

Temperature

Wavelength

Crystal system

Space group

Unit cell dimensions

Volume

$\mathrm{Z}$

Density (calculated)

Absorption coefficient

$\mathrm{F}(000)$

Crystal size

Theta range for data collection

Index ranges

Reflections collected

Independent reflections

Completeness to theta $=25.00^{\circ}$

Absorption correction

Max. and min. transmission

Refinement method

Data / restraints / parameters

Goodness-of-fit on $\mathrm{F}^{2}$

Final R indices [I $>2$ sigma(I)]

$\mathrm{R}$ indices (all data)

Largest diff. peak and hole k1180

$\mathrm{C}_{23} \mathrm{H}_{51} \mathrm{Br} \mathrm{N} \mathrm{O}_{3} \mathrm{P}$

500.53

150(1) K

$0.71073 \AA$

Triclinic

P -1

$\mathrm{a}=6.5523(5) \AA \quad \alpha=87.242(4)^{\circ}$.

$\mathrm{b}=7.3545(4) \AA \quad \beta=89.564(3)^{\circ}$.

$\mathrm{c}=28.873(2) \AA \quad \gamma=77.409(4)^{\circ}$.

1356.32(16) $\AA^{3}$

2

$1.226 \mathrm{Mg} / \mathrm{m}^{3}$

$1.596 \mathrm{~mm}^{-1}$

540

$0.50 \times 0.50 \times 0.02 \mathrm{~mm}^{3}$

2.83 to $25.00^{\circ}$.

$-7<=\mathrm{h}<=7,-8<=\mathrm{k}<=8,-34<=\mathrm{l}<=34$

12148

4737 [R(int) $=0.1147]$

$99.1 \%$

Semi-empirical from equivalents

0.992 and 0.706

Full-matrix least-squares on $\mathrm{F}^{2}$

4737 / 0 / 265

1.079

$\mathrm{R} 1=0.0767, \mathrm{wR} 2=0.1501$

$\mathrm{R} 1=0.1429, \mathrm{wR} 2=0.1819$

0.500 and -0.610 e. $\AA^{-3}$ 
Table A 2. Atomic coordinates ( $\mathrm{x} 10^{4}$ ) and equivalent isotropic displacement parameters $\left(\AA^{2} \mathrm{x}\right.$ $10^{3}$ ) for 34.

\begin{tabular}{|c|c|c|c|c|}
\hline & $\mathrm{x}$ & $\mathrm{y}$ & $\mathrm{z}$ & $\mathrm{U}(\mathrm{eq})$ \\
\hline $\operatorname{Br}(1)$ & 6398(1) & 3573(1) & 6468(1) & $56(1)$ \\
\hline $\mathrm{P}(1)$ & 6397(3) & $7024(2)$ & $4707(1)$ & $40(1)$ \\
\hline $\mathrm{O}(1)$ & 7347(7) & 4952(6) & $4696(2)$ & $44(1)$ \\
\hline $\mathrm{O}(2)$ & 4221(7) & 7436(6) & $4912(2)$ & $45(1)$ \\
\hline $\mathrm{O}(3)$ & 6408(7) & 7948(6) & $4210(2)$ & $49(1)$ \\
\hline $\mathrm{N}(1)$ & 9810(8) & $8204(7)$ & $6300(2)$ & $37(1)$ \\
\hline $\mathrm{C}(1)$ & $8069(10)$ & 8120(8) & $5022(2)$ & $38(2)$ \\
\hline $\mathrm{C}(2)$ & $8050(11)$ & 7605(9) & $5546(2)$ & $39(2)$ \\
\hline $\mathrm{C}(3)$ & $9370(10)$ & 8708(8) & 5787(2) & $38(2)$ \\
\hline $\mathrm{C}(4)$ & 7742(10) & 8344(9) & 6553(2) & $36(2)$ \\
\hline $\mathrm{C}(5)$ & 7936(10) & 8286(9) & 7081(2) & $40(2)$ \\
\hline $\mathrm{C}(6)$ & $5862(11)$ & 8014(9) & 7289(2) & $42(2)$ \\
\hline $\mathrm{C}(7)$ & $5683(11)$ & 8235(9) & 7807(2) & $42(2)$ \\
\hline $\mathrm{C}(8)$ & 3732(11) & 7685(9) & 8010(2) & $42(2)$ \\
\hline C(9) & $3324(11)$ & 8125(9) & $8512(2)$ & $44(2)$ \\
\hline$C(10)$ & $1422(11)$ & 7501(9) & 8712(2) & $44(2)$ \\
\hline C(11) & $907(11)$ & 8011(9) & $9210(2)$ & $43(2)$ \\
\hline$C(12)$ & $-985(11)$ & 7345(9) & $9403(2)$ & $44(2)$ \\
\hline $\mathrm{C}(13)$ & $-1505(11)$ & 7846(9) & 9896(2) & $42(2)$ \\
\hline$C(14)$ & $-3389(11)$ & $7170(10)$ & 10092(2) & $45(2)$ \\
\hline$C(15)$ & $-3944(11)$ & 7691(9) & 10584(2) & $44(2)$ \\
\hline$C(16)$ & $-5802(11)$ & 6978(10) & 10779(2) & $44(2)$ \\
\hline$C(17)$ & $-6376(11)$ & 7491(9) & 11272(2) & $43(2)$ \\
\hline C(18) & $-8234(11)$ & 6791(10) & 11463(2) & $44(2)$ \\
\hline C(19) & $-8853(11)$ & 7298(9) & 11952(2) & $43(2)$ \\
\hline$C(20)$ & $-10742(11)$ & $6602(10)$ & $12125(2)$ & $45(2)$ \\
\hline$C(21)$ & $-11409(12)$ & $7187(10)$ & $12608(2)$ & $52(2)$ \\
\hline$C(22)$ & $11117(11)$ & 6252(9) & 6355(2) & $46(2)$ \\
\hline$C(23)$ & 11004(10) & 9534(9) & 6473(3) & $48(2)$ \\
\hline
\end{tabular}


Table A 3. Bond lengths $[\AA]$ and angles $\left[{ }^{\circ}\right]$ for 34 .

\begin{tabular}{ll}
\hline $\mathrm{P}(1)-\mathrm{O}(2)$ & $1.514(5)$ \\
$\mathrm{P}(1)-\mathrm{O}(1)$ & $1.517(4)$ \\
$\mathrm{P}(1)-\mathrm{O}(3)$ & $1.559(5)$ \\
$\mathrm{P}(1)-\mathrm{C}(1)$ & $1.773(7)$ \\
$\mathrm{O}(1)-\mathrm{H}(1 \mathrm{O})$ & 0.8400 \\
$\mathrm{O}(3)-\mathrm{H}(3 \mathrm{O})$ & 0.8402 \\
$\mathrm{~N}(1)-\mathrm{C}(23)$ & $1.486(8)$ \\
$\mathrm{N}(1)-\mathrm{C}(22)$ & $1.505(7)$ \\
$\mathrm{N}(1)-\mathrm{C}(4)$ & $1.522(8)$ \\
$\mathrm{N}(1)-\mathrm{C}(3)$ & $1.523(8)$ \\
$\mathrm{C}(1)-\mathrm{C}(2)$ & $1.544(8)$ \\
$\mathrm{C}(1)-\mathrm{H}(1 \mathrm{~A})$ & 0.9900 \\
$\mathrm{C}(1)-\mathrm{H}(1 \mathrm{~B})$ & 0.9900 \\
$\mathrm{C}(2)-\mathrm{C}(3)$ & $1.503(9)$ \\
$\mathrm{C}(2)-\mathrm{H}(2 \mathrm{~A})$ & 0.9900 \\
$\mathrm{C}(2)-\mathrm{H}(2 \mathrm{~B})$ & 0.9900 \\
$\mathrm{C}(3)-\mathrm{H}(3 \mathrm{~A})$ & 0.9900 \\
$\mathrm{C}(3)-\mathrm{H}(3 \mathrm{~B})$ & 0.9900 \\
$\mathrm{C}(4)-\mathrm{C}(5)$ & $1.528(9)$ \\
$\mathrm{C}(4)-\mathrm{H}(4 \mathrm{~A})$ & 0.9900 \\
$\mathrm{C}(4)-\mathrm{H}(4 \mathrm{~B})$ & 0.9900 \\
$\mathrm{C}(5)-\mathrm{C}(6)$ & $1.530(9)$ \\
$\mathrm{C}(5)-\mathrm{H}(5 \mathrm{~A})$ & 0.9900 \\
$\mathrm{C}(5)-\mathrm{H}(5 \mathrm{~B})$ & 0.9900 \\
$\mathrm{C}(6)-\mathrm{C}(7)$ & $1.513(9)$ \\
$\mathrm{C}(6)-\mathrm{H}(6 \mathrm{~A})$ & 0.9900 \\
$\mathrm{C}(6)-\mathrm{H}(6 \mathrm{~B})$ & 0.9900 \\
$\mathrm{C}(7)-\mathrm{C}(8)$ & $1.527(9)$ \\
$\mathrm{C}(7)-\mathrm{H}(7 \mathrm{~A})$ & 0.9900 \\
$\mathrm{C}(7)-\mathrm{H}(7 \mathrm{~B})$ & 0.9900 \\
$\mathrm{C}(8)-\mathrm{C}(9)$ & $1.511(9)$ \\
$\mathrm{C}(8)-\mathrm{H}(8 \mathrm{~A})$ & 0.9900 \\
$\mathrm{C}(8)-\mathrm{H}(8 \mathrm{~B})$ & 0.9900 \\
322 &
\end{tabular}




\begin{tabular}{|c|c|}
\hline C(9)-C(10) & $1.520(9)$ \\
\hline $\mathrm{C}(9)-\mathrm{H}(9 \mathrm{~A})$ & 0.9900 \\
\hline C(9)-H(9B) & 0.9900 \\
\hline$C(10)-C(11)$ & $1.520(9)$ \\
\hline $\mathrm{C}(10)-\mathrm{H}(10 \mathrm{~A})$ & 0.9900 \\
\hline $\mathrm{C}(10)-\mathrm{H}(10 \mathrm{~B})$ & 0.9900 \\
\hline $\mathrm{C}(11)-\mathrm{C}(12)$ & $1.522(9)$ \\
\hline $\mathrm{C}(11)-\mathrm{H}(11 \mathrm{~A})$ & 0.9900 \\
\hline $\mathrm{C}(11)-\mathrm{H}(11 \mathrm{~B})$ & 0.9900 \\
\hline $\mathrm{C}(12)-\mathrm{C}(13)$ & $1.504(9)$ \\
\hline $\mathrm{C}(12)-\mathrm{H}(12 \mathrm{~A})$ & 0.9900 \\
\hline $\mathrm{C}(12)-\mathrm{H}(12 \mathrm{~B})$ & 0.9900 \\
\hline$C(13)-C(14)$ & $1.523(9)$ \\
\hline $\mathrm{C}(13)-\mathrm{H}(13 \mathrm{~A})$ & 0.9900 \\
\hline $\mathrm{C}(13)-\mathrm{H}(13 \mathrm{~B})$ & 0.9900 \\
\hline$C(14)-C(15)$ & $1.512(9)$ \\
\hline $\mathrm{C}(14)-\mathrm{H}(14 \mathrm{~A})$ & 0.9900 \\
\hline $\mathrm{C}(14)-\mathrm{H}(14 \mathrm{~B})$ & 0.9900 \\
\hline$C(15)-C(16)$ & $1.522(9)$ \\
\hline $\mathrm{C}(15)-\mathrm{H}(15 \mathrm{~A})$ & 0.9900 \\
\hline $\mathrm{C}(15)-\mathrm{H}(15 \mathrm{~B})$ & 0.9900 \\
\hline $\mathrm{C}(16)-\mathrm{C}(17)$ & $1.513(9)$ \\
\hline $\mathrm{C}(16)-\mathrm{H}(16 \mathrm{~A})$ & 0.9900 \\
\hline $\mathrm{C}(16)-\mathrm{H}(16 \mathrm{~B})$ & 0.9900 \\
\hline $\mathrm{C}(17)-\mathrm{C}(18)$ & $1.512(9)$ \\
\hline $\mathrm{C}(17)-\mathrm{H}(17 \mathrm{~A})$ & 0.9900 \\
\hline $\mathrm{C}(17)-\mathrm{H}(17 \mathrm{~B})$ & 0.9900 \\
\hline $\mathrm{C}(18)-\mathrm{C}(19)$ & $1.507(9)$ \\
\hline $\mathrm{C}(18)-\mathrm{H}(18 \mathrm{~A})$ & 0.9900 \\
\hline $\mathrm{C}(18)-\mathrm{H}(18 \mathrm{~B})$ & 0.9900 \\
\hline C(19)-C(20) & $1.511(9)$ \\
\hline $\mathrm{C}(19)-\mathrm{H}(19 \mathrm{~A})$ & 0.9900 \\
\hline C(19)-H(19B) & 0.9900 \\
\hline $\mathrm{C}(20)-\mathrm{C}(21)$ & $1.515(9)$ \\
\hline $\mathrm{C}(20)-\mathrm{H}(20 \mathrm{~A})$ & 0.9900 \\
\hline
\end{tabular}




$\begin{array}{ll}\mathrm{C}(20)-\mathrm{H}(20 \mathrm{~B}) & 0.9900 \\ \mathrm{C}(21)-\mathrm{H}(21 \mathrm{~A}) & 0.9800 \\ \mathrm{C}(21)-\mathrm{H}(21 \mathrm{~B}) & 0.9800 \\ \mathrm{C}(21)-\mathrm{H}(21 \mathrm{C}) & 0.9800 \\ \mathrm{C}(22)-\mathrm{H}(22 \mathrm{~A}) & 0.9800 \\ \mathrm{C}(22)-\mathrm{H}(22 \mathrm{~B}) & 0.9800 \\ \mathrm{C}(22)-\mathrm{H}(22 \mathrm{C}) & 0.9800 \\ \mathrm{C}(23)-\mathrm{H}(23 \mathrm{~A}) & 0.9800 \\ \mathrm{C}(23)-\mathrm{H}(23 \mathrm{~B}) & 0.9800 \\ \mathrm{C}(23)-\mathrm{H}(23 \mathrm{C}) & 0.9800 \\ & \\ \mathrm{O}(2)-\mathrm{P}(1)-\mathrm{O}(1) & 112.9(3) \\ \mathrm{O}(2)-\mathrm{P}(1)-\mathrm{O}(3) & 111.1(3) \\ \mathrm{O}(1)-\mathrm{P}(1)-\mathrm{O}(3) & 109.7(3) \\ \mathrm{O}(2)-\mathrm{P}(1)-\mathrm{C}(1) & 110.2(3) \\ \mathrm{O}(1)-\mathrm{P}(1)-\mathrm{C}(1) & 109.4(3) \\ \mathrm{O}(3)-\mathrm{P}(1)-\mathrm{C}(1) & 103.0(3) \\ \mathrm{P}(1)-\mathrm{O}(1)-\mathrm{H}(1 \mathrm{O}) & 121.5 \\ \mathrm{P}(1)-\mathrm{O}(3)-\mathrm{H}(3 \mathrm{O}) & 123.6 \\ \mathrm{C}(23)-\mathrm{N}(1)-\mathrm{C}(22) & 109.3(5) \\ \mathrm{C}(23)-\mathrm{N}(1)-\mathrm{C}(4) & 111.5(5) \\ \mathrm{C}(22)-\mathrm{N}(1)-\mathrm{C}(4) & 110.4(5) \\ \mathrm{C}(23)-\mathrm{N}(1)-\mathrm{C}(3) & 107.3(5) \\ \mathrm{C}(22)-\mathrm{N}(1)-\mathrm{C}(3) & 109.4(5) \\ \mathrm{C}(4)-\mathrm{N}(1)-\mathrm{C}(3) & 108.9(5) \\ \mathrm{C}(2)-\mathrm{C}(1)-\mathrm{P}(1) & 111.8(4) \\ \mathrm{C}(2)-\mathrm{C}(1)-\mathrm{H}(1 \mathrm{~A}) & 109.2 \\ \mathrm{P}(1)-\mathrm{C}(1)-\mathrm{H}(1 \mathrm{~A}) & 109.2 \\ \mathrm{C}(2)-\mathrm{C}(1)-\mathrm{H}(1 \mathrm{~B}) & 109.2 \\ \mathrm{P}(1)-\mathrm{C}(1)-\mathrm{H}(1 \mathrm{~B}) & 109.2 \\ \mathrm{H}(1 \mathrm{~A})-\mathrm{C}(1)-\mathrm{H}(1 \mathrm{~B}) & 107.9 \\ \mathrm{C}(3)-\mathrm{C}(2)-\mathrm{C}(1) & 108.0(5) \\ \mathrm{C}(3)-\mathrm{C}(2)-\mathrm{H}(2 \mathrm{~A}) & 110.1 \\ \mathrm{C}(1)-\mathrm{C}(2)-\mathrm{H}(2 \mathrm{~A}) & 110.1 \\ \mathrm{C}(3)-\mathrm{C}(2)-\mathrm{H}(2 \mathrm{~B}) & 110.1 \\ \text { 324 } & \\ & \end{array}$




$\begin{array}{ll}\mathrm{C}(1)-\mathrm{C}(2)-\mathrm{H}(2 \mathrm{~B}) & 110.1 \\ \mathrm{H}(2 \mathrm{~A})-\mathrm{C}(2)-\mathrm{H}(2 \mathrm{~B}) & 108.4 \\ \mathrm{C}(2)-\mathrm{C}(3)-\mathrm{N}(1) & 116.3(5) \\ \mathrm{C}(2)-\mathrm{C}(3)-\mathrm{H}(3 \mathrm{~A}) & 108.2 \\ \mathrm{~N}(1)-\mathrm{C}(3)-\mathrm{H}(3 \mathrm{~A}) & 108.2 \\ \mathrm{C}(2)-\mathrm{C}(3)-\mathrm{H}(3 \mathrm{~B}) & 108.2 \\ \mathrm{~N}(1)-\mathrm{C}(3)-\mathrm{H}(3 \mathrm{~B}) & 108.2 \\ \mathrm{H}(3 \mathrm{~A})-\mathrm{C}(3)-\mathrm{H}(3 \mathrm{~B}) & 107.4 \\ \mathrm{~N}(1)-\mathrm{C}(4)-\mathrm{C}(5) & 114.0(5) \\ \mathrm{N}(1)-\mathrm{C}(4)-\mathrm{H}(4 \mathrm{~A}) & 108.8 \\ \mathrm{C}(5)-\mathrm{C}(4)-\mathrm{H}(4 \mathrm{~A}) & 108.8 \\ \mathrm{~N}(1)-\mathrm{C}(4)-\mathrm{H}(4 \mathrm{~B}) & 108.8 \\ \mathrm{C}(5)-\mathrm{C}(4)-\mathrm{H}(4 \mathrm{~B}) & 108.8 \\ \mathrm{H}(4 \mathrm{~A})-\mathrm{C}(4)-\mathrm{H}(4 \mathrm{~B}) & 107.6 \\ \mathrm{C}(4)-\mathrm{C}(5)-\mathrm{C}(6) & 108.1(5) \\ \mathrm{C}(4)-\mathrm{C}(5)-\mathrm{H}(5 \mathrm{~A}) & 110.1 \\ \mathrm{C}(6)-\mathrm{C}(5)-\mathrm{H}(5 \mathrm{~A}) & 110.1 \\ \mathrm{C}(4)-\mathrm{C}(5)-\mathrm{H}(5 \mathrm{~B}) & 110.1 \\ \mathrm{C}(6)-\mathrm{C}(5)-\mathrm{H}(5 \mathrm{~B}) & 110.1 \\ \mathrm{H}(5 \mathrm{~A})-\mathrm{C}(5)-\mathrm{H}(5 \mathrm{~B}) & 108.4 \\ \mathrm{C}(7)-\mathrm{C}(6)-\mathrm{C}(5) & 114.4(6) \\ \mathrm{C}(7)-\mathrm{C}(6)-\mathrm{H}(6 \mathrm{~A}) & 108.7 \\ \mathrm{C}(5)-\mathrm{C}(6)-\mathrm{H}(6 \mathrm{~A}) & 108.7 \\ \mathrm{C}(7)-\mathrm{C}(6)-\mathrm{H}(6 \mathrm{~B}) & 108.7 \\ \mathrm{C}(5)-\mathrm{C}(6)-\mathrm{H}(6 \mathrm{~B}) & 108.7 \\ \mathrm{H}(6 \mathrm{~A})-\mathrm{C}(6)-\mathrm{H}(6 \mathrm{~B}) & 107.6 \\ \mathrm{C}(6)-\mathrm{C}(7)-\mathrm{C}(8) & 112.6(6) \\ \mathrm{C}(6)-\mathrm{C}(7)-\mathrm{H}(7 \mathrm{~A}) & 109.1 \\ \mathrm{C}(8)-\mathrm{C}(7)-\mathrm{H}(7 \mathrm{~A}) & 109.1 \\ \mathrm{C}(6)-\mathrm{C}(7)-\mathrm{H}(7 \mathrm{~B}) & 109.1 \\ \mathrm{C}(8)-\mathrm{C}(7)-\mathrm{H}(7 \mathrm{~B}) & 109.1 \\ \mathrm{H}(7 \mathrm{~A})-\mathrm{C}(7)-\mathrm{H}(7 \mathrm{~B}) & 107.8 \\ \mathrm{C}(9)-\mathrm{C}(8)-\mathrm{C}(7) & 114.6(6) \\ \mathrm{C}(9)-\mathrm{C}(8)-\mathrm{H}(8 \mathrm{~A}) & 108.6 \\ \mathrm{C}(7)-\mathrm{C}(8)-\mathrm{H}(8 \mathrm{~A}) & 108.6 \\ \text { 325 } & \\ & \end{array}$




$\begin{array}{ll}\mathrm{C}(9)-\mathrm{C}(8)-\mathrm{H}(8 \mathrm{~B}) & 108.6 \\ \mathrm{C}(7)-\mathrm{C}(8)-\mathrm{H}(8 \mathrm{~B}) & 108.6 \\ \mathrm{H}(8 \mathrm{~A})-\mathrm{C}(8)-\mathrm{H}(8 \mathrm{~B}) & 107.6 \\ \mathrm{C}(8)-\mathrm{C}(9)-\mathrm{C}(10) & 113.7(6) \\ \mathrm{C}(8)-\mathrm{C}(9)-\mathrm{H}(9 \mathrm{~A}) & 108.8 \\ \mathrm{C}(10)-\mathrm{C}(9)-\mathrm{H}(9 \mathrm{~A}) & 108.8 \\ \mathrm{C}(8)-\mathrm{C}(9)-\mathrm{H}(9 \mathrm{~B}) & 108.8 \\ \mathrm{C}(10)-\mathrm{C}(9)-\mathrm{H}(9 \mathrm{~B}) & 108.8 \\ \mathrm{H}(9 \mathrm{~A})-\mathrm{C}(9)-\mathrm{H}(9 \mathrm{~B}) & 107.7 \\ \mathrm{C}(9)-\mathrm{C}(10)-\mathrm{C}(11) & 114.9(6) \\ \mathrm{C}(9)-\mathrm{C}(10)-\mathrm{H}(10 \mathrm{~A}) & 108.5 \\ \mathrm{C}(11)-\mathrm{C}(10)-\mathrm{H}(10 \mathrm{~A}) & 108.5 \\ \mathrm{C}(9)-\mathrm{C}(10)-\mathrm{H}(10 \mathrm{~B}) & 108.5 \\ \mathrm{C}(11)-\mathrm{C}(10)-\mathrm{H}(10 \mathrm{~B}) & 108.5 \\ \mathrm{H}(10 \mathrm{~A})-\mathrm{C}(10)-\mathrm{H}(10 \mathrm{~B}) & 107.5 \\ \mathrm{C}(10)-\mathrm{C}(11)-\mathrm{C}(12) & 113.8(6) \\ \mathrm{C}(10)-\mathrm{C}(11)-\mathrm{H}(11 \mathrm{~A}) & 108.8 \\ \mathrm{C}(12)-\mathrm{C}(11)-\mathrm{H}(11 \mathrm{~A}) & 108.8 \\ \mathrm{C}(10)-\mathrm{C}(11)-\mathrm{H}(11 \mathrm{~B}) & 108.8 \\ \mathrm{C}(12)-\mathrm{C}(11)-\mathrm{H}(11 \mathrm{~B}) & 108.8 \\ \mathrm{H}(11 \mathrm{~A})-\mathrm{C}(11)-\mathrm{H}(11 \mathrm{~B}) & 107.7 \\ \mathrm{C}(13)-\mathrm{C}(12)-\mathrm{C}(11) & 114.1(6) \\ \mathrm{C}(13)-\mathrm{C}(12)-\mathrm{H}(12 \mathrm{~A}) & 108.7 \\ \mathrm{C}(11)-\mathrm{C}(12)-\mathrm{H}(12 \mathrm{~A}) & 108.7 \\ \mathrm{C}(13)-\mathrm{C}(12)-\mathrm{H}(12 \mathrm{~B}) & 108.7 \\ \mathrm{C}(11)-\mathrm{C}(12)-\mathrm{H}(12 \mathrm{~B}) & 108.7 \\ \mathrm{H}(12 \mathrm{~A})-\mathrm{C}(12)-\mathrm{H}(12 \mathrm{~B}) & 107.6 \\ \mathrm{C}(12)-\mathrm{C}(13)-\mathrm{C}(14) & 114.2(6) \\ \mathrm{C}(12)-\mathrm{C}(13)-\mathrm{H}(13 \mathrm{~A}) & 108.7 \\ \mathrm{C}(14)-\mathrm{C}(13)-\mathrm{H}(13 \mathrm{~A}) & 108.7 \\ \mathrm{C}(12)-\mathrm{C}(13)-\mathrm{H}(13 \mathrm{~B}) & 108.7 \\ \mathrm{C}(14)-\mathrm{C}(13)-\mathrm{H}(13 \mathrm{~B}) & 108.7 \\ \mathrm{H}(13 \mathrm{~A})-\mathrm{C}(13)-\mathrm{H}(13 \mathrm{~B}) & 107.6 \\ \mathrm{C}(15)-\mathrm{C}(14)-\mathrm{C}(13) & 114.6(6) \\ \mathrm{C}(15)-\mathrm{C}(14)-\mathrm{H}(14 \mathrm{~A}) & 108.6 \\ \text { 326} & \\ & \end{array}$




\begin{tabular}{|c|c|}
\hline$C(13)-C($ & 108.6 \\
\hline $\mathrm{C}(15)-\mathrm{C}(14)-\mathrm{H}(14 \mathrm{~B})$ & 108.6 \\
\hline $\mathrm{C}(13)-\mathrm{C}(14)-\mathrm{H}(14 \mathrm{~B})$ & 08.6 \\
\hline $\mathrm{H}(14 \mathrm{~A})-\mathrm{C}(14)-\mathrm{H}(14 \mathrm{~B})$ & 07.6 \\
\hline$C(14)-C(15)-C(16)$ & (b) \\
\hline $\mathrm{C}(14)-\mathrm{C}(15)-\mathrm{H}(15 \mathrm{~A})$ & 08.7 \\
\hline $\mathrm{C}(16)-\mathrm{C}(15)-\mathrm{H}(15 \mathrm{~A})$ & 108.7 \\
\hline $\mathrm{C}(14)-\mathrm{C}(15)-\mathrm{H}(15 \mathrm{~B})$ & 8.7 \\
\hline $\mathrm{C}(16)-\mathrm{C}(15)-\mathrm{H}(15 \mathrm{~B})$ & 108.7 \\
\hline H(15A)-C(15)-H(15B) & 107.6 \\
\hline$C(17)-C(16)-C(15)$ & $114.8(6)$ \\
\hline $\mathrm{C}(17)-\mathrm{C}(16)-\mathrm{H}(16 \mathrm{~A})$ & 108.6 \\
\hline $\mathrm{C}(15)-\mathrm{C}(16)-\mathrm{H}(16 \mathrm{~A})$ & 108.6 \\
\hline $\mathrm{C}(17)-\mathrm{C}(16)-\mathrm{H}(16 \mathrm{~B})$ & 108.6 \\
\hline $\mathrm{C}(15)-\mathrm{C}(16)-\mathrm{H}(16 \mathrm{~B})$ & 108.6 \\
\hline $\mathrm{H}(16 \mathrm{~A})-\mathrm{C}(16)-\mathrm{H}(16 \mathrm{~B})$ & 107.5 \\
\hline$C(18)-C(17)-C(16)$ & $114.5(6)$ \\
\hline $\mathrm{C}(18)-\mathrm{C}(17)-\mathrm{H}(17 \mathrm{~A})$ & 108.6 \\
\hline $\mathrm{C}(16)-\mathrm{C}(17)-\mathrm{H}(17 \mathrm{~A})$ & 108.6 \\
\hline $\mathrm{C}(18)-\mathrm{C}(17)-\mathrm{H}(17 \mathrm{~B})$ & 108.6 \\
\hline $\mathrm{C}(16)-\mathrm{C}(17)-\mathrm{H}(17 \mathrm{~B})$ & 108.6 \\
\hline $\mathrm{H}(17 \mathrm{~A})-\mathrm{C}(17)-\mathrm{H}(17 \mathrm{~B})$ & 107.6 \\
\hline$C(19)-C(18)-C(17)$ & $115.7(6)$ \\
\hline $\mathrm{C}(19)-\mathrm{C}(18)-\mathrm{H}(18 \mathrm{~A})$ & 108.4 \\
\hline $\mathrm{C}(17)-\mathrm{C}(18)-\mathrm{H}(18 \mathrm{~A})$ & 108.4 \\
\hline $\mathrm{C}(19)-\mathrm{C}(18)-\mathrm{H}(18 \mathrm{~B})$ & 108.4 \\
\hline $\mathrm{C}(17)-\mathrm{C}(18)-\mathrm{H}(18 \mathrm{~B})$ & 108.4 \\
\hline H(18A)-C(18)-H(18B) & 107.4 \\
\hline$C(18)-C(19)-C(20)$ & 113.8( \\
\hline $\mathrm{C}(18)-\mathrm{C}(19)-\mathrm{H}(19 \mathrm{~A})$ & 108.8 \\
\hline $\mathrm{C}(20)-\mathrm{C}(19)-\mathrm{H}(19 \mathrm{~A})$ & 108.8 \\
\hline $\mathrm{C}(18)-\mathrm{C}(19)-\mathrm{H}(19 \mathrm{~B})$ & 108.8 \\
\hline $\mathrm{C}(20)-\mathrm{C}(19)-\mathrm{H}(19 \mathrm{~B})$ & 108.8 \\
\hline H(19A)-C(19)-H(19B) & 107.7 \\
\hline$C(19)-C(20)-C(21)$ & $113.4(6)$ \\
\hline
\end{tabular}




$\begin{array}{ll}\mathrm{C}(19)-\mathrm{C}(20)-\mathrm{H}(20 \mathrm{~A}) & 108.9 \\ \mathrm{C}(21)-\mathrm{C}(20)-\mathrm{H}(20 \mathrm{~A}) & 108.9 \\ \mathrm{C}(19)-\mathrm{C}(20)-\mathrm{H}(20 \mathrm{~B}) & 108.9 \\ \mathrm{C}(21)-\mathrm{C}(20)-\mathrm{H}(20 \mathrm{~B}) & 108.9 \\ \mathrm{H}(20 \mathrm{~A})-\mathrm{C}(20)-\mathrm{H}(20 \mathrm{~B}) & 107.7 \\ \mathrm{C}(20)-\mathrm{C}(21)-\mathrm{H}(21 \mathrm{~A}) & 109.5 \\ \mathrm{C}(20)-\mathrm{C}(21)-\mathrm{H}(21 \mathrm{~B}) & 109.5 \\ \mathrm{H}(21 \mathrm{~A})-\mathrm{C}(21)-\mathrm{H}(21 \mathrm{~B}) & 109.5 \\ \mathrm{C}(20)-\mathrm{C}(21)-\mathrm{H}(21 \mathrm{C}) & 109.5 \\ \mathrm{H}(21 \mathrm{~A})-\mathrm{C}(21)-\mathrm{H}(21 \mathrm{C}) & 109.5 \\ \mathrm{H}(21 \mathrm{~B})-\mathrm{C}(21)-\mathrm{H}(21 \mathrm{C}) & 109.5 \\ \mathrm{~N}(1)-\mathrm{C}(22)-\mathrm{H}(22 \mathrm{~A}) & 109.5 \\ \mathrm{~N}(1)-\mathrm{C}(22)-\mathrm{H}(22 \mathrm{~B}) & 109.5 \\ \mathrm{H}(22 \mathrm{~A})-\mathrm{C}(22)-\mathrm{H}(22 \mathrm{~B}) & 109.5 \\ \mathrm{~N}(1)-\mathrm{C}(22)-\mathrm{H}(22 \mathrm{C}) & 109.5 \\ \mathrm{H}(22 \mathrm{~A})-\mathrm{C}(22)-\mathrm{H}(22 \mathrm{C}) & 109.5 \\ \mathrm{H}(22 \mathrm{~B})-\mathrm{C}(22)-\mathrm{H}(22 \mathrm{C}) & 109.5 \\ \mathrm{~N}(1)-\mathrm{C}(23)-\mathrm{H}(23 \mathrm{~A}) & 109.5 \\ \mathrm{~N}(1)-\mathrm{C}(23)-\mathrm{H}(23 \mathrm{~B}) & 109.5 \\ \mathrm{H}(23 \mathrm{~A})-\mathrm{C}(23)-\mathrm{H}(23 \mathrm{~B}) & 109.5 \\ \text { N(1)-C(23)-H(23C) } & 109.5 \\ \mathrm{H}(23 \mathrm{~A})-\mathrm{C}(23)-\mathrm{H}(23 \mathrm{C}) & 109.5 \\ \mathrm{H}(23 \mathrm{~B})-\mathrm{C}(23)-\mathrm{H}(23 \mathrm{C}) & 109.5 \\ & \end{array}$

Symmetry transformations used to generate equivalent atoms: 
Table A 4. Anisotropic displacement parameters $\left(\AA^{2} \times 10^{3}\right)$ for 34. The anisotropic displacement factor exponent takes the form: $-2 p^{2}\left[h^{2} a^{* 2} U^{11}+\ldots+2 h k a^{*} b^{*} U^{12}\right]$

\begin{tabular}{lllllll}
\hline & $\mathrm{U} 11$ & $\mathrm{U} 22$ & $\mathrm{U} 33$ & $\mathrm{U} 23$ & $\mathrm{U} 13$ & $\mathrm{U} 12$ \\
\hline $\mathrm{Br}(1)$ & $47(1)$ & $43(1)$ & $77(1)$ & $-9(1)$ & $-14(1)$ & $-7(1)$ \\
$\mathrm{P}(1)$ & $38(1)$ & $37(1)$ & $44(1)$ & $0(1)$ & $4(1)$ & $-9(1)$ \\
$\mathrm{O}(1)$ & $39(3)$ & $40(2)$ & $52(3)$ & $-4(2)$ & $8(2)$ & $-5(2)$ \\
$\mathrm{O}(2)$ & $36(3)$ & $39(2)$ & $58(3)$ & $-4(2)$ & $10(2)$ & $-7(2)$ \\
$\mathrm{O}(3)$ & $60(3)$ & $54(3)$ & $39(3)$ & $7(2)$ & $-4(2)$ & $-25(2)$ \\
$\mathrm{N}(1)$ & $36(3)$ & $36(3)$ & $38(4)$ & $-3(2)$ & $-1(3)$ & $-4(2)$ \\
$\mathrm{C}(1)$ & $42(4)$ & $38(4)$ & $36(4)$ & $-2(3)$ & $4(3)$ & $-11(3)$ \\
$\mathrm{C}(2)$ & $39(4)$ & $45(4)$ & $32(4)$ & $2(3)$ & $1(3)$ & $-12(3)$ \\
$\mathrm{C}(3)$ & $41(4)$ & $34(3)$ & $40(4)$ & $2(3)$ & $4(3)$ & $-11(3)$ \\
$\mathrm{C}(4)$ & $30(4)$ & $42(4)$ & $35(4)$ & $-6(3)$ & $6(3)$ & $-9(3)$ \\
$\mathrm{C}(5)$ & $34(4)$ & $44(4)$ & $42(5)$ & $-3(3)$ & $3(3)$ & $-9(3)$ \\
$\mathrm{C}(6)$ & $45(5)$ & $45(4)$ & $34(4)$ & $0(3)$ & $5(3)$ & $-11(3)$ \\
$\mathrm{C}(7)$ & $46(5)$ & $47(4)$ & $35(4)$ & $-4(3)$ & $6(3)$ & $-12(3)$ \\
$\mathrm{C}(8)$ & $45(4)$ & $46(4)$ & $38(4)$ & $-8(3)$ & $7(3)$ & $-17(3)$ \\
$\mathrm{C}(9)$ & $50(5)$ & $50(4)$ & $32(4)$ & $-2(3)$ & $2(3)$ & $-16(3)$ \\
$\mathrm{C}(10)$ & $48(5)$ & $51(4)$ & $36(4)$ & $-2(3)$ & $6(3)$ & $-21(3)$ \\
$\mathrm{C}(11)$ & $46(5)$ & $48(4)$ & $39(4)$ & $-7(3)$ & $5(3)$ & $-18(3)$ \\
$\mathrm{C}(12)$ & $46(5)$ & $52(4)$ & $37(4)$ & $-8(3)$ & $6(3)$ & $-16(3)$ \\
$\mathrm{C}(13)$ & $41(4)$ & $55(4)$ & $36(4)$ & $-5(3)$ & $5(3)$ & $-22(3)$ \\
$\mathrm{C}(14)$ & $45(5)$ & $57(4)$ & $39(4)$ & $-2(3)$ & $3(3)$ & $-25(4)$ \\
$\mathrm{C}(15)$ & $47(5)$ & $52(4)$ & $36(4)$ & $-1(3)$ & $3(3)$ & $-16(3)$ \\
$\mathrm{C}(16)$ & $47(5)$ & $53(4)$ & $35(4)$ & $0(3)$ & $4(3)$ & $-19(3)$ \\
$\mathrm{C}(17)$ & $41(4)$ & $49(4)$ & $42(5)$ & $-5(3)$ & $5(3)$ & $-16(3)$ \\
$\mathrm{C}(18)$ & $46(5)$ & $55(4)$ & $36(4)$ & $-8(3)$ & $3(3)$ & $-19(3)$ \\
$\mathrm{C}(19)$ & $43(4)$ & $46(4)$ & $42(5)$ & $-4(3)$ & $7(3)$ & $-16(3)$ \\
$\mathrm{C}(20)$ & $40(4)$ & $59(4)$ & $39(5)$ & $-1(3)$ & $0(3)$ & $-15(3)$ \\
$\mathrm{C}(21)$ & $52(5)$ & $60(5)$ & $42(5)$ & $0(4)$ & $1(4)$ & $-12(4)$ \\
$\mathrm{C}(22)$ & $39(4)$ & $46(4)$ & $44(5)$ & $-1(3)$ & $-2(4)$ & $9(3)$ \\
$\mathrm{C}(23)$ & $30(4)$ & $56(4)$ & $58(5)$ & $-8(4)$ & $1(4)$ & $-14(3)$ \\
\hline
\end{tabular}


Table A 5. Hydrogen coordinates ( x $\left.10^{4}\right)$ and isotropic displacement parameters $\left(\AA^{2} \times 10^{3}\right)$ for 34 .

\begin{tabular}{|c|c|c|c|c|}
\hline & $\mathrm{x}$ & $\mathrm{y}$ & $\mathrm{z}$ & $\mathrm{U}(\mathrm{eq})$ \\
\hline $\mathrm{H}(1 \mathrm{O})$ & 6719 & 4152 & 4808 & 66 \\
\hline $\mathrm{H}(3 \mathrm{O})$ & 5700 & 7712 & 3988 & 74 \\
\hline $\mathrm{H}(1 \mathrm{~A})$ & 9514 & 7739 & 4903 & 46 \\
\hline $\mathrm{H}(1 \mathrm{~B})$ & 7617 & 9490 & 4971 & 46 \\
\hline $\mathrm{H}(2 \mathrm{~A})$ & 6600 & 7907 & 5665 & 46 \\
\hline $\mathrm{H}(2 \mathrm{~B})$ & 8622 & 6253 & 5604 & 46 \\
\hline $\mathrm{H}(3 \mathrm{~A})$ & 8671 & 10044 & 5754 & 46 \\
\hline $\mathrm{H}(3 \mathrm{~B})$ & 10726 & 8555 & 5623 & 46 \\
\hline $\mathrm{H}(4 \mathrm{~A})$ & 7109 & 7304 & 6465 & 43 \\
\hline $\mathrm{H}(4 \mathrm{~B})$ & 6779 & 9525 & 6450 & 43 \\
\hline $\mathrm{H}(5 \mathrm{~A})$ & 8245 & 9466 & 7182 & 48 \\
\hline $\mathrm{H}(5 \mathrm{~B})$ & 9089 & 7244 & 7187 & 48 \\
\hline $\mathrm{H}(6 \mathrm{~A})$ & 4706 & 8927 & 7134 & 50 \\
\hline $\mathrm{H}(6 \mathrm{~B})$ & 5683 & 6751 & 7221 & 50 \\
\hline $\mathrm{H}(7 \mathrm{~A})$ & 5646 & 9550 & 7873 & 51 \\
\hline $\mathrm{H}(7 \mathrm{~B})$ & 6937 & 7451 & 7961 & 51 \\
\hline $\mathrm{H}(8 \mathrm{~A})$ & 2504 & 8337 & 7824 & 50 \\
\hline $\mathrm{H}(8 \mathrm{~B})$ & 3869 & 6329 & 7980 & 50 \\
\hline $\mathrm{H}(9 \mathrm{~A})$ & 3127 & 9488 & 8541 & 52 \\
\hline $\mathrm{H}(9 \mathrm{~B})$ & 4569 & 7511 & 8698 & 52 \\
\hline $\mathrm{H}(10 \mathrm{~A})$ & 1656 & 6129 & 8696 & 52 \\
\hline $\mathrm{H}(10 \mathrm{~B})$ & 195 & 8060 & 8515 & 52 \\
\hline $\mathrm{H}(11 \mathrm{~A})$ & 645 & 9384 & 9227 & 52 \\
\hline $\mathrm{H}(11 \mathrm{~B})$ & 2135 & 7466 & 9408 & 52 \\
\hline $\mathrm{H}(12 \mathrm{~A})$ & -2212 & 7889 & 9205 & 53 \\
\hline $\mathrm{H}(12 \mathrm{~B})$ & -721 & 5972 & 9387 & 53 \\
\hline $\mathrm{H}(13 \mathrm{~A})$ & -1777 & 9219 & 9912 & 51 \\
\hline $\mathrm{H}(13 \mathrm{~B})$ & -275 & 7309 & 10094 & 51 \\
\hline $\mathrm{H}(14 \mathrm{~A})$ & -4613 & 7691 & 9890 & 54 \\
\hline
\end{tabular}




\begin{tabular}{|c|c|c|c|c|}
\hline $\mathrm{H}(14 \mathrm{~B})$ & -3107 & 5795 & 10079 & 54 \\
\hline $\mathrm{H}(15 \mathrm{~A})$ & -4255 & 9067 & 10596 & 53 \\
\hline $\mathrm{H}(15 \mathrm{~B})$ & -2712 & 7191 & 10785 & 53 \\
\hline $\mathrm{H}(16 \mathrm{~A})$ & -7030 & 7477 & 10578 & 53 \\
\hline $\mathrm{H}(16 \mathrm{~B})$ & -5487 & 5602 & 10767 & 53 \\
\hline $\mathrm{H}(17 \mathrm{~A})$ & -5152 & 6983 & 11475 & 51 \\
\hline $\mathrm{H}(17 \mathrm{~B})$ & -6681 & 8867 & 11285 & 51 \\
\hline $\mathrm{H}(18 \mathrm{~A})$ & -9448 & 7288 & 11257 & 53 \\
\hline $\mathrm{H}(18 \mathrm{~B})$ & -7919 & 5415 & 11451 & 53 \\
\hline $\mathrm{H}(19 \mathrm{~A})$ & -9151 & 8672 & 11967 & 51 \\
\hline $\mathrm{H}(19 \mathrm{~B})$ & -7658 & 6775 & 12161 & 51 \\
\hline $\mathrm{H}(20 \mathrm{~A})$ & -10424 & 5223 & 12123 & 54 \\
\hline $\mathrm{H}(20 \mathrm{~B})$ & -11923 & 7080 & 11908 & 54 \\
\hline $\mathrm{H}(21 \mathrm{~A})$ & -12606 & 6661 & 12704 & 77 \\
\hline $\mathrm{H}(21 \mathrm{~B})$ & -11806 & 8551 & 12610 & 77 \\
\hline $\mathrm{H}(21 \mathrm{C})$ & -10245 & 6728 & 12825 & 77 \\
\hline $\mathrm{H}(22 \mathrm{~A})$ & 12343 & 6131 & 6152 & 69 \\
\hline $\mathrm{H}(22 \mathrm{~B})$ & 10284 & 5361 & 6269 & 69 \\
\hline $\mathrm{H}(22 \mathrm{C})$ & 11577 & 5991 & 6678 & 69 \\
\hline $\mathrm{H}(23 \mathrm{~A})$ & 12217 & 9546 & 6274 & 71 \\
\hline $\mathrm{H}(23 \mathrm{~B})$ & 11479 & 9146 & 6791 & 71 \\
\hline $\mathrm{H}(23 \mathrm{C})$ & 10102 & 10787 & 6470 & 71 \\
\hline
\end{tabular}


Table A 6. Hydrogen bonds for 34 [ $\AA$ and $\left.{ }^{\circ}\right]$.

\begin{tabular}{lcccc}
\hline D-H...A & $d(D-H)$ & $d(H . . . A)$ & $d(D . . A)$ & $<($ DHA $)$ \\
\hline $\mathrm{O}(1)-H(1 O) . . . \mathrm{O}(2) \# 1$ & 0.84 & 1.62 & $2.449(6)$ & 171.1 \\
$\mathrm{O}(3)-\mathrm{H}(3 \mathrm{O}) \ldots \mathrm{Br}(1) \# 1$ & 0.84 & 2.29 & $3.102(5)$ & 163.7 \\
\hline
\end{tabular}

Symmetry transformations used to generate equivalent atoms:

$\# 1-\mathrm{x}+1,-\mathrm{y}+1,-\mathrm{z}+1$

Table A 7. Crystal data and structure refinement for $\mathbf{6 6 .}$

\begin{tabular}{|c|c|c|}
\hline Identification code & \multicolumn{2}{|l|}{ cu_d12327_0m } \\
\hline Empirical formula & \multicolumn{2}{|l|}{ C23 H50 Br N O } \\
\hline Formula weight & \multicolumn{2}{|l|}{436.55} \\
\hline Temperature & \multicolumn{2}{|l|}{$147(2) \mathrm{K}$} \\
\hline Wavelength & \multicolumn{2}{|l|}{$1.54178 \AA$} \\
\hline Crystal system & \multicolumn{2}{|l|}{ Triclinic } \\
\hline Space group & \multicolumn{2}{|l|}{$P-1$} \\
\hline \multirow[t]{3}{*}{ Unit cell dimensions } & $\mathrm{a}=7.3945(3) \AA$ & $\mathrm{a}=87.482(2)^{\circ}$. \\
\hline & $\mathrm{b}=8.2701(4) \AA$ & $\mathrm{b}=81.558(2)^{\circ}$ \\
\hline & $c=22.9009(10) \AA$ & $\mathrm{g}=65.369(2)^{\circ}$. \\
\hline Volume & \multicolumn{2}{|l|}{$1259.01(10) \AA^{3}$} \\
\hline $\mathrm{Z}$ & \multicolumn{2}{|l|}{2} \\
\hline Density (calculated) & \multicolumn{2}{|l|}{$1.152 \mathrm{Mg} / \mathrm{m}^{3}$} \\
\hline Absorption coefficient & \multicolumn{2}{|l|}{$2.284 \mathrm{~mm}^{-1}$} \\
\hline$F(000)$ & \multicolumn{2}{|l|}{476} \\
\hline Crystal size & \multicolumn{2}{|c|}{$0.36 \times 0.22 \times 0.05 \mathrm{~mm}^{3}$} \\
\hline Theta range for data collection & \multicolumn{2}{|l|}{5.89 to $66.36^{\circ}$} \\
\hline Index ranges & \multicolumn{2}{|c|}{$-7<=\mathrm{h}<=8,-9<=\mathrm{k}<=8,-27<=\mathrm{l}<=26$} \\
\hline Reflections collected & \multicolumn{2}{|l|}{12902} \\
\hline Independent reflections & \multicolumn{2}{|c|}{$4231[\mathrm{R}(\mathrm{int})=0.0240]$} \\
\hline Completeness to theta $=66.36^{\circ}$ & \multicolumn{2}{|l|}{$95.8 \%$} \\
\hline Absorption correction & \multicolumn{2}{|c|}{ Semi-empirical from equivalents } \\
\hline
\end{tabular}


Max. and min. transmission

Refinement method

Data / restraints / parameters

Goodness-of-fit on $\mathrm{F}^{2}$

Final R indices [I $>2 \operatorname{sigma(I)]~}$

$\mathrm{R}$ indices (all data)

Largest diff. peak and hole
0.8944 and 0.6183

Full-matrix least-squares on $\mathrm{F}^{2}$

4231 / 0 / 242

1.080

$\mathrm{R} 1=0.0354, \mathrm{wR} 2=0.0934$

$\mathrm{R} 1=0.0360, \mathrm{wR} 2=0.0940$

1.438 and -0.602 e. $\AA^{-3}$

Table A 8. Atomic coordinates ( $\mathrm{x} 10^{4}$ ) and equivalent isotropic displacement parameters $\left(\AA^{2} \times 10^{3}\right)$ for 66.

\begin{tabular}{|c|c|c|c|c|}
\hline & $\mathrm{x}$ & $\mathrm{y}$ & $\mathrm{z}$ & $\mathrm{U}(\mathrm{eq})$ \\
\hline $\operatorname{Br}(1)$ & $3802(1)$ & $3378(1)$ & 911(1) & $41(1)$ \\
\hline $\mathrm{O}(1)$ & $8589(3)$ & $2020(2)$ & $773(1)$ & $46(1)$ \\
\hline $\mathrm{N}(1)$ & 7166(3) & $7645(2)$ & 825(1) & $25(1)$ \\
\hline $\mathrm{C}(1)$ & $8881(4)$ & 2712(3) & $1284(1)$ & $41(1)$ \\
\hline$C(2)$ & $7749(4)$ & 4721(3) & $1355(1)$ & $32(1)$ \\
\hline $\mathrm{C}(3)$ & 8252(3) & 5628(3) & $806(1)$ & $25(1)$ \\
\hline$C(4)$ & 8091(4) & 8338(3) & $302(1)$ & $31(1)$ \\
\hline$C(5)$ & 4997(3) & 8186(3) & $770(1)$ & $35(1)$ \\
\hline$C(6)$ & 7301(3) & $8457(3)$ & 1388(1) & $26(1)$ \\
\hline $\mathrm{C}(7)$ & $9429(3)$ & 8037(3) & $1490(1)$ & $26(1)$ \\
\hline$C(8)$ & $9506(3)$ & 8391(3) & 2134(1) & $27(1)$ \\
\hline $\mathrm{C}(9)$ & 11601(3) & 8136(3) & 2228(1) & $28(1)$ \\
\hline$C(10)$ & 11856(3) & 8220(3) & $2873(1)$ & $29(1)$ \\
\hline $\mathrm{C}(11)$ & 13958(3) & 7976(3) & 2951(1) & $29(1)$ \\
\hline $\mathrm{C}(12)$ & 14259(3) & 8008(3) & $3595(1)$ & $29(1)$ \\
\hline$C(13)$ & 16358(3) & 7773(3) & $3674(1)$ & $29(1)$ \\
\hline$C(14)$ & 16653(3) & 7797(3) & $4317(1)$ & $29(1)$ \\
\hline$C(15)$ & 18749(3) & 7567(3) & $4400(1)$ & $29(1)$ \\
\hline$C(16)$ & 19033(3) & 7591(3) & $5044(1)$ & $29(1)$ \\
\hline $\mathrm{C}(17)$ & 21121(3) & 7367(3) & 5131(1) & $30(1)$ \\
\hline $\mathrm{C}(18)$ & 21393(3) & 7395(3) & $5777(1)$ & $30(1)$ \\
\hline
\end{tabular}




\begin{tabular}{lllll}
$\mathrm{C}(19)$ & $23479(3)$ & $7169(3)$ & $5869(1)$ & $30(1)$ \\
$\mathrm{C}(20)$ & $23740(3)$ & $7210(3)$ & $6515(1)$ & $31(1)$ \\
$\mathrm{C}(21)$ & $25829(3)$ & $6966(3)$ & $6609(1)$ & $32(1)$ \\
$\mathrm{C}(22)$ & $26087(4)$ & $7012(3)$ & $7255(1)$ & $35(1)$ \\
$\mathrm{C}(23)$ & $28183(4)$ & $6750(4)$ & $7345(1)$ & $43(1)$ \\
\hline
\end{tabular}

Table A 9. Bond lengths $[\AA]$ and angles $\left[^{\circ}\right]$ for 66 .

\begin{tabular}{ll}
\hline $\mathrm{O}(1)-\mathrm{C}(1)$ & $1.408(3)$ \\
$\mathrm{O}(1)-\mathrm{H}(1 \mathrm{O})$ & $0.82(4)$ \\
$\mathrm{N}(1)-\mathrm{C}(5)$ & $1.499(3)$ \\
$\mathrm{N}(1)-\mathrm{C}(4)$ & $1.503(3)$ \\
$\mathrm{N}(1)-\mathrm{C}(6)$ & $1.513(3)$ \\
$\mathrm{N}(1)-\mathrm{C}(3)$ & $1.520(3)$ \\
$\mathrm{C}(1)-\mathrm{C}(2)$ & $1.521(3)$ \\
$\mathrm{C}(1)-\mathrm{H}(1 \mathrm{~A})$ & 0.9900 \\
$\mathrm{C}(1)-\mathrm{H}(1 \mathrm{~B})$ & 0.9900 \\
$\mathrm{C}(2)-\mathrm{C}(3)$ & $1.515(3)$ \\
$\mathrm{C}(2)-\mathrm{H}(2 \mathrm{~A})$ & 0.9900 \\
$\mathrm{C}(2)-\mathrm{H}(2 \mathrm{~B})$ & 0.9900 \\
$\mathrm{C}(3)-\mathrm{H}(3 \mathrm{~A})$ & 0.9900 \\
$\mathrm{C}(3)-\mathrm{H}(3 \mathrm{~B})$ & 0.9900 \\
$\mathrm{C}(4)-\mathrm{H}(4 \mathrm{~A})$ & 0.9800 \\
$\mathrm{C}(4)-\mathrm{H}(4 \mathrm{~B})$ & 0.9800 \\
$\mathrm{C}(4)-\mathrm{H}(4 \mathrm{C})$ & 0.9800 \\
$\mathrm{C}(5)-\mathrm{H}(5 \mathrm{~A})$ & 0.9800 \\
$\mathrm{C}(5)-\mathrm{H}(5 B)$ & 0.9800 \\
$\mathrm{C}(5)-\mathrm{H}(5 \mathrm{C})$ & 0.9800 \\
$\mathrm{C}(6)-\mathrm{C}(7)$ & $1.516(3)$ \\
$\mathrm{C}(6)-\mathrm{H}(6 \mathrm{~A})$ & 0.9900 \\
$\mathrm{C}(6)-\mathrm{H}(6 \mathrm{~B})$ & 0.9900 \\
$\mathrm{C}(7)-\mathrm{C}(8)$ & $1.528(3)$ \\
$\mathrm{C}(7)-\mathrm{H}(7 \mathrm{~A})$ & 0.9900 \\
$\mathrm{C}(7)-\mathrm{H}(7 \mathrm{~B})$ & 0.9900 \\
334 & \\
& \\
& \\
& \\
& \\
&
\end{tabular}




\begin{tabular}{|c|c|}
\hline $\mathrm{C}(8)-\mathrm{C}(9)$ & $1.521(3)$ \\
\hline $\mathrm{C}(8)-\mathrm{H}(8 \mathrm{~A})$ & 0.9900 \\
\hline $\mathrm{C}(8)-\mathrm{H}(8 \mathrm{~B})$ & 0.9900 \\
\hline $\mathrm{C}(9)-\mathrm{C}(10)$ & $1.524(3)$ \\
\hline $\mathrm{C}(9)-\mathrm{H}(9 \mathrm{~A})$ & 0.9900 \\
\hline $\mathrm{C}(9)-\mathrm{H}(9 \mathrm{~B})$ & 0.9900 \\
\hline $\mathrm{C}(10)-\mathrm{C}(11)$ & $1.519(3)$ \\
\hline $\mathrm{C}(10)-\mathrm{H}(10 \mathrm{~A})$ & 0.9900 \\
\hline $\mathrm{C}(10)-\mathrm{H}(10 \mathrm{~B})$ & 0.9900 \\
\hline $\mathrm{C}(11)-\mathrm{C}(12)$ & $1.526(3)$ \\
\hline $\mathrm{C}(11)-\mathrm{H}(11 \mathrm{~A})$ & 0.9900 \\
\hline $\mathrm{C}(11)-\mathrm{H}(11 \mathrm{~B})$ & 0.9900 \\
\hline$C(12)-C(13)$ & $1.519(3)$ \\
\hline $\mathrm{C}(12)-\mathrm{H}(12 \mathrm{~A})$ & 0.9900 \\
\hline $\mathrm{C}(12)-\mathrm{H}(12 \mathrm{~B})$ & 0.9900 \\
\hline$C(13)-C(14)$ & $1.522(3)$ \\
\hline $\mathrm{C}(13)-\mathrm{H}(13 \mathrm{~A})$ & 0.9900 \\
\hline $\mathrm{C}(13)-\mathrm{H}(13 \mathrm{~B})$ & 0.9900 \\
\hline$C(14)-C(15)$ & $1.522(3)$ \\
\hline $\mathrm{C}(14)-\mathrm{H}(14 \mathrm{~A})$ & 0.9900 \\
\hline $\mathrm{C}(14)-\mathrm{H}(14 \mathrm{~B})$ & 0.9900 \\
\hline$C(15)-C(16)$ & $1.521(3)$ \\
\hline $\mathrm{C}(15)-\mathrm{H}(15 \mathrm{~A})$ & 0.9900 \\
\hline $\mathrm{C}(15)-\mathrm{H}(15 \mathrm{~B})$ & 0.9900 \\
\hline$C(16)-C(17)$ & $1.519(3)$ \\
\hline$C(16)-H(16 A)$ & 0.9900 \\
\hline $\mathrm{C}(16)-\mathrm{H}(16 \mathrm{~B})$ & 0.9900 \\
\hline $\mathrm{C}(17)-\mathrm{C}(18)$ & $1.523(3)$ \\
\hline $\mathrm{C}(17)-\mathrm{H}(17 \mathrm{~A})$ & 0.9900 \\
\hline $\mathrm{C}(17)-\mathrm{H}(17 \mathrm{~B})$ & 0.9900 \\
\hline C(18)-C(19) & $1.520(3)$ \\
\hline $\mathrm{C}(18)-\mathrm{H}(18 \mathrm{~A})$ & 0.9900 \\
\hline $\mathrm{C}(18)-\mathrm{H}(18 \mathrm{~B})$ & 0.9900 \\
\hline$C(19)-C(20)$ & $1.524(3)$ \\
\hline C(19)-H(19A) & 0.9900 \\
\hline
\end{tabular}




$\begin{array}{ll}\mathrm{C}(19)-\mathrm{H}(19 \mathrm{~B}) & 0.9900 \\ \mathrm{C}(20)-\mathrm{C}(21) & 1.519(3) \\ \mathrm{C}(20)-\mathrm{H}(20 \mathrm{~A}) & 0.9900 \\ \mathrm{C}(20)-\mathrm{H}(20 \mathrm{~B}) & 0.9900 \\ \mathrm{C}(21)-\mathrm{C}(22) & 1.522(3) \\ \mathrm{C}(21)-\mathrm{H}(21 \mathrm{~A}) & 0.9900 \\ \mathrm{C}(21)-\mathrm{H}(21 \mathrm{~B}) & 0.9900 \\ \mathrm{C}(22)-\mathrm{C}(23) & 1.517(3) \\ \mathrm{C}(22)-\mathrm{H}(22 \mathrm{~A}) & 0.9900 \\ \mathrm{C}(22)-\mathrm{H}(22 \mathrm{~B}) & 0.9900 \\ \mathrm{C}(23)-\mathrm{H}(23 \mathrm{~A}) & 0.9800 \\ \mathrm{C}(23)-\mathrm{H}(23 \mathrm{~B}) & 0.9800 \\ \mathrm{C}(23)-\mathrm{H}(23 \mathrm{C}) & 0.9800 \\ & \\ \mathrm{C}(1)-\mathrm{O}(1)-\mathrm{H}(1 \mathrm{O}) & 107(3) \\ \mathrm{C}(5)-\mathrm{N}(1)-\mathrm{C}(4) & 108.15(17) \\ \mathrm{C}(5)-\mathrm{N}(1)-\mathrm{C}(6) & 108.90(16) \\ \mathrm{C}(4)-\mathrm{N}(1)-\mathrm{C}(6) & 109.80(16) \\ \mathrm{C}(5)-\mathrm{N}(1)-\mathrm{C}(3) & 109.48(17) \\ \mathrm{C}(4)-\mathrm{N}(1)-\mathrm{C}(3) & 107.72(15) \\ \mathrm{C}(6)-\mathrm{N}(1)-\mathrm{C}(3) & 112.69(15) \\ \mathrm{O}(1)-\mathrm{C}(1)-\mathrm{C}(2) & 113.7(2) \\ \mathrm{O}(1)-\mathrm{C}(1)-\mathrm{H}(1 \mathrm{~A}) & 108.8 \\ \mathrm{C}(2)-\mathrm{C}(1)-\mathrm{H}(1 \mathrm{~A}) & 108.8 \\ \mathrm{O}(1)-\mathrm{C}(1)-\mathrm{H}(1 \mathrm{~B}) & 108.8 \\ \mathrm{C}(2)-\mathrm{C}(1)-\mathrm{H}(1 \mathrm{~B}) & 108.8 \\ \mathrm{H}(1 \mathrm{~A})-\mathrm{C}(1)-\mathrm{H}(1 \mathrm{~B}) & 107.7 \\ \mathrm{C}(3)-\mathrm{C}(2)-\mathrm{C}(1) & 110.38(19) \\ \mathrm{C}(3)-\mathrm{C}(2)-\mathrm{H}(2 \mathrm{~A}) & 109.6 \\ \mathrm{C}(1)-\mathrm{C}(2)-\mathrm{H}(2 \mathrm{~A}) & 109.6 \\ \mathrm{C}(3)-\mathrm{C}(2)-\mathrm{H}(2 \mathrm{~B}) & 109.6 \\ \mathrm{C}(1)-\mathrm{C}(2)-\mathrm{H}(2 \mathrm{~B}) & 109.6 \\ \mathrm{H}(2 \mathrm{~A})-\mathrm{C}(2)-\mathrm{H}(2 \mathrm{~B}) & 108.1 \\ \mathrm{C}(2)-\mathrm{C}(3)-\mathrm{N}(1) & 114.73(17) \\ \mathrm{C}(2)-\mathrm{C}(3)-\mathrm{H}(3 \mathrm{~A}) & 108.6 \\ 336 & \\ & \end{array}$




\begin{tabular}{|c|c|}
\hline N(1)-C(3)-H(3A) & 108.6 \\
\hline $\mathrm{C}(2)-\mathrm{C}(3)-\mathrm{H}(3 \mathrm{~B})$ & 108.6 \\
\hline N(1)-C(3)-H(3B) & 108.6 \\
\hline H(3A)-C(3)-H(3B) & 107.6 \\
\hline $\mathrm{N}(1)-\mathrm{C}(4)-\mathrm{H}(4 \mathrm{~A})$ & 109.5 \\
\hline $\mathrm{N}(1)-\mathrm{C}(4)-\mathrm{H}(4 \mathrm{~B})$ & 109.5 \\
\hline $\mathrm{H}(4 \mathrm{~A})-\mathrm{C}(4)-\mathrm{H}(4 \mathrm{~B})$ & 109.5 \\
\hline N(1)-C(4)-H(4C) & 109.5 \\
\hline $\mathrm{H}(4 \mathrm{~A})-\mathrm{C}(4)-\mathrm{H}(4 \mathrm{C})$ & 109.5 \\
\hline $\mathrm{H}(4 \mathrm{~B})-\mathrm{C}(4)-\mathrm{H}(4 \mathrm{C})$ & 109.5 \\
\hline $\mathrm{N}(1)-\mathrm{C}(5)-\mathrm{H}(5 \mathrm{~A})$ & 109.5 \\
\hline $\mathrm{N}(1)-\mathrm{C}(5)-\mathrm{H}(5 \mathrm{~B})$ & 109.5 \\
\hline $\mathrm{H}(5 \mathrm{~A})-\mathrm{C}(5)-\mathrm{H}(5 \mathrm{~B})$ & 109.5 \\
\hline $\mathrm{N}(1)-\mathrm{C}(5)-\mathrm{H}(5 \mathrm{C})$ & 109.5 \\
\hline $\mathrm{H}(5 \mathrm{~A})-\mathrm{C}(5)-\mathrm{H}(5 \mathrm{C})$ & 109.5 \\
\hline H(5B)-C(5)-H(5C) & 109.5 \\
\hline $\mathrm{N}(1)-\mathrm{C}(6)-\mathrm{C}(7)$ & $114.18(16)$ \\
\hline $\mathrm{N}(1)-\mathrm{C}(6)-\mathrm{H}(6 \mathrm{~A})$ & 108.7 \\
\hline $\mathrm{C}(7)-\mathrm{C}(6)-\mathrm{H}(6 \mathrm{~A})$ & 108.7 \\
\hline $\mathrm{N}(1)-\mathrm{C}(6)-\mathrm{H}(6 \mathrm{~B})$ & 108.7 \\
\hline $\mathrm{C}(7)-\mathrm{C}(6)-\mathrm{H}(6 \mathrm{~B})$ & 108.7 \\
\hline $\mathrm{H}(6 \mathrm{~A})-\mathrm{C}(6)-\mathrm{H}(6 \mathrm{~B})$ & 107.6 \\
\hline $\mathrm{C}(6)-\mathrm{C}(7)-\mathrm{C}(8)$ & $111.27(17)$ \\
\hline $\mathrm{C}(6)-\mathrm{C}(7)-\mathrm{H}(7 \mathrm{~A})$ & 109.4 \\
\hline $\mathrm{C}(8)-\mathrm{C}(7)-\mathrm{H}(7 \mathrm{~A})$ & 109.4 \\
\hline $\mathrm{C}(6)-\mathrm{C}(7)-\mathrm{H}(7 \mathrm{~B})$ & 109.4 \\
\hline $\mathrm{C}(8)-\mathrm{C}(7)-\mathrm{H}(7 \mathrm{~B})$ & 109.4 \\
\hline H(7A)-C(7)-H(7B) & 108.0 \\
\hline $\mathrm{C}(9)-\mathrm{C}(8)-\mathrm{C}(7)$ & $111.58(17)$ \\
\hline $\mathrm{C}(9)-\mathrm{C}(8)-\mathrm{H}(8 \mathrm{~A})$ & 109.3 \\
\hline $\mathrm{C}(7)-\mathrm{C}(8)-\mathrm{H}(8 \mathrm{~A})$ & 109.3 \\
\hline $\mathrm{C}(9)-\mathrm{C}(8)-\mathrm{H}(8 \mathrm{~B})$ & 109.3 \\
\hline $\mathrm{C}(7)-\mathrm{C}(8)-\mathrm{H}(8 \mathrm{~B})$ & 109.3 \\
\hline H(8A)-C(8)-H(8B) & 108.0 \\
\hline $\mathrm{C}(8)-\mathrm{C}(9)-\mathrm{C}(10)$ & $114.25(17)$ \\
\hline
\end{tabular}




\begin{tabular}{|c|c|}
\hline $\mathrm{C}(8)-\mathrm{C}(9)-\mathrm{H}(9 \mathrm{~A})$ & 108.7 \\
\hline $\mathrm{C}(10)-\mathrm{C}(9)-\mathrm{H}(9 \mathrm{~A})$ & 108.7 \\
\hline $\mathrm{C}(8)-\mathrm{C}(9)-\mathrm{H}(9 \mathrm{~B})$ & 108.7 \\
\hline $\mathrm{C}(10)-\mathrm{C}(9)-\mathrm{H}(9 \mathrm{~B})$ & 108.7 \\
\hline $\mathrm{H}(9 \mathrm{~A})-\mathrm{C}(9)-\mathrm{H}(9 \mathrm{~B})$ & 107.6 \\
\hline $\mathrm{C}(11)-\mathrm{C}(10)-\mathrm{C}(9)$ & $112.93(17)$ \\
\hline $\mathrm{C}(11)-\mathrm{C}(10)-\mathrm{H}(10 \mathrm{~A})$ & 109.0 \\
\hline $\mathrm{C}(9)-\mathrm{C}(10)-\mathrm{H}(10 \mathrm{~A})$ & 109.0 \\
\hline $\mathrm{C}(11)-\mathrm{C}(10)-\mathrm{H}(10 \mathrm{~B})$ & 109.0 \\
\hline $\mathrm{C}(9)-\mathrm{C}(10)-\mathrm{H}(10 \mathrm{~B})$ & 109.0 \\
\hline $\mathrm{H}(10 \mathrm{~A})-\mathrm{C}(10)-\mathrm{H}(10 \mathrm{~B})$ & 107.8 \\
\hline$C(10)-C(11)-C(12)$ & $113.76(17)$ \\
\hline $\mathrm{C}(10)-\mathrm{C}(11)-\mathrm{H}(11 \mathrm{~A})$ & 108.8 \\
\hline $\mathrm{C}(12)-\mathrm{C}(11)-\mathrm{H}(11 \mathrm{~A})$ & 108.8 \\
\hline $\mathrm{C}(10)-\mathrm{C}(11)-\mathrm{H}(11 \mathrm{~B})$ & 108.8 \\
\hline $\mathrm{C}(12)-\mathrm{C}(11)-\mathrm{H}(11 \mathrm{~B})$ & 108.8 \\
\hline $\mathrm{H}(11 \mathrm{~A})-\mathrm{C}(11)-\mathrm{H}(11 \mathrm{~B})$ & 107.7 \\
\hline$C(13)-C(12)-C(11)$ & $113.80(17)$ \\
\hline $\mathrm{C}(13)-\mathrm{C}(12)-\mathrm{H}(12 \mathrm{~A})$ & 108.8 \\
\hline $\mathrm{C}(11)-\mathrm{C}(12)-\mathrm{H}(12 \mathrm{~A})$ & 108.8 \\
\hline $\mathrm{C}(13)-\mathrm{C}(12)-\mathrm{H}(12 \mathrm{~B})$ & 108.8 \\
\hline $\mathrm{C}(11)-\mathrm{C}(12)-\mathrm{H}(12 \mathrm{~B})$ & 108.8 \\
\hline $\mathrm{H}(12 \mathrm{~A})-\mathrm{C}(12)-\mathrm{H}(12 \mathrm{~B})$ & 107.7 \\
\hline$C(12)-C(13)-C(14)$ & $113.56(17)$ \\
\hline $\mathrm{C}(12)-\mathrm{C}(13)-\mathrm{H}(13 \mathrm{~A})$ & 108.9 \\
\hline $\mathrm{C}(14)-\mathrm{C}(13)-\mathrm{H}(13 \mathrm{~A})$ & 108.9 \\
\hline $\mathrm{C}(12)-\mathrm{C}(13)-\mathrm{H}(13 \mathrm{~B})$ & 108.9 \\
\hline $\mathrm{C}(14)-\mathrm{C}(13)-\mathrm{H}(13 \mathrm{~B})$ & 108.9 \\
\hline $\mathrm{H}(13 \mathrm{~A})-\mathrm{C}(13)-\mathrm{H}(13 \mathrm{~B})$ & 107.7 \\
\hline$C(15)-C(14)-C(13)$ & 113.98(18) \\
\hline $\mathrm{C}(15)-\mathrm{C}(14)-\mathrm{H}(14 \mathrm{~A})$ & 108.8 \\
\hline $\mathrm{C}(13)-\mathrm{C}(14)-\mathrm{H}(14 \mathrm{~A})$ & 108.8 \\
\hline $\mathrm{C}(15)-\mathrm{C}(14)-\mathrm{H}(14 \mathrm{~B})$ & 108.8 \\
\hline $\mathrm{C}(13)-\mathrm{C}(14)-\mathrm{H}(14 \mathrm{~B})$ & 108.8 \\
\hline $\mathrm{H}(14 \mathrm{~A})-\mathrm{C}(14)-\mathrm{H}(14 \mathrm{~B})$ & 107.7 \\
\hline
\end{tabular}




$\begin{array}{ll}\mathrm{C}(16)-\mathrm{C}(15)-\mathrm{C}(14) & 113.69(18) \\ \mathrm{C}(16)-\mathrm{C}(15)-\mathrm{H}(15 \mathrm{~A}) & 108.8 \\ \mathrm{C}(14)-\mathrm{C}(15)-\mathrm{H}(15 \mathrm{~A}) & 108.8 \\ \mathrm{C}(16)-\mathrm{C}(15)-\mathrm{H}(15 \mathrm{~B}) & 108.8 \\ \mathrm{C}(14)-\mathrm{C}(15)-\mathrm{H}(15 \mathrm{~B}) & 108.8 \\ \mathrm{H}(15 \mathrm{~A})-\mathrm{C}(15)-\mathrm{H}(15 \mathrm{~B}) & 107.7 \\ \mathrm{C}(17)-\mathrm{C}(16)-\mathrm{C}(15) & 113.99(18) \\ \mathrm{C}(17)-\mathrm{C}(16)-\mathrm{H}(16 \mathrm{~A}) & 108.8 \\ \mathrm{C}(15)-\mathrm{C}(16)-\mathrm{H}(16 \mathrm{~A}) & 108.8 \\ \mathrm{C}(17)-\mathrm{C}(16)-\mathrm{H}(16 \mathrm{~B}) & 108.8 \\ \mathrm{C}(15)-\mathrm{C}(16)-\mathrm{H}(16 \mathrm{~B}) & 108.8 \\ \mathrm{H}(16 \mathrm{~A})-\mathrm{C}(16)-\mathrm{H}(16 \mathrm{~B}) & 107.7 \\ \mathrm{C}(16)-\mathrm{C}(17)-\mathrm{C}(18) & 113.69(18) \\ \mathrm{C}(16)-\mathrm{C}(17)-\mathrm{H}(17 \mathrm{~A}) & 108.8 \\ \mathrm{C}(18)-\mathrm{C}(17)-\mathrm{H}(17 \mathrm{~A}) & 108.8 \\ \mathrm{C}(16)-\mathrm{C}(17)-\mathrm{H}(17 \mathrm{~B}) & 108.8 \\ \mathrm{C}(18)-\mathrm{C}(17)-\mathrm{H}(17 \mathrm{~B}) & 108.8 \\ \mathrm{H}(17 \mathrm{~A})-\mathrm{C}(17)-\mathrm{H}(17 \mathrm{~B}) & 107.7 \\ \mathrm{C}(19)-\mathrm{C}(18)-\mathrm{C}(17) & 114.13(18) \\ \mathrm{C}(19)-\mathrm{C}(18)-\mathrm{H}(18 \mathrm{~A}) & 108.7 \\ \mathrm{C}(17)-\mathrm{C}(18)-\mathrm{H}(18 \mathrm{~A}) & 108.7 \\ \mathrm{C}(19)-\mathrm{C}(18)-\mathrm{H}(18 \mathrm{~B}) & 108.7 \\ \mathrm{C}(17)-\mathrm{C}(18)-\mathrm{H}(18 \mathrm{~B}) & 108.7 \\ \mathrm{H}(18 \mathrm{~A})-\mathrm{C}(18)-\mathrm{H}(18 \mathrm{~B}) & 107.6 \\ \mathrm{C}(18)-\mathrm{C}(19)-\mathrm{C}(20) & 113.93(18) \\ \mathrm{C}(18)-\mathrm{C}(19)-\mathrm{H}(19 \mathrm{~A}) & 108.8 \\ \mathrm{C}(20)-\mathrm{C}(19)-\mathrm{H}(19 \mathrm{~A}) & 108.8 \\ \mathrm{C}(18)-\mathrm{C}(19)-\mathrm{H}(19 \mathrm{~B}) & 108.8 \\ \mathrm{C}(20)-\mathrm{C}(19)-\mathrm{H}(19 \mathrm{~B}) & 108.8 \\ \mathrm{H}(19 \mathrm{~A})-\mathrm{C}(19)-\mathrm{H}(19 \mathrm{~B}) & 107.7 \\ \mathrm{C}(21)-\mathrm{C}(20)-\mathrm{C}(19) & 114.07(18) \\ \mathrm{C}(21)-\mathrm{C}(20)-\mathrm{H}(20 \mathrm{~A}) & 108.7 \\ \mathrm{C}(19)-\mathrm{C}(20)-\mathrm{H}(20 \mathrm{~A}) & 108.7 \\ \mathrm{C}(21)-\mathrm{C}(20)-\mathrm{H}(20 \mathrm{~B}) & 108.7 \\ \mathrm{C}(19)-\mathrm{C}(20)-\mathrm{H}(20 \mathrm{~B}) & 108.7 \\ \text { 339} & \\ & \end{array}$




$\begin{array}{ll}\mathrm{H}(20 \mathrm{~A})-\mathrm{C}(20)-\mathrm{H}(20 \mathrm{~B}) & 107.6 \\ \mathrm{C}(20)-\mathrm{C}(21)-\mathrm{C}(22) & 114.05(19) \\ \mathrm{C}(20)-\mathrm{C}(21)-\mathrm{H}(21 \mathrm{~A}) & 108.7 \\ \mathrm{C}(22)-\mathrm{C}(21)-\mathrm{H}(21 \mathrm{~A}) & 108.7 \\ \mathrm{C}(20)-\mathrm{C}(21)-\mathrm{H}(21 \mathrm{~B}) & 108.7 \\ \mathrm{C}(22)-\mathrm{C}(21)-\mathrm{H}(21 \mathrm{~B}) & 108.7 \\ \mathrm{H}(21 \mathrm{~A})-\mathrm{C}(21)-\mathrm{H}(21 \mathrm{~B}) & 107.6 \\ \mathrm{C}(23)-\mathrm{C}(22)-\mathrm{C}(21) & 113.7(2) \\ \mathrm{C}(23)-\mathrm{C}(22)-\mathrm{H}(22 \mathrm{~A}) & 108.8 \\ \mathrm{C}(21)-\mathrm{C}(22)-\mathrm{H}(22 \mathrm{~A}) & 108.8 \\ \mathrm{C}(23)-\mathrm{C}(22)-\mathrm{H}(22 \mathrm{~B}) & 108.8 \\ \mathrm{C}(21)-\mathrm{C}(22)-\mathrm{H}(22 \mathrm{~B}) & 108.8 \\ \mathrm{H}(22 \mathrm{~A})-\mathrm{C}(22)-\mathrm{H}(22 \mathrm{~B}) & 107.7 \\ \mathrm{C}(22)-\mathrm{C}(23)-\mathrm{H}(23 \mathrm{~A}) & 109.5 \\ \mathrm{C}(22)-\mathrm{C}(23)-\mathrm{H}(23 \mathrm{~B}) & 109.5 \\ \mathrm{H}(23 \mathrm{~A})-\mathrm{C}(23)-\mathrm{H}(23 \mathrm{~B}) & 109.5 \\ \mathrm{C}(22)-\mathrm{C}(23)-\mathrm{H}(23 \mathrm{C}) & 109.5 \\ \mathrm{H}(23 \mathrm{~A})-\mathrm{C}(23)-\mathrm{H}(23 \mathrm{C}) & 109.5 \\ \mathrm{H}(23 \mathrm{~B})-\mathrm{C}(23)-\mathrm{H}(23 \mathrm{C}) & 109.5\end{array}$

Symmetry transformations used to generate equivalent atoms: 
Table A 10. Anisotropic displacement parameters $\left(\AA^{2} \times 10^{3}\right)$ for 66 . The anisotropic displacement factor exponent takes the form: $-2 p^{2}\left[h^{2} a^{* 2} U^{11}+\ldots+2 h k a^{*} b^{*} U^{12}\right]$

\begin{tabular}{lllllll}
\hline & $\mathrm{U} 11$ & $\mathrm{U}^{22}$ & $\mathrm{U}^{33}$ & $\mathrm{U}^{23}$ & $\mathrm{U} 13$ & $\mathrm{U} 12$ \\
\hline $\mathrm{Br}(1)$ & $28(1)$ & $43(1)$ & $48(1)$ & $-13(1)$ & $-9(1)$ & $-9(1)$ \\
$\mathrm{O}(1)$ & $38(1)$ & $32(1)$ & $66(1)$ & $-10(1)$ & $6(1)$ & $-18(1)$ \\
$\mathrm{N}(1)$ & $28(1)$ & $23(1)$ & $20(1)$ & $0(1)$ & $-3(1)$ & $-7(1)$ \\
$\mathrm{C}(1)$ & $39(1)$ & $29(1)$ & $56(2)$ & $10(1)$ & $-7(1)$ & $-16(1)$ \\
$\mathrm{C}(2)$ & $36(1)$ & $29(1)$ & $33(1)$ & $3(1)$ & $-5(1)$ & $-16(1)$ \\
$\mathrm{C}(3)$ & $26(1)$ & $20(1)$ & $28(1)$ & $-2(1)$ & $-3(1)$ & $-9(1)$ \\
$\mathrm{C}(4)$ & $47(1)$ & $31(1)$ & $19(1)$ & $2(1)$ & $-4(1)$ & $-18(1)$ \\
$\mathrm{C}(5)$ & $27(1)$ & $40(1)$ & $32(1)$ & $1(1)$ & $-9(1)$ & $-6(1)$ \\
$\mathrm{C}(6)$ & $32(1)$ & $22(1)$ & $18(1)$ & $-2(1)$ & $-4(1)$ & $-5(1)$ \\
$\mathrm{C}(7)$ & $33(1)$ & $22(1)$ & $20(1)$ & $-2(1)$ & $-3(1)$ & $-9(1)$ \\
$\mathrm{C}(8)$ & $34(1)$ & $25(1)$ & $19(1)$ & $0(1)$ & $-4(1)$ & $-8(1)$ \\
$\mathrm{C}(9)$ & $33(1)$ & $27(1)$ & $20(1)$ & $-1(1)$ & $-4(1)$ & $-7(1)$ \\
$\mathrm{C}(10)$ & $33(1)$ & $29(1)$ & $21(1)$ & $-1(1)$ & $-4(1)$ & $-9(1)$ \\
$\mathrm{C}(11)$ & $33(1)$ & $28(1)$ & $20(1)$ & $0(1)$ & $-3(1)$ & $-8(1)$ \\
$\mathrm{C}(12)$ & $33(1)$ & $30(1)$ & $21(1)$ & $-1(1)$ & $-4(1)$ & $-9(1)$ \\
$\mathrm{C}(13)$ & $32(1)$ & $27(1)$ & $23(1)$ & $-1(1)$ & $-3(1)$ & $-7(1)$ \\
$\mathrm{C}(14)$ & $32(1)$ & $28(1)$ & $23(1)$ & $-2(1)$ & $-3(1)$ & $-9(1)$ \\
$\mathrm{C}(15)$ & $32(1)$ & $26(1)$ & $23(1)$ & $-1(1)$ & $-3(1)$ & $-7(1)$ \\
$\mathrm{C}(16)$ & $31(1)$ & $28(1)$ & $25(1)$ & $-3(1)$ & $-3(1)$ & $-8(1)$ \\
$\mathrm{C}(17)$ & $31(1)$ & $27(1)$ & $26(1)$ & $-1(1)$ & $-4(1)$ & $-7(1)$ \\
$\mathrm{C}(18)$ & $31(1)$ & $27(1)$ & $27(1)$ & $-2(1)$ & $-3(1)$ & $-8(1)$ \\
$\mathrm{C}(19)$ & $30(1)$ & $28(1)$ & $29(1)$ & $-1(1)$ & $-4(1)$ & $-7(1)$ \\
$\mathrm{C}(20)$ & $31(1)$ & $28(1)$ & $30(1)$ & $-4(1)$ & $-4(1)$ & $-9(1)$ \\
$\mathrm{C}(21)$ & $30(1)$ & $28(1)$ & $32(1)$ & $-1(1)$ & $-5(1)$ & $-8(1)$ \\
$\mathrm{C}(22)$ & $34(1)$ & $35(1)$ & $34(1)$ & $-3(1)$ & $-6(1)$ & $-12(1)$ \\
$\mathrm{C}(23)$ & $39(1)$ & $47(1)$ & $44(1)$ & $0(1)$ & $-14(1)$ & $-15(1)$ \\
\hline
\end{tabular}


Table A 11. Hydrogen coordinates ( $\left.x 10^{4}\right)$ and isotropic displacement parameters $\left(\AA^{2} \times 10^{3}\right)$ for 66 .

\begin{tabular}{|c|c|c|c|c|}
\hline & $\mathrm{x}$ & $\mathrm{y}$ & $\mathrm{z}$ & $\mathrm{U}(\mathrm{eq})$ \\
\hline $\mathrm{H}(1 \mathrm{~A})$ & 10334 & 2389 & 1272 & 49 \\
\hline $\mathrm{H}(1 \mathrm{~B})$ & 8444 & 2149 & 1634 & 49 \\
\hline $\mathrm{H}(2 \mathrm{~A})$ & 6285 & 5047 & 1426 & 38 \\
\hline $\mathrm{H}(2 \mathrm{~B})$ & 8118 & 5131 & 1701 & 38 \\
\hline $\mathrm{H}(3 \mathrm{~A})$ & 7921 & 5172 & 462 & 30 \\
\hline $\mathrm{H}(3 \mathrm{~B})$ & 9718 & 5293 & 742 & 30 \\
\hline $\mathrm{H}(4 \mathrm{~A})$ & 7437 & 9641 & 308 & 47 \\
\hline $\mathrm{H}(4 \mathrm{~B})$ & 7909 & 7879 & -63 & 47 \\
\hline $\mathrm{H}(4 \mathrm{C})$ & 9529 & 7944 & 318 & 47 \\
\hline $\mathrm{H}(5 \mathrm{~A})$ & 4300 & 9488 & 778 & 53 \\
\hline $\mathrm{H}(5 \mathrm{~B})$ & 4378 & 7707 & 1100 & 53 \\
\hline $\mathrm{H}(5 \mathrm{C})$ & 4894 & 7718 & 397 & 53 \\
\hline $\mathrm{H}(6 \mathrm{~A})$ & 6639 & 8024 & 1727 & 32 \\
\hline $\mathrm{H}(6 \mathrm{~B})$ & 6548 & 9764 & 1377 & 32 \\
\hline $\mathrm{H}(7 \mathrm{~A})$ & 9956 & 8777 & 1230 & 31 \\
\hline $\mathrm{H}(7 \mathrm{~B})$ & 10297 & 6772 & 1386 & 31 \\
\hline $\mathrm{H}(8 \mathrm{~A})$ & 8541 & 9624 & 2249 & 33 \\
\hline $\mathrm{H}(8 \mathrm{~B})$ & 9097 & 7571 & 2390 & 33 \\
\hline $\mathrm{H}(9 \mathrm{~A})$ & 11925 & 9065 & 2010 & 34 \\
\hline $\mathrm{H}(9 \mathrm{~B})$ & 12582 & 6967 & 2056 & 34 \\
\hline $\mathrm{H}(10 \mathrm{~A})$ & 10874 & 9385 & 3047 & 35 \\
\hline $\mathrm{H}(10 \mathrm{~B})$ & 11552 & 7282 & 3091 & 35 \\
\hline $\mathrm{H}(11 \mathrm{~A})$ & 14245 & 8933 & 2740 & 35 \\
\hline $\mathrm{H}(11 \mathrm{~B})$ & 14939 & 6826 & 2766 & 35 \\
\hline $\mathrm{H}(12 \mathrm{~A})$ & 13274 & 9156 & 3780 & 35 \\
\hline $\mathrm{H}(12 \mathrm{~B})$ & 13977 & 7049 & 3805 & 35 \\
\hline $\mathrm{H}(13 \mathrm{~A})$ & 16639 & 8736 & 3465 & 35 \\
\hline $\mathrm{H}(13 \mathrm{~B})$ & 17345 & 6627 & 3487 & 35 \\
\hline $\mathrm{H}(14 \mathrm{~A})$ & 15661 & 8940 & 4503 & 35 \\
\hline
\end{tabular}




\begin{tabular}{lllll}
$\mathrm{H}(14 \mathrm{~B})$ & 16374 & 6832 & 4524 & 35 \\
$\mathrm{H}(15 \mathrm{~A})$ & 19742 & 6424 & 4214 & 35 \\
$\mathrm{H}(15 \mathrm{~B})$ & 19029 & 8533 & 4194 & 35 \\
$\mathrm{H}(16 \mathrm{~A})$ & 18036 & 8733 & 5230 & 35 \\
$\mathrm{H}(16 \mathrm{~B})$ & 18756 & 6624 & 5250 & 35 \\
$\mathrm{H}(17 \mathrm{~A})$ & 21400 & 8333 & 4925 & 36 \\
$\mathrm{H}(17 \mathrm{~B})$ & 22119 & 6224 & 4946 & 36 \\
$\mathrm{H}(18 \mathrm{~A})$ & 20395 & 8539 & 5961 & 36 \\
$\mathrm{H}(18 B)$ & 21109 & 6431 & 5983 & 36 \\
$\mathrm{H}(19 \mathrm{~A})$ & 23768 & 8128 & 5661 & 37 \\
$\mathrm{H}(19 B)$ & 24478 & 6021 & 5687 & 37 \\
$\mathrm{H}(20 \mathrm{~A})$ & 22750 & 8363 & 6696 & 37 \\
$\mathrm{H}(20 \mathrm{~B})$ & 23437 & 6259 & 6724 & 37 \\
$\mathrm{H}(21 \mathrm{~A})$ & 26134 & 7916 & 6400 & 38 \\
$\mathrm{H}(21 \mathrm{~B})$ & 26819 & 5812 & 6430 & 38 \\
$\mathrm{H}(22 \mathrm{~A})$ & 25108 & 8172 & 7433 & 42 \\
$\mathrm{H}(22 \mathrm{~B})$ & 25771 & 6070 & 7465 & 42 \\
$\mathrm{H}(23 \mathrm{~A})$ & 28269 & 6718 & 7769 & 65 \\
$\mathrm{H}(23 \mathrm{~B})$ & 28465 & 7736 & 7168 & 65 \\
$\mathrm{H}(23 \mathrm{C})$ & 29169 & 5625 & 7156 & 65 \\
$\mathrm{H}(1 \mathrm{O})$ & $2390(50)$ & $778(17)$ & $70(11)$ \\
\hline
\end{tabular}

Table A 12. Torsion angles $\left[^{\circ}\right]$ for 66.

\begin{tabular}{lc}
\hline $\mathrm{O}(1)-\mathrm{C}(1)-\mathrm{C}(2)-\mathrm{C}(3)$ & $-54.0(3)$ \\
$\mathrm{C}(1)-\mathrm{C}(2)-\mathrm{C}(3)-\mathrm{N}(1)$ & $178.88(18)$ \\
$\mathrm{C}(5)-\mathrm{N}(1)-\mathrm{C}(3)-\mathrm{C}(2)$ & $-72.3(2)$ \\
$\mathrm{C}(4)-\mathrm{N}(1)-\mathrm{C}(3)-\mathrm{C}(2)$ & $170.31(18)$ \\
$\mathrm{C}(6)-\mathrm{N}(1)-\mathrm{C}(3)-\mathrm{C}(2)$ & $49.0(2)$ \\
$\mathrm{C}(5)-\mathrm{N}(1)-\mathrm{C}(6)-\mathrm{C}(7)$ & $-178.89(17)$ \\
$\mathrm{C}(4)-\mathrm{N}(1)-\mathrm{C}(6)-\mathrm{C}(7)$ & $-60.6(2)$ \\
$\mathrm{C}(3)-\mathrm{N}(1)-\mathrm{C}(6)-\mathrm{C}(7)$ & $59.4(2)$ \\
$\mathrm{N}(1)-\mathrm{C}(6)-\mathrm{C}(7)-\mathrm{C}(8)$ & $-162.72(16)$ \\
$\mathrm{C}(6)-\mathrm{C}(7)-\mathrm{C}(8)-\mathrm{C}(9)$ & $-174.78(17)$ \\
343 &
\end{tabular}




$\begin{array}{lc}\mathrm{C}(7)-\mathrm{C}(8)-\mathrm{C}(9)-\mathrm{C}(10) & -172.08(17) \\ \mathrm{C}(8)-\mathrm{C}(9)-\mathrm{C}(10)-\mathrm{C}(11) & -179.50(17) \\ \mathrm{C}(9)-\mathrm{C}(10)-\mathrm{C}(11)-\mathrm{C}(12) & -178.52(18) \\ \mathrm{C}(10)-\mathrm{C}(11)-\mathrm{C}(12)-\mathrm{C}(13) & -179.76(18) \\ \mathrm{C}(11)-\mathrm{C}(12)-\mathrm{C}(13)-\mathrm{C}(14) & -179.72(18) \\ \mathrm{C}(12)-\mathrm{C}(13)-\mathrm{C}(14)-\mathrm{C}(15) & -179.82(18) \\ \mathrm{C}(13)-\mathrm{C}(14)-\mathrm{C}(15)-\mathrm{C}(16) & -179.94(18) \\ \mathrm{C}(14)-\mathrm{C}(15)-\mathrm{C}(16)-\mathrm{C}(17) & -179.86(18) \\ \mathrm{C}(15)-\mathrm{C}(16)-\mathrm{C}(17)-\mathrm{C}(18) & 179.90(18) \\ \mathrm{C}(16)-\mathrm{C}(17)-\mathrm{C}(18)-\mathrm{C}(19) & 179.89(18) \\ \mathrm{C}(17)-\mathrm{C}(18)-\mathrm{C}(19)-\mathrm{C}(20) & 179.61(18) \\ \mathrm{C}(18)-\mathrm{C}(19)-\mathrm{C}(20)-\mathrm{C}(21) & 179.43(18) \\ \mathrm{C}(19)-\mathrm{C}(20)-\mathrm{C}(21)-\mathrm{C}(22) & 179.86(19) \\ \mathrm{C}(20)-\mathrm{C}(21)-\mathrm{C}(22)-\mathrm{C}(23) & 179.4(2)\end{array}$

Symmetry transformations used to generate equivalent atoms:

Table A 13. Hydrogen bonds for 66 [ $\AA$ and $\left.{ }^{\circ}\right]$.

\begin{tabular}{lcccc}
\hline D-H...A & d(D-H) & d(H...A & $d(D . . . A)$ & $<$ (DHA) \\
\hline O(1)-H(1O)...Br(1) & $0.82(4)$ & $2.39(4)$ & $3.206(2)$ & $173(4)$ \\
\hline
\end{tabular}

Symmetry transformations used to generate equivalent atoms:

Table A 14. Crystal data and structure refinement for 19.

Identification code

Empirical formula

Formula weight

Temperature

Wavelength

Crystal system

Space group

Unit cell dimensions d12321

$\mathrm{C}_{16} \mathrm{H}_{22} \mathrm{~N} \mathrm{O}_{5} \mathrm{P}$

339.32

150(2) K

$1.54178 \AA$

Monoclinic

P 21/n

$\mathrm{a}=12.9548(6) \AA$ $\alpha=90^{\circ}$. 
Volume

Z

Density (calculated)

Absorption coefficient

$\mathrm{F}(000)$

Crystal size

Theta range for data collection

Index ranges

Reflections collected

Independent reflections

Completeness to theta $=66.38^{\circ}$

Absorption correction

Max. and min. transmission

Refinement method

Data / restraints / parameters

Goodness-of-fit on $\mathrm{F}^{2}$

Final R indices [I $>2 \operatorname{sigma(I)]~}$

$\mathrm{R}$ indices (all data)

Largest diff. peak and hole $\mathrm{b}=8.0163(4) \AA$

$\beta=91.100(2)^{\circ}$.

c $=16.5747(8) \AA$ $\gamma=90^{\circ}$.
1720.96(14) $\AA^{3}$

4

$1.310 \mathrm{Mg} / \mathrm{m}^{3}$

$1.633 \mathrm{~mm}^{-1}$

720

$0.32 \times 0.12 \times 0.09 \mathrm{~mm}^{3}$

4.29 to $66.38^{\circ}$.

$-15<=\mathrm{h}<=15,-9<=\mathrm{k}<=4,-19<=\mathrm{l}<=19$

11311

$2936[\mathrm{R}(\mathrm{int})=0.0258]$

$96.8 \%$

Semi-empirical from equivalents

0.7528 and 0.6679

Full-matrix least-squares on $\mathrm{F}^{2}$

2936 / 0 / 210

1.059

$\mathrm{R} 1=0.0386, \mathrm{wR} 2=0.0996$

$\mathrm{R} 1=0.0398, \mathrm{wR} 2=0.1007$

0.570 and -0.438 e. $\AA^{-}-3$

Table A 15. Atomic coordinates ( $x 10^{4}$ ) and equivalent isotropic displacement parameters $\left(\AA^{2} \times 10^{3}\right)$ for 19 . $U(e q)$ is defined as one third of the trace of the orthogonalized $U^{i j}$ tensor.

\begin{tabular}{lrrrr}
\hline & $\mathrm{x}$ & $\mathrm{y}$ & $\mathrm{z}$ & $\mathrm{U}(\mathrm{eq})$ \\
\hline $\mathrm{P}(1)$ & $5032(1)$ & $2830(1)$ & $1022(1)$ & $25(1)$ \\
$\mathrm{O}(1)$ & $-396(1)$ & $5618(2)$ & $1055(1)$ & $48(1)$ \\
$\mathrm{O}(2)$ & $1813(1)$ & $9911(2)$ & $1635(1)$ & $40(1)$ \\
$\mathrm{O}(3)$ & $5702(1)$ & $2250(2)$ & $375(1)$ & $34(1)$ \\
$\mathrm{O}(4)$ & $4675(1)$ & $1417(2)$ & $1618(1)$ & $30(1)$ \\
$\mathrm{O}(5)$ & $5542(1)$ & $4128(2)$ & $1622(1)$ & $32(1)$ \\
$\mathrm{N}(1)$ & $894(1)$ & $7480(2)$ & $1408(1)$ & $30(1)$
\end{tabular}




\begin{tabular}{lrrrr}
$\mathrm{C}(1)$ & $-83(1)$ & $7026(2)$ & $1120(1)$ & $31(1)$ \\
$\mathrm{C}(2)$ & $-626(1)$ & $8625(2)$ & $933(1)$ & $25(1)$ \\
$\mathrm{C}(3)$ & $-1613(1)$ & $8909(2)$ & $636(1)$ & $31(1)$ \\
$\mathrm{C}(4)$ & $-1919(1)$ & $10561(2)$ & $534(1)$ & $32(1)$ \\
$\mathrm{C}(5)$ & $-1250(2)$ & $11859(2)$ & $718(1)$ & $32(1)$ \\
$\mathrm{C}(6)$ & $-252(1)$ & $11566(2)$ & $1011(1)$ & $29(1)$ \\
$\mathrm{C}(7)$ & $39(1)$ & $9925(2)$ & $1116(1)$ & $24(1)$ \\
$\mathrm{C}(8)$ & $1032(1)$ & $9201(2)$ & $1418(1)$ & $27(1)$ \\
$\mathrm{C}(9)$ & $1673(2)$ & $6275(3)$ & $1682(1)$ & $38(1)$ \\
$\mathrm{C}(10)$ & $2339(1)$ & $5647(2)$ & $1004(1)$ & $29(1)$ \\
$\mathrm{C}(11)$ & $3170(1)$ & $4463(2)$ & $1327(1)$ & $32(1)$ \\
$\mathrm{C}(12)$ & $3907(1)$ & $3914(2)$ & $671(1)$ & $28(1)$ \\
$\mathrm{C}(13)$ & $4434(2)$ & $-244(2)$ & $1316(1)$ & $36(1)$ \\
$\mathrm{C}(14)$ & $5232(2)$ & $-1432(3)$ & $1619(2)$ & $61(1)$ \\
$\mathrm{C}(15)$ & $6535(1)$ & $3712(3)$ & $1986(1)$ & $37(1)$ \\
$\mathrm{C}(16)$ & $7137(2)$ & $5228(3)$ & $2104(2)$ & $74(1)$ \\
\hline
\end{tabular}

Table A 16. Bond lengths $[\AA]$ and angles $\left[{ }^{\circ}\right]$ for 19.

\begin{tabular}{ll}
\hline $\mathrm{P}(1)-\mathrm{O}(3)$ & $1.4687(13)$ \\
$\mathrm{P}(1)-\mathrm{O}(5)$ & $1.5765(13)$ \\
$\mathrm{P}(1)-\mathrm{O}(4)$ & $1.5782(12)$ \\
$\mathrm{P}(1)-\mathrm{C}(12)$ & $1.7837(17)$ \\
$\mathrm{O}(1)-\mathrm{C}(1)$ & $1.204(2)$ \\
$\mathrm{O}(2)-\mathrm{C}(8)$ & $1.210(2)$ \\
$\mathrm{O}(4)-\mathrm{C}(13)$ & $1.454(2)$ \\
$\mathrm{O}(5)-\mathrm{C}(15)$ & $1.450(2)$ \\
$\mathrm{N}(1)-\mathrm{C}(8)$ & $1.390(2)$ \\
$\mathrm{N}(1)-\mathrm{C}(1)$ & $1.393(2)$ \\
$\mathrm{N}(1)-\mathrm{C}(9)$ & $1.463(2)$ \\
$\mathrm{C}(1)-\mathrm{C}(2)$ & $1.492(2)$ \\
$\mathrm{C}(2)-\mathrm{C}(3)$ & $1.381(2)$ \\
$\mathrm{C}(2)-\mathrm{C}(7)$ & $1.381(2)$ \\
$\mathrm{C}(3)-\mathrm{C}(4)$ & $1.392(3)$ \\
346
\end{tabular}




$\begin{array}{ll}\mathrm{C}(3)-\mathrm{H}(3 \mathrm{~A}) & 0.9500 \\ \mathrm{C}(4)-\mathrm{C}(5) & 1.384(3) \\ \mathrm{C}(4)-\mathrm{H}(4 \mathrm{~A}) & 0.9500 \\ \mathrm{C}(5)-\mathrm{C}(6) & 1.392(3) \\ \mathrm{C}(5)-\mathrm{H}(5 \mathrm{~A}) & 0.9500 \\ \mathrm{C}(6)-\mathrm{C}(7) & 1.379(2) \\ \mathrm{C}(6)-\mathrm{H}(6 \mathrm{~A}) & 0.9500 \\ \mathrm{C}(7)-\mathrm{C}(8) & 1.489(2) \\ \mathrm{C}(9)-\mathrm{C}(10) & 1.515(2) \\ \mathrm{C}(9)-\mathrm{H}(9 \mathrm{~A}) & 0.9900 \\ \mathrm{C}(9)-\mathrm{H}(9 \mathrm{~B}) & 0.9900 \\ \mathrm{C}(10)-\mathrm{C}(11) & 1.524(2) \\ \mathrm{C}(10)-\mathrm{H}(10 \mathrm{~A}) & 0.9900 \\ \mathrm{C}(10)-\mathrm{H}(10 \mathrm{~B}) & 0.9900 \\ \mathrm{C}(11)-\mathrm{C}(12) & 1.525(2) \\ \mathrm{C}(11)-\mathrm{H}(11 \mathrm{~A}) & 0.9900 \\ \mathrm{C}(11)-\mathrm{H}(11 \mathrm{~B}) & 0.9900 \\ \mathrm{C}(12)-\mathrm{H}(12 \mathrm{~A}) & 0.9900 \\ \mathrm{C}(12)-\mathrm{H}(12 \mathrm{~B}) & 0.9900 \\ \mathrm{C}(13)-\mathrm{C}(14) & 1.486(3) \\ \mathrm{C}(13)-\mathrm{H}(13 \mathrm{~A}) & 0.9900 \\ \mathrm{C}(13)-\mathrm{H}(13 \mathrm{~B}) & 0.9900 \\ \mathrm{C}(14)-\mathrm{H}(14 \mathrm{~A}) & 0.9800 \\ \mathrm{C}(14)-\mathrm{H}(14 \mathrm{~B}) & 0.9800 \\ \mathrm{C}(14)-\mathrm{H}(14 \mathrm{C}) & 0.9800 \\ \mathrm{C}(15)-\mathrm{C}(16) & 1.455(3) \\ \mathrm{C}(15)-\mathrm{H}(15 \mathrm{~A}) & 0.9900 \\ \mathrm{C}(15)-\mathrm{H}(15 \mathrm{~B}) & 0.9900 \\ \mathrm{C}(16)-\mathrm{H}(16 \mathrm{~A}) & 0.9800 \\ \mathrm{C}(16)-\mathrm{H}(16 \mathrm{~B}) & 0.9800 \\ \mathrm{C}(16)-\mathrm{H}(16 \mathrm{C}) & 0.9800 \\ \mathrm{O}(3)-\mathrm{P}(1)-\mathrm{O}(5) & 115.03(7) \\ \mathrm{O}(3)-\mathrm{P}(1)-\mathrm{O}(4) & 114.48(7) \\ \mathrm{O}(5)-\mathrm{P}(1)-\mathrm{O}(4) & 101.70(7) \\ \mathrm{O}(3)-\mathrm{P}(1)-\mathrm{C}(12) & 114.03(8) \\ 347 & \\ & \end{array}$




\begin{tabular}{|c|c|}
\hline $\mathrm{O}(5)-\mathrm{P}(1)-\mathrm{C}(12)$ & $102.37(8)$ \\
\hline $\mathrm{O}(4)-\mathrm{P}(1)-\mathrm{C}(12)$ & $107.84(8)$ \\
\hline $\mathrm{C}(13)-\mathrm{O}(4)-\mathrm{P}(1)$ & $120.34(11)$ \\
\hline $\mathrm{C}(15)-\mathrm{O}(5)-\mathrm{P}(1)$ & $118.00(11)$ \\
\hline $\mathrm{C}(8)-\mathrm{N}(1)-\mathrm{C}(1)$ & $112.26(14)$ \\
\hline $\mathrm{C}(8)-\mathrm{N}(1)-\mathrm{C}(9)$ & 124.31(16) \\
\hline $\mathrm{C}(1)-\mathrm{N}(1)-\mathrm{C}(9)$ & 123.41(16) \\
\hline $\mathrm{O}(1)-\mathrm{C}(1)-\mathrm{N}(1)$ & $125.33(17)$ \\
\hline $\mathrm{O}(1)-\mathrm{C}(1)-\mathrm{C}(2)$ & $129.12(17)$ \\
\hline $\mathrm{N}(1)-\mathrm{C}(1)-\mathrm{C}(2)$ & $105.54(15)$ \\
\hline$C(3)-C(2)-C(7)$ & $121.56(16)$ \\
\hline $\mathrm{C}(3)-\mathrm{C}(2)-\mathrm{C}(1)$ & $130.18(17)$ \\
\hline$C(7)-C(2)-C(1)$ & 108.26(15) \\
\hline$C(2)-C(3)-C(4)$ & $117.36(17)$ \\
\hline$C(2)-C(3)-H(3 A)$ & 121.3 \\
\hline $\mathrm{C}(4)-\mathrm{C}(3)-\mathrm{H}(3 \mathrm{~A})$ & 121.3 \\
\hline$C(5)-C(4)-C(3)$ & $120.86(16)$ \\
\hline $\mathrm{C}(5)-\mathrm{C}(4)-\mathrm{H}(4 \mathrm{~A})$ & 119.6 \\
\hline $\mathrm{C}(3)-\mathrm{C}(4)-\mathrm{H}(4 \mathrm{~A})$ & 119.6 \\
\hline$C(4)-C(5)-C(6)$ & $121.55(17)$ \\
\hline $\mathrm{C}(4)-\mathrm{C}(5)-\mathrm{H}(5 \mathrm{~A})$ & 119.2 \\
\hline $\mathrm{C}(6)-\mathrm{C}(5)-\mathrm{H}(5 \mathrm{~A})$ & 119.2 \\
\hline$C(7)-C(6)-C(5)$ & $117.08(17)$ \\
\hline$C(7)-C(6)-H(6 A)$ & 121.5 \\
\hline $\mathrm{C}(5)-\mathrm{C}(6)-\mathrm{H}(6 \mathrm{~A})$ & 121.5 \\
\hline$C(6)-C(7)-C(2)$ & $121.59(16)$ \\
\hline$C(6)-C(7)-C(8)$ & $130.32(16)$ \\
\hline$C(2)-C(7)-C(8)$ & $108.09(15)$ \\
\hline $\mathrm{O}(2)-\mathrm{C}(8)-\mathrm{N}(1)$ & $125.22(16)$ \\
\hline $\mathrm{O}(2)-\mathrm{C}(8)-\mathrm{C}(7)$ & $128.94(17)$ \\
\hline $\mathrm{N}(1)-\mathrm{C}(8)-\mathrm{C}(7)$ & $105.84(15)$ \\
\hline $\mathrm{N}(1)-\mathrm{C}(9)-\mathrm{C}(10)$ & $112.86(14)$ \\
\hline $\mathrm{N}(1)-\mathrm{C}(9)-\mathrm{H}(9 \mathrm{~A})$ & 109.0 \\
\hline $\mathrm{C}(10)-\mathrm{C}(9)-\mathrm{H}(9 \mathrm{~A})$ & 109.0 \\
\hline $\mathrm{N}(1)-\mathrm{C}(9)-\mathrm{H}(9 \mathrm{~B})$ & 109.0 \\
\hline
\end{tabular}




\begin{tabular}{|c|c|}
\hline $\mathrm{C}(10)-\mathrm{C}(9)-\mathrm{H}(9 \mathrm{~B})$ & 109.0 \\
\hline $\mathrm{H}(9 \mathrm{~A})-\mathrm{C}(9)-\mathrm{H}(9 \mathrm{~B})$ & 107.8 \\
\hline $\mathrm{C}(9)-\mathrm{C}(10)-\mathrm{C}(11)$ & $110.82(14)$ \\
\hline $\mathrm{C}(9)-\mathrm{C}(10)-\mathrm{H}(10 \mathrm{~A})$ & 109.5 \\
\hline $\mathrm{C}(11)-\mathrm{C}(10)-\mathrm{H}(10 \mathrm{~A})$ & 109.5 \\
\hline $\mathrm{C}(9)-\mathrm{C}(10)-\mathrm{H}(10 \mathrm{~B})$ & 109.5 \\
\hline $\mathrm{C}(11)-\mathrm{C}(10)-\mathrm{H}(10 \mathrm{~B})$ & 109.5 \\
\hline $\mathrm{H}(10 \mathrm{~A})-\mathrm{C}(10)-\mathrm{H}(10 \mathrm{~B})$ & 108.1 \\
\hline$C(10)-C(11)-C(12)$ & $112.22(14)$ \\
\hline $\mathrm{C}(10)-\mathrm{C}(11)-\mathrm{H}(11 \mathrm{~A})$ & 109.2 \\
\hline $\mathrm{C}(12)-\mathrm{C}(11)-\mathrm{H}(11 \mathrm{~A})$ & 109.2 \\
\hline $\mathrm{C}(10)-\mathrm{C}(11)-\mathrm{H}(11 \mathrm{~B})$ & 109.2 \\
\hline $\mathrm{C}(12)-\mathrm{C}(11)-\mathrm{H}(11 \mathrm{~B})$ & 109.2 \\
\hline $\mathrm{H}(11 \mathrm{~A})-\mathrm{C}(11)-\mathrm{H}(11 \mathrm{~B})$ & 107.9 \\
\hline $\mathrm{C}(11)-\mathrm{C}(12)-\mathrm{P}(1)$ & $115.29(12)$ \\
\hline $\mathrm{C}(11)-\mathrm{C}(12)-\mathrm{H}(12 \mathrm{~A})$ & 108.5 \\
\hline $\mathrm{P}(1)-\mathrm{C}(12)-\mathrm{H}(12 \mathrm{~A})$ & 108.5 \\
\hline $\mathrm{C}(11)-\mathrm{C}(12)-\mathrm{H}(12 \mathrm{~B})$ & 108.5 \\
\hline $\mathrm{P}(1)-\mathrm{C}(12)-\mathrm{H}(12 \mathrm{~B})$ & 108.5 \\
\hline $\mathrm{H}(12 \mathrm{~A})-\mathrm{C}(12)-\mathrm{H}(12 \mathrm{~B})$ & 107.5 \\
\hline $\mathrm{O}(4)-\mathrm{C}(13)-\mathrm{C}(14)$ & 109.08(16) \\
\hline $\mathrm{O}(4)-\mathrm{C}(13)-\mathrm{H}(13 \mathrm{~A})$ & 109.9 \\
\hline $\mathrm{C}(14)-\mathrm{C}(13)-\mathrm{H}(13 \mathrm{~A})$ & 109.9 \\
\hline $\mathrm{O}(4)-\mathrm{C}(13)-\mathrm{H}(13 \mathrm{~B})$ & 109.9 \\
\hline $\mathrm{C}(14)-\mathrm{C}(13)-\mathrm{H}(13 \mathrm{~B})$ & 109.9 \\
\hline $\mathrm{H}(13 \mathrm{~A})-\mathrm{C}(13)-\mathrm{H}(13 \mathrm{~B})$ & 108.3 \\
\hline $\mathrm{C}(13)-\mathrm{C}(14)-\mathrm{H}(14 \mathrm{~A})$ & 109.5 \\
\hline $\mathrm{C}(13)-\mathrm{C}(14)-\mathrm{H}(14 \mathrm{~B})$ & 109.5 \\
\hline $\mathrm{H}(14 \mathrm{~A})-\mathrm{C}(14)-\mathrm{H}(14 \mathrm{~B})$ & 109.5 \\
\hline $\mathrm{C}(13)-\mathrm{C}(14)-\mathrm{H}(14 \mathrm{C})$ & 109.5 \\
\hline $\mathrm{H}(14 \mathrm{~A})-\mathrm{C}(14)-\mathrm{H}(14 \mathrm{C})$ & 109.5 \\
\hline $\mathrm{H}(14 \mathrm{~B})-\mathrm{C}(14)-\mathrm{H}(14 \mathrm{C})$ & 109.5 \\
\hline $\mathrm{O}(5)-\mathrm{C}(15)-\mathrm{C}(16)$ & 109.45(18) \\
\hline $\mathrm{O}(5)-\mathrm{C}(15)-\mathrm{H}(15 \mathrm{~A})$ & 109.8 \\
\hline $\mathrm{C}(16)-\mathrm{C}(15)-\mathrm{H}(15 \mathrm{~A})$ & 109.8 \\
\hline
\end{tabular}


$\mathrm{O}(5)-\mathrm{C}(15)-\mathrm{H}(15 \mathrm{~B}) \quad 109.8$

$\mathrm{C}(16)-\mathrm{C}(15)-\mathrm{H}(15 \mathrm{~B}) \quad 109.8$

H(15A)-C(15)-H(15B) 108.2

C(15)-C(16)-H(16A) 109.5

C(15)-C(16)-H(16B) 109.5

H(16A)-C(16)-H(16B) 109.5

C(15)-C(16)-H(16C) 109.5

H(16A)-C(16)-H(16C) 109.5

H(16B)-C(16)-H(16C) 109.5

Symmetry transformations used to generate equivalent atoms:

Table A 17. Anisotropic displacement parameters $\left(\AA^{2} \times 10^{3}\right)$ for 19. The anisotropic displacement factor exponent takes the form: $-2 p^{2}\left[h^{2} a^{* 2} U^{11}+\ldots+2 h k\right.$ a* b* $\left.U^{12}\right]$

\begin{tabular}{lcccccc}
\hline & $\mathrm{U} 11$ & $\mathrm{U}^{22}$ & $\mathrm{U}^{33}$ & $\mathrm{U}^{23}$ & $\mathrm{U} 13$ & $\mathrm{U}$ \\
\hline $\mathrm{P}(1)$ & $22(1)$ & $23(1)$ & $30(1)$ & $0(1)$ & $1(1)$ & $6(1)$ \\
$\mathrm{O}(1)$ & $47(1)$ & $24(1)$ & $71(1)$ & $2(1)$ & $4(1)$ & $4(1)$ \\
$\mathrm{O}(2)$ & $25(1)$ & $55(1)$ & $38(1)$ & $-3(1)$ & $-5(1)$ & $0(1)$ \\
$\mathrm{O}(3)$ & $29(1)$ & $36(1)$ & $38(1)$ & $0(1)$ & $7(1)$ & $10(1)$ \\
$\mathrm{O}(4)$ & $35(1)$ & $23(1)$ & $33(1)$ & $0(1)$ & $3(1)$ & $4(1)$ \\
$\mathrm{O}(5)$ & $26(1)$ & $26(1)$ & $44(1)$ & $-3(1)$ & $-7(1)$ & $5(1)$ \\
$\mathrm{N}(1)$ & $26(1)$ & $33(1)$ & $31(1)$ & $5(1)$ & $2(1)$ & $13(1)$ \\
$\mathrm{C}(1)$ & $29(1)$ & $28(1)$ & $36(1)$ & $2(1)$ & $6(1)$ & $8(1)$ \\
$\mathrm{C}(2)$ & $23(1)$ & $26(1)$ & $27(1)$ & $0(1)$ & $4(1)$ & $5(1)$ \\
$\mathrm{C}(3)$ & $23(1)$ & $36(1)$ & $35(1)$ & $-1(1)$ & $1(1)$ & $2(1)$ \\
$\mathrm{C}(4)$ & $24(1)$ & $44(1)$ & $30(1)$ & $3(1)$ & $2(1)$ & $13(1)$ \\
$\mathrm{C}(5)$ & $36(1)$ & $29(1)$ & $32(1)$ & $4(1)$ & $6(1)$ & $14(1)$ \\
$\mathrm{C}(6)$ & $32(1)$ & $26(1)$ & $30(1)$ & $-1(1)$ & $5(1)$ & $3(1)$ \\
$\mathrm{C}(7)$ & $22(1)$ & $28(1)$ & $22(1)$ & $0(1)$ & $4(1)$ & $4(1)$ \\
$\mathrm{C}(8)$ & $24(1)$ & $37(1)$ & $21(1)$ & $0(1)$ & $3(1)$ & $6(1)$ \\
$\mathrm{C}(9)$ & $34(1)$ & $46(1)$ & $33(1)$ & $10(1)$ & $4(1)$ & $23(1)$ \\
$\mathrm{C}(10)$ & $26(1)$ & $32(1)$ & $28(1)$ & $5(1)$ & $1(1)$ & $10(1)$ \\
$\mathrm{C}(11)$ & $27(1)$ & $37(1)$ & $31(1)$ & $8(1)$ & $4(1)$ & $13(1)$ \\
& & & & & \\
\end{tabular}




$\begin{array}{llllclc}\mathrm{C}(12) & 24(1) & 33(1) & 28(1) & 1(1) & 0(1) & 7(1) \\ \mathrm{C}(13) & 35(1) & 27(1) & 46(1) & -4(1) & 2(1) & -4(1) \\ \mathrm{C}(14) & 67(2) & 30(1) & 86(2) & 1(1) & -6(1) & 10(1) \\ \mathrm{C}(15) & 26(1) & 48(1) & 36(1) & -1(1) & -5(1) & 8(1) \\ \mathrm{C}(16) & 40(1) & 50(2) & 132(3) & -12(2) & -24(2) & -3(1)\end{array}$

Table A 18. Hydrogen coordinates ( $x$ 104) and isotropic displacement parameters $\left(\AA^{2} \times 10^{3}\right)$ for 19.

\begin{tabular}{|c|c|c|c|c|}
\hline & $\mathrm{x}$ & $\mathrm{y}$ & $\mathrm{z}$ & $\mathrm{U}(\mathrm{eq})$ \\
\hline $\mathrm{H}(3 \mathrm{~A})$ & -2066 & 8012 & 506 & 37 \\
\hline $\mathrm{H}(4 \mathrm{~A})$ & -2596 & 10801 & 336 & 39 \\
\hline $\mathrm{H}(5 \mathrm{~A})$ & -1477 & 12976 & 643 & 39 \\
\hline $\mathrm{H}(6 \mathrm{~A})$ & 208 & 12458 & 1133 & 35 \\
\hline $\mathrm{H}(9 \mathrm{~A})$ & 1324 & 5315 & 1934 & 45 \\
\hline H(9B) & 2122 & 6804 & 2099 & 45 \\
\hline $\mathrm{H}(10 \mathrm{~A})$ & 2668 & 6605 & 735 & 35 \\
\hline $\mathrm{H}(10 \mathrm{~B})$ & 1900 & 5059 & 600 & 35 \\
\hline $\mathrm{H}(11 \mathrm{~A})$ & 2836 & 3467 & 1559 & 38 \\
\hline $\mathrm{H}(11 \mathrm{~B})$ & 3568 & 5025 & 1764 & 38 \\
\hline $\mathrm{H}(12 \mathrm{~A})$ & 4128 & 4914 & 370 & 34 \\
\hline $\mathrm{H}(12 \mathrm{~B})$ & 3525 & 3184 & 287 & 34 \\
\hline $\mathrm{H}(13 \mathrm{~A})$ & 4421 & -238 & 718 & 43 \\
\hline $\mathrm{H}(13 \mathrm{~B})$ & 3745 & -592 & 1501 & 43 \\
\hline $\mathrm{H}(14 \mathrm{~A})$ & 5056 & -2563 & 1438 & 91 \\
\hline $\mathrm{H}(14 \mathrm{~B})$ & 5259 & -1401 & 2210 & 91 \\
\hline $\mathrm{H}(14 \mathrm{C})$ & 5906 & -1116 & 1409 & 91 \\
\hline $\mathrm{H}(15 \mathrm{~A})$ & 6909 & 2934 & 1632 & 44 \\
\hline $\mathrm{H}(15 \mathrm{~B})$ & 6434 & 3156 & 2512 & 44 \\
\hline $\mathrm{H}(16 \mathrm{~A})$ & 7791 & 4956 & 2380 & 112 \\
\hline $\mathrm{H}(16 \mathrm{~B})$ & 6748 & 6018 & 2432 & 112 \\
\hline
\end{tabular}


Table A 19. Torsion angles $\left[^{\circ}\right]$ for 19.

\begin{tabular}{|c|c|}
\hline $\mathrm{O}(3)-\mathrm{P}(1)-\mathrm{O}(4)-\mathrm{C}(13)$ & $-37.34(15)$ \\
\hline $\mathrm{O}(5)-\mathrm{P}(1)-\mathrm{O}(4)-\mathrm{C}(13)$ & $-162.02(12)$ \\
\hline $\mathrm{C}(12)-\mathrm{P}(1)-\mathrm{O}(4)-\mathrm{C}(13)$ & $90.73(14)$ \\
\hline $\mathrm{O}(3)-\mathrm{P}(1)-\mathrm{O}(5)-\mathrm{C}(15)$ & $-51.29(15)$ \\
\hline $\mathrm{O}(4)-\mathrm{P}(1)-\mathrm{O}(5)-\mathrm{C}(15)$ & 73.03(14) \\
\hline $\mathrm{C}(12)-\mathrm{P}(1)-\mathrm{O}(5)-\mathrm{C}(15)$ & $-175.52(13)$ \\
\hline $\mathrm{C}(8)-\mathrm{N}(1)-\mathrm{C}(1)-\mathrm{O}(1)$ & $-179.67(18)$ \\
\hline $\mathrm{C}(9)-\mathrm{N}(1)-\mathrm{C}(1)-\mathrm{O}(1)$ & $-0.8(3)$ \\
\hline $\mathrm{C}(8)-\mathrm{N}(1)-\mathrm{C}(1)-\mathrm{C}(2)$ & $-0.44(19)$ \\
\hline $\mathrm{C}(9)-\mathrm{N}(1)-\mathrm{C}(1)-\mathrm{C}(2)$ & 178.46(15) \\
\hline $\mathrm{O}(1)-\mathrm{C}(1)-\mathrm{C}(2)-\mathrm{C}(3)$ & $-0.4(3)$ \\
\hline $\mathrm{N}(1)-\mathrm{C}(1)-\mathrm{C}(2)-\mathrm{C}(3)$ & $-179.58(17)$ \\
\hline $\mathrm{O}(1)-\mathrm{C}(1)-\mathrm{C}(2)-\mathrm{C}(7)$ & $179.2(2)$ \\
\hline $\mathrm{N}(1)-\mathrm{C}(1)-\mathrm{C}(2)-\mathrm{C}(7)$ & $-0.04(18)$ \\
\hline$C(7)-C(2)-C(3)-C(4)$ & $-0.7(3)$ \\
\hline$C(1)-C(2)-C(3)-C(4)$ & 178.83(17) \\
\hline$C(2)-C(3)-C(4)-C(5)$ & $0.6(3)$ \\
\hline$C(3)-C(4)-C(5)-C(6)$ & $-0.1(3)$ \\
\hline$C(4)-C(5)-C(6)-C(7)$ & $-0.5(3)$ \\
\hline$C(5)-C(6)-C(7)-C(2)$ & $0.5(2)$ \\
\hline$C(5)-C(6)-C(7)-C(8)$ & $-179.45(16)$ \\
\hline$C(3)-C(2)-C(7)-C(6)$ & $0.1(3)$ \\
\hline$C(1)-C(2)-C(7)-C(6)$ & $-179.48(15)$ \\
\hline$C(3)-C(2)-C(7)-C(8)$ & $-179.95(15)$ \\
\hline$C(1)-C(2)-C(7)-C(8)$ & $0.46(18)$ \\
\hline $\mathrm{C}(1)-\mathrm{N}(1)-\mathrm{C}(8)-\mathrm{O}(2)$ & $-179.32(16)$ \\
\hline $\mathrm{C}(9)-\mathrm{N}(1)-\mathrm{C}(8)-\mathrm{O}(2)$ & $1.8(3)$ \\
\hline $\mathrm{C}(1)-\mathrm{N}(1)-\mathrm{C}(8)-\mathrm{C}(7)$ & $0.71(19)$ \\
\hline $\mathrm{C}(9)-\mathrm{N}(1)-\mathrm{C}(8)-\mathrm{C}(7)$ & $-178.17(14)$ \\
\hline $\mathrm{C}(6)-\mathrm{C}(7)-\mathrm{C}(8)-\mathrm{O}(2)$ & $-0.7(3)$ \\
\hline
\end{tabular}




\begin{tabular}{lc}
$\mathrm{C}(2)-\mathrm{C}(7)-\mathrm{C}(8)-\mathrm{O}(2)$ & $179.32(17)$ \\
$\mathrm{C}(6)-\mathrm{C}(7)-\mathrm{C}(8)-\mathrm{N}(1)$ & $179.22(17)$ \\
$\mathrm{C}(2)-\mathrm{C}(7)-\mathrm{C}(8)-\mathrm{N}(1)$ & $-0.71(18)$ \\
$\mathrm{C}(8)-\mathrm{N}(1)-\mathrm{C}(9)-\mathrm{C}(10)$ & $-93.4(2)$ \\
$\mathrm{C}(1)-\mathrm{N}(1)-\mathrm{C}(9)-\mathrm{C}(10)$ & $87.8(2)$ \\
$\mathrm{N}(1)-\mathrm{C}(9)-\mathrm{C}(10)-\mathrm{C}(11)$ & $177.22(17)$ \\
$\mathrm{C}(9)-\mathrm{C}(10)-\mathrm{C}(11)-\mathrm{C}(12)$ & $-175.49(16)$ \\
$\mathrm{C}(10)-\mathrm{C}(11)-\mathrm{C}(12)-\mathrm{P}(1)$ & $169.45(13)$ \\
$\mathrm{O}(3)-\mathrm{P}(1)-\mathrm{C}(12)-\mathrm{C}(11)$ & $177.35(13)$ \\
$\mathrm{O}(5)-\mathrm{P}(1)-\mathrm{C}(12)-\mathrm{C}(11)$ & $-57.76(15)$ \\
$\mathrm{O}(4)-\mathrm{P}(1)-\mathrm{C}(12)-\mathrm{C}(11)$ & $49.02(16)$ \\
$\mathrm{P}(1)-\mathrm{O}(4)-\mathrm{C}(13)-\mathrm{C}(14)$ & $111.31(18)$ \\
$\mathrm{P}(1)-\mathrm{O}(5)-\mathrm{C}(15)-\mathrm{C}(16)$ & $146.77(19)$ \\
& \\
\hline
\end{tabular}

Symmetry transformations used to generate equivalent atoms: 
Table A 20. Crystal data and structure refinement for 220.

\begin{tabular}{|c|c|c|}
\hline Identification code & \multicolumn{2}{|l|}{$\mathrm{d} 1317$} \\
\hline Empirical formula & \multicolumn{2}{|l|}{$\mathrm{C}_{28} \mathrm{H}_{35} \mathrm{Br} \mathrm{N}_{4} \mathrm{O}_{4} \mathrm{~S}$} \\
\hline Formula weight & \multicolumn{2}{|l|}{603.57} \\
\hline Temperature & \multicolumn{2}{|l|}{$147(2) \mathrm{K}$} \\
\hline Wavelength & \multicolumn{2}{|l|}{$0.71073 \AA$} \\
\hline Crystal system & \multicolumn{2}{|l|}{ Triclinic } \\
\hline Space group & \multicolumn{2}{|l|}{$\mathrm{P}-1$} \\
\hline \multirow[t]{3}{*}{ Unit cell dimensions } & $\mathrm{a}=8.4368(14) \AA$ & $\alpha=103.268(4)^{\circ}$ \\
\hline & $\mathrm{b}=11.2944(19) \AA$ & $\beta=93.320(4)^{\circ}$. \\
\hline & $c=16.383(3) \AA$ & $\gamma=109.880(4)^{\circ}$ \\
\hline Volume & \multicolumn{2}{|l|}{$1413.1(4) \AA^{3}$} \\
\hline $\mathrm{Z}$ & \multicolumn{2}{|l|}{2} \\
\hline Density (calculated) & \multicolumn{2}{|l|}{$1.419 \mathrm{Mg} / \mathrm{m}^{3}$} \\
\hline Absorption coefficient & \multicolumn{2}{|l|}{$1.569 \mathrm{~mm}^{-1}$} \\
\hline $\mathrm{F}(000)$ & \multicolumn{2}{|l|}{628} \\
\hline Crystal size & \multicolumn{2}{|c|}{$0.48 \times 0.22 \times 0.18 \mathrm{~mm}^{3}$} \\
\hline Theta range for data collection & \multicolumn{2}{|l|}{1.29 to $27.55^{\circ}$. } \\
\hline Index ranges & \multicolumn{2}{|c|}{$-10<=\mathrm{h}<=10,-10<=\mathrm{k}<=14,-21<=\mathrm{l}<=19$} \\
\hline Reflections collected & \multicolumn{2}{|l|}{23597} \\
\hline Independent reflections & \multicolumn{2}{|c|}{$6441[\mathrm{R}$ (int) $=0.0272]$} \\
\hline Completeness to theta $=27.55^{\circ}$ & \multicolumn{2}{|l|}{$99.0 \%$} \\
\hline Absorption correction & \multicolumn{2}{|c|}{ Semi-empirical from equivalents } \\
\hline Max. and min. transmission & \multicolumn{2}{|l|}{0.7456 and 0.6341} \\
\hline Refinement method & \multicolumn{2}{|c|}{ Full-matrix least-squares on $\mathrm{F}^{2}$} \\
\hline Data / restraints / parameters & \multicolumn{2}{|l|}{$6441 / 0$ / 345} \\
\hline Goodness-of-fit on $\mathrm{F}^{2}$ & \multicolumn{2}{|l|}{1.063} \\
\hline Final $R$ indices $[\mathrm{I}>2 \operatorname{sigma}(\mathrm{I})]$ & \multicolumn{2}{|c|}{$\mathrm{R} 1=0.0272, \mathrm{wR} 2=0.0728$} \\
\hline $\mathrm{R}$ indices (all data) & \multicolumn{2}{|c|}{$\mathrm{R} 1=0.0304, \mathrm{wR} 2=0.0744$} \\
\hline Largest diff. peak and hole & \multicolumn{2}{|c|}{0.959 and -0.336 e. $\AA^{-3}$} \\
\hline
\end{tabular}


Table A 21. Atomic coordinates ( $\times 10^{4}$ ) and equivalent isotropic displacement parameters $\left(\AA^{2} \times 10^{3}\right)$ for $\mathbf{2 2 0}$. $U(e q)$ is defined as one third of the trace of the orthogonalized $U^{i j}$ tensor.

\begin{tabular}{lrrrr}
\hline & $\mathrm{x}$ & $\mathrm{y}$ & $\mathrm{z}$ & $\mathrm{U}(\mathrm{eq})$ \\
\hline $\mathrm{Br}(1)$ & & & \\
$\mathrm{S}(1)$ & $2188(1)$ & $3861(1)$ & $3729(1)$ & $27(1)$ \\
$\mathrm{O}(1)$ & $3811(1)$ & $6885(1)$ & $2219(1)$ & $20(1)$ \\
$\mathrm{O}(2)$ & $2067(1)$ & $6168(1)$ & $1839(1)$ & $30(1)$ \\
$\mathrm{O}(3)$ & $4506(2)$ & $8277(1)$ & $2356(1)$ & $28(1)$ \\
$\mathrm{O}(4)$ & $8054(2)$ & $1106(1)$ & $4611(1)$ & $34(1)$ \\
$\mathrm{N}(1)$ & $7236(2)$ & $800(1)$ & $1778(1)$ & $36(1)$ \\
$\mathrm{N}(2)$ & $7134(2)$ & $2839(1)$ & $206(1)$ & $26(1)$ \\
$\mathrm{N}(3)$ & $3995(2)$ & $6607(1)$ & $3134(1)$ & $22(1)$ \\
$\mathrm{N}(4)$ & $8227(2)$ & $5296(1)$ & $3634(1)$ & $20(1)$ \\
$\mathrm{C}(1)$ & $7394(2)$ & $1154(1)$ & $3232(1)$ & $24(1)$ \\
$\mathrm{C}(2)$ & $5114(2)$ & $6259(1)$ & $1571(1)$ & $19(1)$ \\
$\mathrm{C}(3)$ & $6334(2)$ & $7135(2)$ & $1266(1)$ & $22(1)$ \\
$\mathrm{C}(4)$ & $7375(2)$ & $6708(2)$ & $725(1)$ & $25(1)$ \\
$\mathrm{C}(5)$ & $7226(2)$ & $5431(2)$ & $524(1)$ & $22(1)$ \\
$\mathrm{C}(6)$ & $6030(2)$ & $4511(1)$ & $856(1)$ & $18(1)$ \\
$\mathrm{C}(7)$ & $5916(2)$ & $3173(2)$ & $667(1)$ & $21(1)$ \\
$\mathrm{C}(8)$ & $4602(2)$ & $2278(2)$ & $916(1)$ & $26(1)$ \\
$\mathrm{C}(9)$ & $3426(2)$ & $2666(2)$ & $1376(1)$ & $26(1)$ \\
$\mathrm{C}(10)$ & $3556(2)$ & $3942(2)$ & $1604(1)$ & $22(1)$ \\
$\mathrm{C}(11)$ & $4877(2)$ & $4903(1)$ & $1358(1)$ & $18(1)$ \\
$\mathrm{C}(12)$ & $8873(2)$ & $3366(2)$ & $668(1)$ & $36(1)$ \\
$\mathrm{C}(13)$ & $6664(3)$ & $1462(2)$ & $-225(1)$ & $33(1)$ \\
$\mathrm{C}(14)$ & $5422(2)$ & $7415(2)$ & $3809(1)$ & $27(1)$ \\
$\mathrm{C}(15)$ & $7060(2)$ & $7128(2)$ & $3723(1)$ & $26(1)$ \\
$\mathrm{C}(16)$ & $6733(2)$ & $5734(2)$ & $3753(1)$ & $22(1)$ \\
$\mathrm{C}(17)$ & $8737(2)$ & $5276(2)$ & $2781(1)$ & $36(1)$ \\
$\mathrm{C}(18)$ & $9745(2)$ & $6169(2)$ & $4284(1)$ & $28(1)$ \\
$\mathrm{C}(19)$ & $7741(2)$ & $3948(2)$ & $3807(1)$ & $28(1)$ \\
$\mathrm{C}(20)$ & $6248(2)$ & $2904(2)$ & $3194(1)$ & $35(1)$ \\
355 & $5946(2)$ & $1570(2)$ & $3342(1)$ & $31(1)$ \\
& & &
\end{tabular}




\begin{tabular}{lrrrl}
$\mathrm{C}(21)$ & $8292(2)$ & $916(2)$ & $3876(1)$ & $24(1)$ \\
$\mathrm{C}(22)$ & $9505(2)$ & $363(1)$ & $3470(1)$ & $22(1)$ \\
$\mathrm{C}(23)$ & $10692(2)$ & $-51(2)$ & $3816(1)$ & $26(1)$ \\
$\mathrm{C}(24)$ & $11596(2)$ & $-594(2)$ & $3260(1)$ & $30(1)$ \\
$\mathrm{C}(25)$ & $11340(2)$ & $-696(2)$ & $2402(1)$ & $32(1)$ \\
$\mathrm{C}(26)$ & $10162(2)$ & $-259(2)$ & $2060(1)$ & $29(1)$ \\
$\mathrm{C}(27)$ & $9251(2)$ & $260(2)$ & $2613(1)$ & $23(1)$ \\
$\mathrm{C}(28)$ & $7873(2)$ & $753(2)$ & $2444(1)$ & $25(1)$ \\
\hline
\end{tabular}

Table A 22. Bond lengths $[\AA]$ and angles $\left[^{\circ}\right]$ for 220.

\begin{tabular}{ll}
\hline $\mathrm{S}(1)-\mathrm{O}(1)$ & $1.4354(12)$ \\
$\mathrm{S}(1)-\mathrm{O}(2)$ & $1.4360(12)$ \\
$\mathrm{S}(1)-\mathrm{N}(2)$ & $1.6106(14)$ \\
$\mathrm{S}(1)-\mathrm{C}(1)$ & $1.7763(15)$ \\
$\mathrm{O}(3)-\mathrm{C}(21)$ & $1.214(2)$ \\
$\mathrm{O}(4)-\mathrm{C}(28)$ & $1.208(2)$ \\
$\mathrm{N}(1)-\mathrm{C}(6)$ & $1.411(2)$ \\
$\mathrm{N}(1)-\mathrm{C}(12)$ & $1.456(2)$ \\
$\mathrm{N}(1)-\mathrm{C}(11)$ & $1.467(2)$ \\
$\mathrm{N}(2)-\mathrm{C}(13)$ & $1.462(2)$ \\
$\mathrm{N}(2)-\mathrm{H}(2 \mathrm{~N})$ & 0.8800 \\
$\mathrm{~N}(3)-\mathrm{C}(16)$ & $1.484(2)$ \\
$\mathrm{N}(3)-\mathrm{C}(17)$ & $1.499(2)$ \\
$\mathrm{N}(3)-\mathrm{C}(15)$ & $1.5101(18)$ \\
$\mathrm{N}(3)-\mathrm{C}(18)$ & $1.534(2)$ \\
$\mathrm{N}(4)-\mathrm{C}(21)$ & $1.391(2)$ \\
$\mathrm{N}(4)-\mathrm{C}(28)$ & $1.398(2)$ \\
$\mathrm{N}(4)-\mathrm{C}(20)$ & $1.457(2)$ \\
$\mathrm{C}(1)-\mathrm{C}(2)$ & $1.372(2)$ \\
$\mathrm{C}(1)-\mathrm{C}(10)$ & $1.431(2)$ \\
$\mathrm{C}(2)-\mathrm{C}(3)$ & $1.404(2)$ \\
$\mathrm{C}(2)-\mathrm{H}(2 \mathrm{~A})$ & 0.9500 \\
$\mathrm{C}(3)-\mathrm{C}(4)$ & $1.363(2)$ \\
356 & \\
& \\
& \\
&
\end{tabular}




\begin{tabular}{|c|c|}
\hline $\mathrm{C}(3)-\mathrm{H}(3 \mathrm{~A})$ & 0.9500 \\
\hline$C(4)-C(5)$ & $1.416(2)$ \\
\hline $\mathrm{C}(4)-\mathrm{H}(4 \mathrm{~A})$ & 0.9500 \\
\hline C(5)-C(10) & $1.427(2)$ \\
\hline$C(5)-C(6)$ & $1.439(2)$ \\
\hline$C(6)-C(7)$ & $1.377(2)$ \\
\hline$C(7)-C(8)$ & $1.405(2)$ \\
\hline $\mathrm{C}(7)-\mathrm{H}(7 \mathrm{~A})$ & 0.9500 \\
\hline $\mathrm{C}(8)-\mathrm{C}(9)$ & $1.367(2)$ \\
\hline $\mathrm{C}(8)-\mathrm{H}(8 \mathrm{~A})$ & 0.9500 \\
\hline $\mathrm{C}(9)-\mathrm{C}(10)$ & $1.418(2)$ \\
\hline $\mathrm{C}(9)-\mathrm{H}(9 \mathrm{~A})$ & 0.9500 \\
\hline $\mathrm{C}(11)-\mathrm{H}(11 \mathrm{~A})$ & 0.9800 \\
\hline $\mathrm{C}(11)-\mathrm{H}(11 \mathrm{~B})$ & 0.9800 \\
\hline $\mathrm{C}(11)-\mathrm{H}(11 \mathrm{C})$ & 0.9800 \\
\hline $\mathrm{C}(12)-\mathrm{H}(12 \mathrm{~A})$ & 0.9800 \\
\hline $\mathrm{C}(12)-\mathrm{H}(12 \mathrm{~B})$ & 0.9800 \\
\hline $\mathrm{C}(12)-\mathrm{H}(12 \mathrm{C})$ & 0.9800 \\
\hline$C(13)-C(14)$ & $1.530(2)$ \\
\hline $\mathrm{C}(13)-\mathrm{H}(13 \mathrm{~A})$ & 0.9900 \\
\hline $\mathrm{C}(13)-\mathrm{H}(13 \mathrm{~B})$ & 0.9900 \\
\hline $\mathrm{C}(14)-\mathrm{C}(15)$ & $1.516(2)$ \\
\hline $\mathrm{C}(14)-\mathrm{H}(14 \mathrm{~A})$ & 0.9900 \\
\hline $\mathrm{C}(14)-\mathrm{H}(14 \mathrm{~B})$ & 0.9900 \\
\hline $\mathrm{C}(15)-\mathrm{H}(15 \mathrm{~A})$ & 0.9900 \\
\hline $\mathrm{C}(15)-\mathrm{H}(15 \mathrm{~B})$ & 0.9900 \\
\hline $\mathrm{C}(16)-\mathrm{H}(16 \mathrm{~A})$ & 0.9800 \\
\hline $\mathrm{C}(16)-\mathrm{H}(16 \mathrm{~B})$ & 0.9800 \\
\hline $\mathrm{C}(16)-\mathrm{H}(16 \mathrm{C})$ & 0.9800 \\
\hline $\mathrm{C}(17)-\mathrm{H}(17 \mathrm{~A})$ & 0.9800 \\
\hline $\mathrm{C}(17)-\mathrm{H}(17 \mathrm{~B})$ & 0.9800 \\
\hline $\mathrm{C}(17)-\mathrm{H}(17 \mathrm{C})$ & 0.9800 \\
\hline C(18)-C(19) & $1.512(2)$ \\
\hline $\mathrm{C}(18)-\mathrm{H}(18 \mathrm{~A})$ & 0.9900 \\
\hline $\mathrm{C}(18)-\mathrm{H}(18 \mathrm{~B})$ & 0.9900 \\
\hline
\end{tabular}




$\begin{array}{lc}\mathrm{C}(19)-\mathrm{C}(20) & 1.519(2) \\ \mathrm{C}(19)-\mathrm{H}(19 \mathrm{~A}) & 0.9900 \\ \mathrm{C}(19)-\mathrm{H}(19 \mathrm{~B}) & 0.9900 \\ \mathrm{C}(20)-\mathrm{H}(20 \mathrm{~A}) & 0.9900 \\ \mathrm{C}(20)-\mathrm{H}(20 \mathrm{~B}) & 0.9900 \\ \mathrm{C}(21)-\mathrm{C}(22) & 1.484(2) \\ \mathrm{C}(22)-\mathrm{C}(27) & 1.381(2) \\ \mathrm{C}(22)-\mathrm{C}(23) & 1.385(2) \\ \mathrm{C}(23)-\mathrm{C}(24) & 1.389(2) \\ \mathrm{C}(23)-\mathrm{H}(23 \mathrm{~A}) & 0.9500 \\ \mathrm{C}(24)-\mathrm{C}(25) & 1.383(3) \\ \mathrm{C}(24)-\mathrm{H}(24 \mathrm{~A}) & 0.9500 \\ \mathrm{C}(25)-\mathrm{C}(26) & 1.393(2) \\ \mathrm{C}(25)-\mathrm{H}(25 \mathrm{~A}) & 0.9500 \\ \mathrm{C}(26)-\mathrm{C}(27) & 1.378(2) \\ \mathrm{C}(26)-\mathrm{H}(26 \mathrm{~A}) & 0.9500 \\ \mathrm{C}(27)-\mathrm{C}(28) & 1.487(2) \\ \mathrm{O}(1)-\mathrm{S}(1)-\mathrm{O}(2) & 119.72(7) \\ \mathrm{O}(1)-\mathrm{S}(1)-\mathrm{N}(2) & 106.44(7) \\ \mathrm{O}(2)-\mathrm{S}(1)-\mathrm{N}(2) & 106.73(7) \\ \mathrm{O}(1)-\mathrm{S}(1)-\mathrm{C}(1) & 107.91(7) \\ \mathrm{O}(2)-\mathrm{S}(1)-\mathrm{C}(1) & 106.14(7) \\ \mathrm{N}(2)-\mathrm{S}(1)-\mathrm{C}(1) & 109.69(7) \\ \mathrm{C}(6)-\mathrm{N}(1)-\mathrm{C}(12) & 116.00(14) \\ \mathrm{C}(6)-\mathrm{N}(1)-\mathrm{C}(11) & 115.17(13) \\ \mathrm{C}(12)-\mathrm{N}(1)-\mathrm{C}(11) & 110.27(14) \\ \mathrm{C}(13)-\mathrm{N}(2)-\mathrm{S}(1) & 123.19(11) \\ \mathrm{C}(13)-\mathrm{N}(2)-\mathrm{H}(2 \mathrm{~N}) & 118.4 \\ \mathrm{~S}(1)-\mathrm{N}(2)-\mathrm{H}(2 \mathrm{~N}) & 118.4 \\ \mathrm{C}(16)-\mathrm{N}(3)-\mathrm{C}(17) & 108.20(13) \\ \mathrm{C}(16)-\mathrm{N}(3)-\mathrm{C}(15) & 112.47(13) \\ \mathrm{C}(17)-\mathrm{N}(3)-\mathrm{C}(15) & 111.09(12) \\ \mathrm{C}(16)-\mathrm{N}(3)-\mathrm{C}(18) & 111.72(14) \\ \mathrm{C}(17)-\mathrm{N}(3)-\mathrm{C}(18) & 105.45(12) \\ \mathrm{C}(15)-\mathrm{N}(3)-\mathrm{C}(18) & 107.70(11) \\ 358 & \end{array}$




$\begin{array}{ll}\mathrm{C}(21)-\mathrm{N}(4)-\mathrm{C}(28) & 111.74(13) \\ \mathrm{C}(21)-\mathrm{N}(4)-\mathrm{C}(20) & 123.86(15) \\ \mathrm{C}(28)-\mathrm{N}(4)-\mathrm{C}(20) & 123.70(14) \\ \mathrm{C}(2)-\mathrm{C}(1)-\mathrm{C}(10) & 121.51(14) \\ \mathrm{C}(2)-\mathrm{C}(1)-\mathrm{S}(1) & 116.72(11) \\ \mathrm{C}(10)-\mathrm{C}(1)-\mathrm{S}(1) & 121.75(11) \\ \mathrm{C}(1)-\mathrm{C}(2)-\mathrm{C}(3) & 120.15(14) \\ \mathrm{C}(1)-\mathrm{C}(2)-\mathrm{H}(2 \mathrm{~A}) & 119.9 \\ \mathrm{C}(3)-\mathrm{C}(2)-\mathrm{H}(2 \mathrm{~A}) & 119.9 \\ \mathrm{C}(4)-\mathrm{C}(3)-\mathrm{C}(2) & 120.37(14) \\ \mathrm{C}(4)-\mathrm{C}(3)-\mathrm{H}(3 \mathrm{~A}) & 119.8 \\ \mathrm{C}(2)-\mathrm{C}(3)-\mathrm{H}(3 \mathrm{~A}) & 119.8 \\ \mathrm{C}(3)-\mathrm{C}(4)-\mathrm{C}(5) & 120.96(14) \\ \mathrm{C}(3)-\mathrm{C}(4)-\mathrm{H}(4 \mathrm{~A}) & 119.5 \\ \mathrm{C}(5)-\mathrm{C}(4)-\mathrm{H}(4 \mathrm{~A}) & 119.5 \\ \mathrm{C}(4)-\mathrm{C}(5)-\mathrm{C}(10) & 119.58(13) \\ \mathrm{C}(4)-\mathrm{C}(5)-\mathrm{C}(6) & 120.87(13) \\ \mathrm{C}(10)-\mathrm{C}(5)-\mathrm{C}(6) & 119.50(13) \\ \mathrm{C}(7)-\mathrm{C}(6)-\mathrm{N}(1) & 123.29(14) \\ \mathrm{C}(7)-\mathrm{C}(6)-\mathrm{C}(5) & 118.87(14) \\ \mathrm{N}(1)-\mathrm{C}(6)-\mathrm{C}(5) & 117.80(13) \\ \mathrm{C}(6)-\mathrm{C}(7)-\mathrm{C}(8) & 120.89(14) \\ \mathrm{C}(6)-\mathrm{C}(7)-\mathrm{H}(7 \mathrm{~A}) & 119.6 \\ \mathrm{C}(8)-\mathrm{C}(7)-\mathrm{H}(7 \mathrm{~A}) & 119.6 \\ \mathrm{C}(9)-\mathrm{C}(8)-\mathrm{C}(7) & 121.53(15) \\ \mathrm{C}(9)-\mathrm{C}(8)-\mathrm{H}(8 \mathrm{~A}) & 119.2 \\ \mathrm{C}(7)-\mathrm{C}(8)-\mathrm{H}(8 \mathrm{~A}) & 119.2 \\ \mathrm{C}(8)-\mathrm{C}(9)-\mathrm{C}(10) & 119.92(14) \\ \mathrm{C}(8)-\mathrm{C}(9)-\mathrm{H}(9 \mathrm{~A}) & 120.0 \\ \mathrm{C}(10)-\mathrm{C}(9)-\mathrm{H}(9 \mathrm{~A}) & 120.0 \\ \mathrm{C}(9)-\mathrm{C}(10)-\mathrm{C}(5) & 119.06(13) \\ \mathrm{C}(9)-\mathrm{C}(10)-\mathrm{C}(1) & 123.77(14) \\ \mathrm{C}(5)-\mathrm{C}(10)-\mathrm{C}(1) & 117.15(13) \\ \mathrm{N}(1)-\mathrm{C}(11)-\mathrm{H}(11 \mathrm{~A}) & 109.5 \\ \mathrm{~N}(1)-\mathrm{C}(11)-\mathrm{H}(11 \mathrm{~B}) & 109.5 \\ \text { 359 } & \\ & \end{array}$




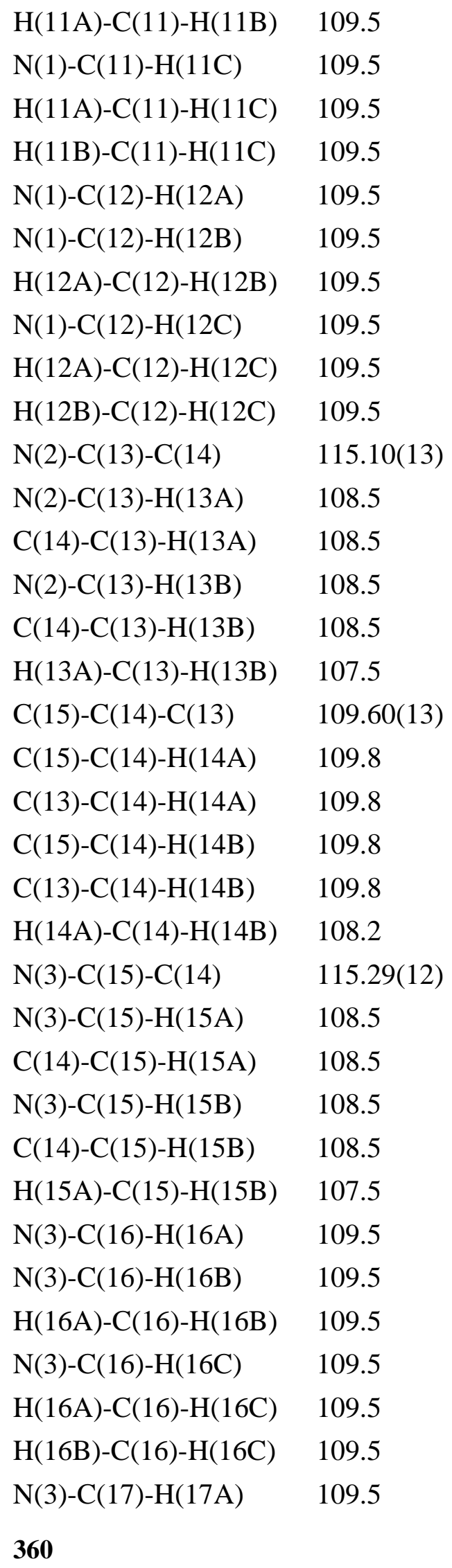




\begin{tabular}{ll} 
N(3)-C(17)-H(17B) & 109.5 \\
H(17A)-C(17)-H(17B) & 109.5 \\
N(3)-C(17)-H(17C) & 109.5 \\
H(17A)-C(17)-H(17C) & 109.5 \\
H(17B)-C(17)-H(17C) & 109.5 \\
C(19)-C(18)-N(3) & $113.79(14)$ \\
C(19)-C(18)-H(18A) & 108.8 \\
N(3)-C(18)-H(18A) & 108.8 \\
C(19)-C(18)-H(18B) & 108.8 \\
N(3)-C(18)-H(18B) & 108.8 \\
H(18A)-C(18)-H(18B) & 107.7 \\
C(18)-C(19)-C(20) & $111.13(15)$ \\
C(18)-C(19)-H(19A) & 109.4 \\
C(20)-C(19)-H(19A) & 109.4 \\
C(18)-C(19)-H(19B) & 109.4 \\
C(20)-C(19)-H(19B) & 109.4 \\
H(19A)-C(19)-H(19B) & 108.0 \\
N(4)-C(20)-C(19) & $113.48(14)$ \\
N(4)-C(20)-H(20A) & 108.9 \\
C(19)-C(20)-H(20A) & 108.9 \\
N(4)-C(20)-H(20B) & 108.9 \\
C(19)-C(20)-H(20B) & 108.9 \\
H(20A)-C(20)-H(20B) & 107.7 \\
O(3)-C(21)-N(4) & $125.03(15)$ \\
O(3)-C(21)-C(22) & $128.86(15)$ \\
N(4)-C(21)-C(22) & $106.07(14)$ \\
C(27)-C(22)-C(23) & $121.48(15)$ \\
C(27)-C(22)-C(21) & $108.16(13)$ \\
C(23)-C(22)-C(21) & $130.33(15)$ \\
C(22)-C(23)-C(24) & $117.00(16)$ \\
C(22)-C(23)-H(23A) & 121.5 \\
C(24)-C(23)-H(23A) & 121.5 \\
C(25)-C(24)-C(23) & $121.38(15)$ \\
C(25)-C(24)-H(24A) & 119.3 \\
C(23)-C(24)-H(24A) & 119.3 \\
& \\
\hline
\end{tabular}




$\begin{array}{ll}\mathrm{C}(24)-\mathrm{C}(25)-\mathrm{C}(26) & 121.31(16) \\ \mathrm{C}(24)-\mathrm{C}(25)-\mathrm{H}(25 \mathrm{~A}) & 119.3 \\ \mathrm{C}(26)-\mathrm{C}(25)-\mathrm{H}(25 \mathrm{~A}) & 119.3 \\ \mathrm{C}(27)-\mathrm{C}(26)-\mathrm{C}(25) & 117.04(16) \\ \mathrm{C}(27)-\mathrm{C}(26)-\mathrm{H}(26 \mathrm{~A}) & 121.5 \\ \mathrm{C}(25)-\mathrm{C}(26)-\mathrm{H}(26 \mathrm{~A}) & 121.5 \\ \mathrm{C}(26)-\mathrm{C}(27)-\mathrm{C}(22) & 121.78(15) \\ \mathrm{C}(26)-\mathrm{C}(27)-\mathrm{C}(28) & 129.97(16) \\ \mathrm{C}(22)-\mathrm{C}(27)-\mathrm{C}(28) & 108.23(14) \\ \mathrm{O}(4)-\mathrm{C}(28)-\mathrm{N}(4) & 124.86(15) \\ \mathrm{O}(4)-\mathrm{C}(28)-\mathrm{C}(27) & 129.38(16) \\ \mathrm{N}(4)-\mathrm{C}(28)-\mathrm{C}(27) & 105.75(14)\end{array}$

Symmetry transformations used to generate equivalent atoms:

Table A 23. Anisotropic displacement parameters $\left(\AA^{2} \times 10^{3}\right)$ for 222. The anisotropic displacement factor exponent takes the form: $-2 \mathrm{p}^{2}\left[\mathrm{~h}^{2} \mathrm{a}^{* 2} \mathrm{U}^{11}+\ldots+2 \mathrm{~h} \mathrm{k} \mathrm{a}^{*} \mathrm{~b}^{*} \mathrm{U}^{12}\right]$

\begin{tabular}{lcccccc}
\hline & $\mathrm{U} 11$ & $\mathrm{U}^{22}$ & $\mathrm{U}^{33}$ & $\mathrm{U}^{23}$ & $\mathrm{U} 13$ & $\mathrm{U} 12$ \\
\hline $\mathrm{Br}(1)$ & $23(1)$ & $28(1)$ & $34(1)$ & $13(1)$ & $13(1)$ & $10(1)$ \\
$\mathrm{S}(1)$ & $18(1)$ & $20(1)$ & $27(1)$ & $8(1)$ & $6(1)$ & $10(1)$ \\
$\mathrm{O}(1)$ & $18(1)$ & $36(1)$ & $36(1)$ & $11(1)$ & $2(1)$ & $11(1)$ \\
$\mathrm{O}(2)$ & $31(1)$ & $21(1)$ & $39(1)$ & $12(1)$ & $13(1)$ & $15(1)$ \\
$\mathrm{O}(3)$ & $39(1)$ & $37(1)$ & $27(1)$ & $5(1)$ & $8(1)$ & $16(1)$ \\
$\mathrm{O}(4)$ & $38(1)$ & $41(1)$ & $33(1)$ & $14(1)$ & $-1(1)$ & $18(1)$ \\
$\mathrm{N}(1)$ & $34(1)$ & $22(1)$ & $23(1)$ & $2(1)$ & $11(1)$ & $12(1)$ \\
$\mathrm{N}(2)$ & $23(1)$ & $21(1)$ & $24(1)$ & $7(1)$ & $7(1)$ & $9(1)$ \\
$\mathrm{N}(3)$ & $15(1)$ & $22(1)$ & $23(1)$ & $5(1)$ & $2(1)$ & $6(1)$ \\
$\mathrm{N}(4)$ & $22(1)$ & $19(1)$ & $31(1)$ & $5(1)$ & $2(1)$ & $8(1)$ \\
$\mathrm{C}(1)$ & $17(1)$ & $20(1)$ & $20(1)$ & $4(1)$ & $2(1)$ & $8(1)$ \\
$\mathrm{C}(2)$ & $23(1)$ & $17(1)$ & $25(1)$ & $5(1)$ & $3(1)$ & $6(1)$ \\
$\mathrm{C}(3)$ & $24(1)$ & $22(1)$ & $25(1)$ & $8(1)$ & $9(1)$ & $4(1)$ \\
$\mathrm{C}(4)$ & $23(1)$ & $23(1)$ & $20(1)$ & $5(1)$ & $7(1)$ & $7(1)$ \\
$\mathrm{C}(5)$ & $20(1)$ & $18(1)$ & $15(1)$ & $3(1)$ & $2(1)$ & $5(1)$ \\
362 & & & & & &
\end{tabular}




$\begin{array}{lllllll}\mathrm{C}(6) & 25(1) & 20(1) & 16(1) & 3(1) & 3(1) & 8(1) \\ \mathrm{C}(7) & 34(1) & 17(1) & 25(1) & 4(1) & 6(1) & 8(1) \\ \mathrm{C}(8) & 27(1) & 20(1) & 28(1) & 8(1) & 9(1) & 3(1) \\ \mathrm{C}(9) & 21(1) & 21(1) & 22(1) & 5(1) & 6(1) & 5(1) \\ \mathrm{C}(10) & 18(1) & 18(1) & 16(1) & 4(1) & 0(1) & 5(1) \\ \mathrm{C}(11) & 29(1) & 33(1) & 48(1) & 7(1) & 16(1) & 13(1) \\ \mathrm{C}(12) & 50(1) & 25(1) & 25(1) & 2(1) & 13(1) & 18(1) \\ \mathrm{C}(13) & 33(1) & 20(1) & 27(1) & -1(1) & 0(1) & 13(1) \\ \mathrm{C}(14) & 23(1) & 19(1) & 32(1) & 6(1) & -1(1) & 5(1) \\ \mathrm{C}(15) & 17(1) & 20(1) & 30(1) & 7(1) & 5(1) & 8(1) \\ \mathrm{C}(16) & 32(1) & 61(1) & 20(1) & 10(1) & 7(1) & 23(1) \\ \mathrm{C}(17) & 20(1) & 35(1) & 25(1) & 6(1) & -2(1) & 9(1) \\ \mathrm{C}(18) & 25(1) & 26(1) & 35(1) & 9(1) & 4(1) & 11(1) \\ \mathrm{C}(19) & 24(1) & 27(1) & 52(1) & 6(1) & -2(1) & 12(1) \\ \mathrm{C}(20) & 19(1) & 23(1) & 48(1) & 6(1) & 3(1) & 8(1) \\ \mathrm{C}(21) & 24(1) & 17(1) & 29(1) & 3(1) & 1(1) & 5(1) \\ \mathrm{C}(22) & 21(1) & 15(1) & 28(1) & 6(1) & 3(1) & 4(1) \\ \mathrm{C}(23) & 25(1) & 25(1) & 30(1) & 10(1) & 2(1) & 8(1) \\ \mathrm{C}(24) & 23(1) & 27(1) & 45(1) & 14(1) & 6(1) & 11(1) \\ \mathrm{C}(25) & 28(1) & 30(1) & 40(1) & 7(1) & 10(1) & 14(1) \\ \mathrm{C}(26) & 29(1) & 28(1) & 28(1) & 6(1) & 6(1) & 9(1) \\ \mathrm{C}(27) & 22(1) & 17(1) & 28(1) & 6(1) & 1(1) & 5(1) \\ \mathrm{C}(28) & 24(1) & 19(1) & 31(1) & 7(1) & 1(1) & 7(1)\end{array}$


Table A 24. Hydrogen coordinates ( $\left.x 10^{4}\right)$ and isotropic displacement parameters $\left(\AA^{2} \times 10^{3}\right)$ for 220.

\begin{tabular}{|c|c|c|c|c|}
\hline & $\mathrm{x}$ & $\mathrm{y}$ & $\mathrm{z}$ & $\mathrm{U}(\mathrm{eq})$ \\
\hline $\mathrm{H}(2 \mathrm{~N})$ & 3199 & 5936 & 3233 & $67(8)$ \\
\hline $\mathrm{H}(2 \mathrm{~A})$ & 6476 & 8033 & 1421 & 27 \\
\hline $\mathrm{H}(3 \mathrm{~A})$ & 8188 & 7313 & 498 & 29 \\
\hline $\mathrm{H}(4 \mathrm{~A})$ & 7936 & 5155 & 156 & 27 \\
\hline $\mathrm{H}(7 \mathrm{~A})$ & 4489 & 1386 & 774 & 31 \\
\hline $\mathrm{H}(8 \mathrm{~A})$ & 2520 & 2027 & 1531 & 32 \\
\hline $\mathrm{H}(9 \mathrm{~A})$ & 2763 & 4185 & 1927 & 26 \\
\hline $\mathrm{H}(11 \mathrm{~A})$ & 9169 & 4290 & 960 & 54 \\
\hline $\mathrm{H}(11 \mathrm{~B})$ & 9671 & 3279 & 267 & 54 \\
\hline $\mathrm{H}(11 \mathrm{C})$ & 8941 & 2880 & 1085 & 54 \\
\hline $\mathrm{H}(12 \mathrm{~A})$ & 5499 & 1121 & -529 & 50 \\
\hline $\mathrm{H}(12 \mathrm{~B})$ & 6727 & 977 & 193 & 50 \\
\hline $\mathrm{H}(12 \mathrm{C})$ & 7451 & 1361 & -629 & 50 \\
\hline $\mathrm{H}(13 \mathrm{~A})$ & 5064 & 7294 & 4358 & 32 \\
\hline $\mathrm{H}(13 \mathrm{~B})$ & 5676 & 8343 & 3823 & 32 \\
\hline $\mathrm{H}(14 \mathrm{~A})$ & 7446 & 7252 & 3179 & 31 \\
\hline $\mathrm{H}(14 \mathrm{~B})$ & 7968 & 7739 & 4190 & 31 \\
\hline $\mathrm{H}(15 \mathrm{~A})$ & 6379 & 5637 & 4308 & 27 \\
\hline $\mathrm{H}(15 \mathrm{~B})$ & 5768 & 5143 & 3307 & 27 \\
\hline $\mathrm{H}(16 \mathrm{~A})$ & 9041 & 6151 & 2694 & 54 \\
\hline $\mathrm{H}(16 \mathrm{~B})$ & 7786 & 4659 & 2348 & 54 \\
\hline $\mathrm{H}(16 \mathrm{C})$ & 9722 & 5004 & 2739 & 54 \\
\hline $\mathrm{H}(17 \mathrm{~A})$ & 10124 & 7052 & 4206 & 42 \\
\hline $\mathrm{H}(17 \mathrm{~B})$ & 10670 & 5830 & 4218 & 42 \\
\hline $\mathrm{H}(17 \mathrm{C})$ & 9434 & 6195 & 4854 & 42 \\
\hline $\mathrm{H}(18 \mathrm{~A})$ & 8740 & 3676 & 3777 & 33 \\
\hline $\mathrm{H}(18 \mathrm{~B})$ & 7460 & 4018 & 4390 & 33 \\
\hline $\mathrm{H}(19 \mathrm{~A})$ & 6473 & 2882 & 2605 & 42 \\
\hline $\mathrm{H}(19 \mathrm{~B})$ & 5211 & 3118 & 3265 & 42 \\
\hline
\end{tabular}




\begin{tabular}{lrrrr}
$\mathrm{H}(20 \mathrm{~A})$ & 5683 & 1591 & 3925 & 37 \\
$\mathrm{H}(20 \mathrm{~B})$ & 4940 & 917 & 2944 & 37 \\
$\mathrm{H}(23 \mathrm{~A})$ & 10880 & 32 & 4408 & 32 \\
$\mathrm{H}(24 \mathrm{~A})$ & 12407 & -903 & 3474 & 36 \\
$\mathrm{H}(25 \mathrm{~A})$ & 11979 & -1071 & 2038 & 39 \\
$\mathrm{H}(26 \mathrm{~A})$ & 9995 & -316 & 1472 & 35 \\
\hline
\end{tabular}

Table A 25. Torsion angles $\left[^{\circ}\right]$ for 220.

\begin{tabular}{lc}
\hline $\mathrm{O}(1)-\mathrm{S}(1)-\mathrm{N}(2)-\mathrm{C}(13)$ & $-162.99(12)$ \\
$\mathrm{O}(2)-\mathrm{S}(1)-\mathrm{N}(2)-\mathrm{C}(13)$ & $-34.05(13)$ \\
$\mathrm{C}(1)-\mathrm{S}(1)-\mathrm{N}(2)-\mathrm{C}(13)$ & $80.51(13)$ \\
$\mathrm{O}(1)-\mathrm{S}(1)-\mathrm{C}(1)-\mathrm{C}(2)$ & $123.02(12)$ \\
$\mathrm{O}(2)-\mathrm{S}(1)-\mathrm{C}(1)-\mathrm{C}(2)$ & $-6.47(14)$ \\
$\mathrm{N}(2)-\mathrm{S}(1)-\mathrm{C}(1)-\mathrm{C}(2)$ & $-121.42(12)$ \\
$\mathrm{O}(1)-\mathrm{S}(1)-\mathrm{C}(1)-\mathrm{C}(10)$ & $-55.43(14)$ \\
$\mathrm{O}(2)-\mathrm{S}(1)-\mathrm{C}(1)-\mathrm{C}(10)$ & $175.08(12)$ \\
$\mathrm{N}(2)-\mathrm{S}(1)-\mathrm{C}(1)-\mathrm{C}(10)$ & $60.13(14)$ \\
$\mathrm{C}(10)-\mathrm{C}(1)-\mathrm{C}(2)-\mathrm{C}(3)$ & $0.7(2)$ \\
$\mathrm{S}(1)-\mathrm{C}(1)-\mathrm{C}(2)-\mathrm{C}(3)$ & $-177.76(12)$ \\
$\mathrm{C}(1)-\mathrm{C}(2)-\mathrm{C}(3)-\mathrm{C}(4)$ & $-2.5(2)$ \\
$\mathrm{C}(2)-\mathrm{C}(3)-\mathrm{C}(4)-\mathrm{C}(5)$ & $-0.2(2)$ \\
$\mathrm{C}(3)-\mathrm{C}(4)-\mathrm{C}(5)-\mathrm{C}(10)$ & $4.6(2)$ \\
$\mathrm{C}(3)-\mathrm{C}(4)-\mathrm{C}(5)-\mathrm{C}(6)$ & $-177.81(15)$ \\
$\mathrm{C}(12)-\mathrm{N}(1)-\mathrm{C}(6)-\mathrm{C}(7)$ & $18.4(2)$ \\
$\mathrm{C}(11)-\mathrm{N}(1)-\mathrm{C}(6)-\mathrm{C}(7)$ & $-112.54(18)$ \\
$\mathrm{C}(12)-\mathrm{N}(1)-\mathrm{C}(6)-\mathrm{C}(5)$ & $-159.28(14)$ \\
$\mathrm{C}(11)-\mathrm{N}(1)-\mathrm{C}(6)-\mathrm{C}(5)$ & $69.78(19)$ \\
$\mathrm{C}(4)-\mathrm{C}(5)-\mathrm{C}(6)-\mathrm{C}(7)$ & $-172.13(15)$ \\
$\mathrm{C}(10)-\mathrm{C}(5)-\mathrm{C}(6)-\mathrm{C}(7)$ & $5.4(2)$ \\
$\mathrm{C}(4)-\mathrm{C}(5)-\mathrm{C}(6)-\mathrm{N}(1)$ & \\
$\mathrm{C}(10)-\mathrm{C}(5)-\mathrm{C}(6)-\mathrm{N}(1)$ & \\
$\mathrm{N}(1)-\mathrm{C}(6)-\mathrm{C}(7)-\mathrm{C}(8)$ & \\
365 & \\
& \\
& \\
& \\
& \\
&
\end{tabular}




$\begin{array}{lc}\mathrm{C}(5)-\mathrm{C}(6)-\mathrm{C}(7)-\mathrm{C}(8) & -2.5(2) \\ \mathrm{C}(6)-\mathrm{C}(7)-\mathrm{C}(8)-\mathrm{C}(9) & -1.0(3) \\ \mathrm{C}(7)-\mathrm{C}(8)-\mathrm{C}(9)-\mathrm{C}(10) & 1.5(2) \\ \mathrm{C}(8)-\mathrm{C}(9)-\mathrm{C}(10)-\mathrm{C}(5) & 1.5(2) \\ \mathrm{C}(8)-\mathrm{C}(9)-\mathrm{C}(10)-\mathrm{C}(1) & -179.79(15) \\ \mathrm{C}(4)-\mathrm{C}(5)-\mathrm{C}(10)-\mathrm{C}(9) & 172.67(14) \\ \mathrm{C}(6)-\mathrm{C}(5)-\mathrm{C}(10)-\mathrm{C}(9) & -4.9(2) \\ \mathrm{C}(4)-\mathrm{C}(5)-\mathrm{C}(10)-\mathrm{C}(1) & -6.2(2) \\ \mathrm{C}(6)-\mathrm{C}(5)-\mathrm{C}(10)-\mathrm{C}(1) & 176.25(13) \\ \mathrm{C}(2)-\mathrm{C}(1)-\mathrm{C}(10)-\mathrm{C}(9) & -175.17(15) \\ \mathrm{S}(1)-\mathrm{C}(1)-\mathrm{C}(10)-\mathrm{C}(9) & 3.2(2) \\ \mathrm{C}(2)-\mathrm{C}(1)-\mathrm{C}(10)-\mathrm{C}(5) & 3.6(2) \\ \mathrm{S}(1)-\mathrm{C}(1)-\mathrm{C}(10)-\mathrm{C}(5) & -178.03(11) \\ \mathrm{S}(1)-\mathrm{N}(2)-\mathrm{C}(13)-\mathrm{C}(14) & -80.18(16) \\ \mathrm{N}(2)-\mathrm{C}(13)-\mathrm{C}(14)-\mathrm{C}(15) & -61.73(19) \\ \mathrm{C}(16)-\mathrm{N}(3)-\mathrm{C}(15)-\mathrm{C}(14) & -64.16(18) \\ \mathrm{C}(17)-\mathrm{N}(3)-\mathrm{C}(15)-\mathrm{C}(14) & 57.29(18) \\ \mathrm{C}(18)-\mathrm{N}(3)-\mathrm{C}(15)-\mathrm{C}(14) & 172.30(14) \\ \mathrm{C}(13)-\mathrm{C}(14)-\mathrm{C}(15)-\mathrm{N}(3) & 177.27(13) \\ \mathrm{C}(16)-\mathrm{N}(3)-\mathrm{C}(18)-\mathrm{C}(19) & -60.96(18) \\ \mathrm{C}(17)-\mathrm{N}(3)-\mathrm{C}(18)-\mathrm{C}(19) & -178.28(14) \\ \mathrm{C}(15)-\mathrm{N}(3)-\mathrm{C}(18)-\mathrm{C}(19) & 63.03(18) \\ \mathrm{N}(3)-\mathrm{C}(18)-\mathrm{C}(19)-\mathrm{C}(20) & 174.26(14) \\ \mathrm{C}(21)-\mathrm{N}(4)-\mathrm{C}(20)-\mathrm{C}(19) & 117.72(18) \\ \mathrm{C}(28)-\mathrm{N}(4)-\mathrm{C}(20)-\mathrm{C}(19) & -72.7(2) \\ \mathrm{C}(18)-\mathrm{C}(19)-\mathrm{C}(20)-\mathrm{N}(4) & -60.4(2) \\ \mathrm{C}(28)-\mathrm{N}(4)-\mathrm{C}(21)-\mathrm{O}(3) & -175.77(15) \\ \mathrm{C}(20)-\mathrm{N}(4)-\mathrm{C}(21)-\mathrm{O}(3) & -5.1(3) \\ \mathrm{C}(28)-\mathrm{N}(4)-\mathrm{C}(21)-\mathrm{C}(22) & 2.47(17) \\ \mathrm{C}(20)-\mathrm{N}(4)-\mathrm{C}(21)-\mathrm{C}(22) & 173.17(13) \\ \mathrm{O}(3)-\mathrm{C}(21)-\mathrm{C}(22)-\mathrm{C}(27) & 176.46(16) \\ \mathrm{N}(4)-\mathrm{C}(21)-\mathrm{C}(22)-\mathrm{C}(27) & -1.69(17) \\ \mathrm{O}(3)-\mathrm{C}(21)-\mathrm{C}(22)-\mathrm{C}(23) & -1.5(3) \\ \mathrm{N}(4)-\mathrm{C}(21)-\mathrm{C}(22)-\mathrm{C}(23) & \\ \mathrm{C}(27)-\mathrm{C}(22)-\mathrm{C}(23)-\mathrm{C}(24) & \\ 366 & \end{array}$




$\begin{array}{lc}\mathrm{C}(21)-\mathrm{C}(22)-\mathrm{C}(23)-\mathrm{C}(24) & 176.70(15) \\ \mathrm{C}(22)-\mathrm{C}(23)-\mathrm{C}(24)-\mathrm{C}(25) & 1.0(2) \\ \mathrm{C}(23)-\mathrm{C}(24)-\mathrm{C}(25)-\mathrm{C}(26) & -0.1(3) \\ \mathrm{C}(24)-\mathrm{C}(25)-\mathrm{C}(26)-\mathrm{C}(27) & -0.9(3) \\ \mathrm{C}(25)-\mathrm{C}(26)-\mathrm{C}(27)-\mathrm{C}(22) & 0.9(2) \\ \mathrm{C}(25)-\mathrm{C}(26)-\mathrm{C}(27)-\mathrm{C}(28) & -177.21(16) \\ \mathrm{C}(23)-\mathrm{C}(22)-\mathrm{C}(27)-\mathrm{C}(26) & 0.0(2) \\ \mathrm{C}(21)-\mathrm{C}(22)-\mathrm{C}(27)-\mathrm{C}(26) & -178.13(15) \\ \mathrm{C}(23)-\mathrm{C}(22)-\mathrm{C}(27)-\mathrm{C}(28) & 178.53(14) \\ \mathrm{C}(21)-\mathrm{C}(22)-\mathrm{C}(27)-\mathrm{C}(28) & 0.36(17) \\ \mathrm{C}(21)-\mathrm{N}(4)-\mathrm{C}(28)-\mathrm{O}(4) & 176.72(16) \\ \mathrm{C}(20)-\mathrm{N}(4)-\mathrm{C}(28)-\mathrm{O}(4) & 6.0(3) \\ \mathrm{C}(21)-\mathrm{N}(4)-\mathrm{C}(28)-\mathrm{C}(27) & -2.26(17) \\ \mathrm{C}(20)-\mathrm{N}(4)-\mathrm{C}(28)-\mathrm{C}(27) & -172.97(14) \\ \mathrm{C}(26)-\mathrm{C}(27)-\mathrm{C}(28)-\mathrm{O}(4) & 0.5(3) \\ \mathrm{C}(22)-\mathrm{C}(27)-\mathrm{C}(28)-\mathrm{O}(4) & -177.81(17) \\ \mathrm{C}(26)-\mathrm{C}(27)-\mathrm{C}(28)-\mathrm{N}(4) & 179.43(16) \\ \mathrm{C}(22)-\mathrm{C}(27)-\mathrm{C}(28)-\mathrm{N}(4) & 1.10(17) \\ & \end{array}$

Symmetry transformations used to generate equivalent atoms:

Table A 26. Hydrogen bonds for 220 [ $\AA$ and ${ }^{\circ}$ ].

\begin{tabular}{lcccc}
\hline D-H...A & $d(D-H)$ & $d(H . . A)$ & $d(D . . A)$ & $<(D H A)$ \\
\hline $\mathrm{N}(2)-H(2 N) \ldots \operatorname{Br}(1)$ & 0.88 & 2.54 & $3.3518(14)$ & 152.9
\end{tabular}

Symmetry transformations used to generate equivalent atoms: 


\subsection{Appendix 1.2 - NMR Spectra}

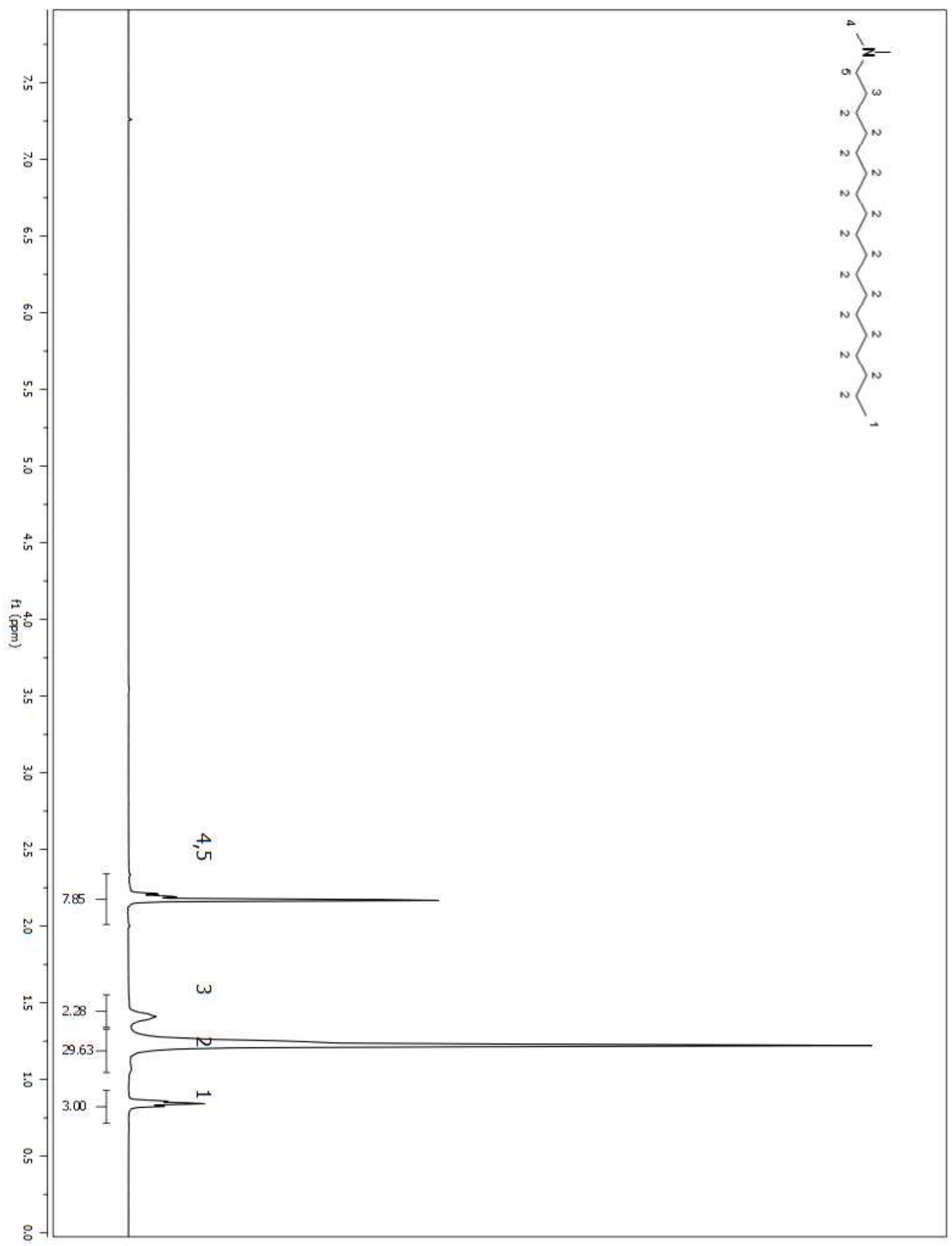

Figure A 1. ${ }^{1} \mathrm{H}$ NMR spectrum of compound 2 in $\mathrm{CDCl}_{3}$ 


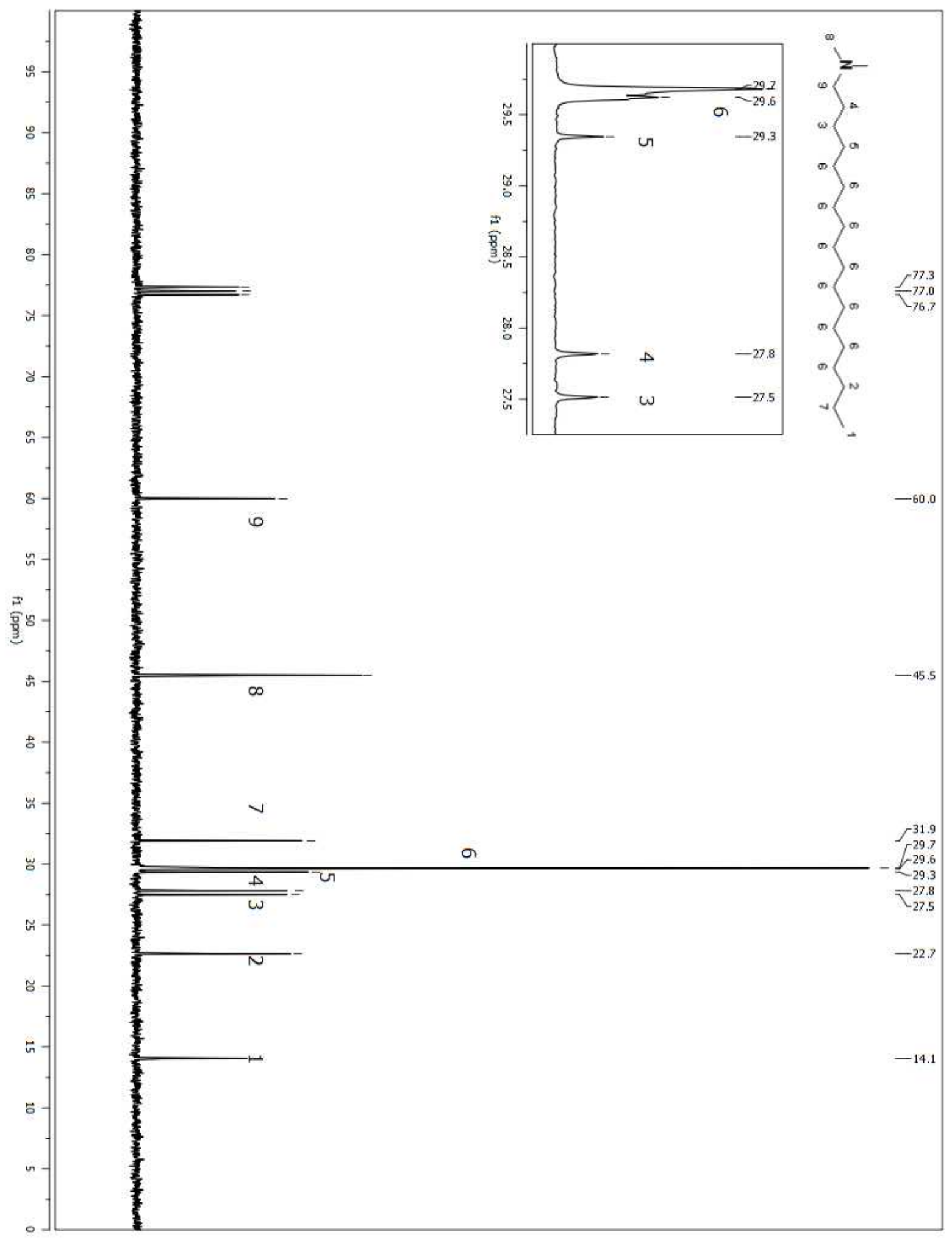

Figure A 2. ${ }^{13} \mathrm{C}$ NMR spectrum of compound 2 in $\mathrm{CDCl}_{3}$ 


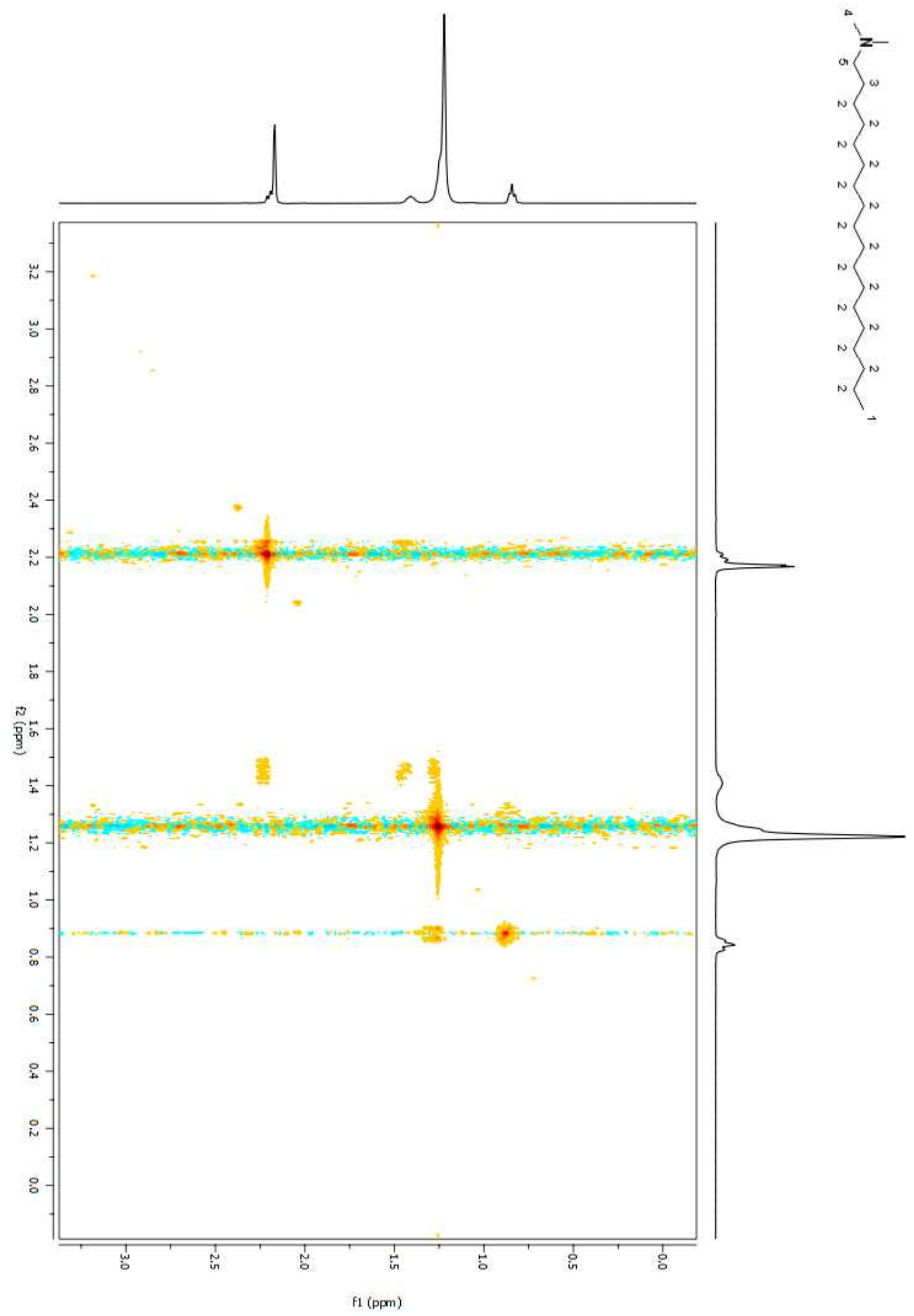

Figure A 3. COSY 2D NMR spectrum of compound 2 in $\mathrm{CDCl}_{3}$ 


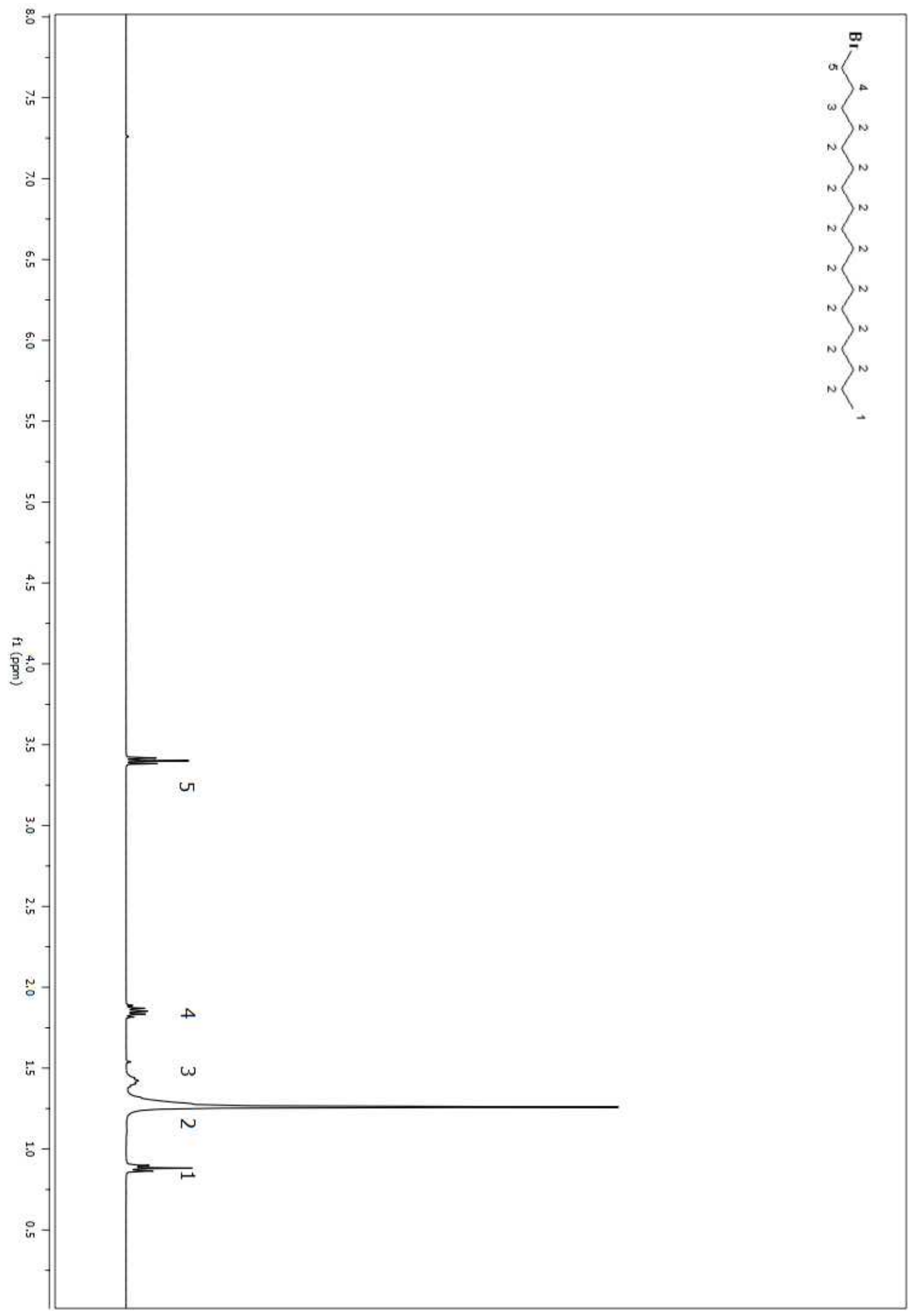

Figure A 4. ${ }^{1} \mathrm{H}$ NMR spectrum of purified 46 in $\mathrm{CDCl}_{3}$ 


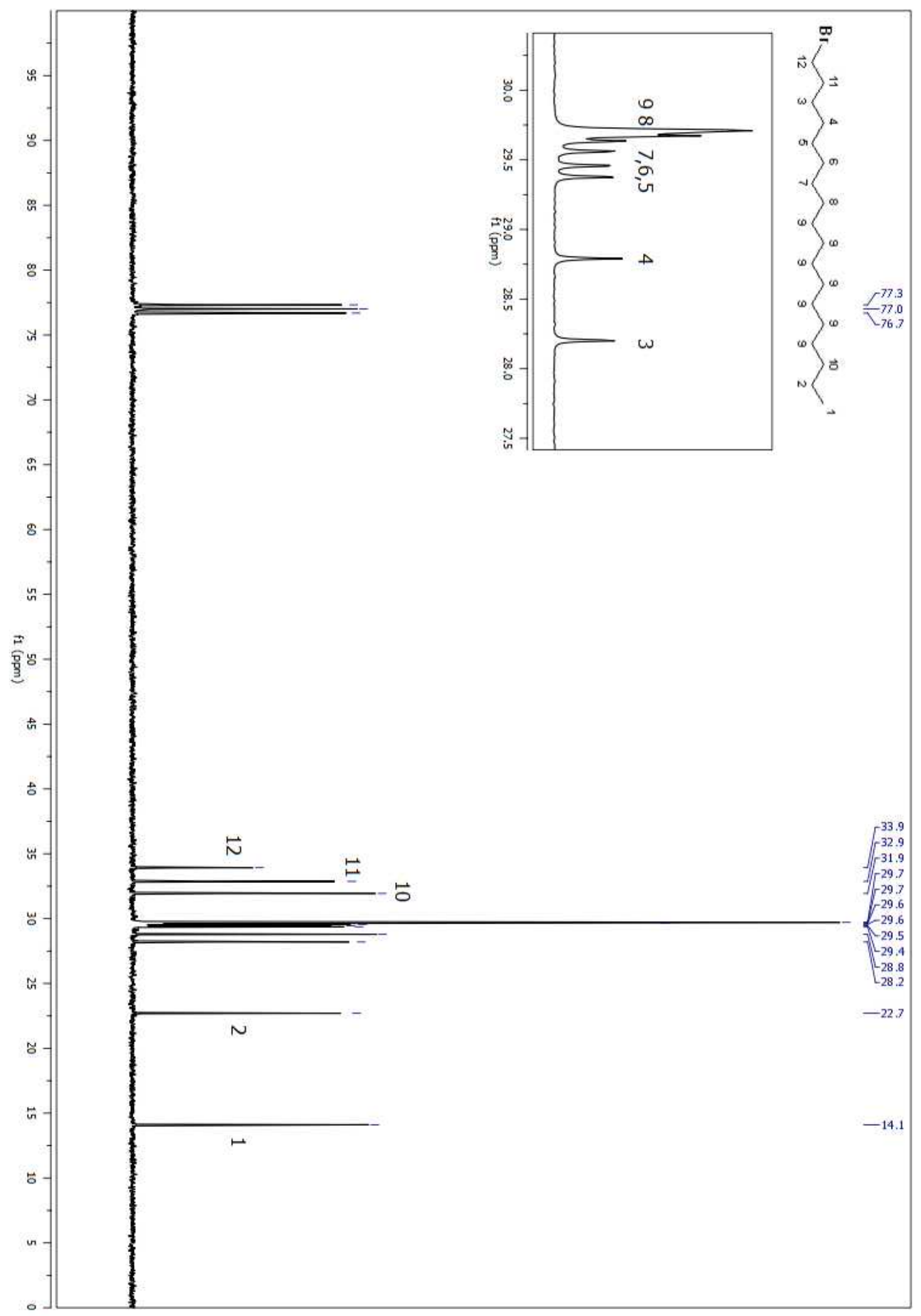

Figure A 5. ${ }^{13} \mathrm{C}$ NMR spectrum of purified 46 in $\mathrm{CDCl}_{3}$ 


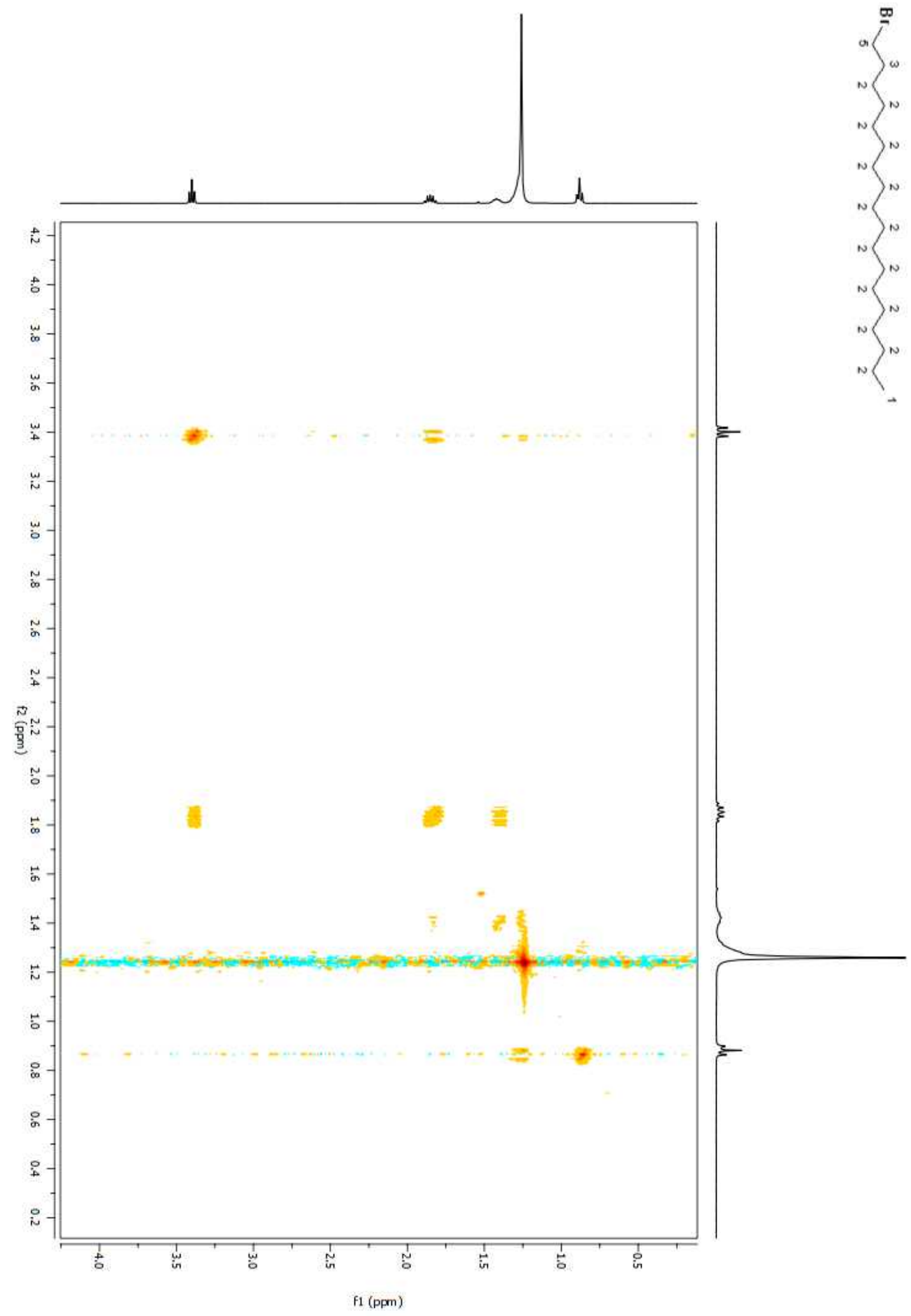

Figure A 6. COSY 2D NMR spectrum of purified 46 in $\mathrm{CDCl}_{3}$ 


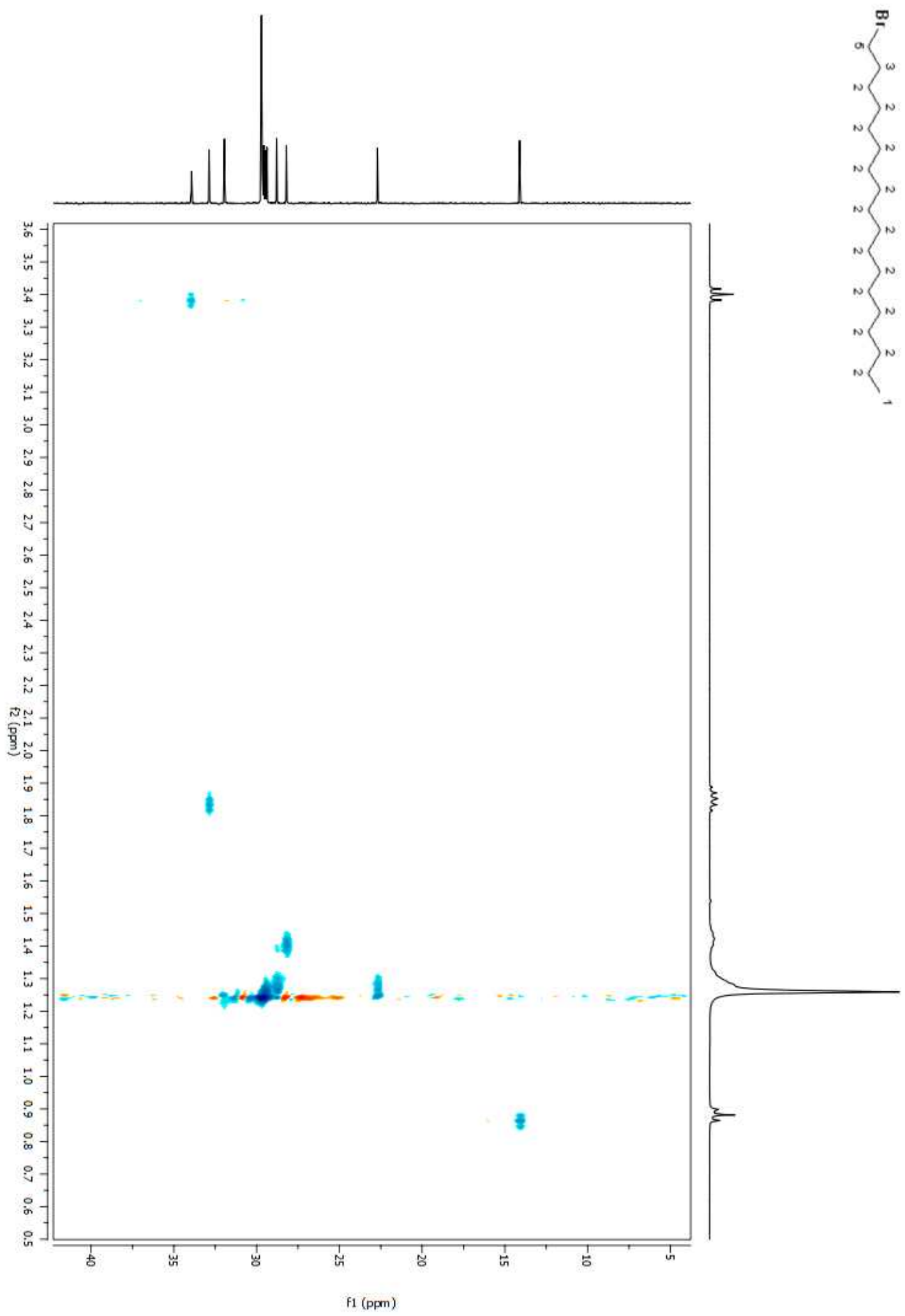

Figure A 7. HSQC 2D NMR spectrum of purified 46 in $\mathrm{CDCl}_{3}$ 


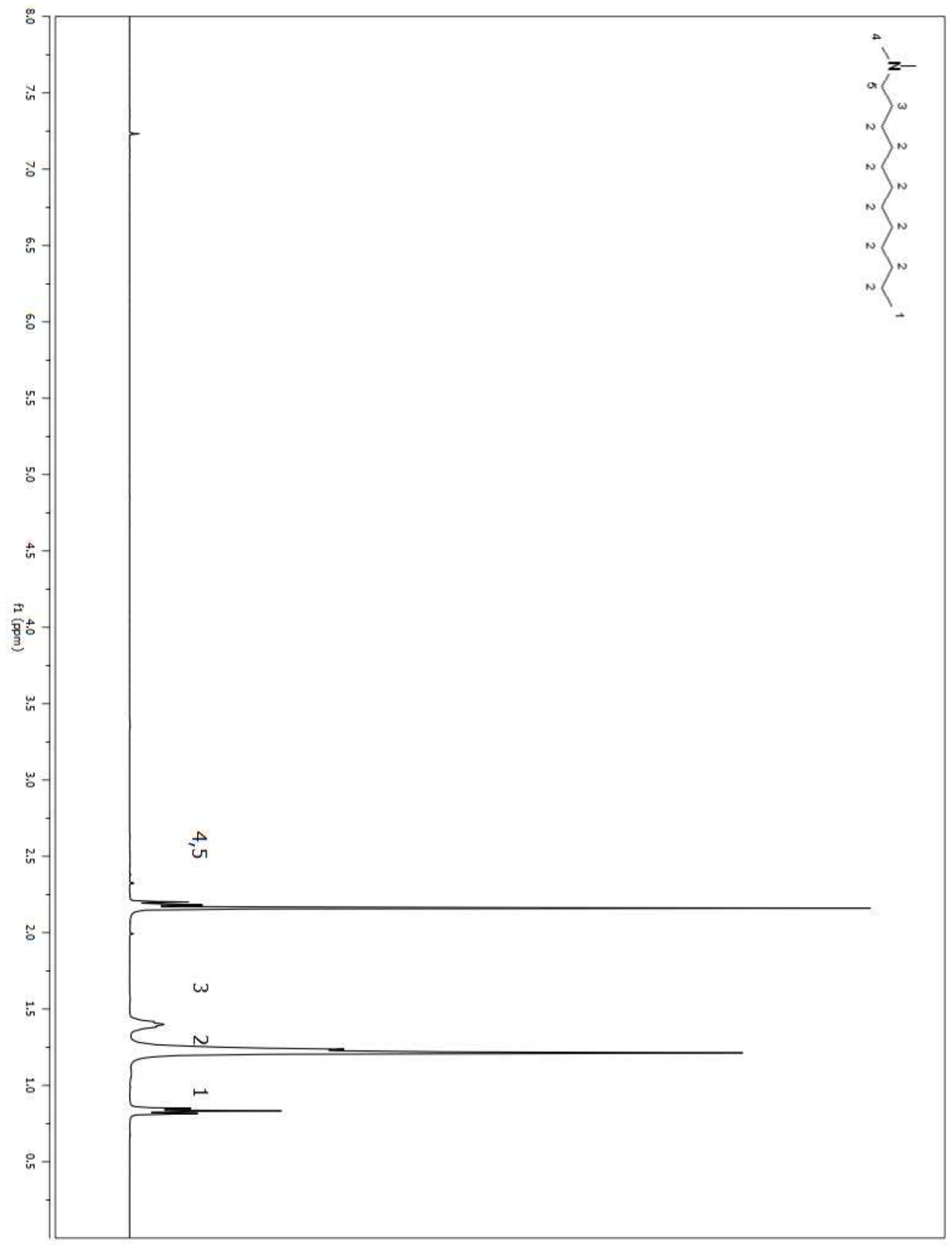

Figure A 8. ${ }^{1} \mathrm{H}$ NMR spectrum of compound 22 in $\mathrm{CDCl}_{3}$ 


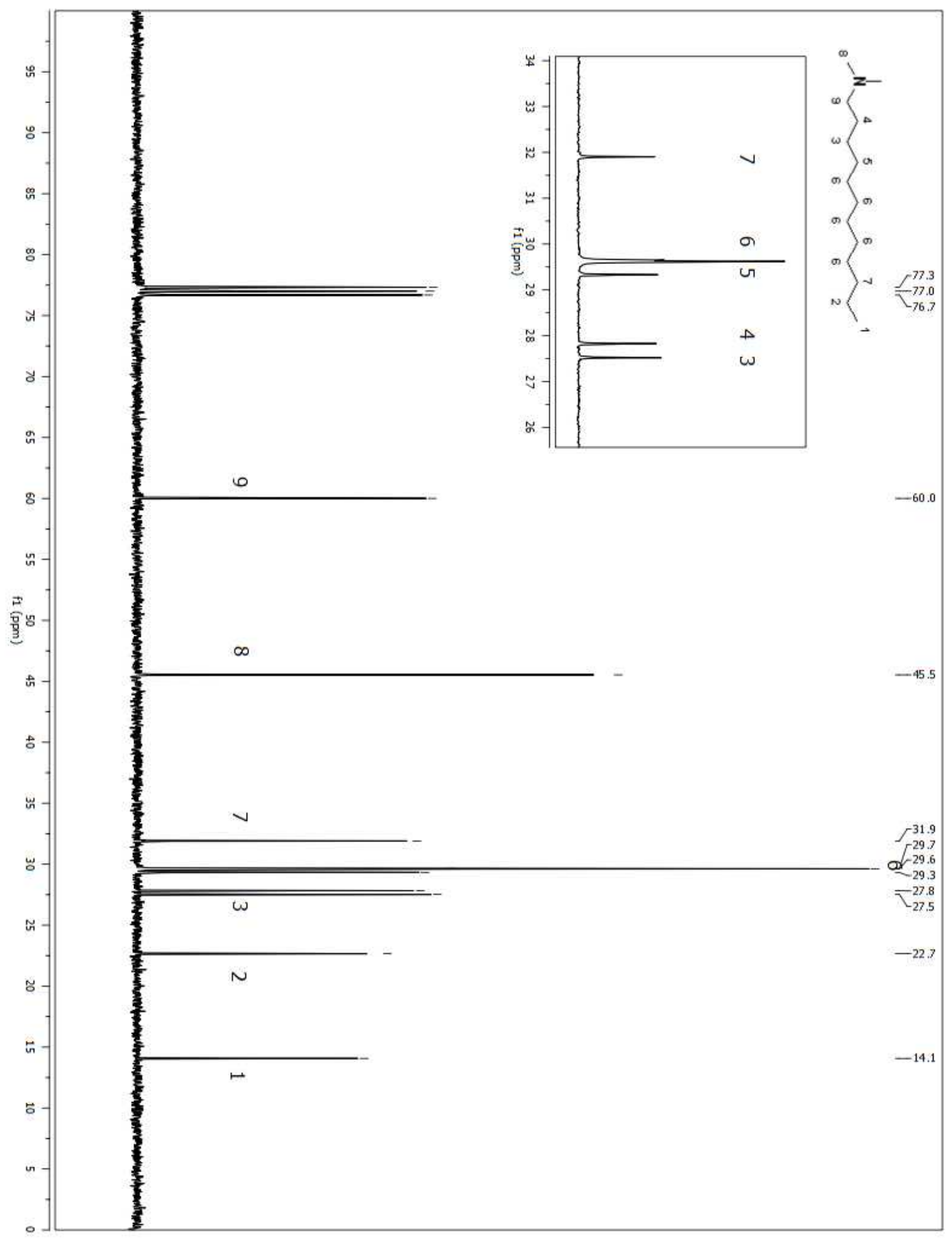

Figure A 9. ${ }^{13} \mathrm{C}$ NMR spectrum of compound 22 in $\mathrm{CDCl}_{3}$ 


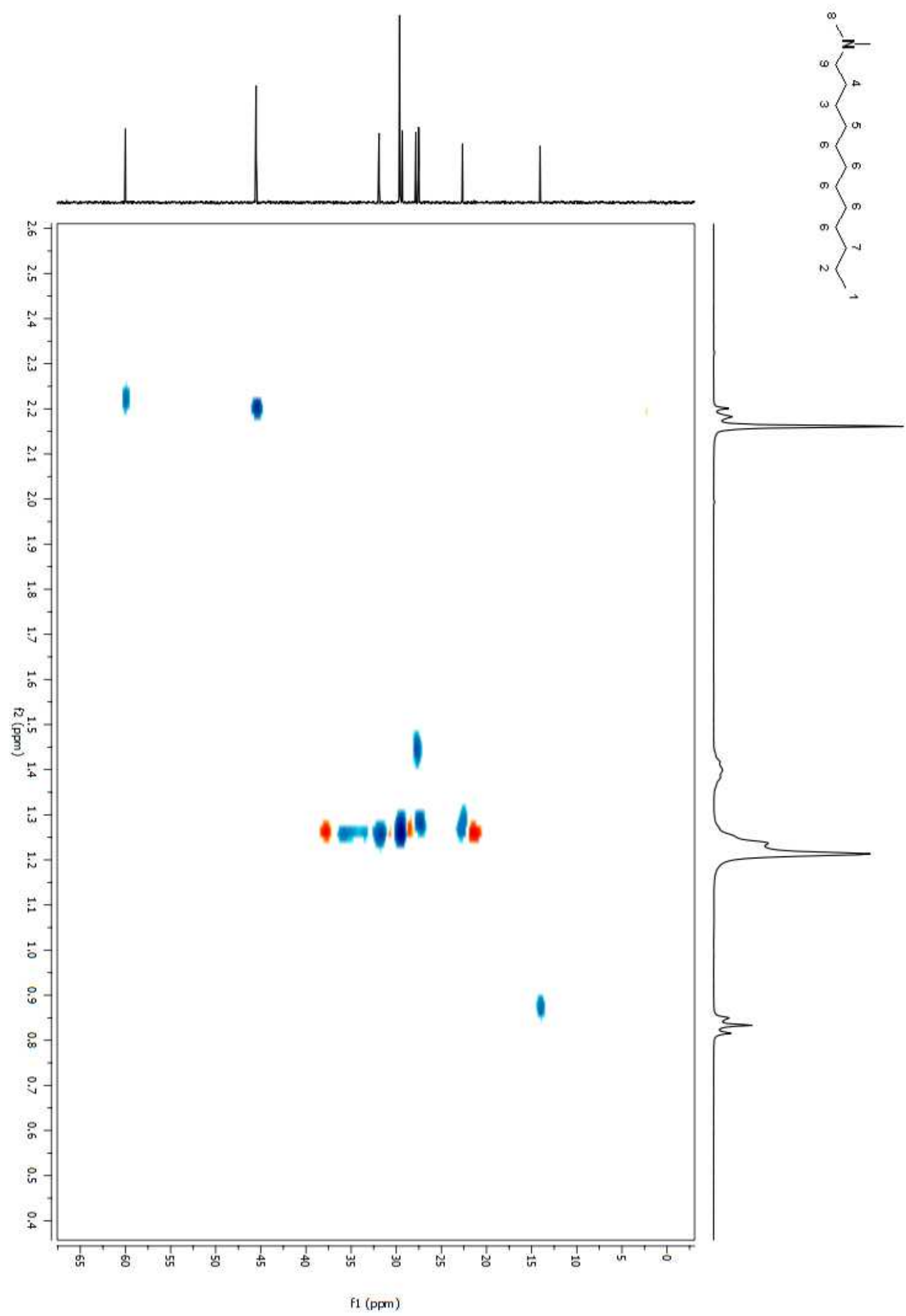

Figure A 10. HSQC 2D NMR spectrum of compound 22 in $\mathrm{CDCl}_{3}$ 


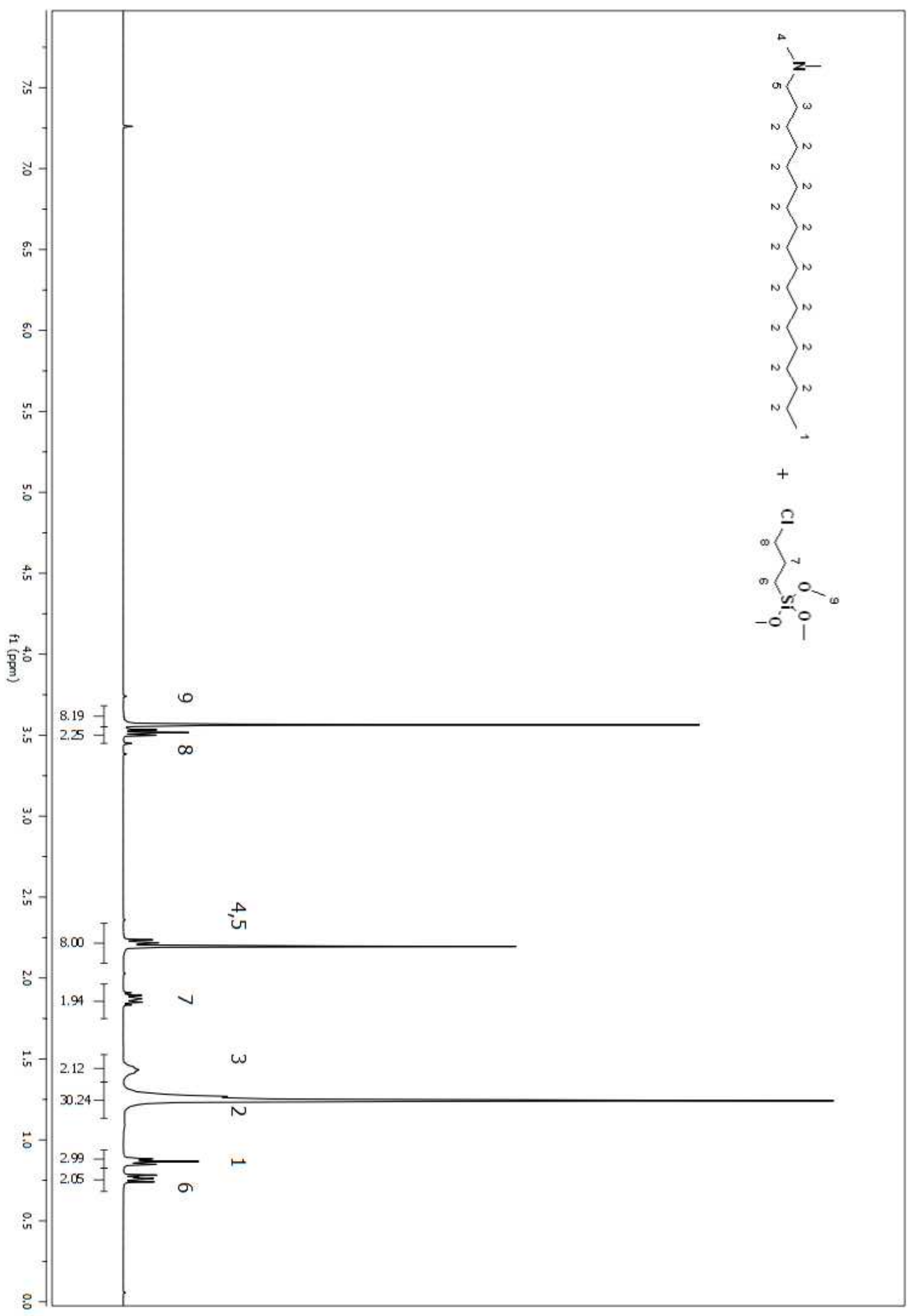

Figure A 11. ${ }^{1} \mathrm{H}$ NMR spectrum of compound $\mathbf{1}+2$ in $\mathrm{CDCl}_{3}$ 


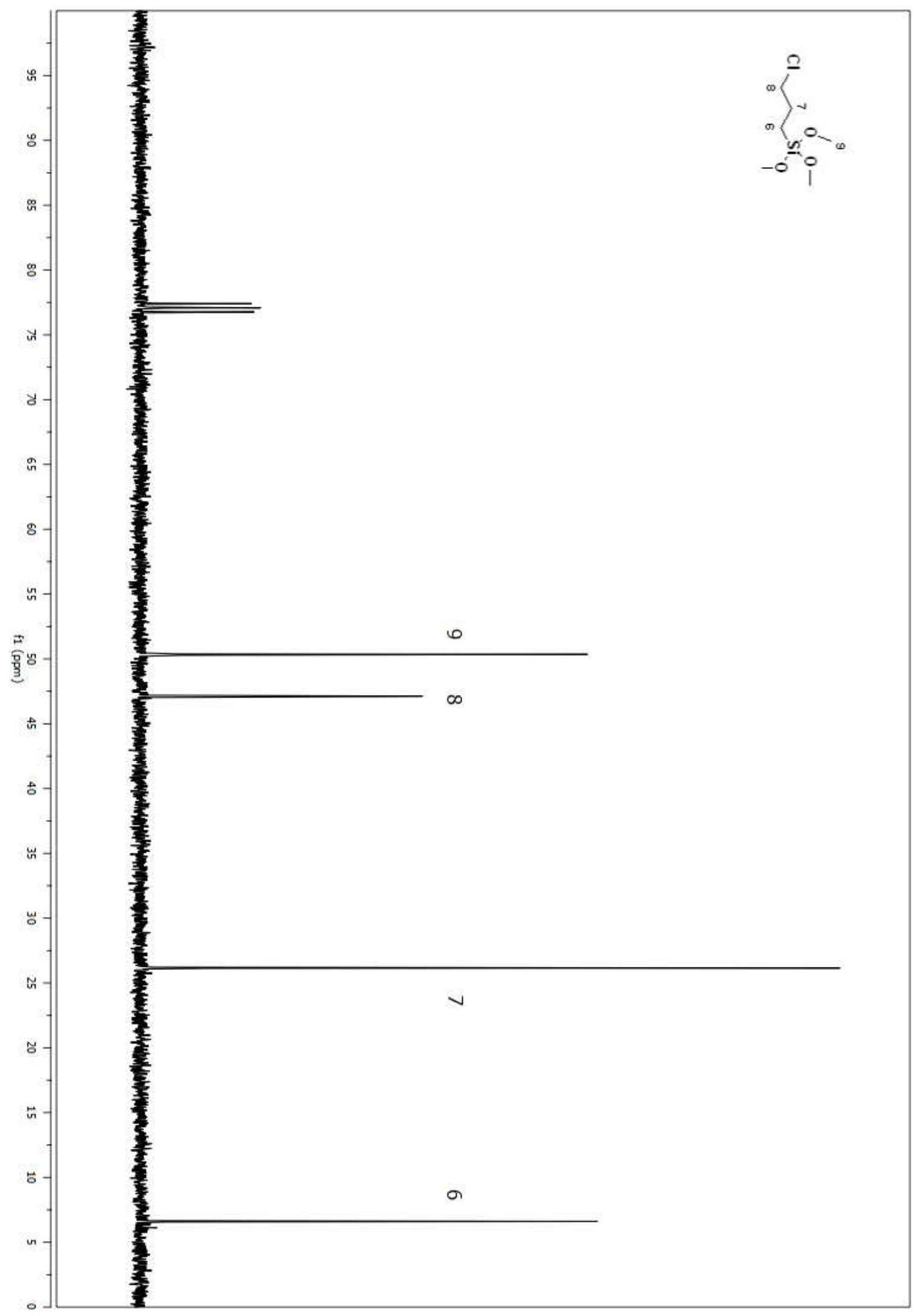

Figure A 12. ${ }^{13} \mathrm{C}$ NMR spectrum of compound 1 in $\mathrm{CDCl}_{3}$ 


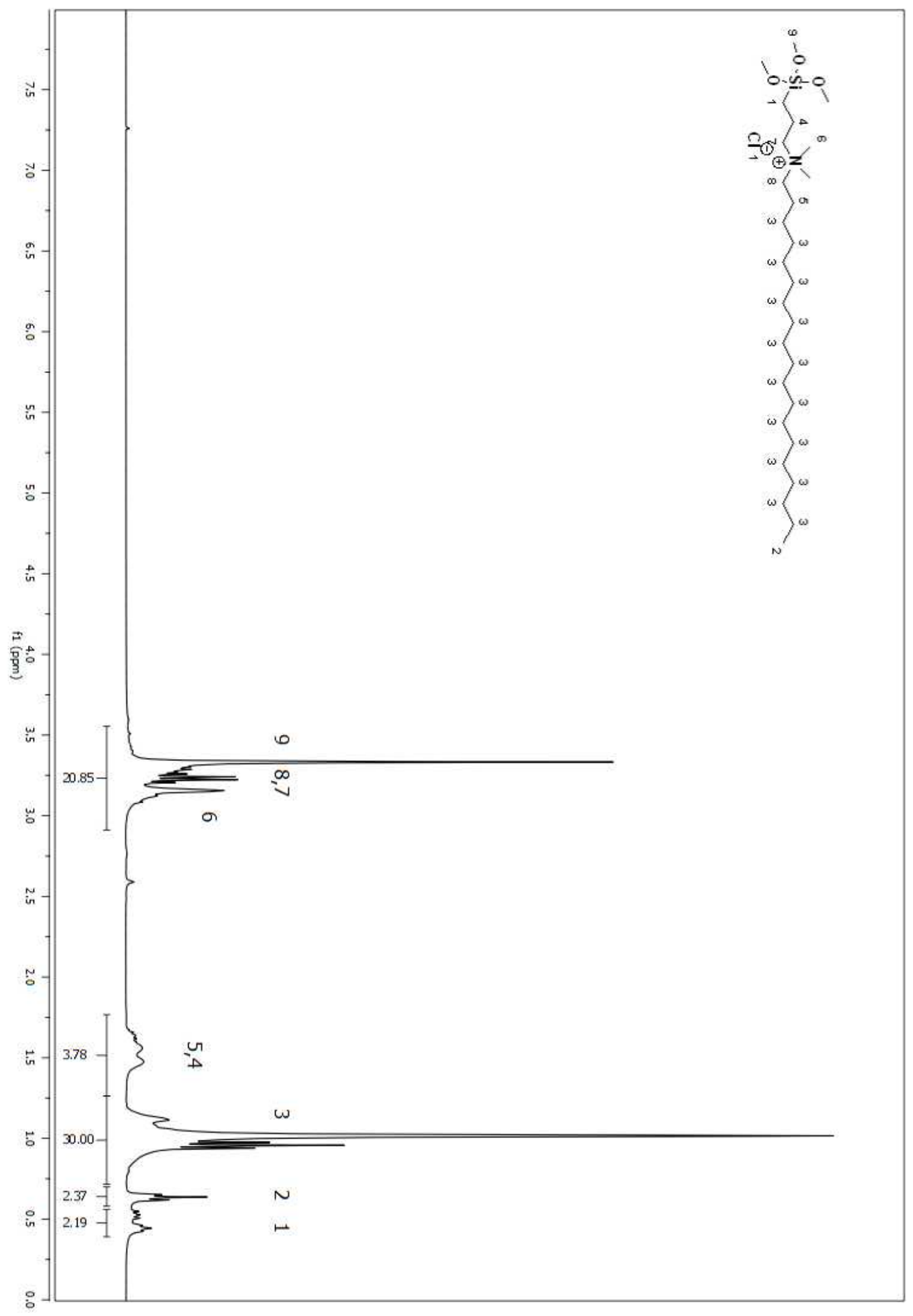

Figure A 13. ${ }^{1} \mathrm{H}$ NMR spectrum of compound 3 in $\mathrm{CDCl}_{3}$ (Table 2.1, entry i) 380 


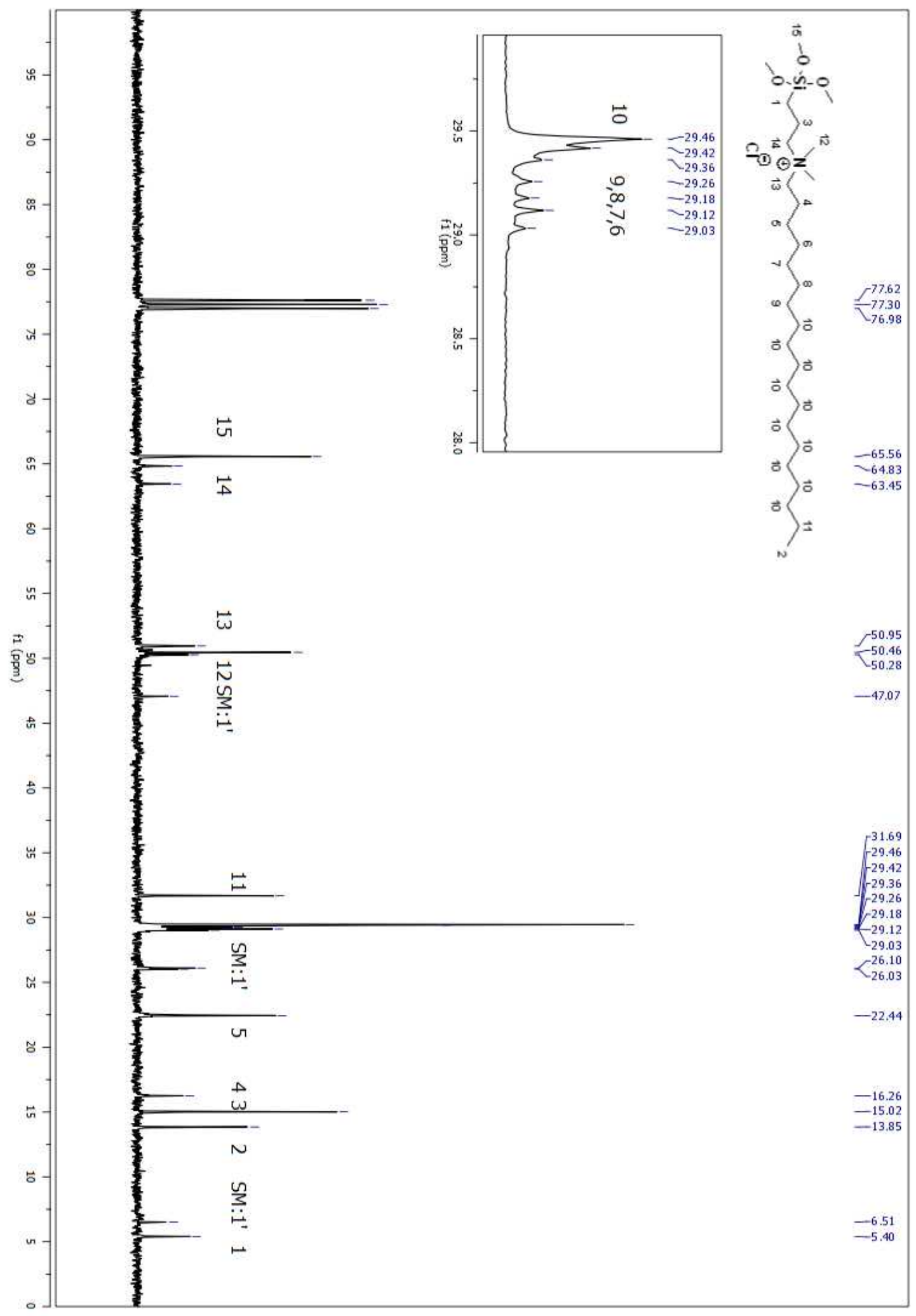

Figure A 14. ${ }^{13} \mathrm{C}$ NMR spectrum of compound 3 in $\mathrm{CDCl}_{3}$ (Table 2.1, entry i) 


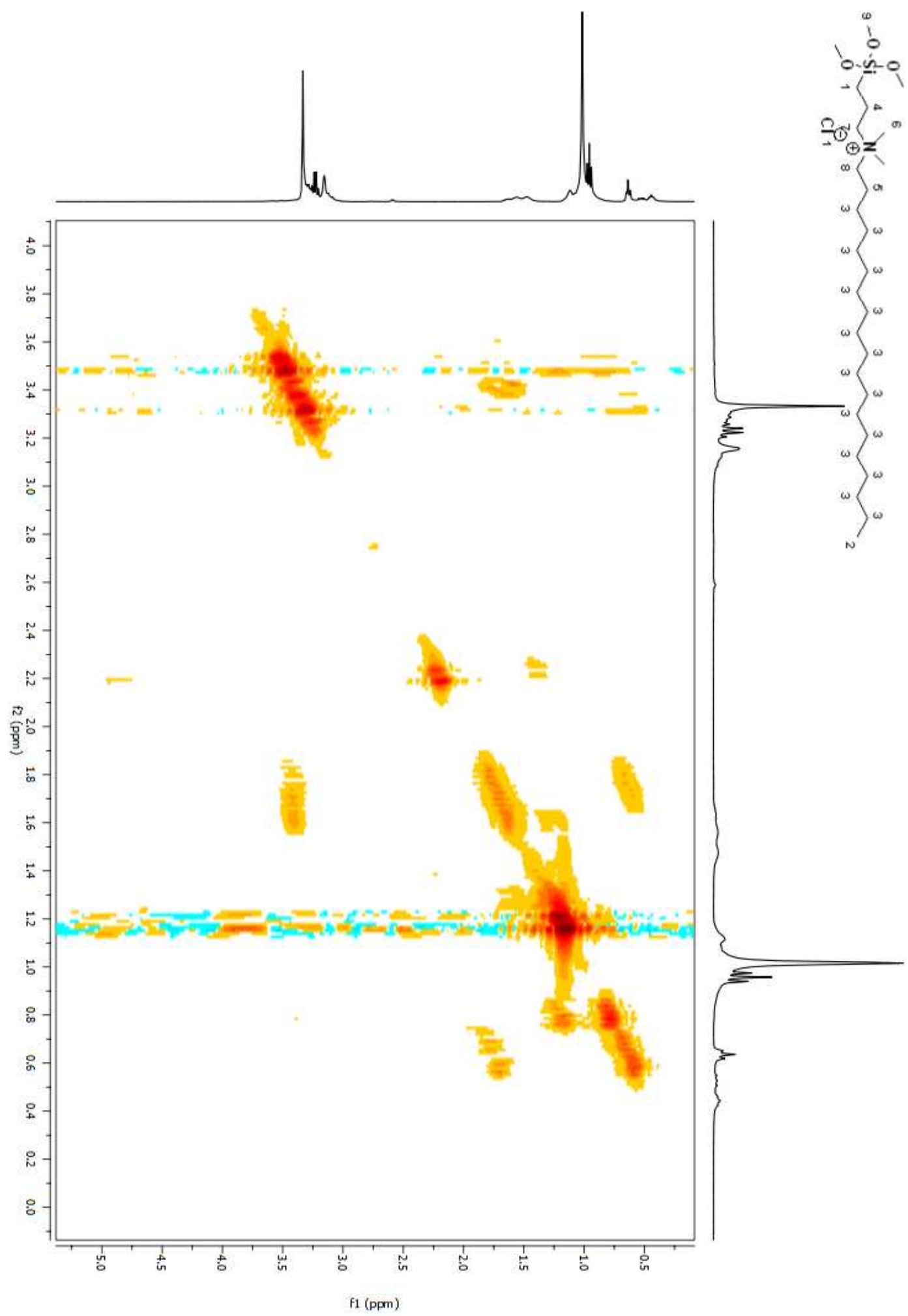

Figure A 15. COSY 2D NMR spectrum of compound 3 in $\mathrm{CDCl}_{3}$ (Table 2.1, entry i) 


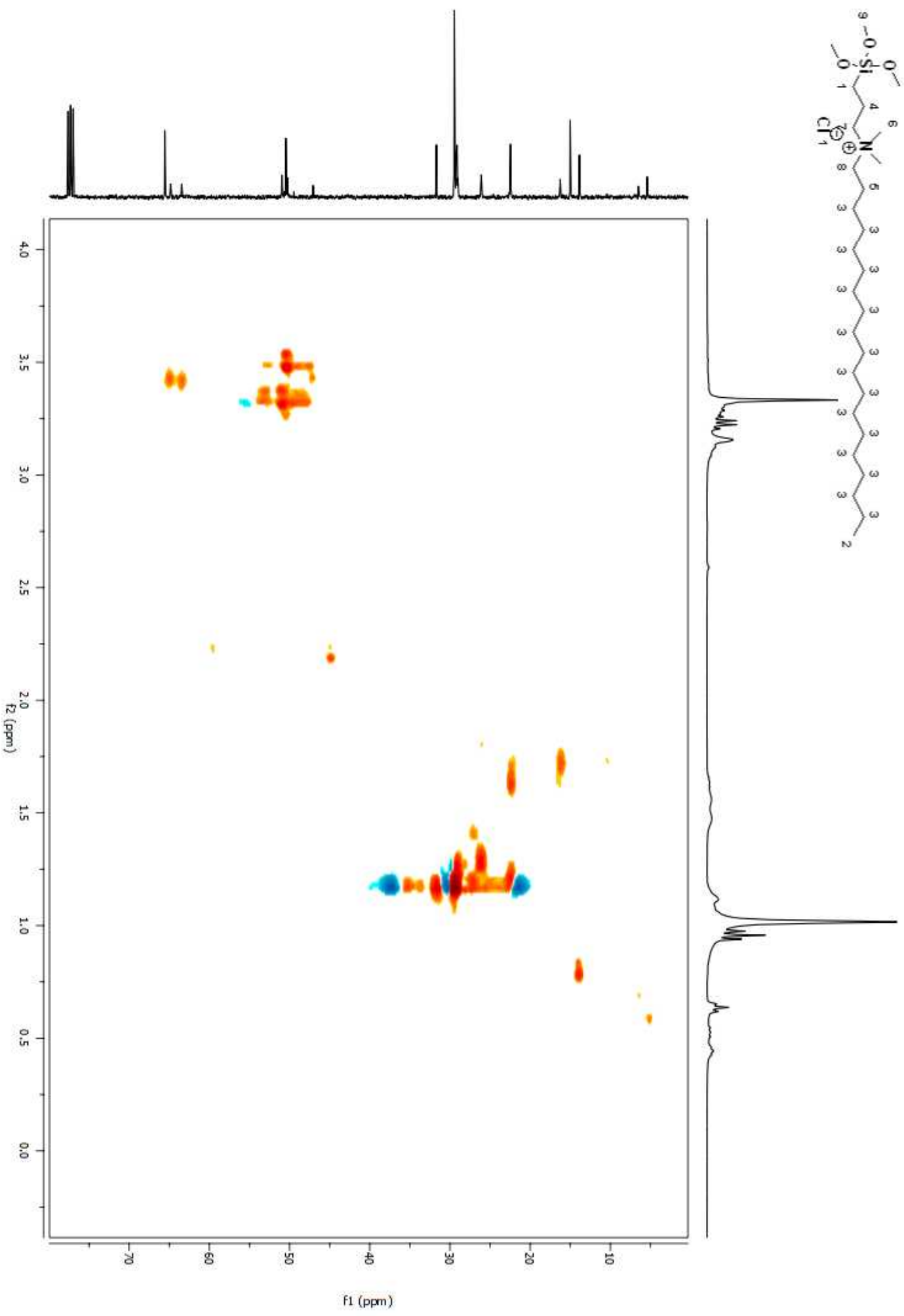

Figure A 16. HSQC 2D NMR spectrum of compound 3 in $\mathrm{CDCl}_{3}$ (Table 2.1, entry i) 


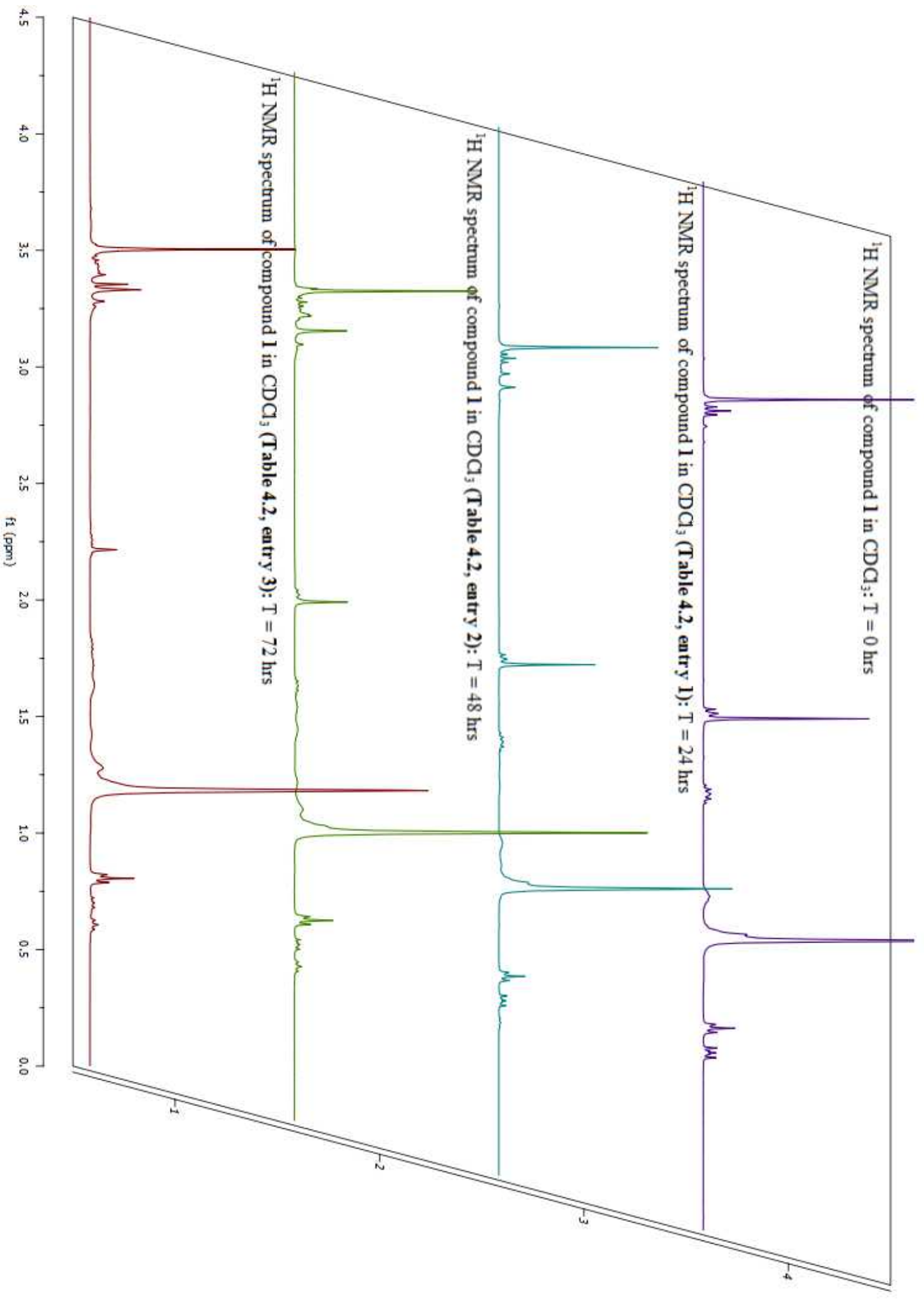

Figure A 17. ${ }^{1} \mathrm{H}$ stacked NMR spectra of compound 3 in $\mathrm{CDCl}_{3}$ (Table 2.1, entry i-iii) 384 


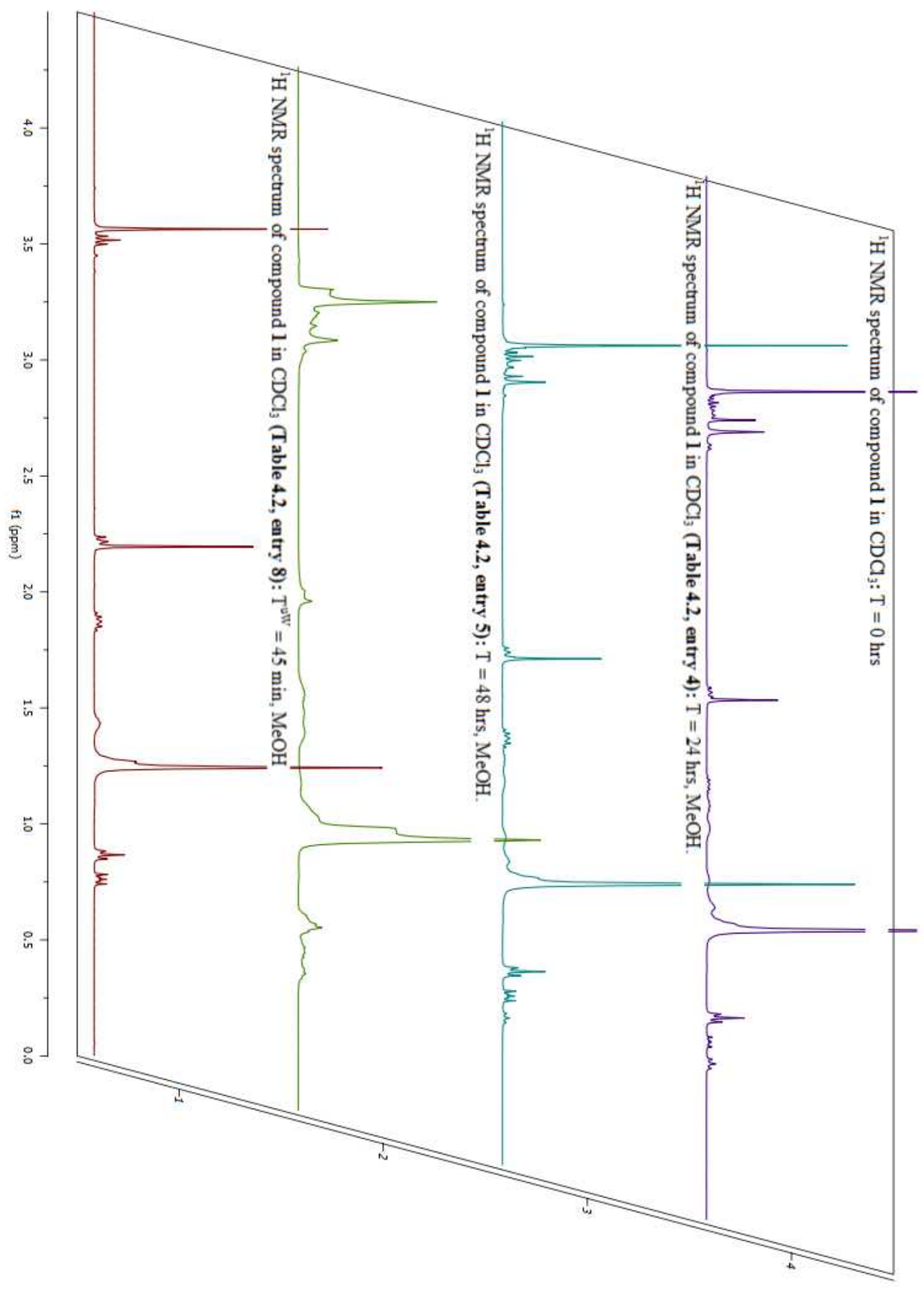

Figure A 18. ${ }^{1} \mathrm{H}$ stacked NMR spectra of compound 3 in $\mathrm{CDCl}_{3}$ (Table 2.1, entry iv-v, viii) 


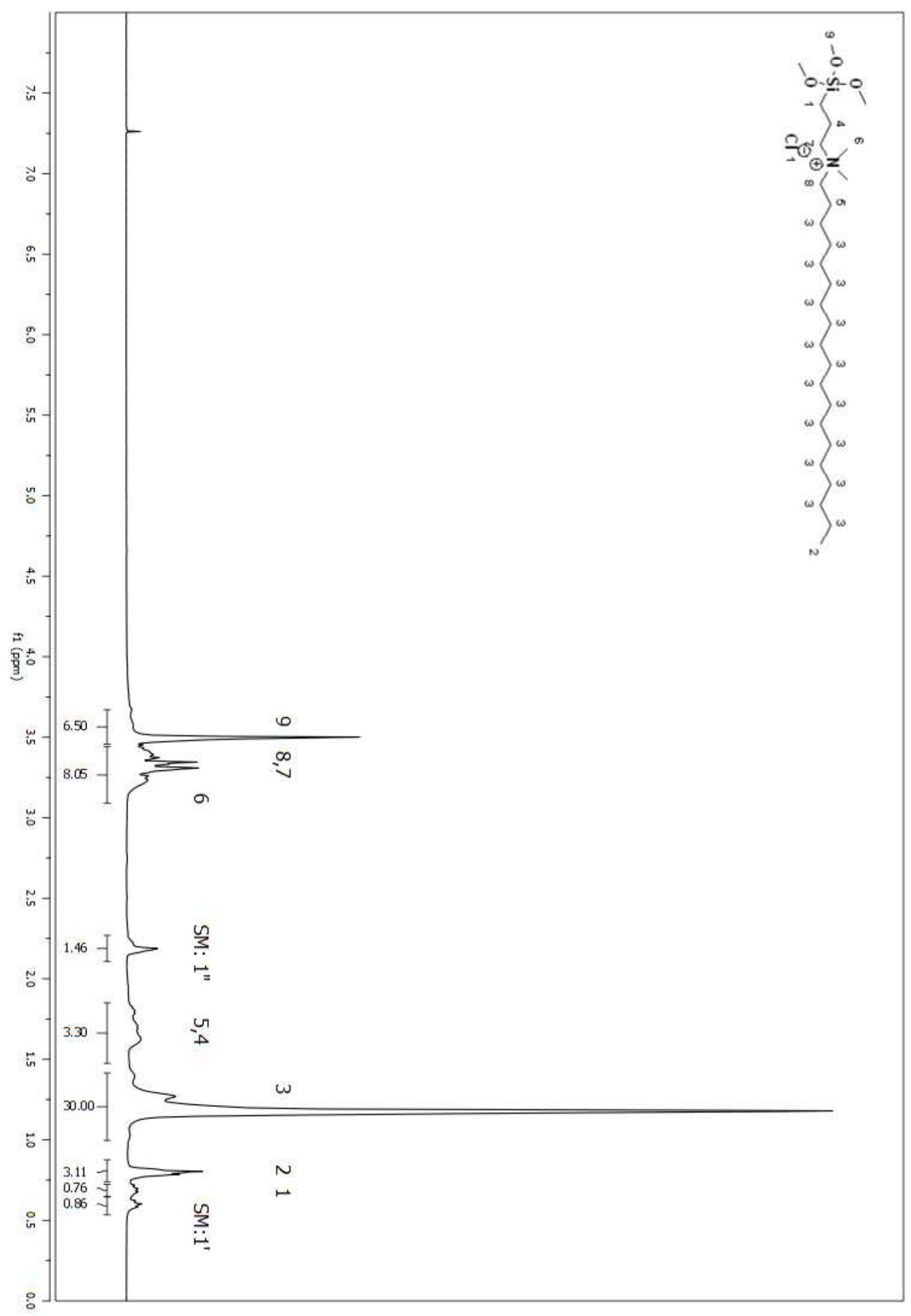

Figure A 19. ${ }^{1} \mathrm{H}$ NMR spectrum of compound 3 in $\mathrm{CDCl}_{3}$ (Table 2.2, entry iii) 


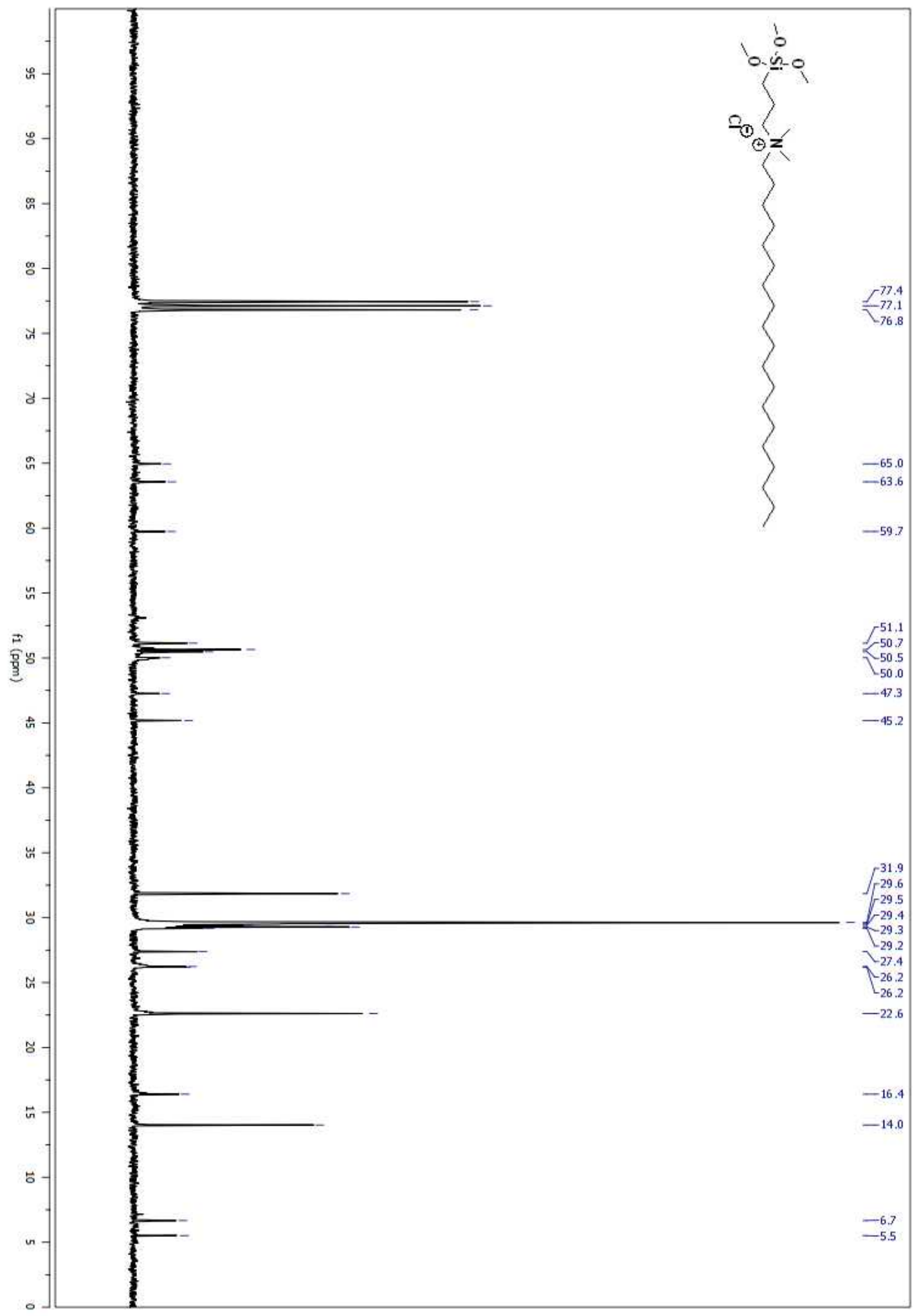

Figure A 20. ${ }^{13} \mathrm{C}$ NMR spectrum of compound 3 in $\mathrm{CDCl}_{3}$ (Table 2.2, entry iii) 


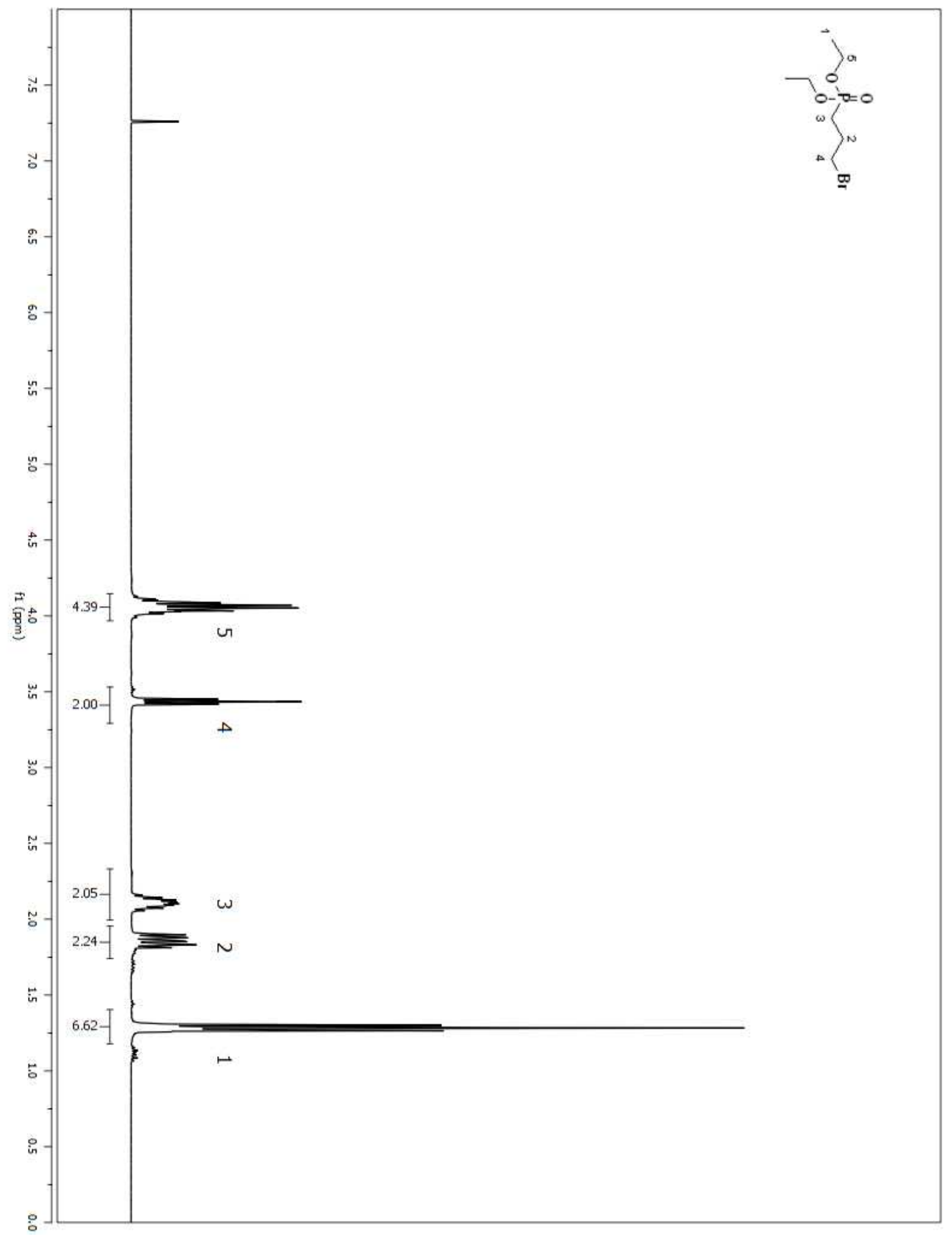

Figure A 21. ${ }^{1} \mathrm{H}$ NMR spectrum of compound 12 in $\mathrm{CDCl}_{3}$ (Table 2.3, entry iii) 


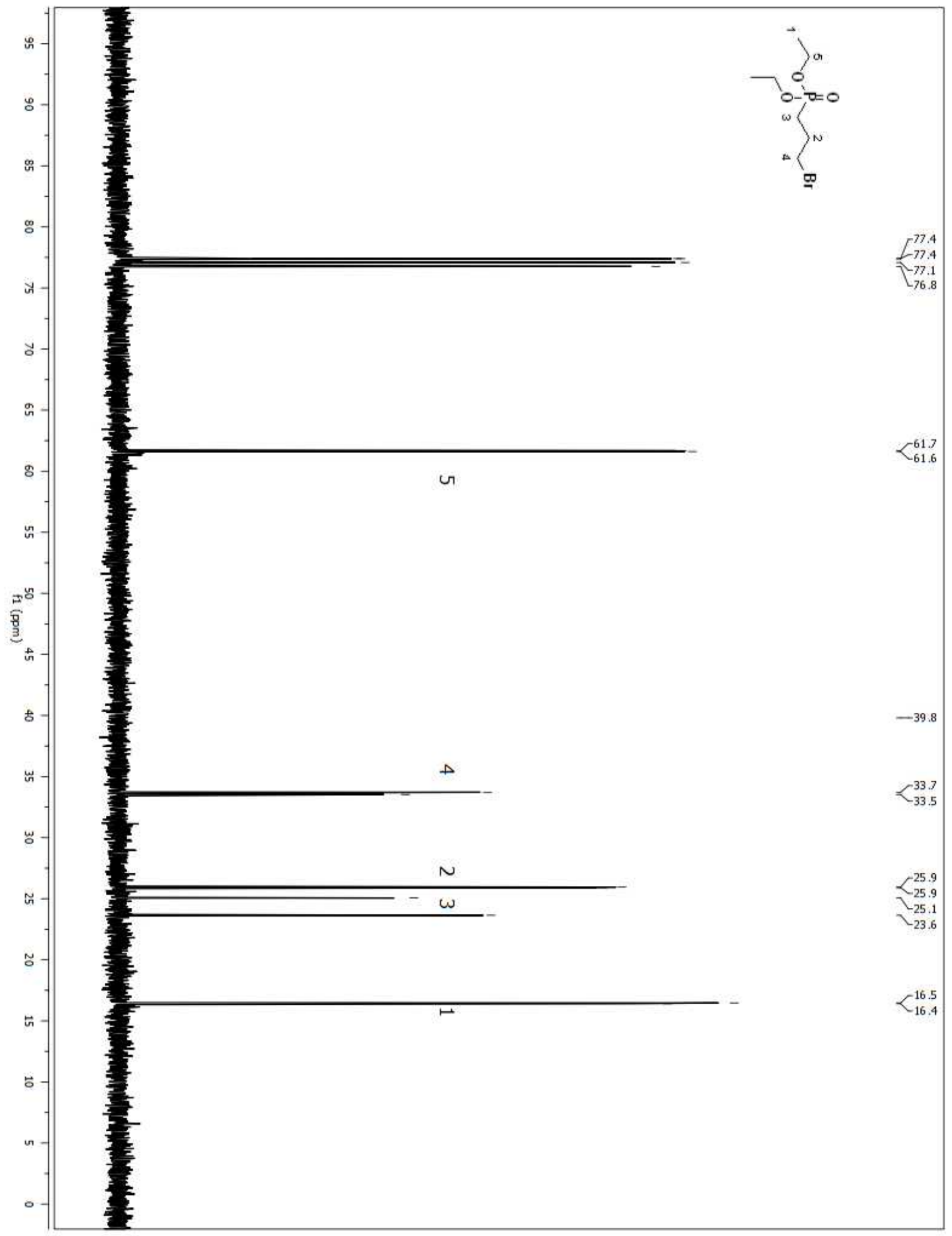

Figure A 22. ${ }^{13} \mathrm{C}$ NMR spectrum of compound 12 in $\mathrm{CDCl}_{3}$ (Table 2.3, entry iii) 


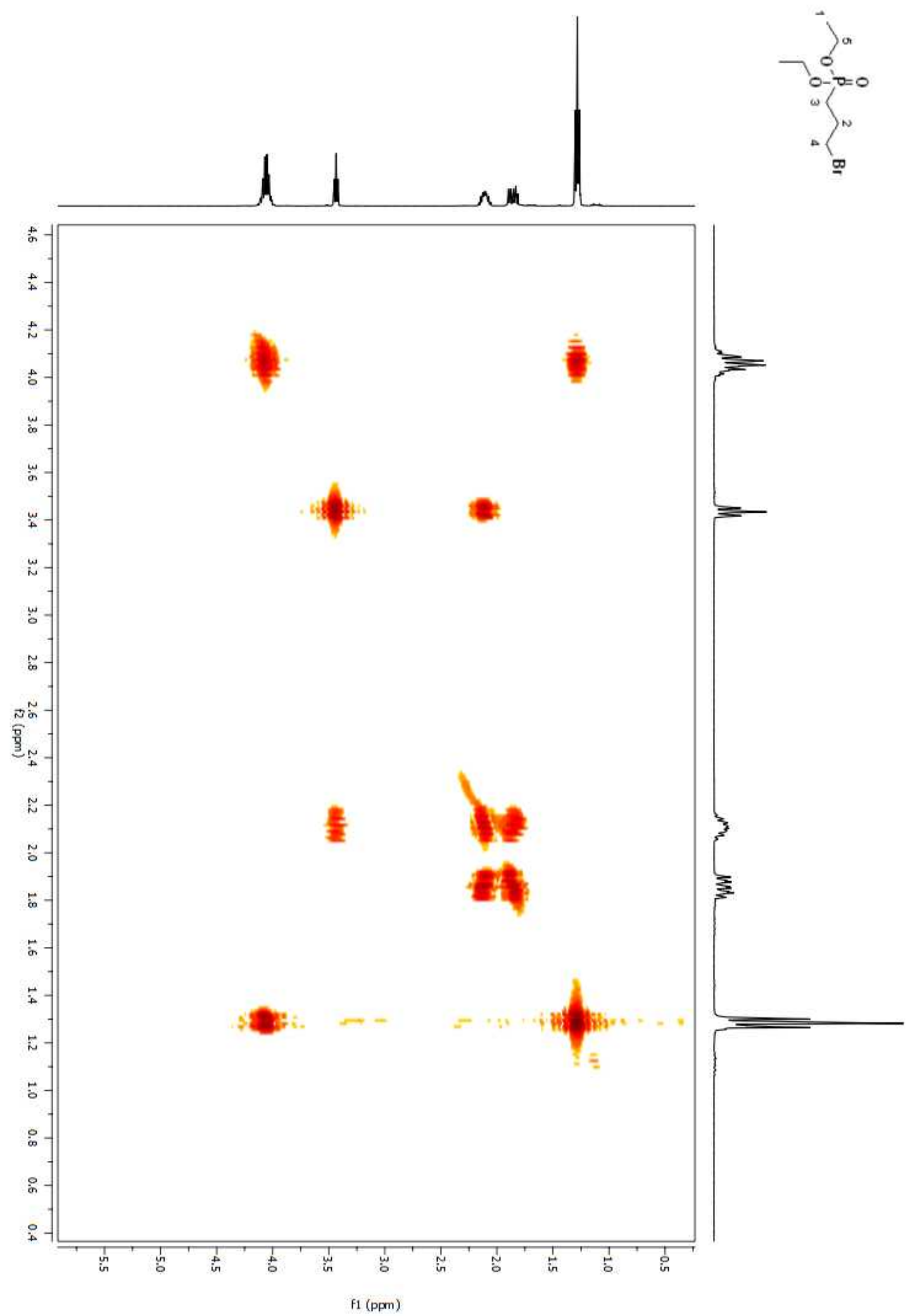

Figure A 23. COSY 2D NMR spectrum of compound 12 in $\mathrm{CDCl}_{3}$ (Table 2.3, entry iii) 390 


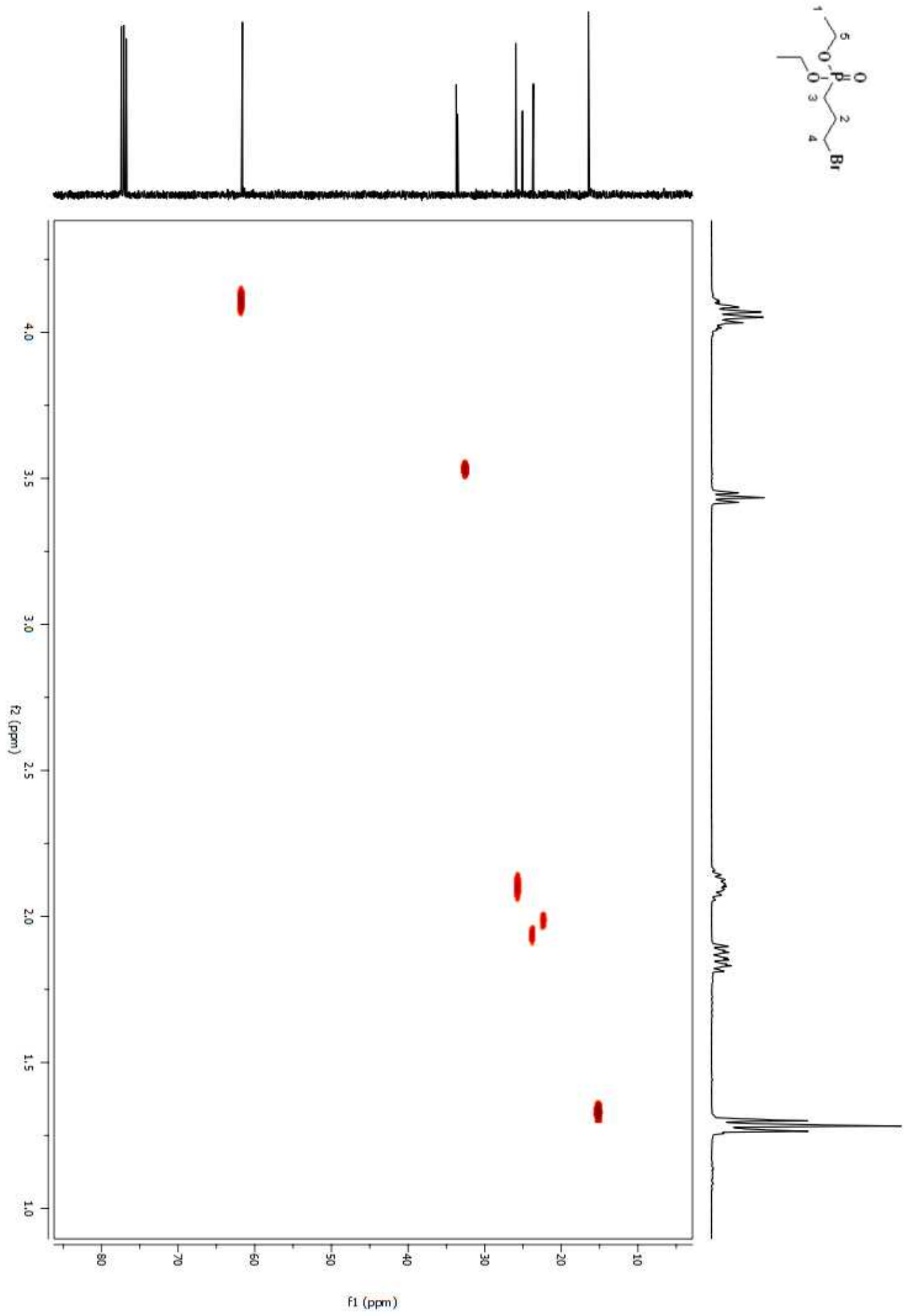

Figure A 24. HSQC 2D NMR spectrum of compound 12 in $\mathrm{CDCl}_{3}$ (Table 2.3, entry iii) 


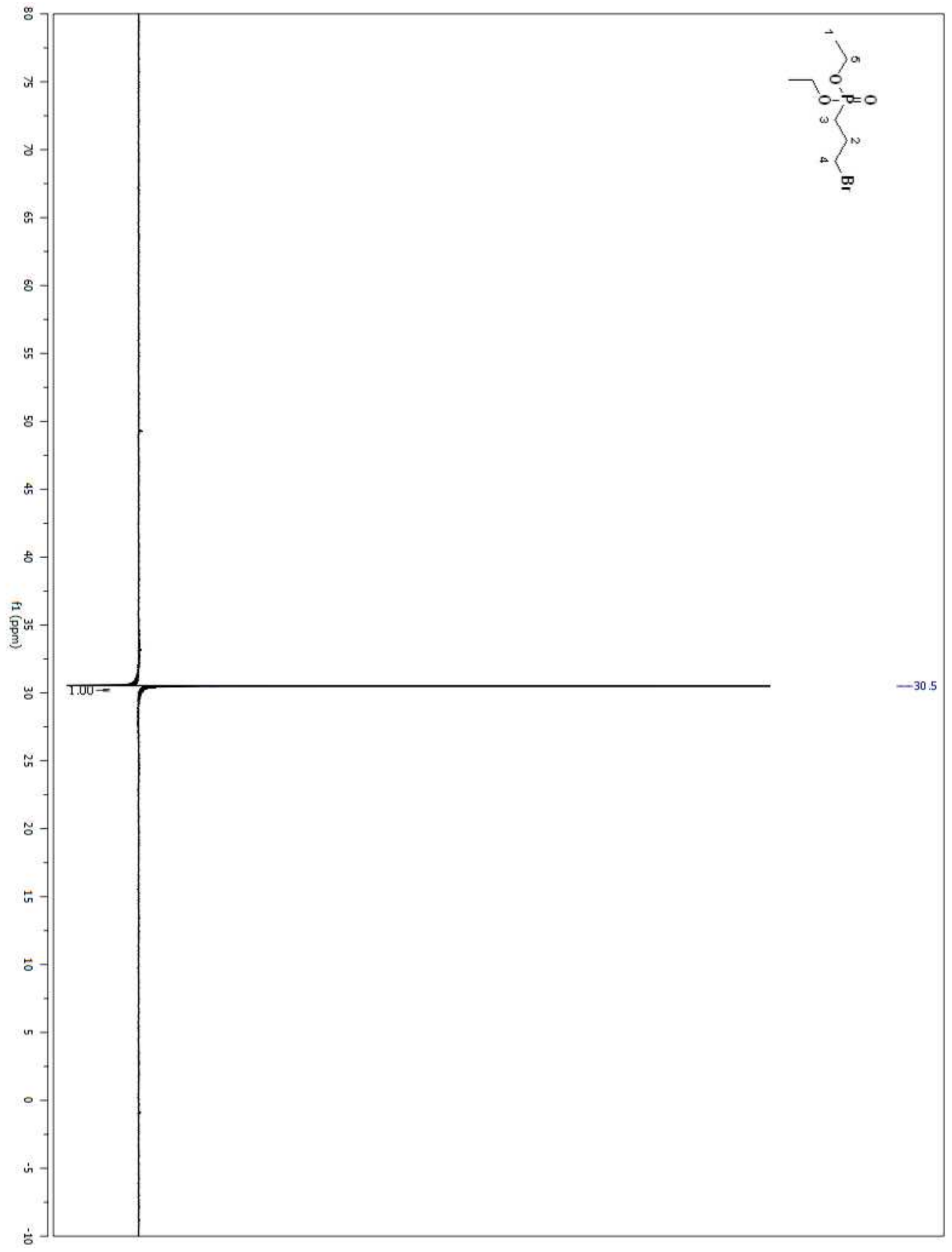

Figure A 25. ${ }^{31} \mathrm{P}$ NMR spectrum of compound 12 in $\mathrm{CDCl}_{3}$ (Table 2.3, entry iii) 


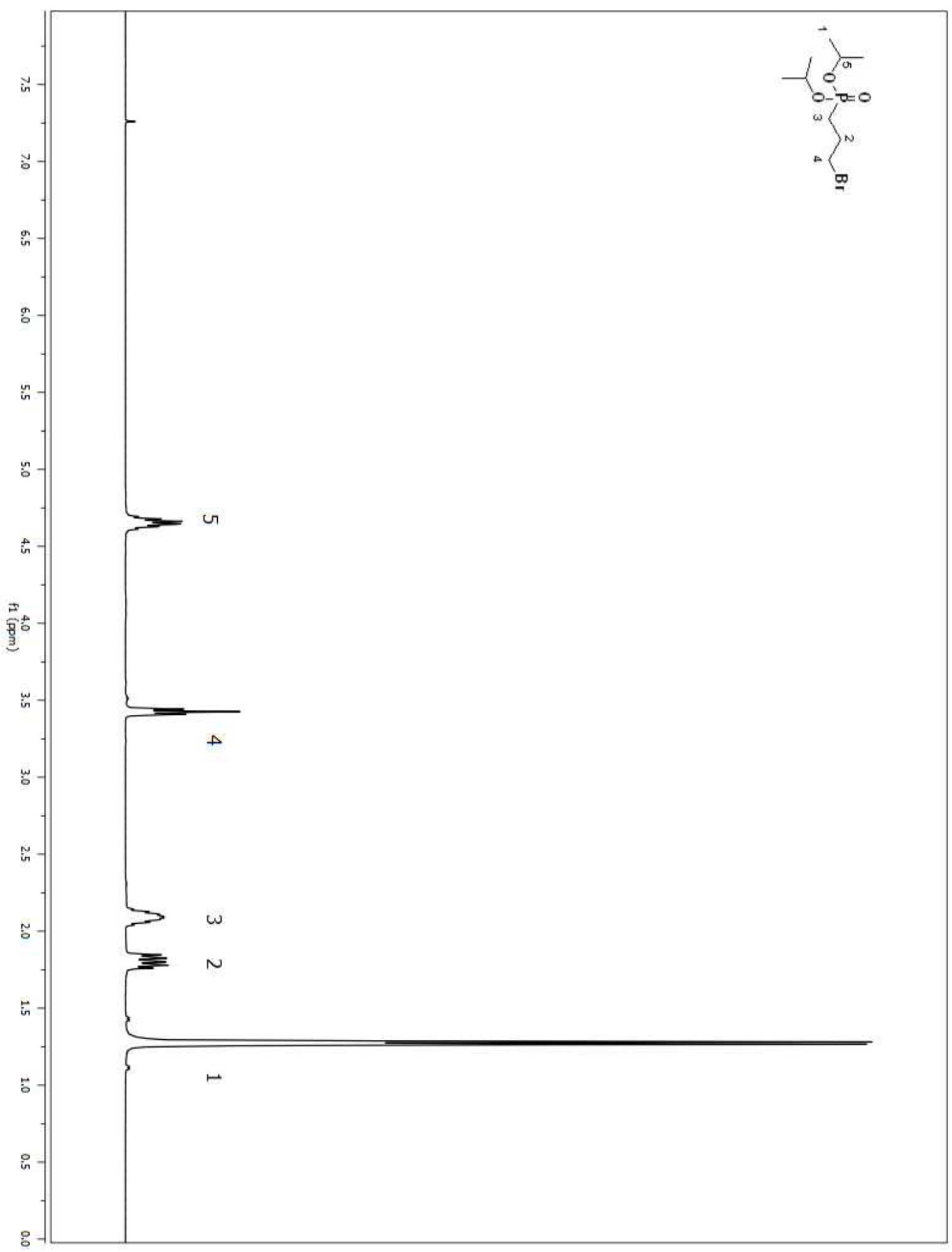

Figure A 26. ${ }^{1} \mathrm{H}$ NMR spectrum of compound 13 in $\mathrm{CDCl}_{3}$ (Table 2.3, entry vii) 


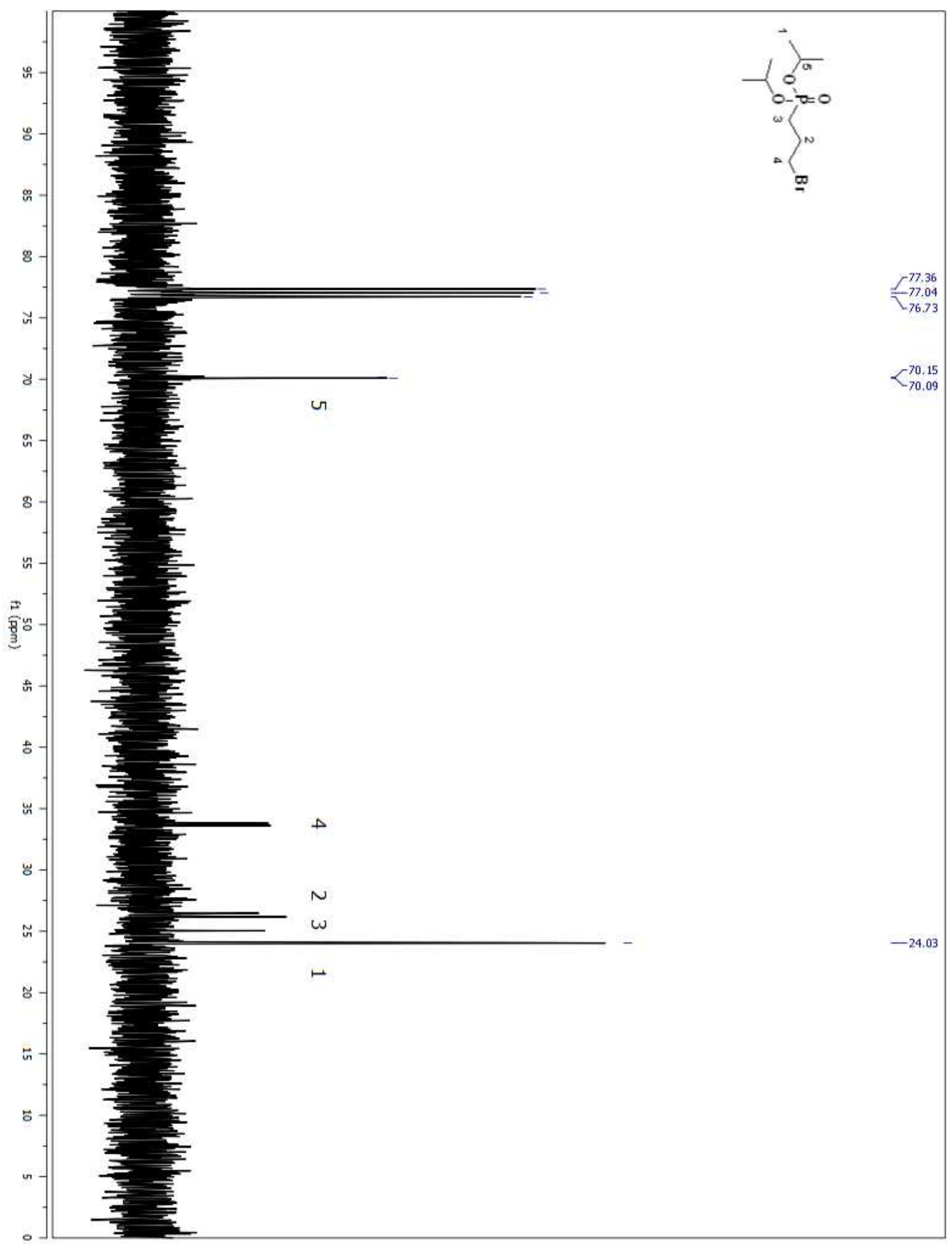

Figure A 27. ${ }^{13} \mathrm{C}$ NMR spectrum of compound 13 in $\mathrm{CDCl}_{3}$ (Table 2.3, entry vii) 


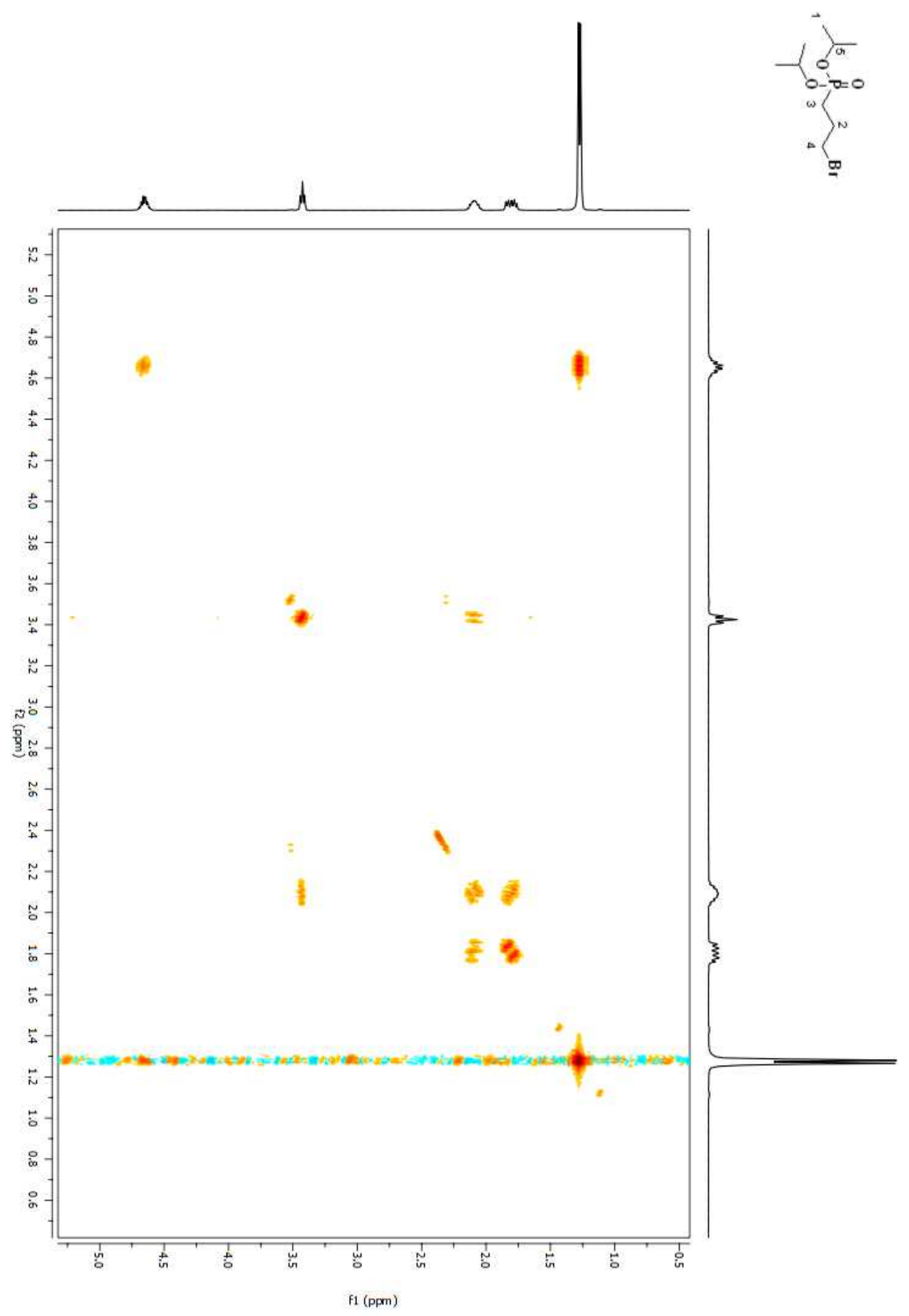

Figure A 28. COSY 2D NMR spectrum of compound 13 in $\mathrm{CDCl}_{3}$ (Table 2.3, entry vii) 


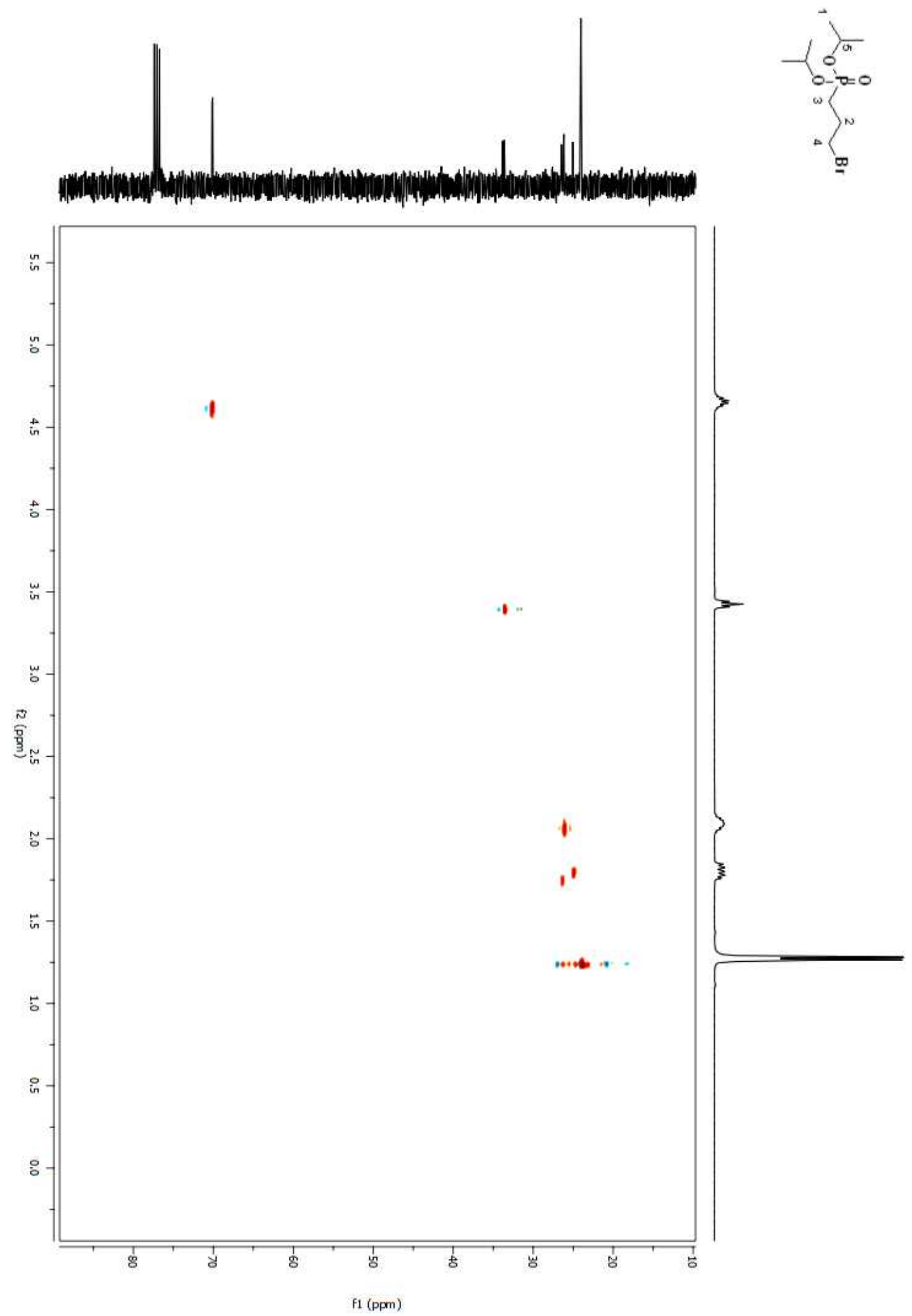

Figure A 29. HSQC 2D NMR spectrum of compound 13 in $\mathrm{CDCl}_{3}$ (Table 2.3, entry vii) 396 


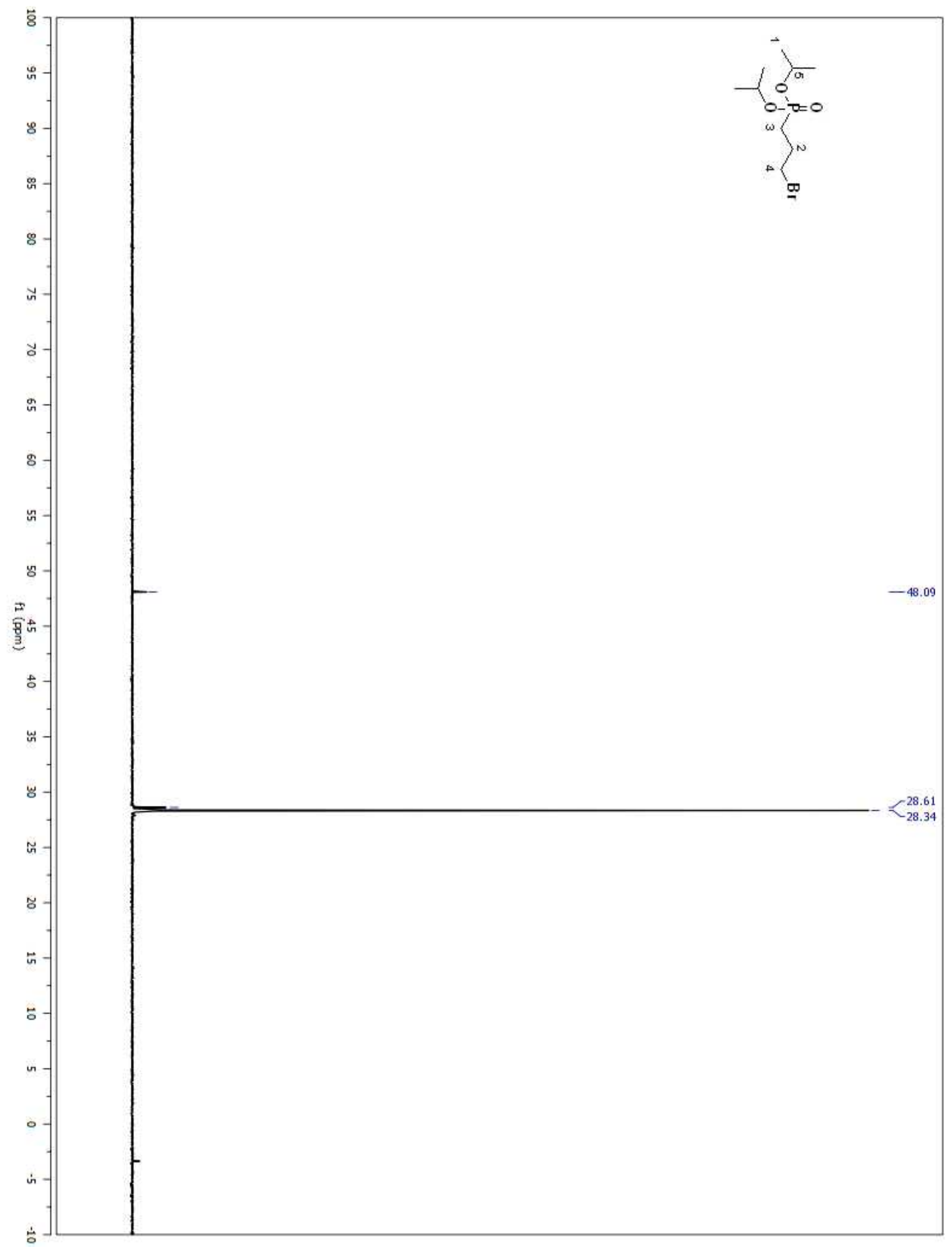

Figure A 30. ${ }^{31} \mathrm{P}$ NMR spectrum of compound 13 in $\mathrm{CDCl}_{3}$ (Table 2.3, entry vii) 


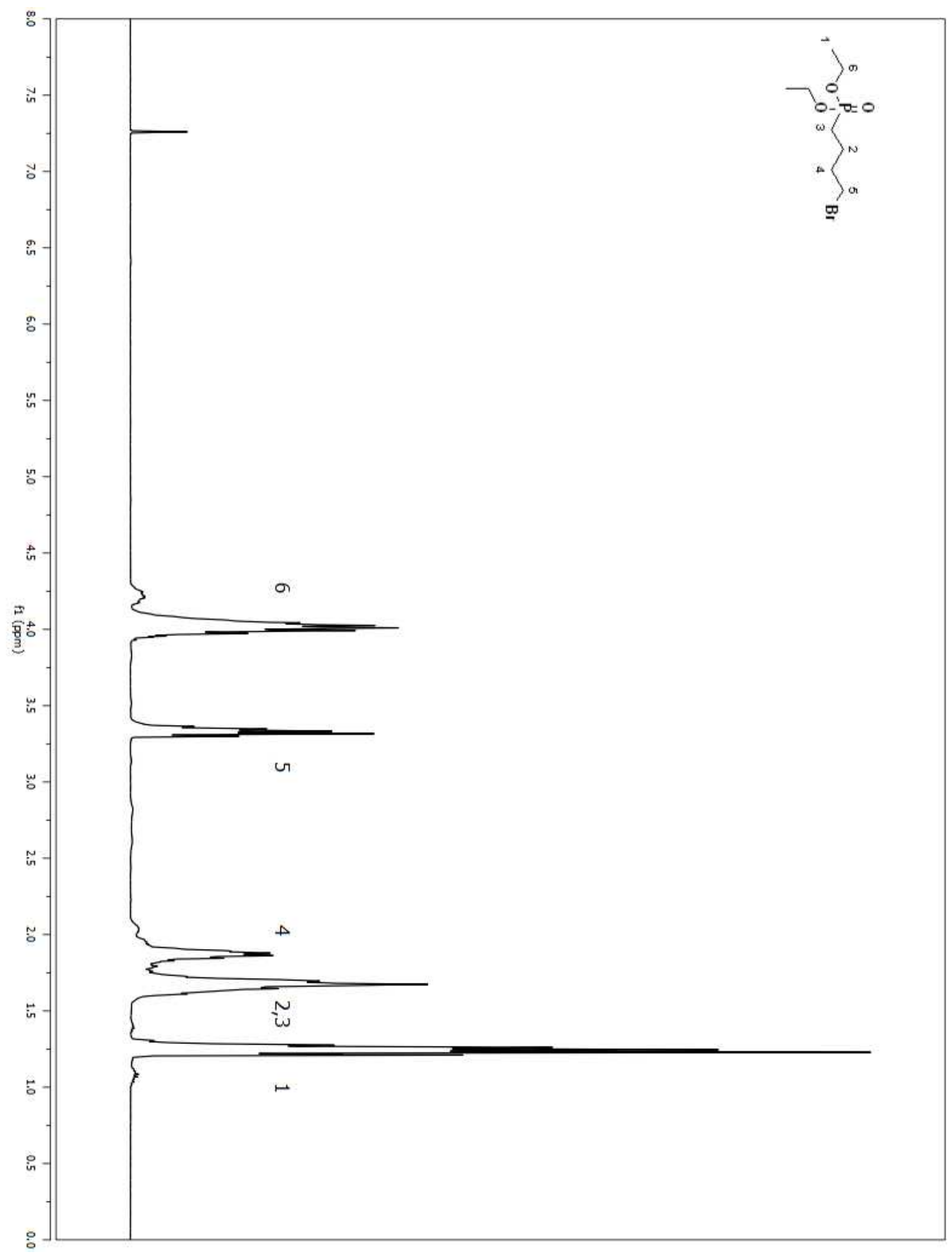

Figure A 31. ${ }^{1} \mathrm{H}$ NMR spectrum of compound 14 in $\mathrm{CDCl}_{3}$ (Table 2.3, entry ix) 


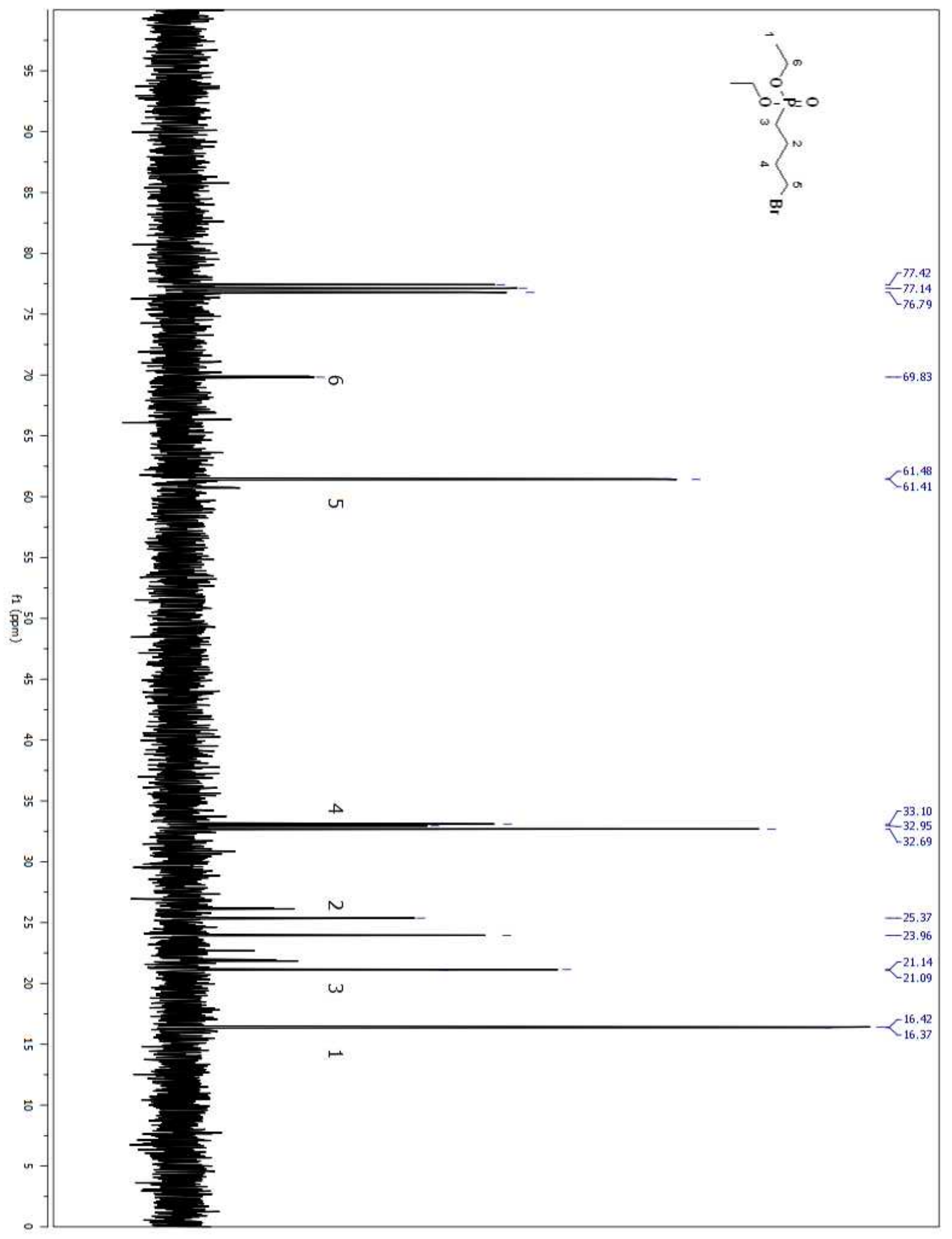

Figure A 32. ${ }^{13} \mathrm{C}$ NMR spectrum of compound 14 in $\mathrm{CDCl}_{3}$ (Table 2.3, entry ix) 


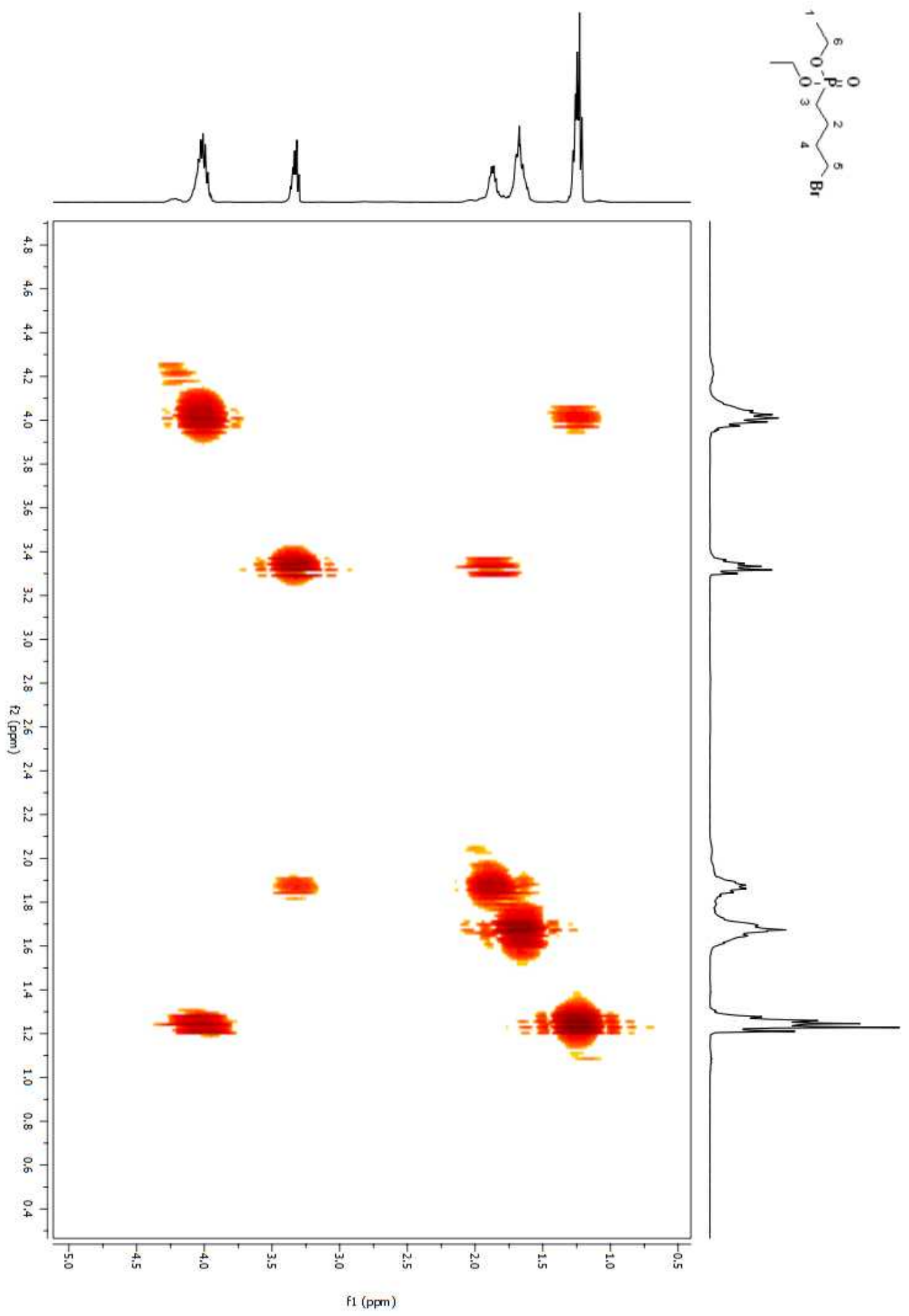

Figure A 33. COSY 2D NMR spectrum of compound 14 in $\mathrm{CDCl}_{3}$ (Table 2.3, entry ix)

400 


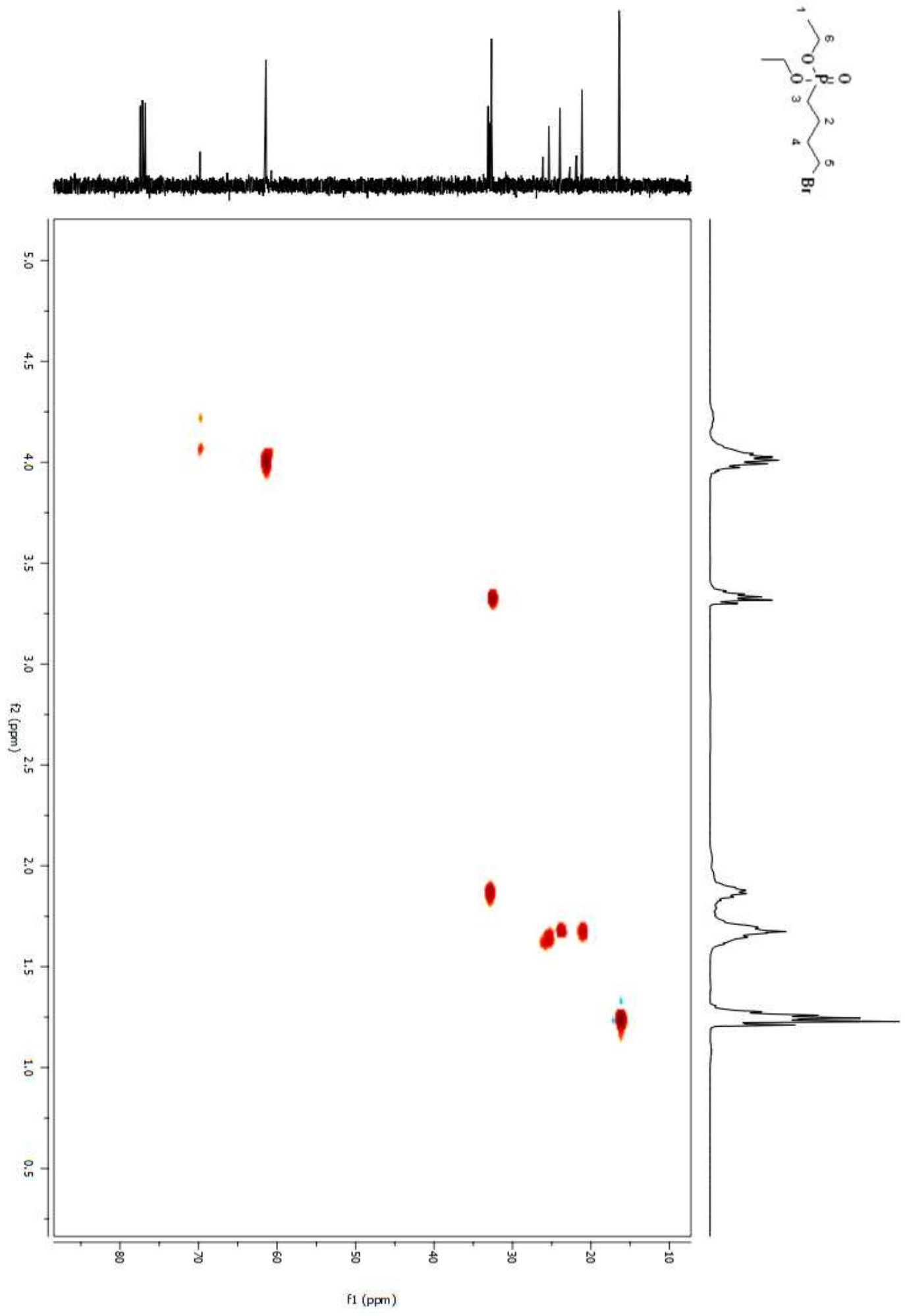

Figure A 34. HSQC 2D NMR spectrum of compound 14 in $\mathrm{CDCl}_{3}$ (Table 2.3, entry ix) 


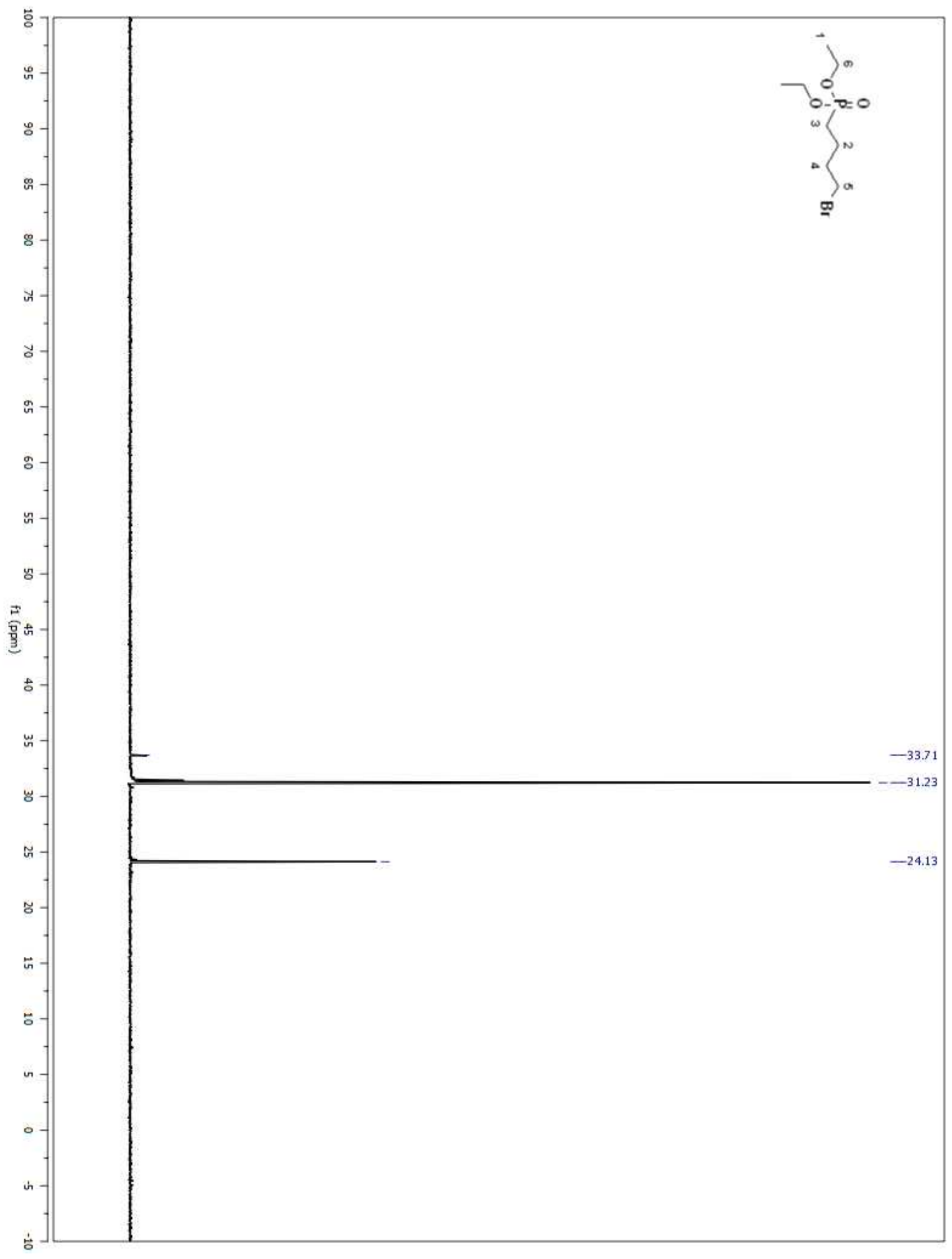

Figure A 35. ${ }^{31} \mathrm{P}$ NMR spectrum of compound 14 in $\mathrm{CDCl}_{3}$ (Table 2.3, entry ix) 


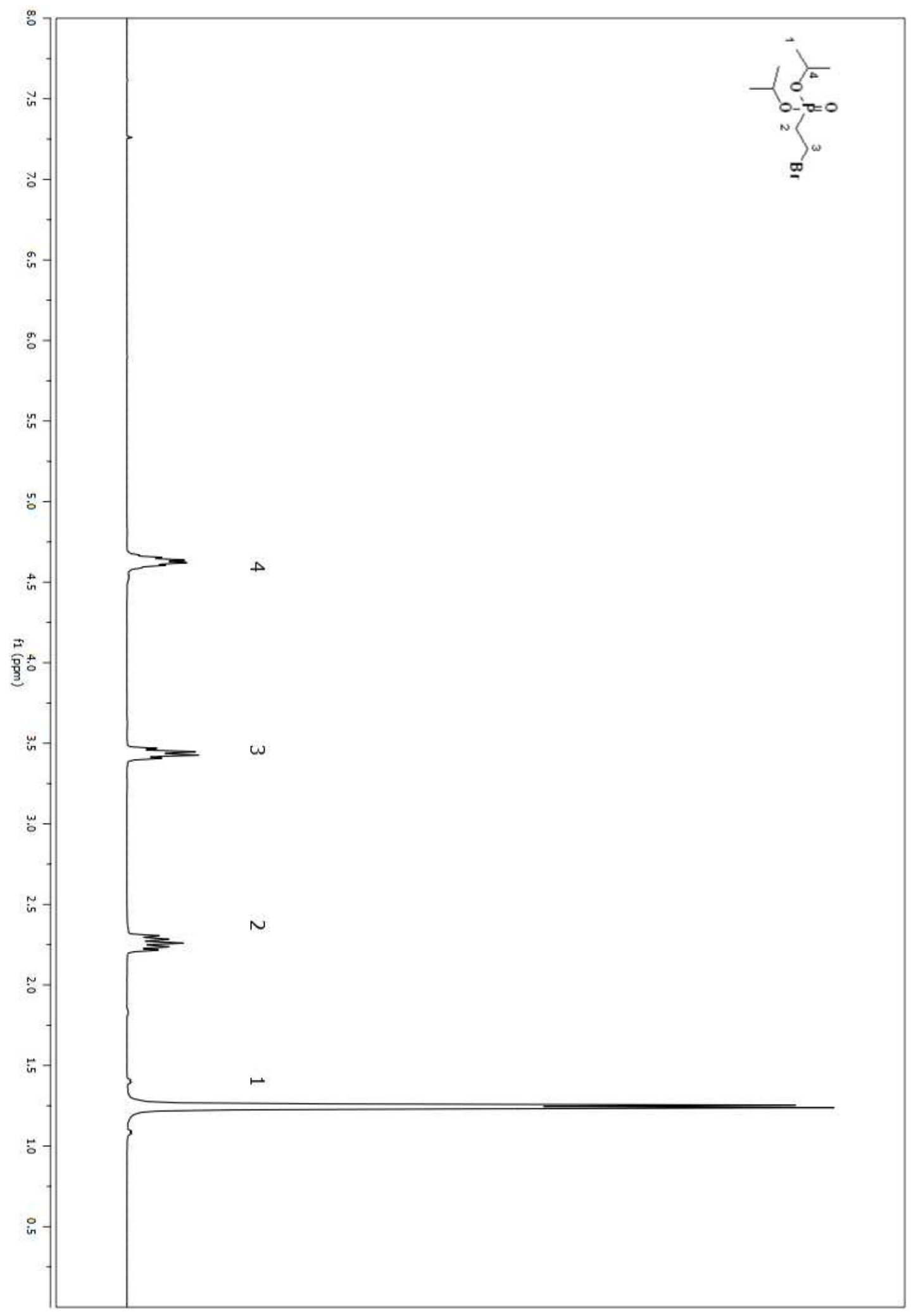

Figure A 36. ${ }^{1} \mathrm{H}$ NMR spectrum of compound 16 in $\mathrm{CDCl}_{3}$ (Table 2.3, entry xv) 


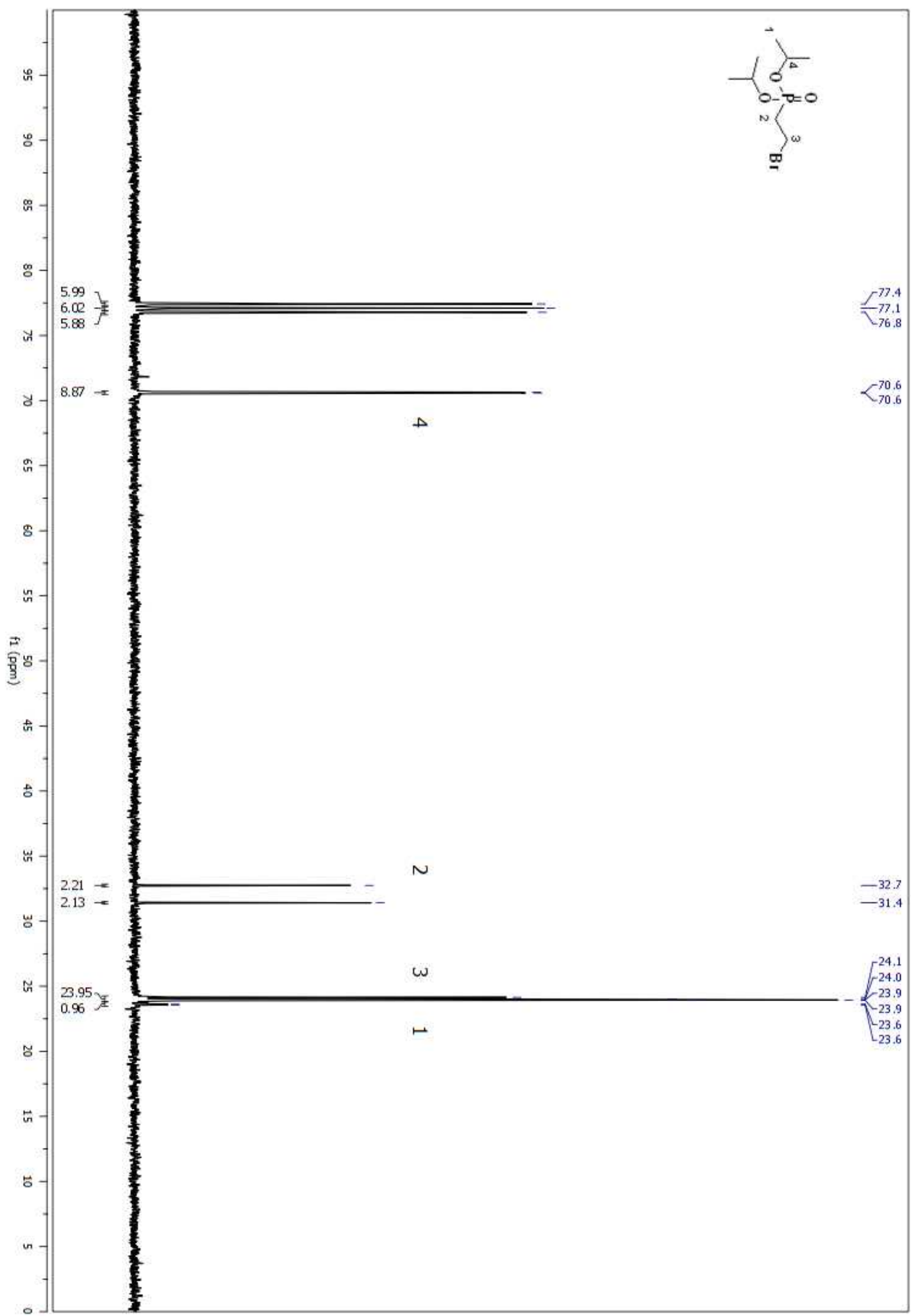

Figure A 37. ${ }^{13} \mathrm{C}$ NMR spectrum of compound 16 in $\mathrm{CDCl}_{3}$ (Table 2.3, entry xv) 


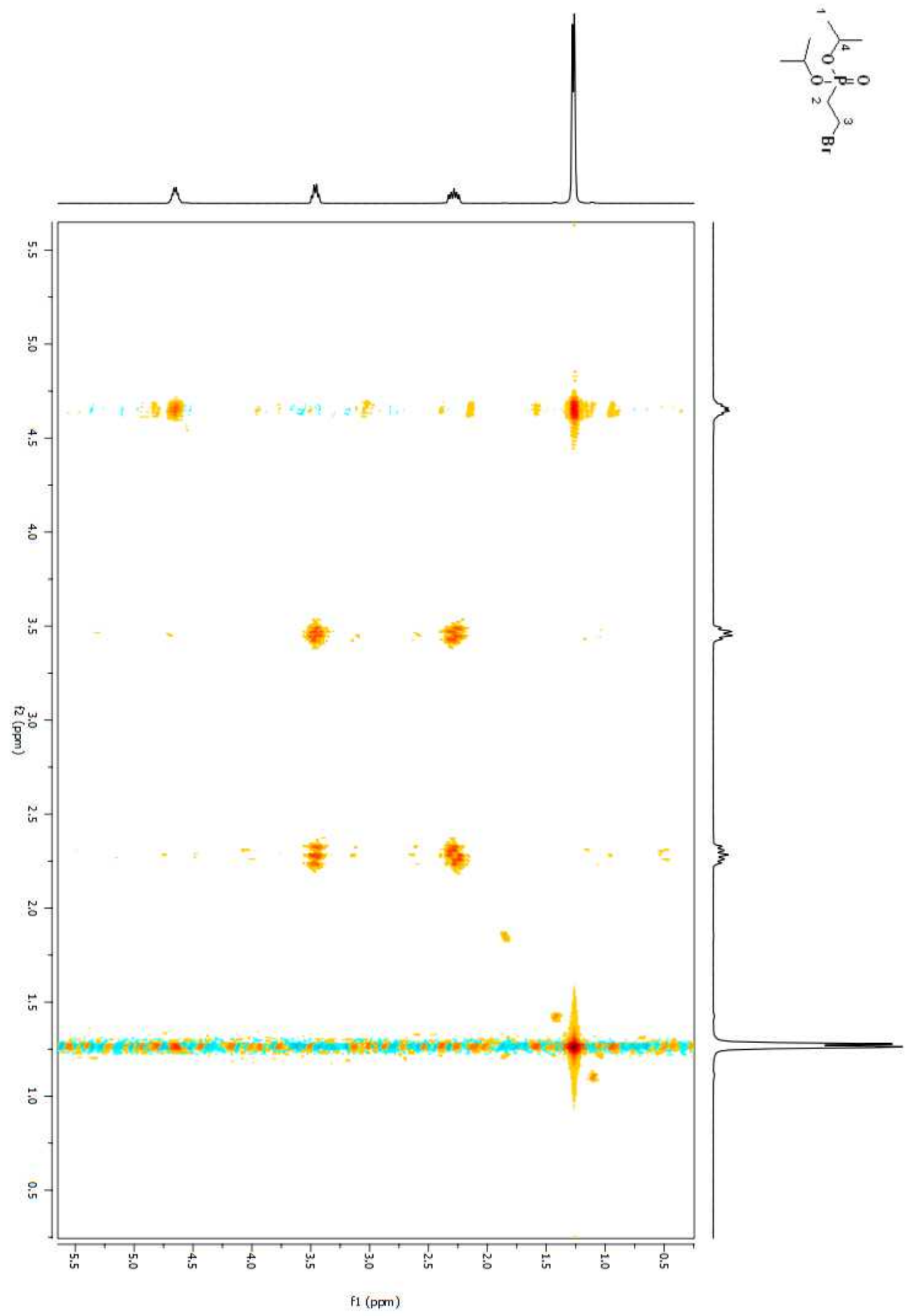

Figure A 38. COSY 2D NMR spectrum of compound 16 in $\mathrm{CDCl}_{3}$ (Table 2.3, entry xv) 


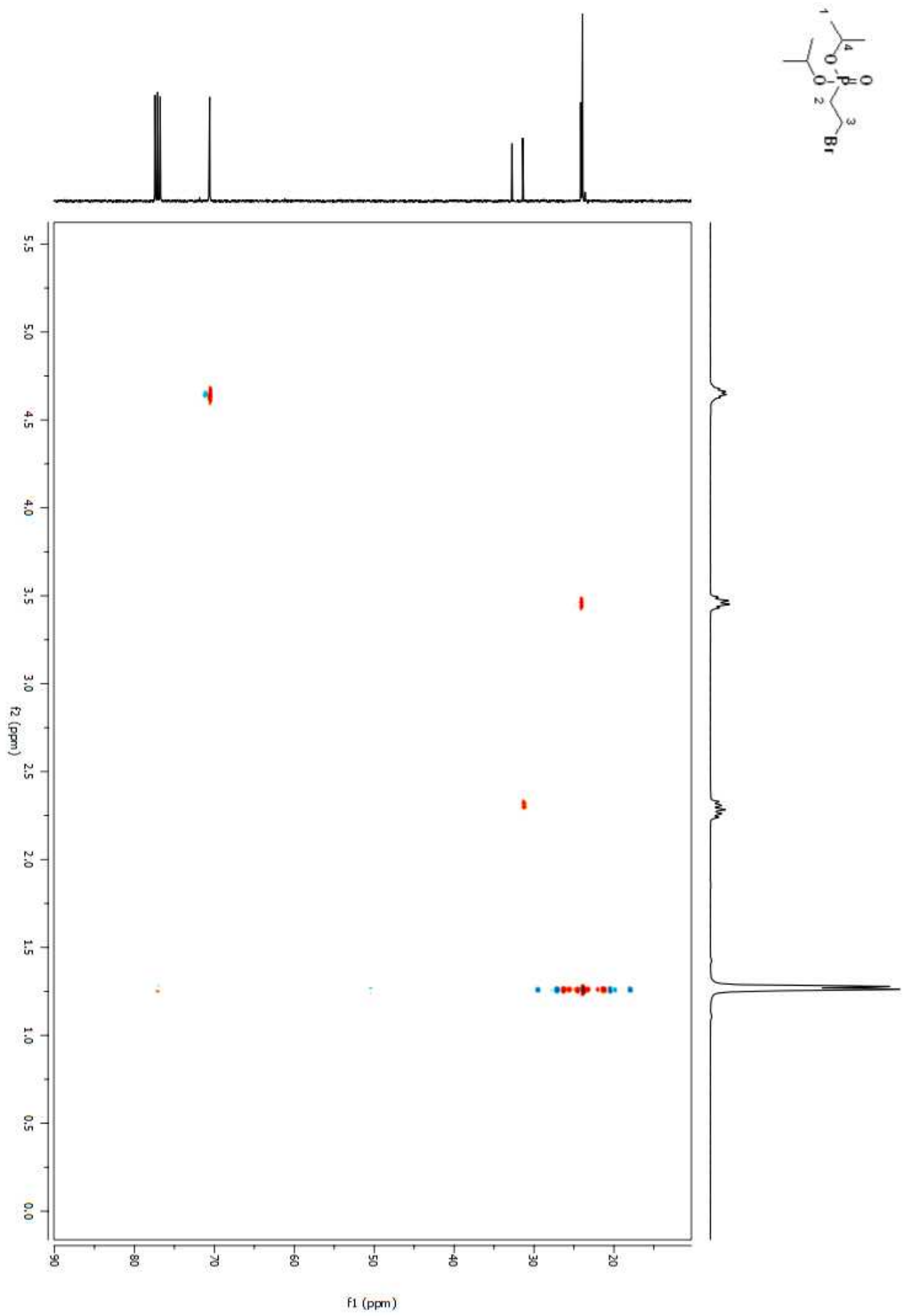

Figure A 39. HSQC 2D NMR spectrum of compound 16 in $\mathrm{CDCl}_{3}$ (Table 2.3, entry xv) 


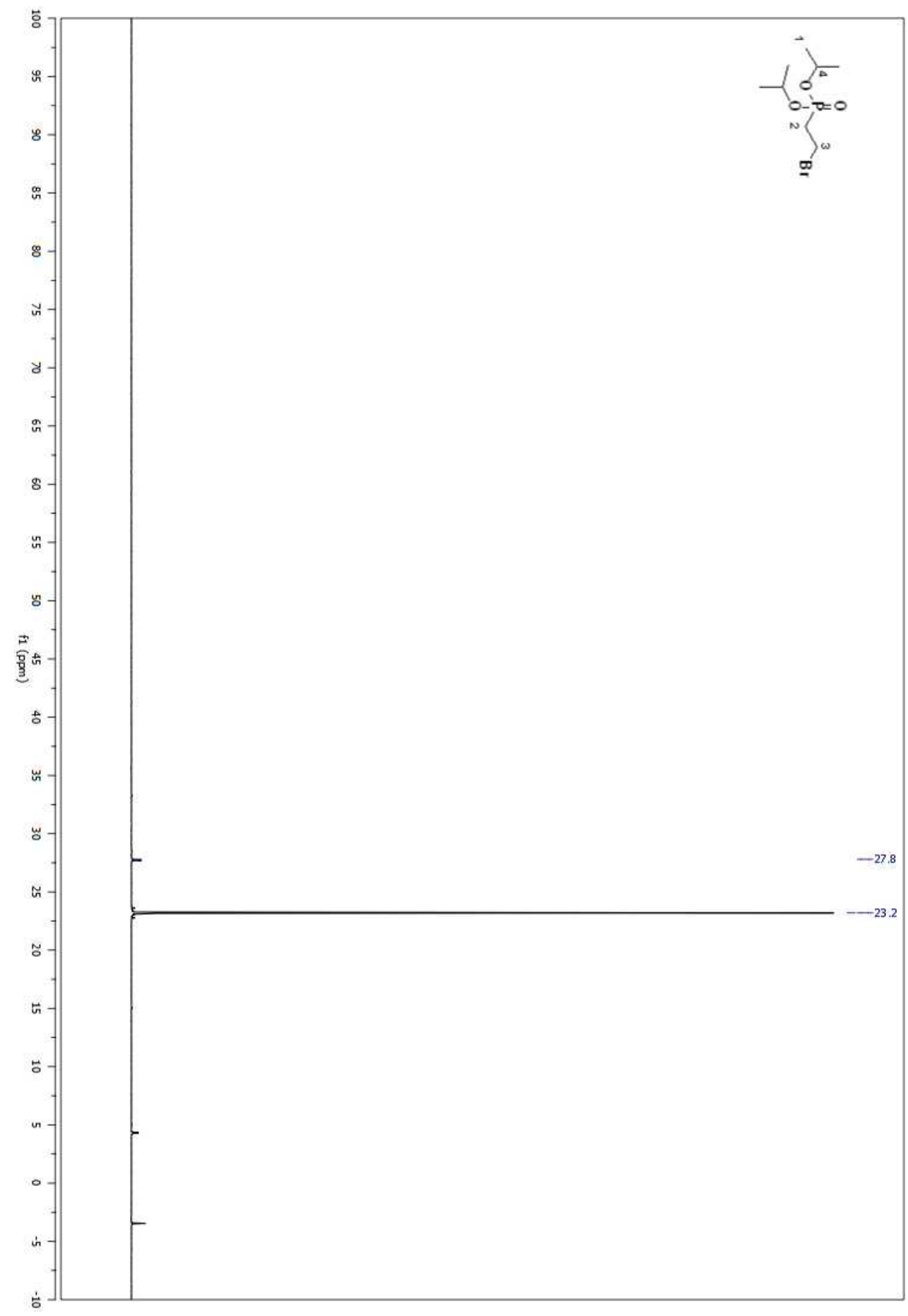

Figure A $40 .{ }^{31} \mathrm{P}$ NMR spectrum of compound 16 in $\mathrm{CDCl}_{3}$ (Table 4.3, entry xv) 


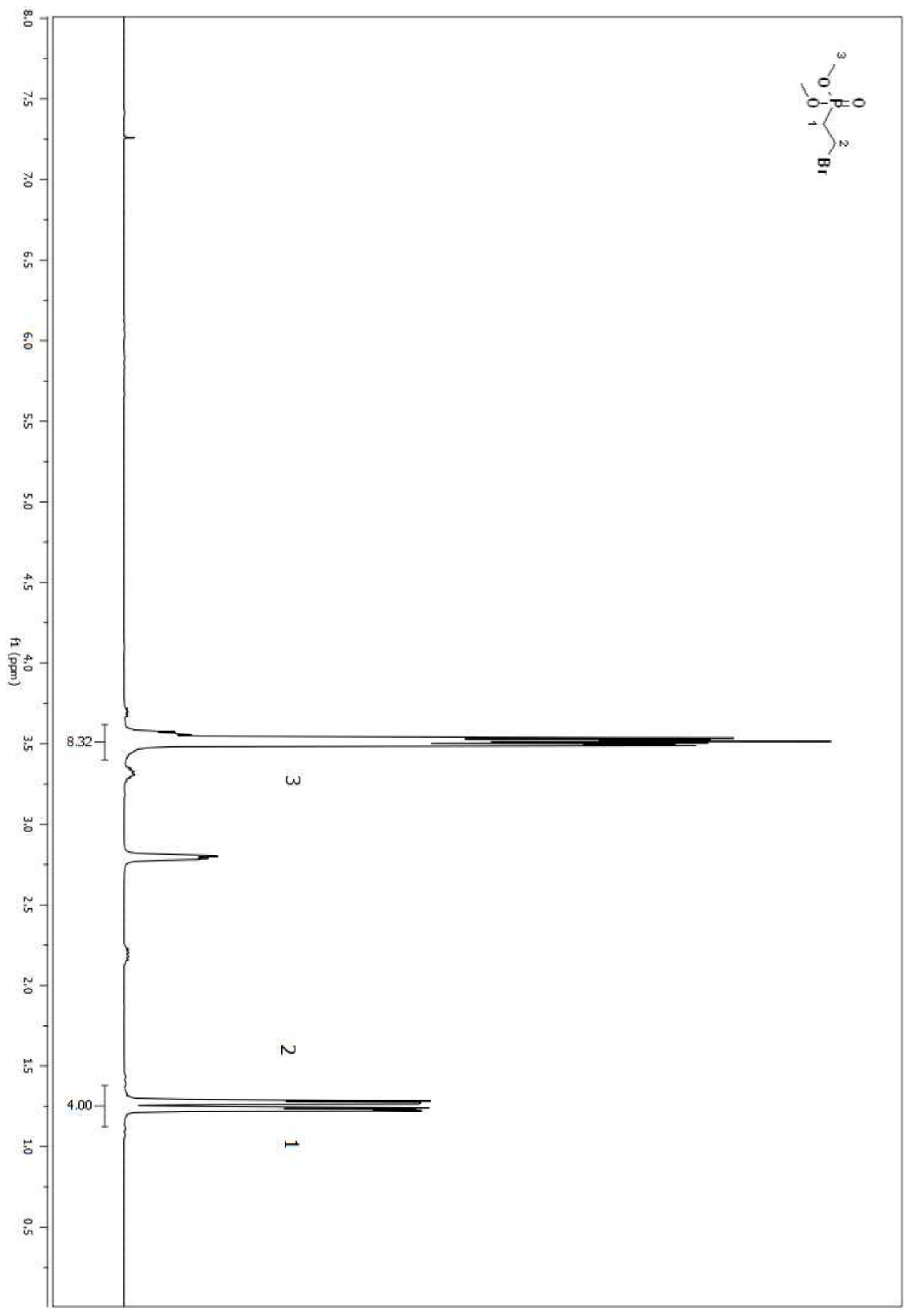

Figure A 41. ${ }^{1} \mathrm{H}$ NMR spectrum of compound 17 in $\mathrm{CDCl}_{3}$ (Table 2.3, entry xvi) 


$$
E
$$




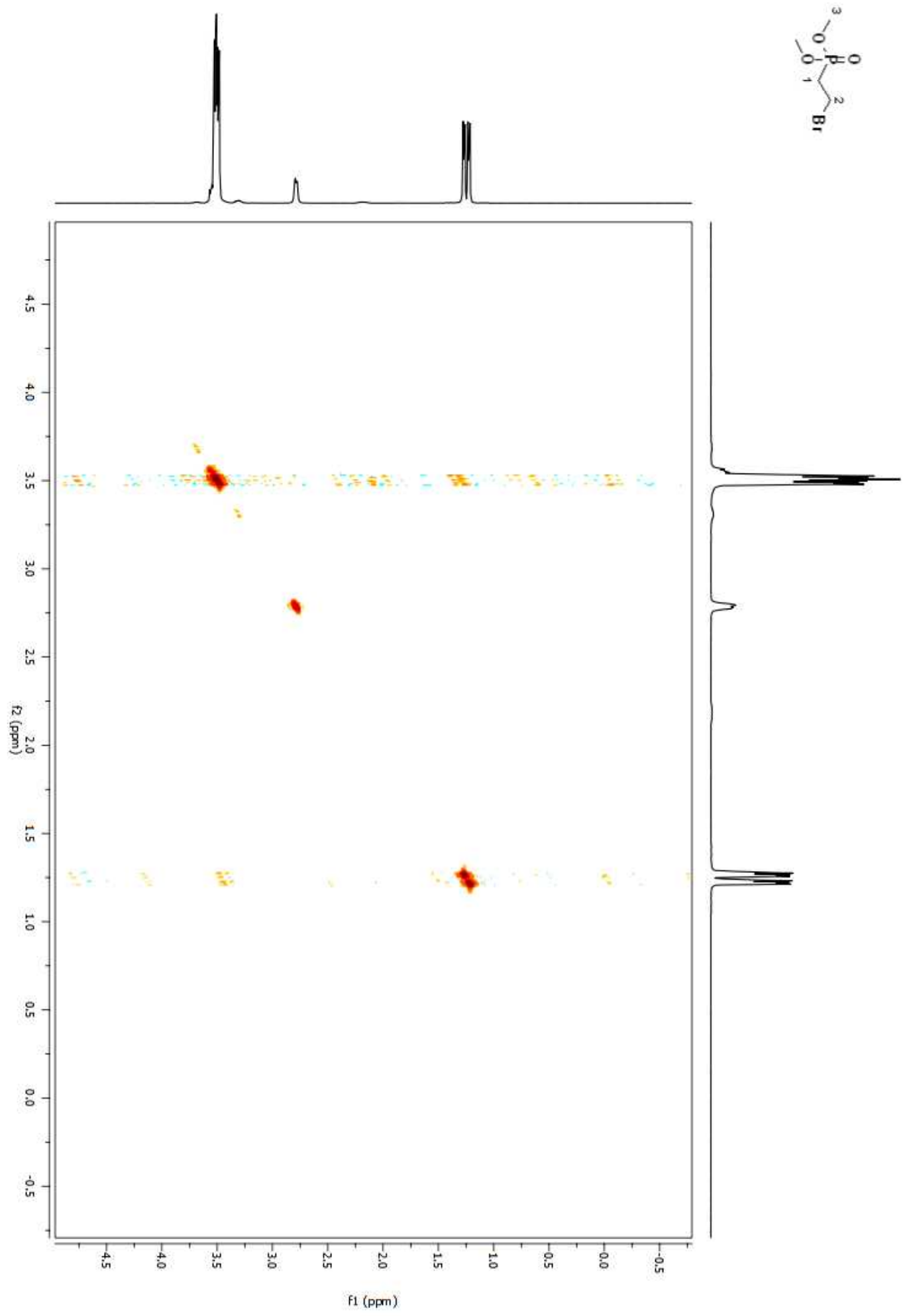

Figure A 43. COSY 2D NMR spectrum of compound 17 in $\mathrm{CDCl}_{3}$ (Table 2.3, entry xvi) 


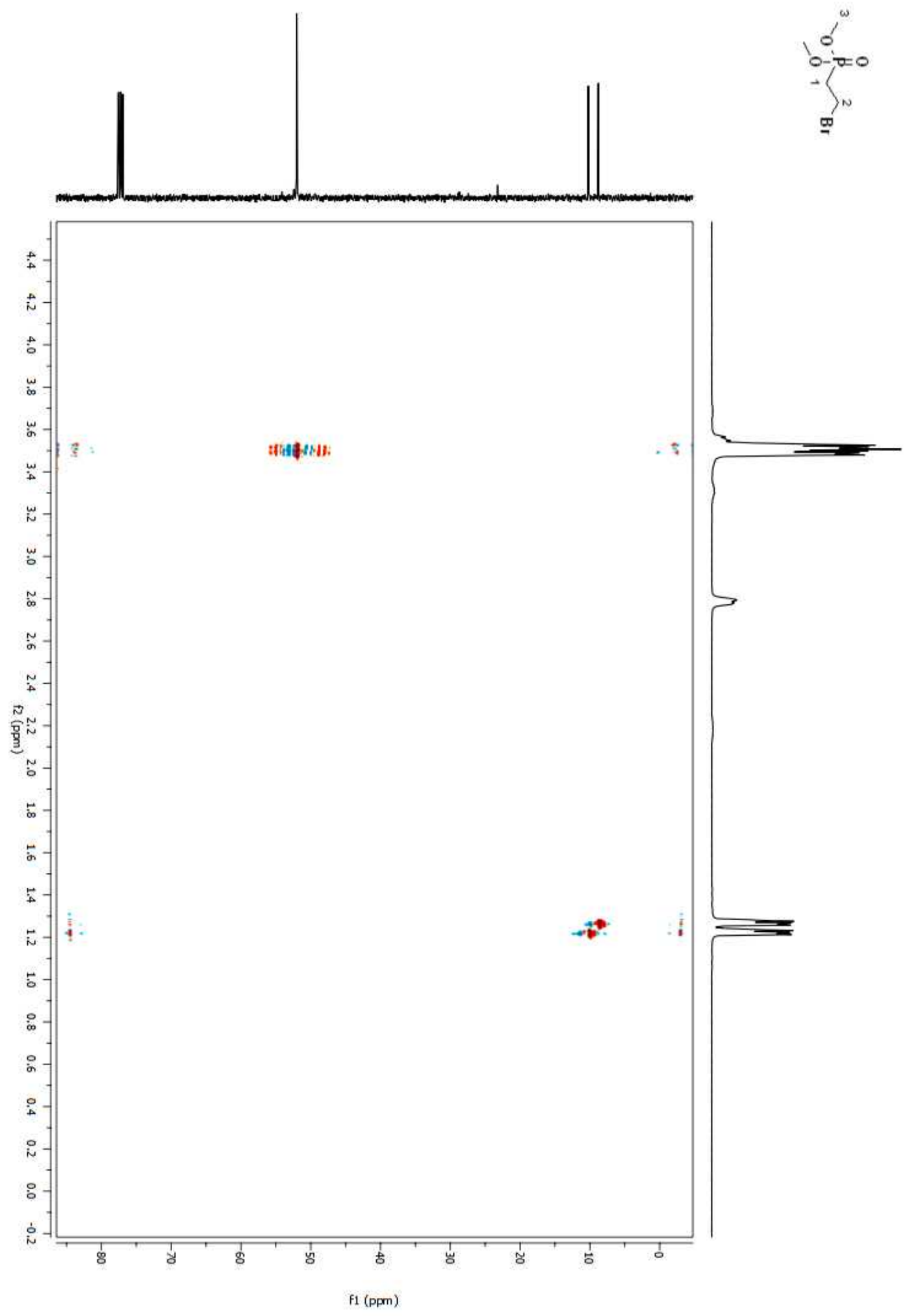

Figure A 44. HSQC 2D NMR spectrum of compound 17 in $\mathrm{CDCl}_{3}$ (Table 2.3, entry xvi) 


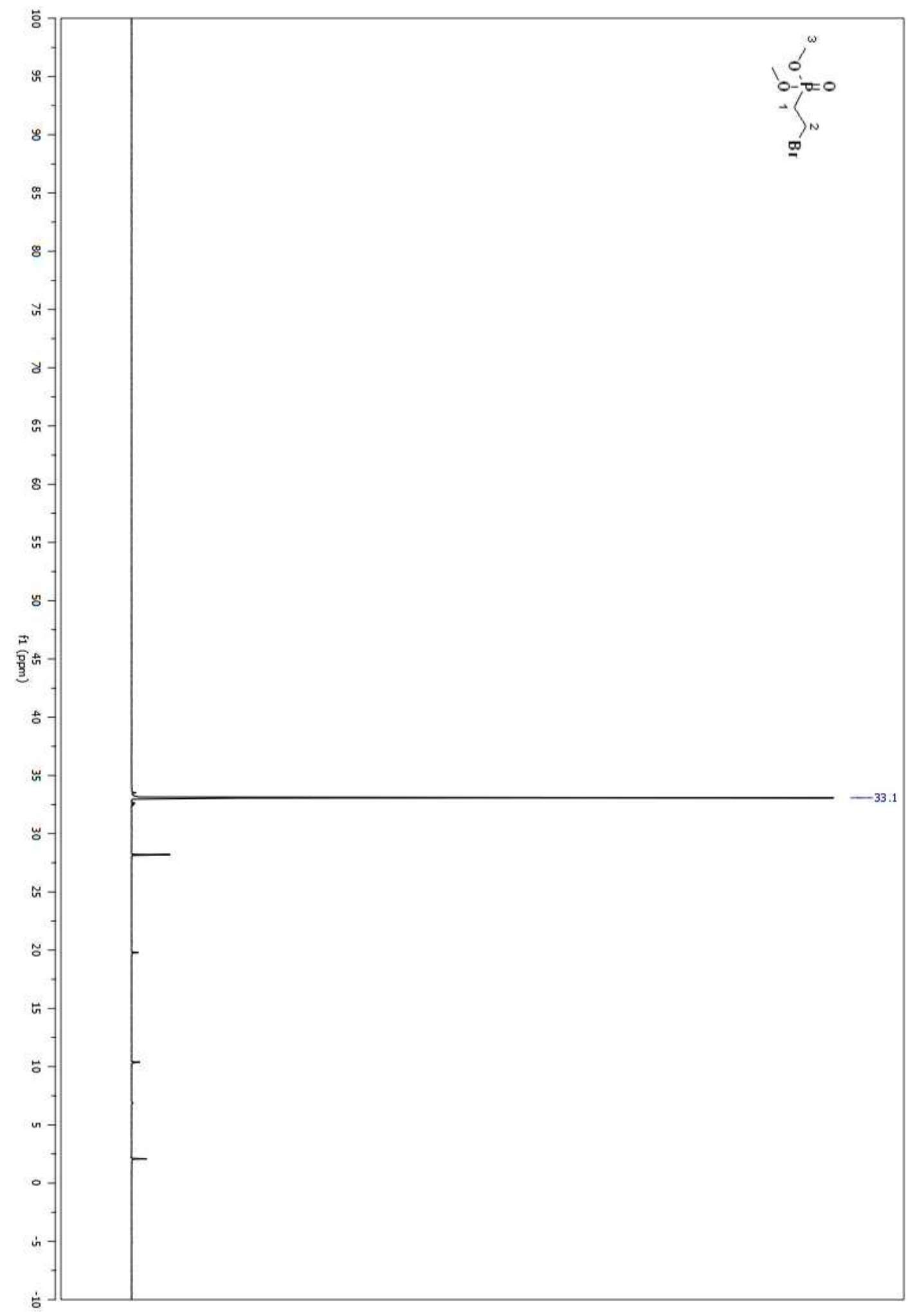

Figure A 45. ${ }^{31} \mathrm{P}$ NMR spectrum of compound 17 in $\mathrm{CDCl}_{3}$ (Table 2.3, entry xvi) 


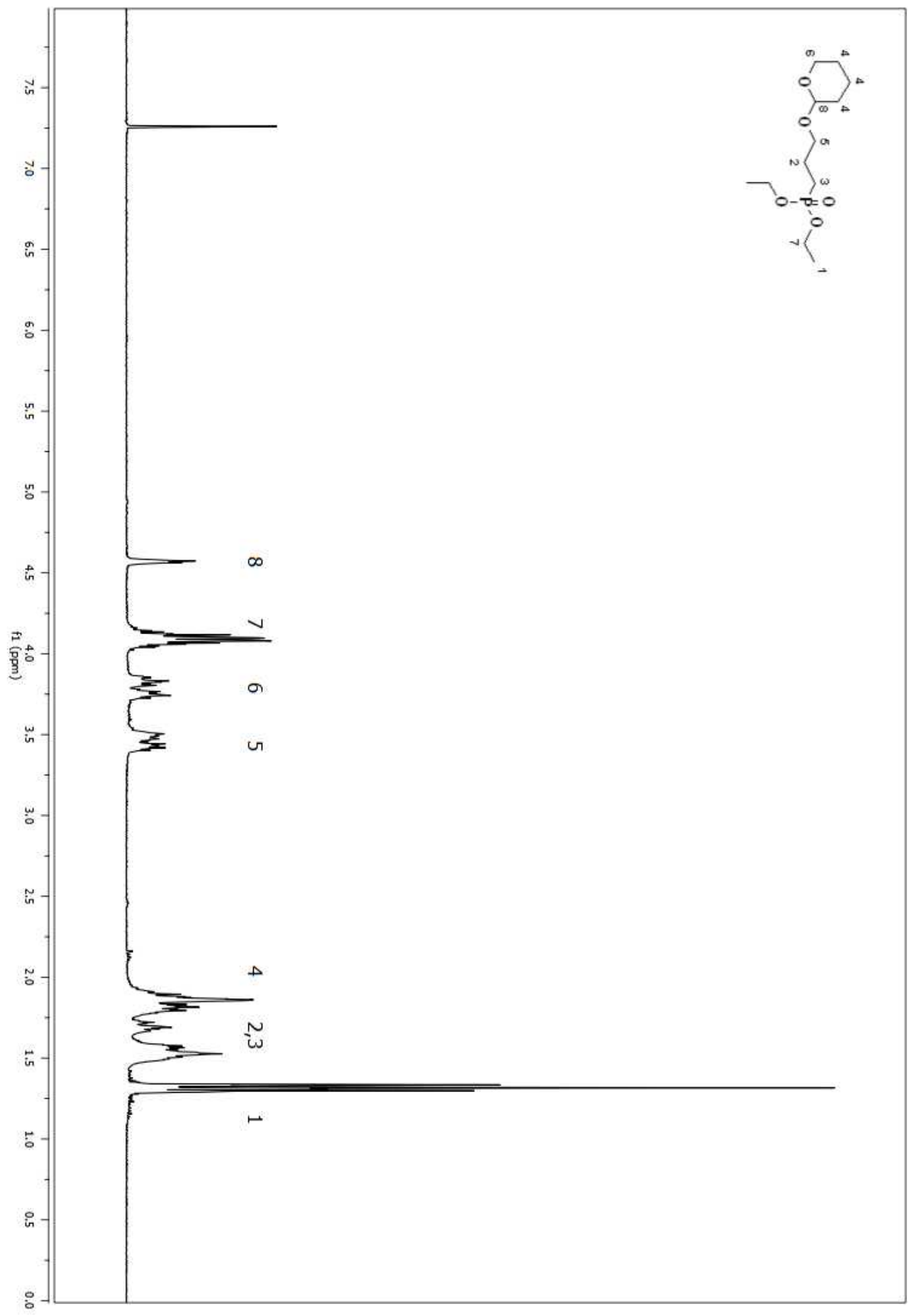

Figure A 46. ${ }^{1} \mathrm{H}$ NMR spectrum of compound 18 in $\mathrm{CDCl}_{3}$ (Table 2.3, entry xvii) 


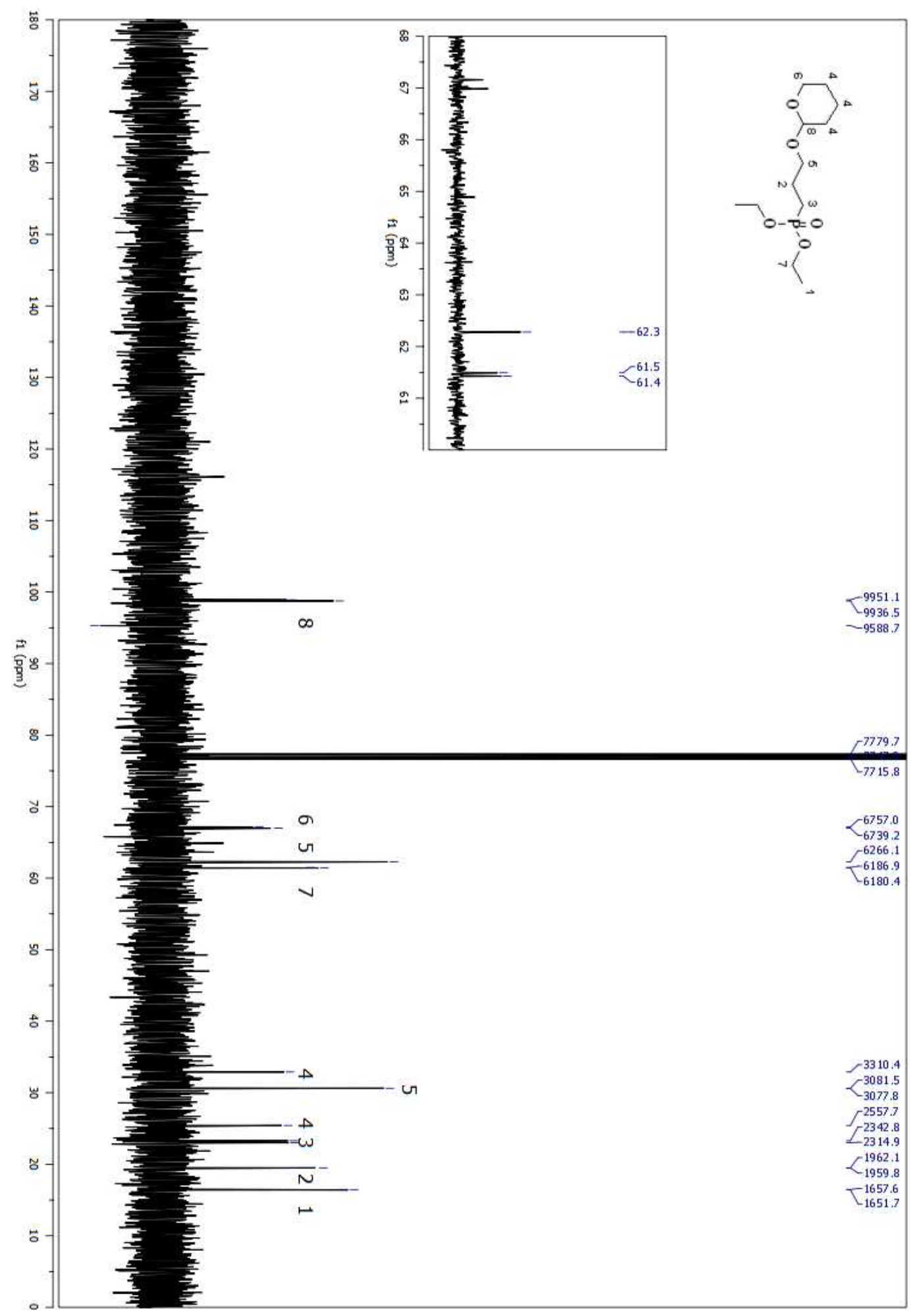

Figure A 47. ${ }^{13} \mathrm{C}$ NMR spectrum of compound 18 in $\mathrm{CDCl}_{3}$ (Table 2.3, entry xvii) 


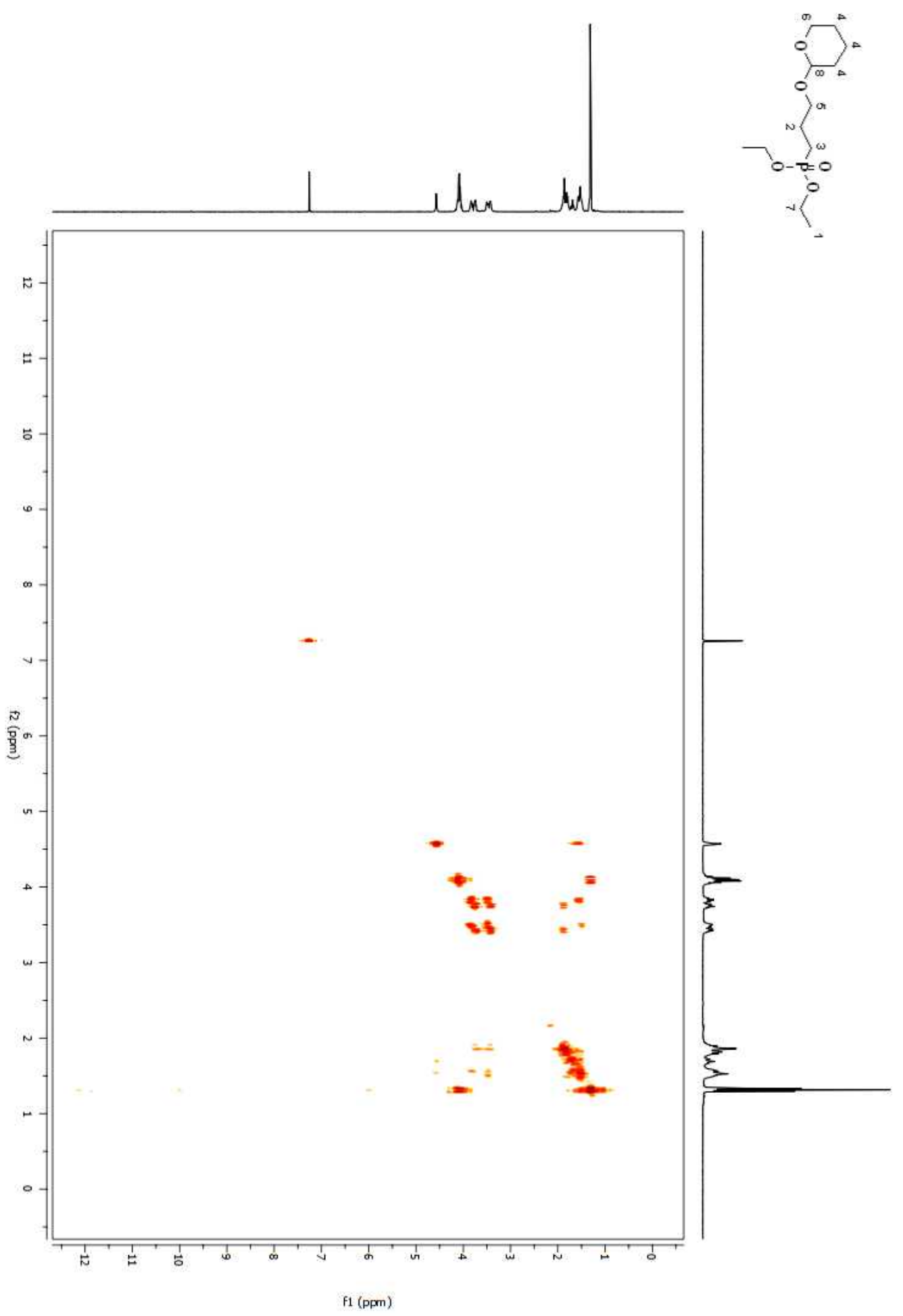

Figure A 48. COSY 2D NMR spectrum of compound 18 in $\mathrm{CDCl}_{3}$ (Table 2.3, entry xvii) 


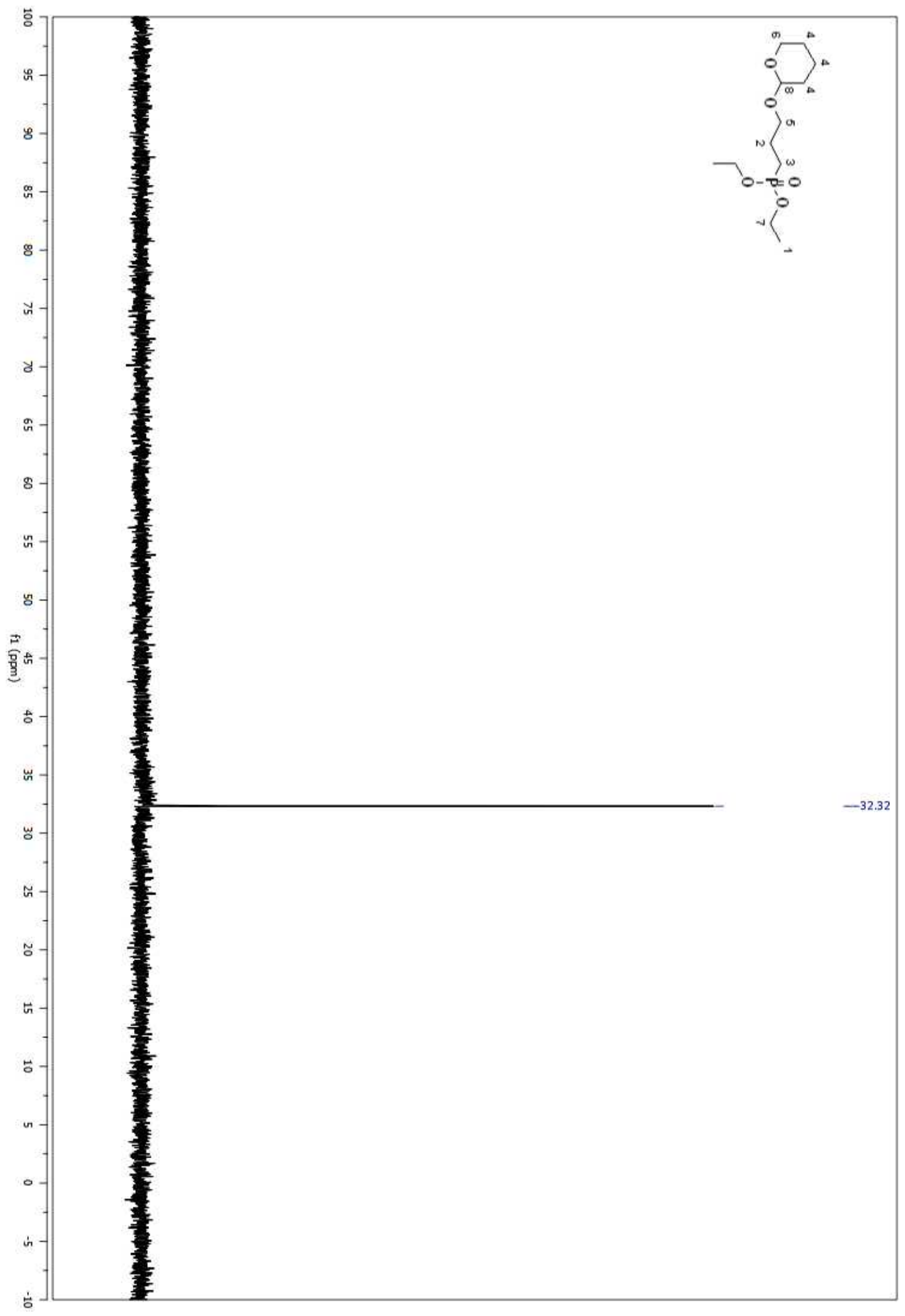

Figure A 49. ${ }^{31} \mathrm{P}$ NMR spectrum of compound 18 in $\mathrm{CDCl}_{3}$ (Table 4.3, entry xvii) 


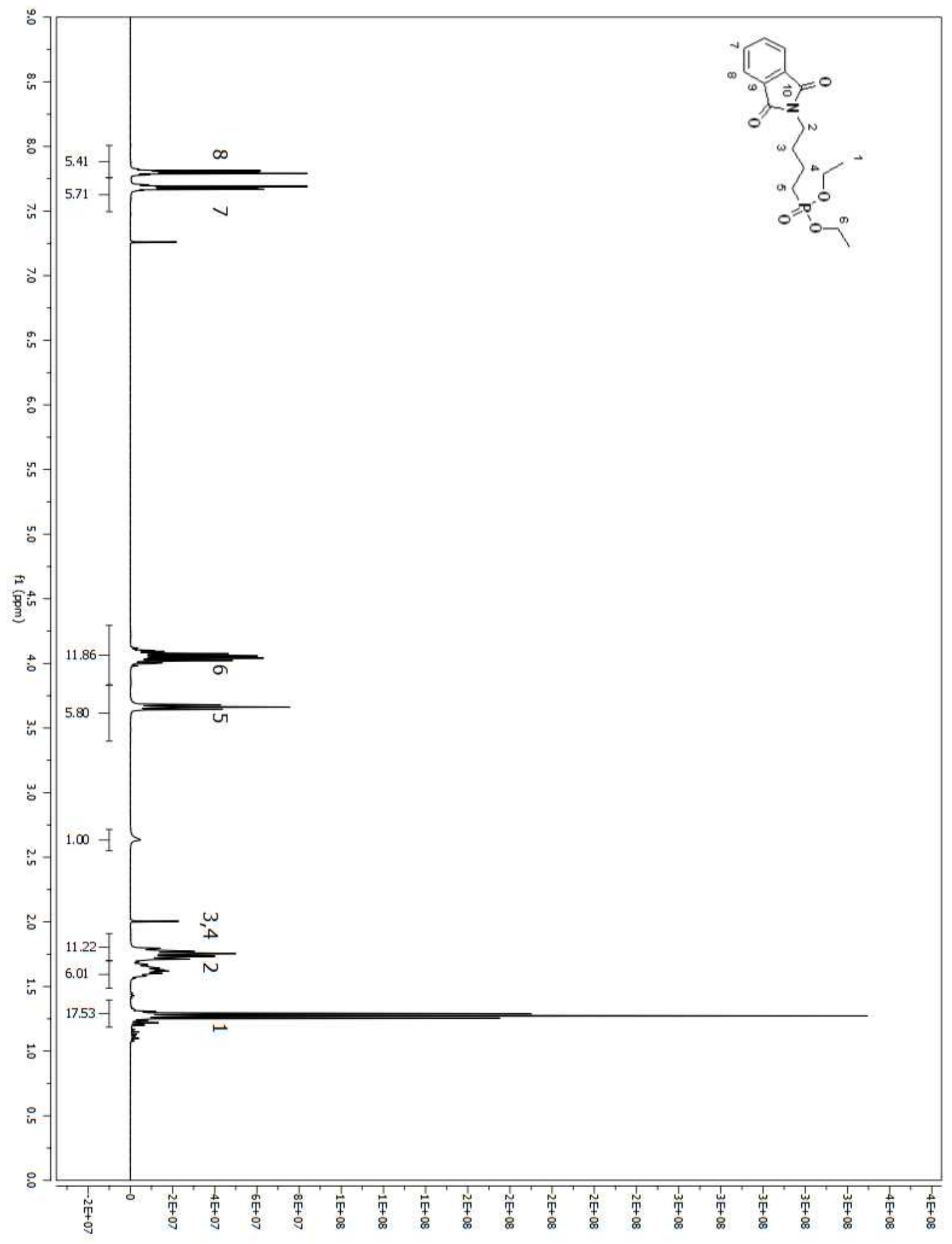

Figure A 50. ${ }^{1} \mathrm{H}$ NMR spectrum of compound 19 in $\mathrm{CDCl}_{3}$ (Table 2.3, entry xviii) 


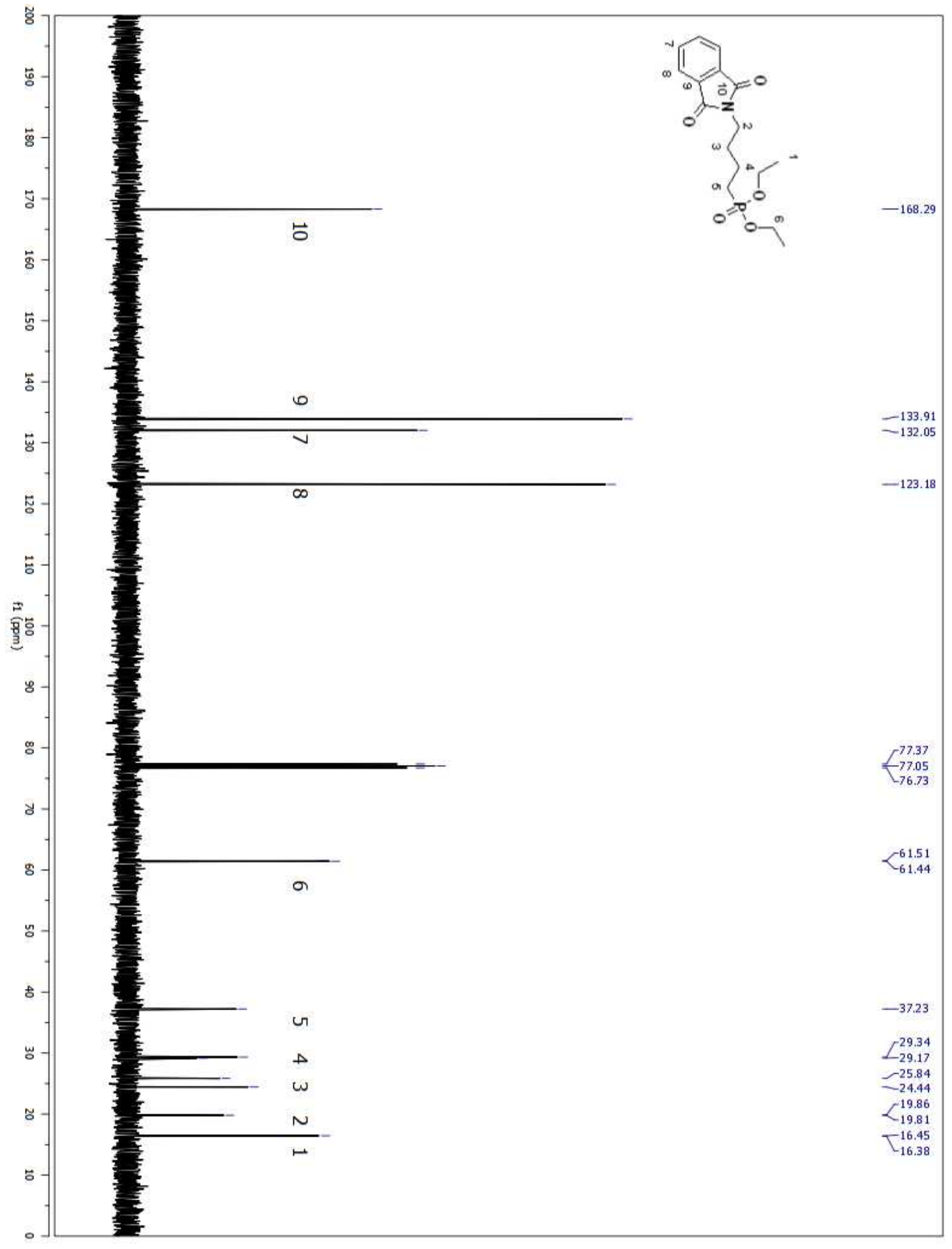

Figure A 51. ${ }^{13} \mathrm{C}$ NMR spectrum of compound 19 in $\mathrm{CDCl}_{3}$ (Table 2.3, entry xviii) 


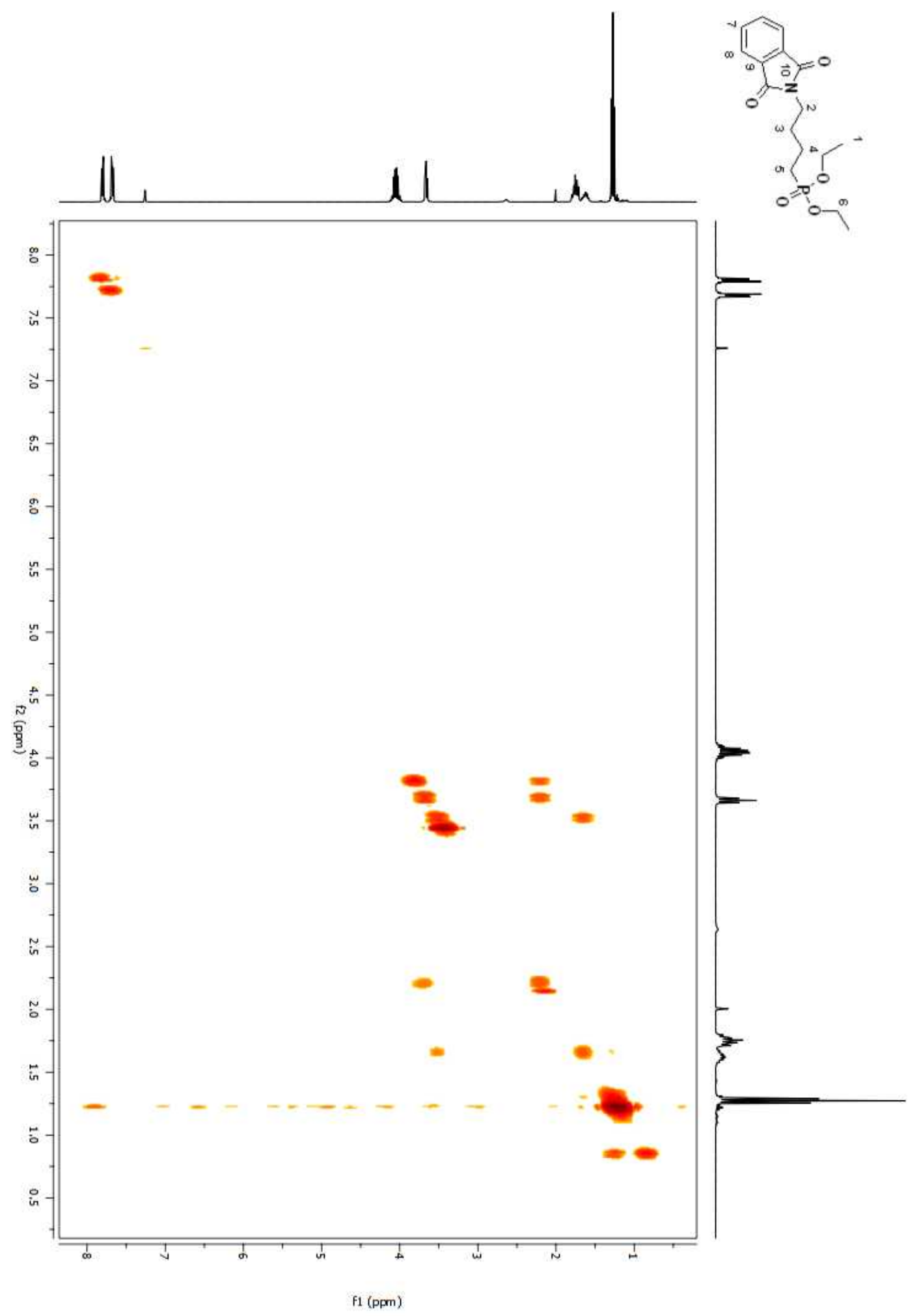

Figure A 52. COSY 2D NMR spectrum of compound 19 in $\mathrm{CDCl}_{3}$ (Table 2.3, entry xviii) 


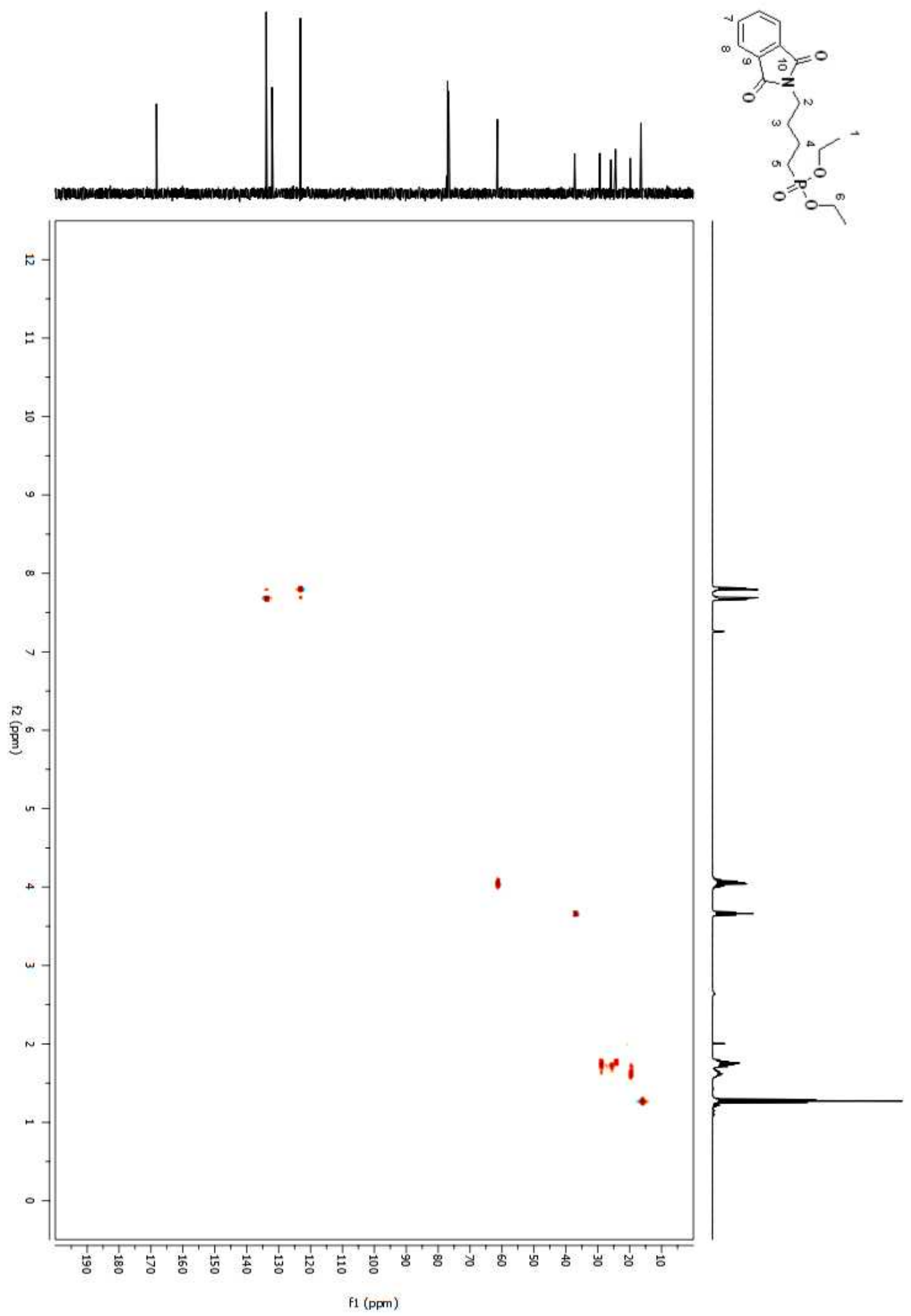

Figure A 53. HSQC 2D NMR spectrum of compound 19 in $\mathrm{CDCl}_{3}$ (Table 2.3, entry xviii) 


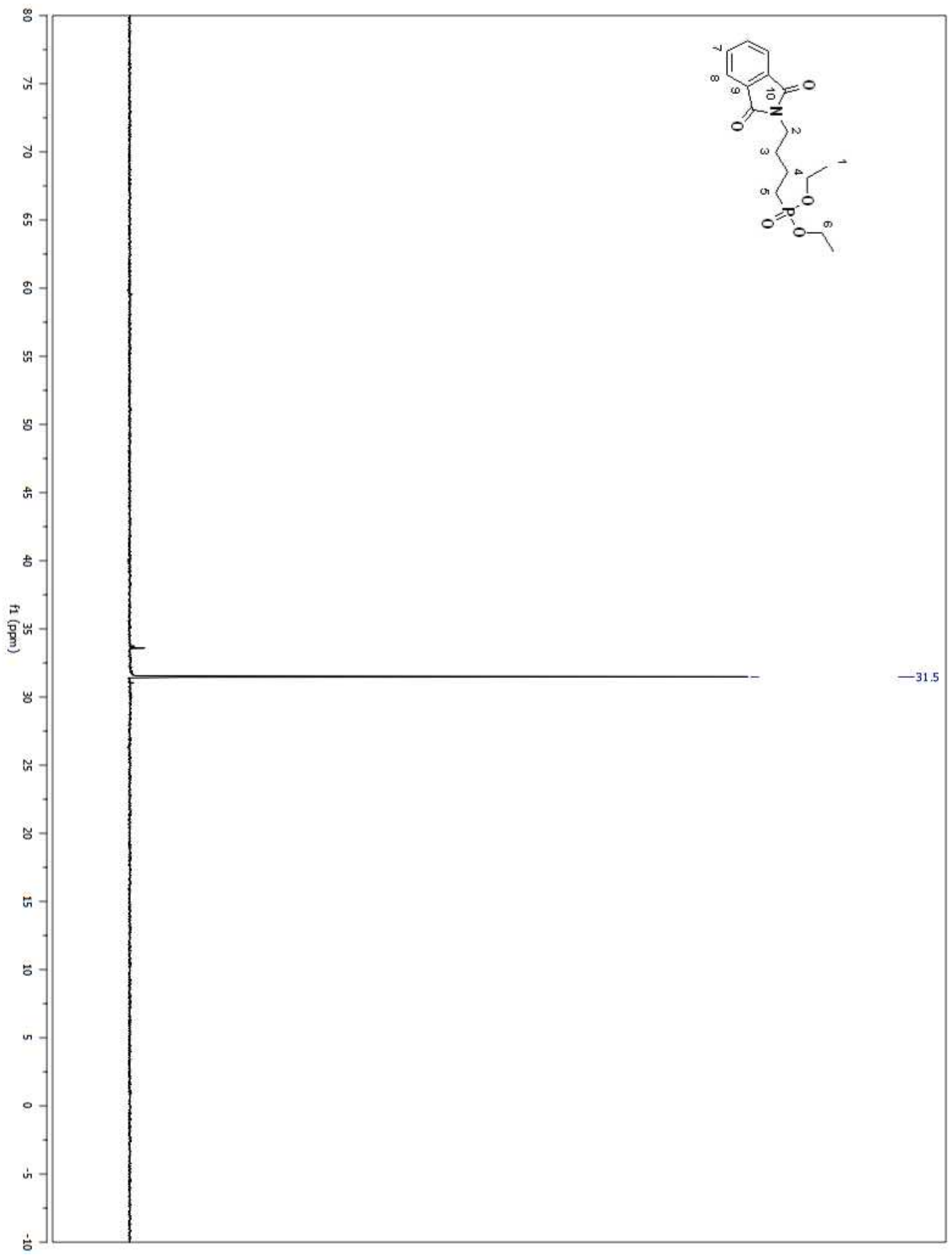

Figure A 54. ${ }^{31} \mathrm{P}$ NMR spectrum of compound 19 in $\mathrm{CDCl}_{3}$ (Table 2.3, entry xviii) 421 


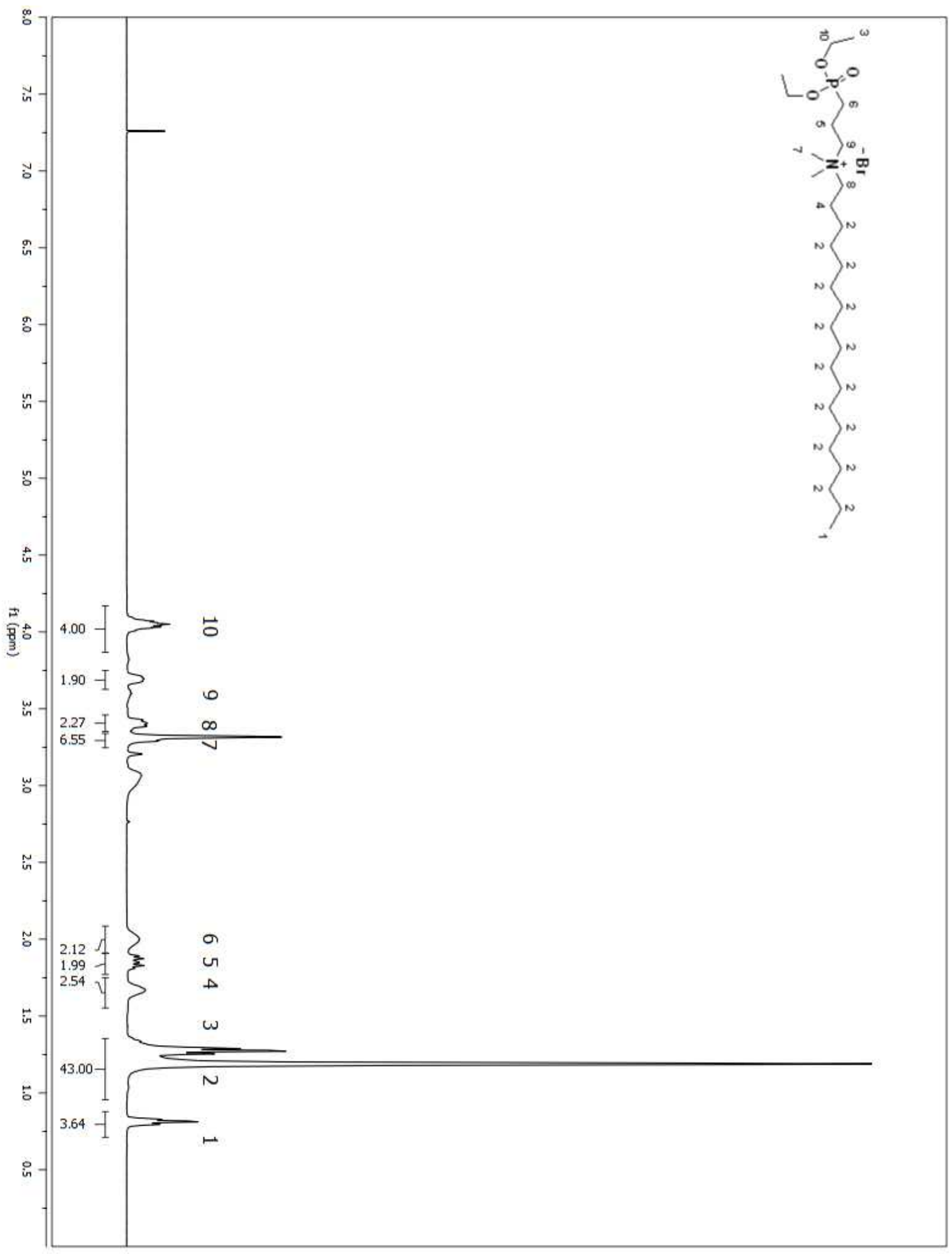

Figure A 55. ${ }^{1} \mathrm{H}$ NMR spectrum of compound 26 in $\mathrm{CDCl}_{3}$ (Table 2.4, entry i) 


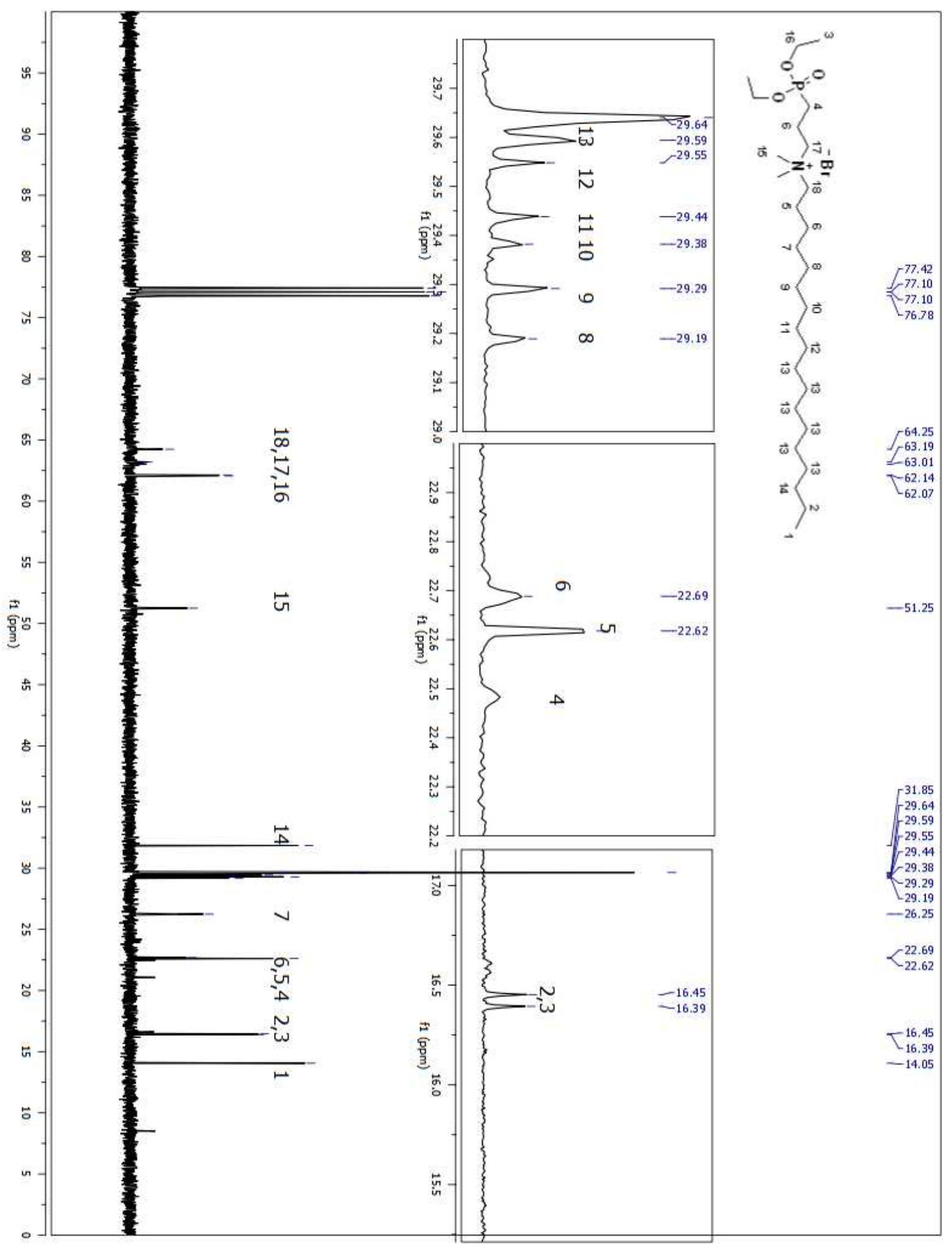

Figure A 56. ${ }^{13} \mathrm{C}$ NMR spectrum of compound 26 in $\mathrm{CDCl}_{3}$ (Table 2.4, entry i) 


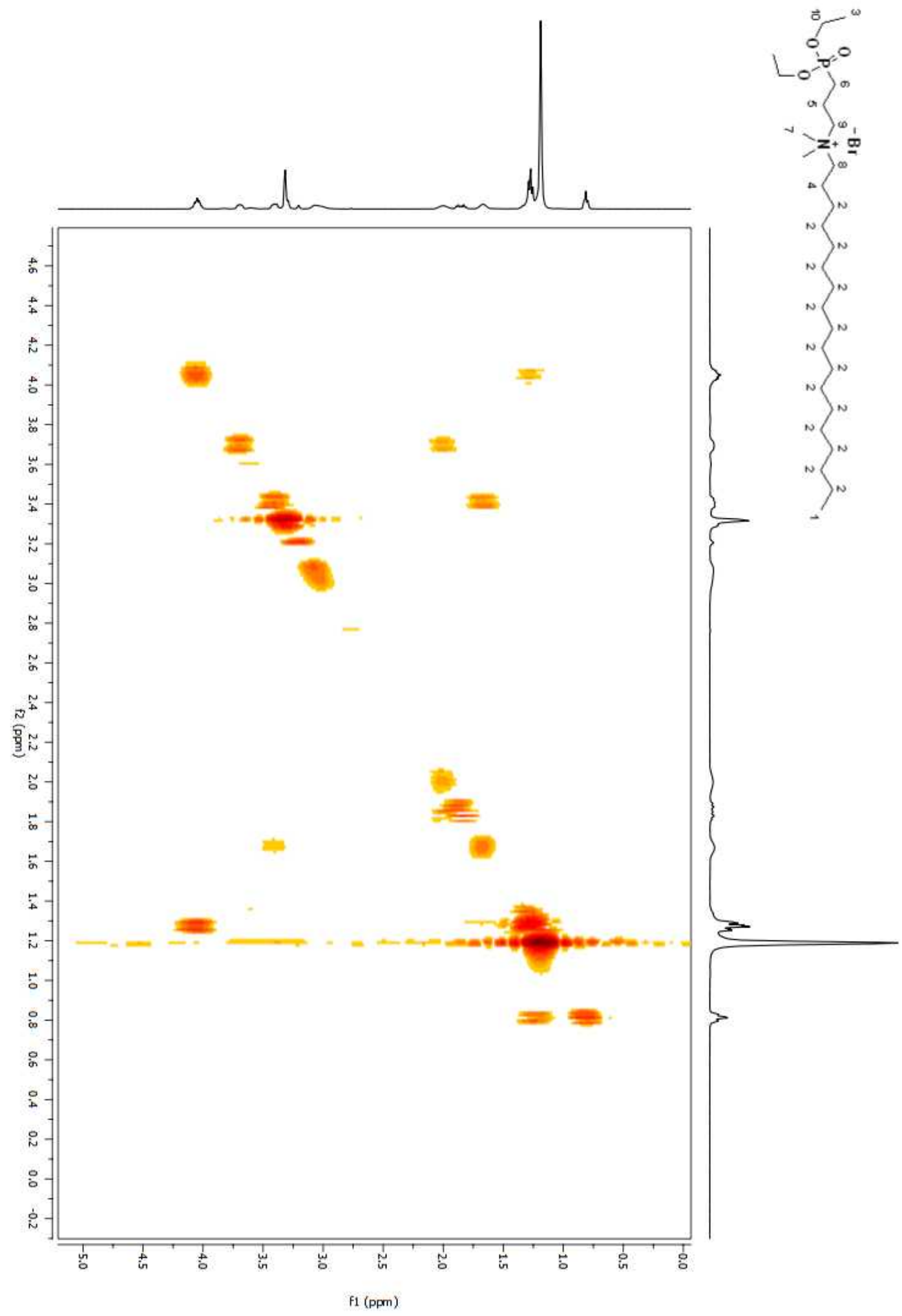

Figure A 57. COSY 2D NMR spectrum of compound 26 in $\mathrm{CDCl}_{3}$ (Table 2.4, entry i) 


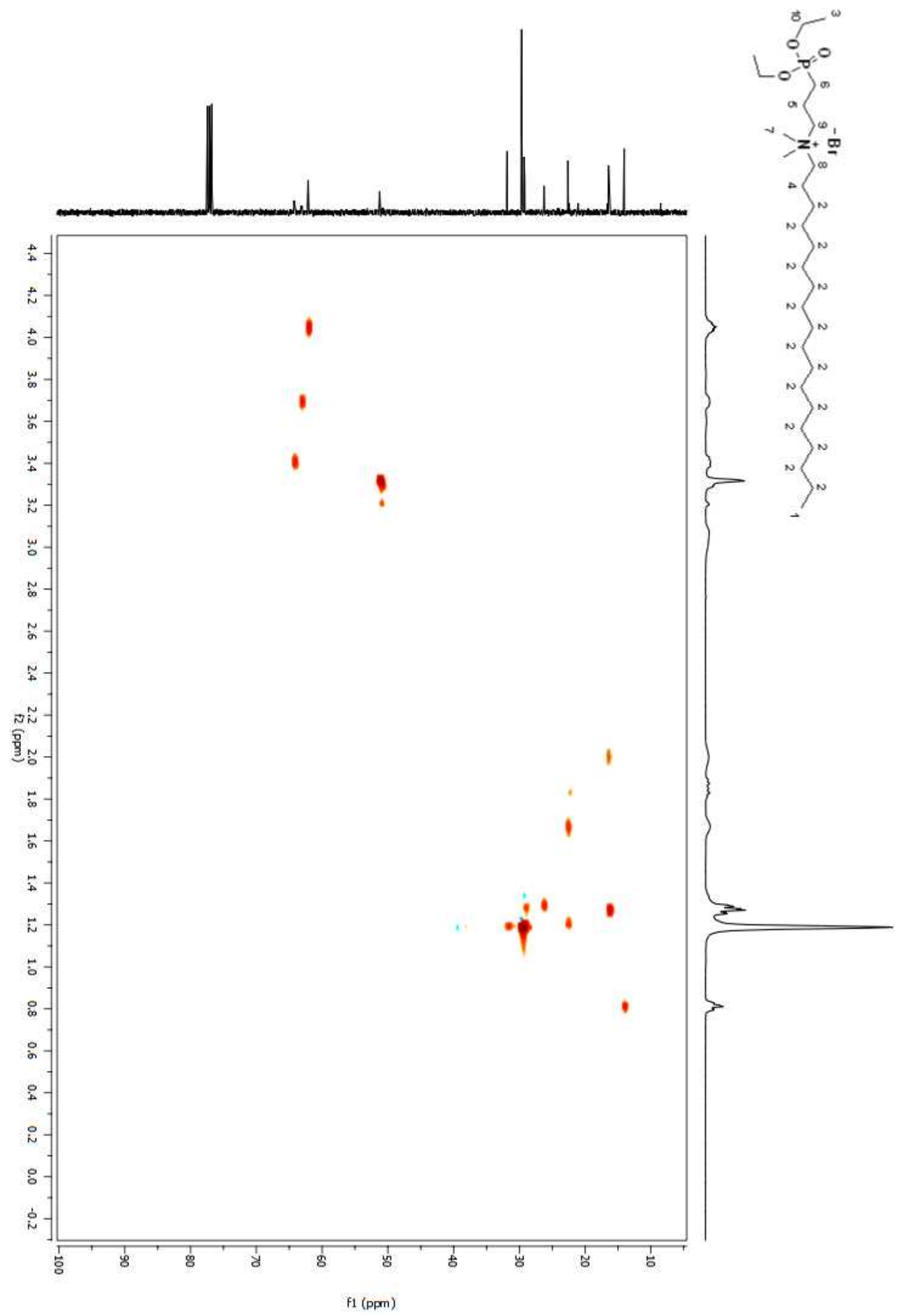

Figure A 58. HSQC 2D NMR spectrum of compound 26 in $\mathrm{CDCl}_{3}$ (Table 2.4, entry i) 


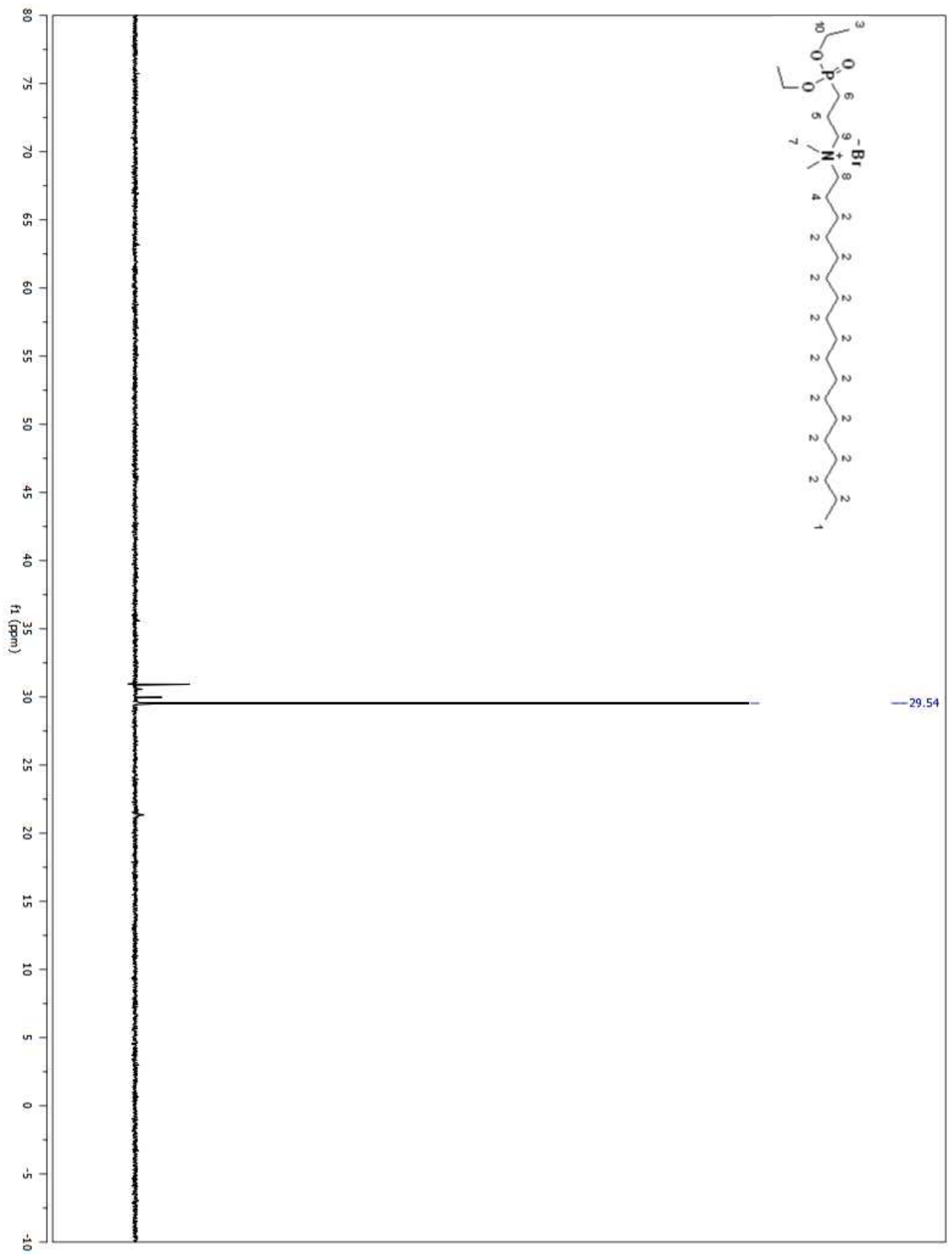

Figure A 59. ${ }^{31} \mathrm{P}$ NMR spectrum of compound 26 in $\mathrm{CDCl}_{3}$ (Table 2.4, entry i) 


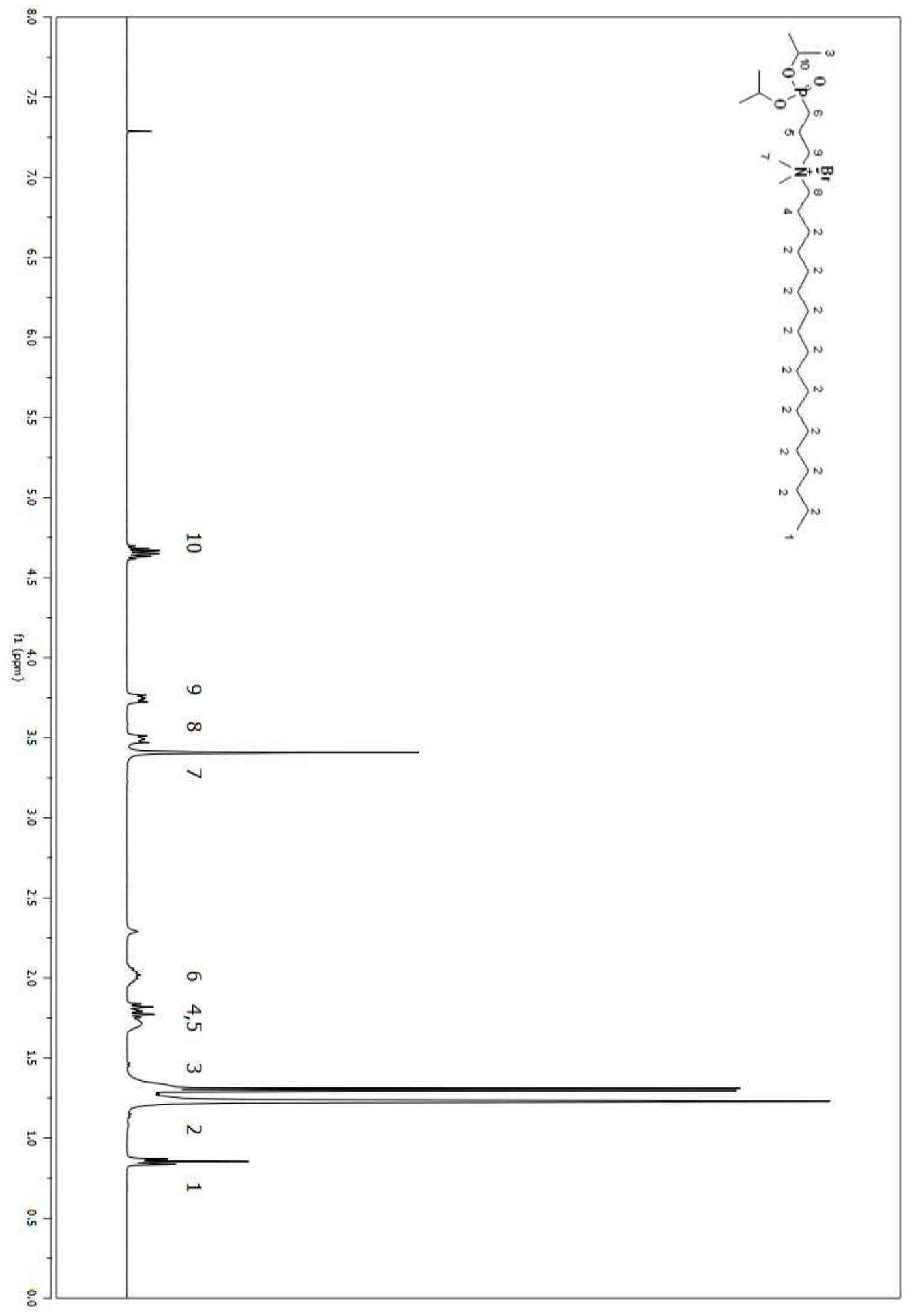

Figure A 60. ${ }^{1} \mathrm{H}$ NMR spectrum of compound 27 in $\mathrm{CDCl}_{3}$ (Table 4.4, entry iv) 


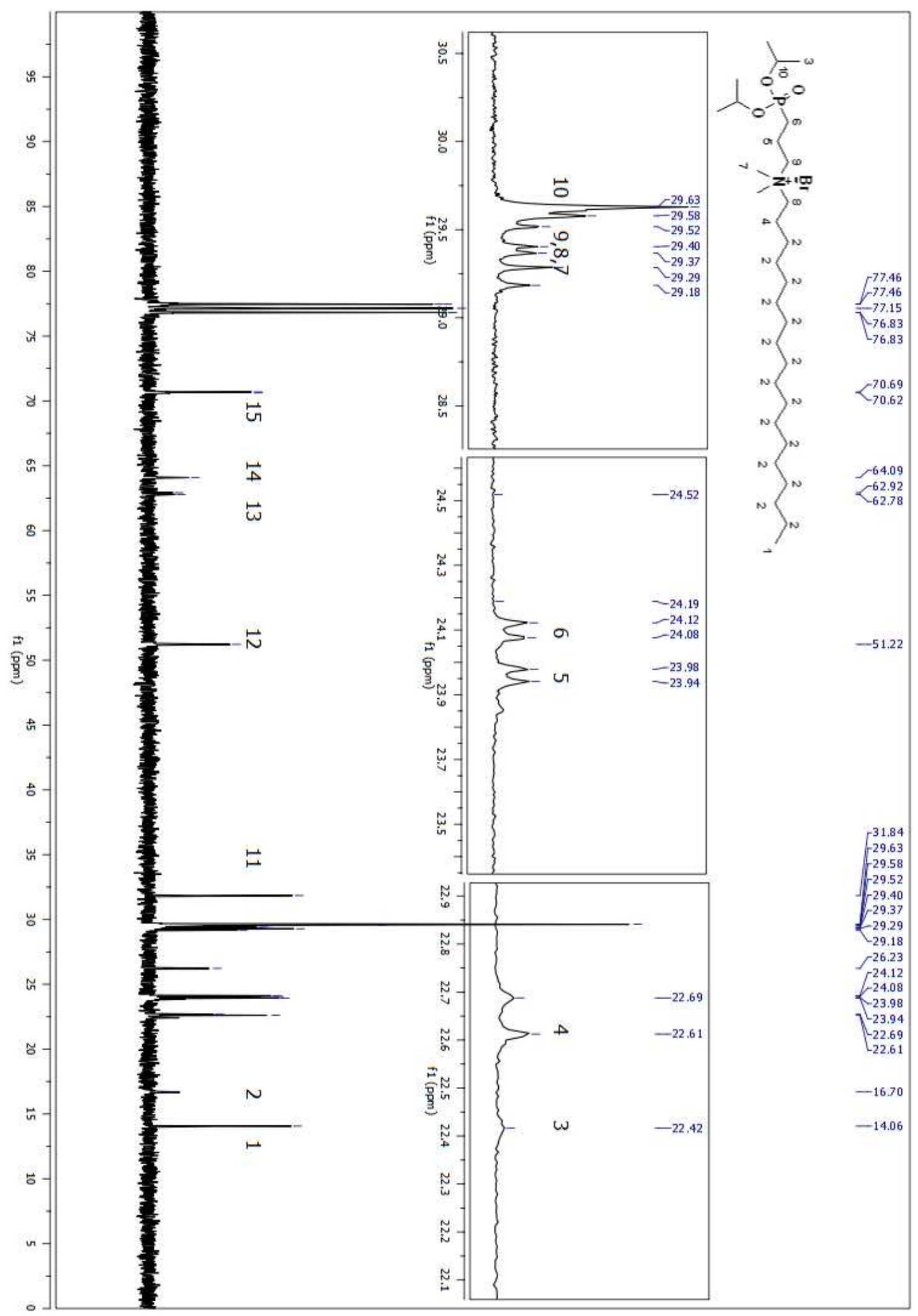

Figure A 61. ${ }^{13} \mathrm{C}$ NMR spectrum of compound 27 in $\mathrm{CDCl}_{3}$ (Table 2.4, entry iv) 


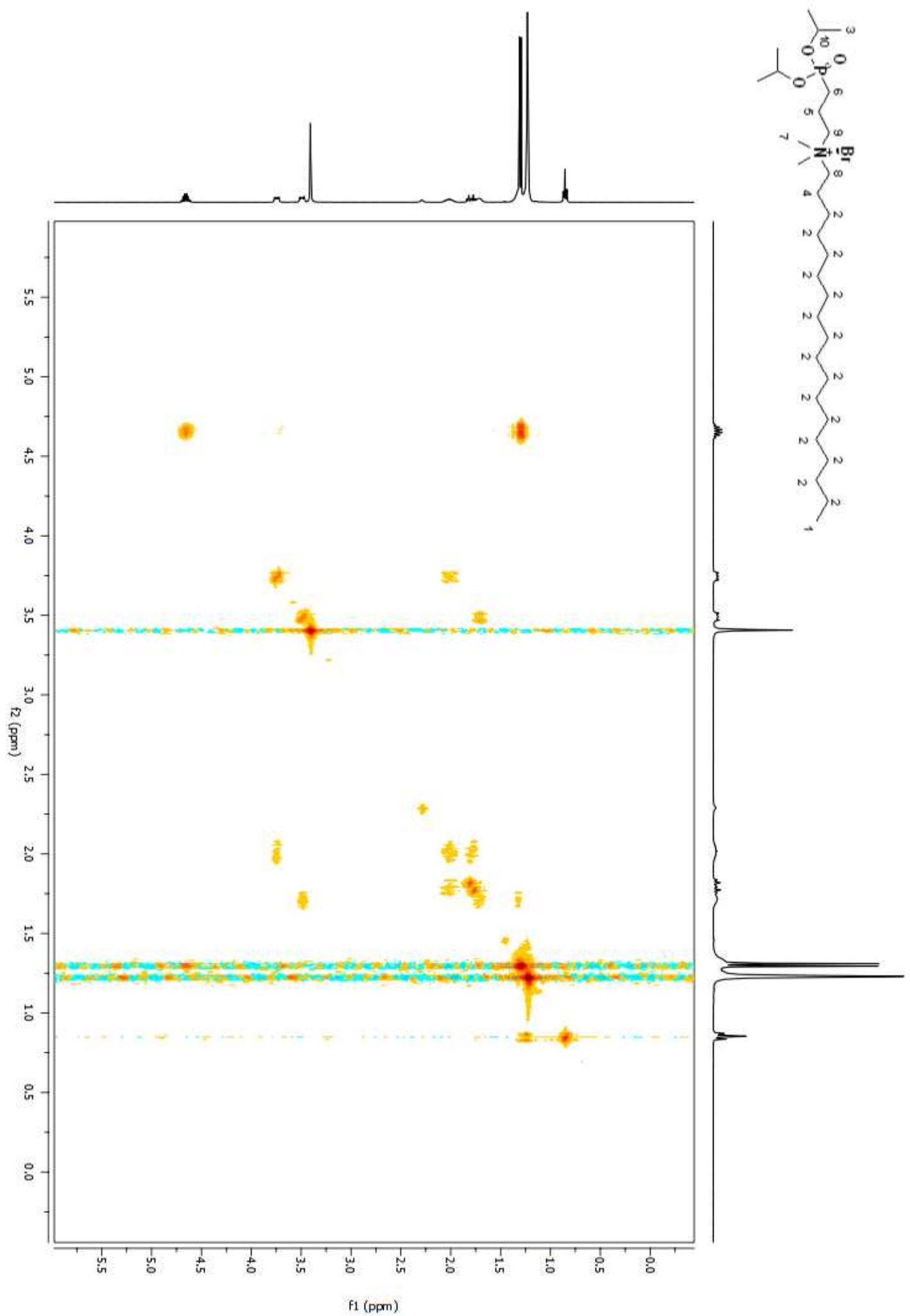

Figure A 62. COSY 2D NMR spectrum of compound 27 in $\mathrm{CDCl}_{3}$ (Table 2.4, entry iv) 429 


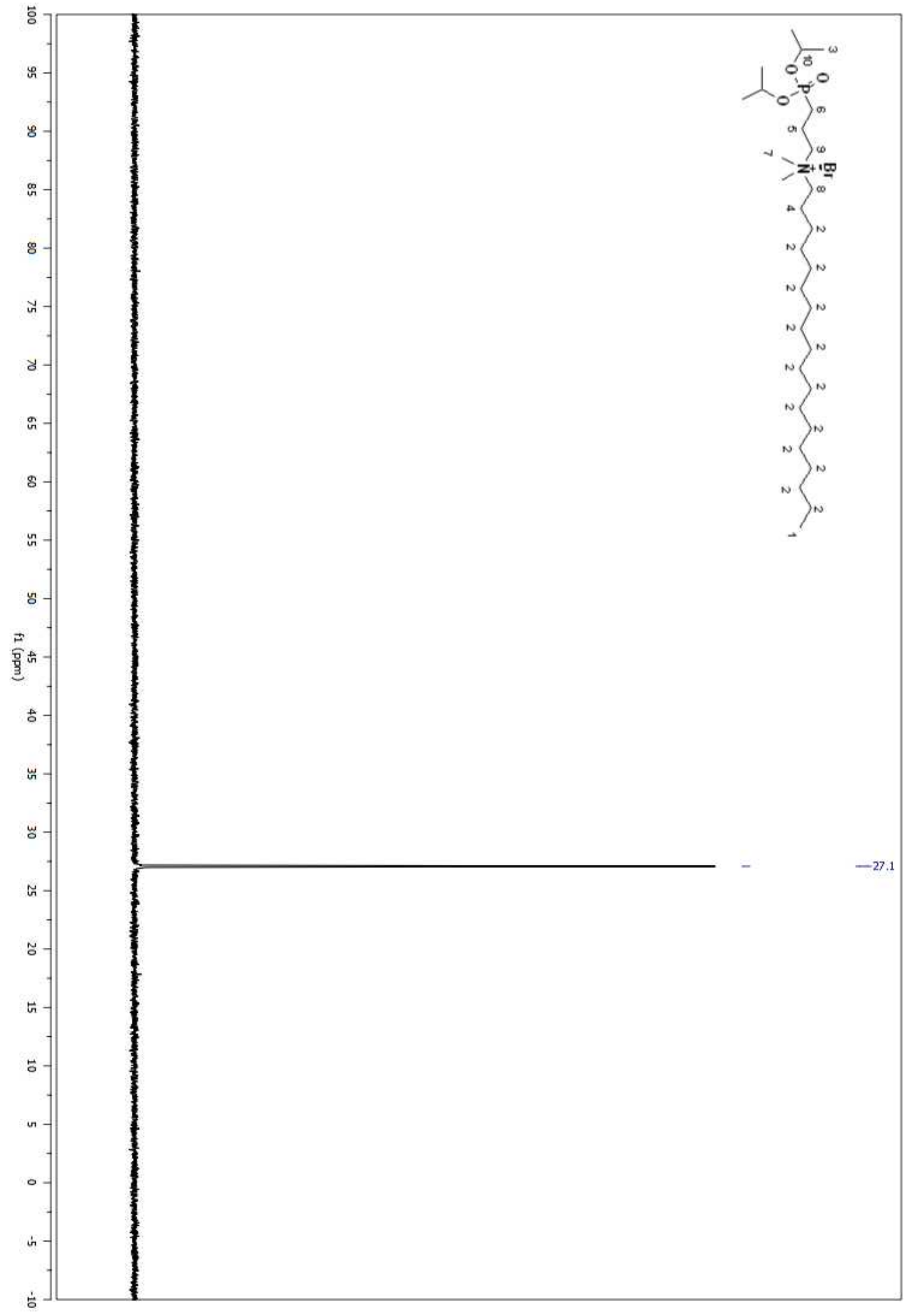

Figure A 63. ${ }^{31} \mathrm{P}$ NMR spectrum of compound 27 in $\mathrm{CDCl}_{3}$ (Table 2.4, entry iv) 


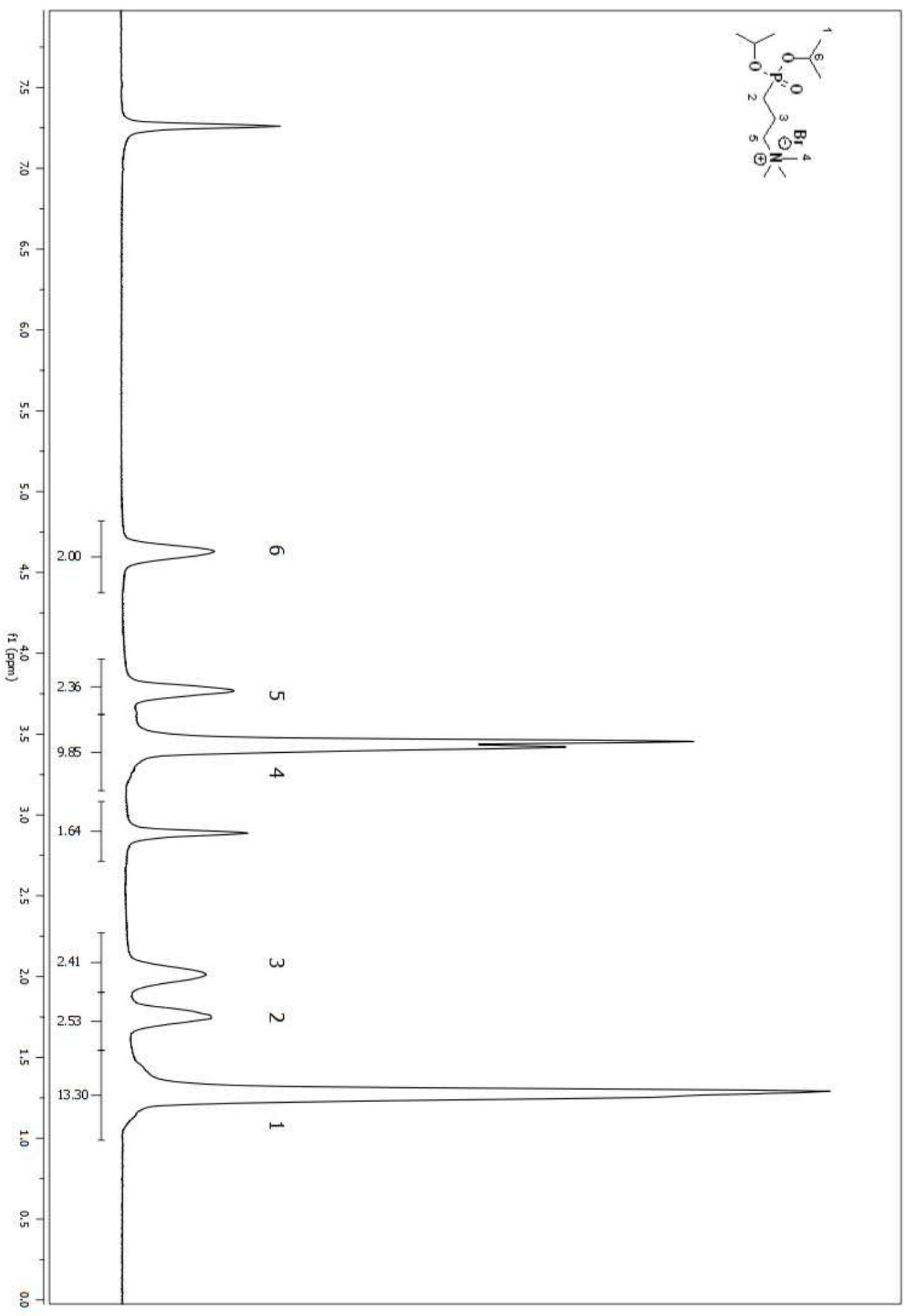

Figure A 64. ${ }^{1} \mathrm{H}$ NMR spectrum of compound 28 in $\mathrm{CDCl}_{3}$ (Table 2.4, entry vii) 


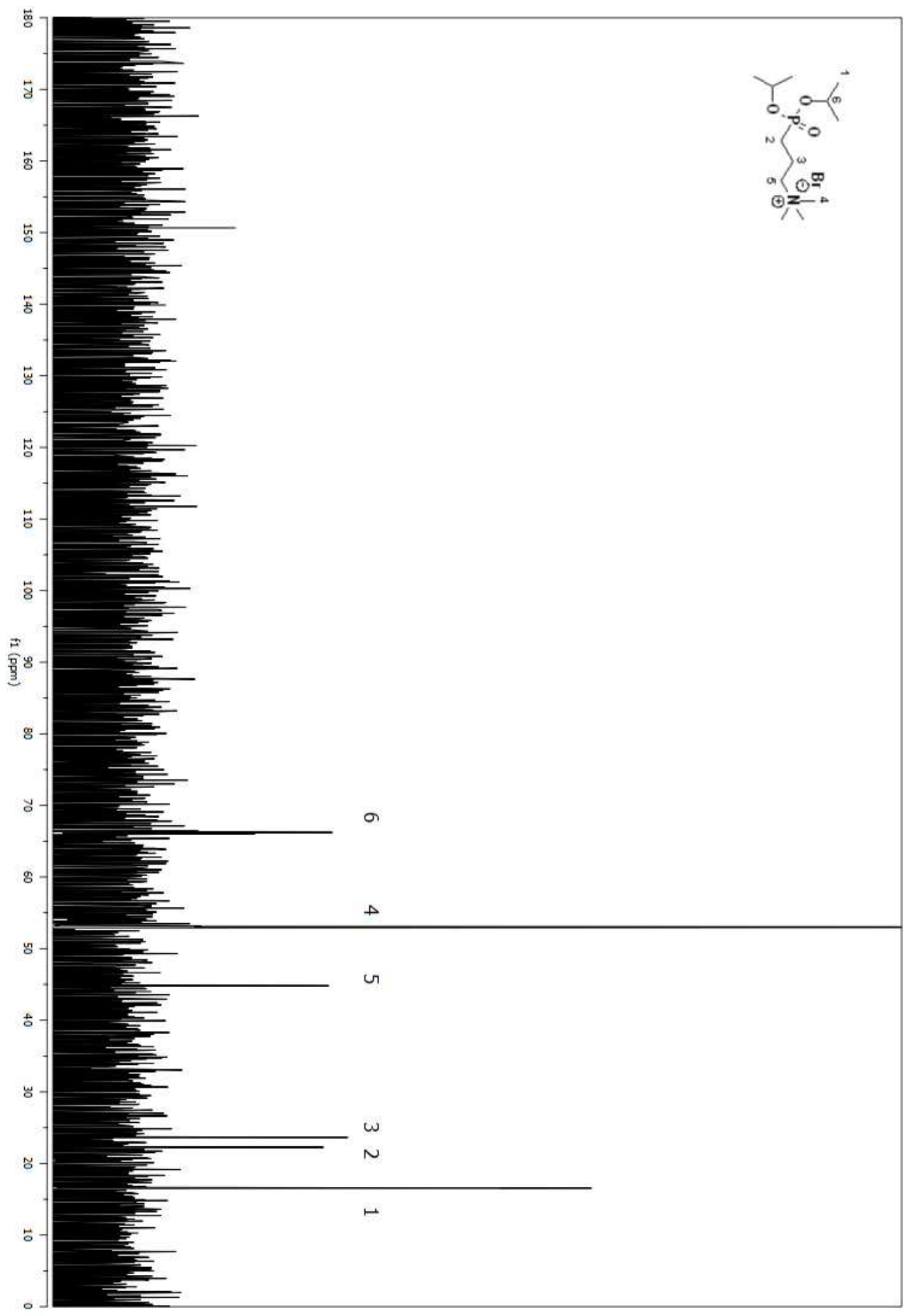

Figure A 65. ${ }^{13} \mathrm{C}$ NMR spectrum of compound 28 in $\mathrm{CDCl}_{3}$ (Table 2.4, entry vii) 


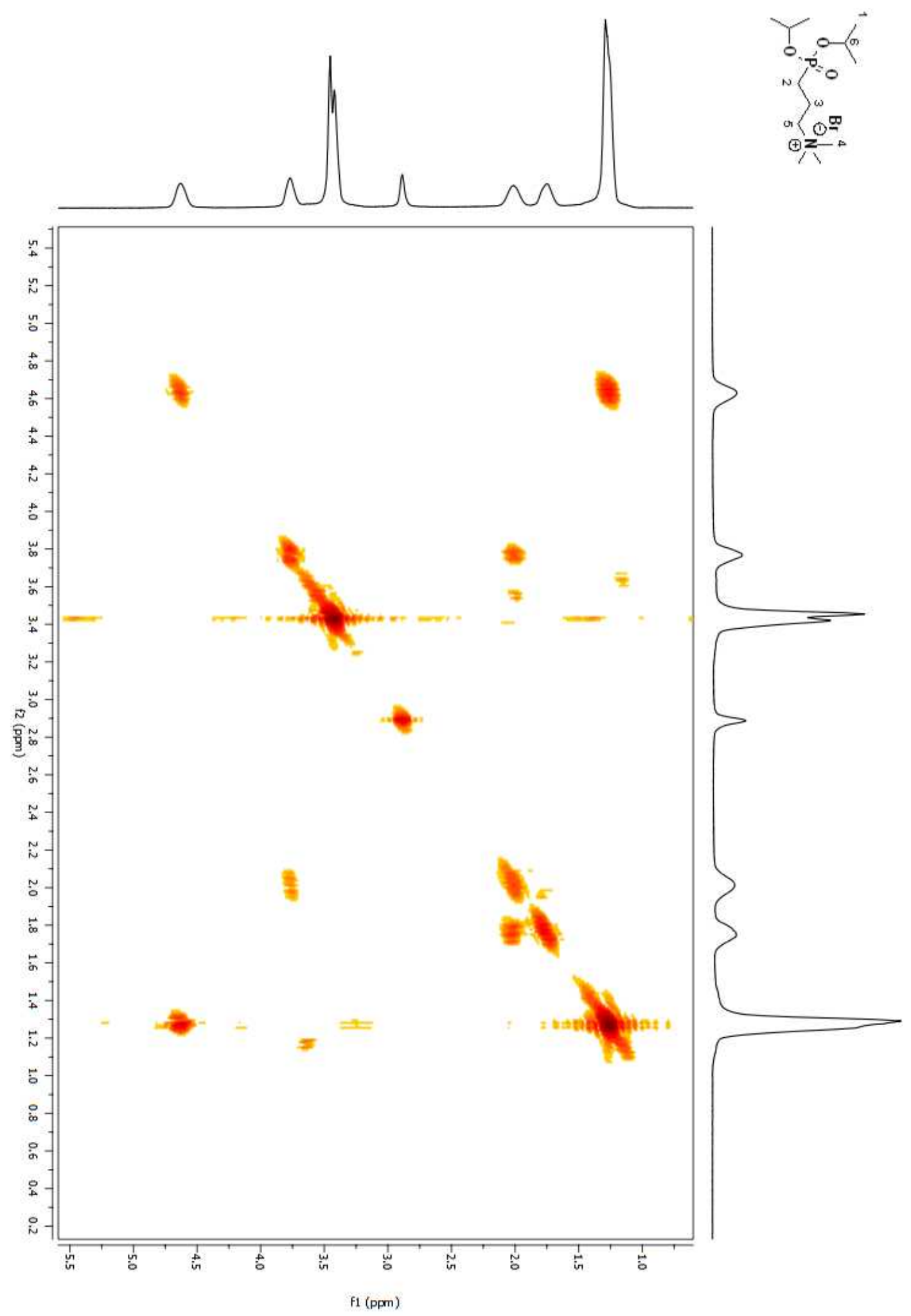

Figure A 66. COSY 2D NMR spectrum of compound 28 in $\mathrm{CDCl}_{3}$ (Table 2.4, entry vii) 


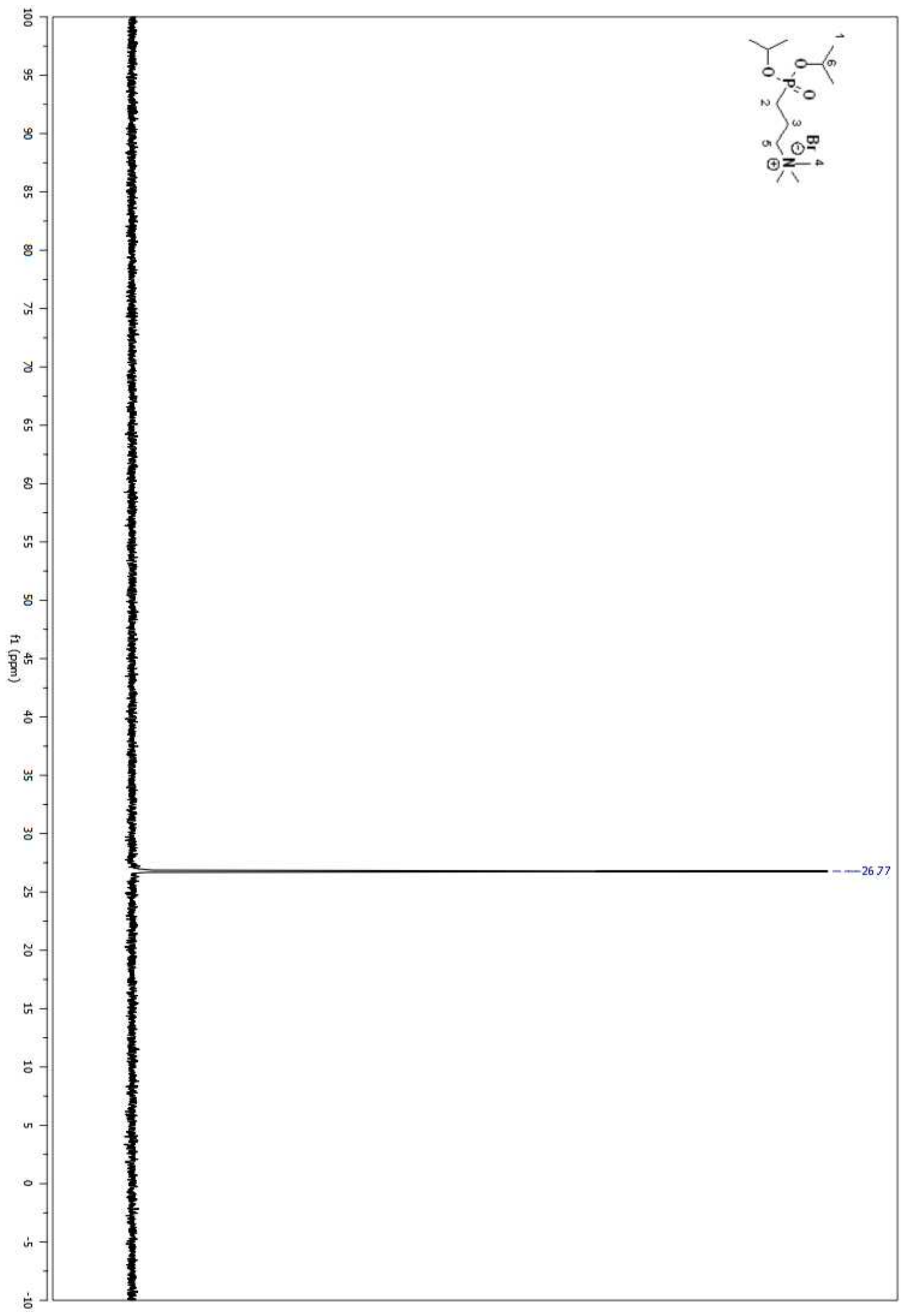

Figure A 67. ${ }^{31} \mathrm{P}$ NMR spectrum of compound 28 in $\mathrm{CDCl}_{3}$ (Table 2.4, entry vii) 


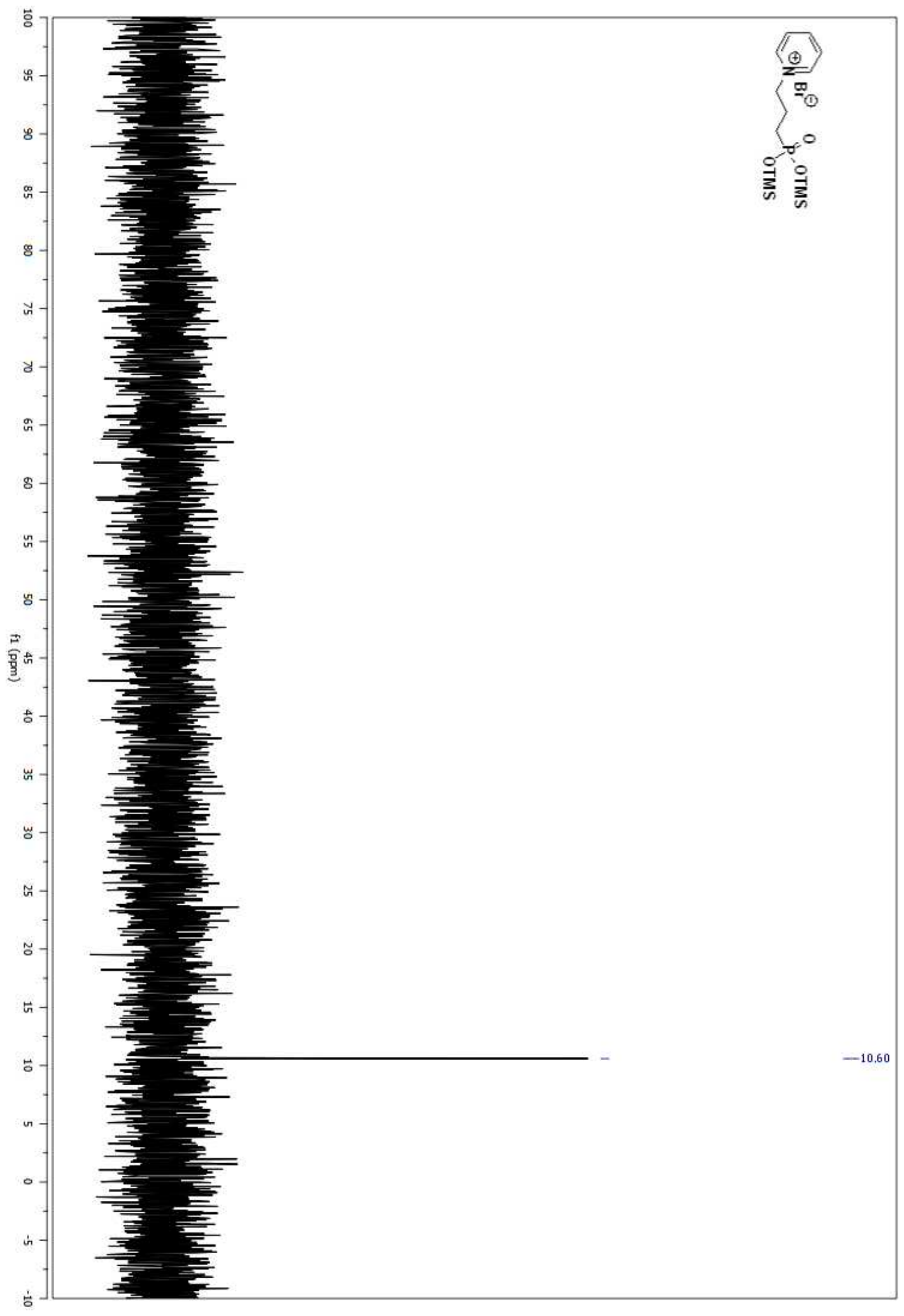

Figure A 68. ${ }^{31} \mathrm{P}$ NMR spectrum of compound 29 in $\mathrm{CDCl}_{3}$ (Table 2.4, entry viii) 


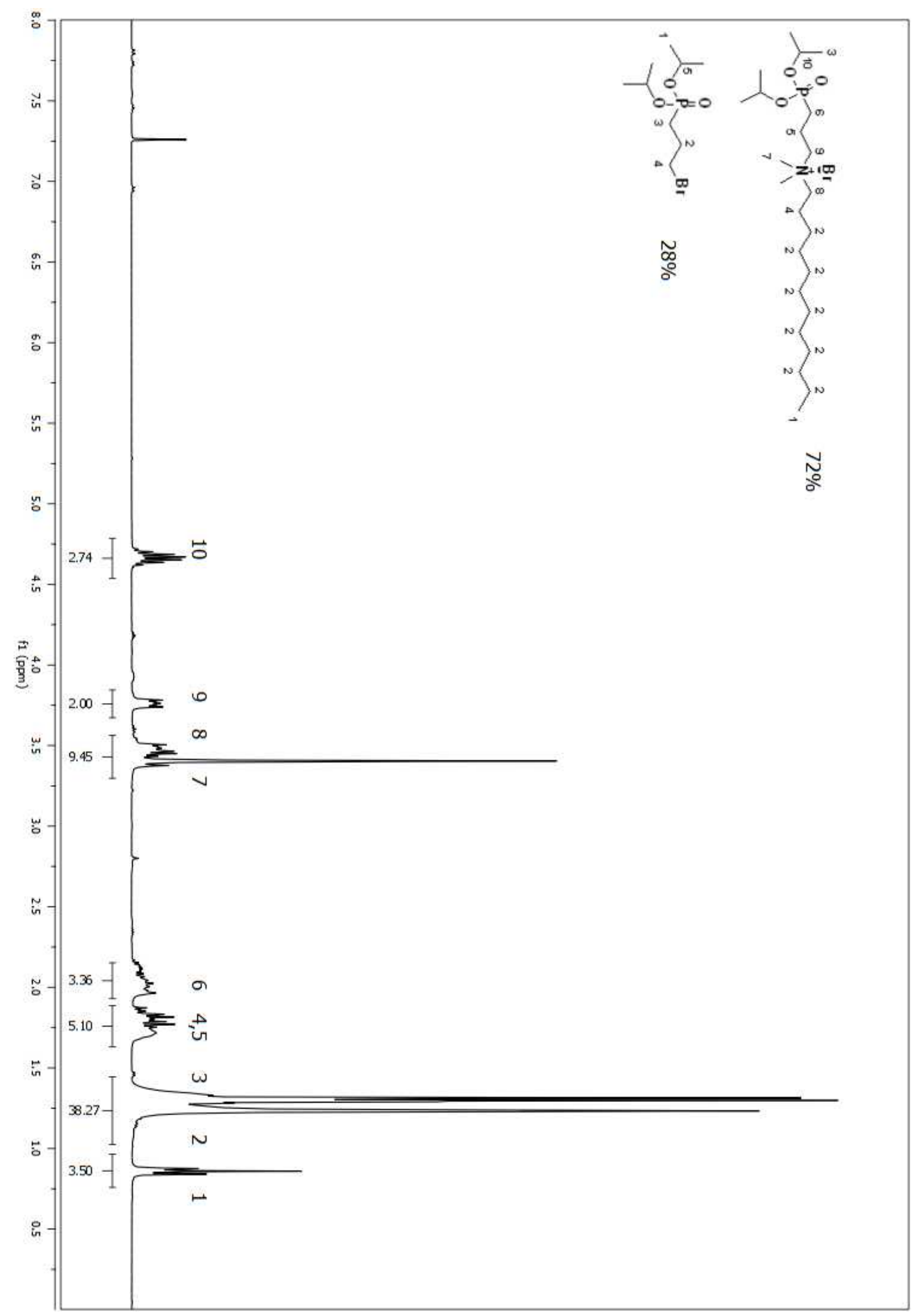

Figure A 69. ${ }^{1} \mathrm{H}$ NMR spectrum of compound 30 in $\mathrm{CDCl}_{3}$ (Table 2.4, entry ix) 


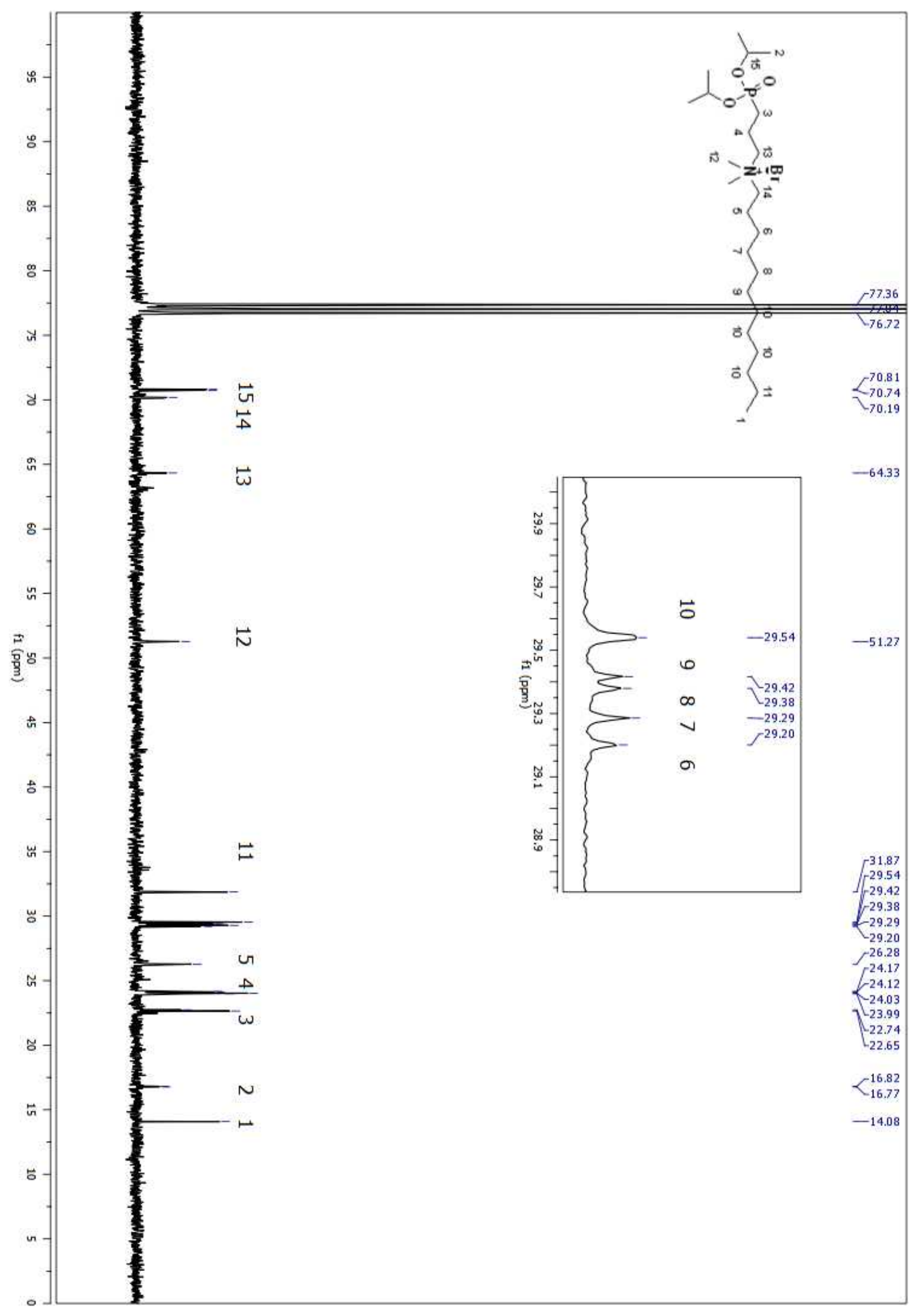

Figure A 70. ${ }^{13} \mathrm{C}$ NMR spectrum of compound 30 in $\mathrm{CDCl}_{3}$ (Table 2.4, entry ix) 


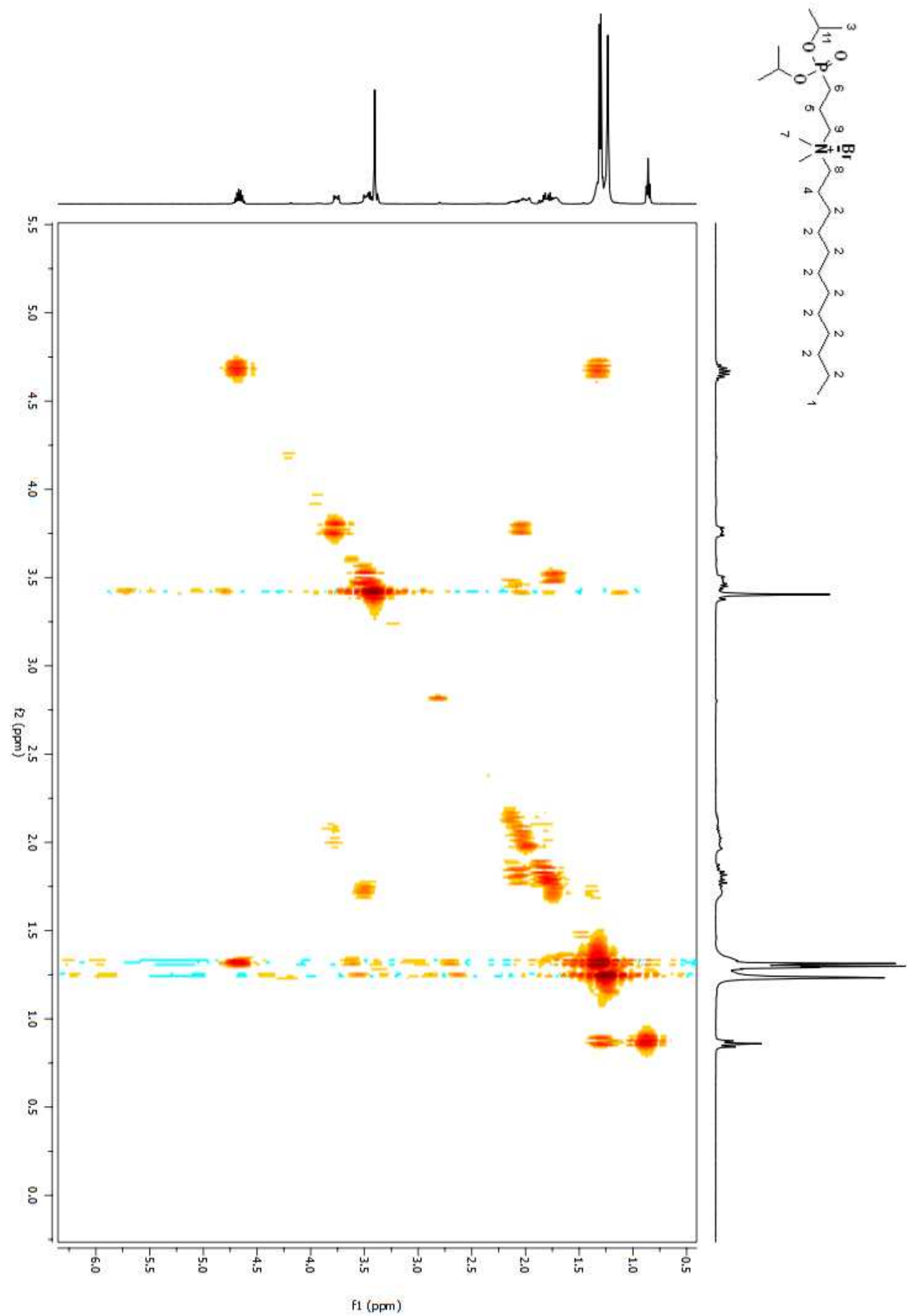

Figure A 71. COSY 2D NMR spectrum of compound 30 in $\mathrm{CDCl}_{3}$ (Table 4.4, entry ix) 


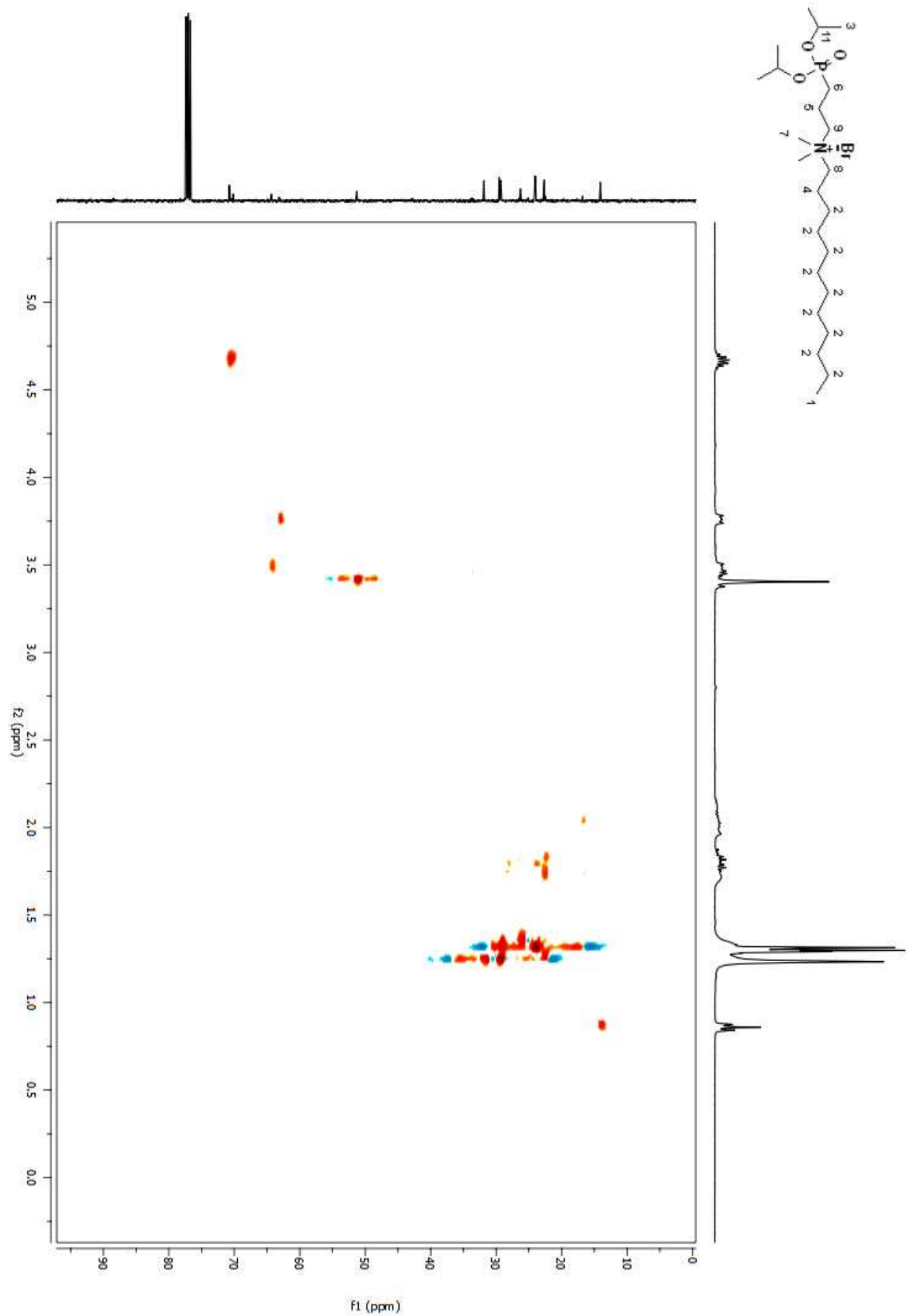

Figure A 72. HSQC 2D NMR spectrum of compound 30 in $\mathrm{CDCl}_{3}$ (Table 2.4, entry ix) 


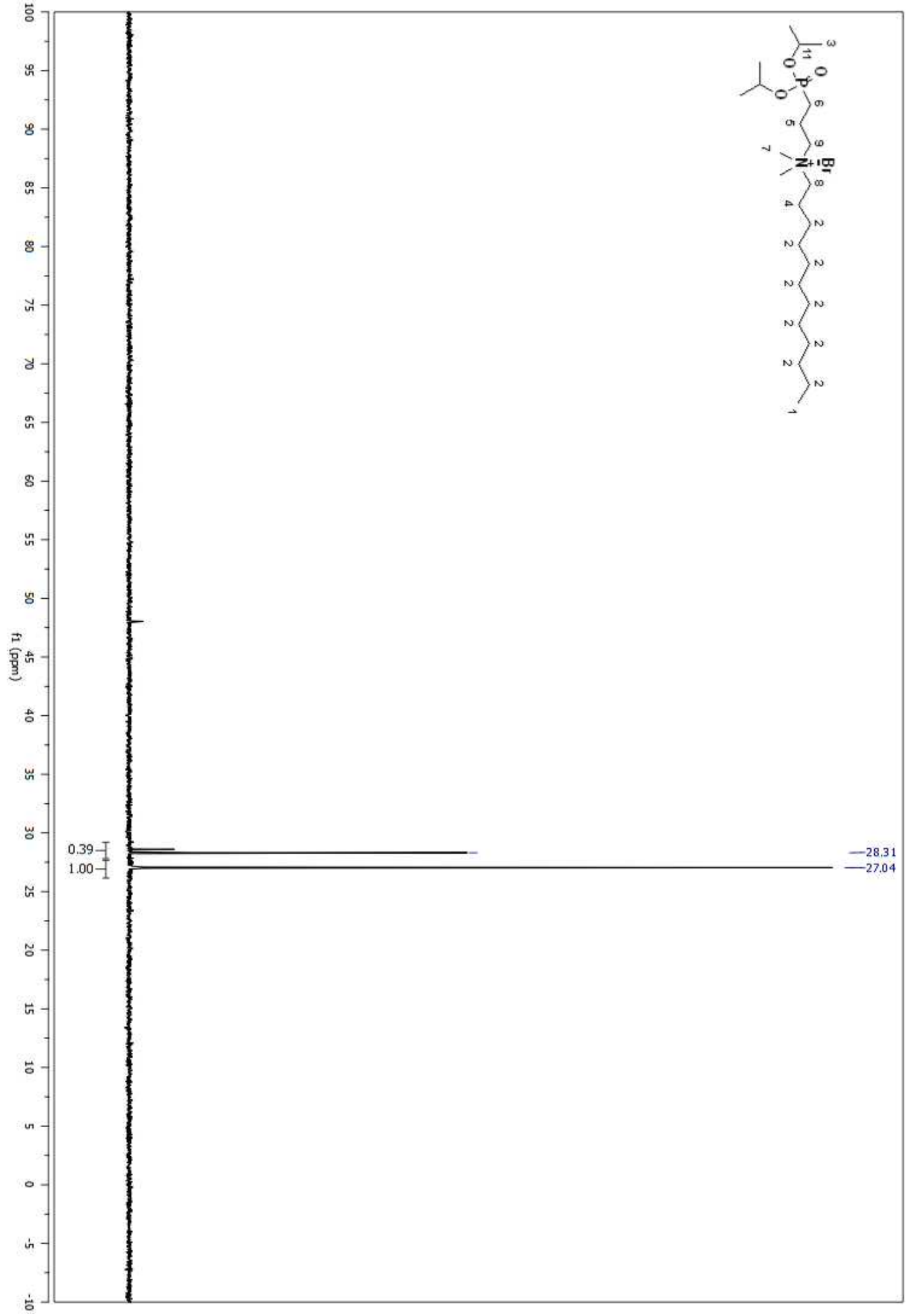

Figure A 73. ${ }^{31} \mathrm{P}$ NMR spectrum of compound 30 in $\mathrm{CDCl}_{3}$ (Table 2.4, entry ix) 


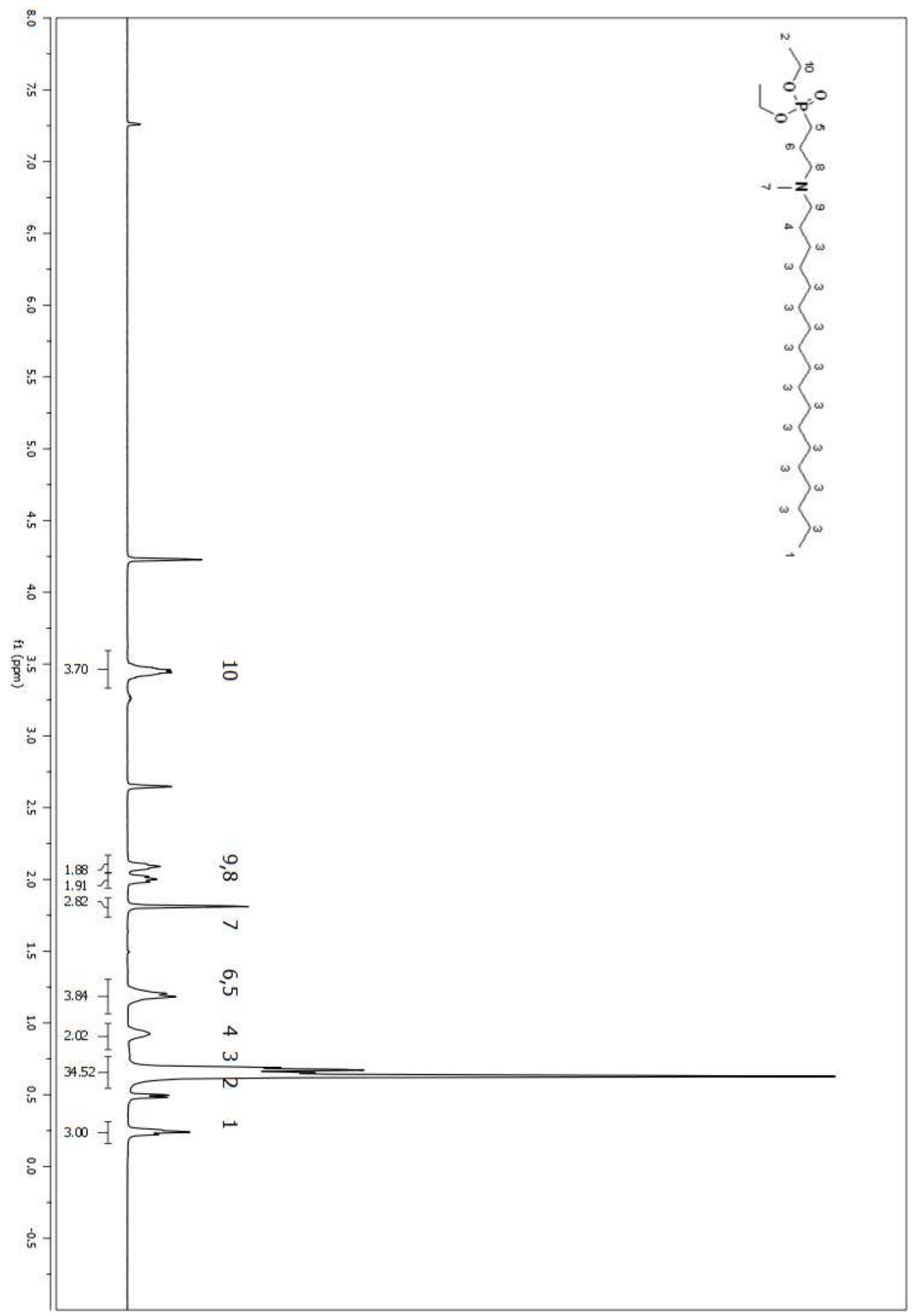

Figure A 74. ${ }^{1} \mathrm{H}$ NMR spectrum of compound 31 in $\mathrm{CDCL}_{3}$ (Table 2.4, entry $\mathrm{x}$ ) 


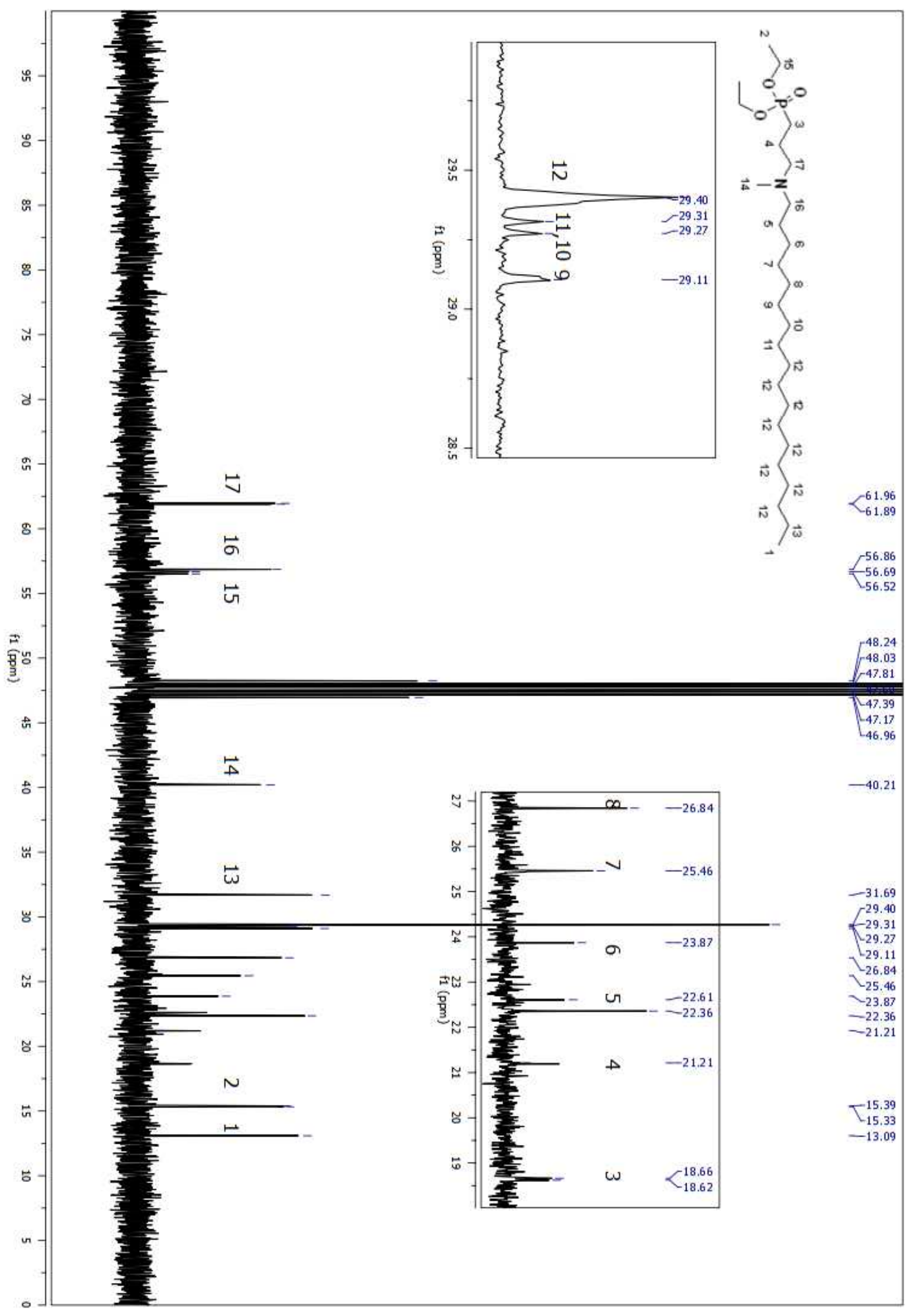

Figure A 75. ${ }^{13} \mathrm{C}$ NMR spectrum of compound 31 in $\mathrm{CDCL}_{3}$ (Table 2.4, entry x) 


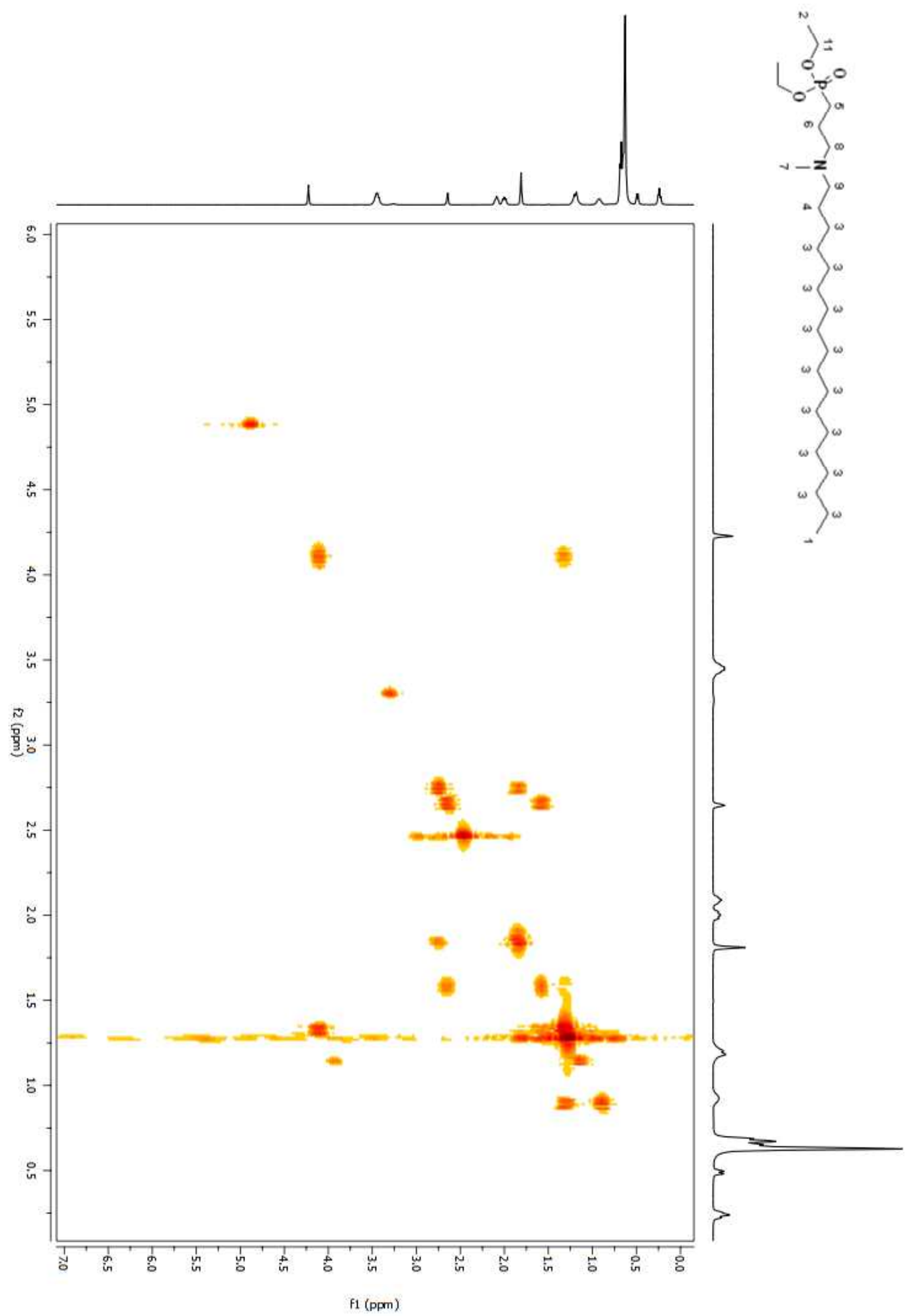

Figure A 76. COSY 2D NMR spectrum of compound 31 in $\mathrm{CDCL}_{3}$ (Table 2.4, entry $\mathbf{x}$ ) 


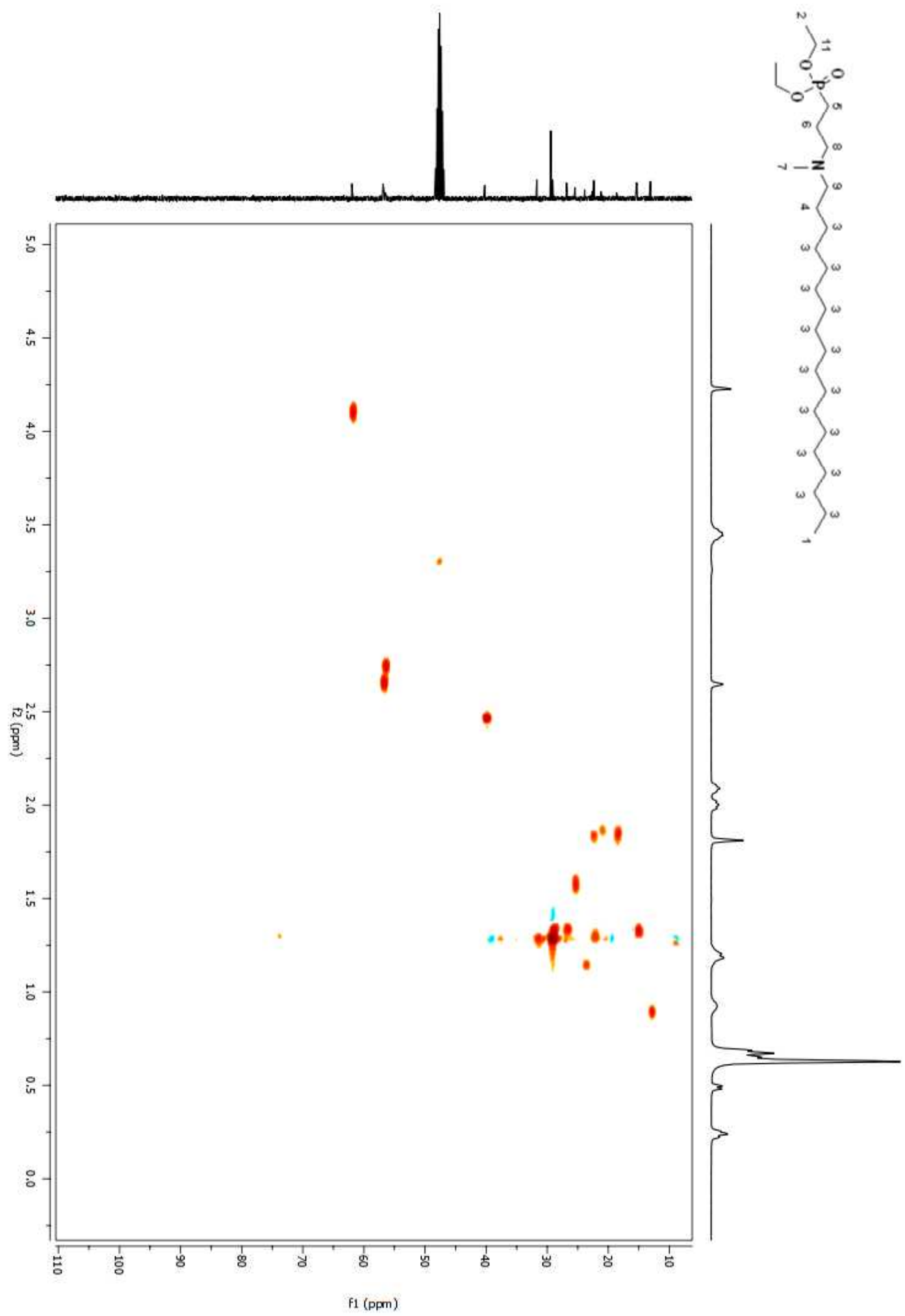

Figure A 77. HSQC 2D NMR spectrum of compound 31 in $\mathrm{CDCL}_{3}$ (Table 4.4, entry x) 


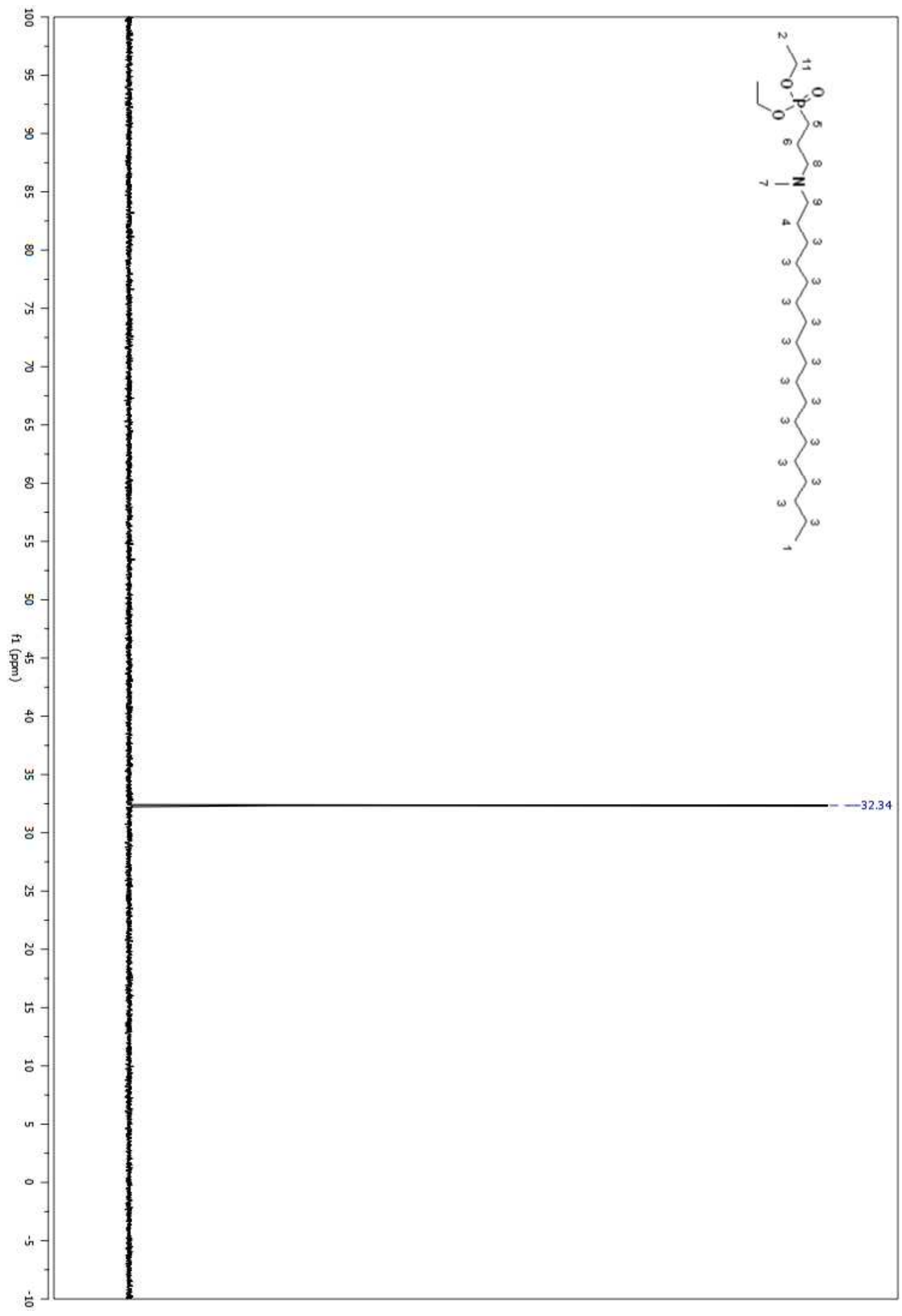

Figure A 78. ${ }^{31} \mathrm{P}$ NMR spectrum of compound 31 in $\mathrm{CDCL}_{3}$ (Table 2.4, entry $\mathrm{x}$ ) 


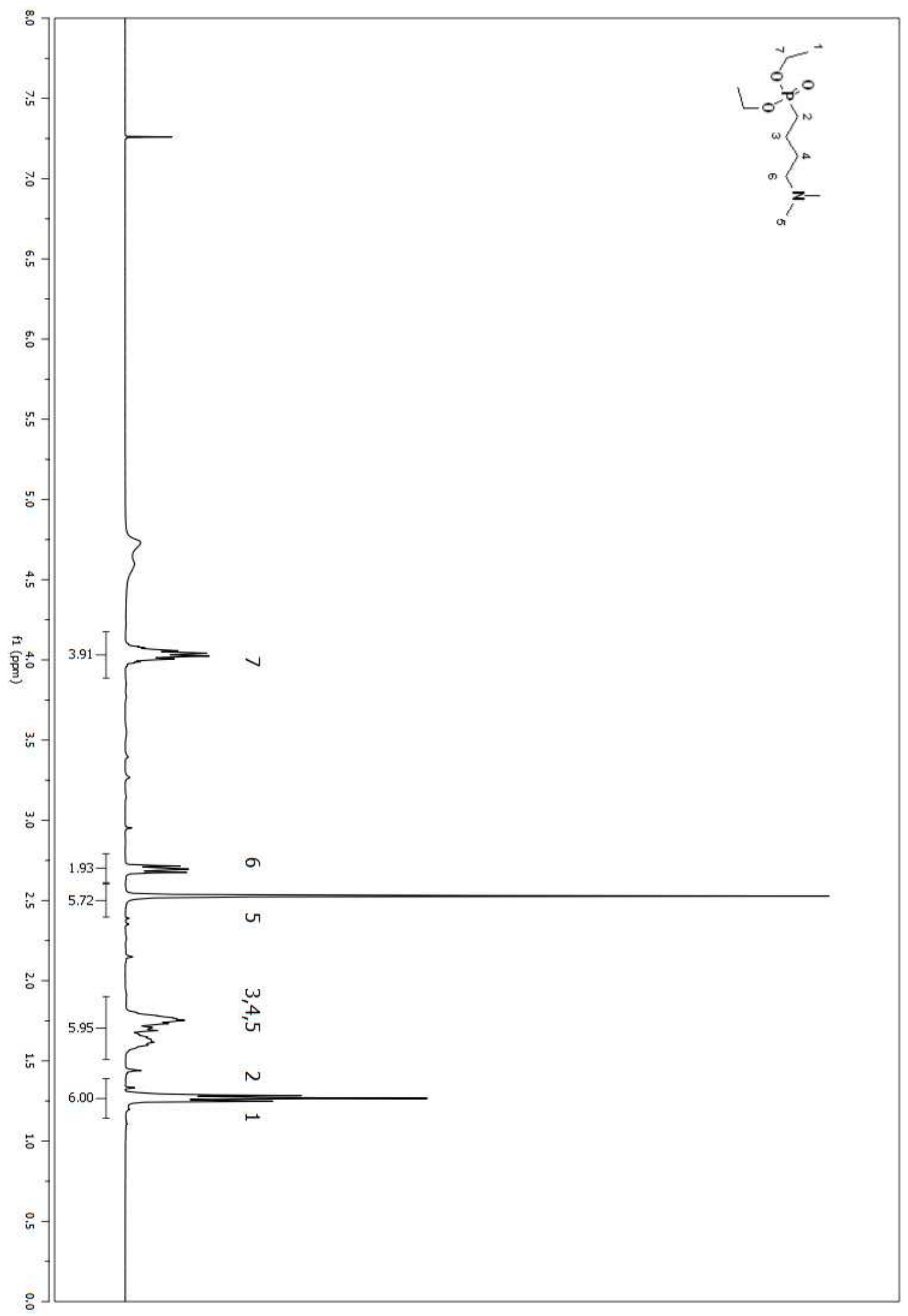

Figure A 79. ${ }^{1} \mathrm{H}$ NMR spectrum of compound 32 in $\mathrm{CDCl}_{3}$ (Table 2.4, entry xi) 


$$
1
$$




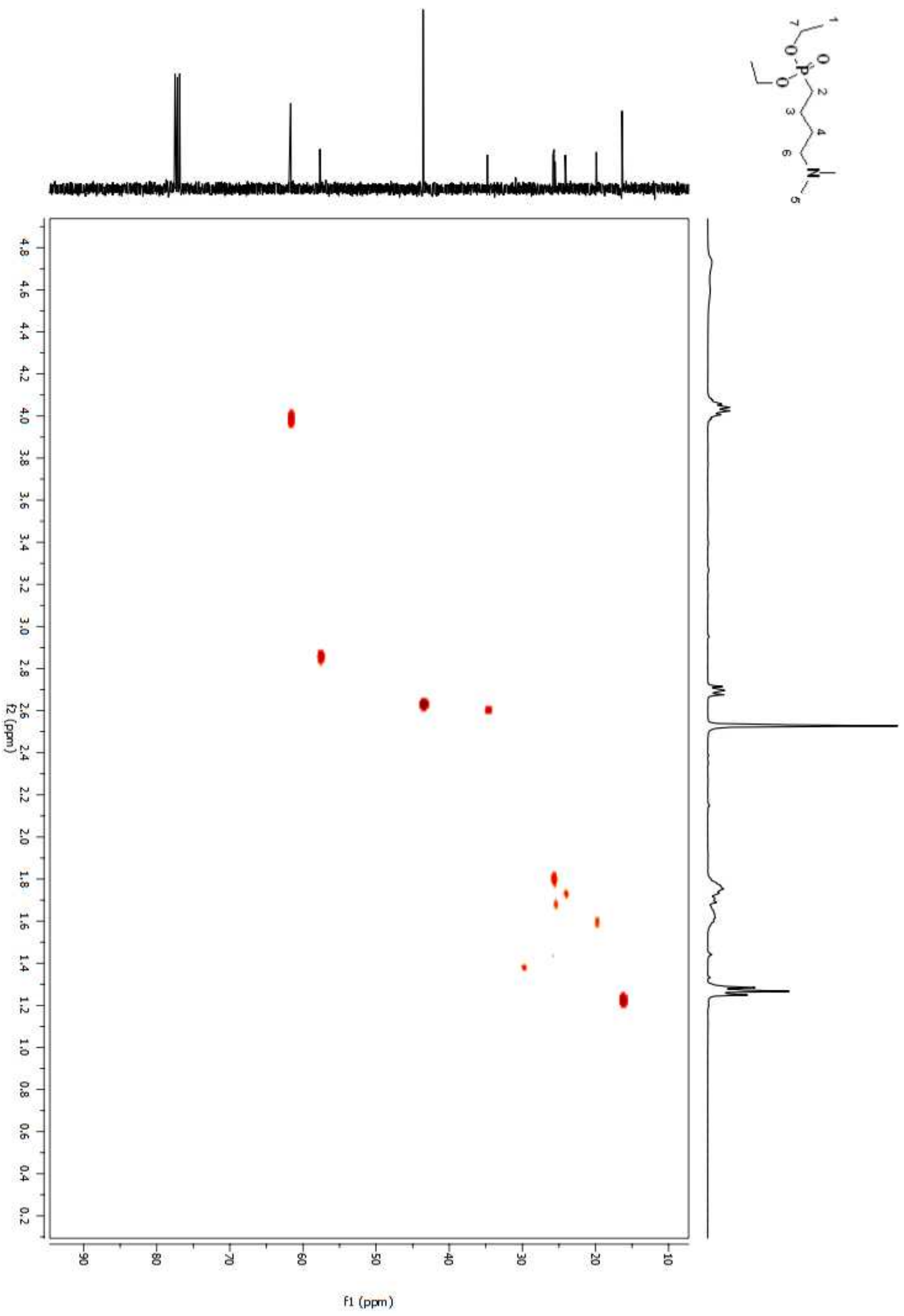

Figure A 81. HSQC 2D NMR spectrum of compound 32 in $\mathrm{CDCl}_{3}$ (Table 2.4, entry xi) 


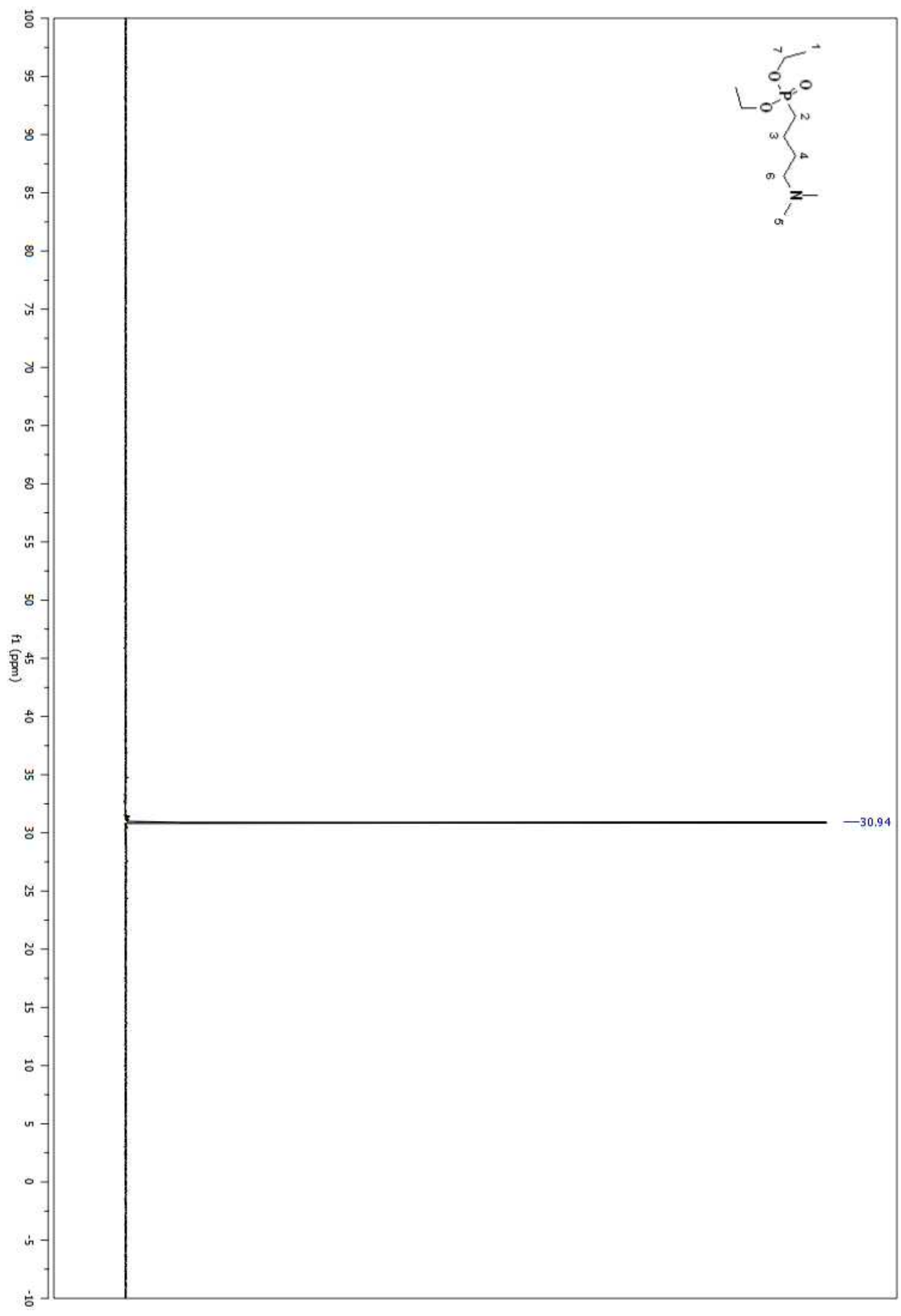

Figure A 82. ${ }^{31} \mathrm{P}$ NMR spectrum of compound 32 in $\mathrm{CDCl}_{3}$ (Table 2.4, entry xi) 


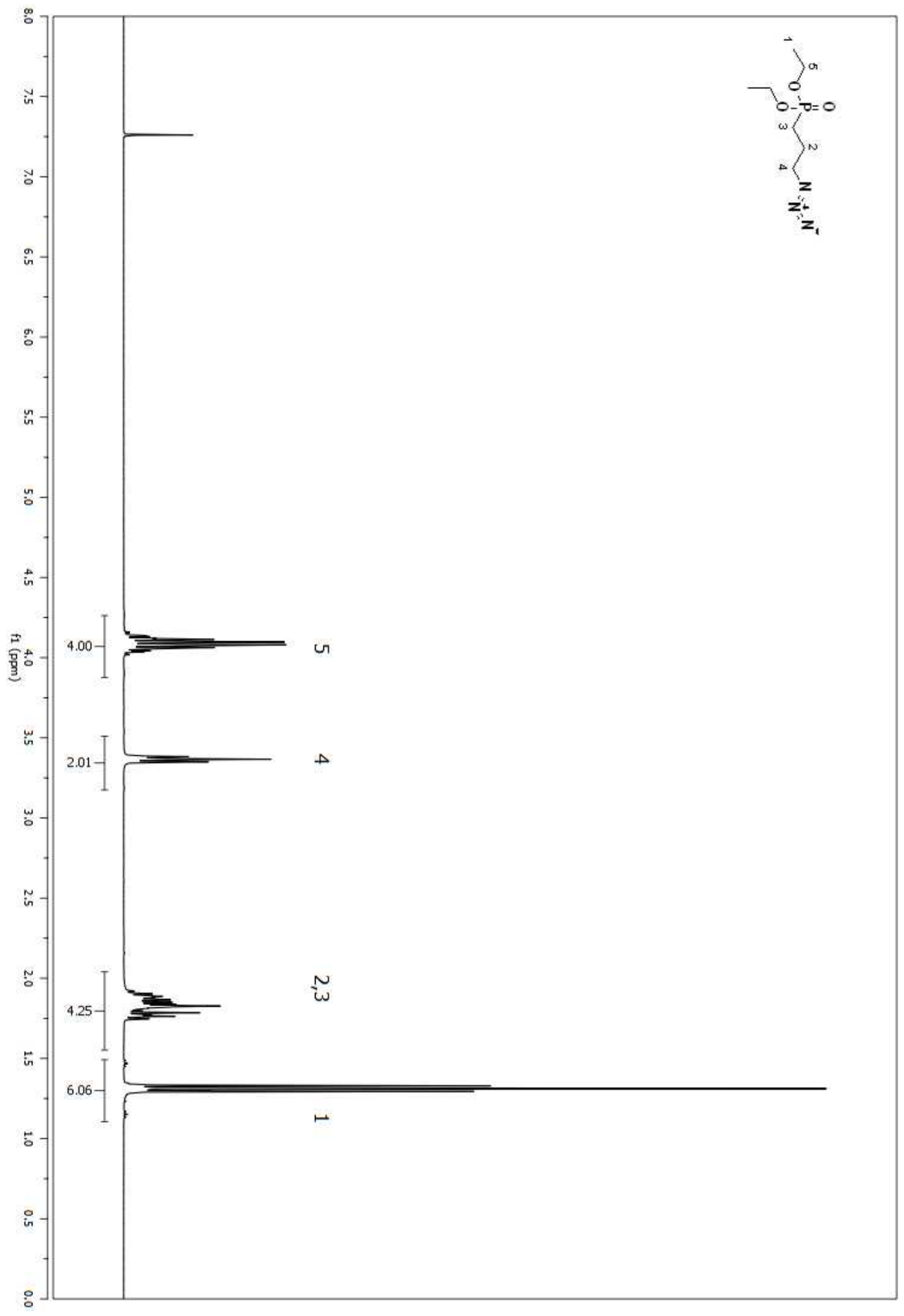

Figure A 83. ${ }^{1} \mathrm{H}$ NMR spectrum of compound 33 in $\mathrm{CDCL}_{3}$ (Table 2.4, entry xii) 


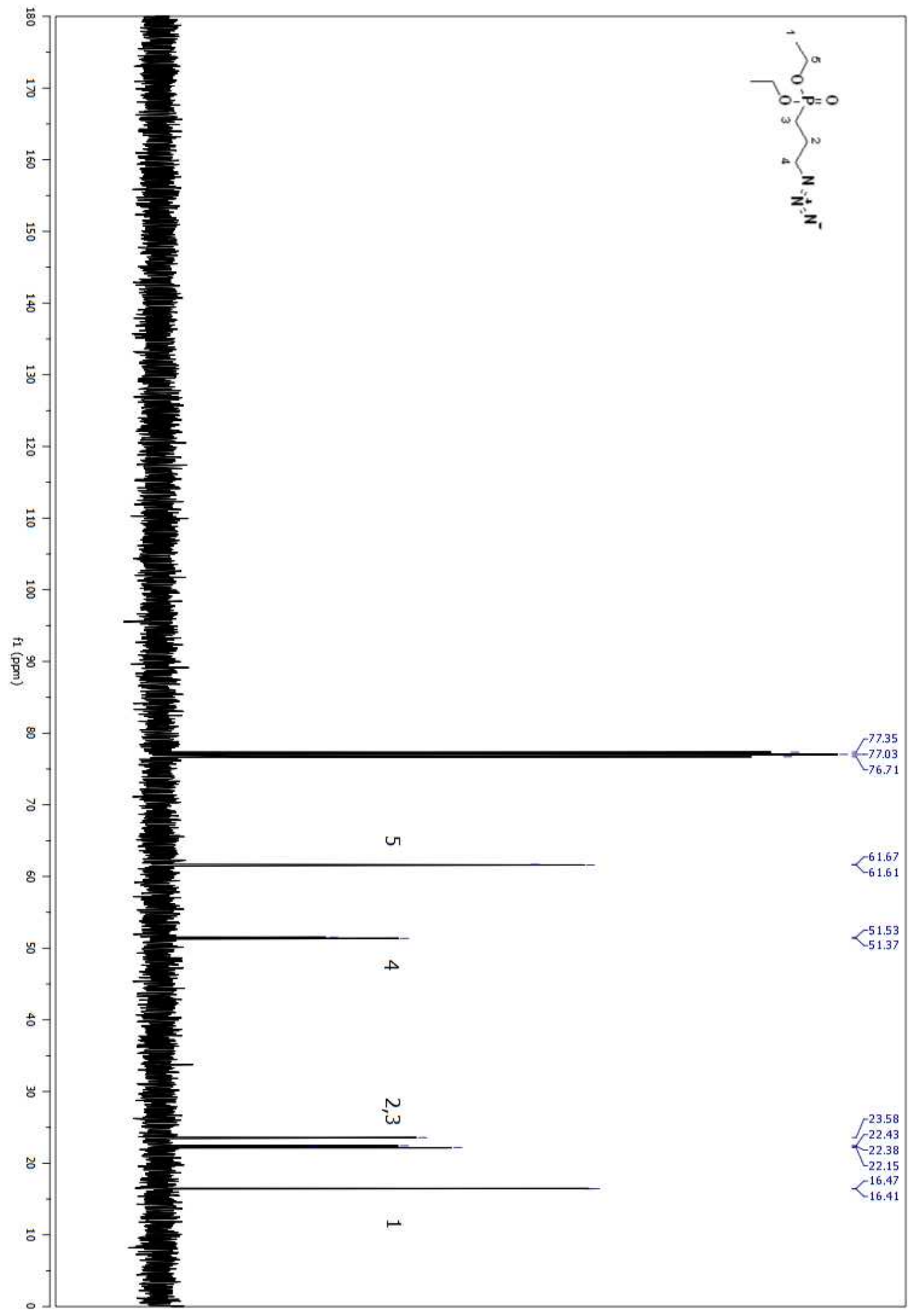

Figure A 84. ${ }^{13} \mathrm{C}$ NMR spectrum of compound 33 in $\mathrm{CDCL}_{3}$ (Table 2.4, entry xii) 


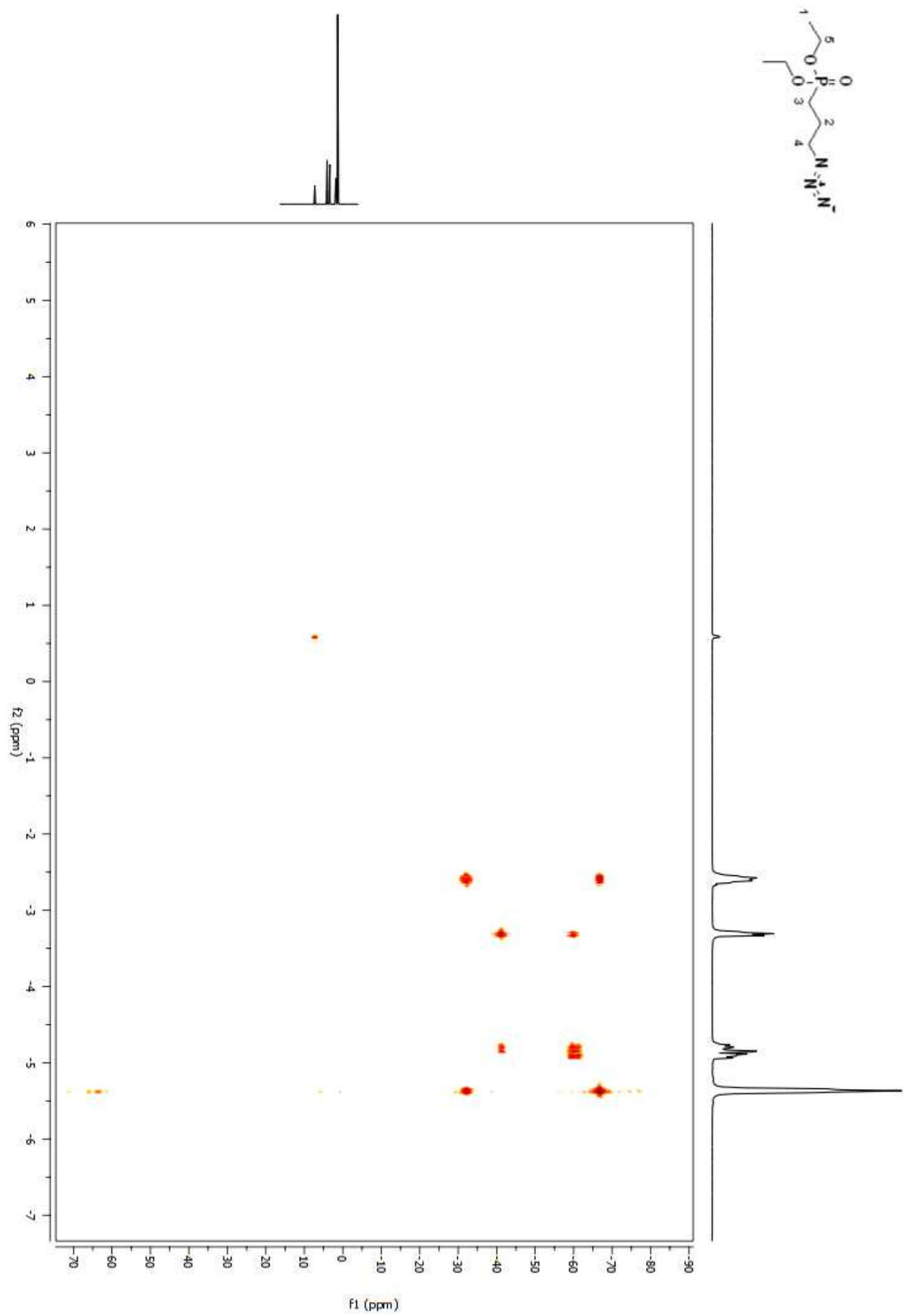

Figure A 85. COSY 2D NMR spectrum of compound 33 in $\mathrm{CDCl}_{3}$ (Table 2.4, entry xii) 


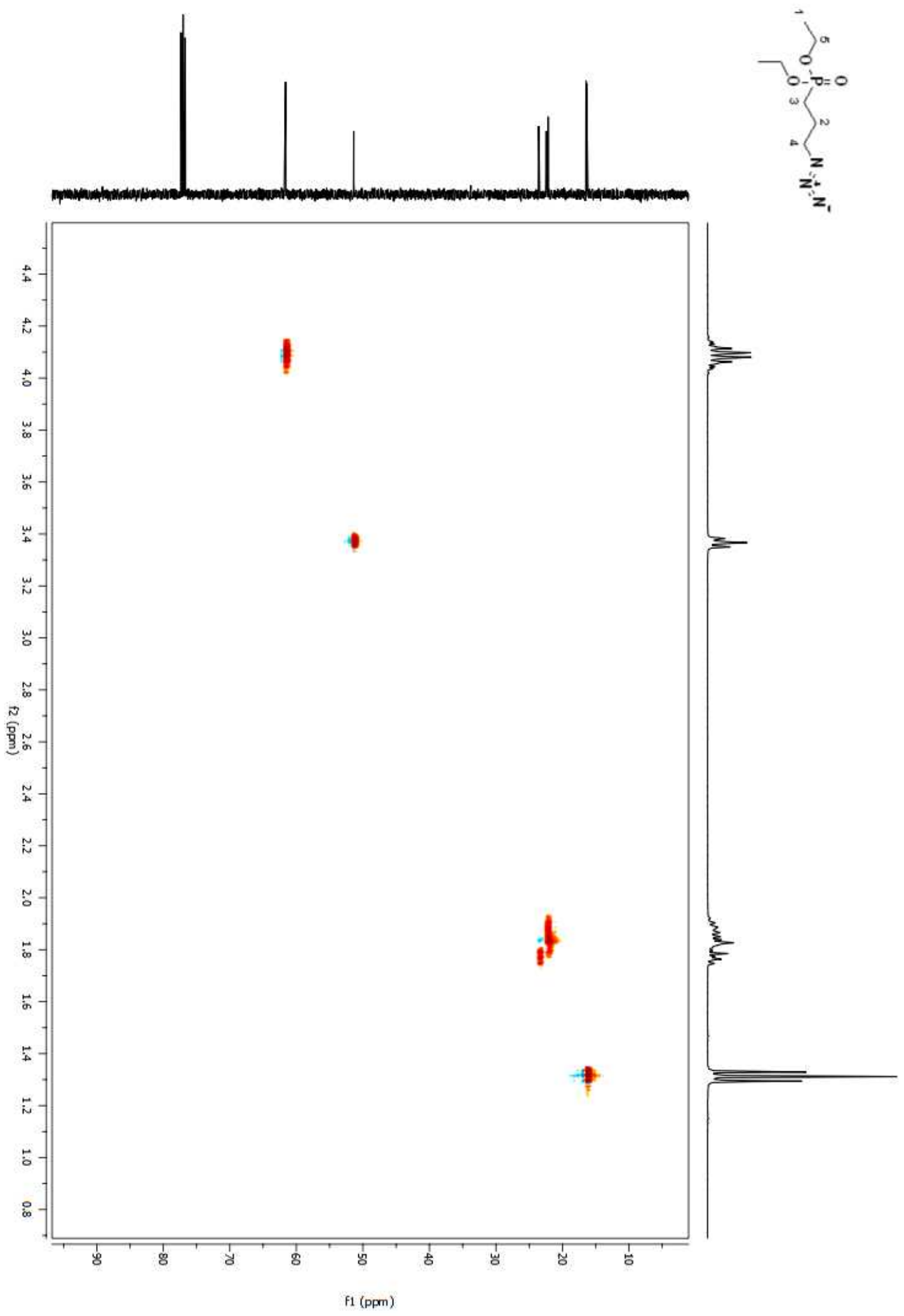

Figure A 86. HSQC 2D NMR spectrum of compound 33 in $\mathrm{CDCl}_{3}$ (Table 2.4, entry xii) 453 


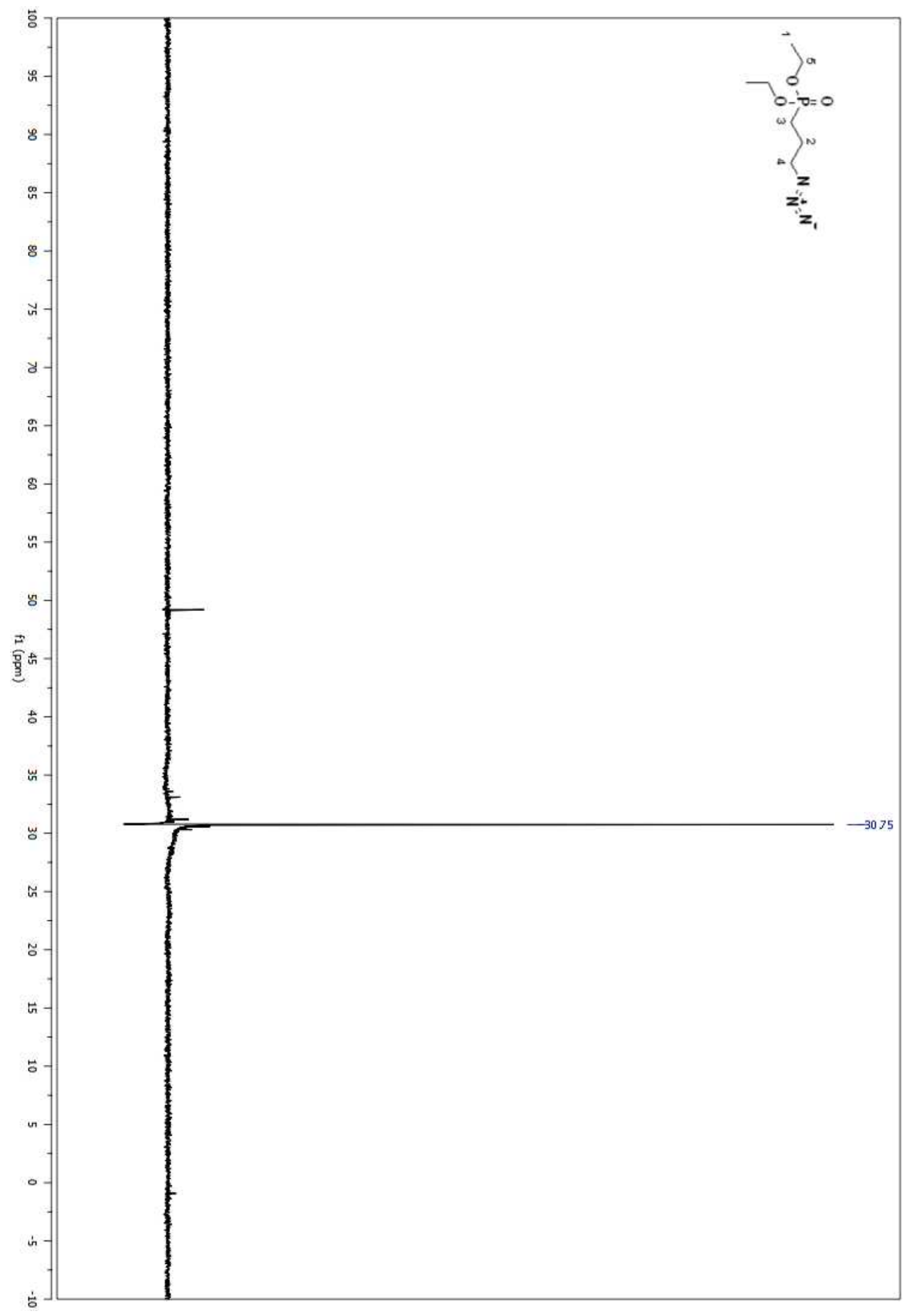

Figure A 87. ${ }^{31} \mathrm{P}$ NMR spectrum of compound 33 in $\mathrm{CDCL}_{3}$ (Table 2.4, entry xii) 454 


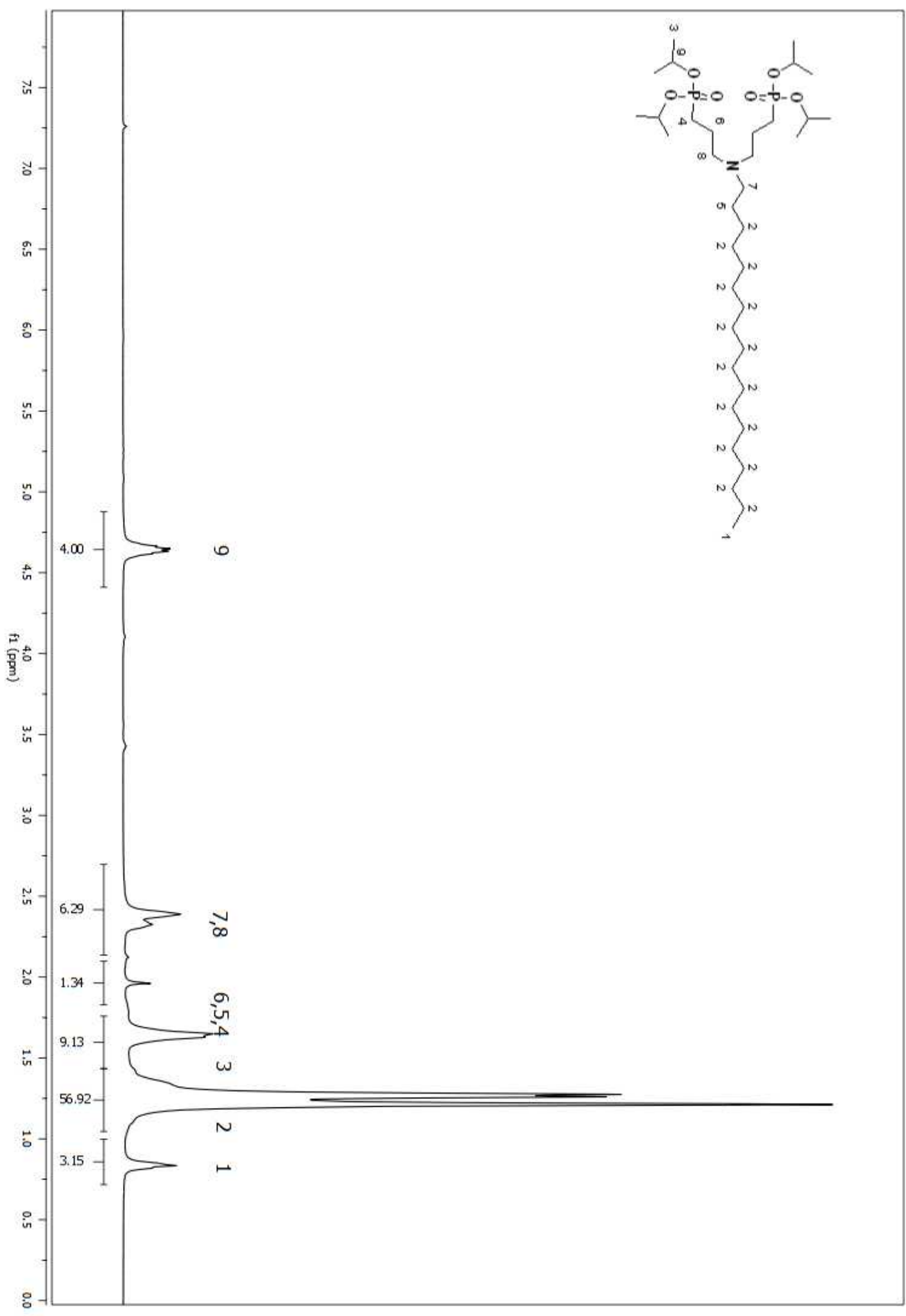

Figure A 88. ${ }^{1} \mathrm{H}$ NMR spectrum of compound 39 in $\mathrm{CDCl}_{3}$ 


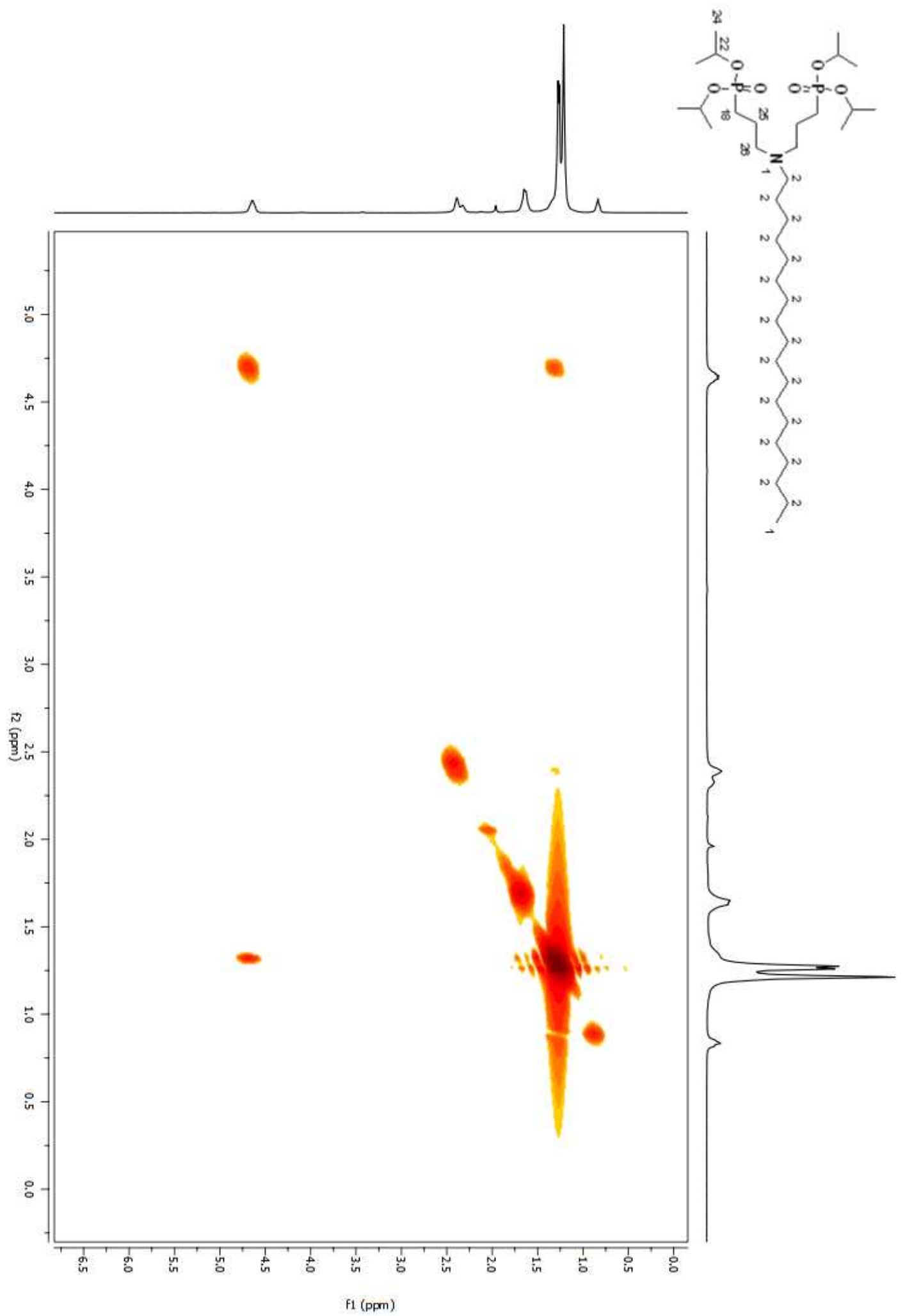

Figure A 89. COSY 2D NMR spectrum of compound 39 in $\mathrm{CDCl}_{3}$ 


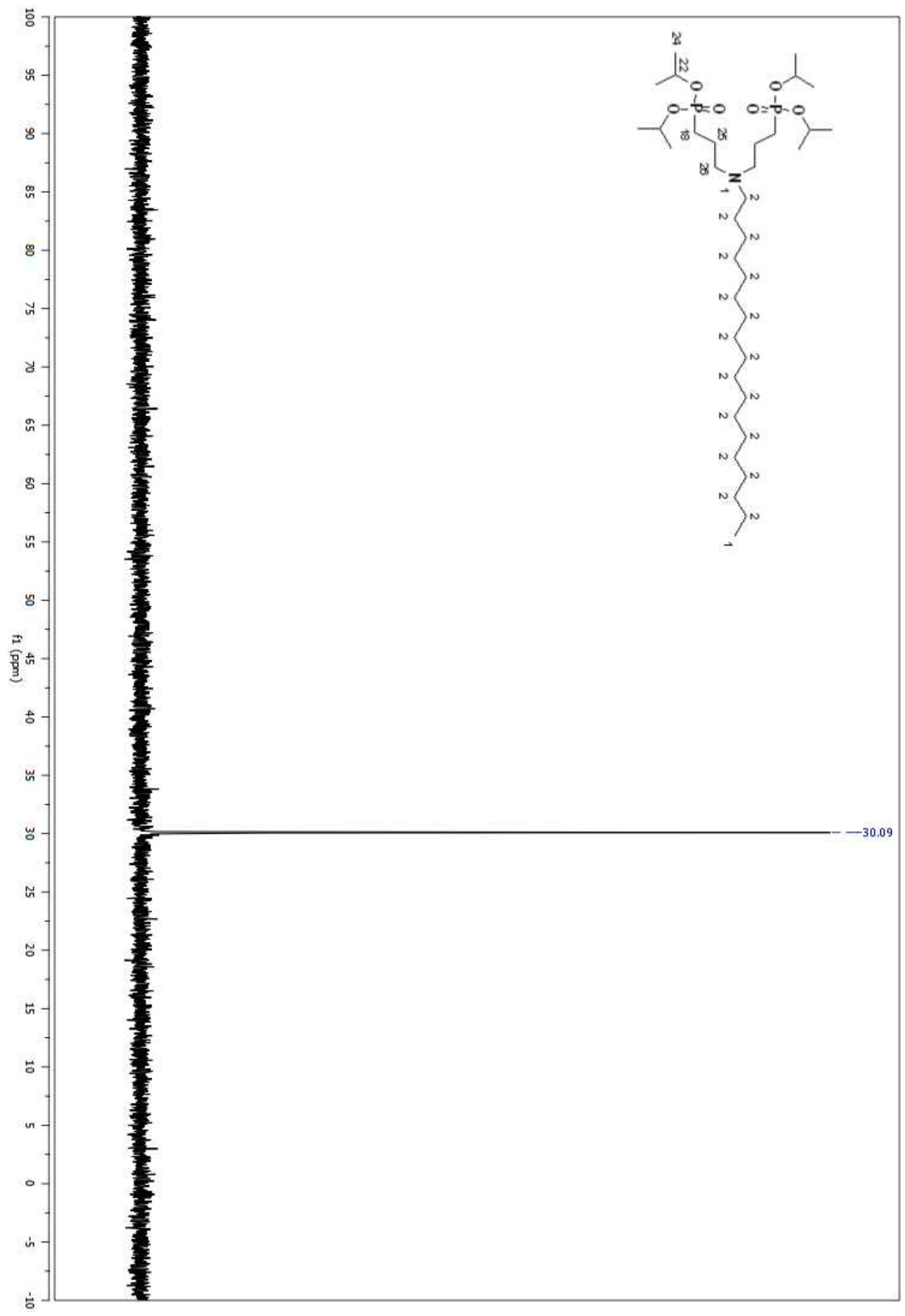

Figure A 90. ${ }^{31} \mathrm{P}$ NMR spectrum of compound 39 in $\mathrm{CDCl}_{3}$ 


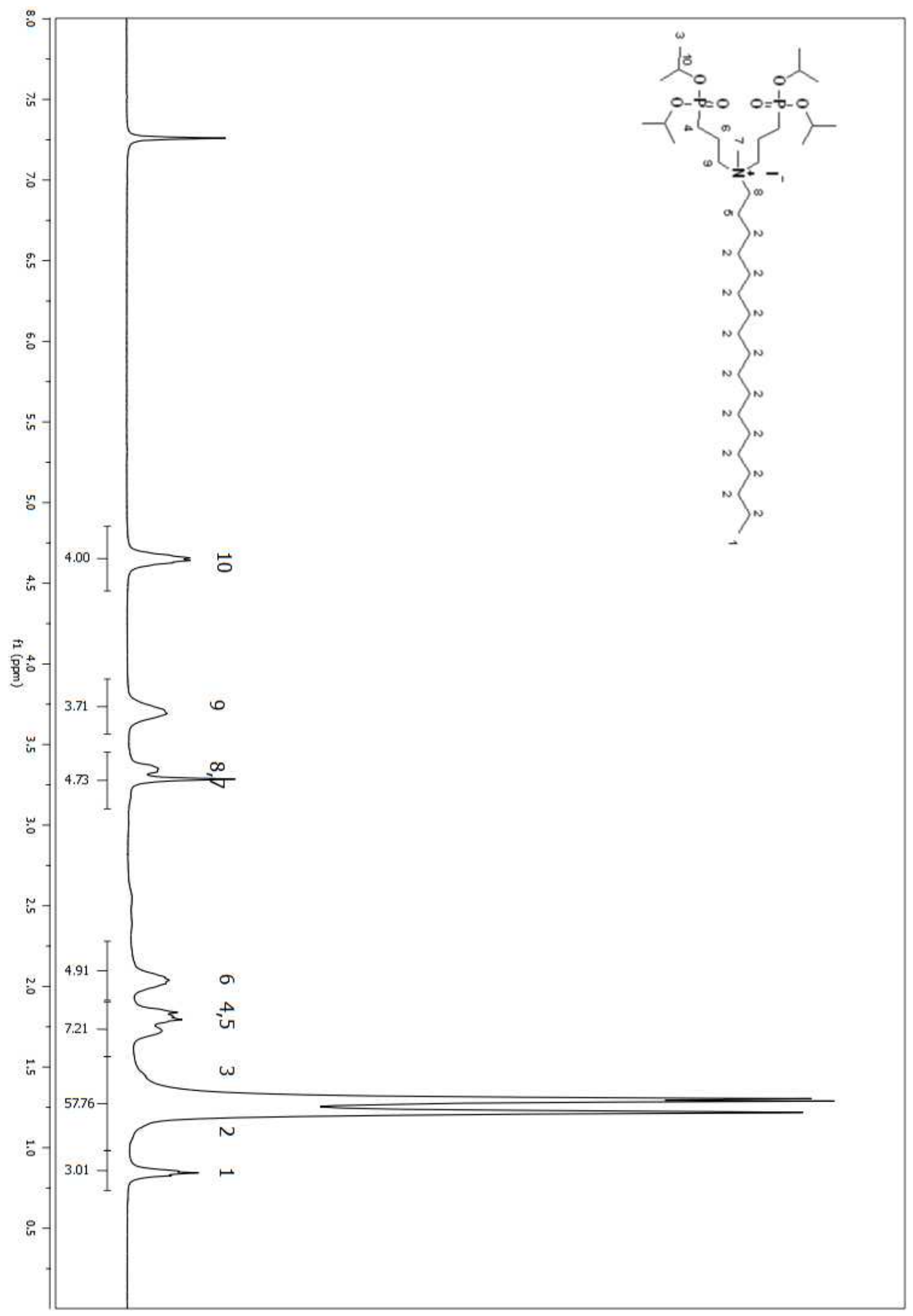

Figure A 91. ${ }^{1} \mathrm{H}$ NMR spectrum of compound 40 in $\mathrm{CDCl}_{3}$ 


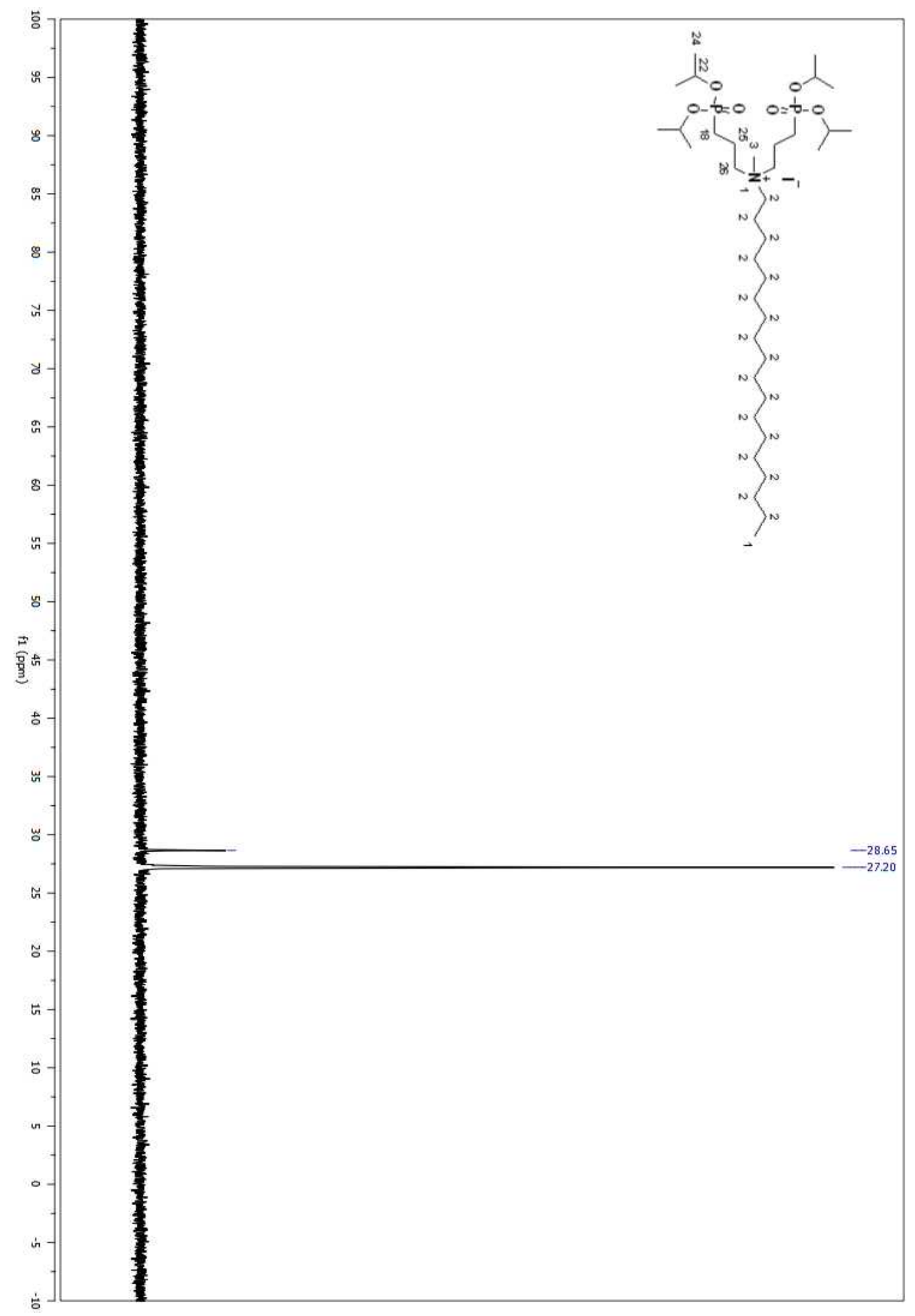

Figure A 92. ${ }^{31} \mathrm{P}$ NMR spectrum of compound 40 in $\mathrm{CDCl}_{3}(\mathrm{BEFORE} \mathrm{COLUMN})$ 


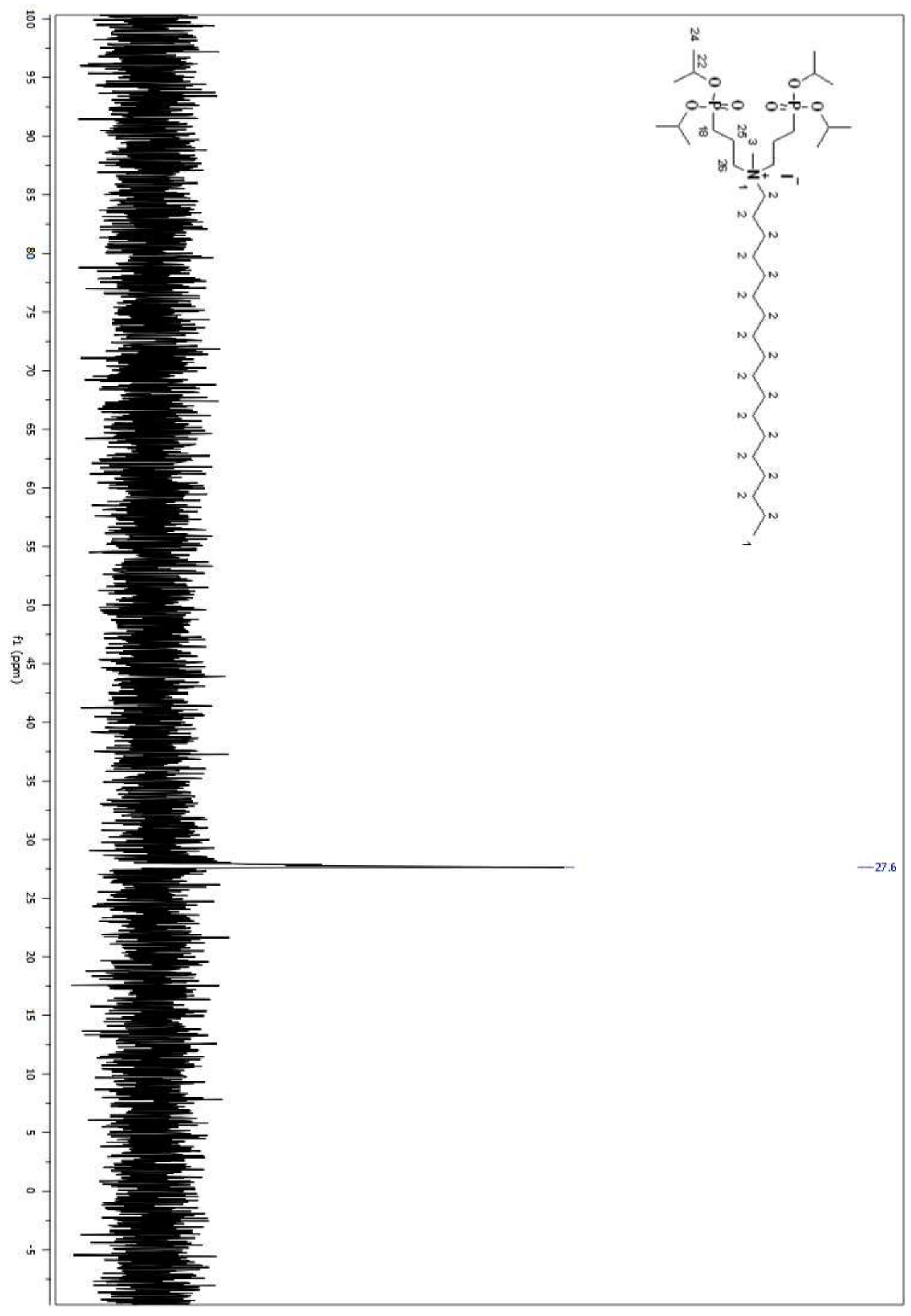

Figure A 93. ${ }^{31} \mathrm{P}$ NMR spectrum of compound 40 in $\mathrm{CDCl}_{3}$ (AFTER COLUMN) 


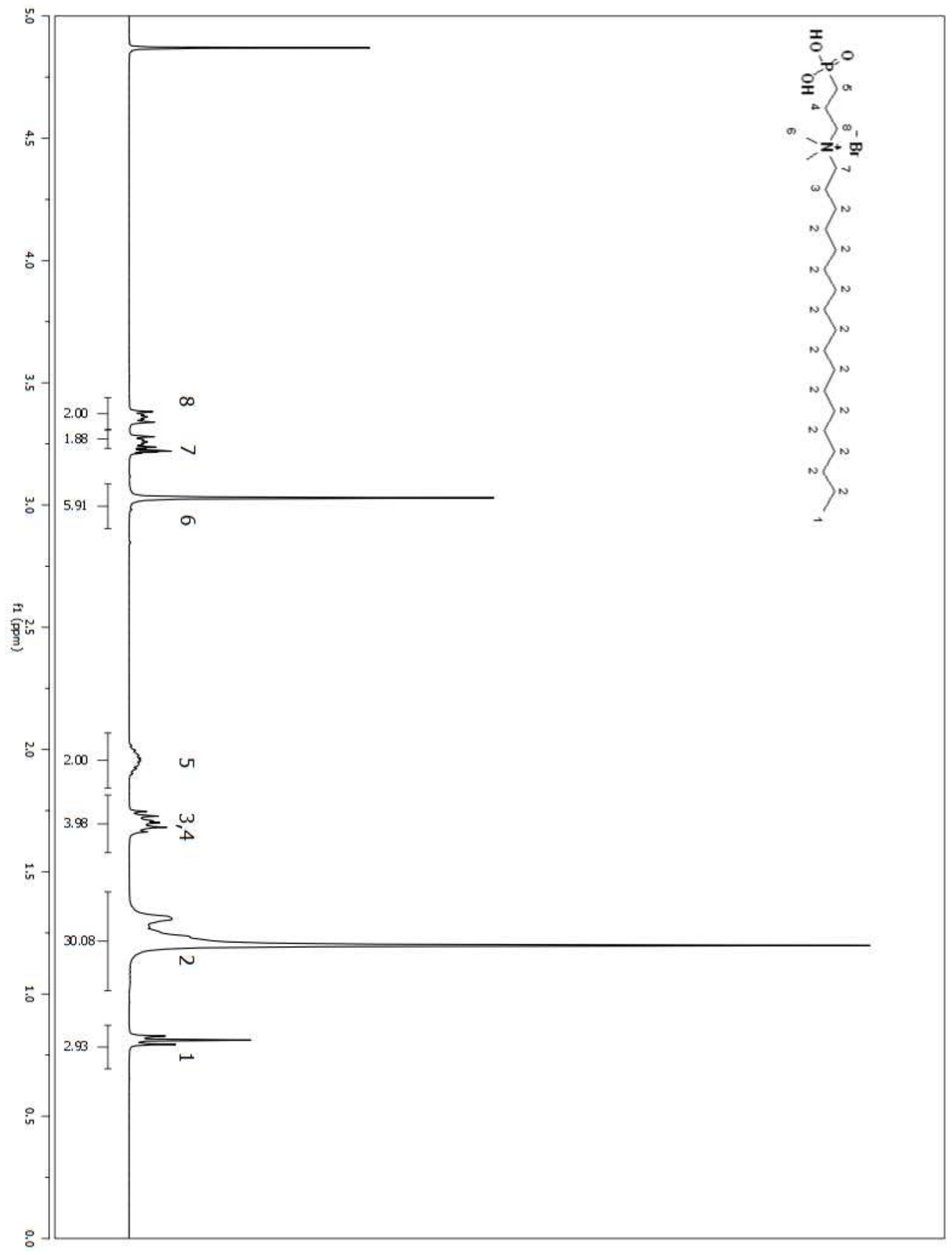

Figure A 94. ${ }^{1} \mathrm{H}$ NMR spectrum of compound 34 in MeOD (Table 2.5, entry i) 


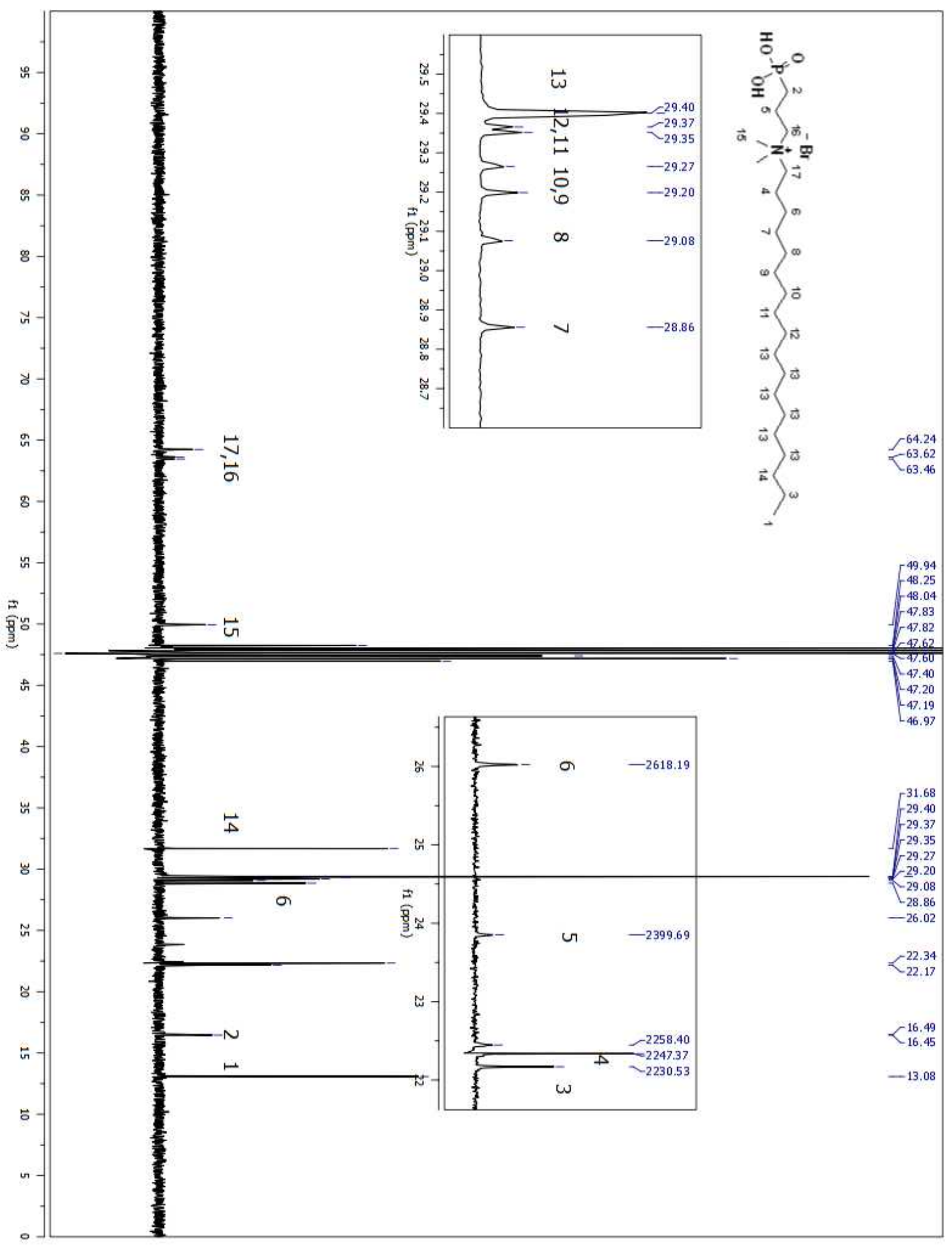

Figure A 95. ${ }^{13} \mathrm{C}$ NMR spectrum of compound 34 in MeOD (Table 2.5, entry i) 


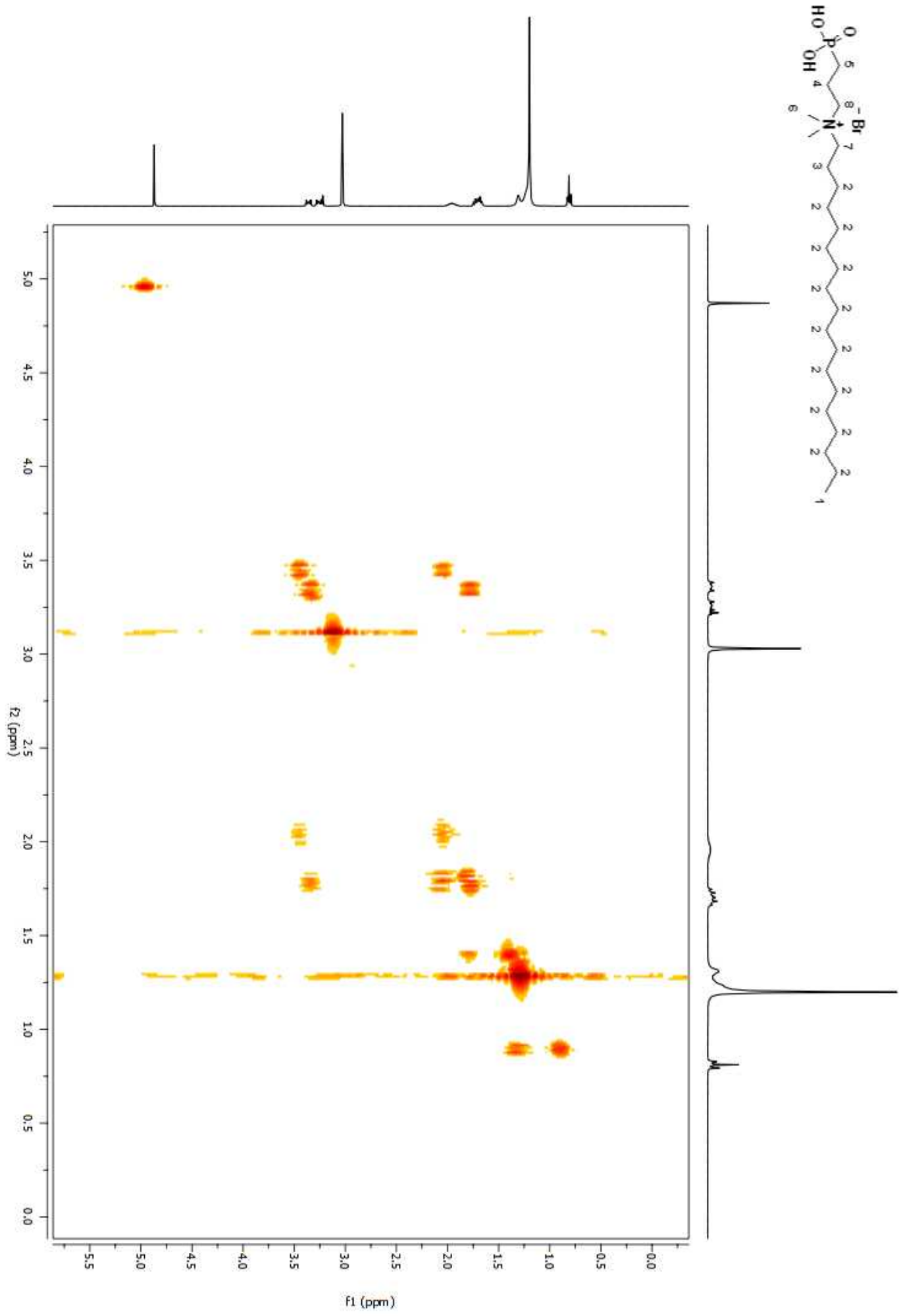

Figure A 96. ${ }^{1} \mathrm{H}$ NMR spectrum of compound 34 in MeOD (Table 2.5, entry i) 


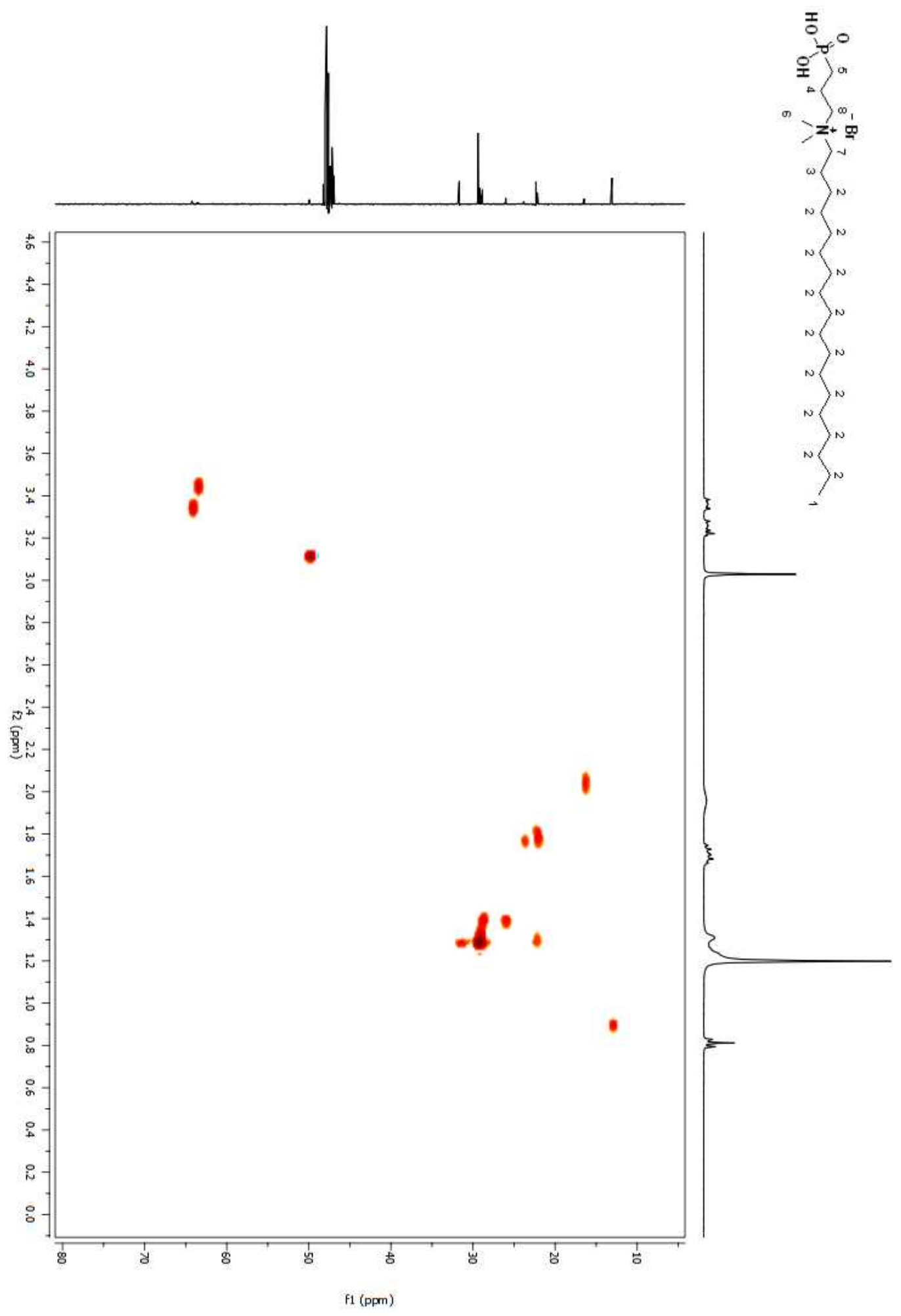

Figure A 97. ${ }^{1} \mathrm{H}$ NMR spectrum of compound 34 in MeOD (Table 2.5, entry i) 


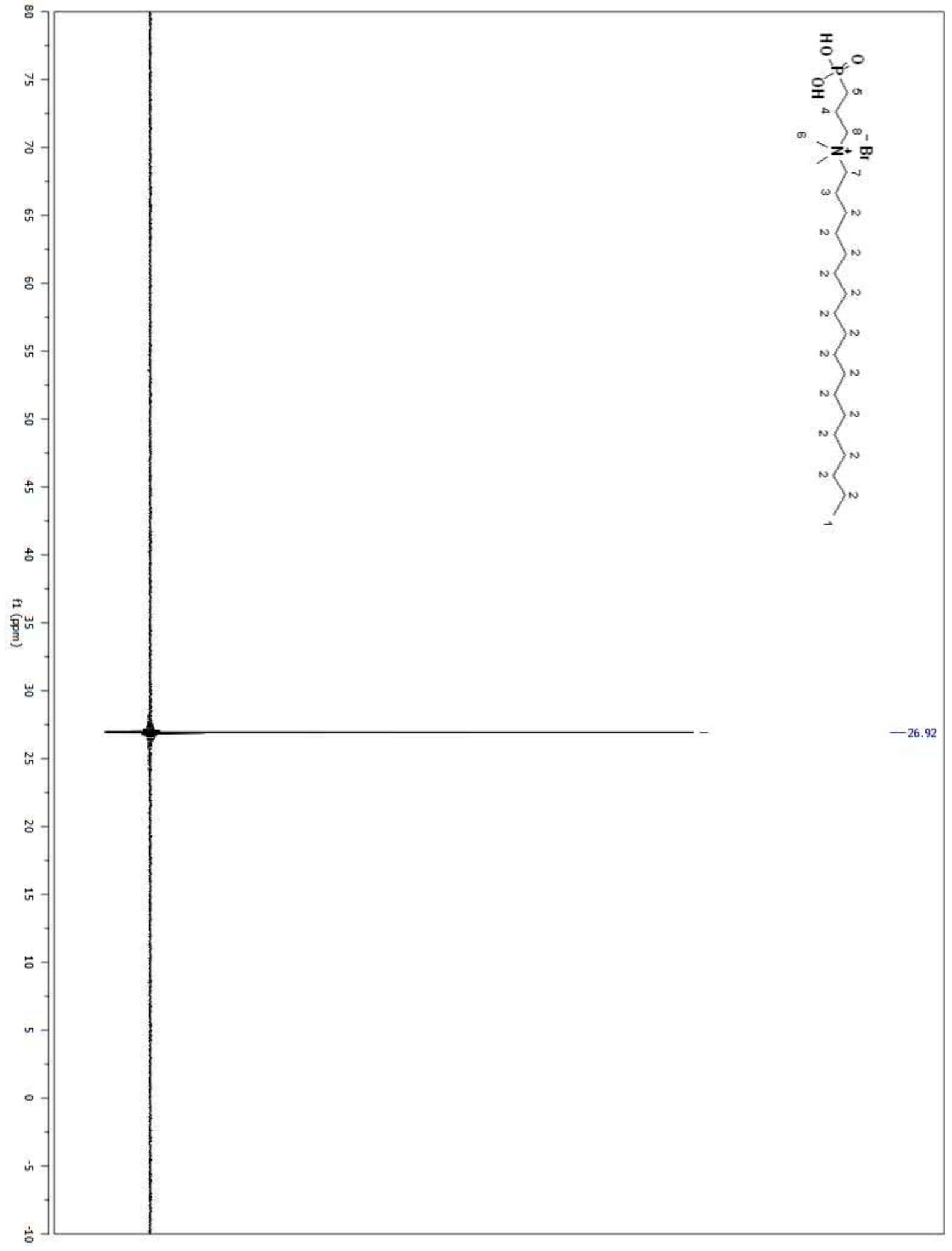

Figure A 98. ${ }^{31} \mathrm{P}$ NMR spectrum of compound 34 in MeOD (Table 2.5, entry i) 465 


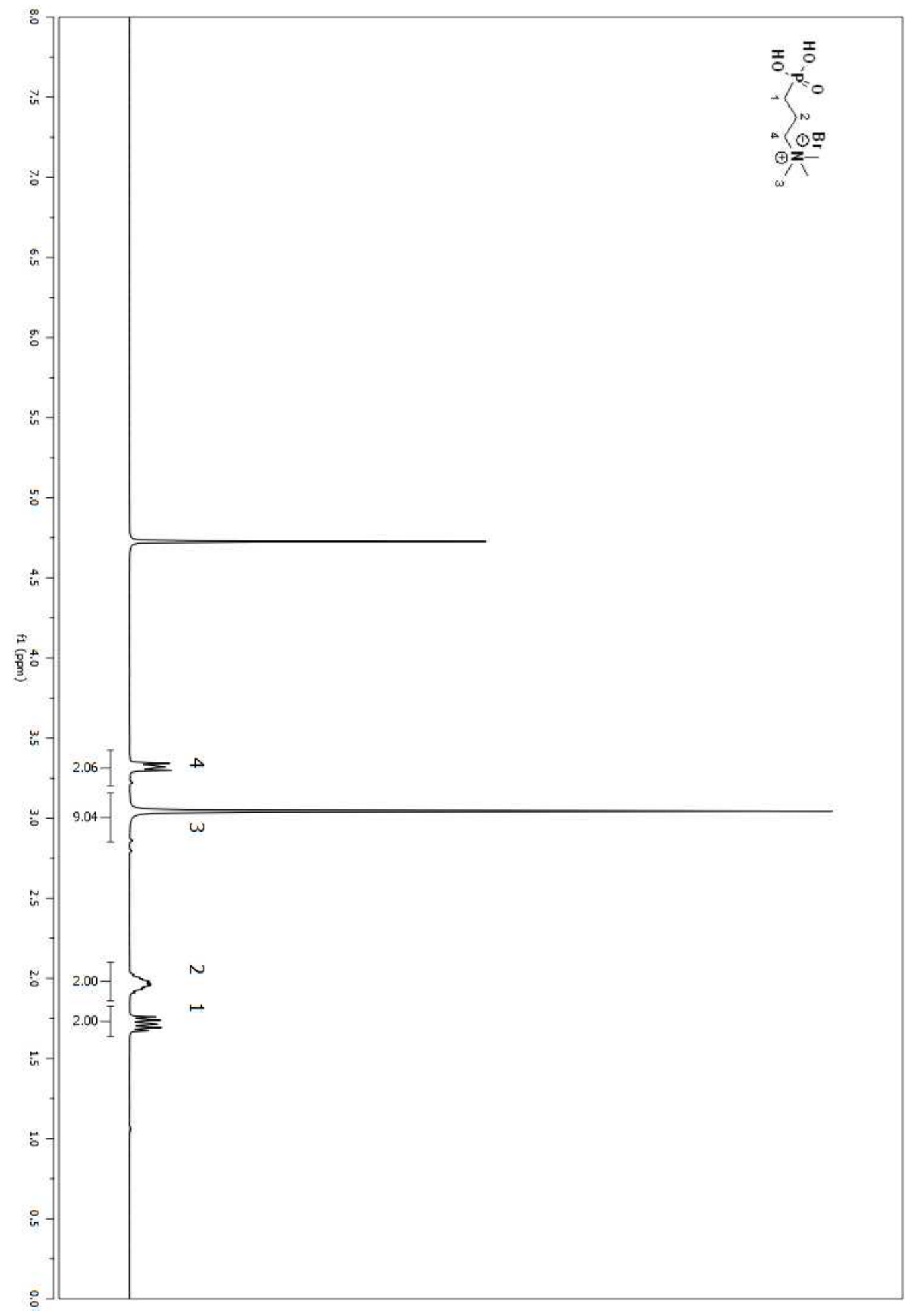

Figure A 99. ${ }^{1} \mathrm{H}$ NMR spectrum of compound 35 in $\mathrm{D}_{2} \mathrm{O}$ (Table 4.5, entry ix) 


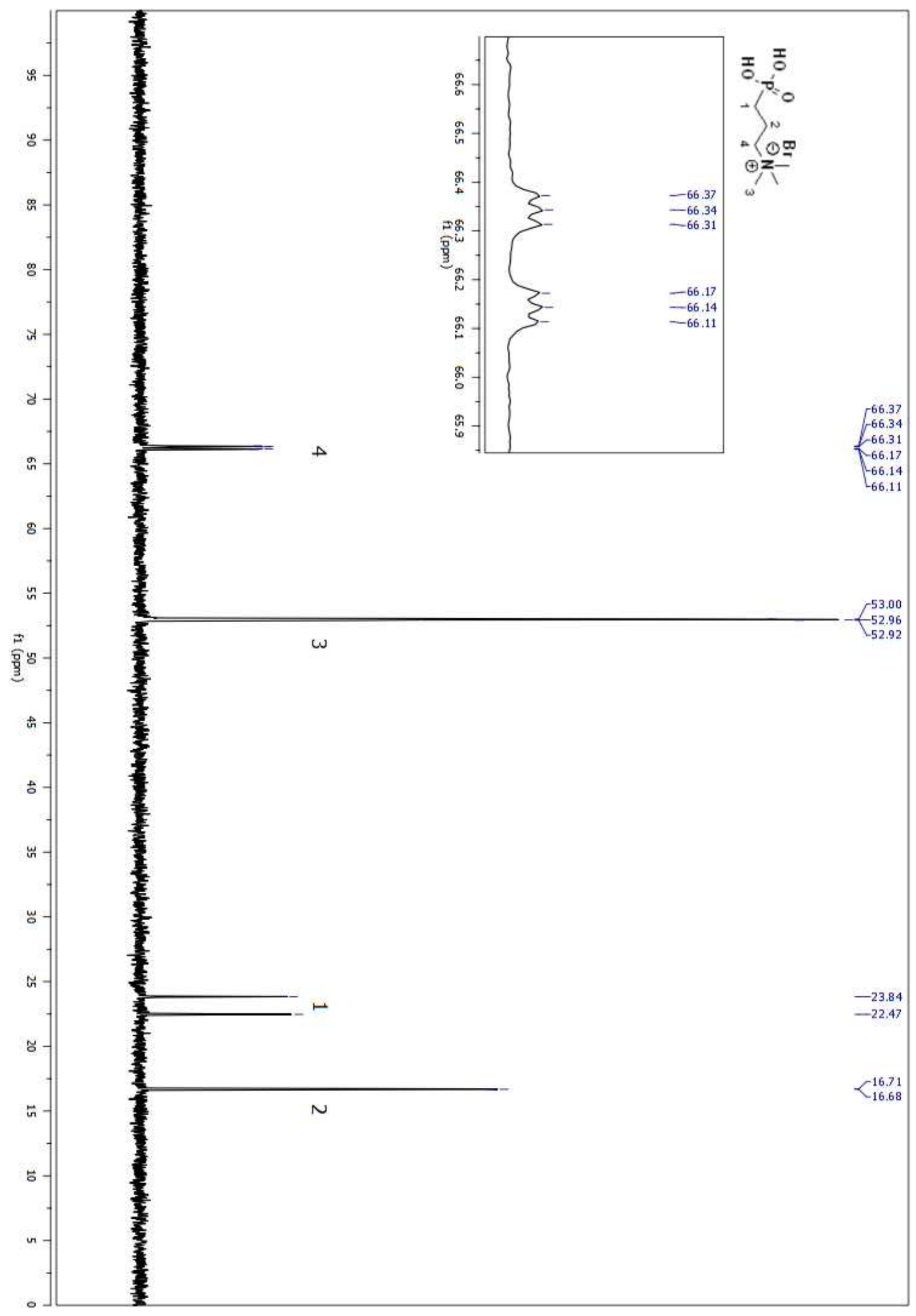

Figure A 100. ${ }^{13} \mathrm{C}$ NMR spectrum of compound 35 in $\mathrm{D}_{2} \mathrm{O}$ (Table 2.5, entry ix) 


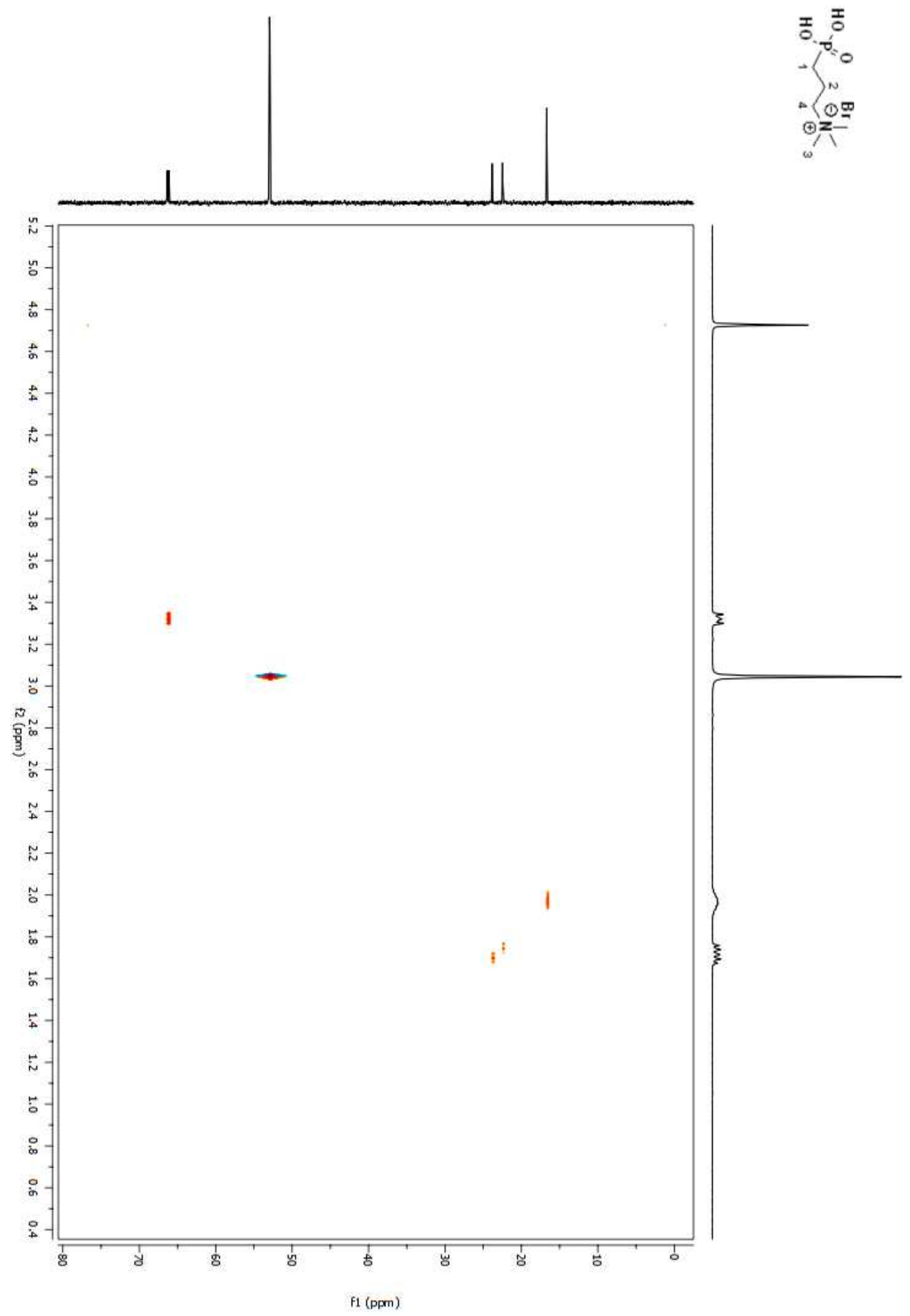

Figure A 101. HSQC spectrum of compound 35 in $\mathrm{D}_{2} \mathrm{O}$ (Table 2.5, entry xi) 


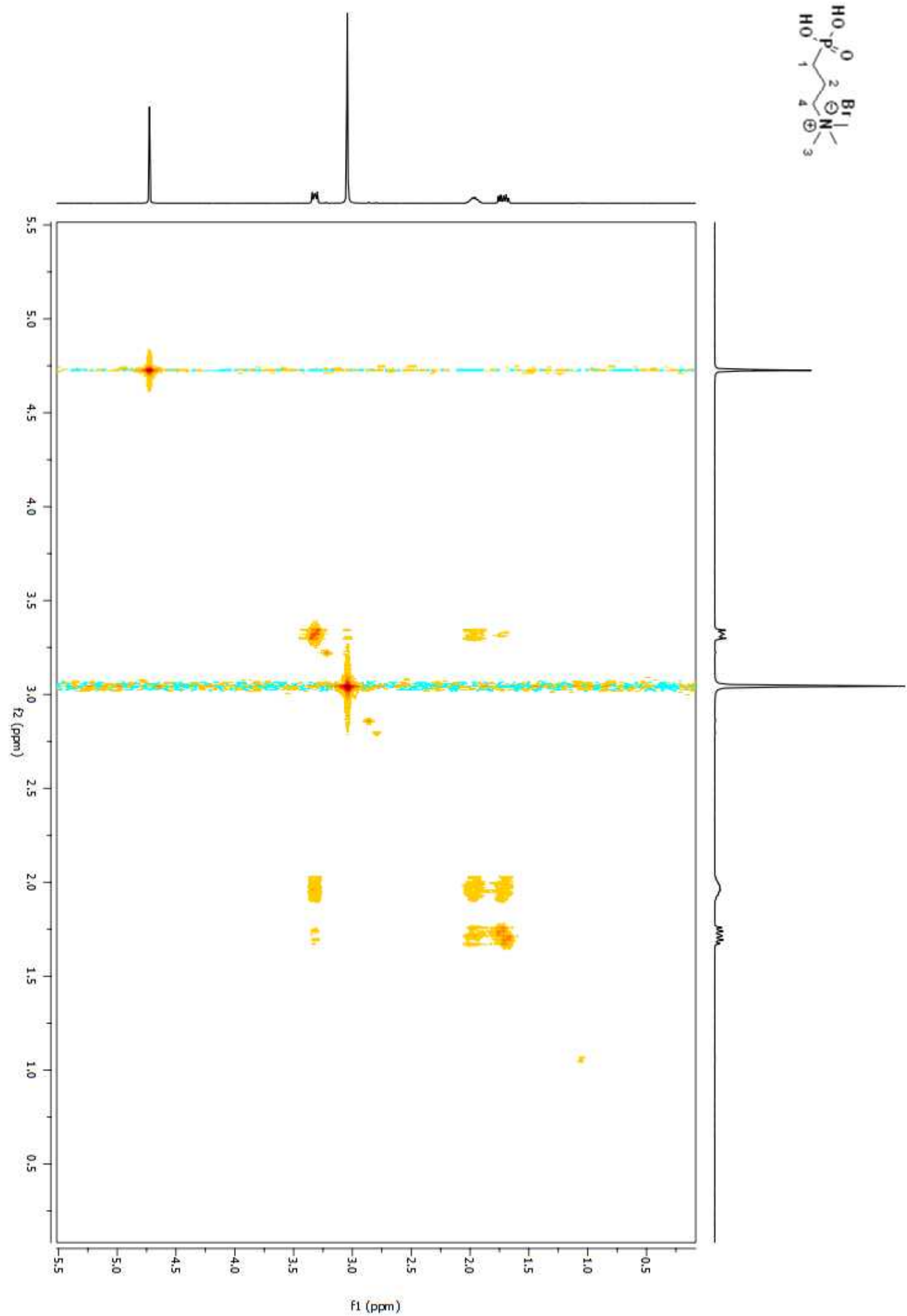

Figure A 102. COSY spectrum of compound 35 in $\mathrm{D}_{2} \mathrm{O}$ (Table 2.5, entry ix) 


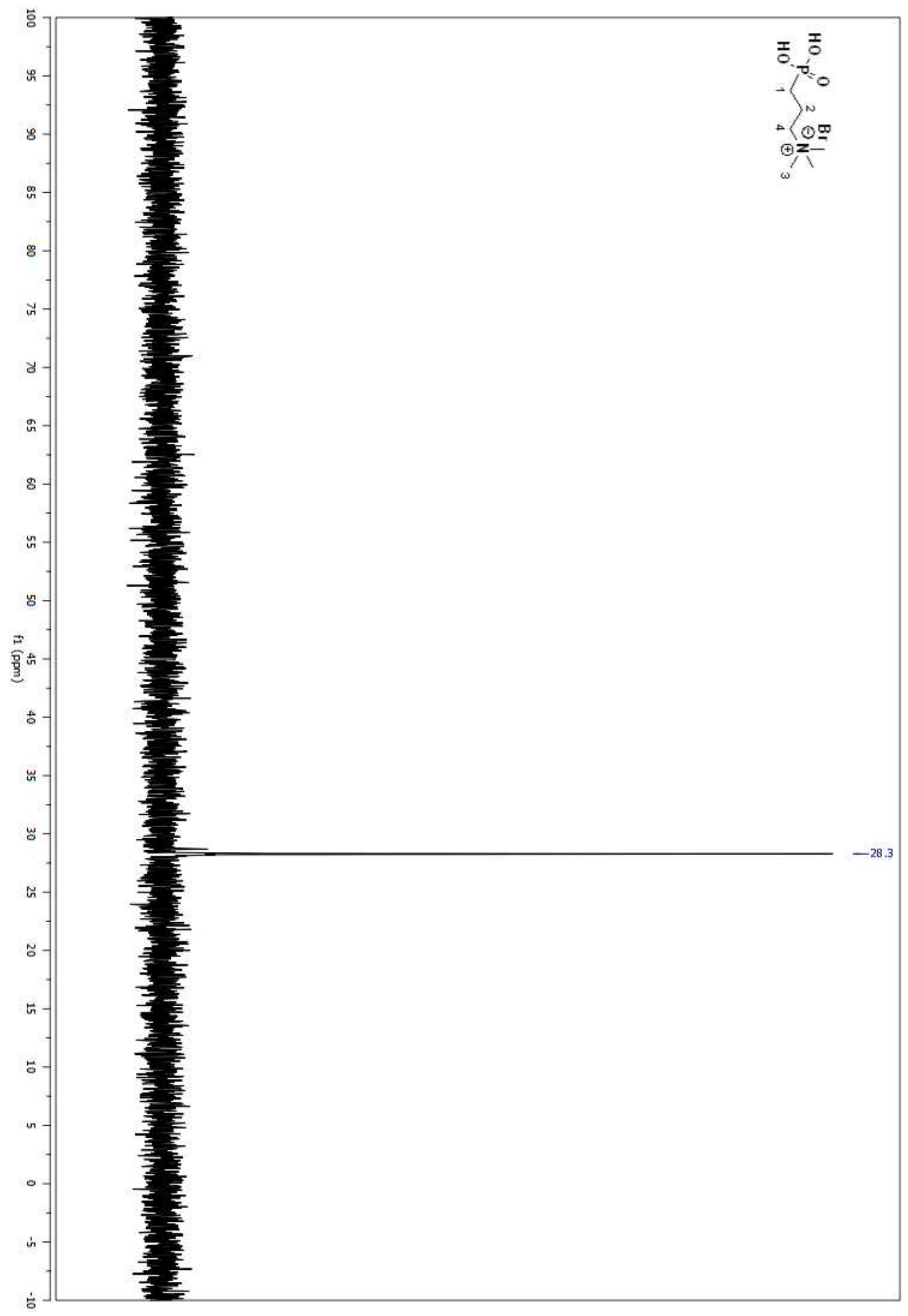

Figure A 103. ${ }^{31} \mathrm{P}$ spectrum of compound 35 in $\mathrm{D}_{2} \mathrm{O}$ (Table 2.5, entry ix) 


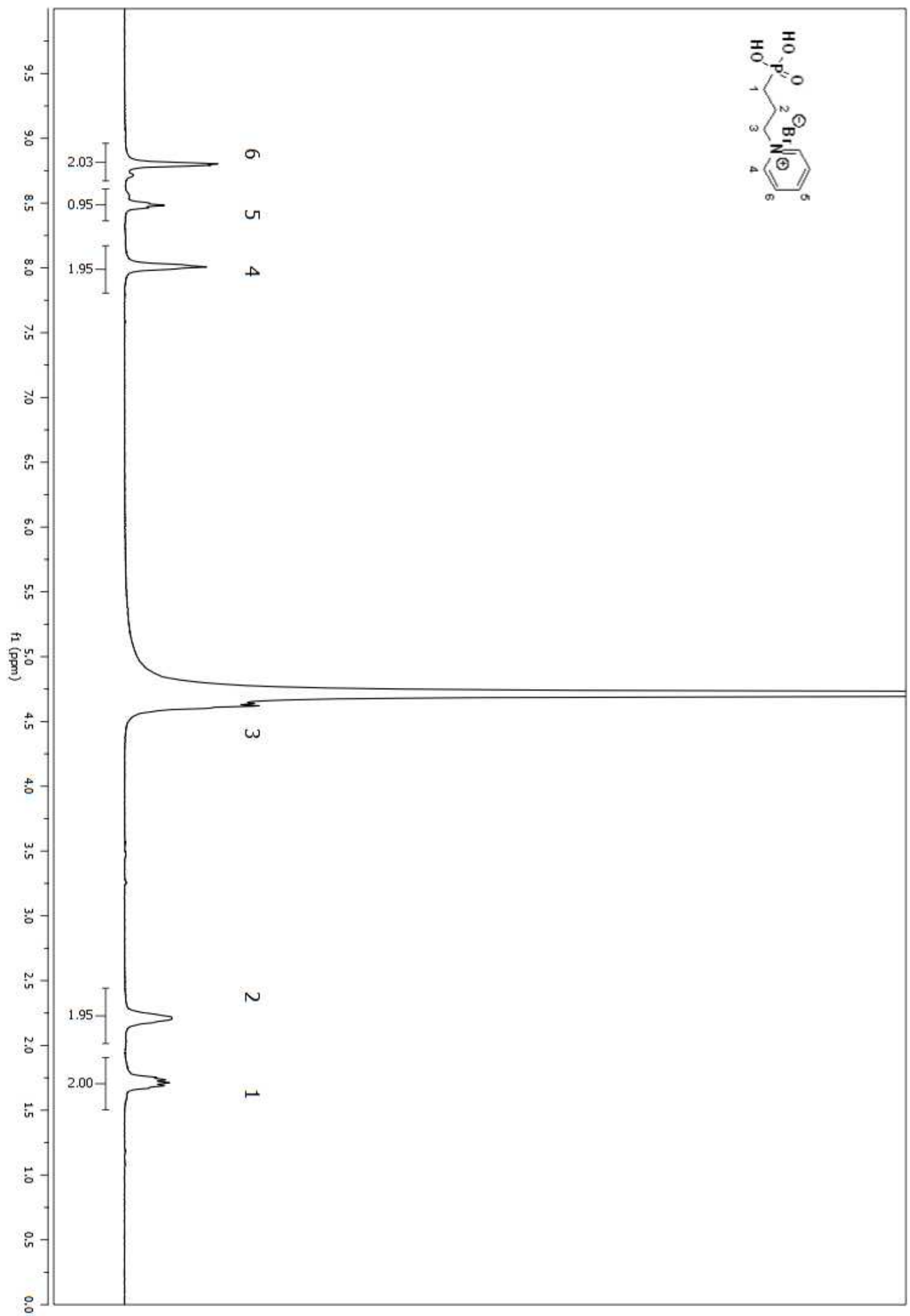

Figure A 104. ${ }^{1} \mathrm{H}$ NMR spectrum of compound 35 in $\mathrm{D}_{2} \mathrm{O}$ (Table 2.5, entry ix) 


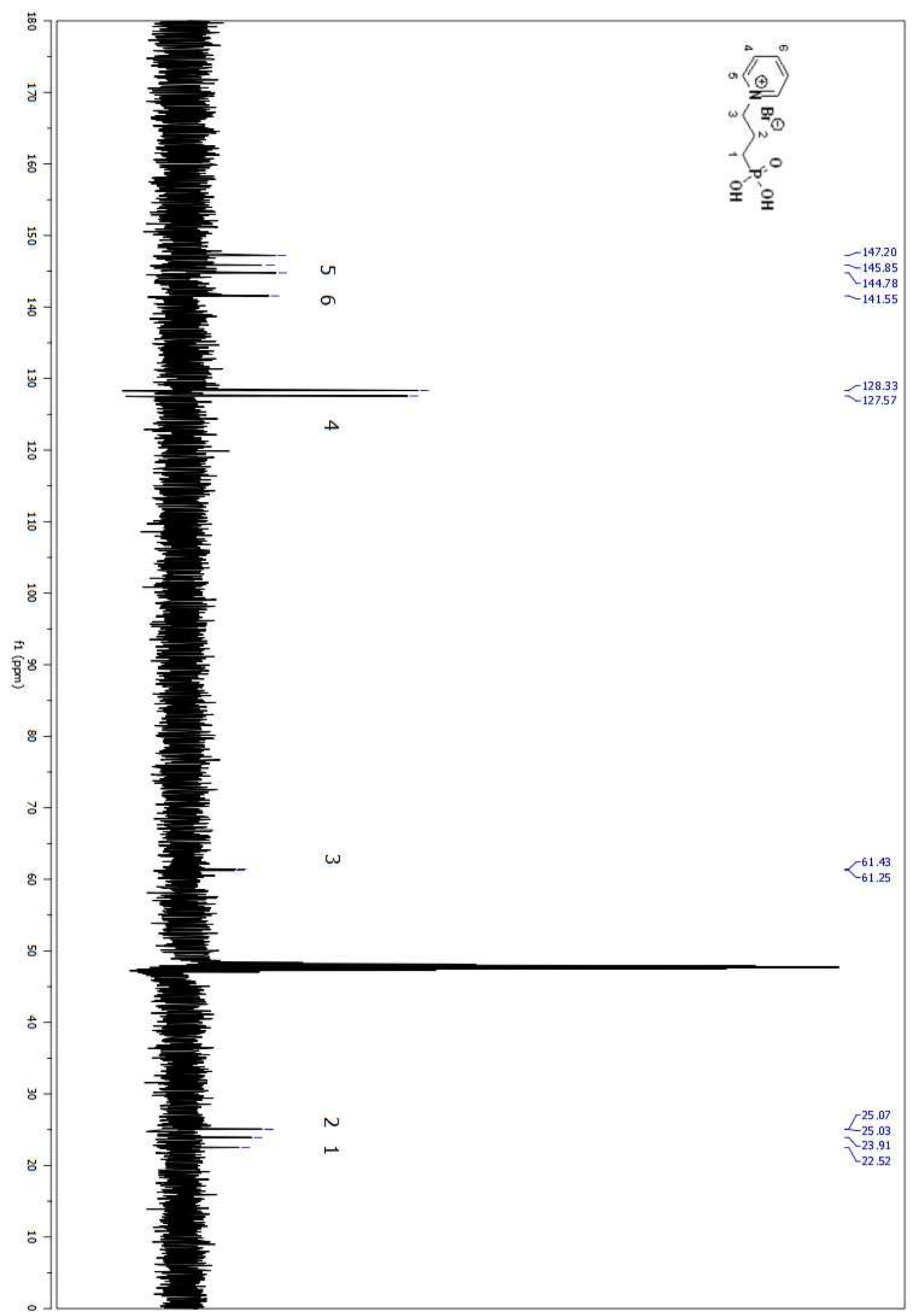

Figure A 105. ${ }^{13} \mathrm{C}$ NMR spectrum of compound 36 in $\mathrm{D}_{2} \mathrm{O}$ (Table 2.5, entry $\mathbf{x}$ ) 


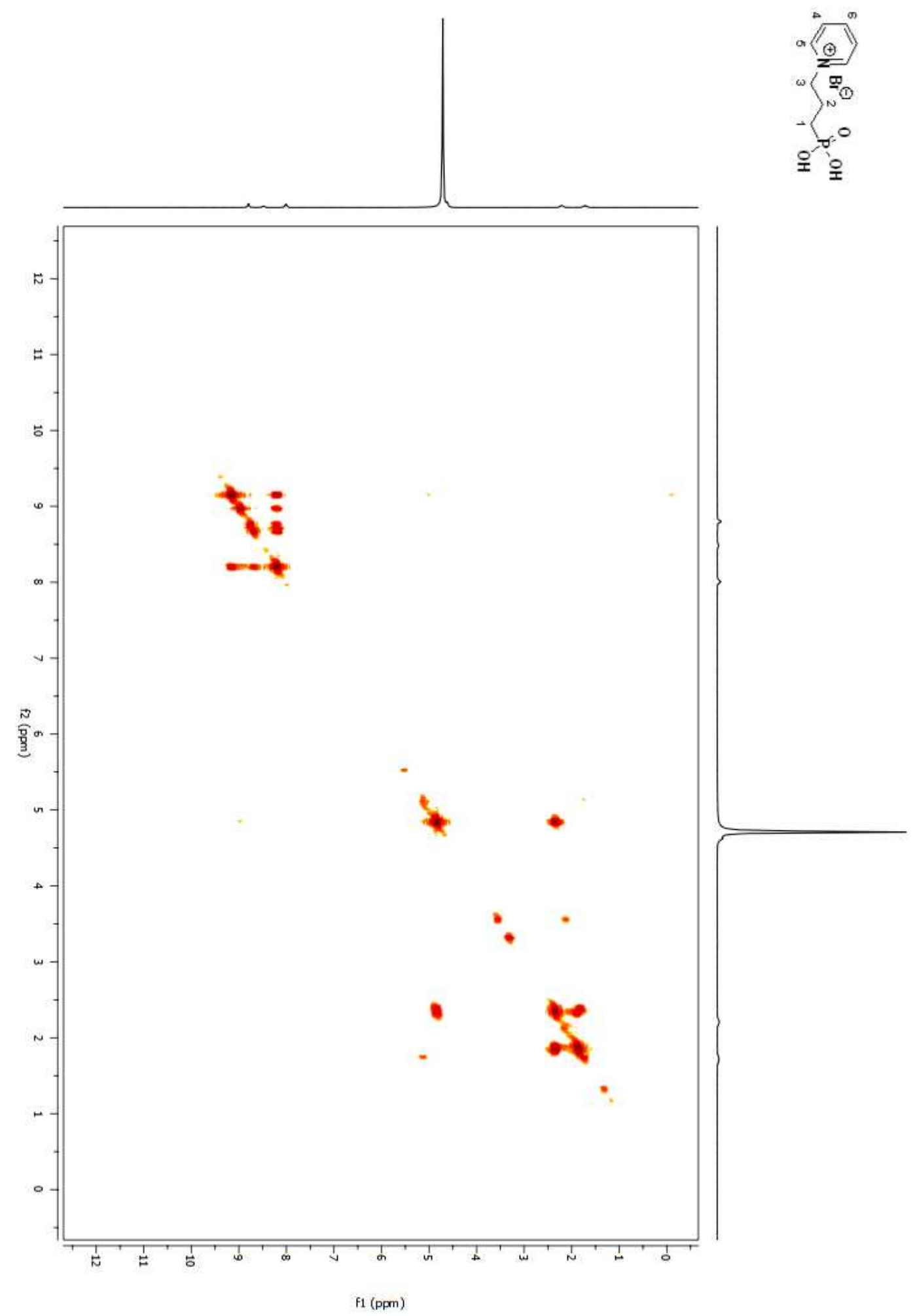

Figure A 106. ${ }^{1} \mathrm{H}$ NMR spectrum of compound 36 in $\mathrm{D}_{2} \mathrm{O}$ (Table 2.5, entry $\mathrm{x}$ ) 


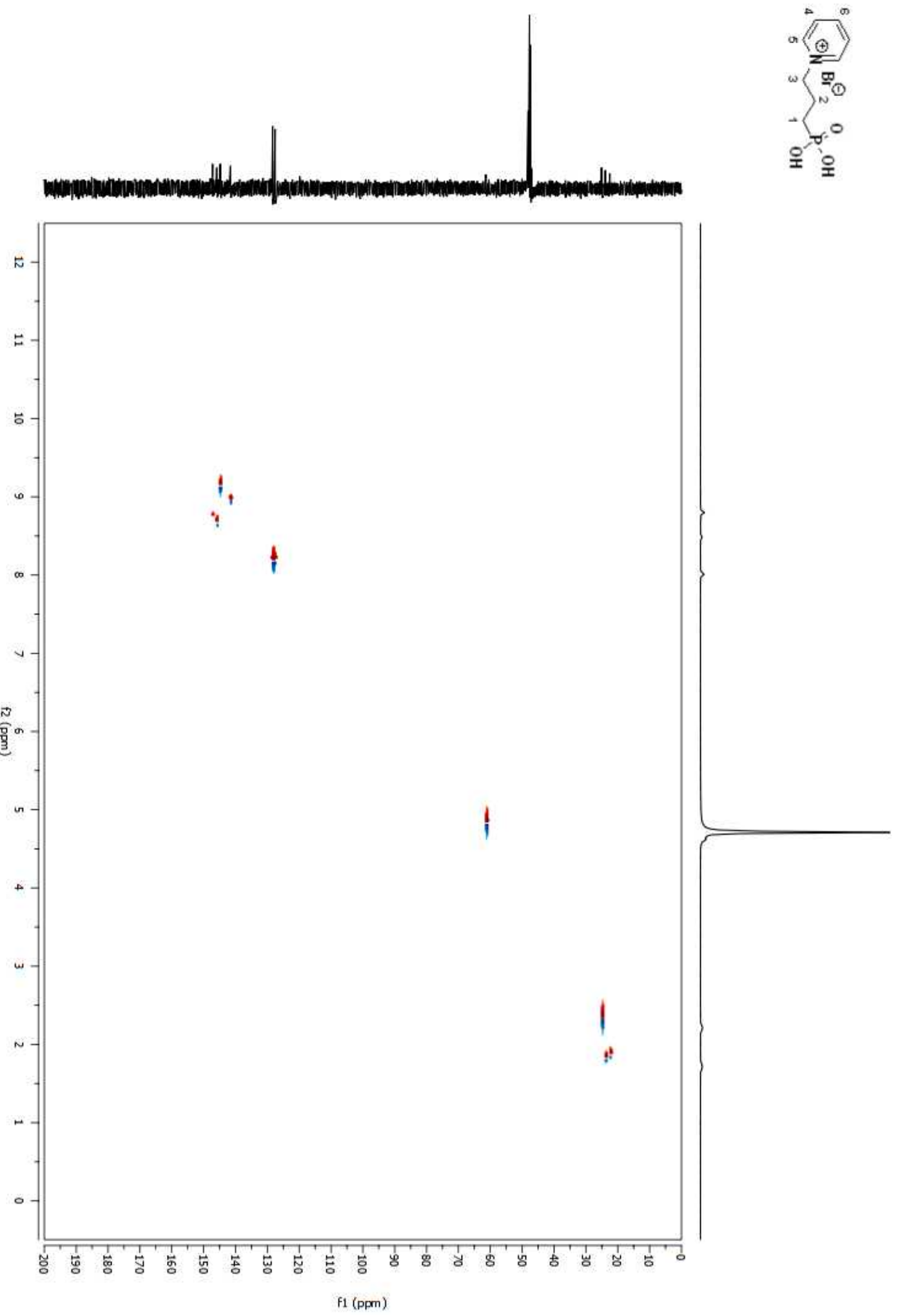

Figure A 107. HSQC NMR spectrum of compound 36 in $\mathrm{D}_{2} \mathrm{O}$ (Table 2.5, entry x) 


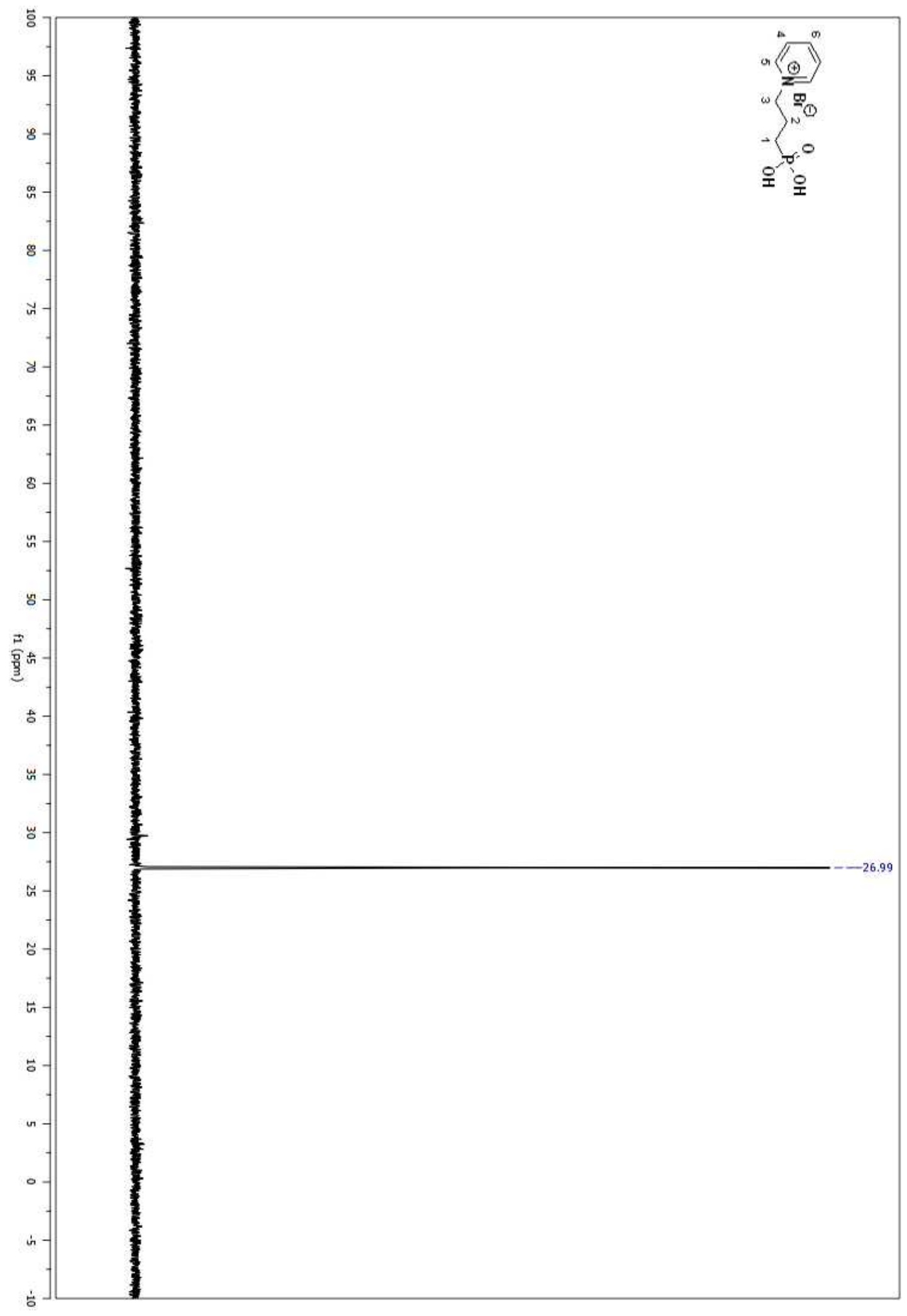

Figure A 108. ${ }^{31} \mathrm{P}$ NMR spectrum of compound 36 in $\mathrm{D}_{2} \mathrm{O}$ (Table 2.5, entry $\mathbf{x}$ ) 


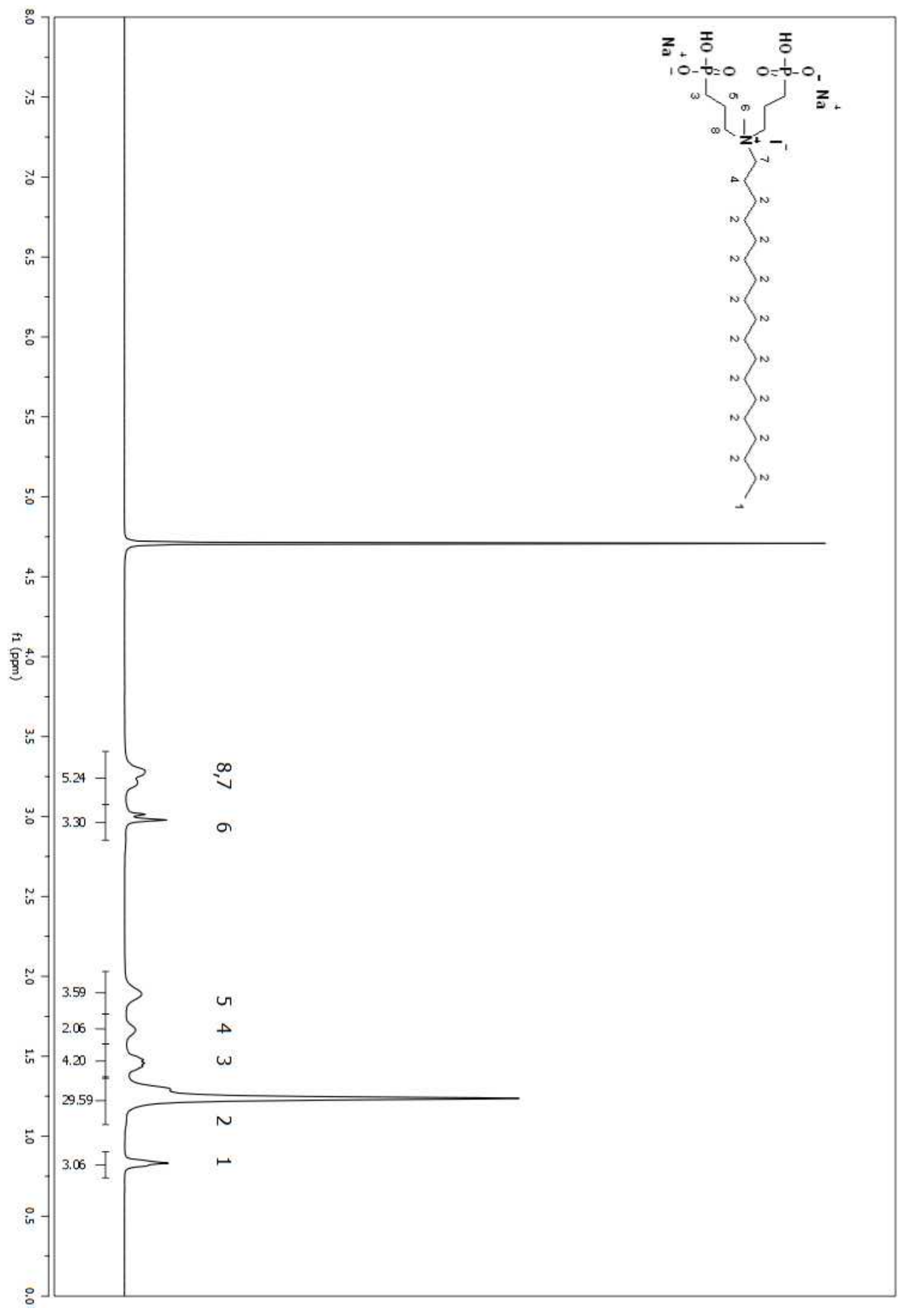

Figure A 109. ${ }^{1} \mathrm{H}$ NMR spectrum of compound 42 in $\mathrm{D}_{2} \mathrm{O}$. 


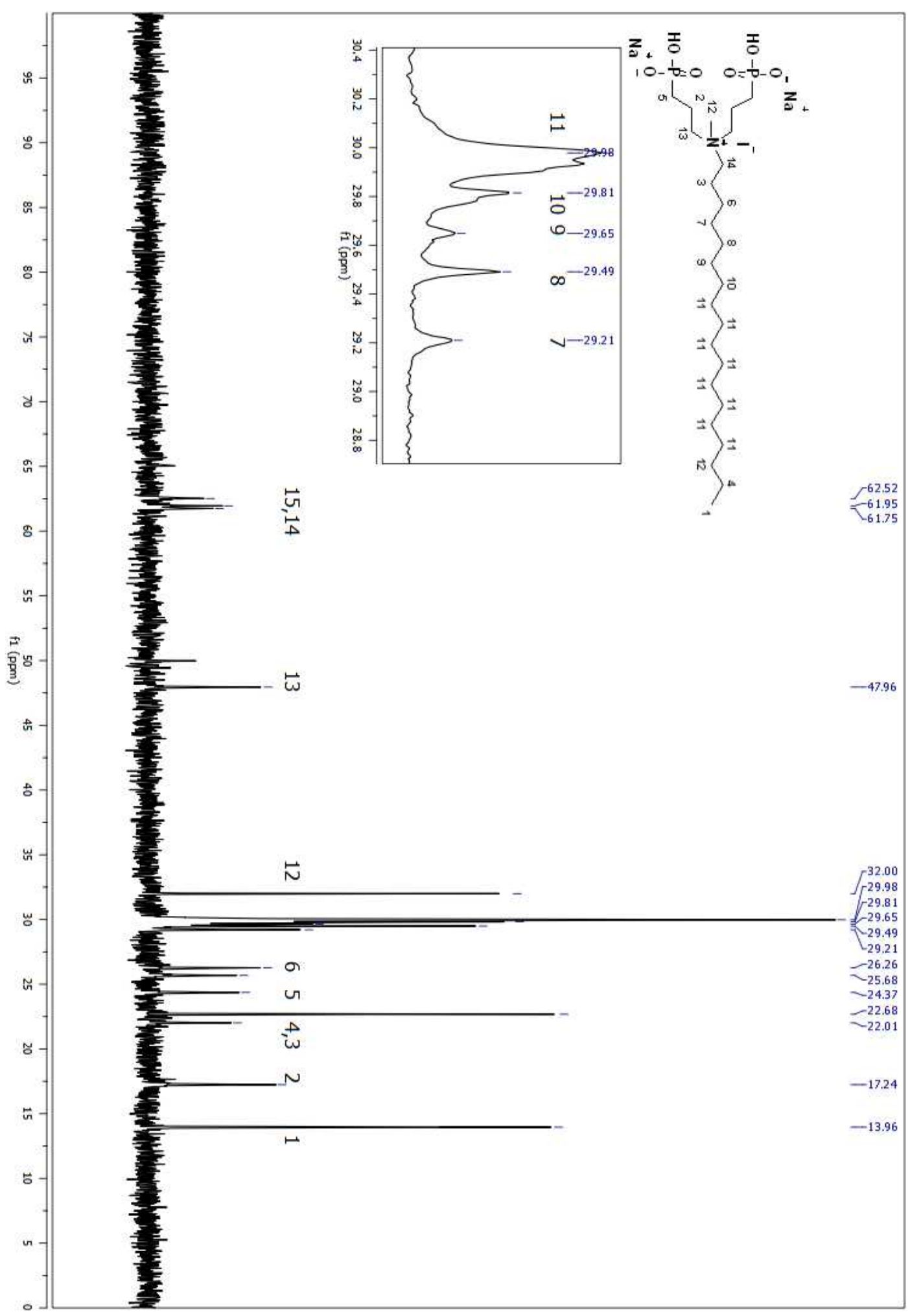

Figure A 110. ${ }^{13} \mathrm{C}$ NMR spectrum of compound 42 in $\mathrm{D}_{2} \mathrm{O}$. 


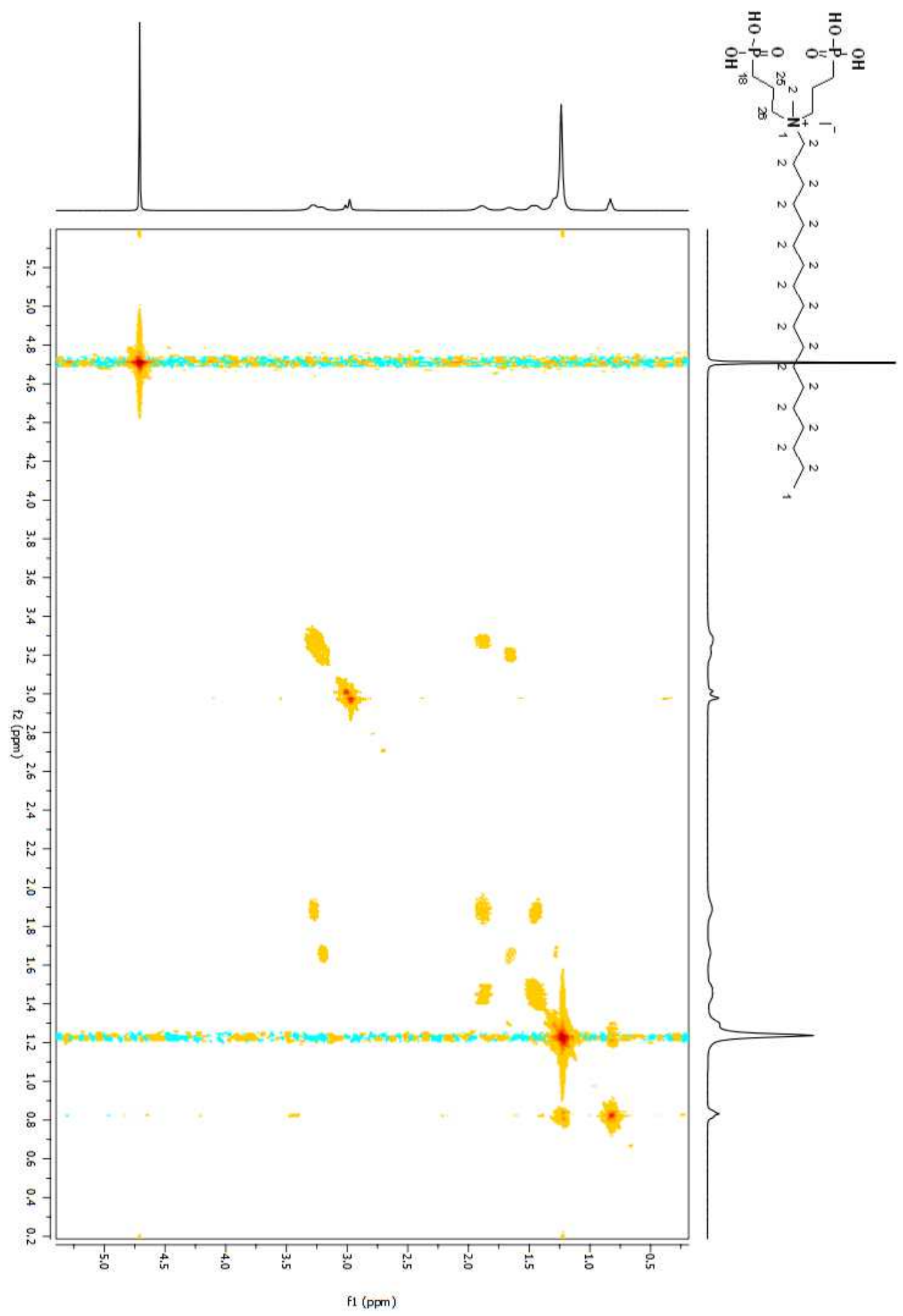

Figure A 111. COSY 2D NMR spectrum of compound 42 in $\mathrm{D}_{2} \mathrm{O}$. 


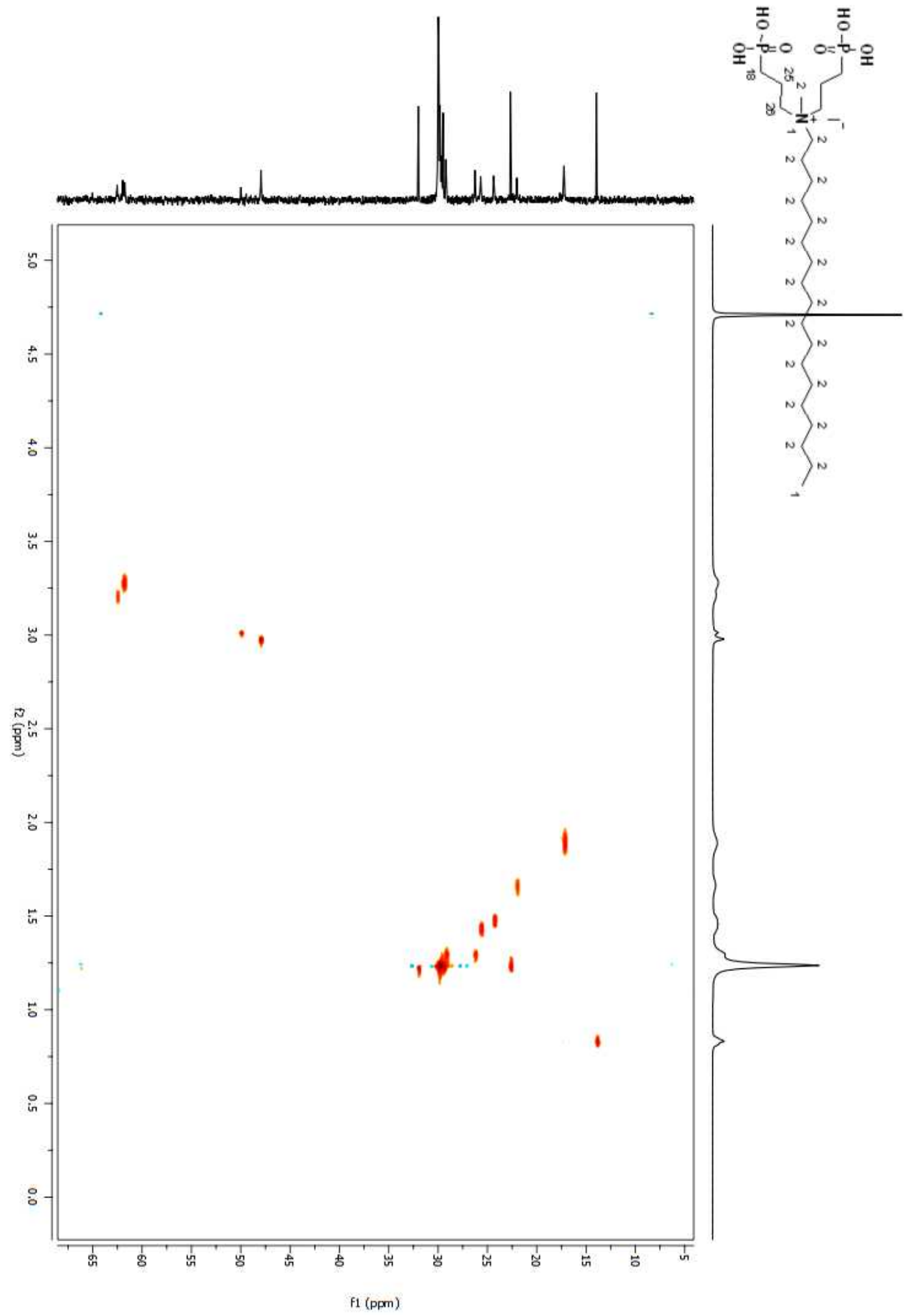

Figure A 112. HSQC 2D NMR spectrum of compound 42 in $\mathrm{D}_{2} \mathrm{O}$. 


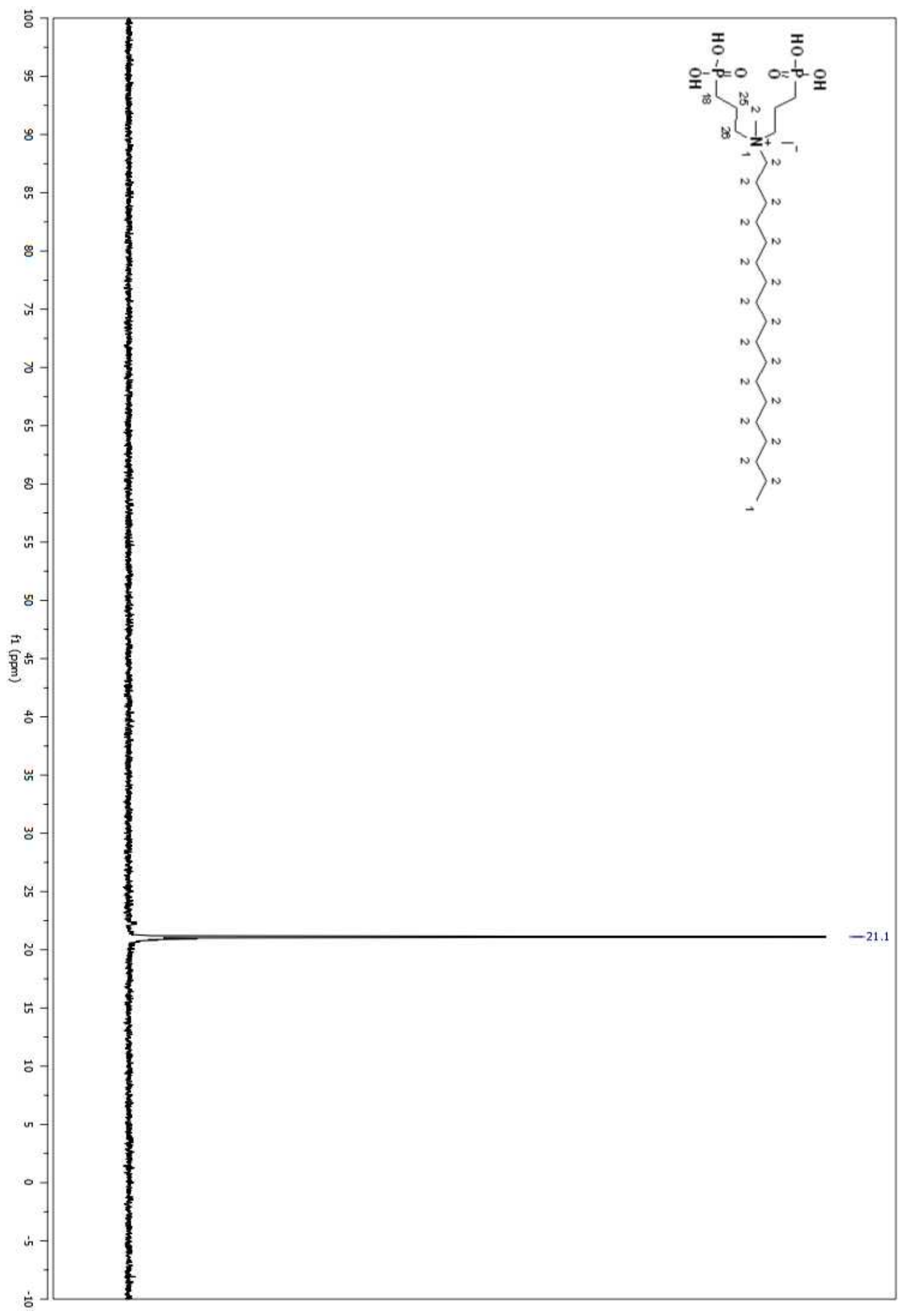

Figure A 113. ${ }^{31} \mathrm{P}$ NMR spectrum of compound 42 in $\mathrm{D}_{2} \mathrm{O}$. 


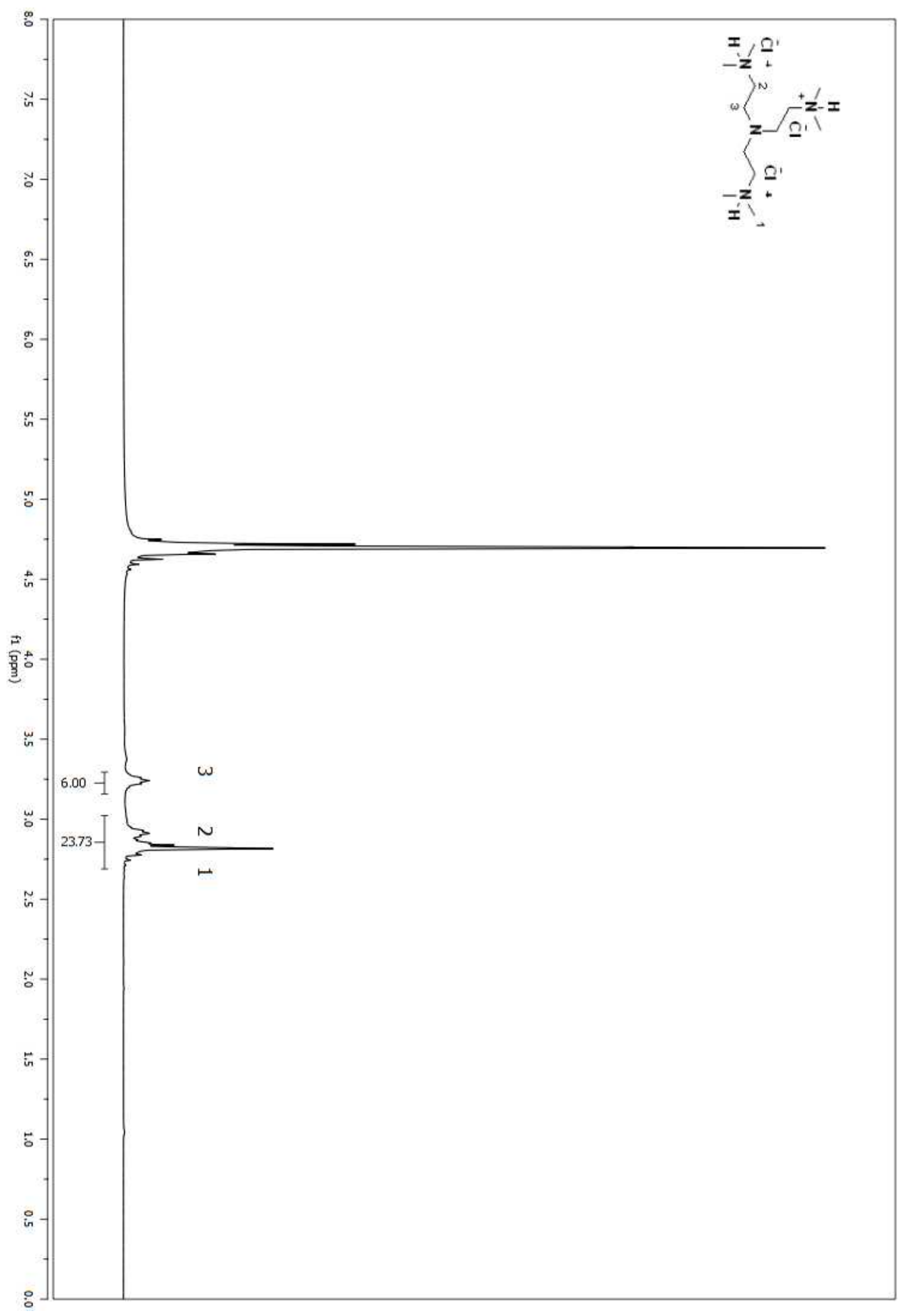

Figure A 114. ${ }^{1} \mathrm{H}$ NMR spectrum of compound 44 in $\mathrm{D}_{2} \mathrm{O}$. 


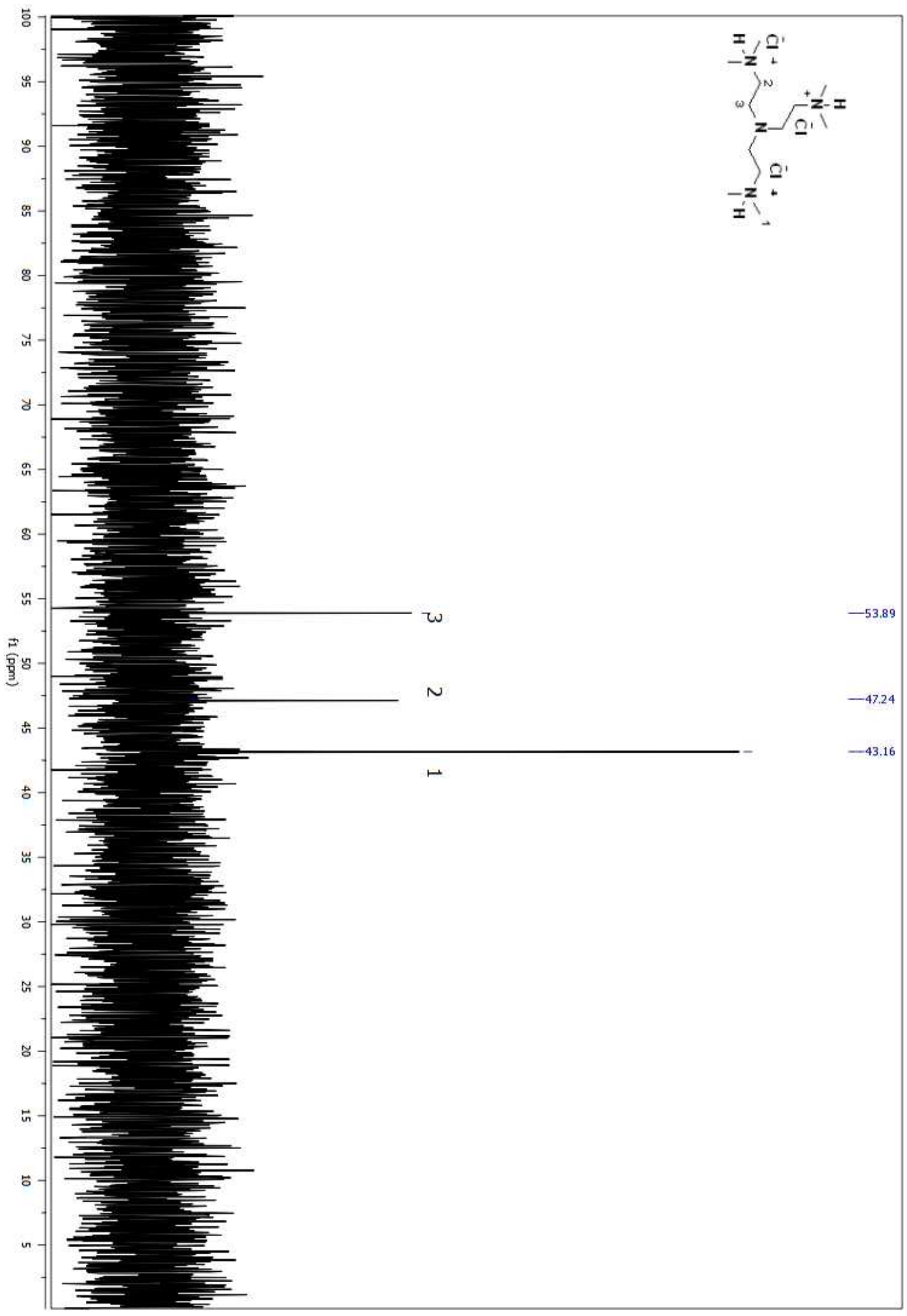

Figure A 115. ${ }^{13} \mathrm{C}$ NMR spectrum of compound 44 in $\mathrm{D}_{2} \mathrm{O}$. 


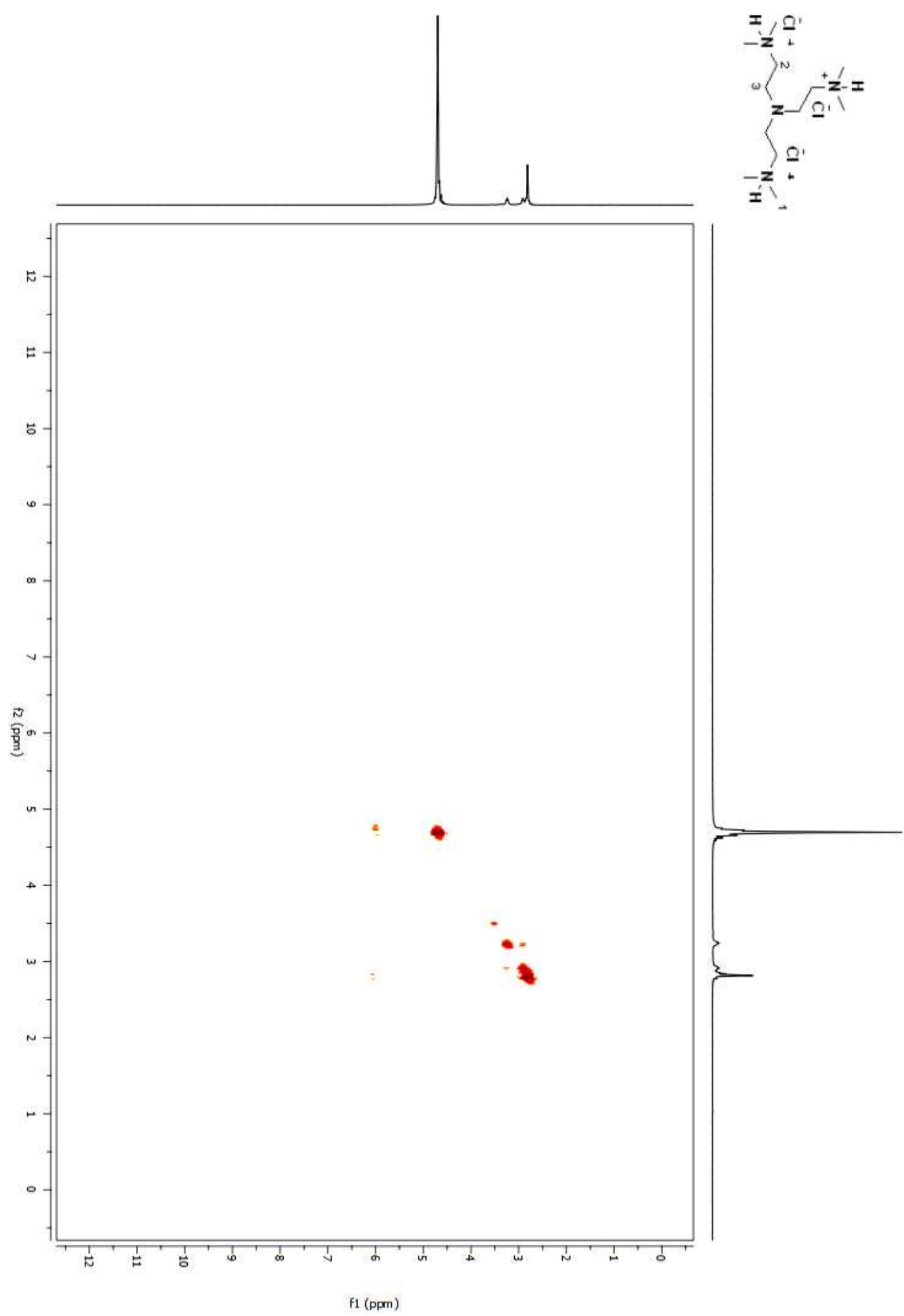

Figure A 116. COSY 2D NMR spectrum of compound 44 in $\mathrm{D}_{2} \mathrm{O}$. 


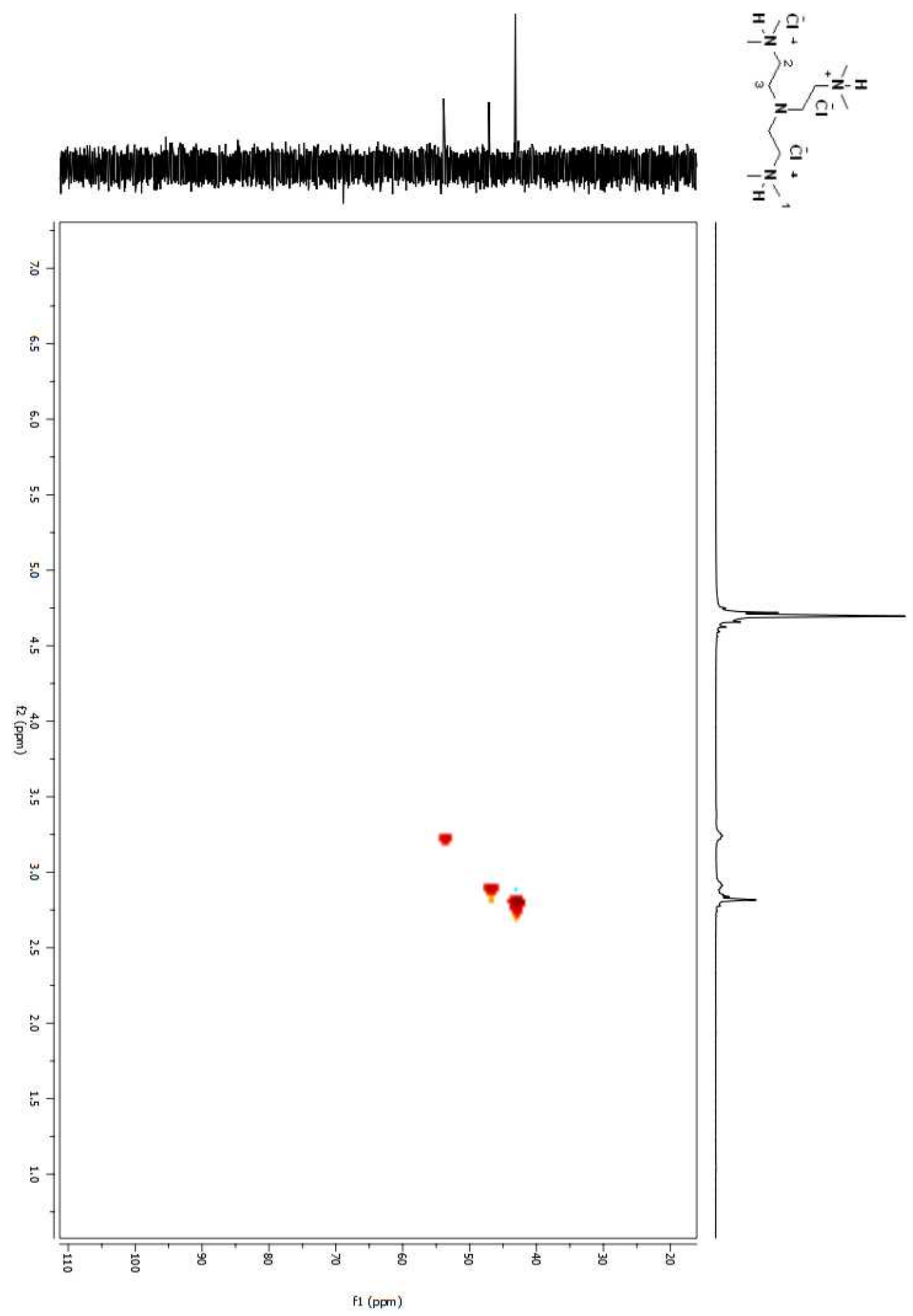

Figure A 117. HSQC 2D NMR spectrum of compound 44 in $\mathrm{D}_{2} \mathrm{O}$. 


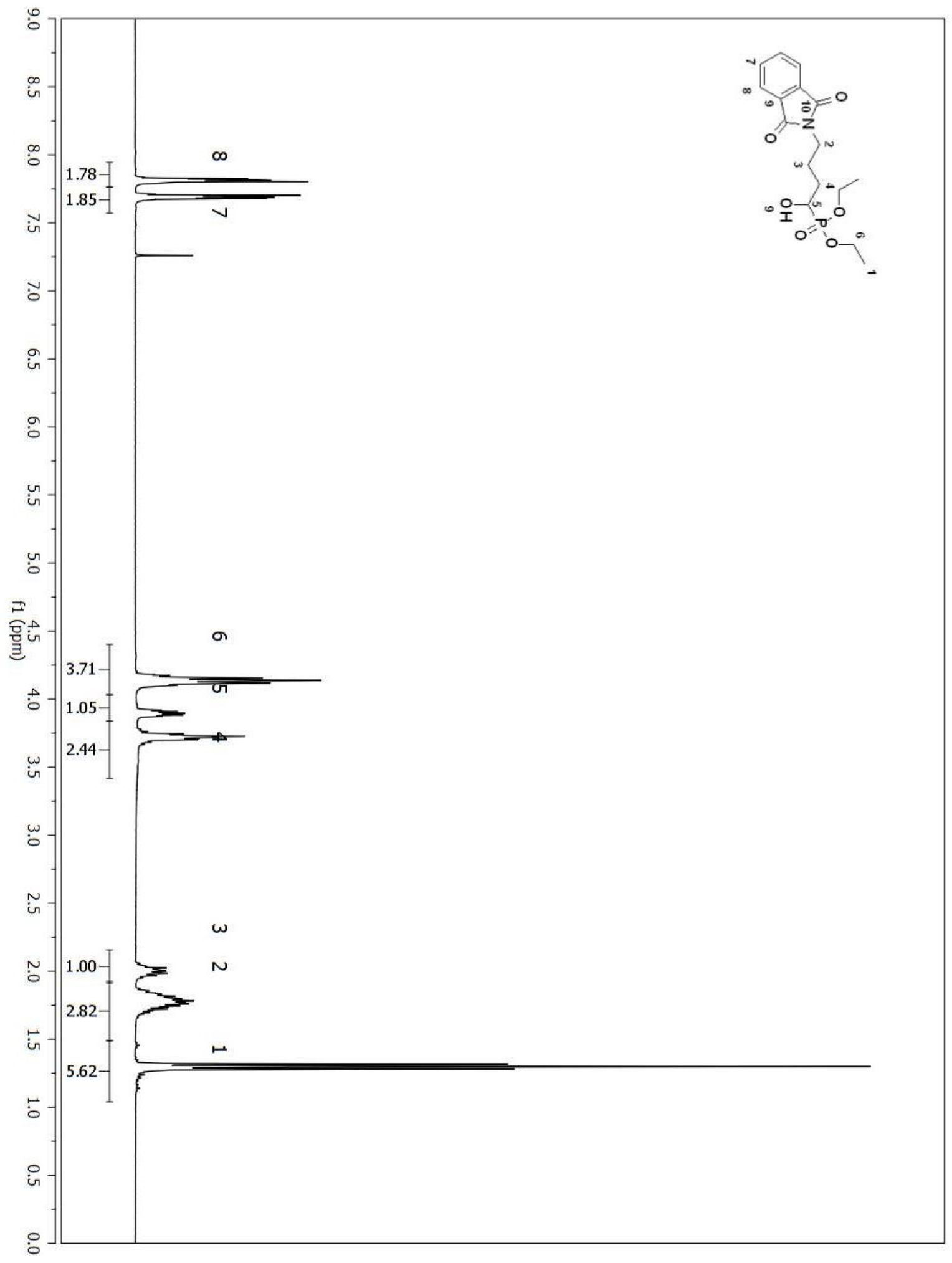

Figure A 118. ${ }^{1} \mathrm{H}$ NMR spectrum of compound (51) in $\mathrm{CDCl}_{3}$. 


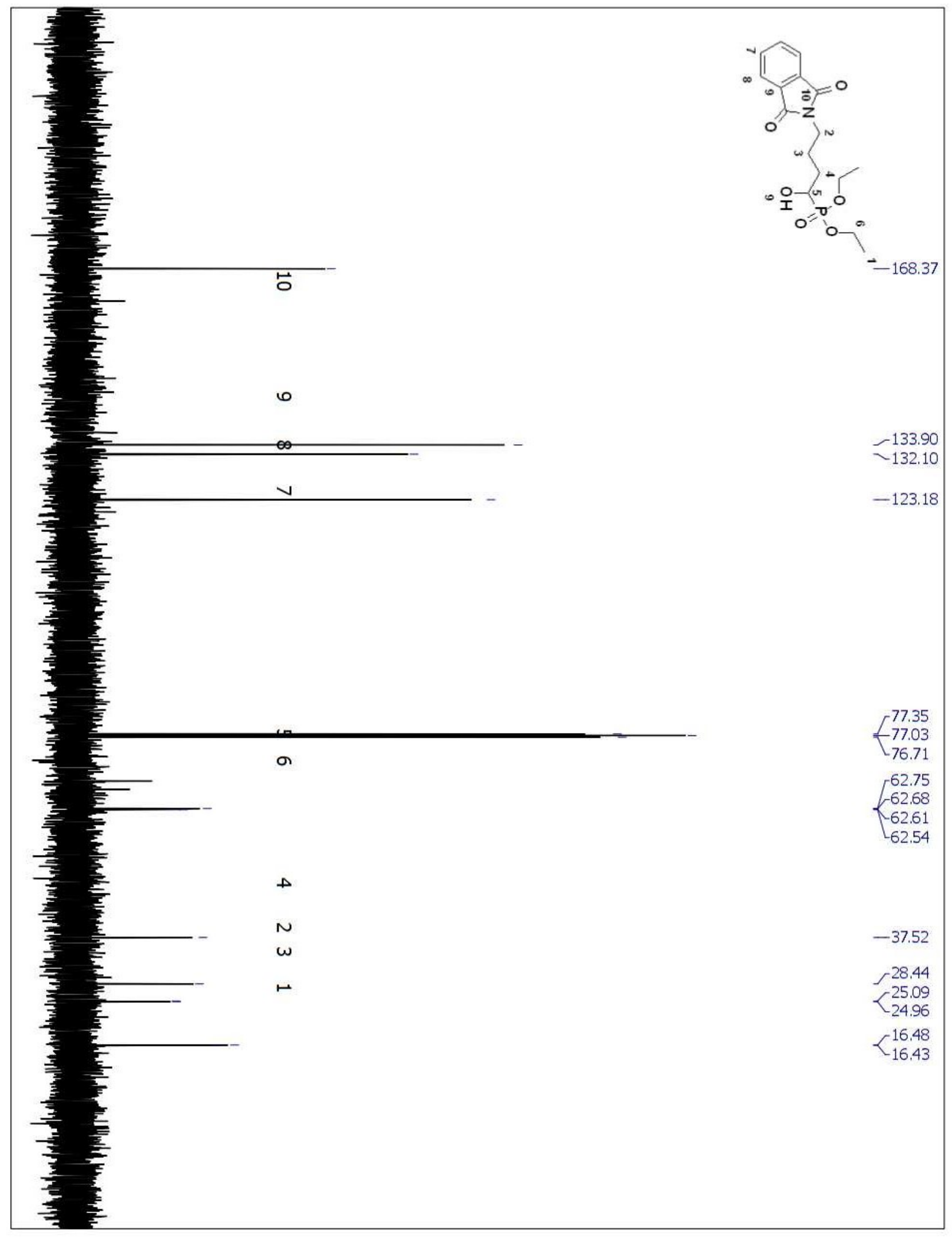

Figure A 119. ${ }^{13} \mathrm{C}$ NMR spectrum of compound (51) in $\mathrm{CDCl}_{3}$. 


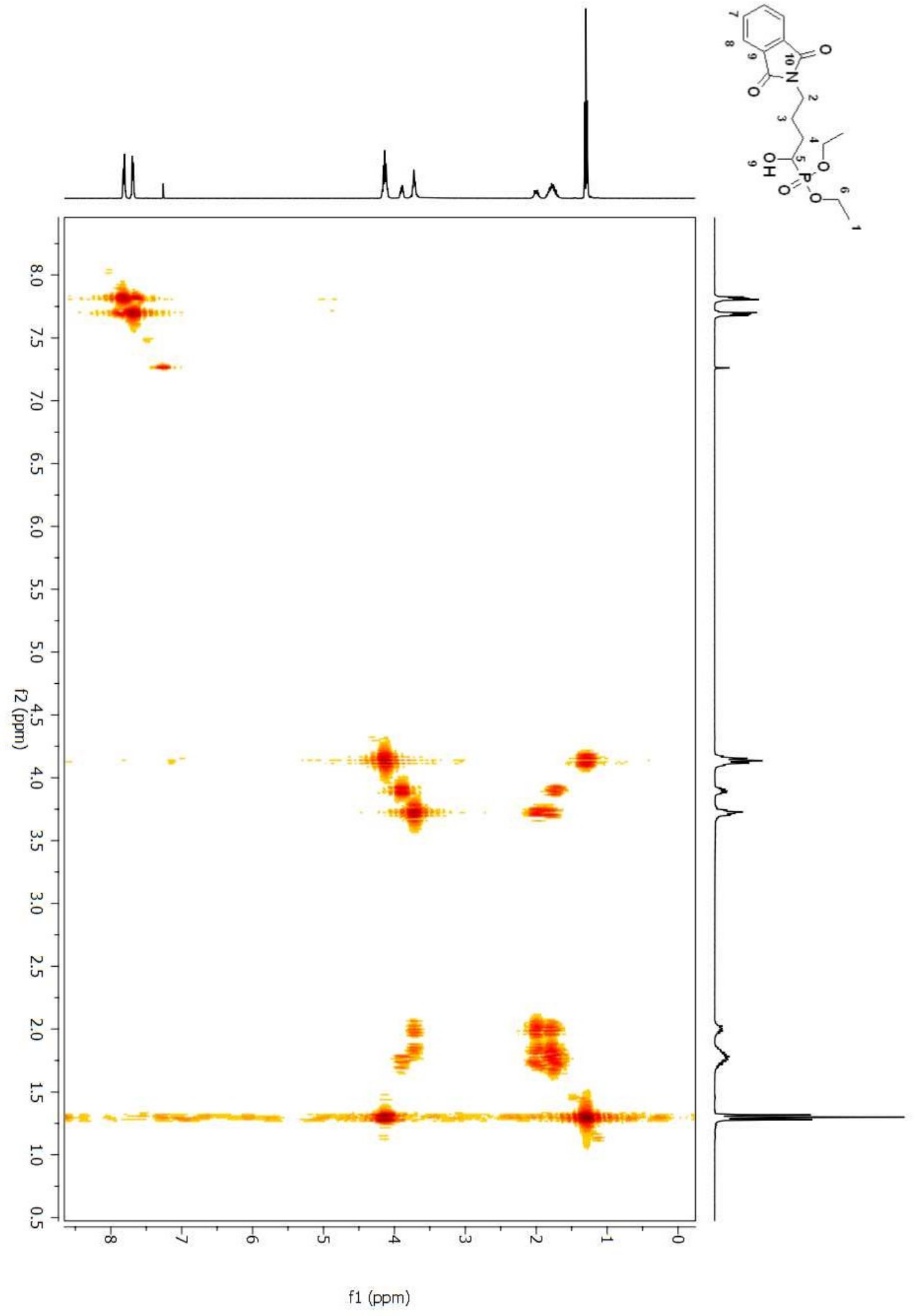

Figure A 120. COSY 2D NMR spectrum of compound (51) in $\mathrm{CDCl}_{3}$. 


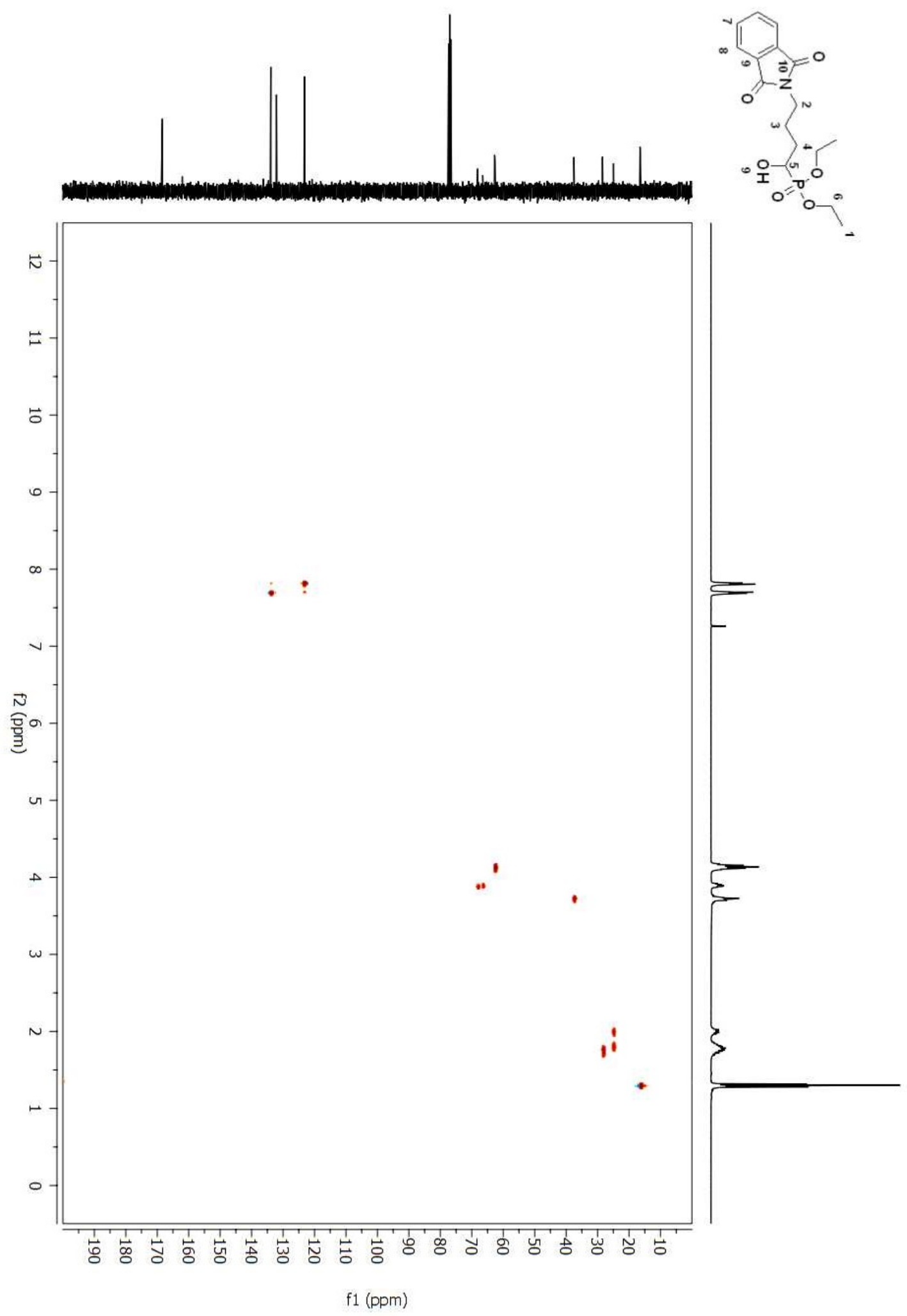

Figure A 121. HSQC 2D NMR spectrum of compound (51) in $\mathrm{CDCl}_{3}$. 


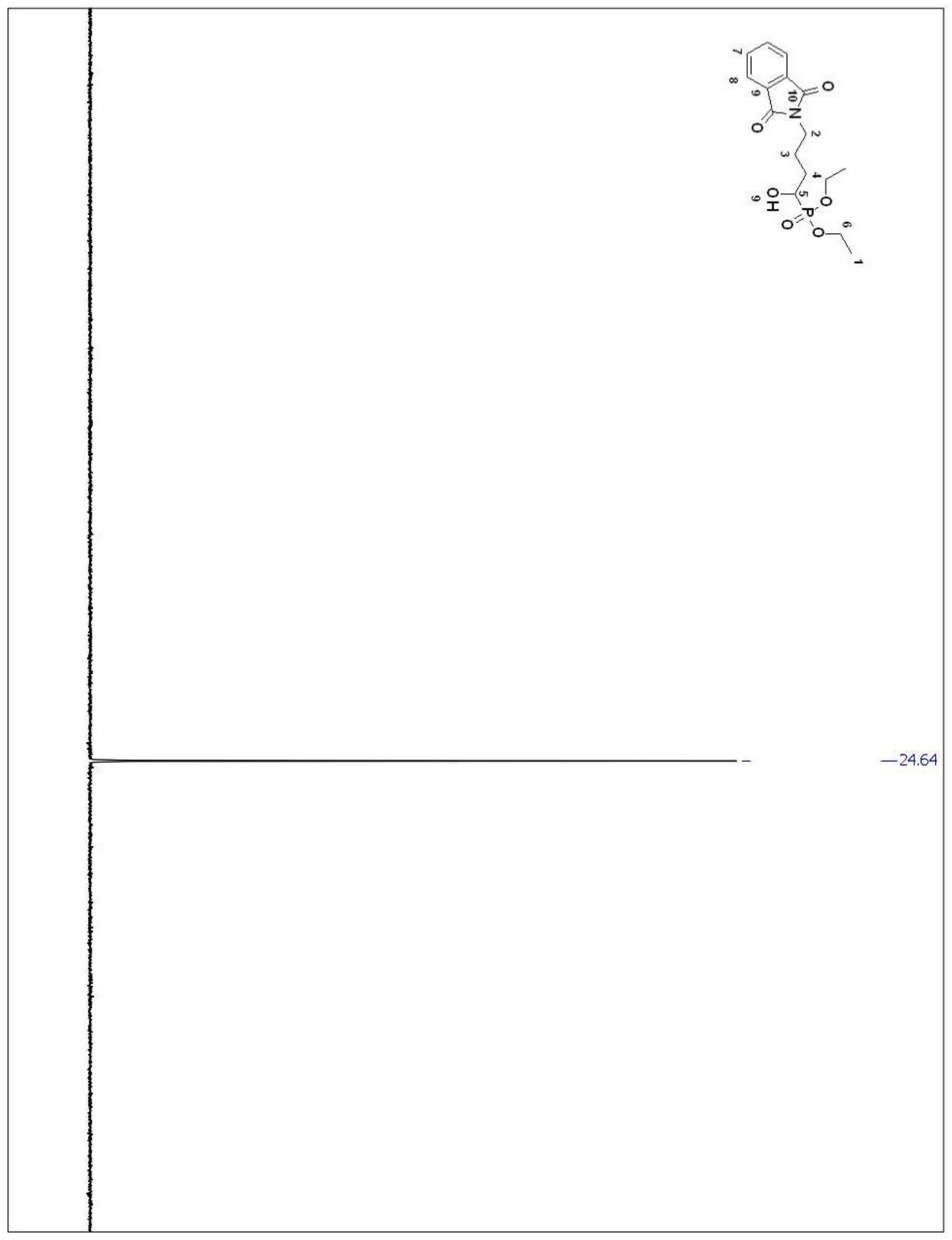

Figure A 122. ${ }^{31} \mathrm{P}$ NMR spectrum of compound (51) in $\mathrm{CDCl}_{3}$. 


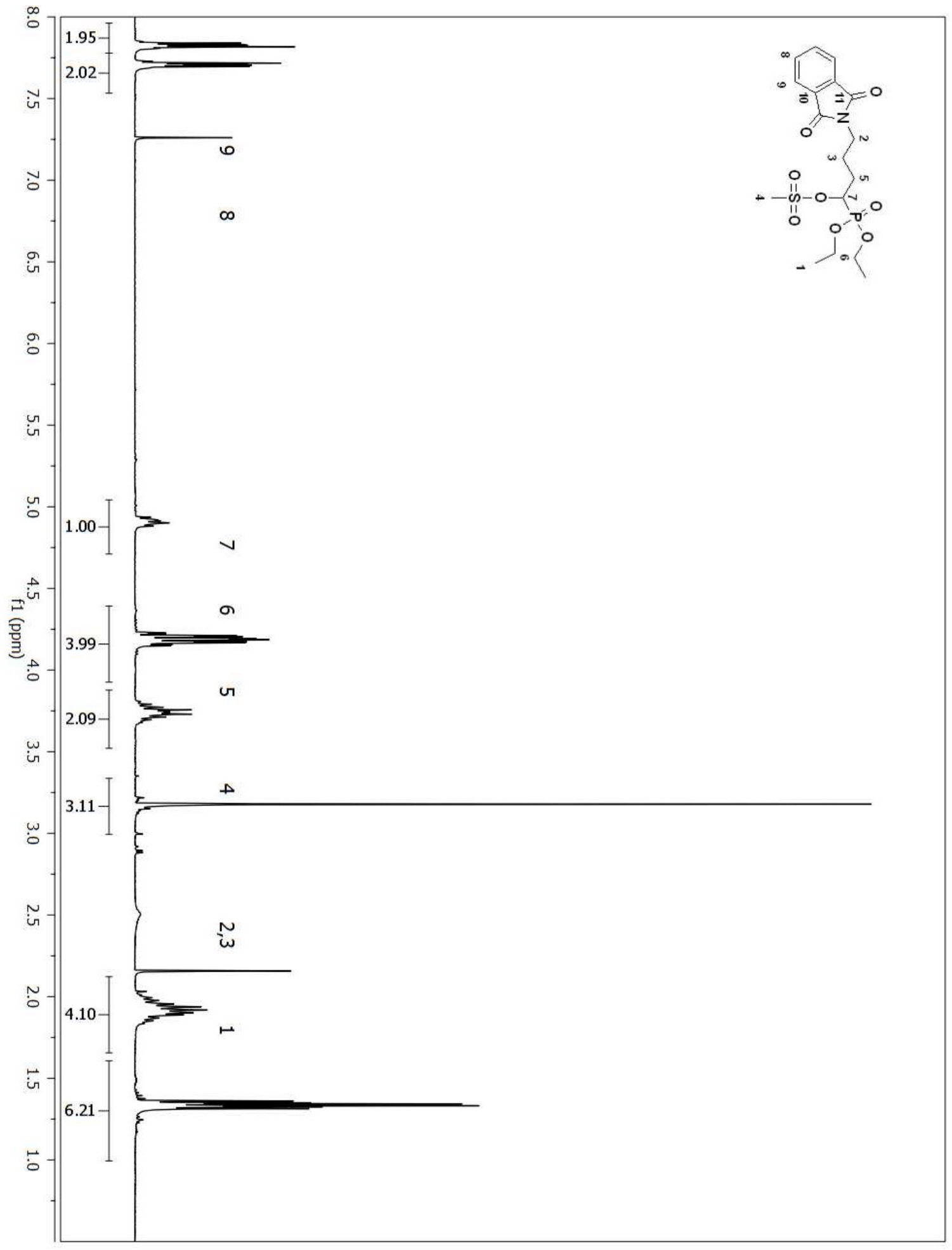

Figure A 123. ${ }^{1} \mathrm{H}$ NMR spectrum of compound (52) in $\mathrm{CDCl}_{3}$. 


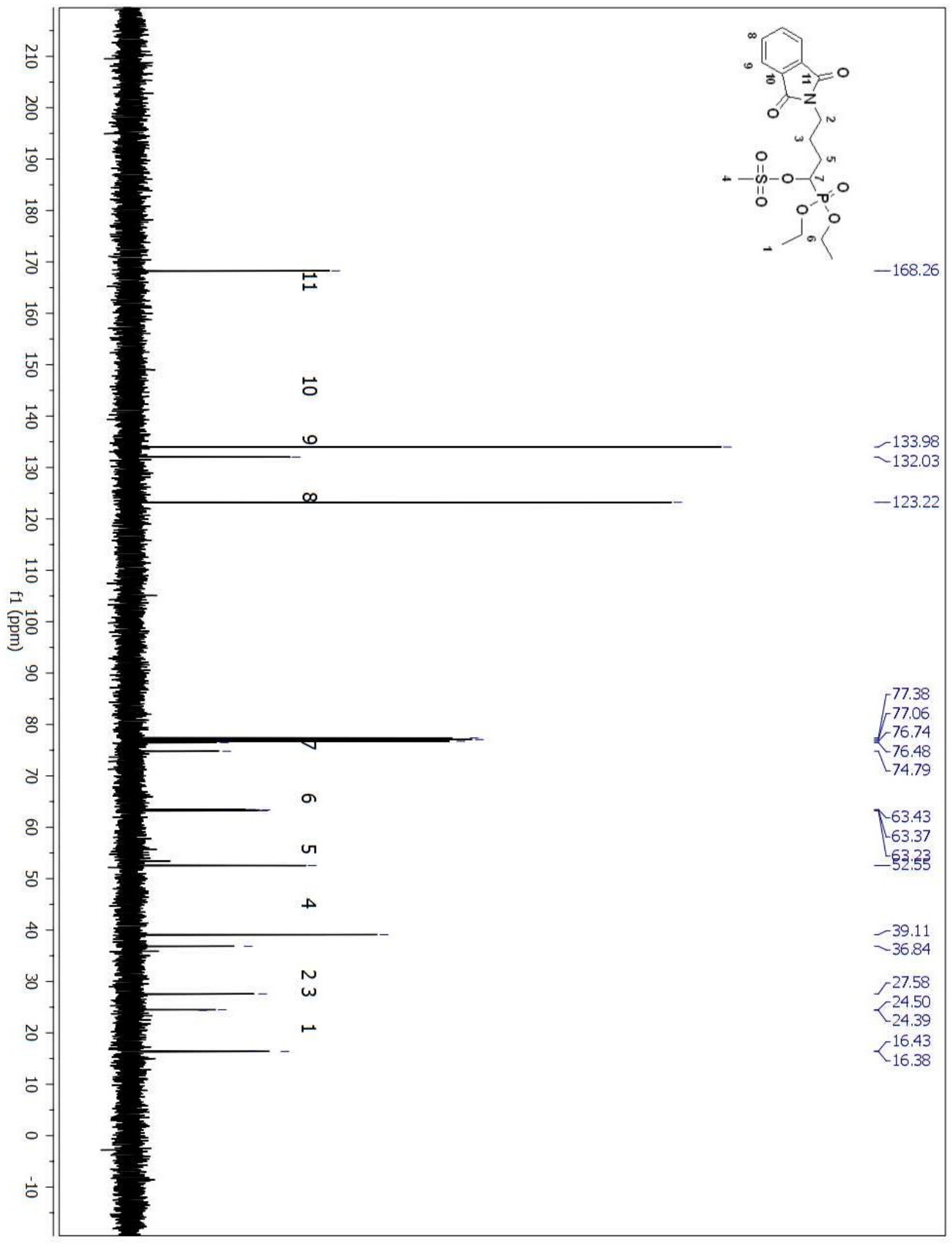

Figure A 124. ${ }^{13} \mathrm{C}$ NMR spectrum of compound (52) in $\mathrm{CDCl}_{3}$. 


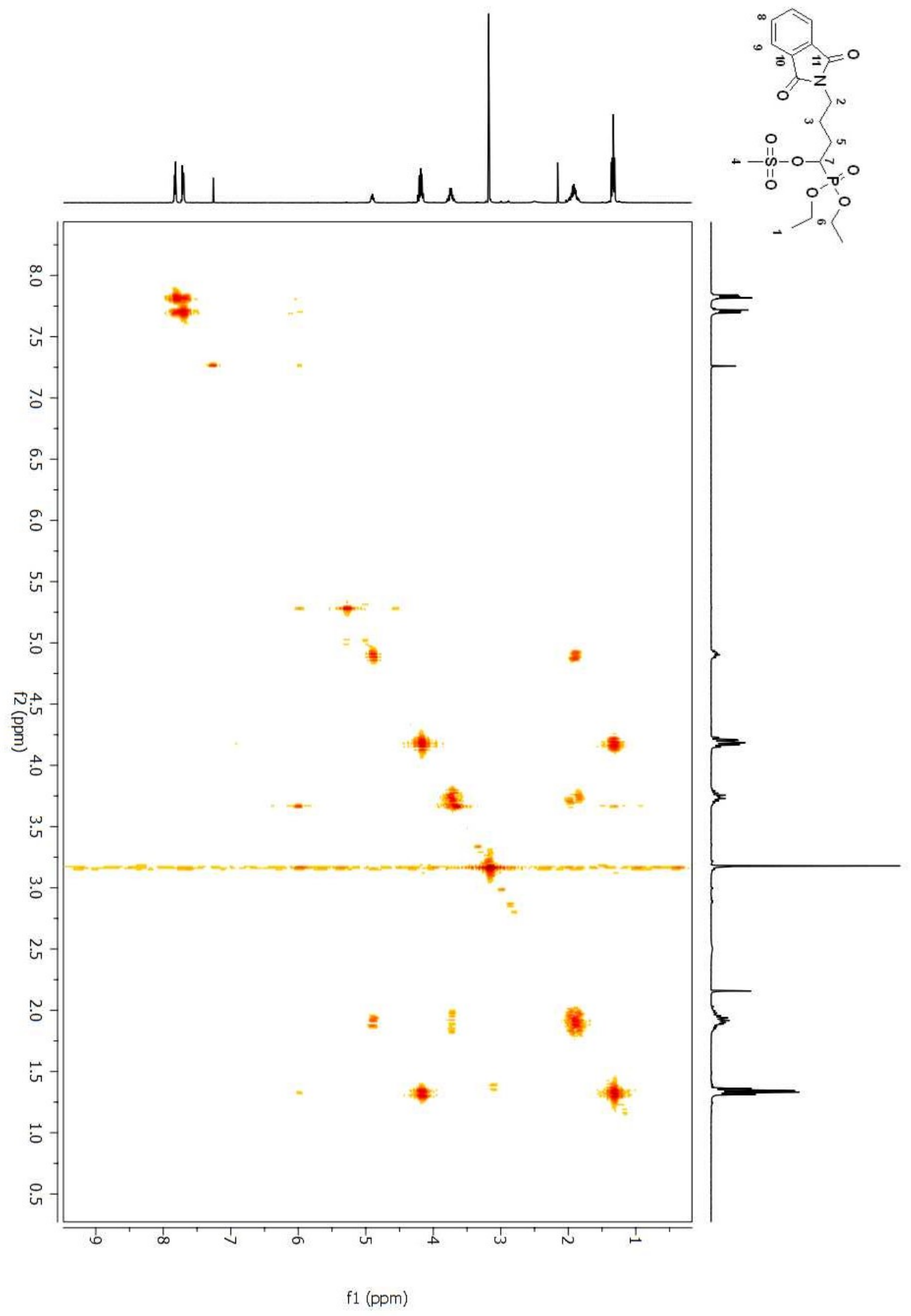

Figure A 125. COSY 2D NMR spectrum of compound (52) in $\mathrm{CDCl}_{3}$. 


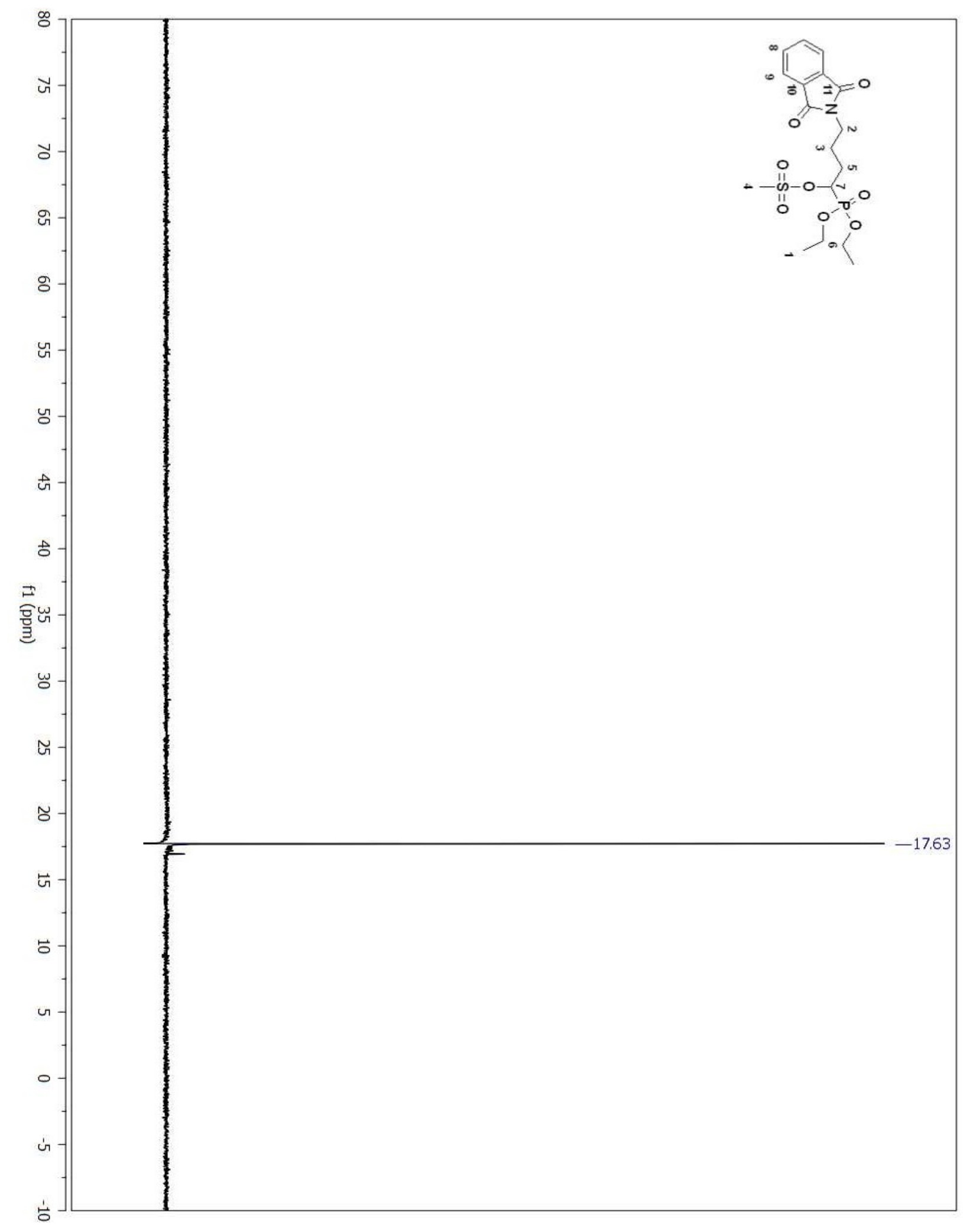

Figure A 126. ${ }^{31} \mathrm{P}$ NMR spectrum of compound (52) in $\mathrm{CDCl}_{3}$. 


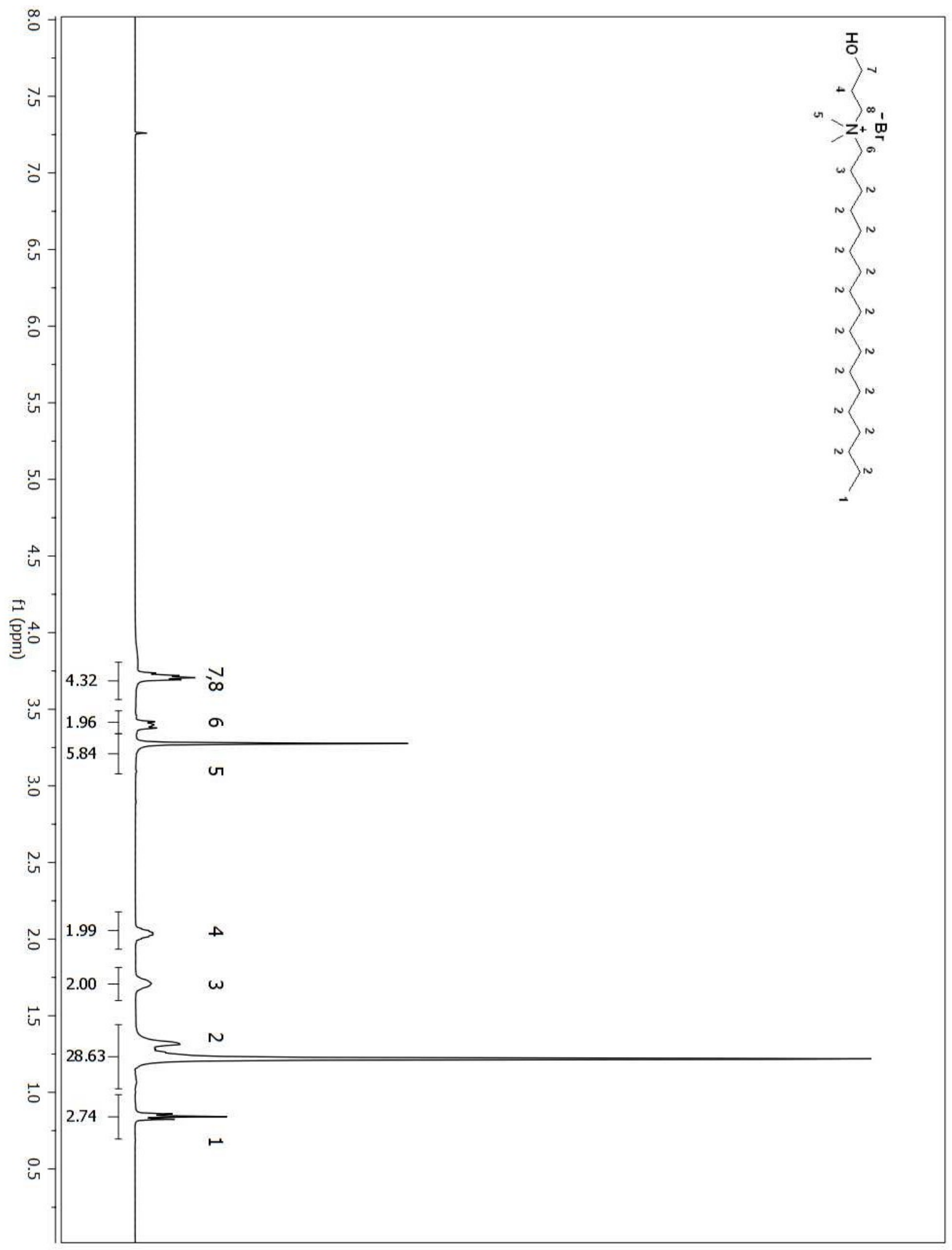

Figure A 127. ${ }^{1} \mathrm{H}$ NMR spectrum of compound (66) in $\mathrm{CDCl}_{3}$. 


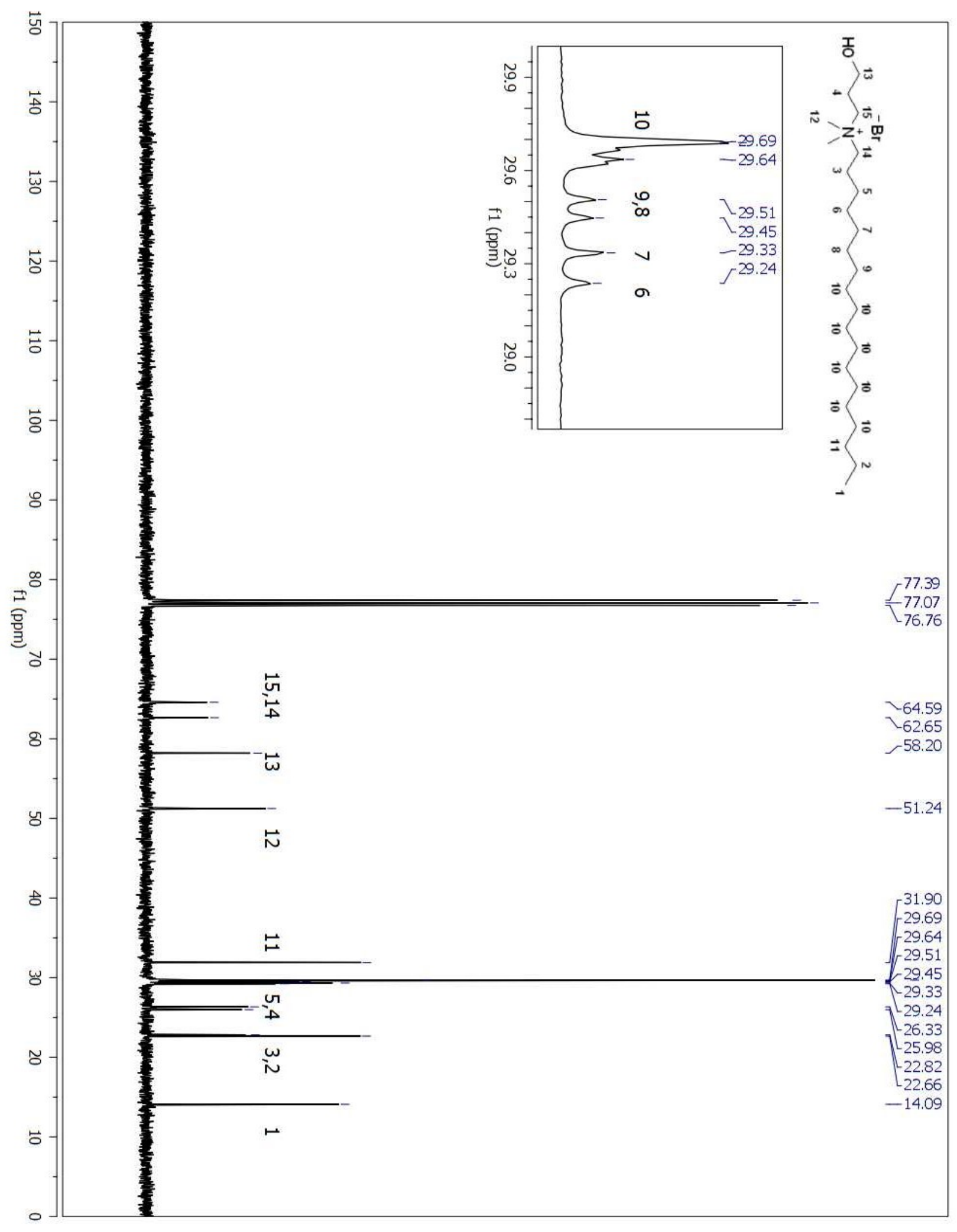

Figure A 128. ${ }^{13} \mathrm{C}$ NMR spectrum of compound (66) in $\mathrm{CDCl}_{3}$. 495 


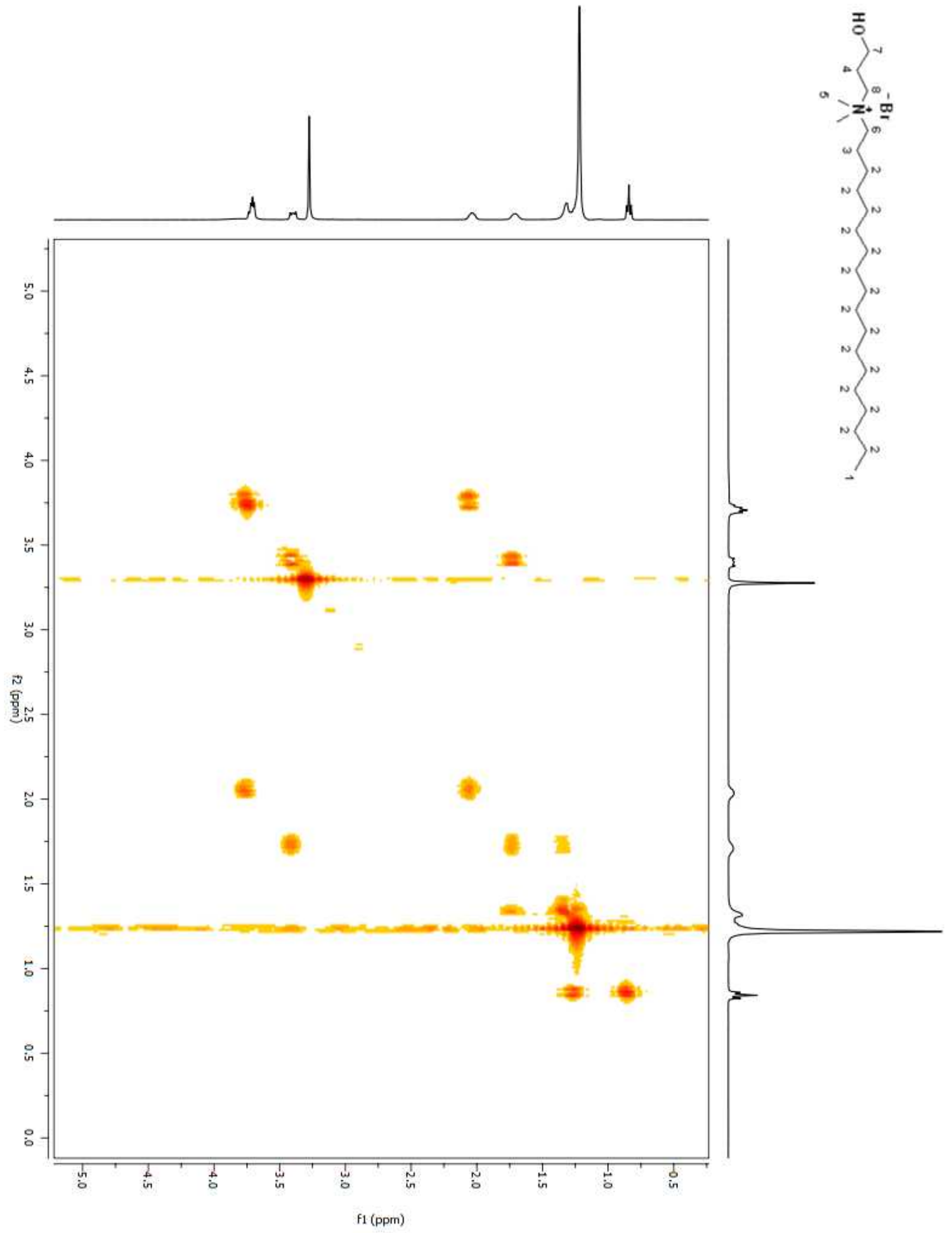

Figure A 129. COSY 2D NMR spectrum of compound (66) in $\mathrm{CDCl}_{3}$. 


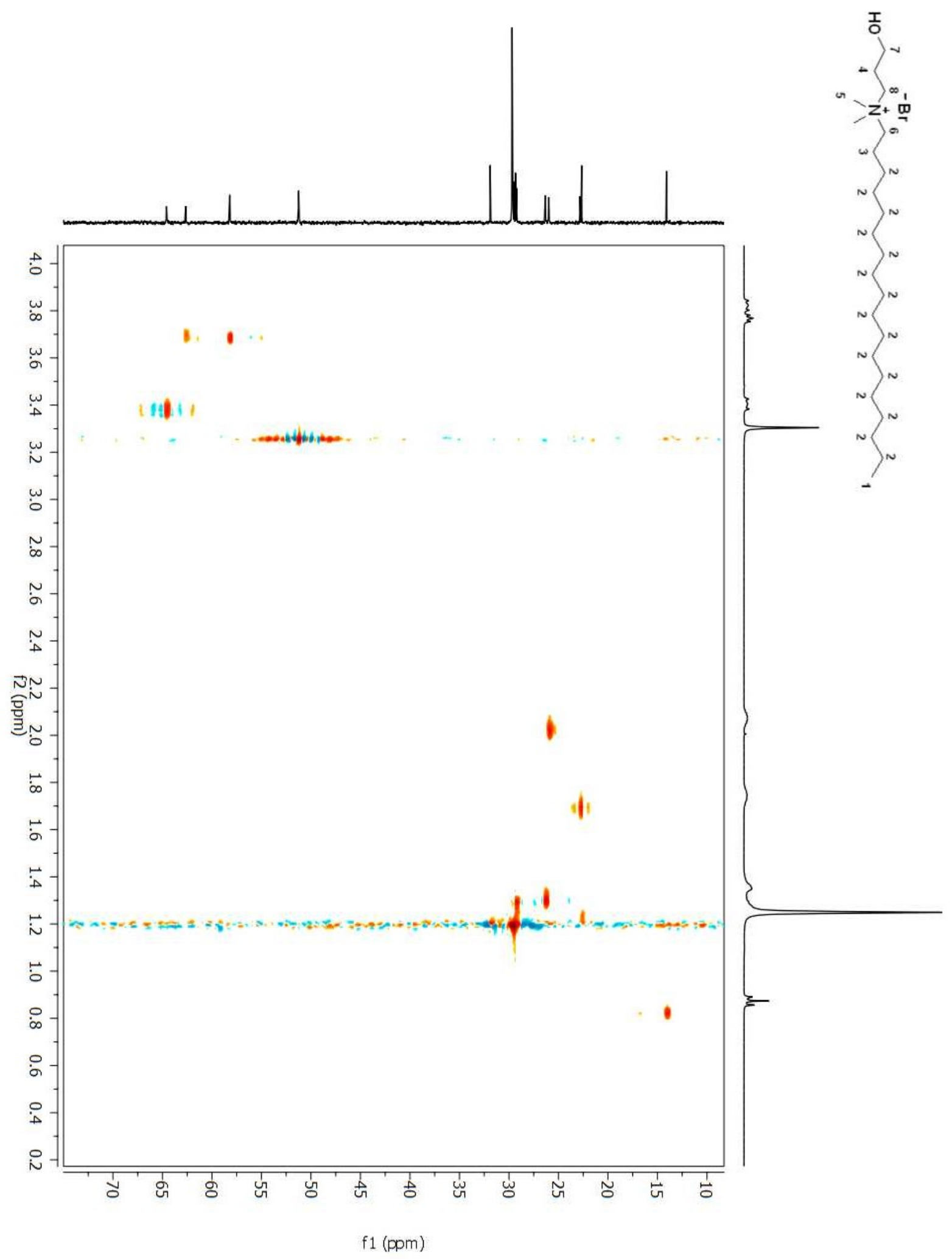

Figure A 130. HSQC 2D NMR spectrum of compound (66) in $\mathrm{CDCl}_{3}$. 


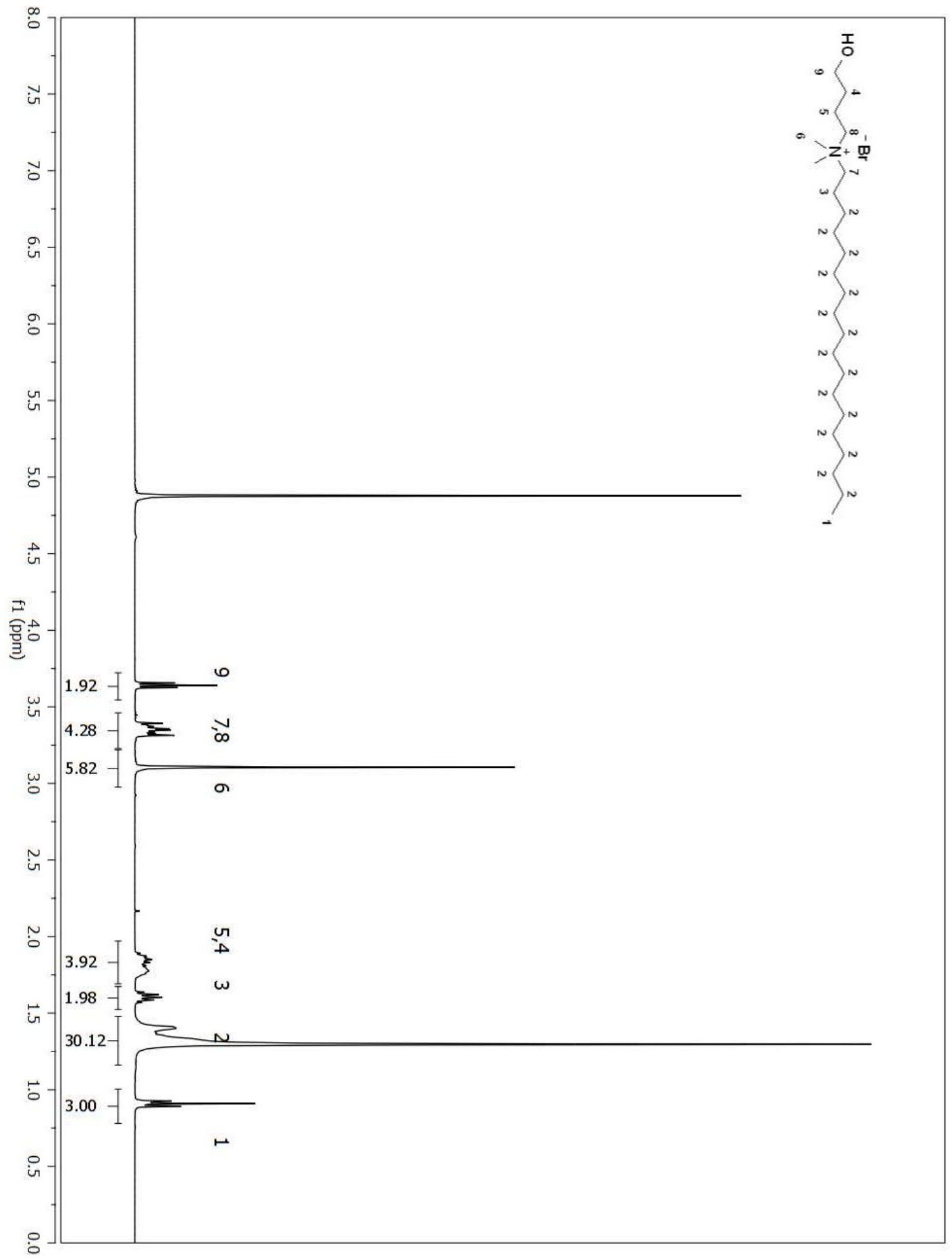

Figure A 131. ${ }^{1} \mathrm{H}$ NMR spectrum of compound (67) in MeOD. 


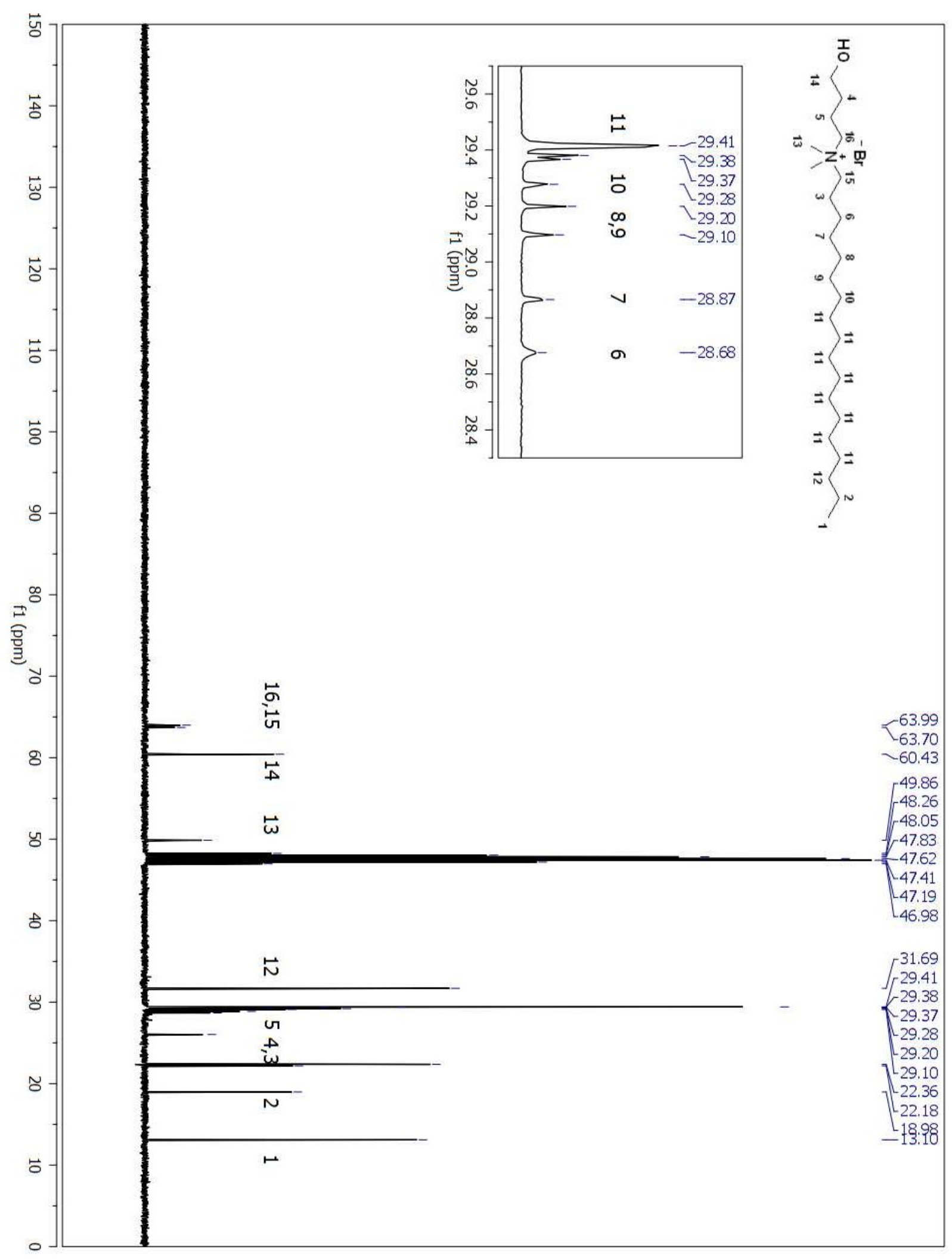

Figure A 132. ${ }^{13} \mathrm{C}$ NMR spectrum of compound (67) in MeOD. 


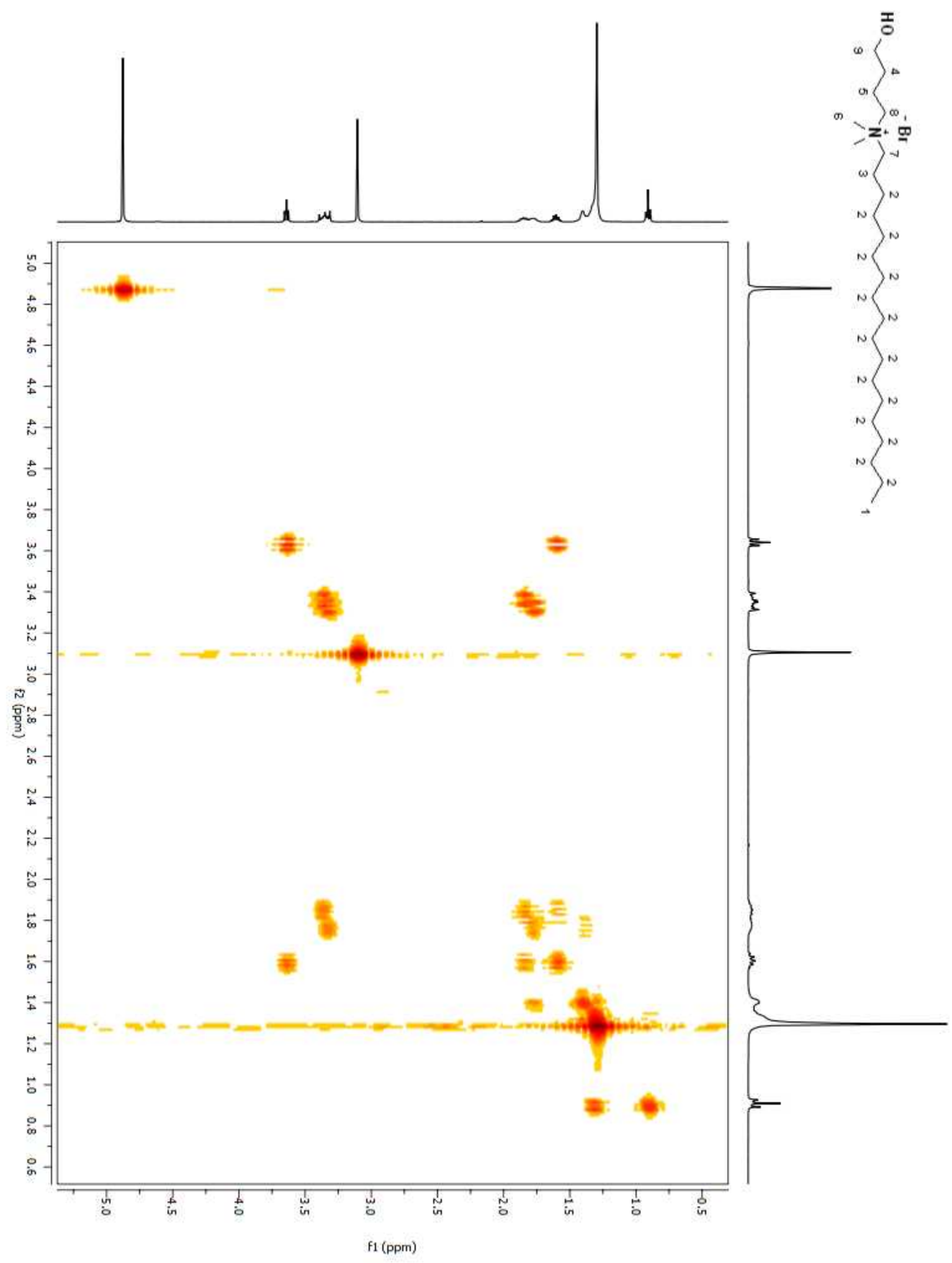

Figure A 133. COSY 2D NMR spectrum of compound (67) in MeOD. 


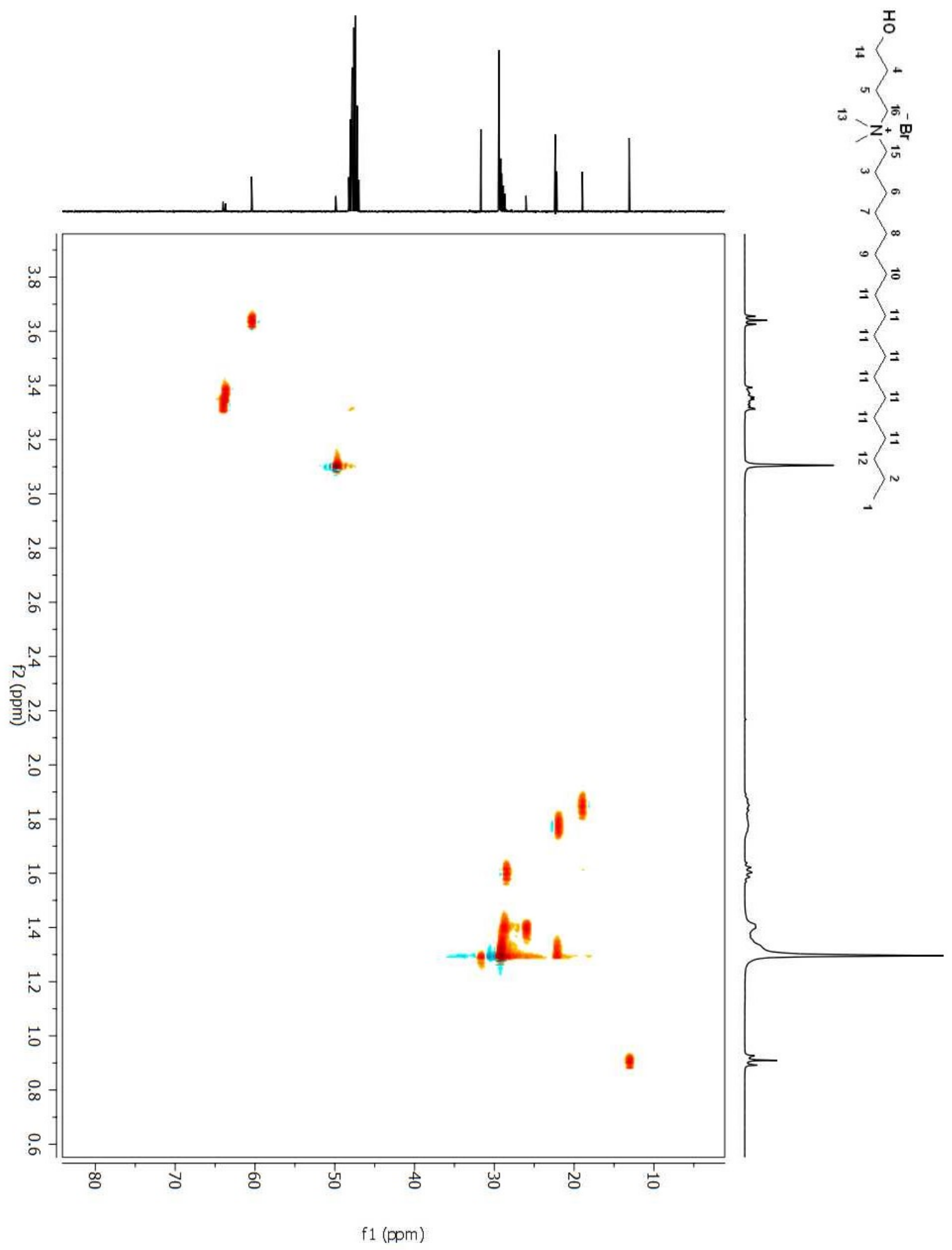

Figure A 134. HSQC 2D NMR spectrum of compound (67) in MeOD. 


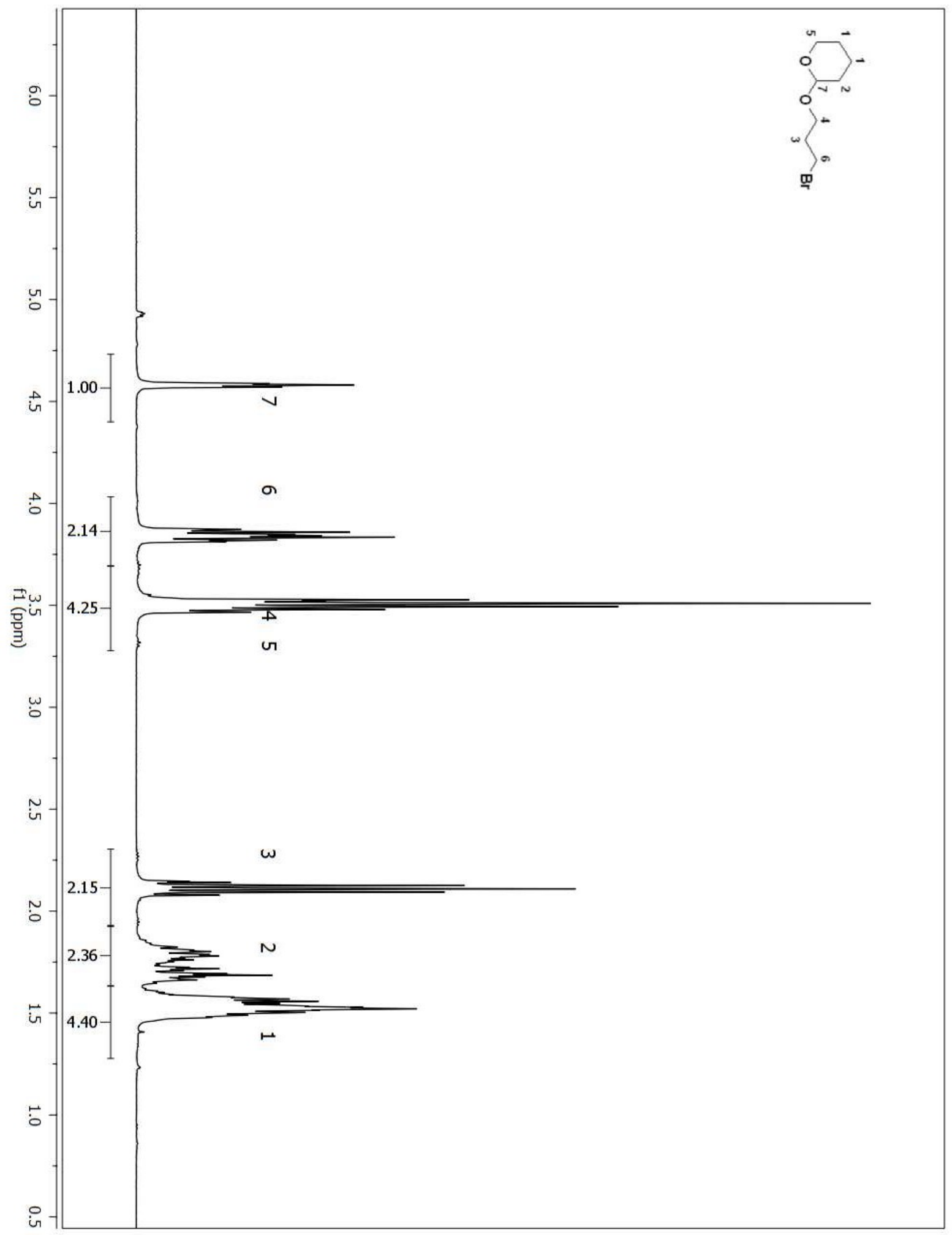

Figure A 135. ${ }^{1} \mathrm{H}$ NMR spectrum of compound (9) in $\mathrm{CDCl}_{3}$. 


$$
E
$$




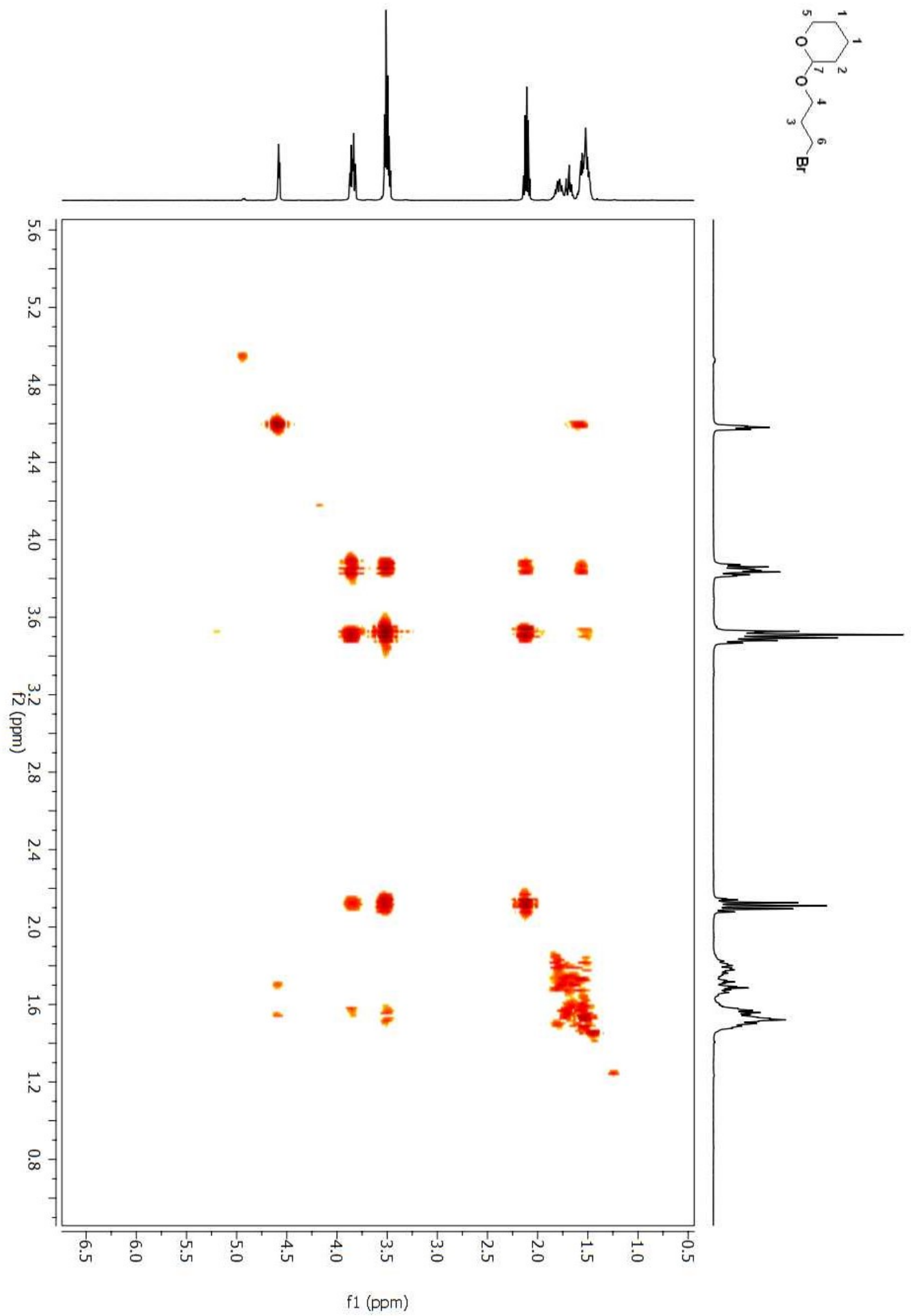

Figure A 137. COSY 2D NMR spectrum of compound (9) in $\mathrm{CDCl}_{3}$. 


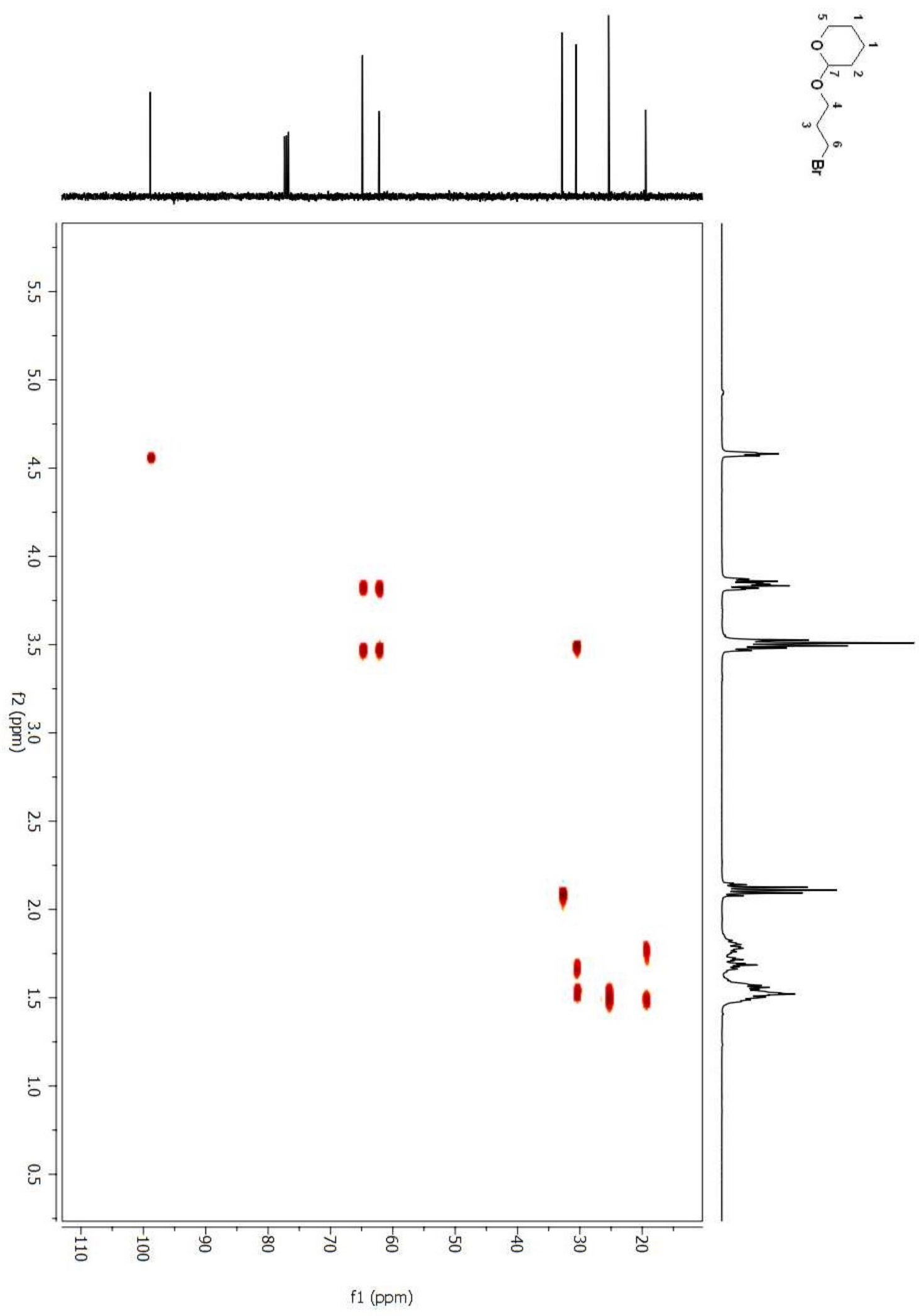

Figure A 138. HSQC 2D NMR spectrum of compound (9) in $\mathrm{CDCl}_{3}$. 505 


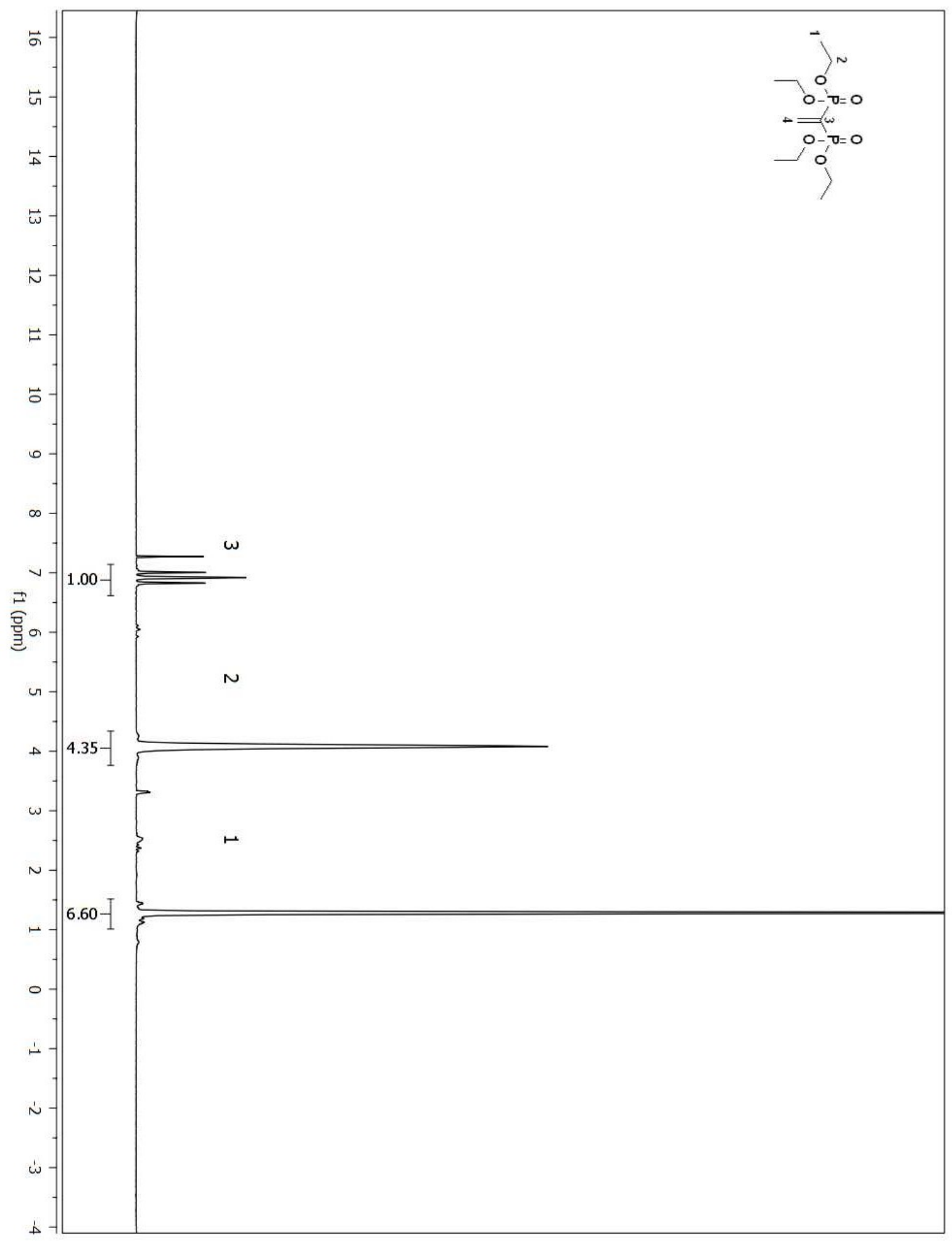

Figure A 139. ${ }^{1} \mathrm{H}$ NMR spectrum of compound (68) in $\mathrm{CDCl}_{3}$. 


$$
1
$$




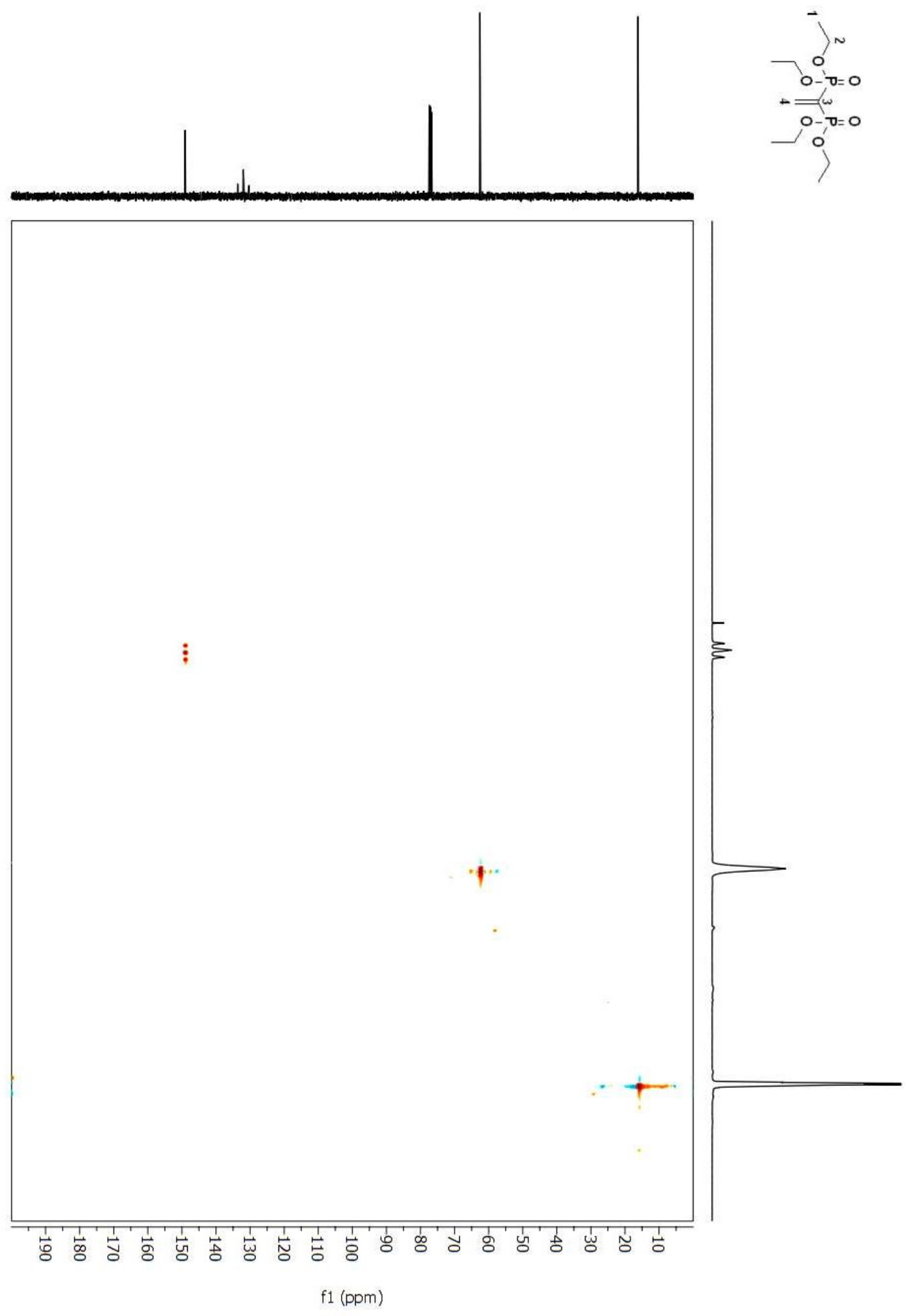

Figure A 141. HSQC 2D NMR spectrum of compound (68) in $\mathrm{CDCl}_{3}$. 


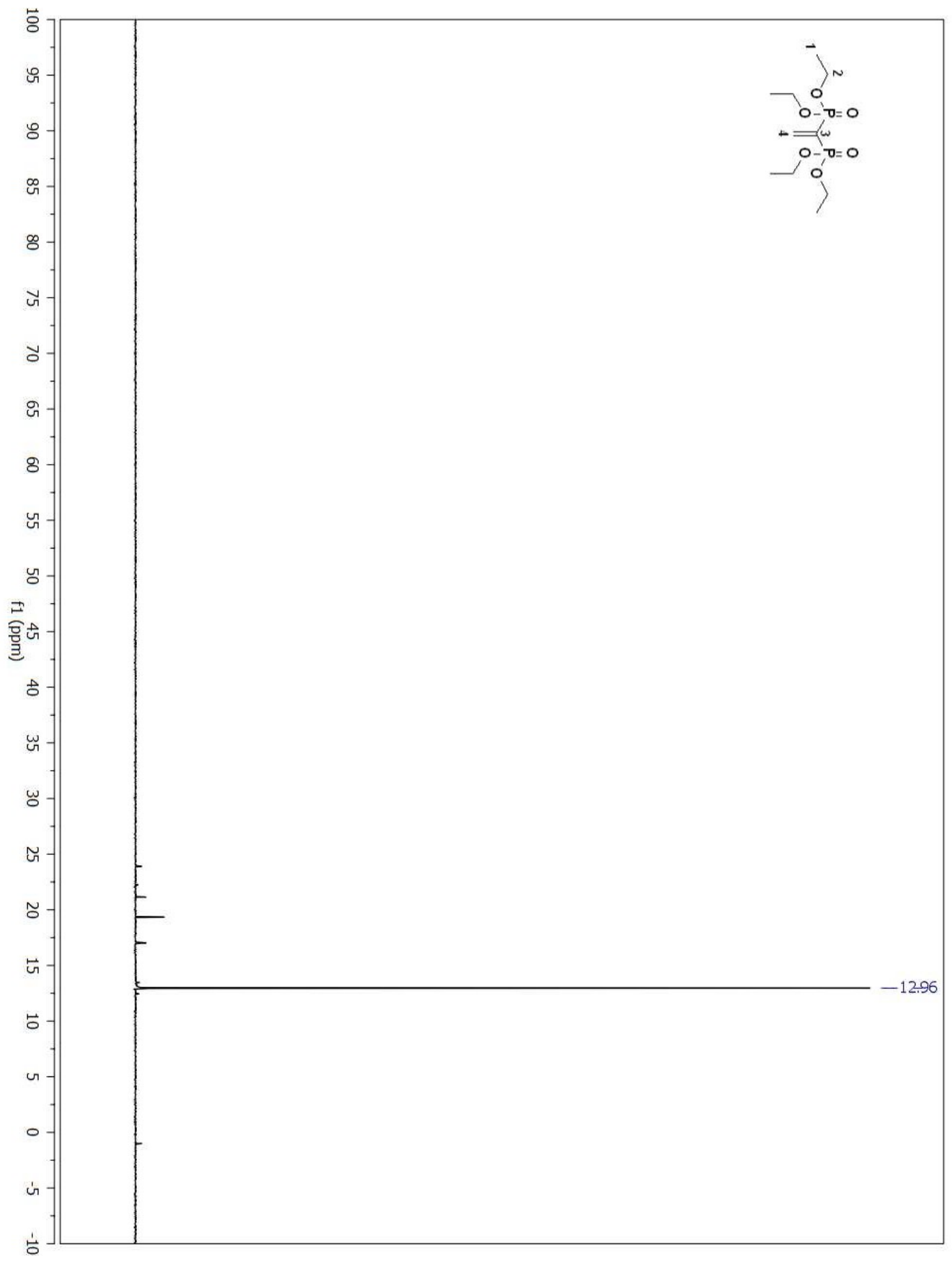

Figure A 142. ${ }^{31} \mathrm{P}$ NMR spectrum of compound (68) in $\mathrm{CDCl}_{3}$. 


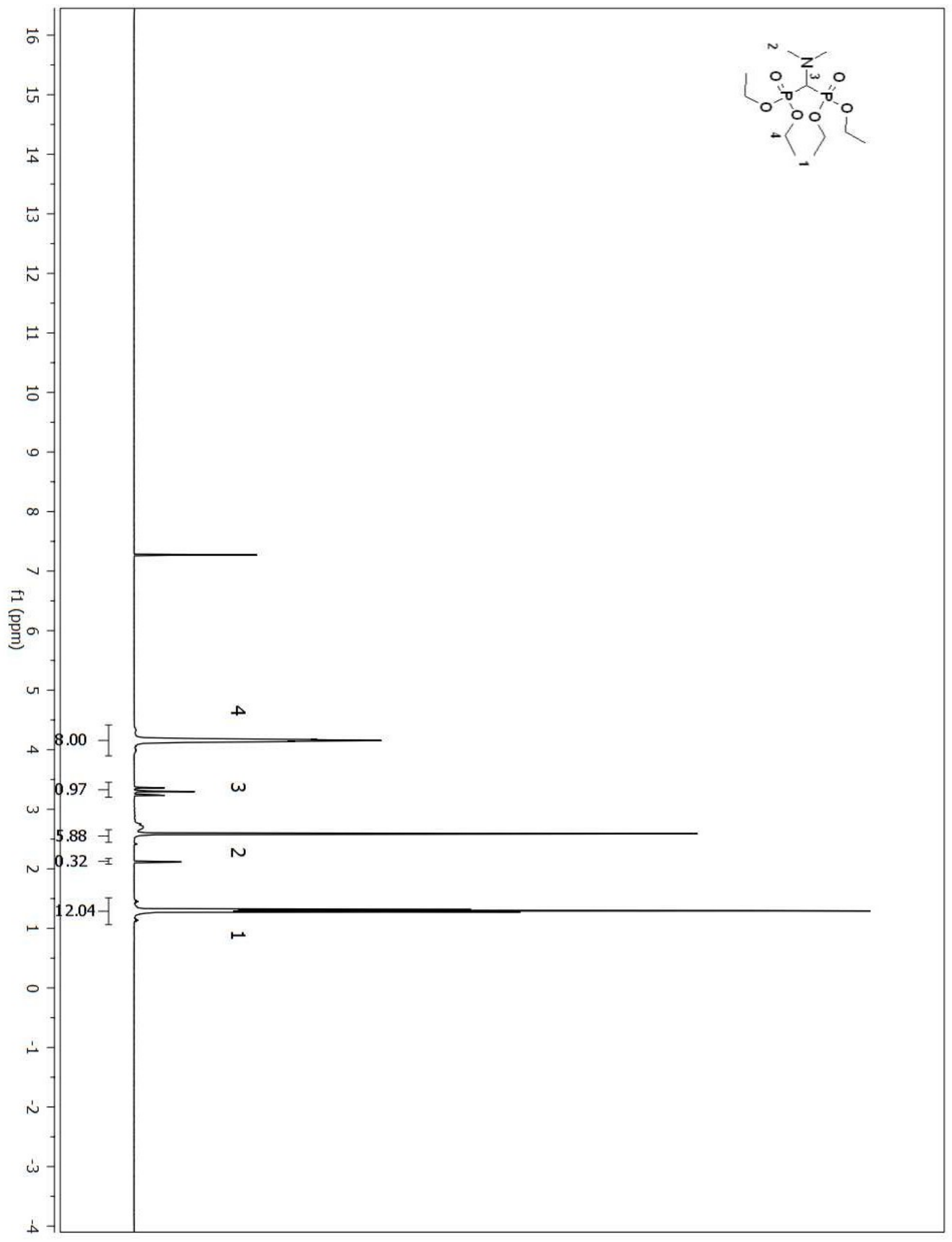

Figure A 143. ${ }^{1} \mathrm{H}$ NMR spectrum of compound (105) in $\mathrm{CDCl}_{3}$. 


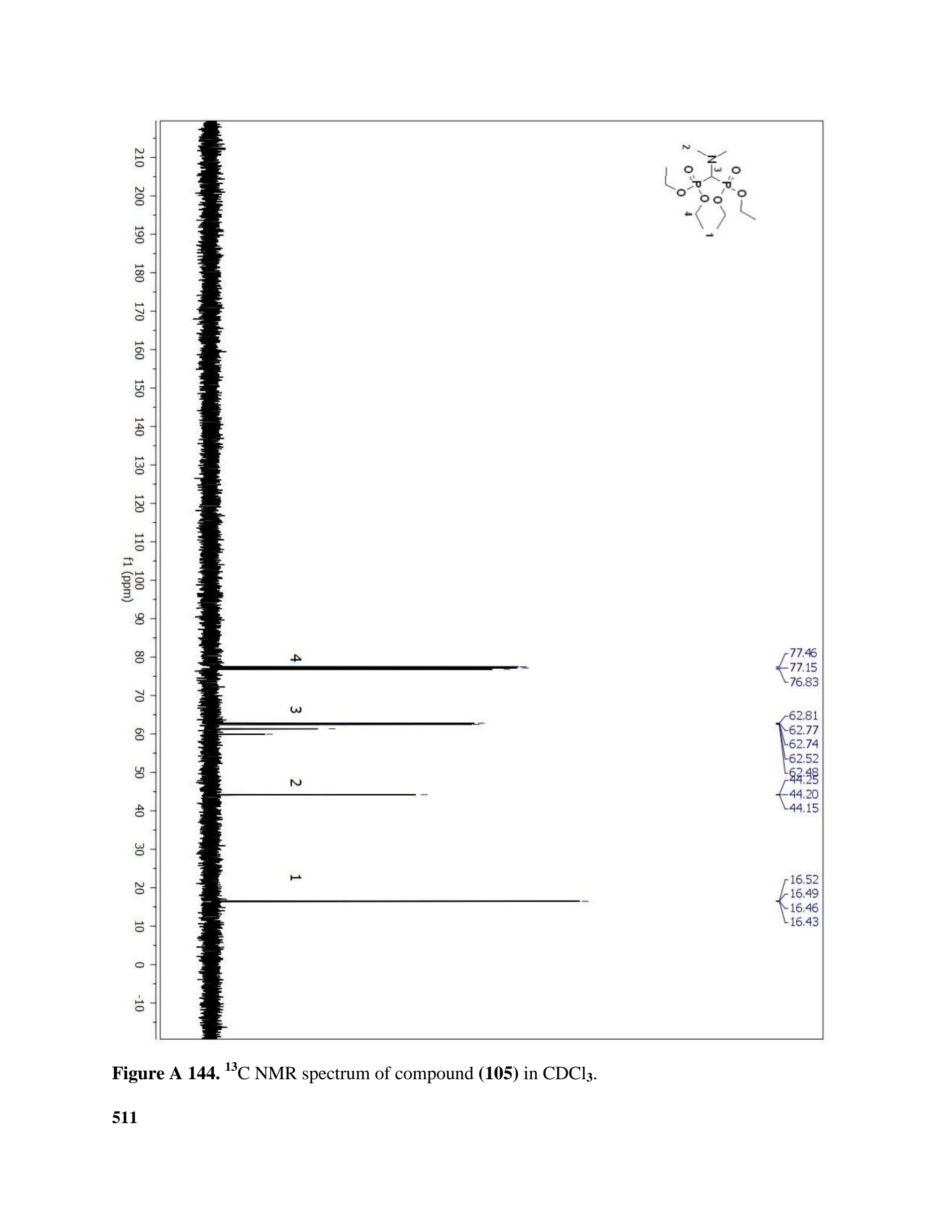




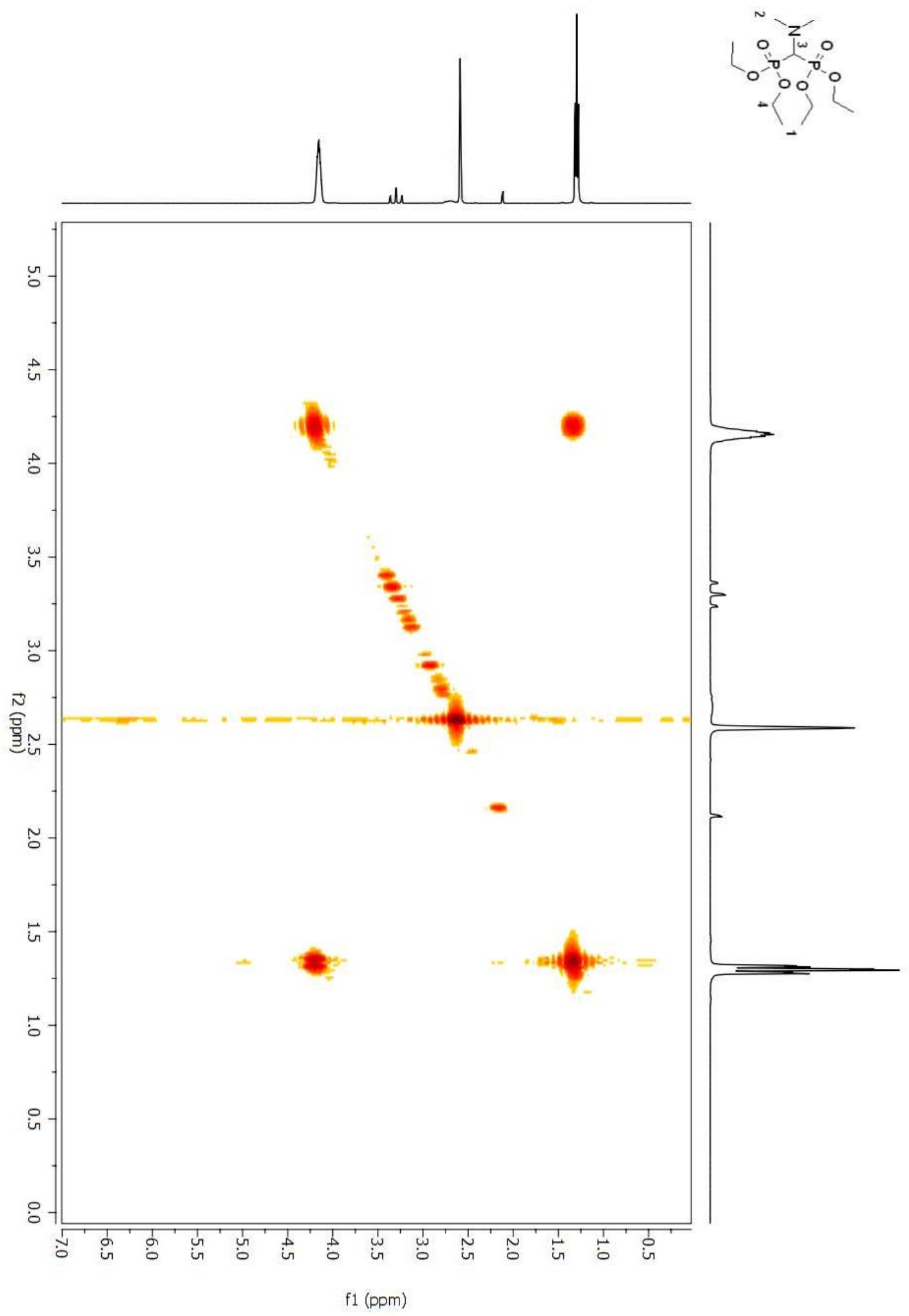

Figure A 145. COSY 2D NMR spectrum of compound (105) in $\mathrm{CDCl}_{3}$. 


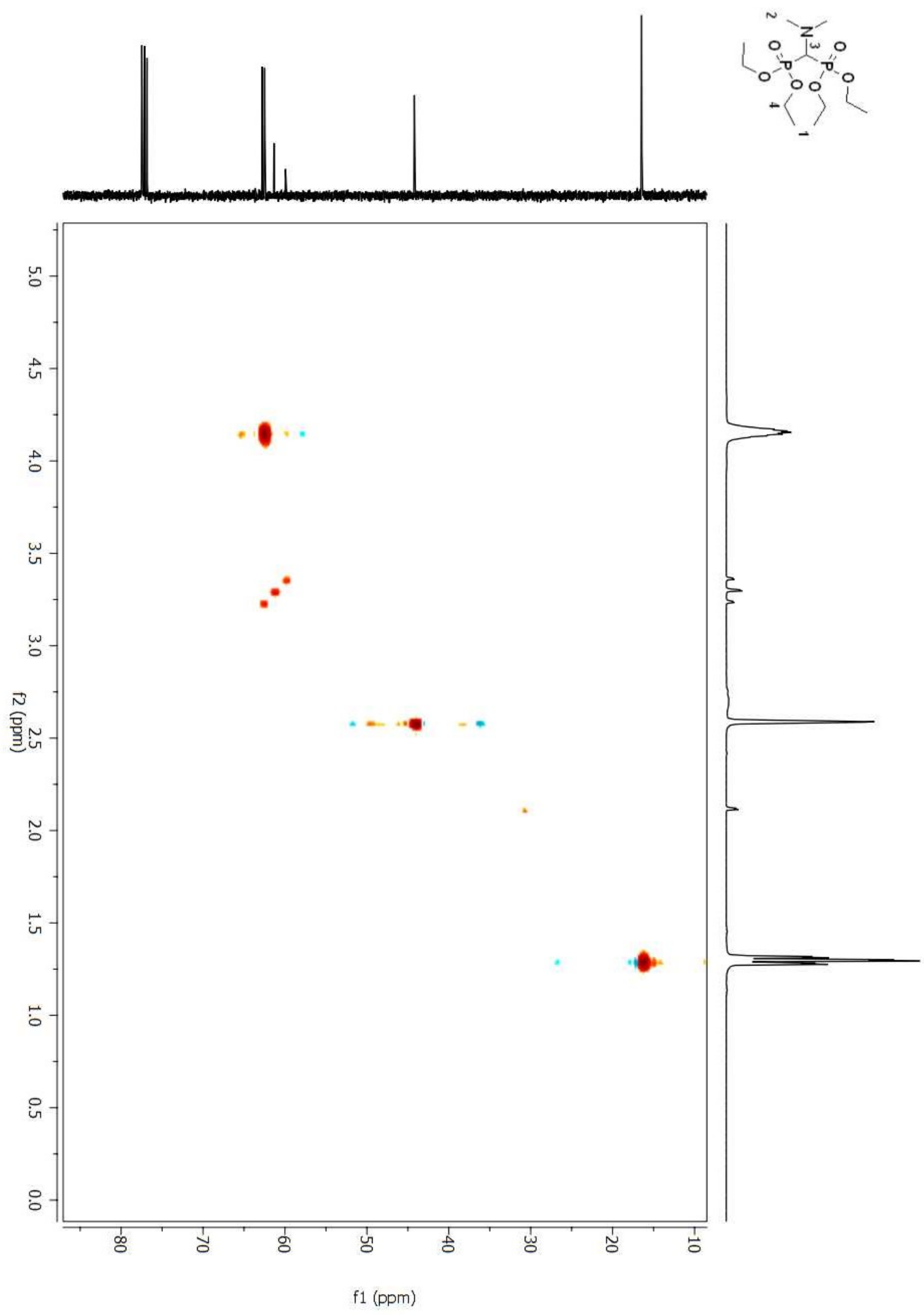

Figure A 146. HSQC 2D NMR spectrum of compound (105) in $\mathrm{CDCl}_{3}$. 


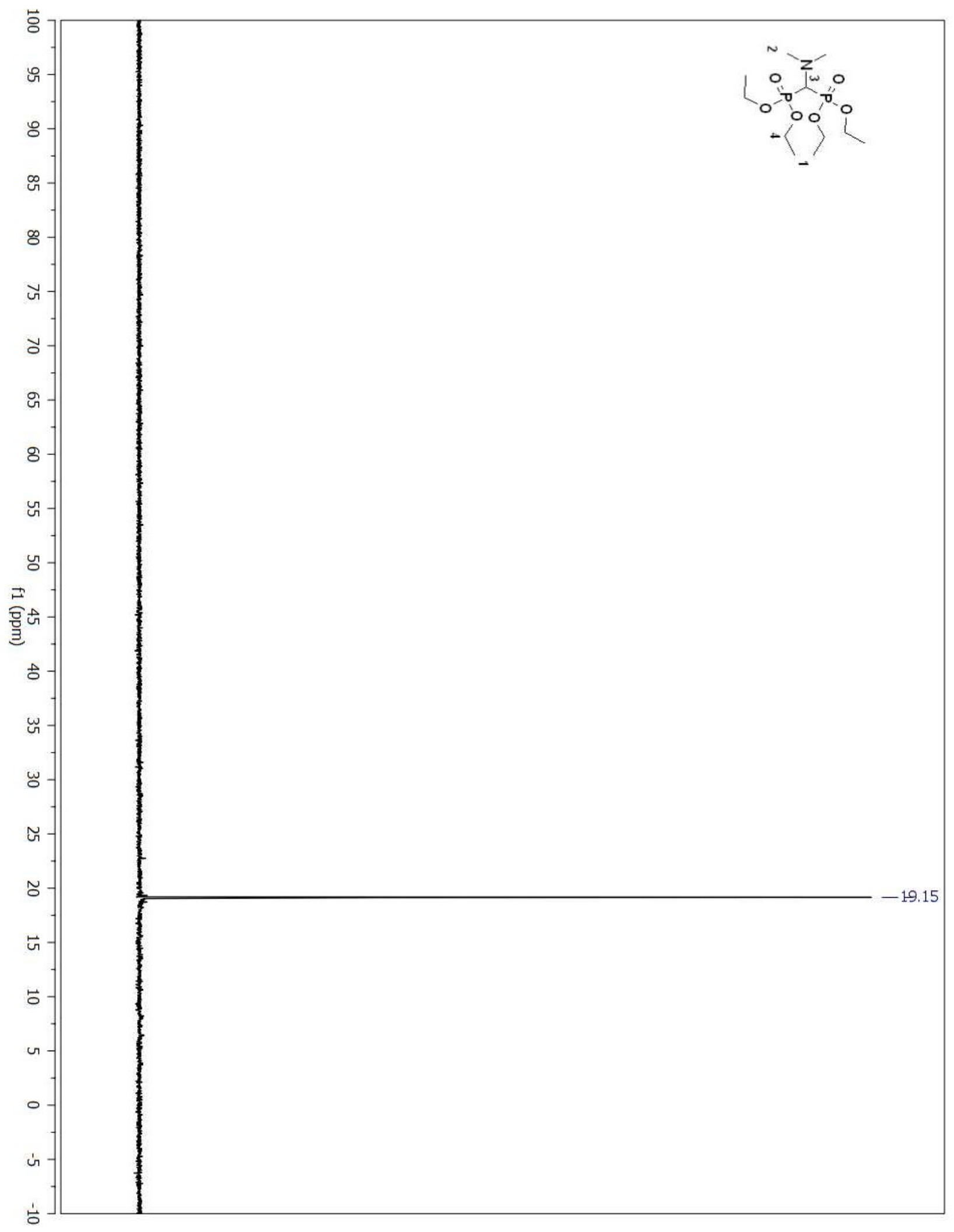

Figure A 147. ${ }^{31} \mathrm{P}$ NMR spectrum of compound (105) in $\mathrm{CDCl}_{3}$. 514 


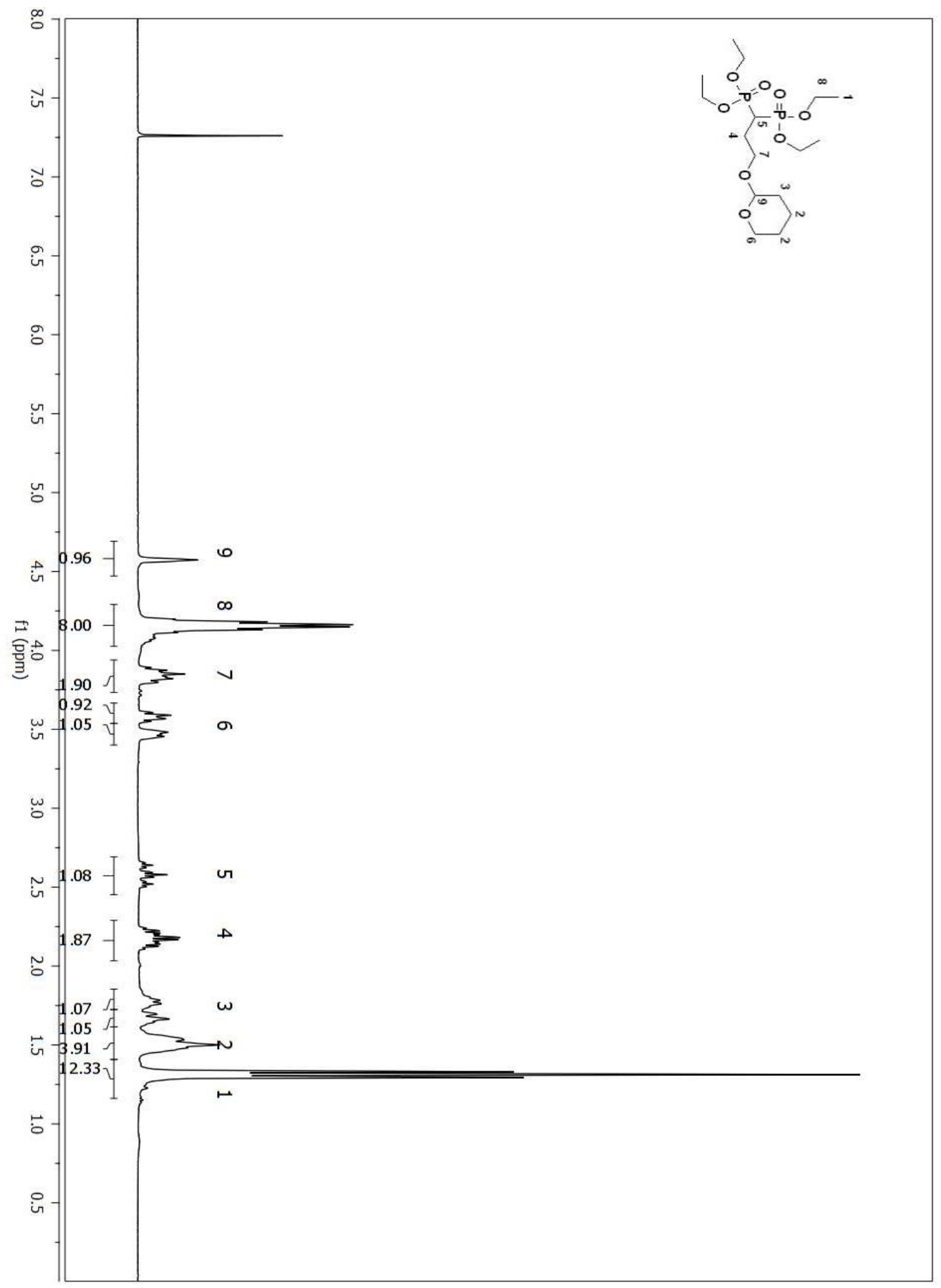

Figure A 148. ${ }^{1} \mathrm{H}$ NMR spectrum of compound (77) in $\mathrm{CDCl}_{3}$.

515 


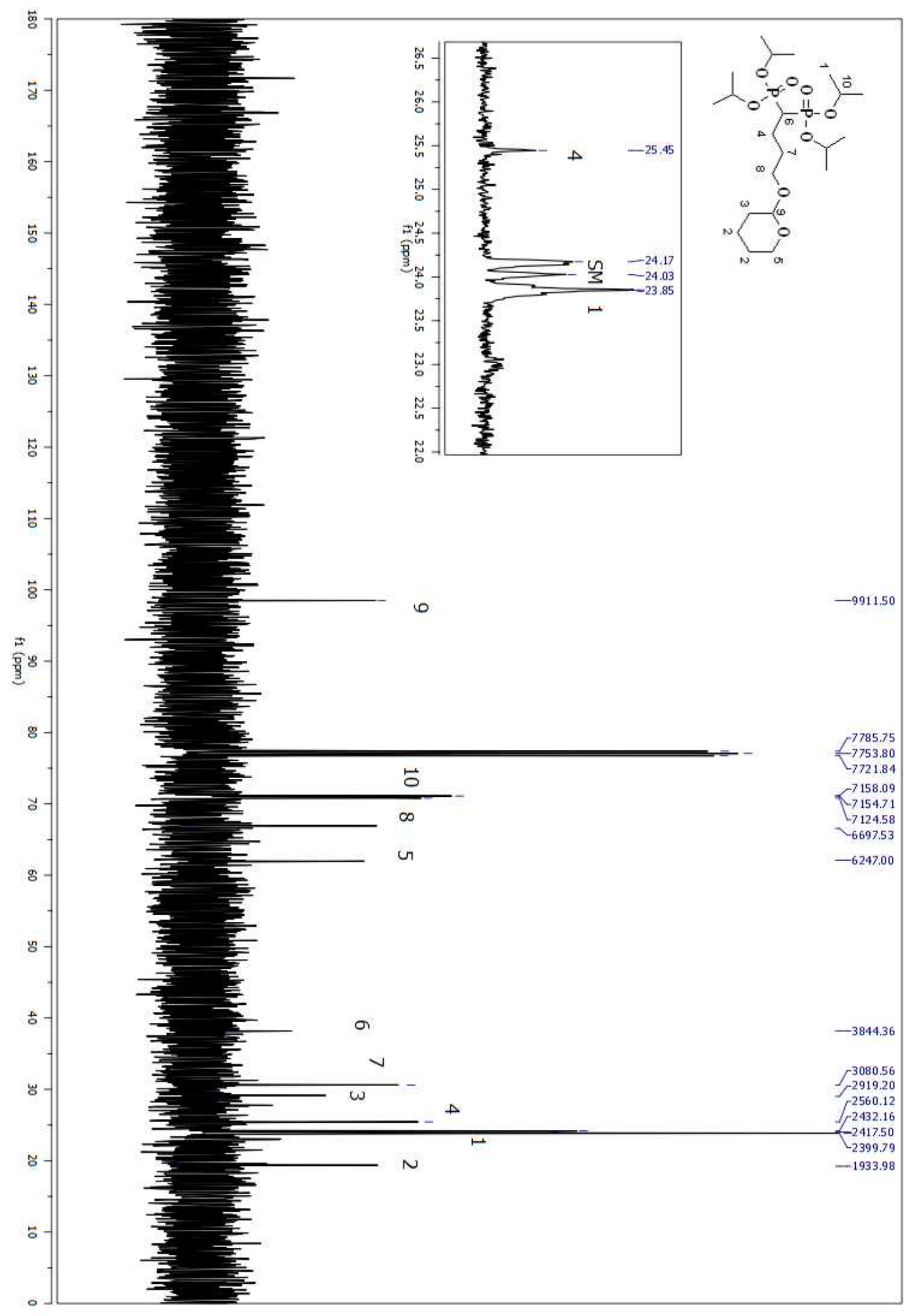

Figure A 149. ${ }^{13} \mathrm{C}$ NMR spectrum of compound (77) in $\mathrm{CDCl}_{3}$. 


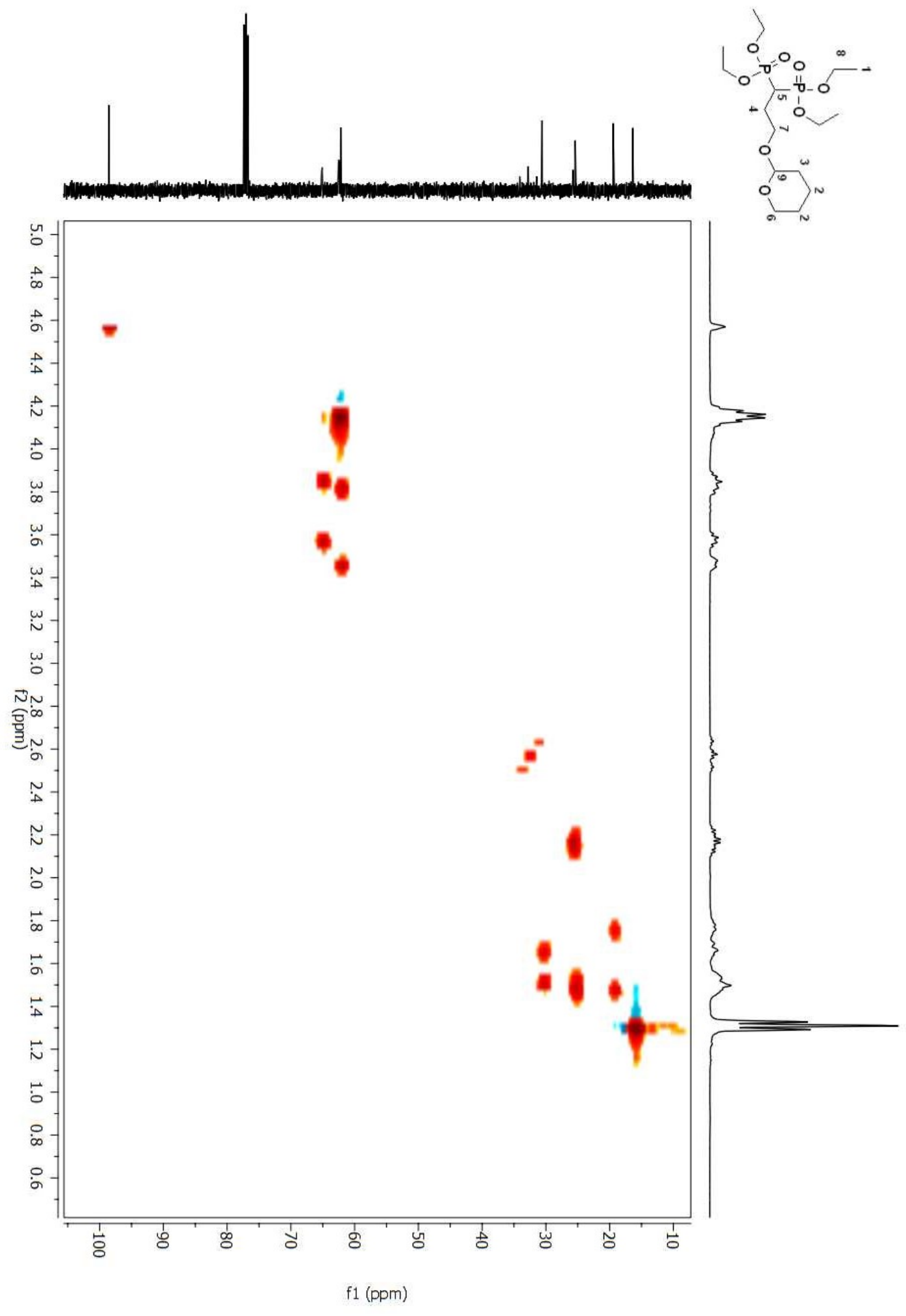

Figure A 150. HSQC 2D NMR spectrum of compound (77) in $\mathrm{CDCl}_{3}$. 


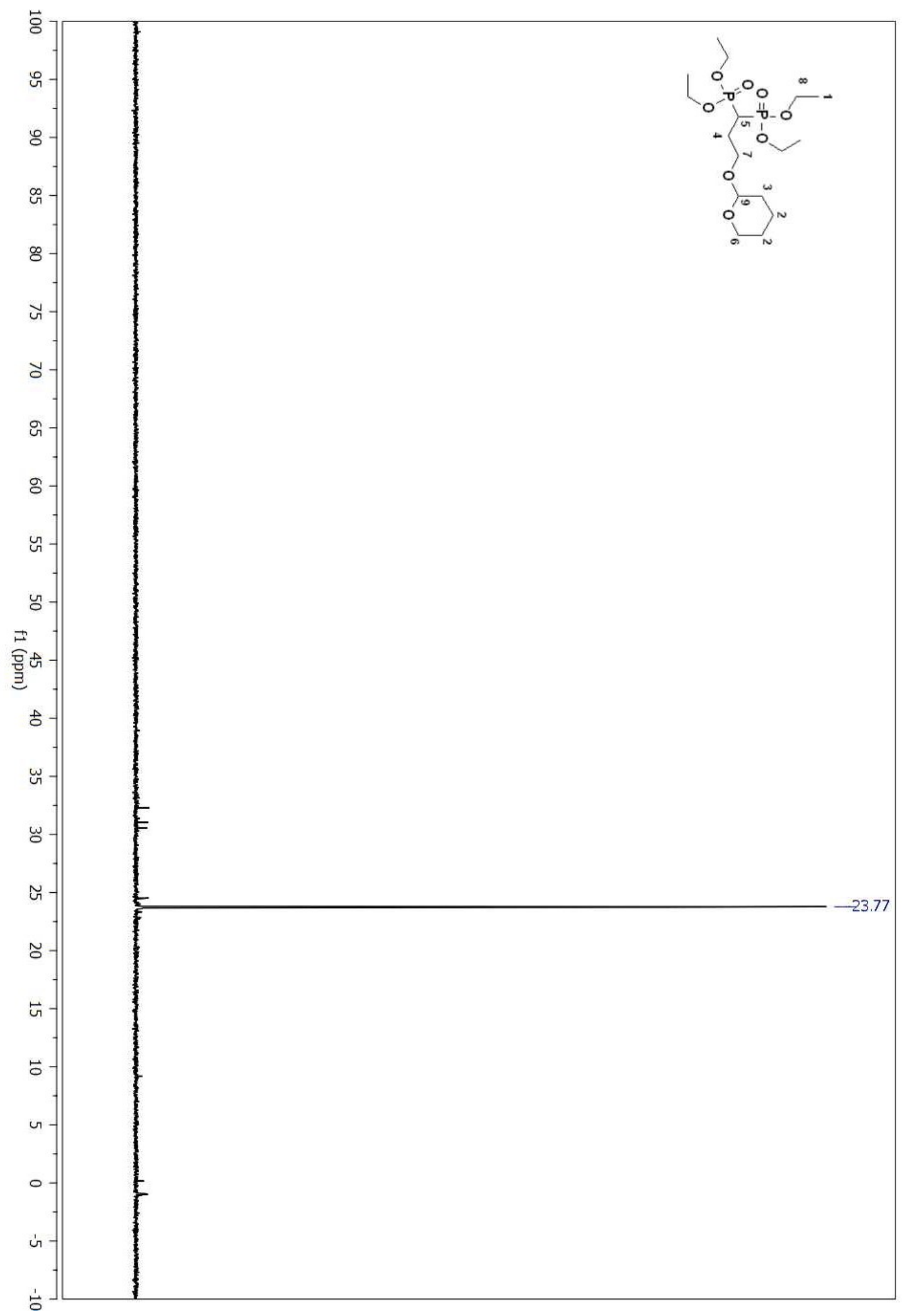

Figure A 151. ${ }^{31} \mathrm{P}$ NMR spectrum of compound (77) in $\mathrm{CDCl}_{3}$. 


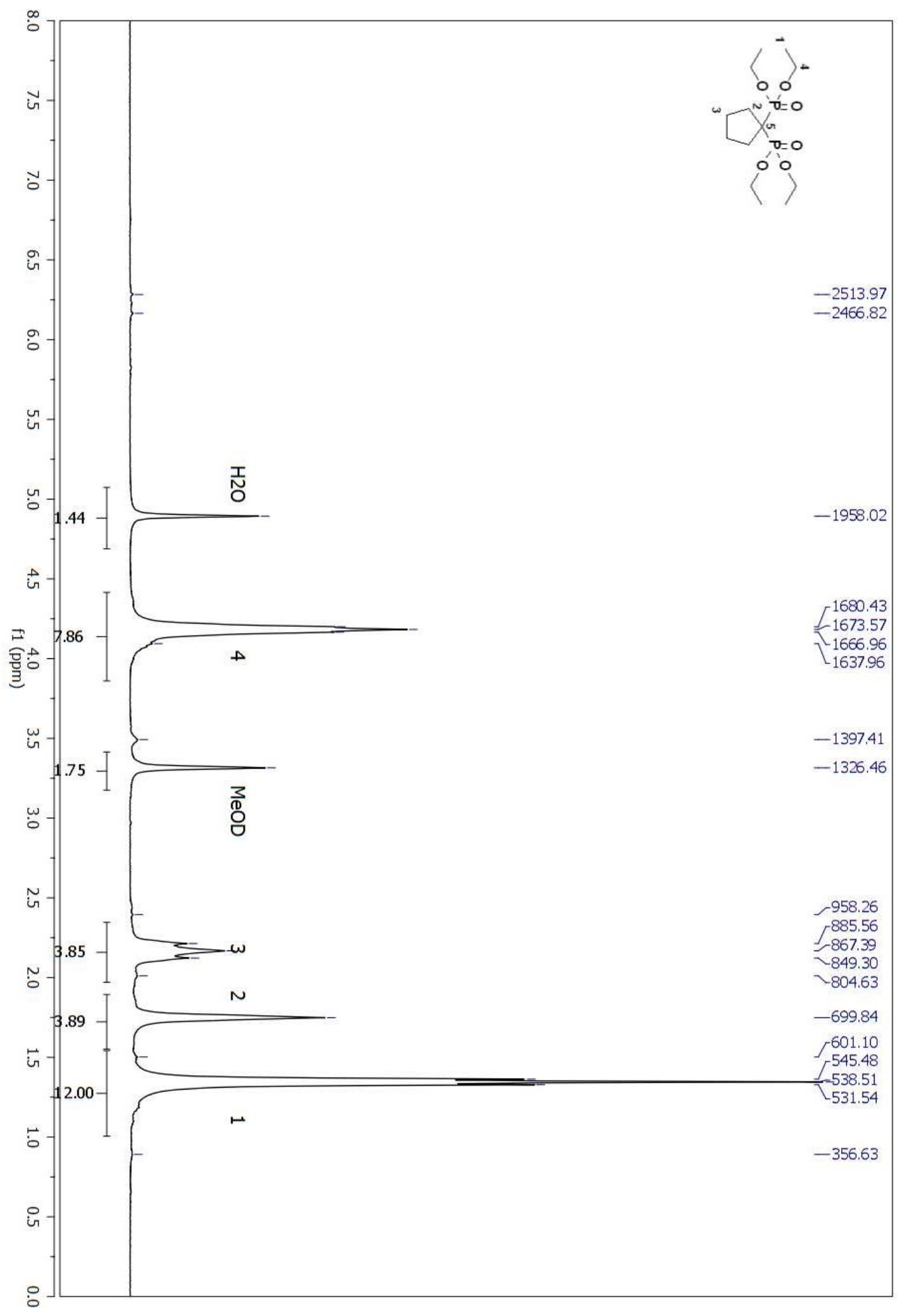

Figure A 152. ${ }^{1} \mathrm{H}$ NMR spectrum of compound (97) in MeOD. 


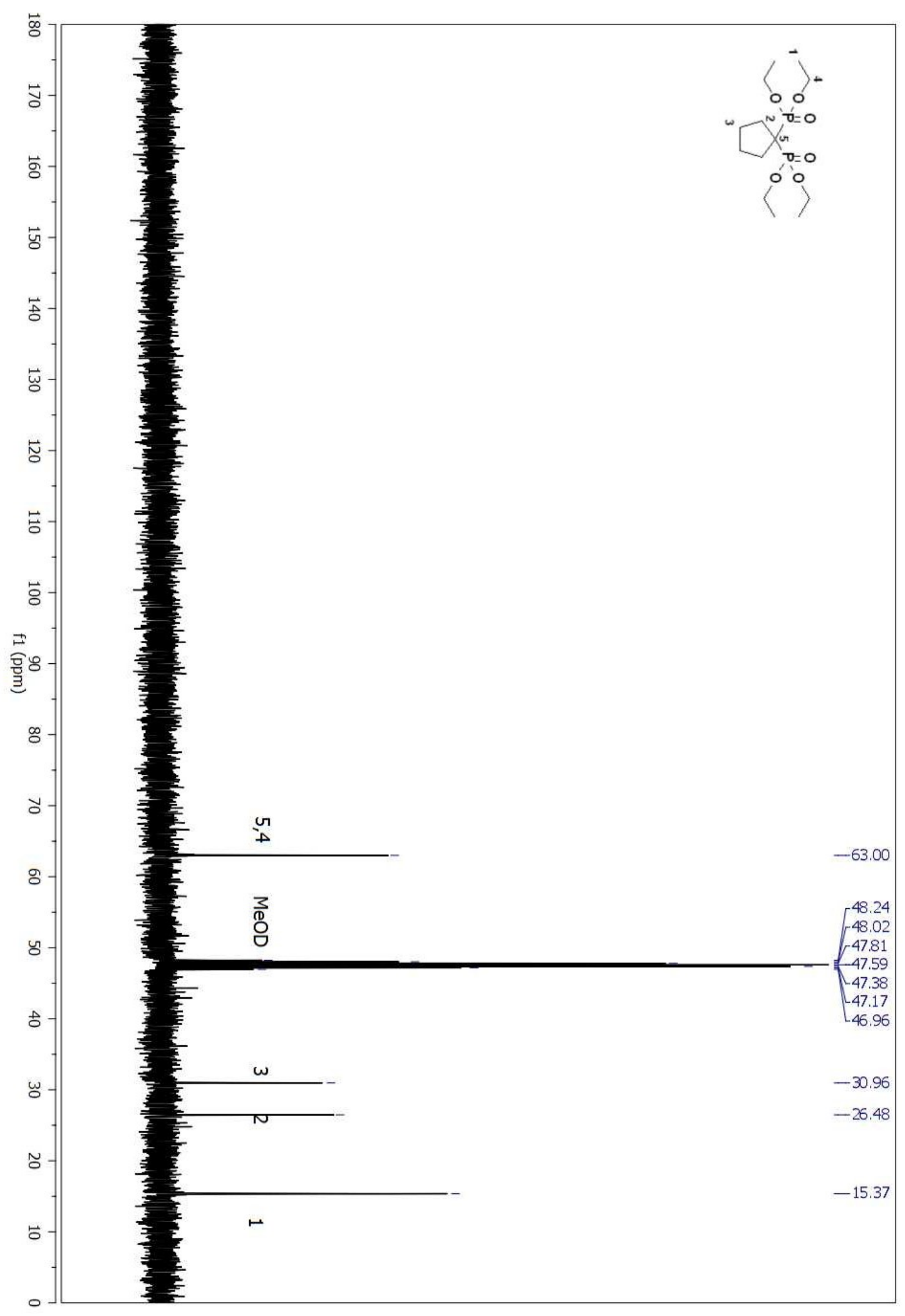

Figure A 153. ${ }^{13} \mathrm{C}$ NMR spectrum of compound (97) in MeOD. 


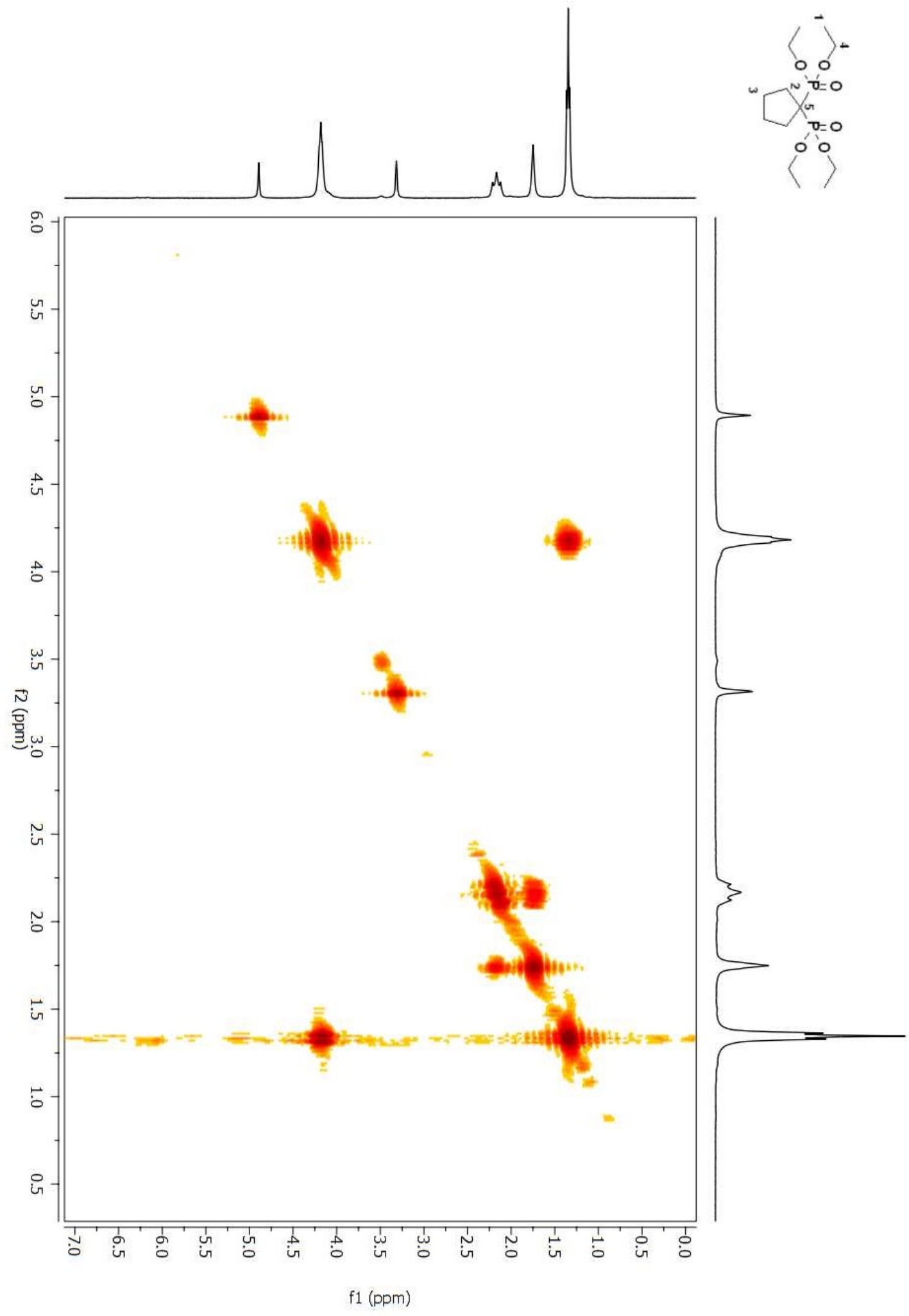

Figure A 154. COSY 2D NMR spectrum of compound (97) in MeOD. 


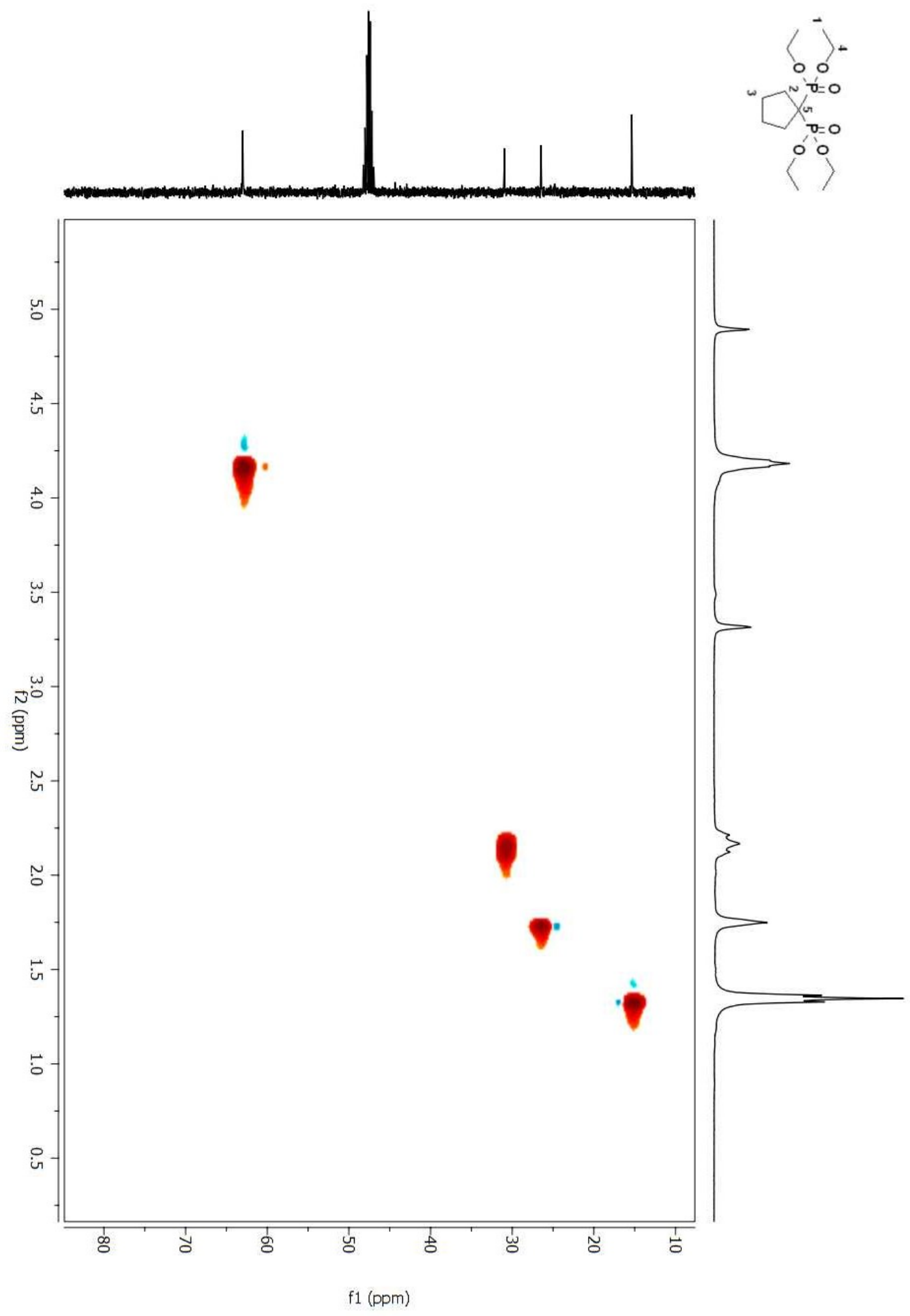

Figure A 155. HSQC 2D NMR spectrum of compound (97) in MeOD. 


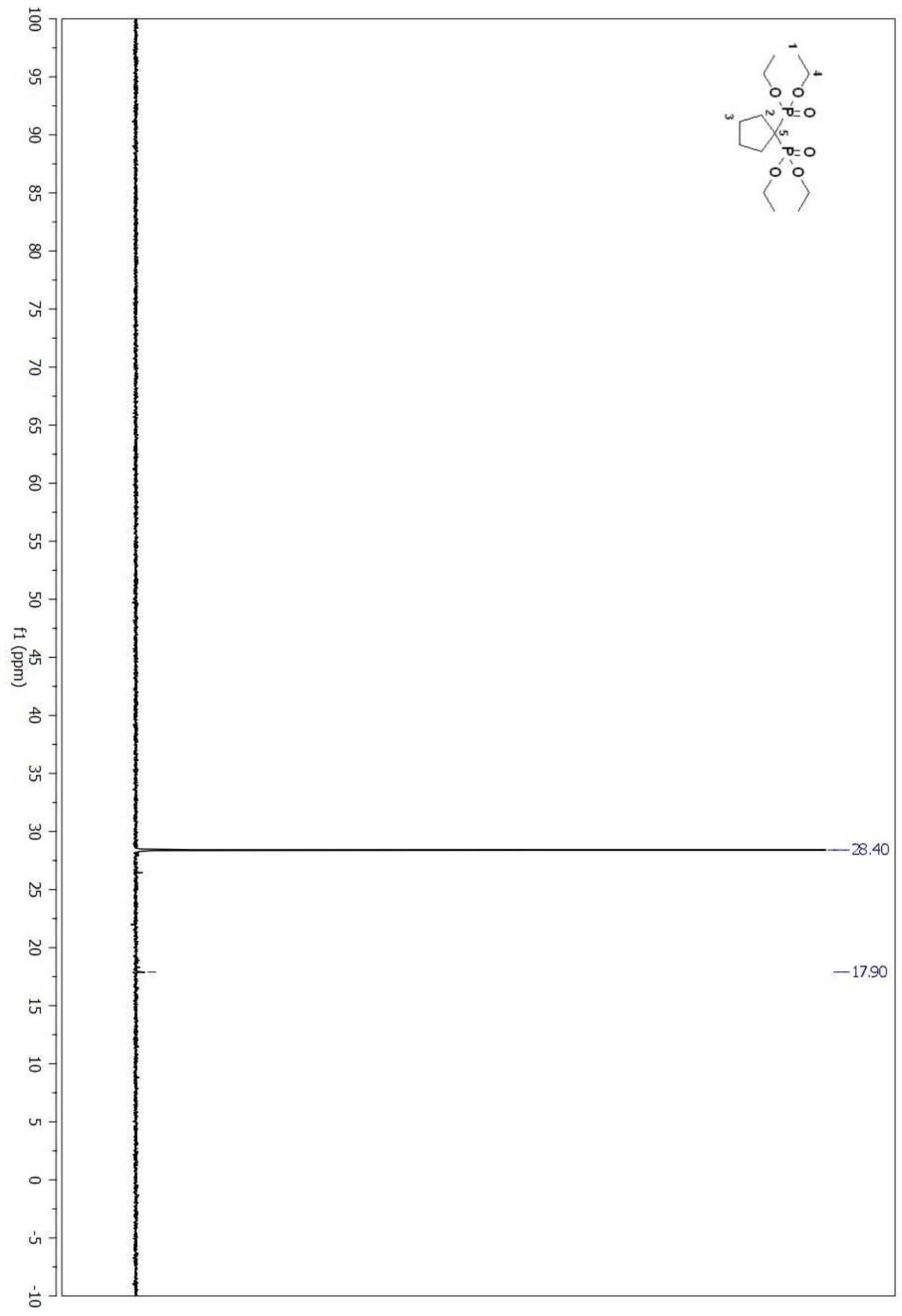

Figure A 156. ${ }^{31} \mathrm{P}$ NMR spectrum of compound (97) in MeOD. 


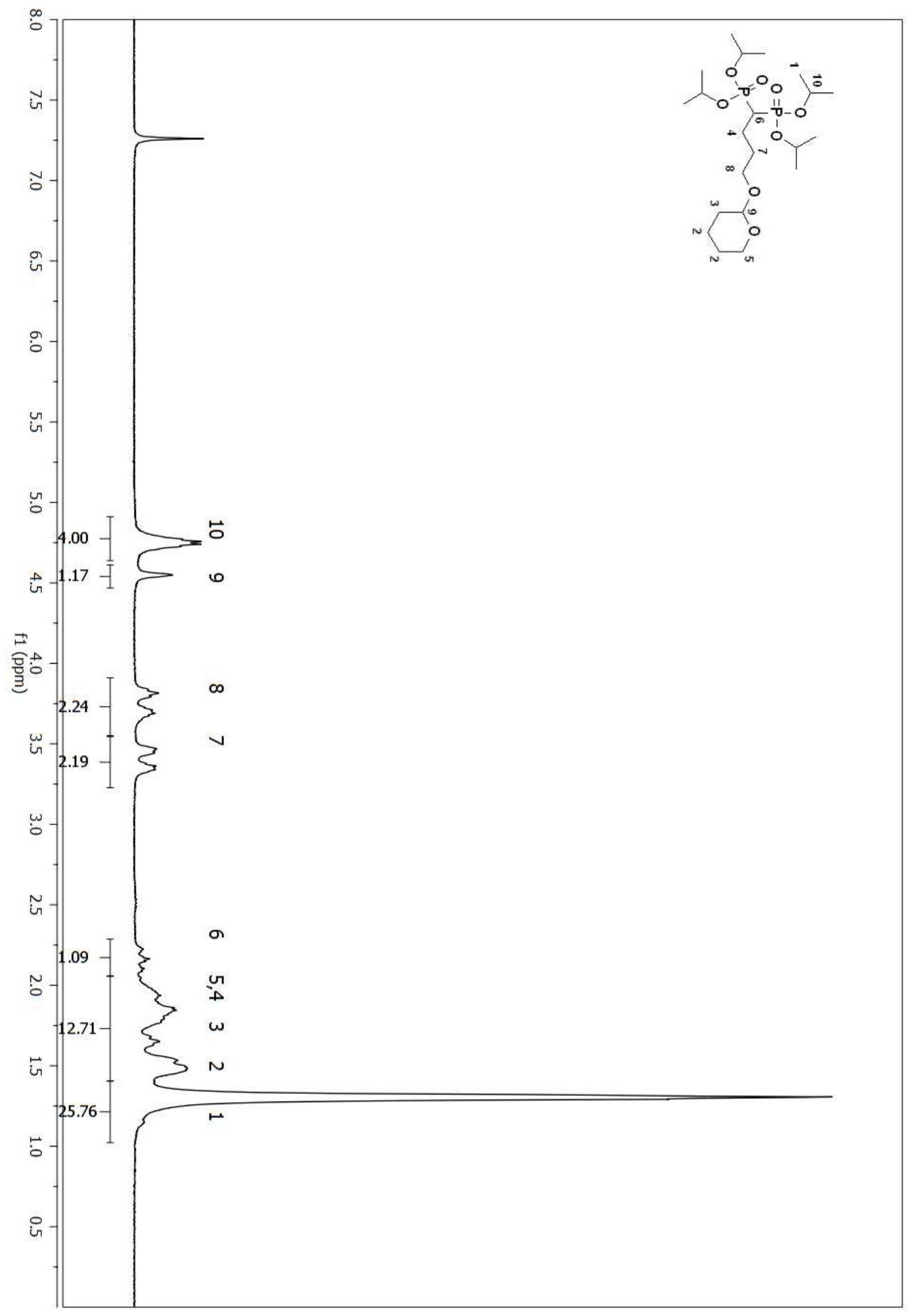

Figure A 157. ${ }^{1} \mathrm{H}$ NMR spectrum of compound (95) in $\mathrm{CDCl}_{3}$. 


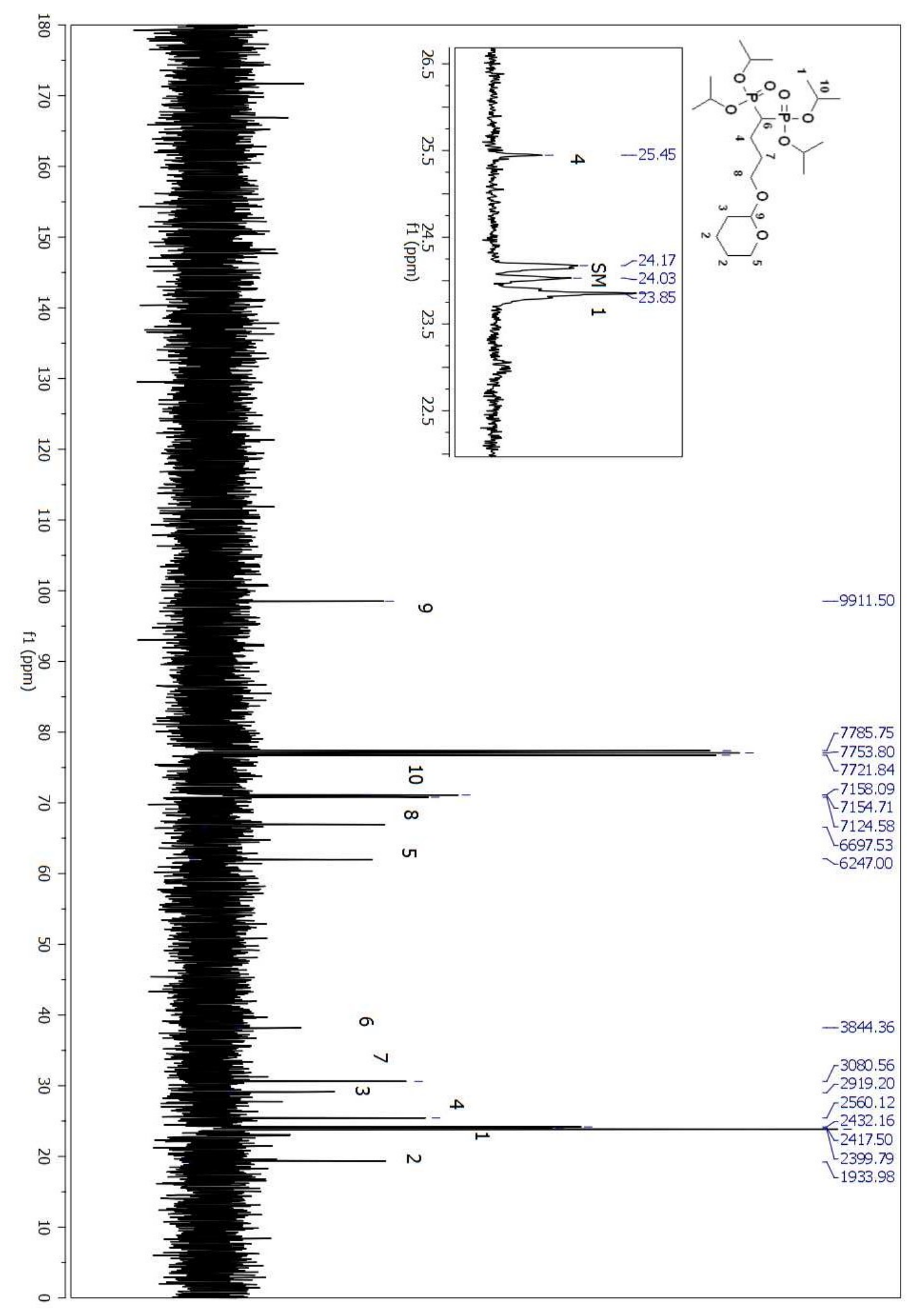

Figure A 158. ${ }^{13} \mathrm{C}$ NMR spectrum of compound (95) in $\mathrm{CDCl}_{3}$. 


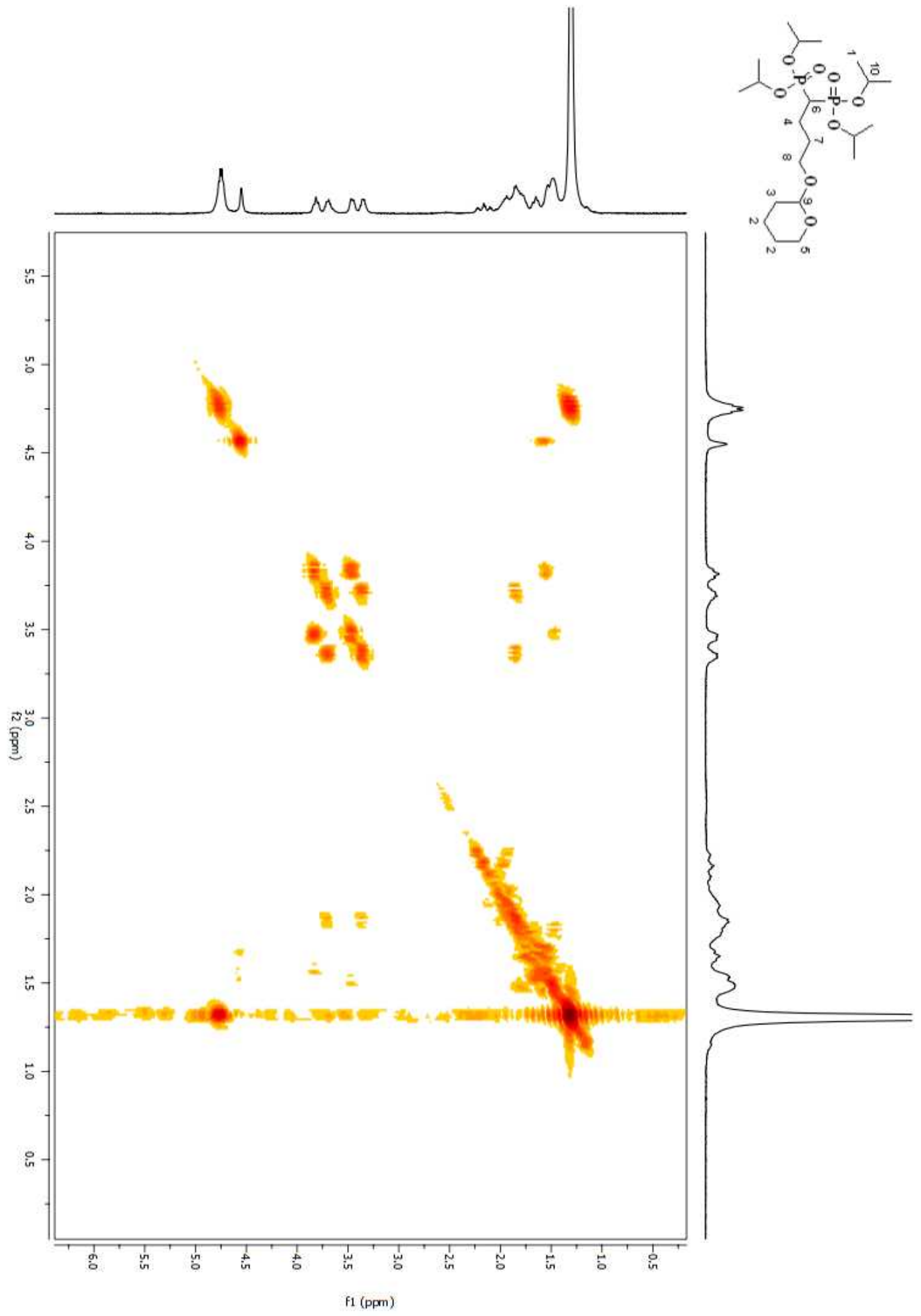

Figure A 159. COSY 2D NMR spectrum of compound (95) in $\mathrm{CDCl}_{3}$. 


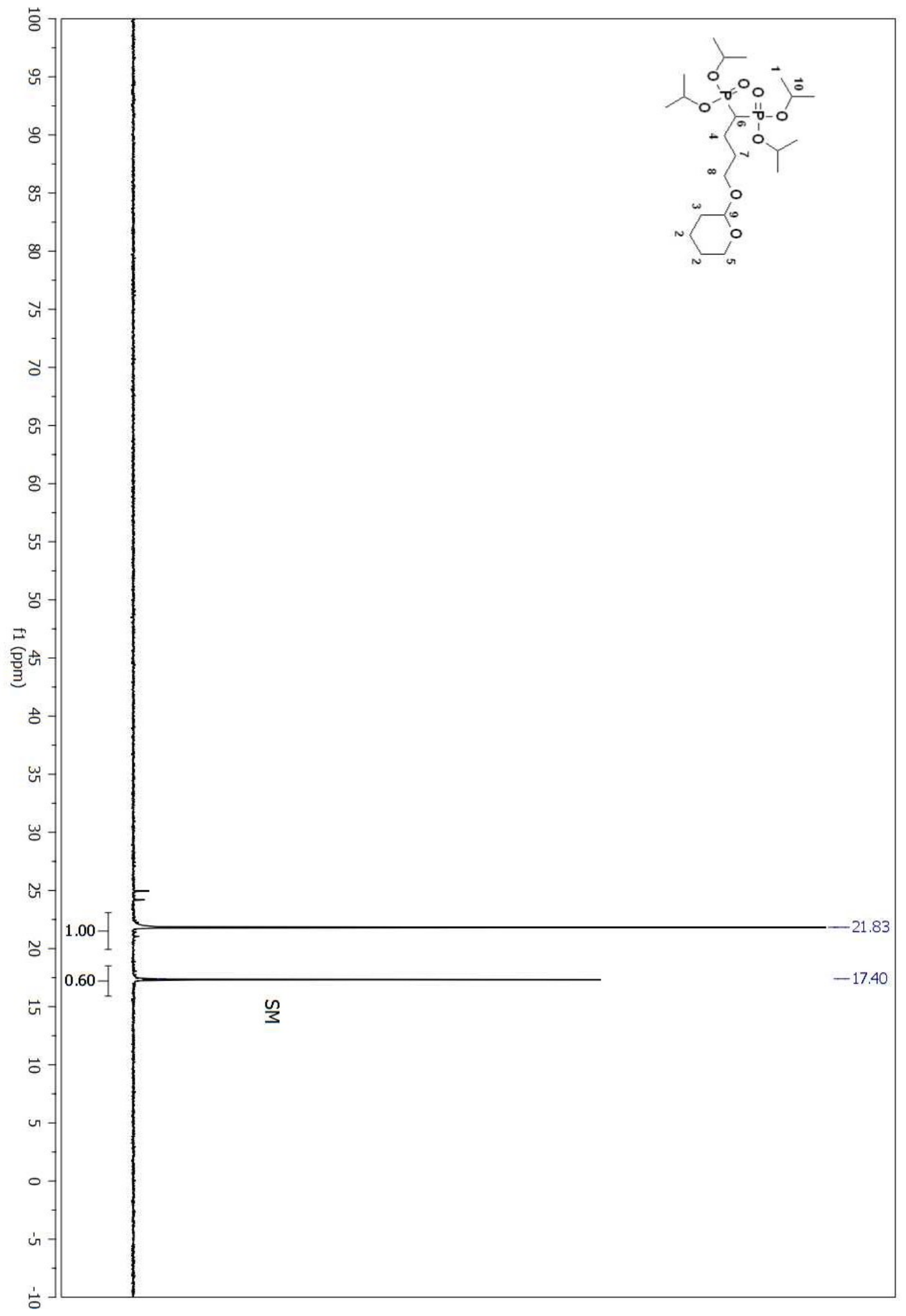

Figure A 160. ${ }^{31} \mathrm{P}$ NMR spectrum of compound (95) in $\mathrm{CDCl}_{3}$. 


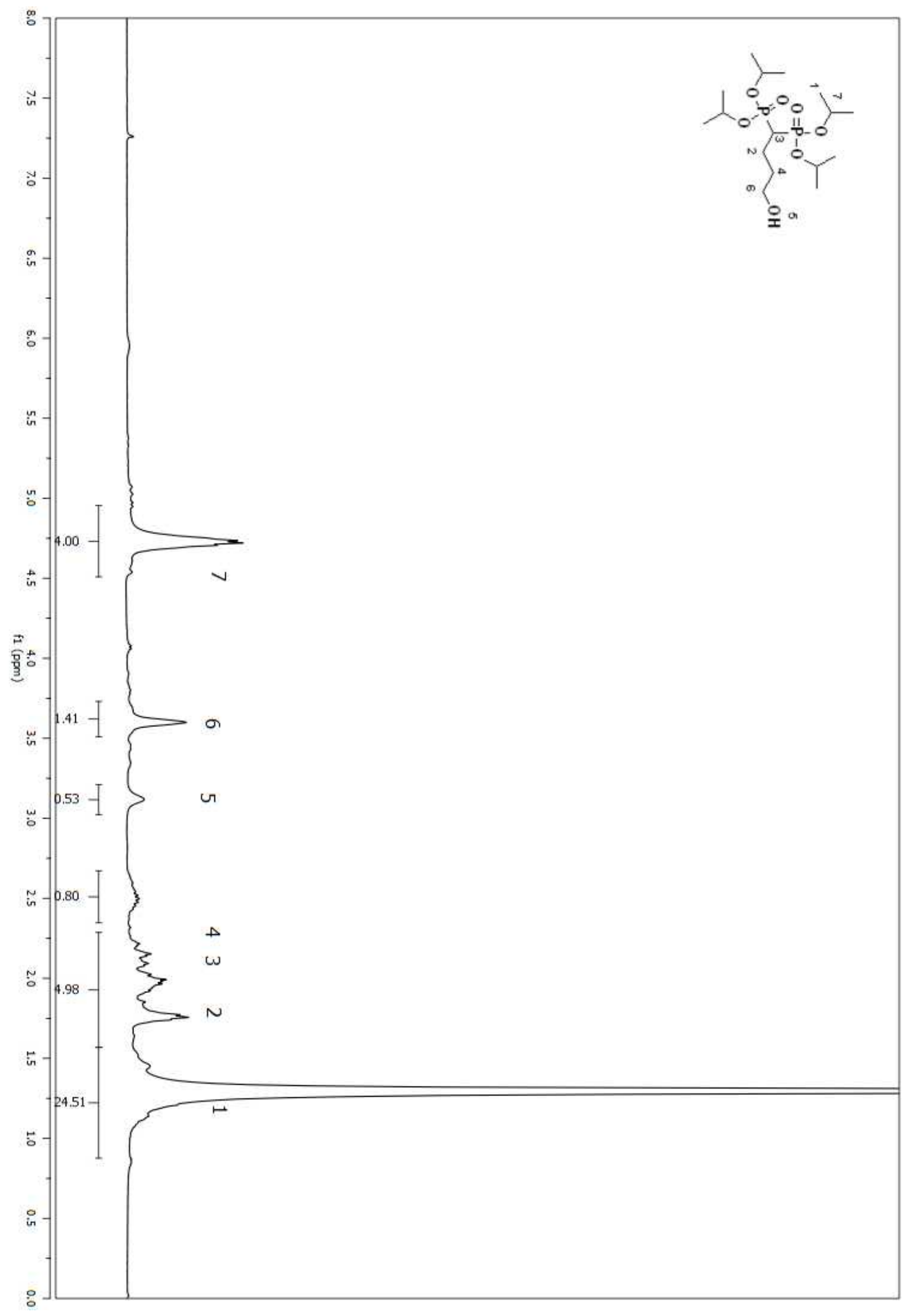

Figure A 161. ${ }^{1} \mathrm{H}$ NMR spectrum of compound (98) in $\mathrm{CDCl}_{3}$. 


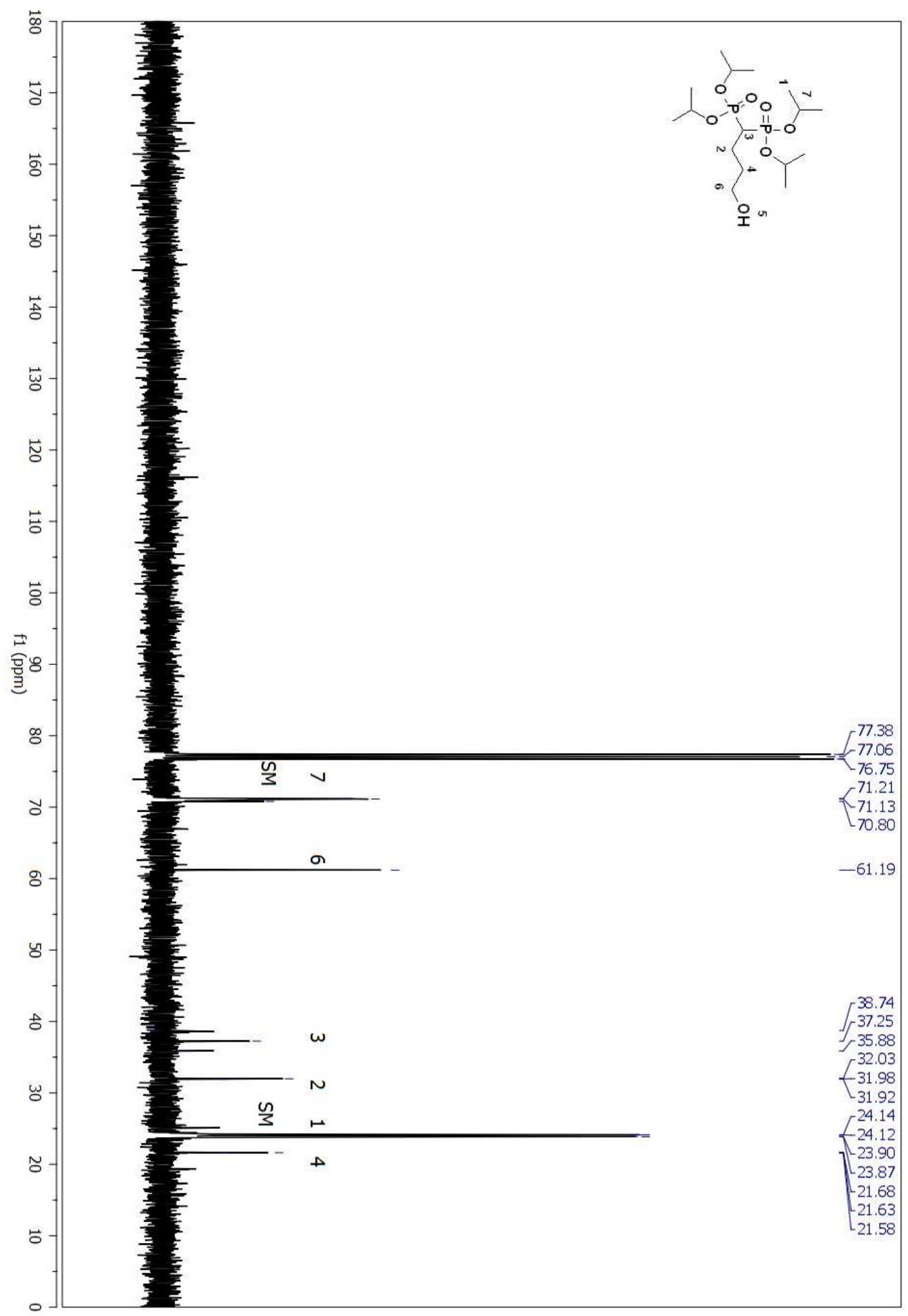

Figure A 162. ${ }^{13} \mathrm{C}$ NMR spectrum of compound (98) in $\mathrm{CDCl}_{3}$. 


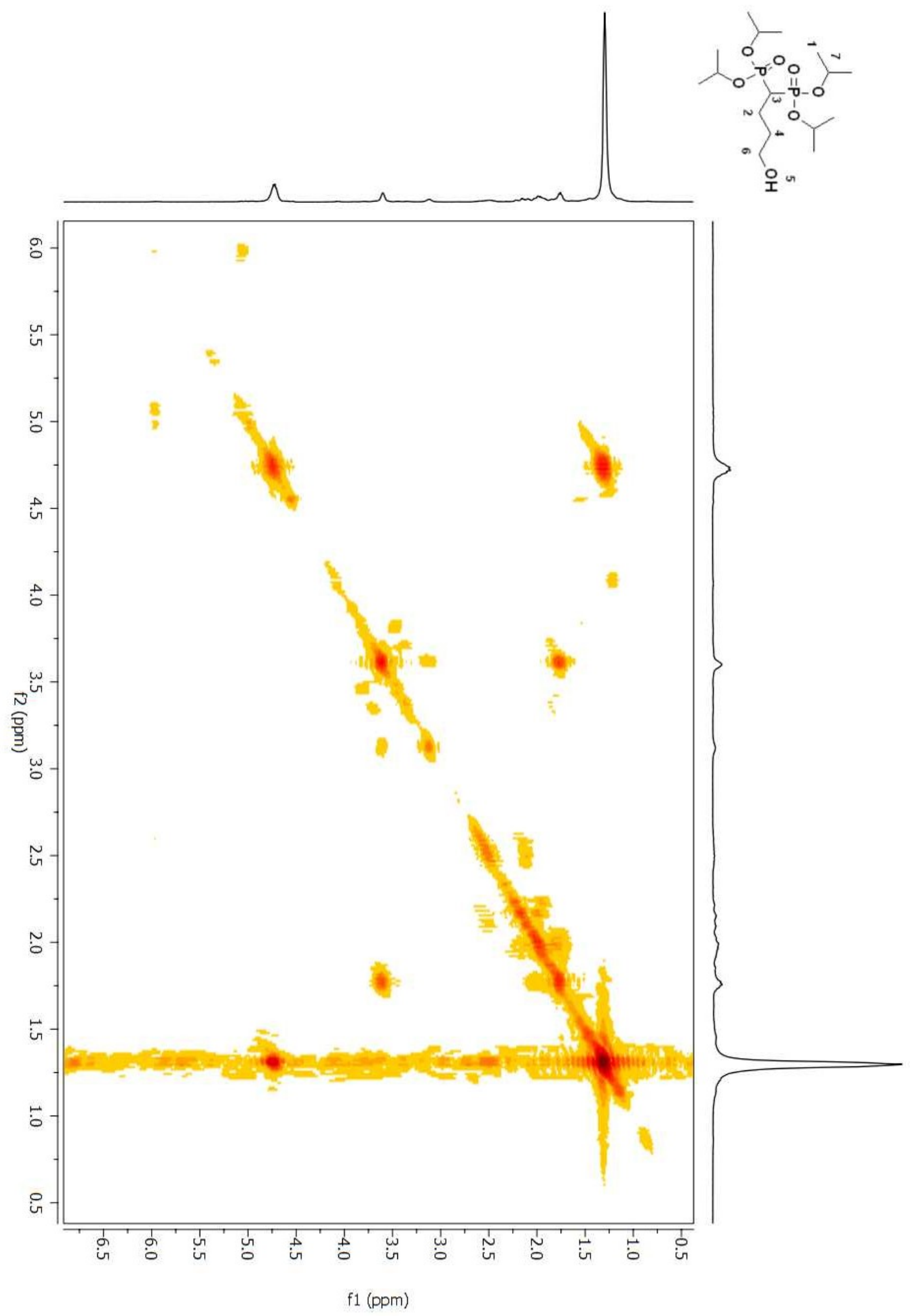

Figure A 163. COSY 2D NMR spectrum of compound (98) in $\mathrm{CDCl}_{3}$. 


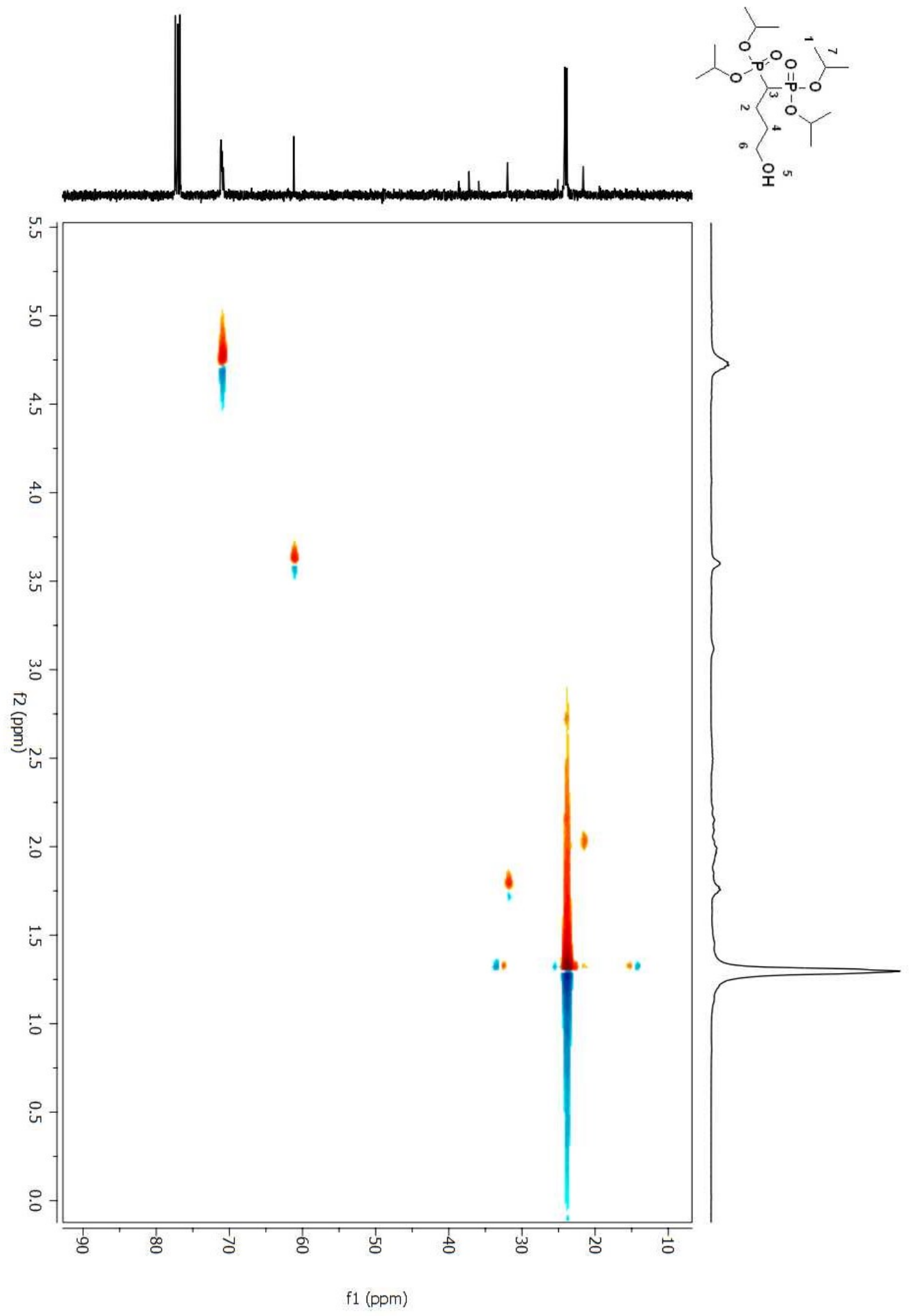

Figure A 164. HSQC 2D NMR spectrum of compound (98) in $\mathrm{CDCl}_{3}$. 


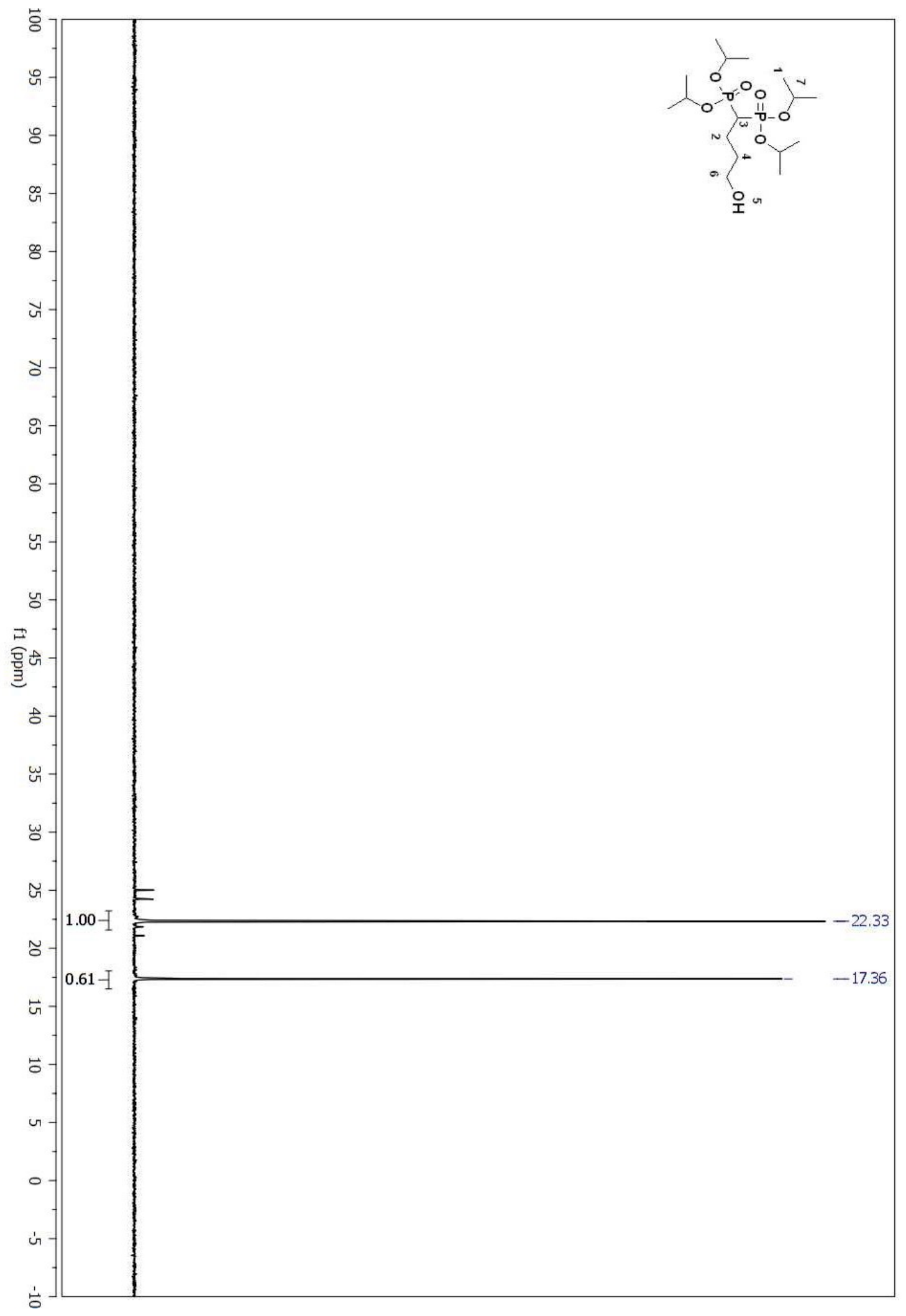

Figure A 165. ${ }^{31} \mathrm{P}$ NMR spectrum of compound (98) in $\mathrm{CDCl}_{3}$. 


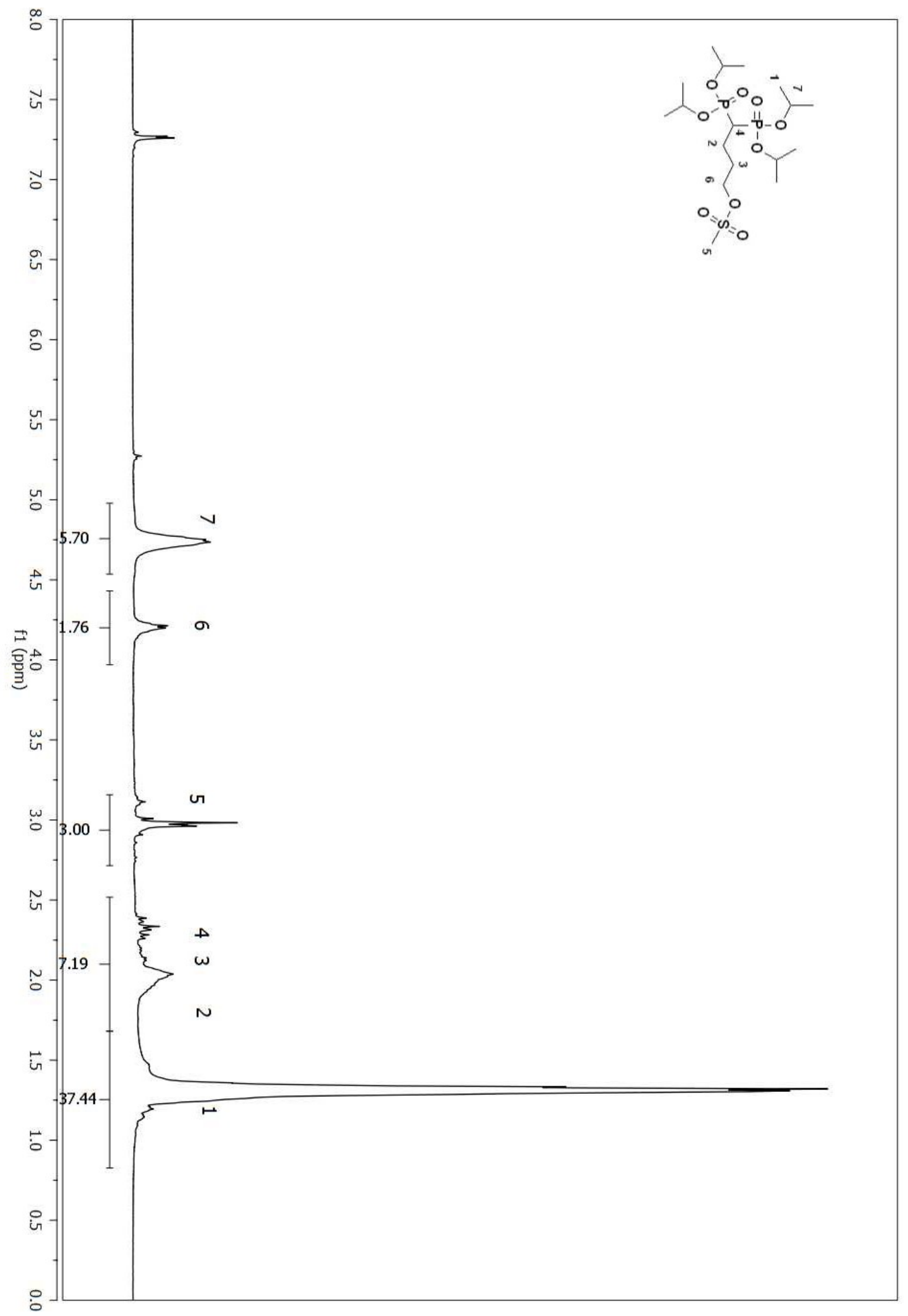

Figure A 166. ${ }^{1} \mathrm{H}$ NMR spectrum of compound (99) in $\mathrm{CDCl}_{3}$. 
1 


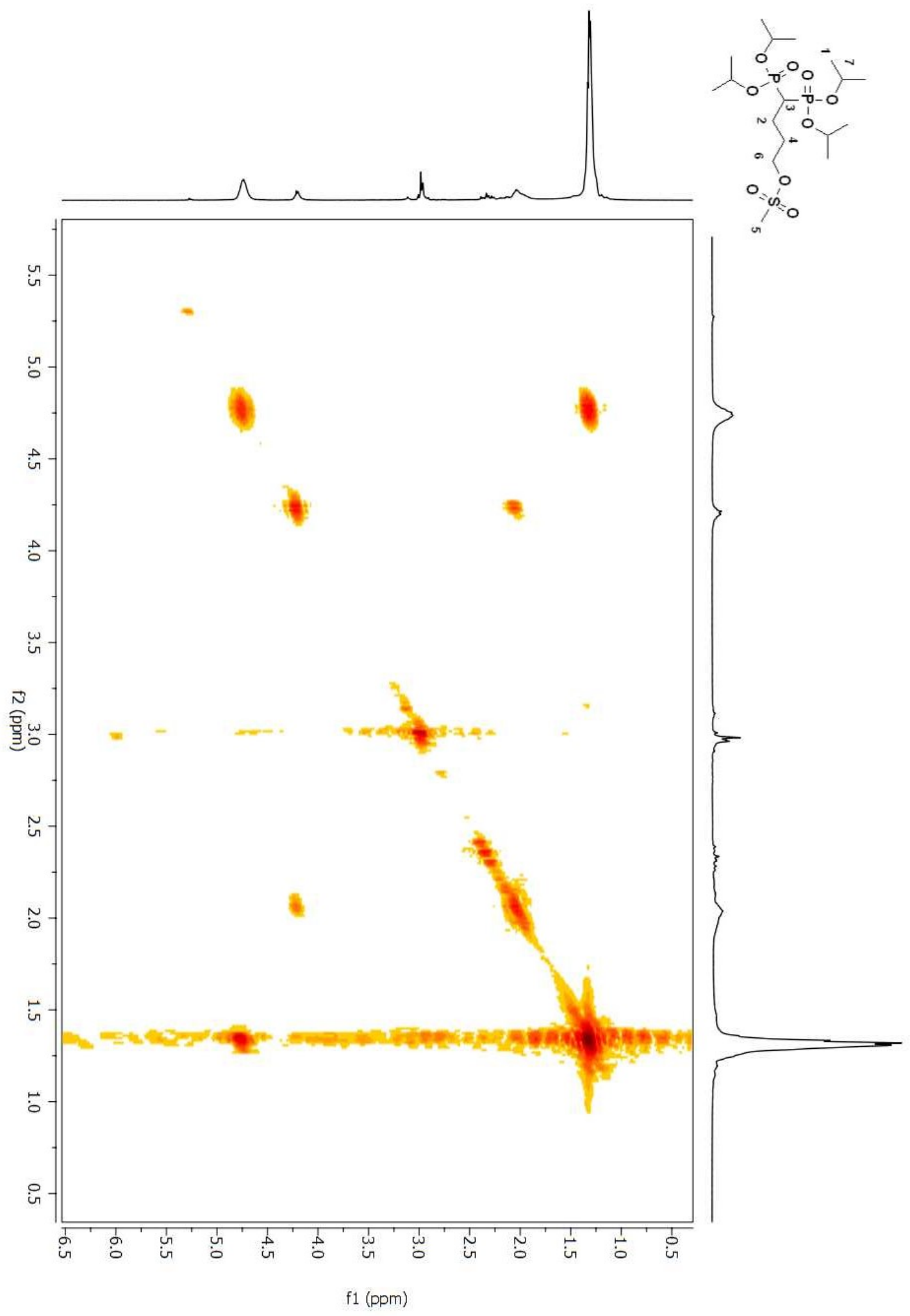

Figure A 168. COSY 2D NMR spectrum of compound (99) in $\mathrm{CDCl}_{3}$. 


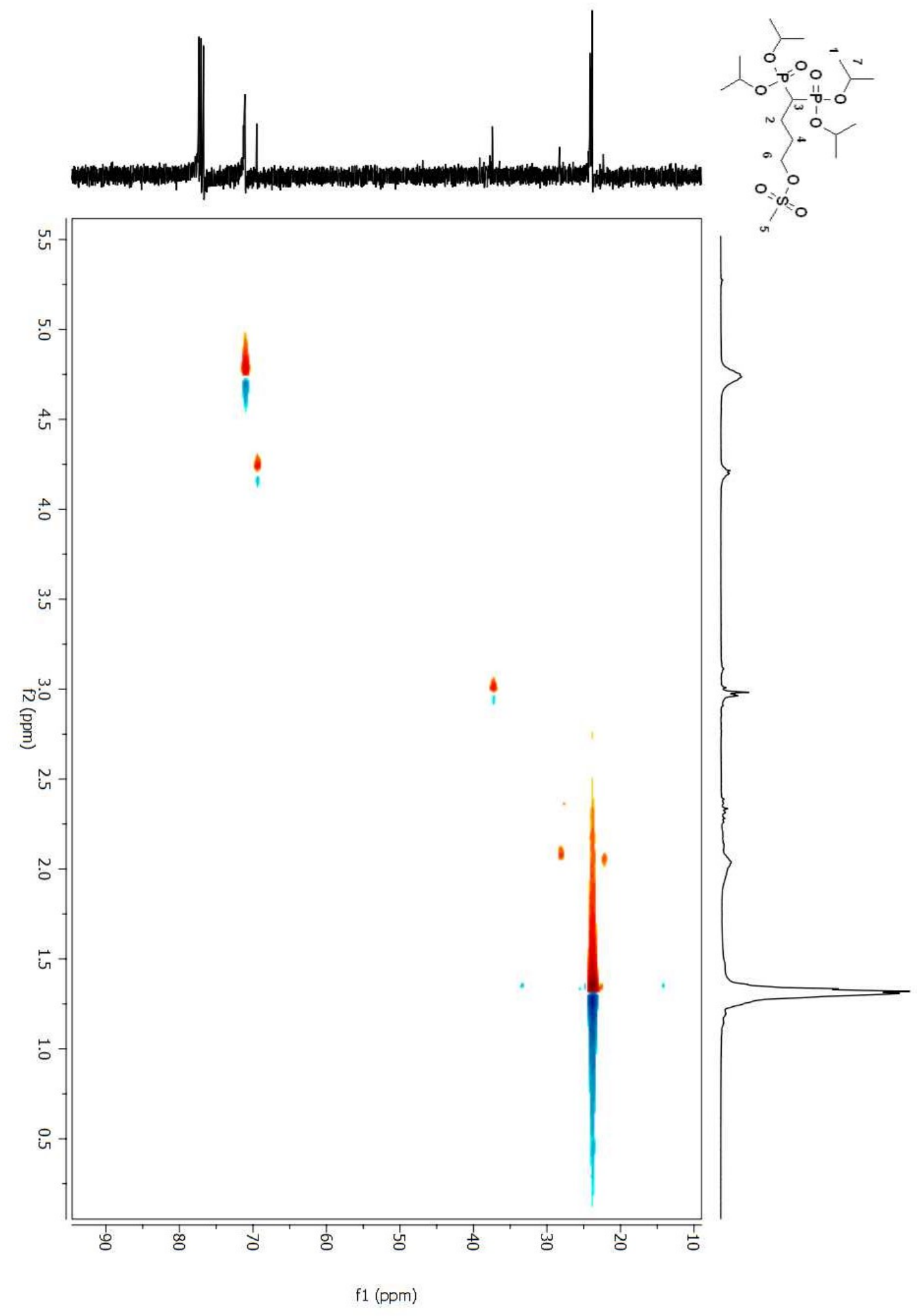

Figure A 169. HSQC 2D NMR spectrum of compound (99) in $\mathrm{CDCl}_{3}$. 


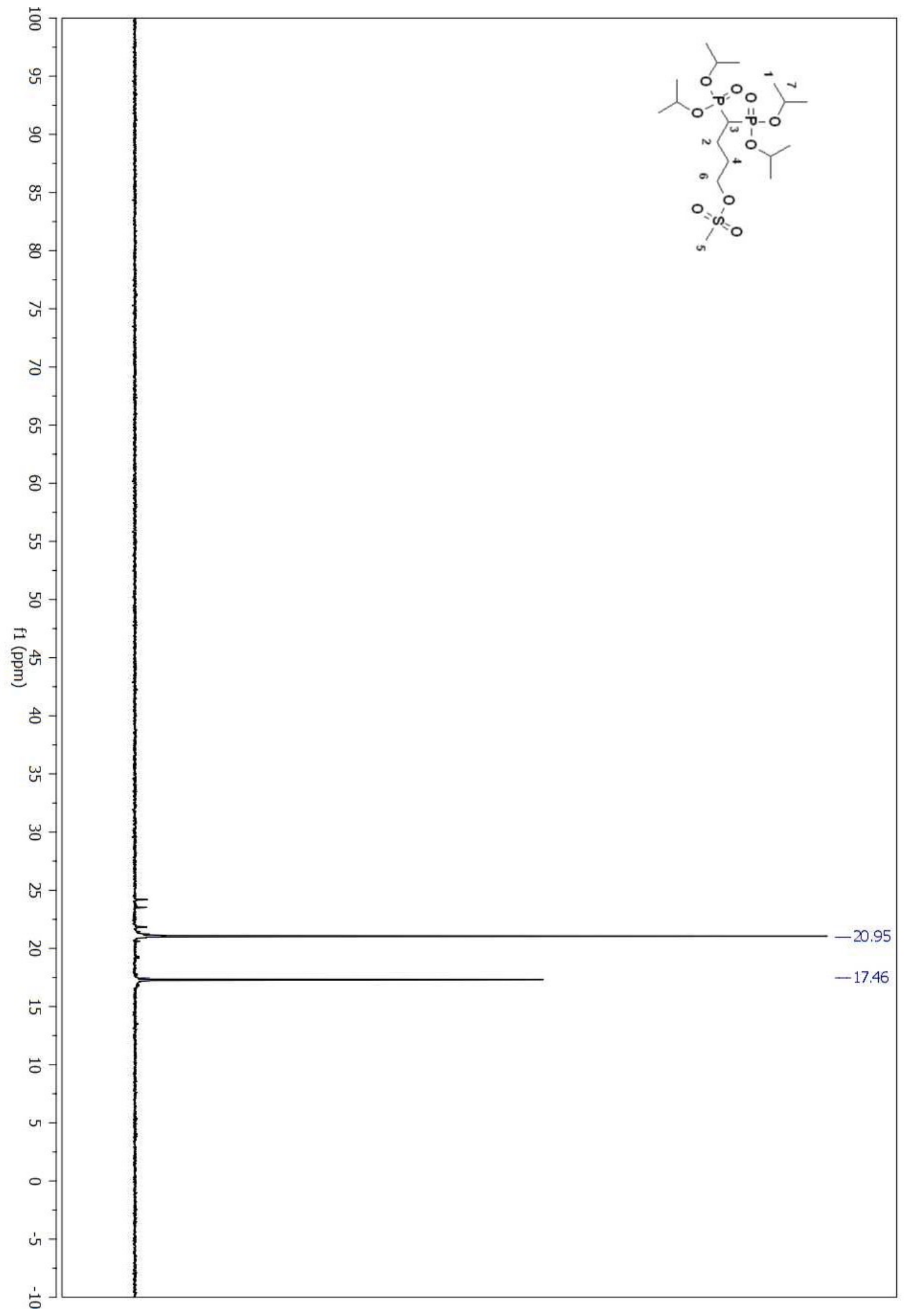

Figure A 170. ${ }^{31} \mathrm{P}$ NMR spectrum of compound (99) in $\mathrm{CDCl}_{3}$. 


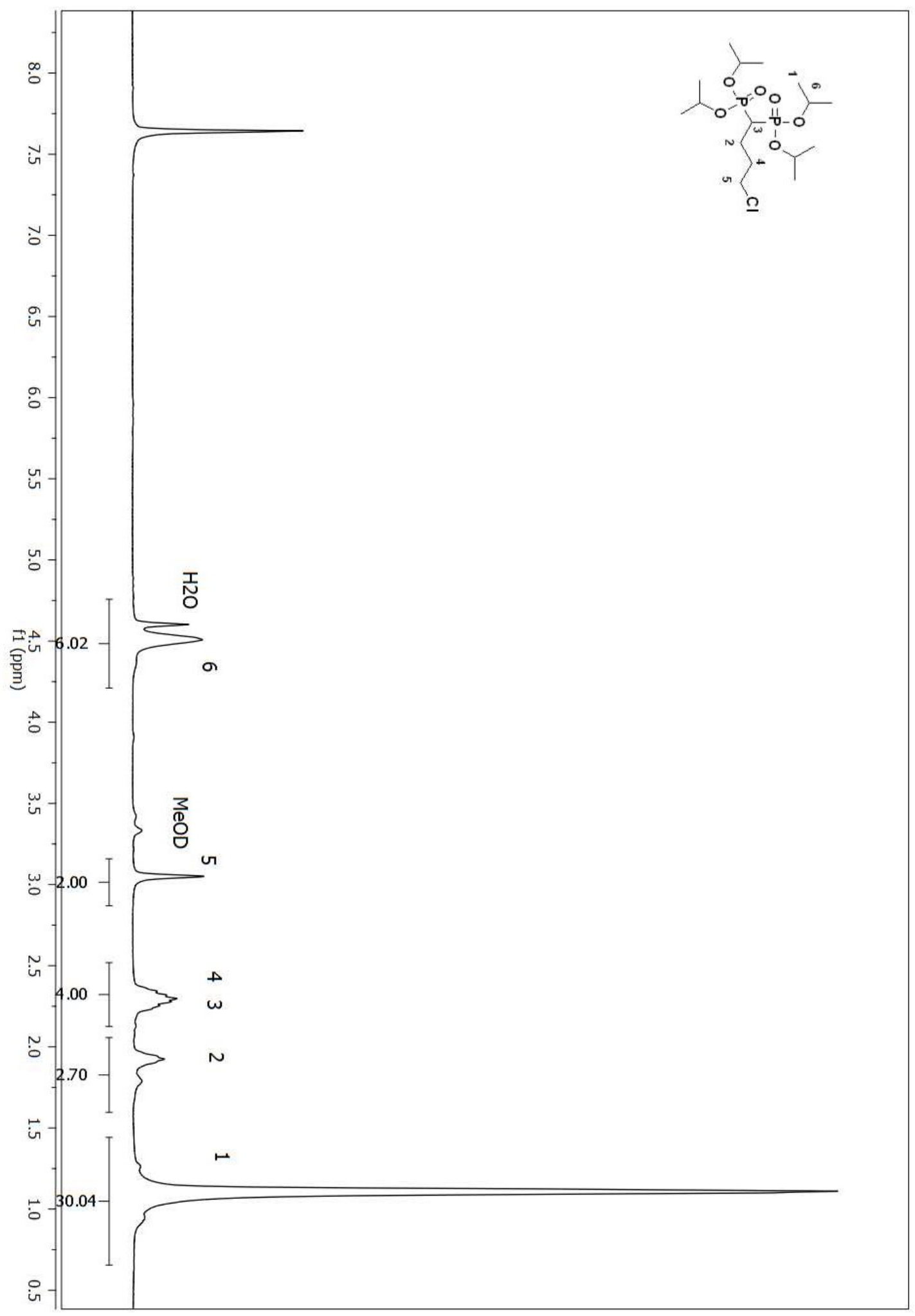

Figure A 171. ${ }^{1} \mathrm{H}$ NMR spectrum of compound (91) in MeOD. 


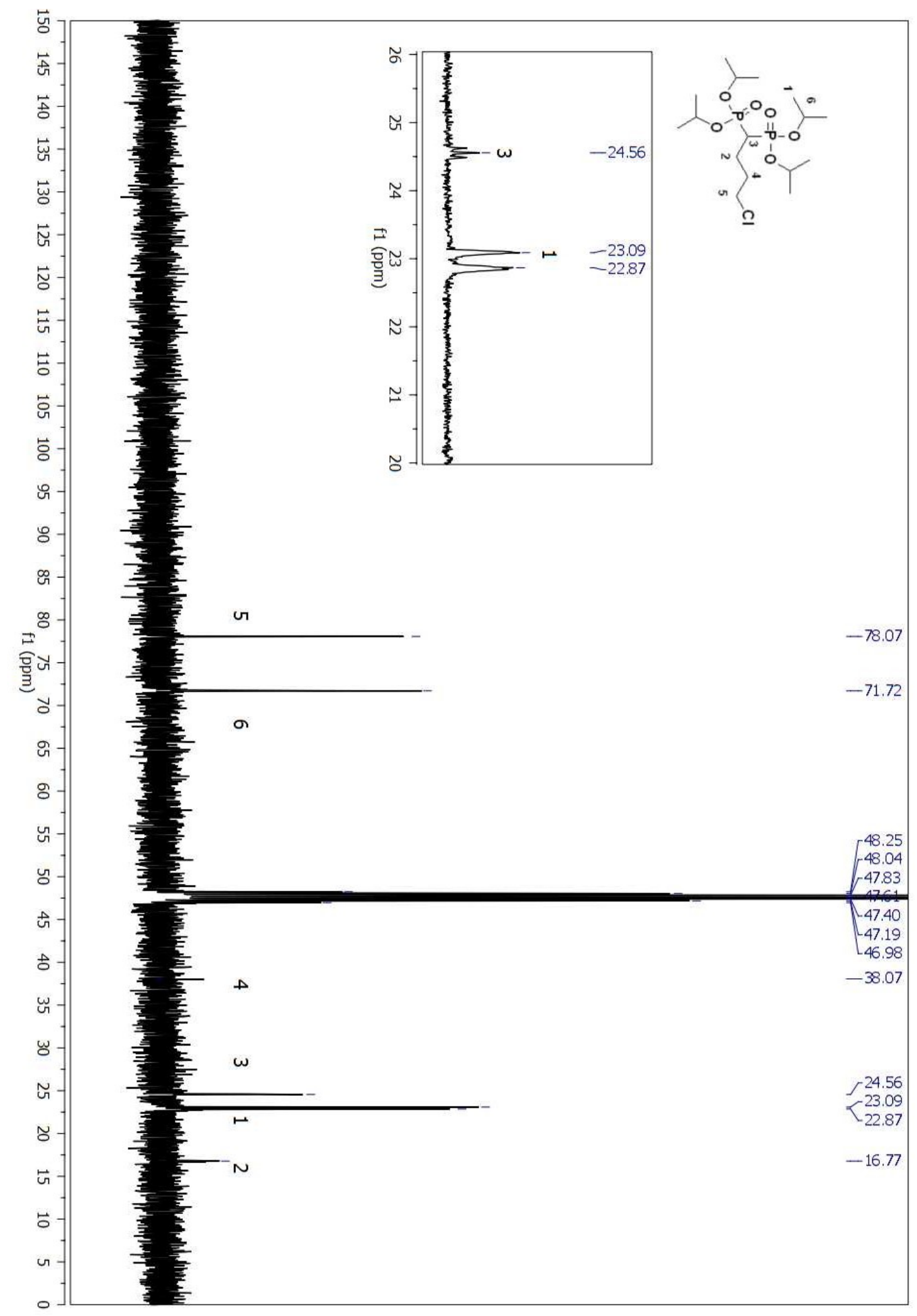

Figure A 172. ${ }^{13} \mathrm{C}$ NMR spectrum of compound (91) in MeOD. 


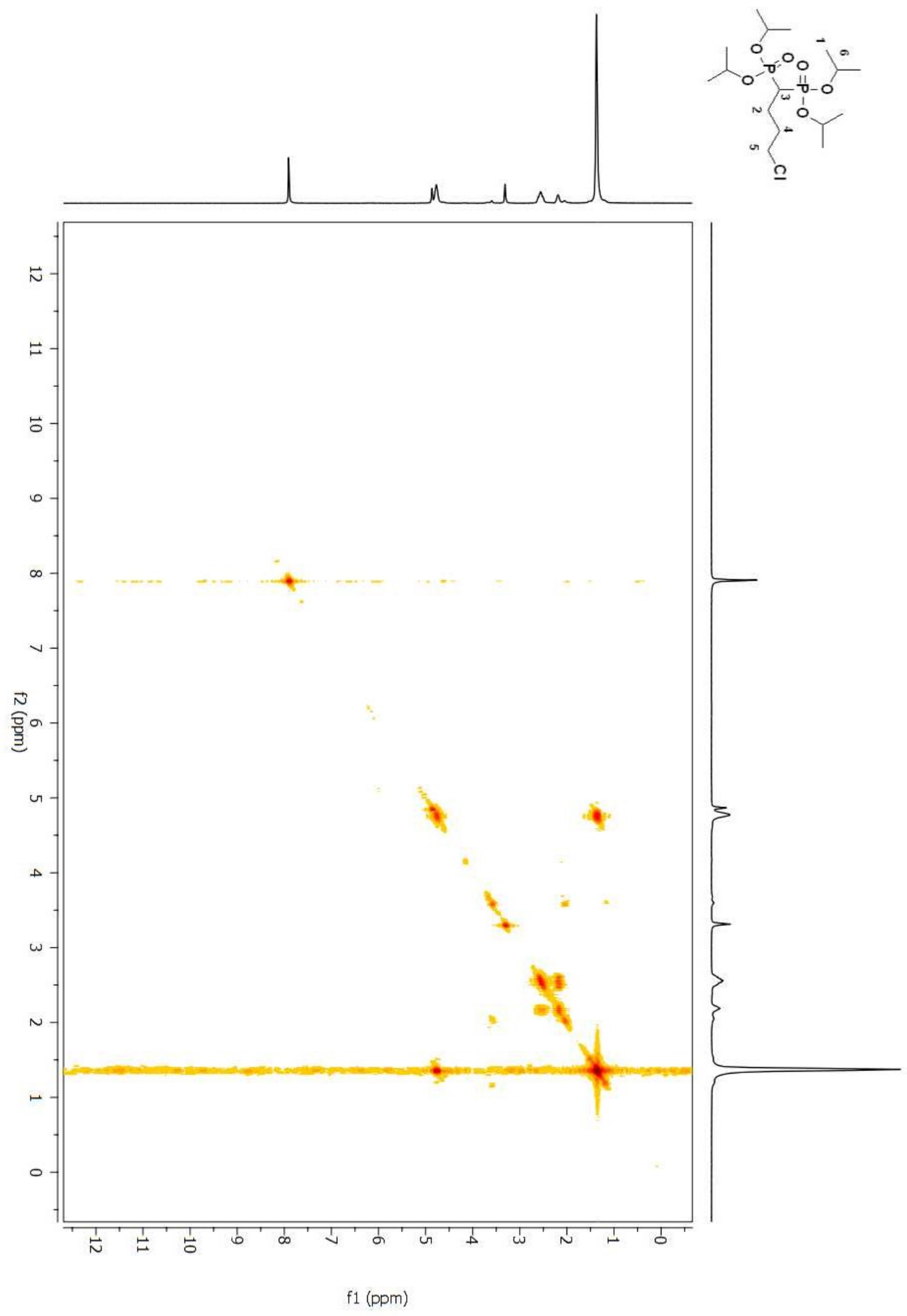

Figure A 173. COSY 2D NMR spectrum of compound (91) in MeOD. 


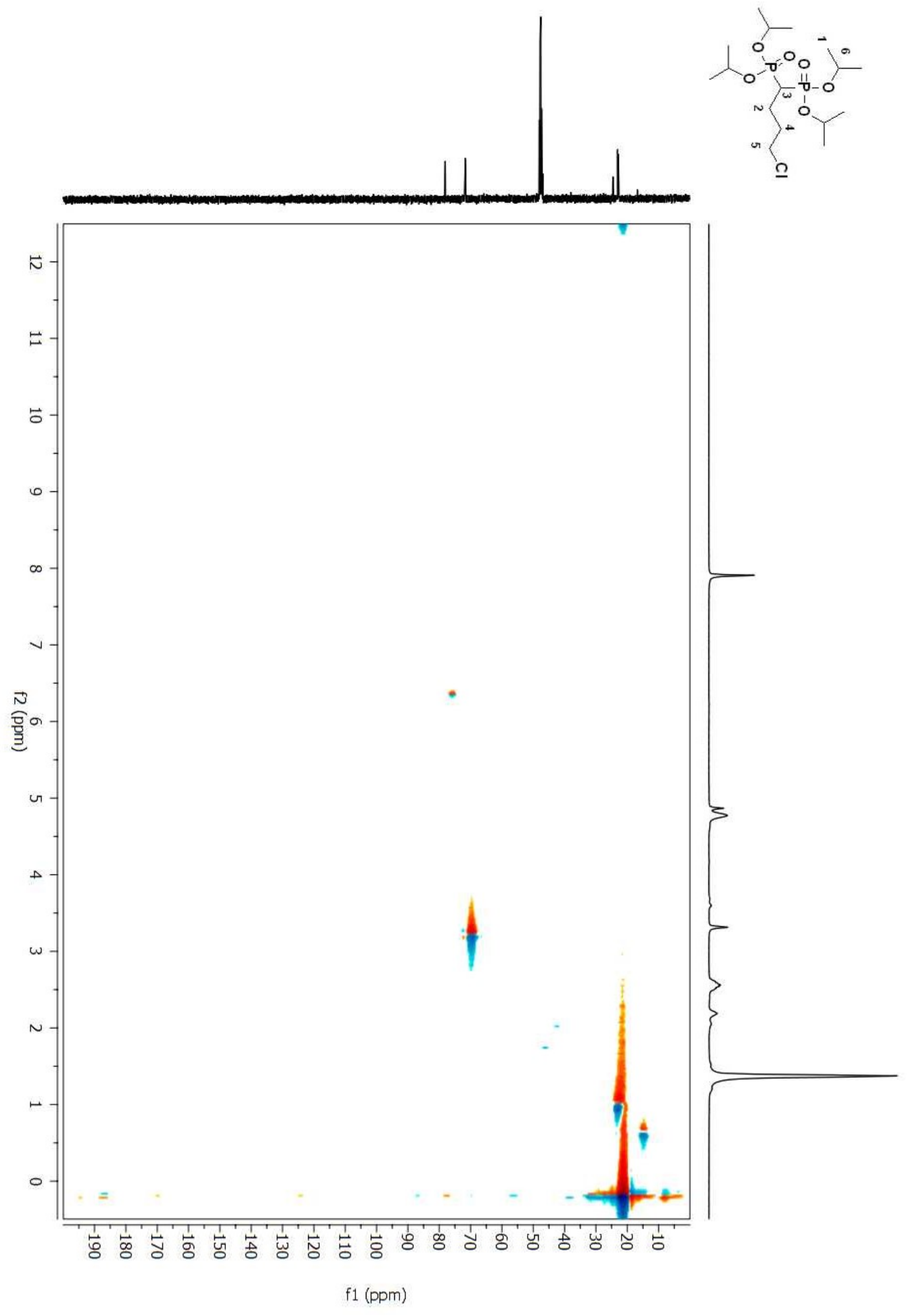

Figure A 174. HSQC 2D NMR spectrum of compound (91) in MeOD. 


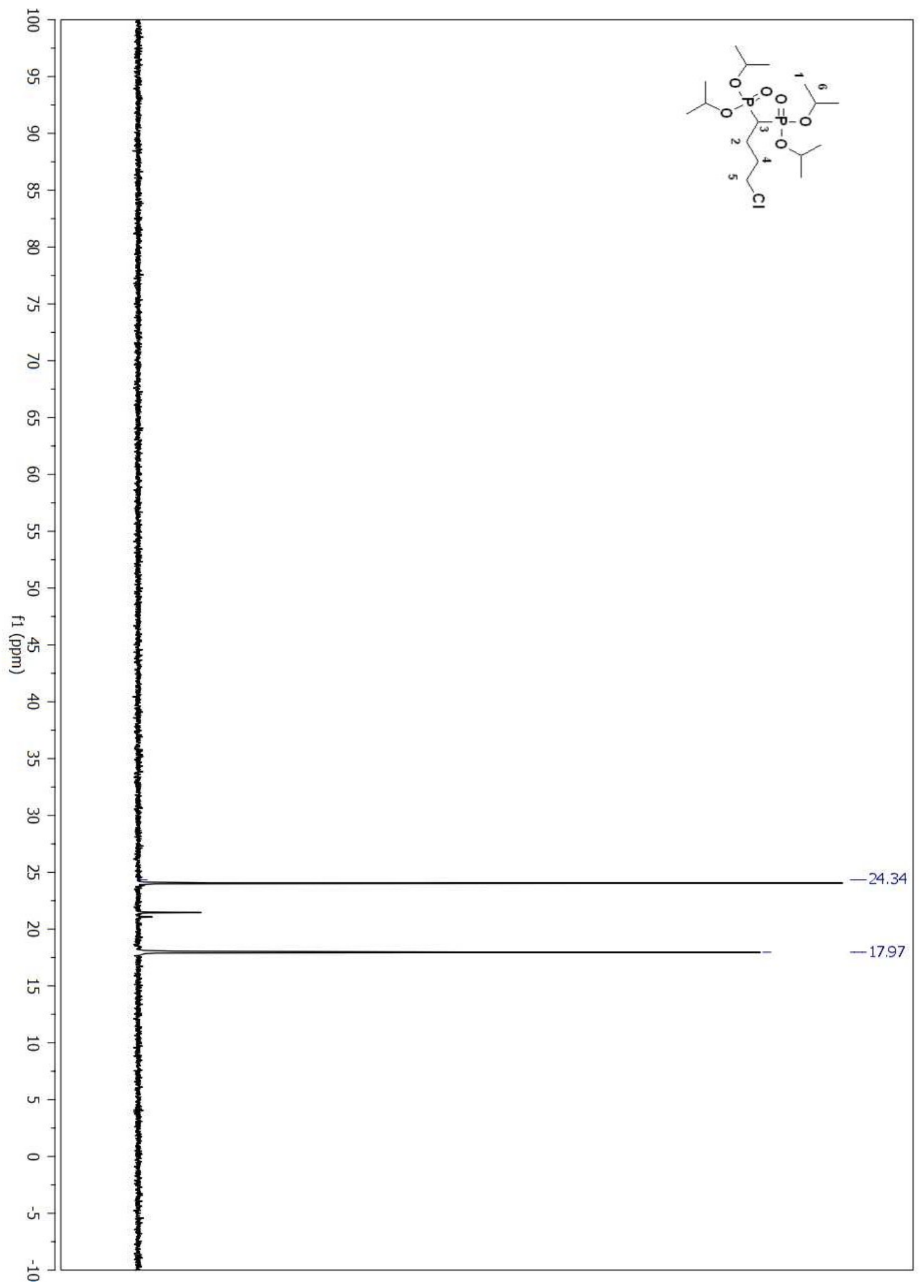

Figure A 175. ${ }^{31} \mathrm{P}$ NMR spectrum of compound (91) in MeOD. (CRUDE) 


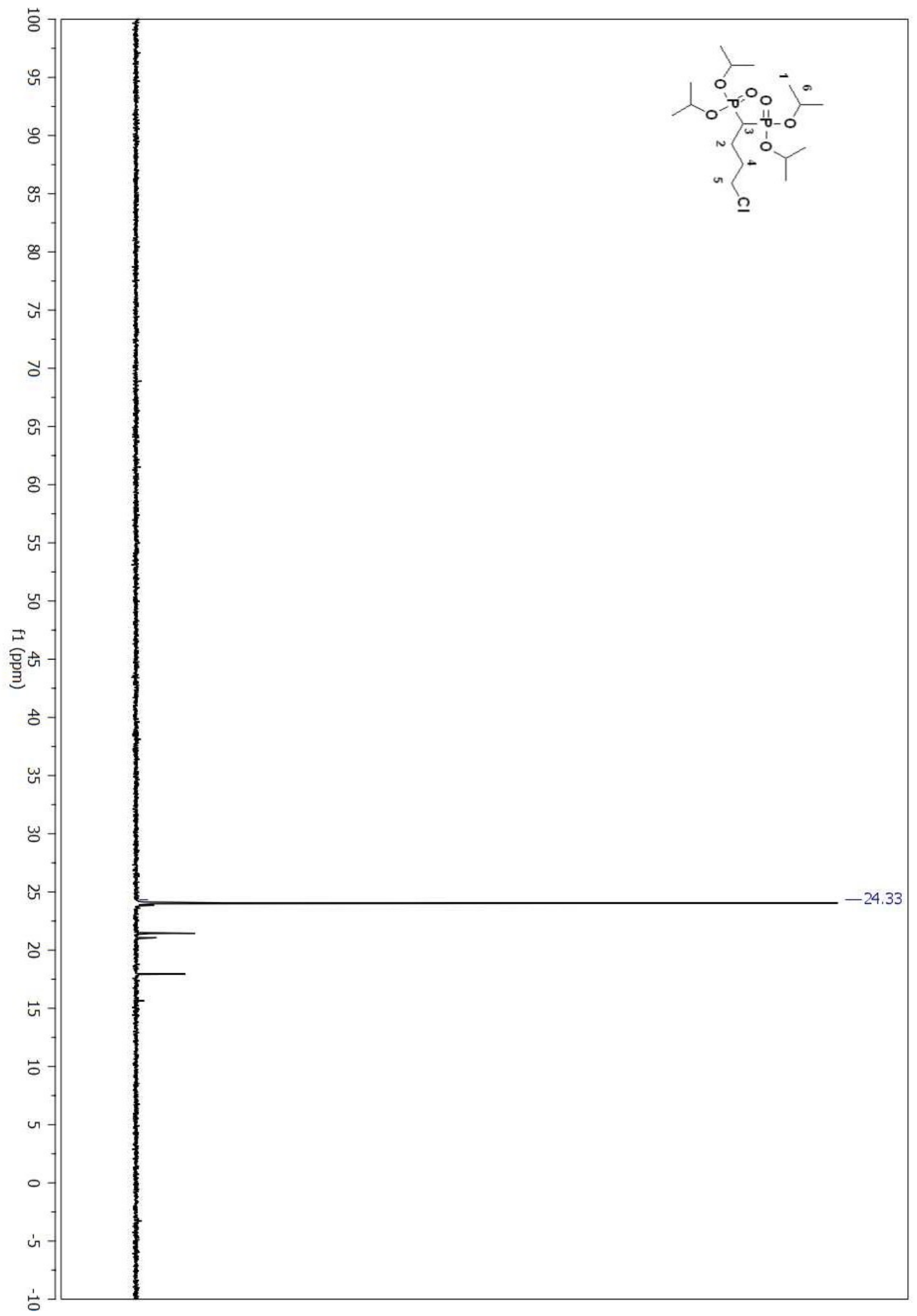

Figure A 176. ${ }^{31} \mathrm{P}$ NMR spectrum of compound (91) in MeOD. (COLUMNED FRACTION) 543 


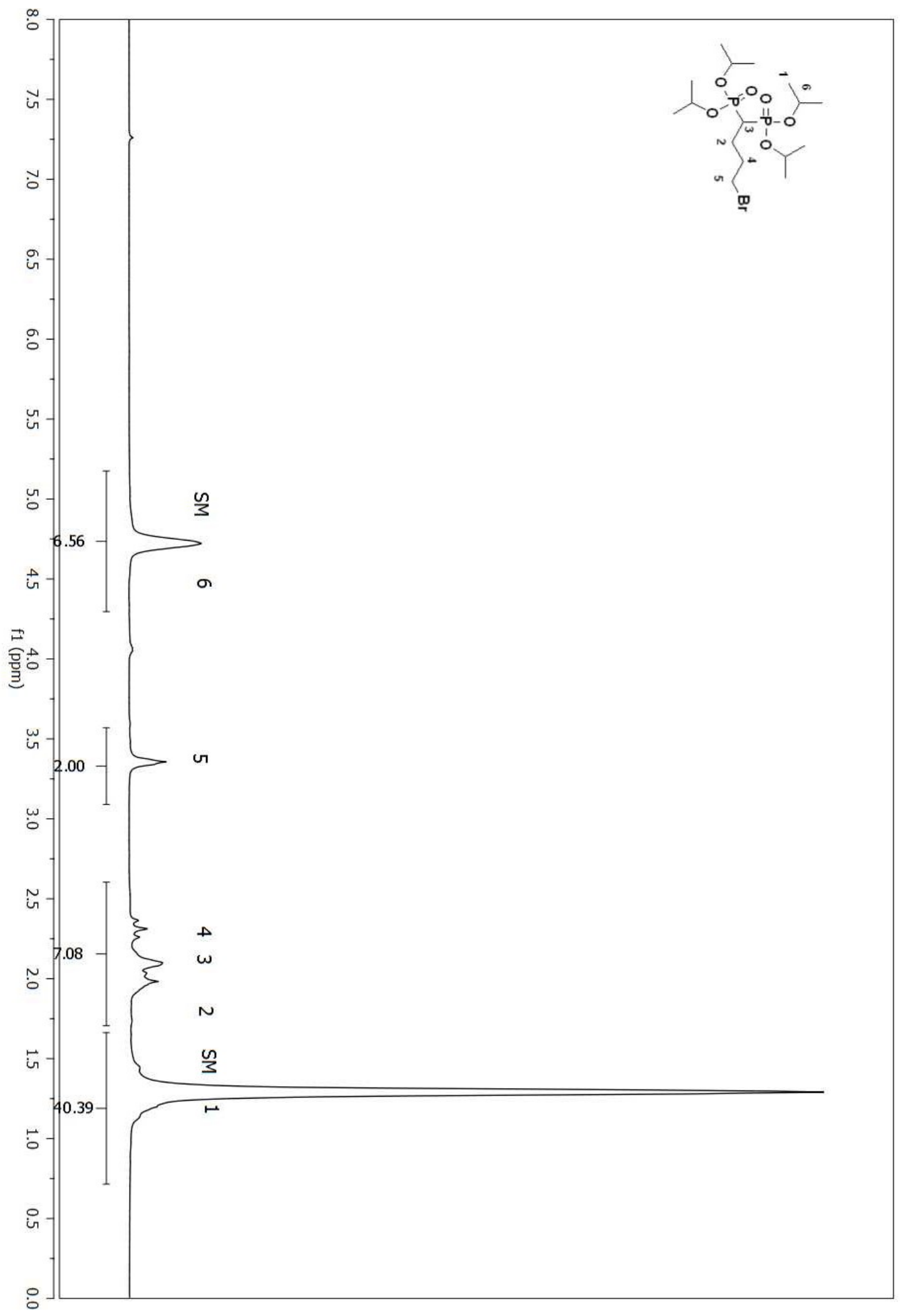

Figure A 177. ${ }^{1} \mathrm{H}$ NMR spectrum of compound (87) in $\mathrm{CDCl}_{3}$. 


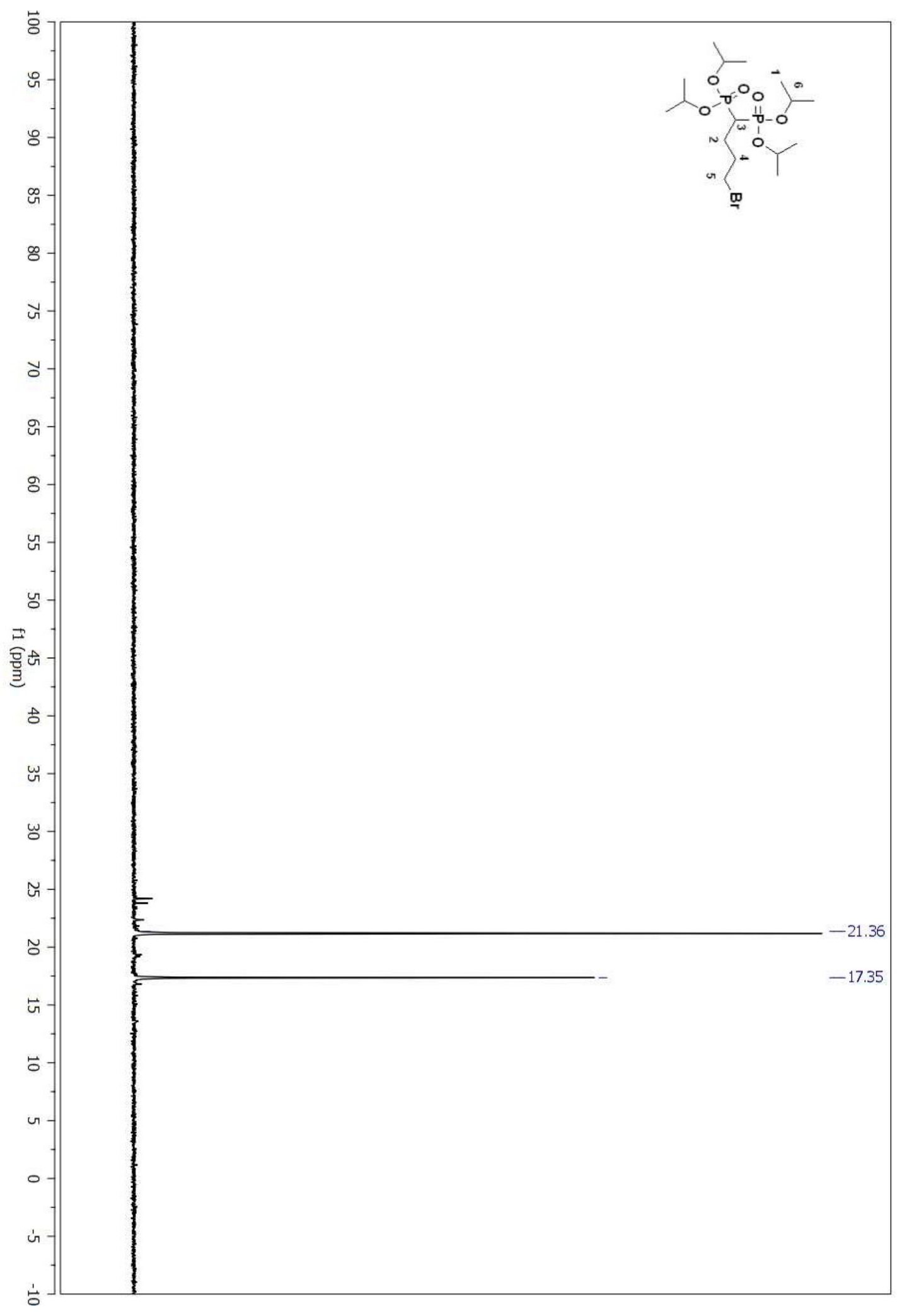

Figure A 178. ${ }^{31} \mathrm{P}$ NMR spectrum of compound (87) in $\mathrm{CDCl}_{3}$. 


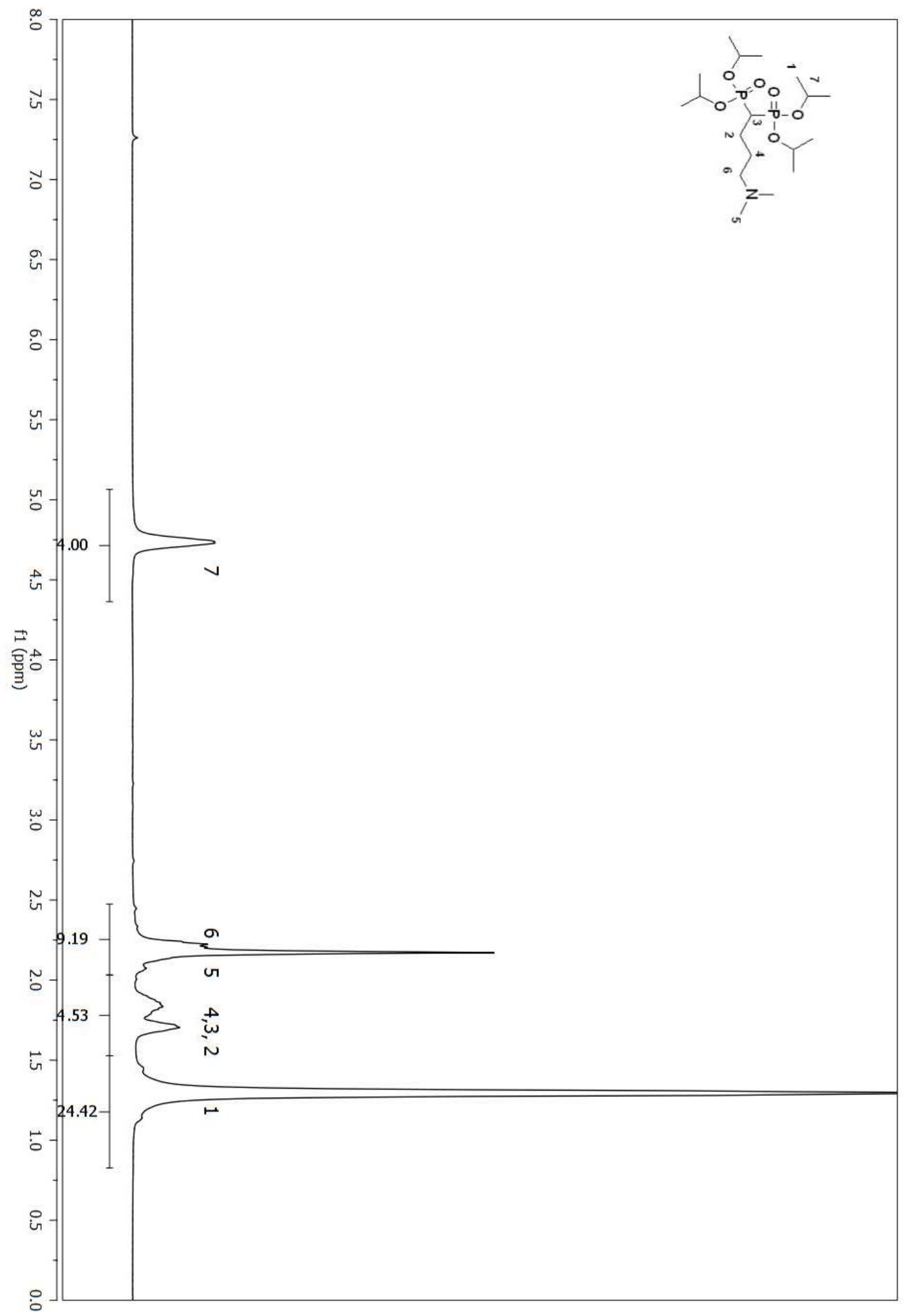

Figure A 179. ${ }^{1} \mathrm{H}$ NMR spectrum of compound (87) in $\mathrm{CDCl}_{3}$. 


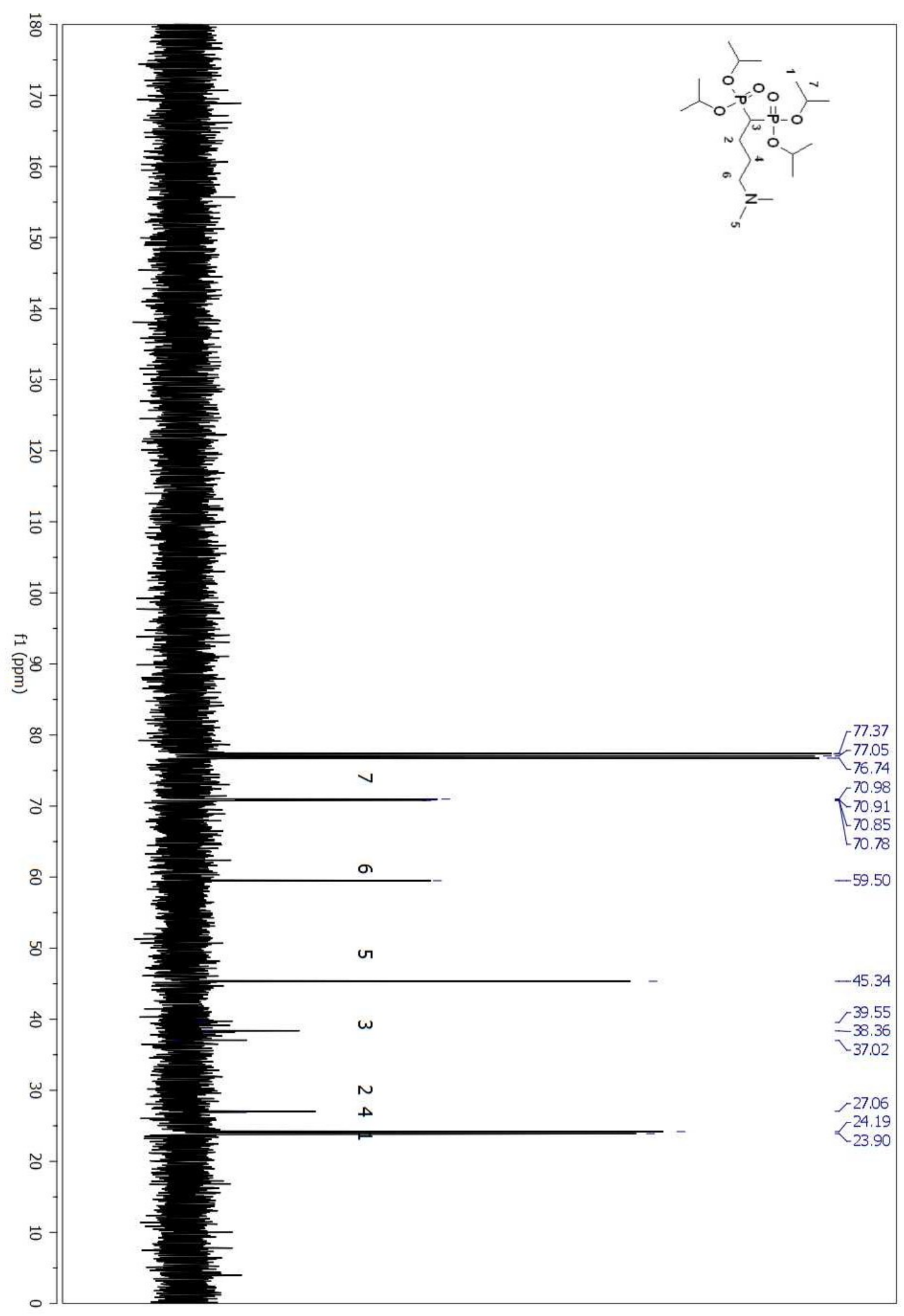

Figure A 180. ${ }^{13} \mathrm{C}$ NMR spectrum of compound (87) in $\mathrm{CDCl}_{3}$. 


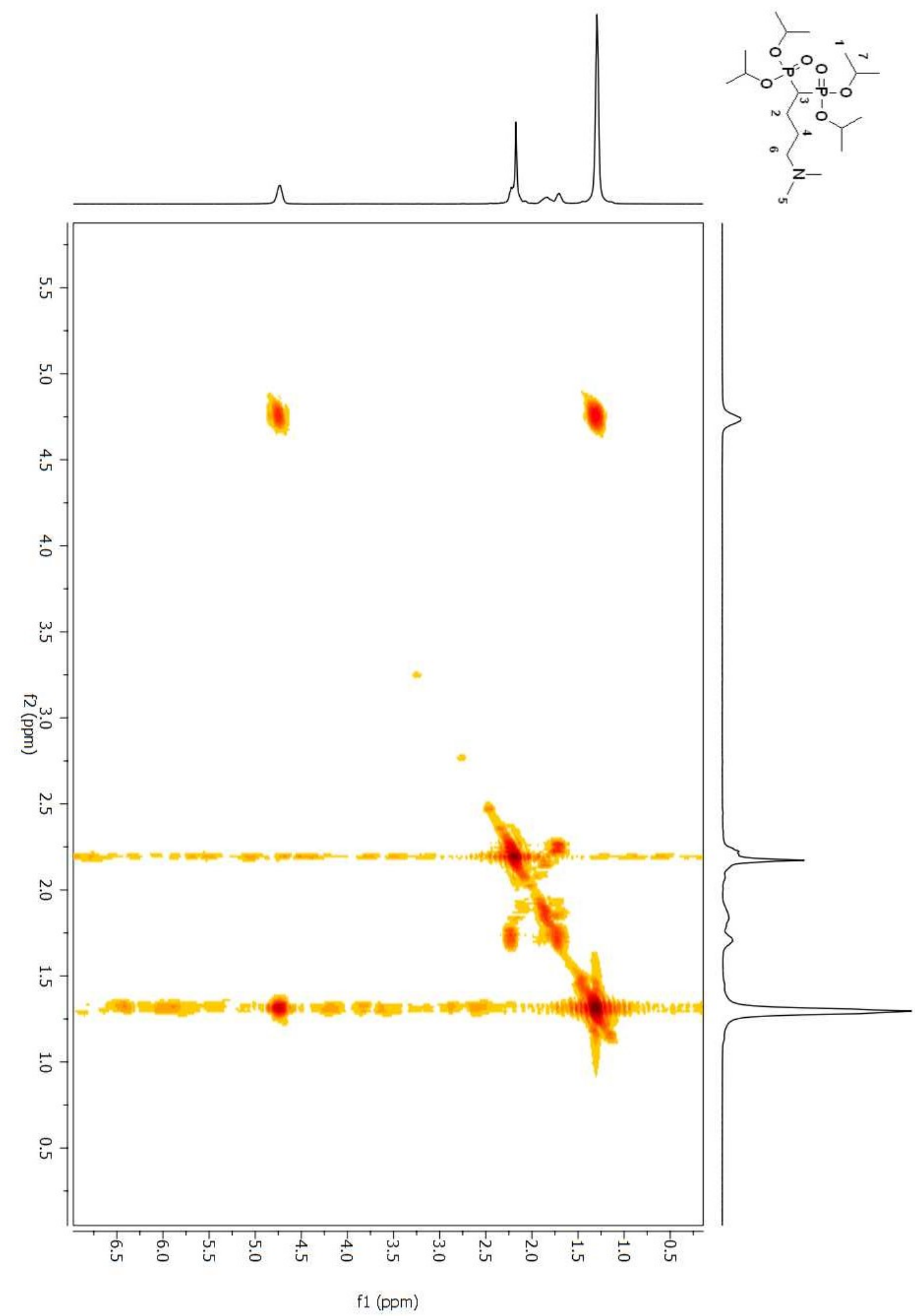

Figure A 181. COSY 2D NMR spectrum of compound (96) in $\mathrm{CDCl}_{3}$. 


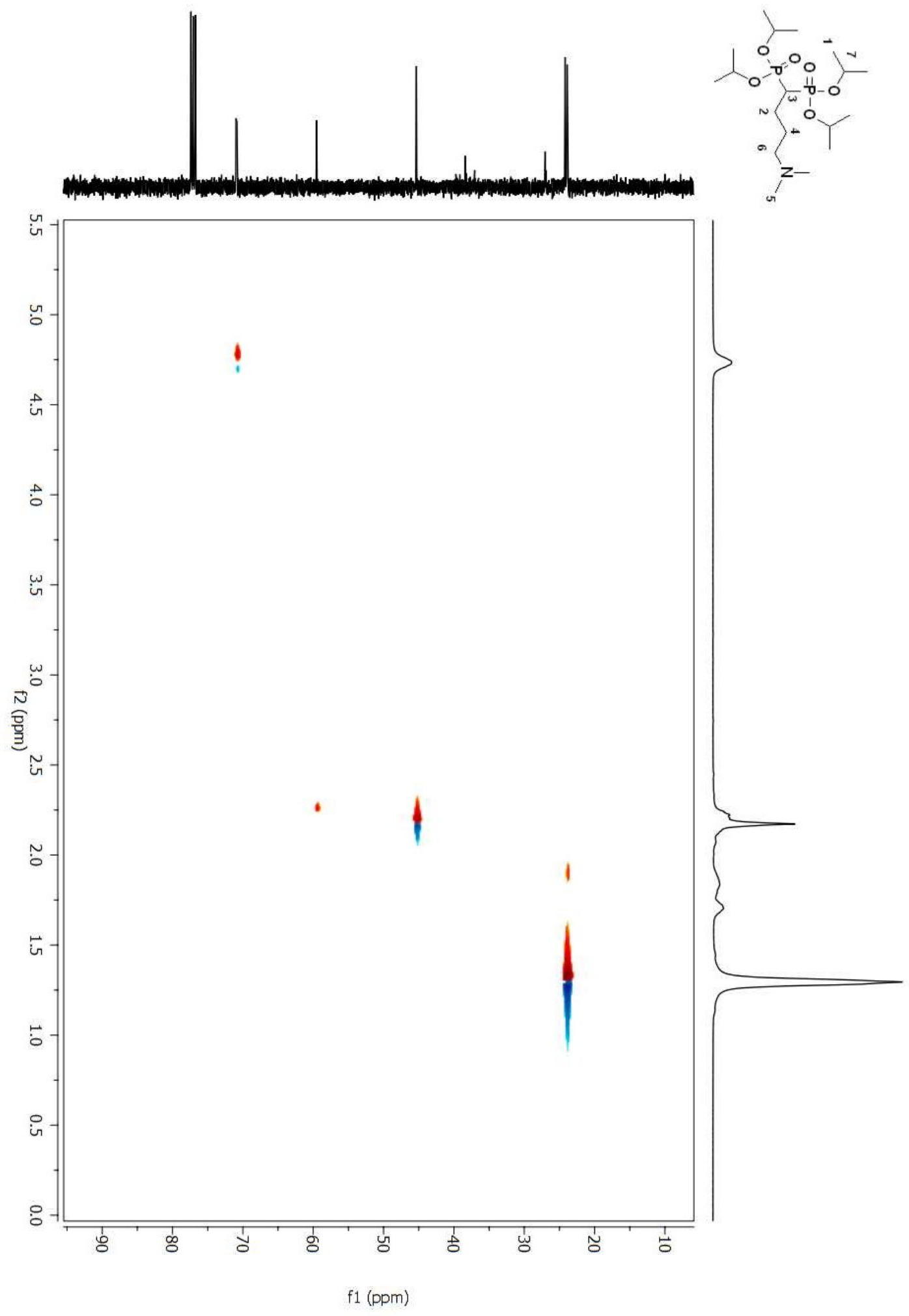

Figure A 182. HSQC 2D NMR spectrum of compound (96) in $\mathrm{CDCl}_{3}$. 


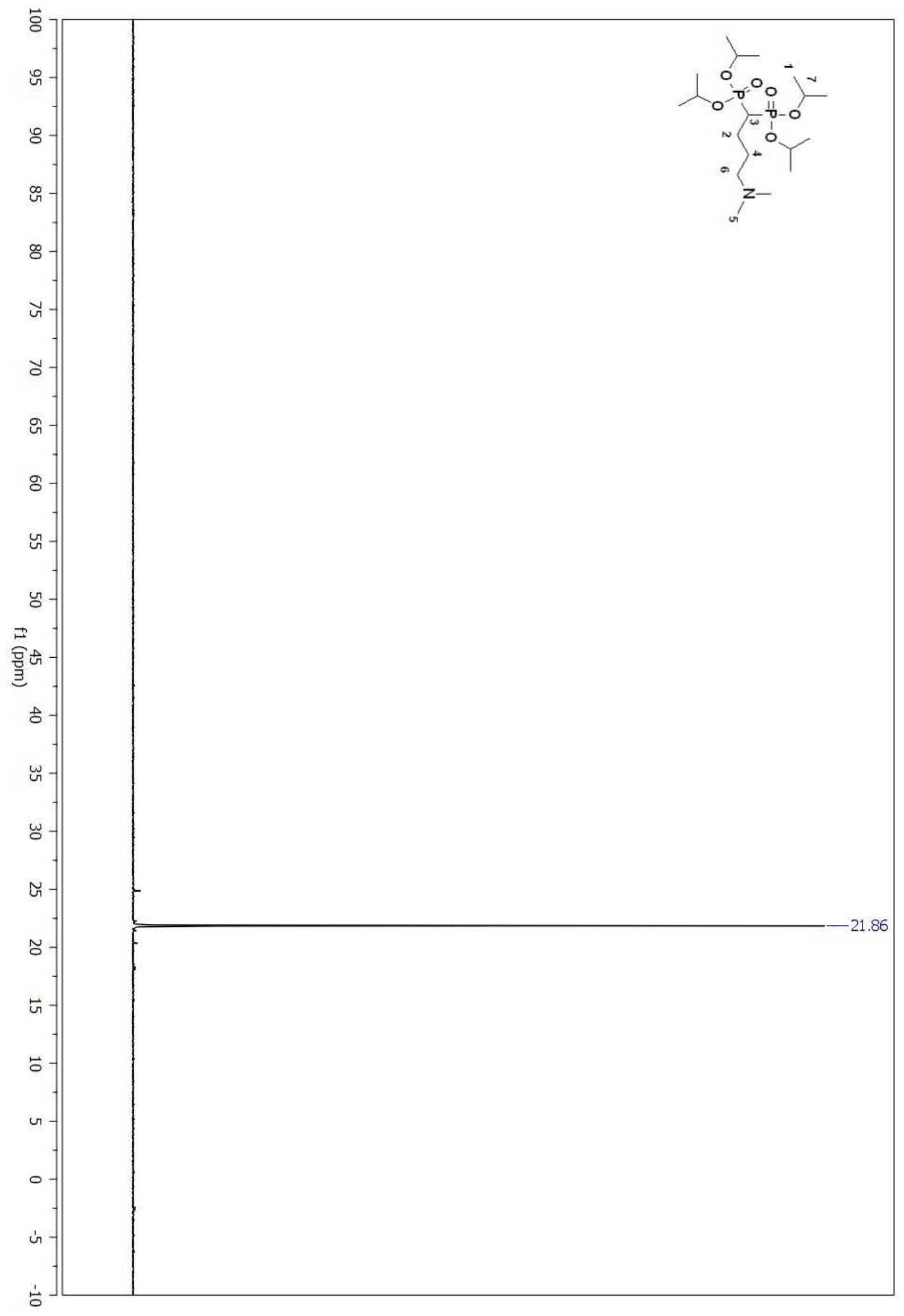

Figure A 183. ${ }^{31} \mathrm{P}$ NMR spectrum of compound (96) in $\mathrm{CDCl}_{3}$. 


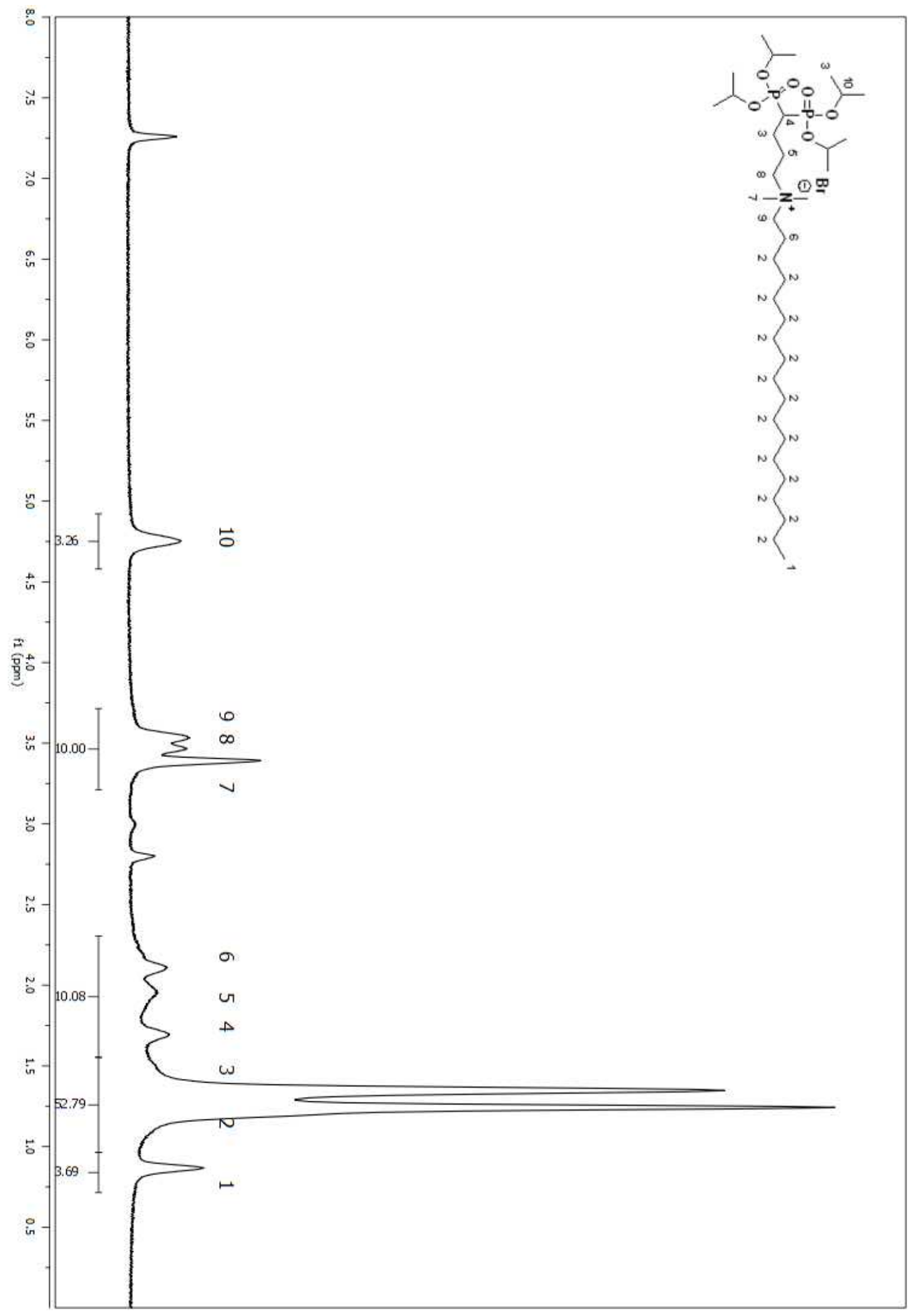

Figure A 184. ${ }^{1} \mathrm{H}$ NMR spectrum of compound (93) in $\mathrm{CDCl}_{3}$. 


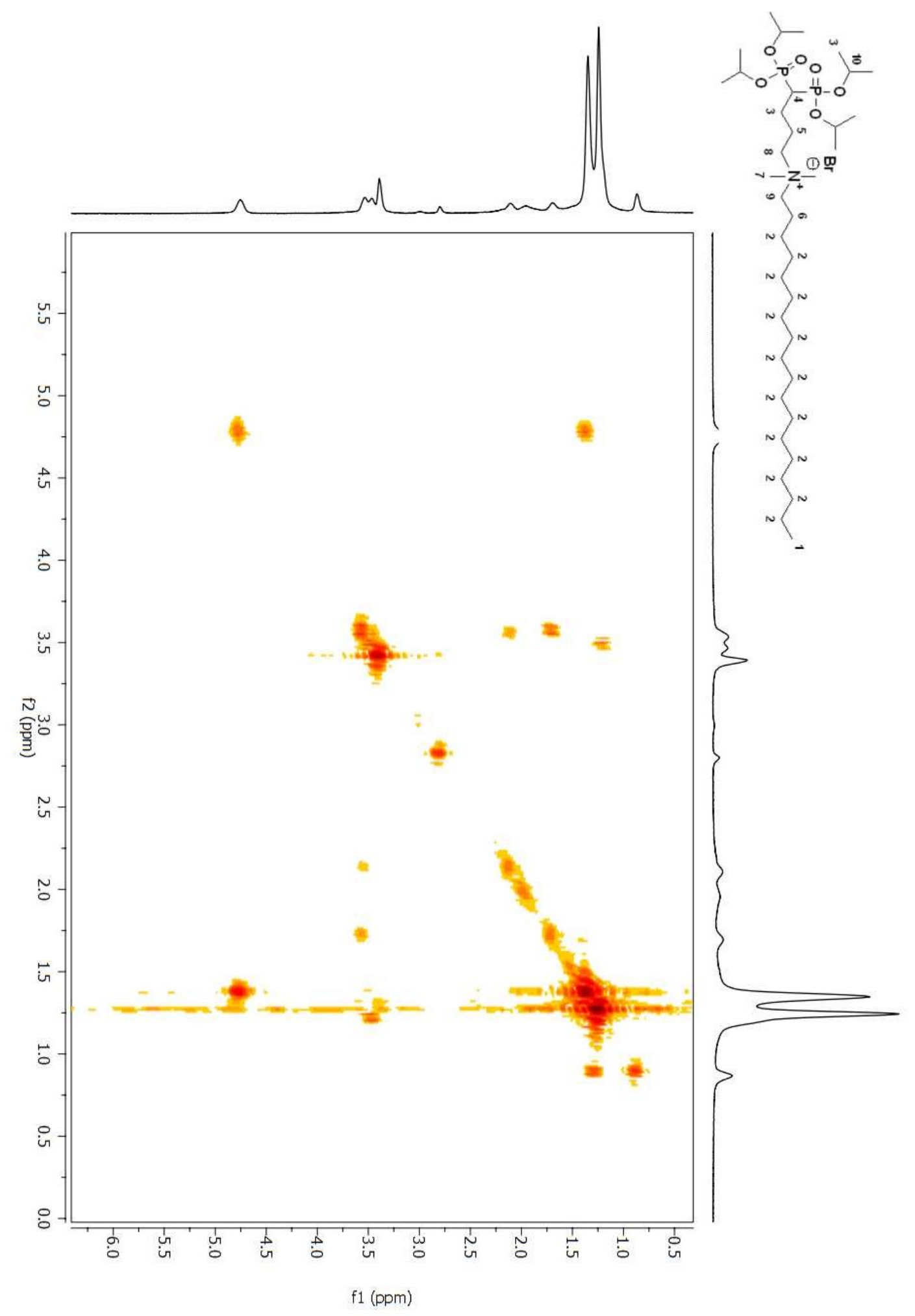

Figure A 185. COSY 2D NMR spectrum of compound (93) in $\mathrm{CDCl}_{3}$. 


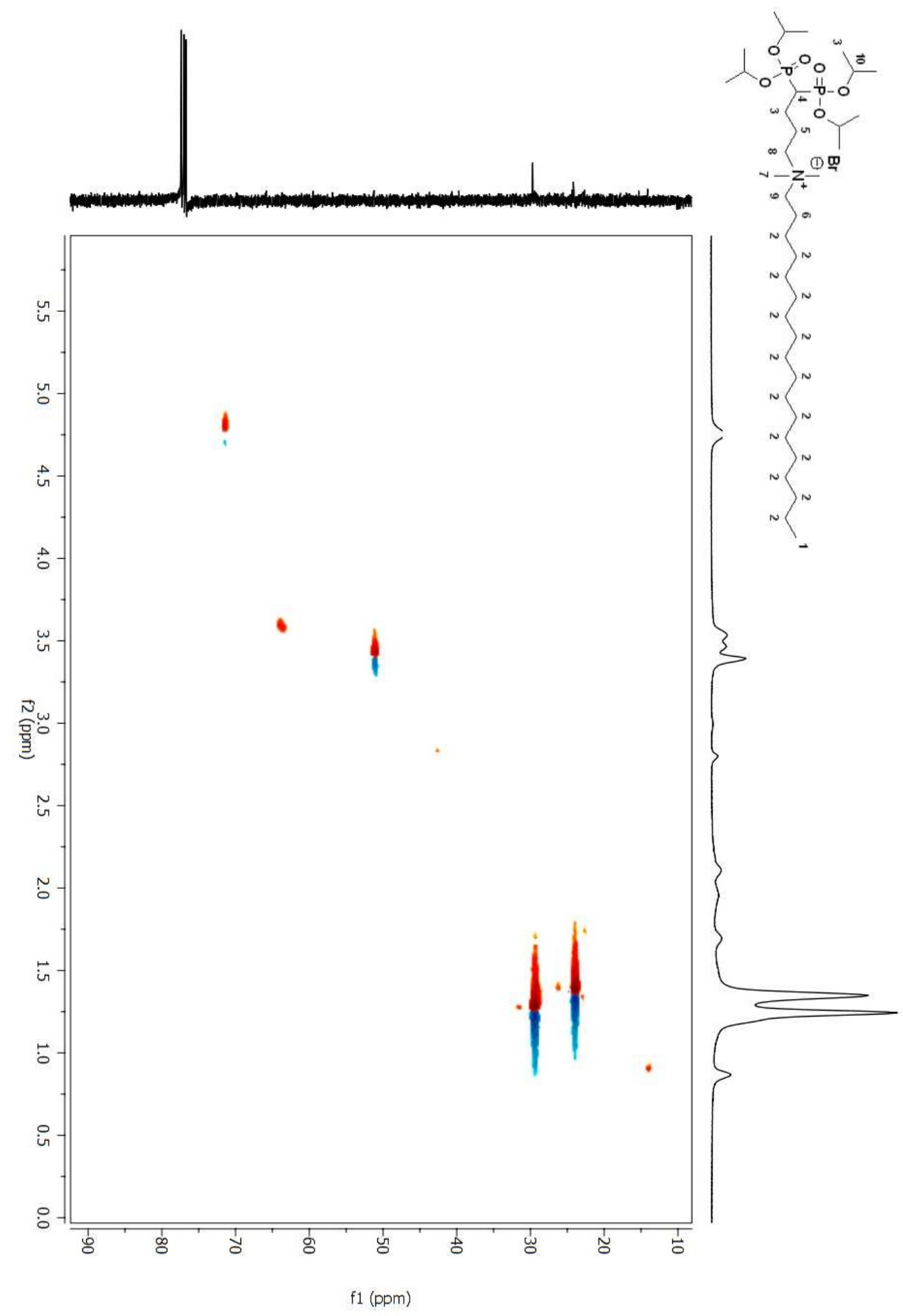

Figure A 186. HSQC 2D NMR spectrum of compound (93) in $\mathrm{CDCl}_{3}$. 


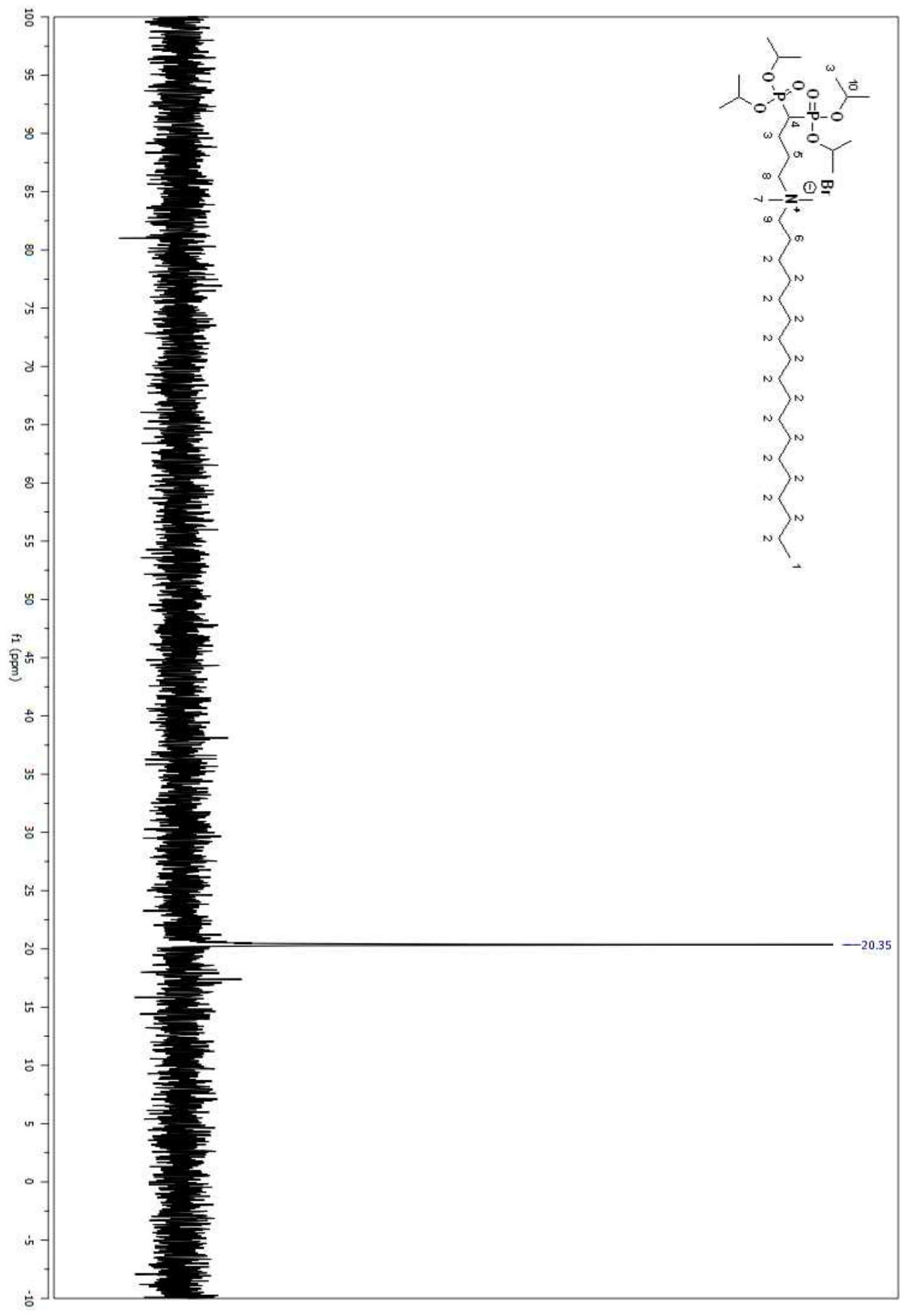

Figure A 187. ${ }^{31} \mathrm{P}$ NMR spectrum of compound (93) in $\mathrm{CDCl}_{3}$. 


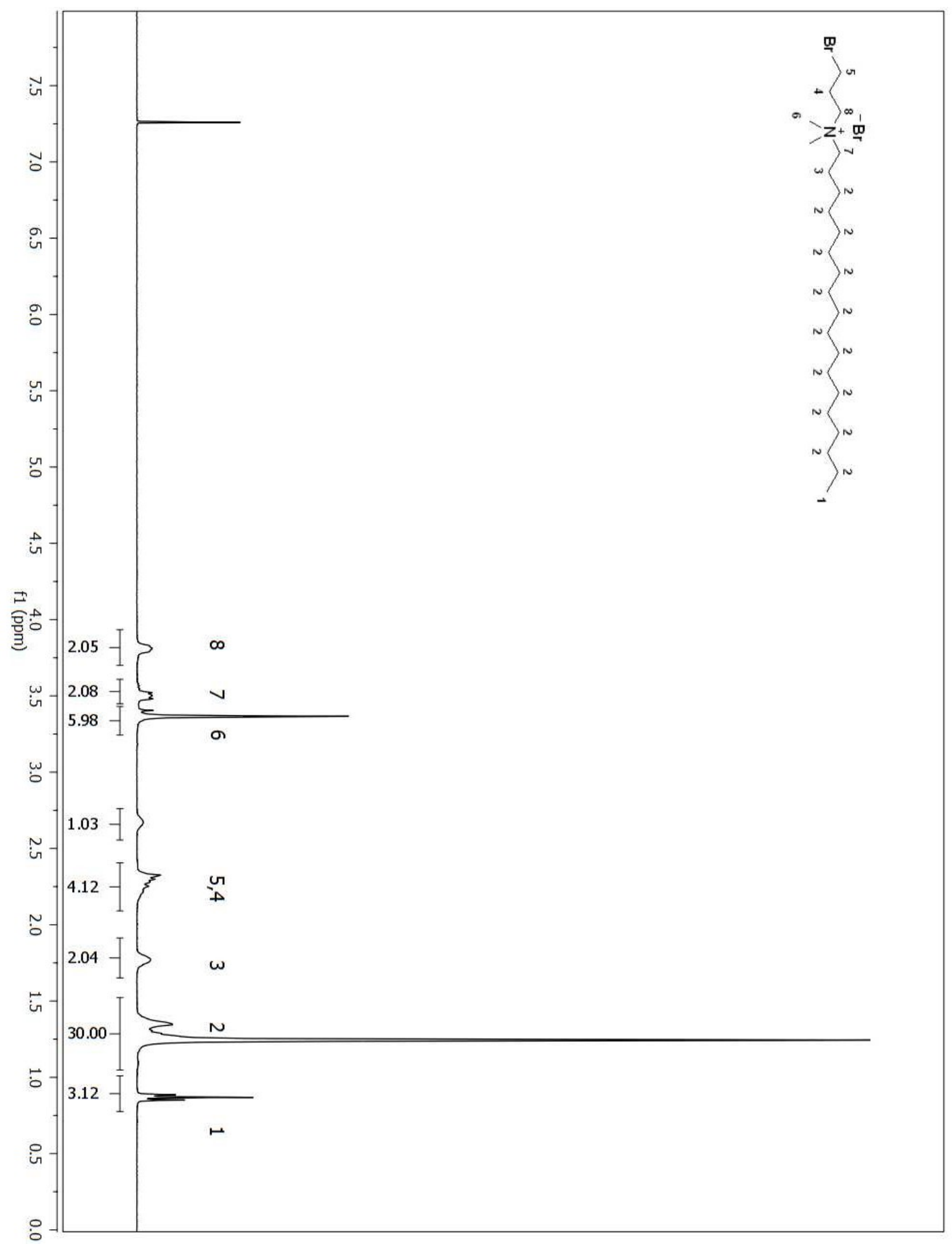

Figure A 188. ${ }^{1} \mathrm{H}$ NMR spectrum of compound (83) in $\mathrm{CDCl}_{3}$. 


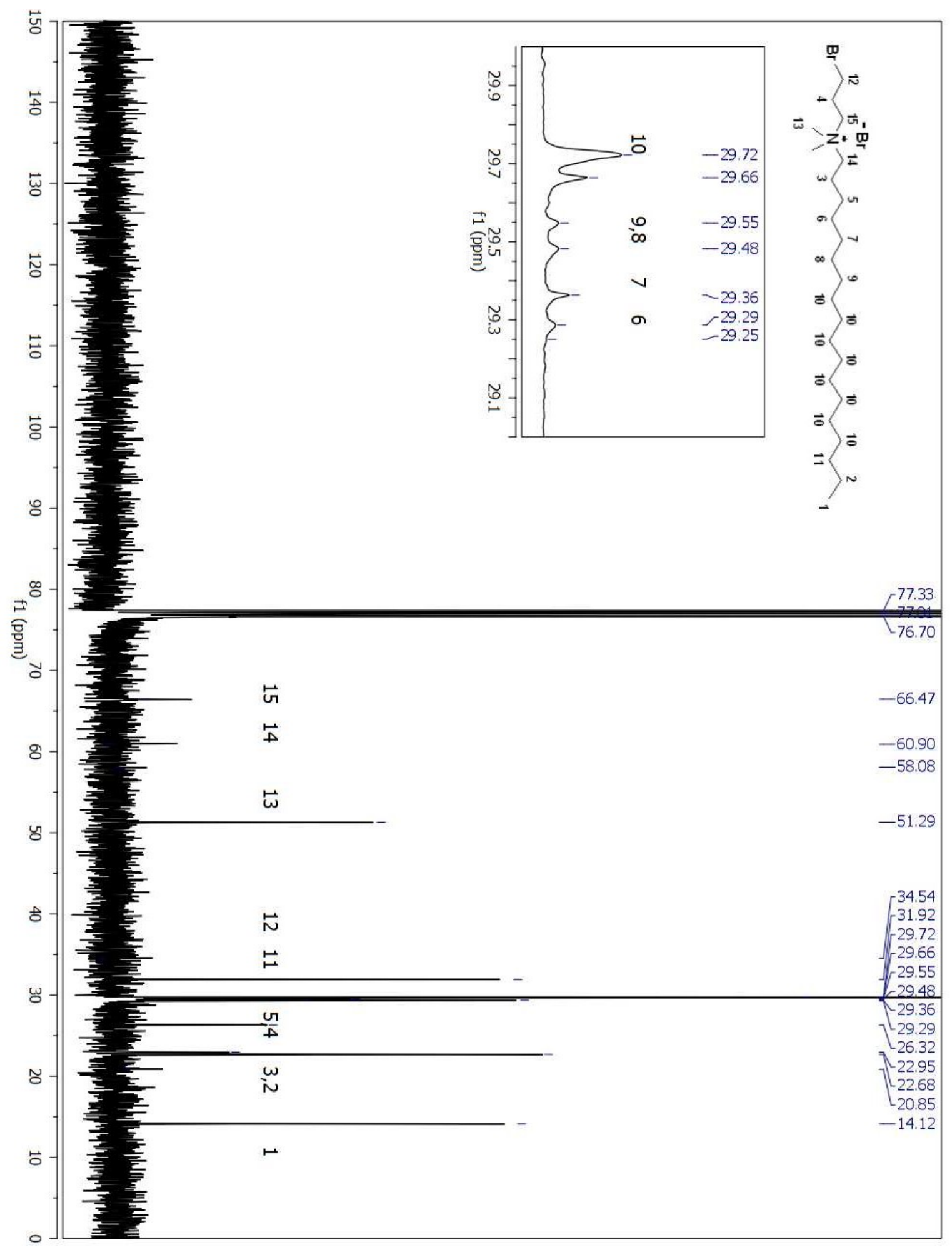

Figure A 189. ${ }^{13} \mathrm{C}$ NMR spectrum of compound (83) in $\mathrm{CDCl}_{3}$. 


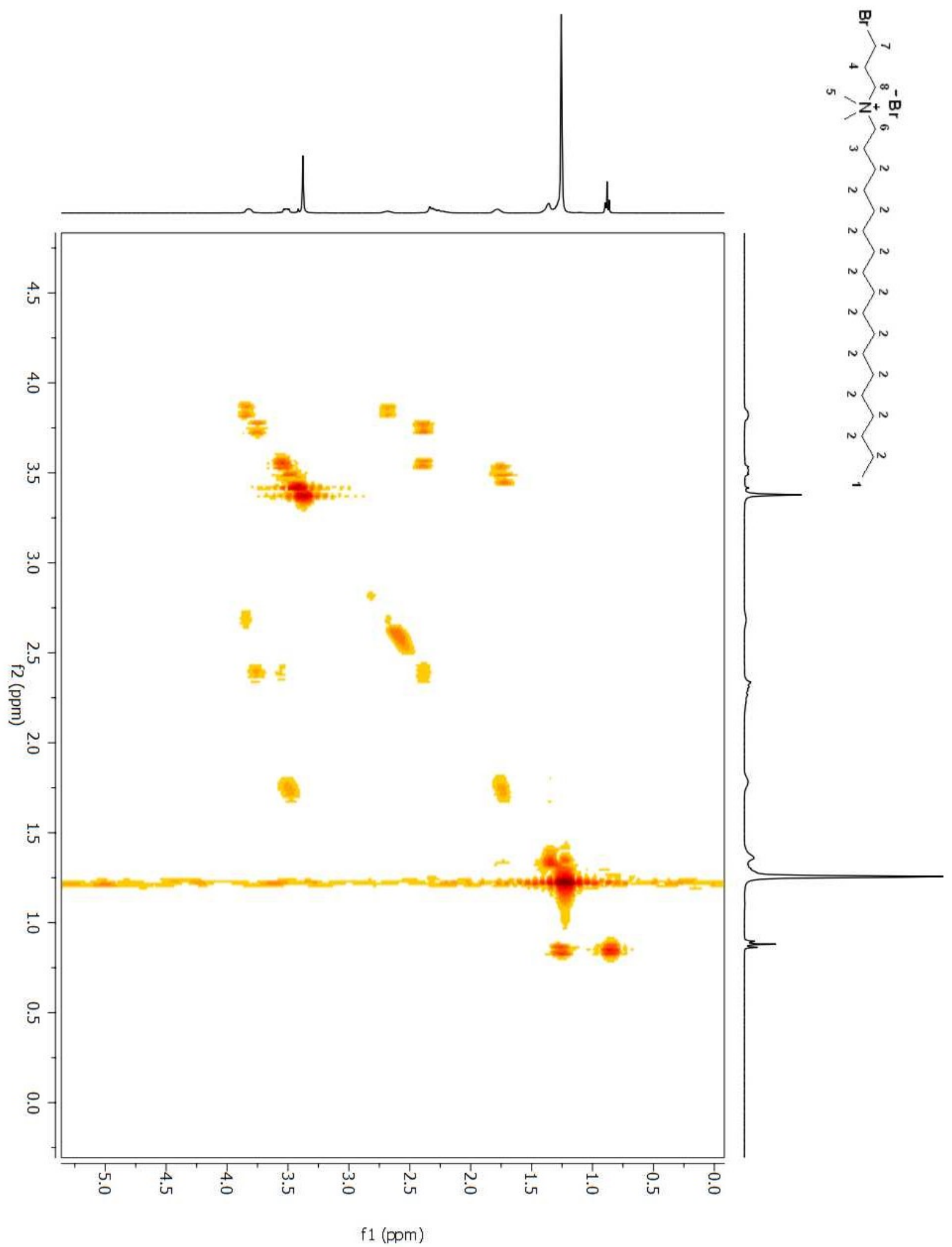

Figure A 190. COSY 2D NMR spectrum of compound (83) in $\mathrm{CDCl}_{3}$. 


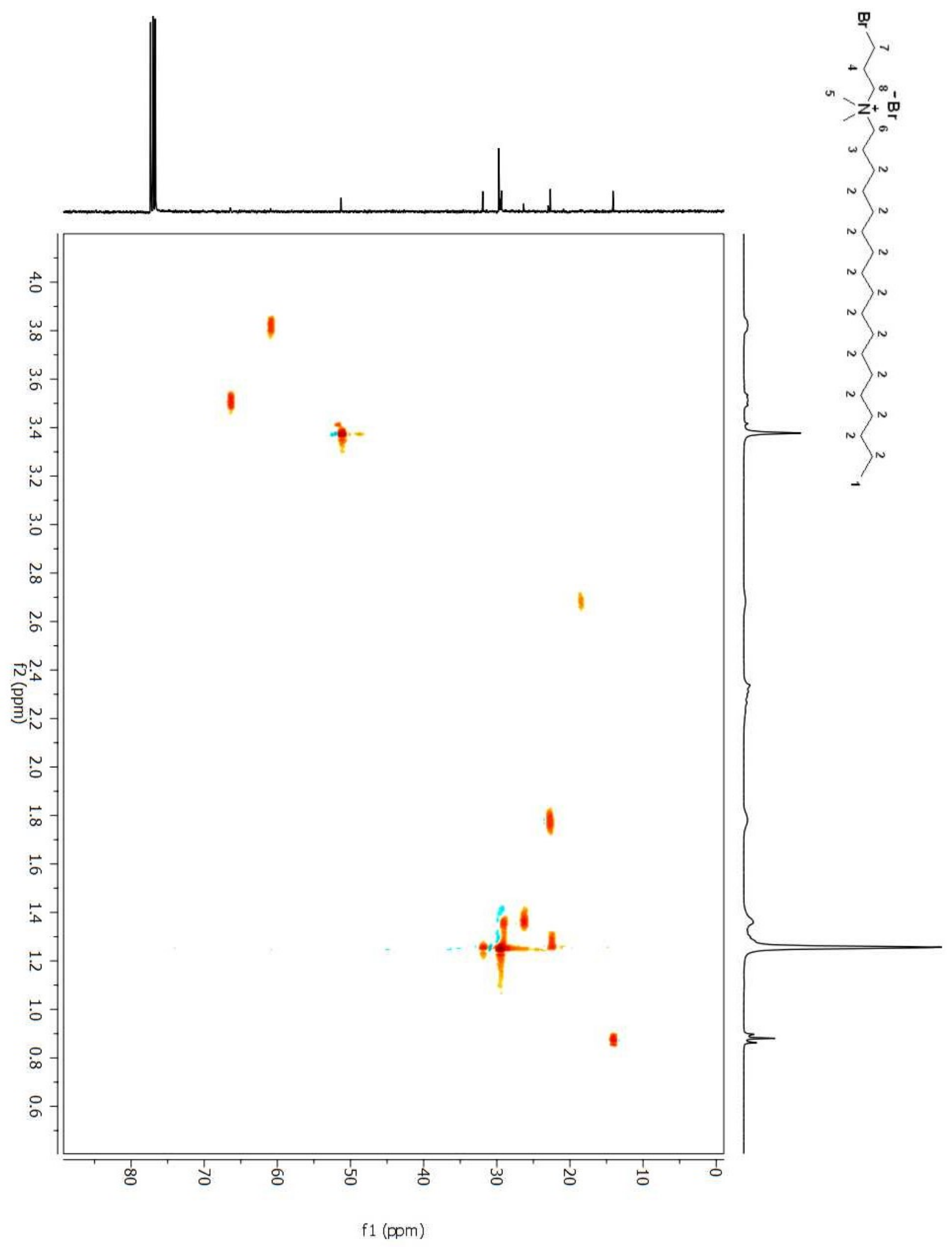

Figure A 191. HSQC 2D NMR spectrum of compound (83) in $\mathrm{CDCl}_{3}$. 


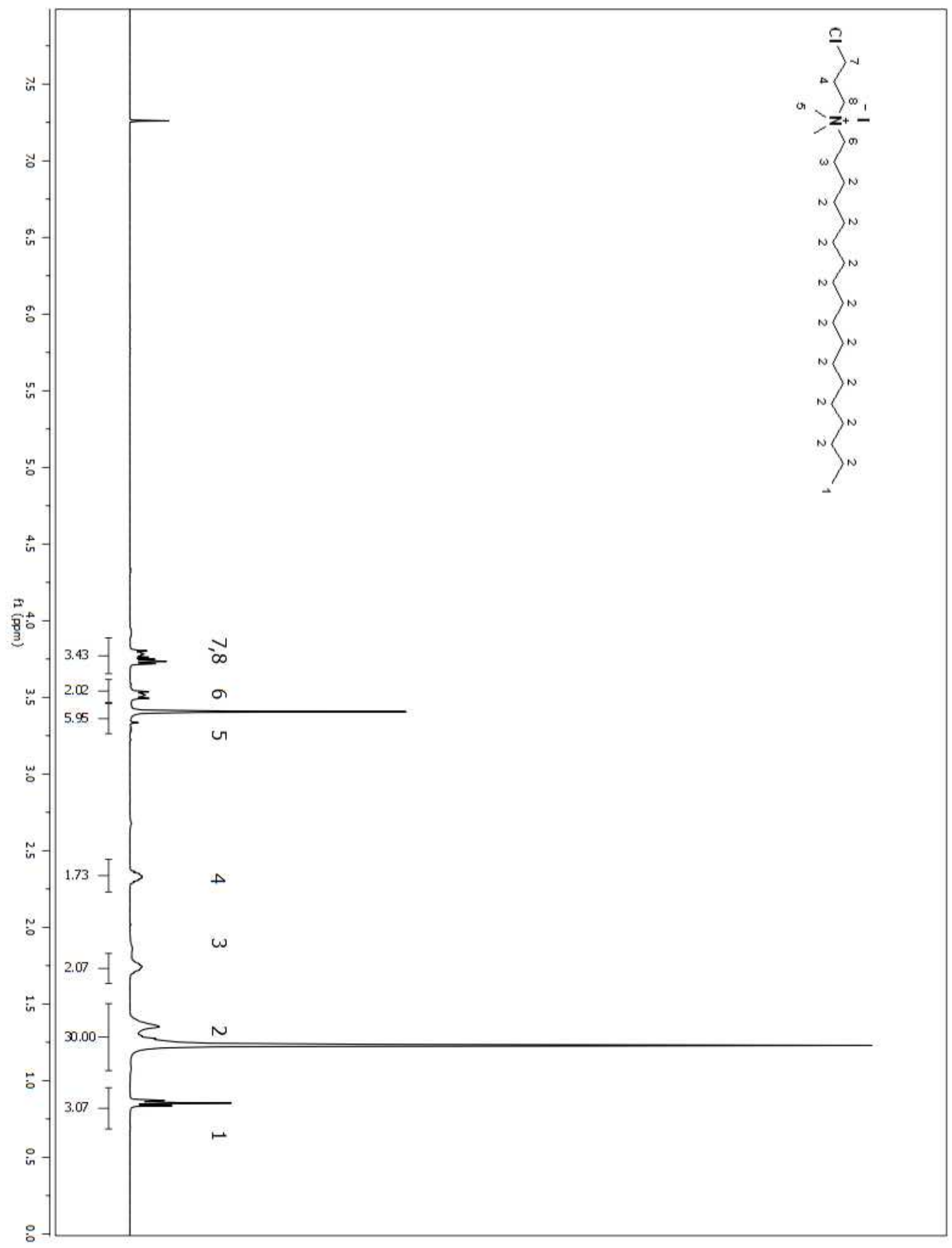

Figure A 192. ${ }^{1} \mathrm{H}$ NMR spectrum of compound (102) in $\mathrm{CDCl}_{3}$. 


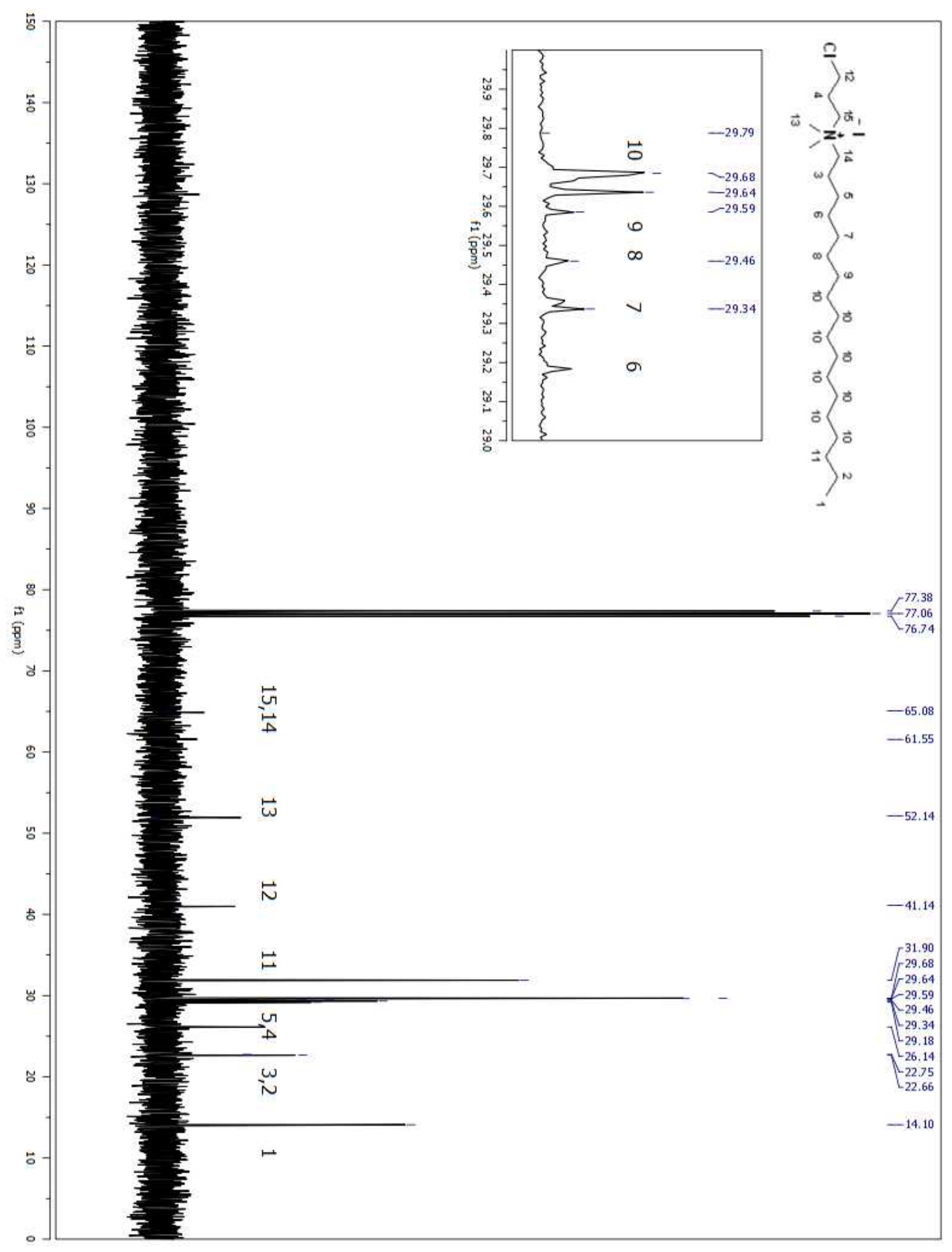

Figure A 193. ${ }^{13} \mathrm{C}$ NMR spectrum of compound (102) in $\mathrm{CDCl}_{3}$. 


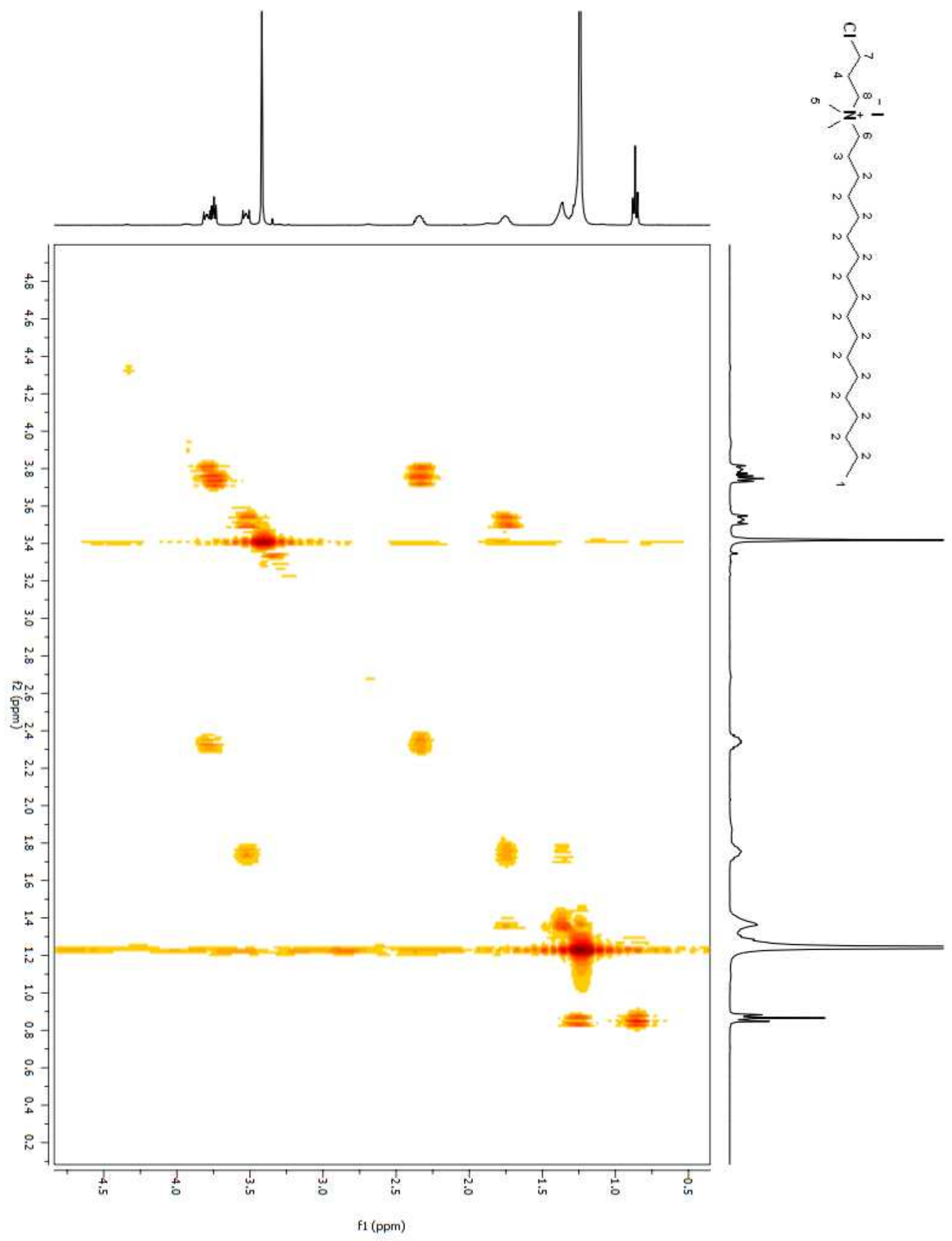

Figure A 194. COSY 2D NMR spectrum of compound (102) in $\mathrm{CDCl}_{3}$. 


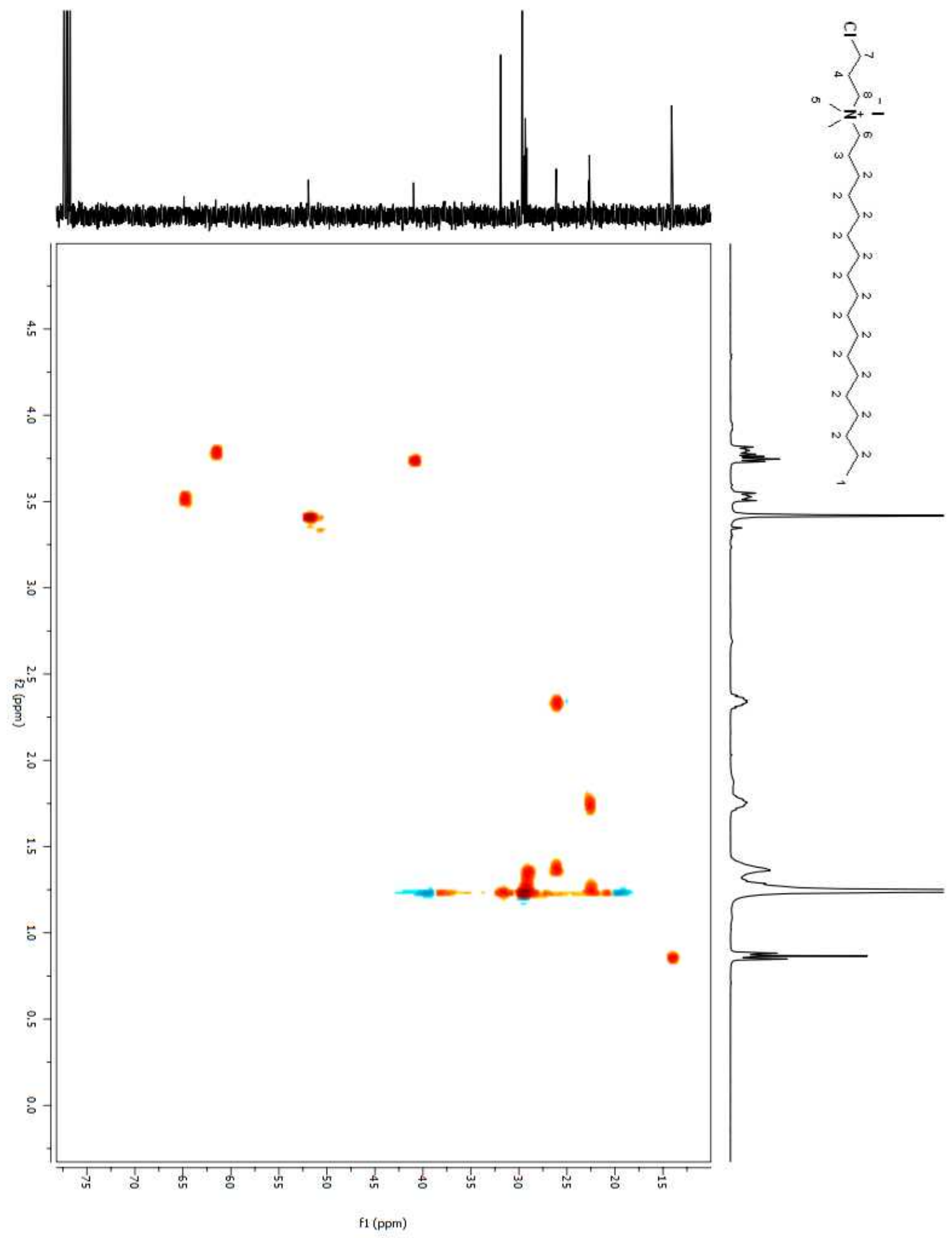

Figure A 195. HSQC 2D NMR spectrum of compound (102) in $\mathrm{CDCl}_{3}$. 


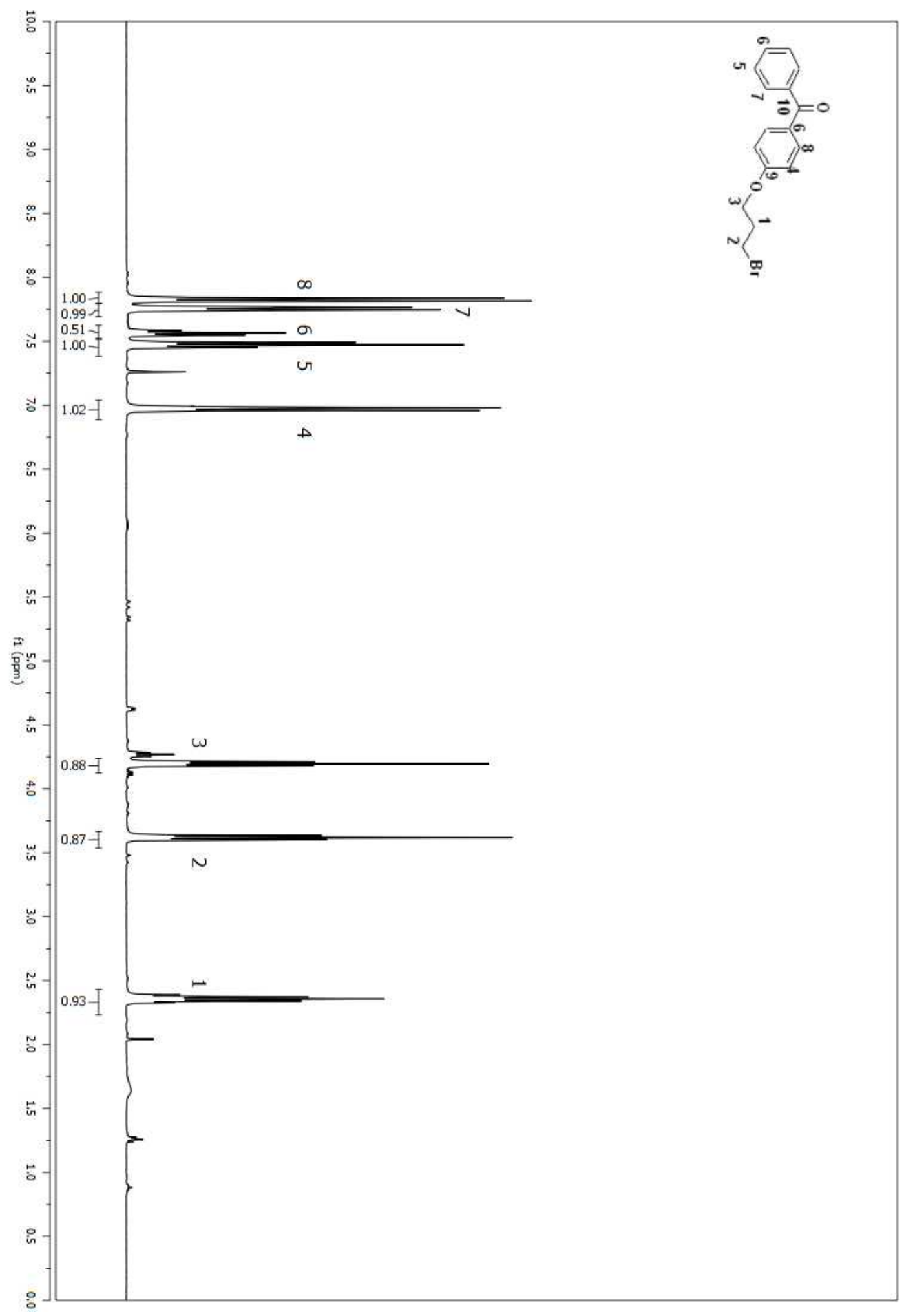

Figure A 196. ${ }^{1} \mathrm{H}$ NMR spectrum of compound (202) in $\mathrm{CDCl}_{3}$. 


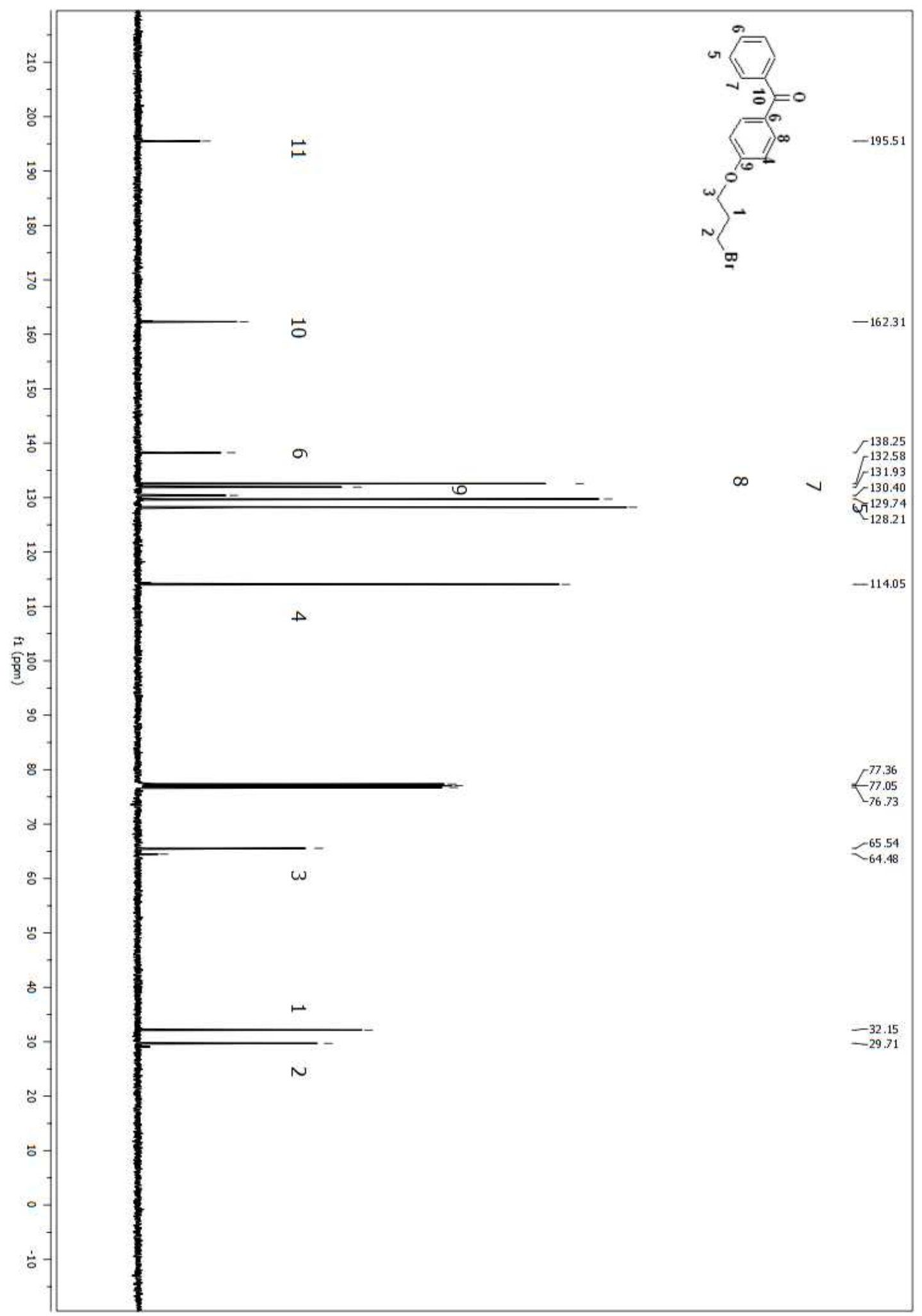

Figure A 197. ${ }^{13} \mathrm{C}$ NMR spectrum of compound (202) in $\mathrm{CDCl}_{3}$. 


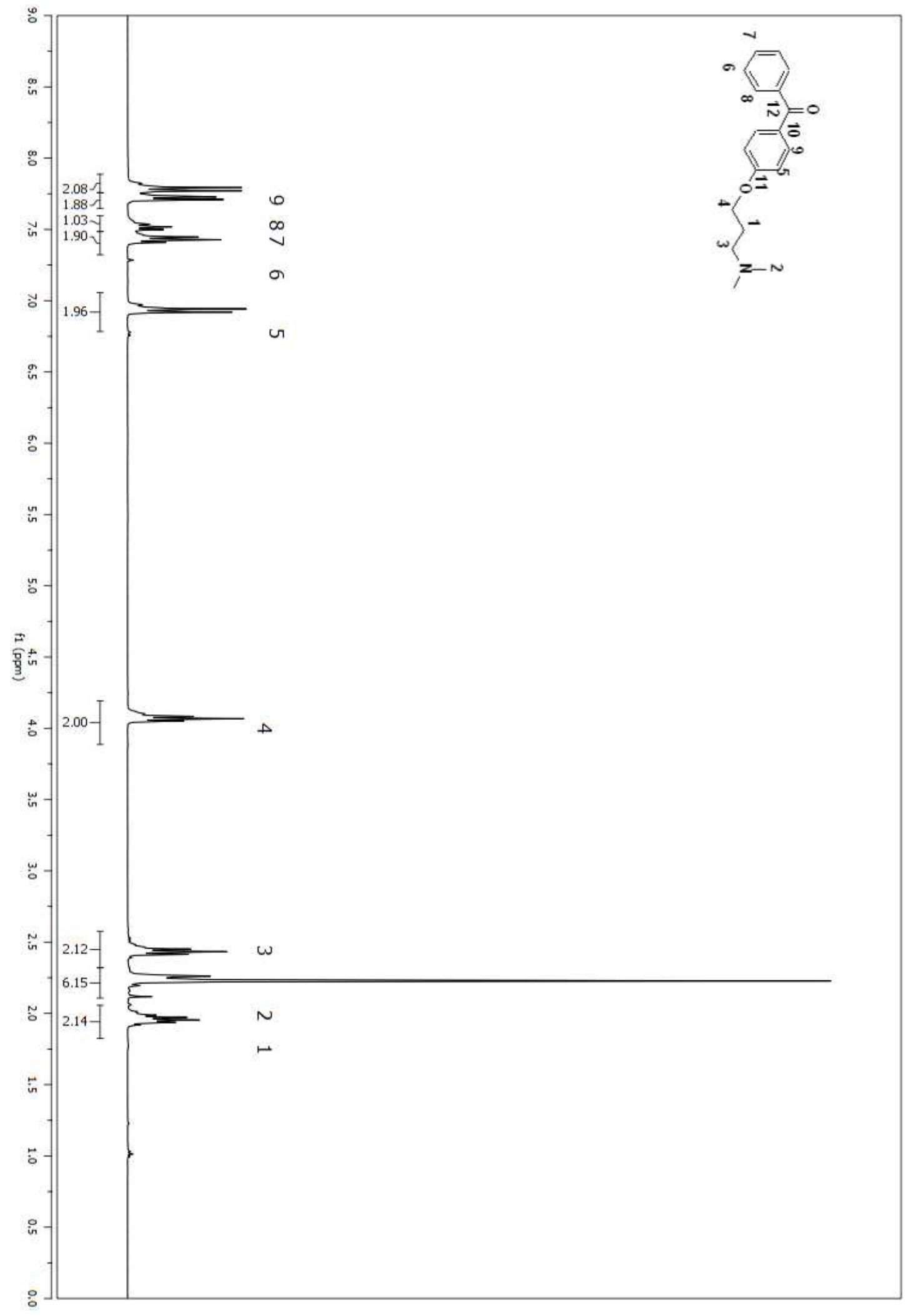

Figure A 198. ${ }^{1} \mathrm{H}$ NMR spectrum of compound (204) in $\mathrm{CDCl}_{3}$. 


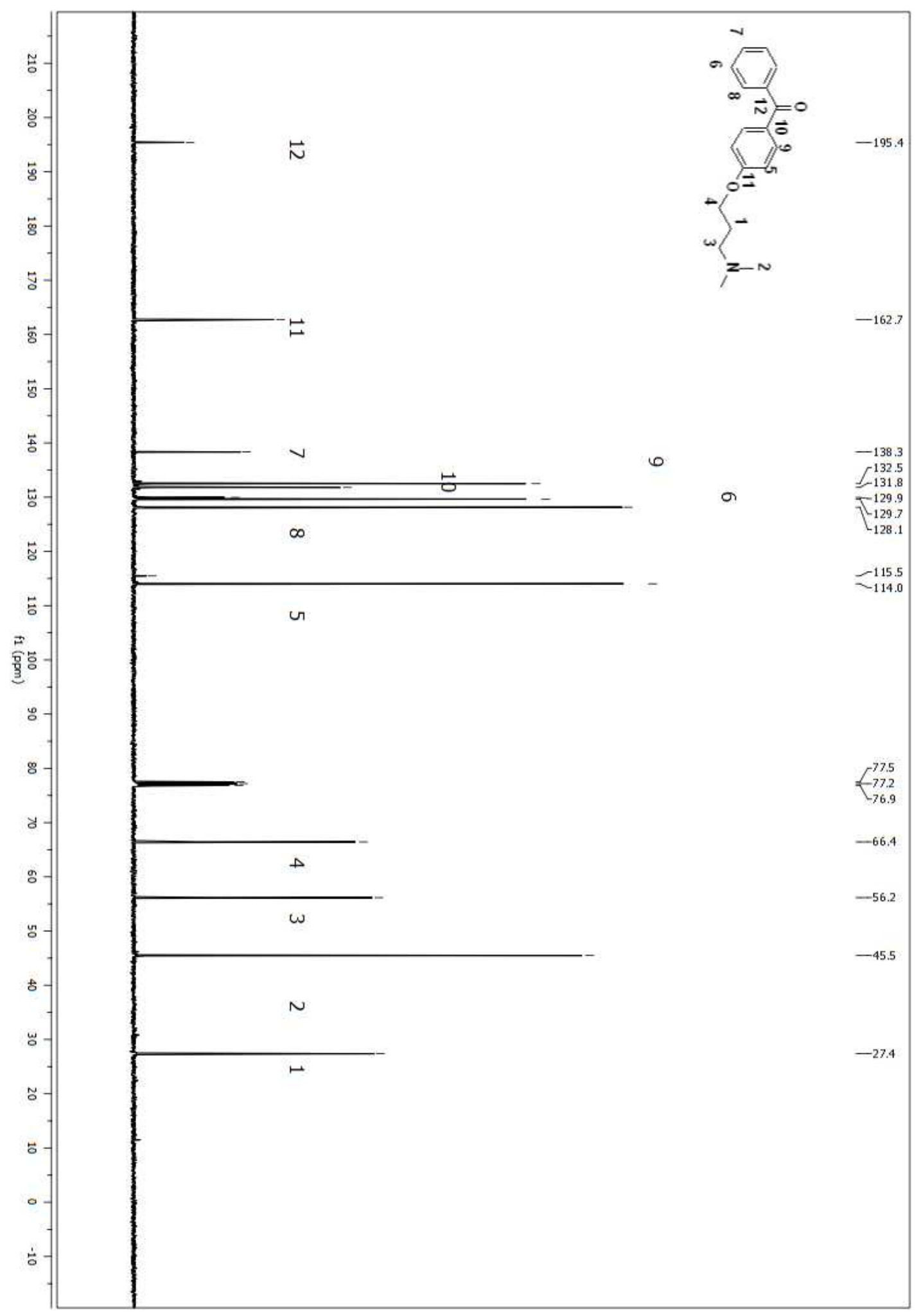

Figure A 199. ${ }^{13} \mathrm{C}$ NMR spectrum of compound (204) in $\mathrm{CDCl}_{3}$. 


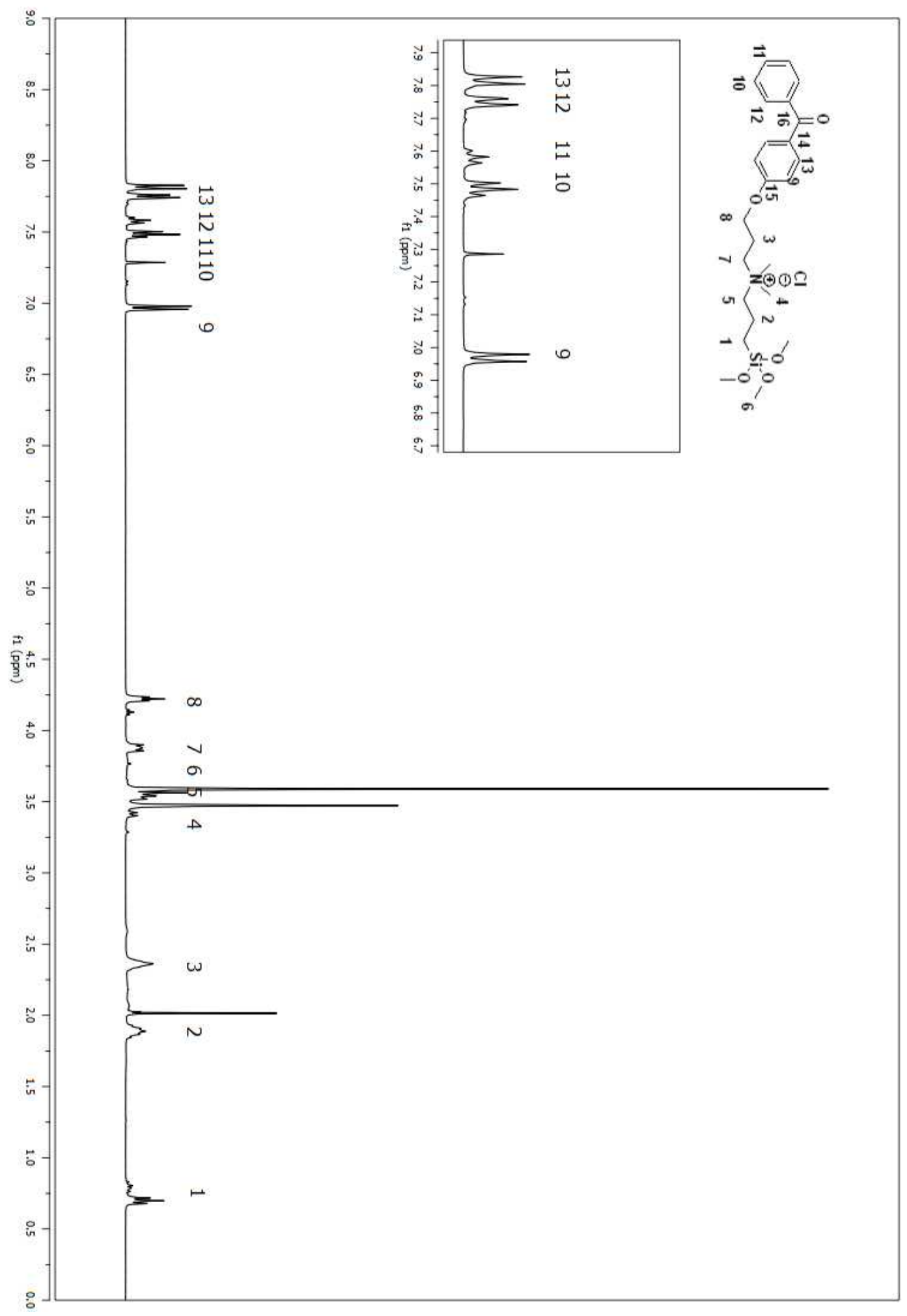

Figure A 200. ${ }^{1} \mathrm{H}$ NMR spectrum of compound (206) in $\mathrm{CDCl}_{3}$. 


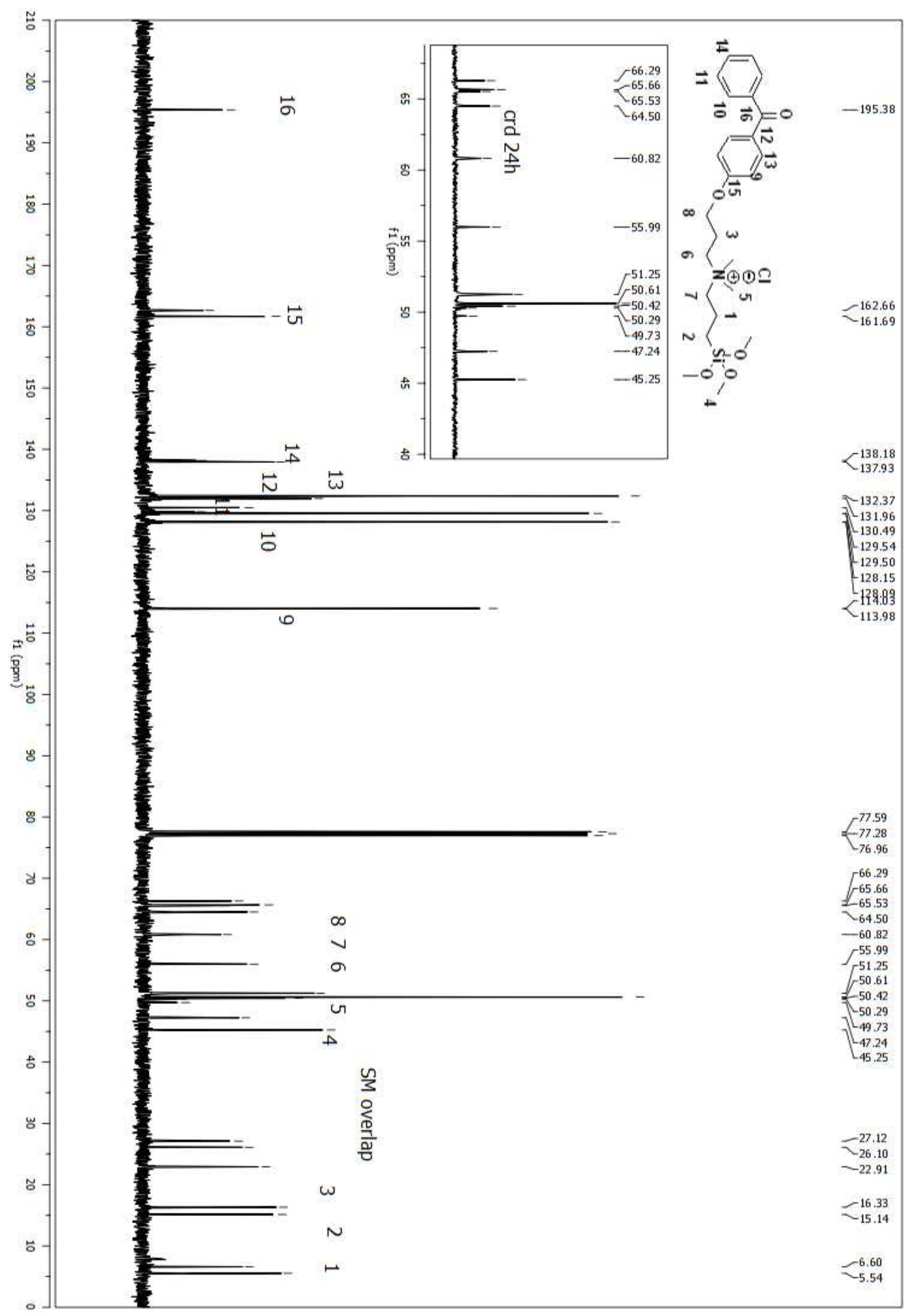

Figure A 201. ${ }^{13} \mathrm{C}$ NMR spectrum of compound (206) in $\mathrm{CDCl}_{3}$. 


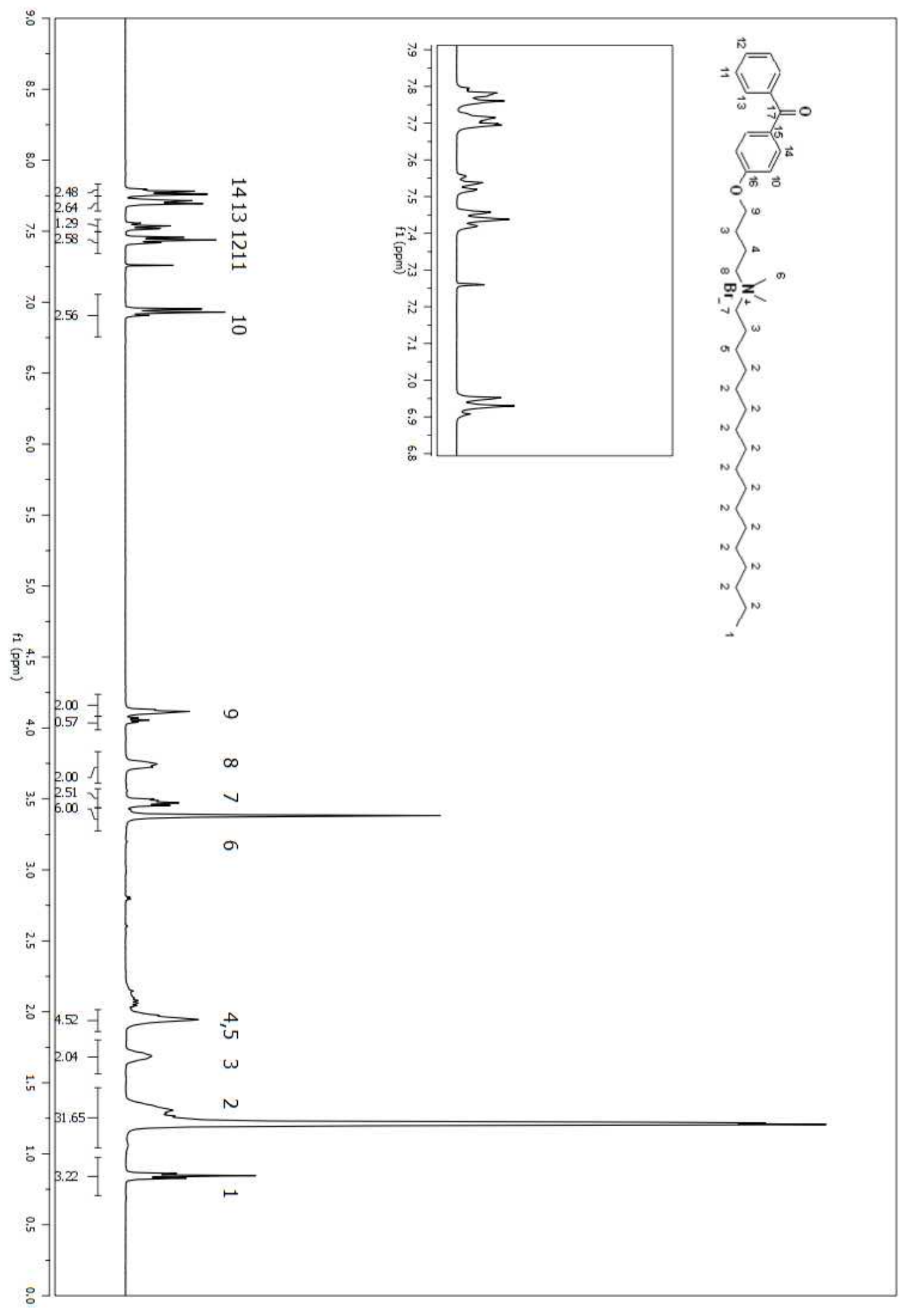

Figure A 202. ${ }^{1} \mathrm{H}$ NMR spectrum of compound (205) in $\mathrm{CDCl}_{3}$. 


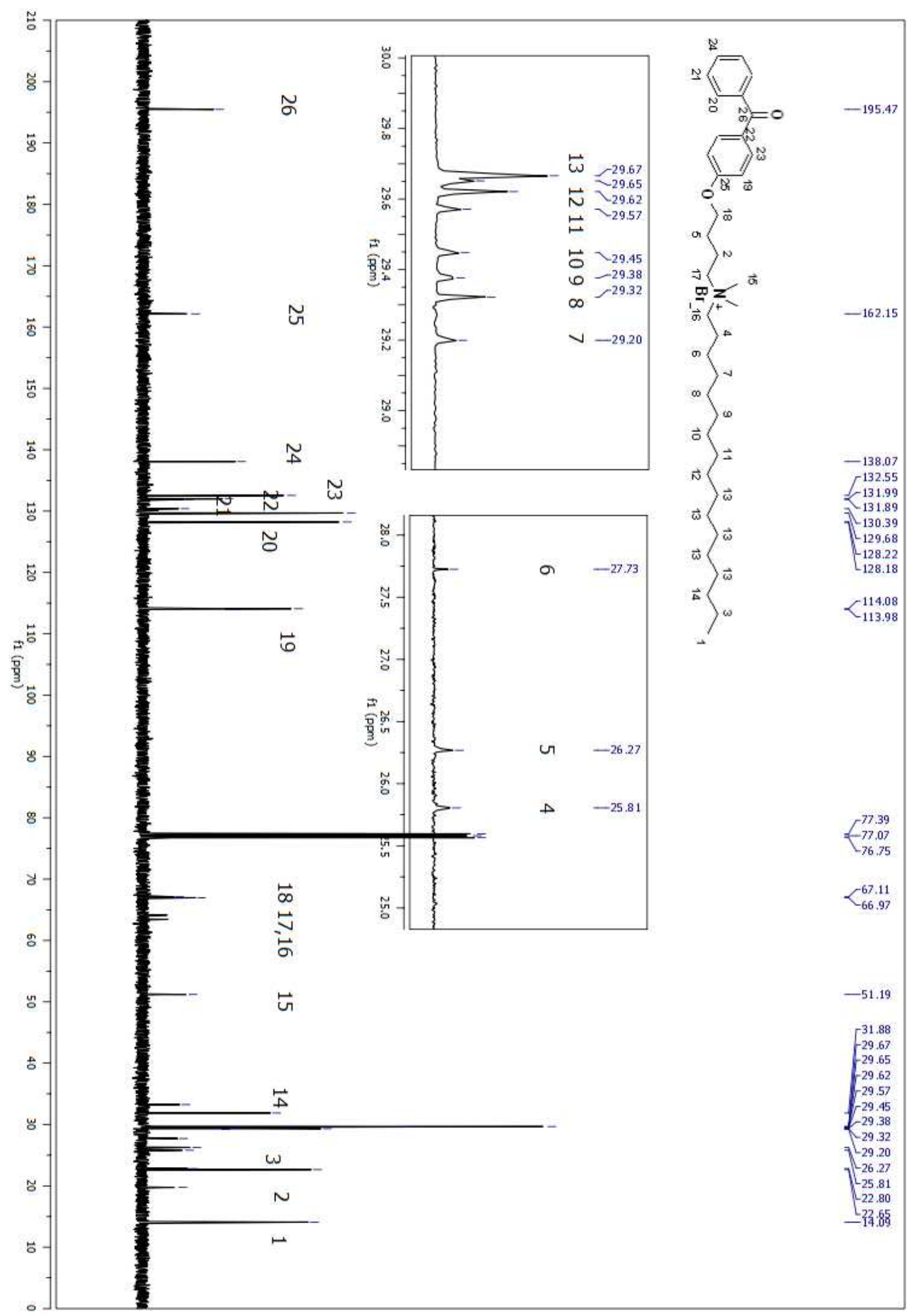

Figure A 203. ${ }^{13} \mathrm{C}$ NMR spectrum of compound (205) in $\mathrm{CDCl}_{3}$. 


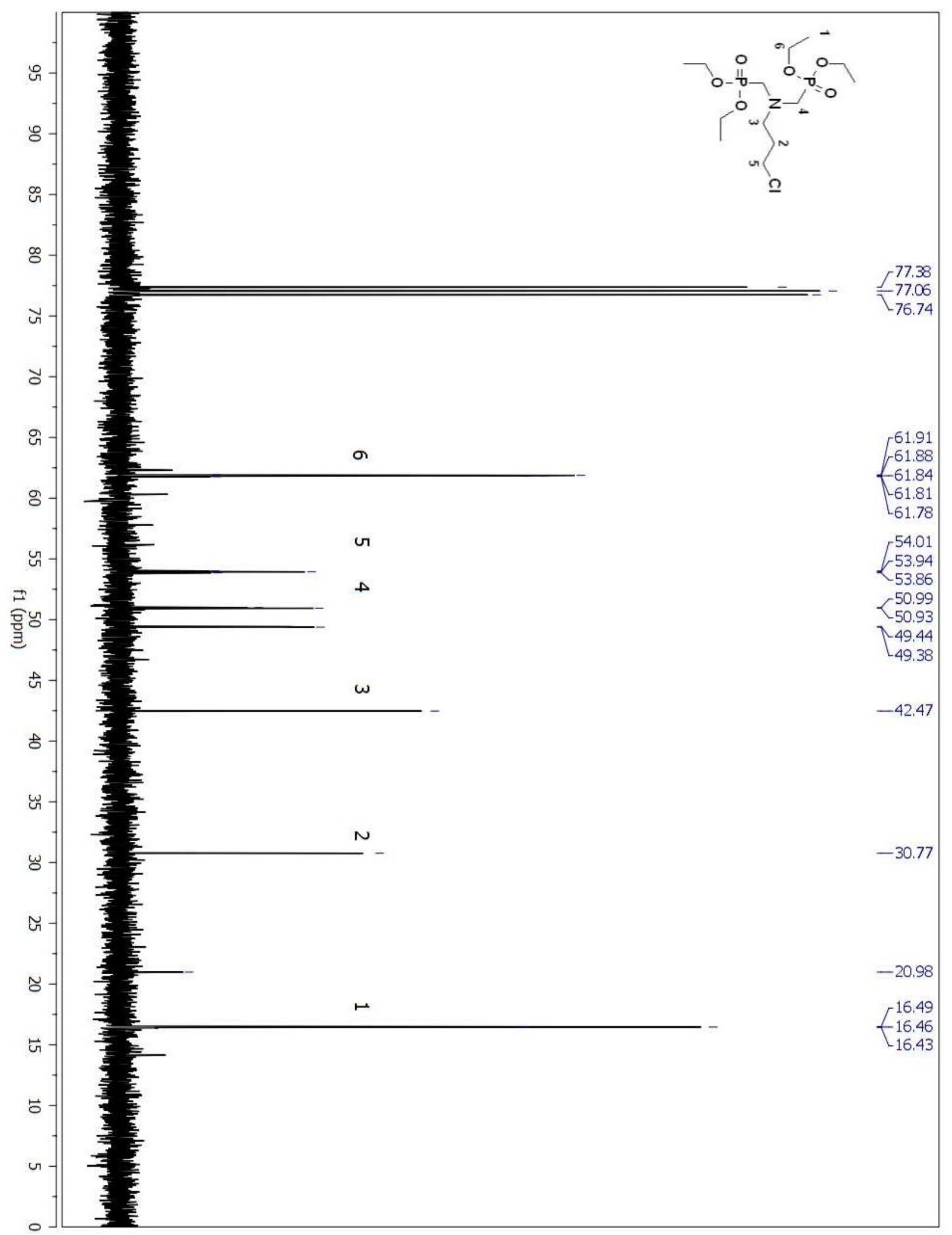

Figure A 204. ${ }^{13} \mathrm{C}$ NMR spectrum of compound (114) in $\mathrm{CDCl}_{3}$. 


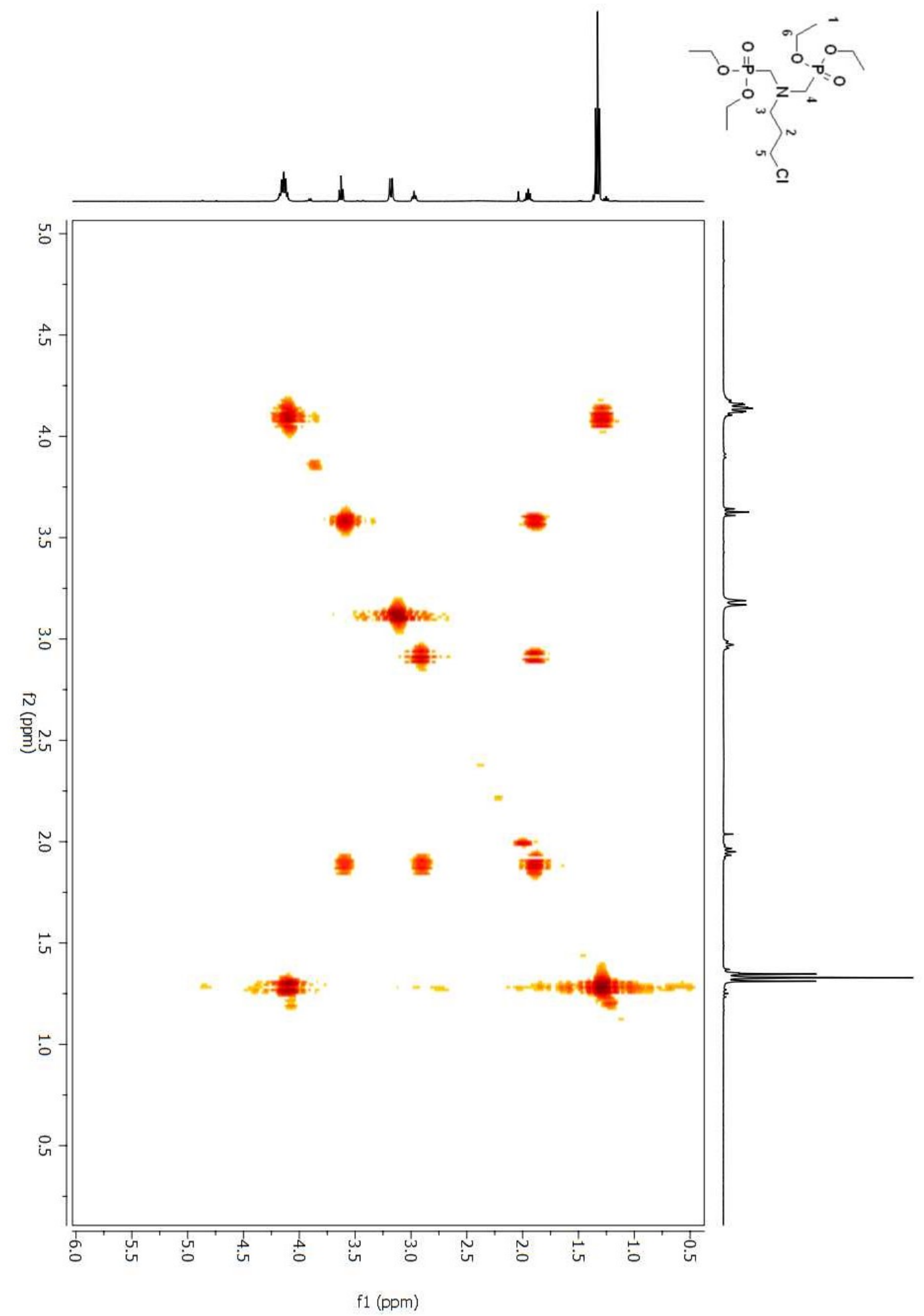

Figure A 205. COSY 2D NMR spectrum of compound (114) in $\mathrm{CDCl}_{3}$. 


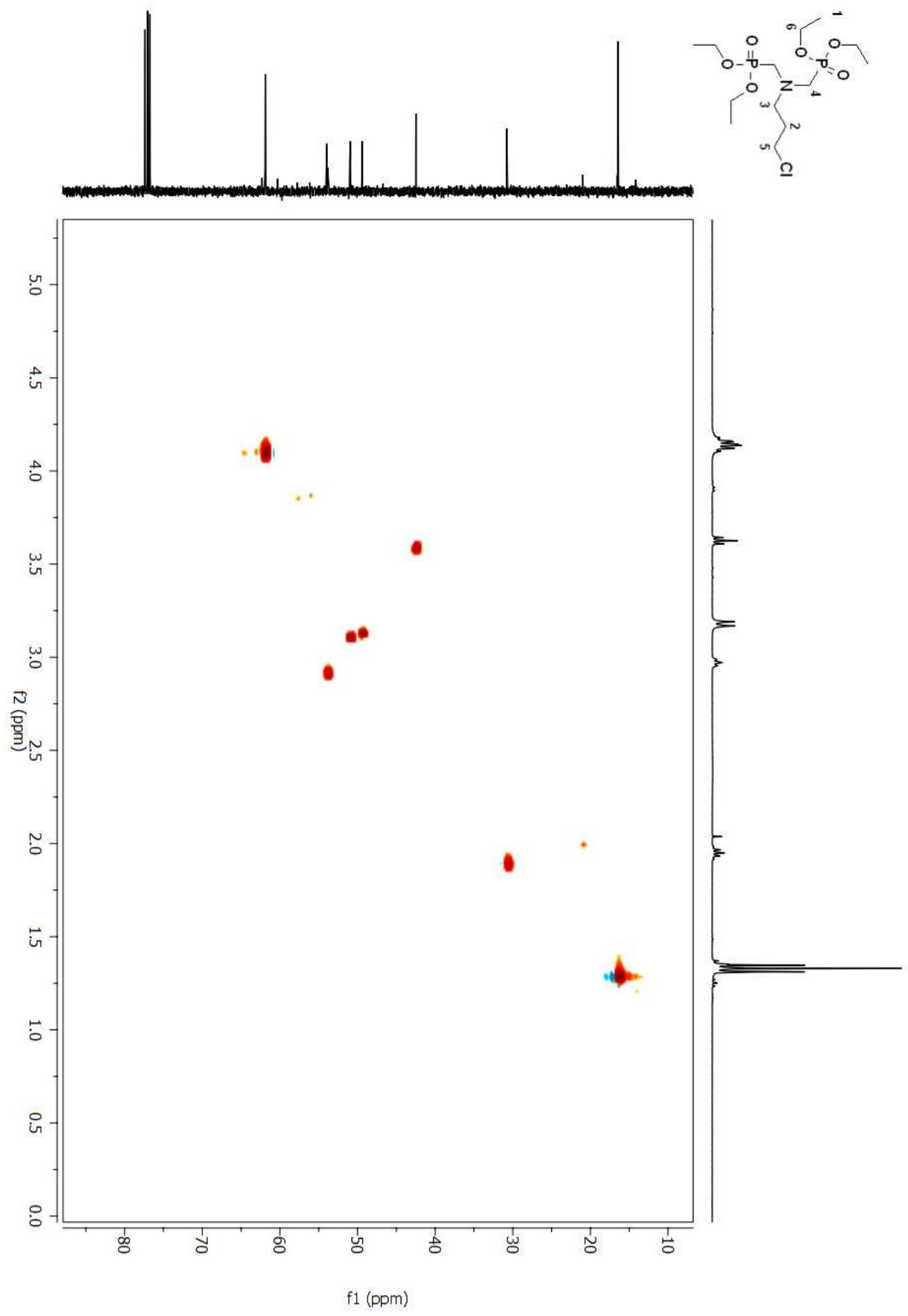

Figure A 206. HSQC 2D NMR spectrum of compound (114) in $\mathrm{CDCl}_{3}$. 


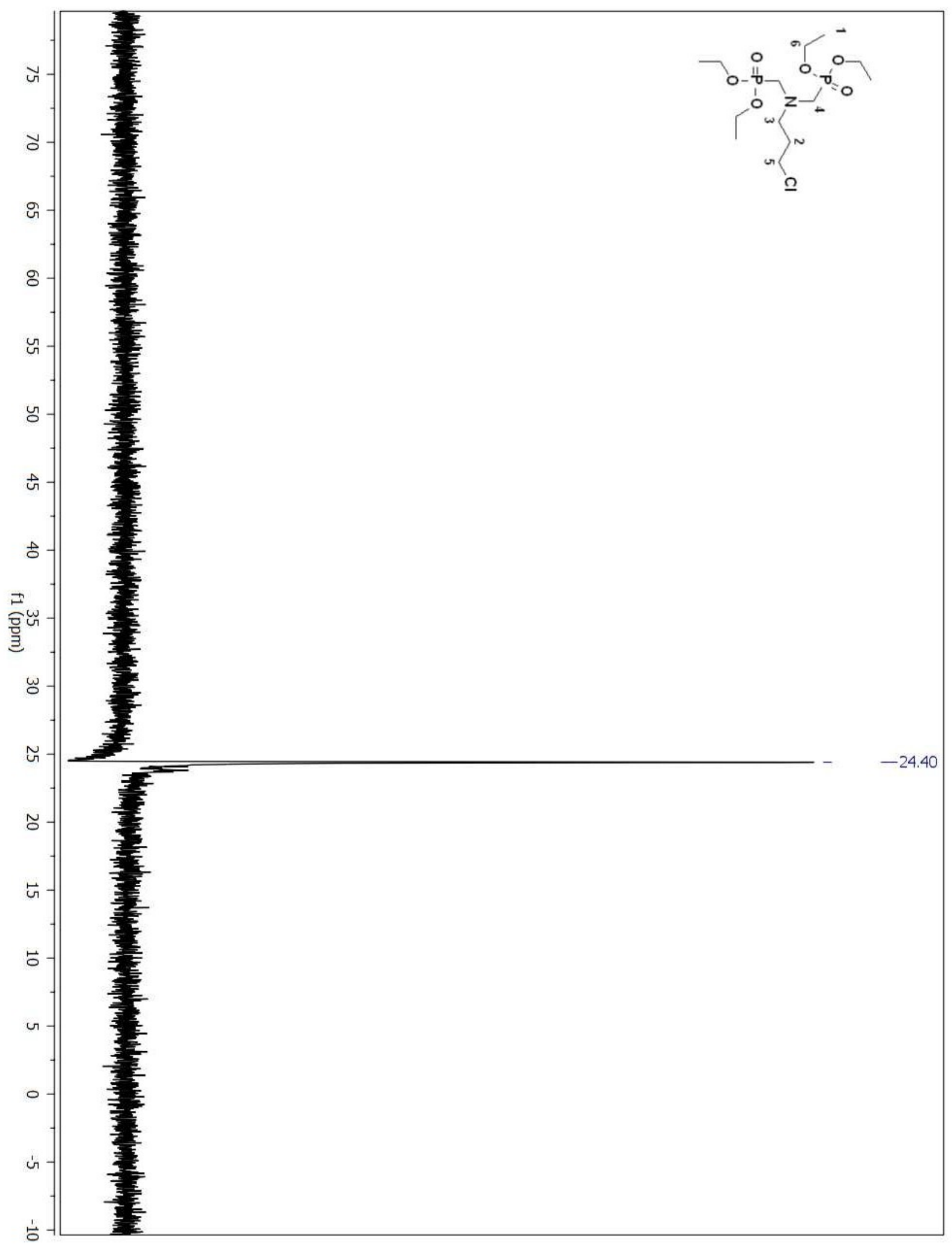

Figure A 207. ${ }^{31} \mathrm{P}$ NMR spectrum of compound (114) in $\mathrm{CDCl}_{3}$. 


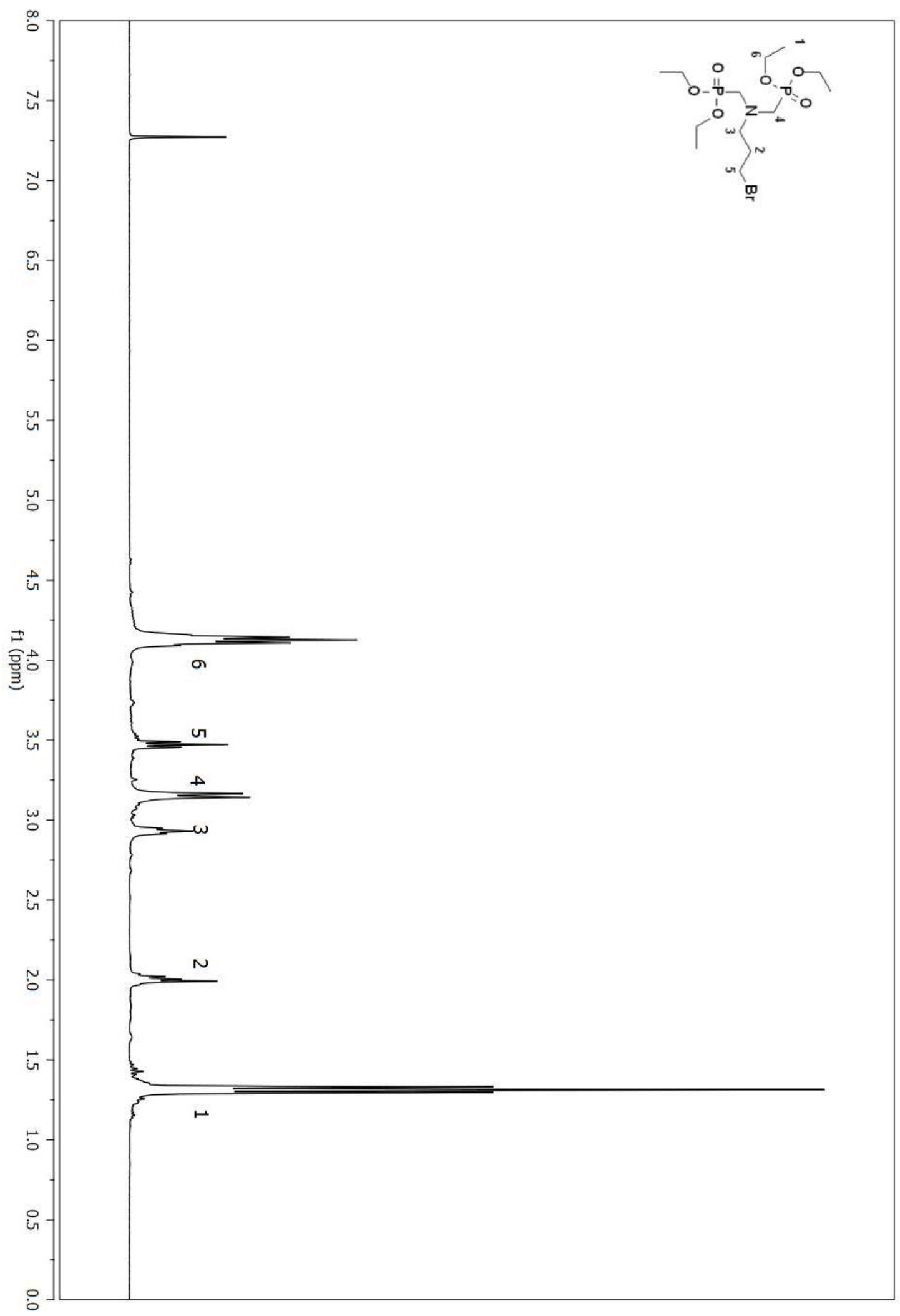

Figure A 208. ${ }^{1} \mathrm{H}$ NMR spectrum of compound (116) in $\mathrm{CDCl}_{3}$. 


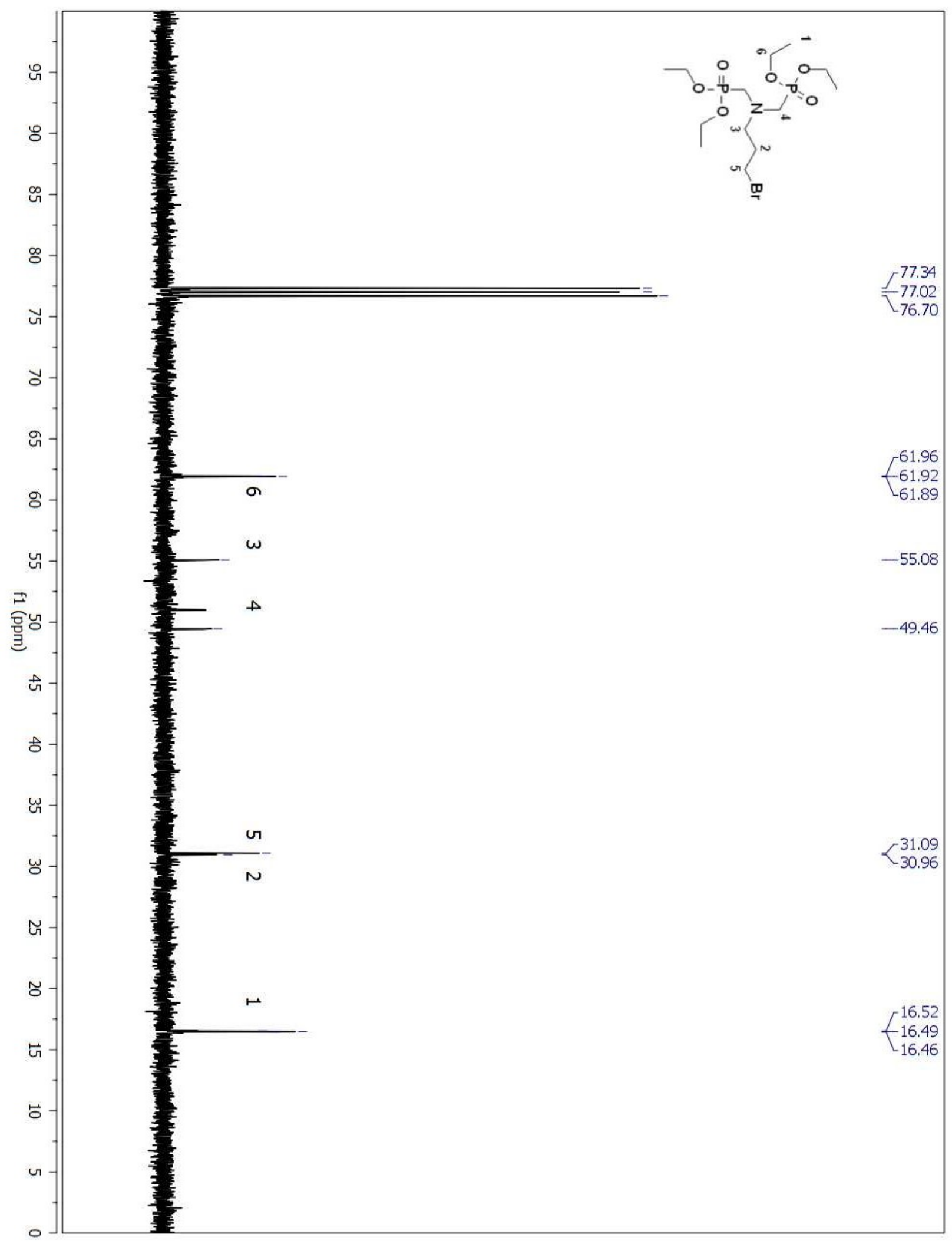

Figure A 209. ${ }^{13} \mathrm{C}$ NMR spectrum of compound (116) in $\mathrm{CDCl}_{3}$. 


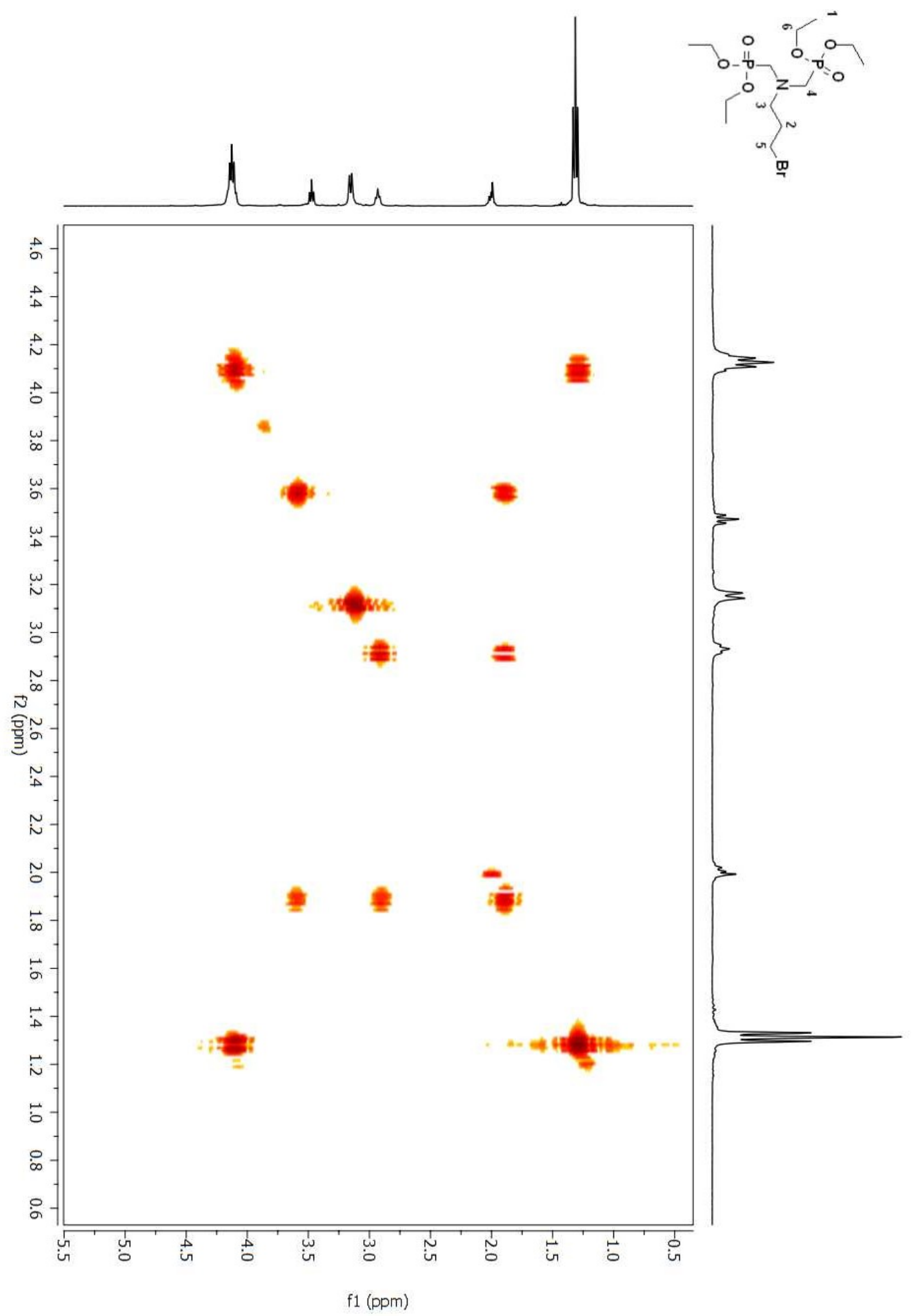

Figure A 210. COSY 2D NMR spectrum of compound (116) in $\mathrm{CDCl}_{3}$. 


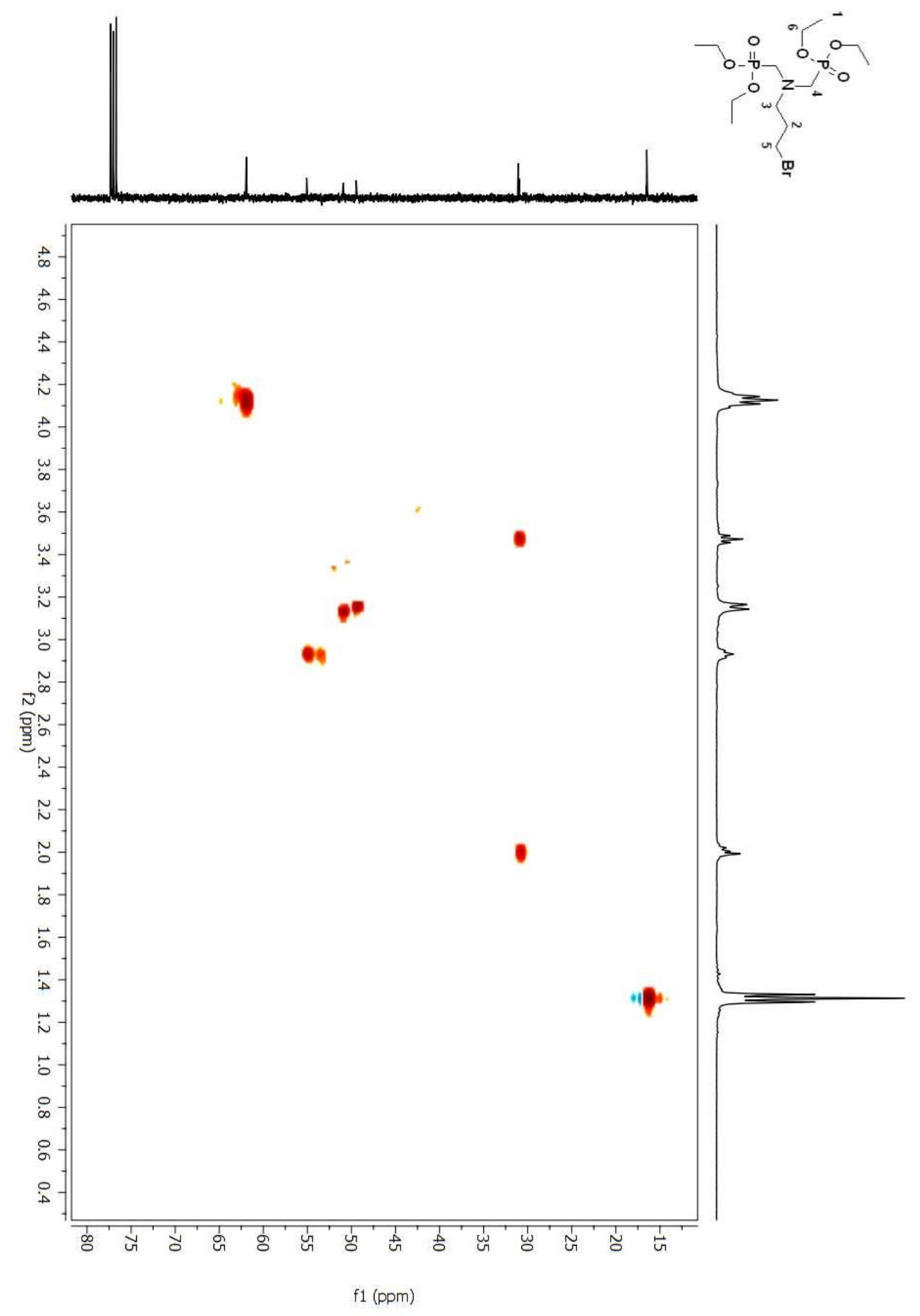

Figure A 211. HSQC 2D NMR spectrum of compound (116) in $\mathrm{CDCl}_{3}$. 


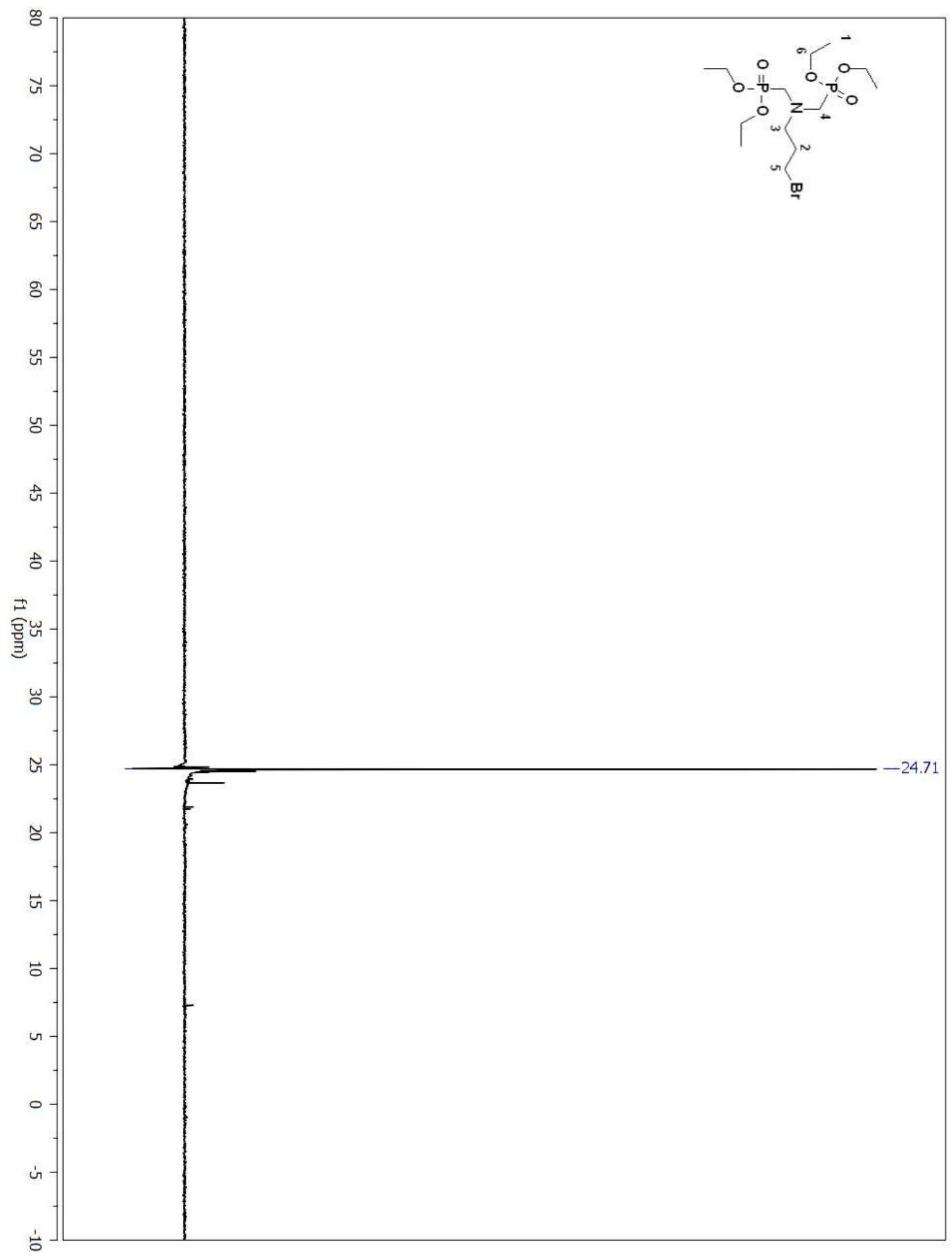

Figure A 212. ${ }^{31} \mathrm{P}$ NMR spectrum of compound (116) in $\mathrm{CDCl}_{3}$. 


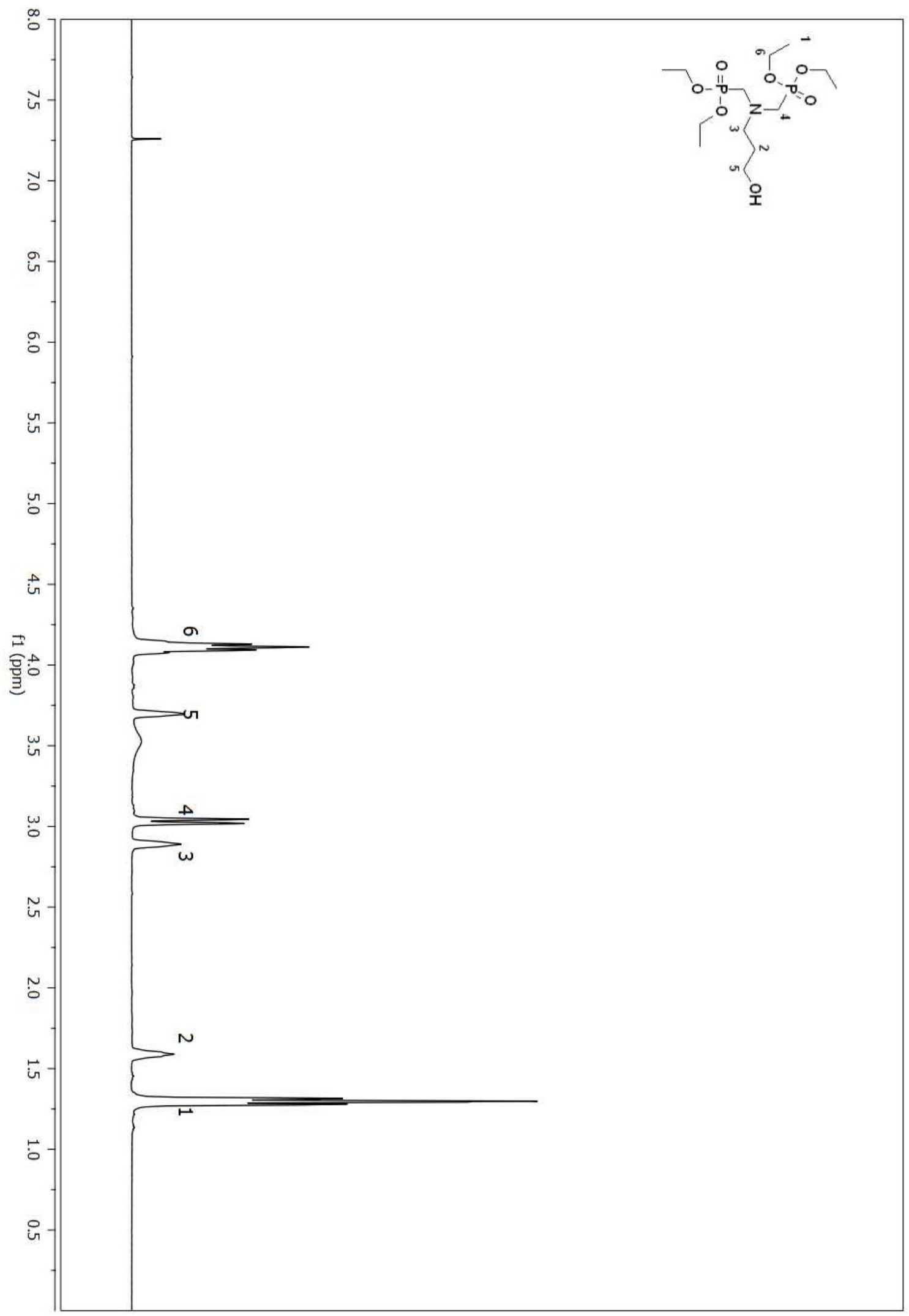

Figure A 213. ${ }^{1} \mathrm{H}$ NMR spectrum of compound (117) in $\mathrm{CDCl}_{3}$. 


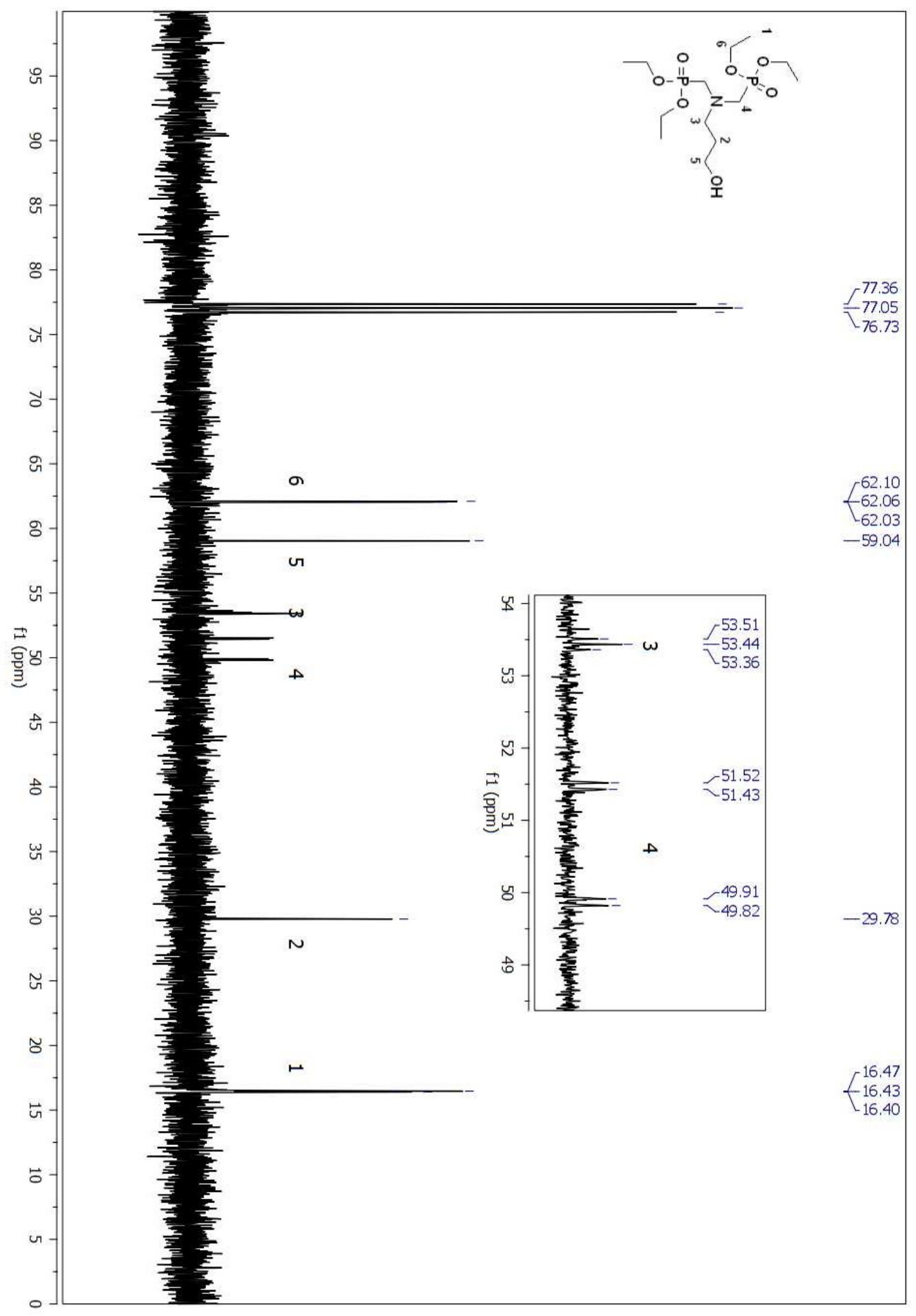

Figure A 214. ${ }^{13} \mathrm{C}$ NMR spectrum of compound (117) in $\mathrm{CDCl}_{3}$. 581 


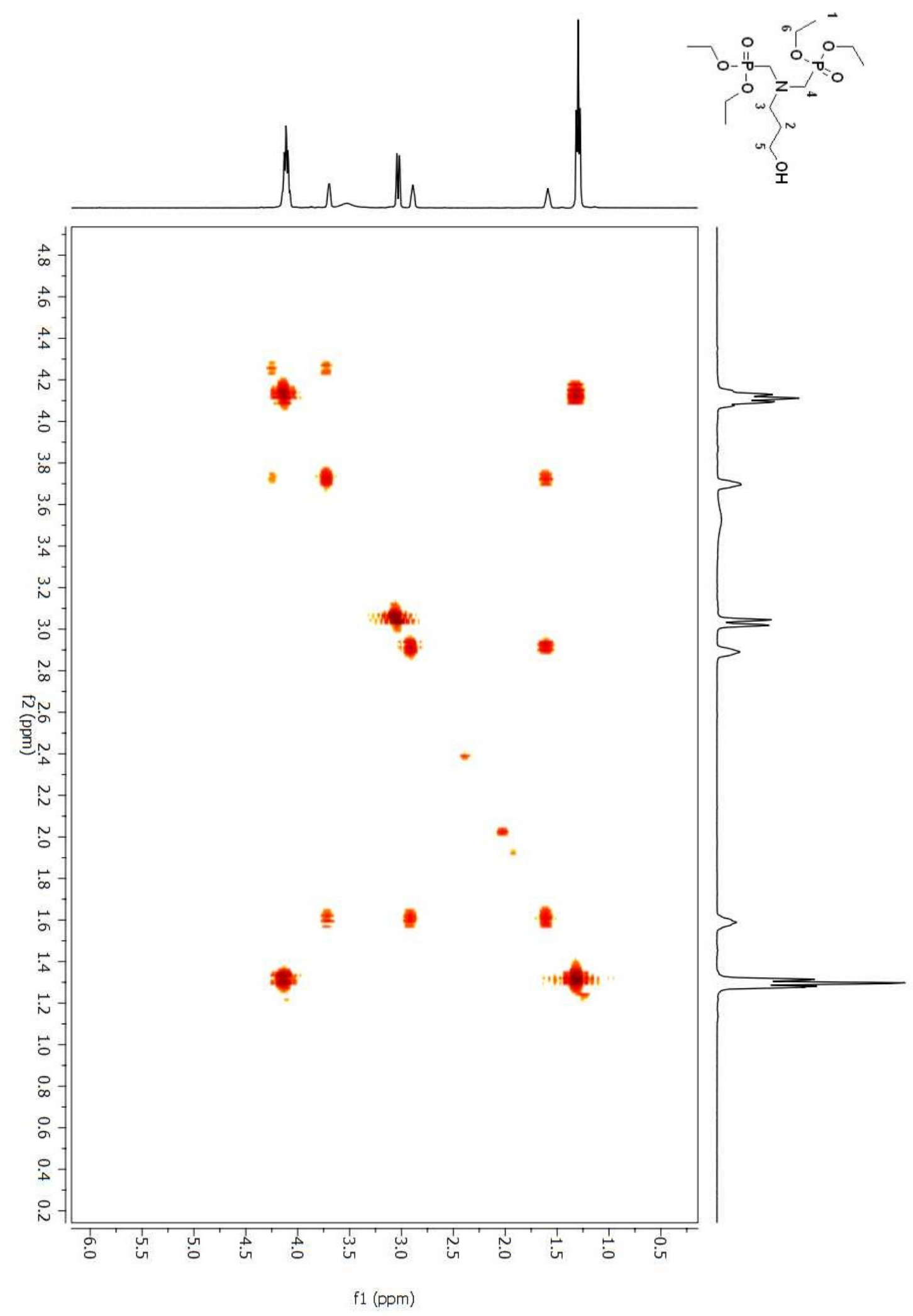

Figure A 215. COSY 2D NMR spectrum of compound (117) in $\mathrm{CDCl}_{3}$. 


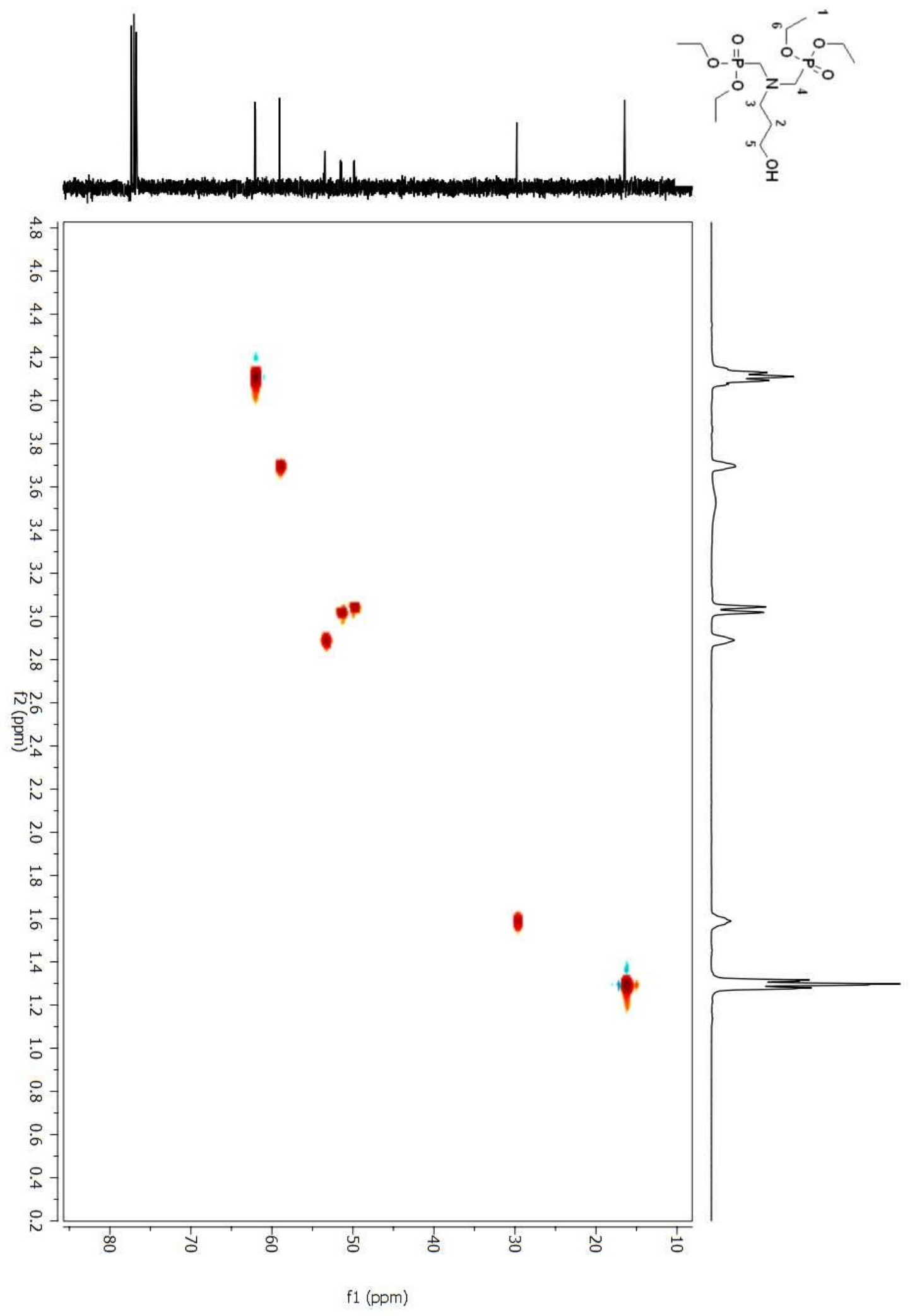

Figure A 216. HSQC $2 \mathrm{D}$ NMR spectrum of compound (117) in $\mathrm{CDCl}_{3}$. 


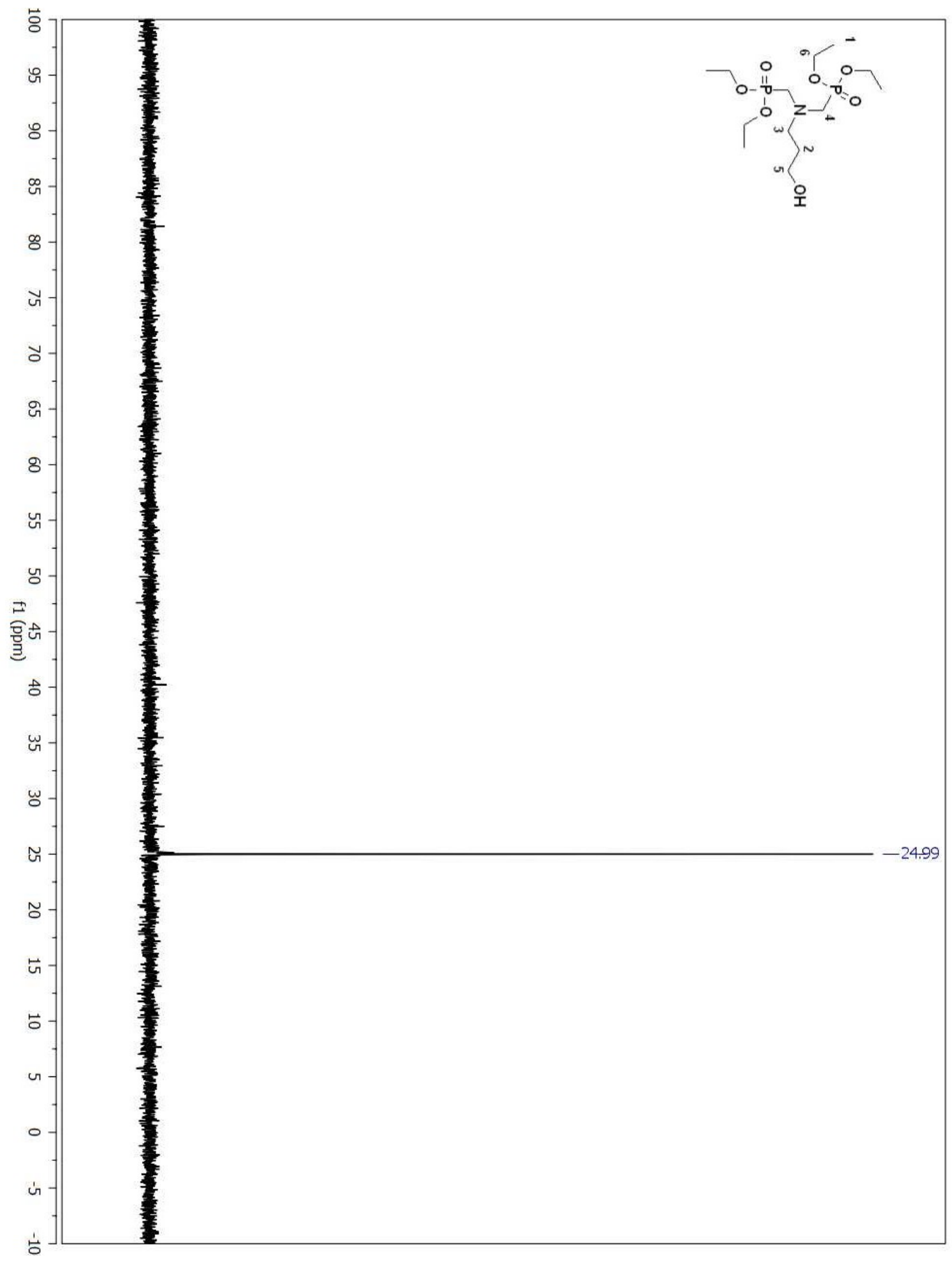

Figure A 217. ${ }^{31} \mathrm{P}$ NMR spectrum of compound (117) in $\mathrm{CDCl}_{3}$. 


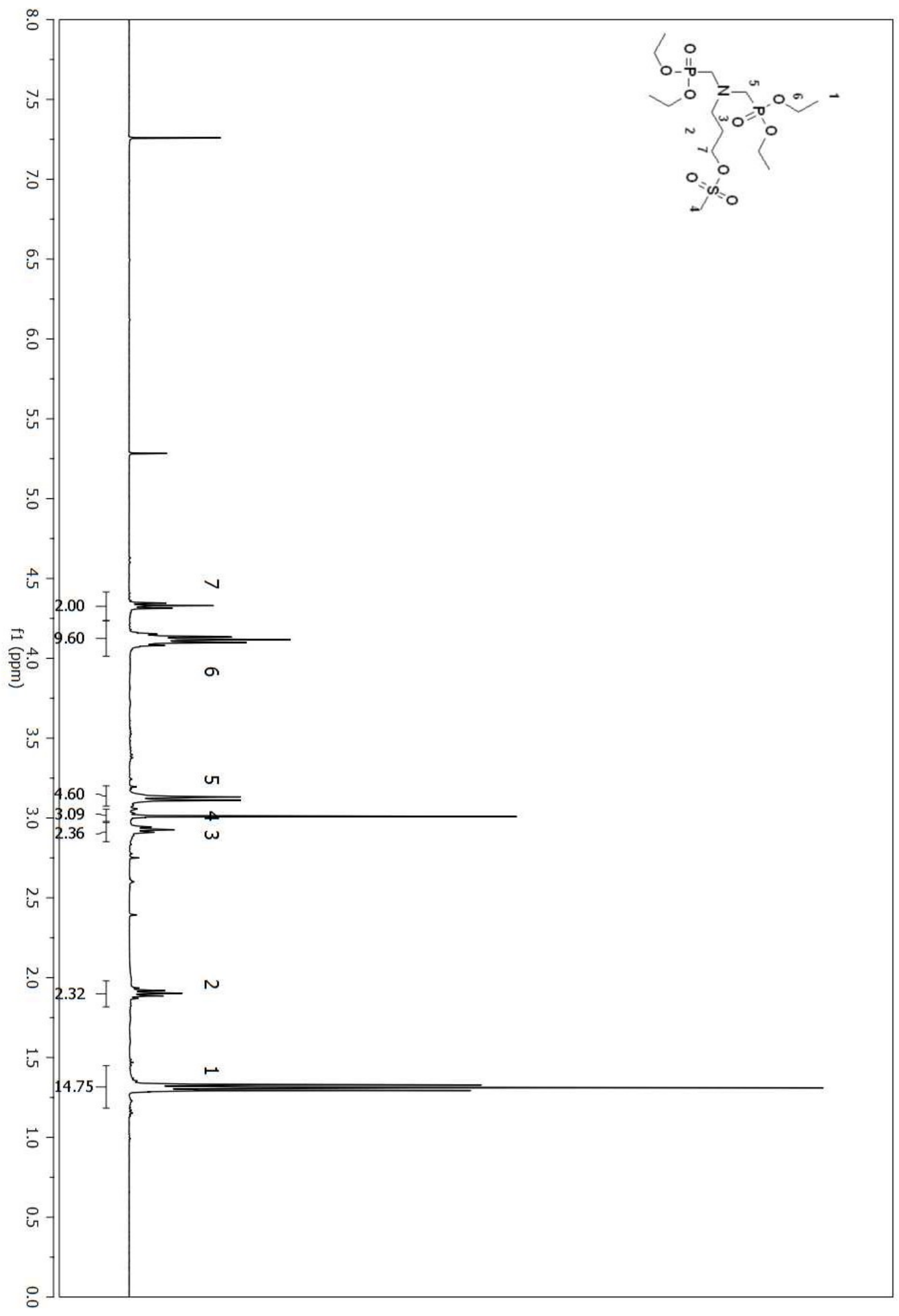

Figure A 218. ${ }^{1} \mathrm{H}$ NMR spectrum of compound (118) in $\mathrm{CDCl}_{3}$. 


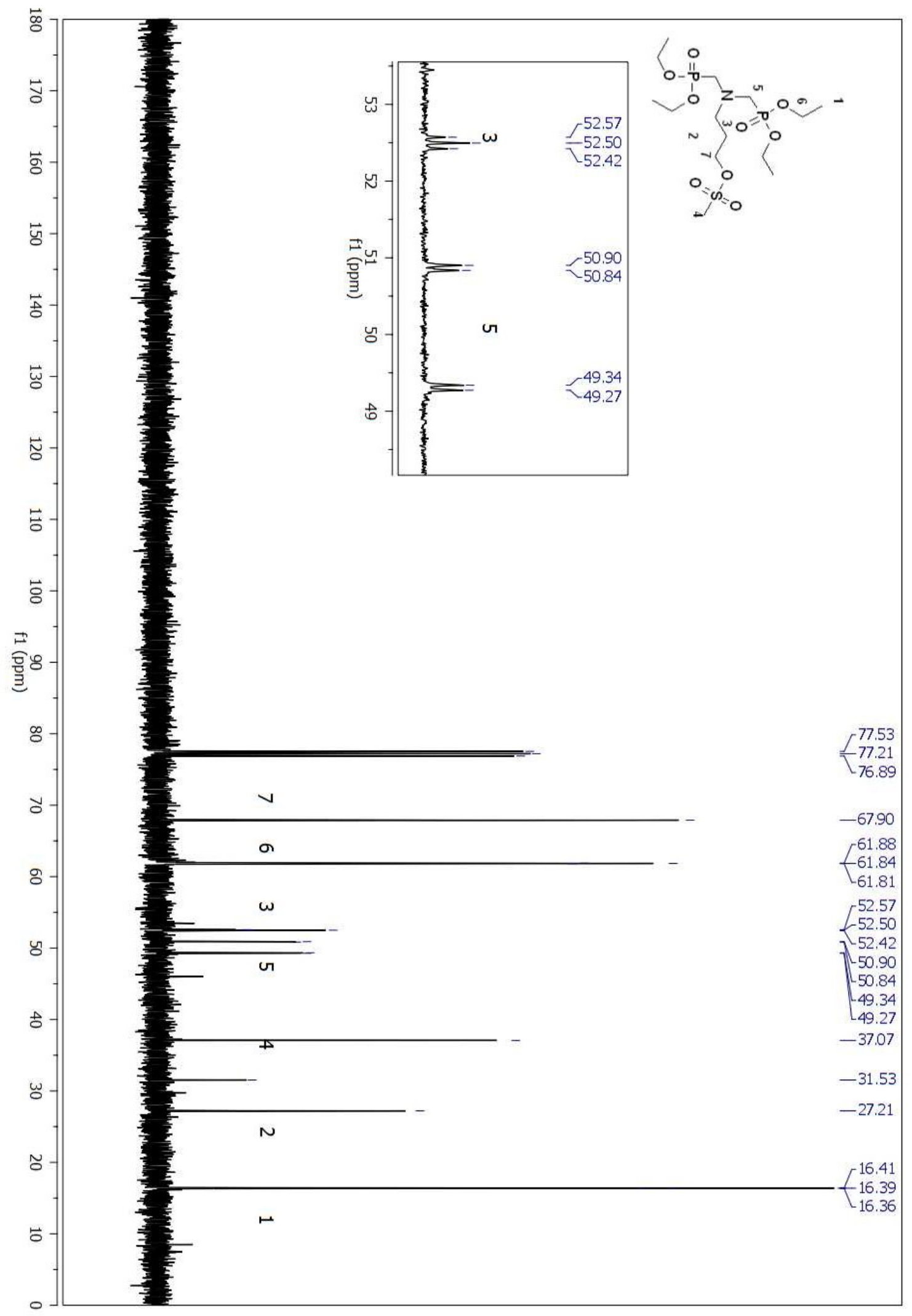

Figure A 219. ${ }^{13} \mathrm{C}$ NMR spectrum of compound (118) in $\mathrm{CDCl}_{3}$. 


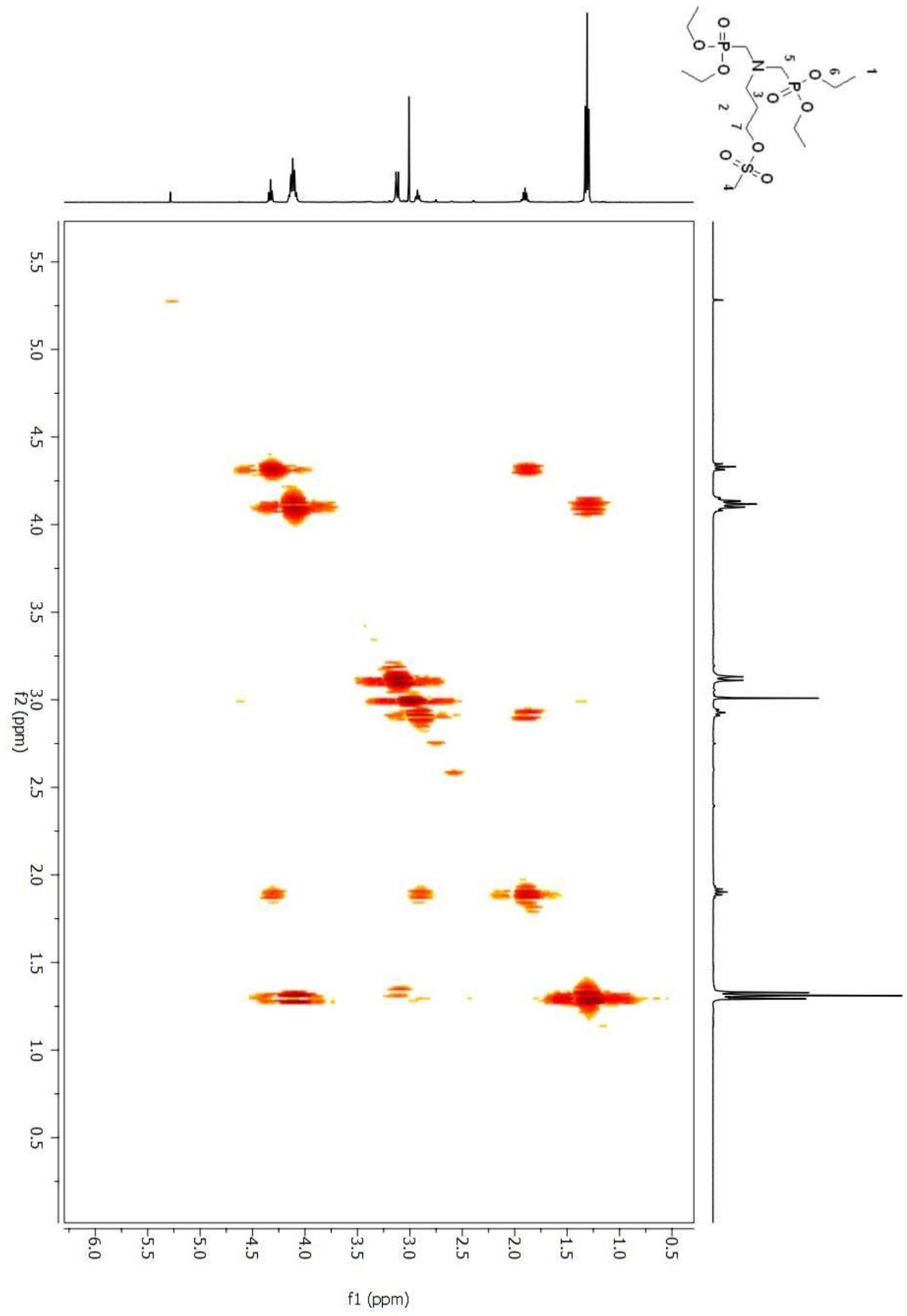

Figure A 220. COSY 2D NMR spectrum of compound (118) in $\mathrm{CDCl}_{3}$. 


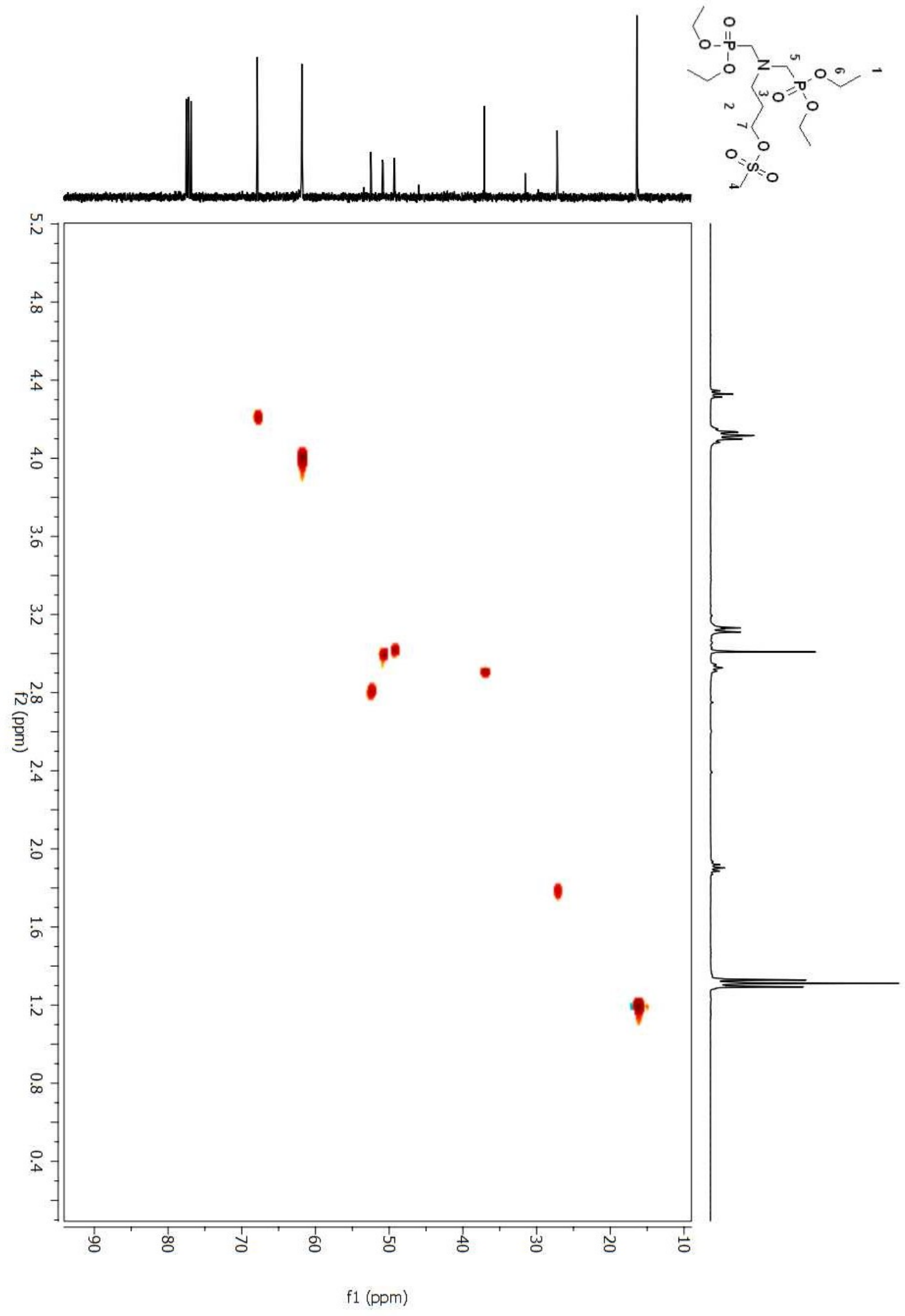

Figure A 221. HSQC 2D NMR spectrum of compound (118) in $\mathrm{CDCl}_{3}$. 


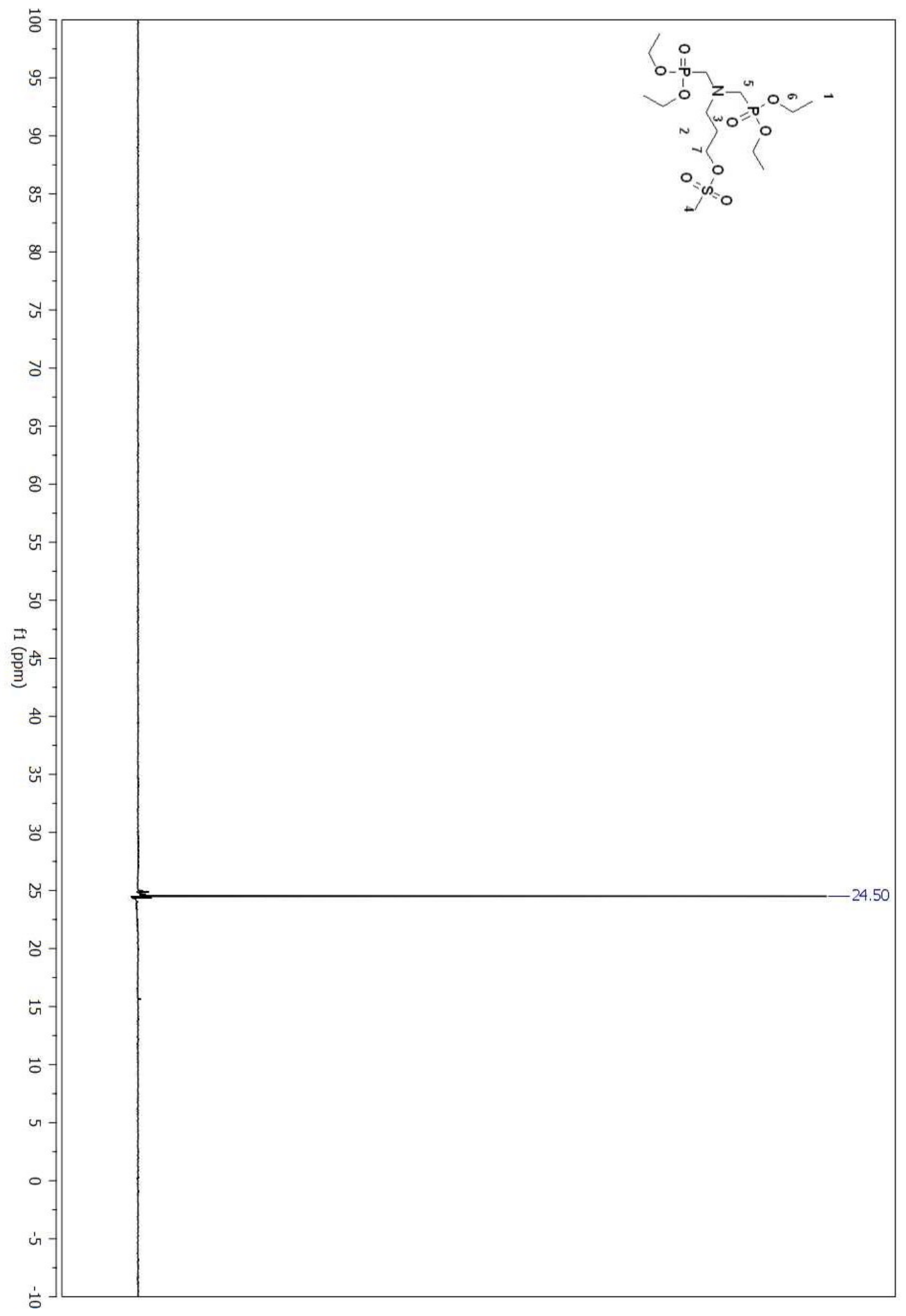

Figure A 222. ${ }^{31} \mathrm{P}$ NMR spectrum of compound (118) in $\mathrm{CDCl}_{3}$. 589 


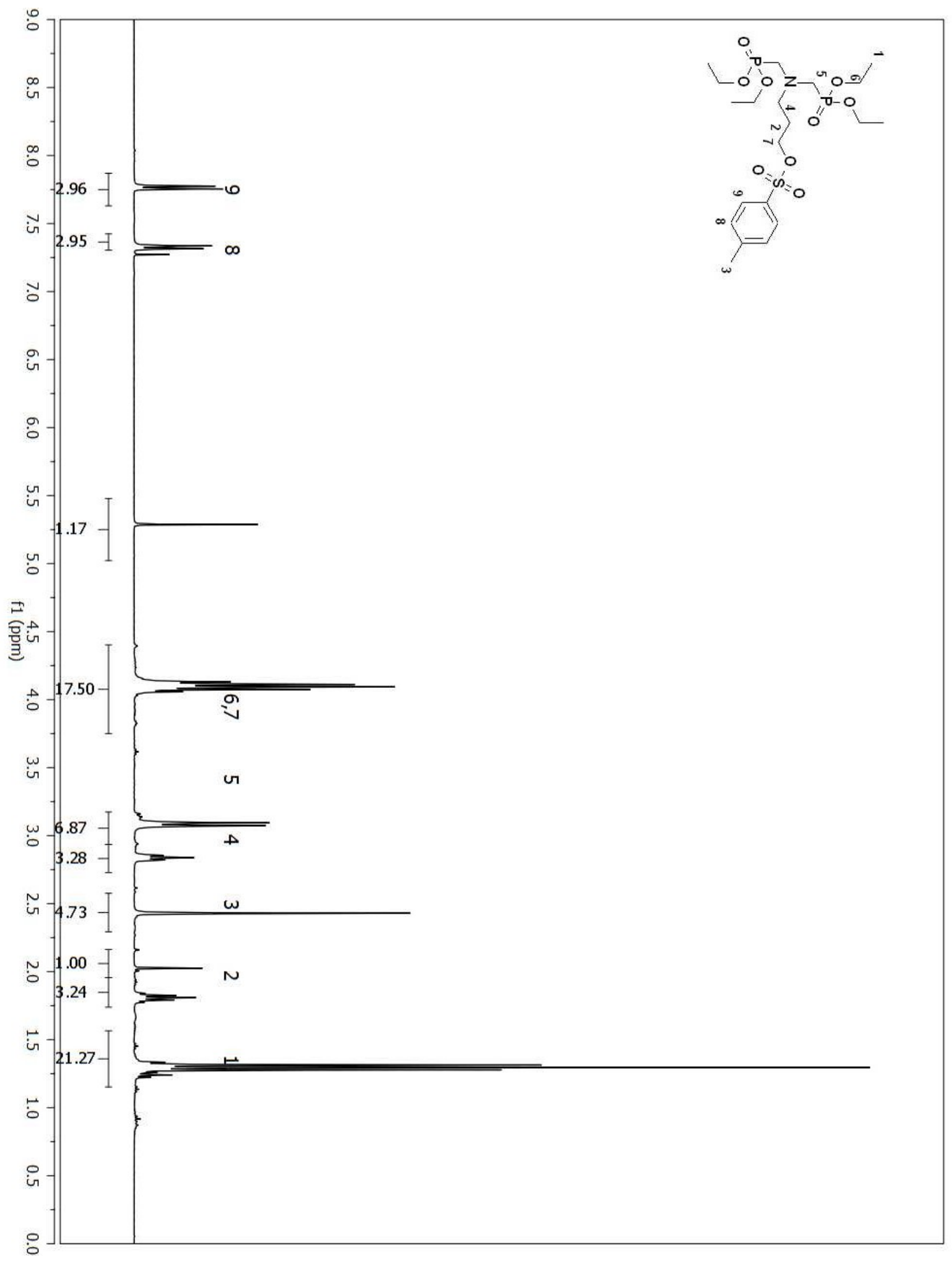

Figure A 223. ${ }^{1} \mathrm{H}$ NMR spectrum of compound (119) in $\mathrm{CDCl}_{3}$. 


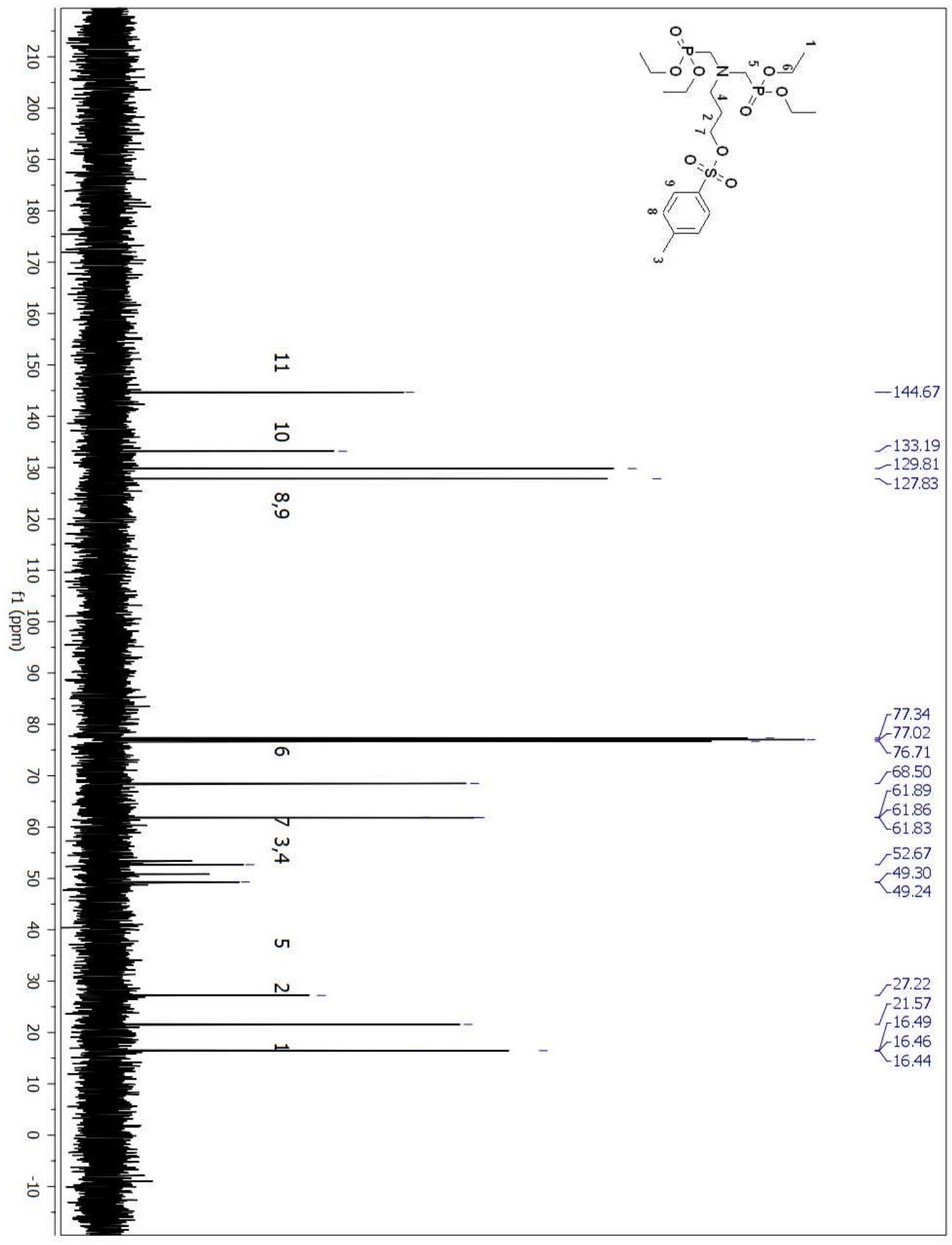

Figure A 224. ${ }^{13} \mathrm{C}$ NMR spectrum of compound (119) in $\mathrm{CDCl}_{3}$. 591 


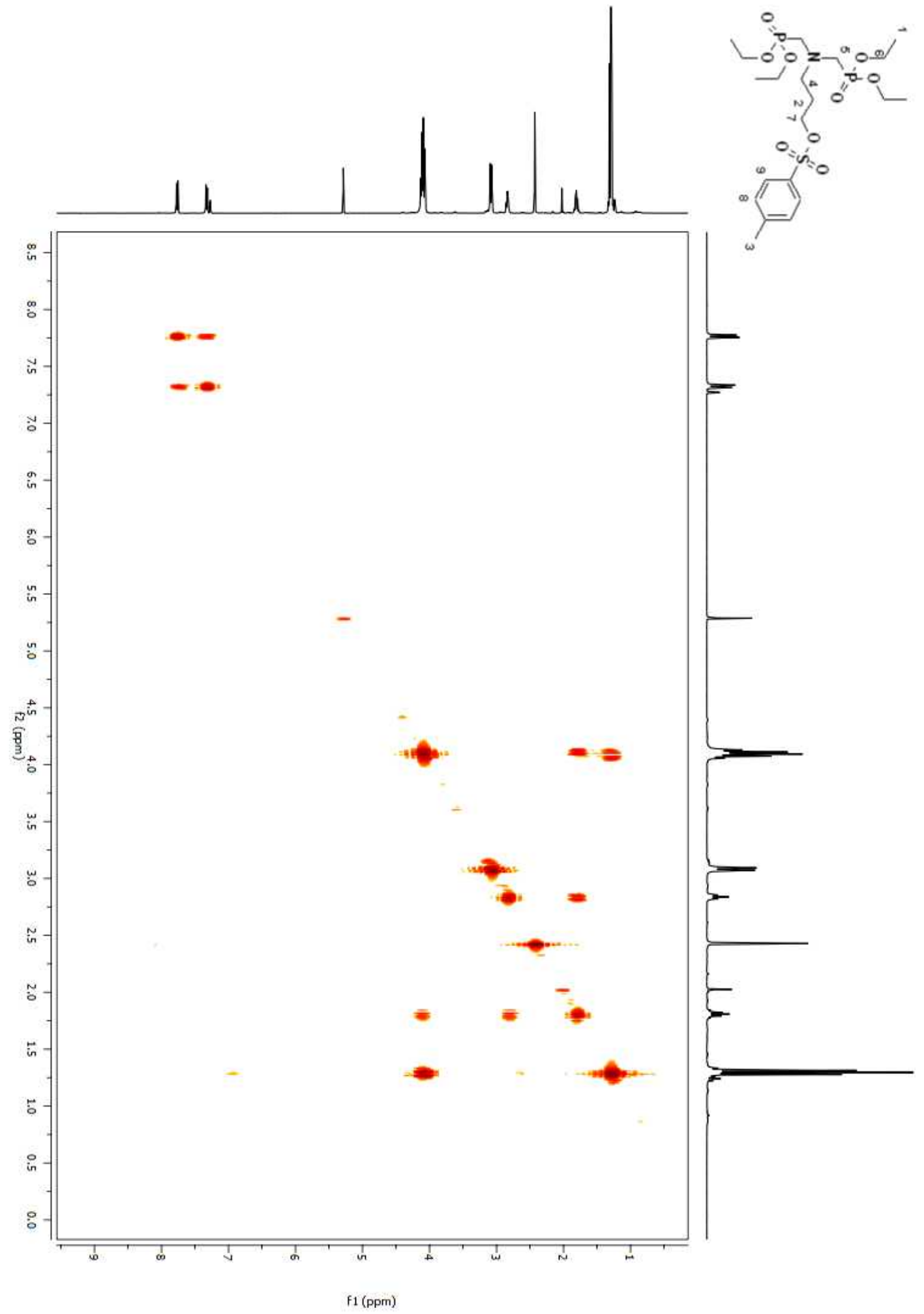

Figure A 225. COSY 2D NMR spectrum of compound (119) in $\mathrm{CDCl}_{3}$. 


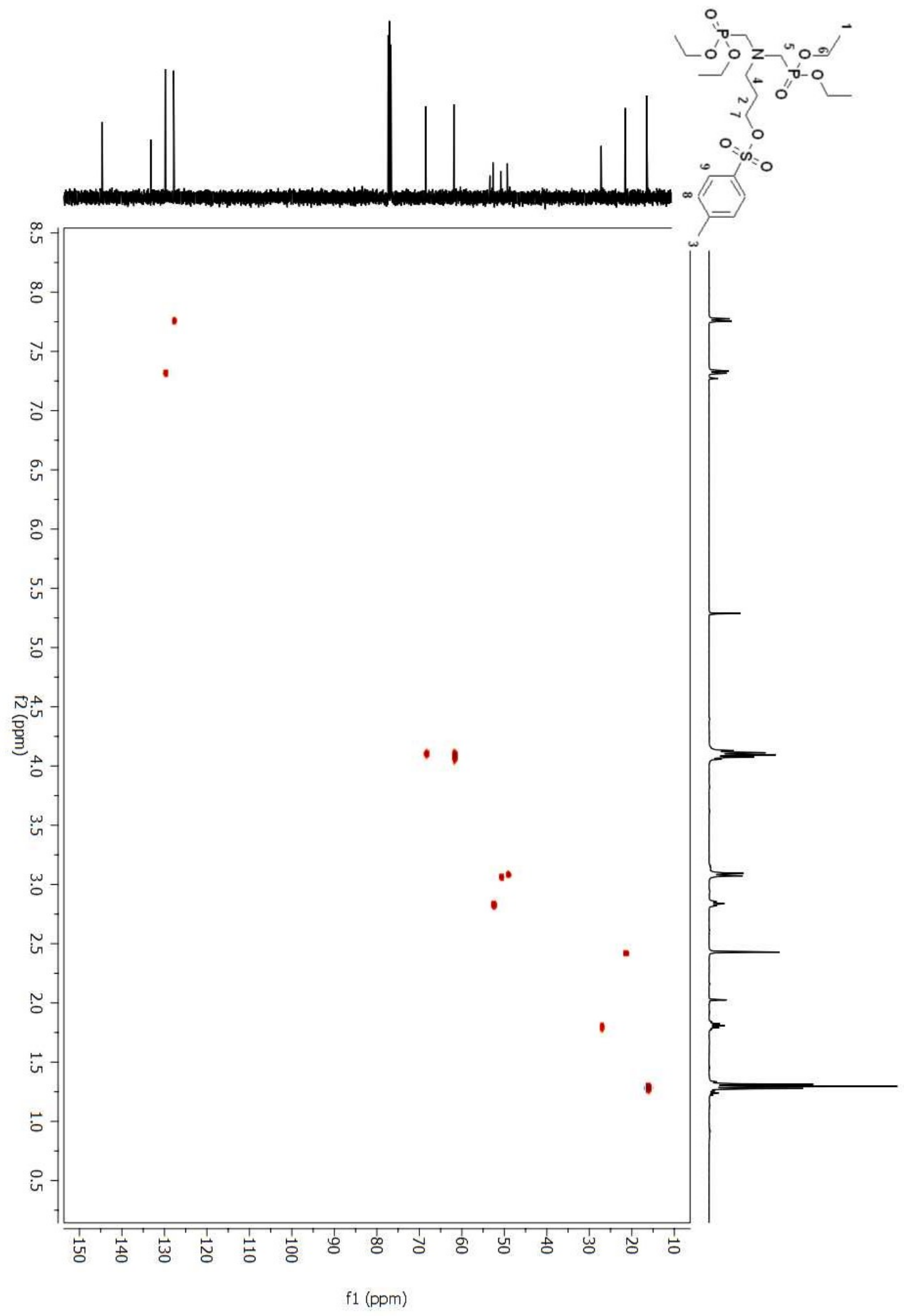

Figure A 226. HSQC 2D NMR spectrum of compound (119) in $\mathrm{CDCl}_{3}$. 593 


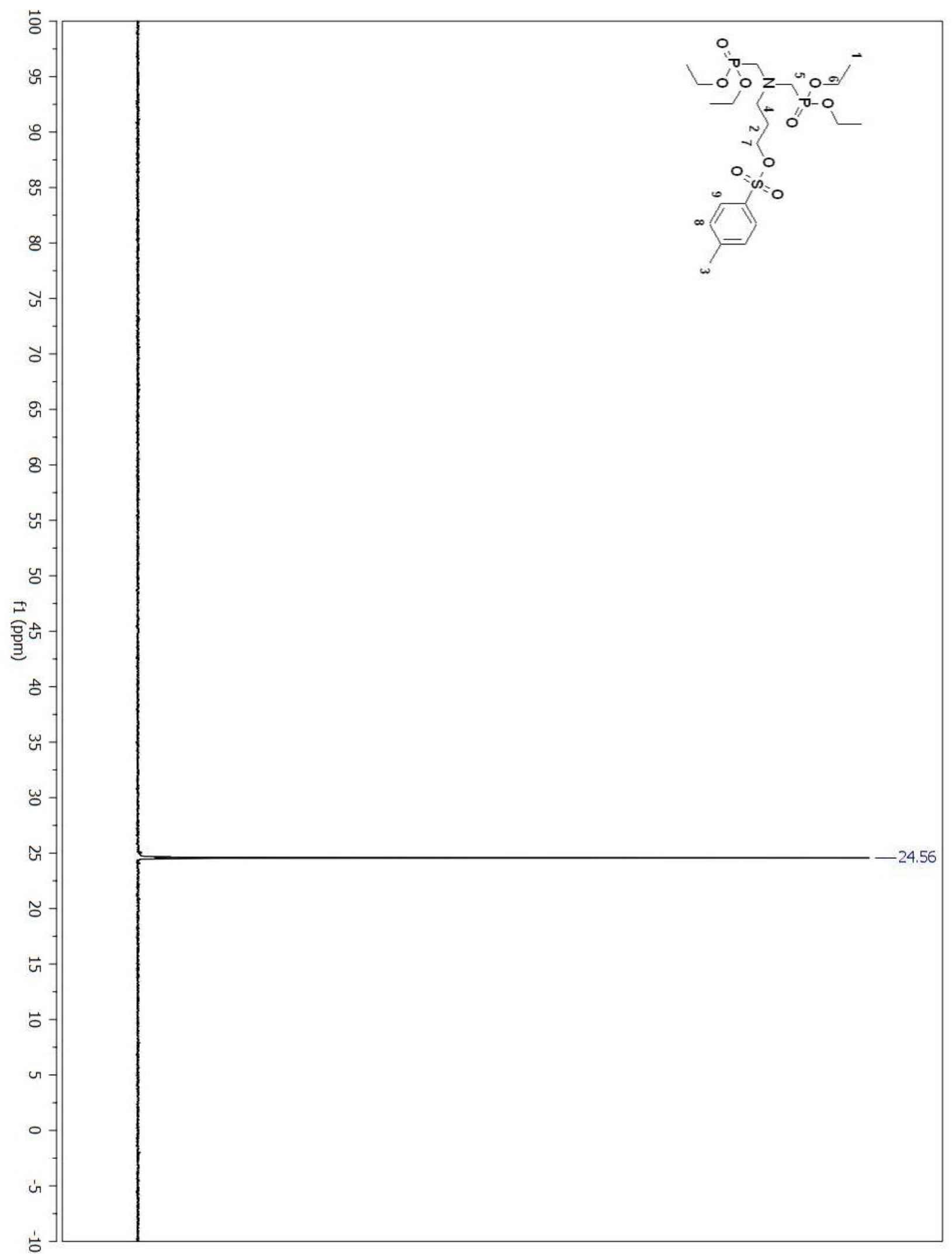

Figure A 227. ${ }^{31} \mathrm{P}$ NMR spectrum of compound (119) in $\mathrm{CDCl}_{3}$. 


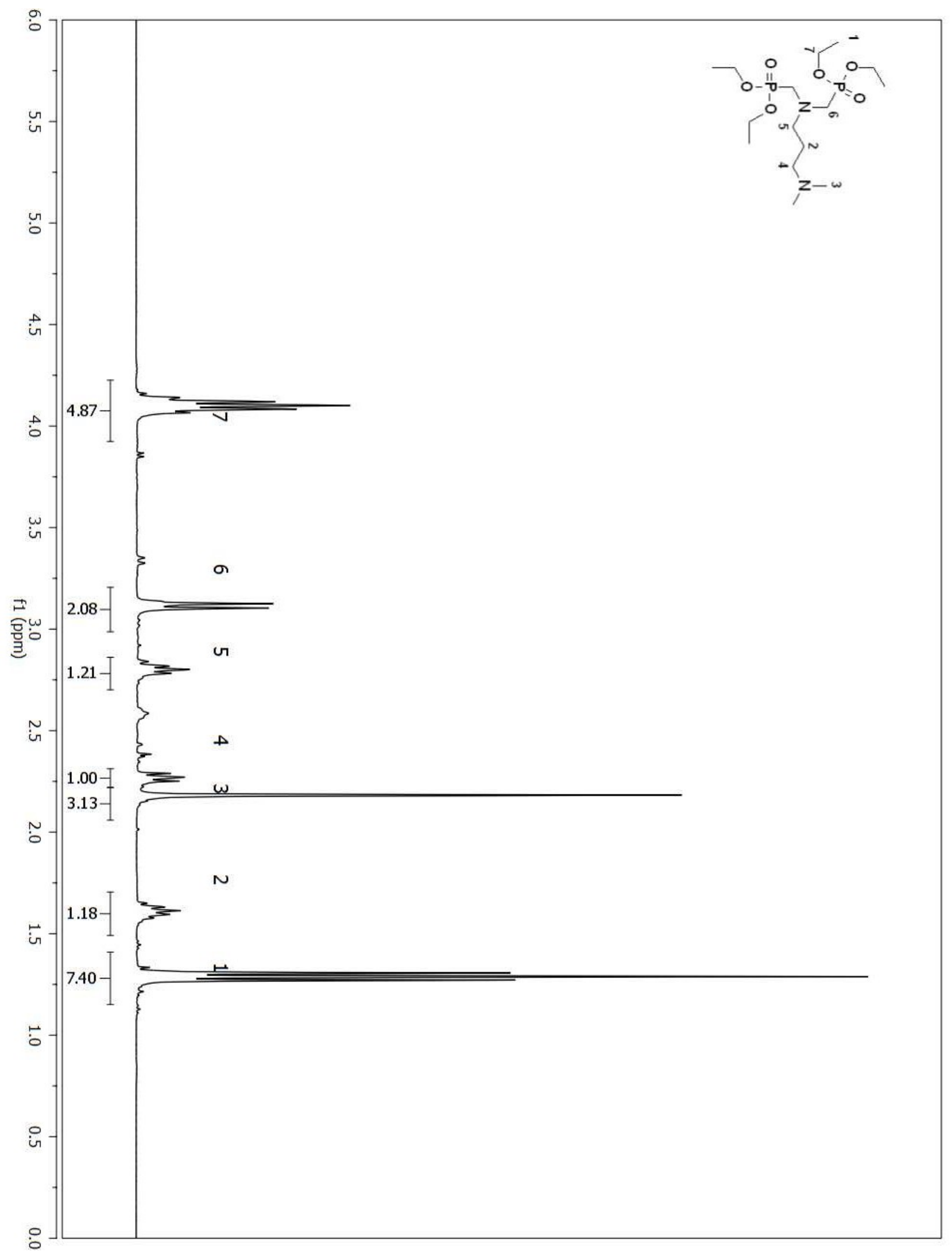

Figure A 228. ${ }^{1} \mathrm{H}$ NMR spectrum of compound (113) in $\mathrm{CDCl}_{3}$. 


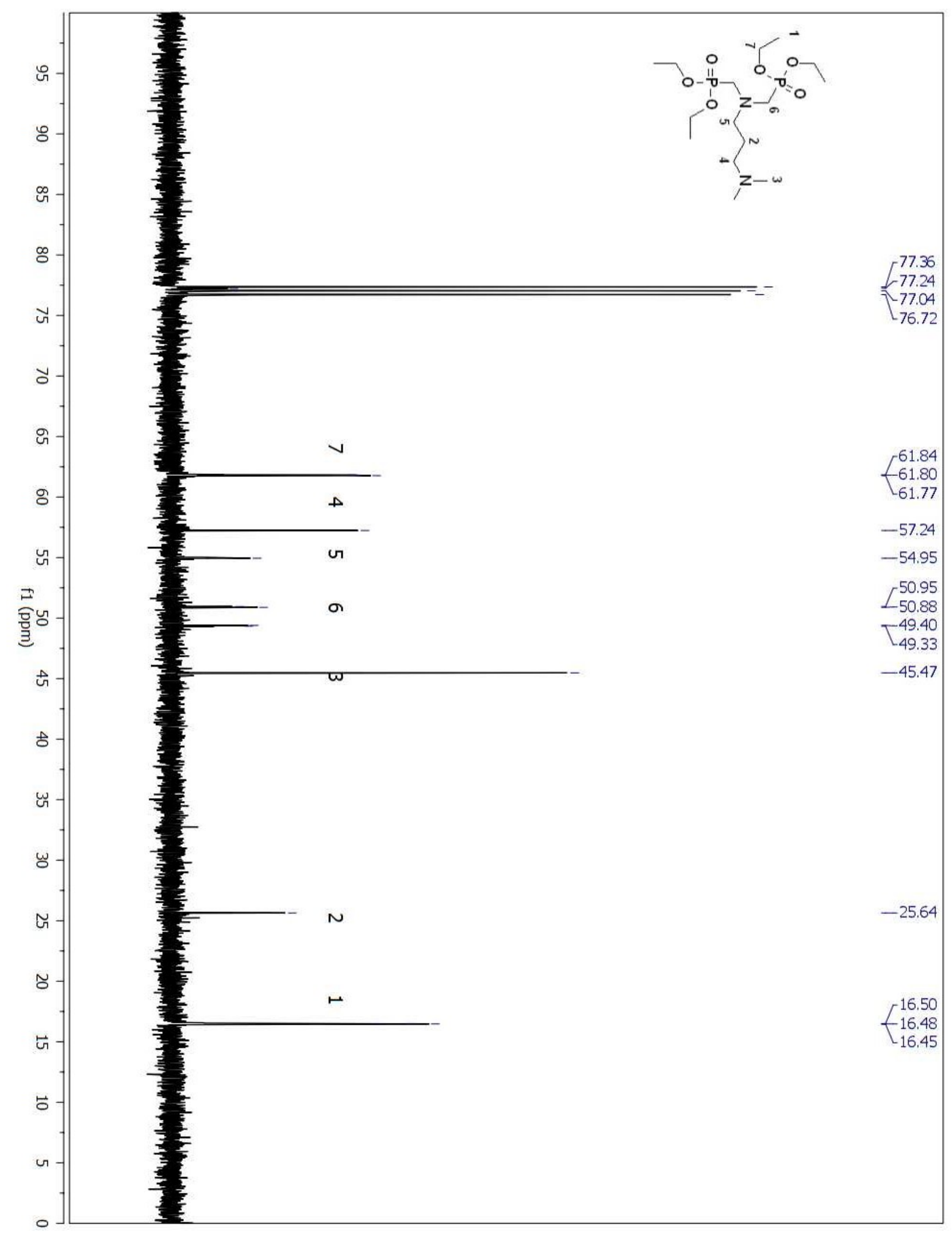

Figure A 229. ${ }^{13} \mathrm{C}$ NMR spectrum of compound (113) in $\mathrm{CDCl}_{3}$. 


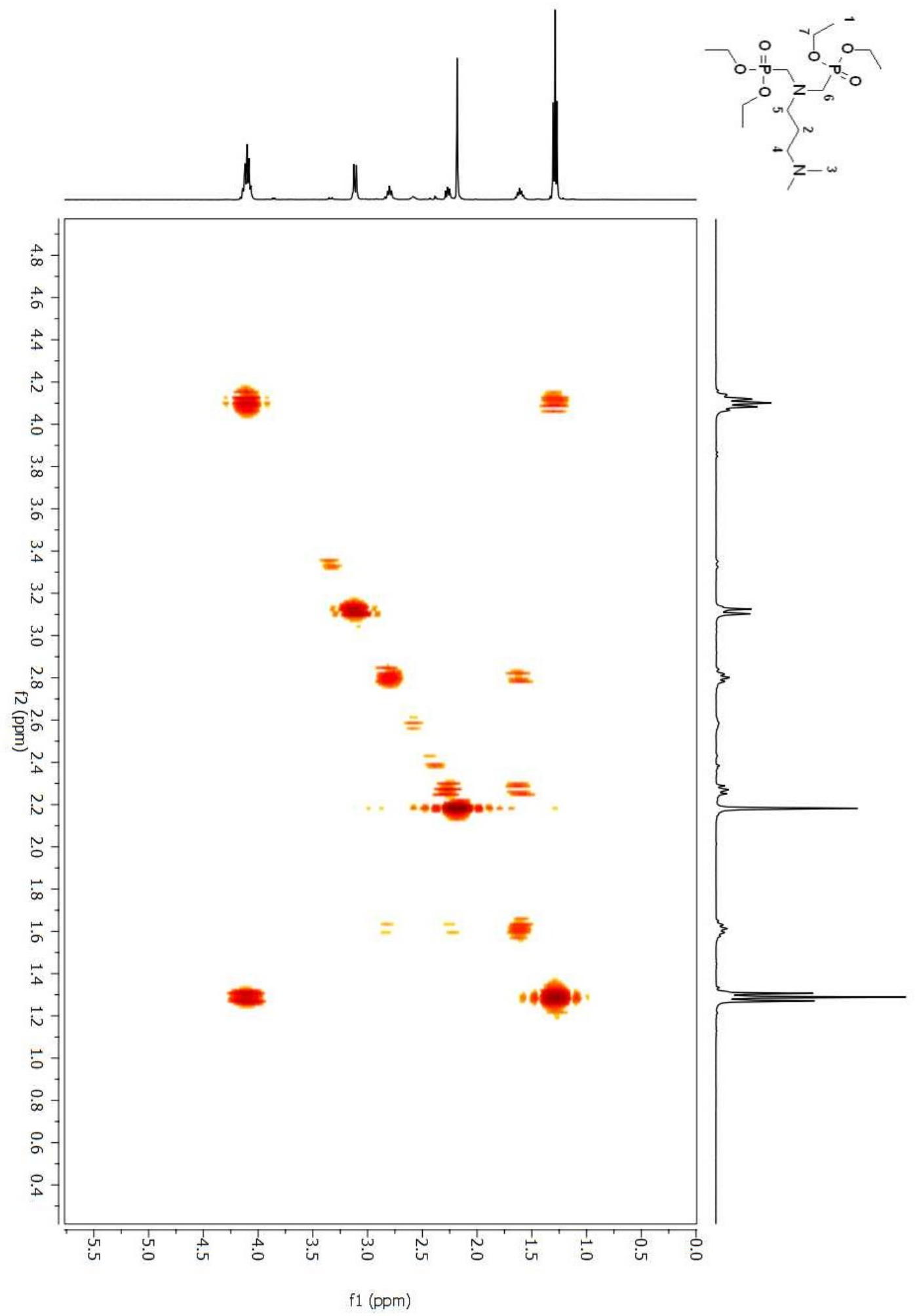

Figure A 230. COSY 2D NMR spectrum of compound (113) in $\mathrm{CDCl}_{3}$. 


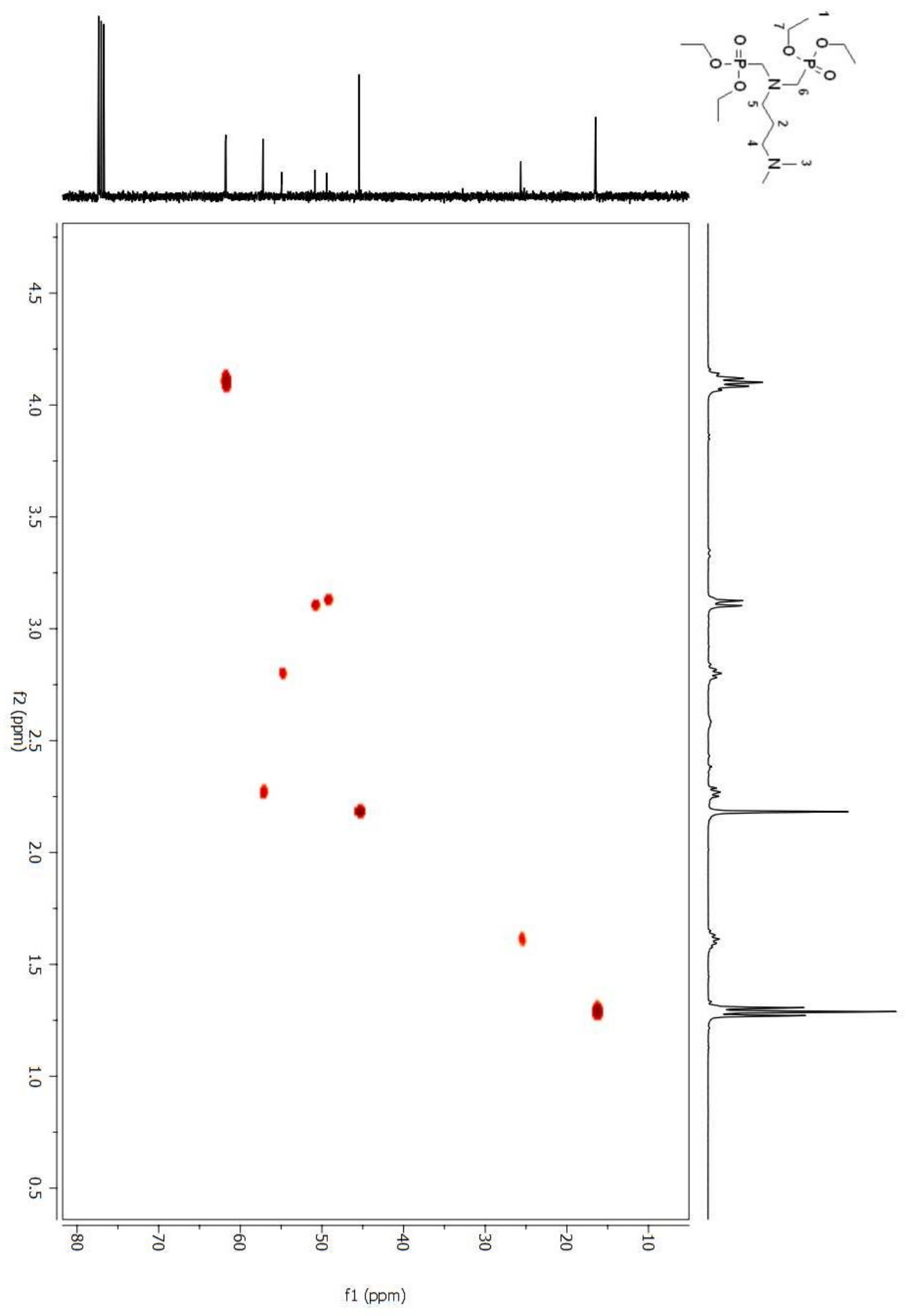

Figure A 231. HSQC 2D NMR spectrum of compound (113) in $\mathrm{CDCl}_{3}$. 


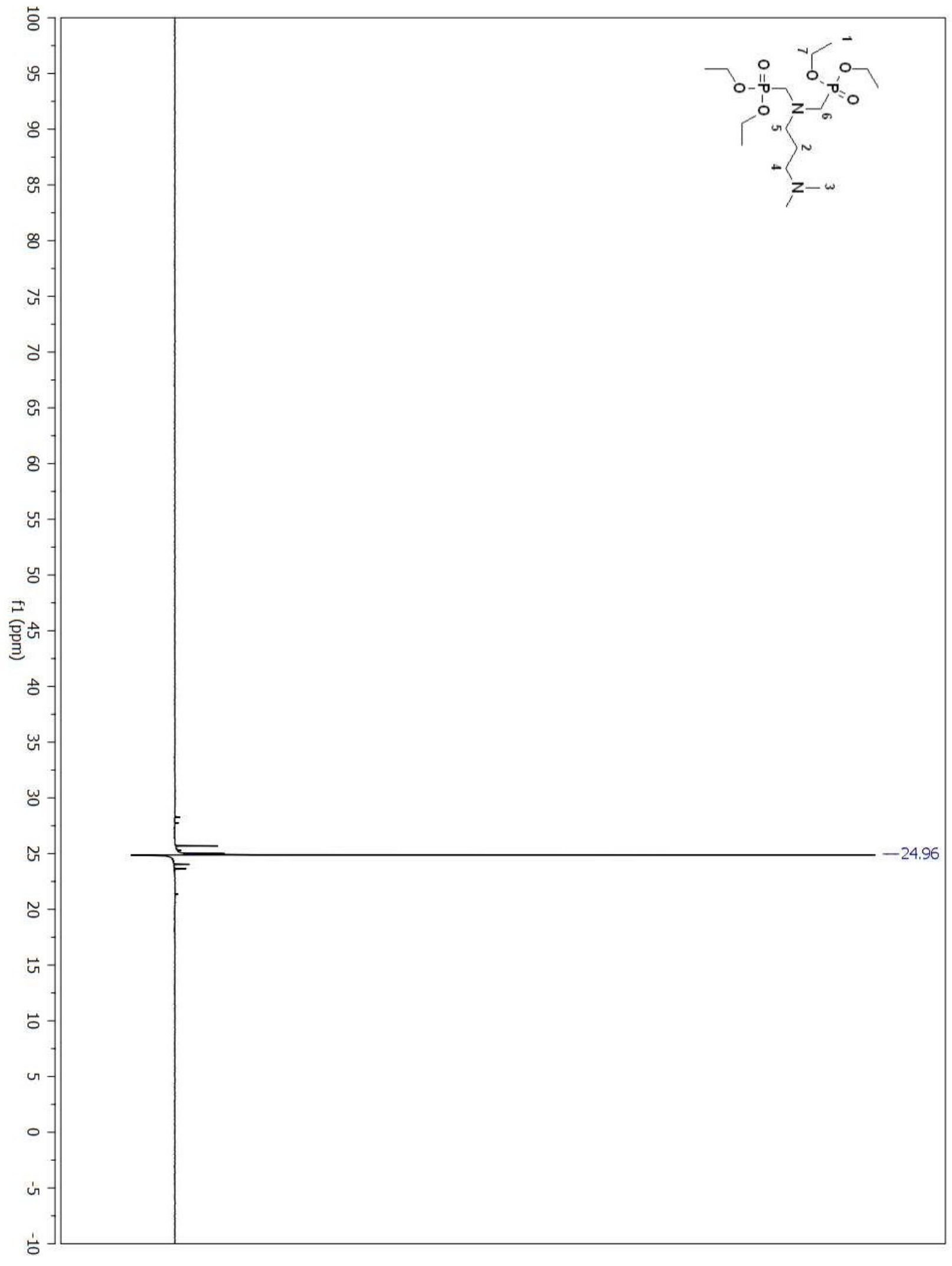

Figure A 232. ${ }^{31} \mathrm{P}$ NMR spectrum of compound (113) in $\mathrm{CDCl}_{3}$. 


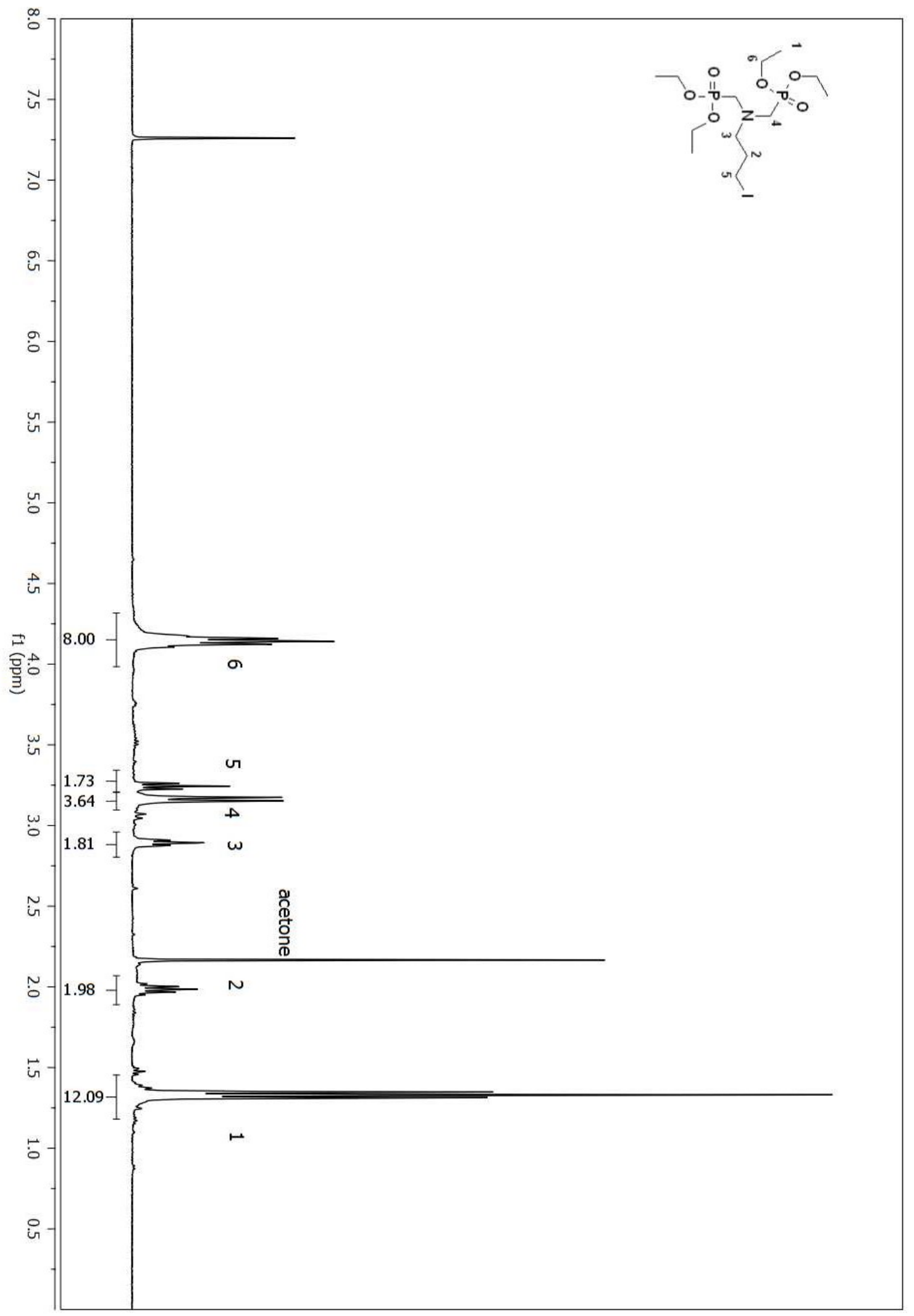

Figure A 233. ${ }^{1} \mathrm{H}$ NMR spectrum of compound (120) in $\mathrm{CDCl}_{3}$. 600 


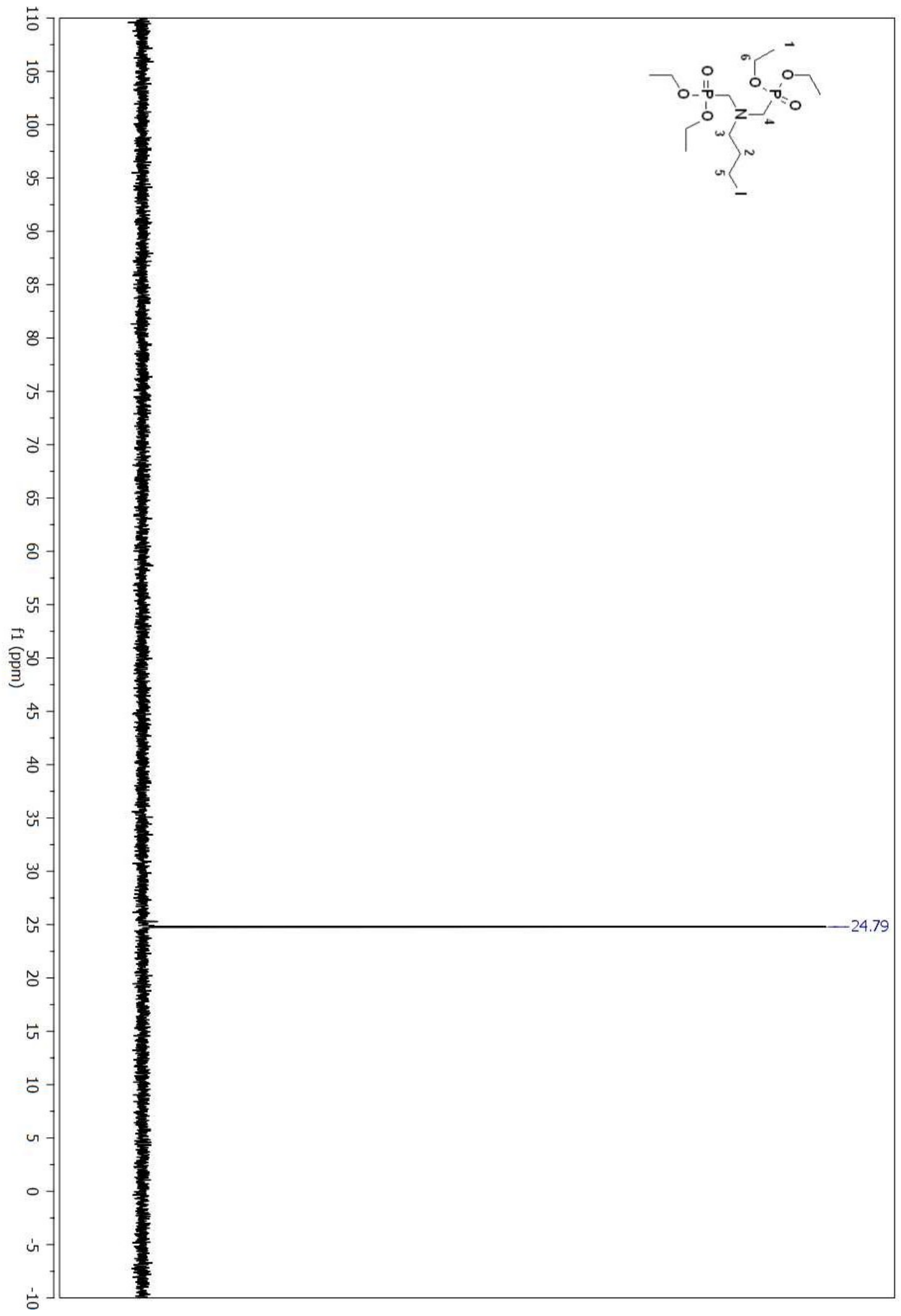

Figure A 234. ${ }^{31} \mathrm{P}$ NMR spectrum of compound (120) in $\mathrm{CDCl}_{3}$. 


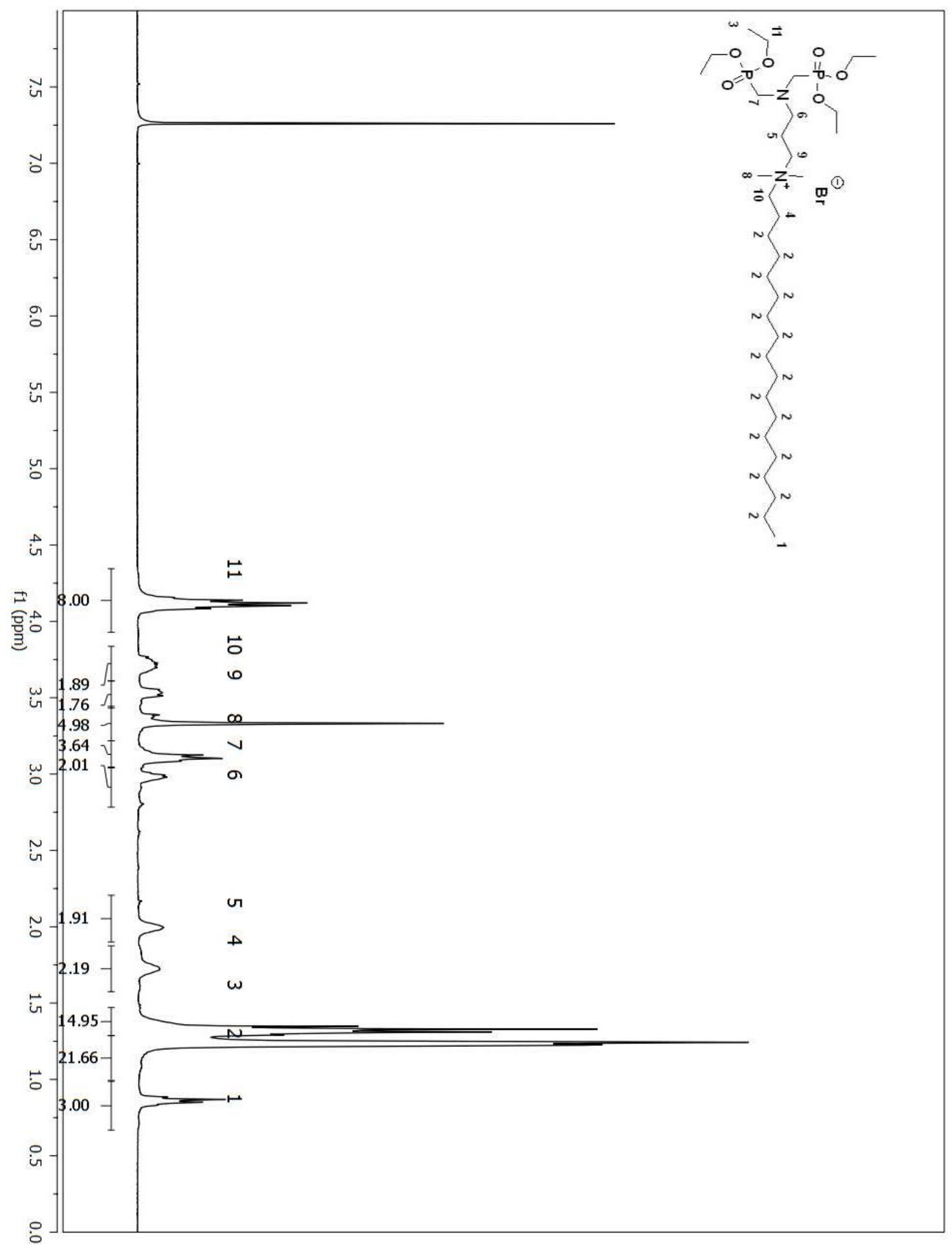

Figure A 235. ${ }^{1} \mathrm{H}$ NMR spectrum of compound (121) in $\mathrm{CDCl}_{3}$. 


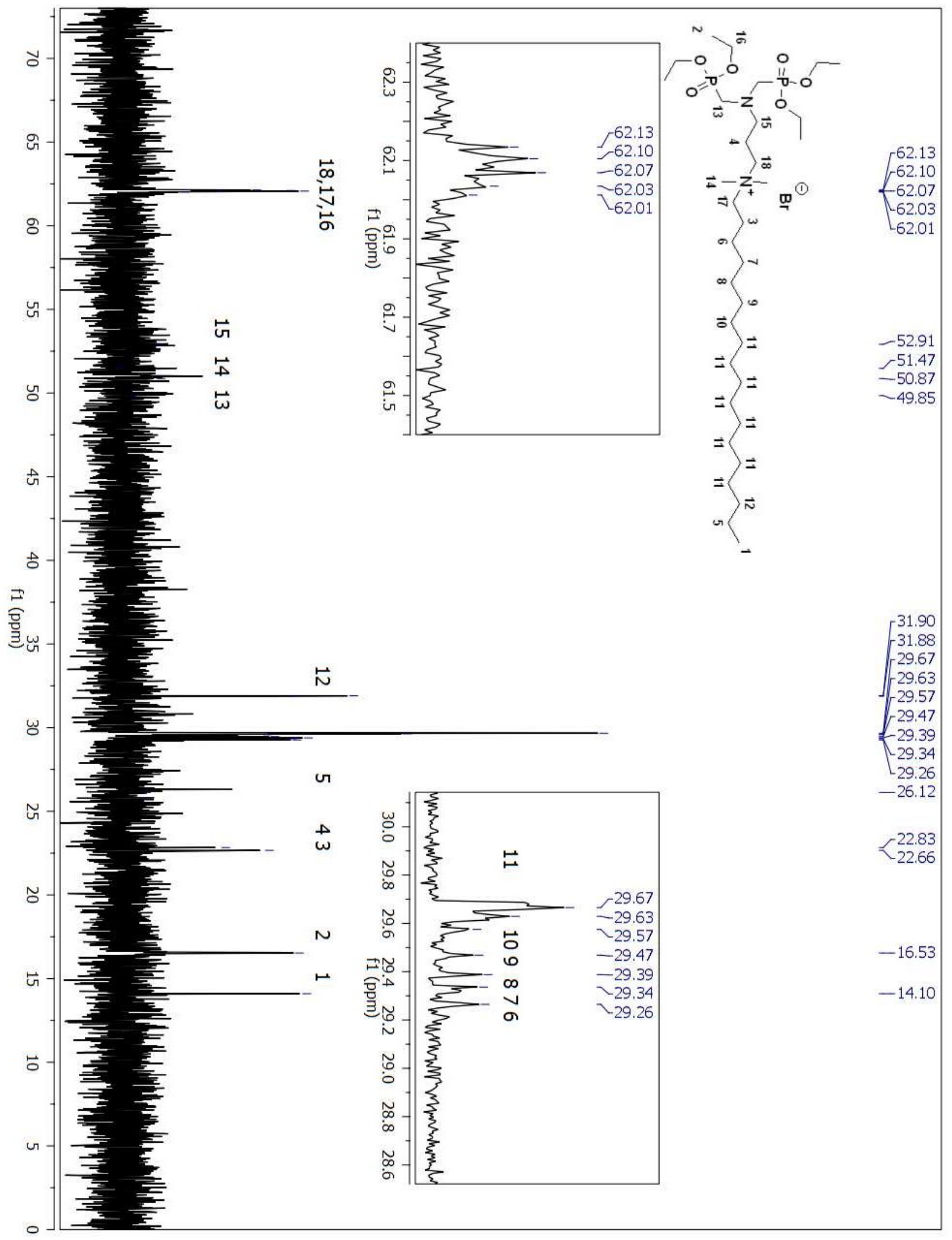

Figure A 236. ${ }^{13} \mathrm{C}$ NMR spectrum of compound (121) in $\mathrm{CDCl}_{3}$. 


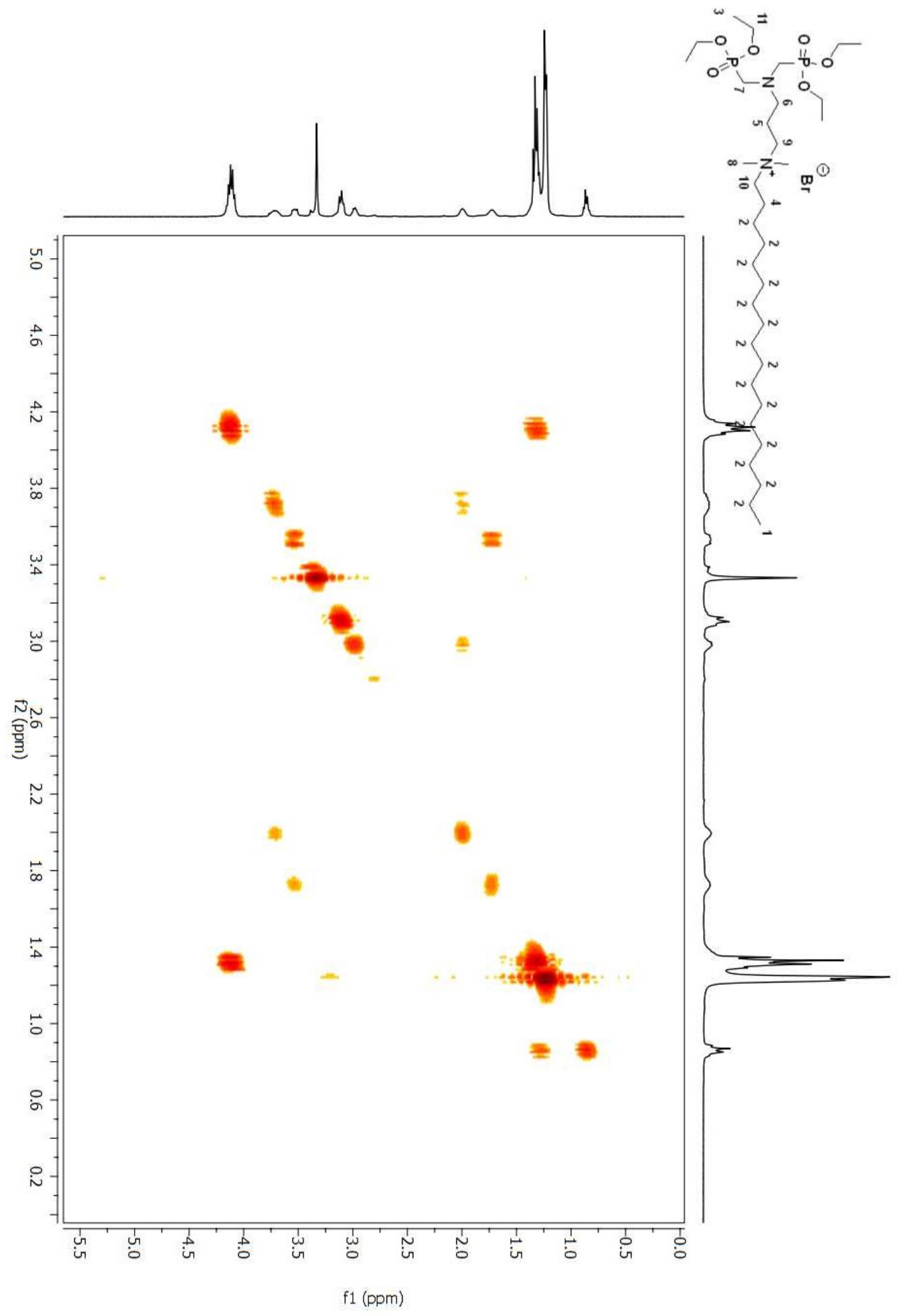

Figure A 237. COSY 2D NMR spectrum of compound (121) in $\mathrm{CDCl}_{3}$. 


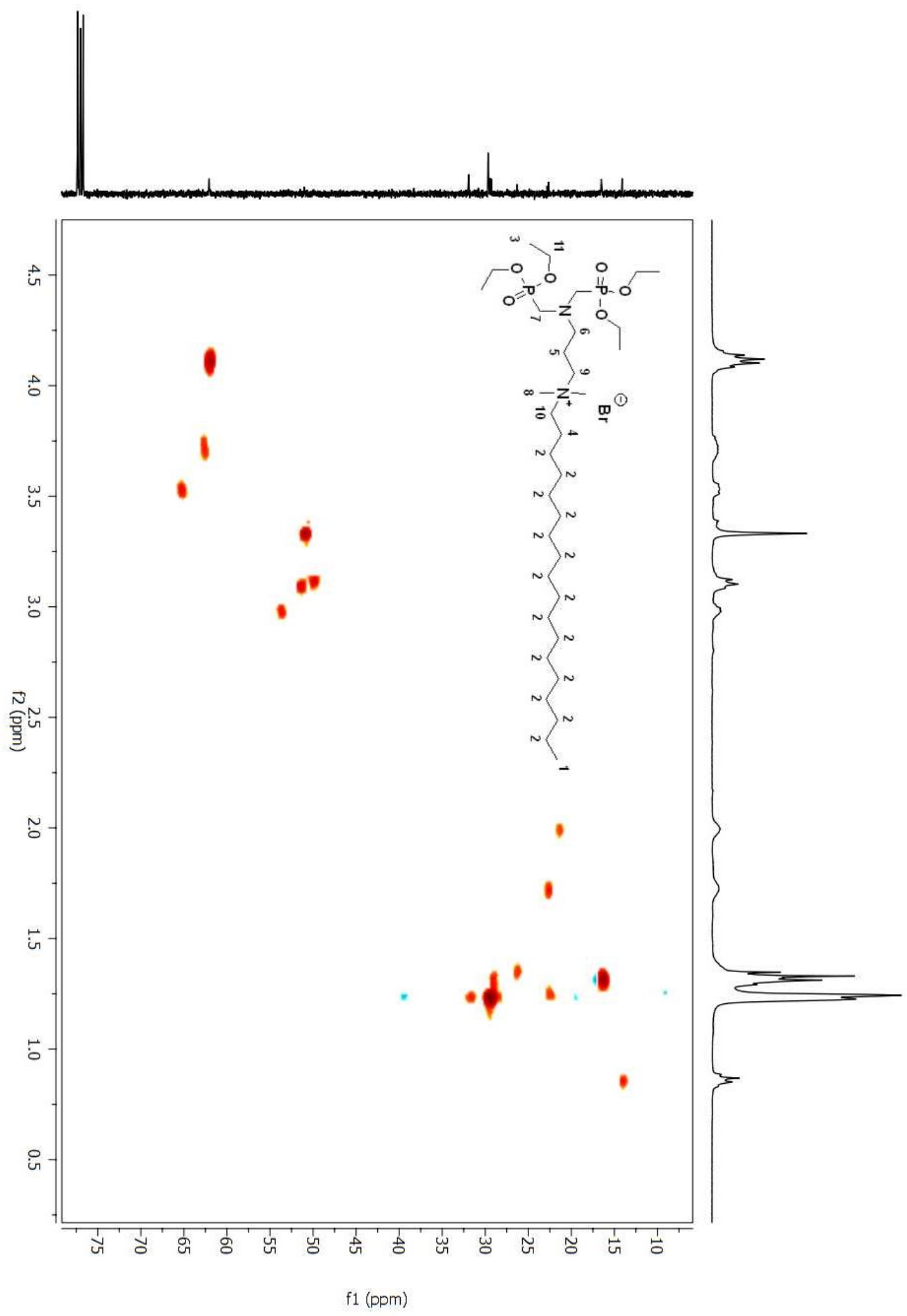

Figure A 238. HSQC 2D NMR spectrum of compound (121) in $\mathrm{CDCl}_{3}$. 605 


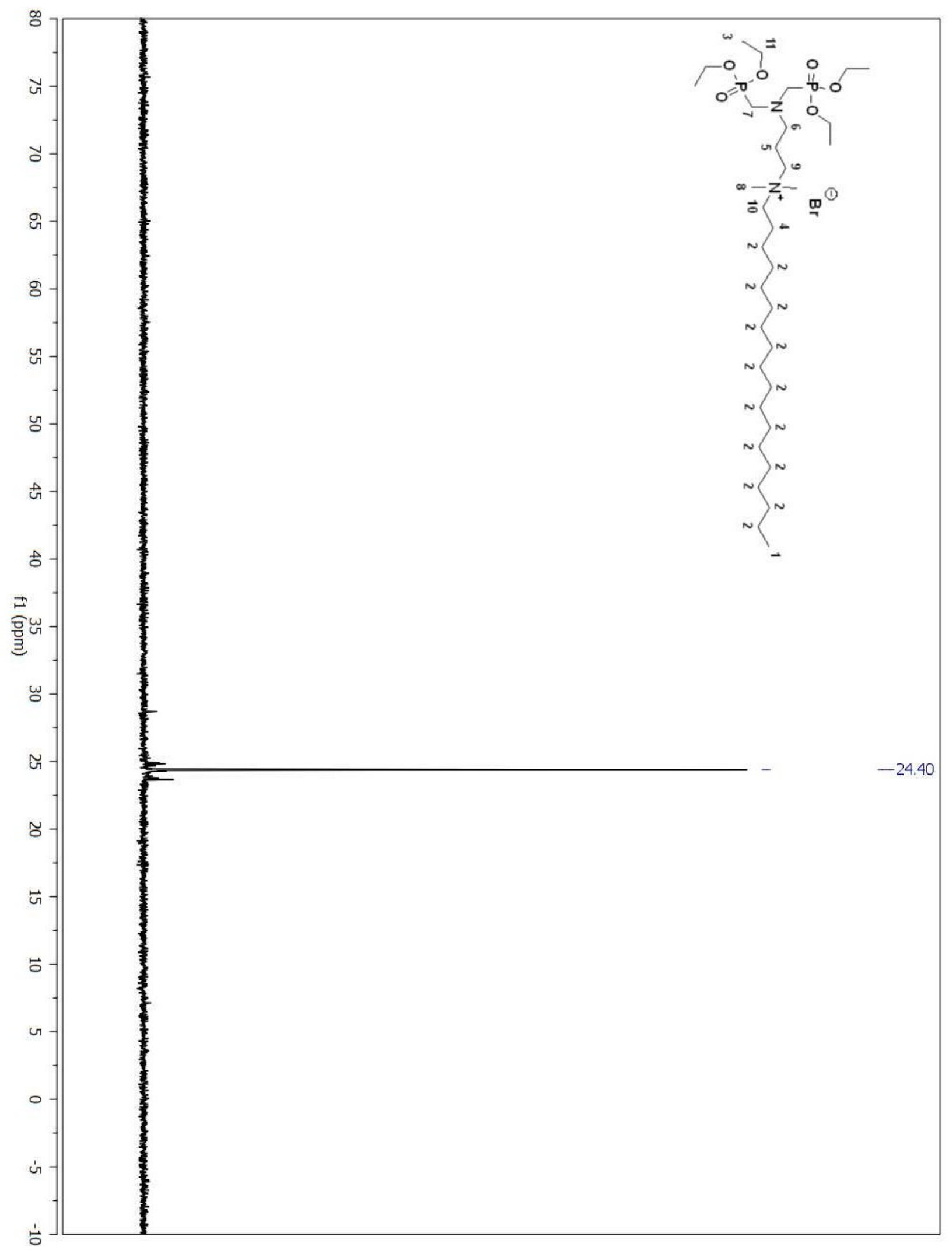

Figure A 239. ${ }^{31} \mathrm{P}$ NMR spectrum of compound (121) in $\mathrm{CDCl}_{3}$. 606 


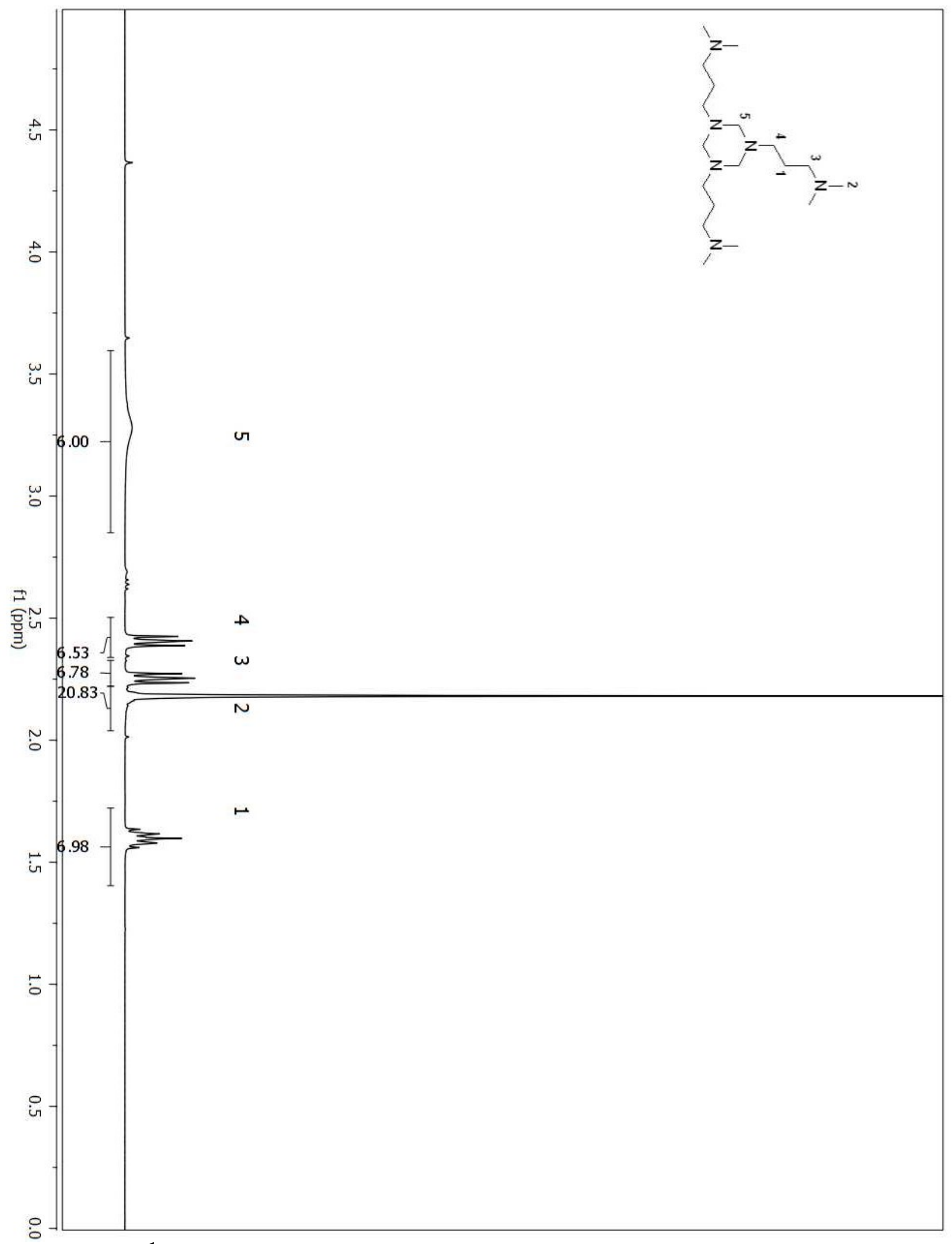

Figure A 240. ${ }^{1} \mathrm{H}$ NMR spectrum of compound (123) in $\mathrm{CDCl}_{3}$. 607 


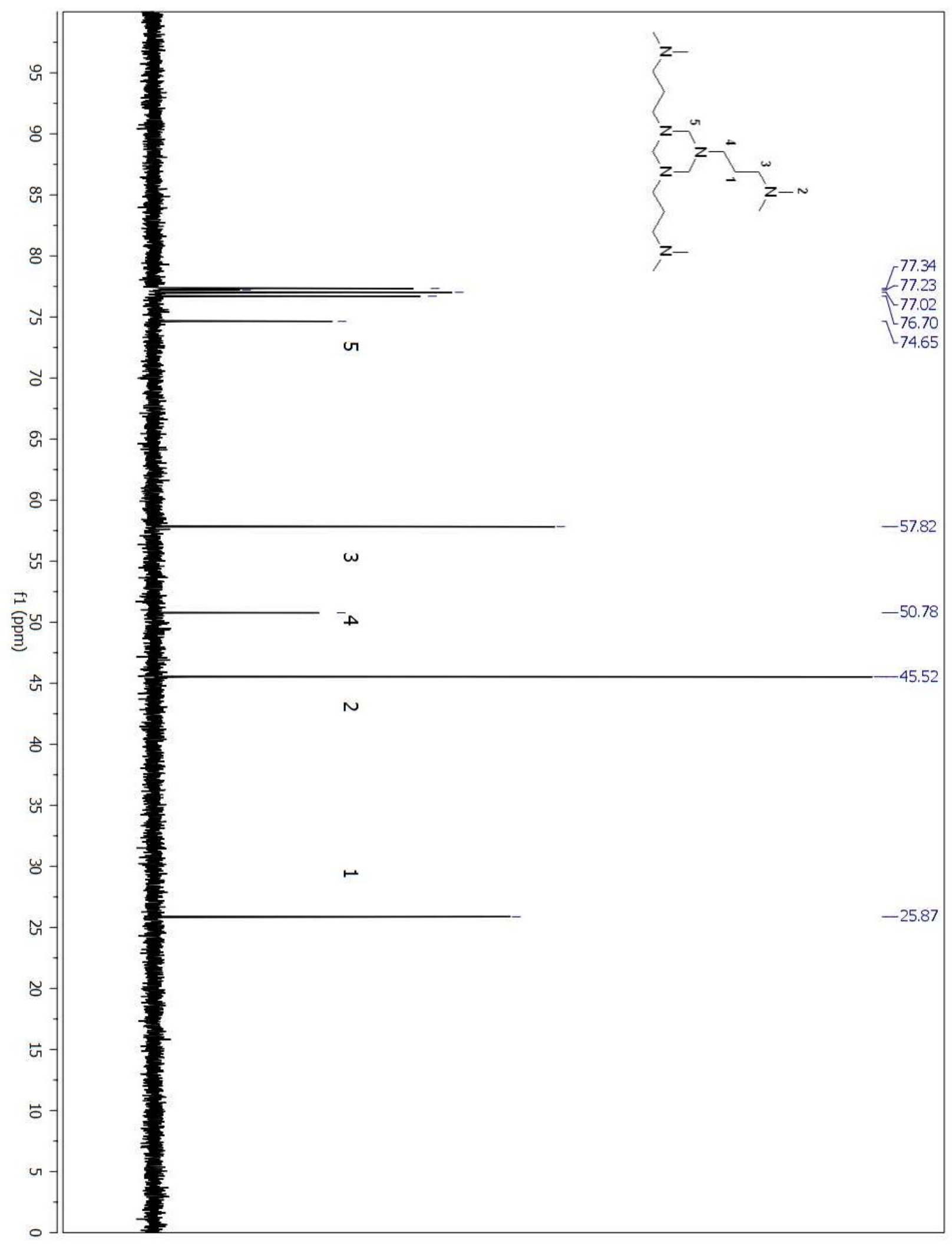

Figure A 241. ${ }^{13} \mathrm{C}$ NMR spectrum of compound (123) in $\mathrm{CDCl}_{3}$. 


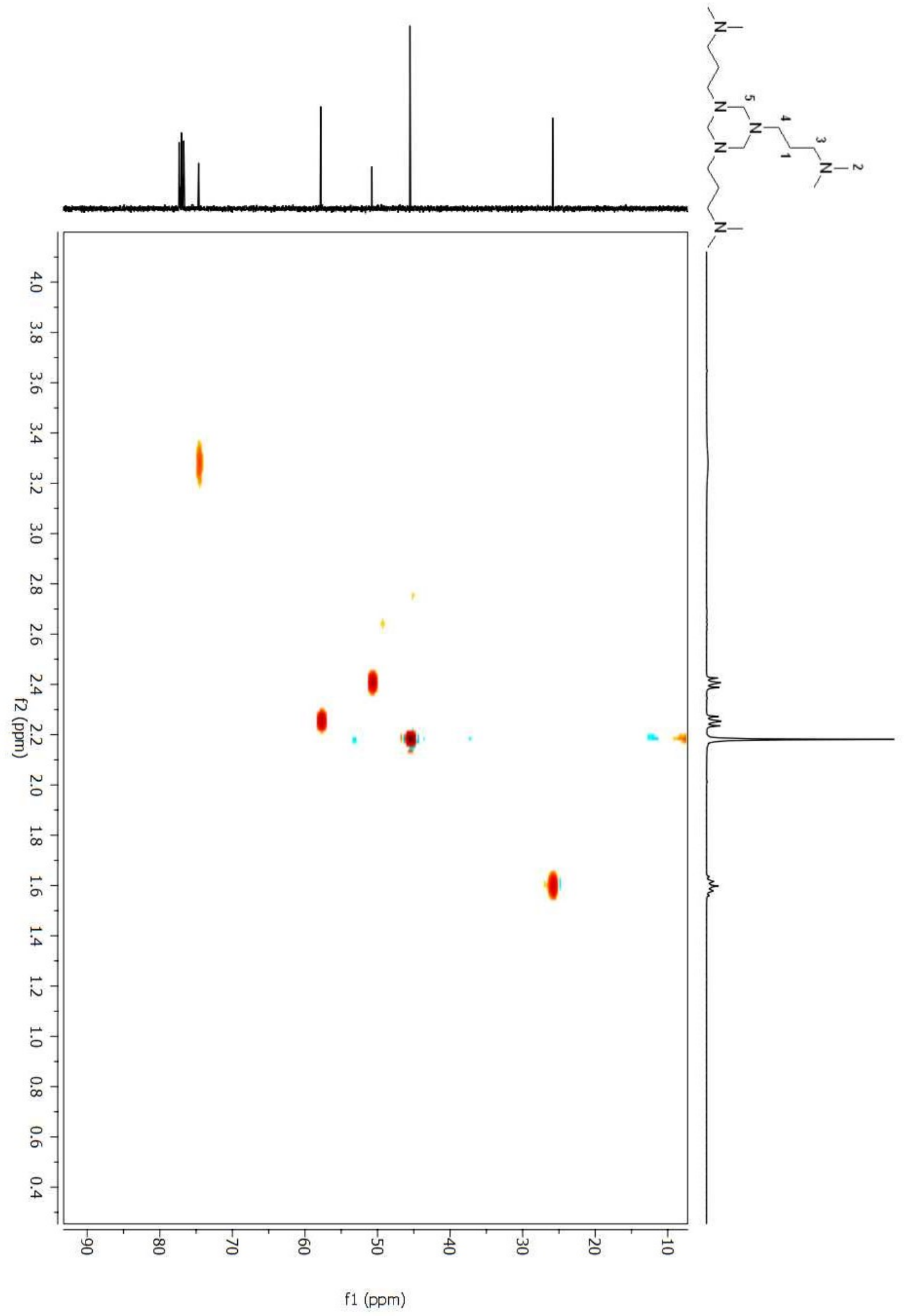

Figure A 242. HSQC 2D NMR spectrum of compound (123) in $\mathrm{CDCl}_{3}$. 609 


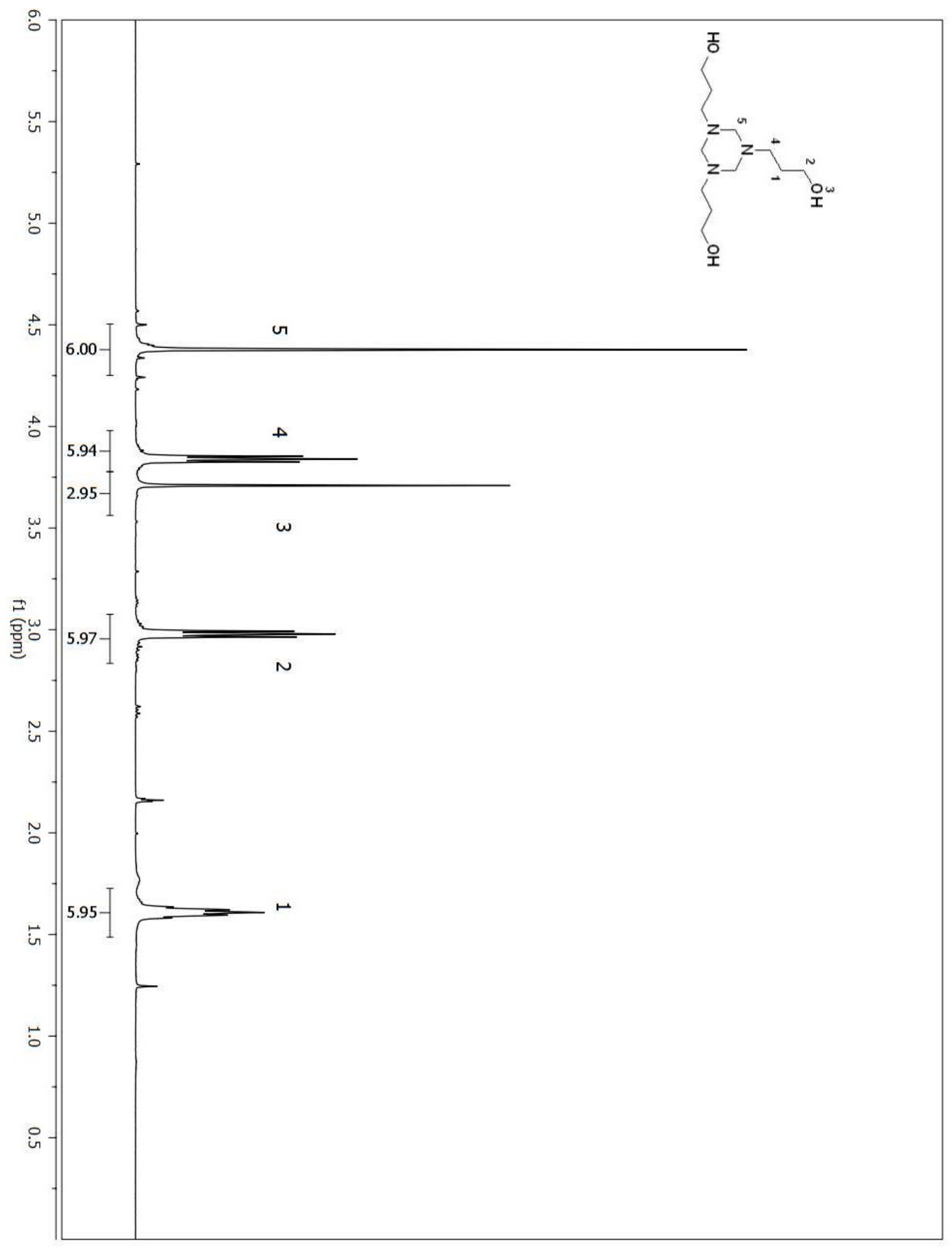

Figure A 243. ${ }^{1} \mathrm{H}$ NMR spectrum of compound (122) in $\mathrm{CDCl}_{3}$. 610 


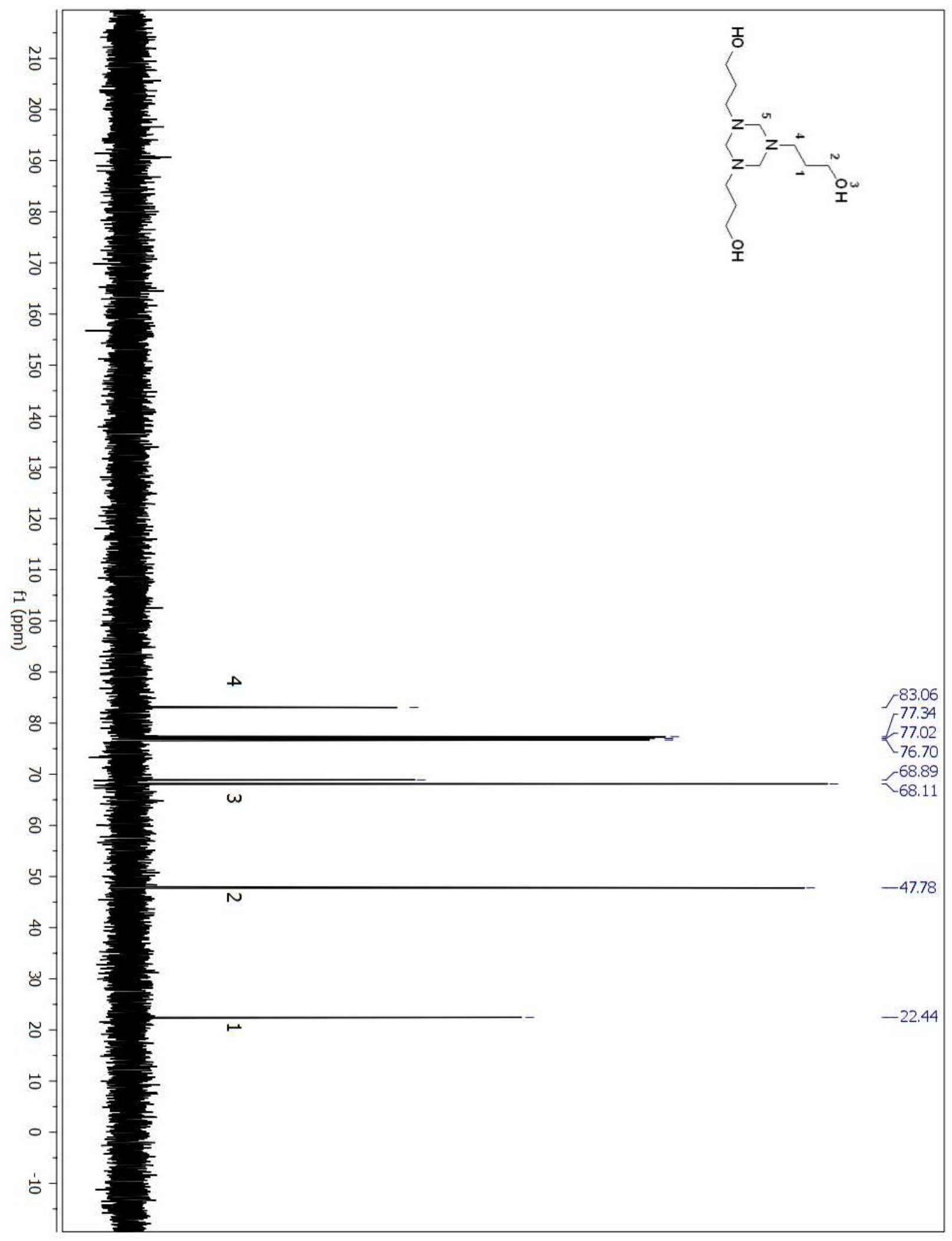

Figure A 244. ${ }^{13} \mathrm{C}$ NMR spectrum of compound (122) in $\mathrm{CDCl}_{3}$. 


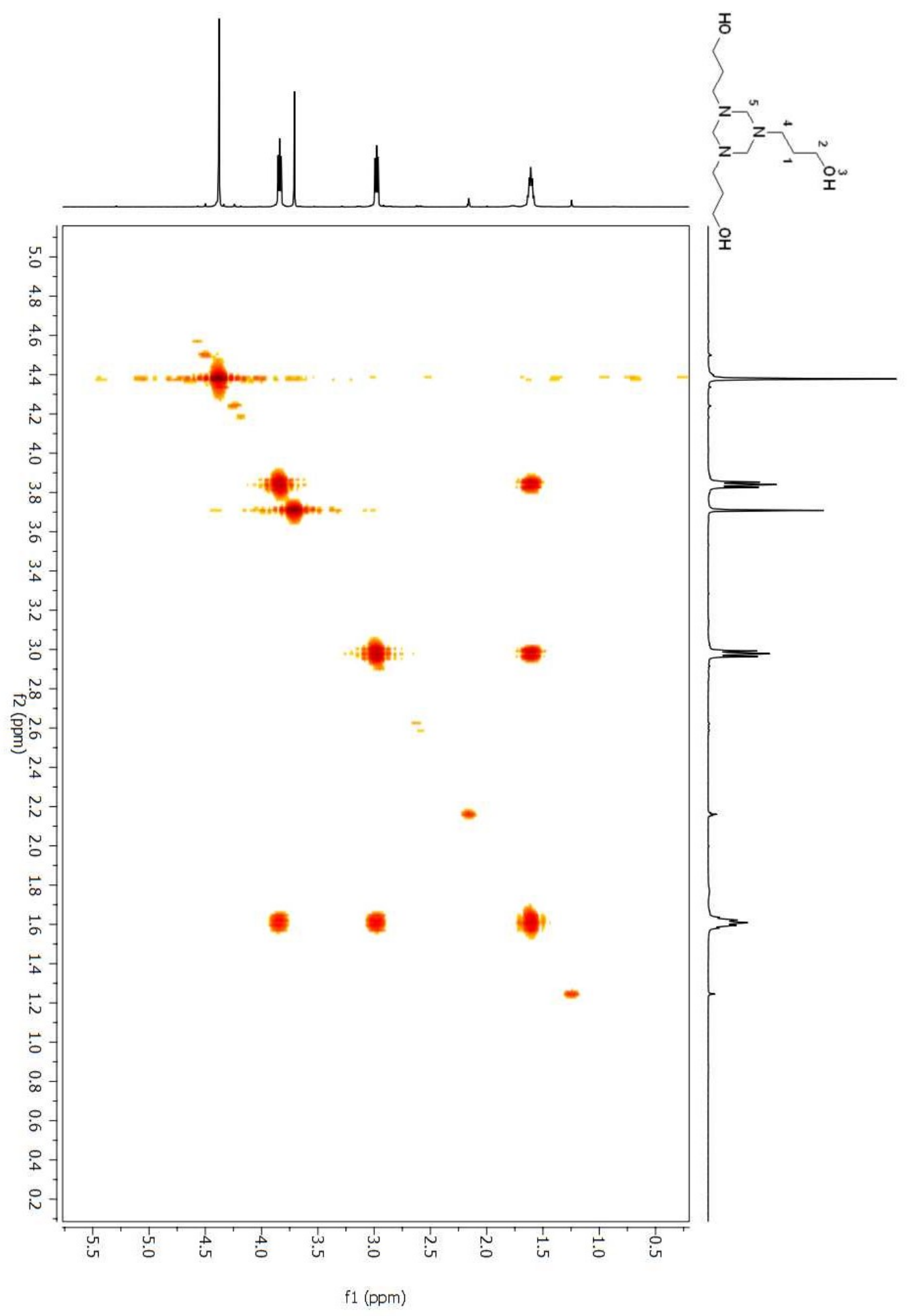

Figure A 245. COSY 2D NMR spectrum of compound (122) in $\mathrm{CDCl}_{3}$. 


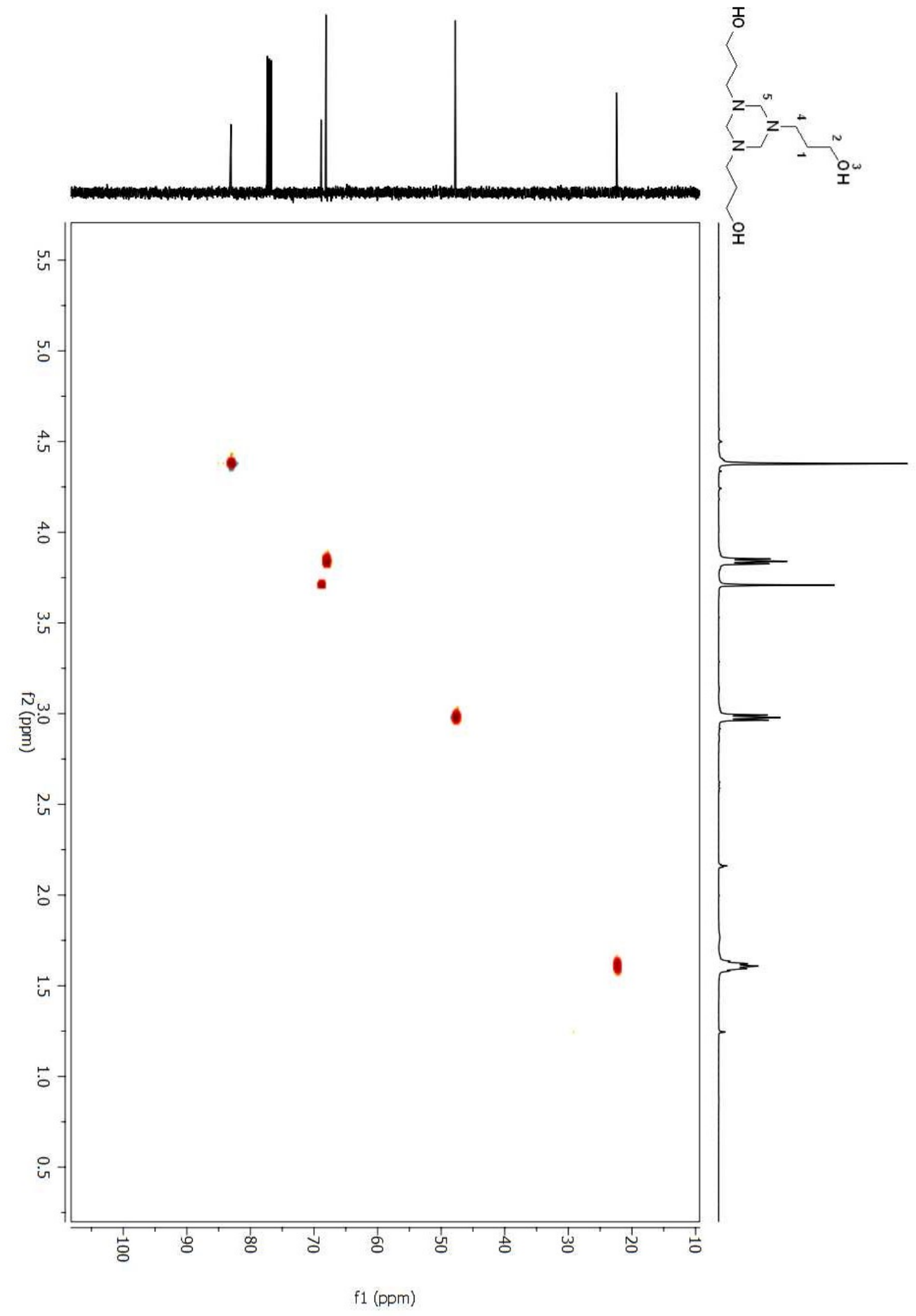

Figure A 246. HSQC 2D NMR spectrum of compound (122) in $\mathrm{CDCl}_{3}$. 


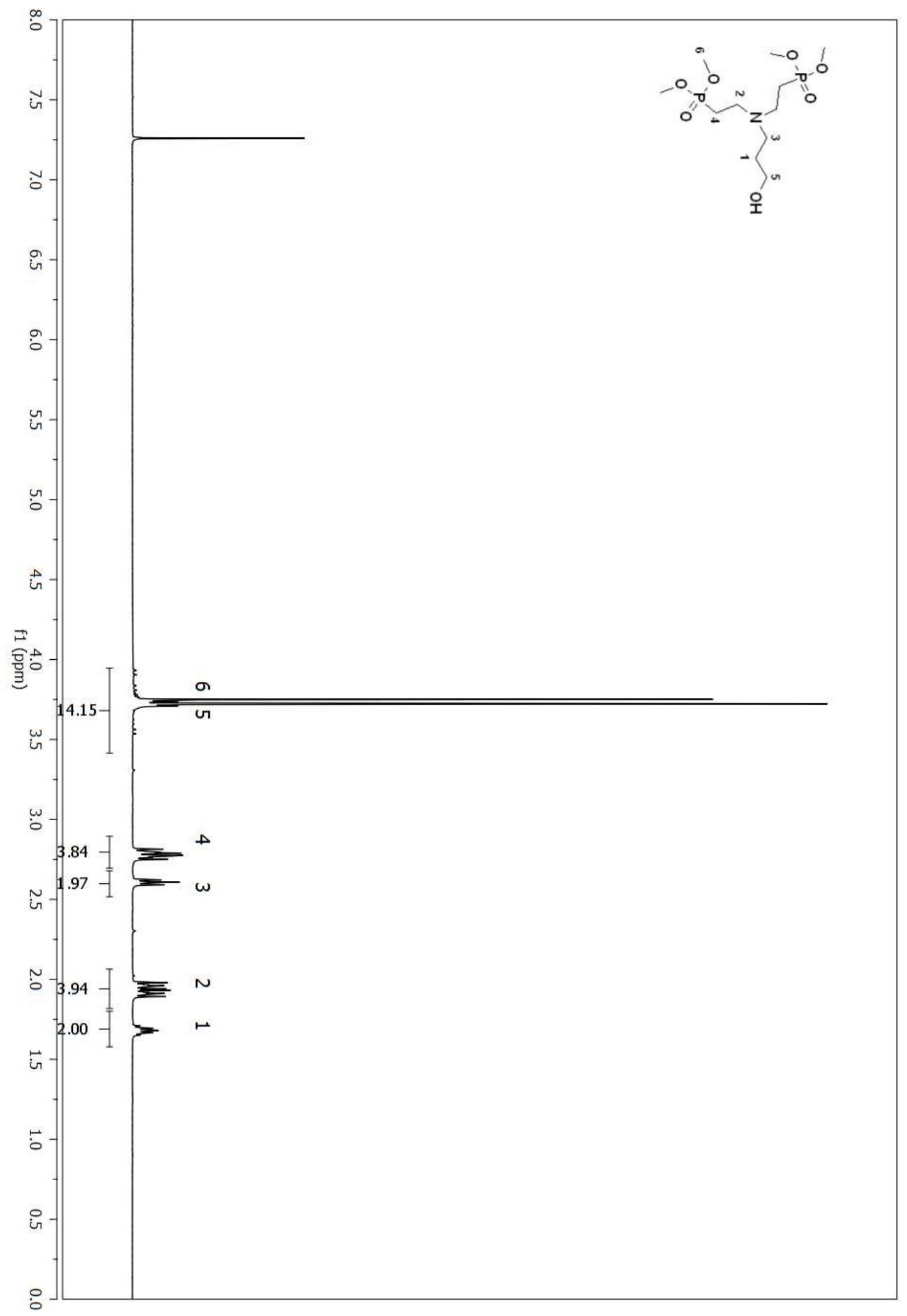

Figure A 247. ${ }^{1} \mathrm{H}$ NMR spectrum of compound (135) in $\mathrm{CDCl}_{3}$. 614 


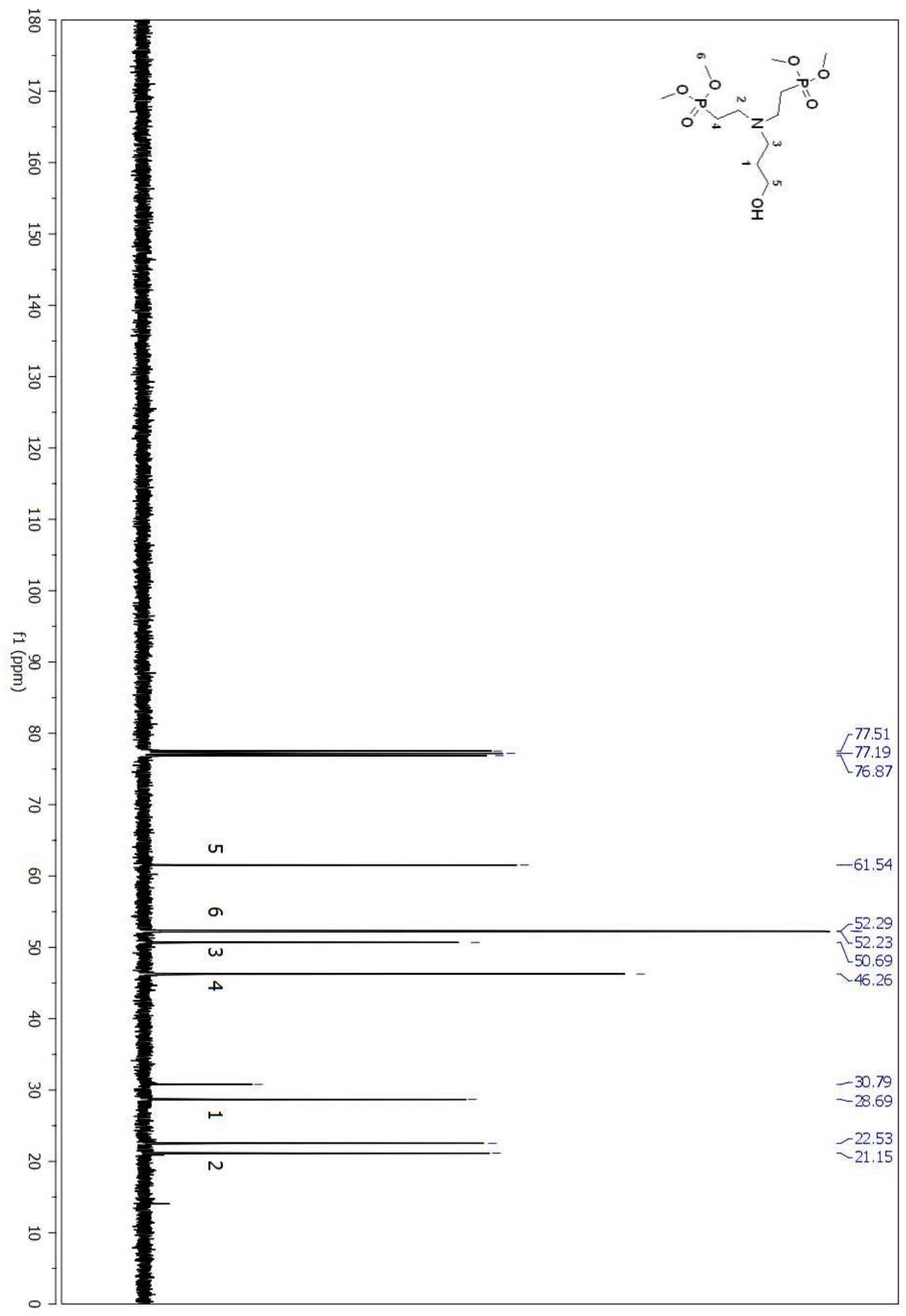

Figure A 248. ${ }^{13} \mathrm{C}$ NMR spectrum of compound (135) in $\mathrm{CDCl}_{3}$. 


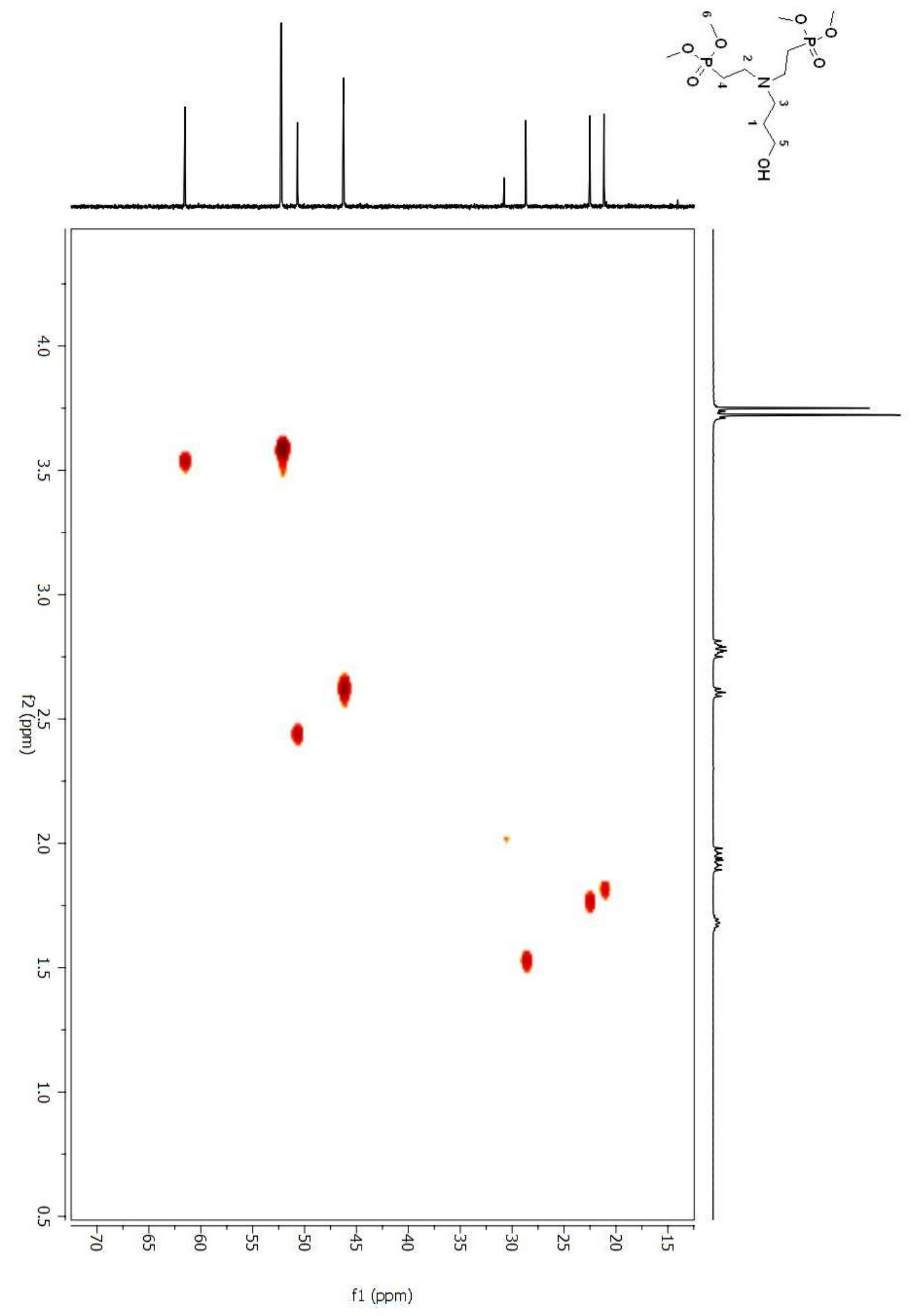

Figure A 249. HSQC 2D NMR spectrum of compound (135) in $\mathrm{CDCl}_{3}$. 616 


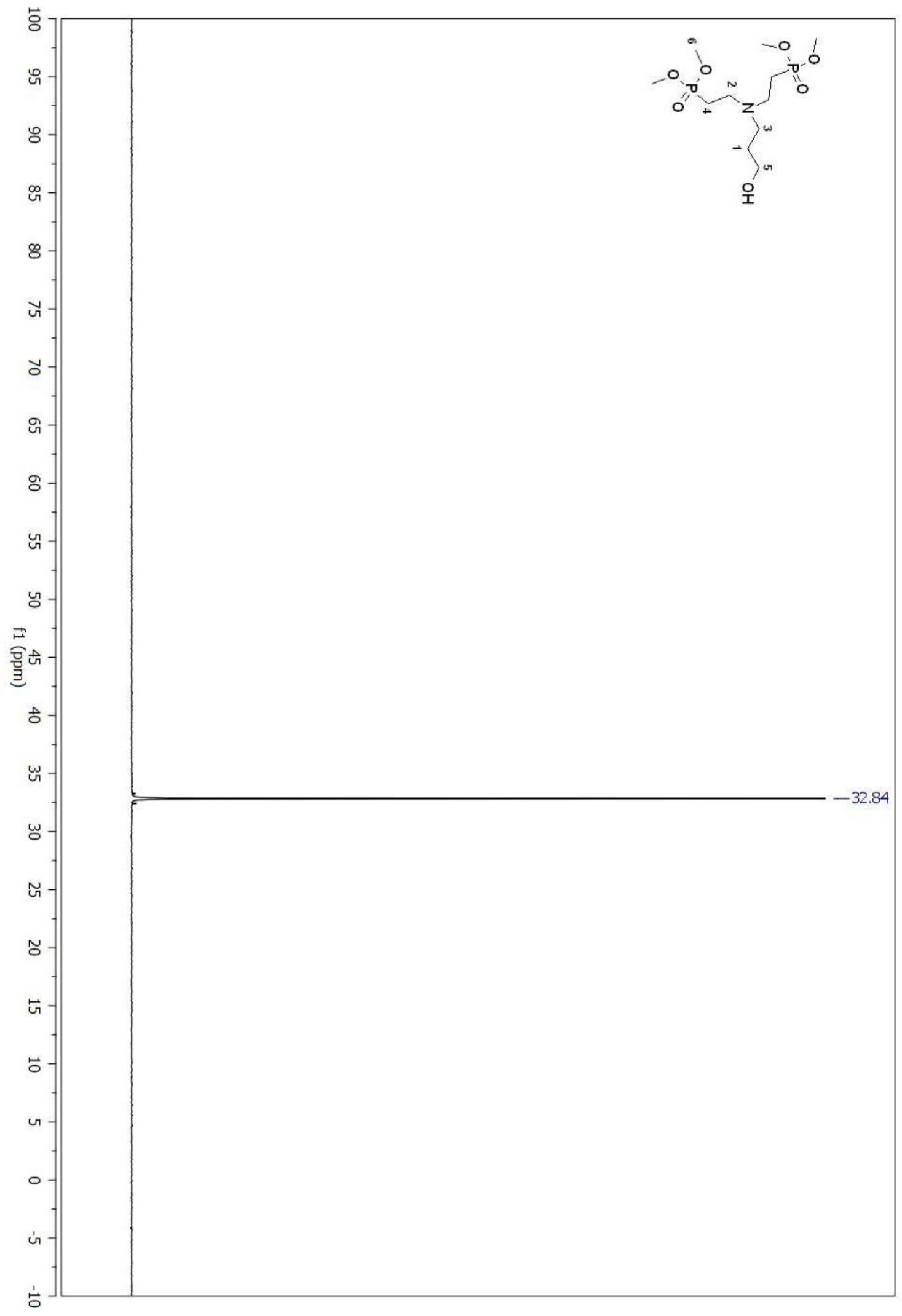

Figure A 250. ${ }^{31} \mathrm{P}$ NMR spectrum of compound (135) in $\mathrm{CDCl}_{3}$. 


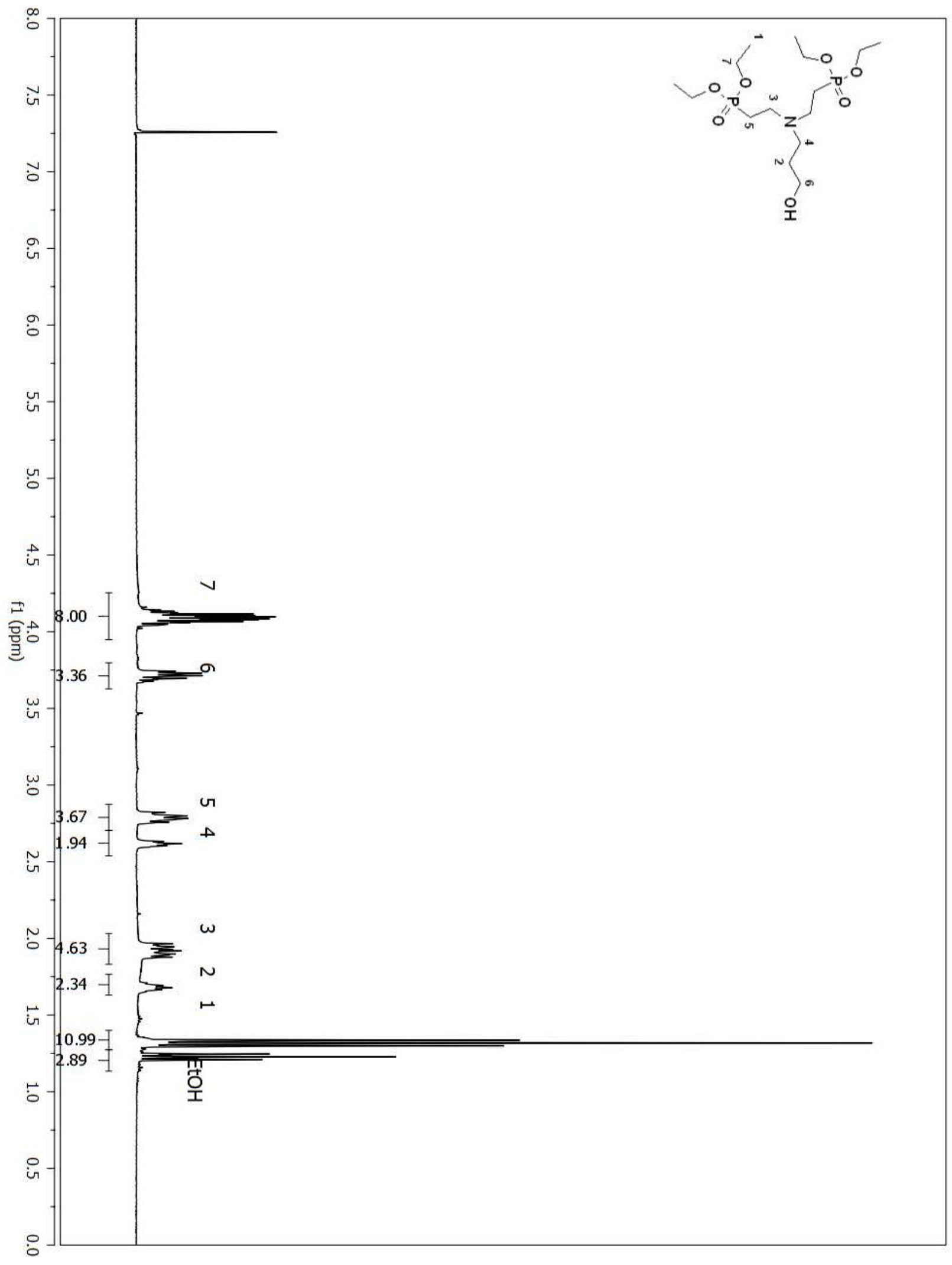

Figure A 251. ${ }^{1} \mathrm{H}$ NMR spectrum of compound (136) in $\mathrm{CDCl}_{3}$. 


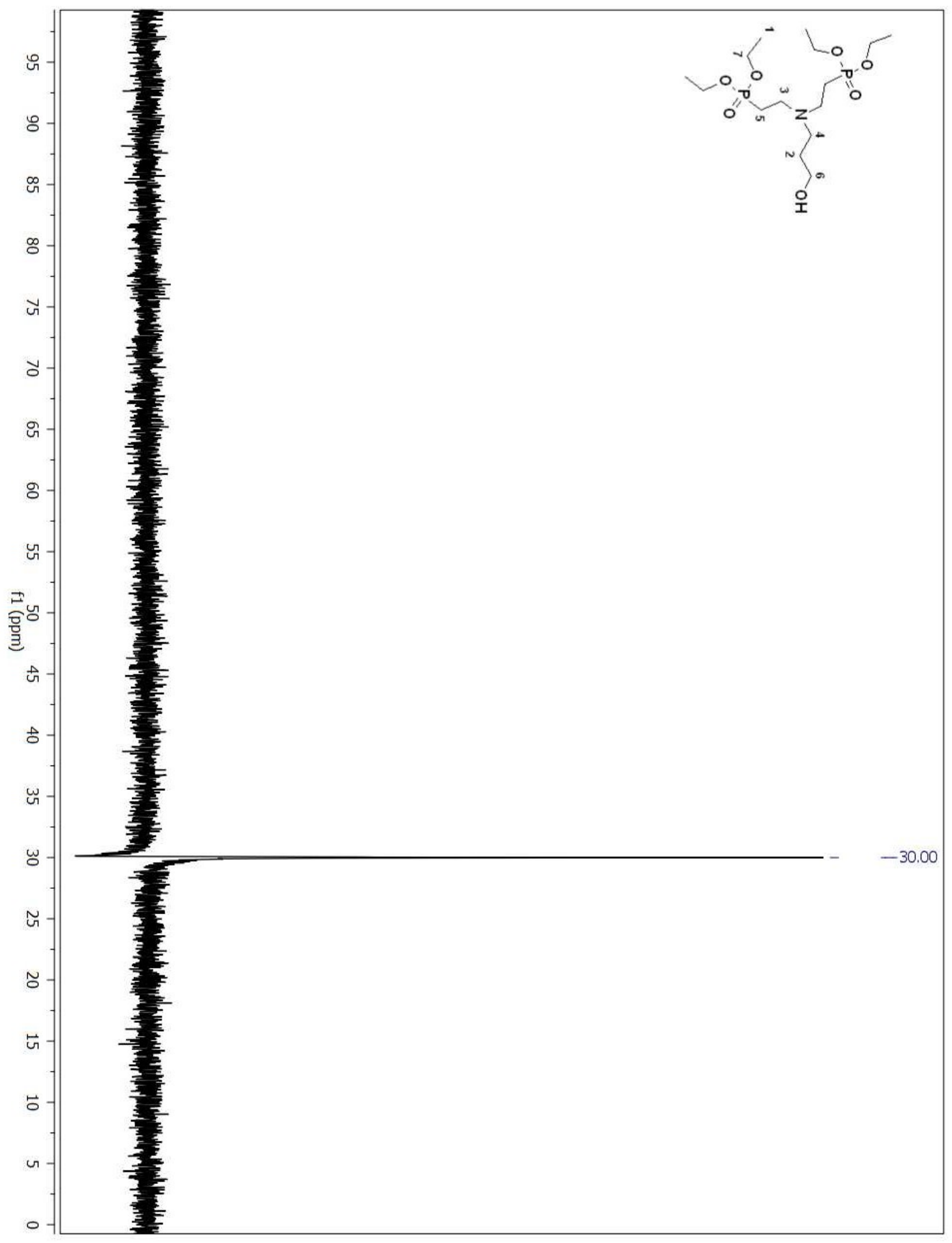

Figure A 252. ${ }^{31} \mathrm{P}$ NMR spectrum of compound (136) in $\mathrm{CDCl}_{3}$. 


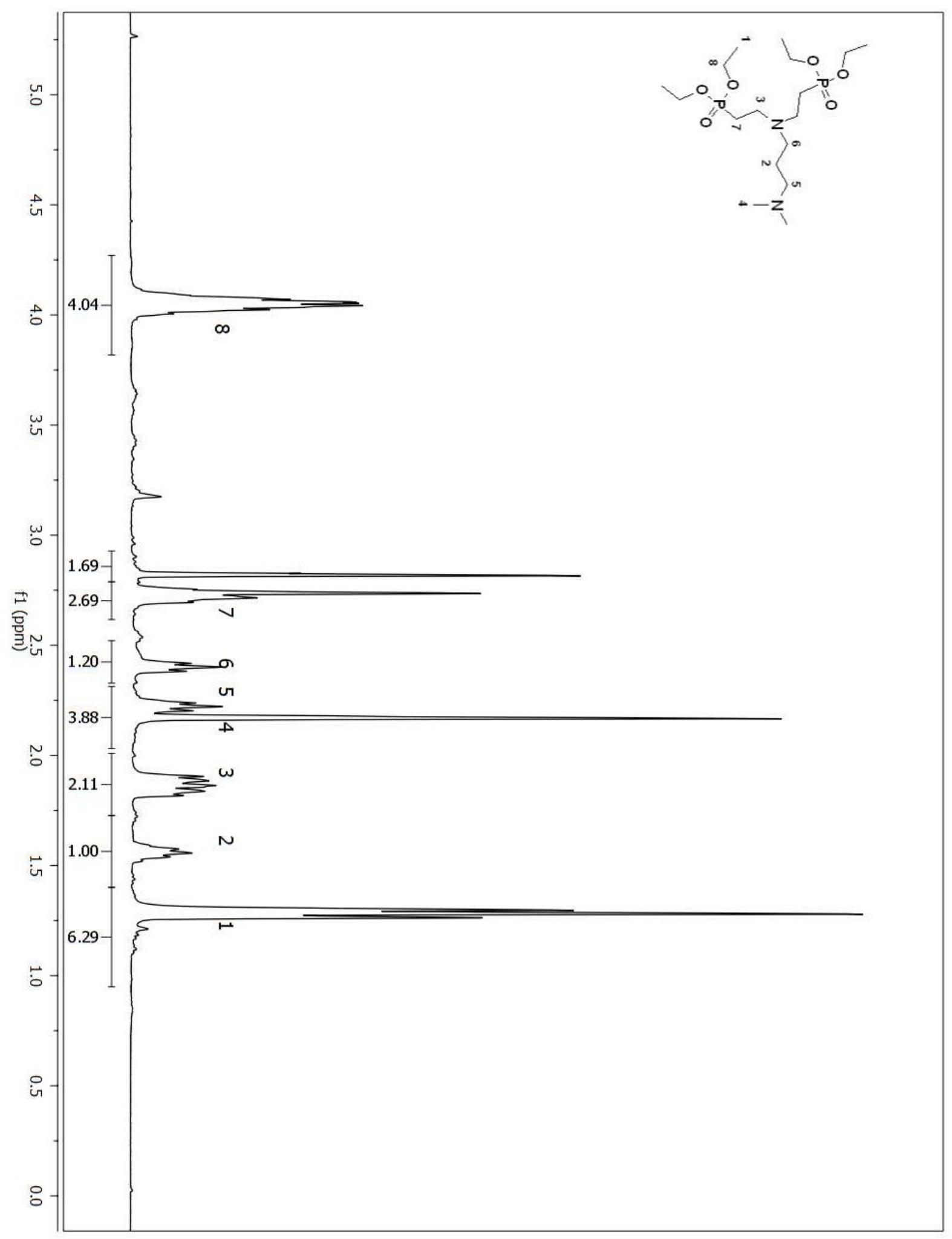

Figure A 253. ${ }^{1} \mathrm{H}$ NMR spectrum of compound (131) in $\mathrm{CDCl}_{3}$.

620 


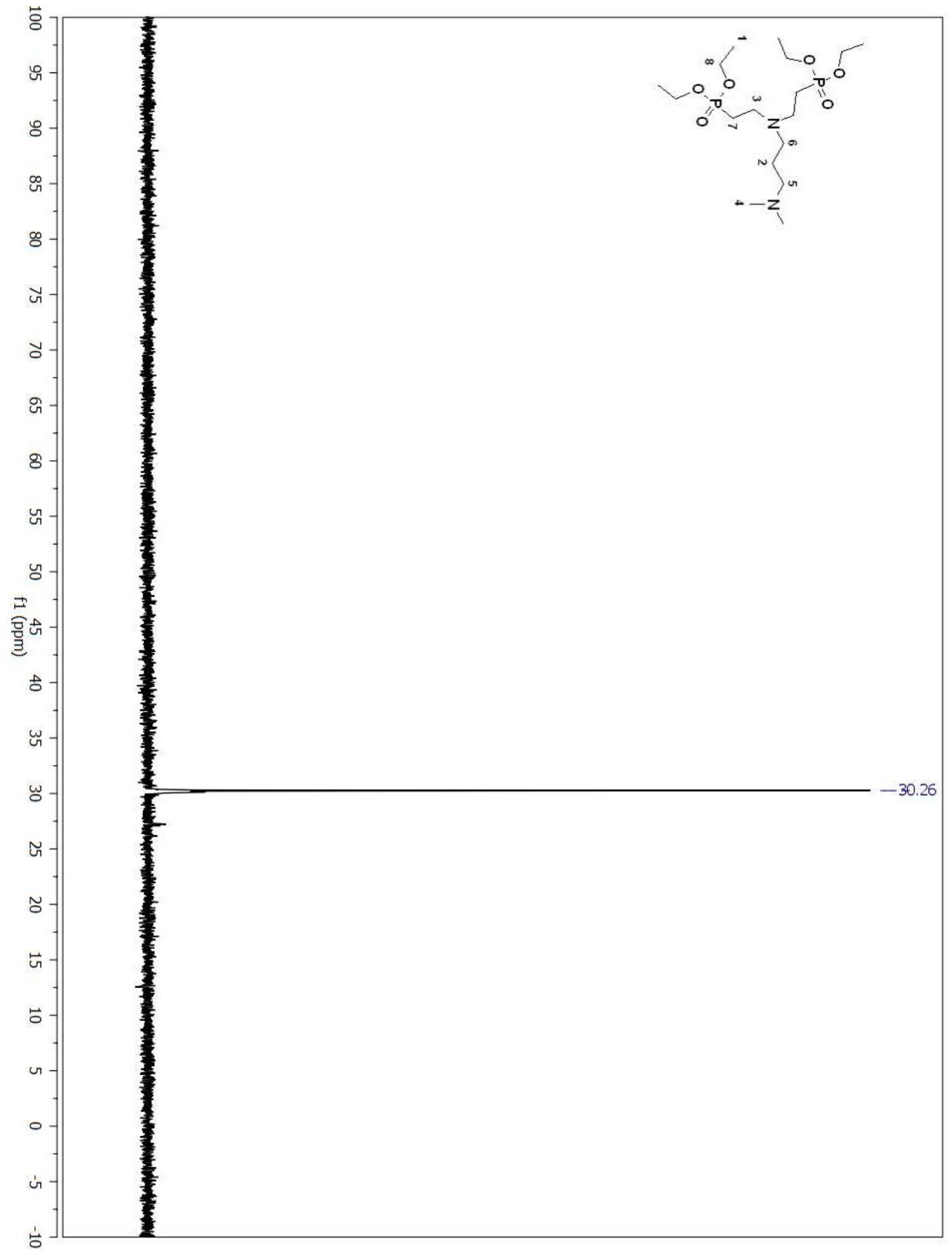

Figure A 254. ${ }^{31} \mathrm{P}$ NMR spectrum of compound (131) in $\mathrm{CDCl}_{3}$. 


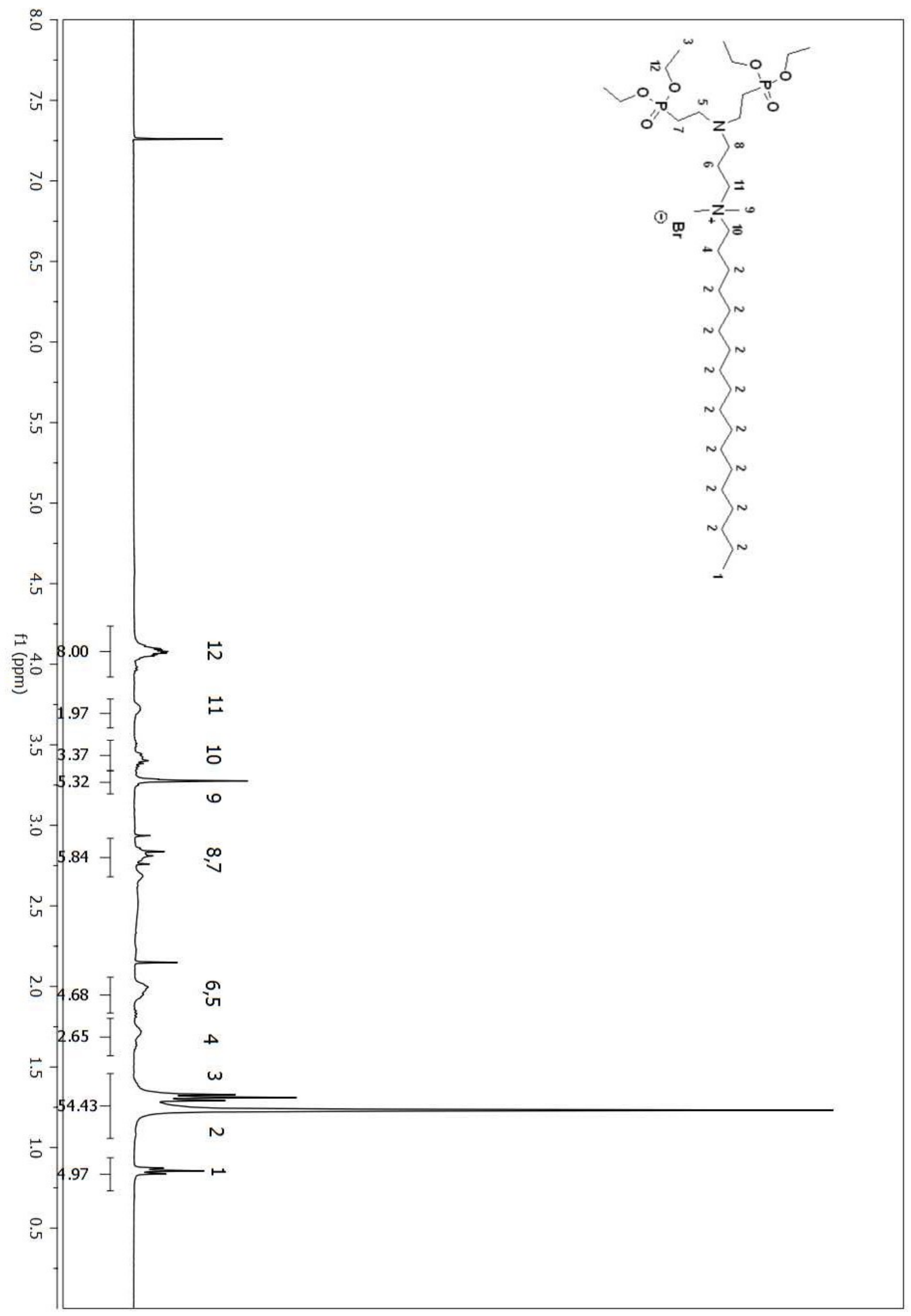

Figure A 255. ${ }^{1} \mathrm{H}$ NMR spectrum of compound (140) in $\mathrm{CDCl}_{3}$. 


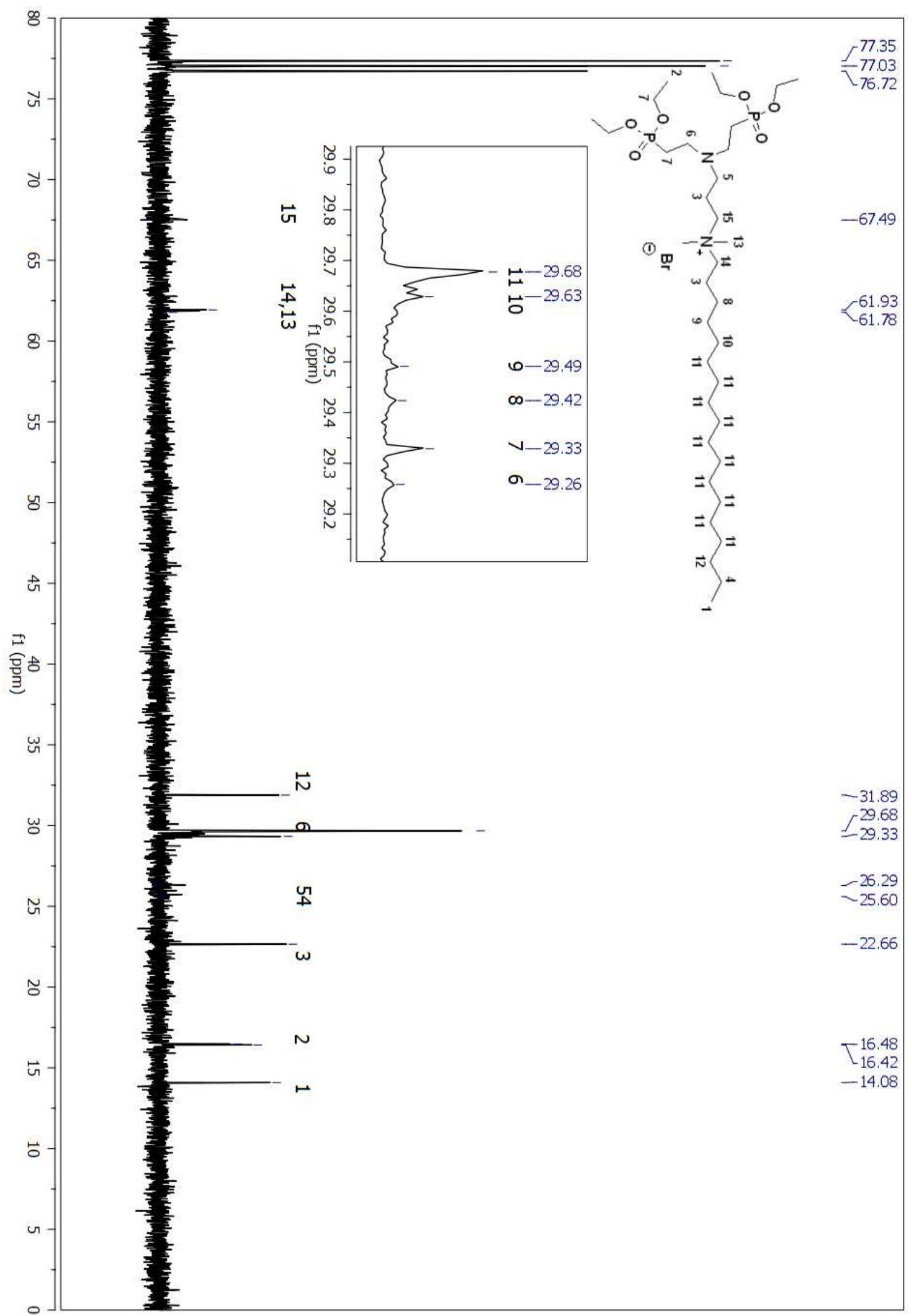

Figure A 256. ${ }^{13} \mathrm{C}$ NMR spectrum of compound (140) in $\mathrm{CDCl}_{3}$. 623 


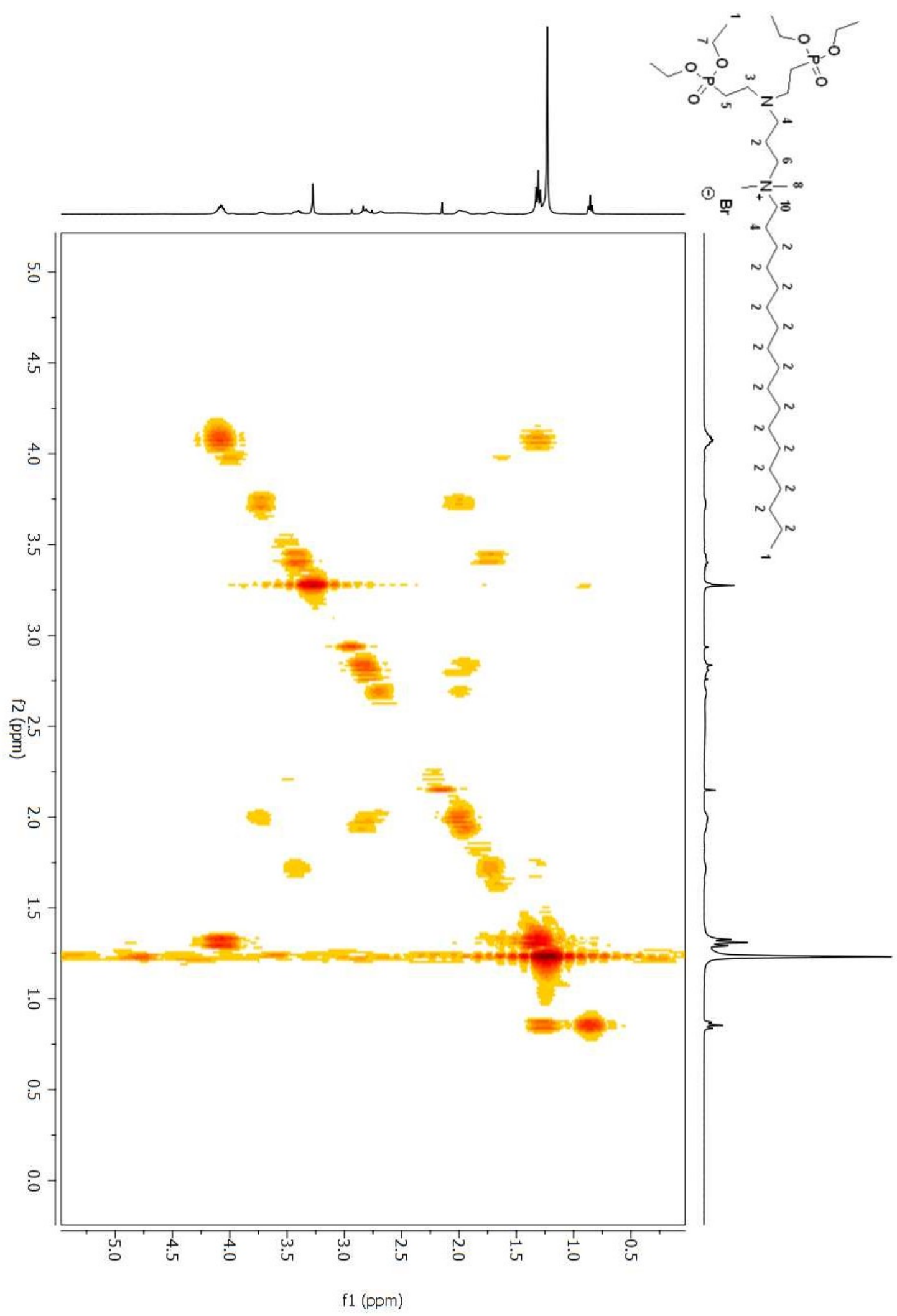

Figure A 257. COSY 2D NMR spectrum of compound (140) in $\mathrm{CDCl}_{3}$. 624 


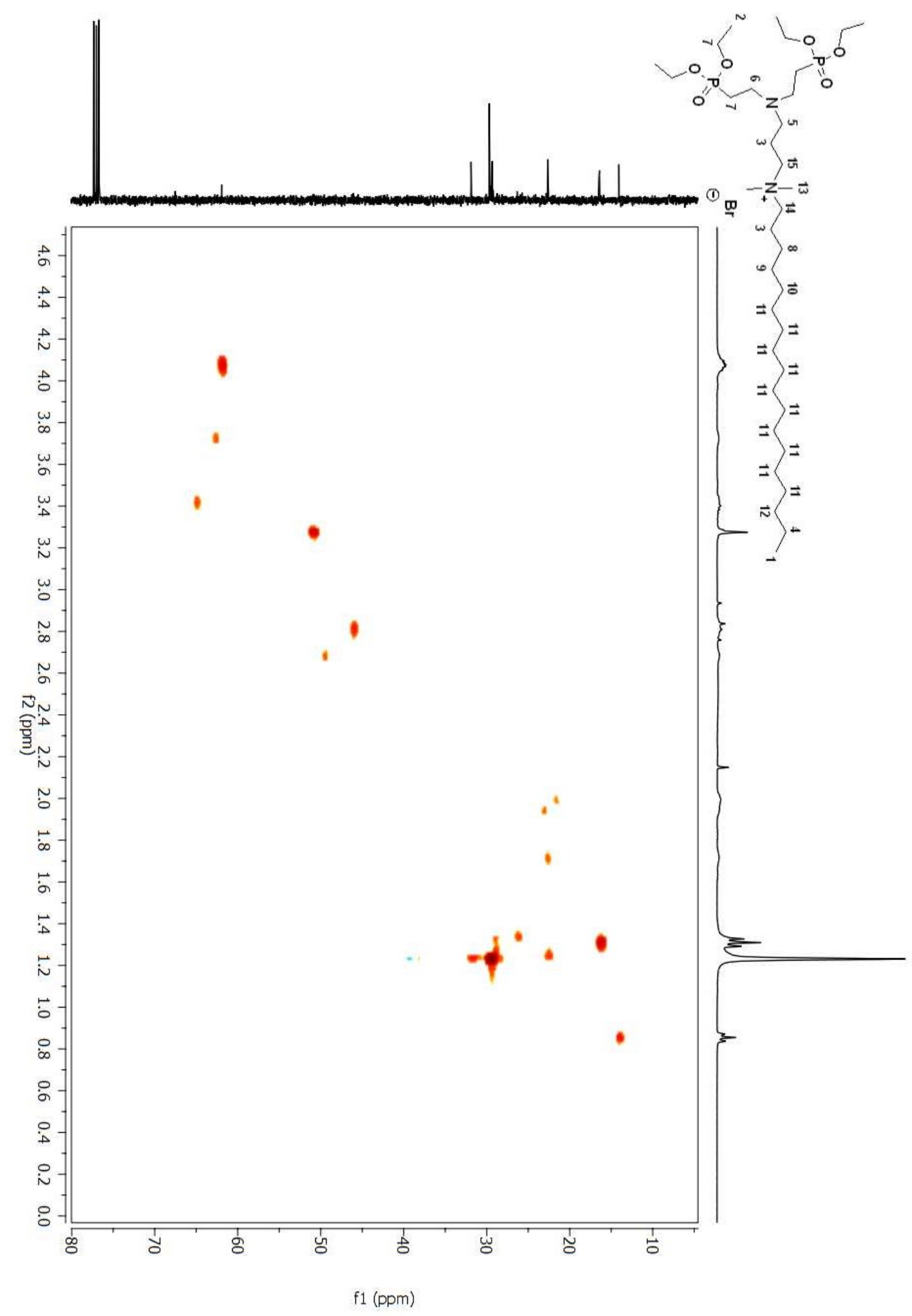

Figure A 258. HSQC 2D NMR spectrum of compound (140) in $\mathrm{CDCl}_{3}$. 625 


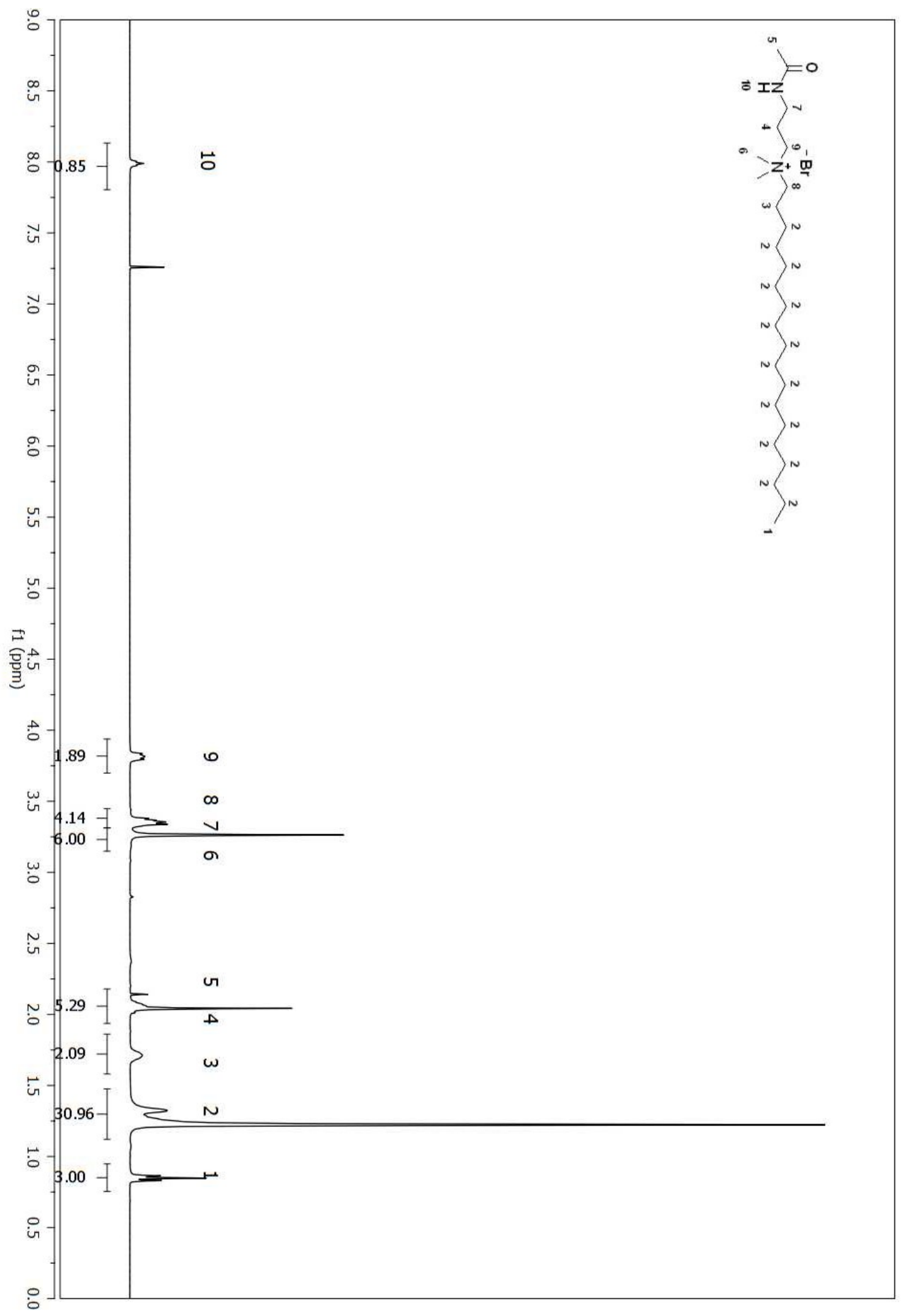

Figure A 259. ${ }^{1} \mathrm{H}$ NMR spectrum of compound (153) in $\mathrm{CDCl}_{3}$. 626 


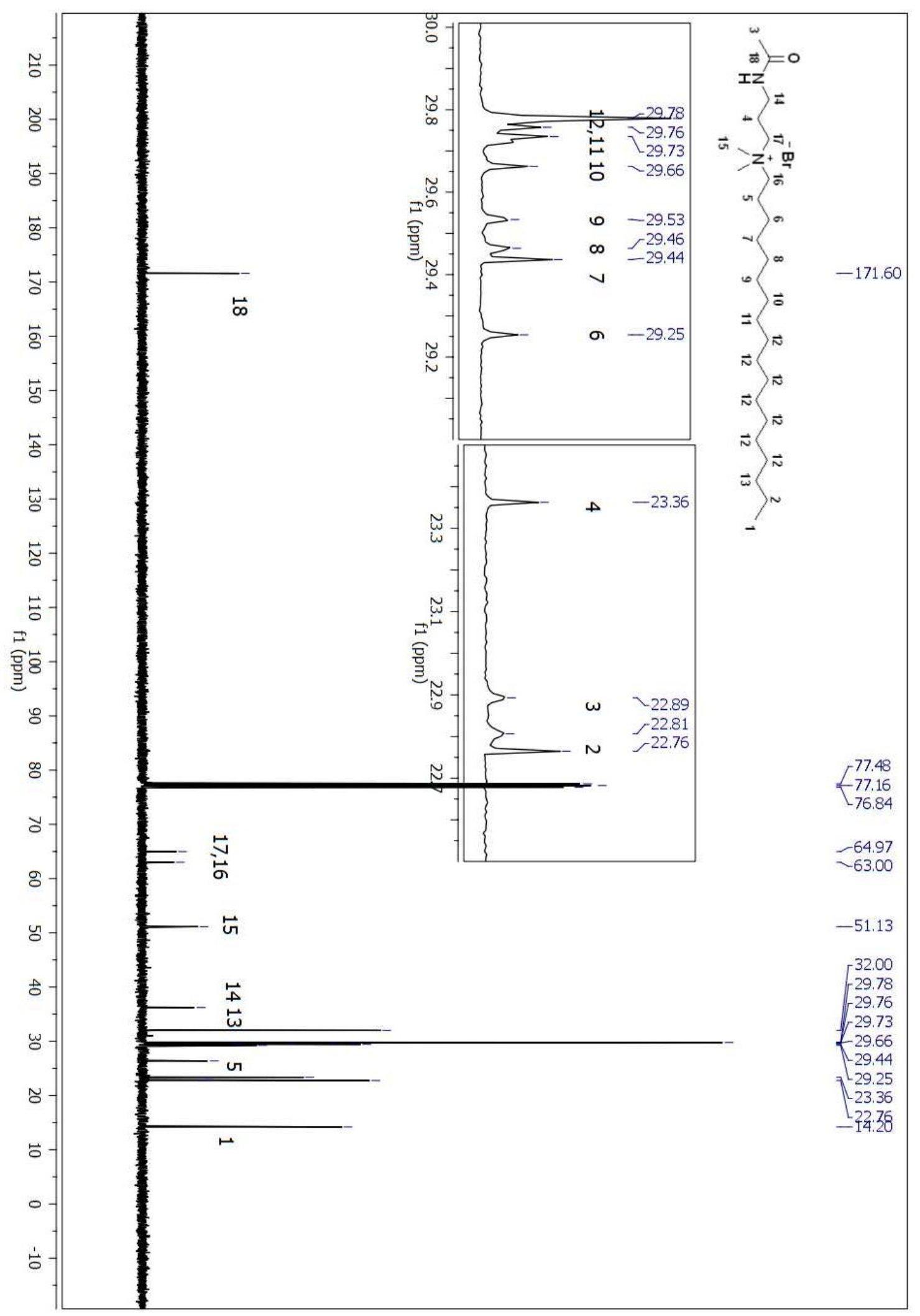

Figure A 260. ${ }^{13} \mathrm{C}$ NMR spectrum of compound (153) in $\mathrm{CDCl}_{3}$. 


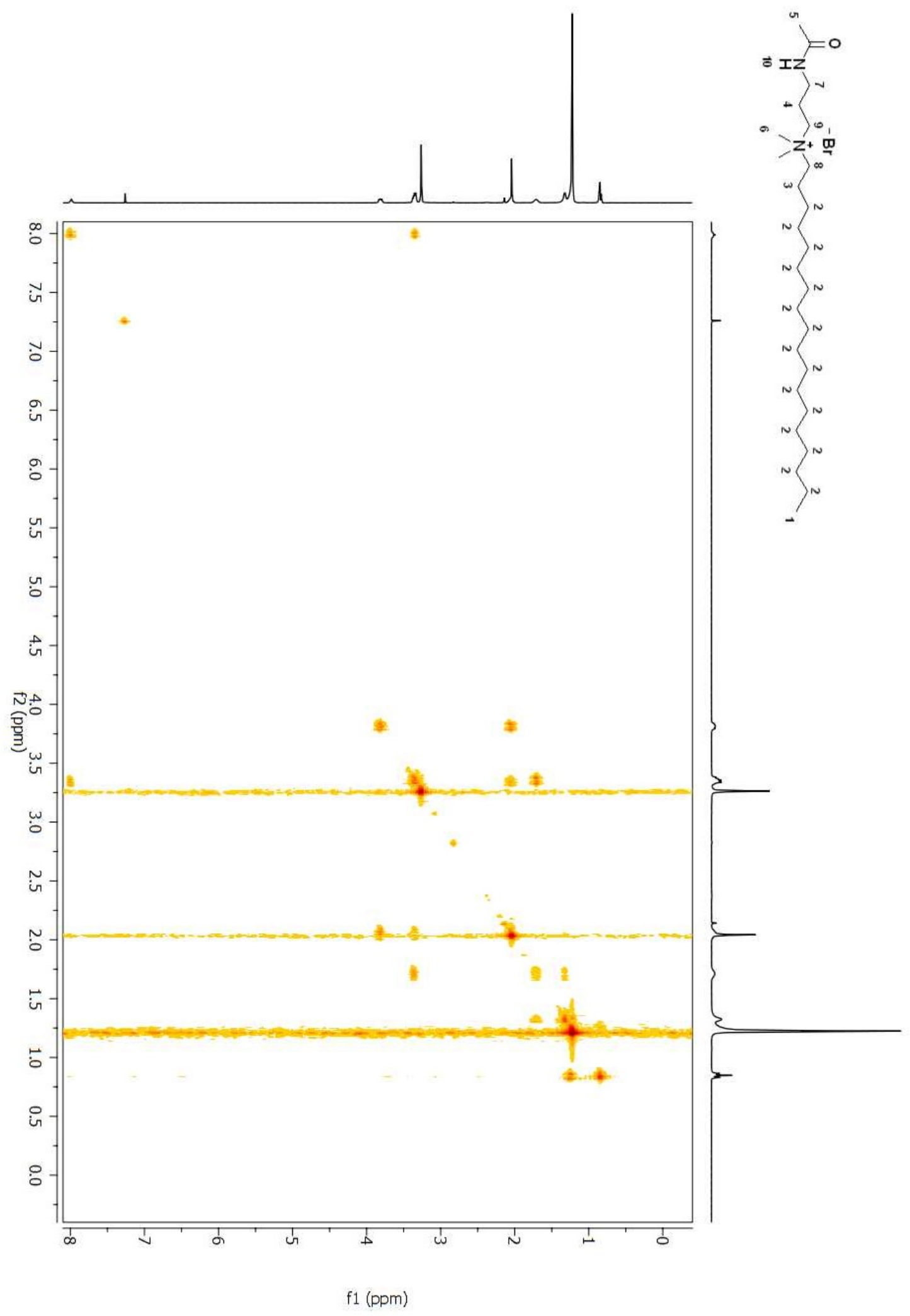

Figure A 261. ${ }^{13} \mathrm{C}$ NMR spectrum of compound (153) in $\mathrm{CDCl}_{3}$. 


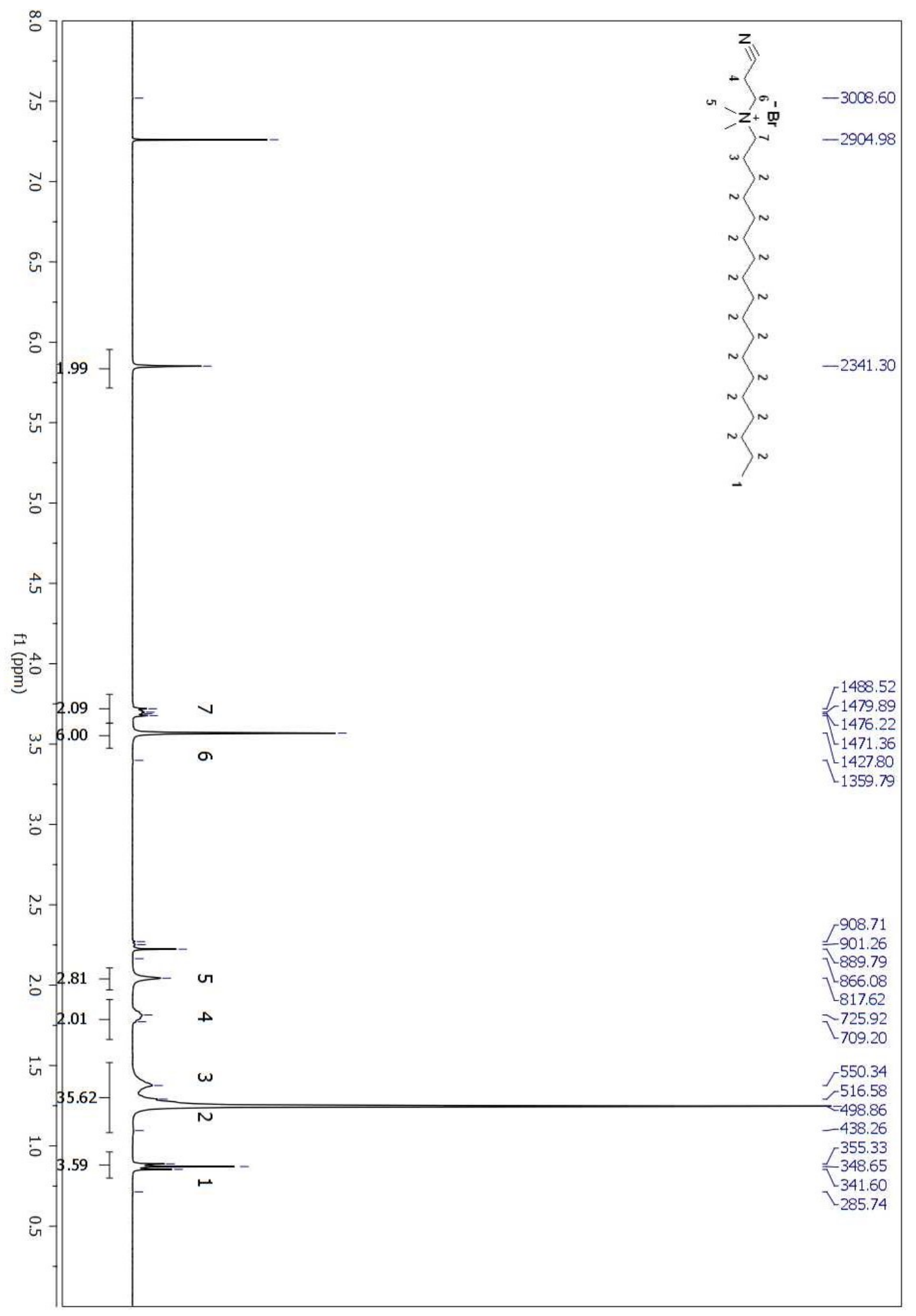

Figure A 262. ${ }^{1} \mathrm{H}$ NMR spectrum of compound (154) in $\mathrm{CDCl}_{3}$. 


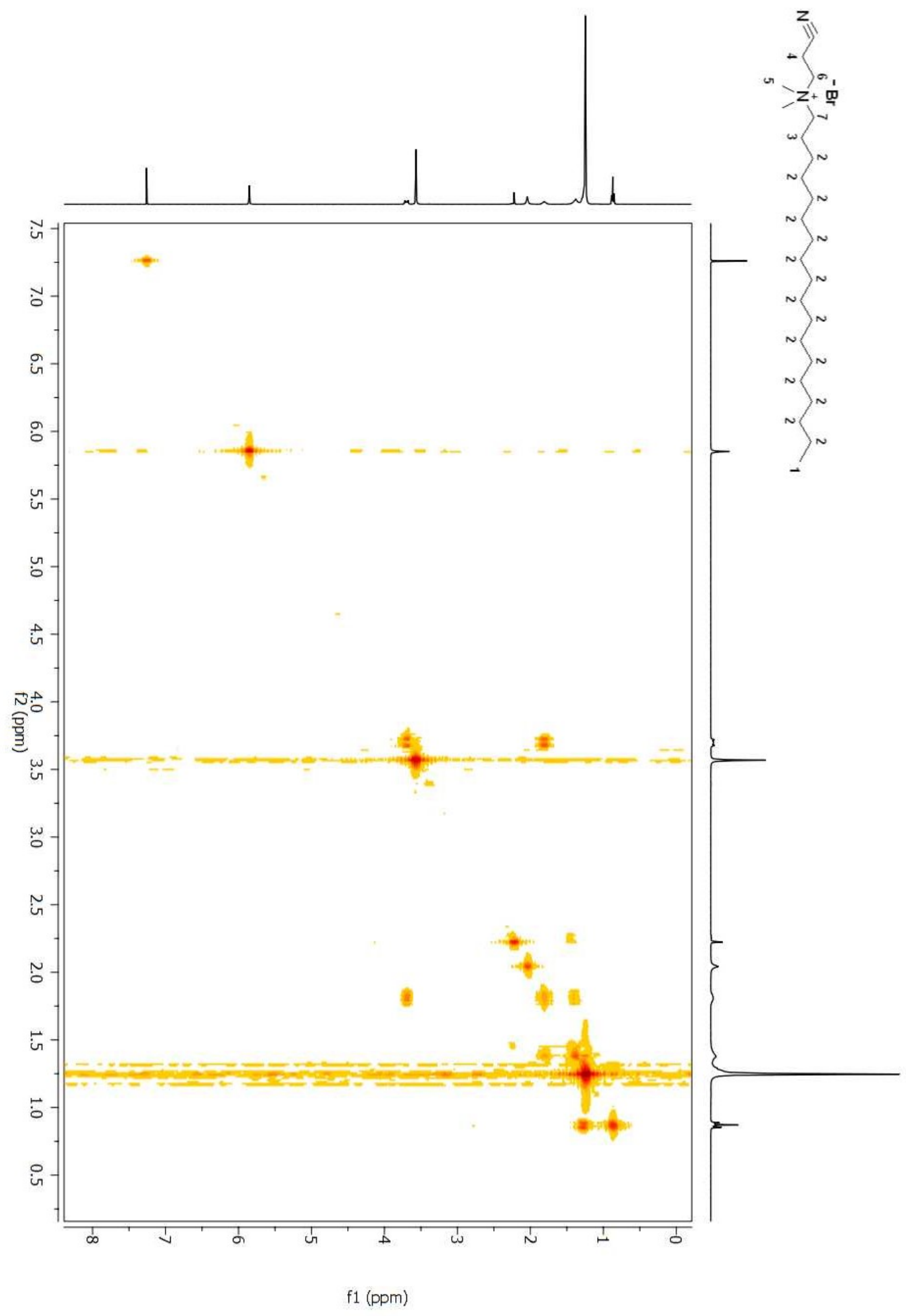

Figure A 263. COSY 2D NMR spectrum of compound (154) in $\mathrm{CDCl}_{3}$. 


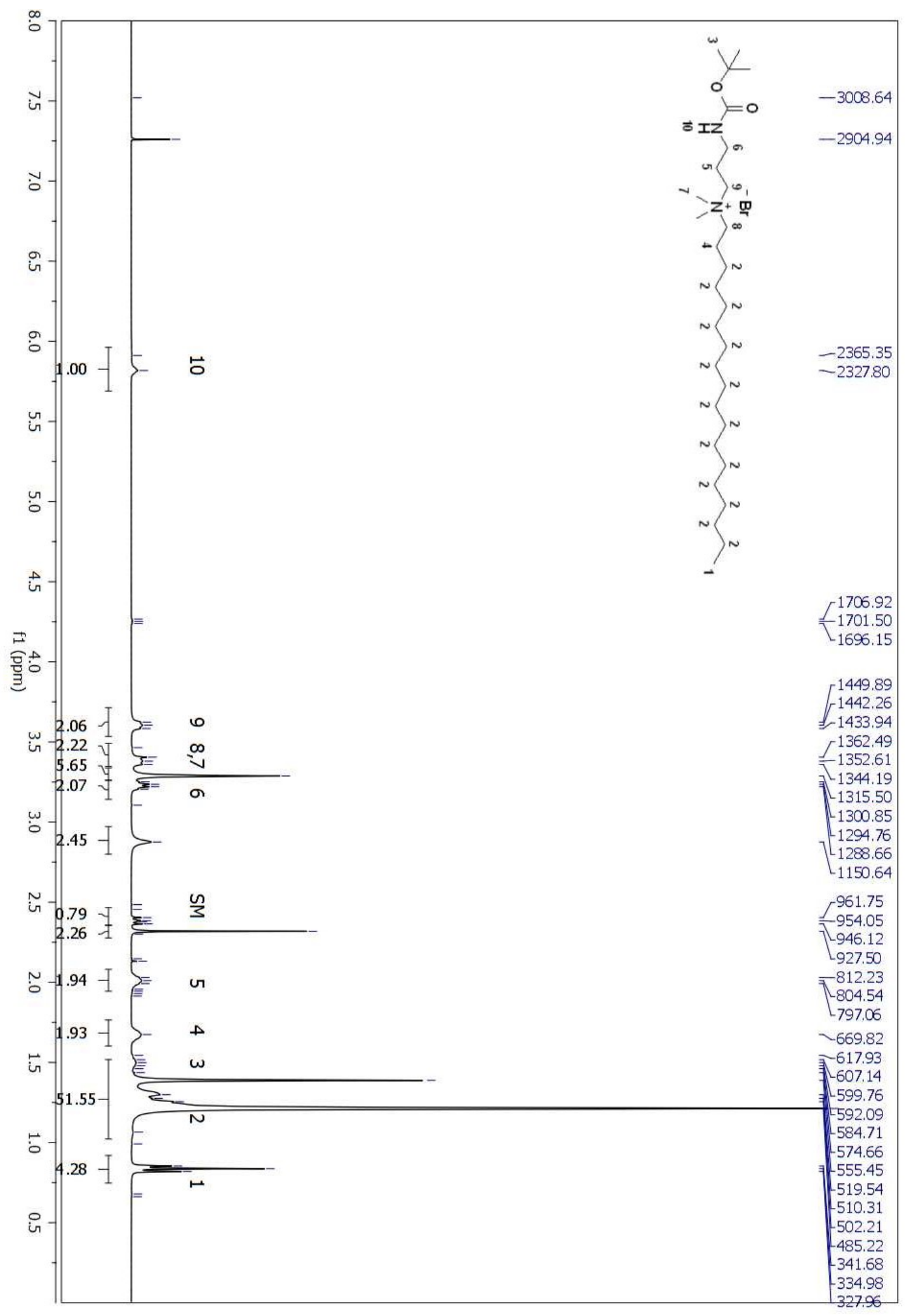

Figure A 264. ${ }^{1} \mathrm{H}$ NMR spectrum of compound (155) in $\mathrm{CDCl}_{3}$. 


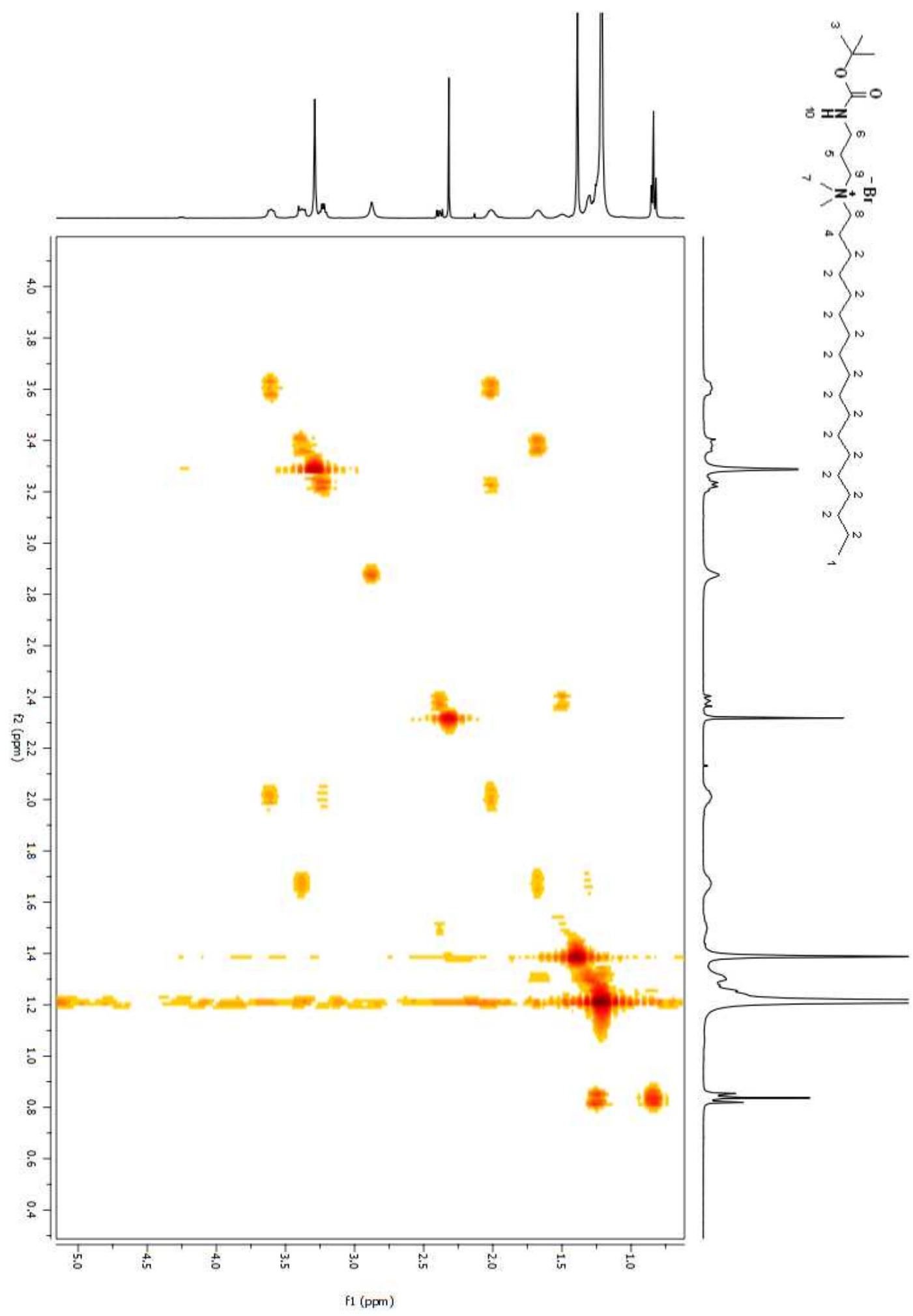

Figure A 265. COSY 2D NMR spectrum of compound (155) in $\mathrm{CDCl}_{3}$. 


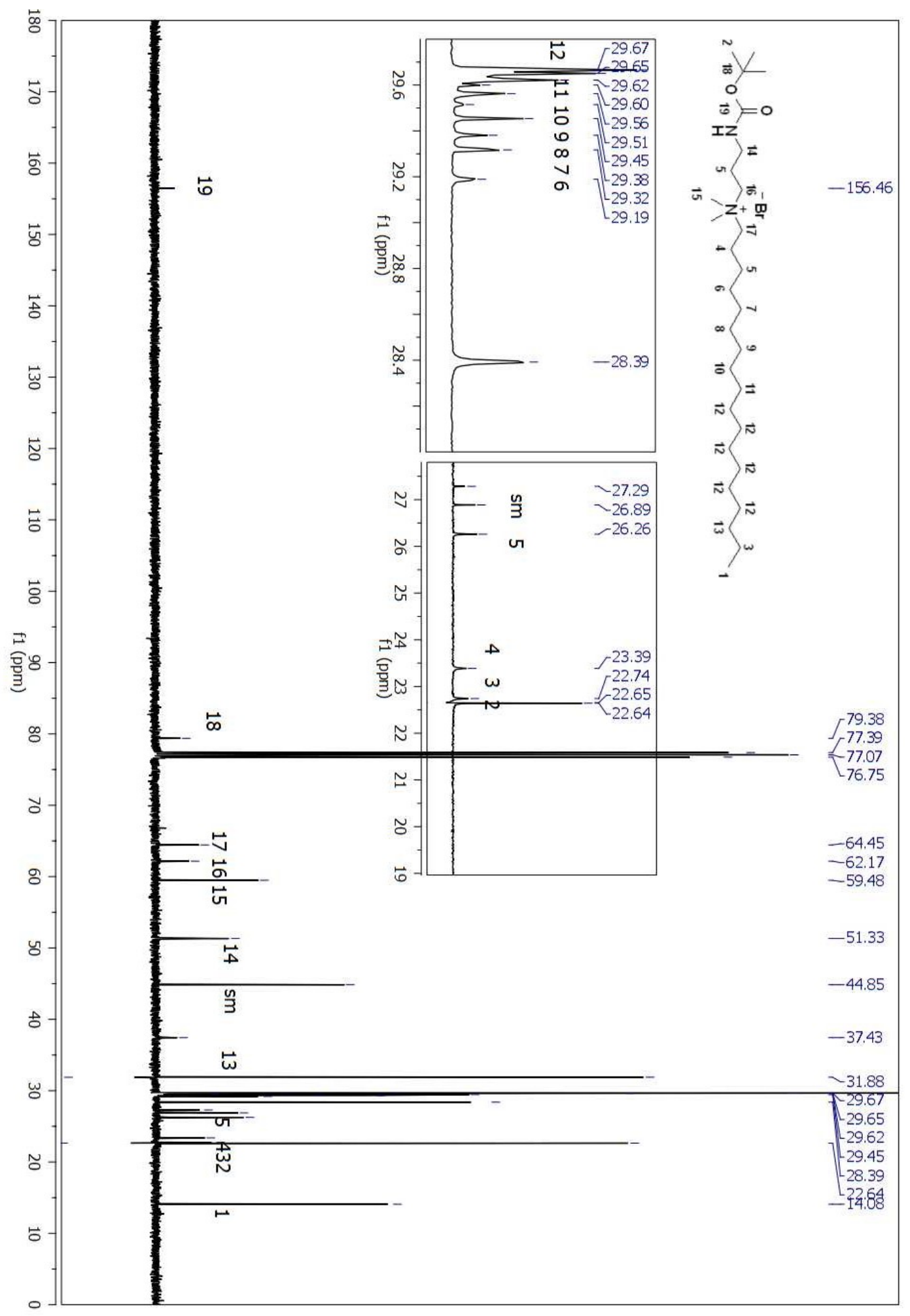

Figure A 266. ${ }^{13} \mathrm{H}$ NMR spectrum of compound (155) in $\mathrm{CDCl}_{3}$. 


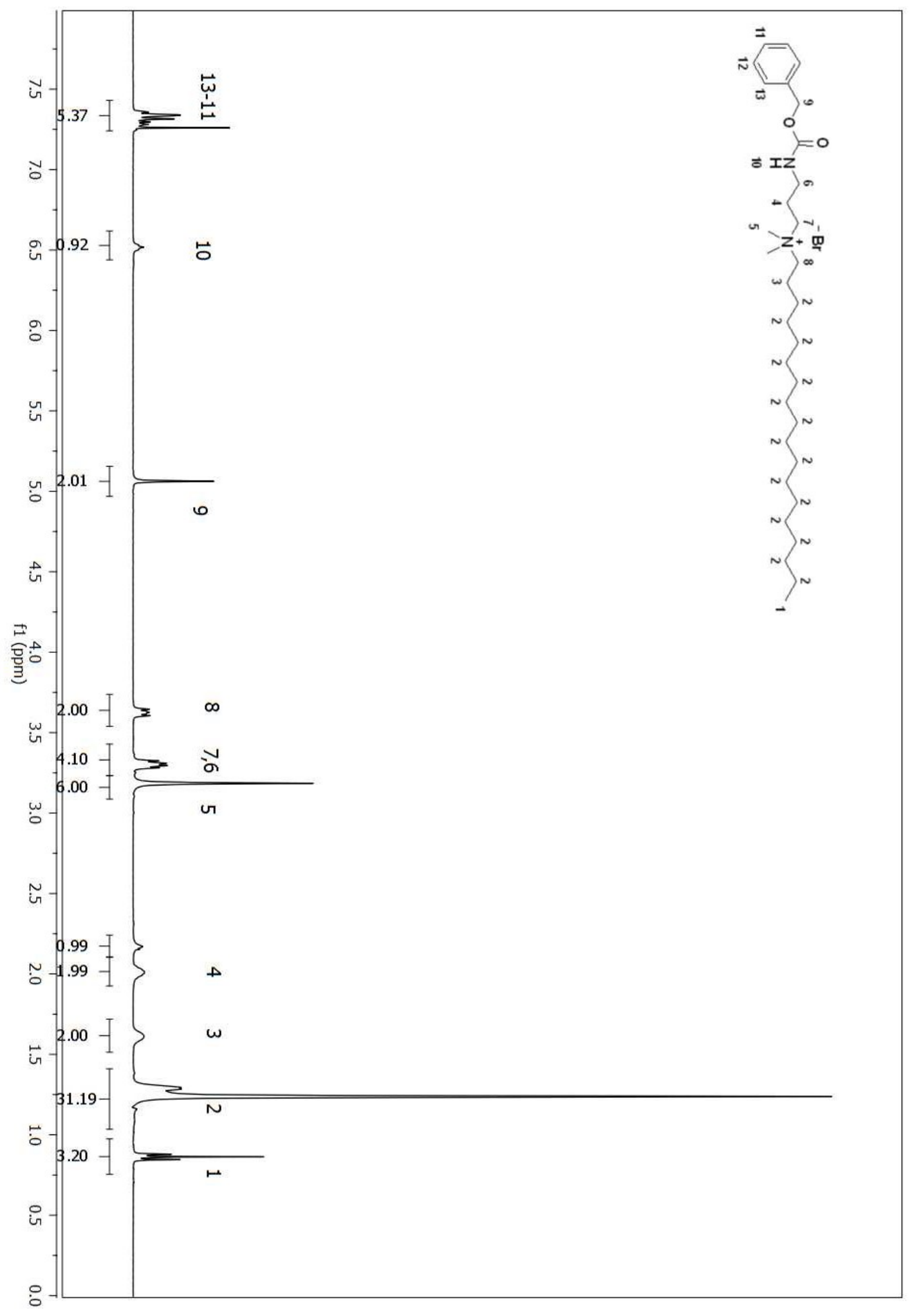

Figure A 267. ${ }^{1} \mathrm{H}$ NMR spectrum of compound (156) in $\mathrm{CDCl}_{3}$. 


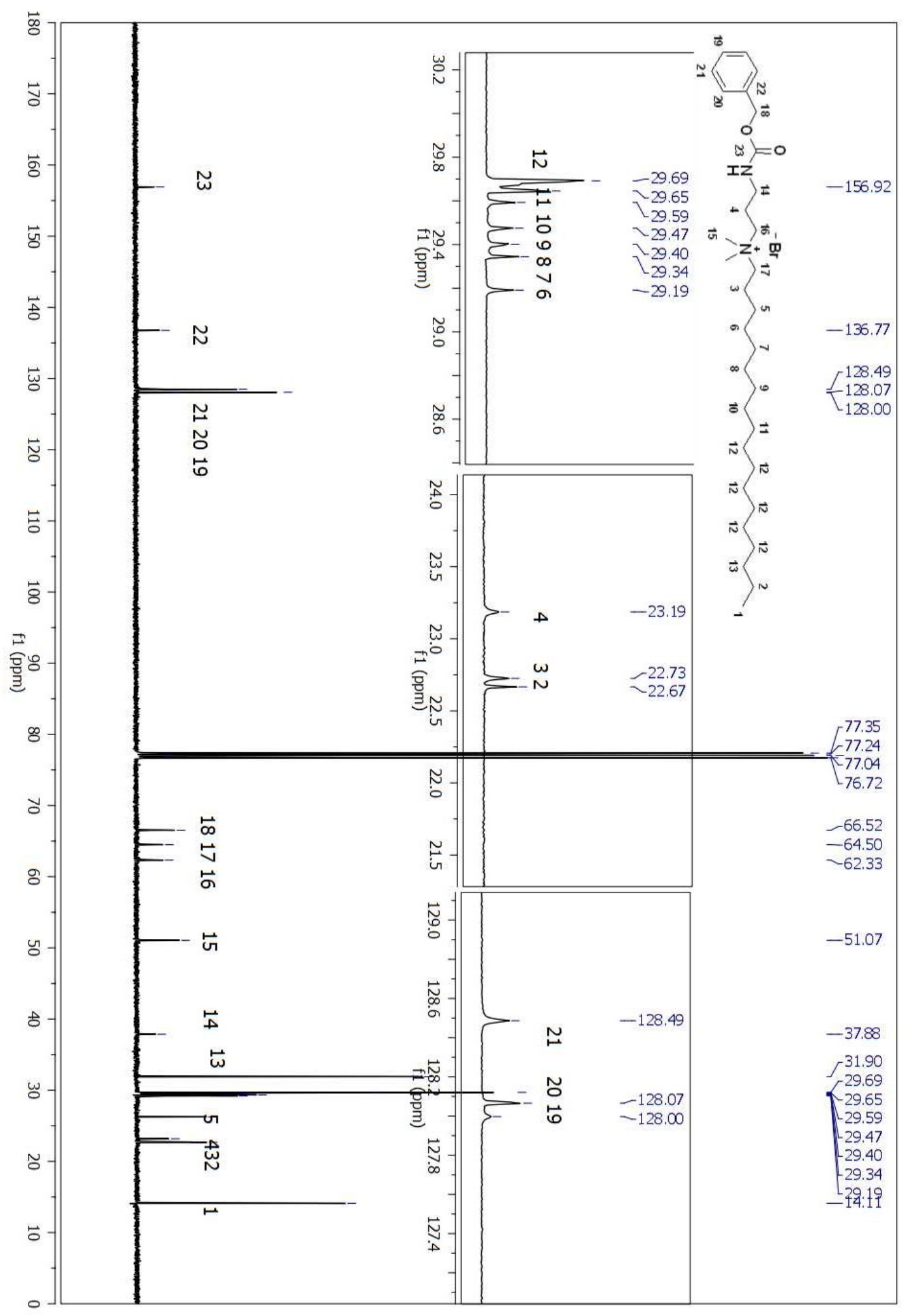

Figure A 268. ${ }^{13} \mathrm{C}$ NMR spectrum of compound (156) in $\mathrm{CDCl}_{3}$. 


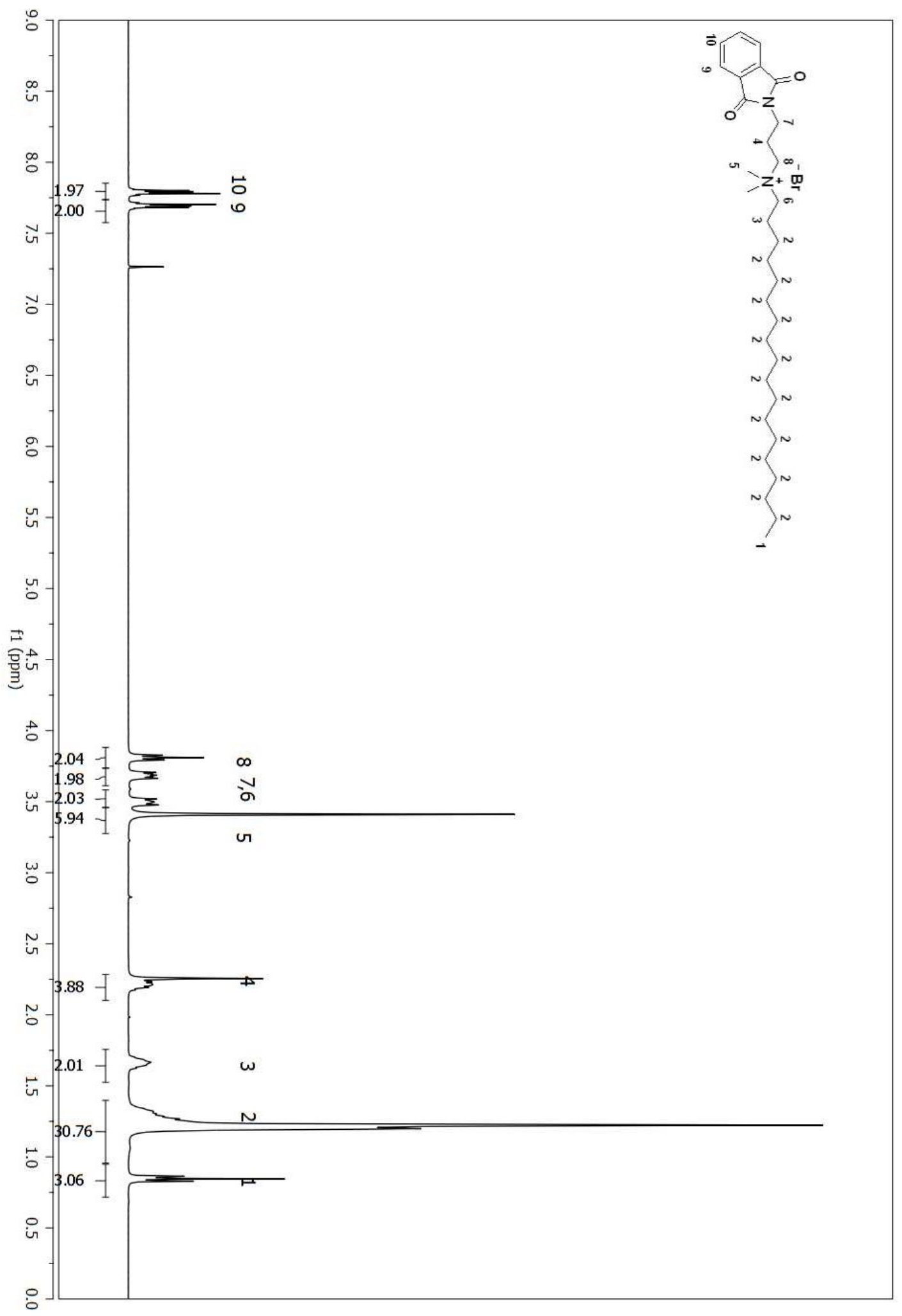

Figure A 269. ${ }^{1} \mathrm{H}$ NMR spectrum of compound (152) in $\mathrm{CDCl}_{3}$. 


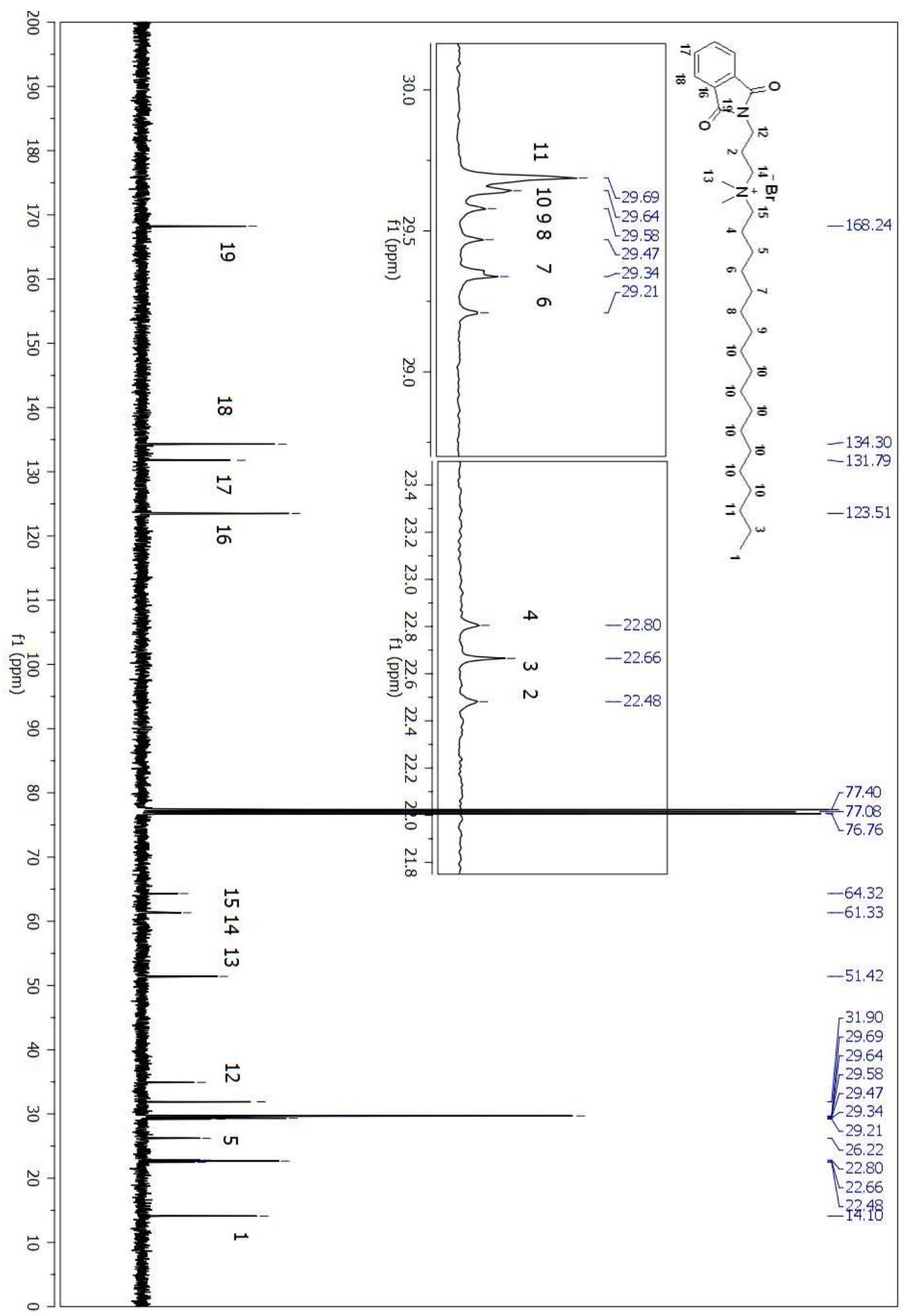

Figure A 270. ${ }^{13} \mathrm{C}$ NMR spectrum of compound (152) in $\mathrm{CDCl}_{3}$. 


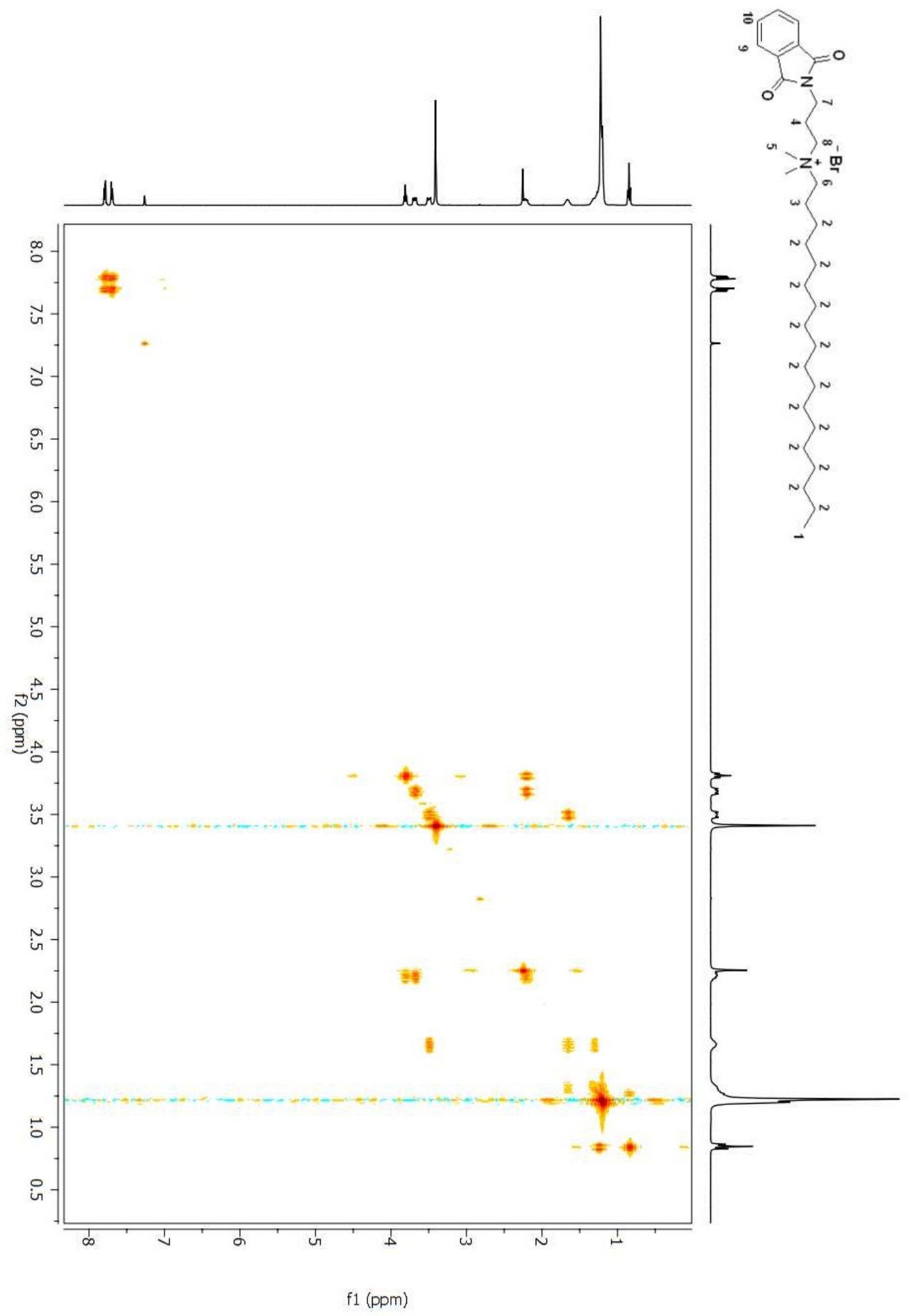

Figure A 271. COSY 2D NMR spectrum of compound (152) in $\mathrm{CDCl}_{3}$. 


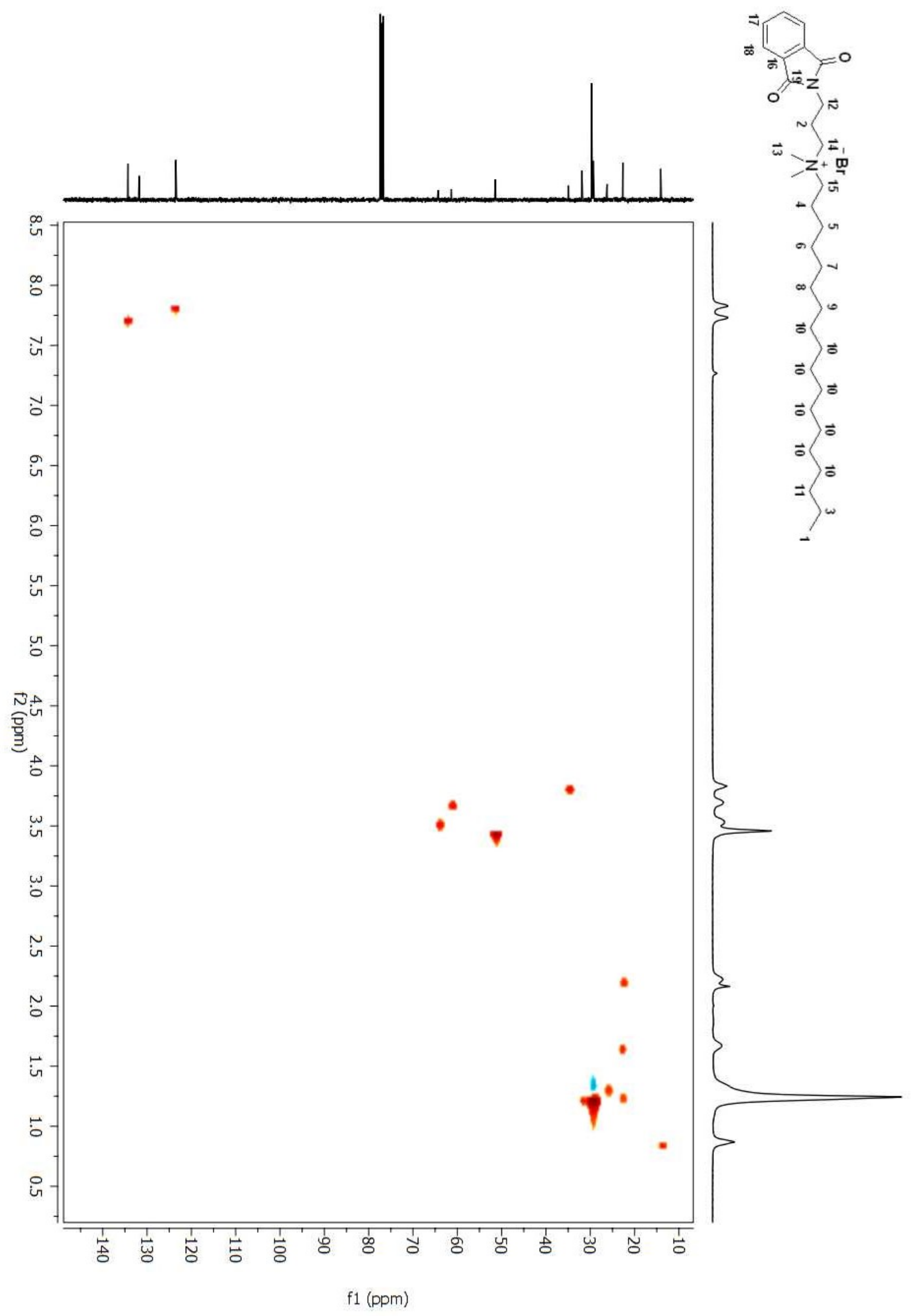

Figure A 272. HSQC 2D NMR spectrum of compound (152) in $\mathrm{CDCl}_{3}$. 


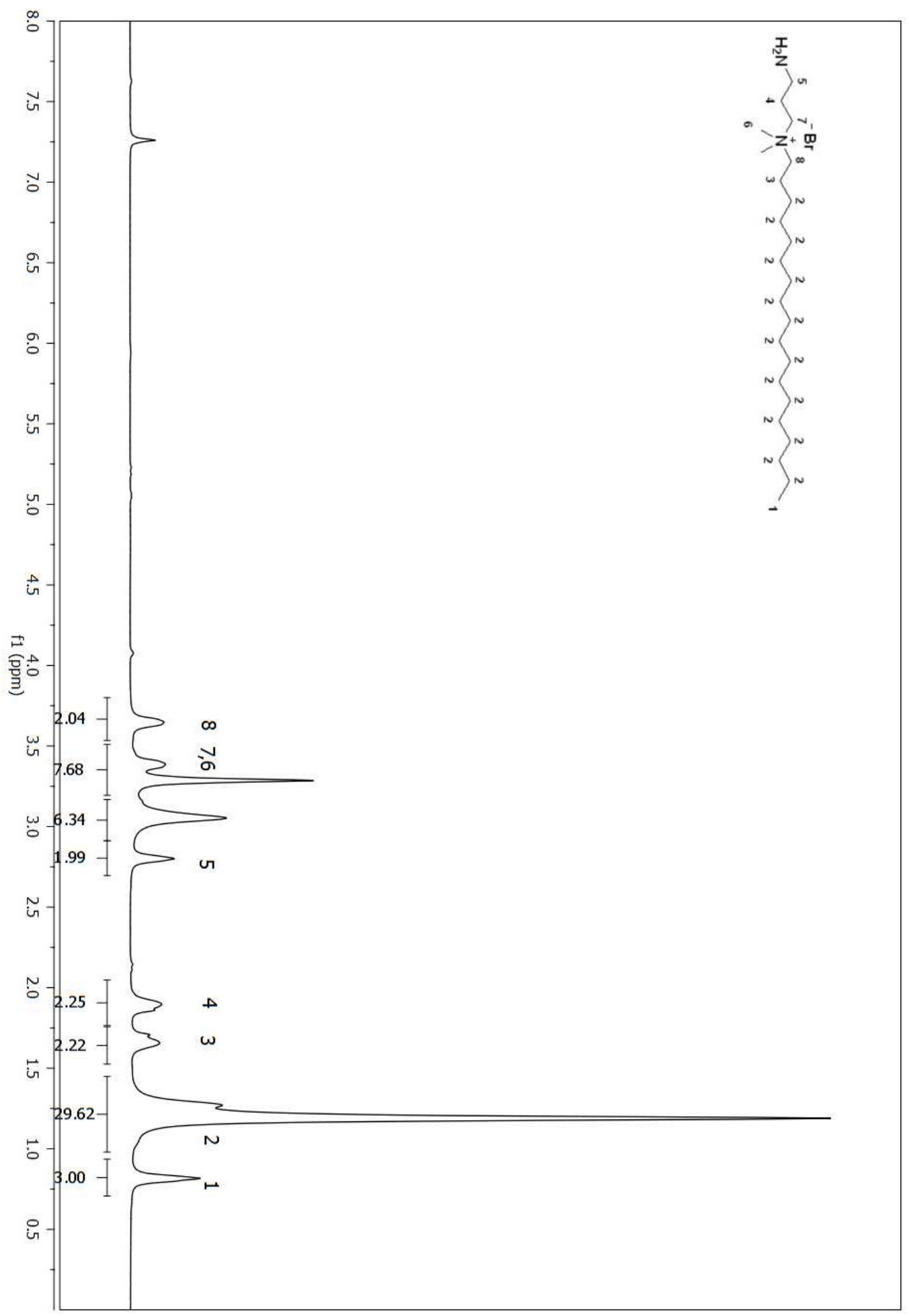

Figure A 273. ${ }^{1} \mathrm{H}$ NMR spectrum of compound (144) from (152) in $\mathrm{CDCl}_{3}$. 


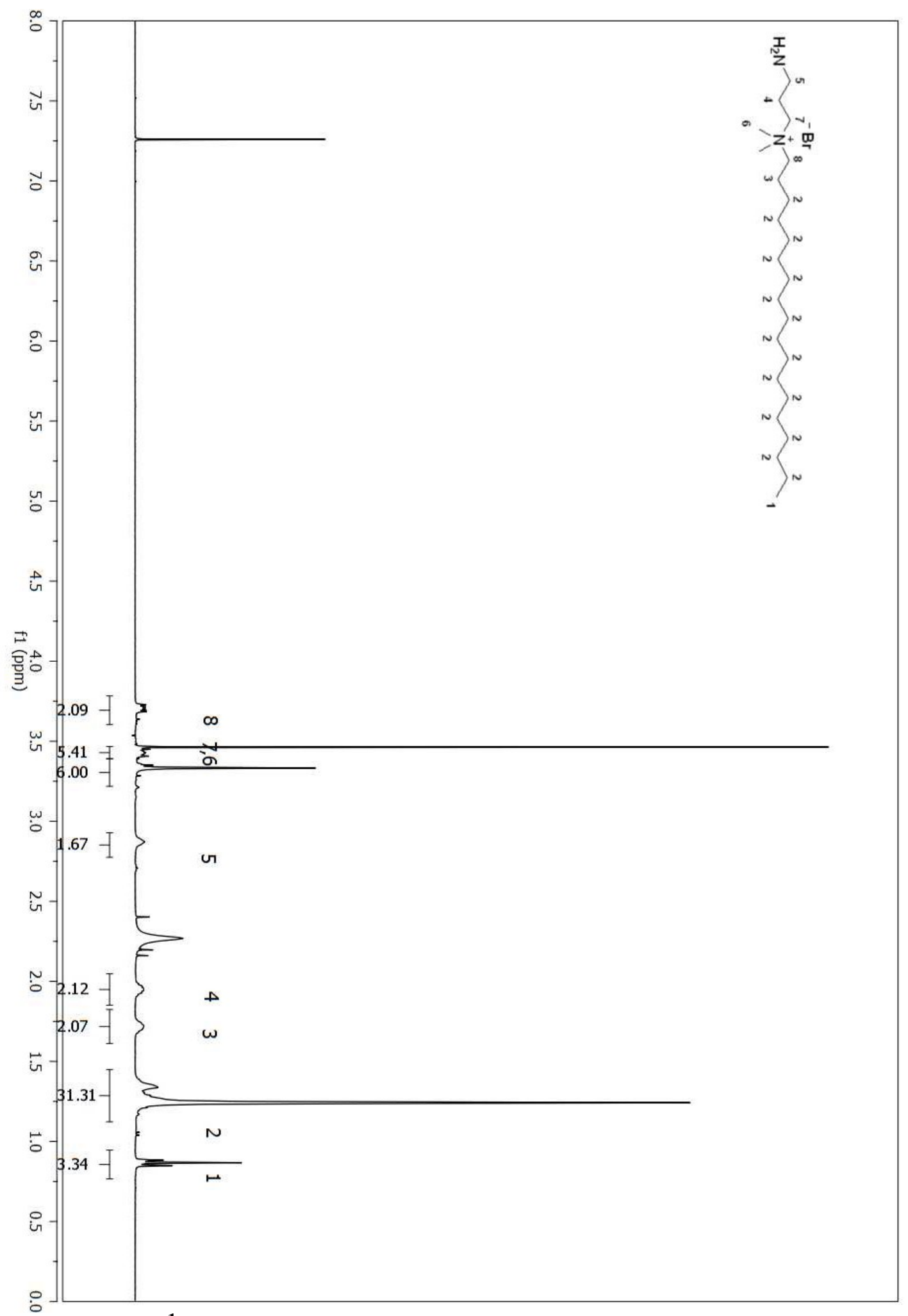

Figure A 274. ${ }^{1} \mathrm{H}$ NMR spectrum of compound (144) from (156) in $\mathrm{CDCl}_{3}$. 


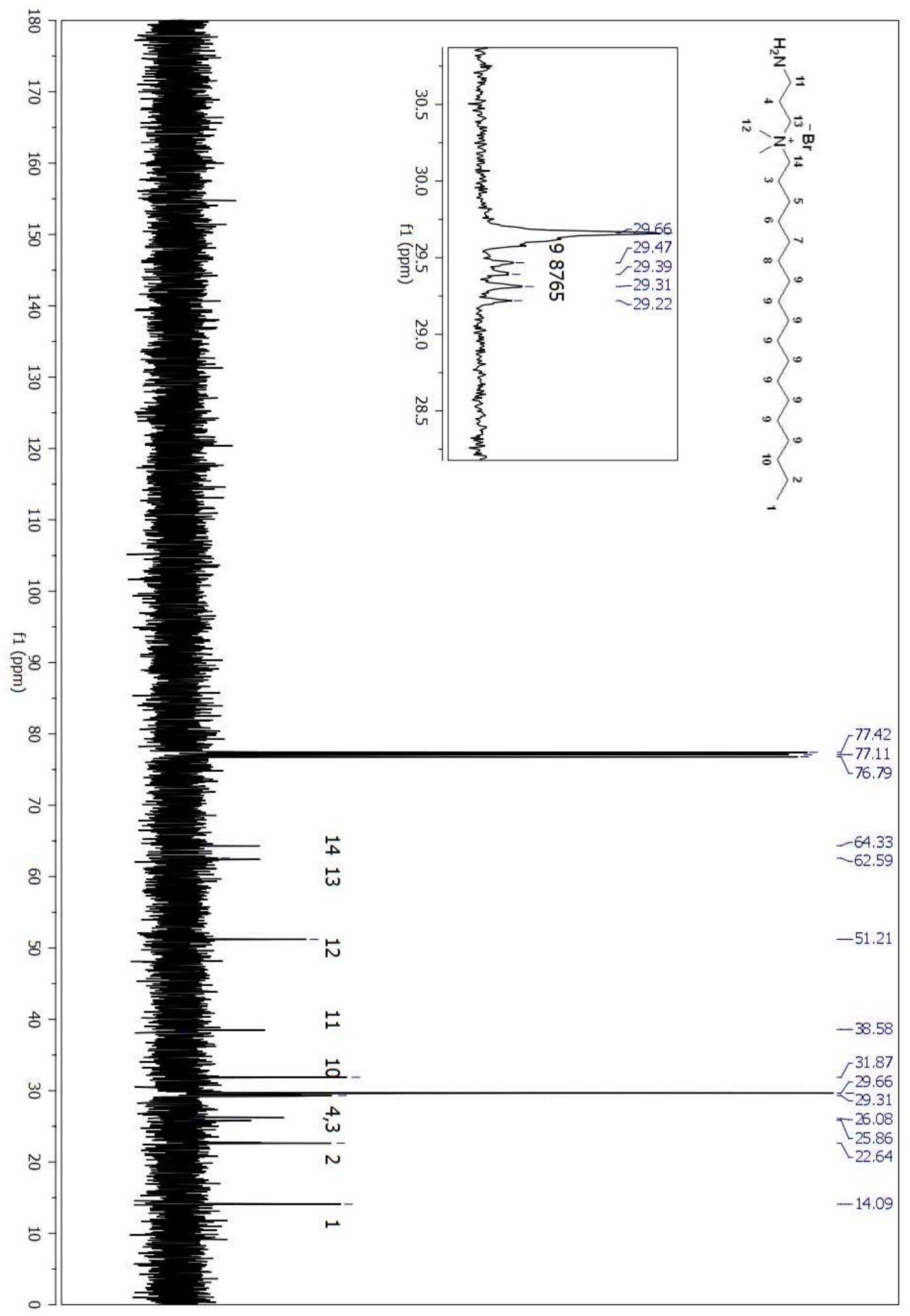

Figure A 275. ${ }^{13} \mathrm{C}$ NMR spectrum of compound (144) from (152) in $\mathrm{CDCl}_{3}$. 


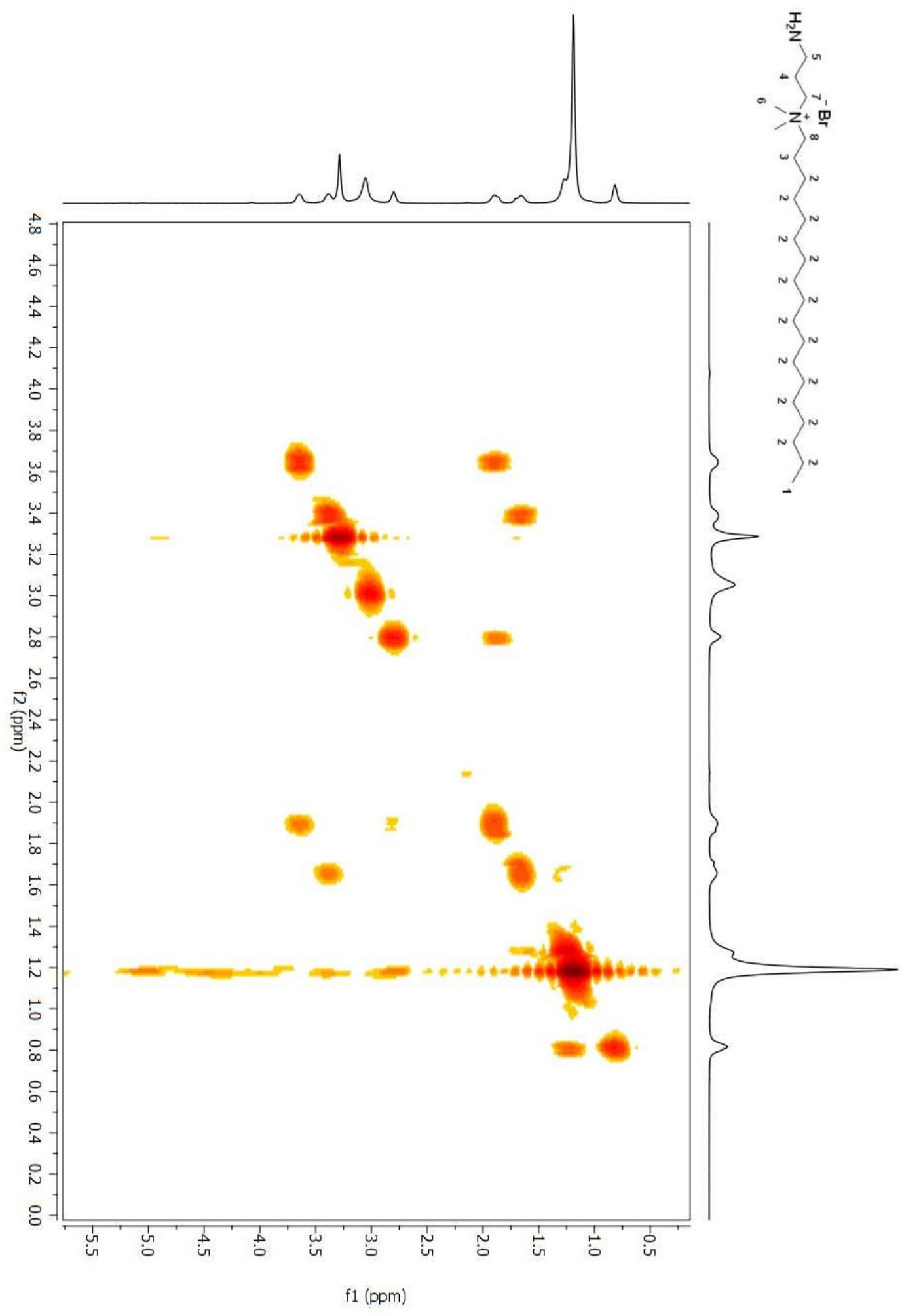

Figure A 276. COSY 2D NMR spectrum of compound (144) from (152) in $\mathrm{CDCl}_{3}$. 


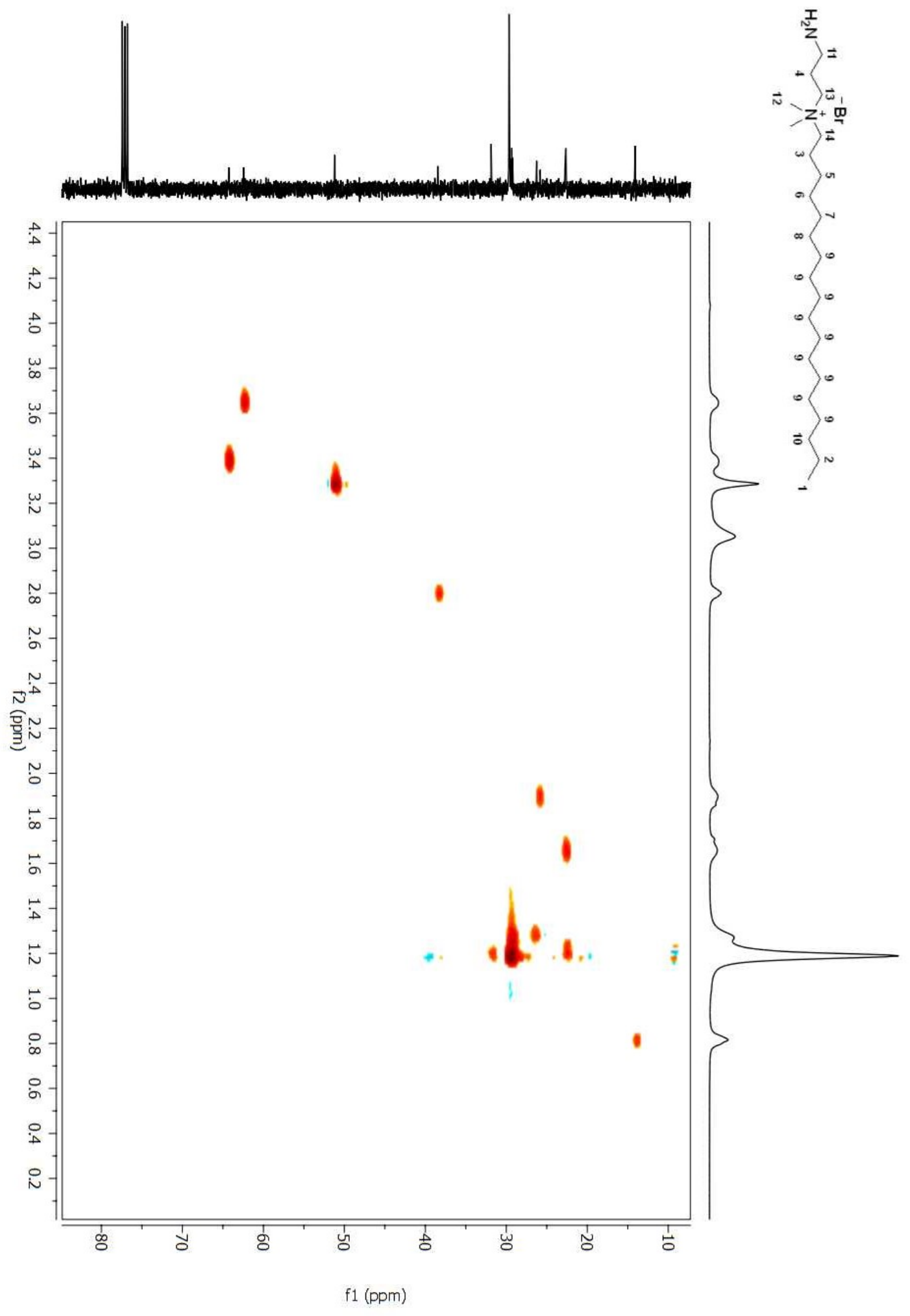

Figure A 277. HSQC 2D spectrum of compound (144) from (152) in $\mathrm{CDCl}_{3}$ 


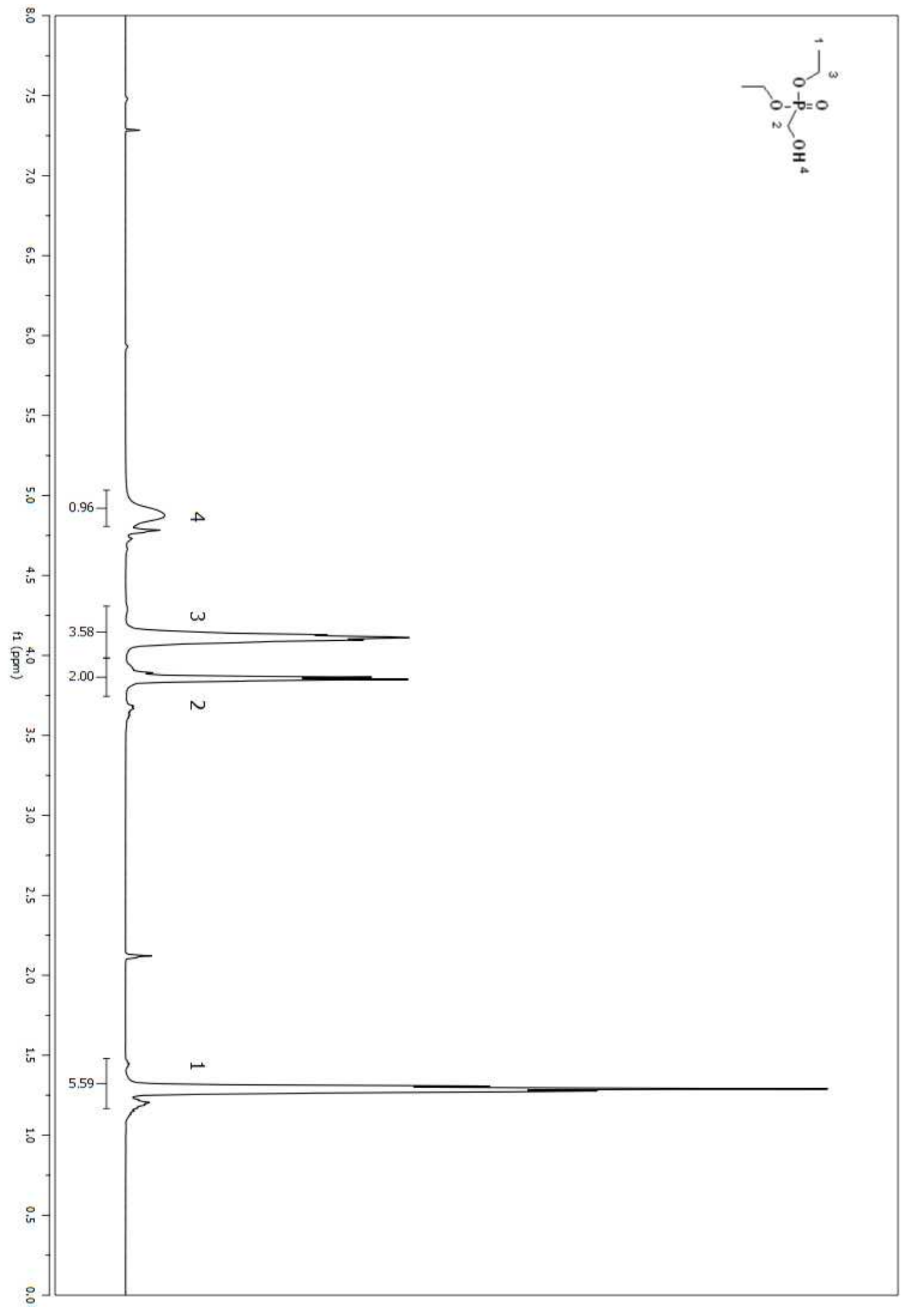

Figure A 278. ${ }^{1} \mathrm{H}$ NMR spectrum of compound (169) in $\mathrm{CDCl}_{3}$. 


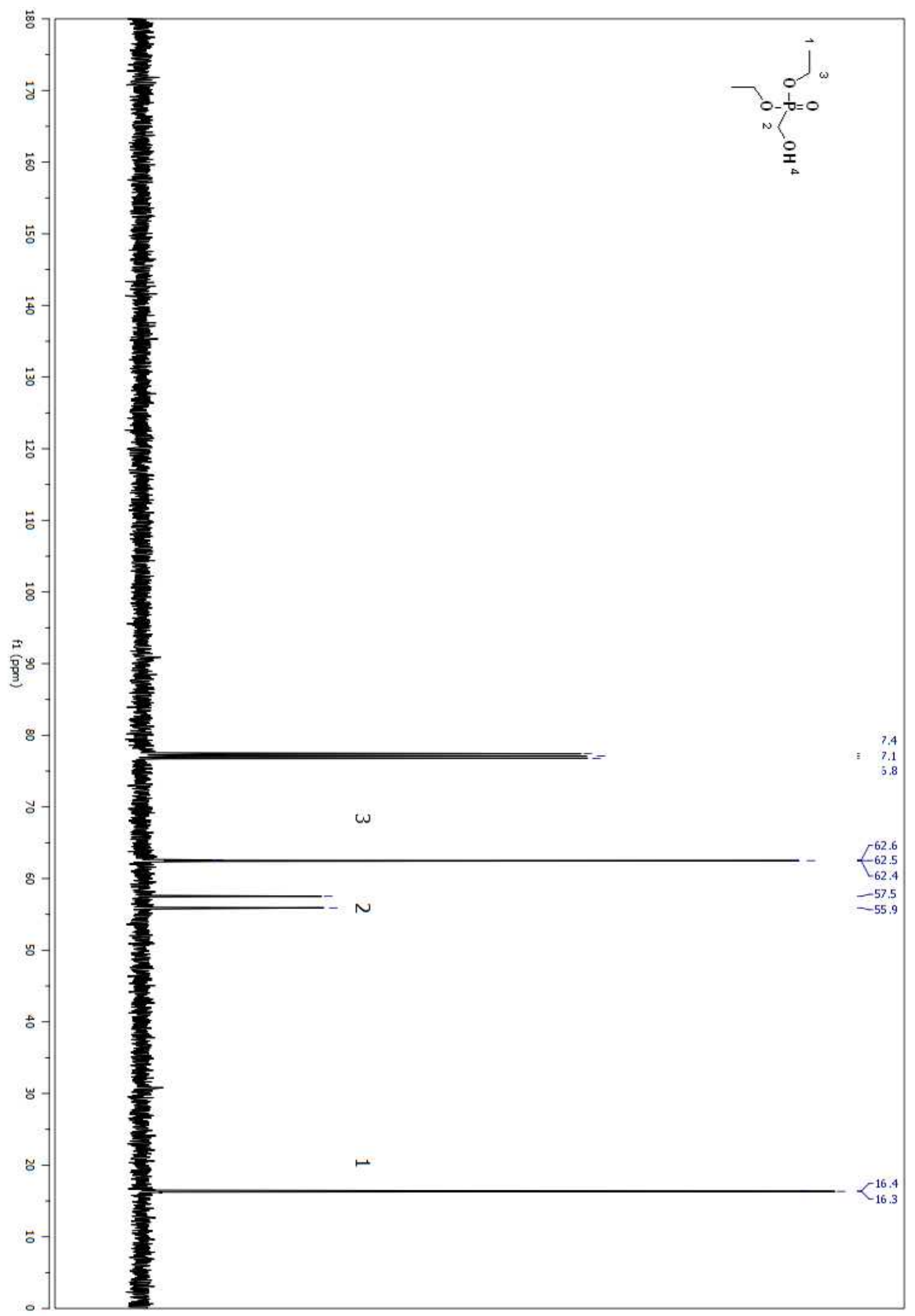

Figure A 279. ${ }^{13} \mathrm{C}$ NMR spectrum of compound (169) in $\mathrm{CDCl}_{3}$. 646 


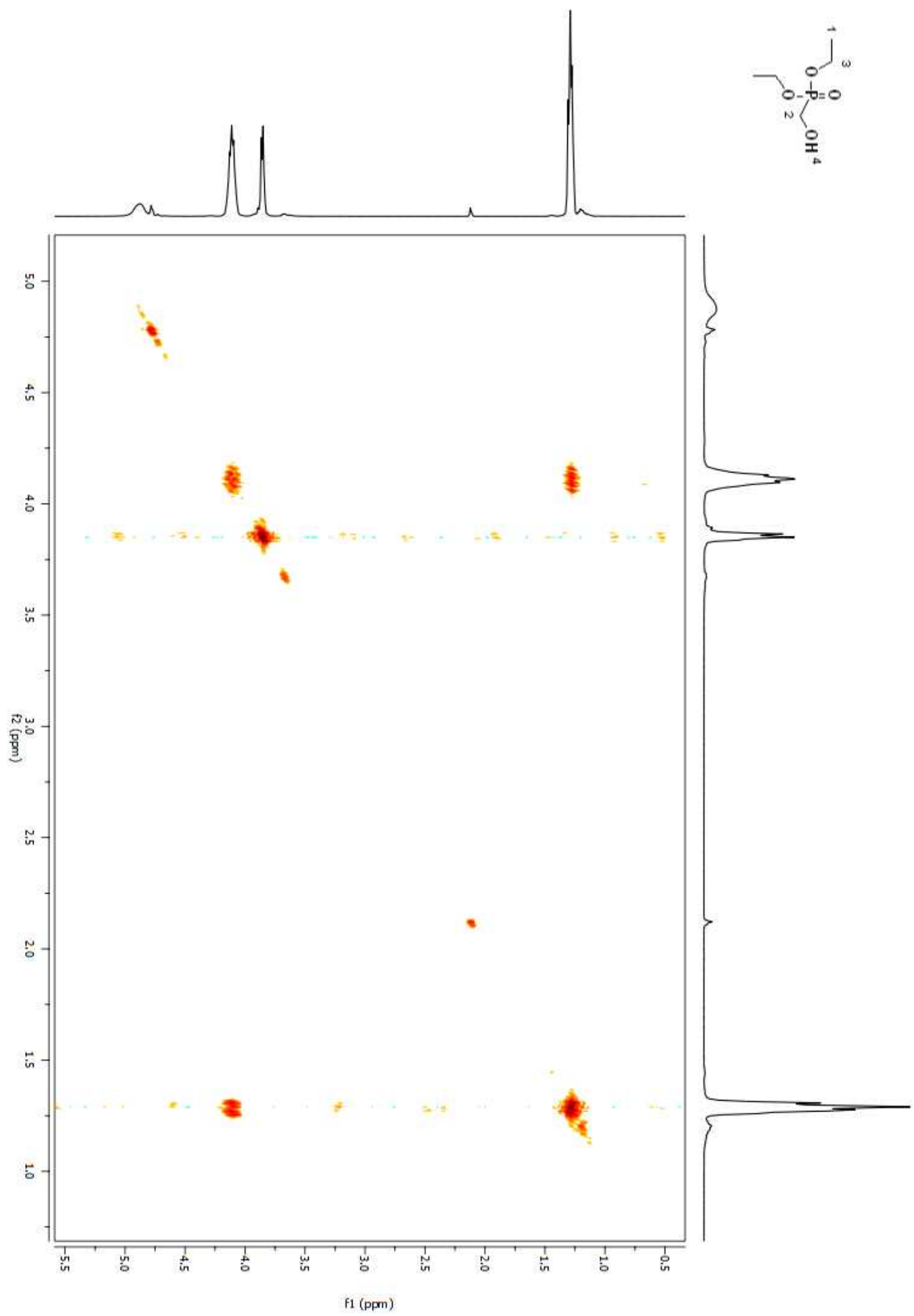

Figure A 280. COSY 2D NMR spectrum of compound (169) in $\mathrm{CDCl}_{3}$. 


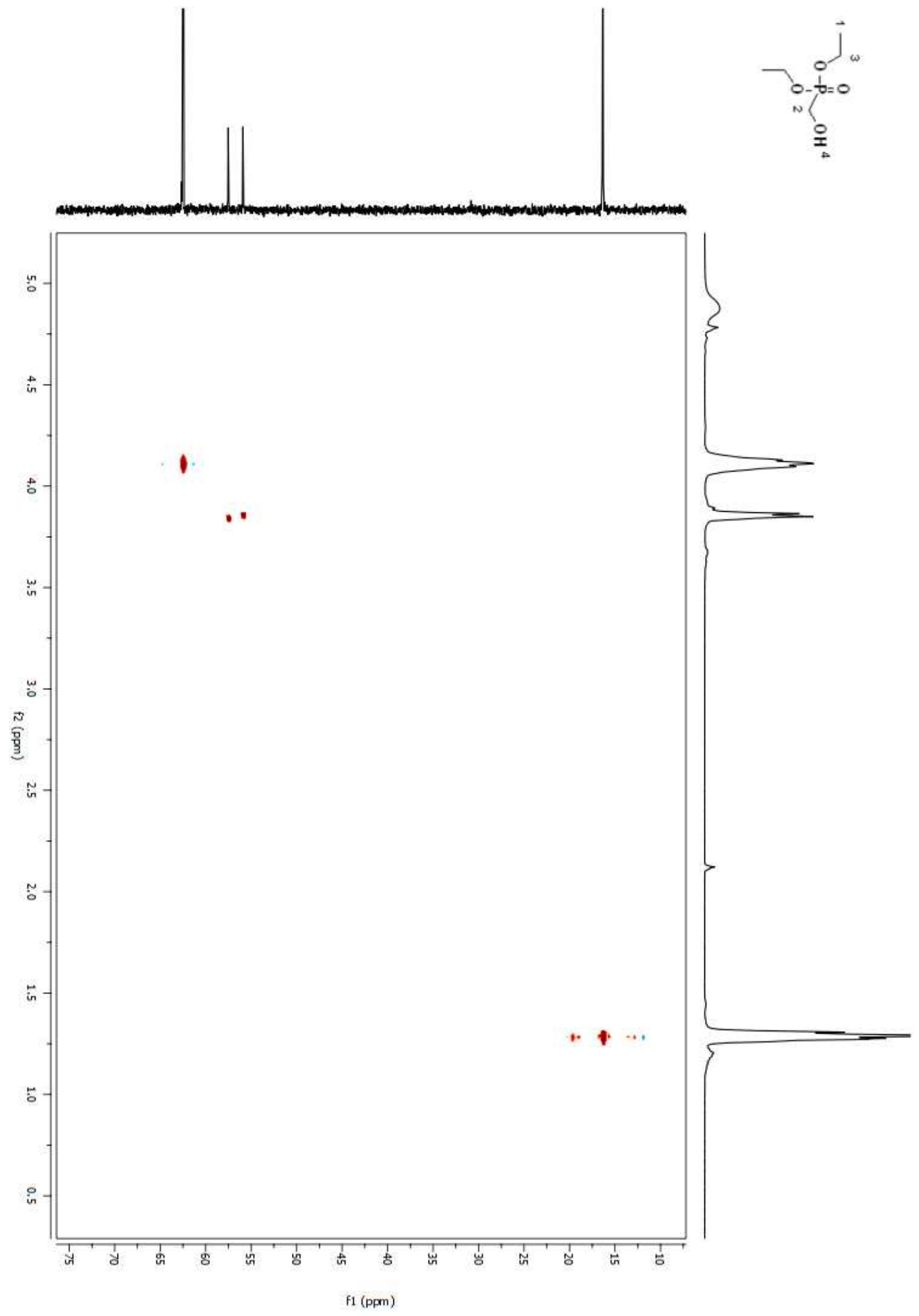

Figure A 281. HSQC 2D NMR spectrum of compound (169) in $\mathrm{CDCl}_{3}$. 


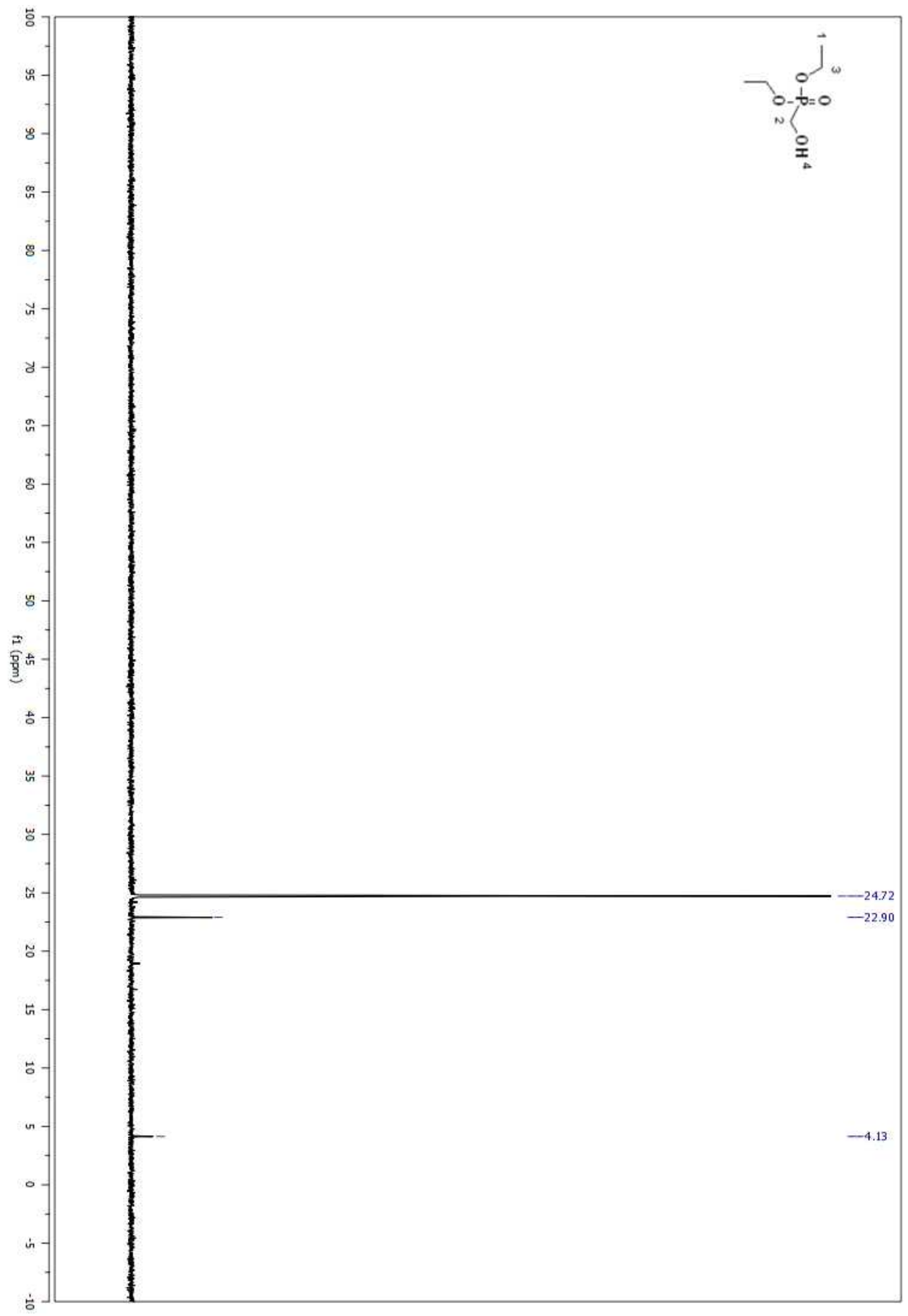

Figure A 282. ${ }^{31} \mathrm{P}$ NMR spectrum of compound (169) in $\mathrm{CDCl}_{3}$. 


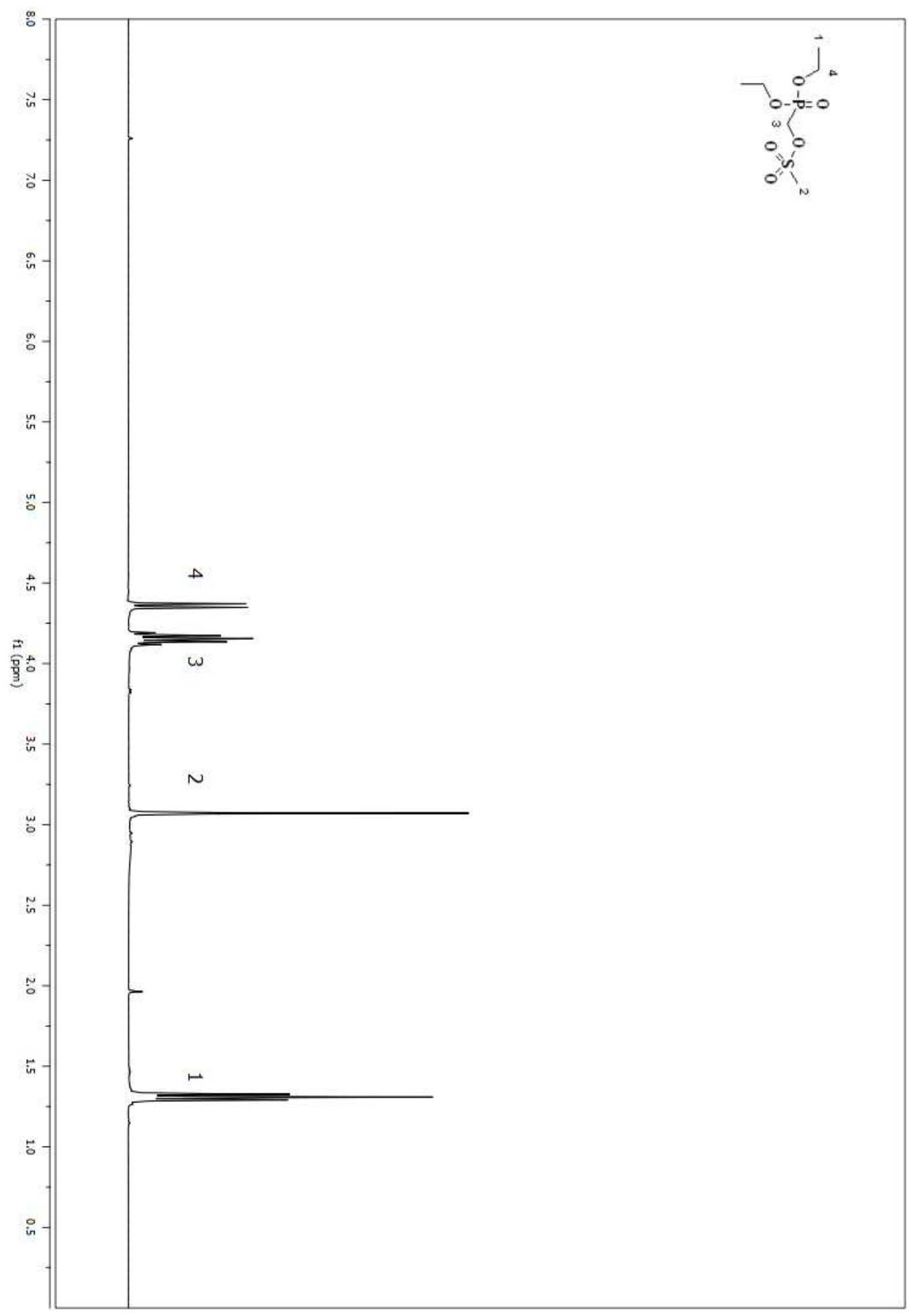

Figure A 283. ${ }^{1} \mathrm{H}$ NMR spectrum of compound (158) in $\mathrm{CDCl}_{3}$. 


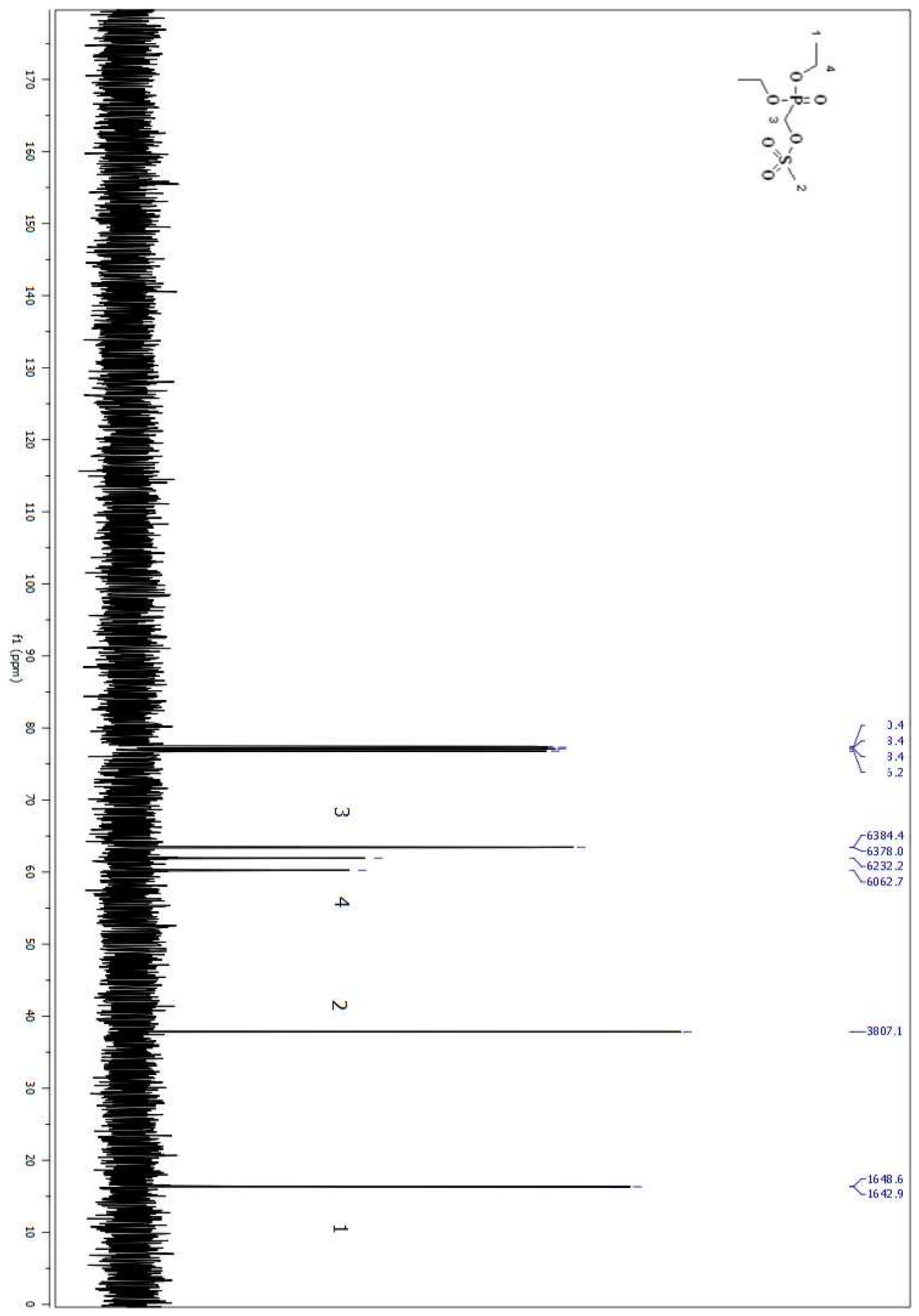

Figure A 284. ${ }^{13} \mathrm{C}$ NMR spectrum of compound (158) in $\mathrm{CDCl}_{3}$. 


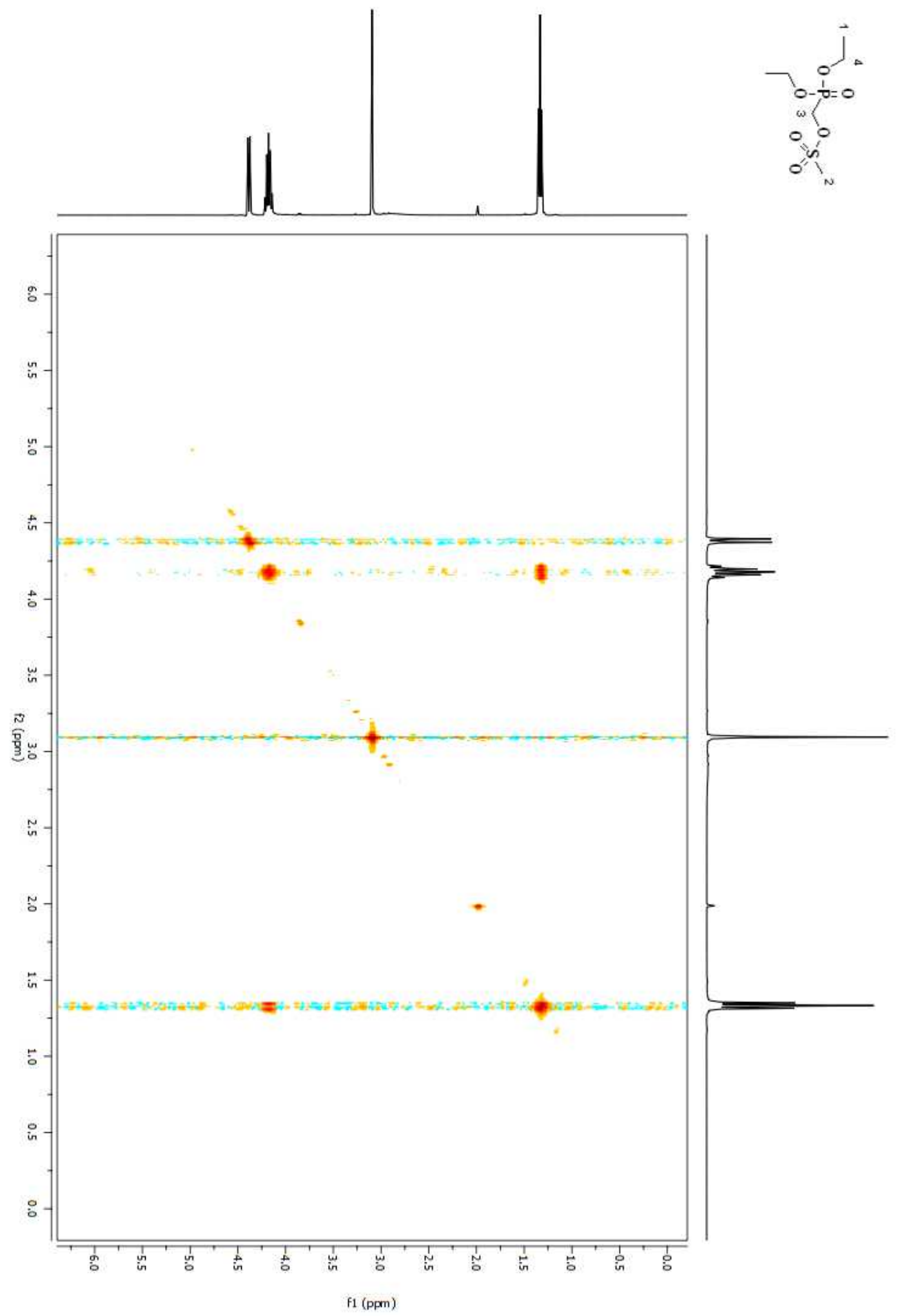

Figure A 285. COSY 2D NMR spectrum of compound (158) in $\mathrm{CDCl}_{3}$. 


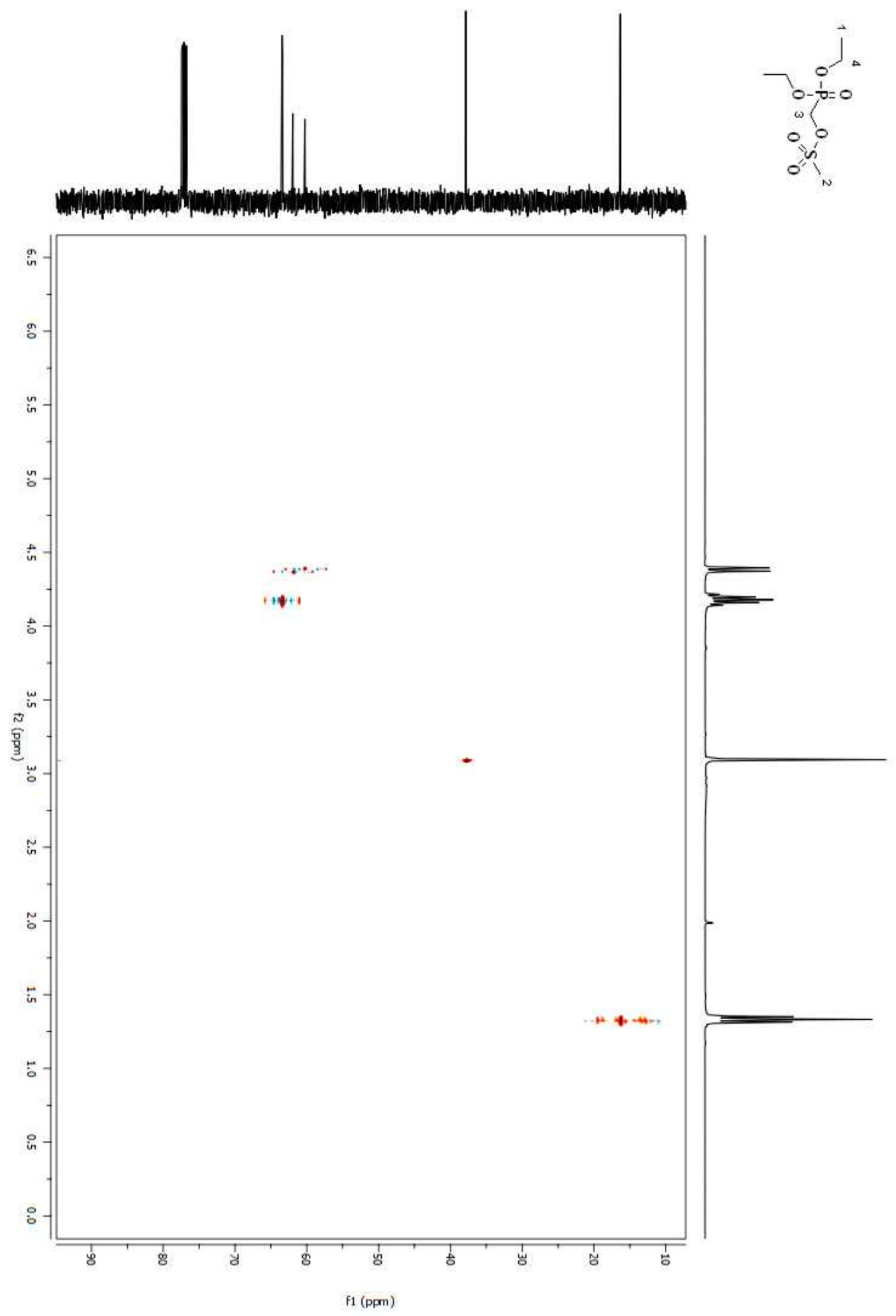

Figure A 286. HSQC 2D NMR spectrum of compound (158) in $\mathrm{CDCl}_{3}$. 


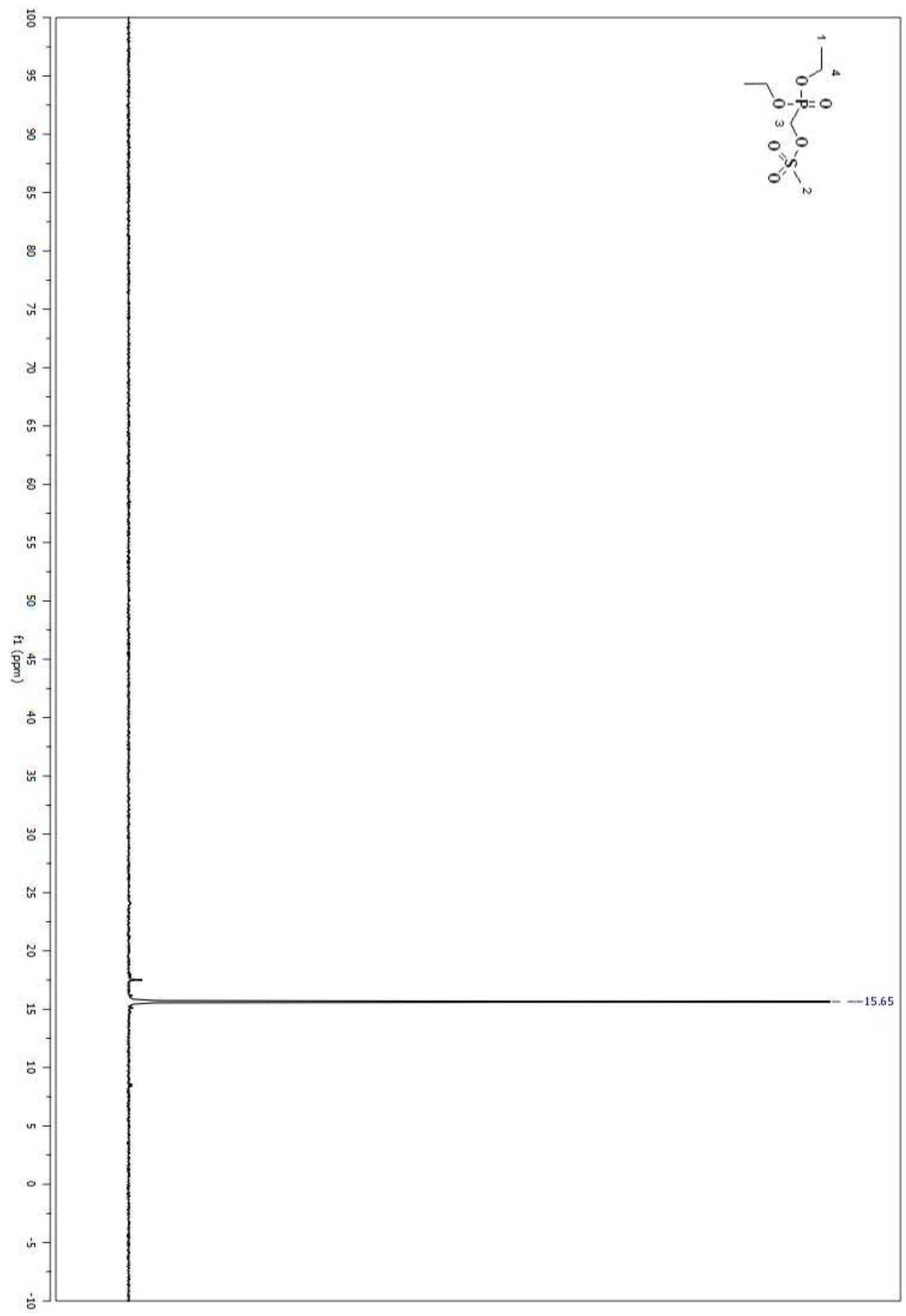

Figure A 287. ${ }^{31} \mathrm{P}$ NMR spectrum of compound (158) in $\mathrm{CDCl}_{3}$. 


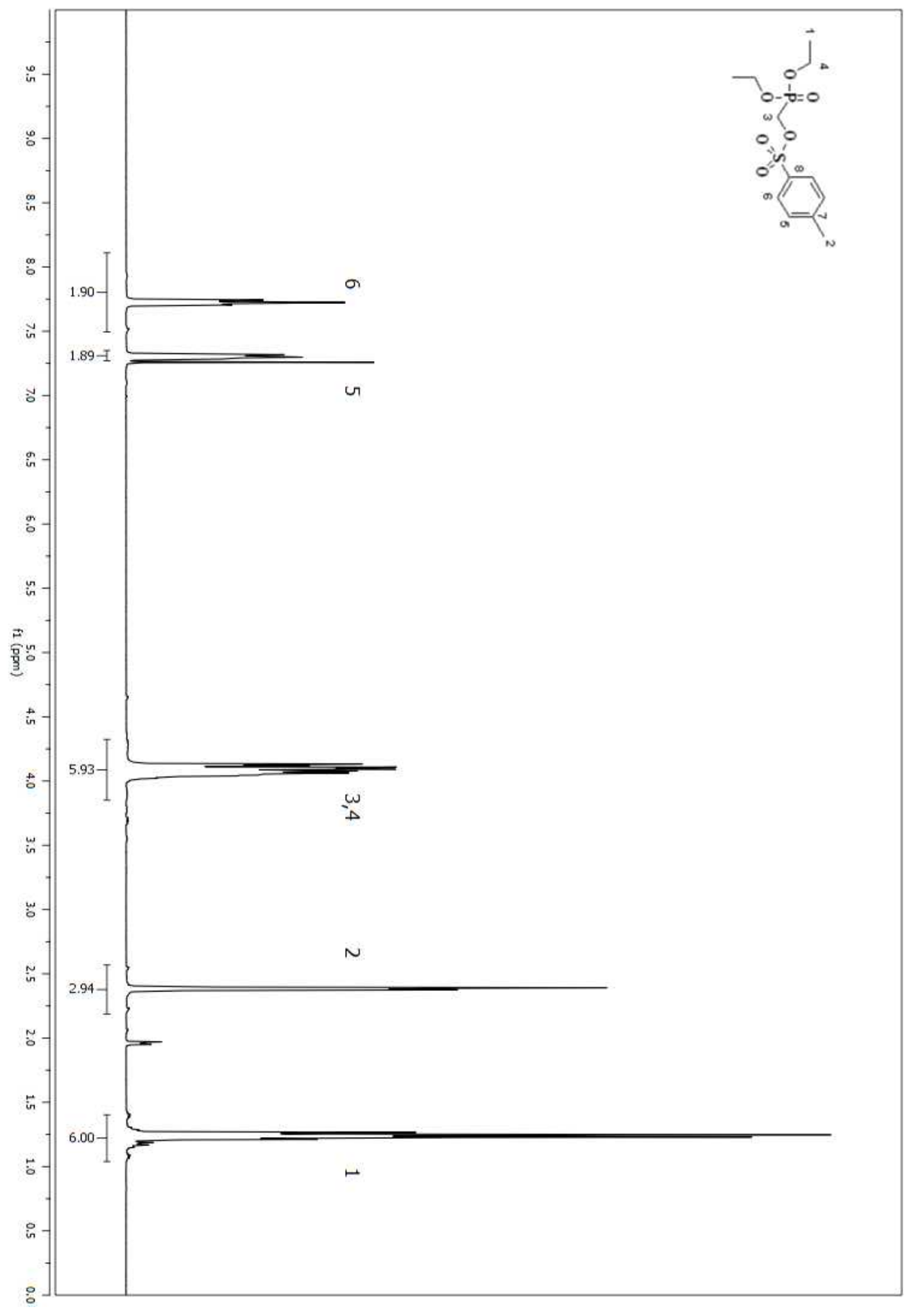

Figure A 288. ${ }^{1} \mathrm{H}$ NMR spectrum of compound (170) in $\mathrm{CDCl}_{3}$. 


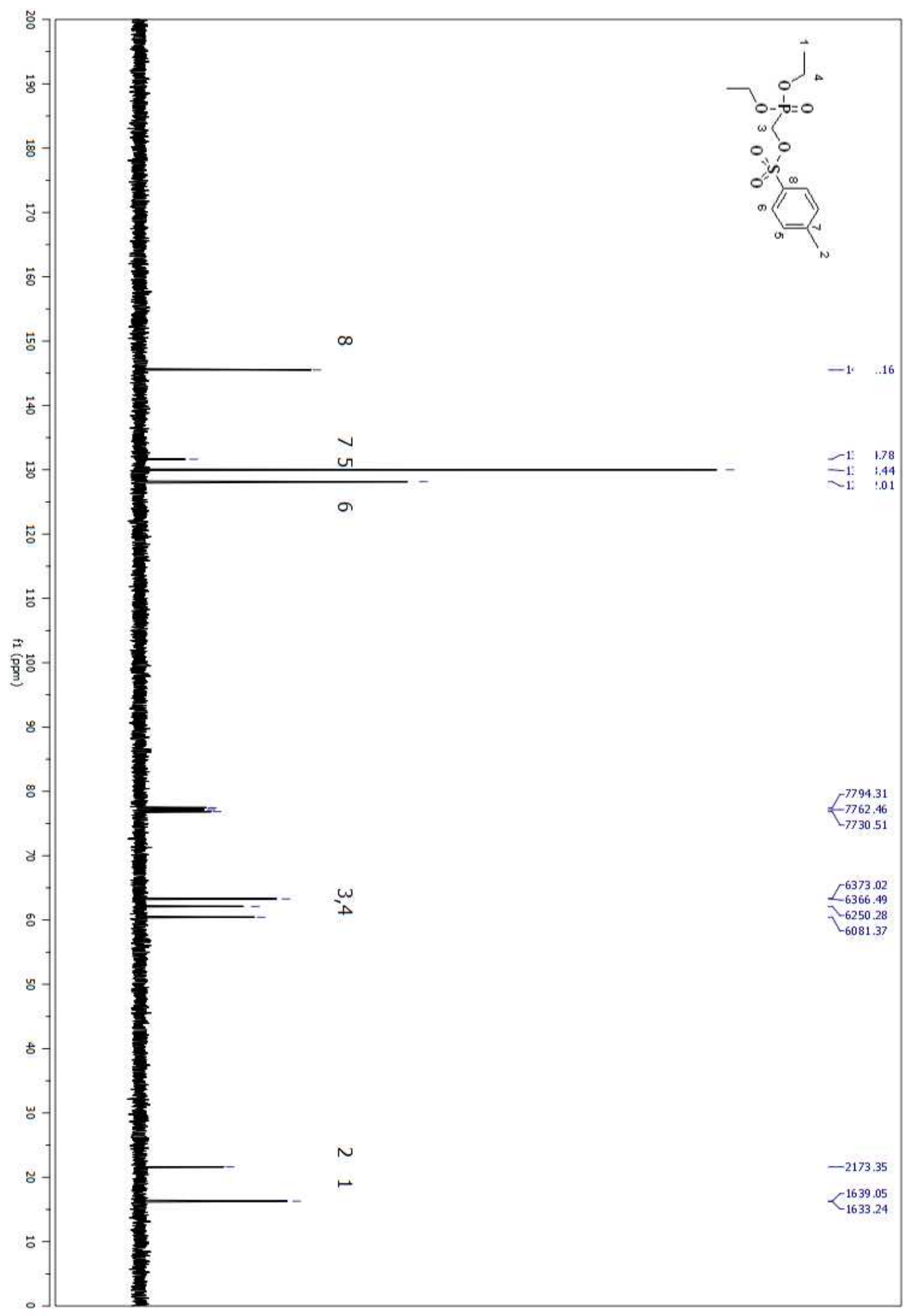

Figure A 289. ${ }^{13} \mathrm{C}$ NMR spectrum of compound (170) in $\mathrm{CDCl}_{3}$. 


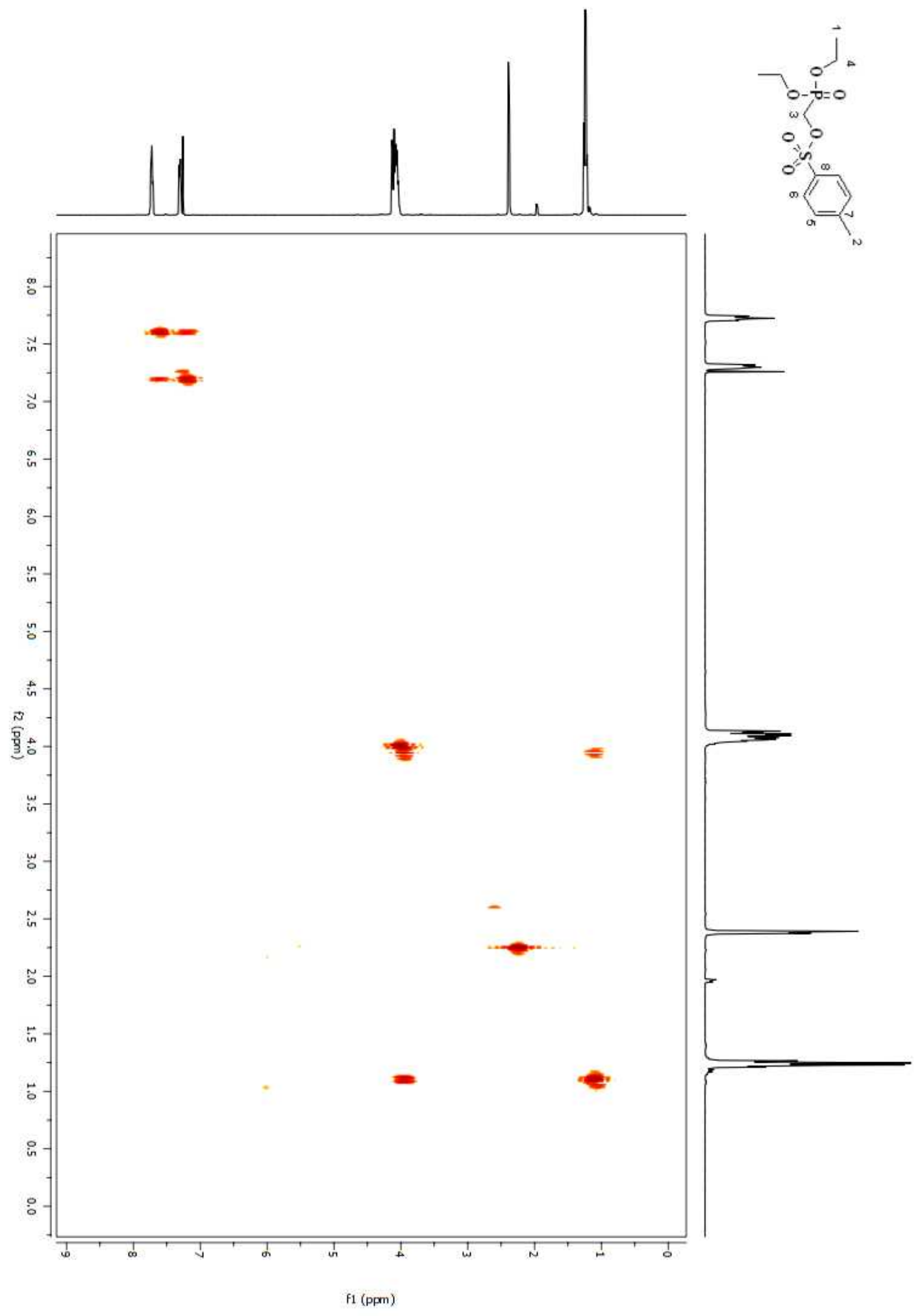

Figure A 290. COSY 2D NMR spectrum of compound (170) in $\mathrm{CDCl}_{3}$. 


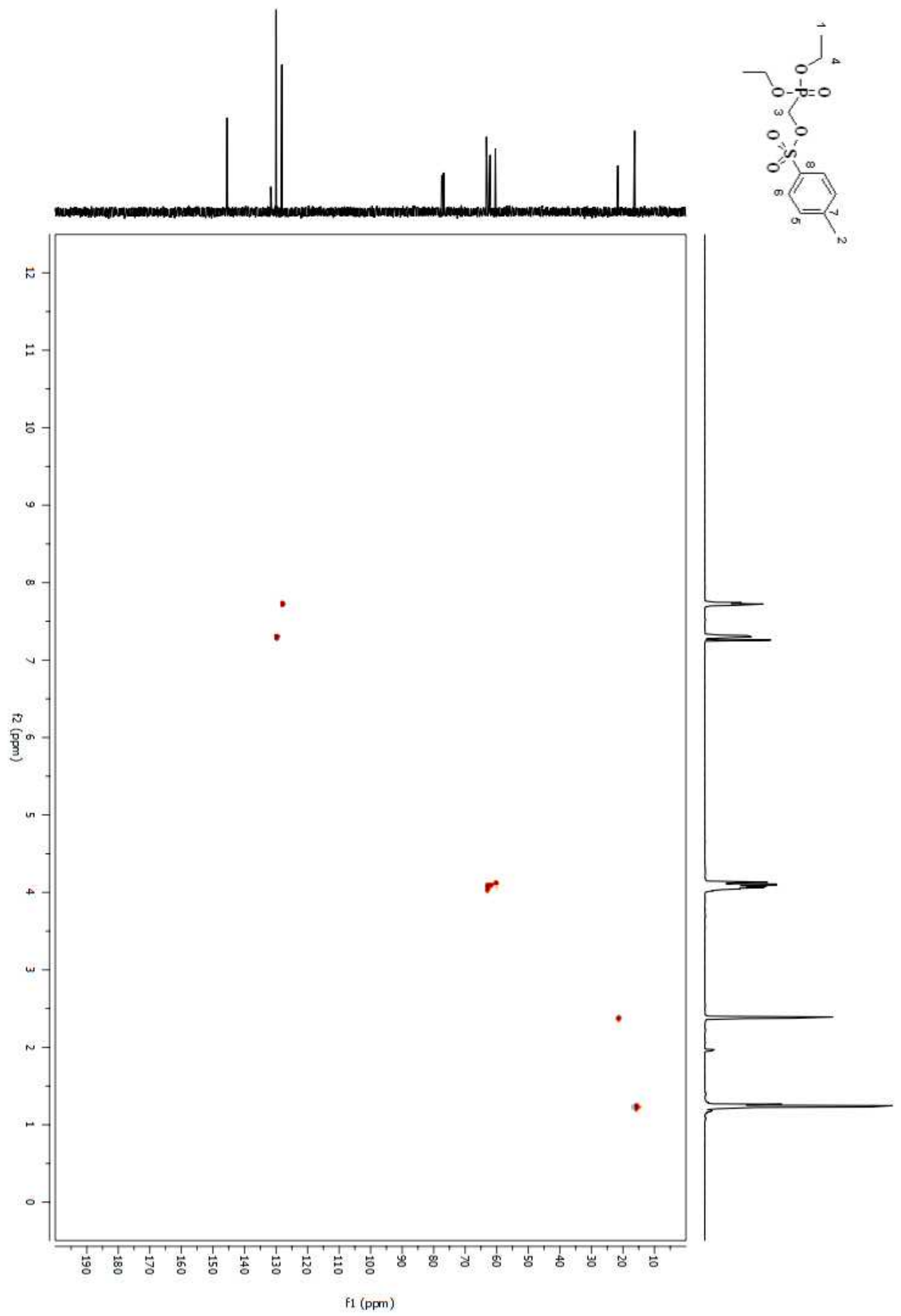

Figure A 291. HSQC 2D NMR spectrum of compound (170) in $\mathrm{CDCl}_{3}$. 


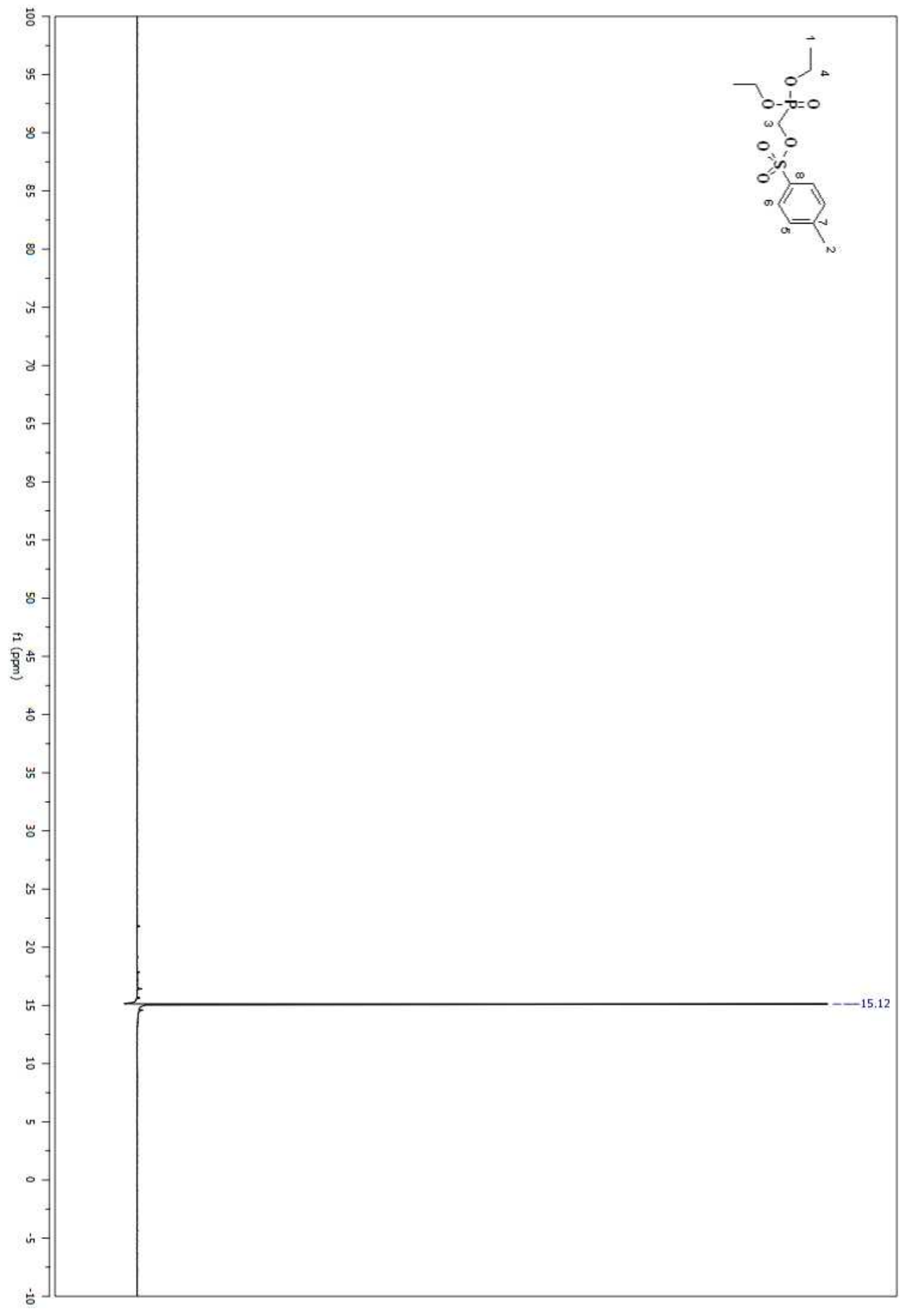

Figure A 292. ${ }^{31} \mathrm{P}$ NMR spectrum of compound (170) in $\mathrm{CDCl}_{3}$. 


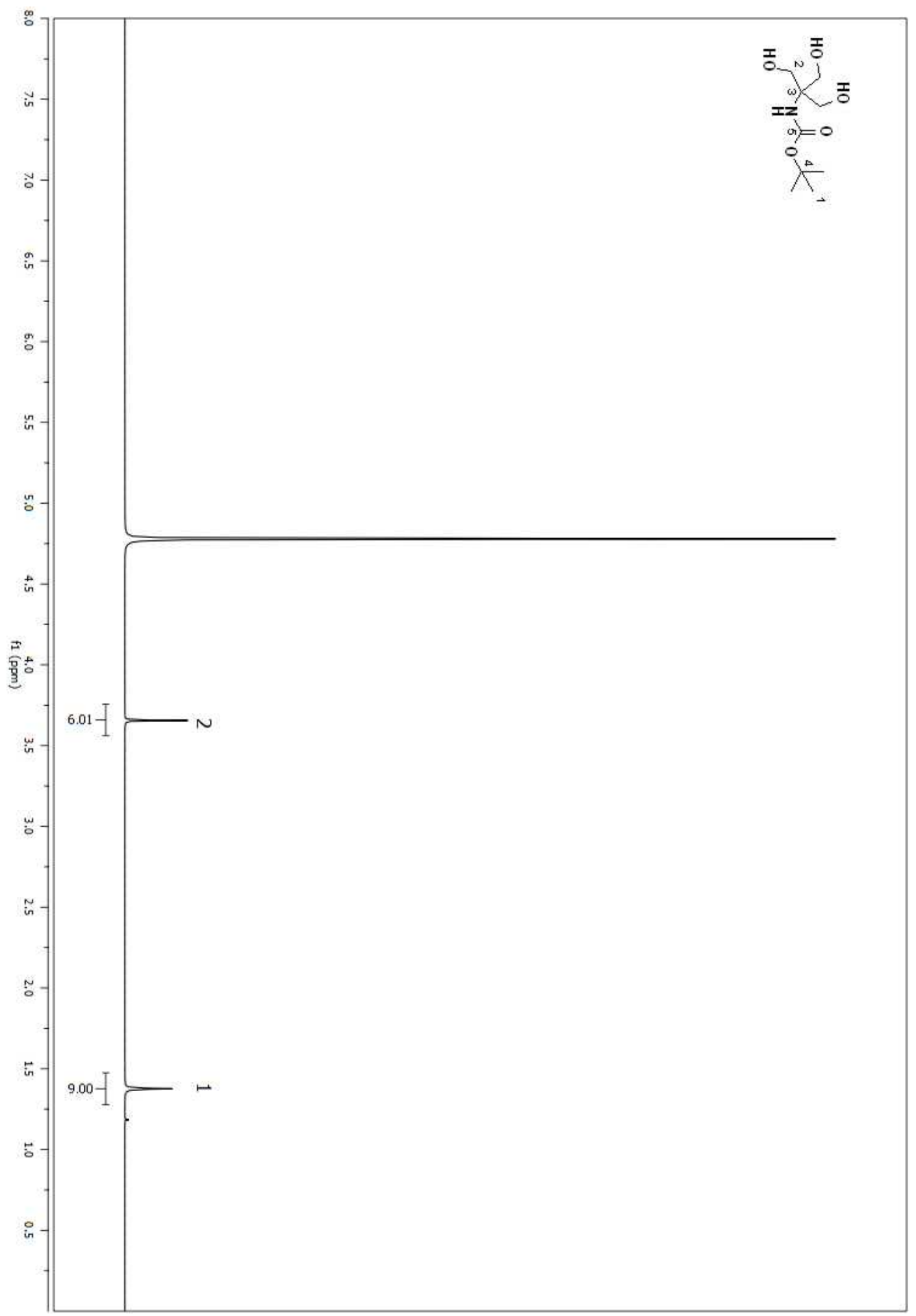

Figure A 293. ${ }^{1} \mathrm{H}$ NMR spectrum of compound (157) in $\mathrm{D}_{2} \mathrm{O}$. 


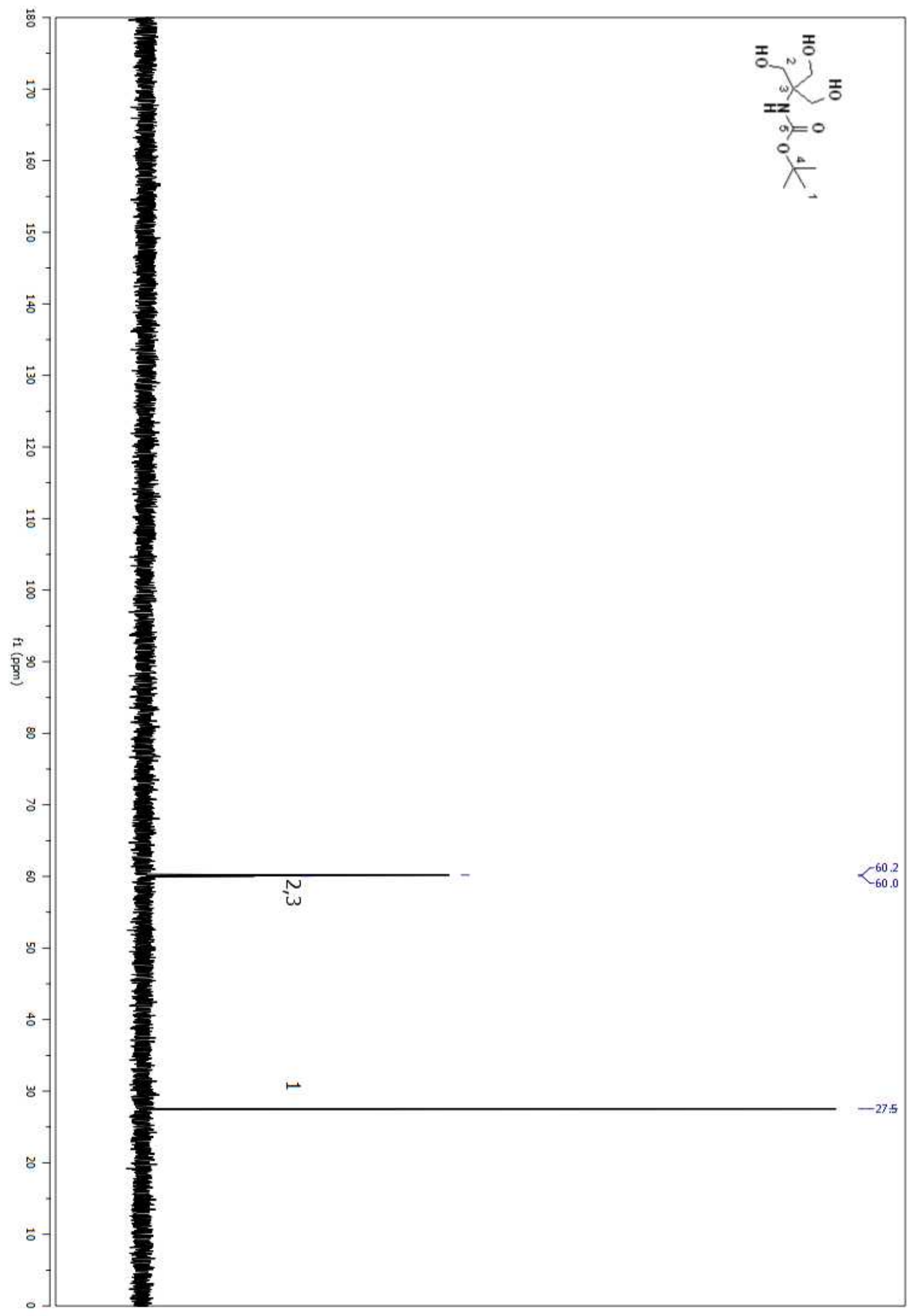

Figure A 294. ${ }^{13} \mathrm{C}$ NMR spectrum of compound (157) in $\mathrm{D}_{2} \mathrm{O}$. 661 


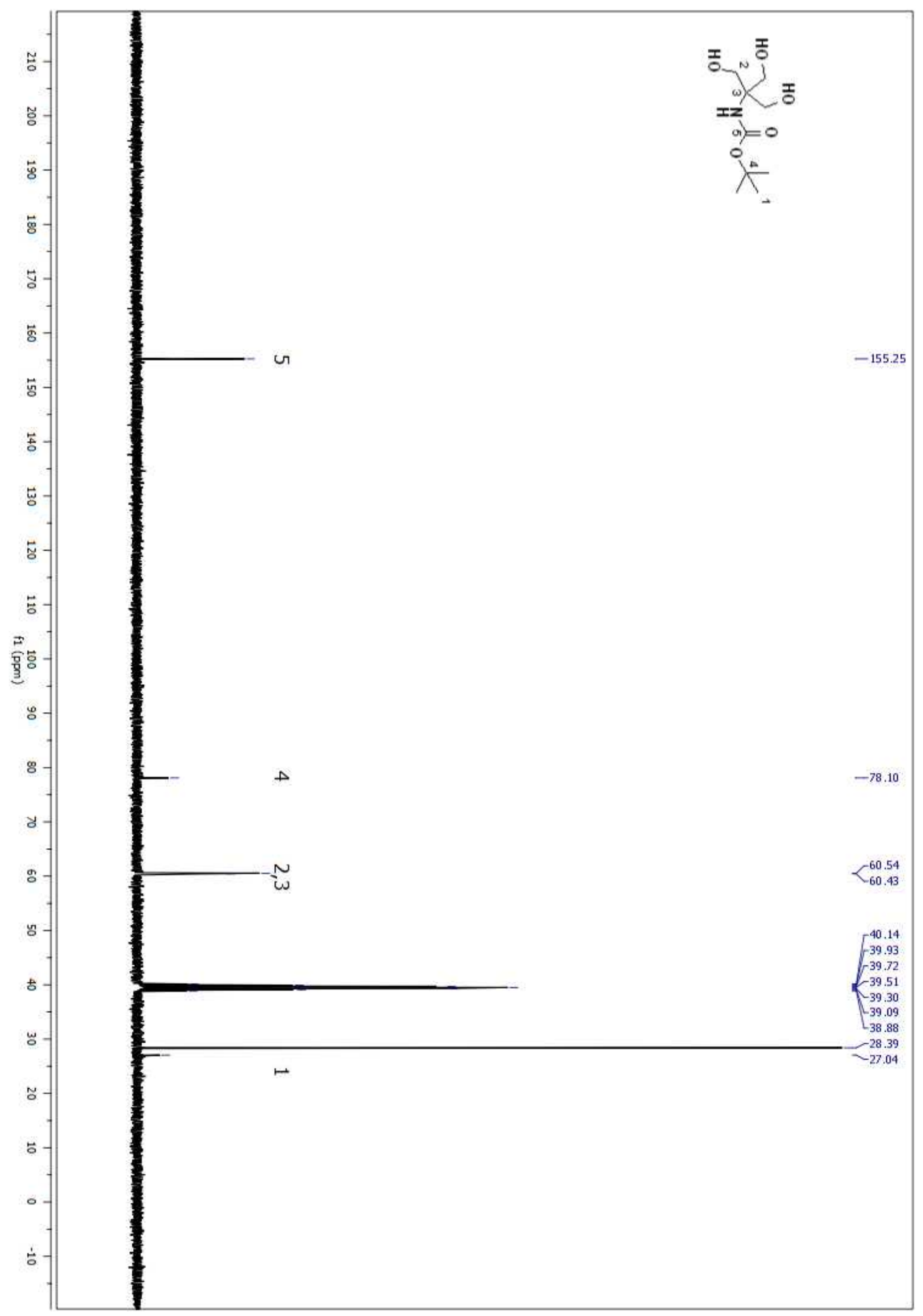

Figure A 295. ${ }^{13} \mathrm{C}$ NMR spectrum of compound (157) in DMSO-d6. 


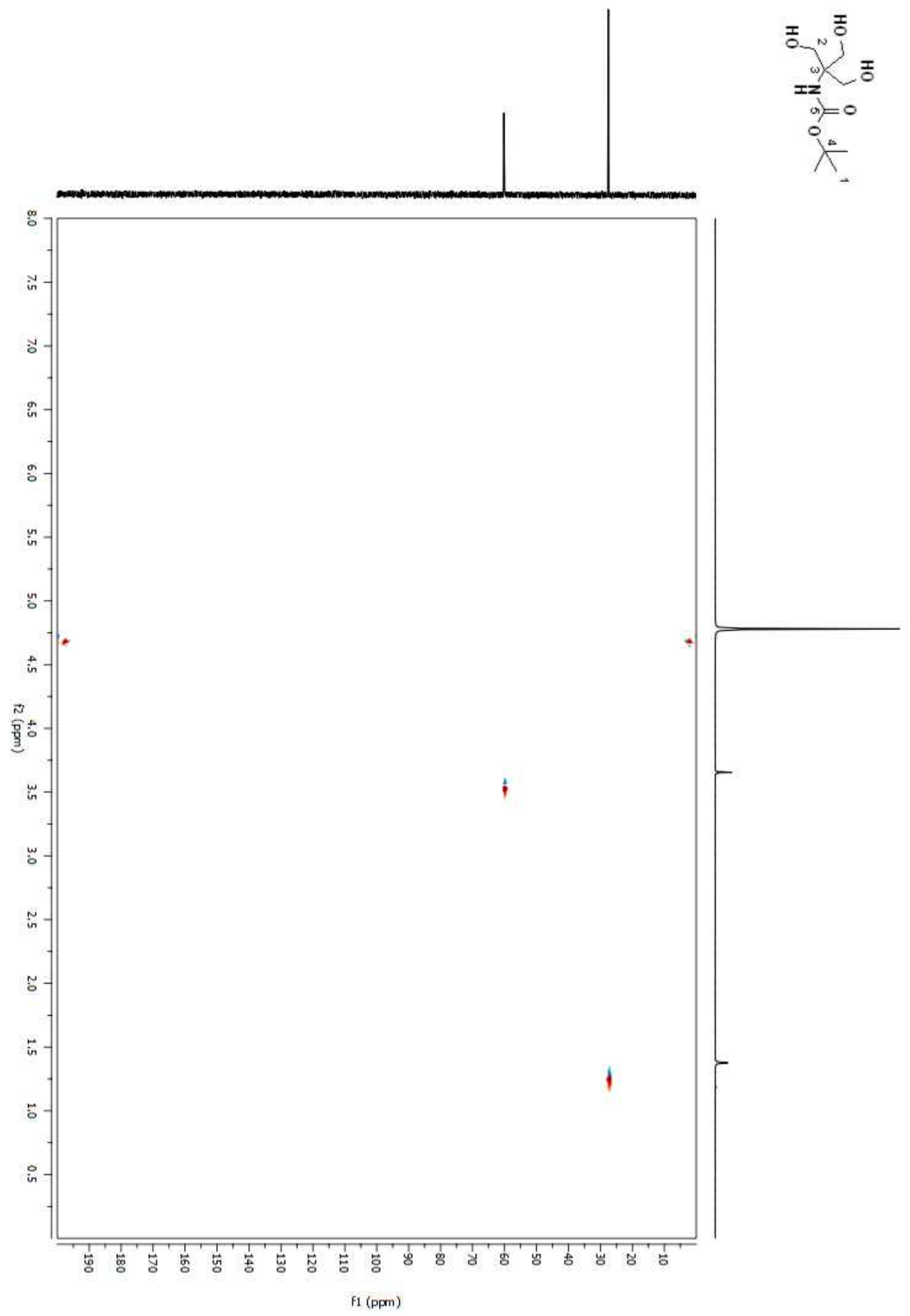

Figure A 296. HSQC 2D NMR spectrum of compound (157) in $\mathrm{CDCl}_{3}$. 


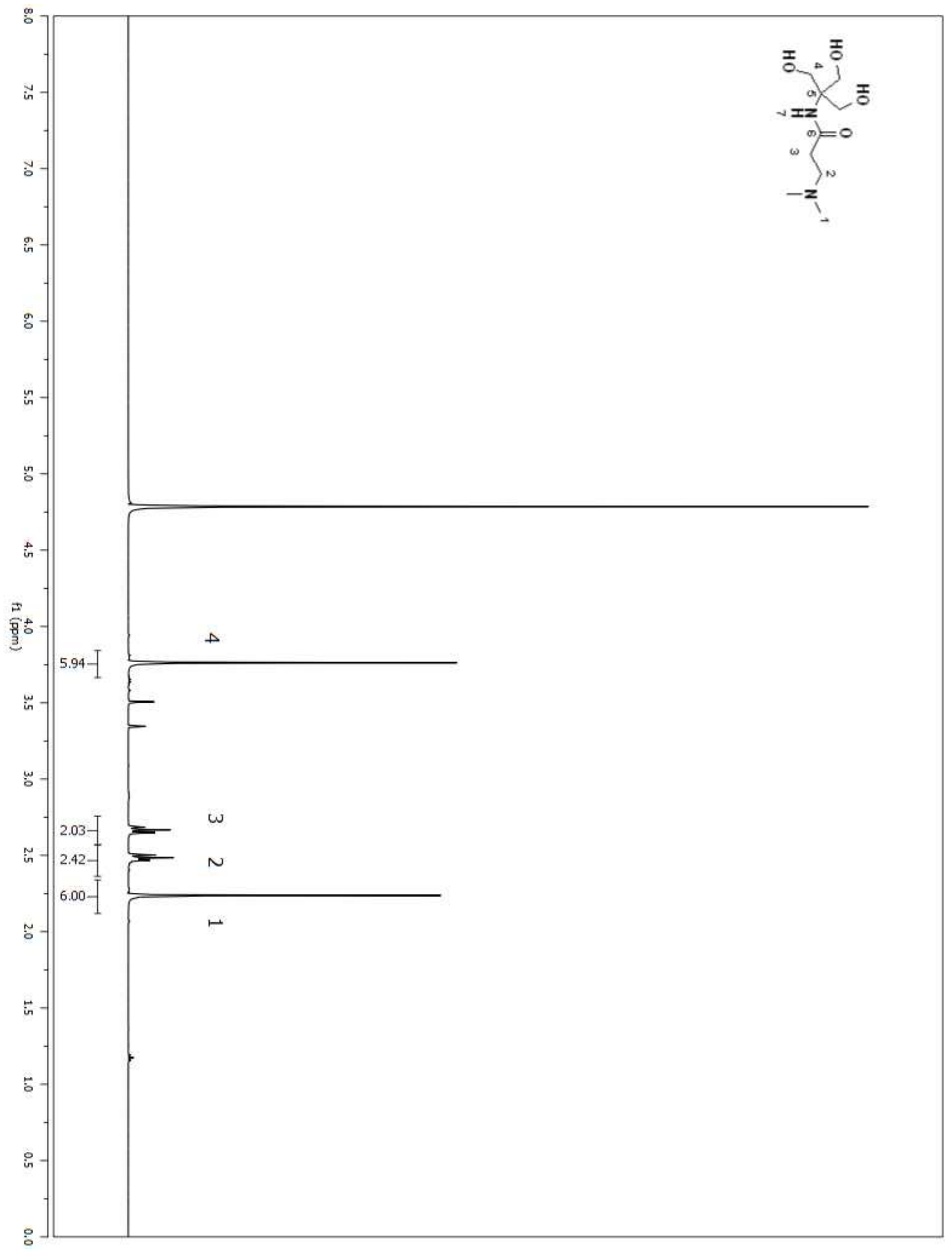

Figure A 297. ${ }^{1} \mathrm{H}$ NMR spectrum of compound (163) in $\mathrm{D}_{2} \mathrm{O}$. 


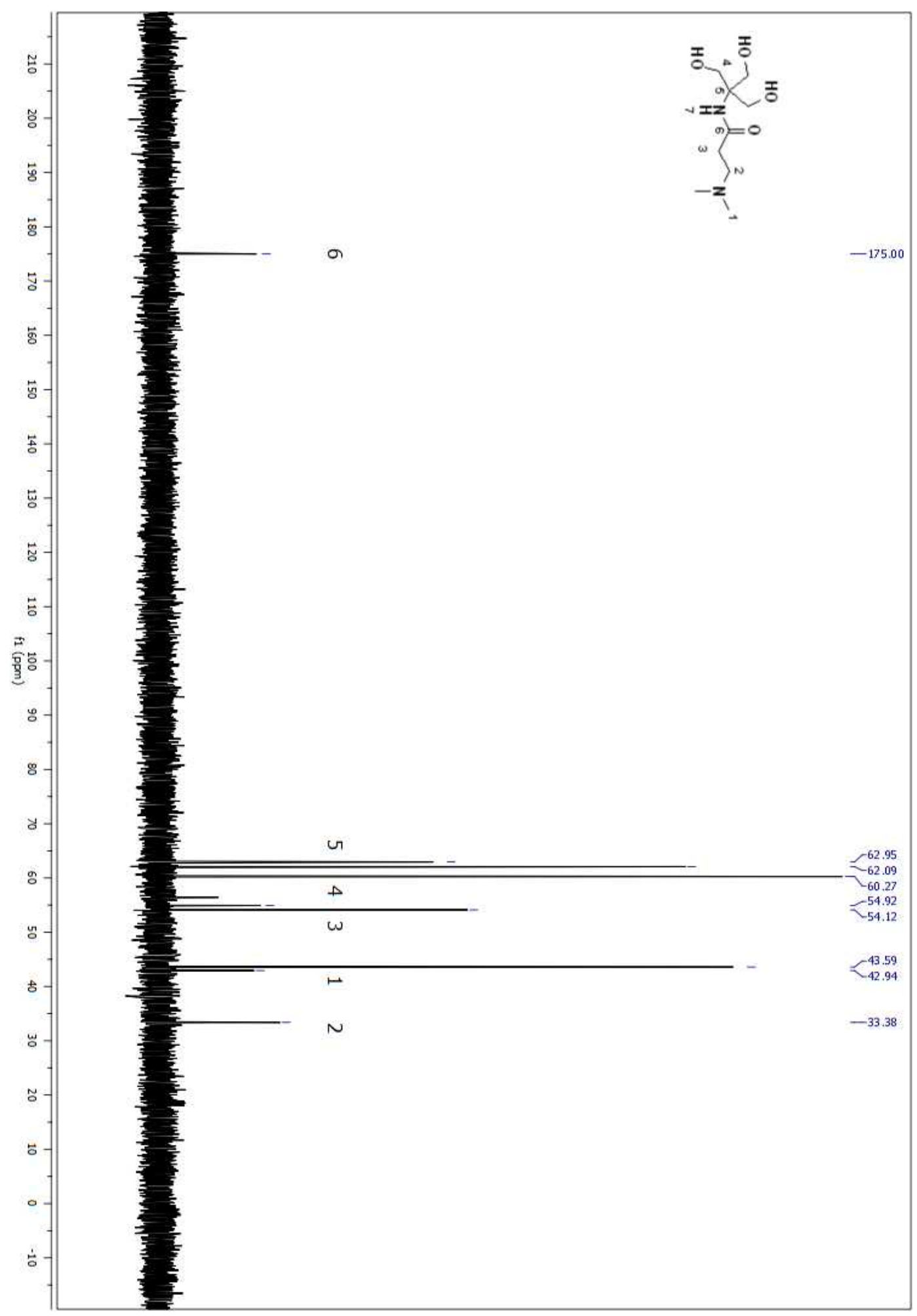

Figure A 298. ${ }^{13} \mathrm{C}$ NMR spectrum of compound (163) in $\mathrm{D}_{2} \mathrm{O}$. 


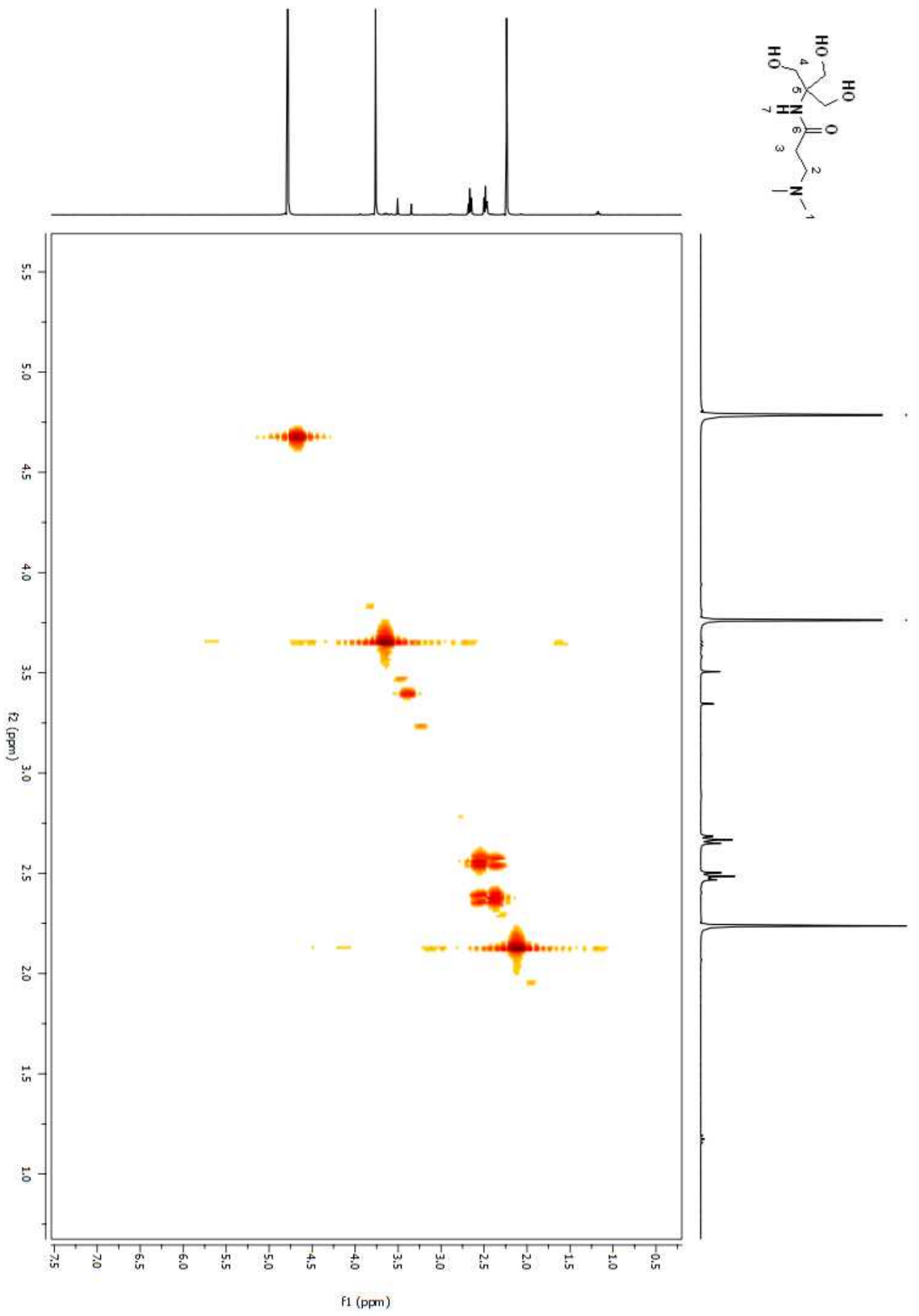

Figure A 299. COSY 2D NMR spectrum of compound (163) in $\mathrm{CDCl}_{3}$. 


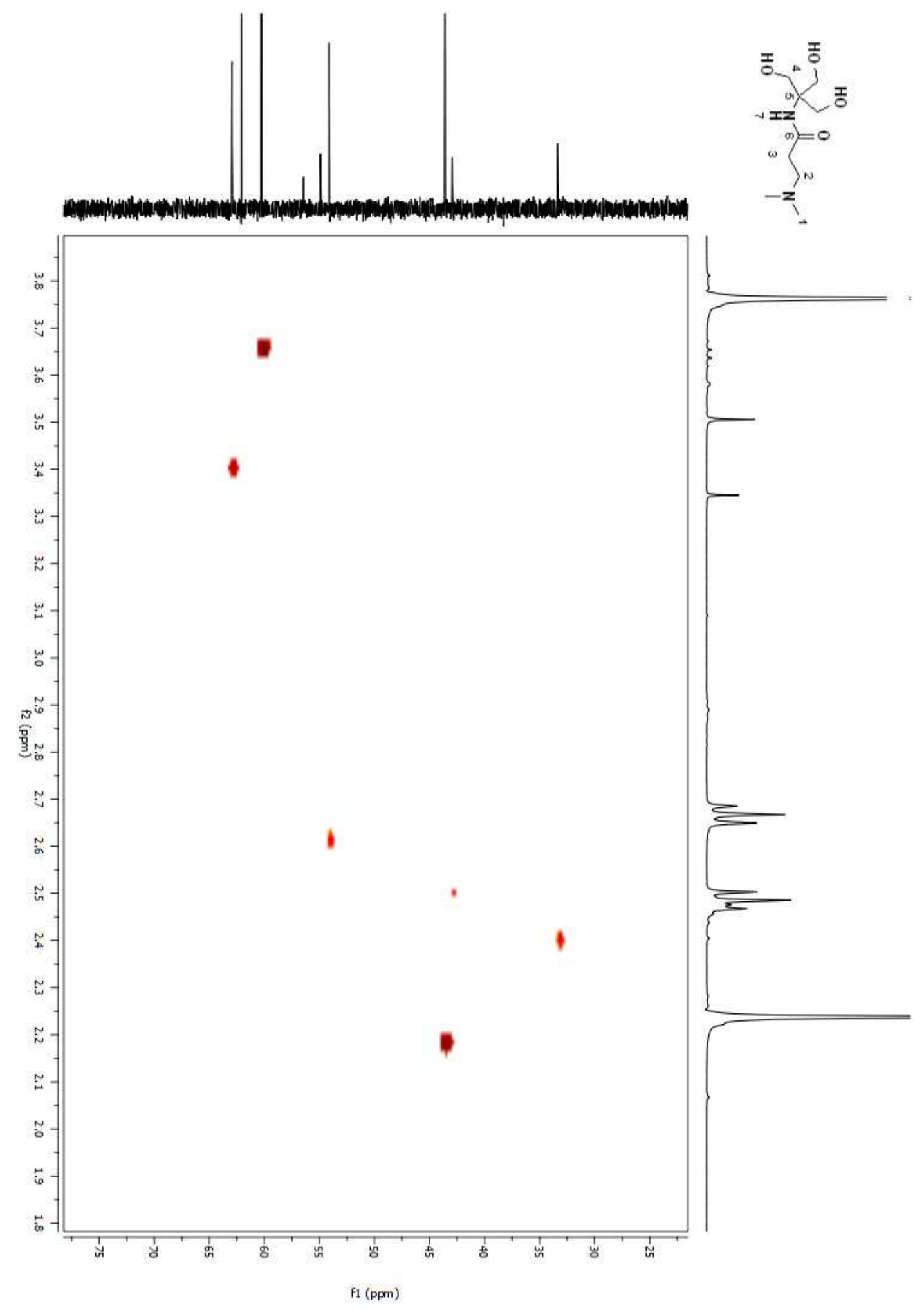

Figure A 300. HSQC 2D NMR spectrum of compound (163) in $\mathrm{CDCl}_{3}$. 


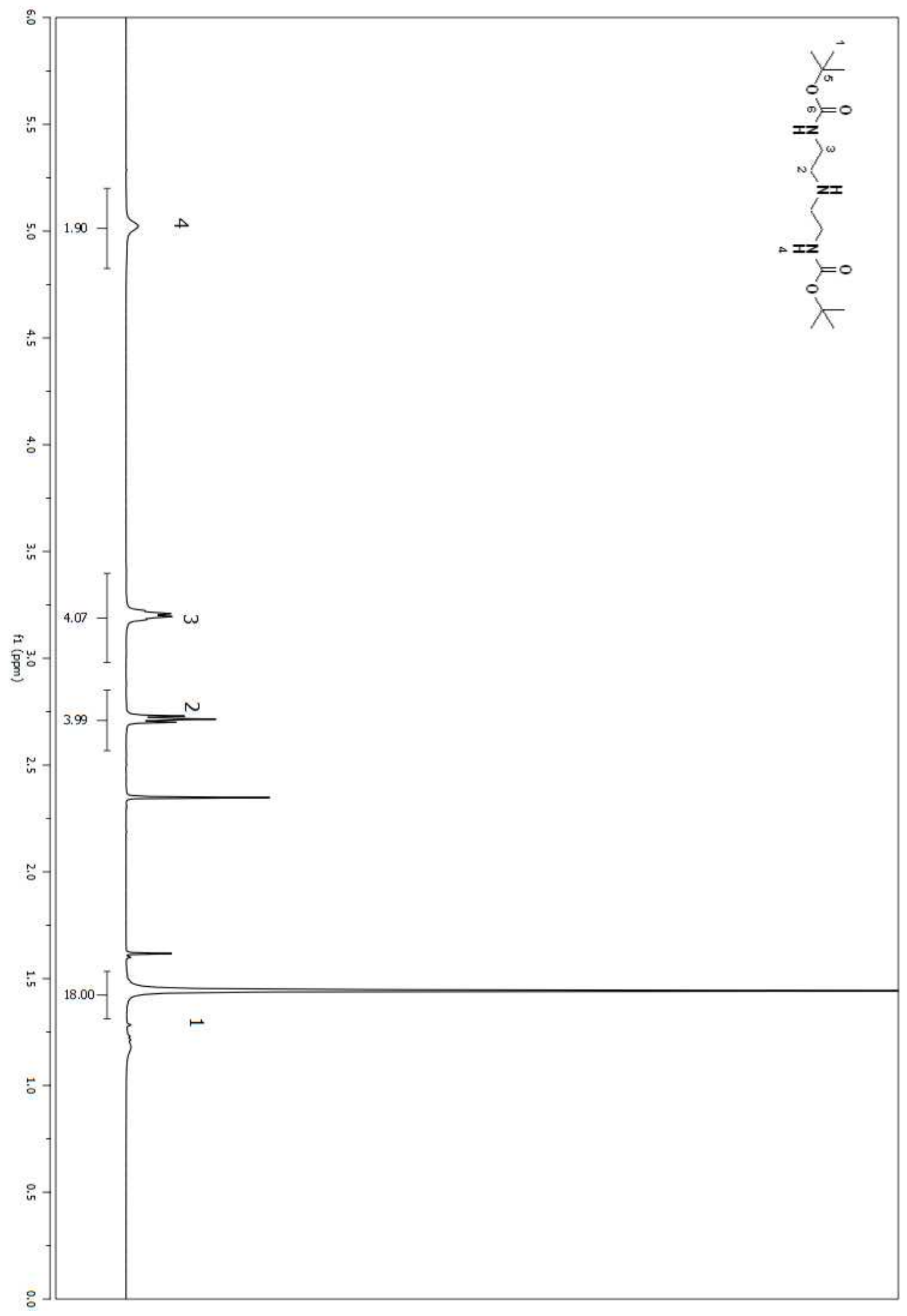

Figure A 301. ${ }^{1} \mathrm{H}$ NMR spectrum of compound (176) in $\mathrm{CDCl}_{3}$. 


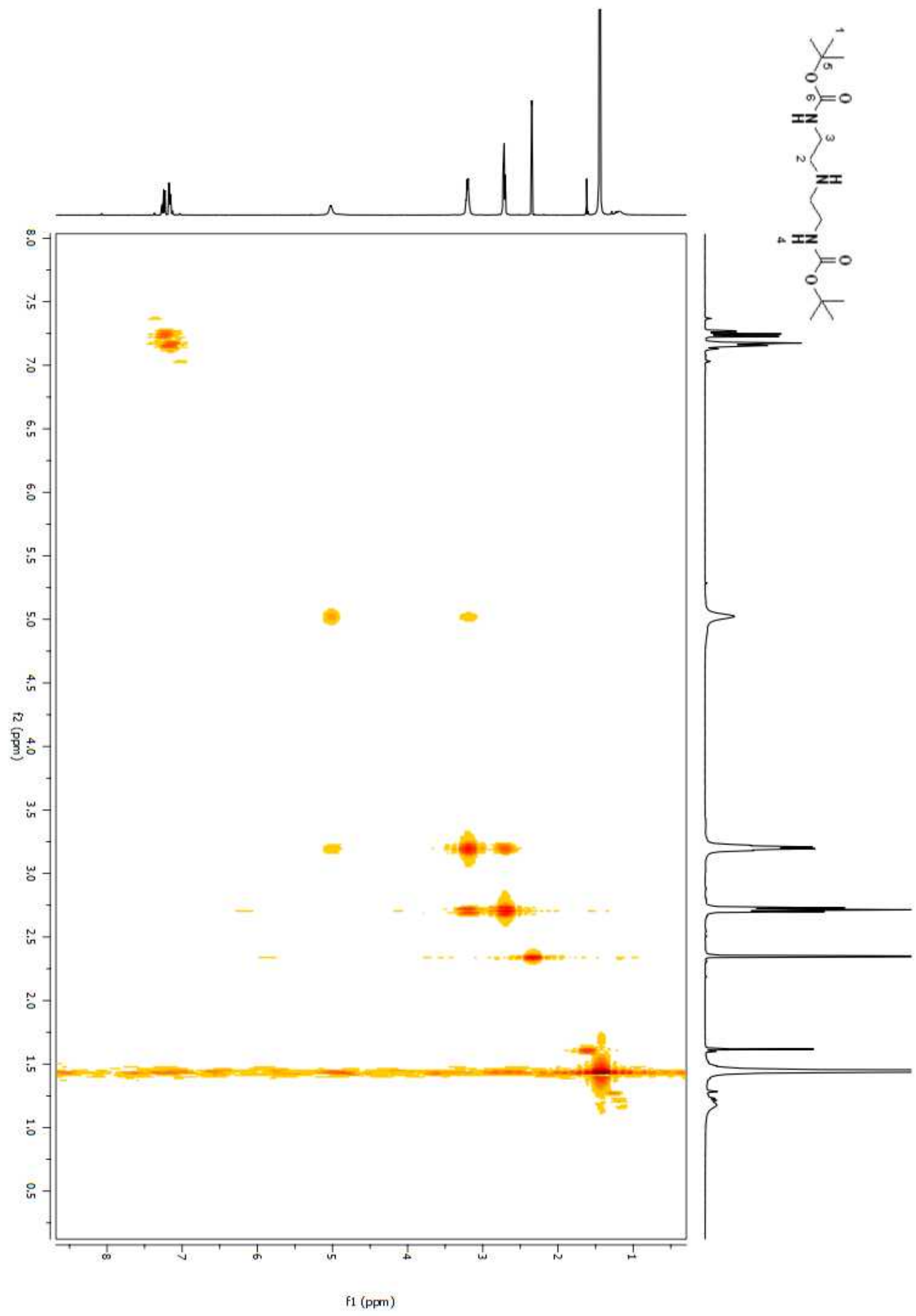

Figure A 302. COSY 2D NMR spectrum of compound (176) in $\mathrm{CDCl}_{3}$. 


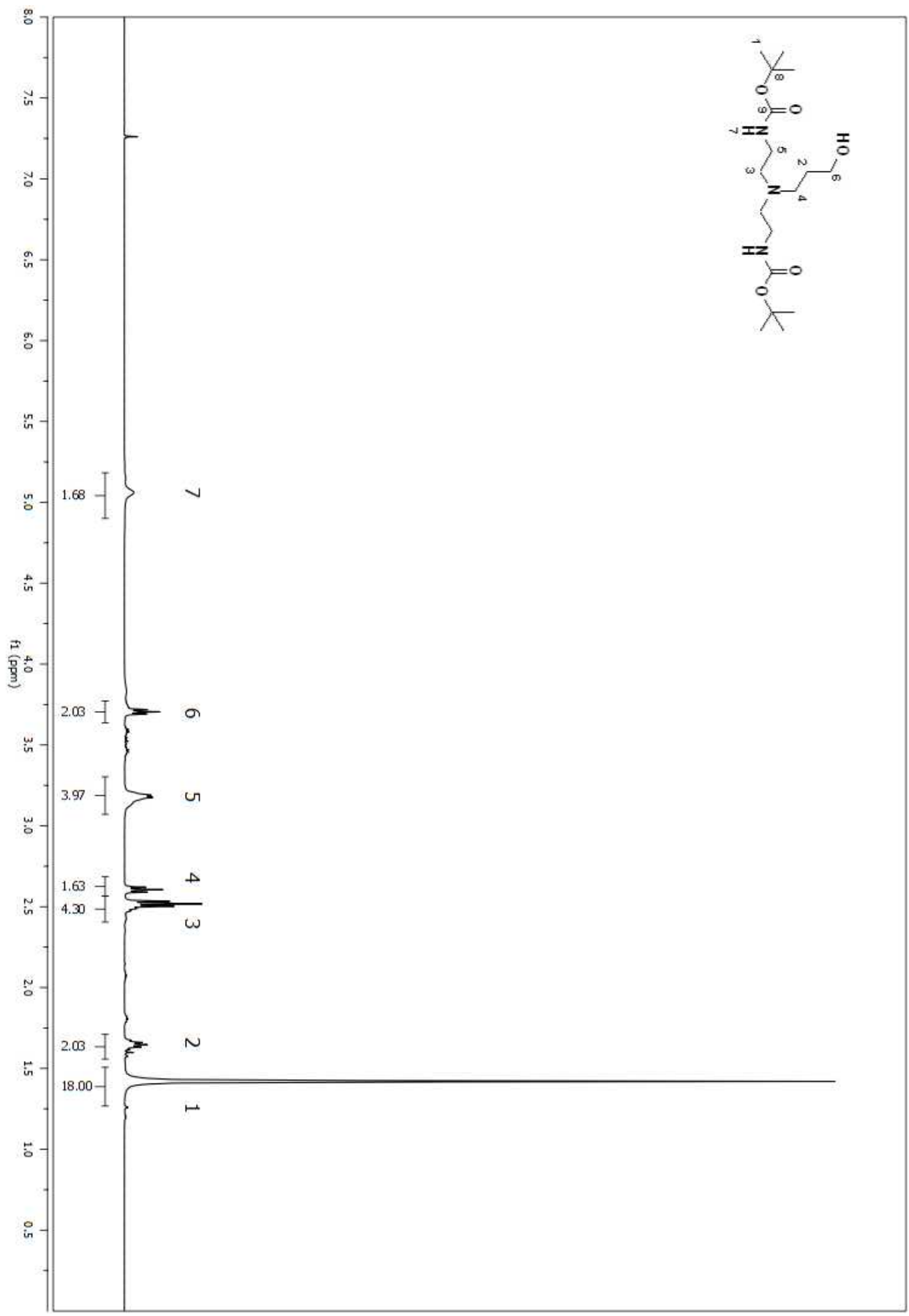

Figure A 303. ${ }^{1} \mathrm{H}$ NMR spectrum of compound (177) in $\mathrm{CDCl}_{3}$. 


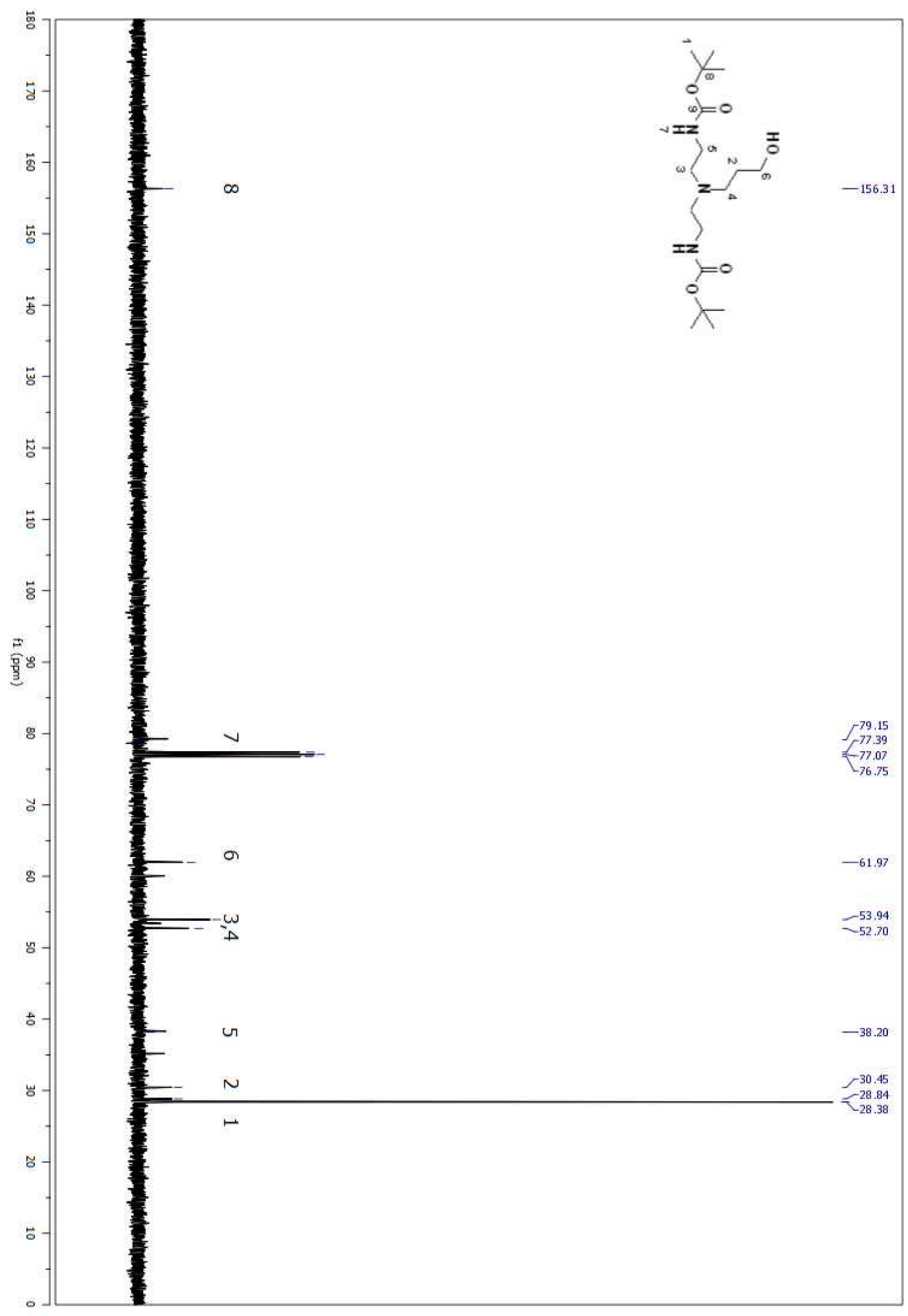

Figure A 304. ${ }^{13} \mathrm{C}$ NMR spectrum of compound (177) in $\mathrm{CDCl}_{3}$. 


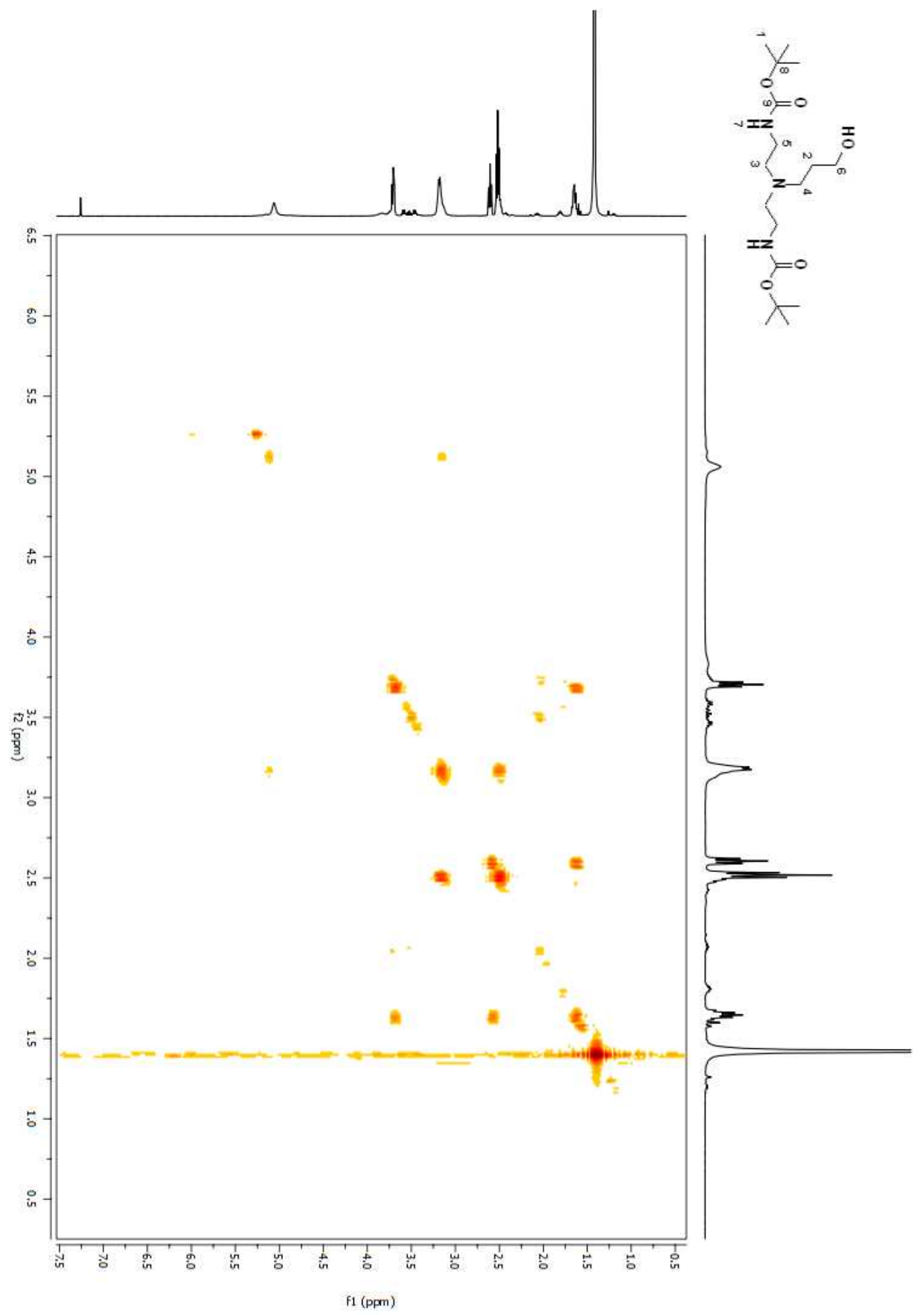

Figure A 305. COSY 2D NMR spectrum of compound (177) in $\mathrm{CDCl}_{3}$. 


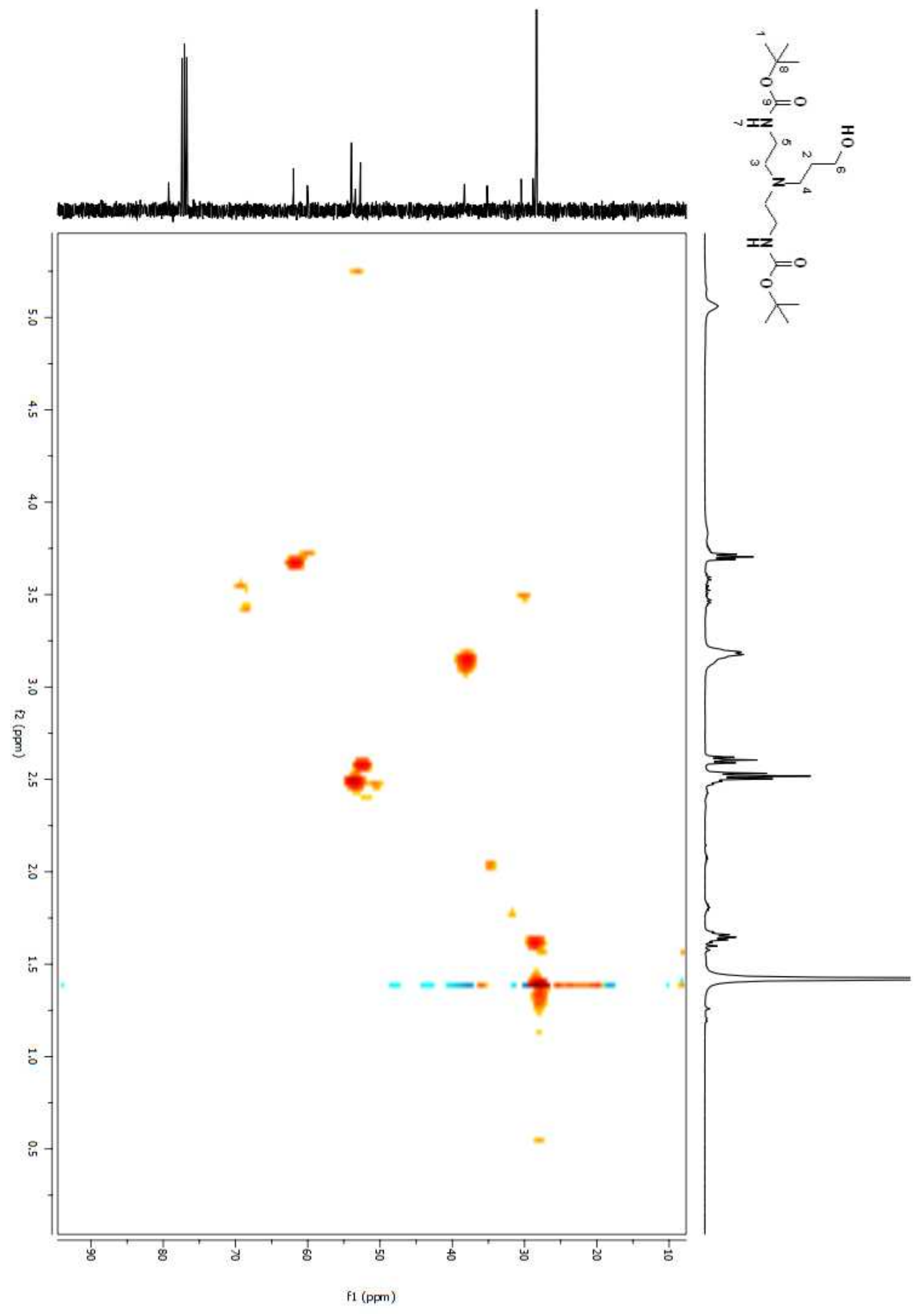

Figure A 306. HSQC 2D NMR spectrum of compound (177) in $\mathrm{CDCl}_{3}$. 


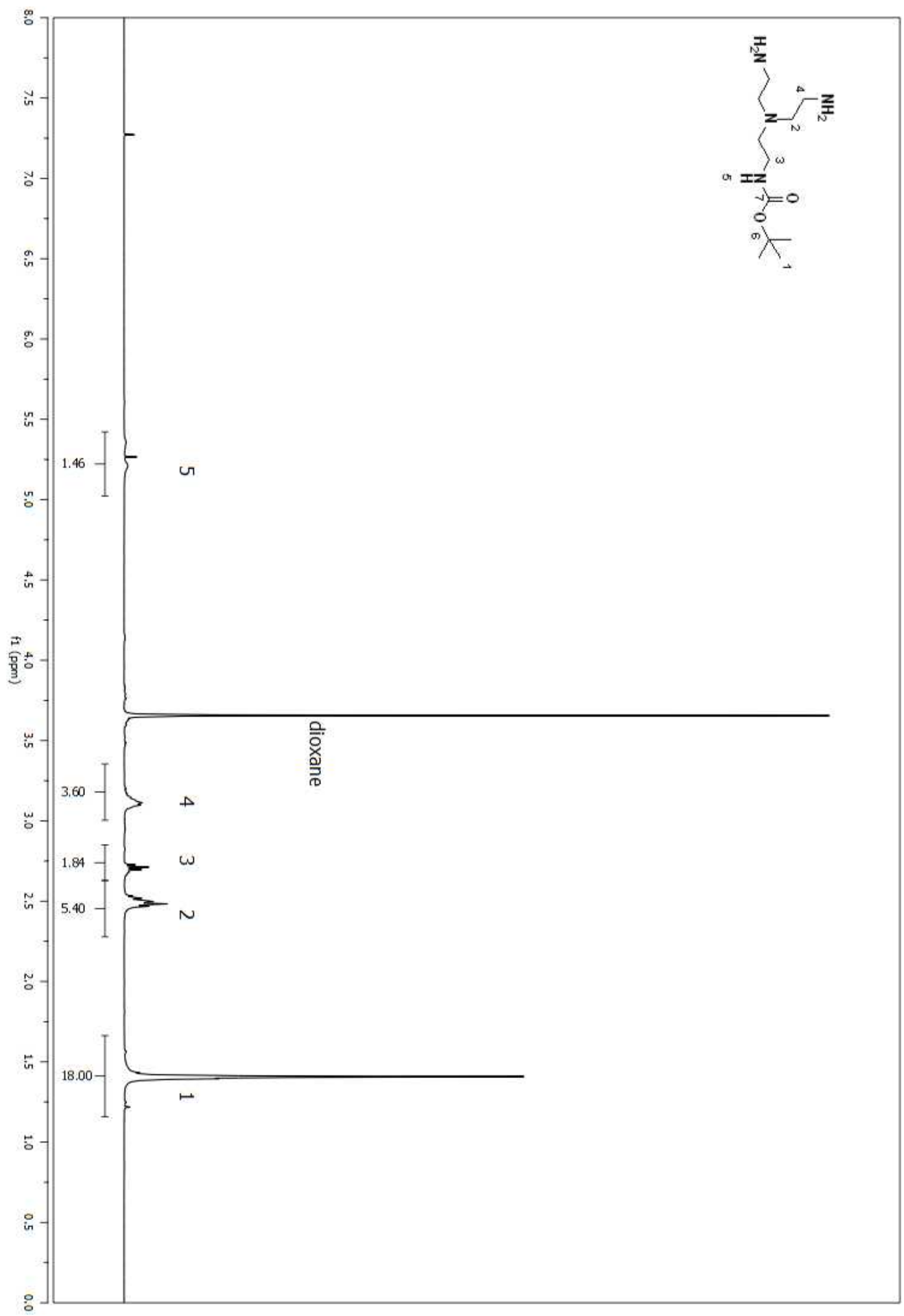

Figure A 307. ${ }^{1} \mathrm{H}$ NMR spectrum of compound (181) in $\mathrm{CDCl}_{3}$. 


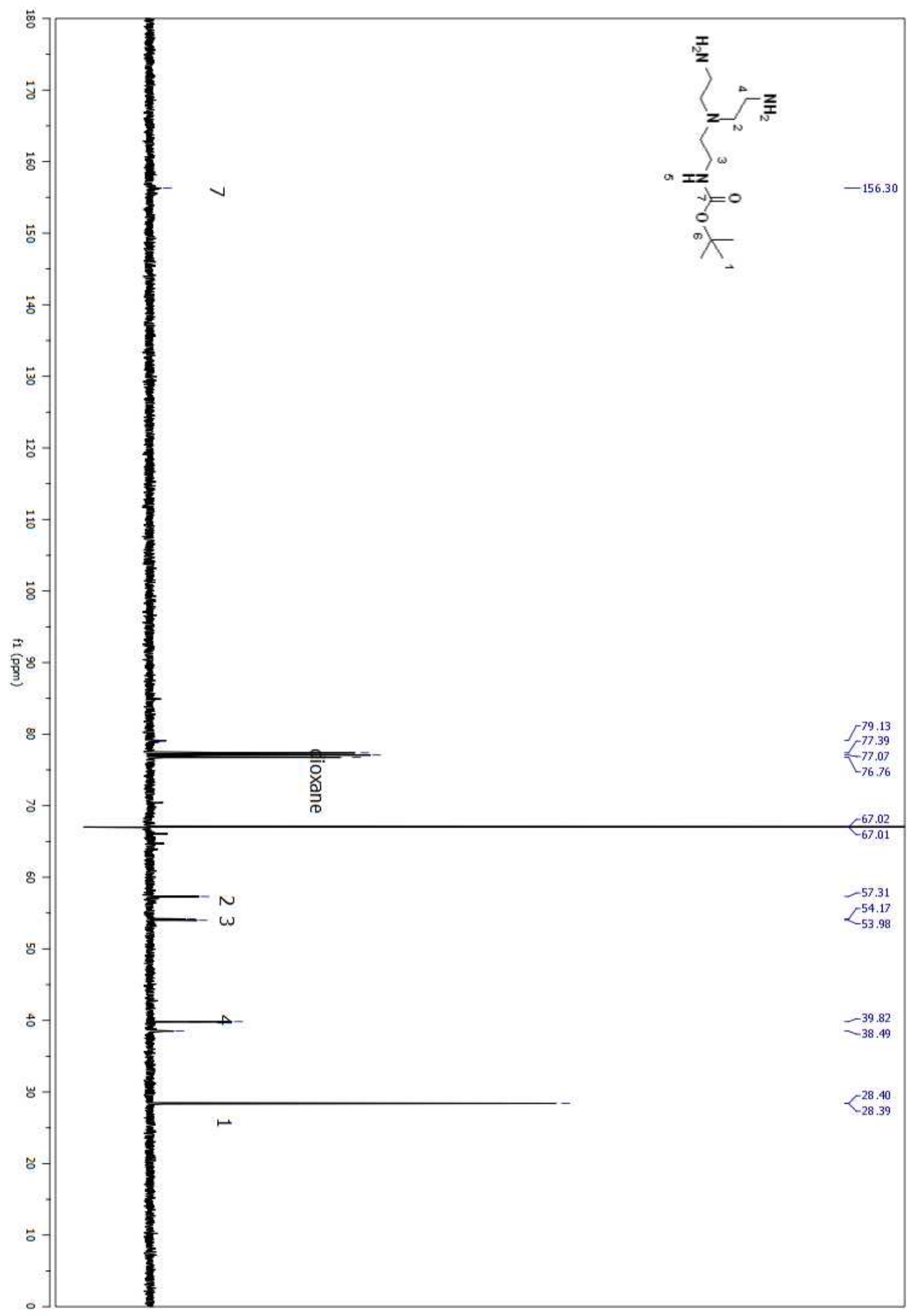

Figure A 308. ${ }^{13} \mathrm{C}$ NMR spectrum of compound (181) in $\mathrm{CDCl}_{3}$. 


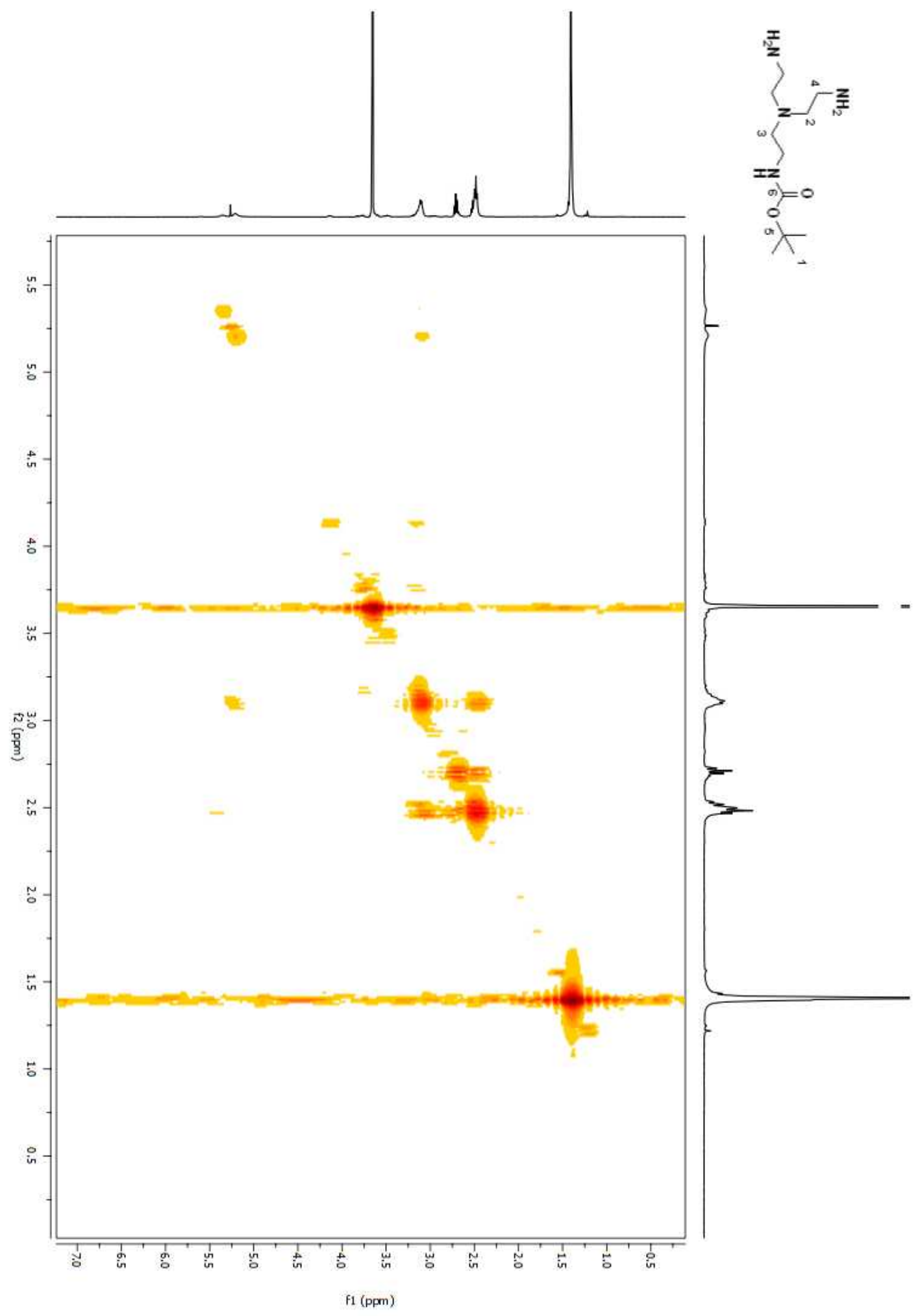

Figure A 309. COSY 2D NMR spectrum of compound (181) in $\mathrm{CDCl}_{3}$. 


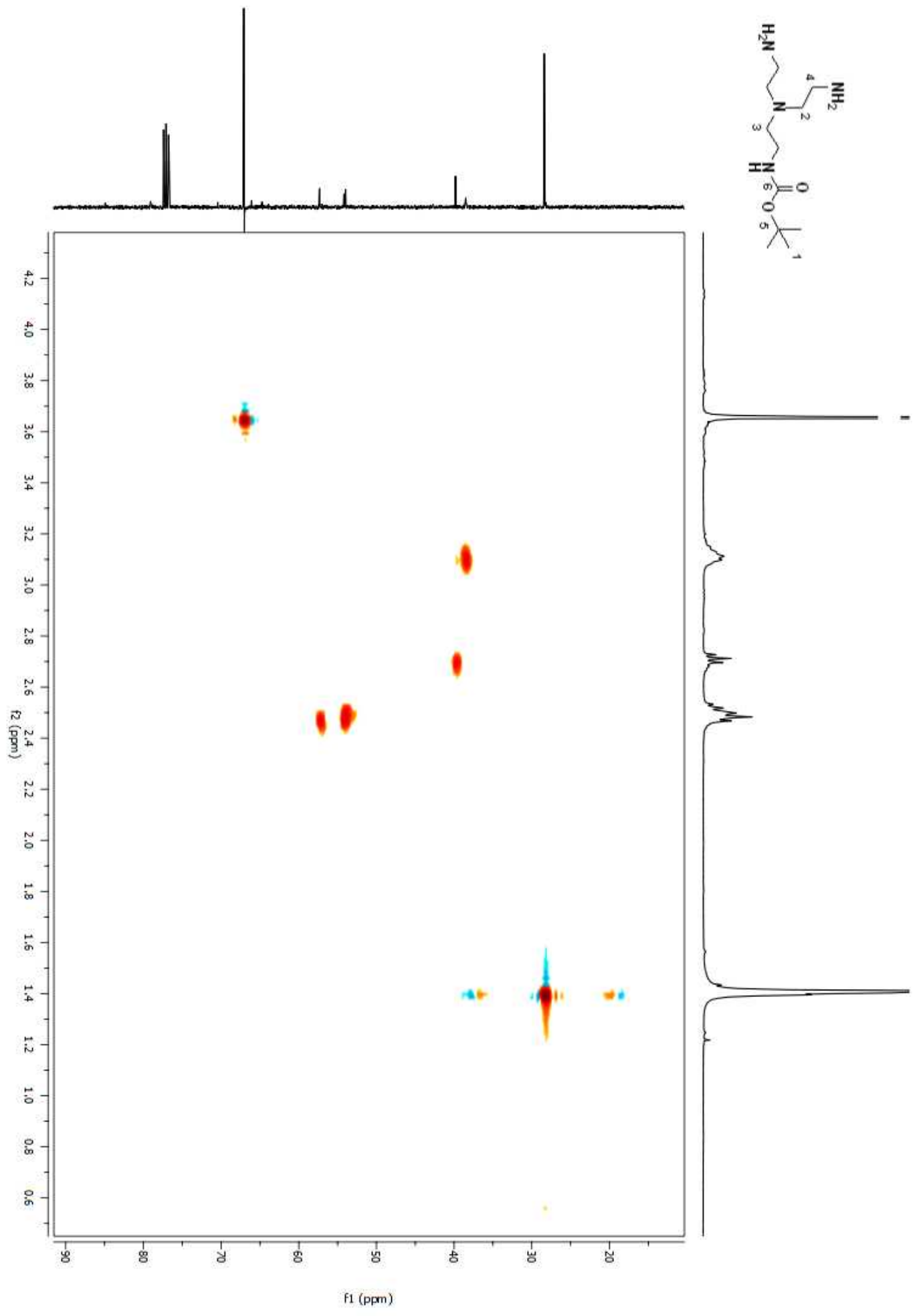

Figure A 310. HSQC 2D NMR spectrum of compound (181) in $\mathrm{CDCl}_{3}$. 


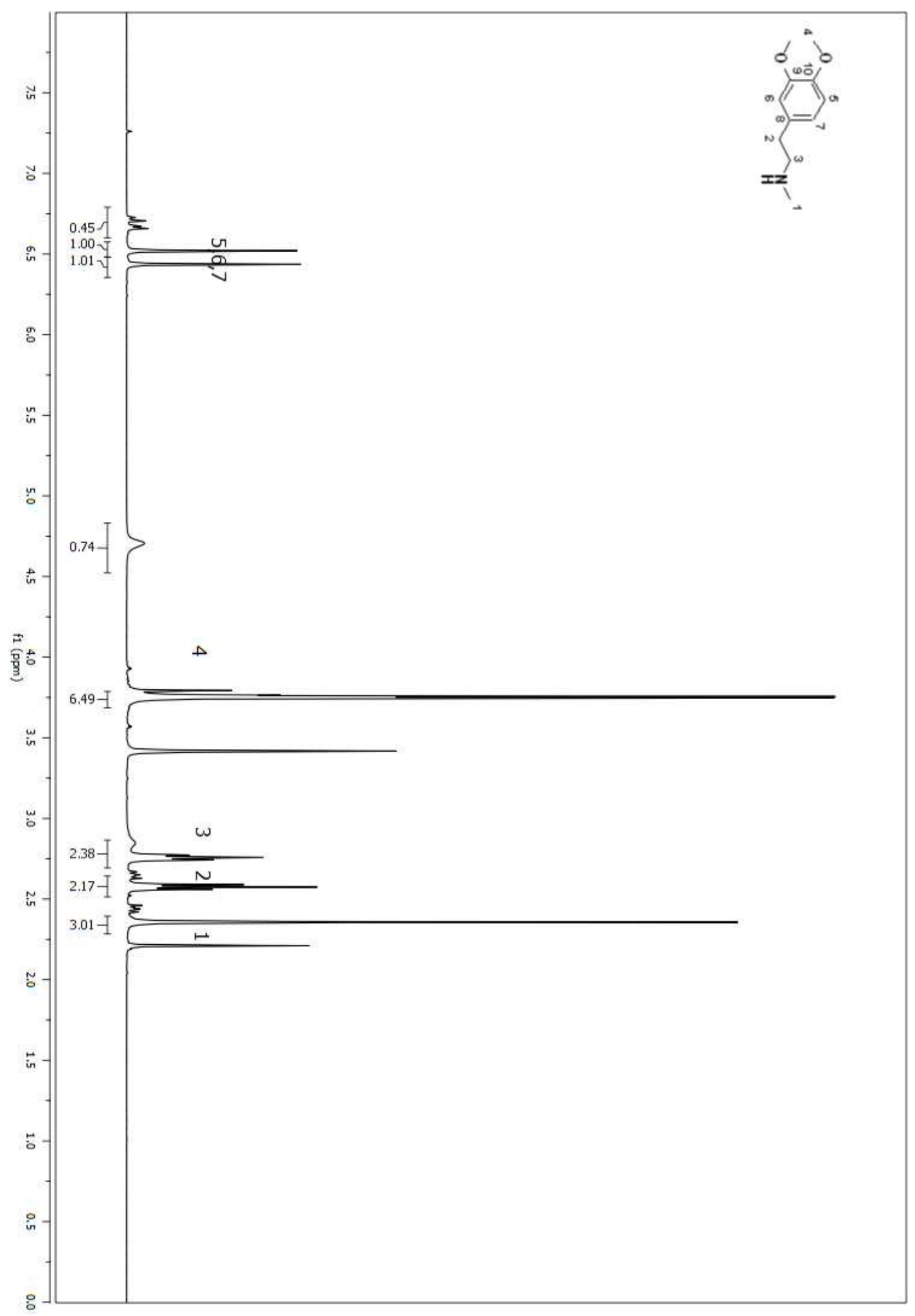

Figure A 311. ${ }^{1} \mathrm{H}$ NMR spectrum of compound (185) in $\mathrm{CDCl}_{3}$. (CRUDE) 


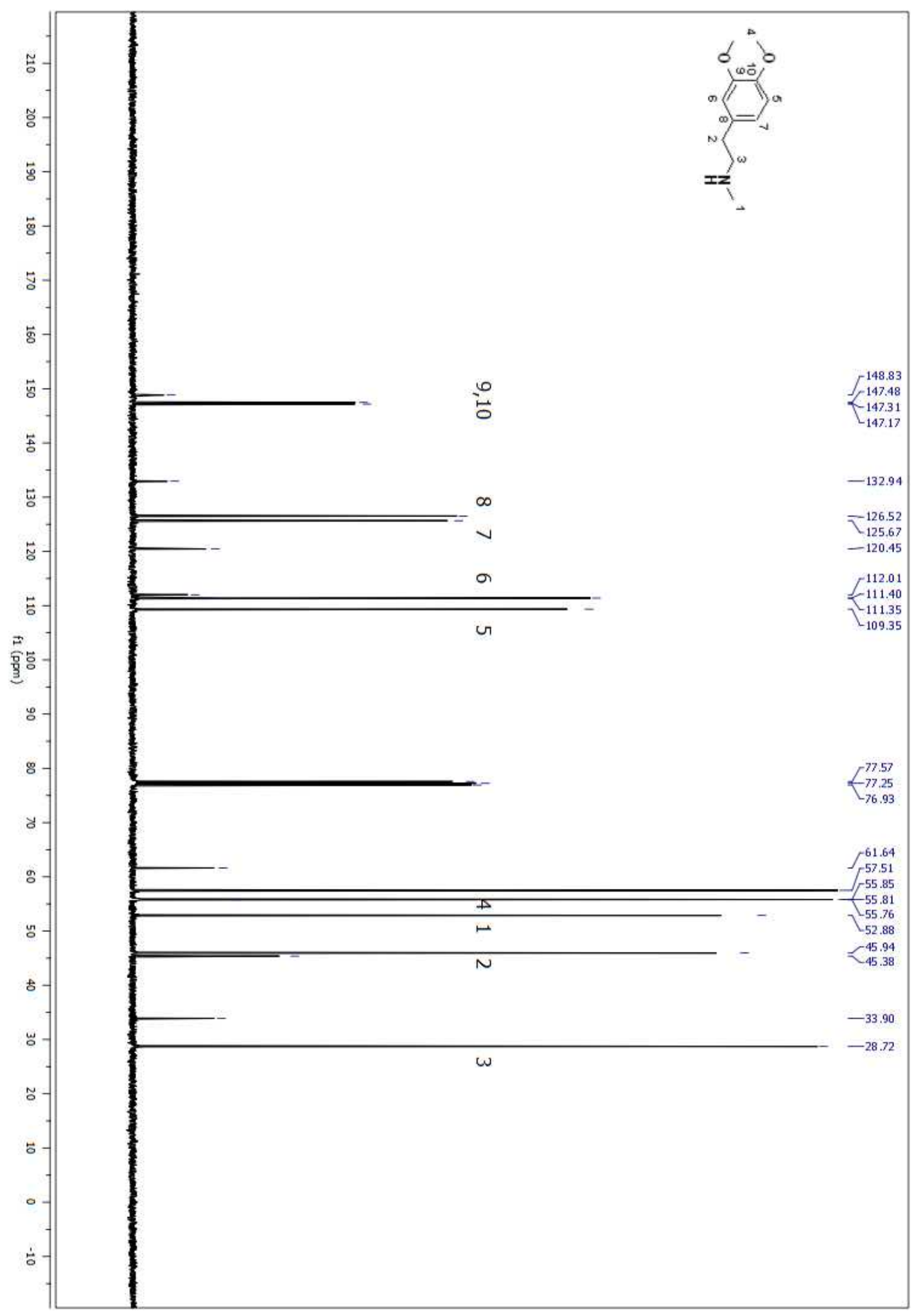

Figure A 312. ${ }^{13} \mathrm{C}$ NMR spectrum of compound (185) in $\mathrm{CDCl}_{3}$.(CRUDE) 


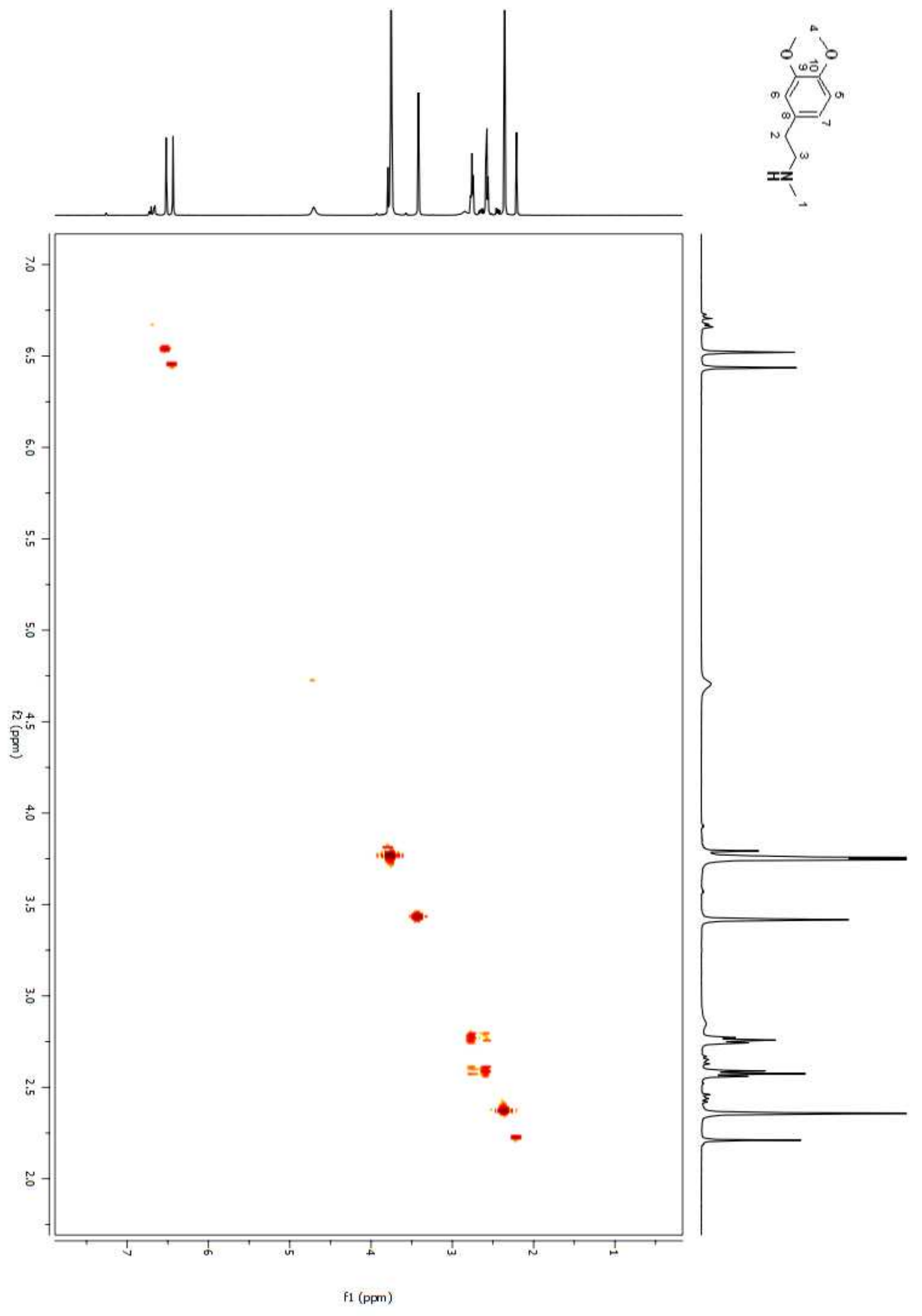

Figure A 313. COSY 2D NMR spectrum of compound (185) in $\mathrm{CDCl}_{3}$. 


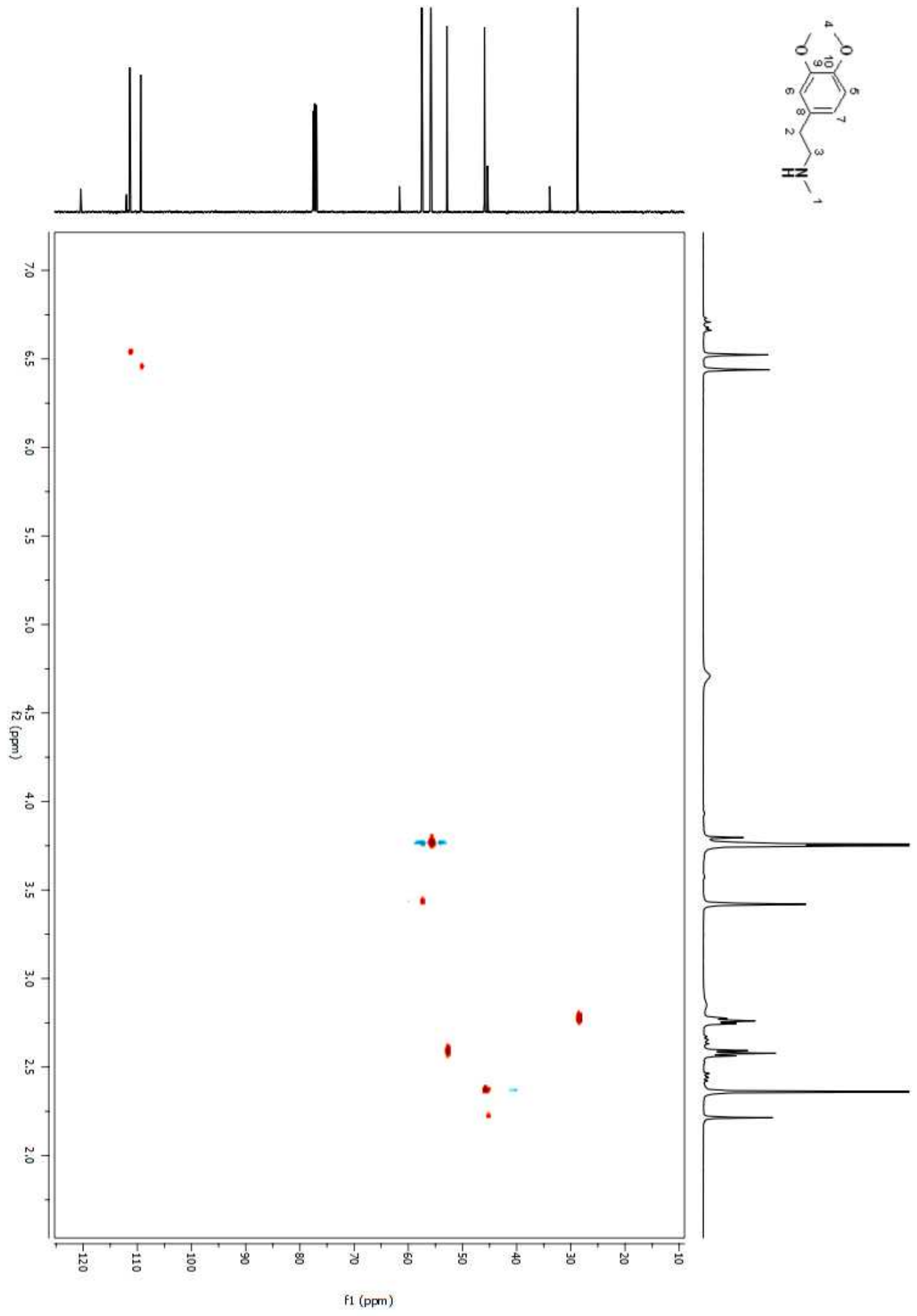

Figure A 314. HSQC 2D NMR spectrum of compound (185) in $\mathrm{CDCl}_{3}$. 


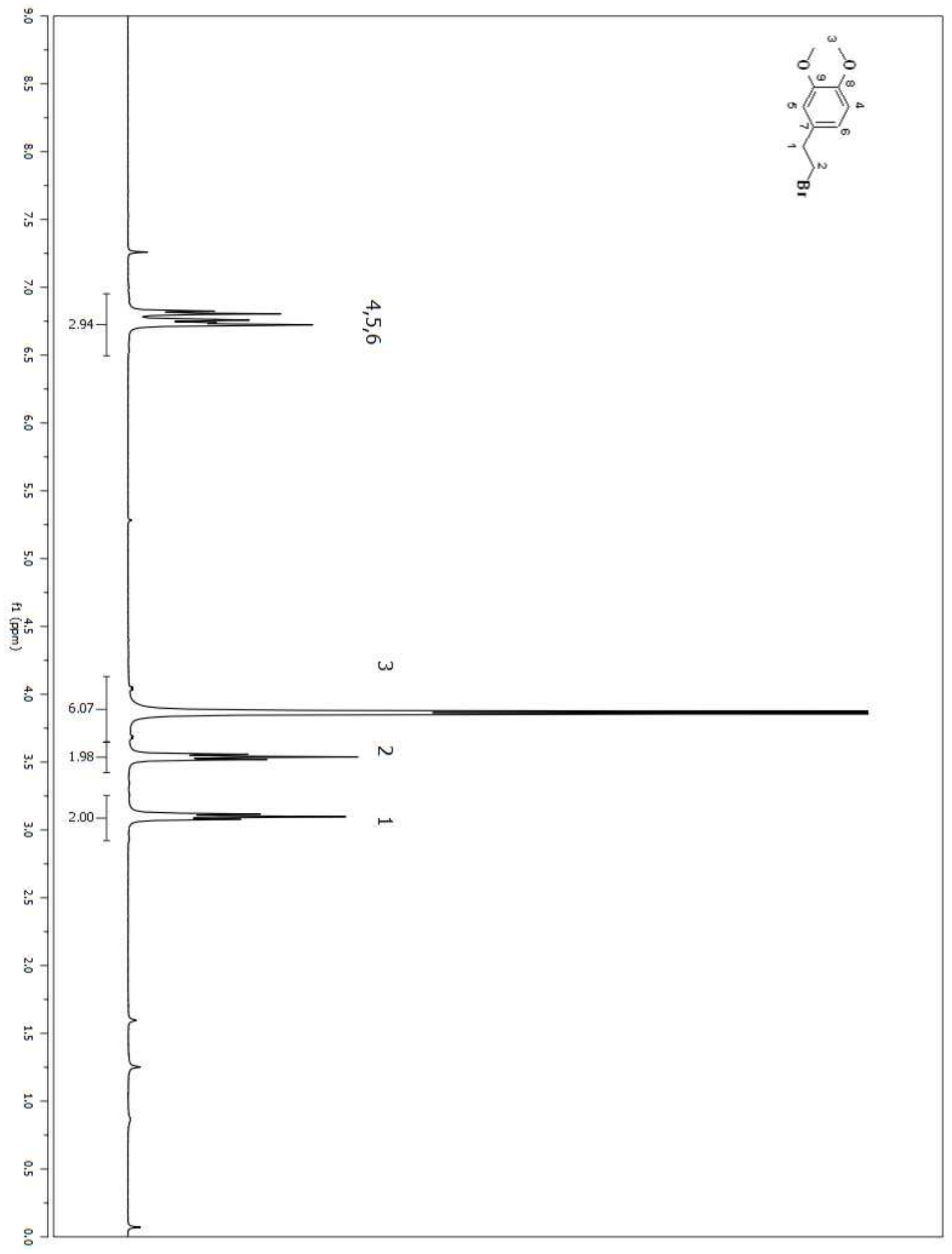

Figure A 315. ${ }^{1} \mathrm{H}$ NMR spectrum of compound (188) in $\mathrm{CDCl}_{3}$. 


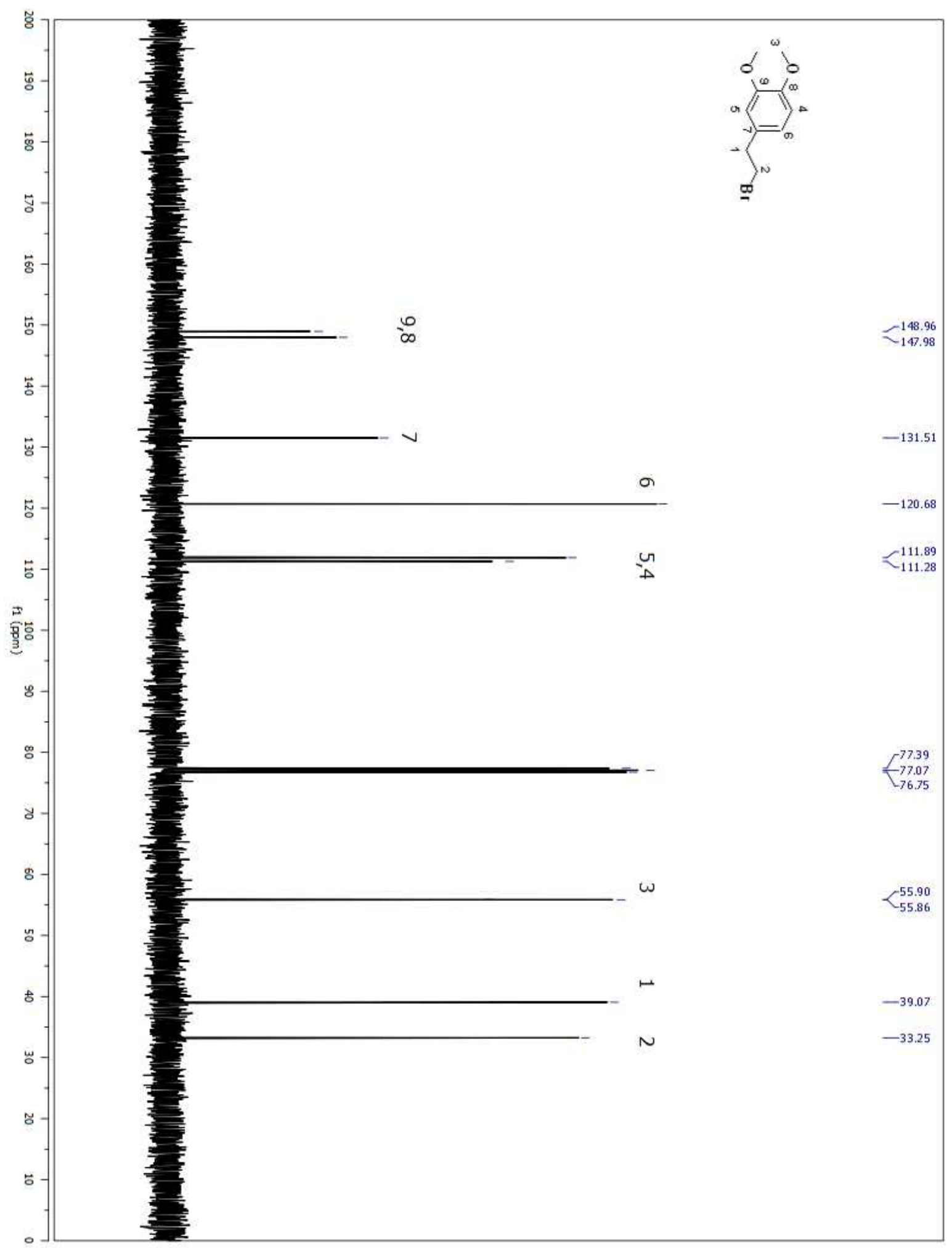

Figure A 316. ${ }^{13} \mathrm{C}$ NMR spectrum of compound (188) in $\mathrm{CDCl}_{3}$. 


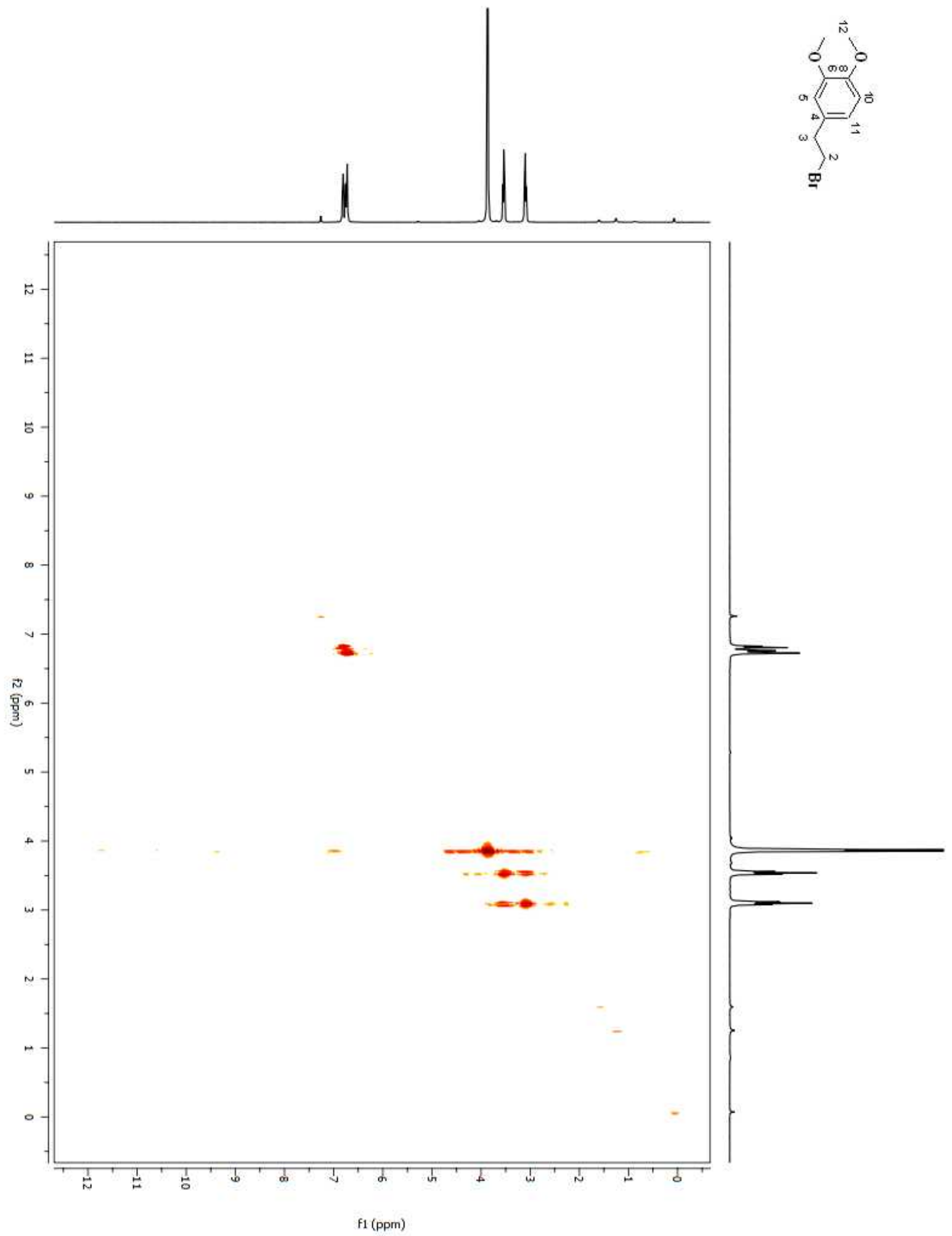

Figure A 317. COSY 2D NMR spectrum of compound (188) in $\mathrm{CDCl}_{3}$. 


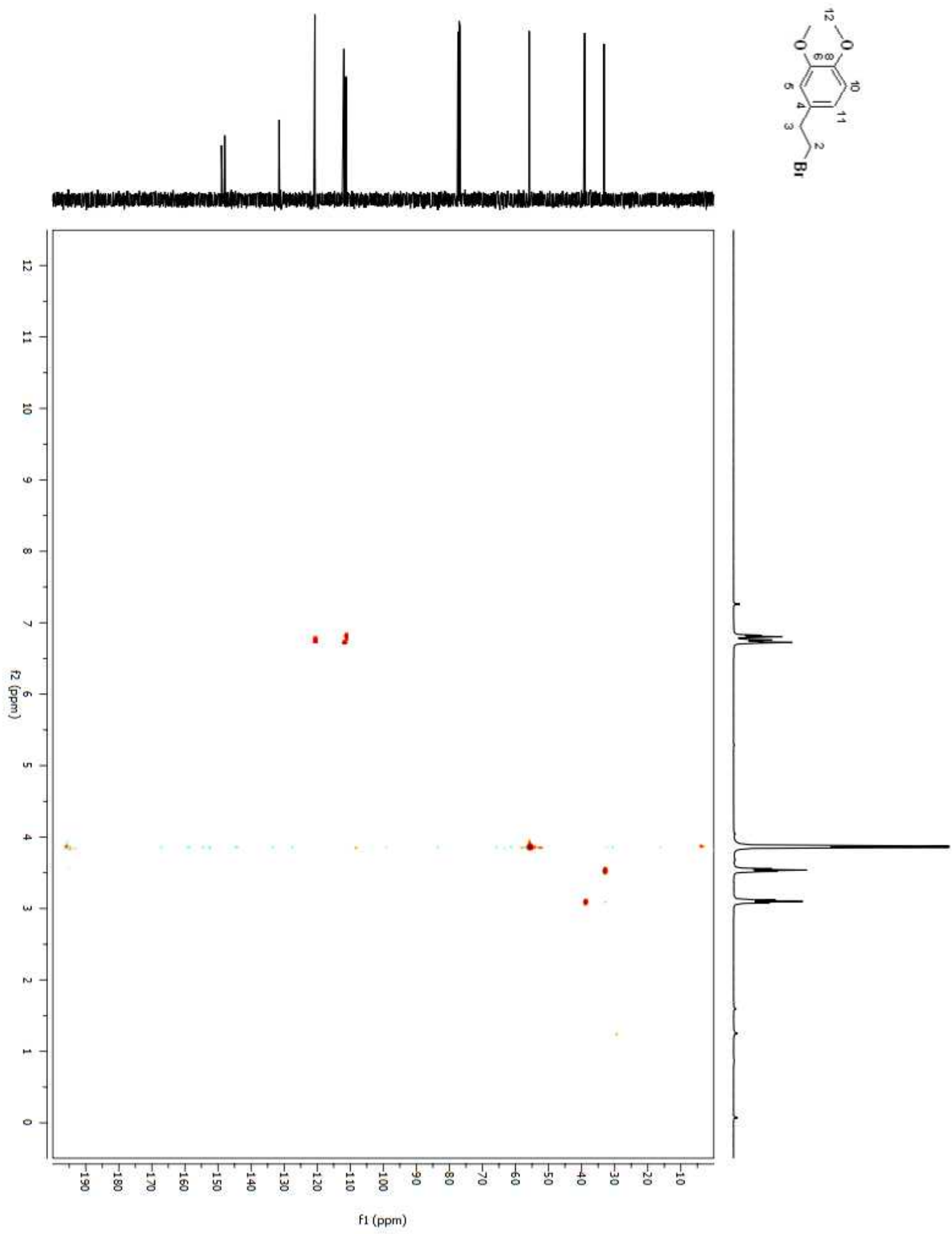

Figure A 318. HSQC 2D NMR spectrum of compound (188) in $\mathrm{CDCl}_{3}$. 


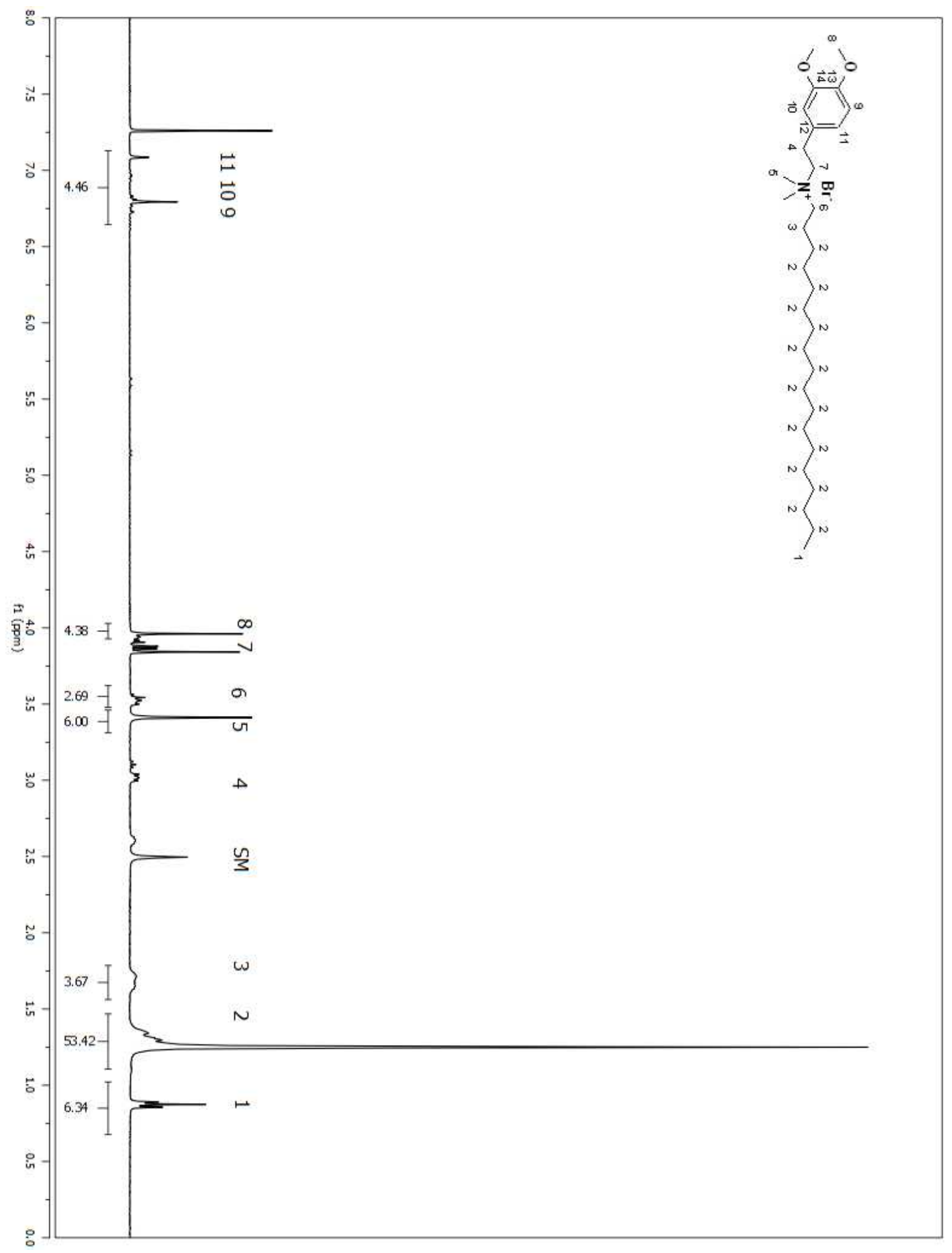

Figure A 319. ${ }^{1} \mathrm{H}$ NMR spectrum of compound (189) in $\mathrm{CDCL}_{3}$.(CRUDE) 


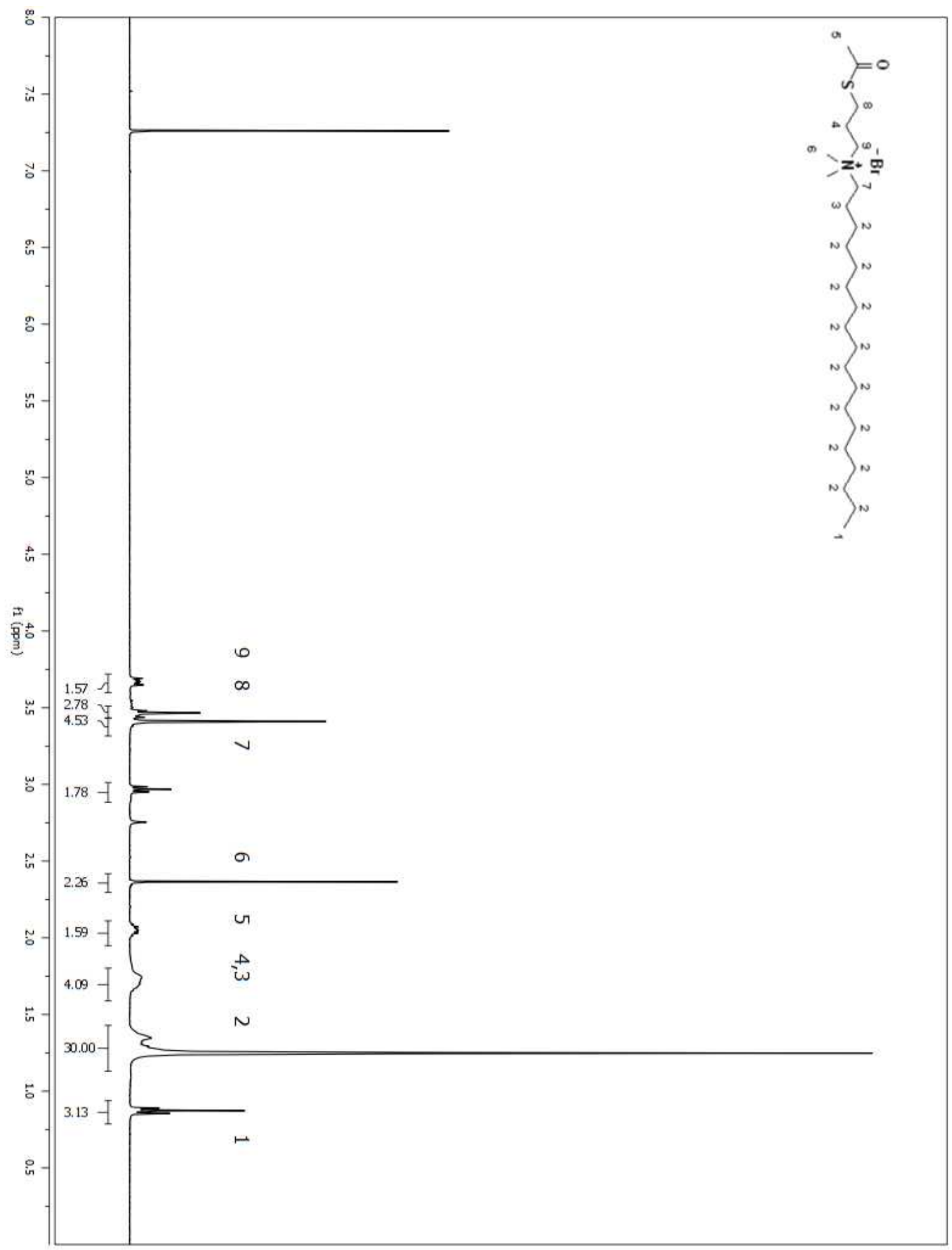

Figure A 320. ${ }^{1} \mathrm{H}$ NMR spectrum of compound (192) in $\mathrm{CDCl}_{3}$. 


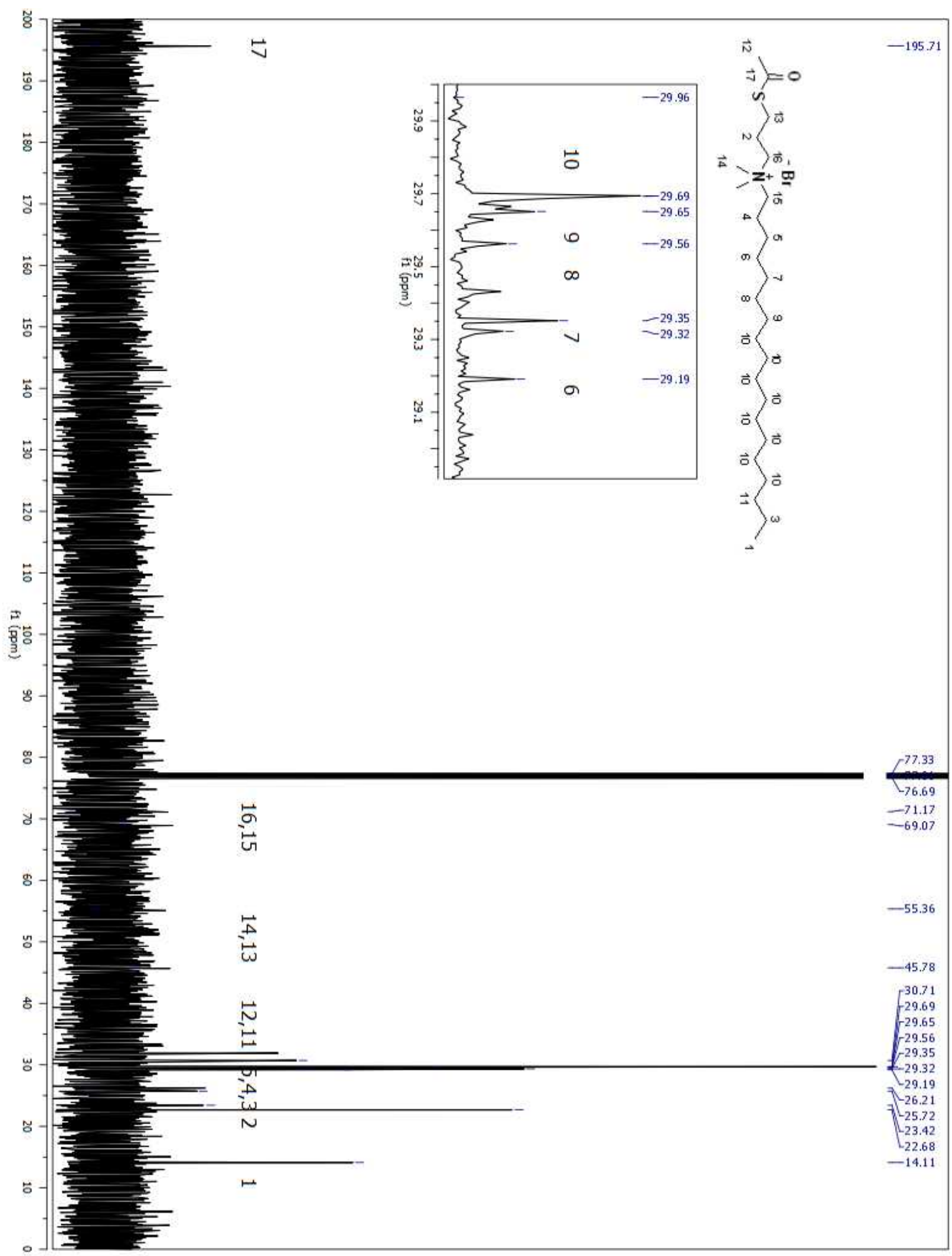

Figure A 321. ${ }^{13} \mathrm{C}$ NMR spectrum of compound (192) in $\mathrm{CDCl}_{3}$. 


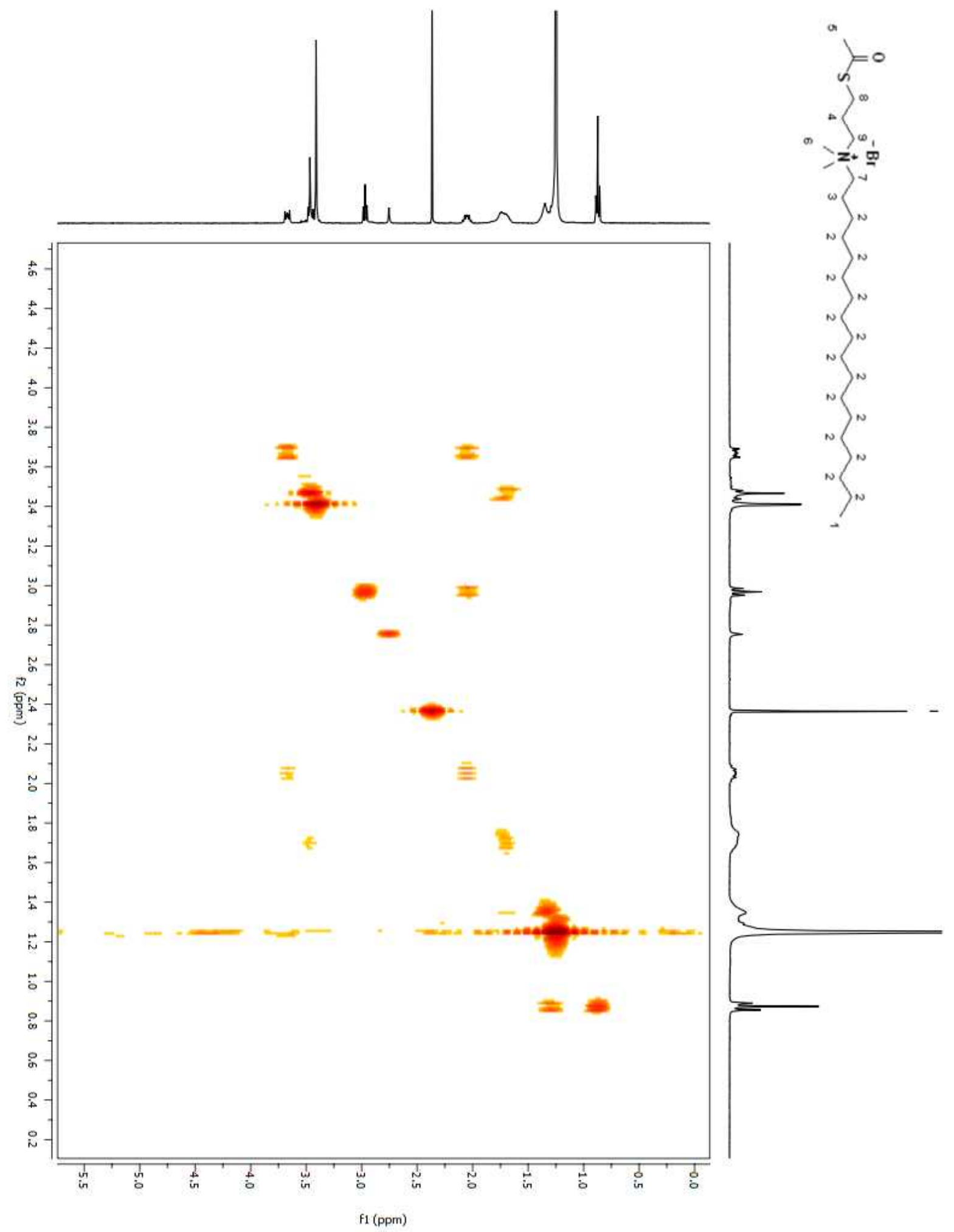

Figure A 322. COSY 2D NMR spectrum of compound (192) in $\mathrm{CDCl}_{3}$. 


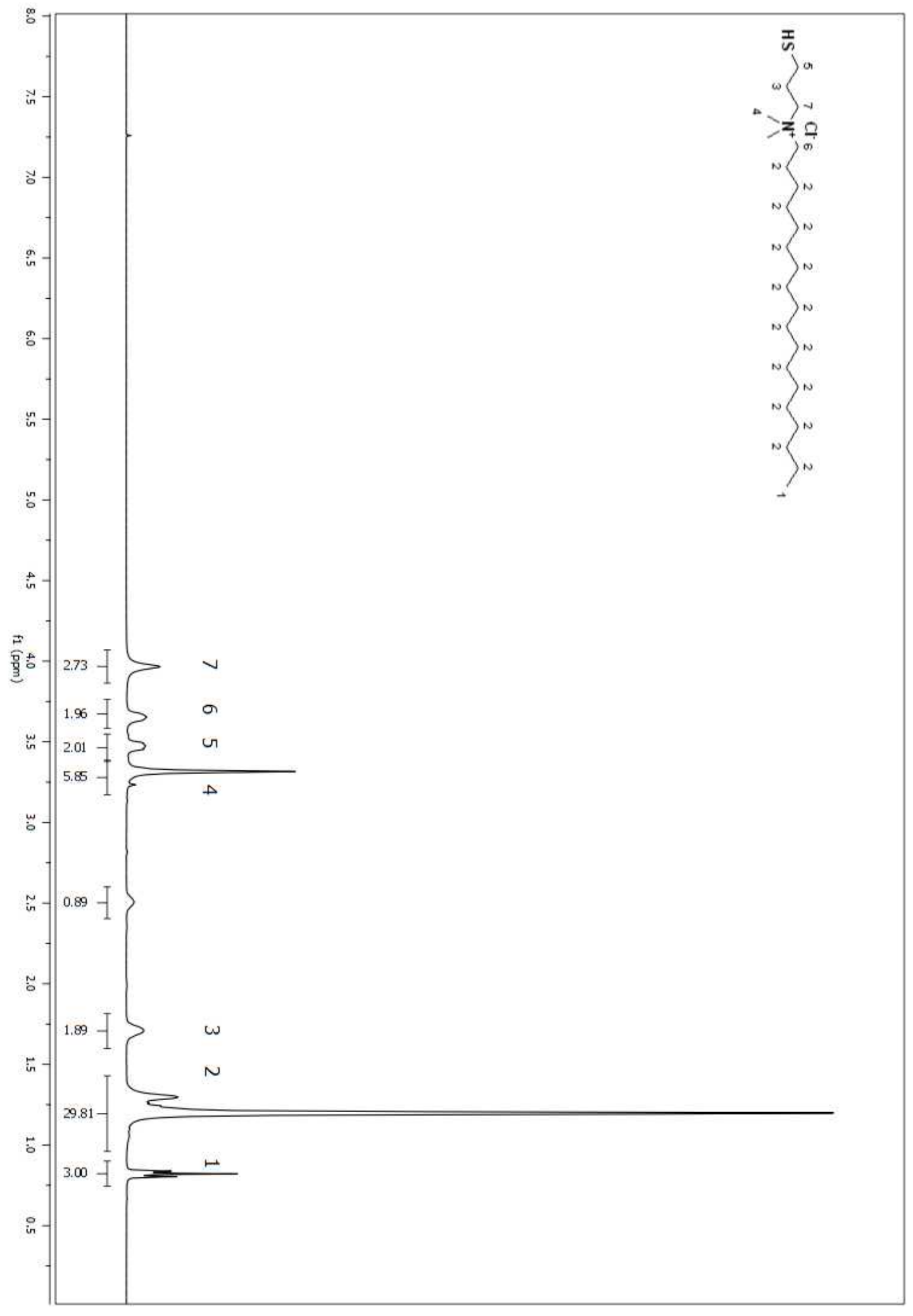

Figure A 323. ${ }^{1} \mathrm{H}$ NMR spectrum of compound (194) in $\mathrm{CDCl}_{3}$. 


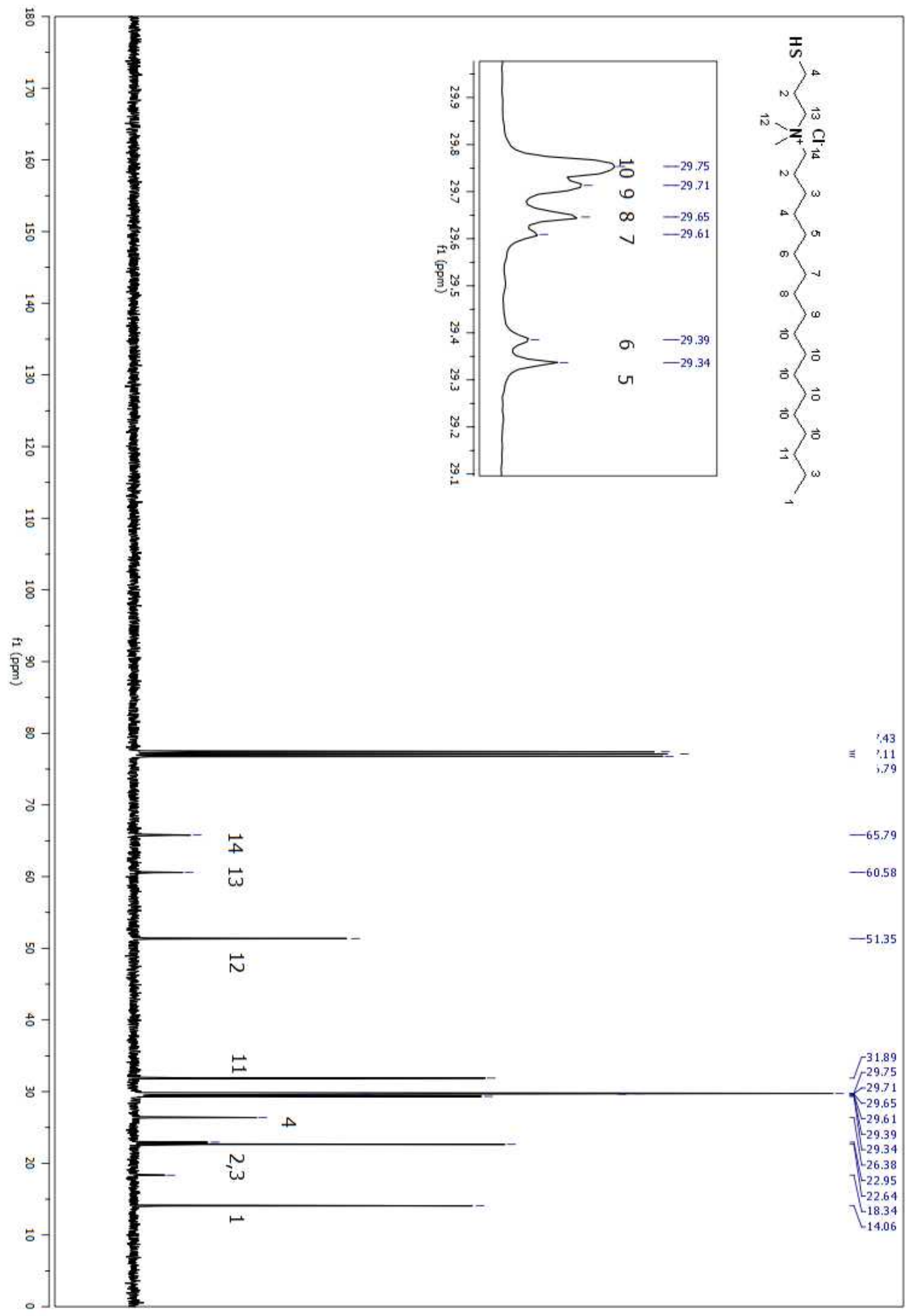

Figure A 324. ${ }^{1} \mathrm{H}$ NMR spectrum of compound (194) in $\mathrm{CDCl}_{3}$. 


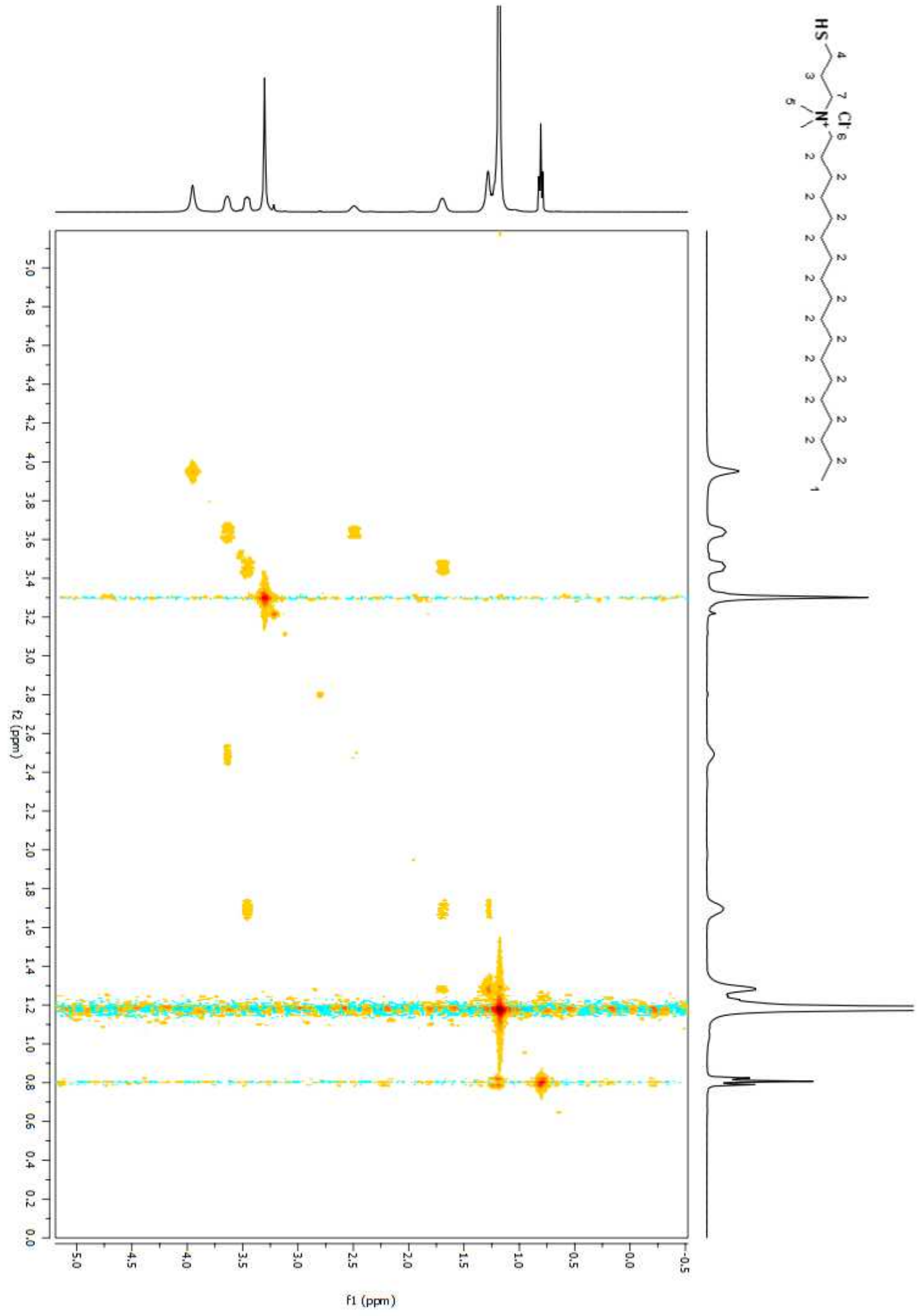

Figure A 325. COSY 2D NMR spectrum of compound (194) in $\mathrm{CDCl}_{3}$. 


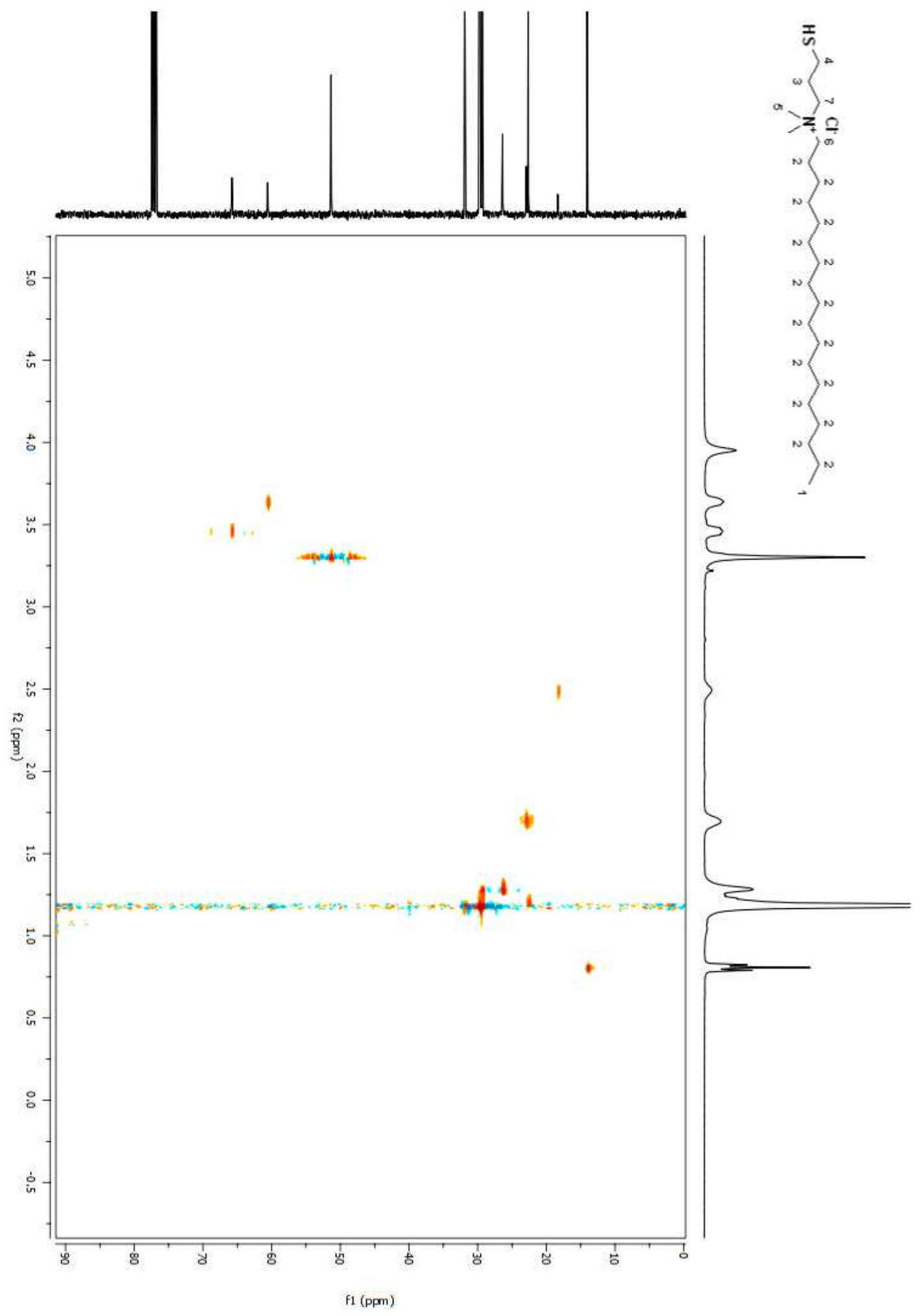

Figure A 326. HSQC 2D NMR spectrum of compound (194) in $\mathrm{CDCl}_{3}$. 


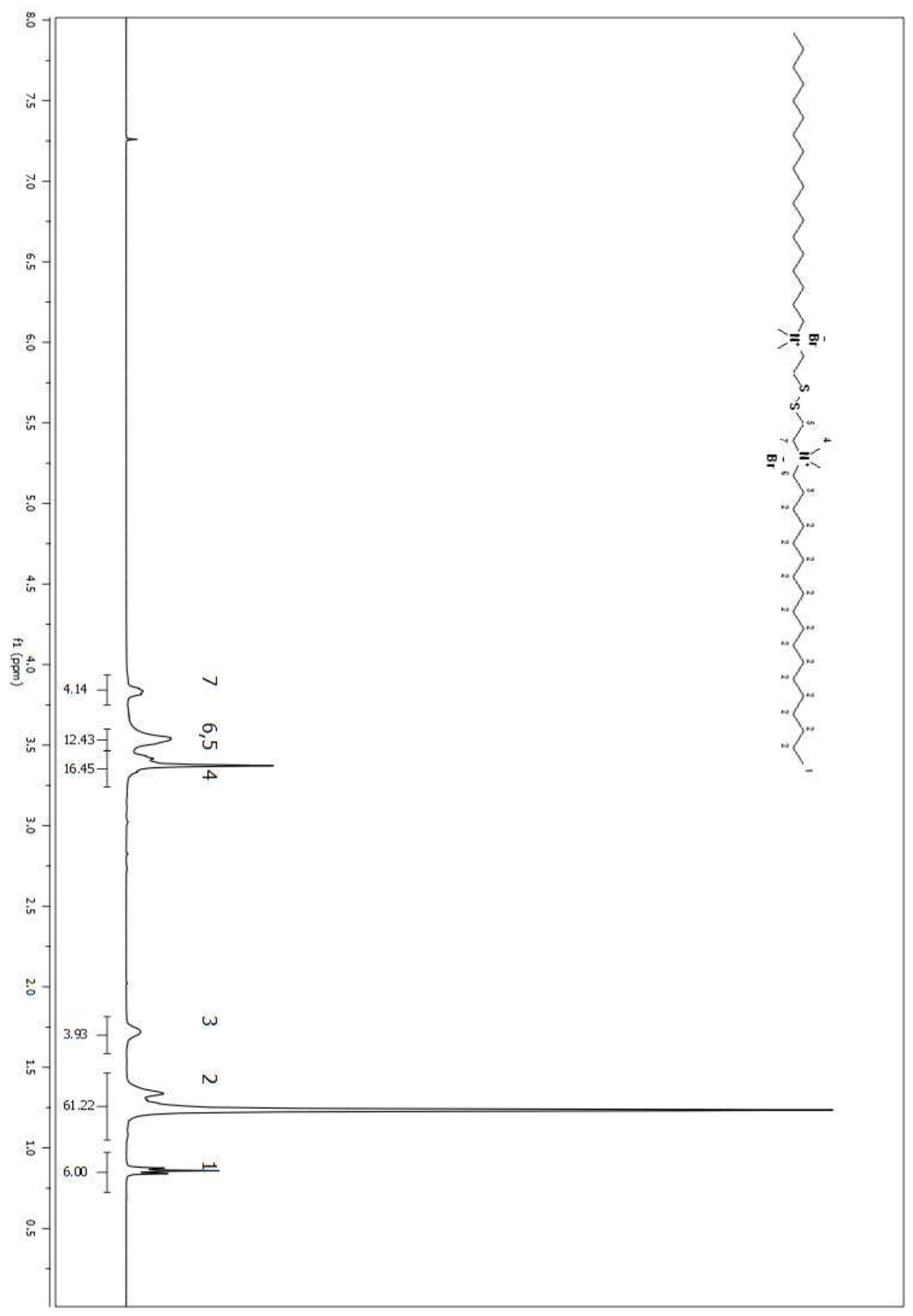

Figure A 327. ${ }^{1} \mathrm{H}$ NMR spectrum of compound (198) in $\mathrm{CDCl}_{3}$. 


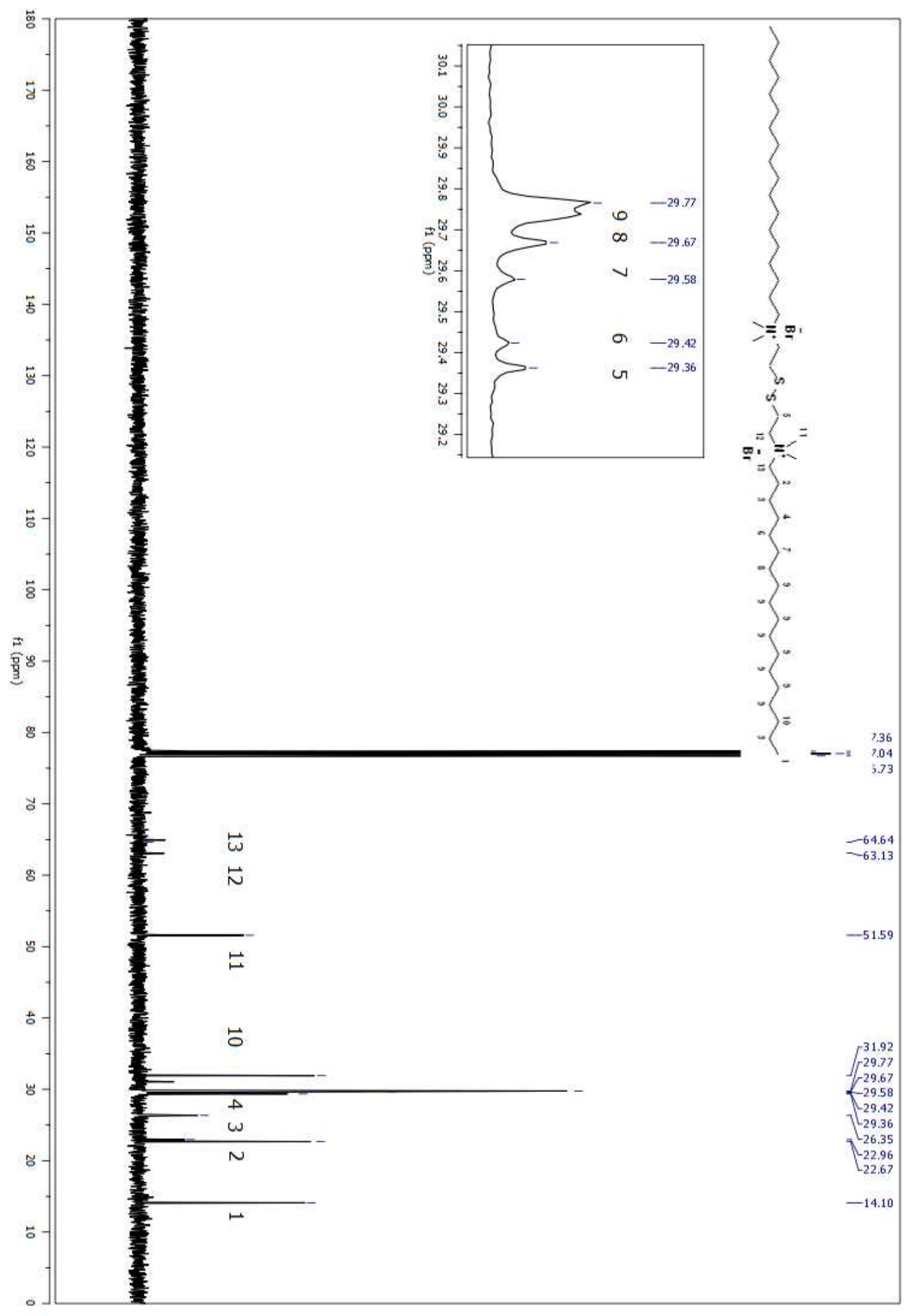

Figure A 328. ${ }^{13} \mathrm{C}$ NMR spectrum of compound (198) in $\mathrm{CDCl}_{3}$. 


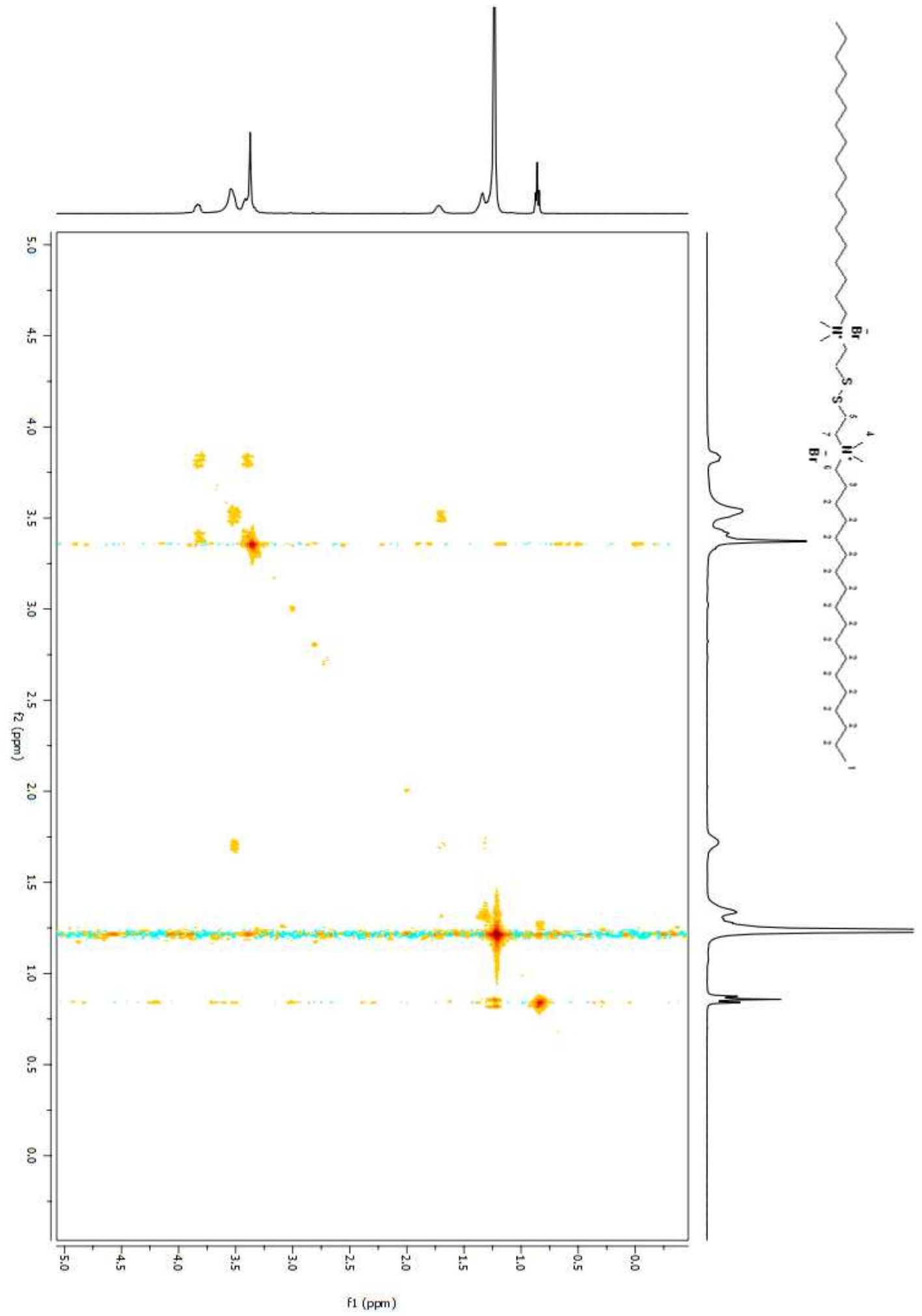

Figure A 329. COSY 2D NMR spectrum of compound (198) in $\mathrm{CDCl}_{3}$. 


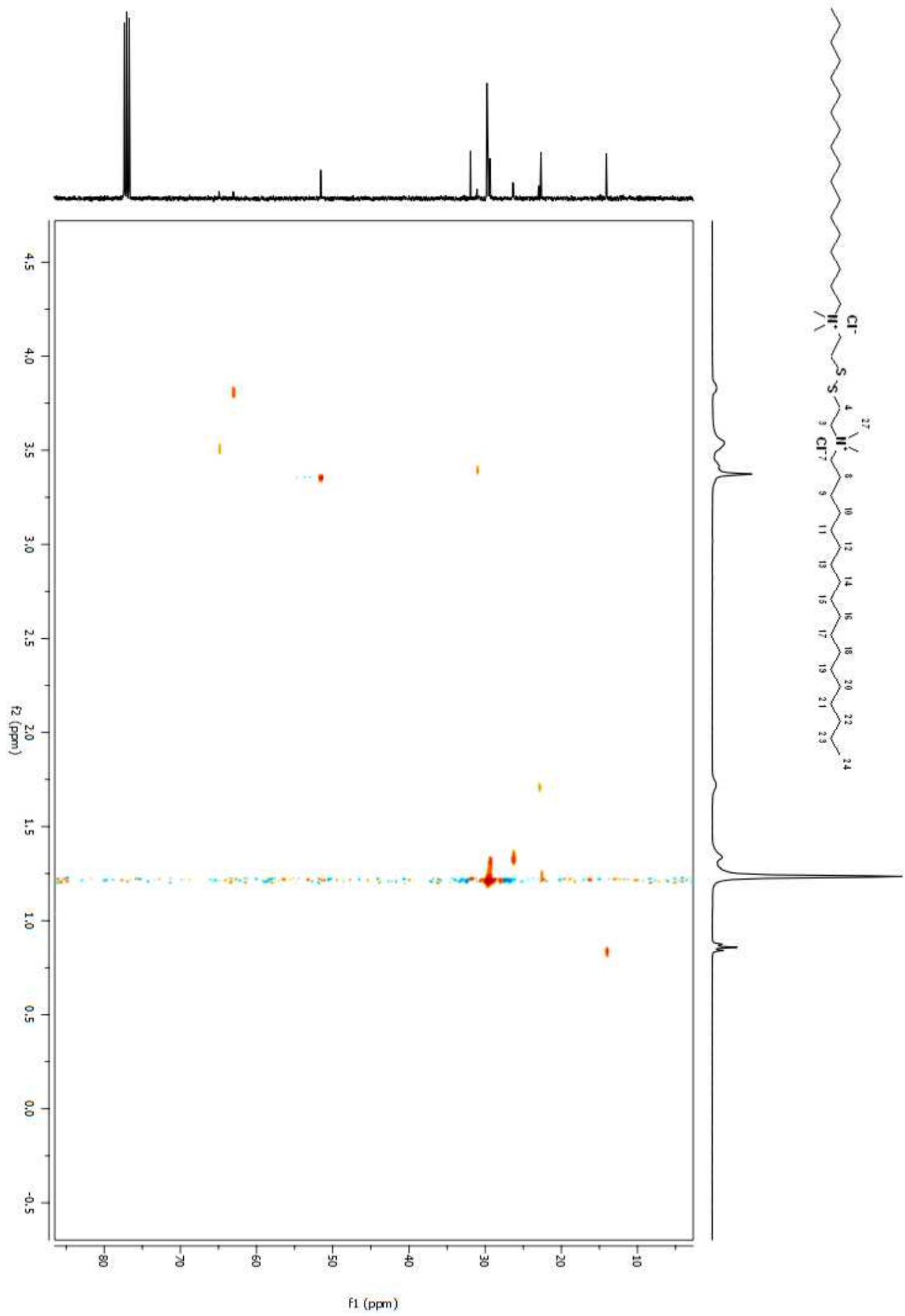

Figure A 330. HSQC 2D NMR spectrum of compound (198) in $\mathrm{CDCl}_{3}$. 


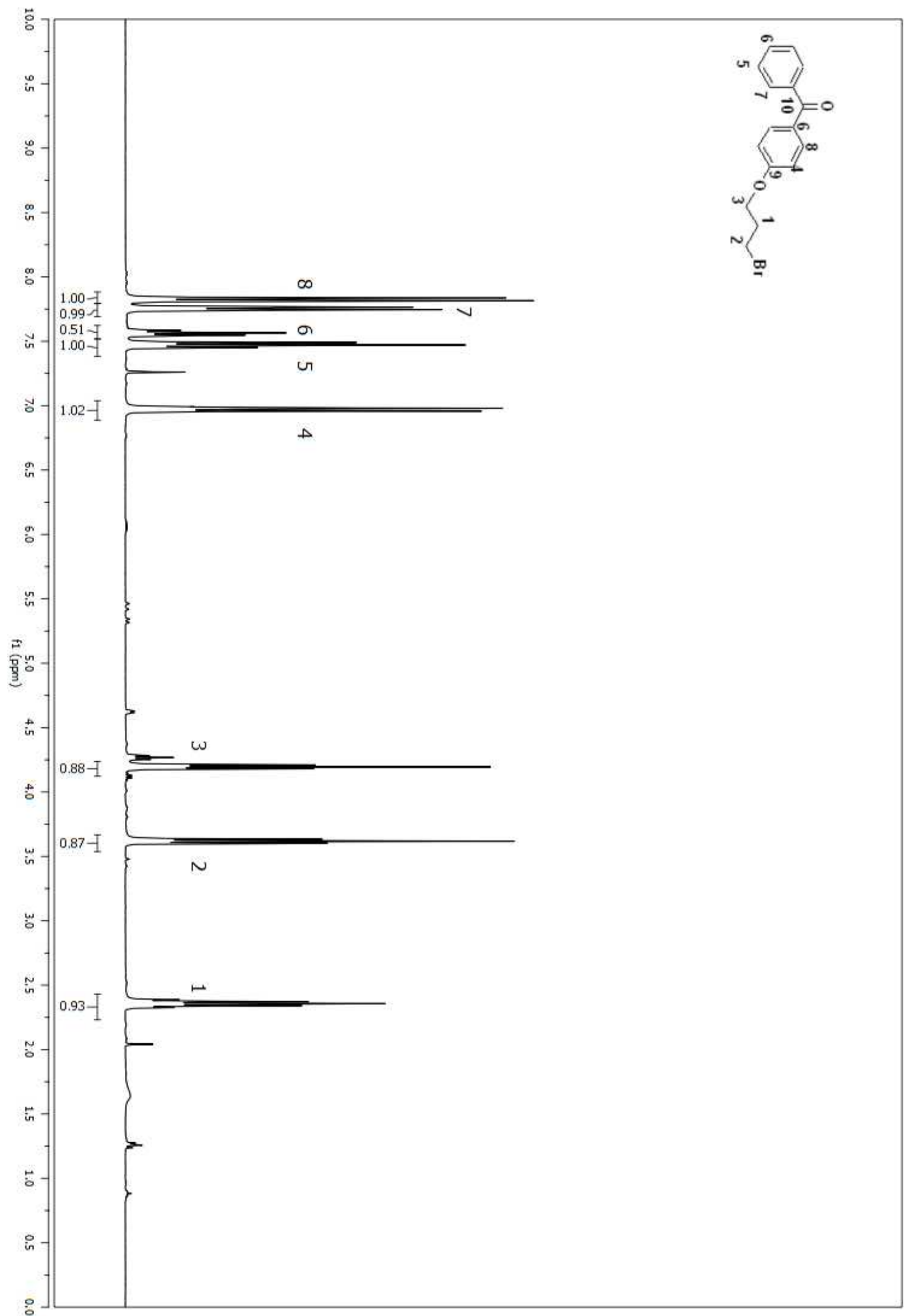

Figure A 331. ${ }^{1} \mathrm{H}$ NMR spectrum of compound (202) in $\mathrm{CDCl}_{3}$. 


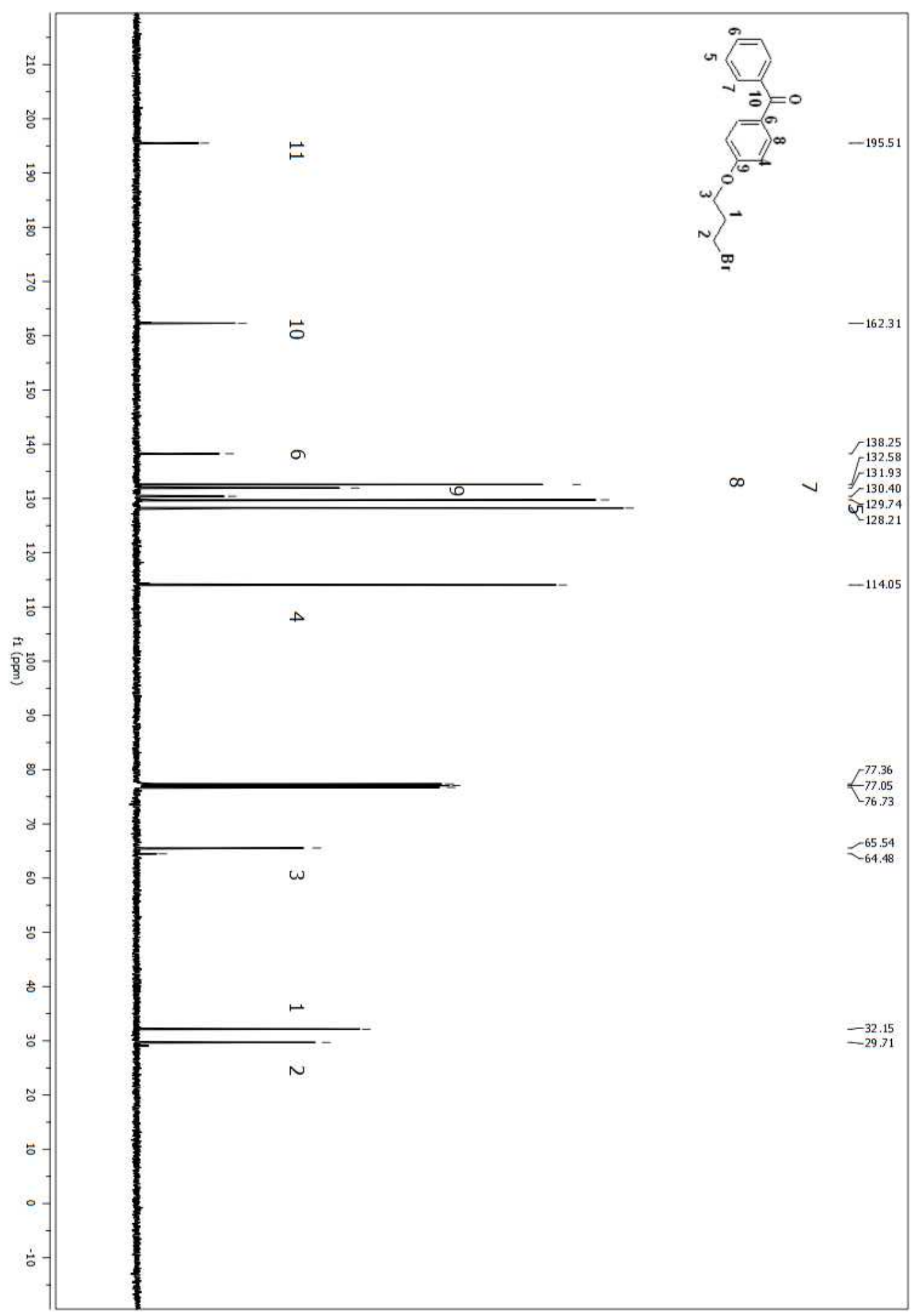

Figure A 332. ${ }^{13} \mathrm{C}$ NMR spectrum of compound (202) in $\mathrm{CDCl}_{3}$. 


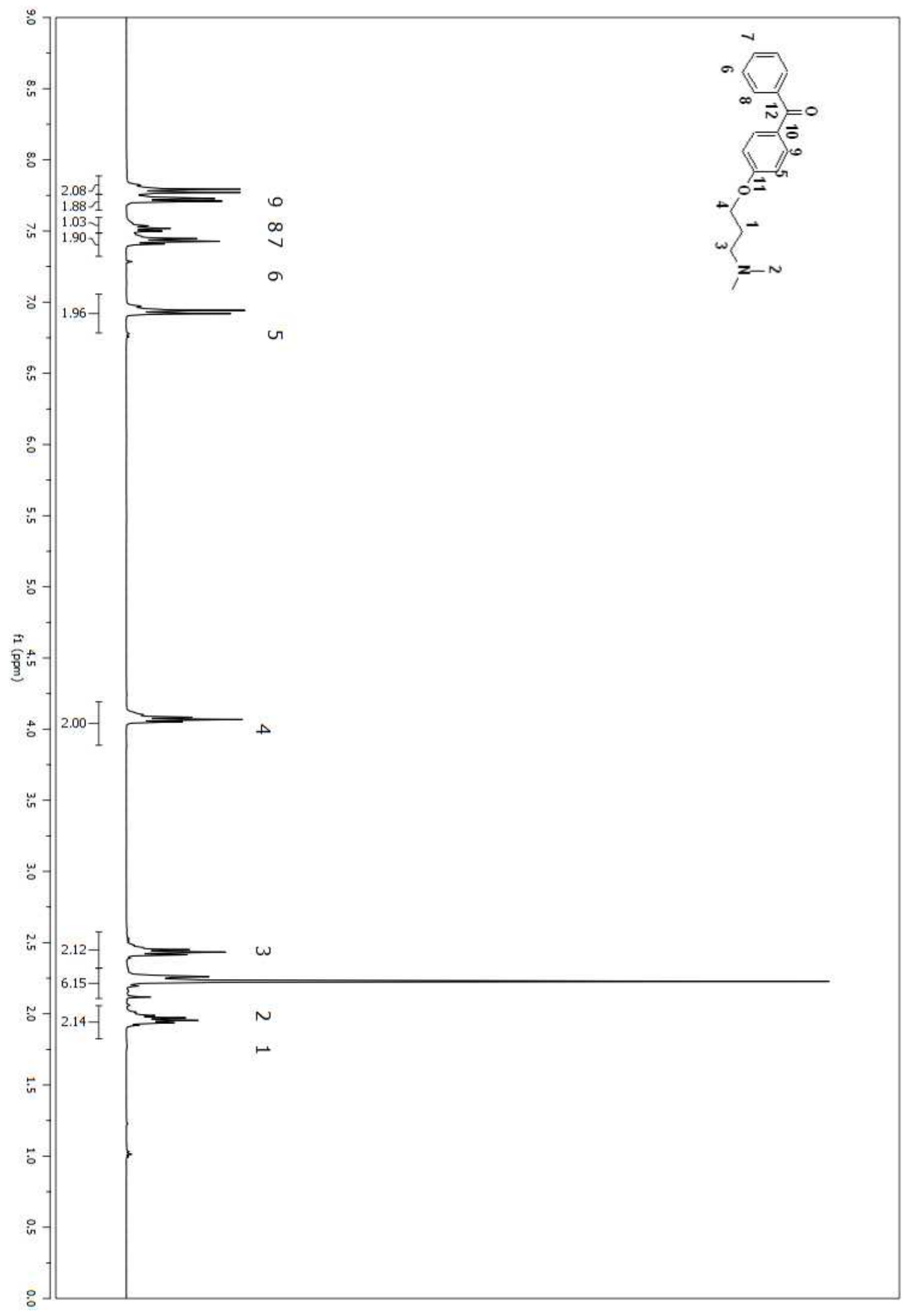

Figure A 333. ${ }^{1} \mathrm{H}$ NMR spectrum of compound (204) in $\mathrm{CDCl}_{3}$. 


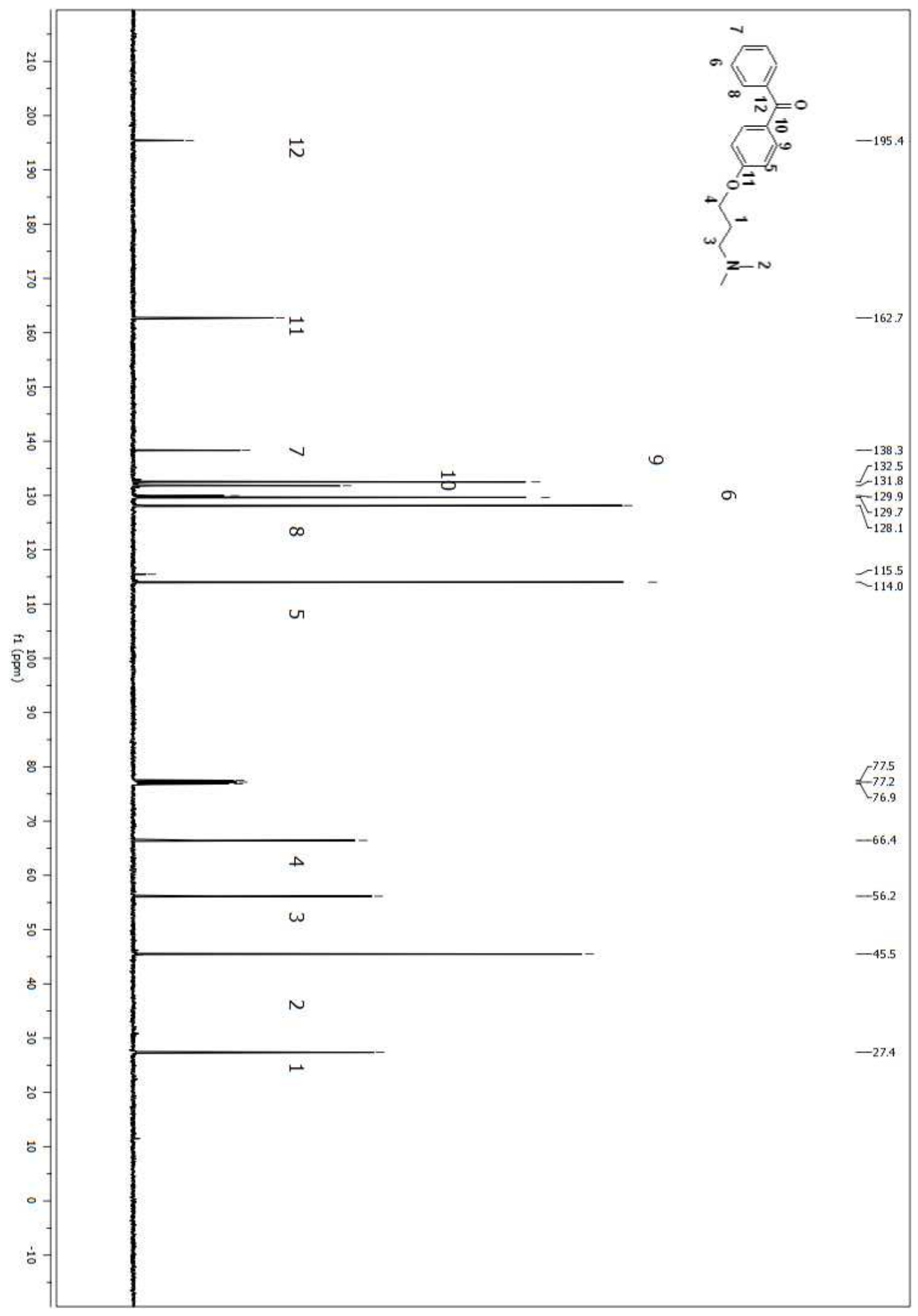

Figure A 334. ${ }^{13} \mathrm{C}$ NMR spectrum of compound (204) in $\mathrm{CDCl}_{3}$. 


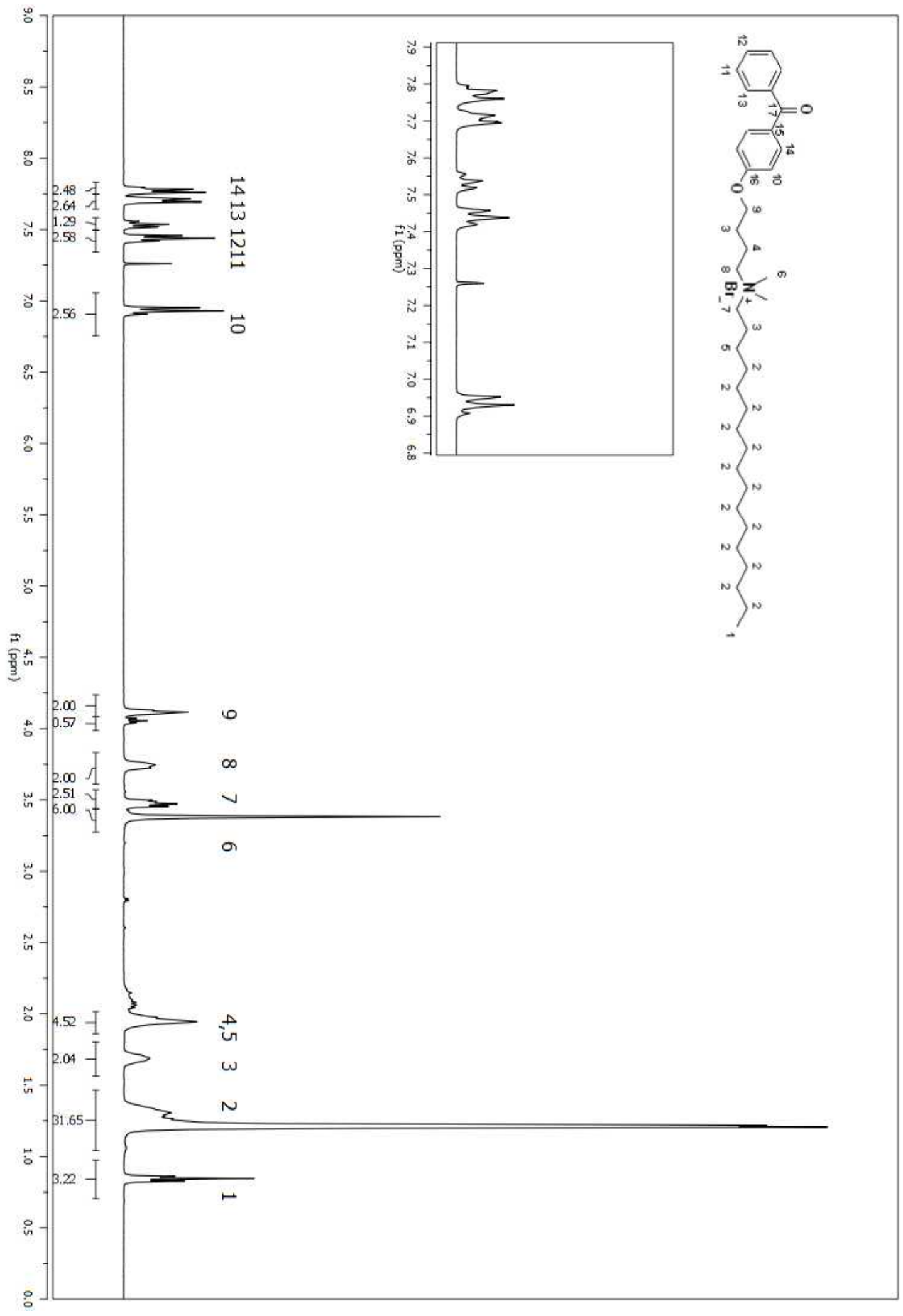

Figure A 335. ${ }^{1} \mathrm{H}$ NMR spectrum of compound (205) in $\mathrm{CDCl}_{3}$. 


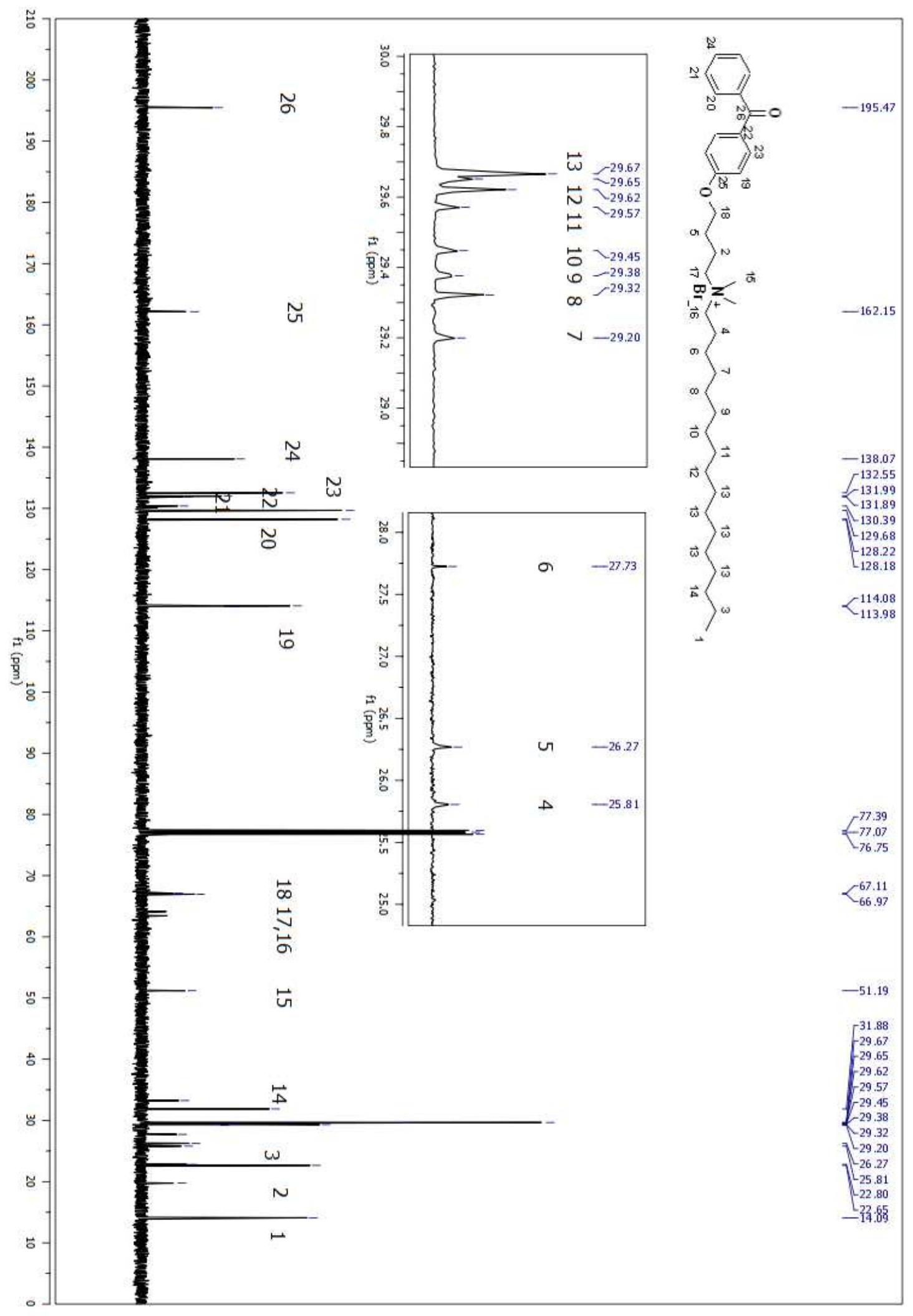

Figure A 336. ${ }^{13} \mathrm{C}$ NMR spectrum of compound (205) in $\mathrm{CDCl}_{3}$. 


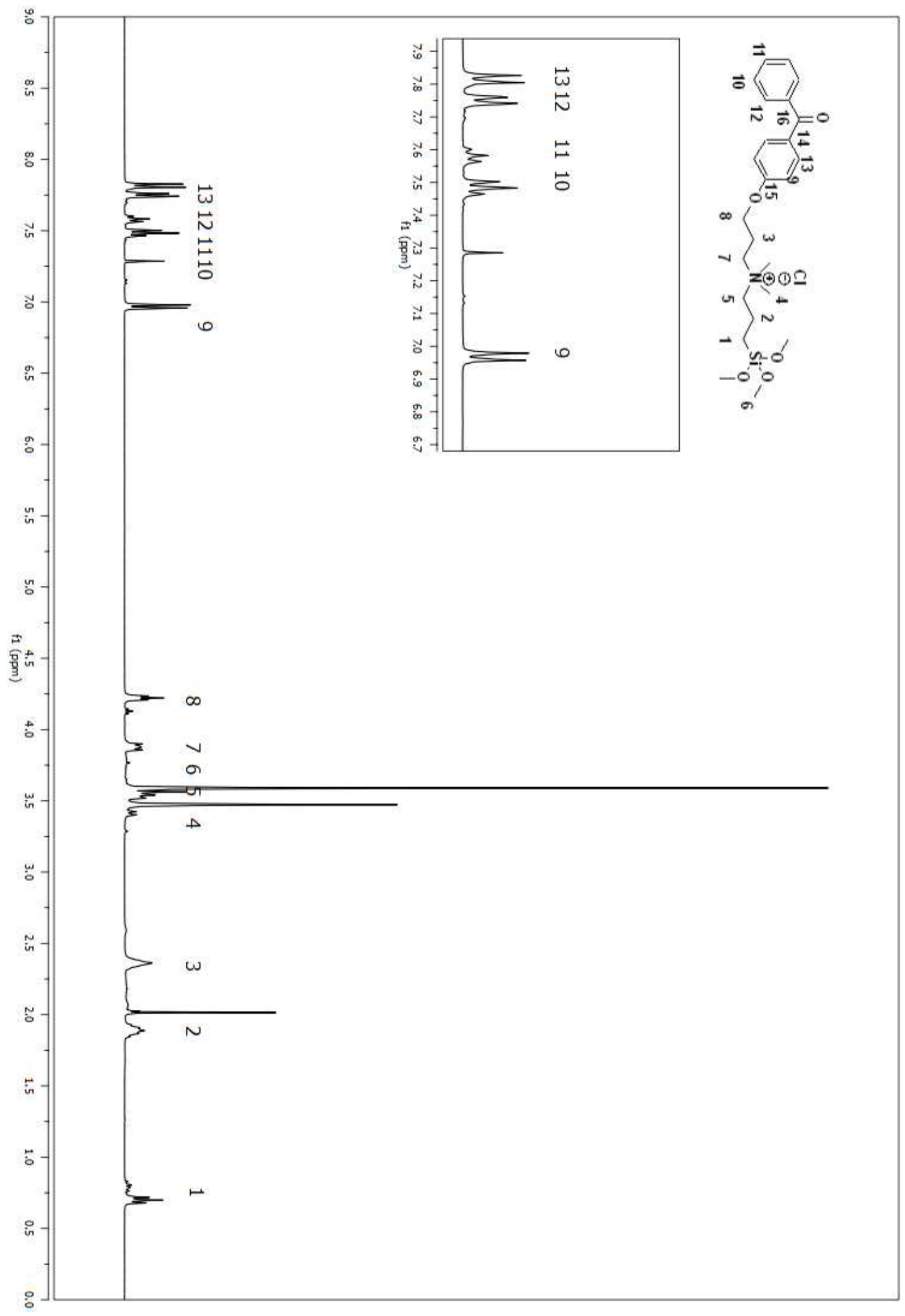

Figure A 337. ${ }^{1} \mathrm{H}$ NMR spectrum of compound (206) in $\mathrm{CDCl}_{3}$. 


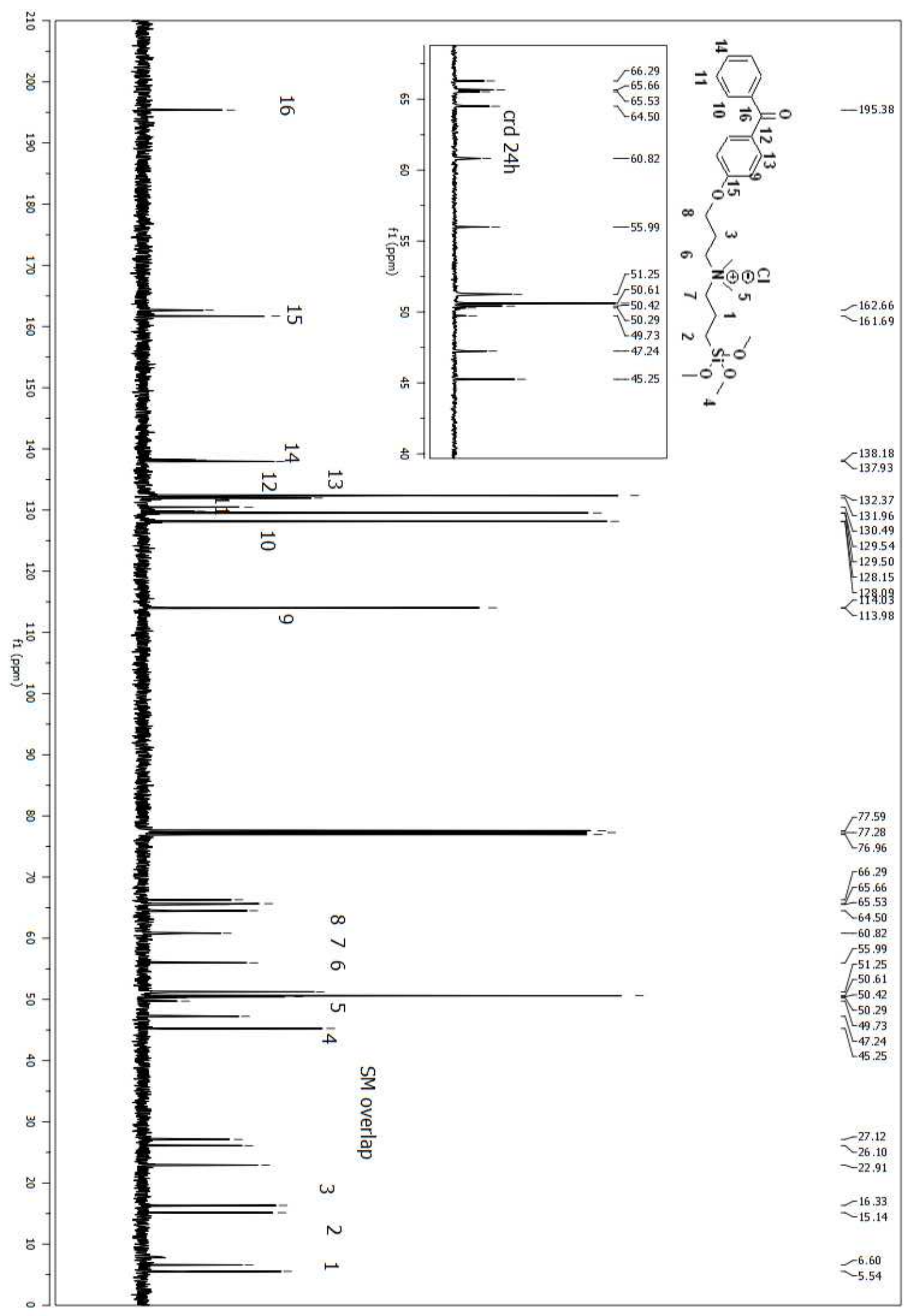

Figure A 338. ${ }^{13} \mathrm{C}$ NMR spectrum of compound (206) in $\mathrm{CDCl}_{3}$. 


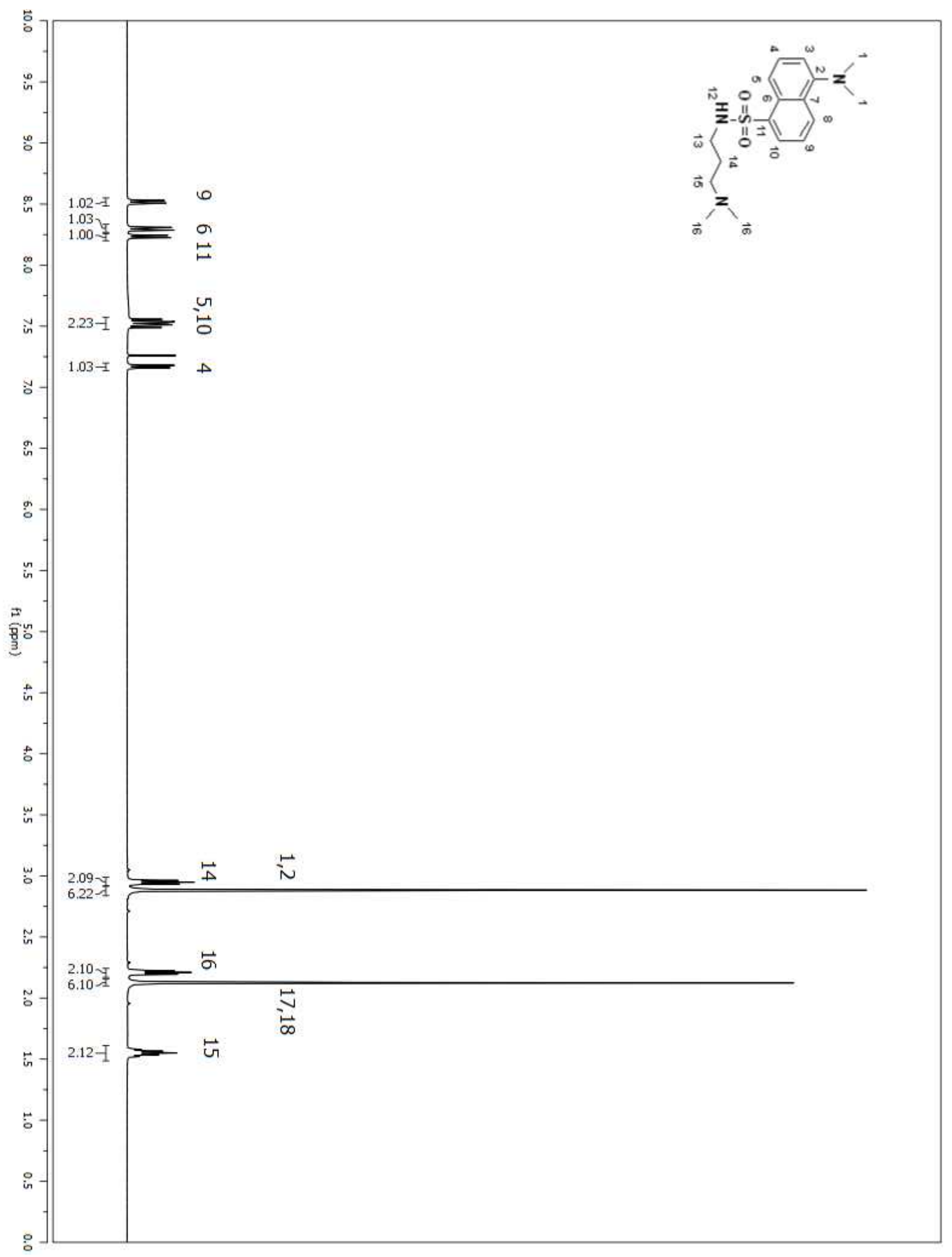

Figure A 339. ${ }^{1} \mathrm{H}$ NMR spectrum of compound (208) in $\mathrm{CDCl}_{3}$. 


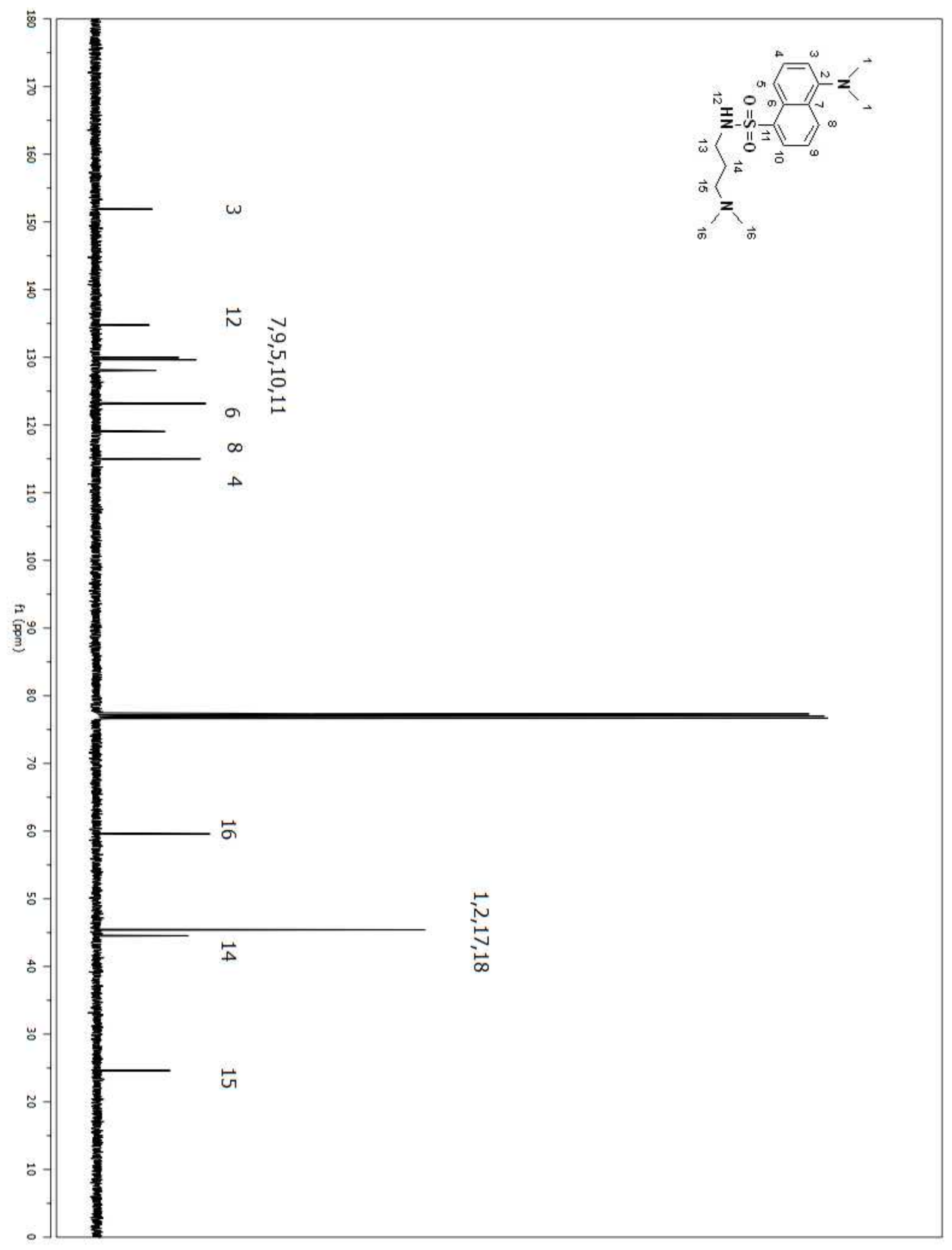

Figure A 340. ${ }^{13} \mathrm{C}$ NMR spectrum of compound (208) in $\mathrm{CDCl}_{3}$. 


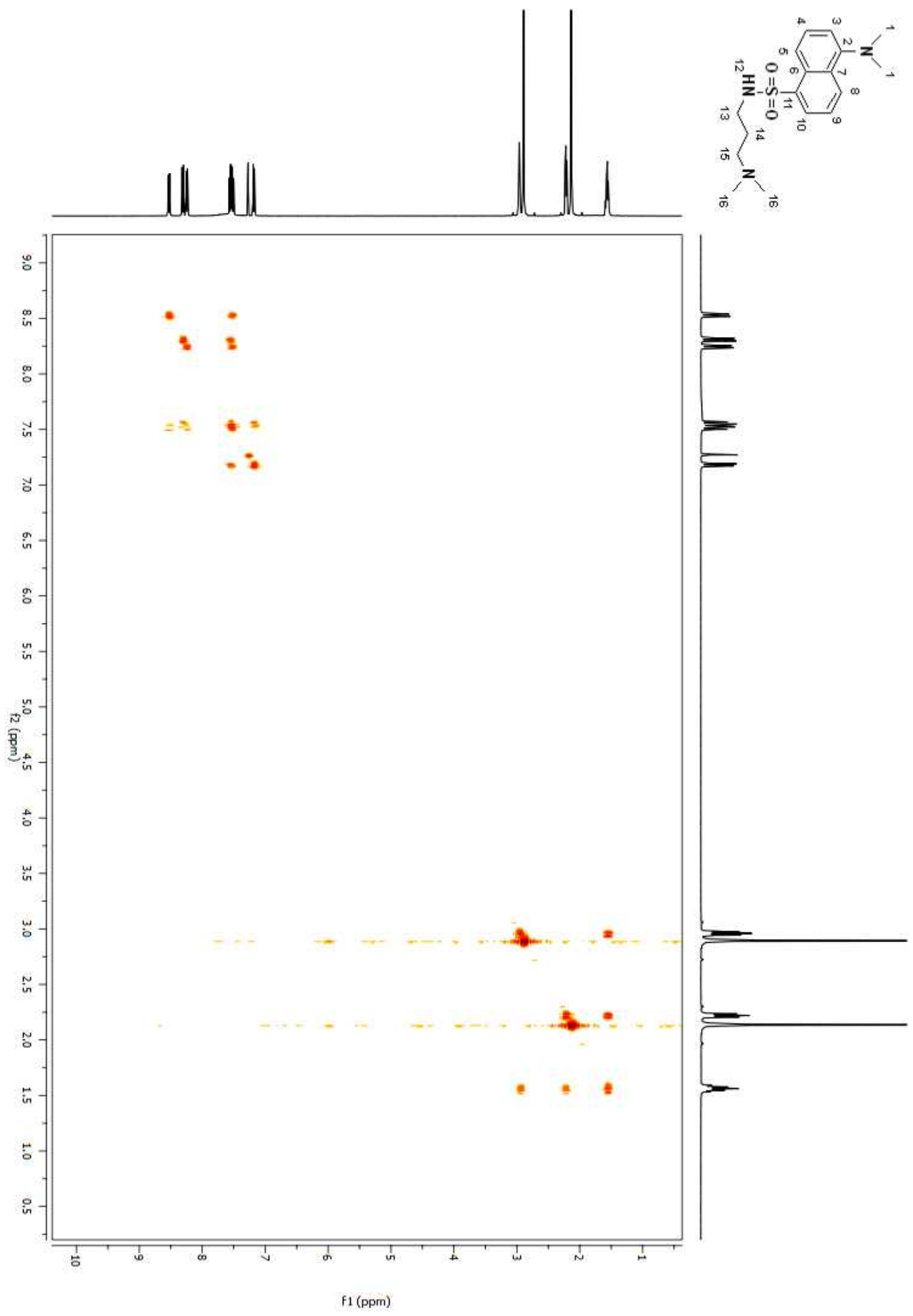

Figure A 341. COSY 2D NMR spectrum of compound (208) in $\mathrm{CDCl}_{3}$. 


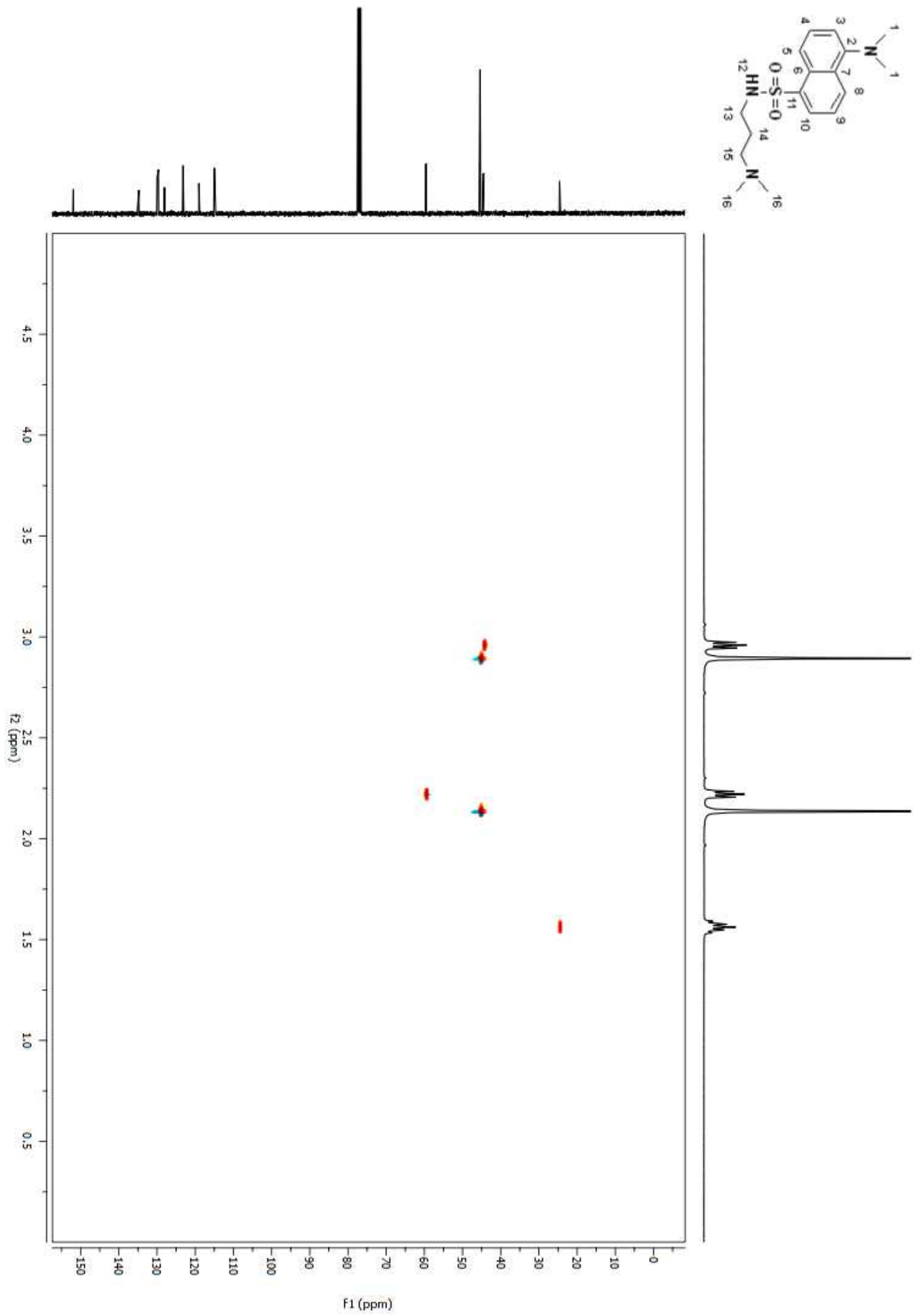

Figure A 342. HSQC 2D NMR spectrum of compound (208) in $\mathrm{CDCl}_{3}$. 


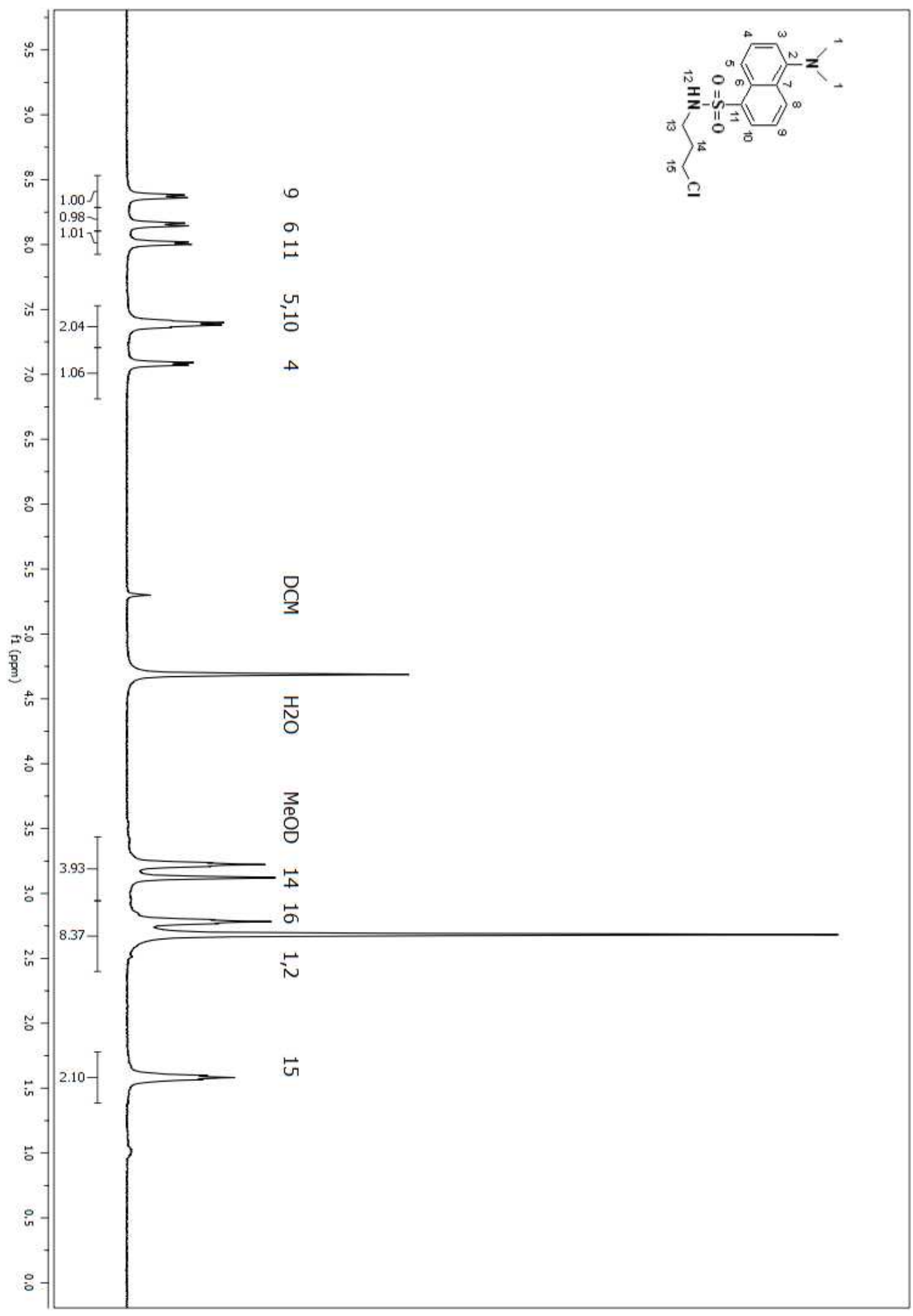

Figure A 343. ${ }^{1} \mathrm{H}$ NMR spectrum of compound (209) in MeOD. 


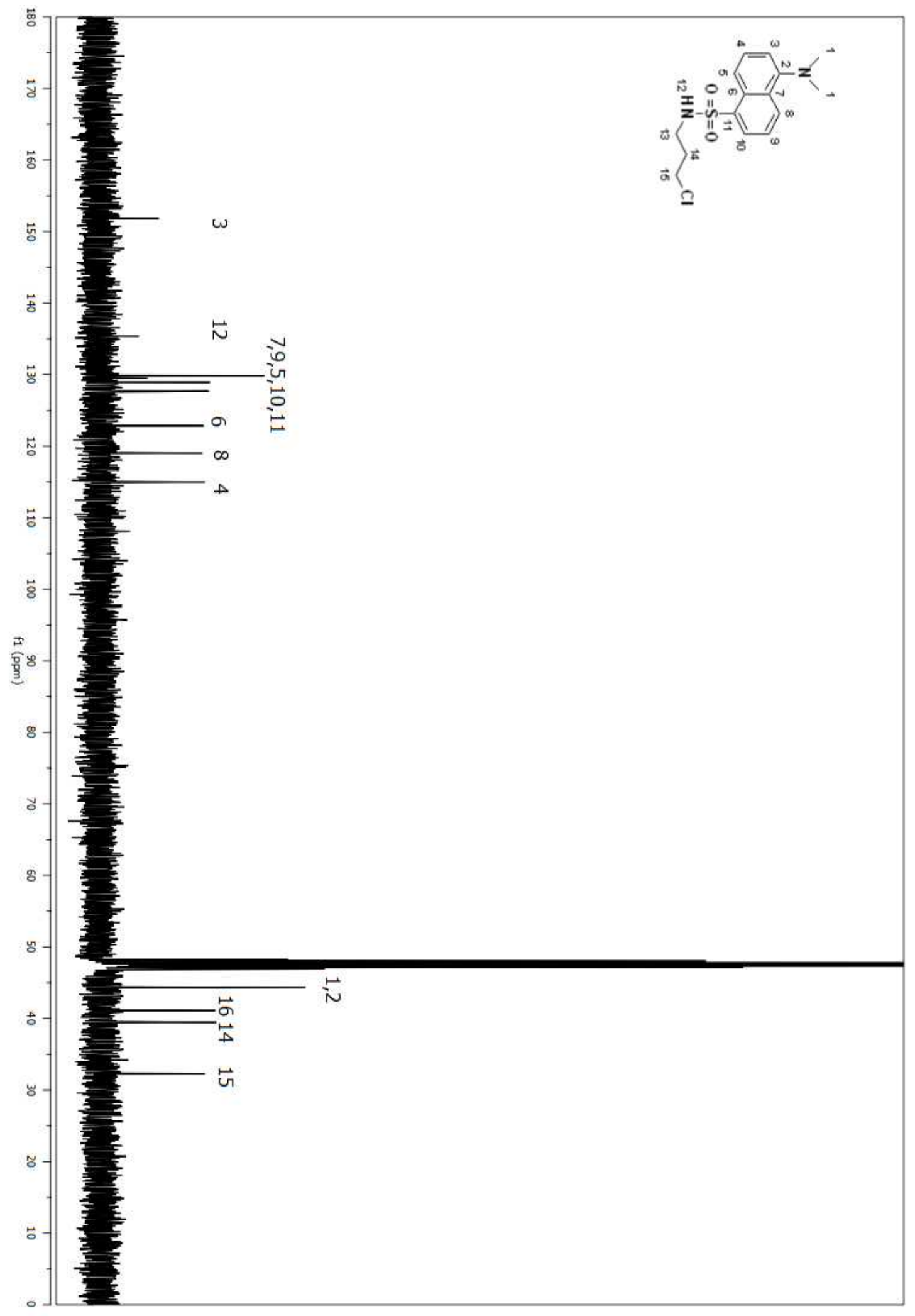

Figure A 344. ${ }^{13} \mathrm{C}$ NMR spectrum of compound (209) in MeOD. 


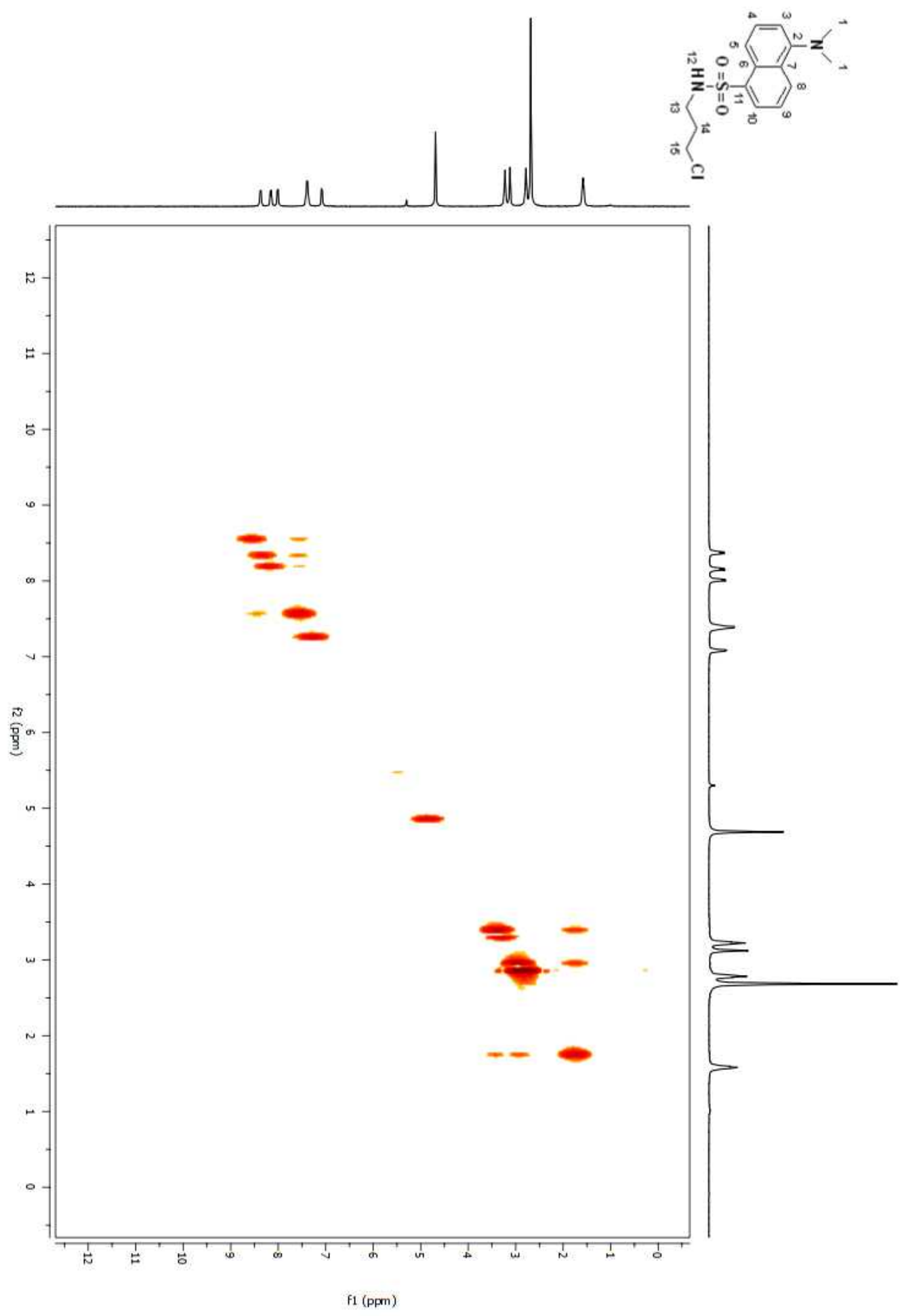

Figure A 345. COSY 2D NMR spectrum of compound (209) in MeOD. 


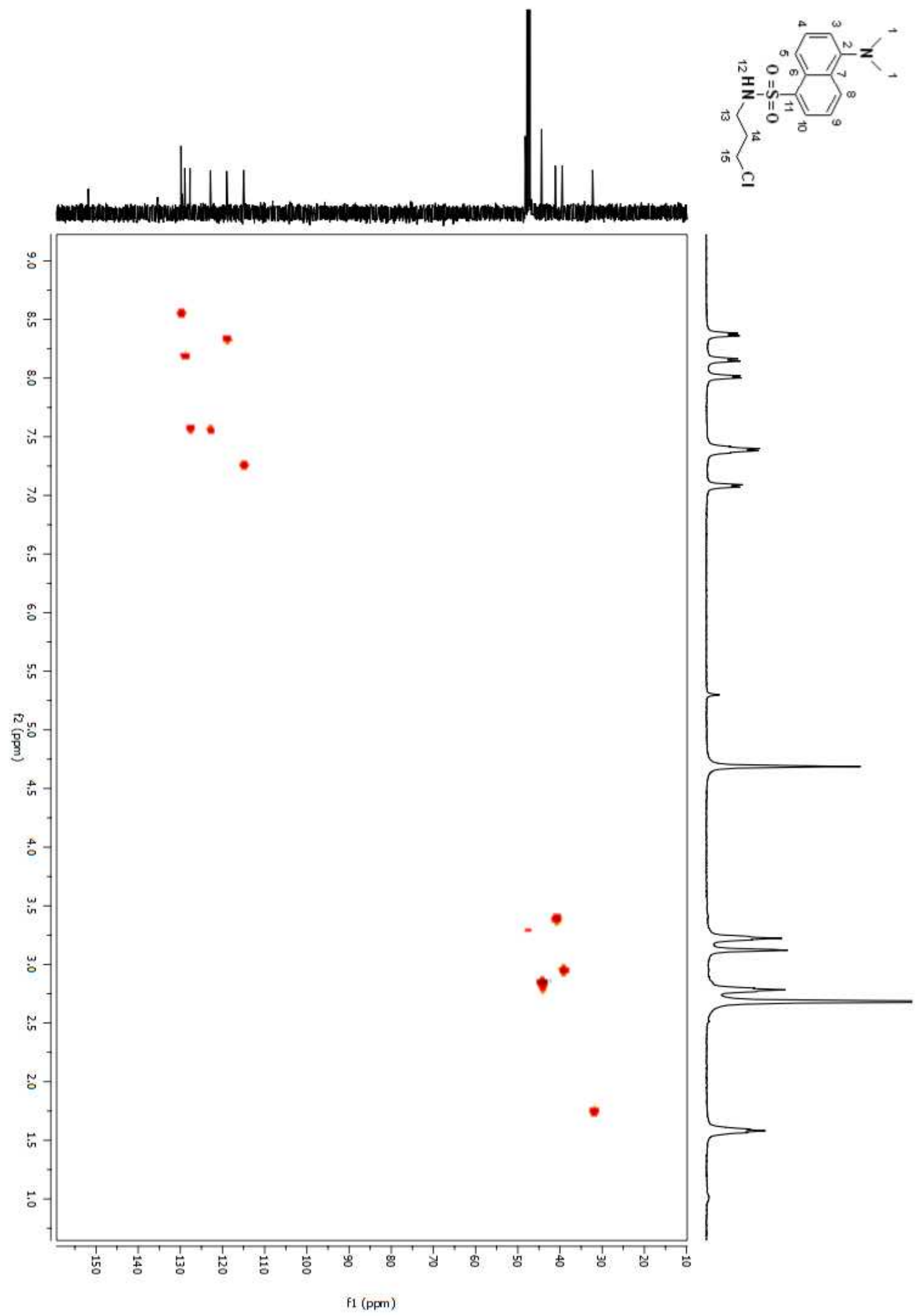

Figure A 346. HSQC 2D NMR spectrum of compound (209) in MeOD. 


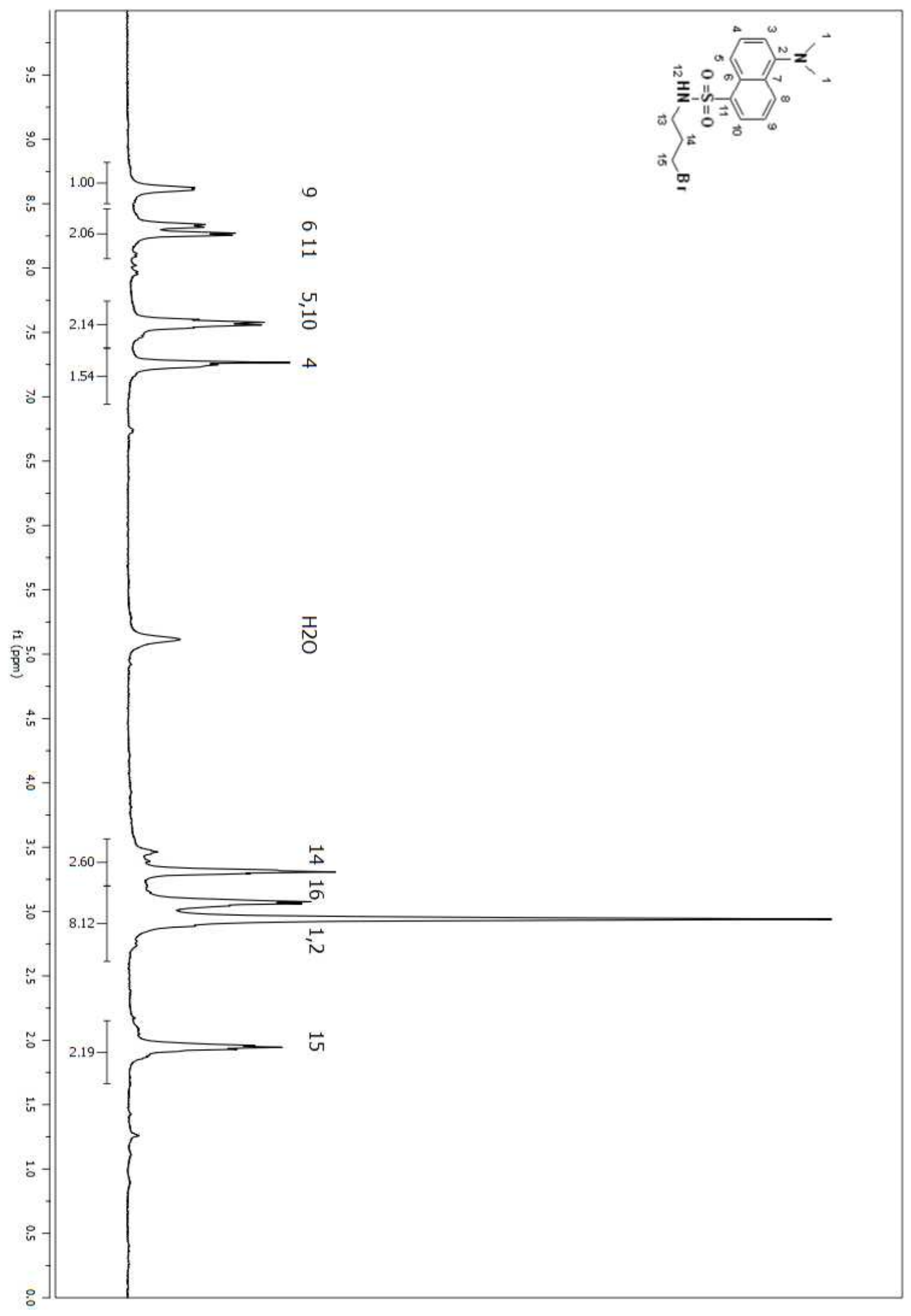

Figure A 347. ${ }^{1} \mathrm{H}$ NMR spectrum of compound (210) in MeOD. 


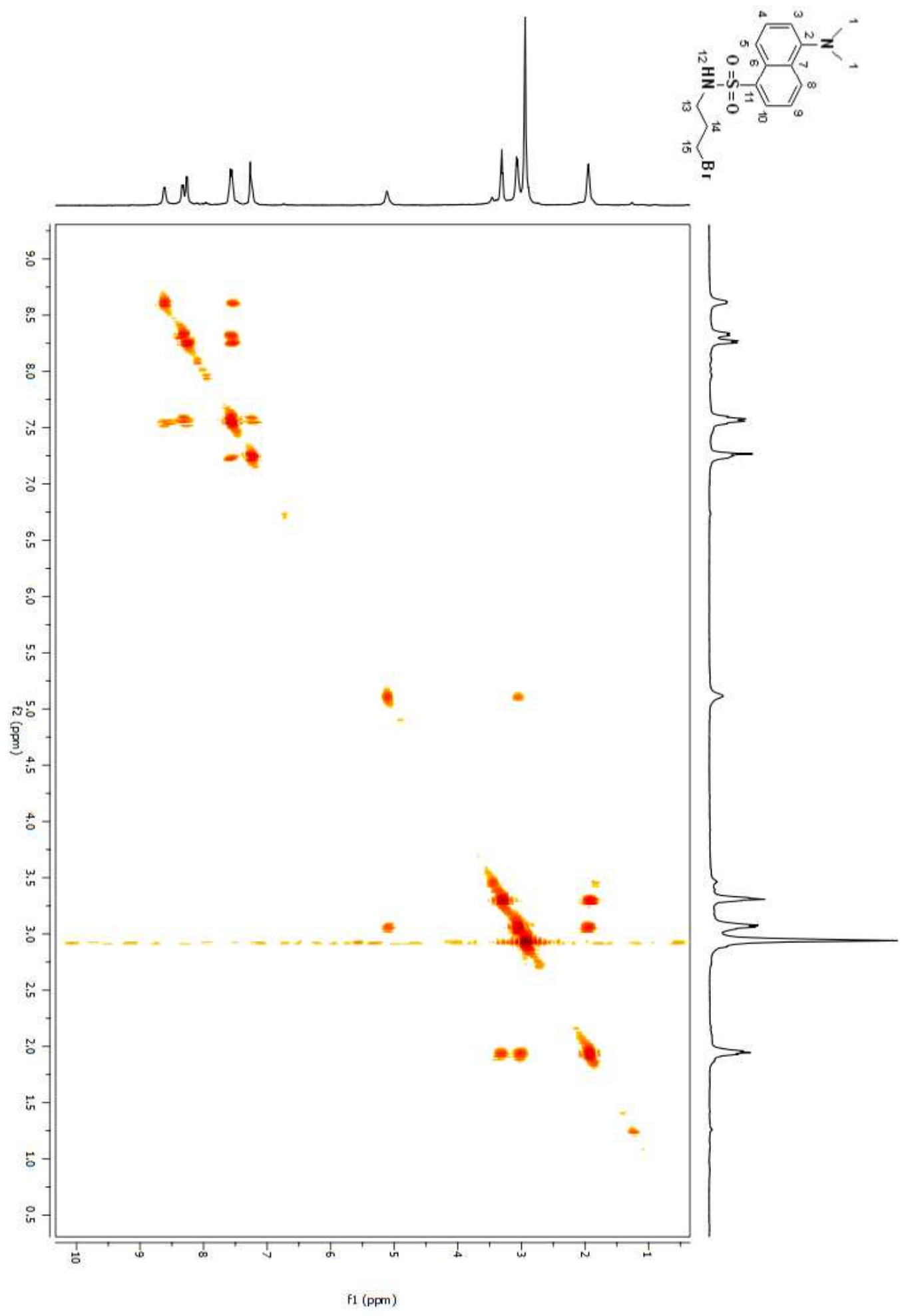

Figure A 348. COSY 2D NMR spectrum of compound (210) in MeOD. 


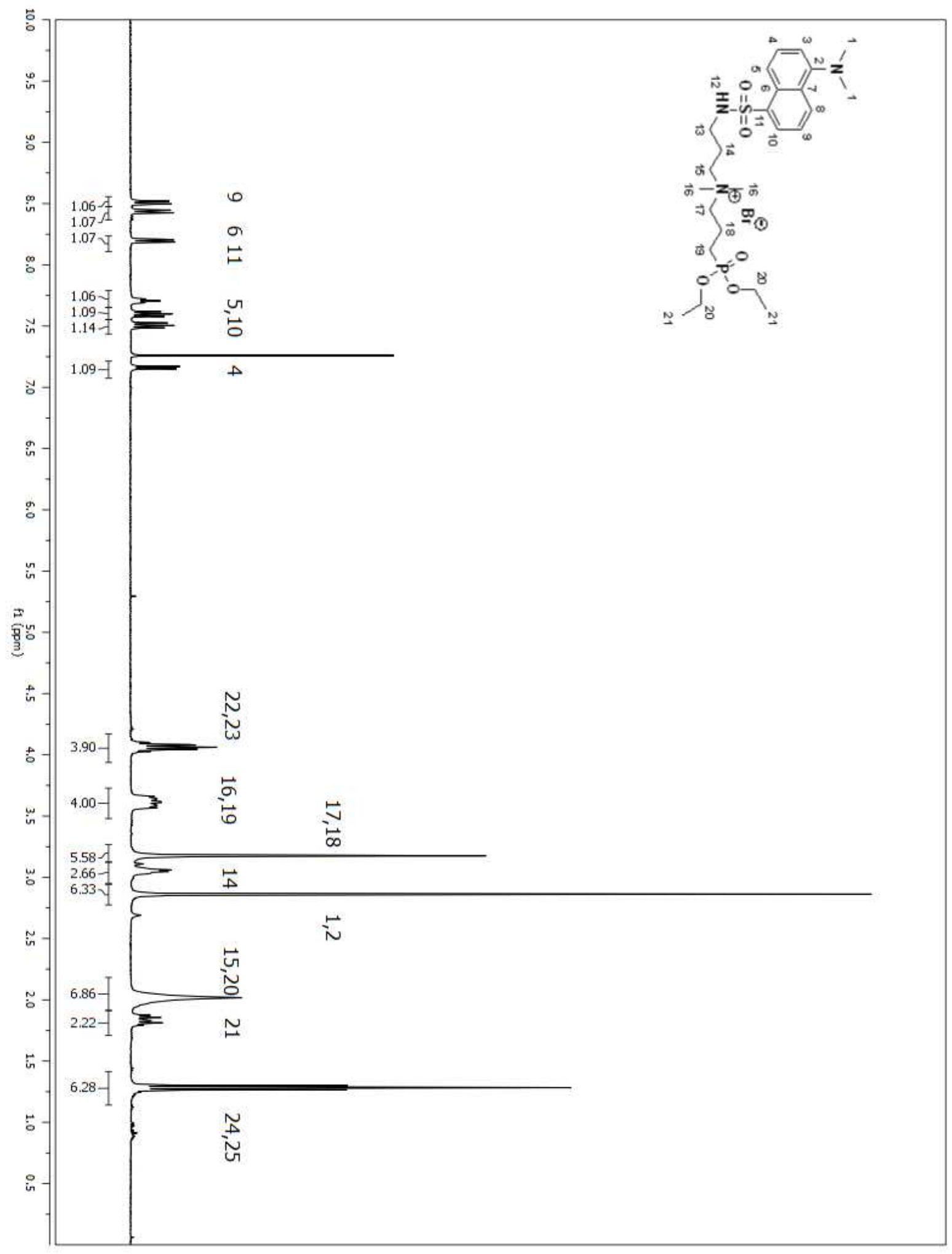

Figure A 349. ${ }^{1} \mathrm{H}$ NMR spectrum of compound (211) in $\mathrm{CDCl}_{3}$. 


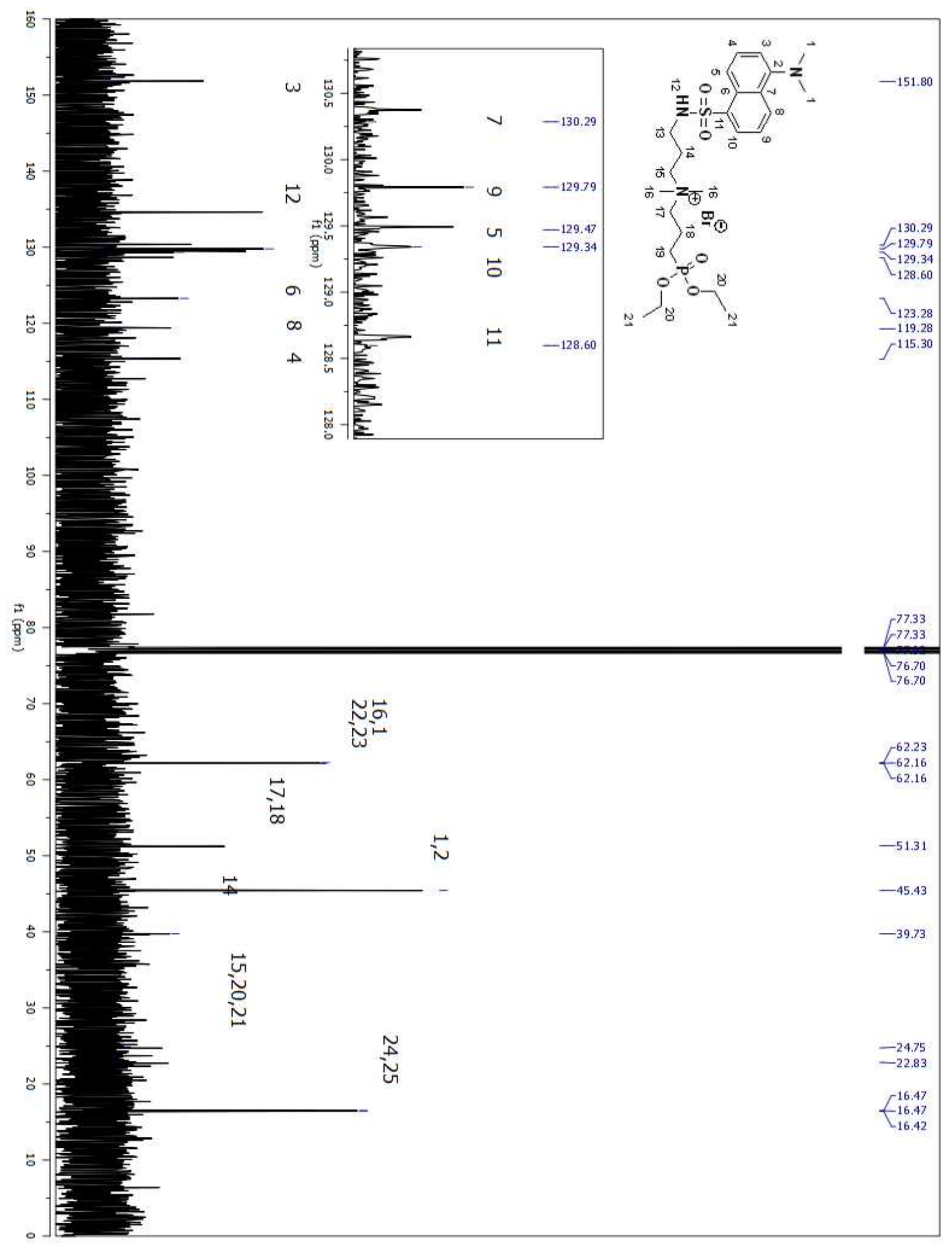

Figure A 350. ${ }^{13} \mathrm{C}$ NMR spectrum of compound (211) in $\mathrm{CDCl}_{3}$. 


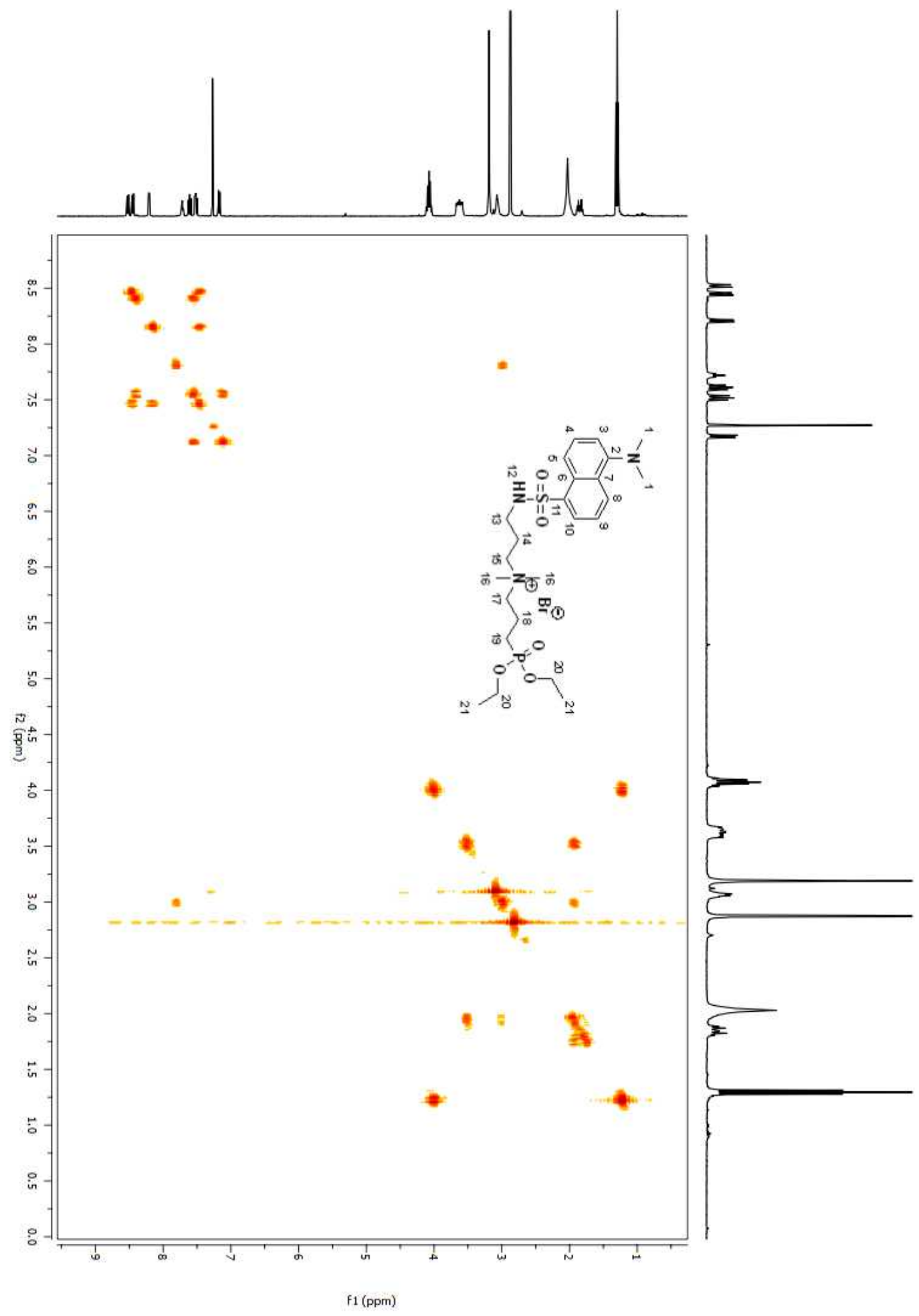

Figure A 351. COSY 2D NMR spectrum of compound (211) in $\mathrm{CDCl}_{3}$. 


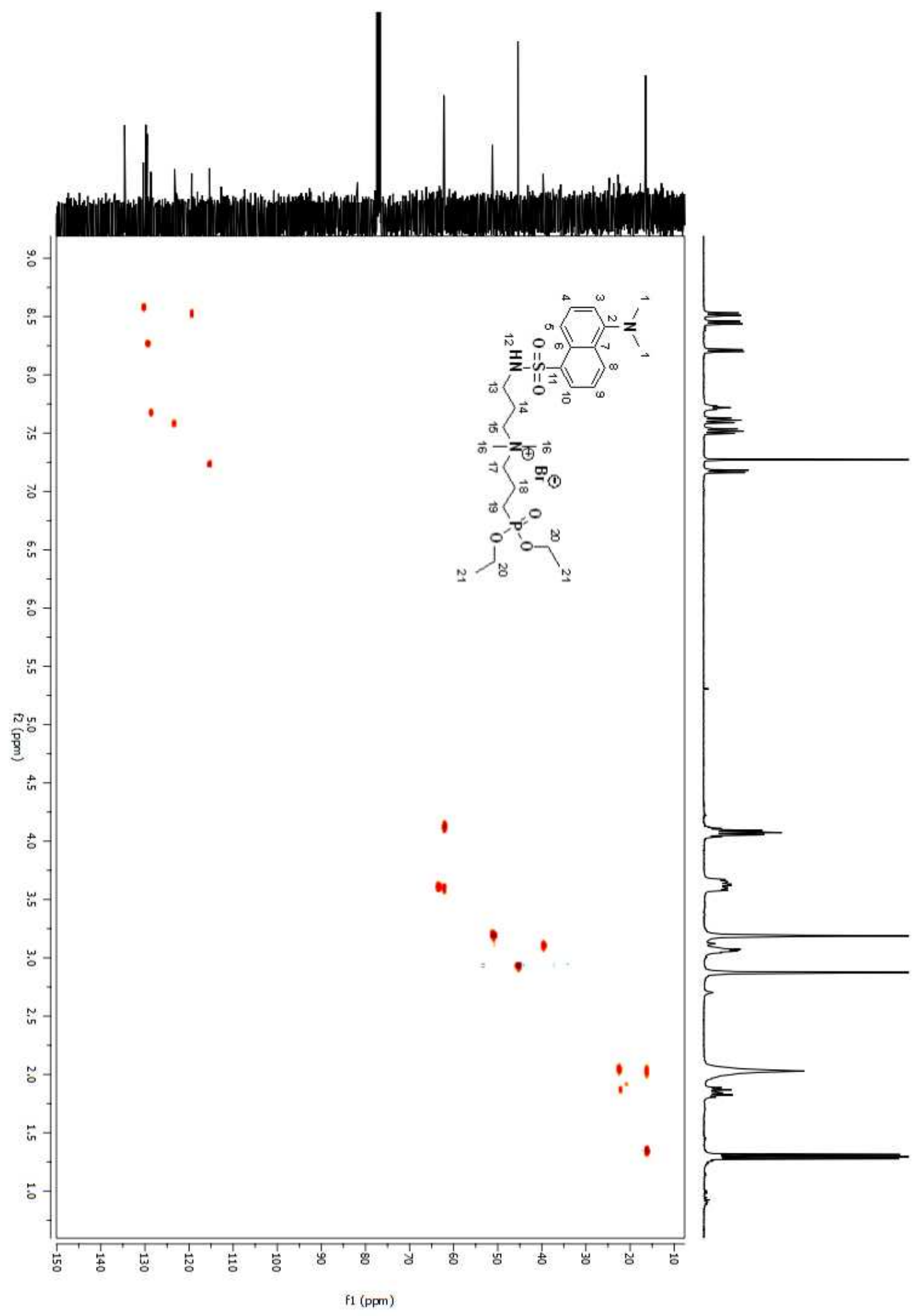

Figure A 352. HSQC 2D NMR spectrum of compound (211) in $\mathrm{CDCl}_{3}$. 


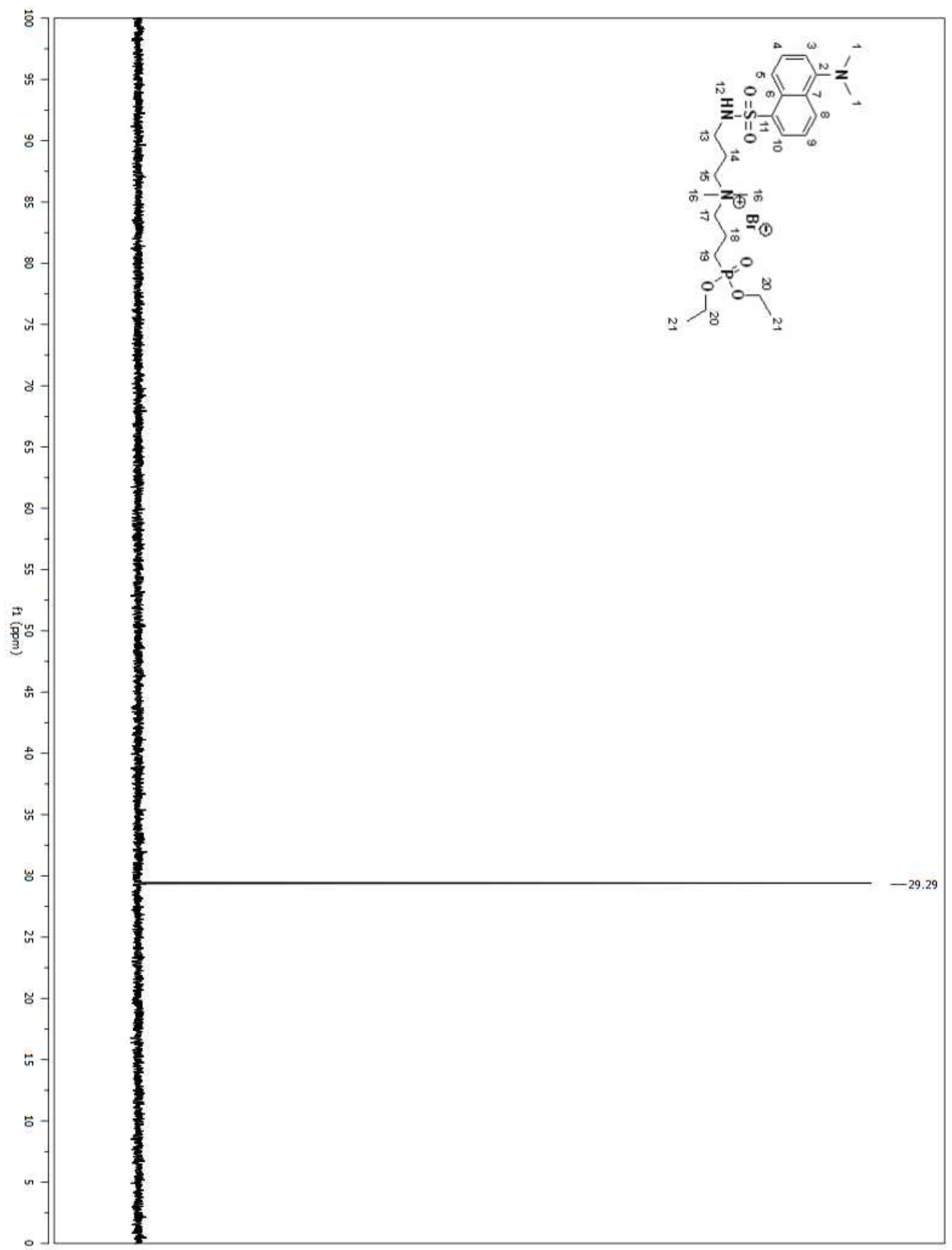

Figure A 353. ${ }^{31} \mathrm{P}$ NMR spectrum of compound (211) in $\mathrm{CDCl}_{3}$. 


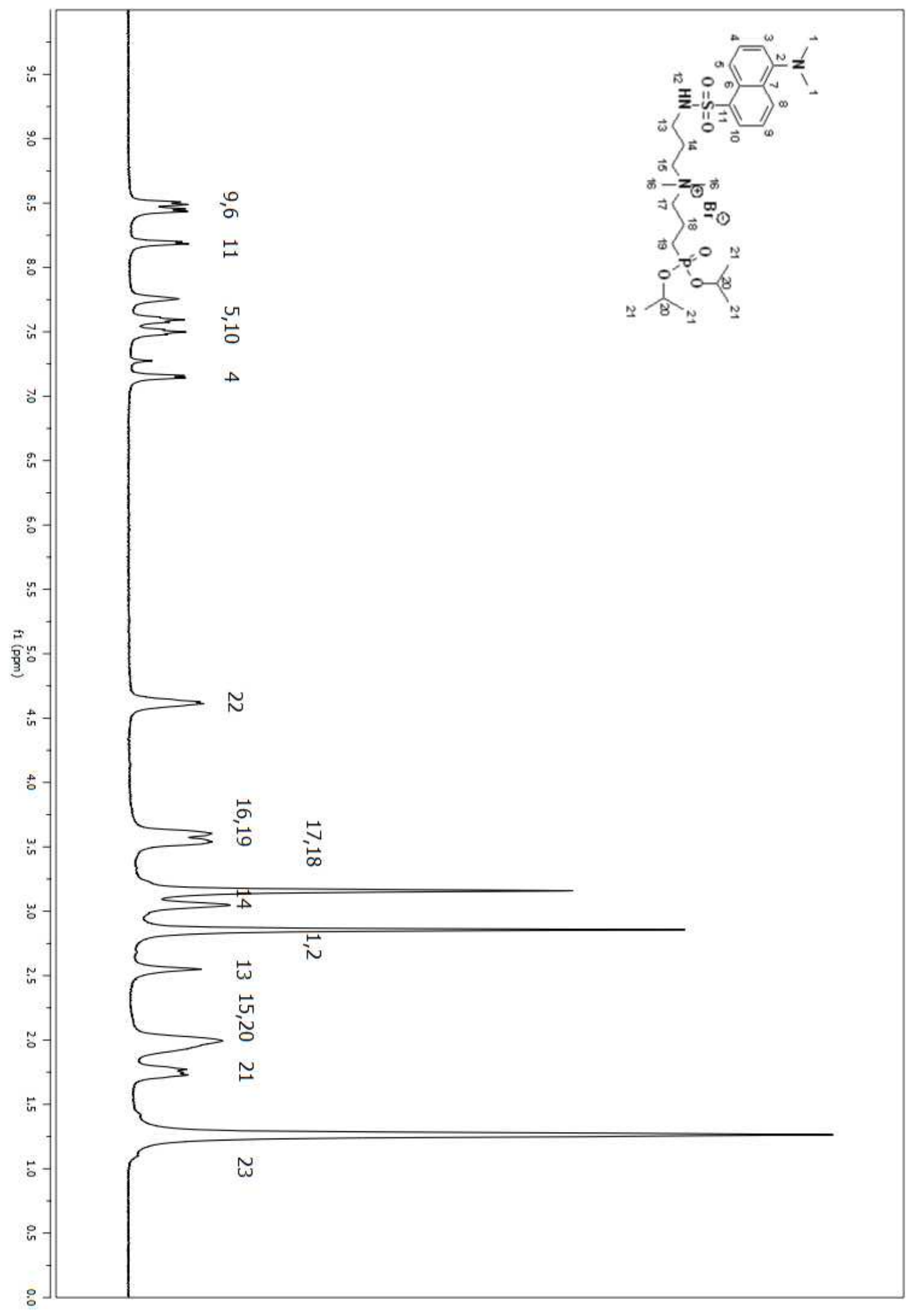

Figure A 354. ${ }^{1} \mathrm{H}$ NMR spectrum of compound (212) in $\mathrm{CDCl}_{3}$. 


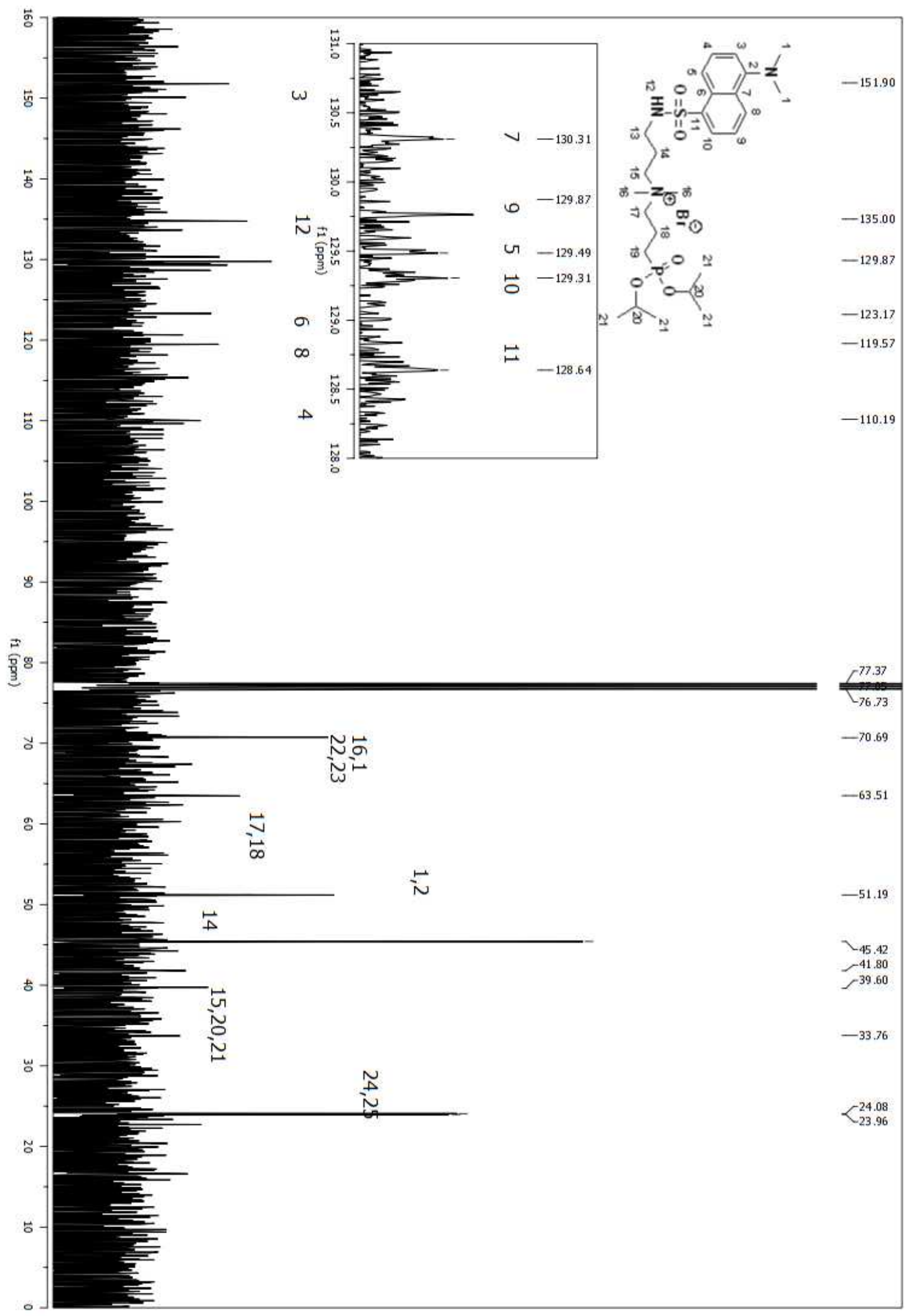

Figure A 355. ${ }^{13} \mathrm{C}$ NMR spectrum of compound (212) in $\mathrm{CDCl}_{3}$. 


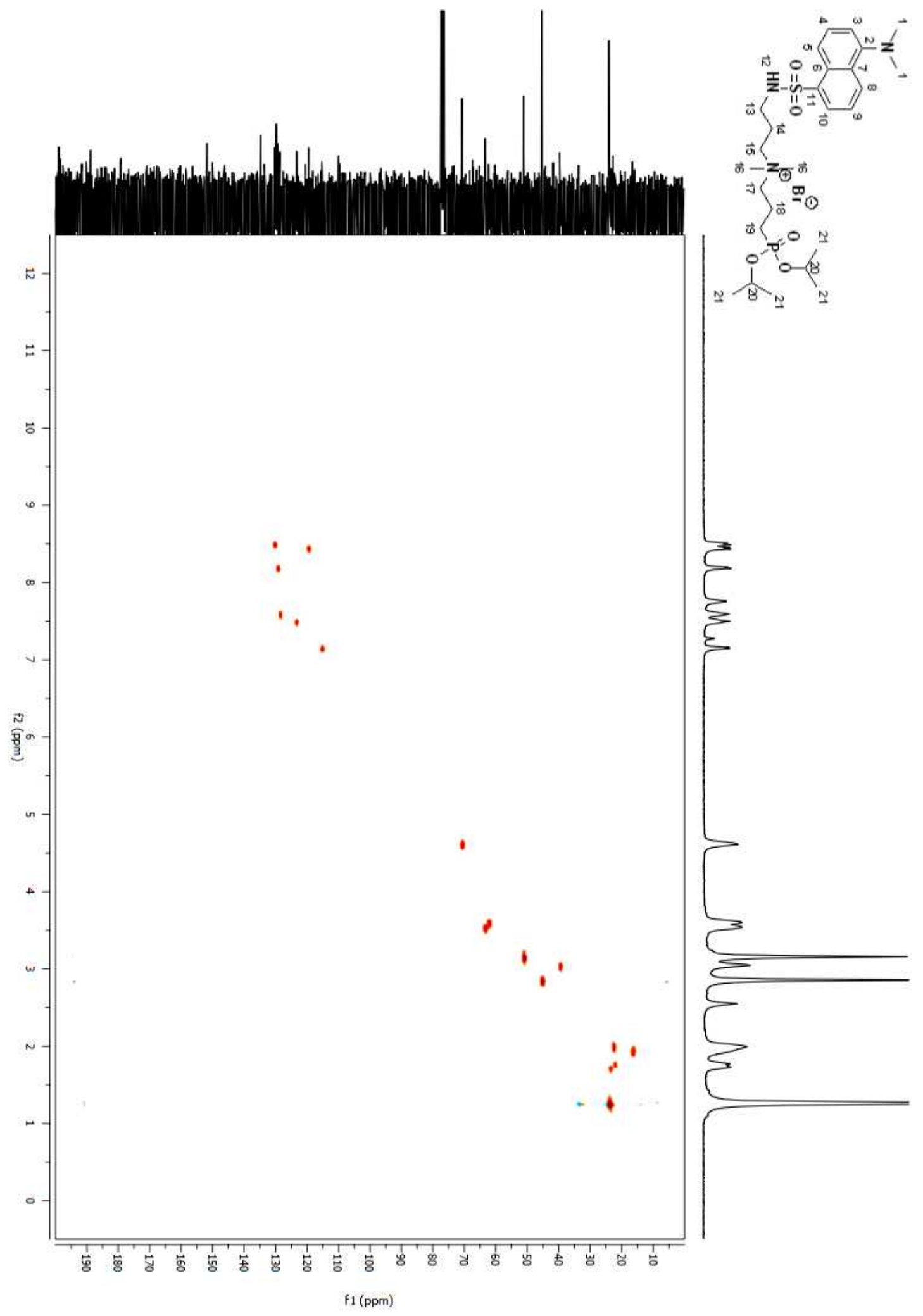

Figure A 356. HSQC 2D NMR spectrum of compound (212) in $\mathrm{CDCl}_{3}$. 


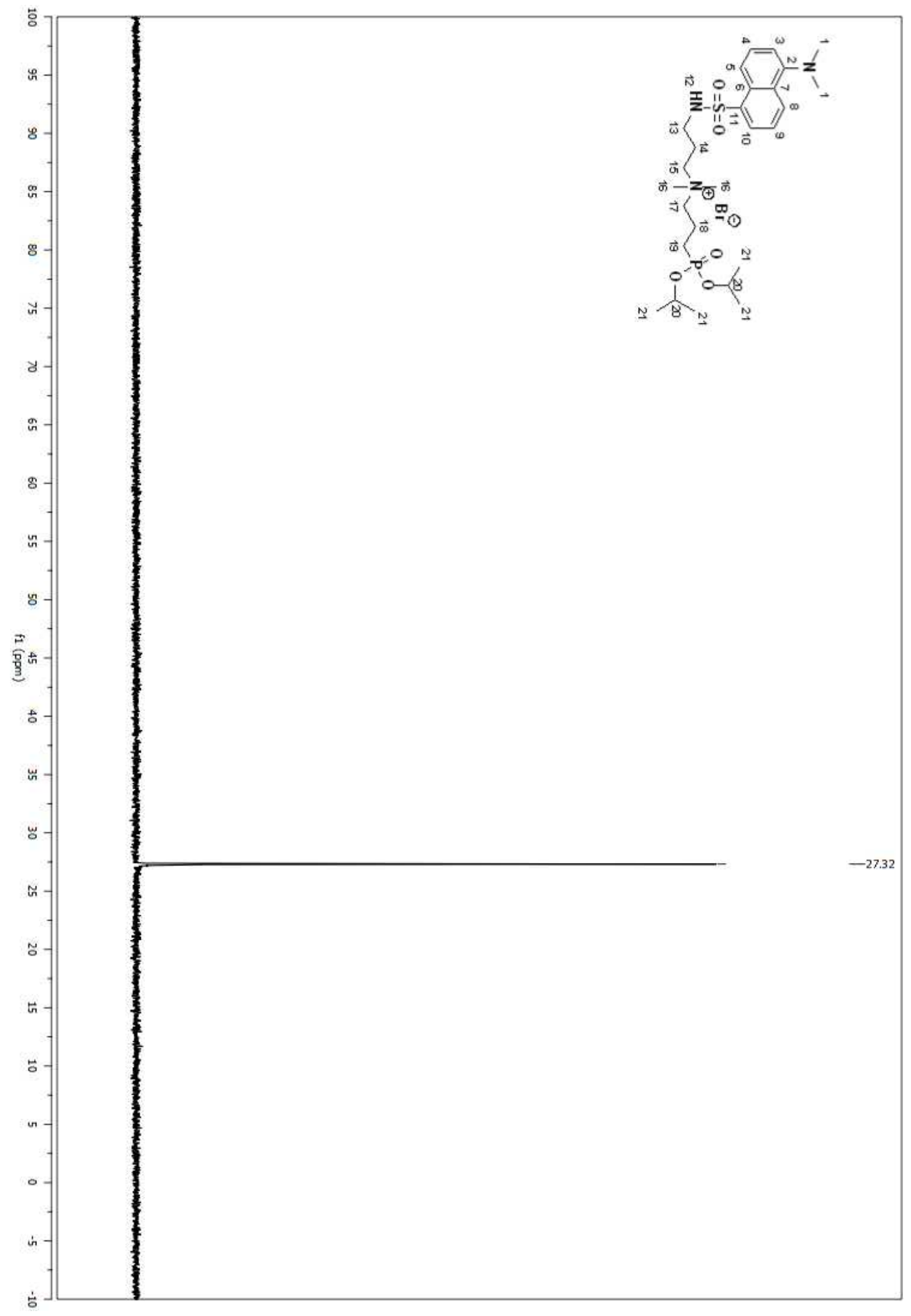

Figure A 357. ${ }^{31} \mathrm{P}$ NMR spectrum of compound (212) in $\mathrm{CDCl}_{3}$. 724 


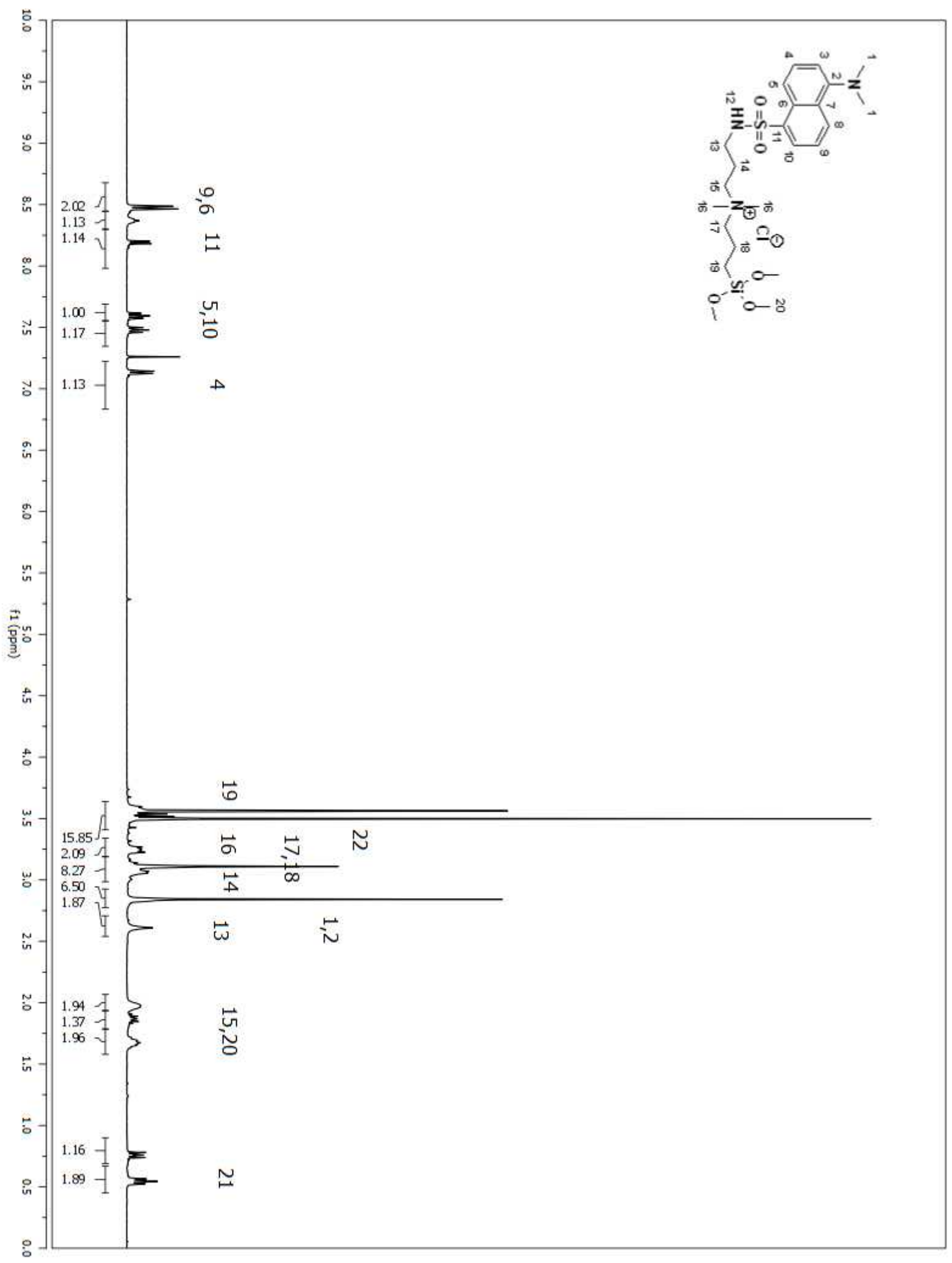

Figure A 358. ${ }^{1} \mathrm{H}$ NMR spectrum of compound (213) in $\mathrm{CDCl}_{3}$. 


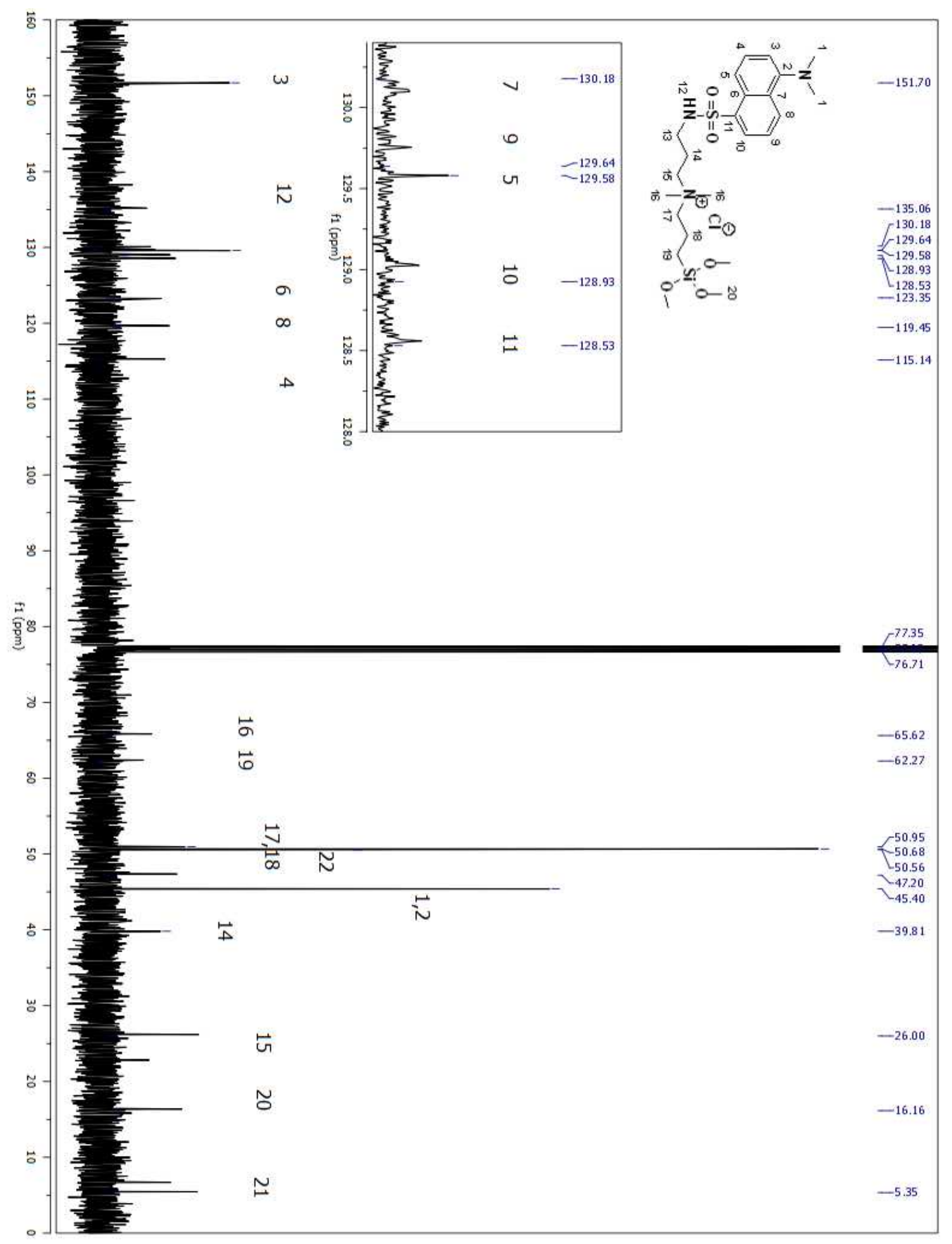

Figure A 359. ${ }^{13} \mathrm{C}$ NMR spectrum of compound (213) in $\mathrm{CDCl}_{3}$. 


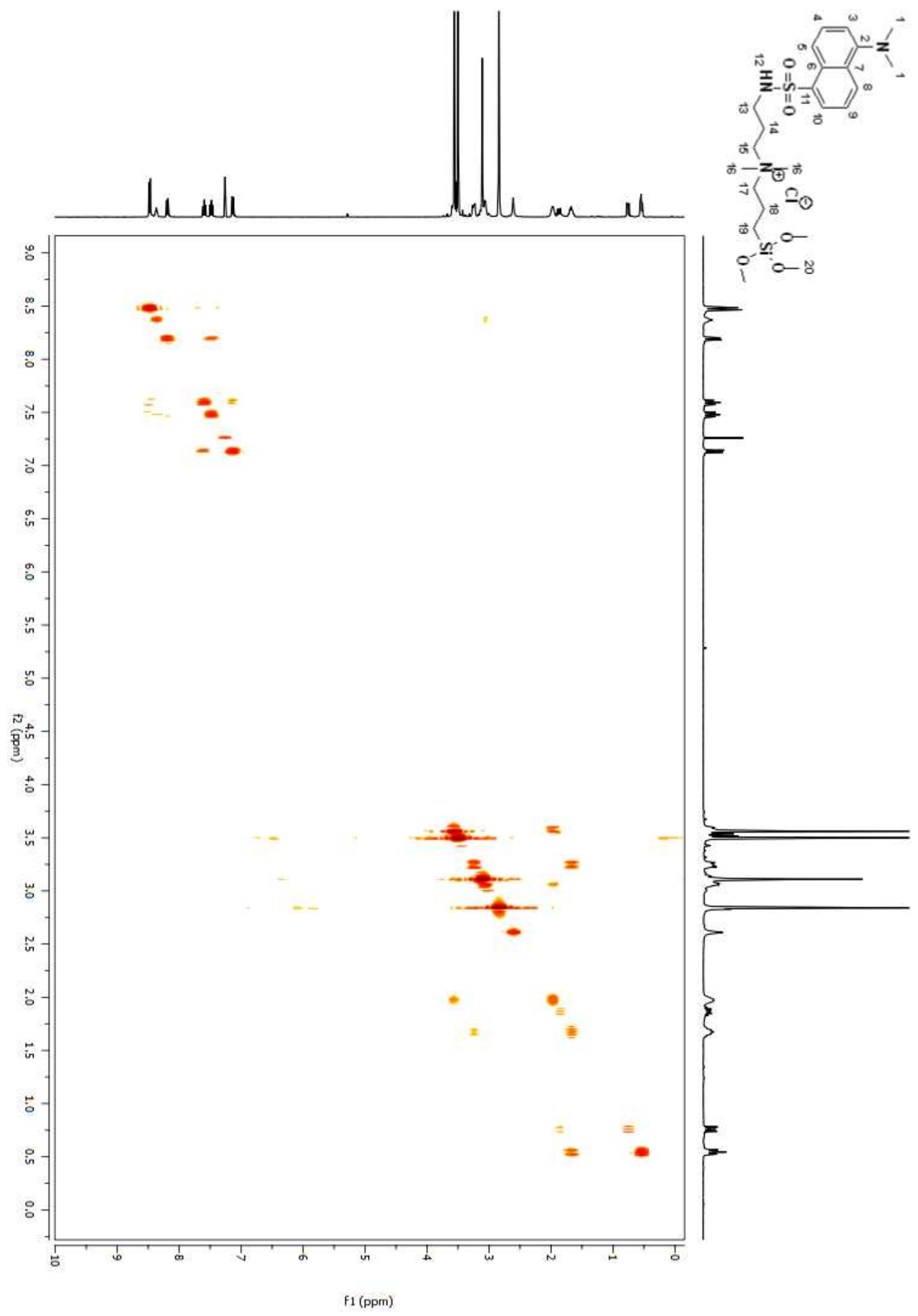

Figure A 360. COSY 2D NMR spectrum of compound (213) in $\mathrm{CDCl}_{3}$. 


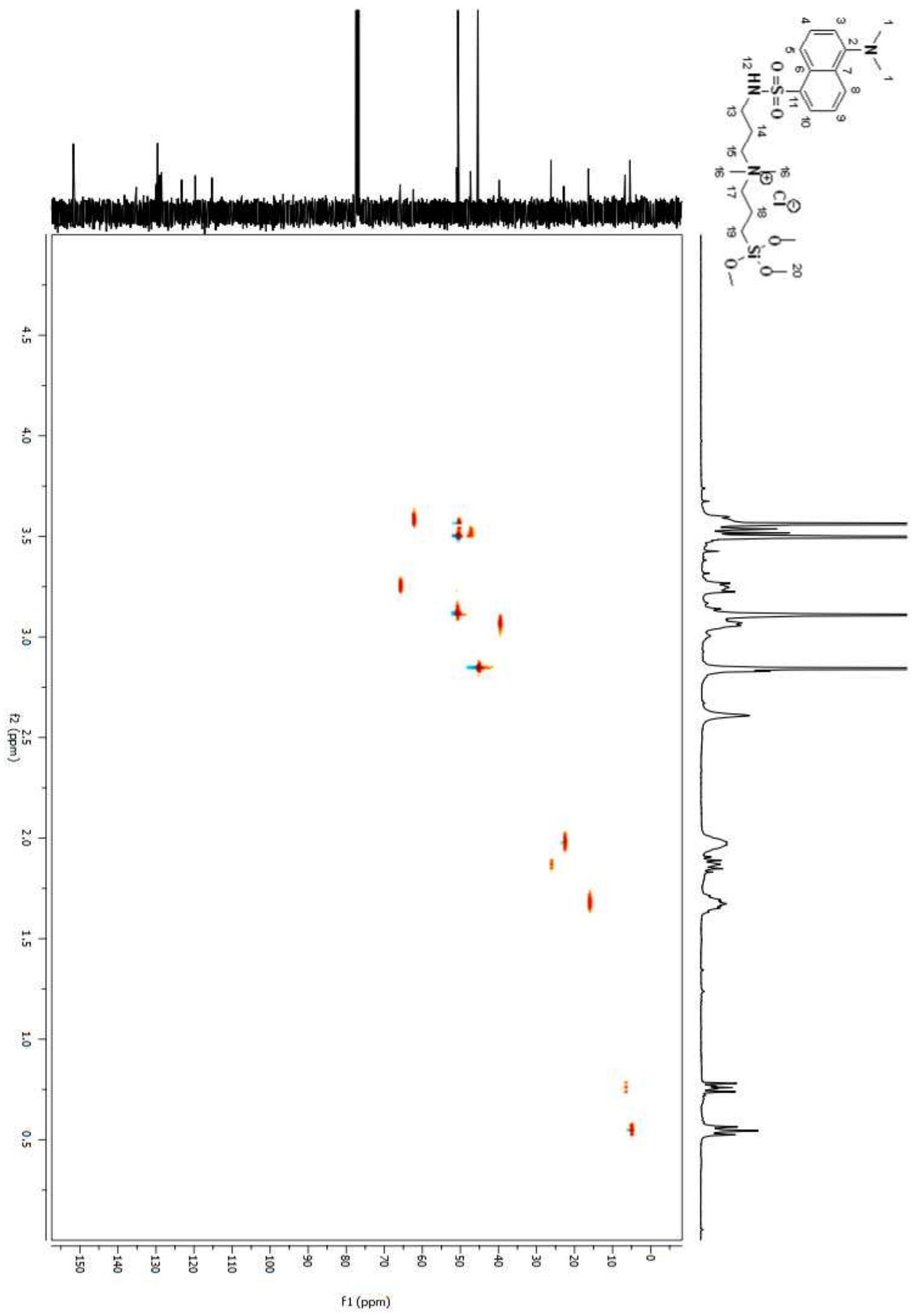

Figure A 361. HSQC 2D NMR spectrum of compound (213) in $\mathrm{CDCl}_{3}$. 


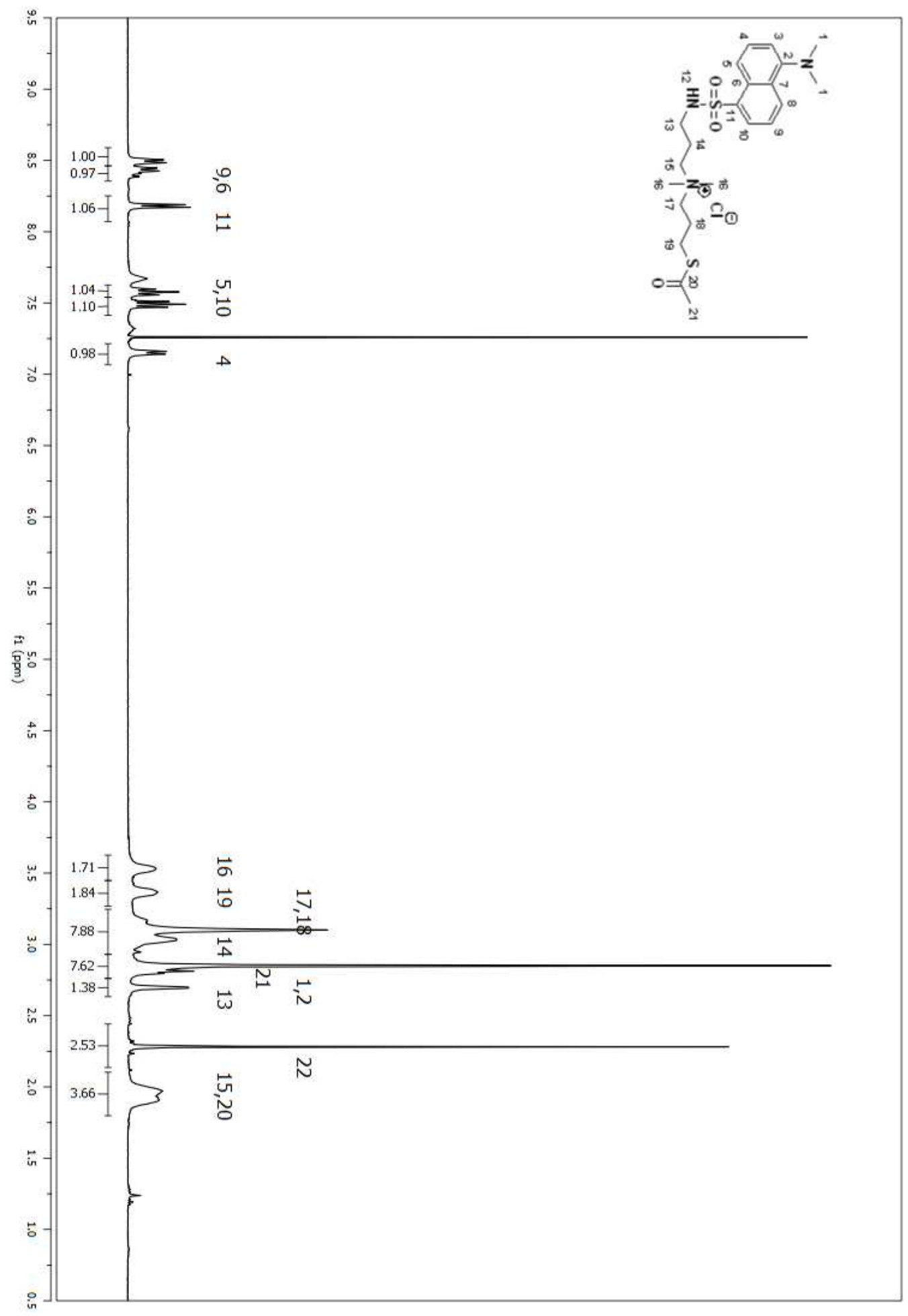

Figure A 362. ${ }^{1} \mathrm{H}$ NMR spectrum of compound (214) in $\mathrm{CDCl}_{3}$. 


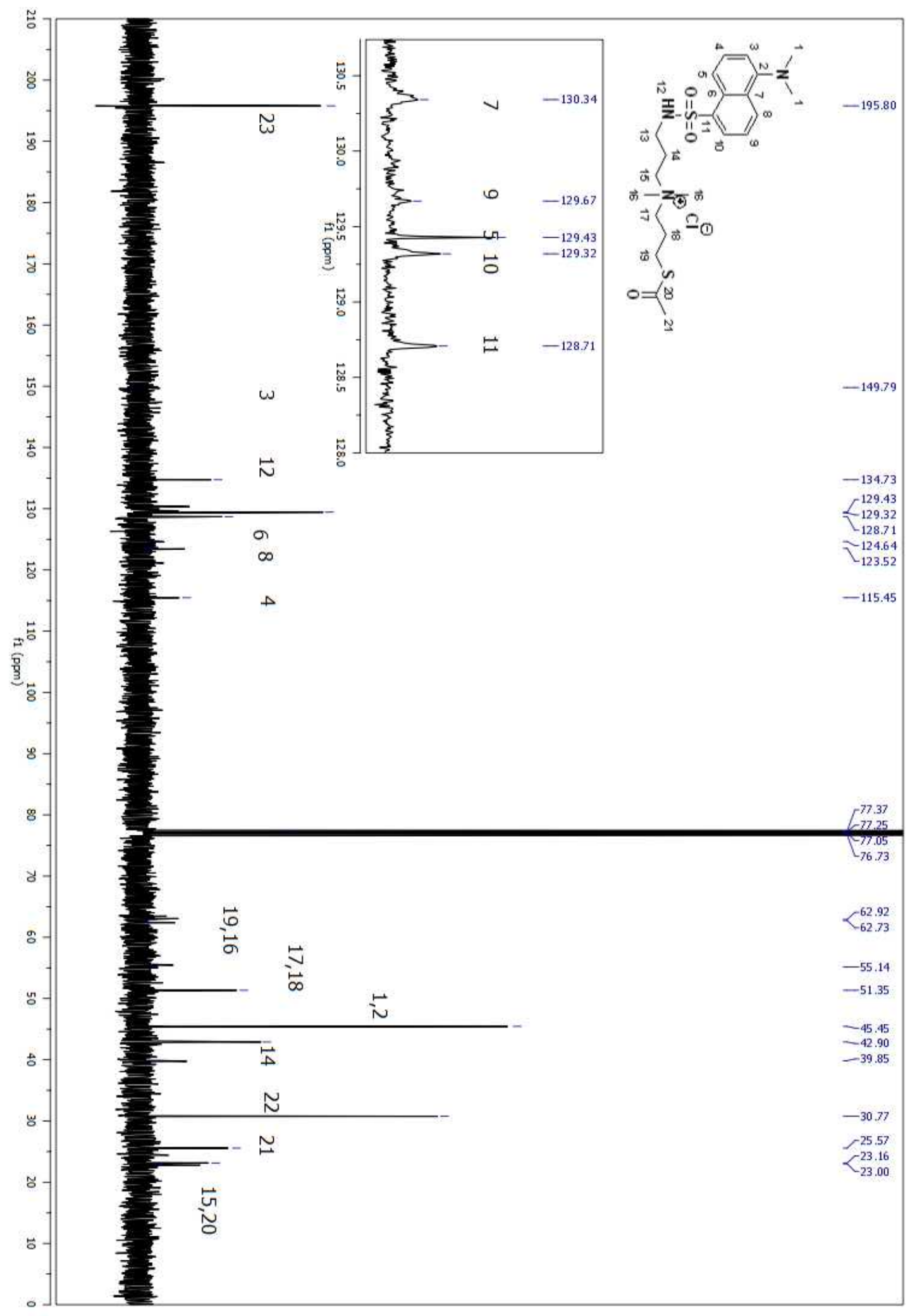

Figure A 363. ${ }^{13} \mathrm{C}$ NMR spectrum of compound (214) in $\mathrm{CDCl}_{3}$. 


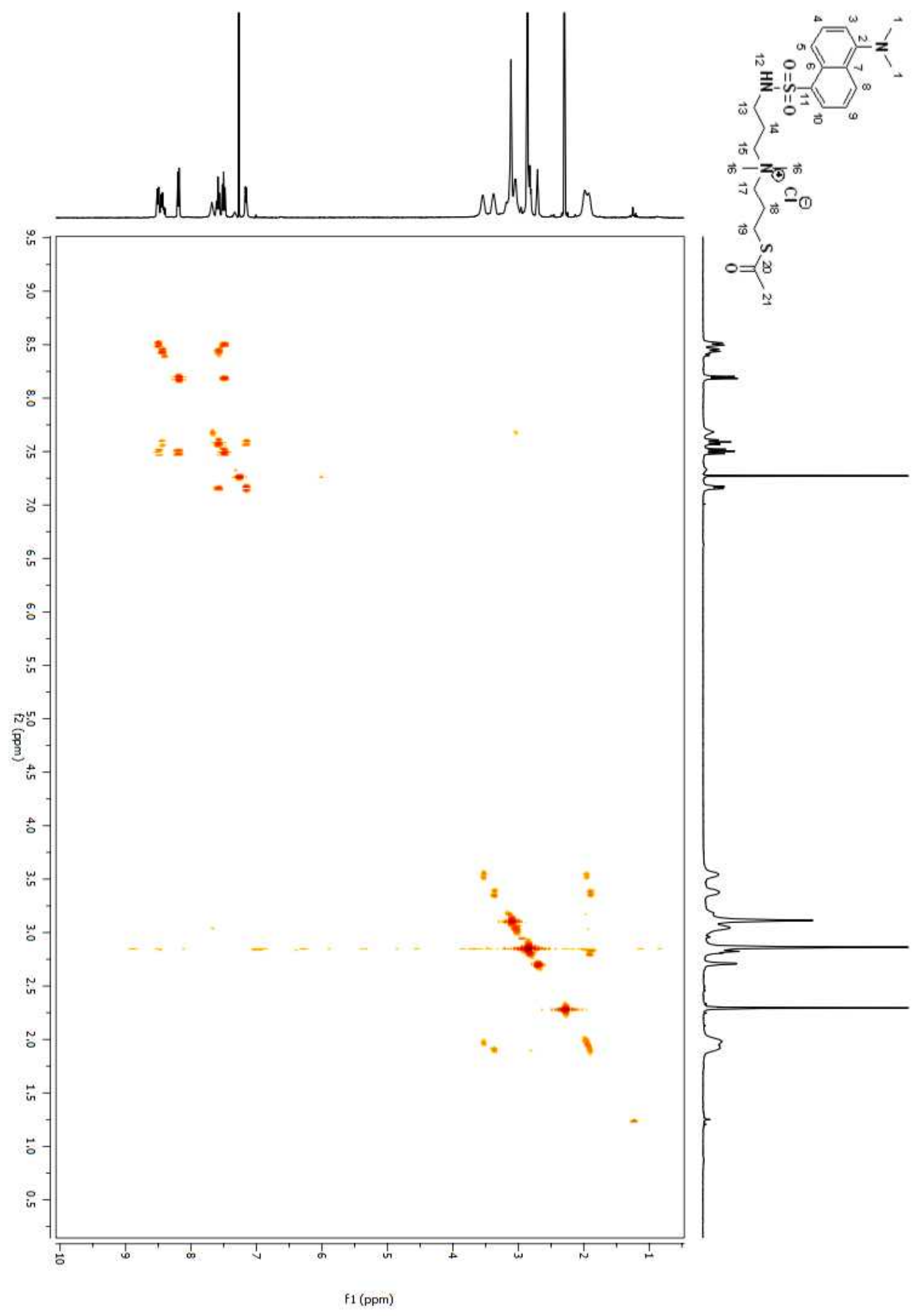

Figure A 364. COSY 2D NMR spectrum of compound (214) in $\mathrm{CDCl}_{3}$. 


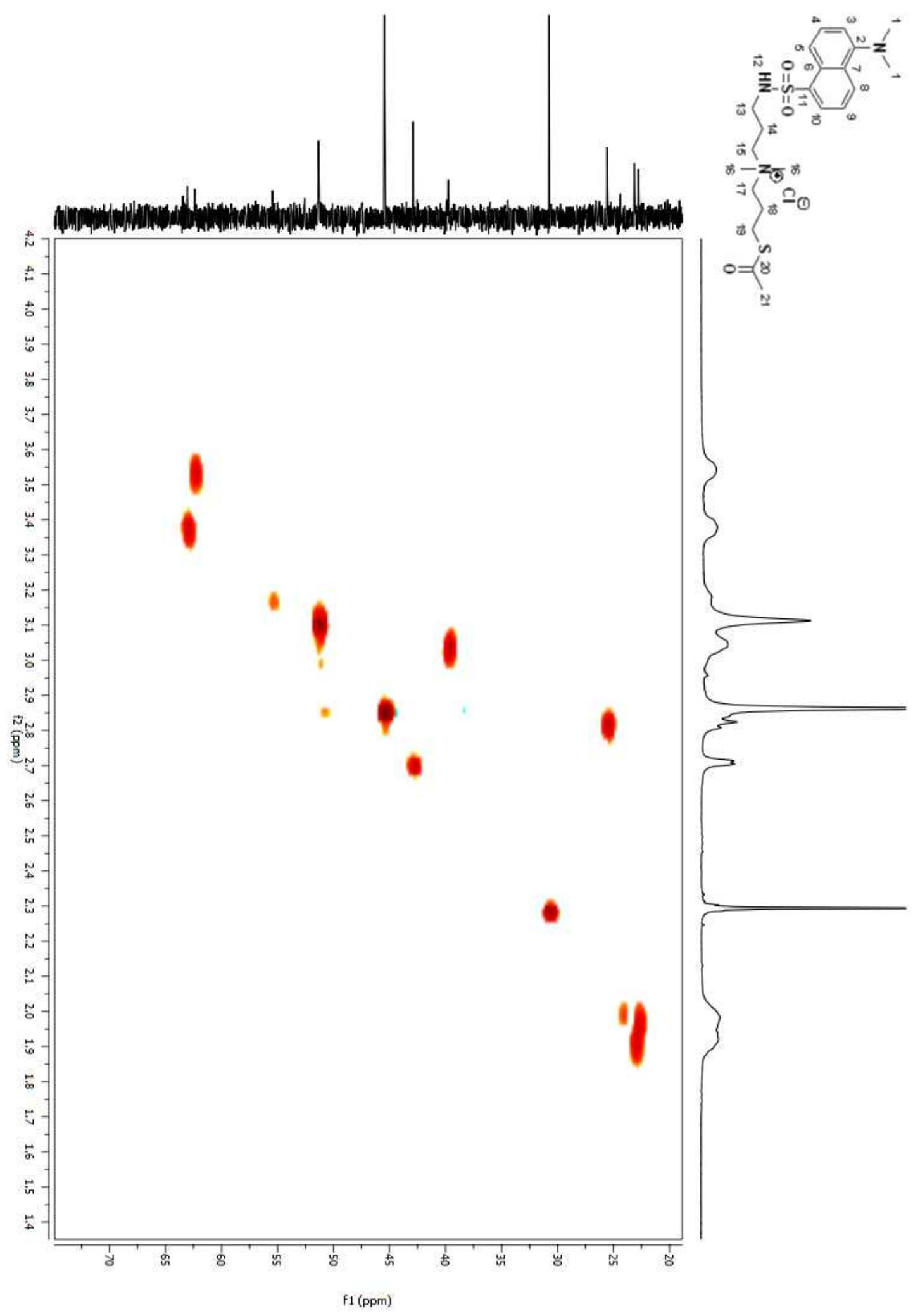

Figure A 365. HSQC 2D NMR spectrum of compound (214) in $\mathrm{CDCl}_{3}$. 


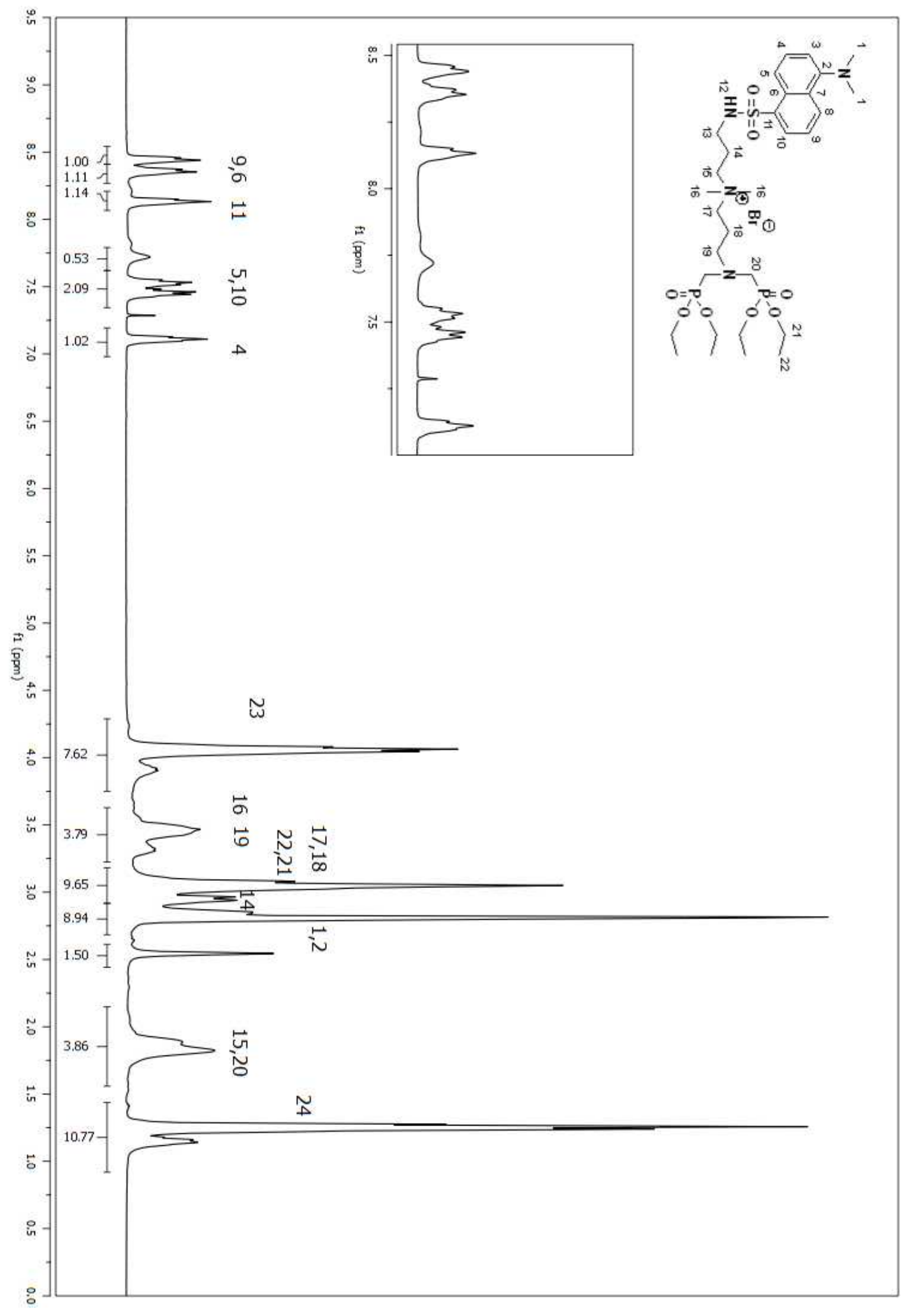

Figure A 366. ${ }^{1} \mathrm{H}$ NMR spectrum of compound (215) in $\mathrm{CDCl}_{3}$. 


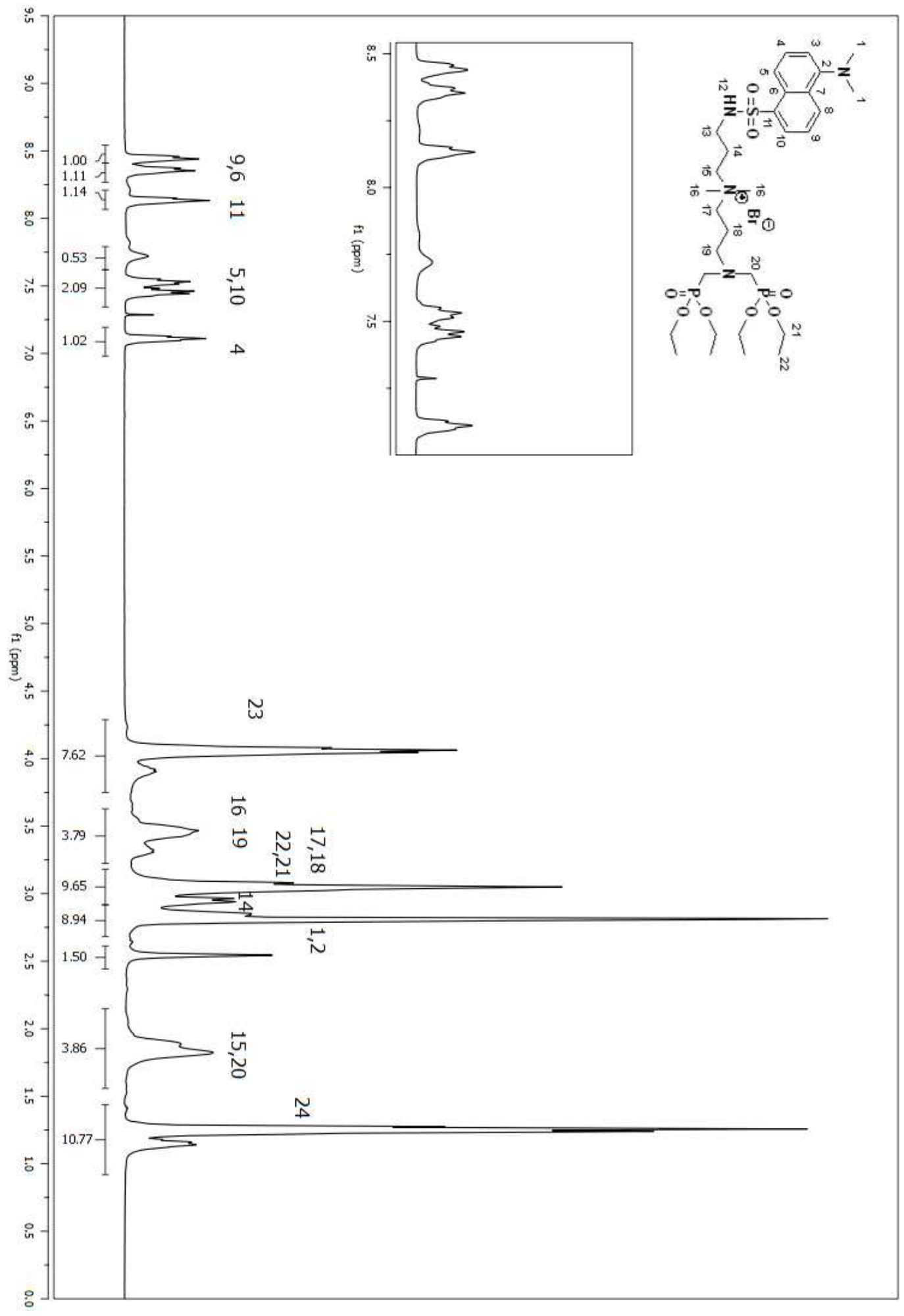

Figure A 367. ${ }^{13} \mathrm{C}$ NMR spectrum of compound (215) in $\mathrm{CDCl}_{3}$. 


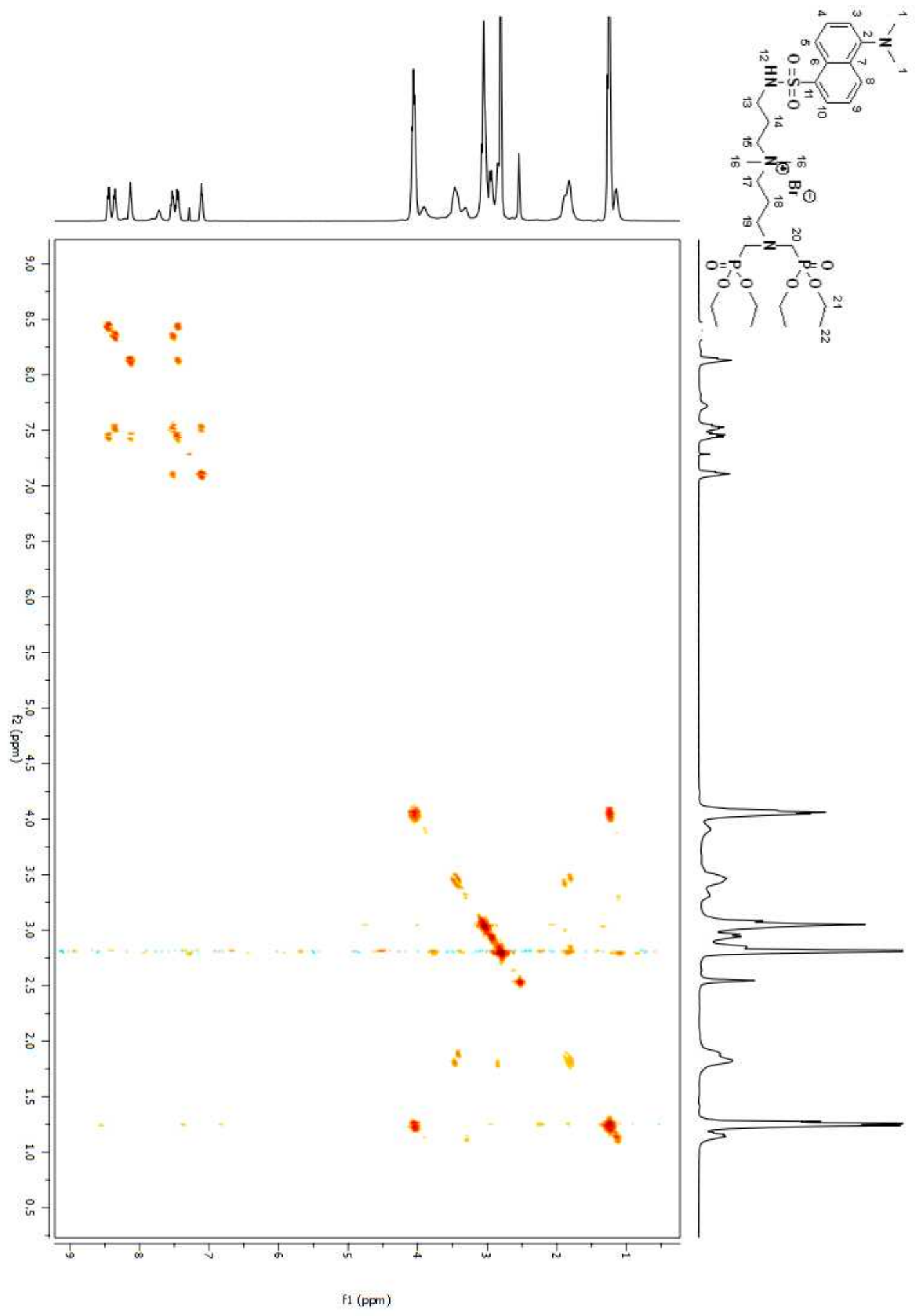

Figure A 368. COSY 2D NMR spectrum of compound (215) in $\mathrm{CDCl}_{3}$. 


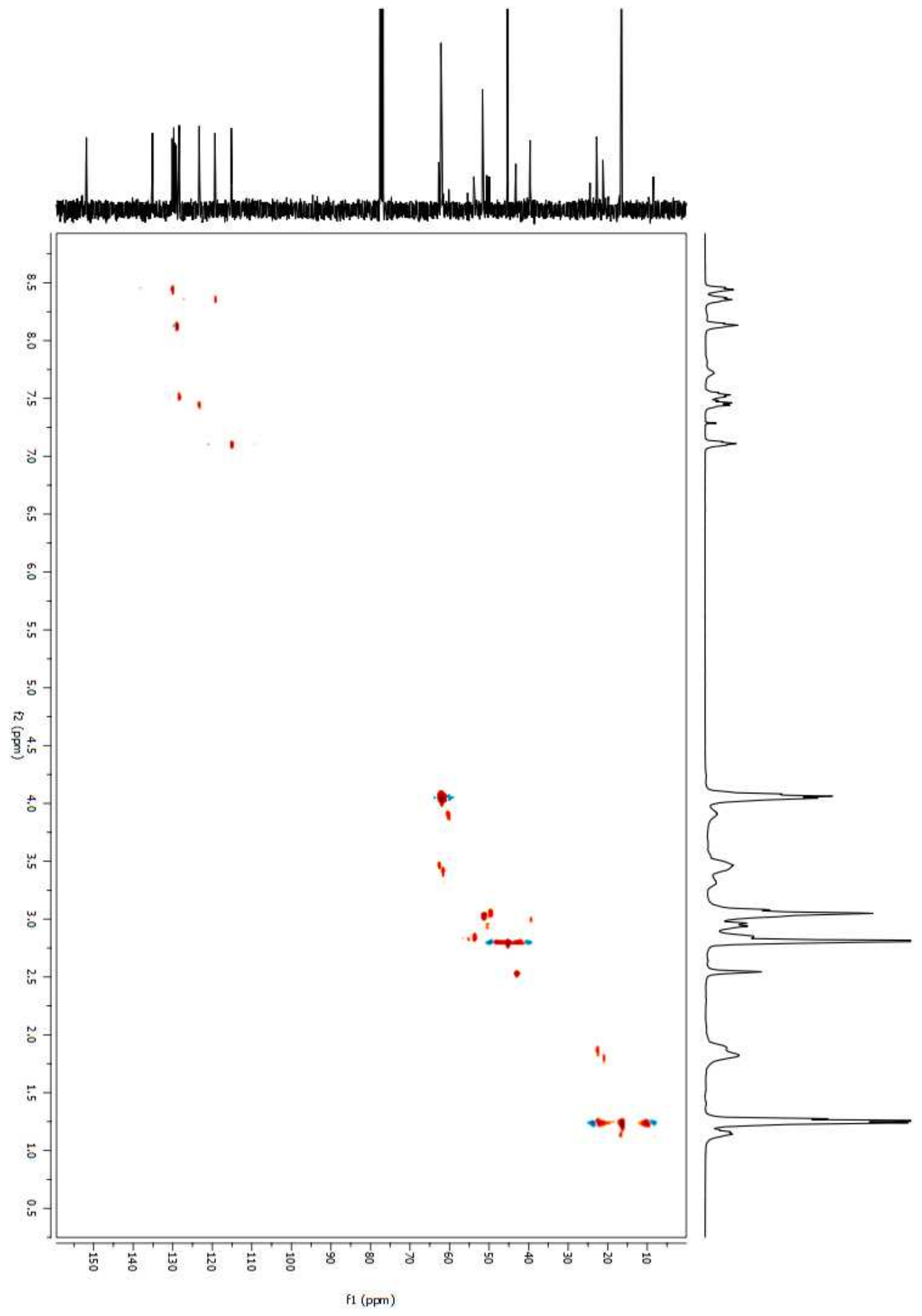

Figure A 369. HSQC 2D NMR spectrum of compound (215) in $\mathrm{CDCl}_{3}$. 


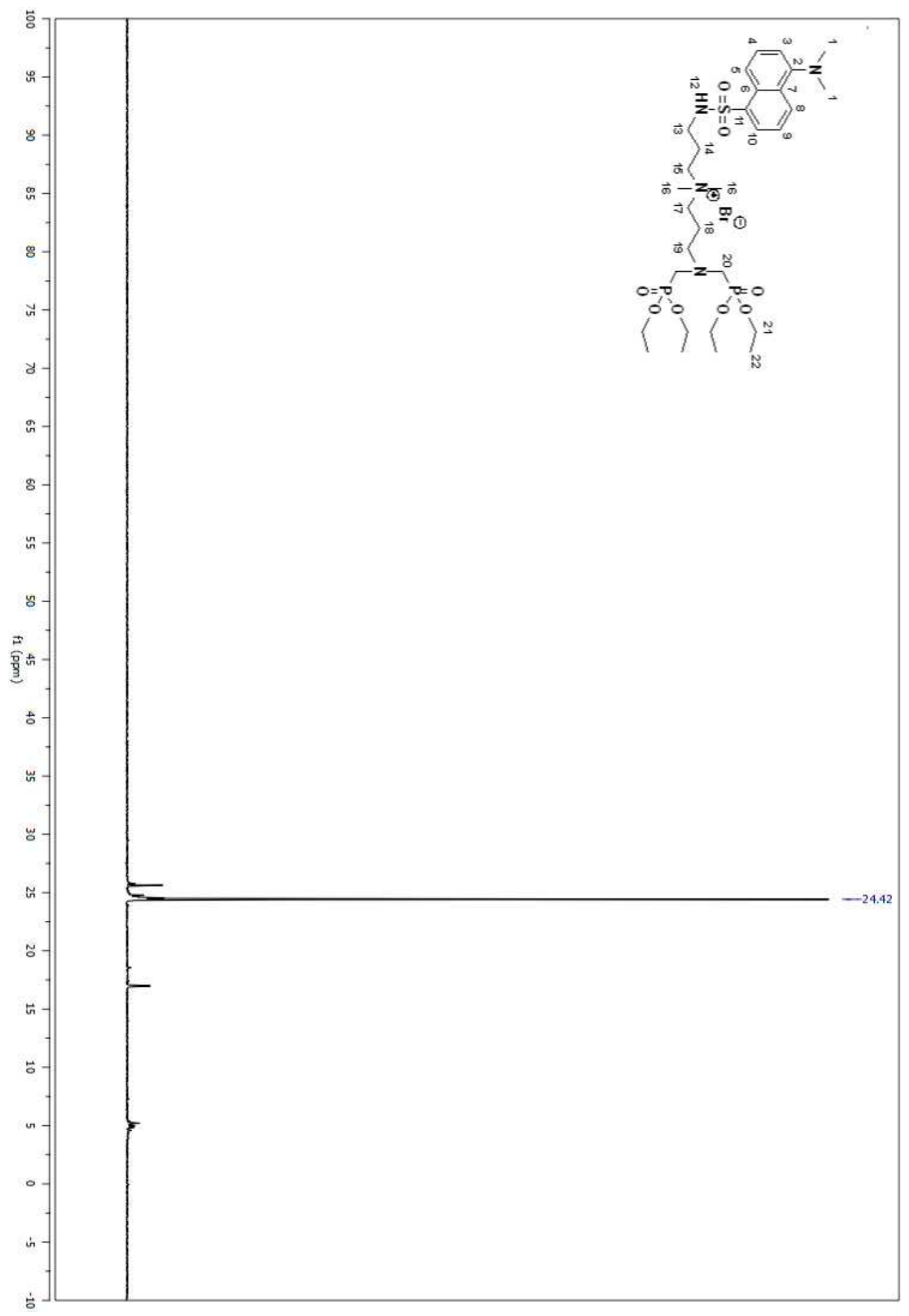

Figure A 370. ${ }^{31} \mathrm{P}$ NMR spectrum of compound (215) in $\mathrm{CDCl}_{3}$ 


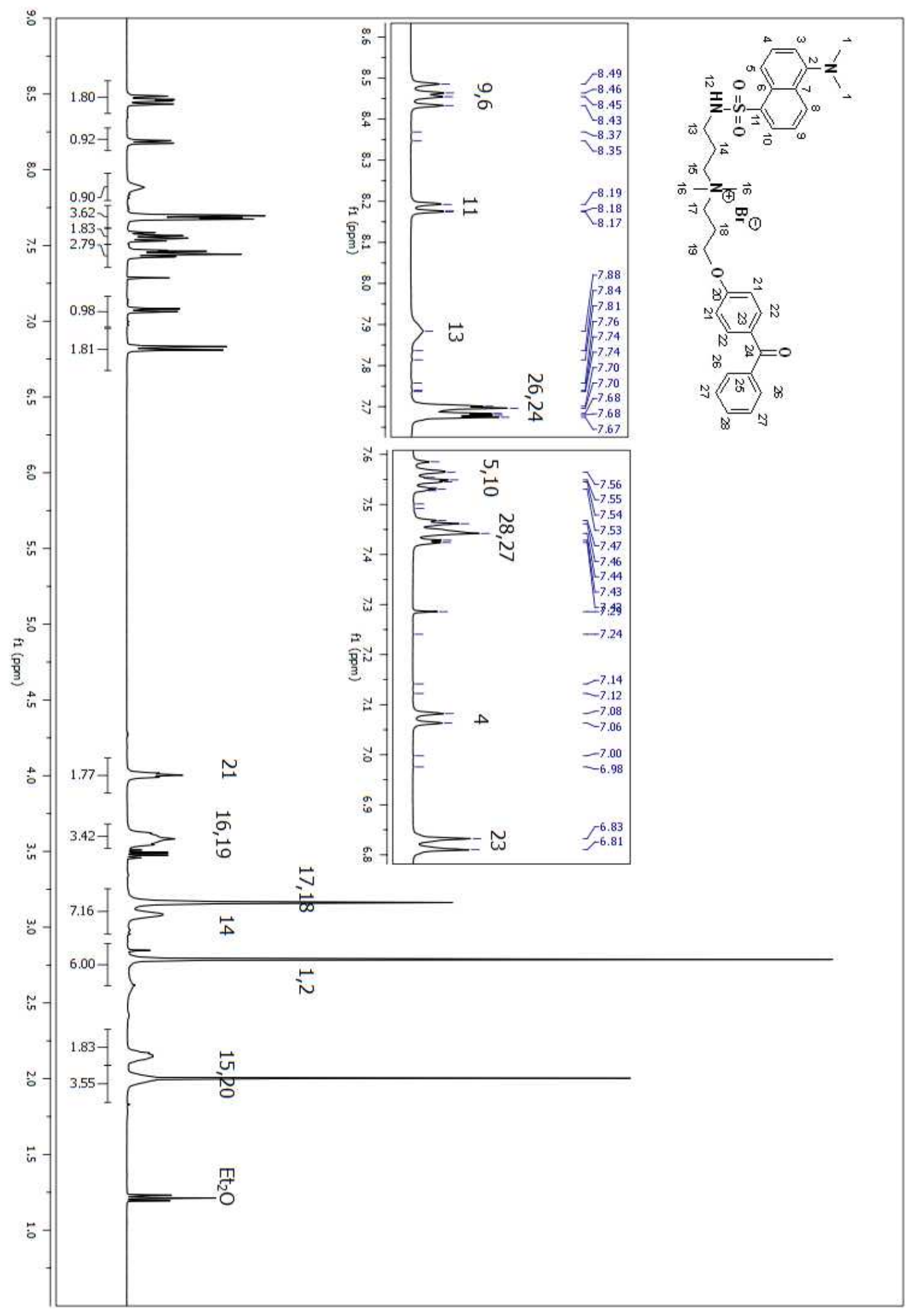

Figure A 371. ${ }^{1} \mathrm{H}$ NMR spectrum of compound (216) in $\mathrm{CDCl}_{3}$. 


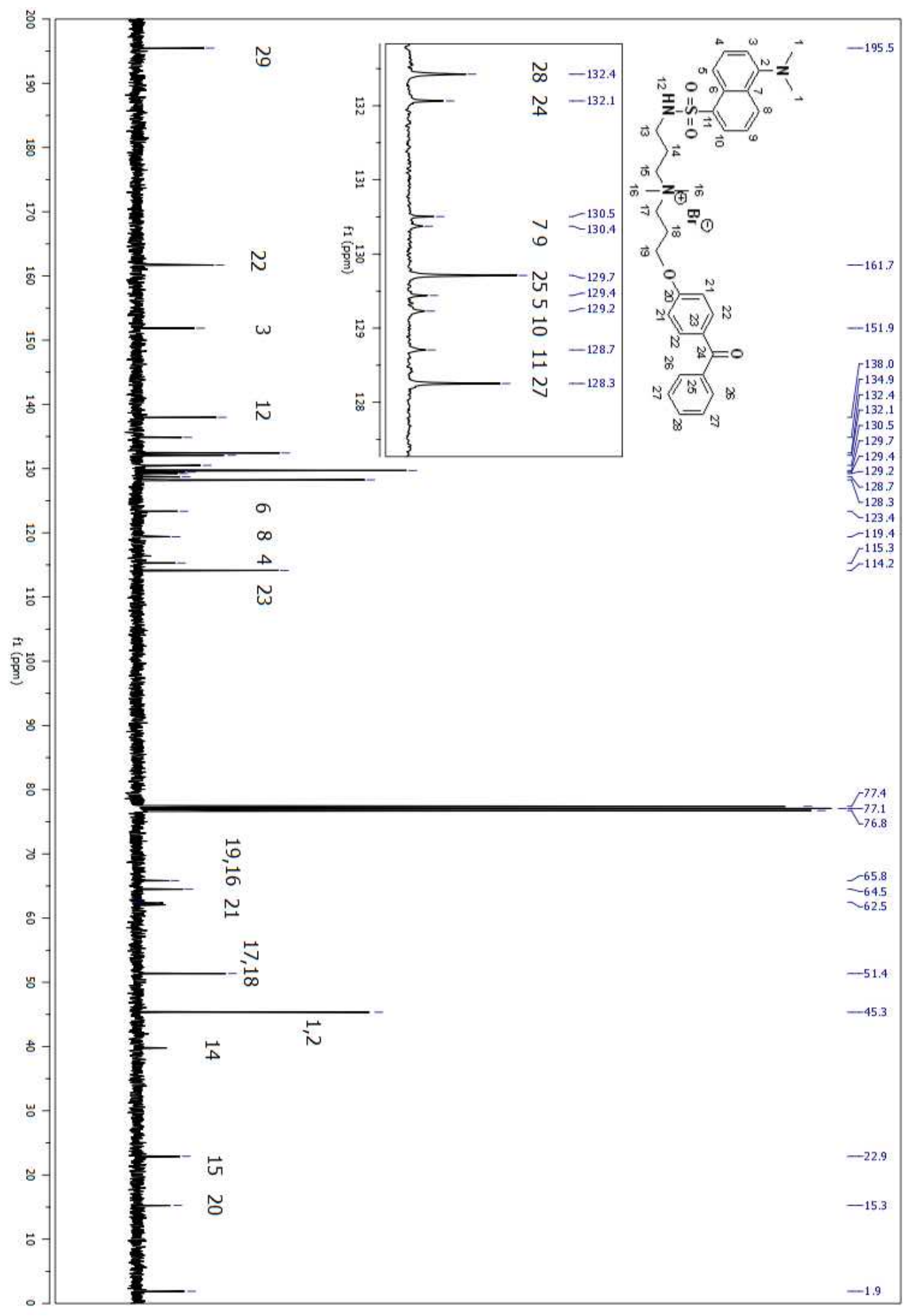

Figure A 372. ${ }^{13} \mathrm{C}$ NMR spectrum of compound (216) in $\mathrm{CDCl}_{3}$. 


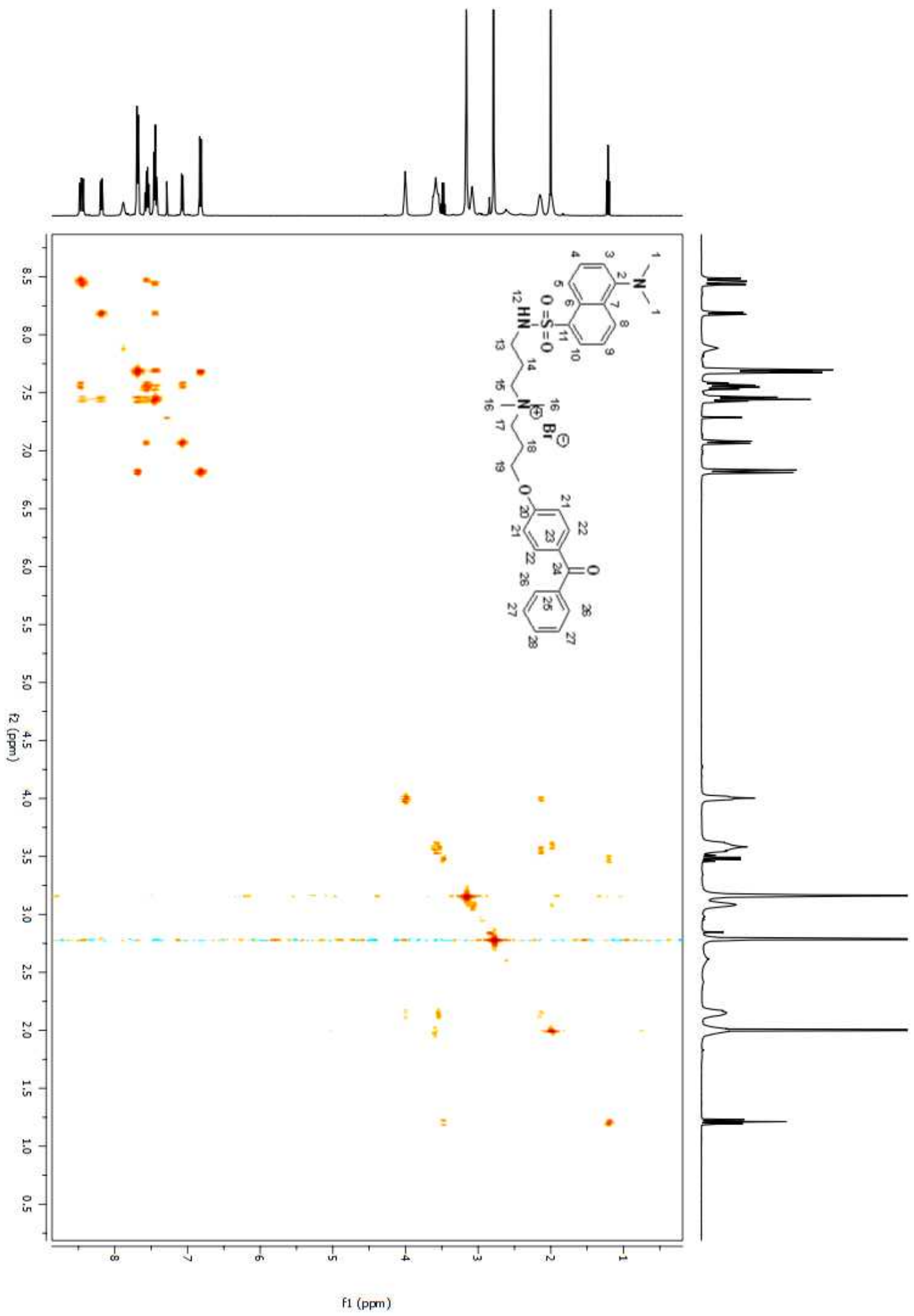

Figure A 373. COSY 2D NMR spectrum of compound (216) in $\mathrm{CDCl}_{3}$. 


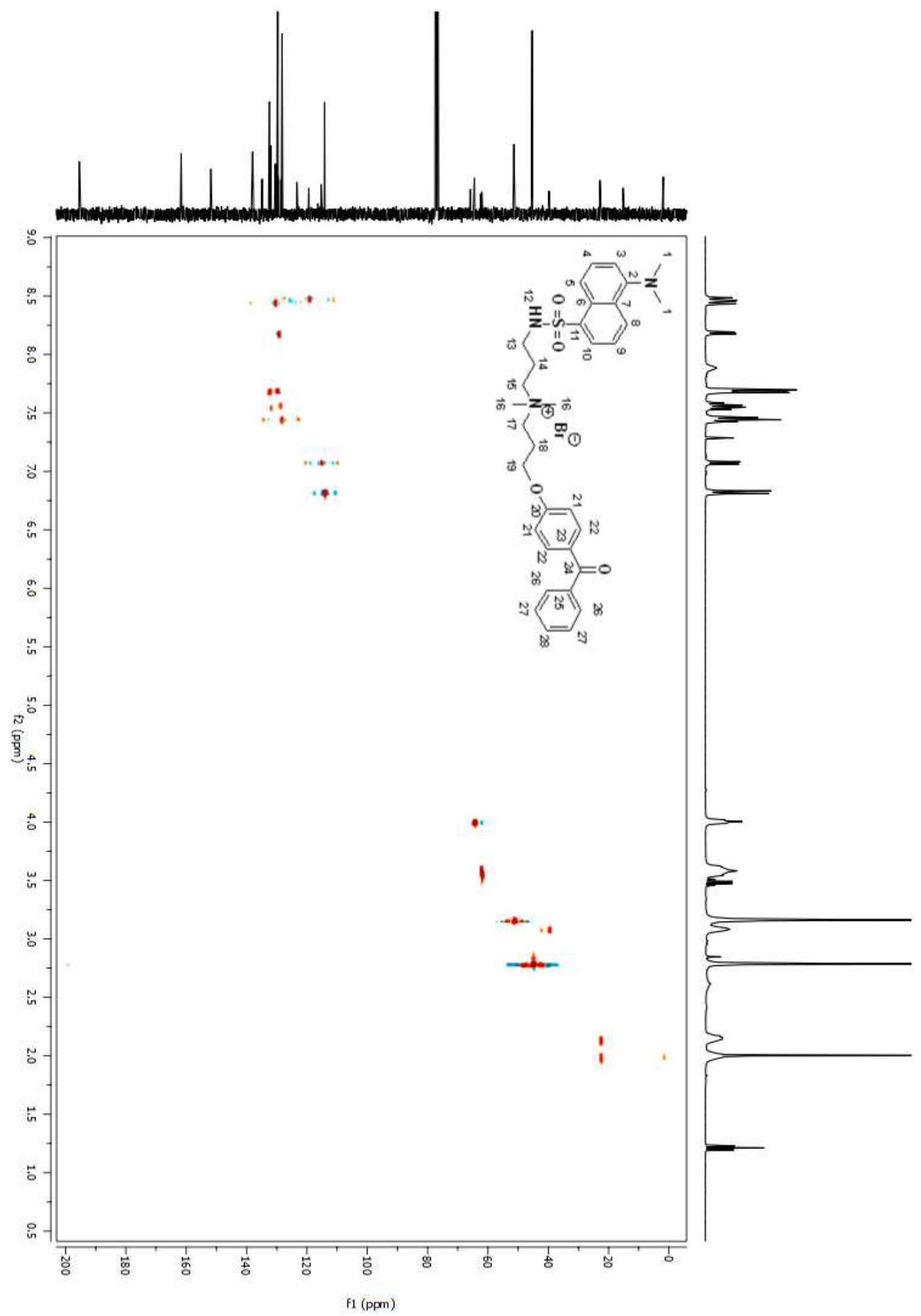

Figure A 374. HSQC 2D NMR spectrum of compound (216) in $\mathrm{CDCl}_{3}$. 


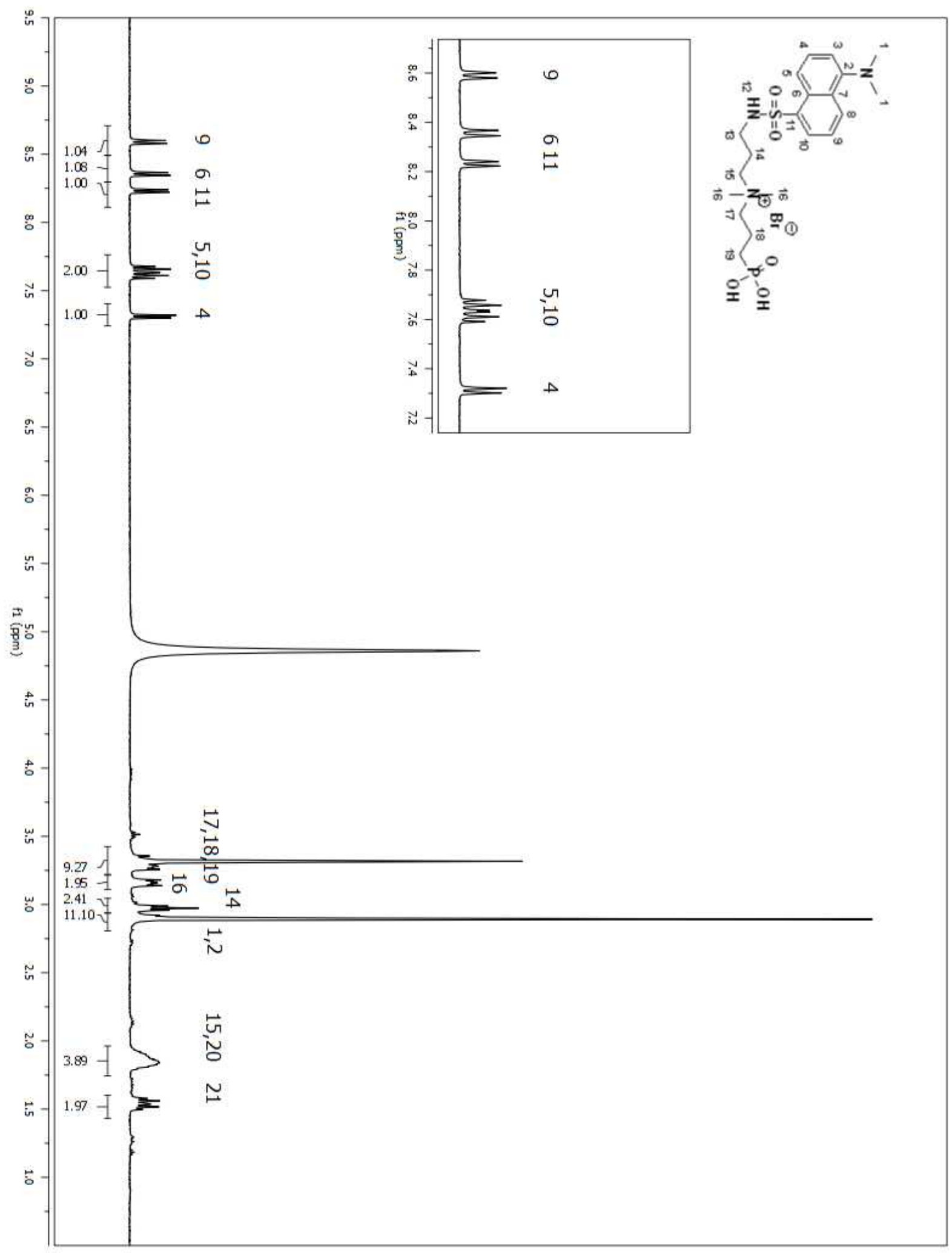

Figure A 375. ${ }^{1} \mathrm{H}$ NMR spectrum of compound (217) in $\mathrm{D}_{2} \mathrm{O}$. 


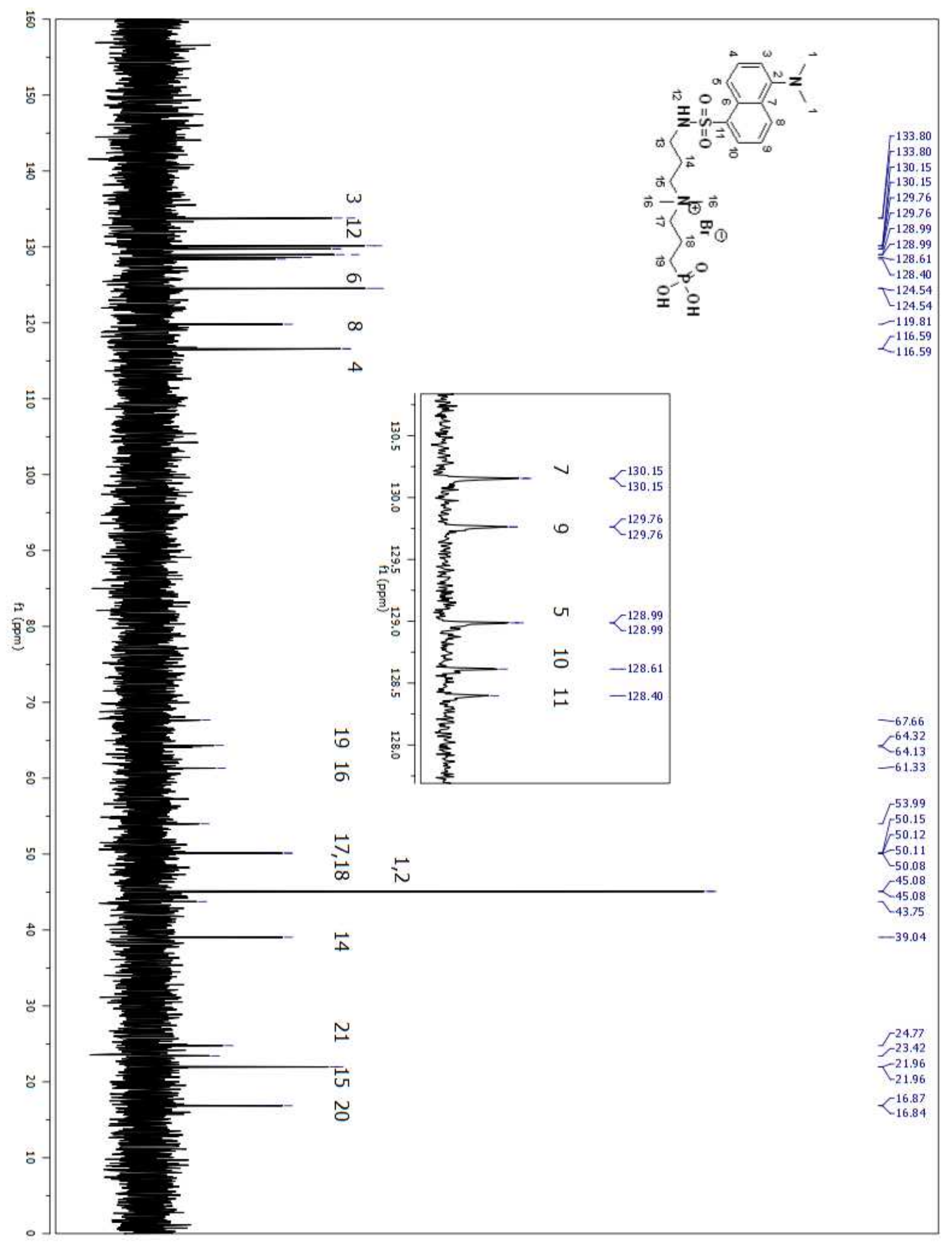

Figure A 376. ${ }^{13} \mathrm{C}$ NMR spectrum of compound (217) in $\mathrm{D}_{2} \mathrm{O}$. 


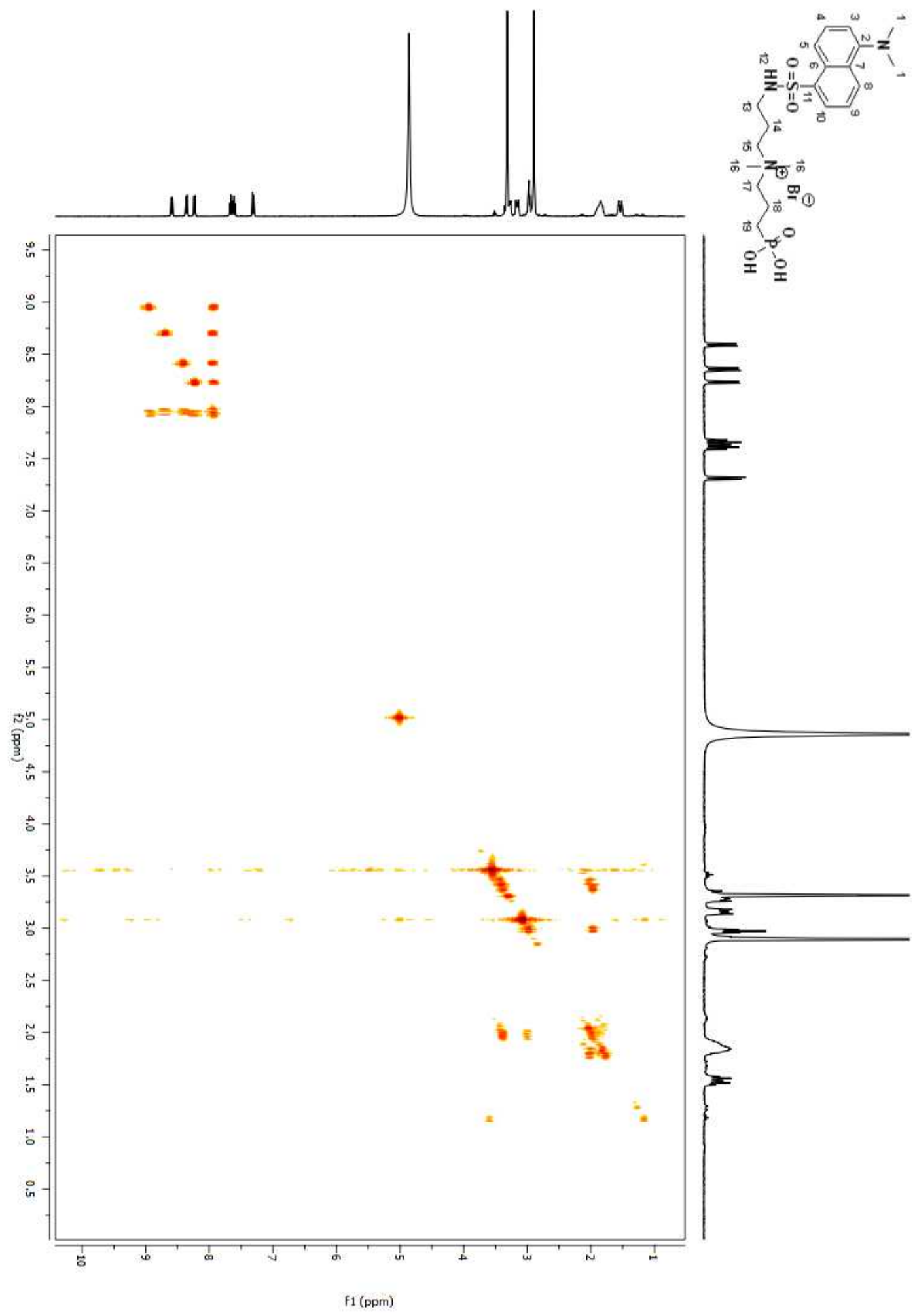

Figure A 377. COSY 2D NMR spectrum of compound (217) in $\mathrm{D}_{2} \mathrm{O}$. 


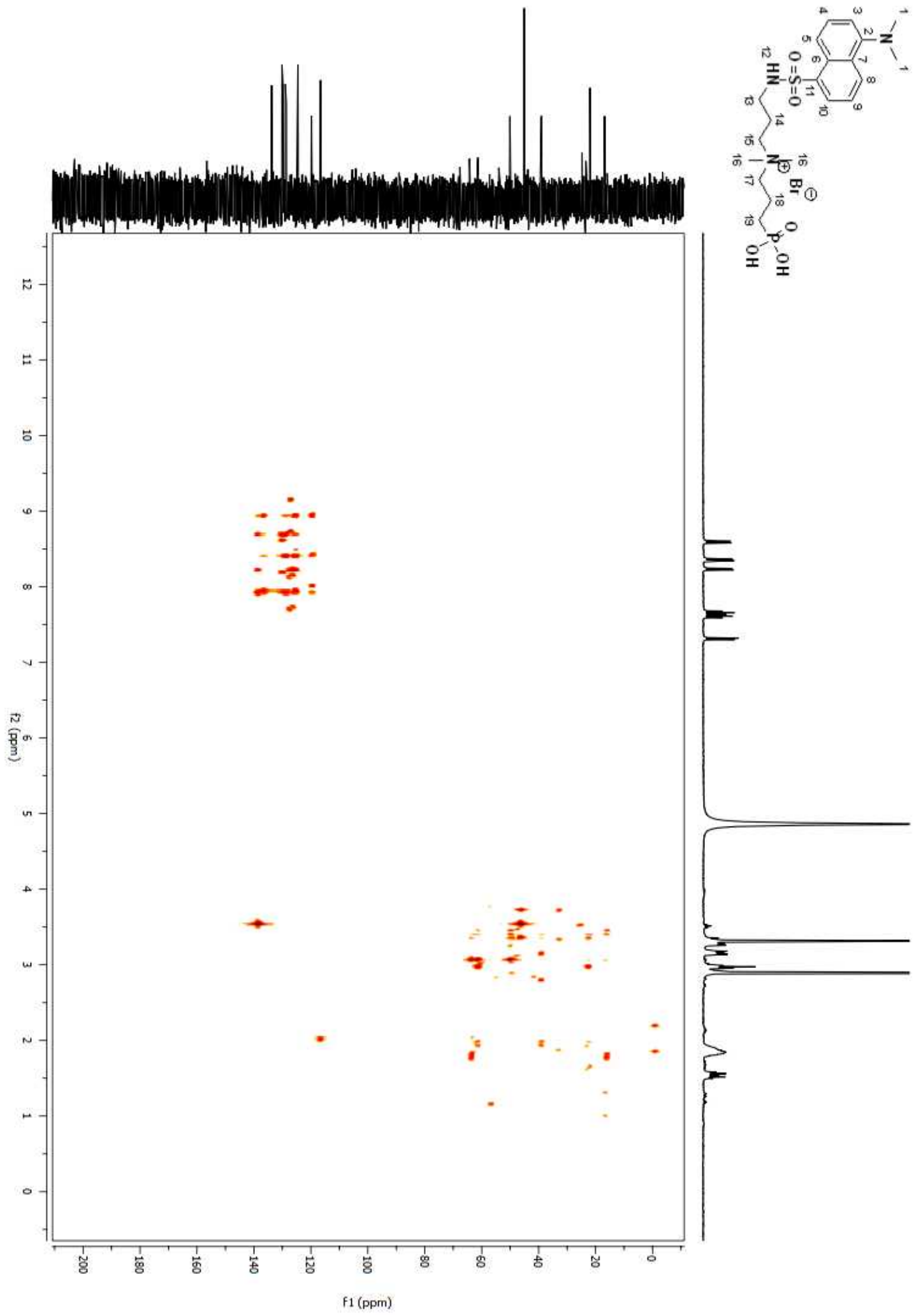

Figure A 378. HSQC 2D NMR spectrum of compound (217) in $\mathrm{D}_{2} \mathrm{O}$. 


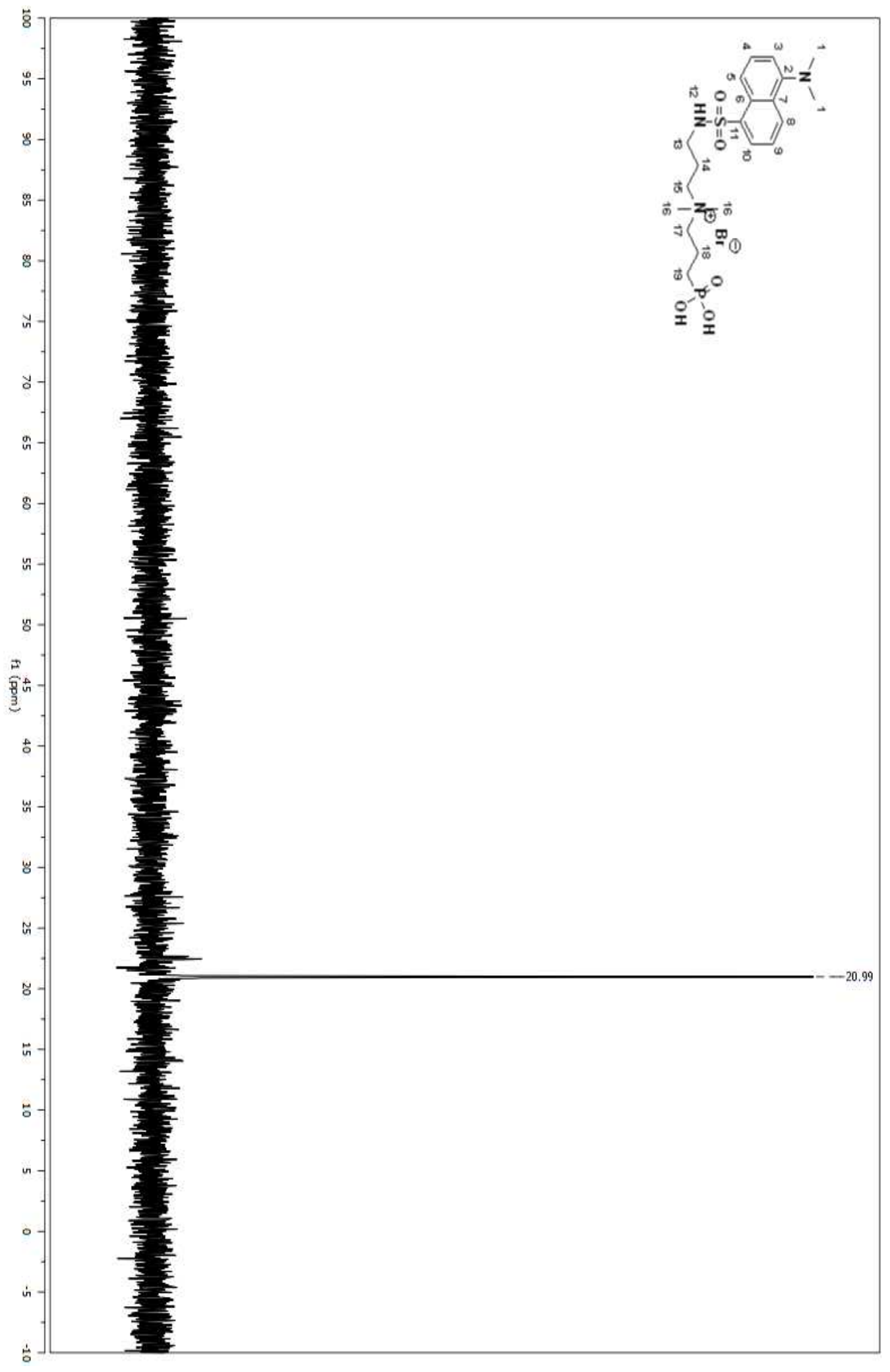

Figure A 379. ${ }^{31} \mathrm{P}$ NMR spectrum of compound in (217) $\mathrm{D}_{2} \mathrm{O}$. 


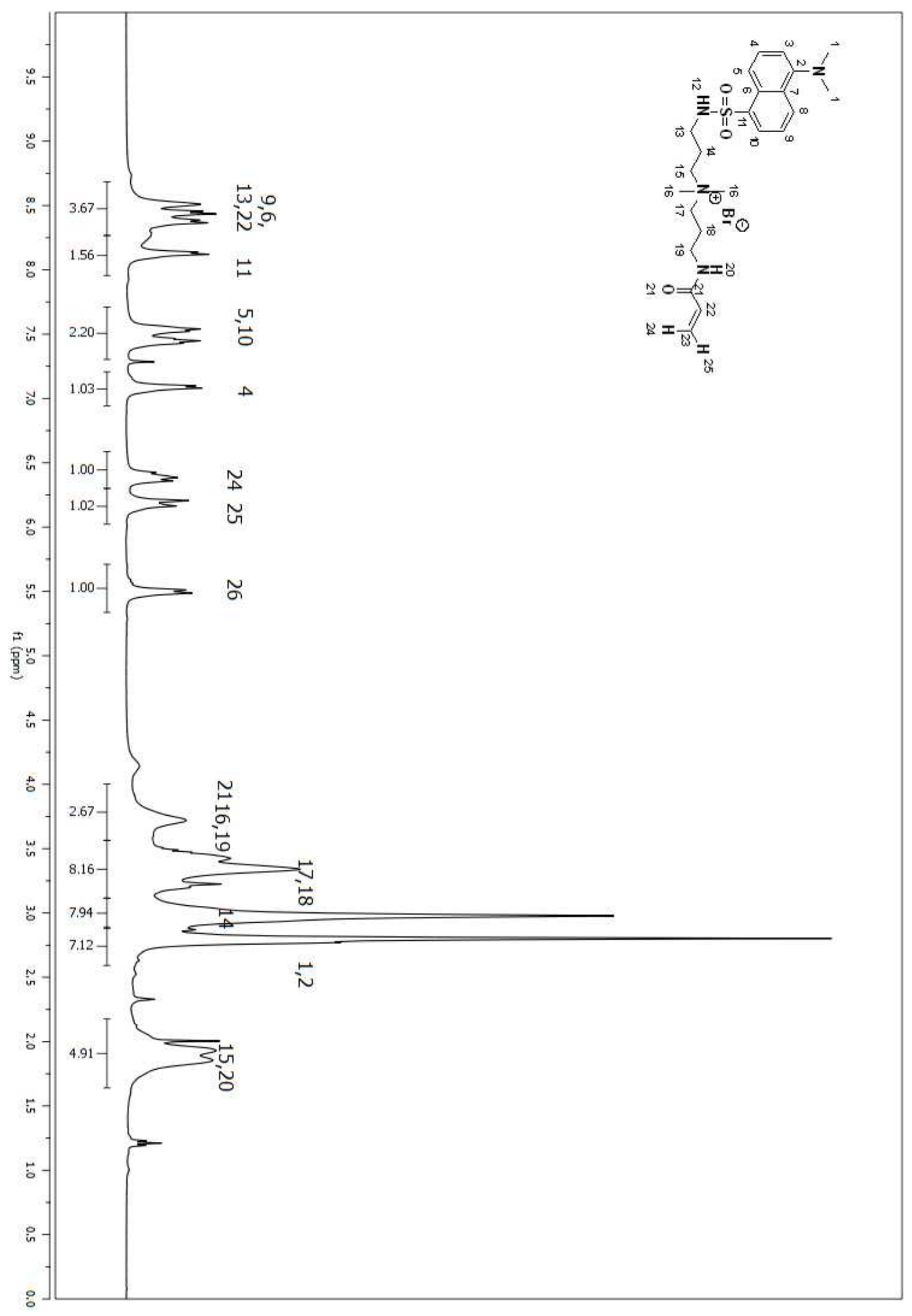

Figure A 380. ${ }^{1} \mathrm{H}$ NMR spectrum of compound (219) in $\mathrm{CDCl}_{3}$. 


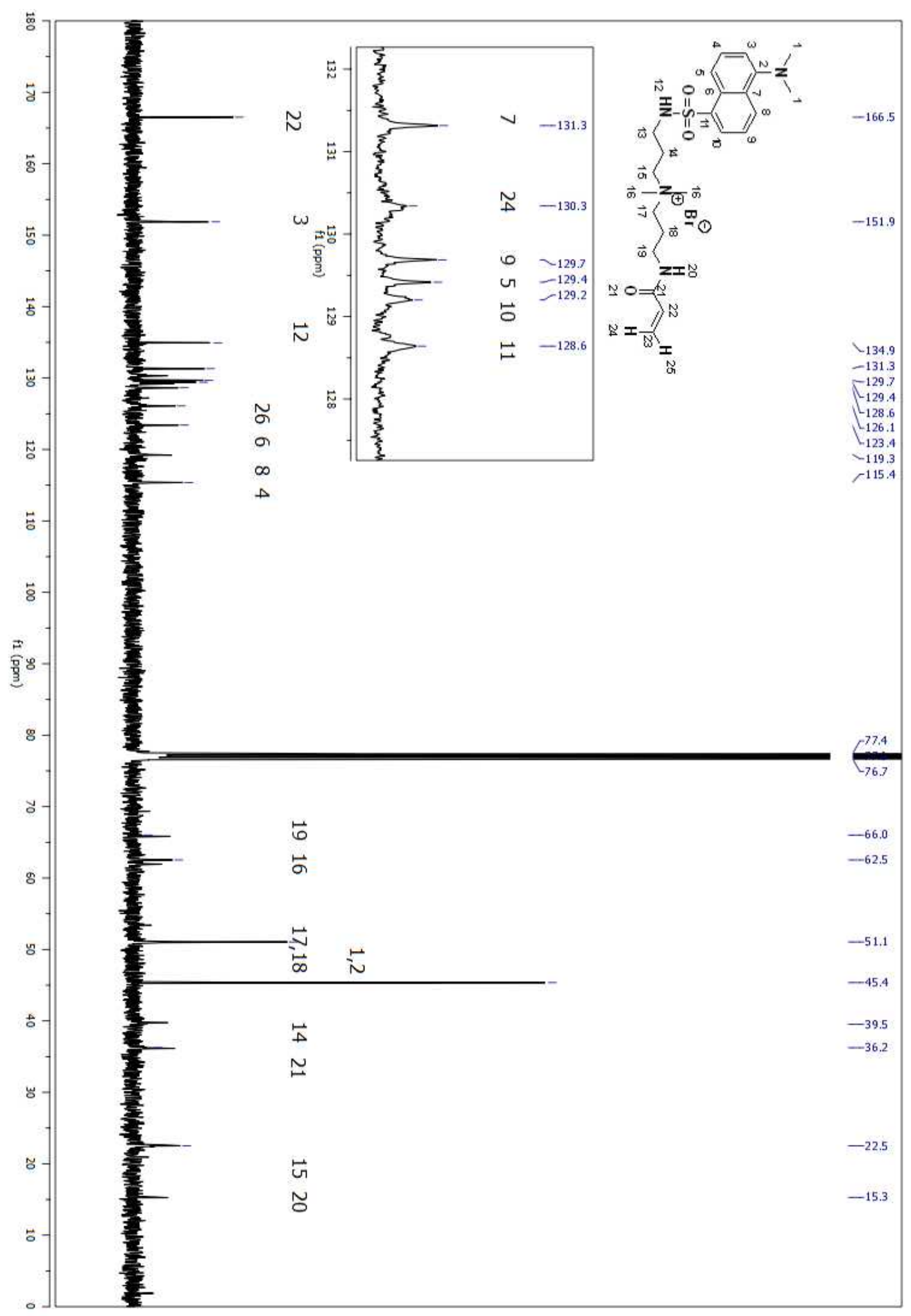

Figure A 381. ${ }^{13} \mathrm{C}$ NMR spectrum of compound (219) in $\mathrm{CDCl}_{3}$. 


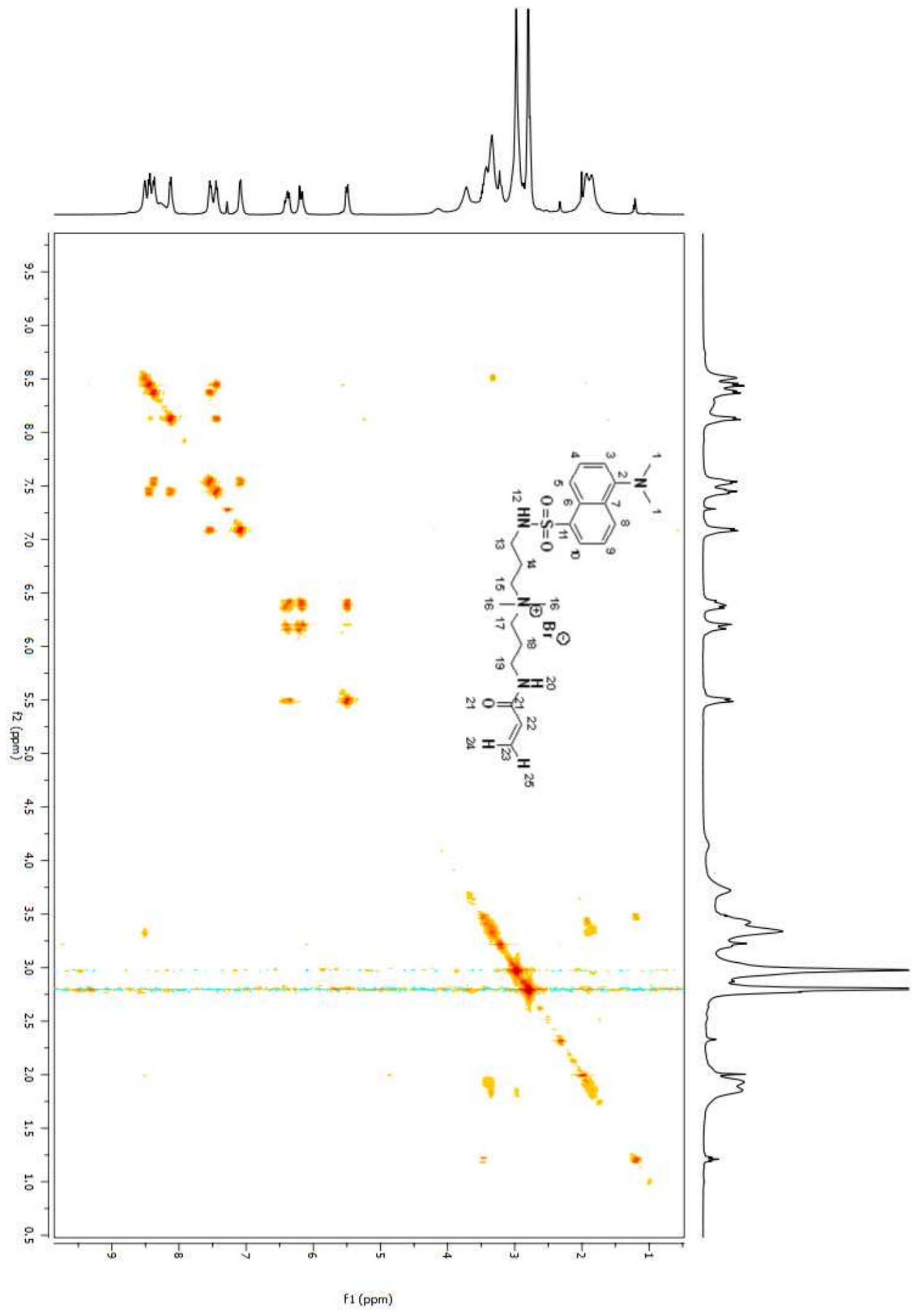

Figure A 382. COSY 2D NMR spectrum of compound (219) in $\mathrm{CDCl}_{3}$. 


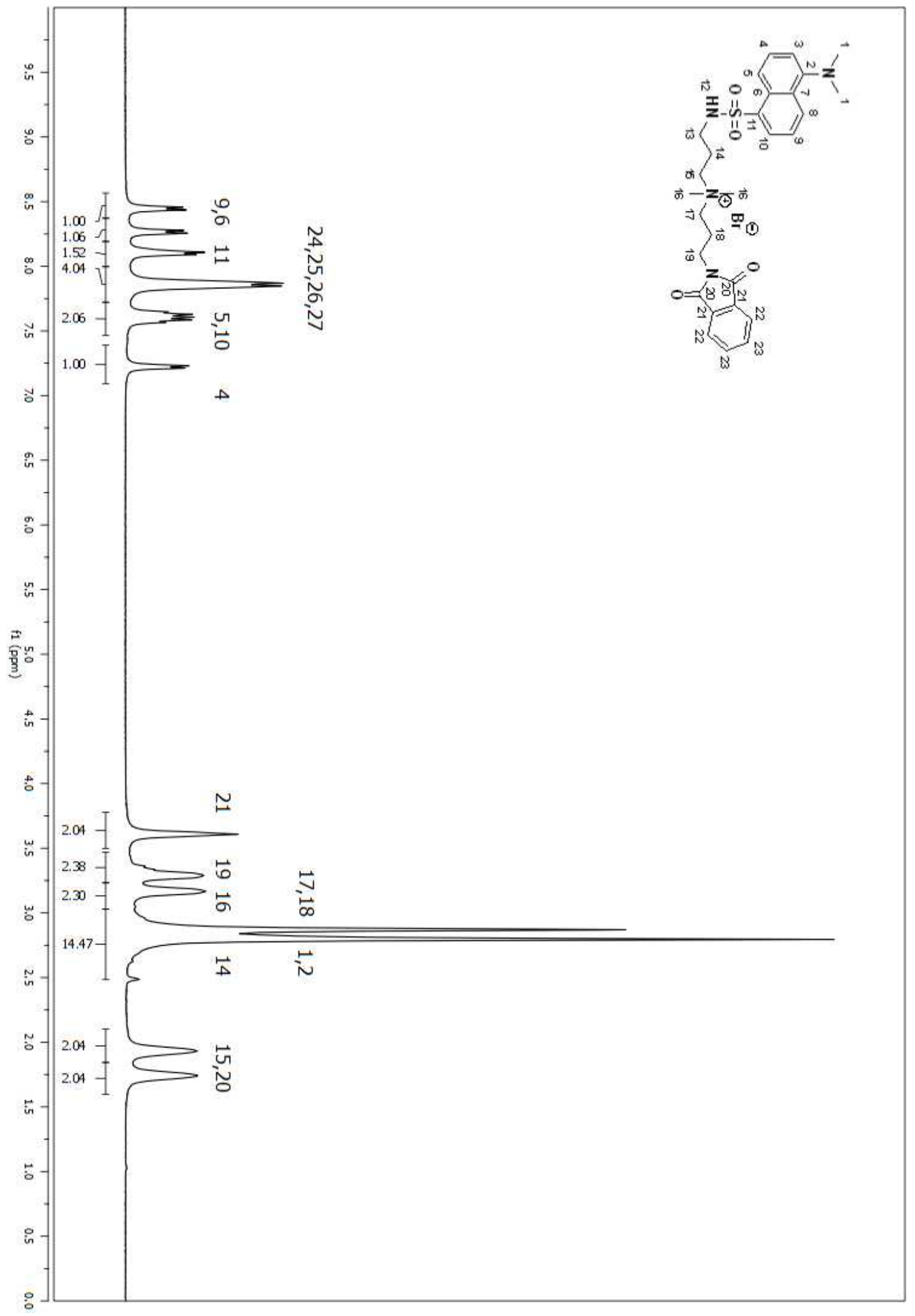

Figure A 383. ${ }^{1} \mathrm{H}$ NMR spectrum of compound (220) in DMSO-d6. 


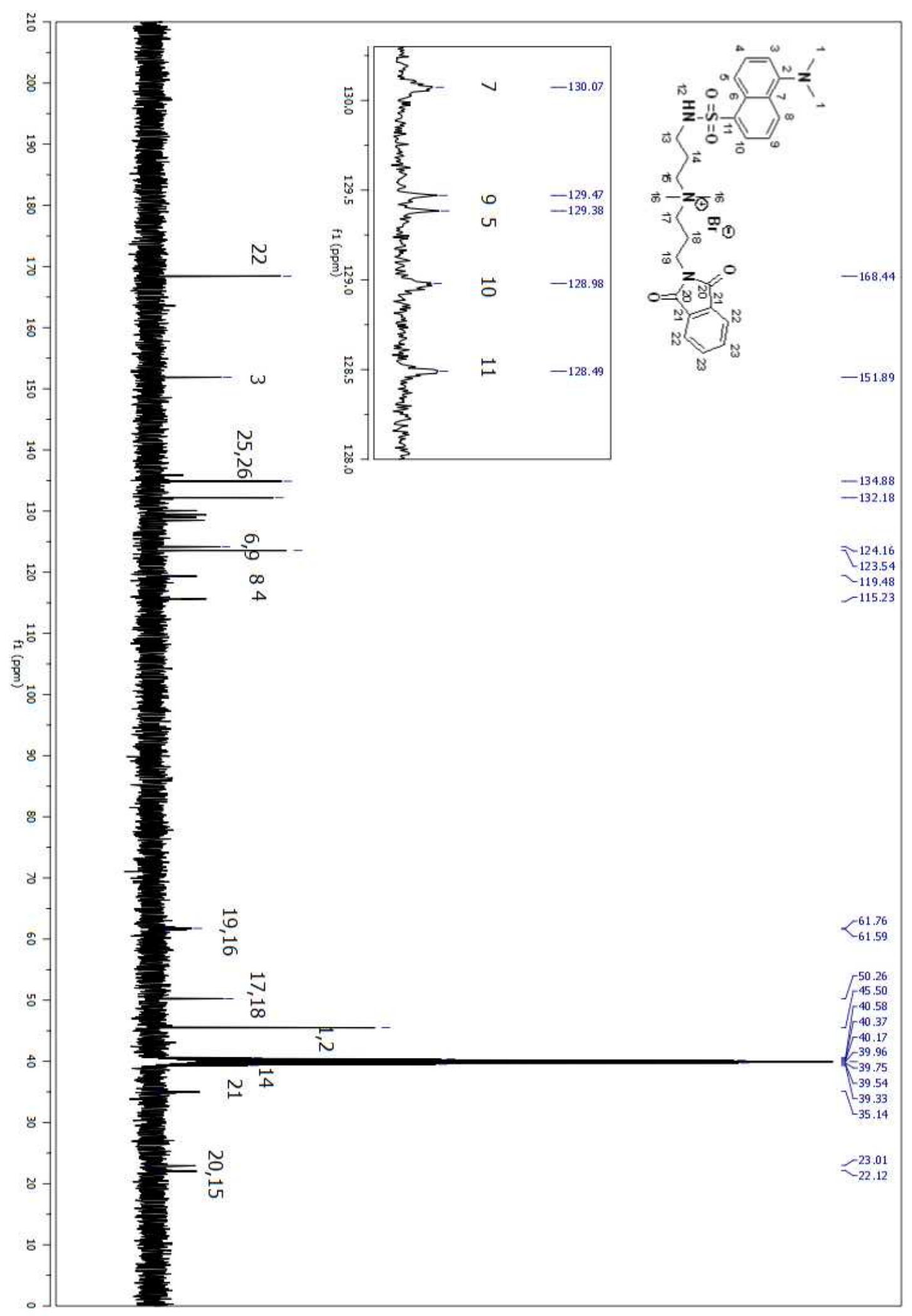

Figure A 384. ${ }^{13} \mathrm{C}$ NMR spectrum of compound (220) in DMSO-d6. 


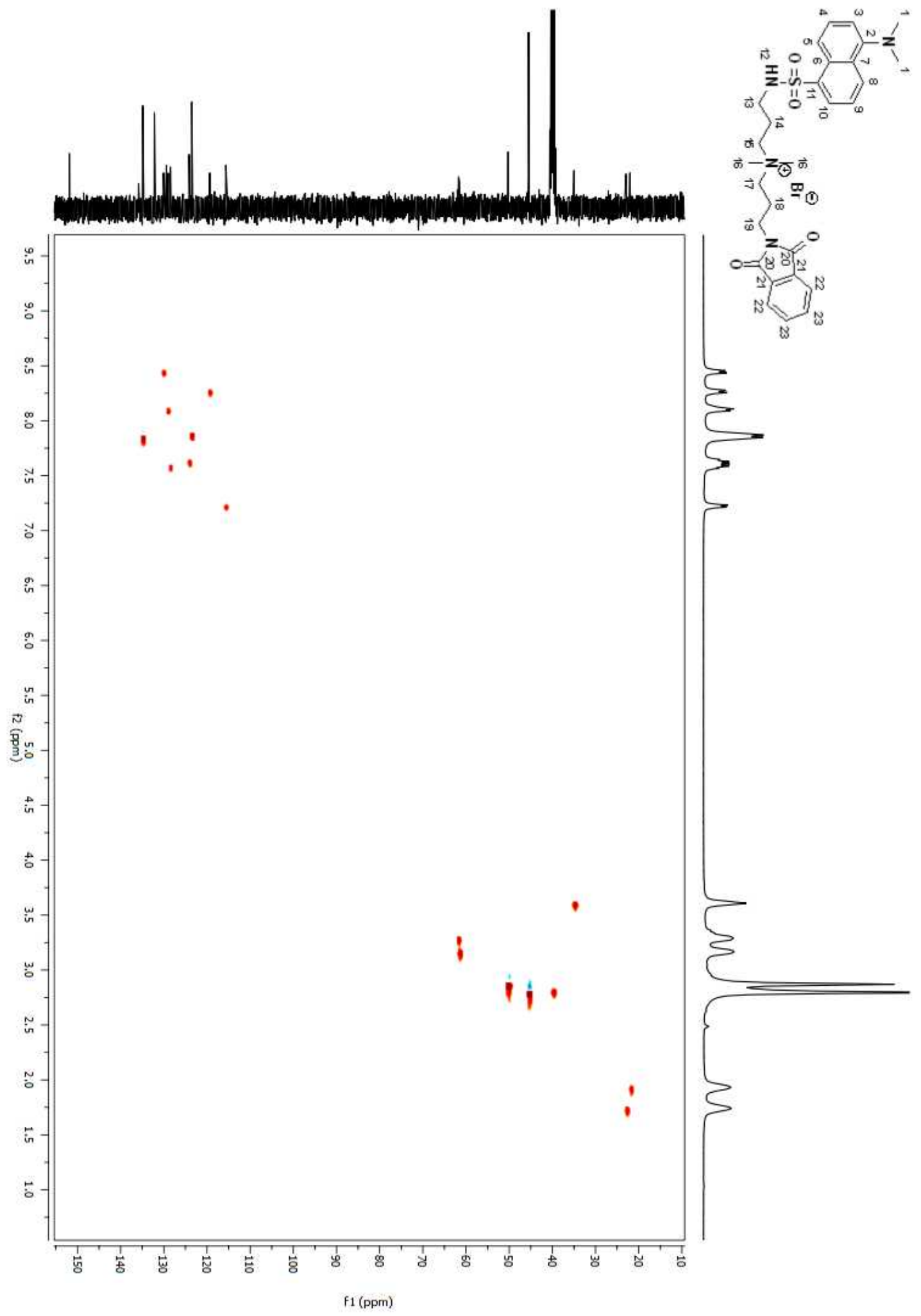

Figure A 385. HSQC 2D NMR spectrum of compound (220) in DMSO-d6. 


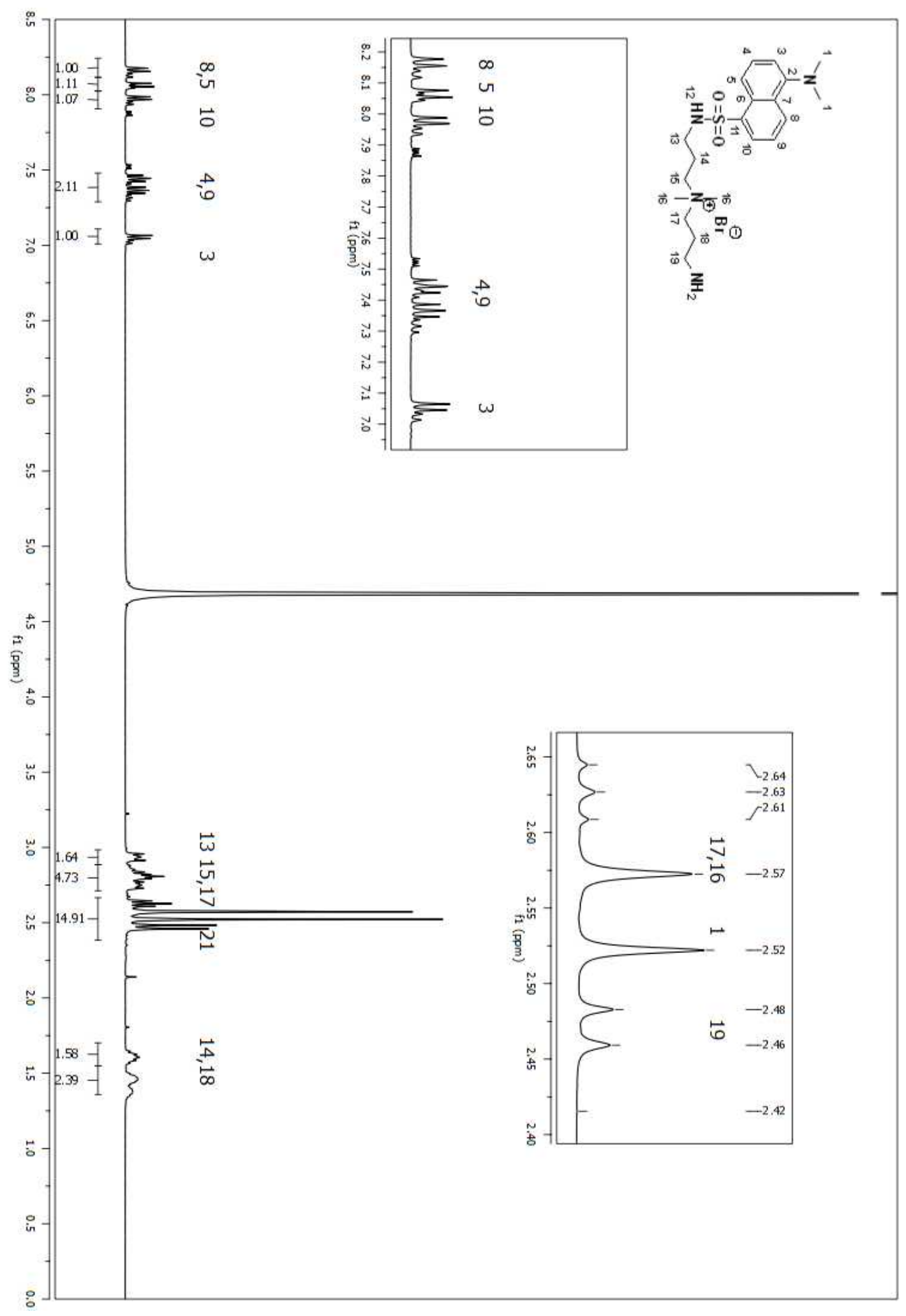

Figure A 386. ${ }^{1} \mathrm{H}$ NMR spectrum of compound (221) in $\mathrm{D}_{2} \mathrm{O}$. 


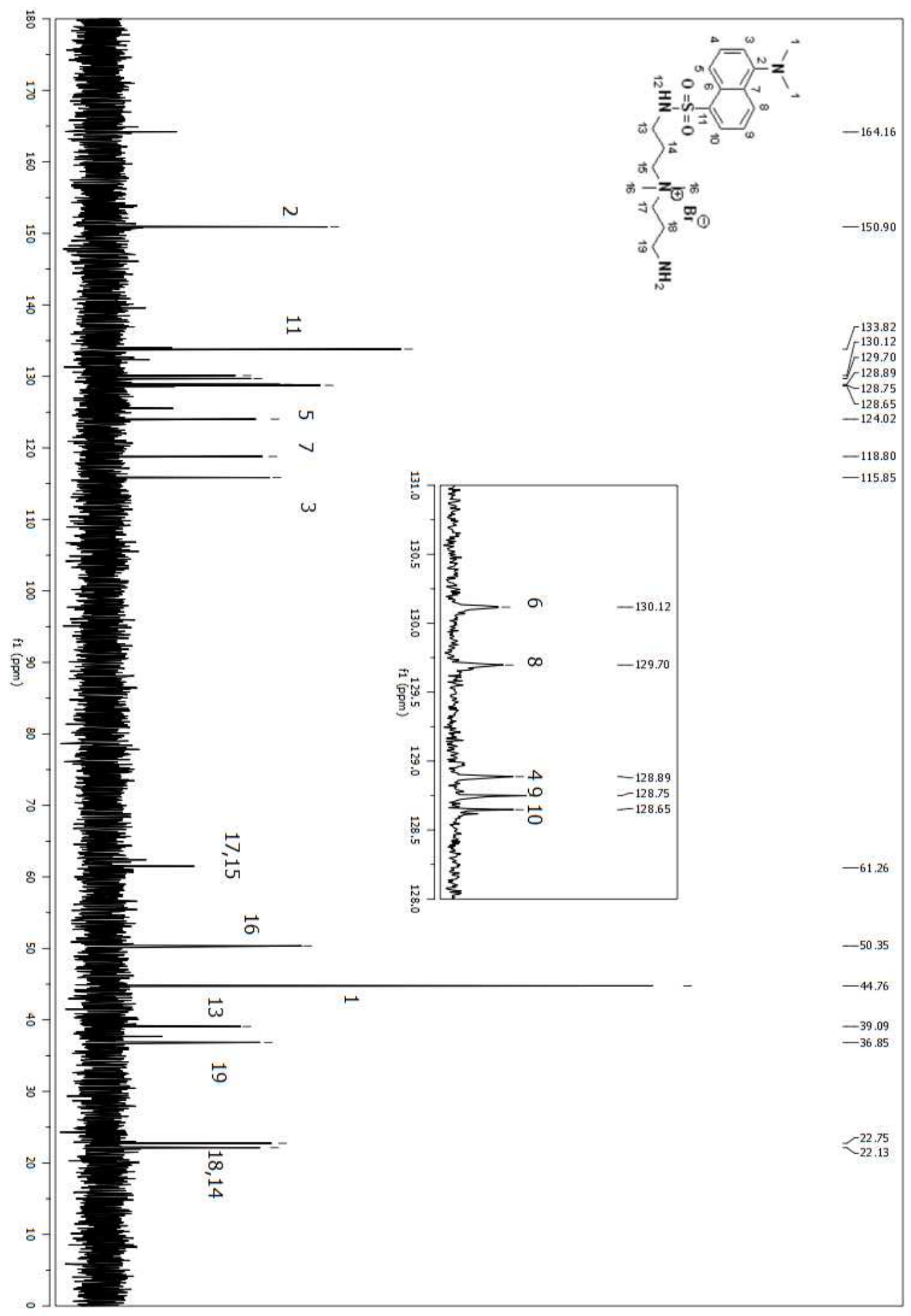

Figure A 387. ${ }^{13} \mathrm{C}$ NMR spectrum of compound (221) in $\mathrm{D}_{2} \mathrm{O}$. 


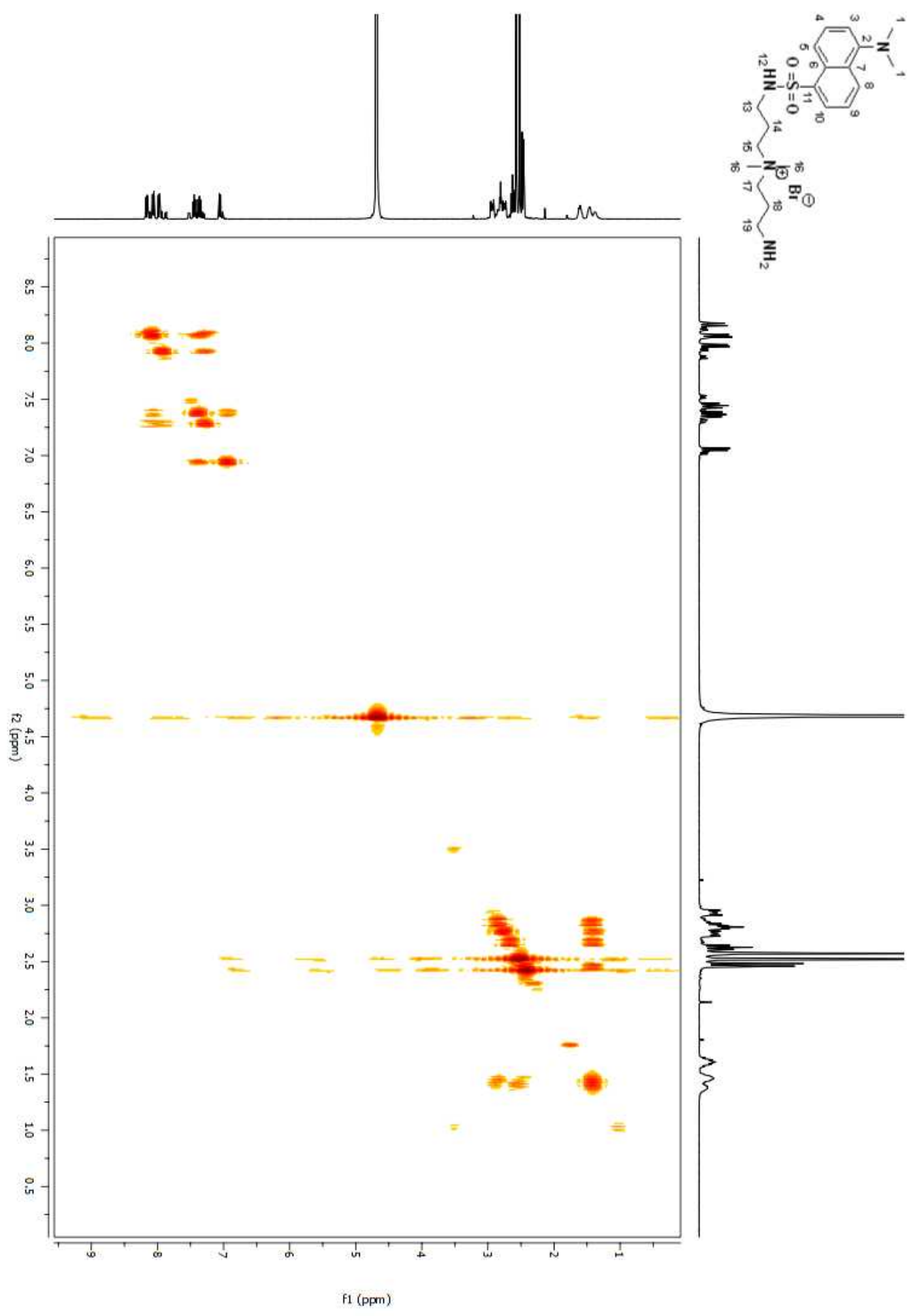

Figure A 388. COSY 2D NMR spectrum of compound (221) in $\mathrm{D}_{2} \mathrm{O}$. 


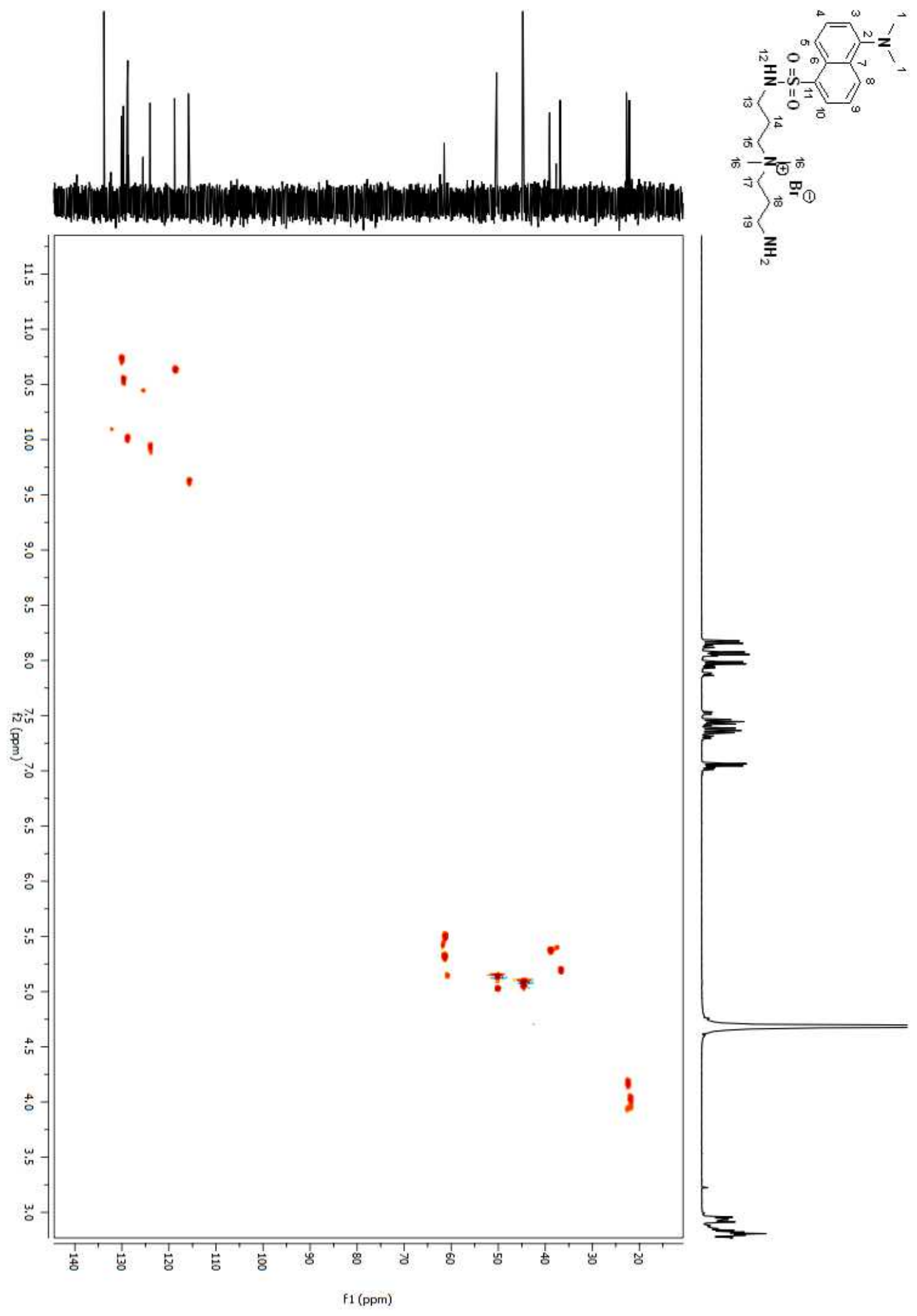

Figure A 389. HSQC 2D NMR spectrum of compound (221) in $\mathrm{D}_{2} \mathrm{O}$. 


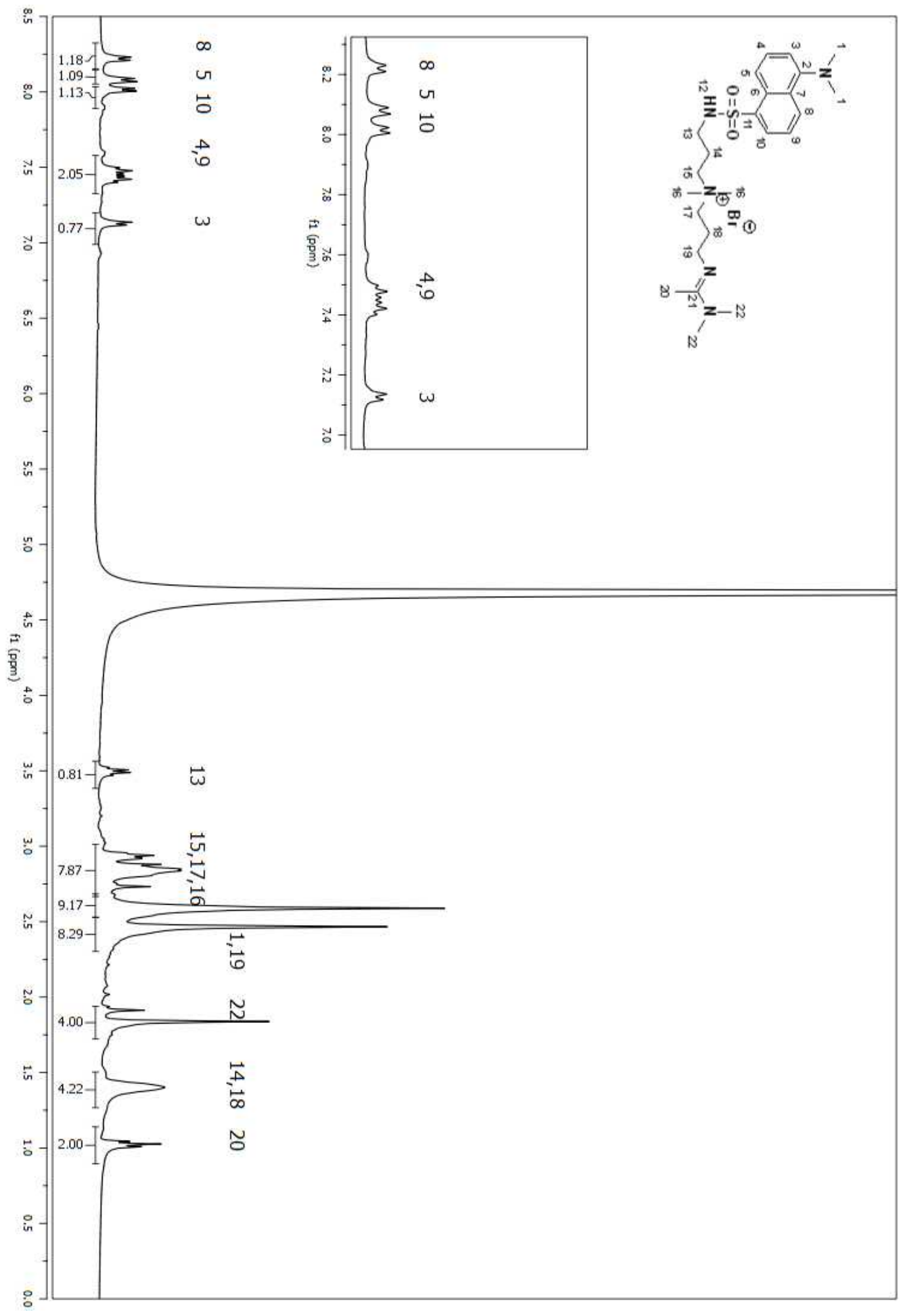

Figure A 390. ${ }^{1} \mathrm{H}$ NMR spectrum of compound (222) in $\mathrm{D}_{2} \mathrm{O}$. 


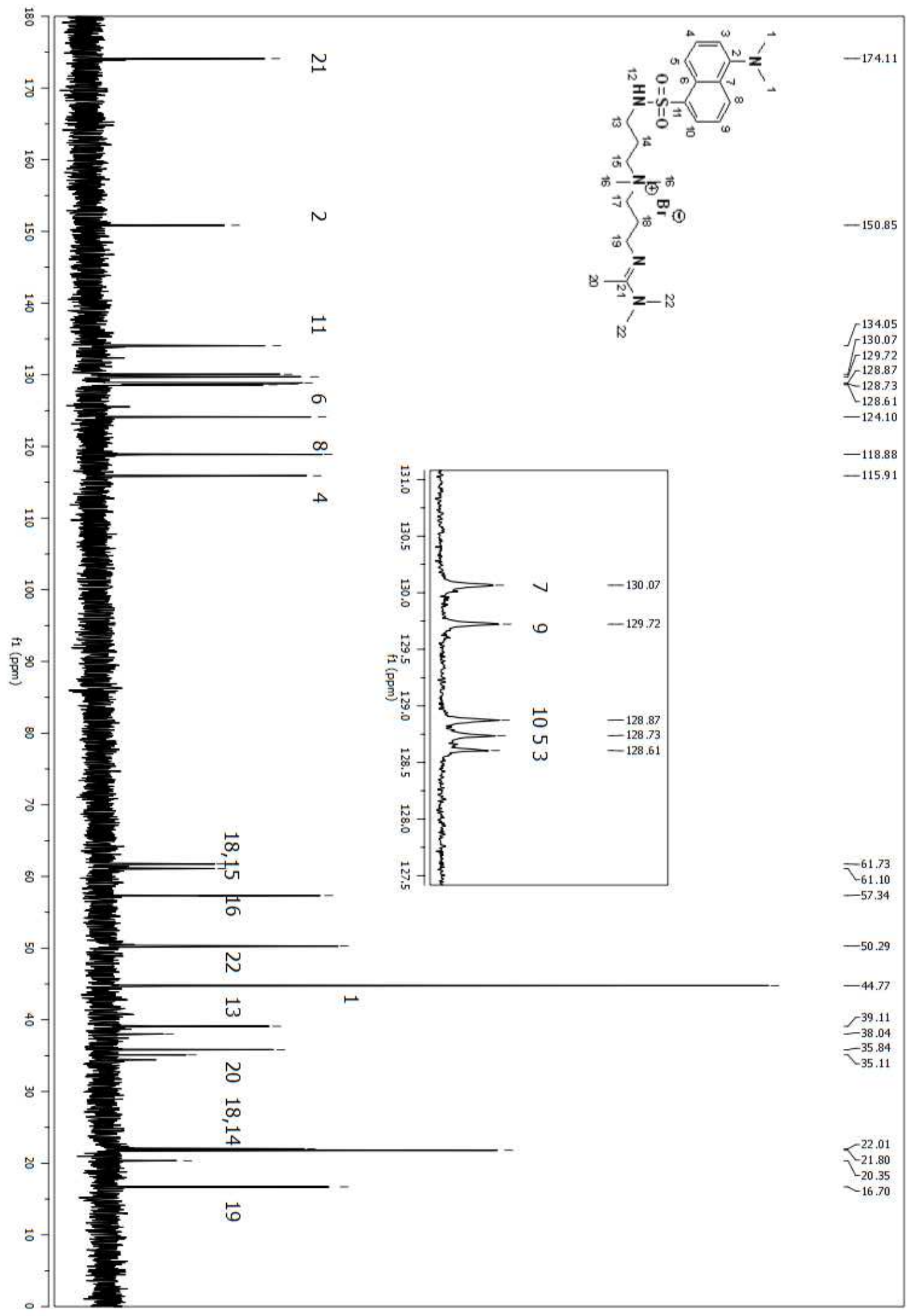

Figure A 391. ${ }^{13} \mathrm{C}$ NMR spectrum of compound (222) in $\mathrm{D}_{2} \mathrm{O}$. 


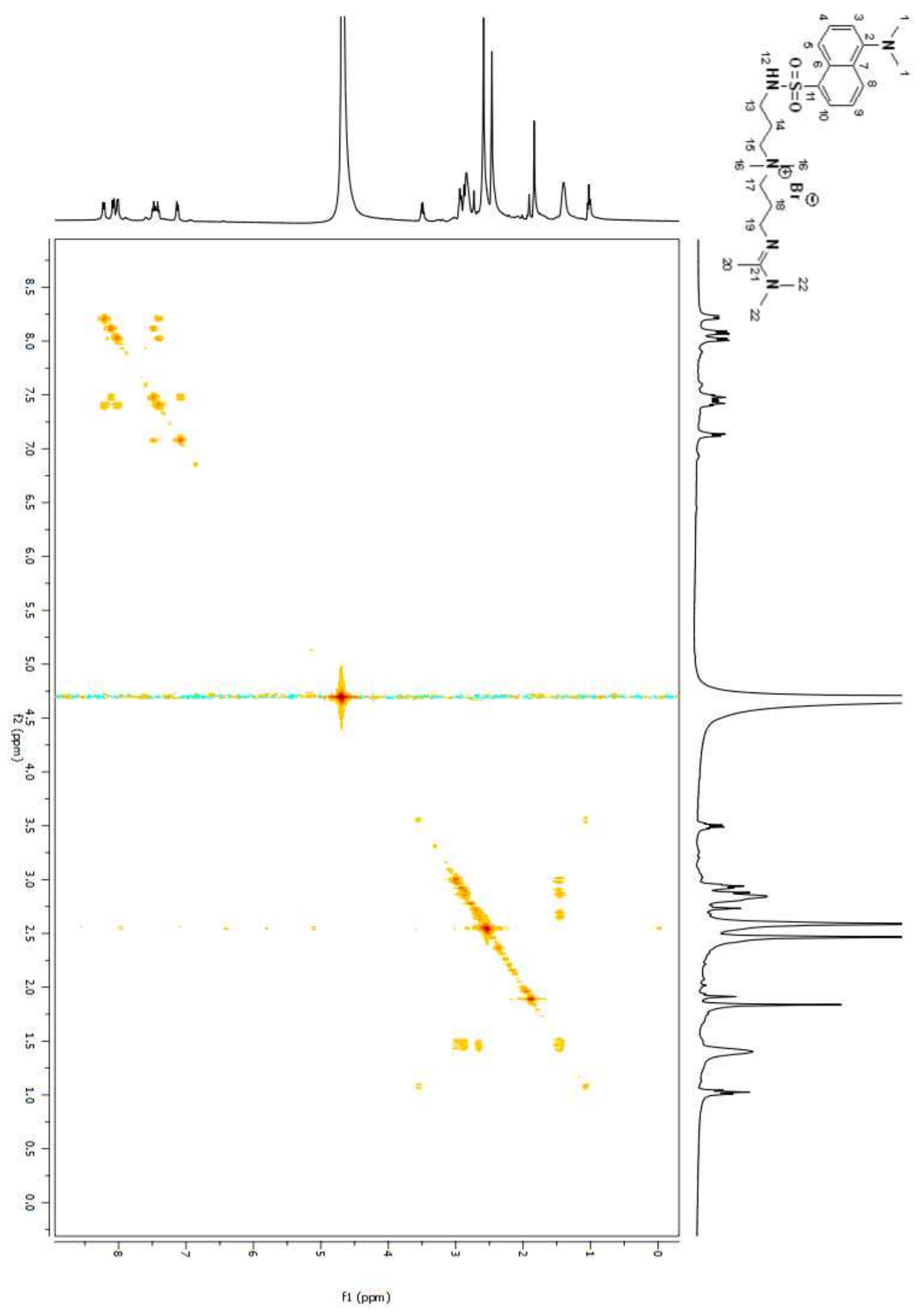

Figure A 392. COSY 2D NMR spectrum of compound (222) in $\mathrm{D}_{2} \mathrm{O}$. 


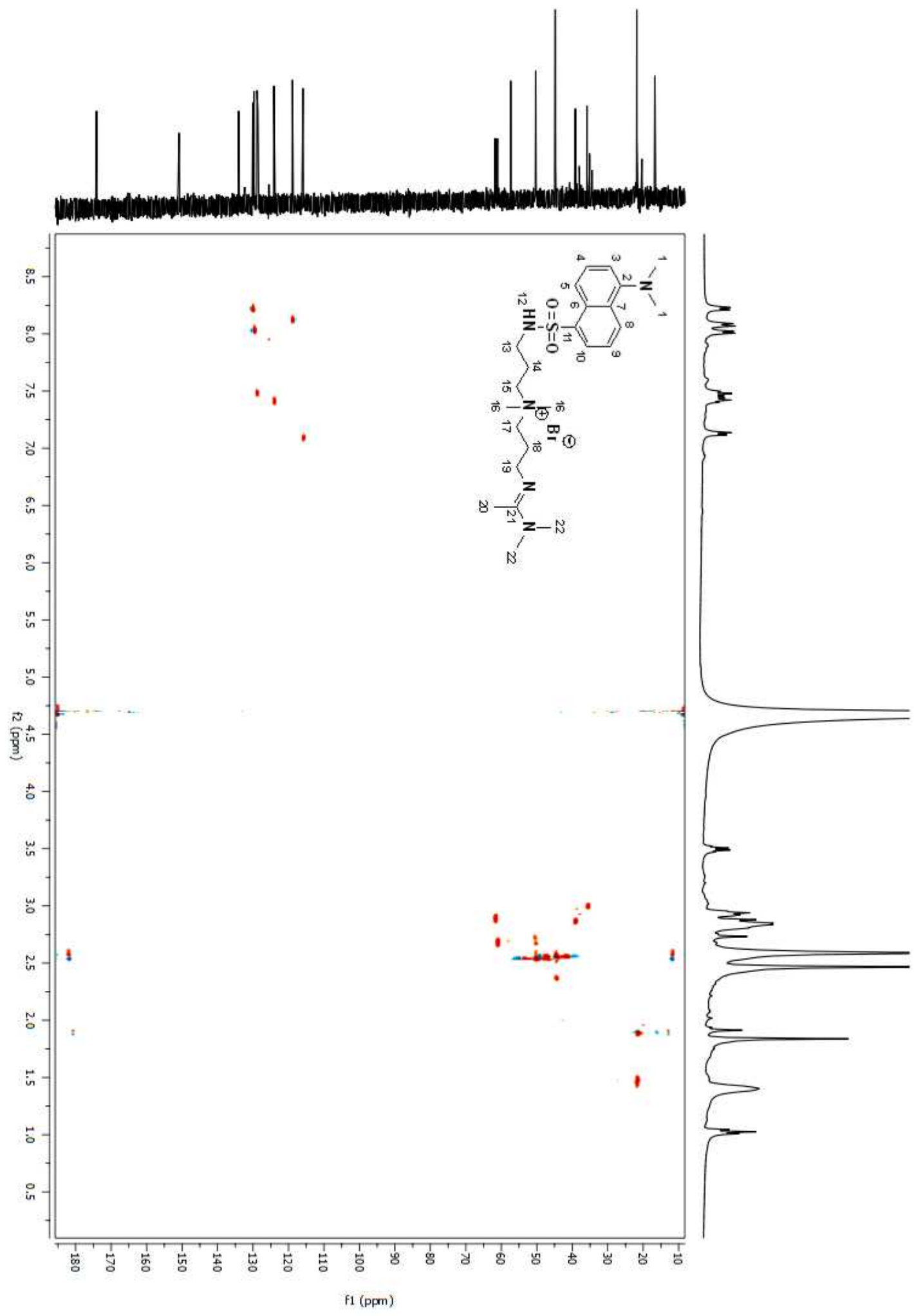

Figure A 393. HSQC 2D NMR spectrum of compound (222) in $\mathrm{D}_{2} \mathrm{O}$. 


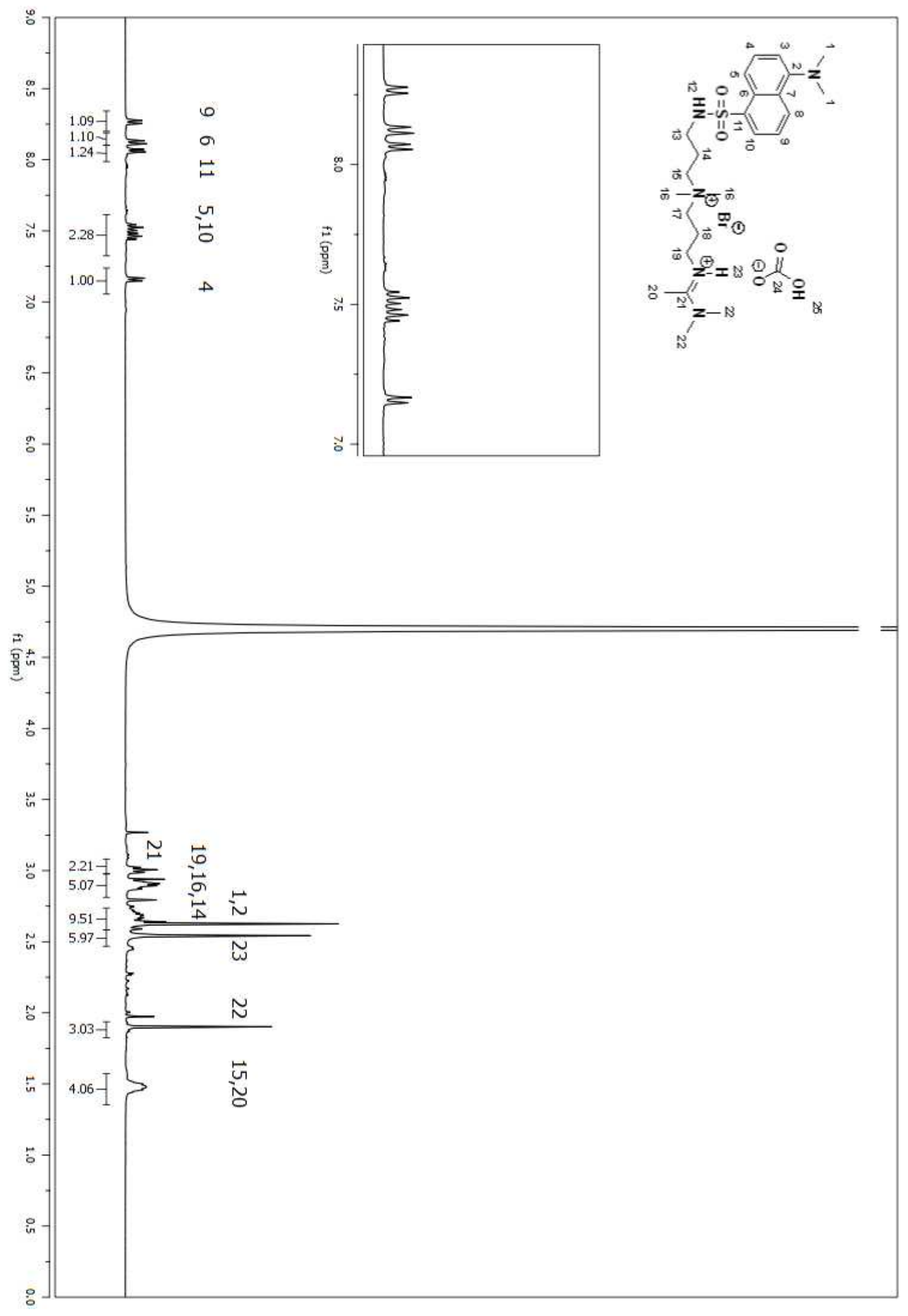

Figure A 394. ${ }^{1} \mathrm{H}$ NMR spectrum of compound (223) in $\mathrm{D}_{2} \mathrm{O}$. 


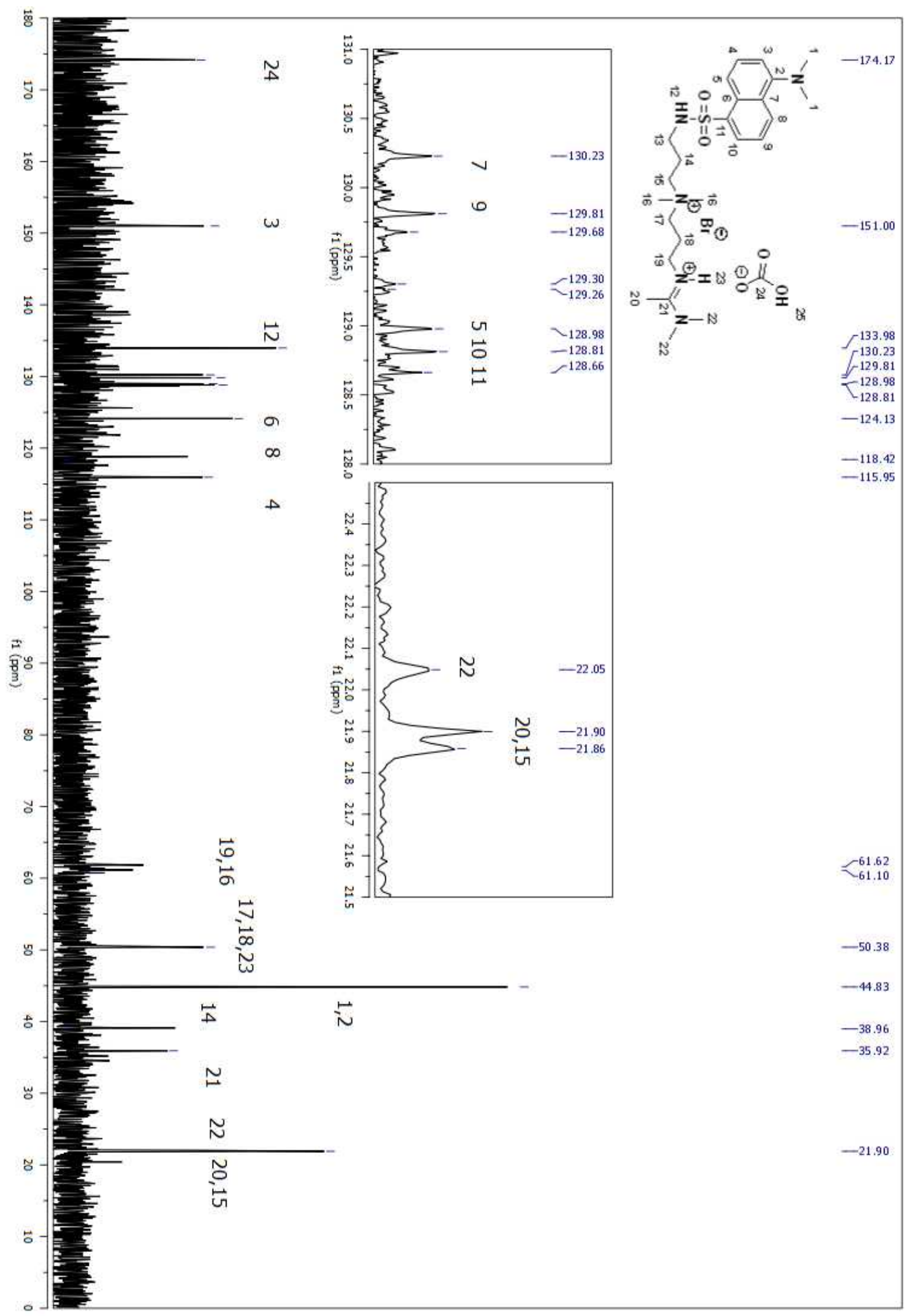

Figure A 395. ${ }^{13} \mathrm{C}$ NMR spectrum of compound (223) in $\mathrm{D}_{2} \mathrm{O}$. 


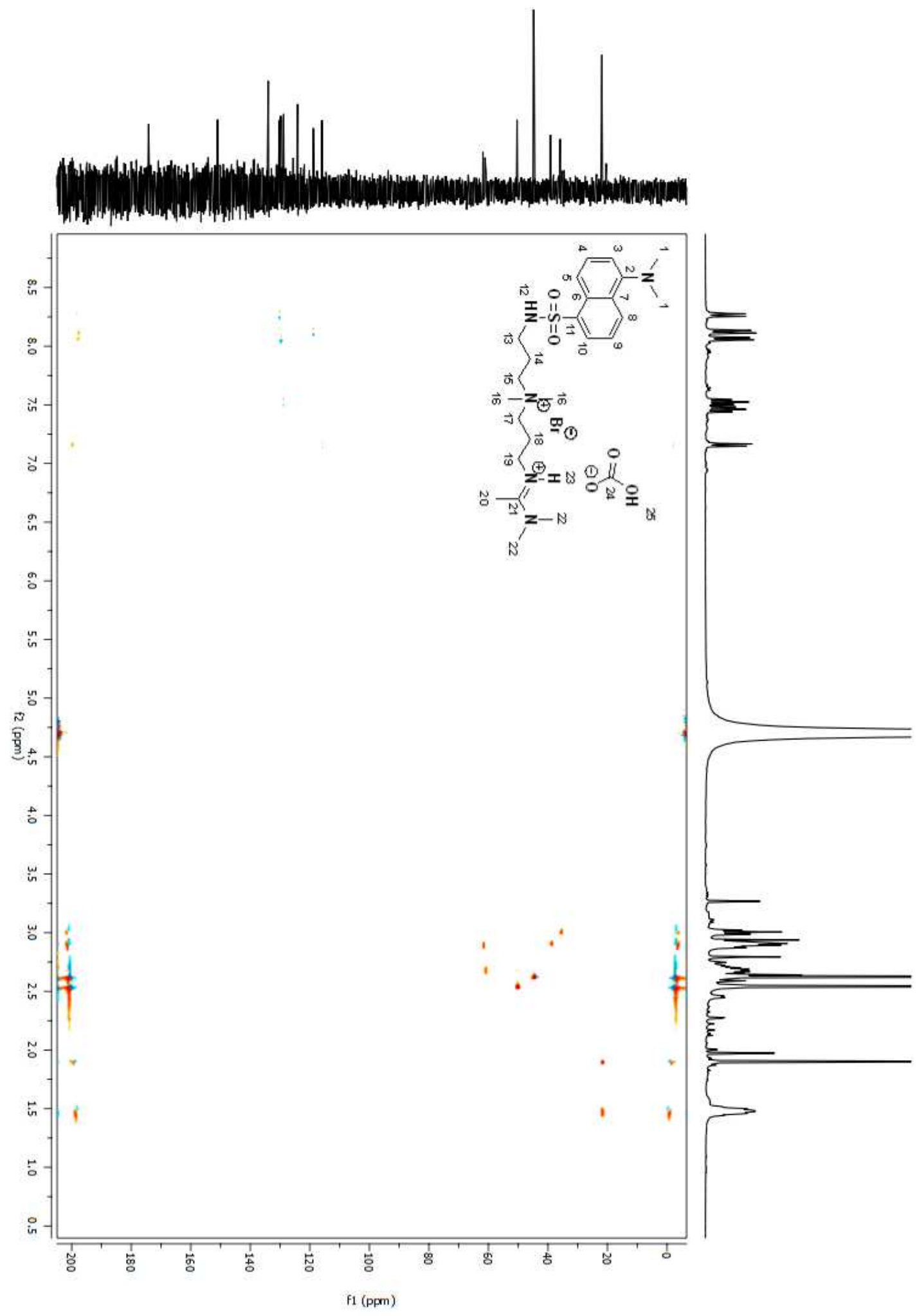

Figure A 396. HSQC 2D NMR spectrum of compound (223) in $\mathrm{D}_{2} \mathrm{O}$. 


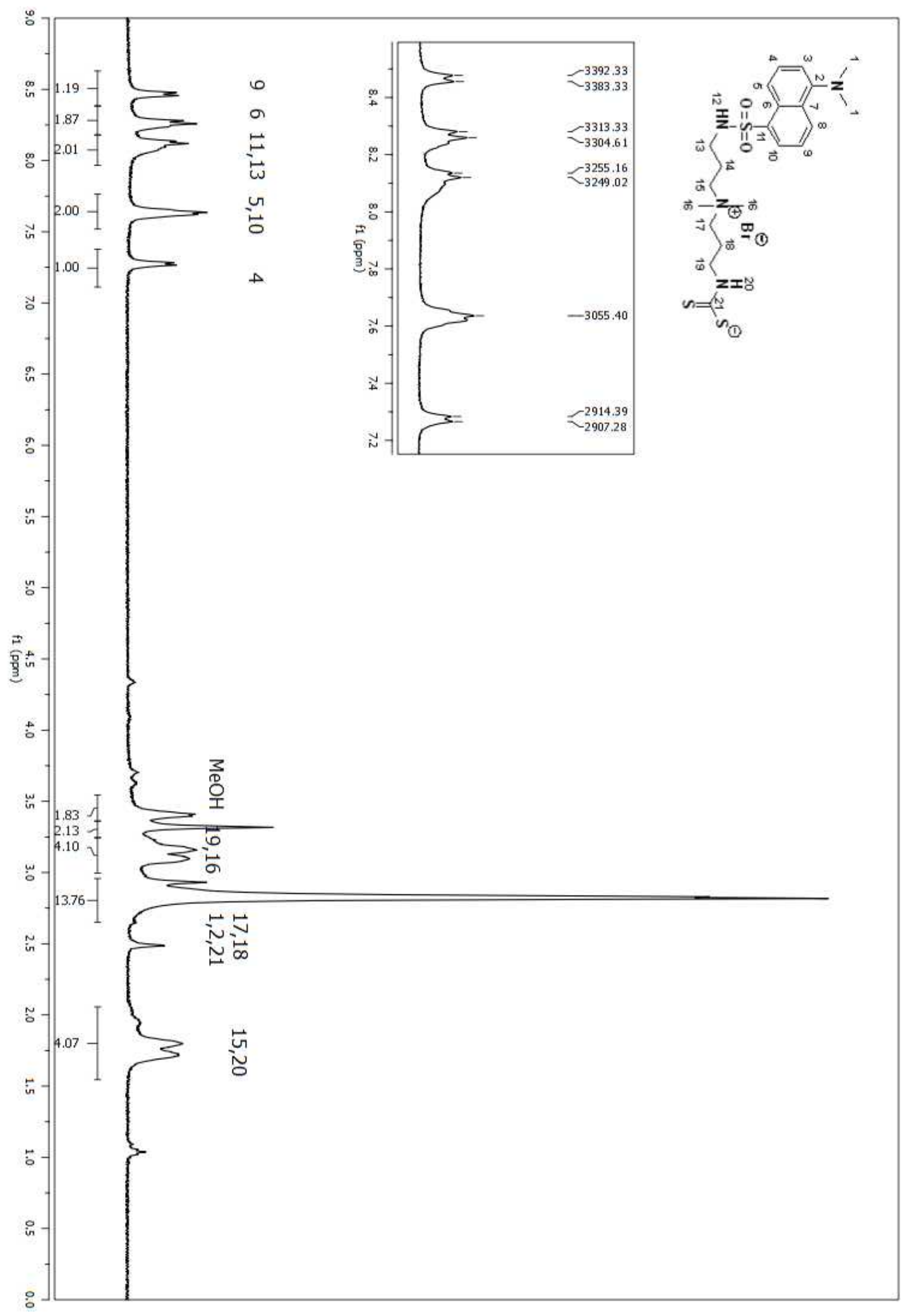

Figure A 397. ${ }^{1} \mathrm{H}$ NMR spectrum of compound (225) in DMSO-d6. 


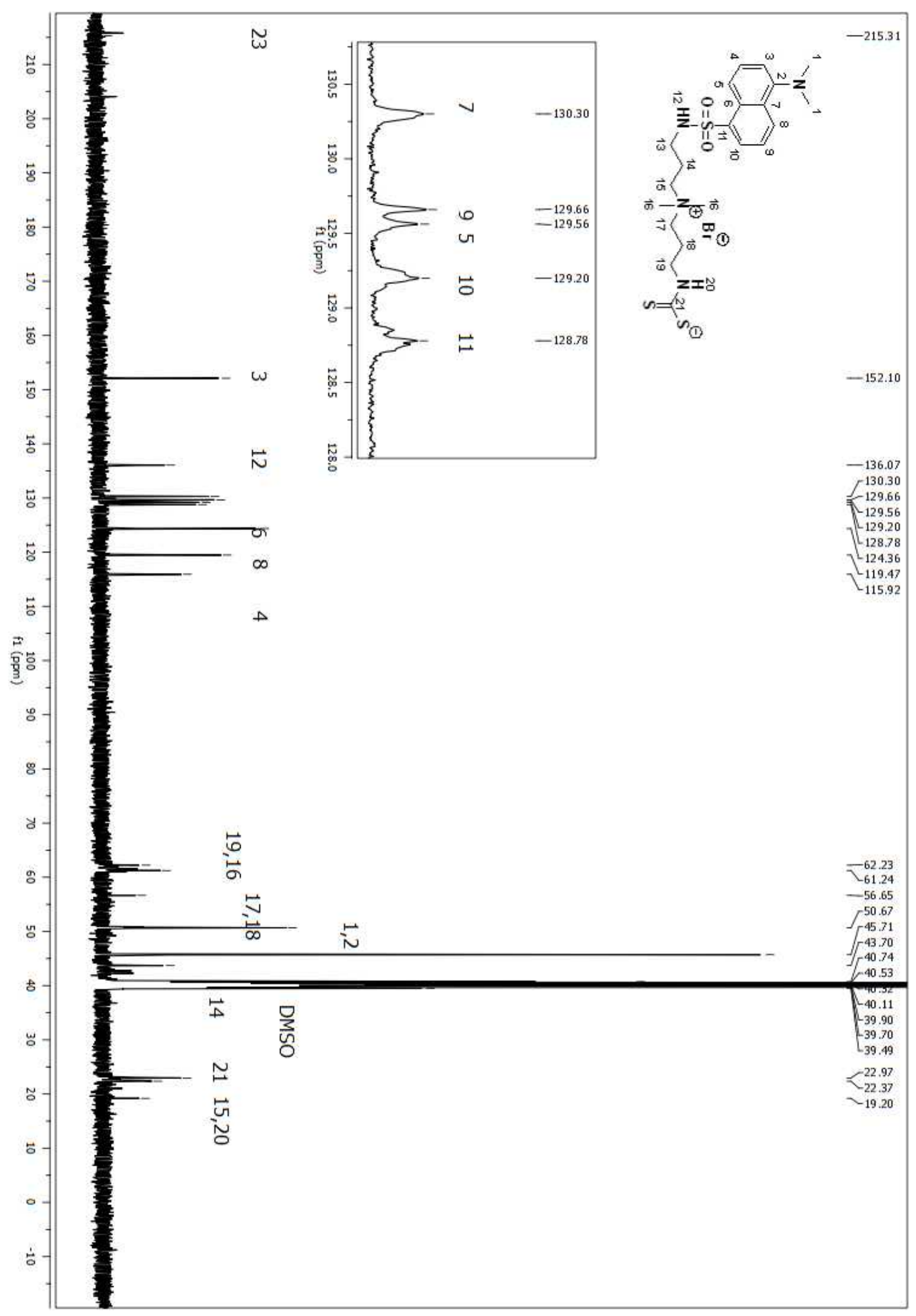

Figure A 398. ${ }^{13} \mathrm{C}$ NMR spectrum of compound (225) in DMSO-d6. 


\subsection{Appendix 1.3 - MS Spectra}

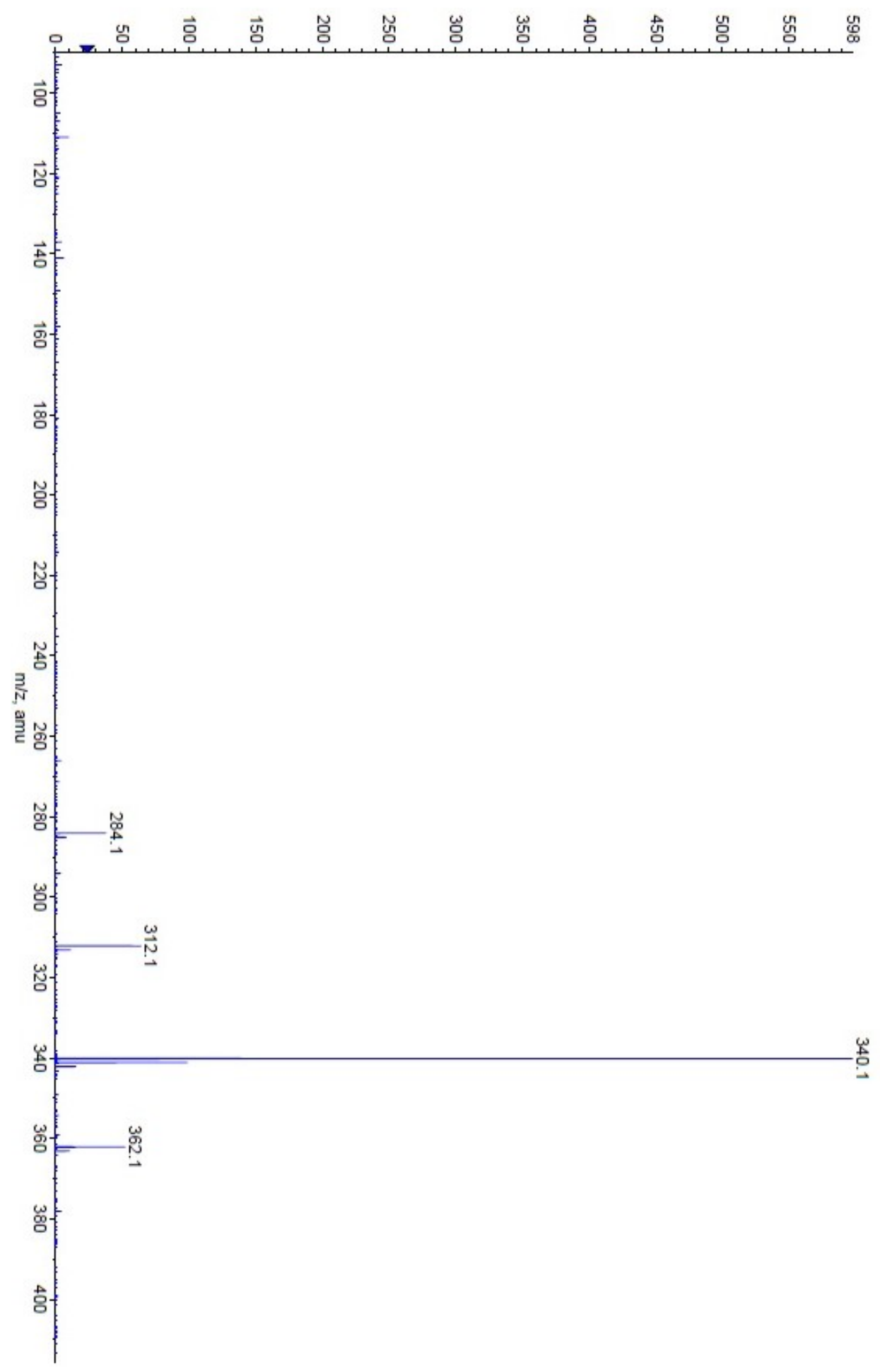

Figure A 399. ESI-TOF spectrum of compound 19. 


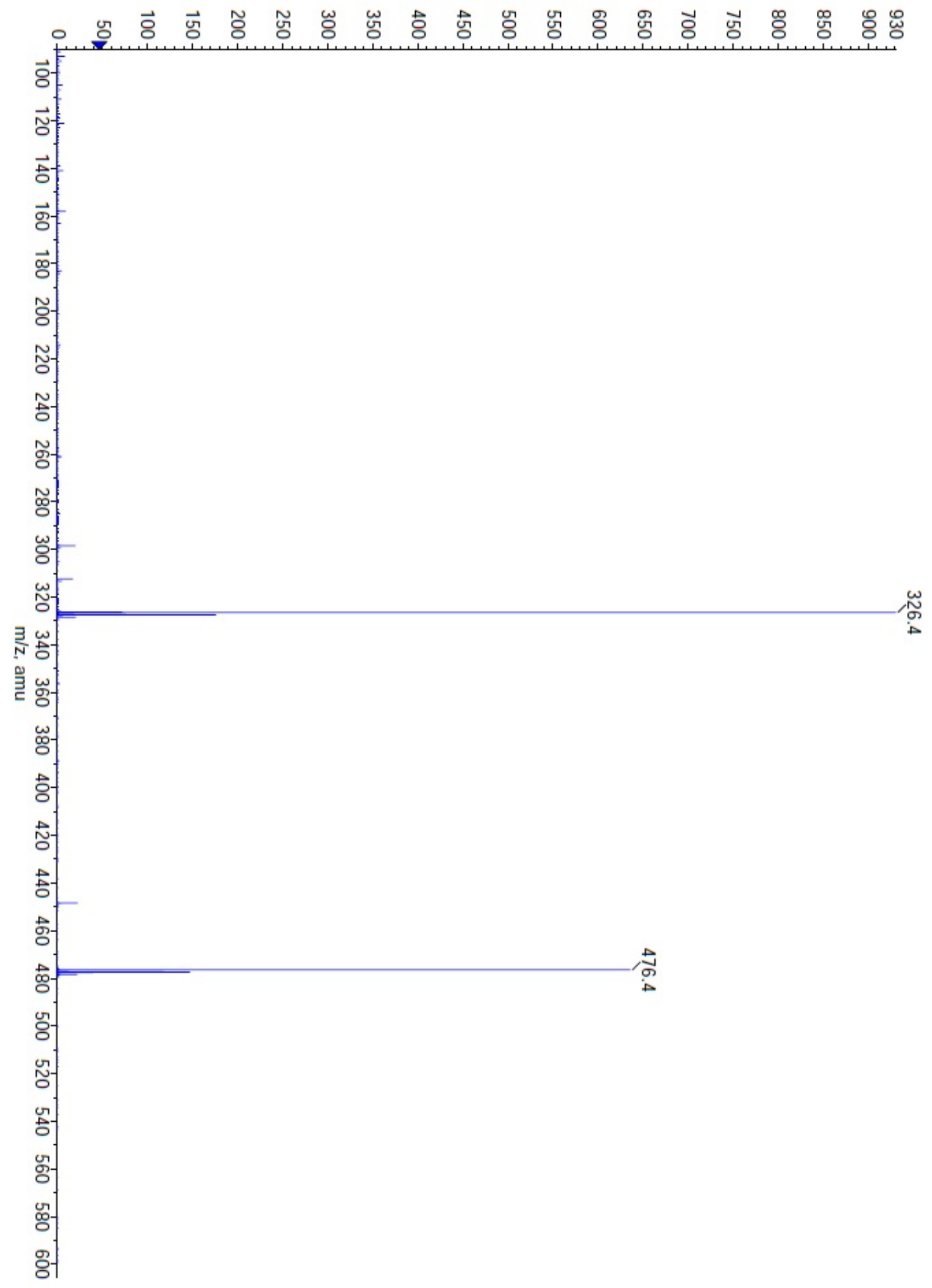

Figure A 400. ESI-TOF spectrum of compound 26.

767 


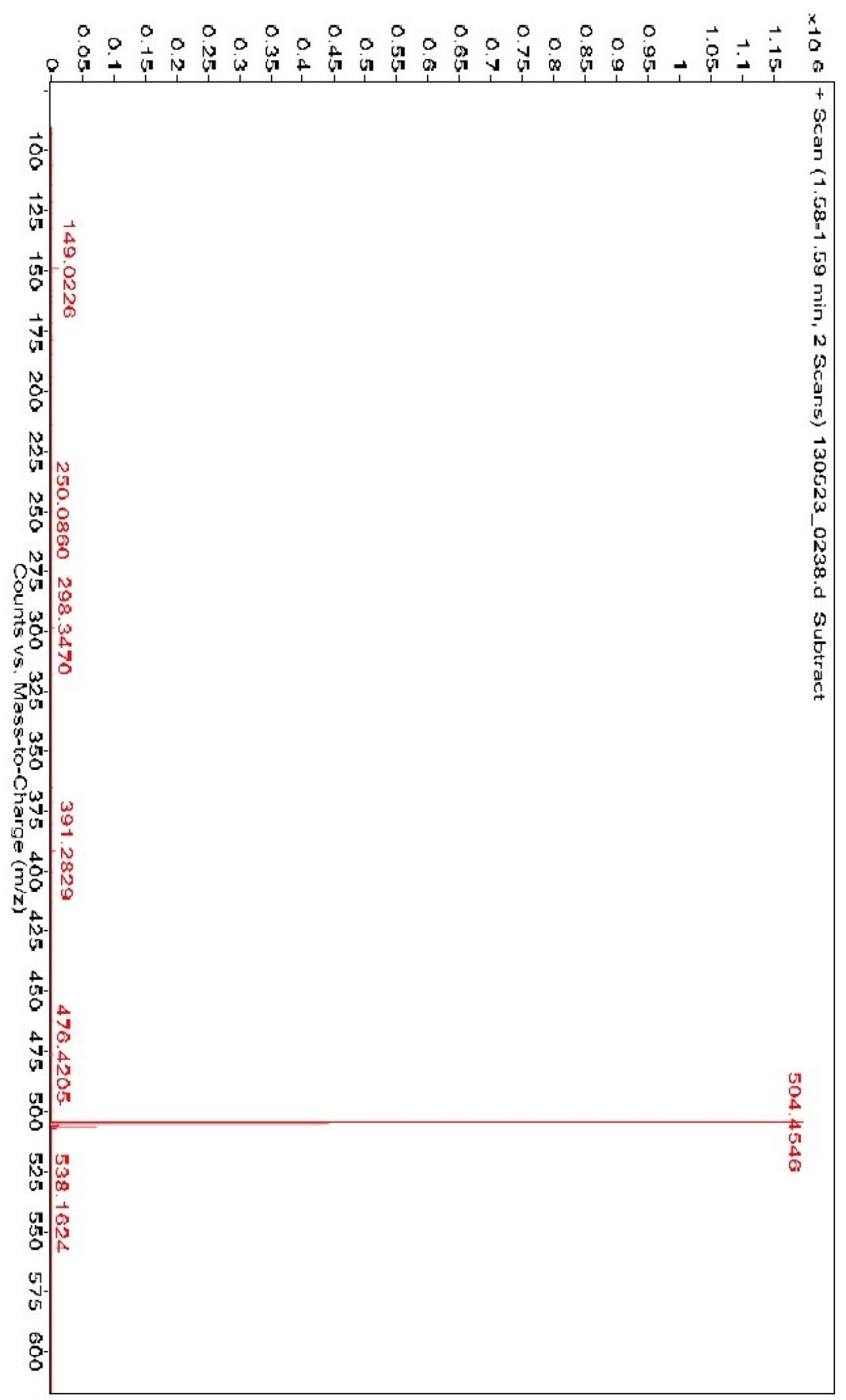

Figure A 401. HRMS ESI-TOF spectrum of compound 27. 


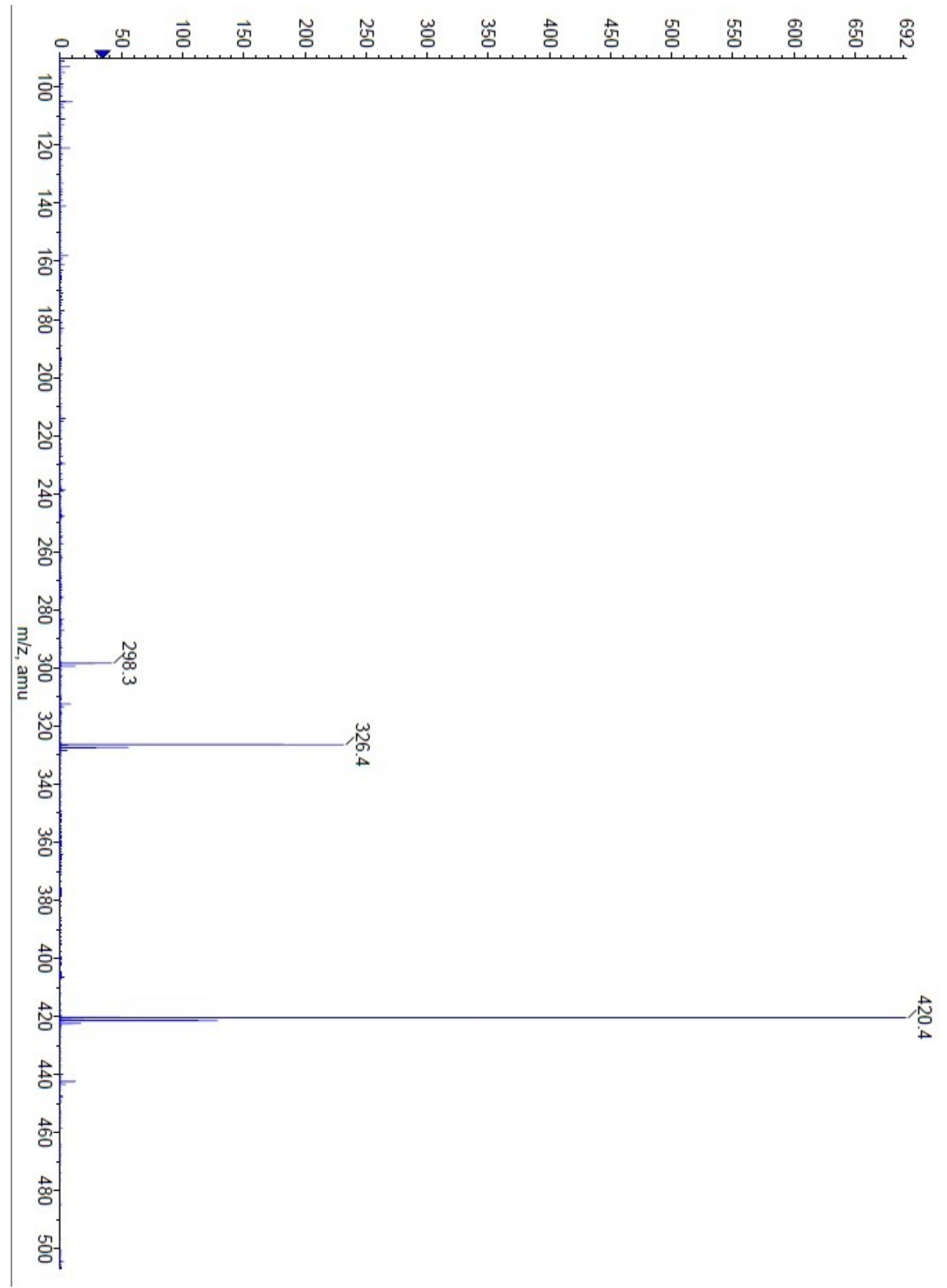

Figure A 402. ESI-TOF spectrum of compound 34.

769 


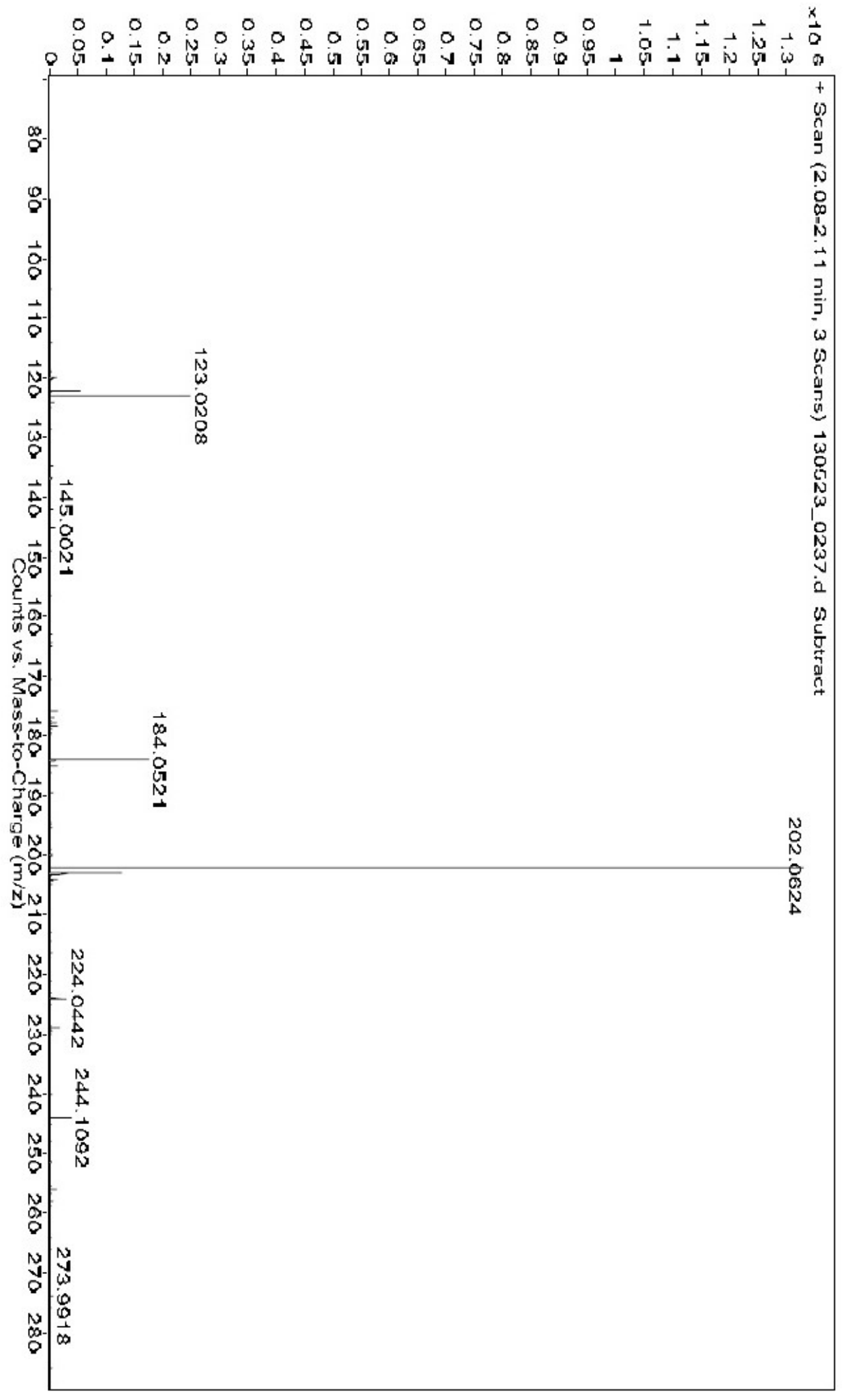

Figure A 403. HRMS ESI-TOF spectrum of compound 36. 


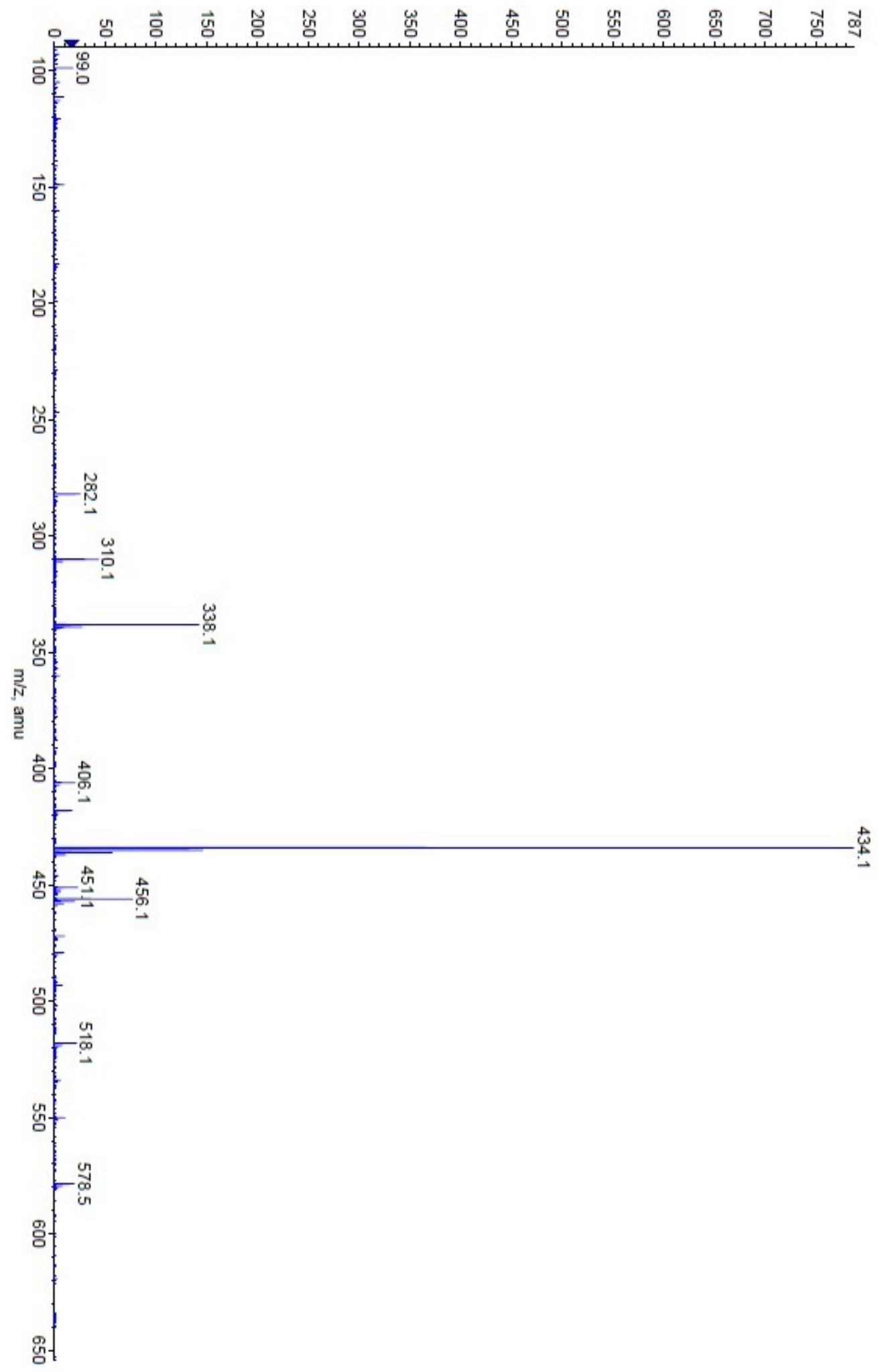

Figure A 404. ESI-TOF spectrum of compound 51. 


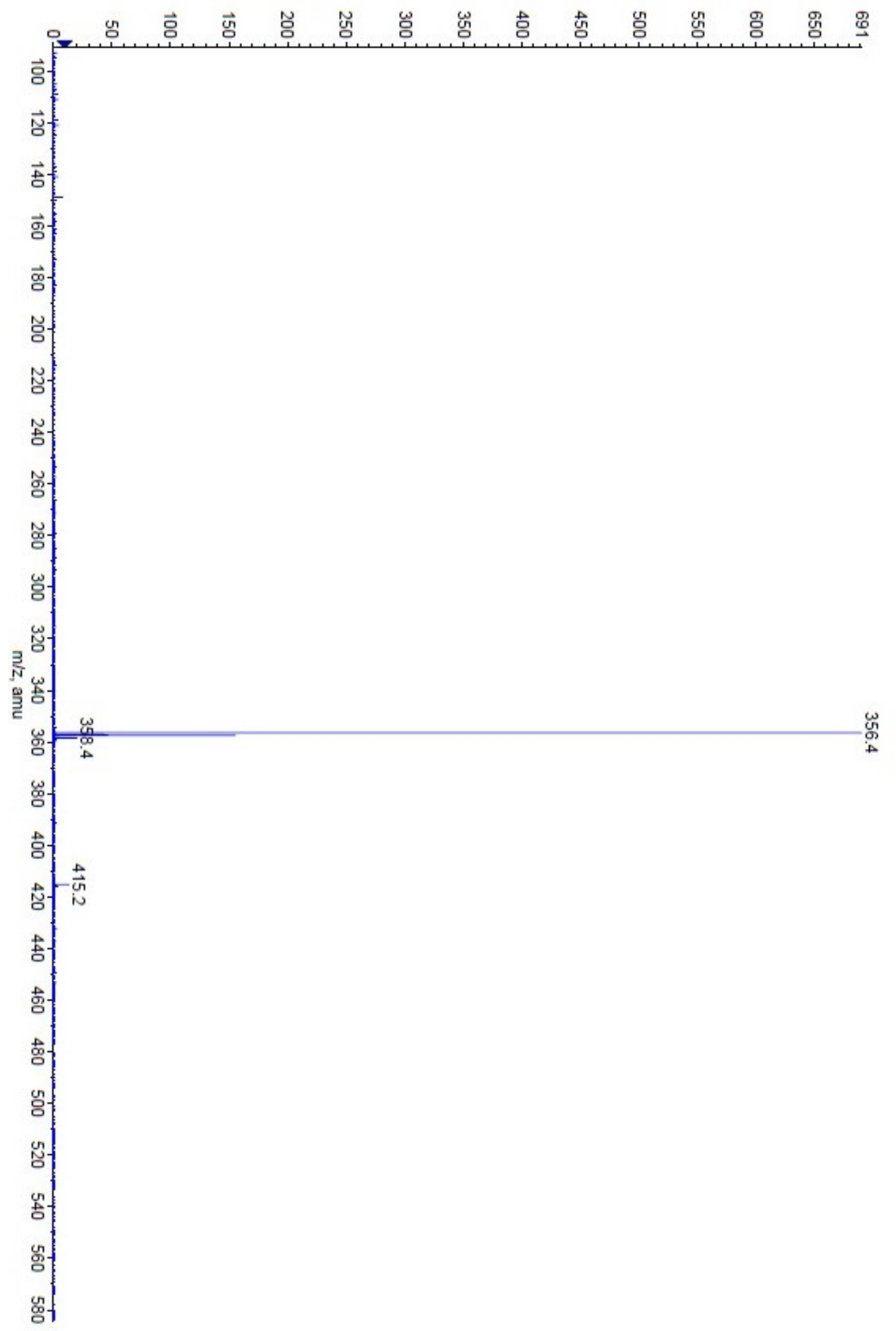

Figure A 405. ESI-TOF spectrum of compound 66. 


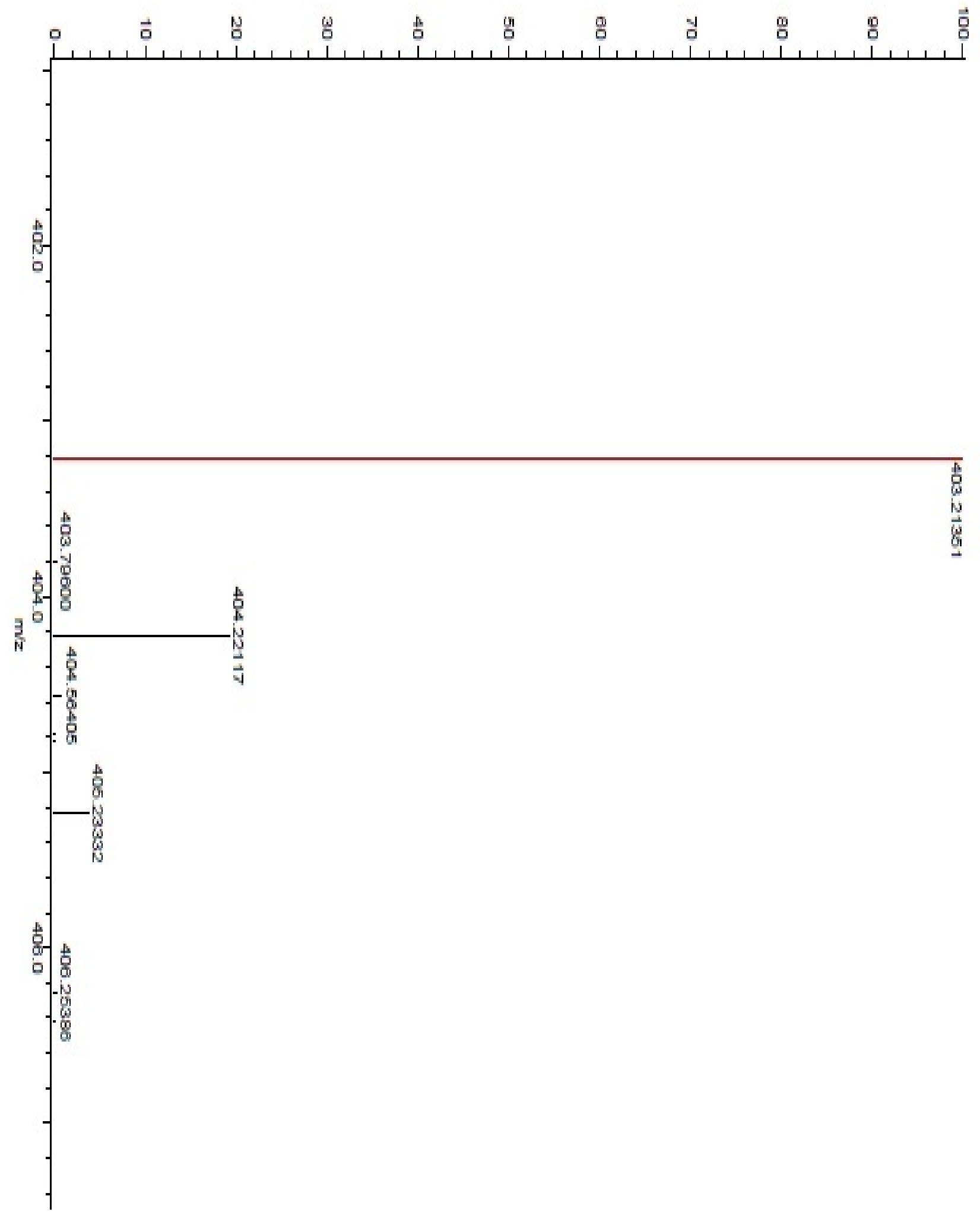

Figure A 406 .DART-HRMS spectrum of compound 113. 


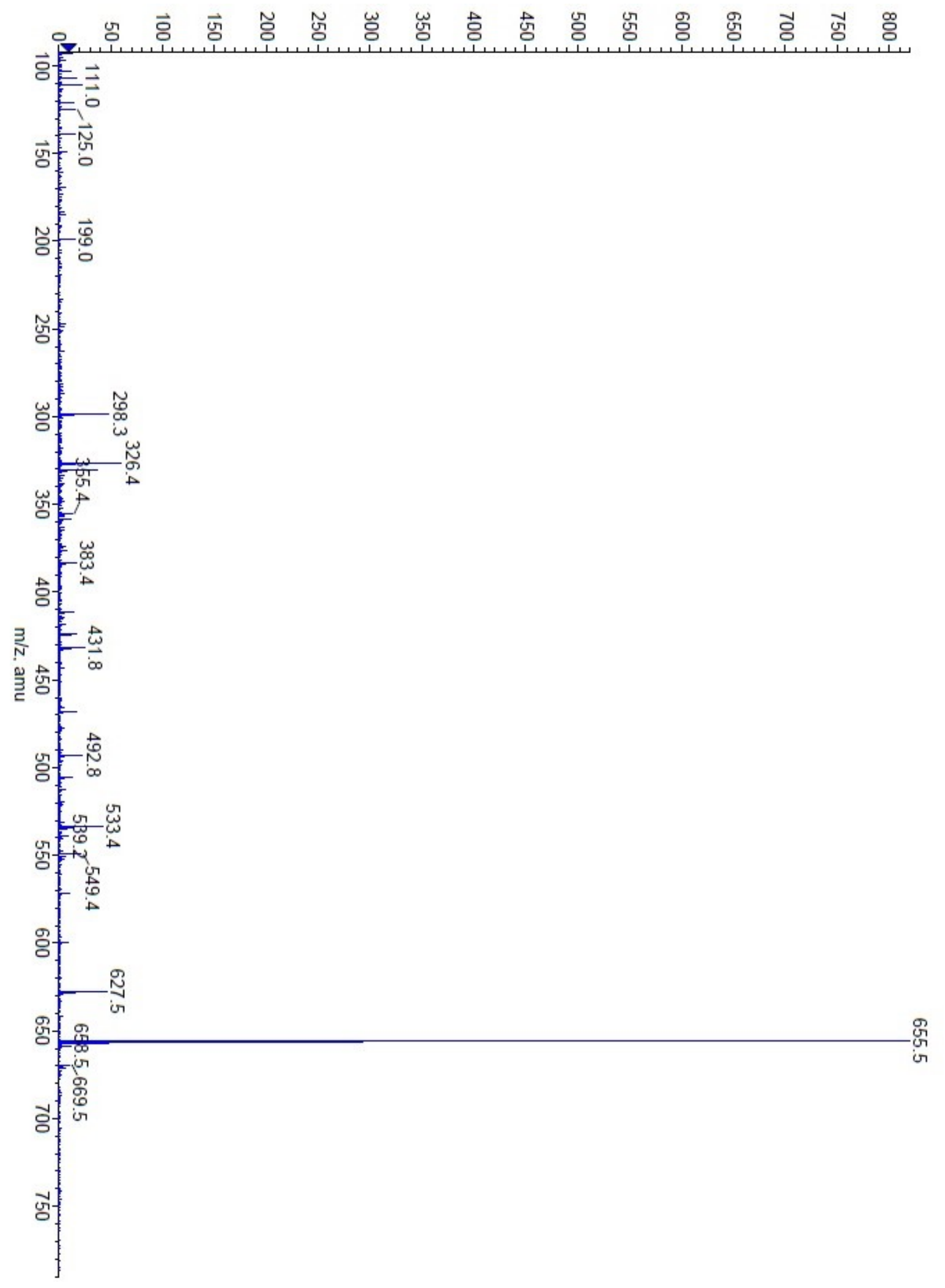

Figure A 407. ESI-TOF spectrum of compound 121. 


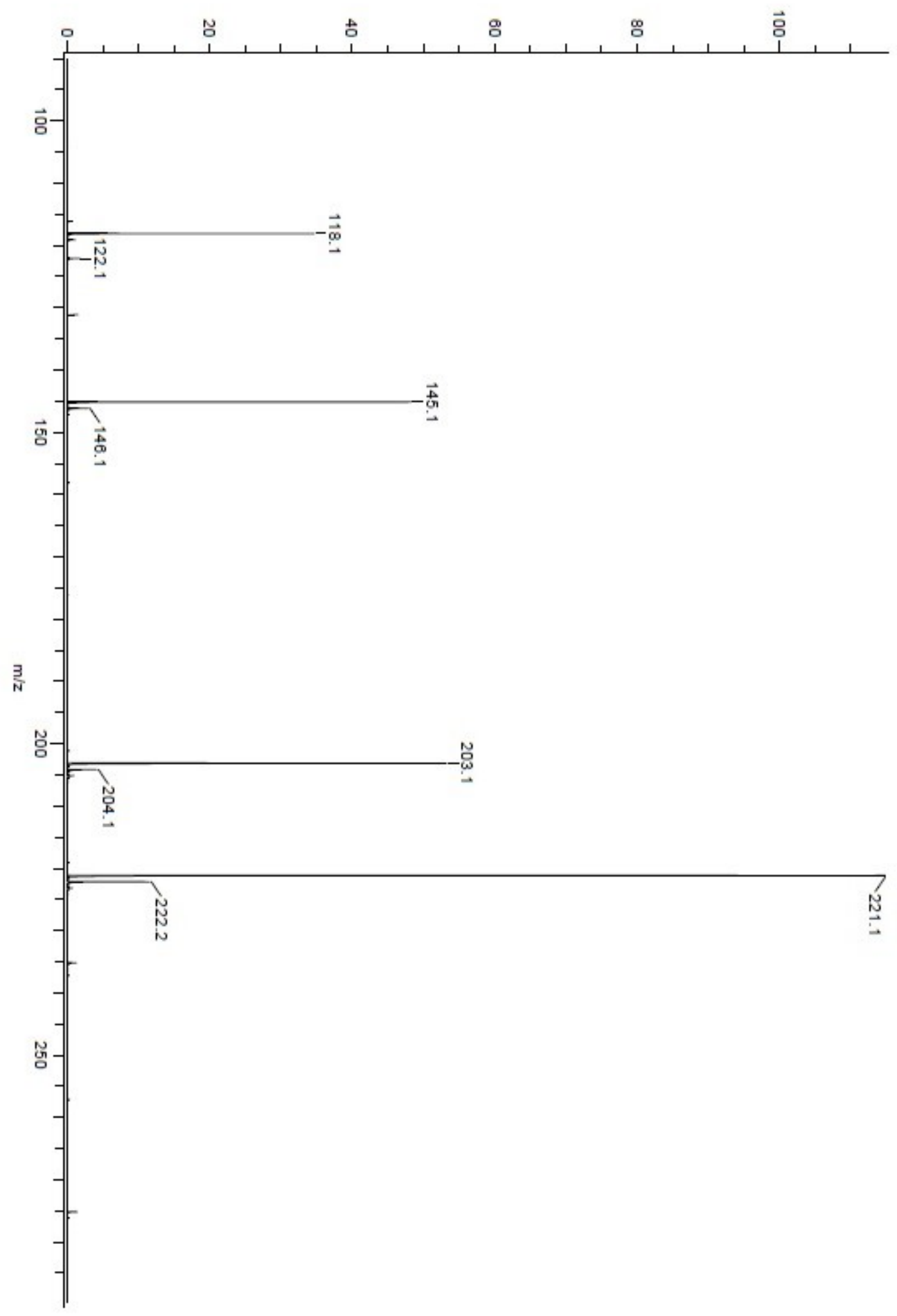

Figure A 408. DART spectrum of compound 163.

775 


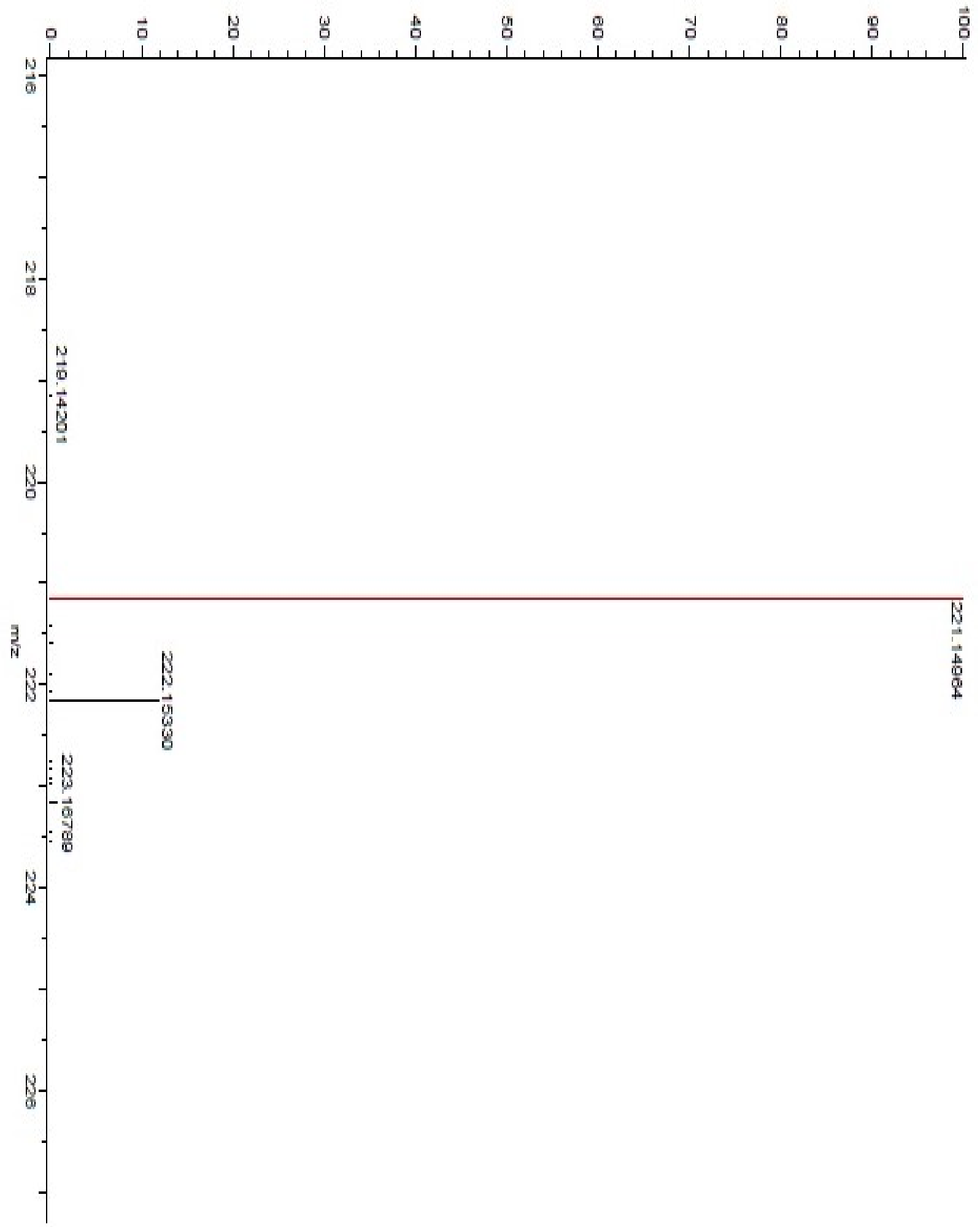

Figure A 409. HRMS-DART spectrum of compound 163. 


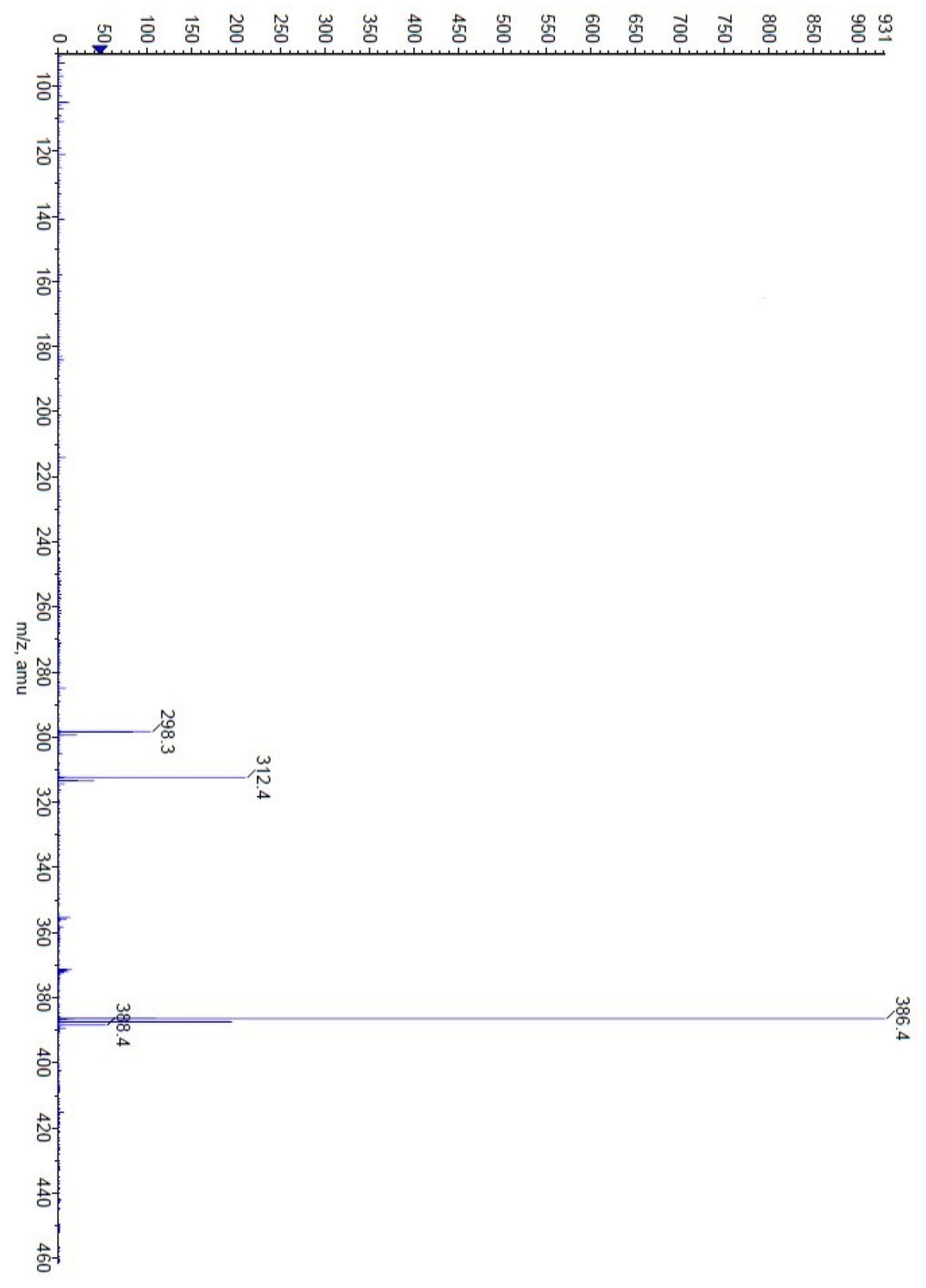

Figure A 410. ESI-TOF spectrum of compound 194. 


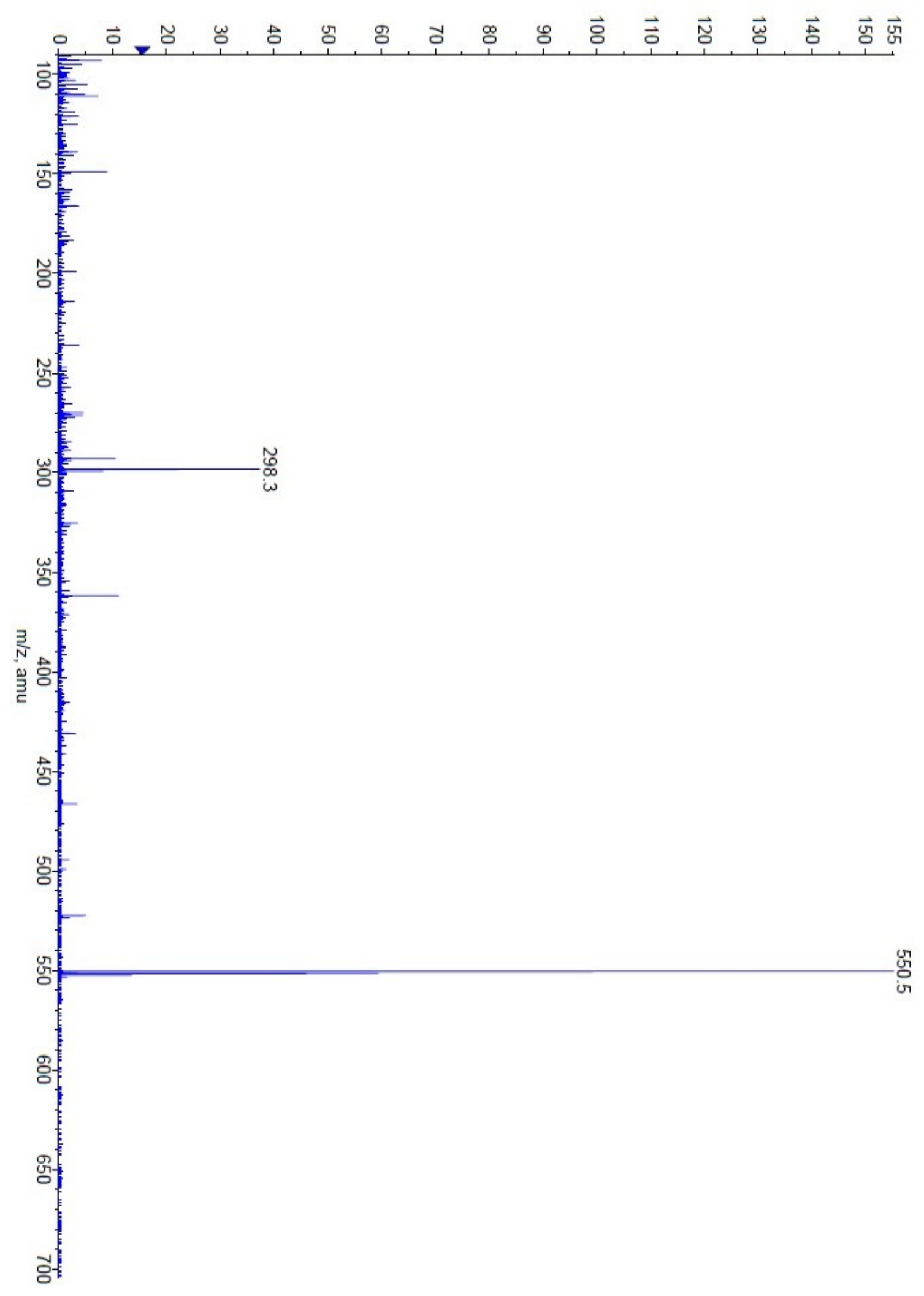

Figure A 411. ESI-TOF spectrum of compound 205.

778 


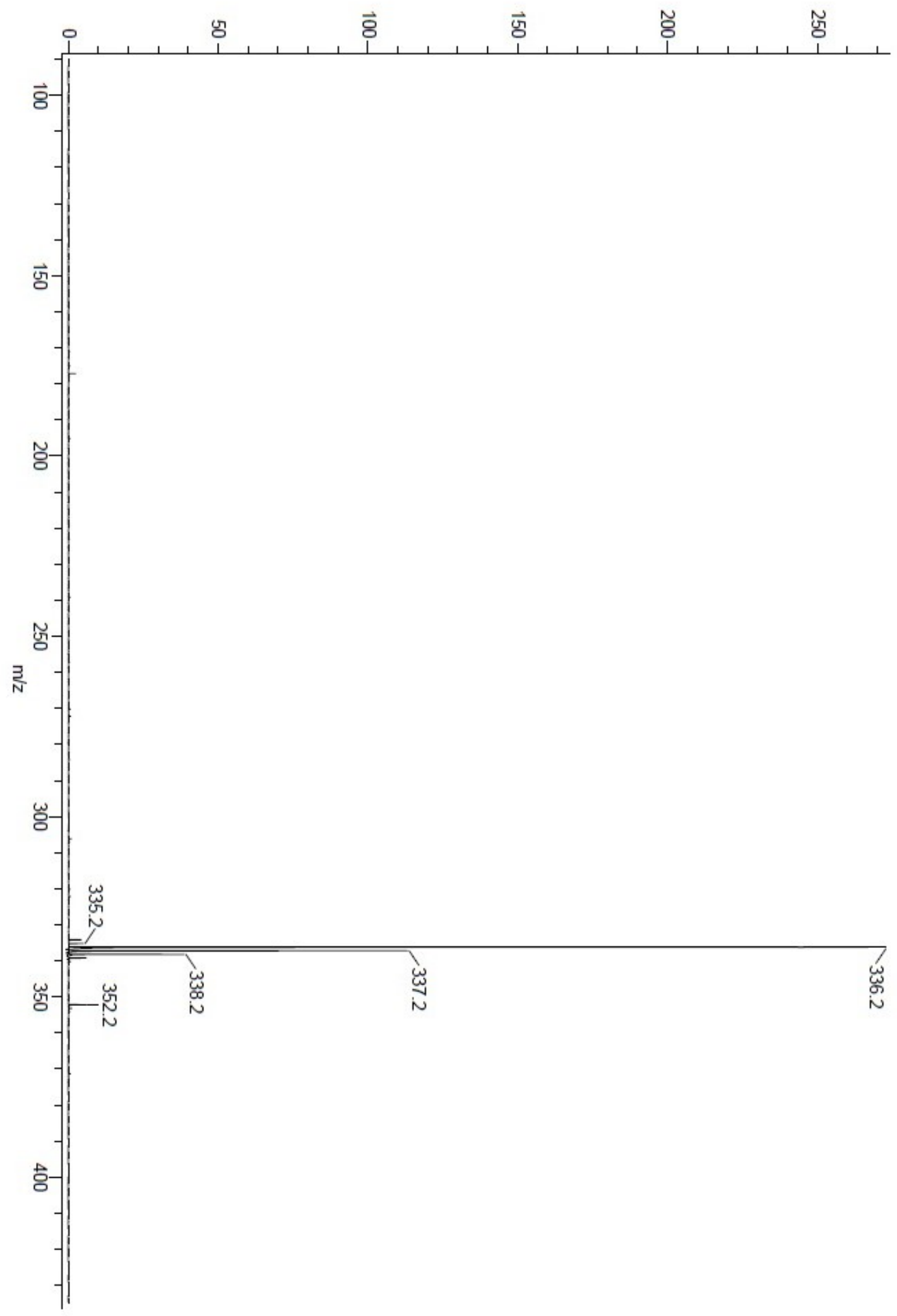

Figure A 412. DART spectrum of compound 208.

779 


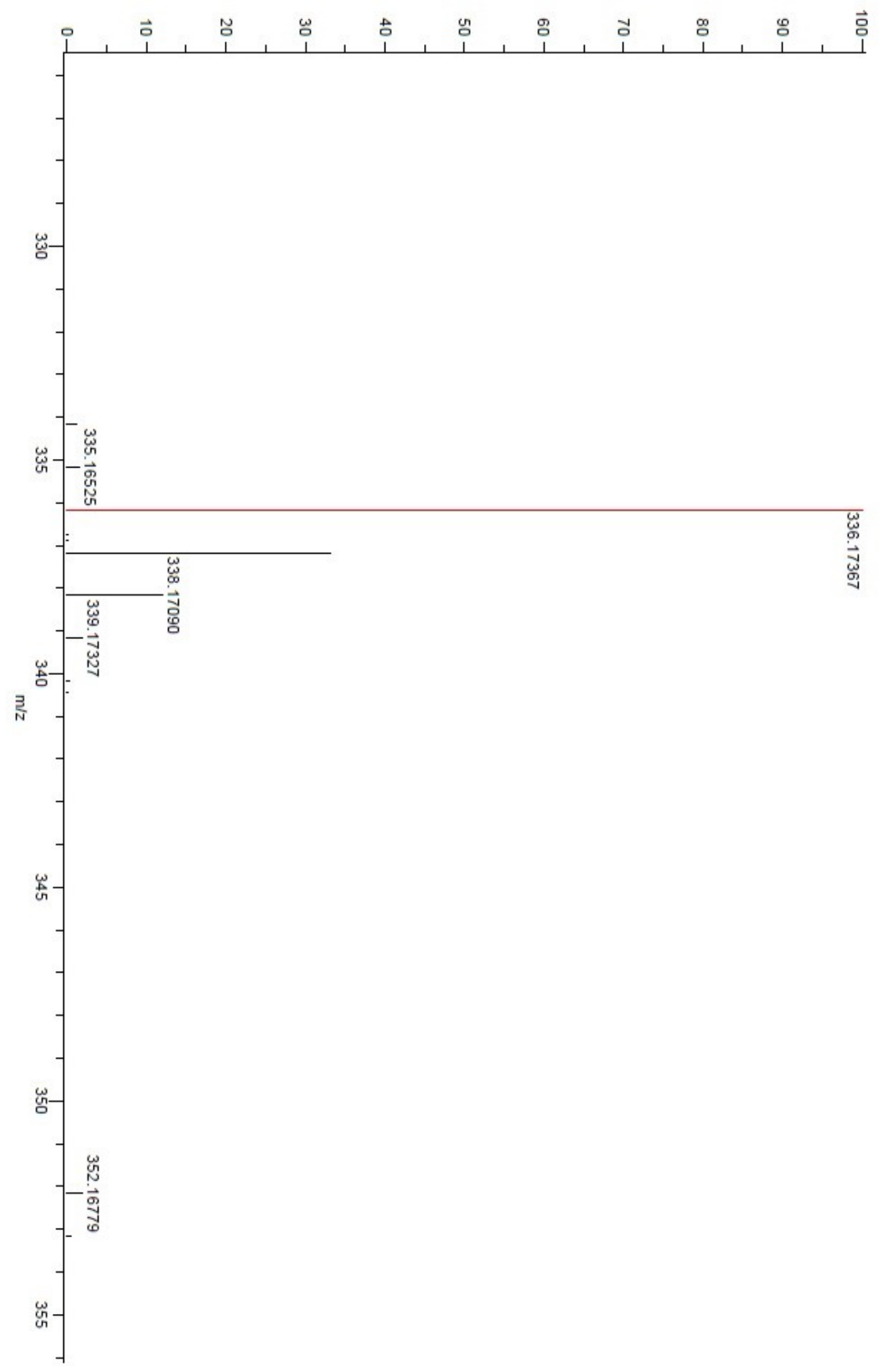

Figure A 413. HRMS-DART spectrum of compound 208. 


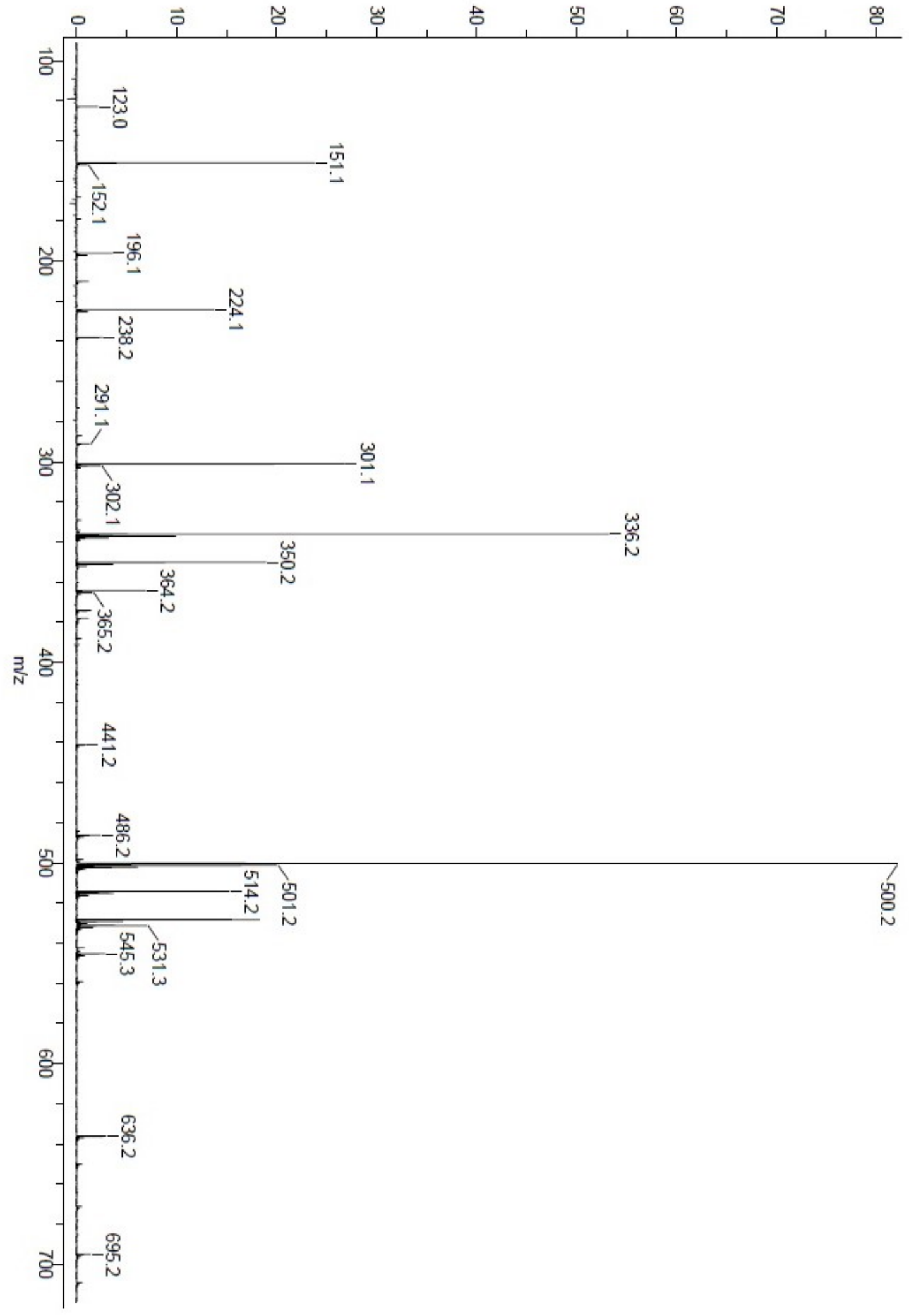

Figure A 414. DART spectrum of compound 211.

781 


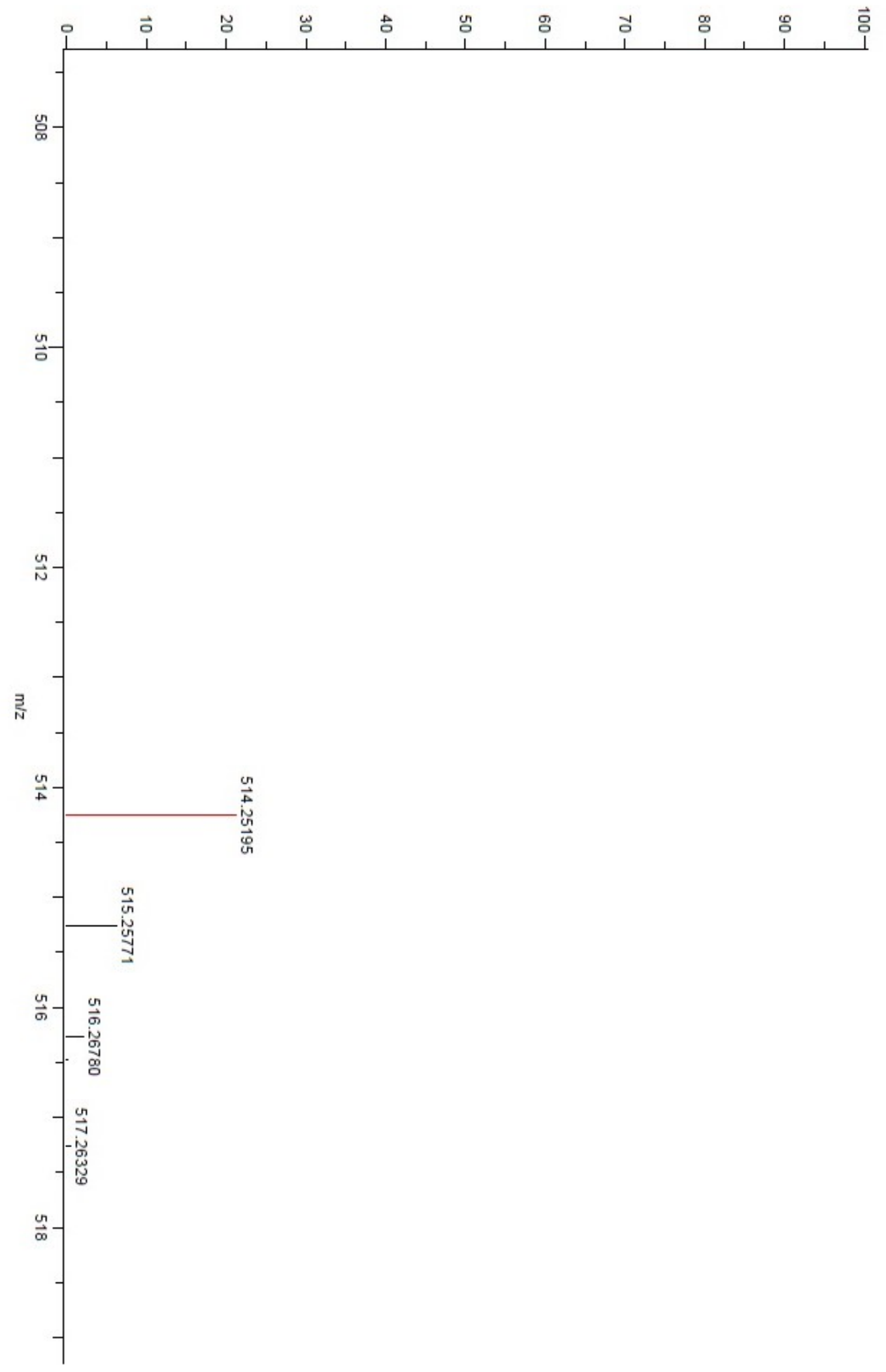

Figure A 415. HRMS-DART spectrum of compound 211.

782 


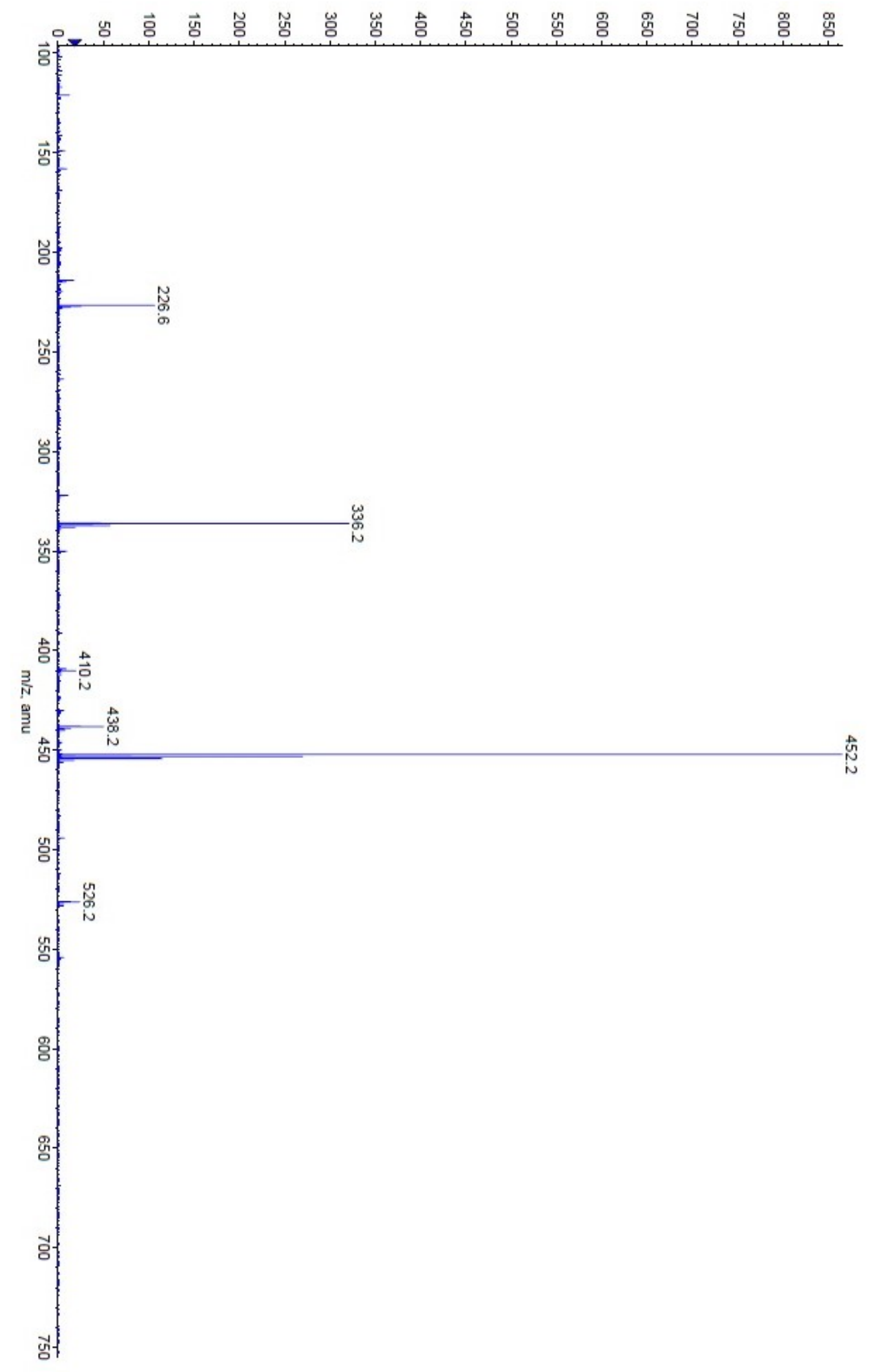

Figure A 416. ESI-TOF spectrum of compound 212.

783 


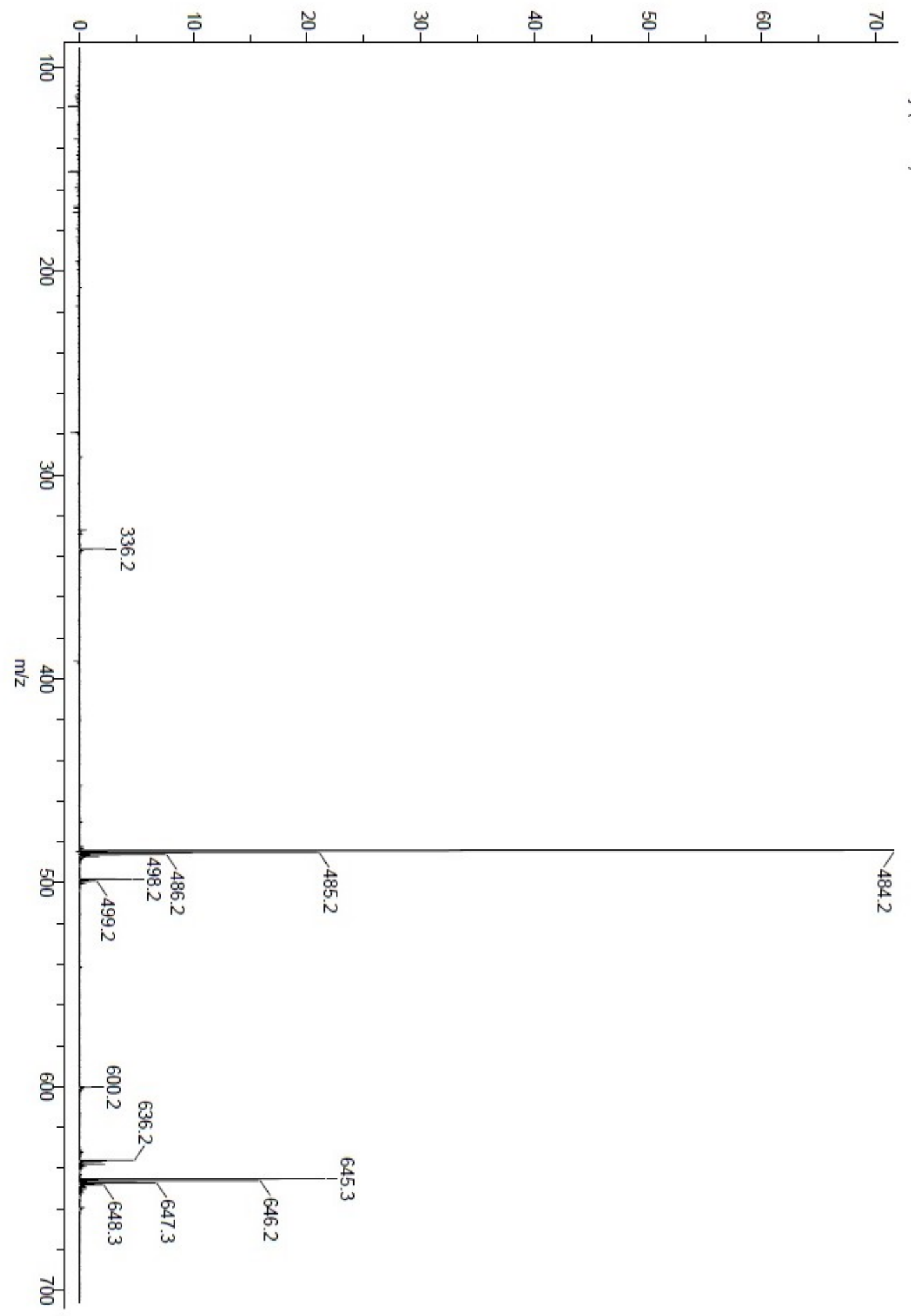

Figure A 417. HRMS-DART spectrum of compound 213. 


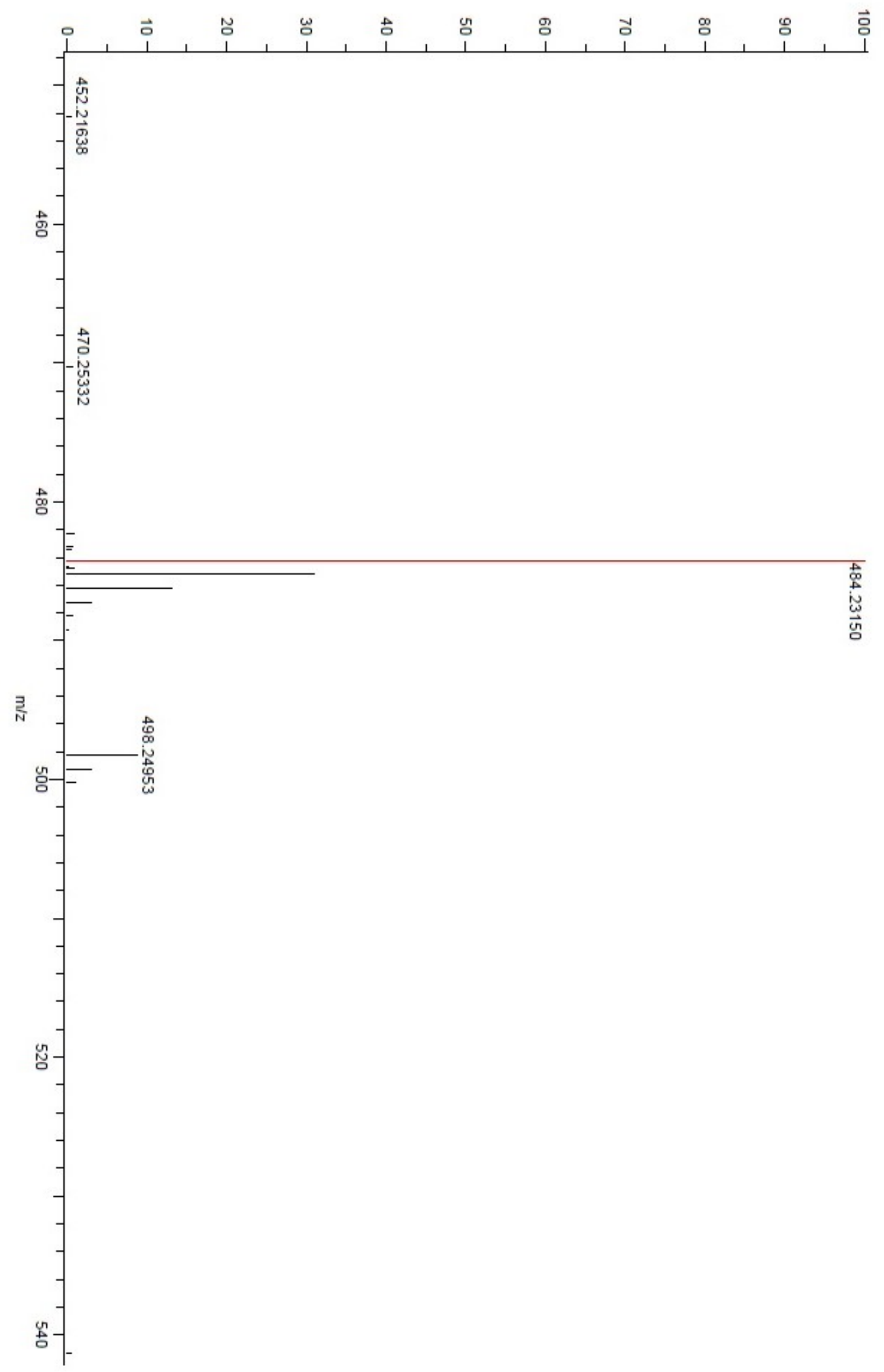

Figure A 418. HRMS-DART spectrum of compound 213.

785 


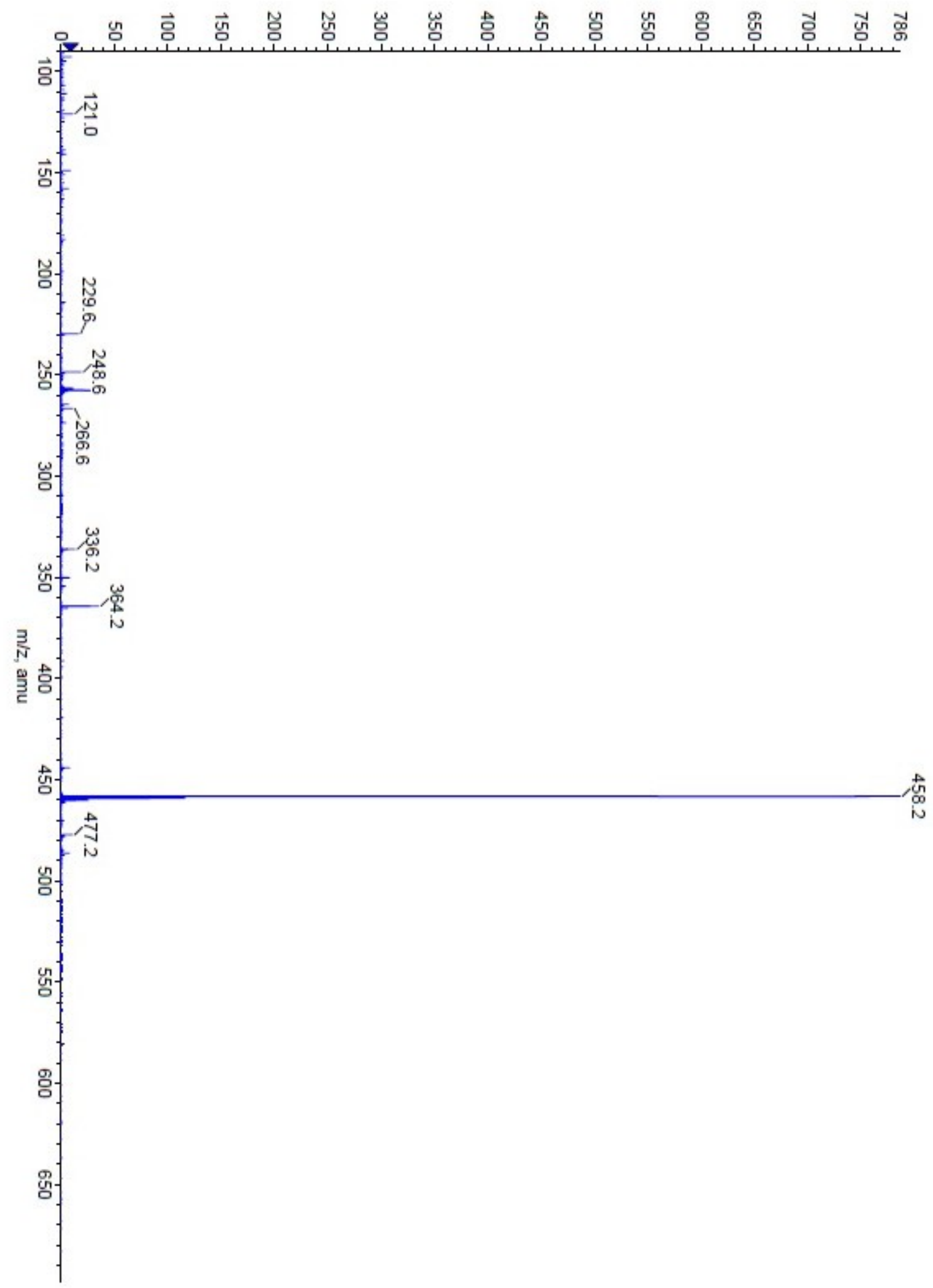

Figure A 419. ESI-TOF spectrum of compound 217. 


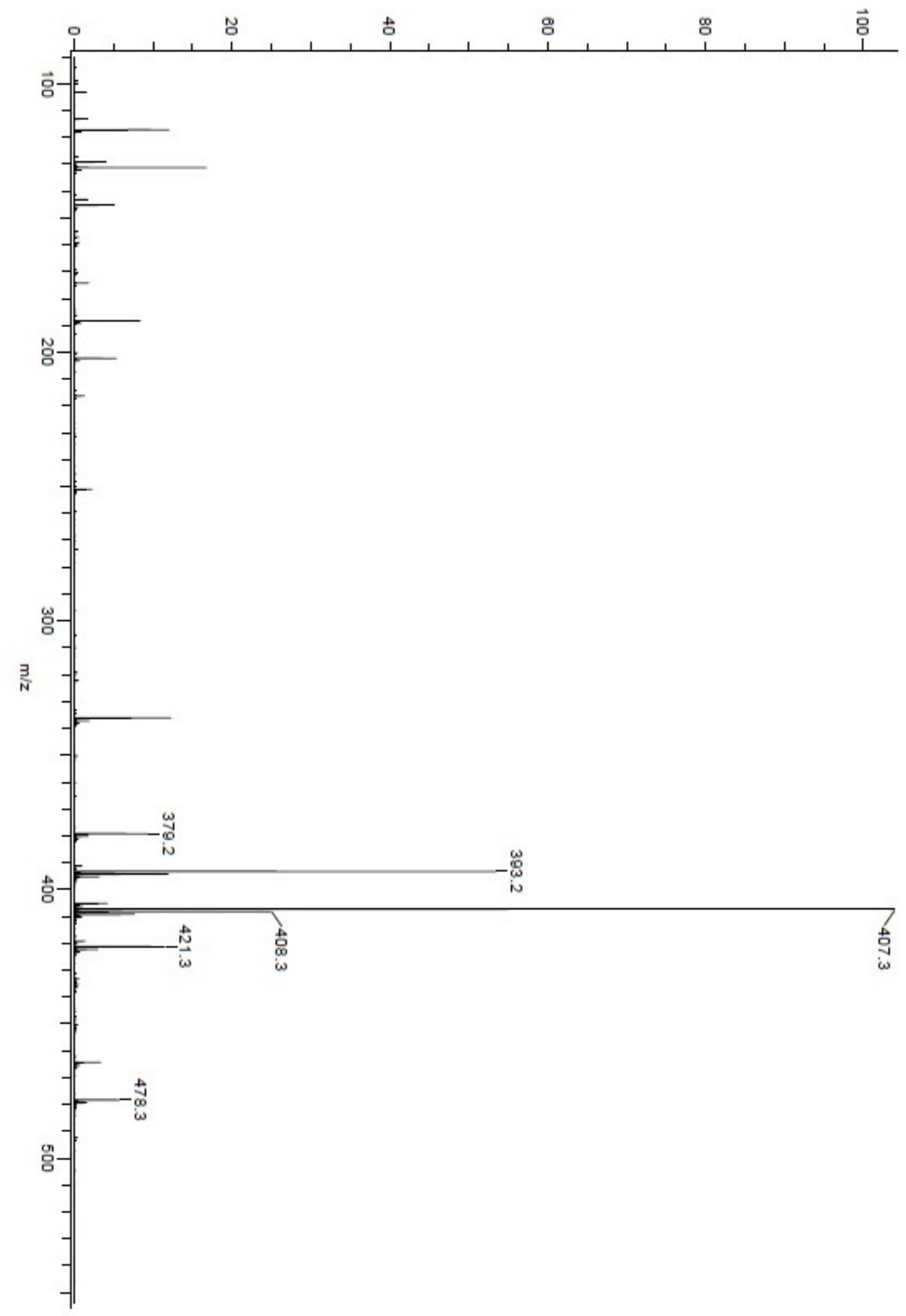

Figure A 420. DART spectrum of compound 221.

787 


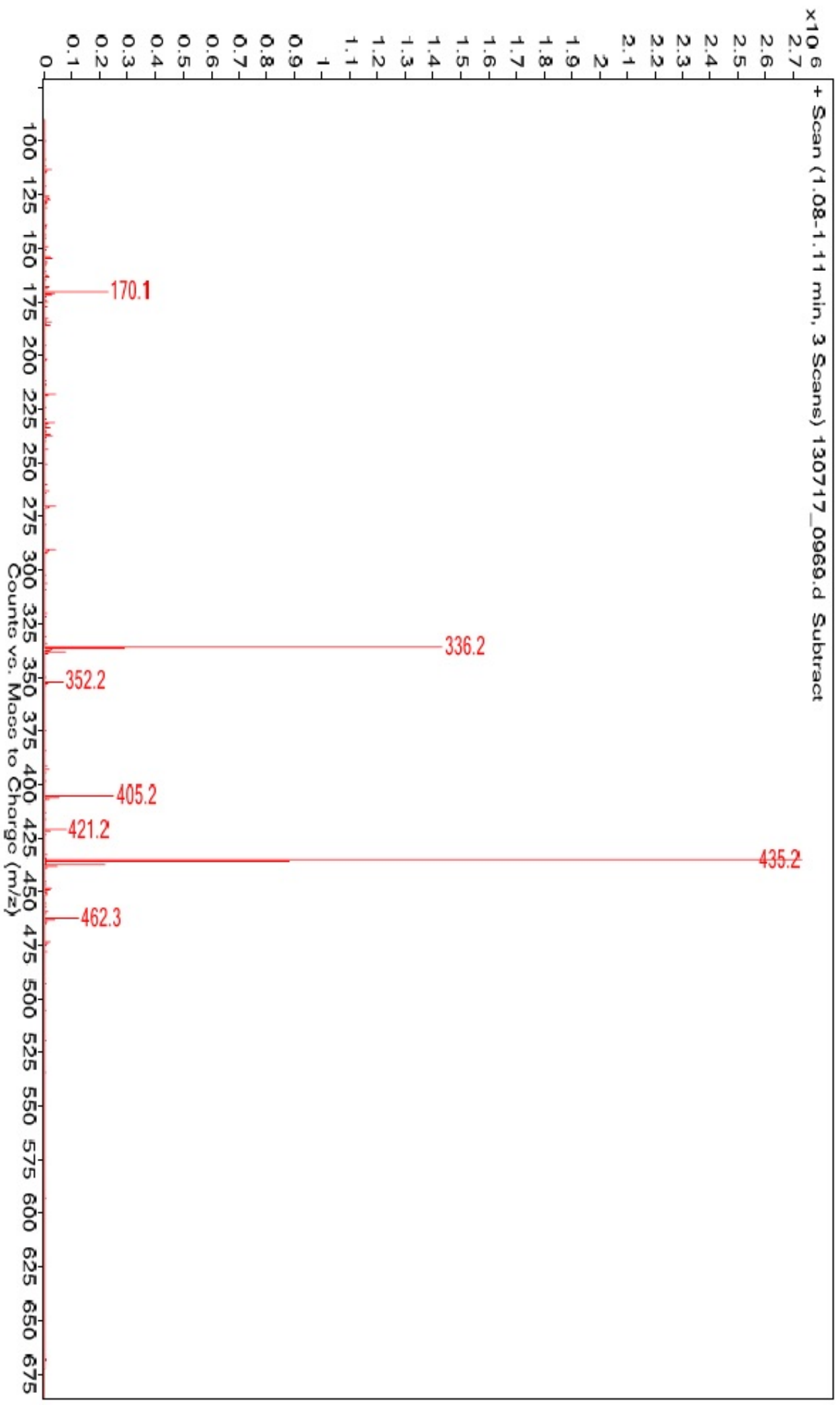

Figure A 421. ESI-TOF spectrum of compound 222. 


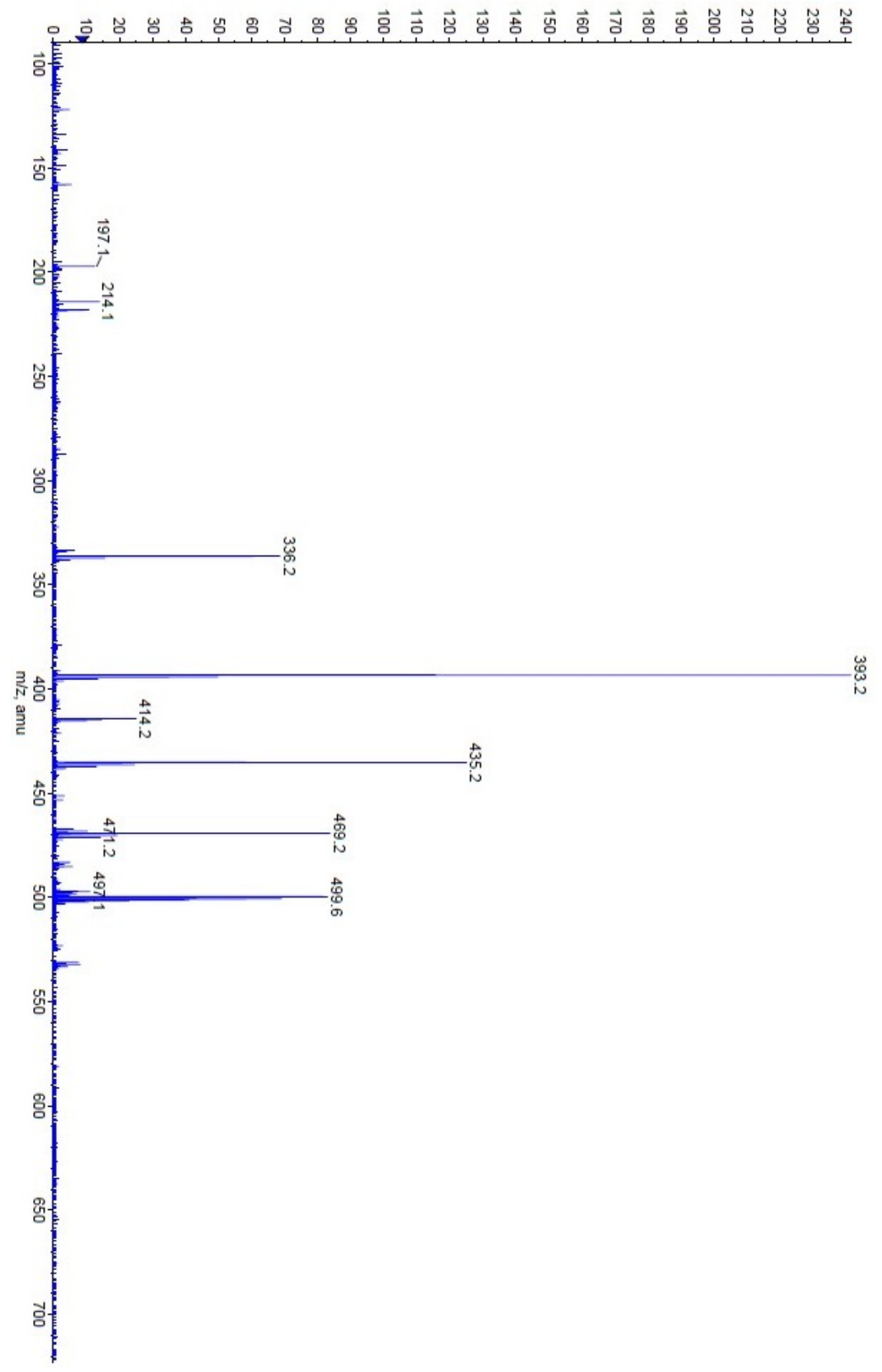

Figure A 422. ESI-TOF spectrum of compound 225. 


\subsection{REFERENCES}

1. Grass, G., Rensing, C., and Solioz., M. Metallic Copper as an Antimicrobial Surface. Appl Environ. Microbiol., 77, 1541-1547 (2011).

2. Chen-Yu, J. H., Eberhardt, D. M., and Kincade, D. H. Antibacterial and Laundering Properties of AMS and PHMB as Finishing Agents on Fabric for Health Care Workers' Uniforms. Cloth. Text. Res. J., 25, 258-272 (2007).

3. Ferreira, L, and Zumbuehl, A. Non-leaching surfaces capable of killing microorganisms on contact. J. Mater. Chem., 19, 7796-7806 (2009).

4. McFee, R. B. Nosocomial or Hospital-acquired Infections: An Overview. Disease-a-Month 55, 422-438 (2009).

5. World Health Organization. Prevention of hospital-acquired infections. A practical guide. 2nd edition (2002). Retrieved August 10, 2013 from http://www.who.int/csr/resources/publications/whocdscsreph200212.pdf.

6. Klevens, R. M. Edwards, J. R., Richards, C. L., Horan, T. C., Gaynes, R. P., Pollock, D. A., and Cardo, D. M. Estimating Health Care-Associated Infections and Deaths in U.S. Hospitals, 2002. Public Health Rep., 122, 160-166 (2007).

7. Hetrick, E. M., and Schoenfisch, M. H. Reducing implant-related infections: active release strategies. Chem. Soc. Rev., 35, 780-789 (2006).

8. Kramer, A., Schwebke, I., and Kampf, G. How long do nosocomial pathogens persist on inanimate surfaces? A systematic review. BMC Infec. Diseas., 6, 1-8 (2006).

9. Page, K., Wilson, M., and Parkin, I. P. Antimicrobial surfaces and their potential in reducing the role of the inanimate environment in the incidence of hospital-acquired infections. J. Mater. Chem., 19, 3819-3831 (2009).

10. Lichter, J. A., Van Vliet, K. J., and Rubner, M. F. Design of Antibacterial Surfaces and Interfaces: Polyelectrolyte Multilayers as a Multifunctional Platform. Macromolecules, 42, 85738586 (2009).

11. Gao, Y., and Cranston, R. Recent Advances in Antimicrobial Treatments of Textiles. Text. Res. J., 78, 60-72 (2008).

12. Tiller, J. C. Antimicrobial Surfaces. Springer, Berlin / Heidelberg, Adv. Polym. Sci., 240, 193-217 (2011).

13. Siedenbiedel, F., and Tiller, J. C. Antimicrobial Polymers in Solution and on Surfaces: Overview and Functional Principles. Polymers, 4, 46-71 (2012). 
14. Denyer, S. P. Mechanisms of action of biocides. Inter. Biodeter., 26, 89-100 (1990).

15. Russell, A. D. Introduction of biocides into clinical practice and the impact on antibioticresistant bacteria. J. Appl. Microbiol., 92, 121S-135S (2002).

16. Perkins, J. J. Principles and Methods of Sterilization in Health Sciences (2nd Ed.), 39-42, (1980).

17. Sharma, V. K., Yngard, R. A., and Lin, Y. Silver nanoparticles: Green synthesis and their antimicrobial activities. Adv. Colloid Interface Sci., 145, 83-96 (2009).

18. Kugel, A., Stafslien, S., and Chisholm, B. J. Antimicrobial coatings produced by "tethering" biocides to the coating matrix: A comprehensive review. Prog. in Org. Coat., 72, 222-252 (2011).

19. Klibanov, A. M. Permanently microbicidal materials coatings. J. Mater. Chem., 17, 24792482 (2007).

20. Simoncic, B., and Tomsic, B. Structures of Novel Antimicrobial Agents for Textiles - A Review. Text. Res. J., 80, 1721-1737 (2010).

21. Caillier, L., de Givenchy, E. T., Levy, R., Vandenberghe, Y., Géribaldi, S., and Guittard, F. Synthesis and antimicrobial properties of polymerizable quaternary ammoniums. Eur. J. Med. Chem., 44, 3201-3208 (2009).

22. Fristrup, C. J., Jankova, K., and Hvilsted, S. Surface-initiated atom transfer radical polymerization-a technique to develop biofunctional coatings. Sof. Mater., 5, 4623-4634 (2009).

23. Pahnke, J., and Rühe, J. Attachment of Polymer Films to Aluminium Surfaces by Photochemically Active Monolayers of Phosphonic Acids. Macromol. Rapid. Commun., 25, 1396-1401 (2004).

24. Prucker, O., Naumann, C., Ruhe, J., Knoll, W., and Frank, C. Photochemical attachment of polymer films to solid surfaces via monolayers of benzophenone derivatives, J. Am. Chem. Soc., 121, 8766-8770 (1999).

25. Dhende, V. P., Samanta, S., Jones, D. M., Hardin, I. R., and Locklin, J. One-Step Photochemical Synthesis of Permanent, Nonleaching, Ultrathin Antimicrobial Coatings for Textiles and Plastics. ACS Appl. Mater. Interfaces., 3, 2830-2837 (2011).

26. Li, B., Franking, R., Landis, E. C., Kim, H., and Hamers, R. J. Photochemical Grafting and Patterning of Biomolecular Layers onto $\mathrm{TiO}_{2}$ Thin Films. ACS Appl. Mater. Interfaces., 1, 1013-1022 (2009).

27. Ye, Q., Zhou, F., and Liu, W. Bioinspired catecholic chemistry for surface modification. Chem. Soc. Rev., 40, 4244-4258 (2011).

791 
28. Barbey, R., Lavanant, L., Paripovic, D., Schuàwer, N., Sugnaux, C., Tugulu, S., and Klok, H. A. Polymer Brushes via Surface-Initiated Controlled Radical Polymerization: Synthesis, Characterization, Properties, and Applications. Chem. Rev., 109, 5437-5527 (2009).

29. Tew, N. W., and Madkour, A. E. Towards self-sterilizing medical devices: controlling infection. Polym. Inter., 57, 6-10 (2008).

30. Dong, H., Huan, J., Koepsel, R., Ye, P., Russell, A. J., and Matyjaszewski, K. Recyclable Antibacterial Magnetic Nanoparticles Grafted with Quaternized Poly(2-(dimethylamino)ethyl methacrylate) Brushes. Biomacromol., 12, 1305-1311 (2011).

31. Faure, E., Lecomte, P., Lenoir, S., Vreuls, C., Weerdt, Van De., Archambeau, C., Martial, J., Jerome, C., Duwez, A. S., and Detrembleur, C. Sustainable and bio-inspired chemistry for robust antibacterial activity of stainless steel. J. Mater. Chem., 21, 7901-7904 (2011).

32. Huang, J., Murata, H., Koepsel, R. R., Russell, A. J. and Matyjaszewski, K. Antibacterial Polypropylene via Surface-Initiated Atom Transfer Radical Polymerization Biomacromolecules. Biomacromol., 8, 1396-1399 (2007).

33. Sweat, D. P., Kim, M., Yu, X., and Gopalan, P. A Single-Component Inimer Containing Cross-Linkable Ultrathin Polymer Coating for Dense Polymer Brush Growth. Langmuir, 29, 3805-3812 (2013).

34. Yang, Y., Hu, H., Li, Y., Wan, L., and Xu, Z. Membrane surface with antibacterial property by grafting polycation. J. Membr. Sci., 376, 132-141 (2011).

35. Isquith, A. J., and McCollum, C. J. Surface kinetic test method for determining rate of kill by an antimicrobial solid. Appl. Environ. Microbiol., 36, 700-704 (1978).

36. Speier, J. L., and Malek, J. R. Destruction of microorganisms by contact with solid surfaces. J. Colloid Interface Sci., 89, 68-76 (1982).

37. Plueddemann, E. P. and Revis, A. Organosilicon quaternary ammonium antimicrobial compounds. Dow Corning Corporation, US 4866192 A (1988).

38. Isquith, A. J., Abbott, E. A., and Walters, P. A. Surface bonded antimicrobial activity of an organosilicon quaternary ammonium chloride. Appl. Microbiol., 2, 859-863 (1972).

39. Pallavicini, P., Taglietti, A., Dacarro, G., Diaz-Fernandez, Y. A., Galli, M., Grisoli, P., Patrini, M., Santucci De Magistris, G., and Zanoni, R. Self-assembled monolayers of silver nanoparticles firmly grafted on glass surfaces: Low $\mathrm{Ag}^{+}$release for an efficient antibacterial activity. J. Colloid. Inter. Sci., 350, 110-116 (2010).

40. Guido, K., Rutzinger, D., and Gallauner, T. Synthesis of hexadentate hexahydro 1,3,5triazine based ligands and their copper (I) complexes. Mont. fuer Chem., 133, 1157-1164 (2002). 792 
41. Gottesman, R., Shukla, S., Perkas, N., Solovyov, L. A., Nitzan, Y., and Gedanken, A. Sonochemical Coating of Paper by Microbiocidal Silver Nanoparticles. Langmuir, 27, 720-726 (2011).

42. Huang, J., Koepsel, R. R., Murata, H., Wu, W., Lee, S.B., Kowalewski, T., Russell, A. J., Matyjaszewski, K. Nonleaching Antibacterial Glass Surfaces via “Grafting Onto": The Effect of the Number of Quaternary Ammonium Groups on Biocidal Activity. Langmuir, 24, 6785-6795 (2008).

43. Sambhy, V., Peterson, B. R., and Sen, A. Multifunctional Silane Polymers for Persistent Surface Derivatization and Their Antimicrobial Properties. Langmuir, 24, 7549-7558 (2008).

44. Bouloussa, O., Rondelez, F. and Semetey, V. A new, simple approach to confer permanent antimicrobial properties to hydroxylated surfaces by surface functionalization. Chem. Commun., 951-953 (2008).

45. Ohlhausen, H. G., and Ludwig, J. H. Solvent-Free Organosilane Quaternary Ammonium Compositions, Method of Making and Use. Resource Development, LLC. US 20110271873 (2011).

46. Aegis. Microbeguard Technology, (2009). Retrieved May 10, 2011 from http://www.nwmoldprevention.com/downloads/microbe_guard_technology.pdf.

47. Allred, G. D., and Liebeskind, L. S. Water-stabilized organosilane compounds and methods for using the same. Emory University, US 5959014 A (1996).

48. Isquith, A. J., Abbott, E. A., and Walters, P. A. Algicidal activity of a surface-bonded organosilicon quaternary ammonium chloride. Appl. Microbiol., 25, 253-256 (1973).

49. Nakagawa, Y., Hayashi, H., Tawaratani, T., Kourai, H., Horie, T., and Shibasaki, I. Disinfection of Water with Quaternary Ammonium Salts Insolubilized on a Porous Glass Surface. Appl. Environ. Microbiol., 47, 513-518 (1984).

50. Battice, D. R., and Hales, M. G. A New Technology for Producing Stabilized Foams Having Antimicrobial Activity. J. Cell. Plast., 21, 332-337 (1985).

51. Murray, P. R., Niles, A. C., and Heeren, R. L. Microbial inhibition on hospital garments treated with Dow Corning 5700 antimicrobial agent. J. Clin. Microbiol., 26, 1884-1886 (1988).

52. Gottenbos, B., van der Mei, H. C., Klatter, F., Nieuwenhuis, P., and Busscher, H. J. In vitro and in vivo antimicrobial activity of covalently coupled quaternary ammonium silane coatings on silicone rubber. Biomaterials, 23, 1417-1423 (2002).

53. Abo El Ola, S. M., Kotek, R., White, W. C., Reeve, J. A., Hauser, P., and Kim, J. H. Unusual polymerization of 3-(trimethoxysilyl)-propyldimethyloctadecyl ammonium chloride on PET substrates. Polymer, 45, 3215-3225 (2004). 
54. Nikawa, H., Ishida, K., Hamada, T., Satoda, T., Murayama, T., Takemoto, T., Tamamoto, M., Tajima, H., Shimoe, S., Fujimoto, H., and Makihira, S. Immobilization of Octadecyl Ammonium Chloride on the Surface of Titanium and Its Effect on Microbial Colonization In Vitro. Dent. Mater. J., 24, 570-582 (2005).

55. Osterhof, J. J., Buijssen, K. J., Busscher, H. J., van der Laan, B.F., and van der Mei, H. C. Effects of quaternary ammonium silane coatings on mixed fungal and bacterial biofilms on tracheoesophageal shunt prostheses. Appl. Environ. Microbiol., 72, 3673-3677 (2006).

56. Andresen, M., Stenstad, P., Møretrø, T., Langsrud, S., Syverud, K., Johansson, L.S., and Stenius, P. Nonleaching Antimicrobial Films Prepared from Surface-Modified Microfibrillated Cellulose. Biomacromol., 8, 2149-2155 (2007).

57. Bouloussa, O., Rondelez, F. and Semetey, V. A new, simple approach to confer permanent antimicrobial properties to hydroxylated surfaces by surface functionalization. Chem. Commun., 951-953 (2008).

58. Sambhy, V., Peterson, B. R., and Sen, A. Multifunctional Silane Polymers for Persistent Surface Derivatization and Their Antimicrobial Properties. Langmuir, 24, 7549-7558 (2008).

59. Saif, M. J., Anwar, J. and Munawar, M. A. A Novel Application of Quaternary Ammonium Compounds as Antibacterial Hybrid Coating on Glass Surfaces. Langmuir, 25, 377379 (2009).

60. Köylü, D., Bilecen, K., Şahin, E., Duman, G., and Taralp, A. Cotton gauze bearing nondiffusible quaternary ammonium salts and featuringanti-microbial activity: An example of single-use articles tailored to self-sterilize. Faculty of Engineering \& Natural Sciences, Materials Science \& Engineering Program, Sabanci University, Istanbul online poster (2010). Retrieved September 01, 2013 from http://people.sabanciuniv.edu/taralp/presentations/ACS2003 \%20NewOrleansPoster\%20Damla.pdf

61. Song, L., and Baney, R. H. Antibacterial evaluation of cotton textile treated by trialkoxysilane compounds with antimicrobial moiety. Text. Res. J., 81, 504-511 (2011).

62. Song, J., Kong, H. and Jang, J. Bacterial adhesion inhibition of the quaternary ammonium functionalized silica nanoparticles. Coll. Surf. B: Biointerfaces 82, 651-656 (2011).

63. Green, J. D., Bickner, S., Carter, P. W., Fulghum, T., Luebke, M., Nordhaus, M. A., and Strathmann, S. Antimicrobial testing for surface-immobilized agents with a surfaceseparated live-dead staining method. Biotechnol. Bioeng., 108, 231-236 (2011).

64. Nanci, A., Wuest, J. D., Peru, L., Brunet, P., Sharma,V., Zalzal, S., McKee, M.D. Chemical modification of titanium surfaces for covalent attachment of biological molecules. $J$. Biomed. Mater. Res., 40, 324-335 (1998). 
65. Gawalt, E. S., Avaltroni, M. J., Koch, N., and Schwartz, J. Self-Assembly and Bonding of Alkanephosphonic Acids on the Native Oxide Surface of Titanium. Langmuir, 17, 5736-5738 (2001).

66. Jampala, S. N., Sarmadi, M., Somers, E. B., Wong, A. C. L., and Denes, F. S. PlasmaEnhanced Synthesis of Bactericidal Quaternary Ammonium Thin Layers on Stainless Steel and Cellulose Surfaces. Langmuir, 24, 8583-8591 (2008).

67. Adden, N., Gamble, L. J., Castner, D. G., Hoffmann, A., Gross, G., and Menzel, H. Phosphonic Acid Monolayers for Binding of Bioactive Molecules to Titanium Surfaces. Langmuir, 22, 8197-8204 (2006).

68. Raman, A., Dubey, M., Gouzman, I. and Gawalt, E. S. Formation of Self-Assembled Monolayers of Alkylphosphonic Acid on the Native Oxide Surface of SS316L. Langmuir, 22, 6469-6472 (2006).

69. Schwartz, J., Avaltroni, M. J., Danahy, M. P., Silverman, B. M., Hanson, E. L., Schwarzbauer, J. E., Midwood, K. S., and Gawalt, E. S. Cell attachment and spreading on metal implant materials. Mat. Sci. Eng: C 23, 395-400 (2003).

70. Mani, G., Johnson, D. M., Marton, D., Dougherty, V. L., Feldman, M. D., Patel, D., Ayon, A. A., and Agrawal, C. M. Stability of Self-Assembled Monolayers on Titanium and Gold. Langmuir, 24, 6774-6784 (2008).

71. Vericat, C., Vela, M. E., Benitez, G., Carro, P., and Salvarezza, R. C. Self-assembled monolayers of thiols and dithiols on gold: new challenges for a well-known system. Chem. Soc. Rev., 39, 1805-1834 (2010).

72. Gawalt, E. S., Avaltroni, M. J., Koch, N., and Schwartz, J. Self-Assembly and Bonding of Alkanephosphonic Acids on the Native Oxide Surface of Titanium. Langmuir, 17, 5736-5738 (2001).

73. Silverman, B. M., Wieghaus, K. A. and Schwartz, J. Comparative Properties of Siloxane vs Phosphonate Monolayers on A Key Titanium Alloy. Langmuir, 21, 225-228 (2005).

74. Lecollinet, G., Delorme, N., Edely, M., Gibaud, A., Bardeau, J. F., Hindré, F., Boury, F., and Portet, D. Self-Assembled Monolayers of Bisphosphonates: Influence of Side Chain Steric Hindrance. Langmuir, 25, 7828-7835 (2009).

75. Hanson, E. L., Schwartz, J., Nickel, B., Koch, N., and Danisman, M. F. Bonding SelfAssembled, Compact Organophosphonate Monolayers to the Native Oxide Surface of Silicon. $J$. Am. Chem. Soc., 125, 16074-16080 (2003).

76. Raman, A., Dubey, M., Gouzman, I., and Gawalt, E. S. Formation of Self-Assembled Monolayers of Alkylphosphonic Acid on the Native Oxide Surface of SS316L. Langmuir, 22, 6469-6472 (2006). 
77. Hoque, E., DeRose, J. A., Bhushan, B., and Mathieu, H. J. Self-Assembled Monolayers on Aluminum and Copper Oxide Surfaces: Surface and Interface Characteristics, Nanotribological Properties, and Chemical Stability, Nano. Sci. Tech., 235-281 (2008).

78. Guerrero, G., Amalric, J., Mutin, P., Sotto, A., and Lavigne, J. Inhibition de l'adhésion bactérienne et prévention de la formation d'un biofilm: utilisation de monocouches autoassemblées organiques sur des surfaces inorganiques. Pathologie Biologie 57, 36-43 (2009).

79. Mutin, P., Amalric, J., Guerrero, G., Ponche, J. P., and Lavigne, J. Surface modification of titanium by Phosphonate monolayers Releasing Bactericidal Species. European Cells and Materials 21, 63 (2011).

80. Mutin, P. H, Guerrero, G., and Amalric, J. P. Preparation of an inorganic substrate having antimicrobial properties. WO 2007080291 (2007).

81. Amalric, J., Mutin, P. H., Guerrero, G., Ponche, A., Sotto, A., and Lavigne, J. P. Phosphonate monolayers functionalized by silver thiolate species as antibacterial nanocoatings on titanium and stainless steel. J. Mater. Chem. 19, 141-149 (2009).

82. Denizot B., Hindre, F. and Portet, D. Geminal bisphosphonates containing at least two quaternary ammonium functions in the backbone for coating of metallic and mineral surfaces for protection against biofilm formation and bacterial contamination. Brevet FR 2878248 (2006).

83. Portet, D., LeCollinet, G., Hindre, F., Bejanin, S., Chappard, D., and Denizot, B. Method of covering self-assembled metal or inorganic monolayer surfaces of gem-bisphosphonic compounds and uses thereof. Surfactis Technologies, Fr., WO2008017721 A3 (2008).

84. Pfaffenroth, C., Winkel, A., Dempwolf, W., Gamble, L.J., Castner, D. G., Stiesch, Meike., and Menzel, H. Self-Assembled Antimicrobial and Biocompatible Copolymer Films on Titanium. Macromol. Biosci., 11, 1515-1525 (2011).

85. Chen, C. and Wickstrom, E. Self-Protecting Bactericidal Titanium Alloy Surface Formed by Covalent Bonding of Daptomycin Bisphosphonates. Bioconjug. Chem., 21, 1978-1986 (2010).

86. Dong, H., Huang, J., Koepsel, R., Ye, P., Russell, A. J., and Matyjaszewski, K. Recyclable Antibacterial Magnetic Nanoparticles Grafted with Quaternized Poly(2(dimethylamino)ethyl methacrylate) Brushes. Biomacromol., 12, 1305-1311 (2011).

87. Kruszewski, K. M., Nistico, L., Longwell, M. J., Hynes, M. J., Maurer, J.A., HallStoodley, L., and Gawalt, E. S. Reducing Staphylococcus aureus biofilm formation on stainless steel 316L using functionalized self-assembled monolayers. Mater. Sci. Eng., C: Mater. Biol. Applic., 33, 2059-2069 (2013). 
88. Dalsin, J. L., Hu, B. H., Lee, B. P., and Messersmith, P. B. Mussel Adhesive Protein Mimetic Polymers for the Preparation of Nonfouling Surfaces. J. Amer. Chem. Soc., 125, 42534258 (2003).

89. Waite, J. H. Surface chemistry: Mussel Power. Nat. Mater., 7, 8-9 (2008).

90. Wach, J., Bonazzi, S. and Gademann, K. Antimicrobial Surfaces through Natural Product Hybrids. Angew. Chem. Int. Ed., 47, 7123-7126 (2008).

91. Yuan, S., Wan, D., L., Liang, B., Pehkonen, S. O., Ting, Y. P., Neoh, K. G., and Kang, E. T. Lysozyme-Coupled Poly(poly(ethylene glycol) methacrylate)-Stainless Steel Hybrids and Their Antifouling and Antibacterial Surfaces. Langmuir, 27, 2761-2774 (2011).

92. Caro, A. Humblot, V., Meathivier, C., Minier, M., Salmain, M., and Pradier, C. M. Grafting of Lysozyme and/or Poly(ethylene glycol) to Prevent Biofilm Growth on Stainless Steel Surfaces. J. Phys. Chem. B., 113, 2101-2109 (2009).

93. Humblot, V., Yala, J. F., Thebault, P., Boukerma, K., Hequet, A., Berjeaud, J. M., and Pradier, C. M. The Antibacterial Activity of Magainin I Immobilized onto Mixed Thiols SelfAssembled Monolayers. Biomaterials, 30, 3503-3512 (2009).

94. Shalev, T., Gopin, A., Bauer., M., Stark, R., and Rahimipour., S. Non-leaching antimicrobial surfaces through polydopamine bio-inspired coating of quaternary ammonium salts or an ultrashort antimicrobial lipopeptide. J. Mater. Chem., 22, 2026-2032 (2012).

95. Appendini, P., and Hotchkiss, J. H. Review of antimicrobial food packaging. Innov. Food Sci. Emerg. Technol., 3, 113-126 (2002).

96. Muñoz-Bonilla, A., and Fernández-García, M. Polymeric materials with antimicrobial activity. Prog. Poly. Sci., 37, 281-339 (2012).

97. Kenawy, E., Worley, S. D. and Broughton, R. The chemistry and applications of antimicrobial polymers: a state-of-the-art review. Biomacromol., 8, 1359-1384 (2007).

98. Harney, M. B., Pant, R. R., Fulmer, P. A., and Wynne, J. H. Surface Self-Concentrating Amphiphilic Quaternary Ammonium Biocides as Coating Additives. ACS App. Mater. Inter., 1, 39-41 (2009).

99. Foucher, D., and Mocella, A. Private Communication. Ryerson University, Toronto, Ontario (2013).

100. Clarkson, N., and Evans, L. V. Further studies investigating a potential non-leaching biocide using the marine fouling diatom Amphora coffeaeformis. Biofouling, 9, 17-30 (1995).

101. Majumdar, P., Crowley, E., Htet, M., Stafslien, S. J., Daniels, J., VanerWal, L., and Chisholm, B. J. Combinatorial Materials Research Applied to the Development of New Surface 797 
Coatings XV: An Investigation of Polysiloxane Anti-Fouling/Fouling-Release Coatings Containing Tethered Quaternary Ammonium Salt Groups. ACS Combinat. Sci., 13, 298-309 (2011).

102. Yagci, M. B. Bolca, S. Heuts, J. P. A. and Ming. W. Self-stratifying antimicrobial polyurethane coatings. Prog. Org. Coat., 72, 305-314 (2011).

103. Stovicek, P. Biocidal compositions based on polymers of dihydroxy quaternary ammonium salts. US 5084096 (1992).

104. Waschinski, C., Zimmermann, J., Salz, U., Hutzler, R., Sadowski, G., and Tiller, J. C. Design of Contact-Active Antimicrobial Acrylate-Based Materials Using Biocidal Macromers. Adv. Mater., 20, 104-108 (2008).

105. Mocella, A. UV Cured Benzophenone Terminated Quaternary Ammonium Antimicrobials for Plastics. $4^{\text {th }}$ Year Undergraduate Thesis. Department of Chemistry and Biology, Ryerson University, Toronto, ON, 1-80 (2012).

106. Yang, Y., Hu, H., Li, Y., Wan, L., and Xu, Z. Membrane surface with antibacterial property by grafting polycation. J. Membr. Sci. 376, 132-141 (2011).

107. Griep-Raming, N., Karger, M., and Menzel, H. Using Benzophenone-Functionalized Phosphonic Acid To Attach Thin Polymer Films to Titanium Surfaces. Langmuir, 20, 1181111814 (2004).

108. Zou, P., Hartleb, W., and Lienkamp, K. It takes walls and knights to defend a castle synthesis of surface coatings from antimicrobial and antibiofouling polymers. J. Mater. Chem. 22, 19579-19589 (2012).

109. Hu, R., Li, G., Jiang, Y., Zhang, Y., Zou, J. J., Wang, L., and Zhang, X. SilverZwitterion Organic-Inorganic Nanocomposite with Antimicrobial and Antiadhesive Capabilities. Langmuir, 29, 3373-3779 (2013).

110. (i) Cheng, G., Xue, H., Zhang, Z., Chen, S., and Jiang, S. A Switchable Biocompatible Polymer Surface with Self-Sterilizing and Nonfouling Capabilities. Angew. Chem. Int. Ed. 47, 8831-8834 (2008). (ii) Cao., Z., Mi, L., Mendiola, J., Ella-Menye, J. R., Zhang, Lei., Xue, Hong., and Jiang, S. Reversibly Switching the Function of a Surface between Attacking and Defending against Bacteria. Angew. Chem. Int. Ed., 51, 2602-2605 (2012). (iii) Yu, Q., Cho, J., Shivapooja, P., Ista, L. K. and López, G. P. Nanopatterned Smart Polymer Surfaces for Controlled Attachment, Killing, and Release of Bacteria. ACS Appl. Mater. Interfaces. XXX, XXX-XXX (2013).

111. Gabriel, G., Maegerlein, J. A., Nelson, C. F., Dabkowski, J. M., Eren, T., Nüsslein, K., and Tew, G. N. Comparison of Facially Amphiphilic versus Segregated Monomers in the Design of Antibacterial Copolymers. Chem. Eur. J. 15, 433-439 (2009). 
112. Gong, S., Niu, L., Kemp, L. K., Yiu, C. K. Y., Ryou, H., Qi, Y., Blizzard, J. D., Nikonov, S., Brackett, M. G., Messer, R. L. W., Wu, C. D., Mao, J., Brister, L. B., Rueggeberg, F. A., Arola, D. D., Pashley, D. H., and Tay, F. R. Quaternary ammonium silanefunctionalized, methacrylate resin composition with antimicrobial activities and self-repair potential. Acta Biomaterialia, 8, 3270-3282 (2012).

113. Gilbert, P., and Moore, L. E. Cationic antiseptics: diversity of action under a common apithet. J Appl. Microbiol., 99, 703-715 (2005).

114. Jansen, A. C., Boucher, C. E., Coetse, E., Kock, J. L. F., vanWyk, P. W. J., Swart, H. C., and Bragg, R. R. The influence of Didecyldimethylammonium Chloride on the morphology and elemental composition of Staphylococcus aureus as determined by NanoSAM. Sci. Res. Ess., 8, 152-160 (2013).

115. Hegstad, K., Langsrud, S., Lunestad, B. T., Scheie, A. A., Sunde, M. and Yazdankhah, S. P. Does the wide use of quaternary ammonium compounds enhance the selection and spread of antimicrobial resistance and thus threaten our health? Microb. Drug Resist., 16, 91-94 (2010).

116. Schaeufele, P. Advances in Quaternary Ammonium Biocides. J. Amer. Oil. Soc. Chem., 61, 387 (1984).

117. Iannou, C. J., Hanlon, G. W., and Denyer, S. P. Action of disinfectant quaternary ammonium compounds against Staphylococcus aureus. Antimicrob. Agents. Chemother., 51, 296-306 (2007).

118. Thebault, P., Taffin de Givency, E., Levy, R., Vandenberghe, Y., Guittard, F., and Géribaldi, S. Preparation and antimicrobial behaviour of quaternary ammonium thiol derivatives able to be grafted on metal surfaces. Eur.J.Med.Chem., 44, 717-724 (2009).

119. Russell, A. D. Mechanism of antimicrobial action of antiseptics and disinfectants: An increasingly important area of investigation. J.Antimicrob.Chemother., 49, 597-599 (2002).

120. McDonnell, G. A., and Russell D. Antiseptics and Disinfectants: Activity, Action, and Resistance. Clin. Microbiol. Rev., 12, 147-179 (1999).

121. Holdsworth, S.R., and Law, C.J. The major facilitator superfamily transporter MdtM contributes to the intrinsic resistance of Escherichia coli to quaternary ammonium compounds. $J$ Antimicrob. Chemother., 68, 831-839 (2013).

122. Tabata, A., Nagamune, H., Maeda, T., Murakami, K., Miyake, Y., and Kourai, H. Correlation between Resistance of Pseudomonas aeruginosa to Quaternary Ammonium Compounds and Expression of Outer Membrane Protein OprR. Antimicrob. Agents Chemother., 47, 2093-2099 (2003). 
123. Mangalappalli-Illathu, A. K., and Korber, D. R. Adaptive Resistance and Differential Protein Expression of Salmonella enterica Serovar Enteritidis Biofilms Exposed to Benzalkonium Chloride. Antimicrob. Agents Chemother., 50, 3588-3596 (2006).

124. Nonaka, T., Hua, L., Ogata, T., and Kurihara, S. Synthesis of water-soluble thermosensitive polymers having phosphonium groups from methacryloyloxyethyl trialkyl phosphonium chlorides-N-isopropylacrylamide copolymers and their functions. J. Appl. Polym. Sci., 87, 386-393 (2003).

125. Waschinski, C. J., Barnert, S., Theobald, A., Schubert, R., Kleinschmidt, F., Hoffmann, A., Saalwächter, K., and Tiller, J. C. Insights in the Antibacterial Action of Poly(methyloxazoline)s with a Biocidal End Group and Varying Satellite Groups. Biomacromol., 9, 1764-1771 (2008).

126. Lewis, K., and Klibanov, A. M. Surpassing nature: rational design of sterile-surface materials. Trends Biotechno.,. 23, 343-348 (2005).

127. Tiller, J. C., Liao, C.J., Lewis, K., and Klibanov, A. M. Designing surfaces that kill bacteria on contact. PNAS., 98, 5981-5985 (2001).

128. Park, D., Wang, J., and Klibanov, A. M. One-Step, Painting-Like Coating Procedures To Make Surfaces Highly and Permanently Bactericidal. Biotechnol. Prog., 22, 584-589 (2006).

129. Lewis, K., and Klibanov, A. M. Surpassing nature: rational design of sterile-surface materials. Trends Biotechnol., 23, 343-348.

130. Murata, H., Koepsel, R. R., Matyjaszewski, K., and Russell, A. J. Permanent, nonleaching antibacterial surfaces-2: How high density cationic surfaces kill bacterial cells. Biomaterials, 28, 4870-4879 (2007).

131. Kuegler, R., Bouloussa, O., and Rondelez, F. Evidence of a charge-density threshold for optimum efficiency of biocidal cationic surfaces. Microbiology Reading, 151, 1341-1348 (2005).

132. Bieser, A. M., Thomann, Y., and Tiller, J. C. Contact-Active Antimicrobial and Potentially Self-Polishing Coatings Based on Cellulose. Macromol. Biosci., 11, 111-121 (2011).

133. Bieser, A. M., and Tiller, J. C. Mechanistic Considerations on Contact-Active Antimicrobial Surfaces with Controlled Functional Group Densities. Macromol. Biosci., 11, 526534 (2011).

134. American Society for Testing and Materials. Minimum Inhibitory Concentration (MIC) Determination. Annual Book of ASTM Standards, 1, 1-10 (2002).

135. Antimicrobial Test laboratories. ISO 22196 Test for Antimicrobial Activity of Plastics. (2013). Retrieved June 10, 2013 from http://www.antimicrobialtestlaboratories.com. 
136. Green, J. D. Science against microbial pathogens: communicating current research and technological advances. Méndez-Vilas Publishing, FORMATEX Microbiology Series $N^{o} 3$, 578585 (2011).

137. Ronan, E. Investigating Microbial Ecology at Solid-Air Interfaces. $4^{\text {th }}$ Year Undergraduate Thesis. Department of Chemistry and Biology, Ryerson University, Toronto, ON, 1-89 (2011).

138. Deng, H. $4^{\text {th }}$ Year Undergraduate Thesis. Department of Chemistry and Biology, Ryerson University, Toronto, ON, 1-78 (2013).

139. Menschutkin, N. Ueber die Einwirkung des Chloracetyls auf phosphorige Saure. Ann. Chem. Pharm., 133, 317-320 (1865).

140. Stanger, K. J., Lee, J. J., and Smith, B. D. Dramatic Acceleration of the Menschutkin Reaction and Distortion of Halide Leaving-Group Order. J. Org. Chem., 72, 9663 - 9668 (2007).

141. Reinheimer, J. D., Harley, J. D., and Meyers, W. W. Solvent Effects in the Menschutkin Reaction. J. Org. Chem., 28, 1575-1579 (1963).

142. Smith, M. March's advanced organic chemistry: reactions, mechanisms, and structure Wiley-Interscience, Hoboken, N.J., 525, (2007).

143. Sommer, H. Z., Lipp, H. I., and Jackson, L. J. Alkylation of amines. General exhaustive alkylation method for the synthesis of quaternary ammonium compounds. J. Org. Chem., 36, 824-828 (1971).

144. Long, B., Nikitin, K., and Fitzmaurice, D. Self-Assembly of a Tripodal Pseudorotaxane on the Surface of a Titanium Dioxide Nanoparticle. J. Am. Chem. Soc,. 125, 5152-5160 (2003).

145. Salvatore, R. Synthesis of secondary amines. Tetrahedron, 57, 7785-7811 (2001).

146. Hayashi, Y., Fujiwara, T., Nagano, Y., and Teramura, K. Preparation of tertiary amines having different substituents from quaternary 2-hydroxyethylammonium salts. Yukagaku, 36, 409-412 (1987).

147. Basu, B., Paul, S., and Nanda, A. K. Highly selective $N$-Alkylation of amines promoted on silica: an efficient and recyclable surface. Green Chem., 11, 115-1120 (2009).

148. Salvatore, R. N., Nagle, A. S., and Jung, K. W. Cesium Effect: High Chemoselectivity in Direct N-Alkylation of Amines. J. Org. Chem., 67, 647-683 (2002).

149. Ju, Y., and Varma, R. S. Aqueous $N$-alkylation of amines using alkyl halides: direct generation of tertiary amines under microwave irradiation. Green Chem., 6, 219-221 (2004). 
150. Ju, Y., and Varma, R. S. An efficient and simple aqueous $N$-heterocyclization of aniline derivatives: microwave-assisted synthesis of $N$-aryl azacycloalkanes. Org. Lett., 7, 2409-2411 (2005).

151. Singh, C. B., Kavala, V., Samal, A. K., and Patel B. K. Aqueous-Mediated N-Alkylation of Amines. Eur. J. Org. Chem., 2007, 1369-1377 (2007).

152. Li, J. J., and Corey, E. J. Name Reactions of Functional Group Transformations. John Wiley and Sons, USA, CH2, 86-92 (2007).

153. Baxter, E. W., and Reitz, A. B. Reductive aminations of carbonyl compounds with borohydride and borane reducing agents. Org. React. (Hoboken, NJ, U. S.) 59, 101 (2002).

154. Abdel-Magid, A. F., and Mehrman, S. J. A Review on the Use of Sodium Triacetoxyborohydride in the Reductive Amination of Ketones and Aldehydes. Org. Process Res. Dev., 10, 971-1031 (2006).

155. Abdel-Magid, A. F., and Maryanoff, C. A. Use of sodium triacetoxyborohydride in reductive amination of ketones and aldehydes. ACS Symp. Ser., 641, 201-216 (1996).

156. Lane, C. F. Sodium cyanoborohydride, a highly selective reducing agent for organic functional groups. Synthesis, 135-46 (1975).

157. Bhattacharyya, S. Borohydride reductions in dichloromethane: a convenient, environmentally compatible procedure for the methylation of amines. Synth. Commun., 25, 20612069 (1995).

158. Da Silva, R. A., Estevam, I. H. S., and Bieber, L. W. Reductive methylation of primary and secondary amines and amino acids by aqueous formaldehyde and zinc. Tetrahedron Lett., 48, 7680-7682 (2007).

159. Cope, A. C., Ciganek, E., Fleckenstein, L. J. and Meisinger, M. A. P. Tertiary Amines from Methiodides and Lithium Aluminum Hydride. J. Am. Chem. Soc., 82, 4651-4655 (1960).

160. Hutchins, R. O., Kandasamy, D., Dux, F., Maryanoff, C. A., Rotstein, D., Goldsmith, B., Burgoyne,W., Cistone, F., Dalessandro, J., and Puglis, J. Nucleophilic borohydride: selective reductive displacement of halides, sulfonate esters, tertiary amines, and $N, N$ disulfonimides with borohydride reagents in polar aprotic solvents. J. Org. Chem., 43, 2259-67 (1978).

161. Saurabh, S., Fadila, L., Moreau, J. P., Hurvois, J. L., Renaudde Weghe, T., and Hierry, R. Synthesis of Alkaloids of Galipea officinalis by Alkylation of an $\alpha$-Amino Nitrile. Eur. J. Org. Chem., 2008, 4622-4631 (2008). 
162. Yoshida, Y., Sakakura, Y., Aso, N., Okada, S., and Tanabe, Y. Practical and efficient methods for sulfonylation of alcohols using $\mathrm{Ts}(\mathrm{Ms}) \mathrm{Cl} / \mathrm{Et}_{3} \mathrm{~N}$ and catalytic $\mathrm{Me}{ }_{3} \mathrm{~N}-\mathrm{HCl}$ as combined base: promising alternative to traditional pyridine. Tetrahedron, 55, 2183-2192 (1999).

163. Olah, G. A., Gupta, B. G., Malhotra, R., and Narang, S. C. Chlorotrimethylsilane/lithium bromide and hexamethyldisilane/pyridinium bromide perbromide: effective and selective reagents for the conversion of alkyl (cycloalkyl and aralkyl) alcohols into bromides. J. Org. Chem., 45, 1638-1639 (1980).

164. Couture, G., Alaaeddine, A., Boschet, F., and Ameduri, B. Polymeric materials as anionexchange membranes for alkaline fuel cells. Prog. Polym. Sci., 36, 1521-1557 (2011).

165. Cai, J. and Wathey, B. A novel traceless solid phase tertiary amine synthesis based on Merrifield resin. Tetrahedron Lett., 42, 1383-1385 (2001).

166. Grovenstein, E., Chandra, S., Collum, C. E., and Davis, W. E. Carbanions. VIII. Products and mechanisms of reaction of allyl- and cyclopropyltrimethylammonium halides and of allyl chloride, alcohol, and p-tolyl sulfide with sodium in liquid ammonia. J. Am. Chem. Soc., 88, 1275-1289 (1966).

167. Ho, T.S. Dequaternization of ammonium salts by nucleophiles. Synth. Commun., 3, 99-100 (1972).

168. Kametani, T., Kigasawa, K., Hiiragi, M., Wagatsuma, N., and Wakisaka, K. Debenzylation of quaternary ammonium salts with thiophenol. Tet. Lett., 8, 635-638 (1969).

169. Hutchins, R. O., Dux, and F. J. Selective demethylation of quaternary salts with lithium propylmercaptide in hexamethylphosphoramide. J. Org. Chem., 38, 1961-1962 (1973).

170. Ho, T. S. Dealkylation of quaternary ammonium salts with 1,4-diazabicyclo[2.2.2]octane. Synthesis, 12, 702 (1972).

171. Bauera, B., Strathmannb, H., Effenbergerc, F. Anion-exchange membranes with improved alkaline stability. Desalination, 79, 125-144 (1992).

172. Cope, A. C., Mehta, and A. S. Mechanism of the Hofmann elimination reaction: an ylide intermediate in the pyrolysis of a highly branched quaternary hydroxide. J. Amer. Chem. Soc., 85, 1949-1952 (1963).

173. William, E. W., and Manning, J. H. Antimicrobial polyalcohols and their derivatives. CA1129851A1 (1982).

174. Battaglini, G. T. Assay of quaternary ammonium antimicrobial compounds by aqueous potentiometric titration. J. Surfact. Deterg., 5, 117-121 (2002). 
175. Jansson, S. O., Modin, R., and Schill, G. Two-phase titration of organic ammonium ions with lauryl sulphate and methyl yellow as indicator. Talanta, 21, 905-918 (1974).

176. Bioshield Technologies. Certification of Limits and Analytical Method for Enforcement of Limits for BST Protectant 50. 1-6 (1997).

177. Mistry, K. New Self-assembled Quaternary Ammonium Fluorescent Compounds. $4^{\text {th }}$ Year Undergraduate Thesis. Department of Chemistry and Biology, Ryerson University, Toronto, ON, 1-37 (2011).

178. Taffa, D., Kathiresan, M. and Walder, L. Tuning the Hydrophilic, Hydrophobic, and Ion Exchange Properties of Mesoporous $\mathrm{TiO}_{2}$. Langmuir, 25, 5371-5379 (2009).

179. Vermeulen, L. A., Fateen, R. Z. and Robinson, P. D. Single Crystal Structure Determination of a New Zirconium $N$-Ethylpyridinium Phosphonate: $\mathrm{Zr}\left(\mathrm{O}_{3} \mathrm{PCH}_{2} \mathrm{CH}_{2} \mathrm{NC}_{5} \mathrm{H}_{5}\right)\left(\mathrm{F}^{-}\right.$ 3. Inorg. Chem., 41, 2310-2312 (2002).

180. Demmer, C. S., Krogsgaard-Larsen, N., and Bunch, L. Review on Modern Advances of Chemical Methods for the Introduction of a Phosphonic Acid Group. Chem. Rev., 111, 79818006 (2011).

181. Jansa, P., Baszczyňski, Procházková, B., Dračínský, M., and Janeba, Z. Microwaveassisted hydrolysis of phosphonate diesters: an efficient protocol for the preparation of phosphonic acids. Green Chem., 14, 2282-2288 (2012).

182. Morita, J. Okamoto, Y., and Sakurai, H. A convenient dealkylation of dialkyl phosphonates by chlorotrimethylsilane in the presence of sodium iodide. Tet. Lett., 19, 25232526 (1978).

183. McKenna, C. The facile dealkylation of phosphonic acid dialkyl esters by bromotrimethylsilane. Tet. Lett., 18, 155-158 (1977).

184. Blackburn, G. M., and Ingleson, D. The dealkylation of phosphate and phosphonate esters by lodotrimethylsilane: a mild and selective procedure. J. Chem. Soc. Perkin Trans., 1, 11501153 (1980).

185. Chowdhury, S., Muni, N. J., Greenwood, N. P., Pepperberg, D. R. and Standaert, R. F. Phosphonic acid analogs of GABA through reductive dealkylation of phosphonic diesters with lithium trialkylborohydrides. Bioorg. Med. Chem. Lett., 17, 3745-3748 (2007).

186. Gauvry, N. M. J. Dealkylation of dialkyl phosphonates with boron tribromide. Synthesis, 4, 553-554 (2001).

187. McKenna, C. E., and Schmidhauser, J. Functional selectivity in phosphonate ester dealkylation with bromotrimethylsilane. J. Chem. Soc. Chem. Commun., 739 (1979). 
188. Mortier, J., Gridnev, I. D., and Guenot, P. Reactions of Phosphonates with Organohaloboranes: New Route to Molecular Borophosphonates. Organometallics, 19, 42664275 (2000).

189. Mortier, J. Recent developments on the mechanism of the reaction of dialkyl phosphonates with haloboranes and their implications for synthesis. Rec. Res. Develop. Org. Chem., 6, 169-176 (2002).

190. Erickson, J. G. Phosphonoammonium compounds. US 2774786 19561218, General Mills Inc. (1956).

191. Frederick, H. Synthetic detergents. NL 7918919551015 (1955).

192. Germanaud, L., Brunel, S., Chevalier, Y., and Le Perchec, P. Synthesis of neutral amphiphilic phosphobetaines with variable interionic distances. Bull. Soc. Chimi. Fra., 4, 699704 (1988).

193. Fu, X. K., and Chen, W. S. Synthesis of dimethyldodecylammonioethylphosphonic acid betaine. Xinan Shifan Daxue Xuebao, Ziran Kexueban, 27, 61-63 (2002).

194. Hüttinger, K. J. and Jung, M. F. Kinetik der Synthese von Trialkyl[-3-(trimethoxysilyl)propyl]-ammoniumchloriden und deren antimikrobielle Wirkung als fixierte Biozide. Chem. Ingen. Tech., 61, 258-259 (1989).

195. Majumdar, P., Crowley, E., Htet, M., Stafslien, S. J., Daniels, J., VanderWal, L and Chisholm, B. J. Combinatorial Materials Research Applied to the Development of New Surface Coatings XIII: An Investigation of Polysiloxane Antimicrobial Coatings Containing Tethered Quaternary Ammonium Salt Groups. J. Comb. Chem., 11, 1115-1127 (2009).

196. Zhu, X. Yang, C., Chen, G., Wan, Y. Microwave-assisted synthesis of siloxane quaternary ammonium salts. Zhongshan Daxue Xuebao, Ziran Kexueban, 48, 56-59 (2009).

197. Hüttinger, K. J., and Zeller, D. Chemisch und verfahrenstechnisch optimierte, trägergebundene Desinfektionsmittel für die Wasserentkeimung mit Tiefenfiltern. Chem. Ingen. Tech., 59, 516-517 (1987).

198. Ragulin, V. V. $\omega$-Haloalkylphosphoryl Compounds: Synthesis and Properties. Russ. J. Gen. Chem., 82, 1928-1937 (2012).

199. Yoshimura, T., Kusano, T., Iwase, H., Shibayama, M., Ogawa, T., and Kurata, H. StarShaped Trimeric Quaternary Ammonium Bromide Surfactants: Adsorption and Aggregation Properties. Langmuir, 28, 9322-9331 (2012).

200. Gourves, J. P., Couthon, H., and Sturtz, G. F. Synthesis of 3,4-dihydro-2H-pyrido[1,2b]isoindol-1-one and 3,4-dihydro-2H-pyrido[1,2-b]pyrrolidin-1-one functionalized at the C(6) 
position by an intramolecular Horner-Wadsworth-Emmons reaction. Eur. J. Org. Chem., 12, 3489-3493 (1999).

201. Smits, J. P., and Wiemer, D. F. Synthesis and Reactivity of Alkyl-1,1,1-trisphosphonate Esters. J. Org. Chem., 76, 8807-8813 (2011).

202. O'Boyle, N. M., Greene, L. M., Bergin, O., Fichet, J. B., McCabe, T., Lloyd, D. G., Zisterer, D. M., and Meegan, M. J. Synthesis, evaluation and structural studies of antiproliferative tubulin-targeting azetidin-2-ones. Bioorg. Med. Chem., 19, 2306-2325 (2011).

203. Zefirov, N. S., and Matveeva, E. D. Catalytic Kabachnik-Fields reaction: new horizons for old reaction. ARKIVOC Special Issue Reviews and Accounts 1, 1-17 (2008).

204. Keglevich, G., Szekrényi, A., Szöllősy, A., and Drahos, L. Synthesis of Bis(phosphonatomethyl), Bis(phosphinatomethyl), and Bis(phosphinoxidomethyl)amines, as Well as Related Ring Bis(phosphine) Platinum Complexes. Synth. Commun., 41, 2265-2272 (2011).

205. Tibhe, G. D., Reyes-González, M. A., Cativiela, C., and Ordóñez, M. Microwaveassisted High Diastereoselective Synthesis of $\alpha$-Aminophosphonates under Solvent and Catalyst Free-conditions. J. Mex. Chem. Soc., 56, 183-187 (2012).

206. Ishikawa, S., Tajima, M. and Mochizuki, M. Synthesis and properties of bifunctional chloroalkyl nitrosamines with an intercalating moiety. Bioorg. Med. Chem., 12, 3791-3796 (2004).

207. Mutin, P. H., Guerrero, G. and Vioux, A. J. Hybrid materials from organophosphorus coupling molecules. Mater. Chem., 15, 3761-3768 (2005).

208. Matveeva, E., Petrovskii, P. V., and Odinets, I. L. Efficient synthesis of racemic $\beta$ aminophosphonates via aza-Michael reaction in water. Tet. Lett., 49, 6129-6133 (2008).

209. Tiasto, F. Preparation of bisphosphonoamine ligands for the synthesis of technetium nitride complexes for radiodiagnostic imaging. PCT Int Appl. 2007083395 (2007).

210. Wang, J., Liang, Y. L., and Qu, J. Boiling water-catalyzed neutral and selective $N$-Boc deprotection. Chem. Commun., 5144-5146 (2009).

211. Simonyan, G. S., Arutyunyan, R. S., Beileryan, N. M. and Grigoryan, E. A. Synthesis of New Surface-Active $N^{2}$-Amino Amides, Agents for Fixation of Dyes on Cotton Fibers, from N-Tri(hydroxymethyl)methyl]acrylamide. Russ. J. Appl .Chem.,76, 258-260 (2003).

212. (i) Borgman, R. J., McPhillips, J. J., and Stitzel, R. E. Synthesis and Pharmacology of Centrally Acting Dopamine Derivatives and Analogs in Relation to Parkinson's Disease. J. Med. Chem., 16, 630-633 (1973). (ii) Louafi, F., Hurvois, J., Chibani, A., and Roisnel, T. Synthesis 
of Tetrahydroisoquinoline Alkaloids via Anodic Cyanation as the Key Step. J. Org. Chem., 75, 5721-5724 (2010).

213. Haslegrave, J. A., and Sullivan, D. S. Nitrogen and sulfur-containing corrosion inhibitors. Exxon Chemical Patents Inc. (Linden, NJ). United States Patent 4673436 (1987).

214. Asakawa, T., Shimizu, Y., Ozawa, T., Ohta, A., and Miyagishi, S. Aqueous solution properties of disulfide linked gemini and cleaved monomeric thiol surfactants. J. Oleo Sci., 57, 243-249 (2008).

215. Thebault, P., Taffin de Givenchy, E., Levy, R., Vandenberghe,Y., Guittard, F., and Géribaldi, S. Contact-active microbicidal gold surfaces using immobilization of quaternary ammonium thiol derivatives. Eur. J. Med. Chem., 44, 4227-4234 (2009).

215. Harjani, J. R., Liang, C., and Jessop, P. G. A Synthesis of Acetamidines. J. Org. Chem., 76, 1683-1691 (2011).

216. Allen, F. H., Kennard, O., Watson, D. G., Brammer, L. and Orpen, A. G. Tables of Bond Lengths Determined by X-Ray and Neutron Diffraction. J. Chem. Soc. Perkin. Trans., 2, S1-S19 (1897).

217. Andrews, J. M. Determination of minimum inhibitory concentrations. J. Antimicrob. Chemother., 48, 5-16 (2001).

218. Wang, X. Xue, Y., Wang, Y. Preparation and application of organosilicon quaternary ammonium salt composition. Faming Zhuanli Shenqing, CN 102391299, A 20120328 (2012).

219. Li, F., Shishkin, E., Mastro, M. A., Hite, J. K., Eddy, C. R., Edgar, J. H., and Ito, T. Photopolymerization of Self-Assembled Monolayers of Diacetylenic Alkylphosphonic Acids on Group-III Nitride Substrates. Langmuir, 26, 10725-10730 (2010).

220. Baber, A., de Vries, J. G., Orpen, A. G., Pringle, P. G., and von der Luehea, K. Allosteric effects in asymmetric hydrogenation catalysis. Asymmetric induction as a function of the substrate and the backbone flexibility of $\mathrm{C} 1$-symmetric diphosphines in rhodium-catalysed hydrogenations. Dalton Trans., 4821-4828 (2006).

221. Viornery, C., Pechy, P., Boegli, M., Aronsson, B. O., Descouts, P., and Gratzel, M. Synthesis of new polyphosphonic acids. Phos. Sul. Silic. Rel. Elem., 177, 231-241 (2002).

223. Villemin, D. Simeon, F. Decreus, H., and Jaffres, P. A. Rapid and efficient Arbuzov reaction under microwave irradiation. Phos. Sul. Silic. Rel. Elem., 133, 209-213 (1998).

224. Jansa, P. Holy, A. Dracinsky, M. Baszczynski, O. Cesnek, M., and Janeba, Z. Efficient and green' microwave-assisted synthesis of haloalkylphosphonates via the Michaelis-Arbuzov reaction. Green Chem., 13, 882-888 (2011). 
225. Sun, L. J., Cao, J. Q., Ge, C. H., Zhang, X. D., Tong, J. and Ma, Y. C. Synthesis and characterization of alkyl substituted dimethyl phosphonates. Liaoning Daxue Xuebao, Ziran Kexueban, 31, 78-80 (2004).

226. Voigt, M. Klaumunzer, M., Ebel, A., Werner, F., Yang, G., Marczak, R., Spiecker, E., Guldi, D. M., Hirsch, A., and Peukert, W. Surface Functionalization of ZnO Nanorods with C60 Derivatives Carrying Phosphonic Acid Functionalities. J. Phys. Chem. C., 115, 5561-5565 (2011).

227. Hara, T., Durell, S. R., Myers, M. C., and Appella, D. H. Probing the Structural Requirements of Peptoids That Inhibit HDM2-p53 Interactions. J. Am. Chem. Soc., 128, 19952004 (2006).

228. (i) Chevalier, Y., Germanaud, L., Brunel, S., Storet, Y., Berthelon, B., LePerchec, P., and Gallot, B. Zwitterionic amphiphiles: synthesis and physical properties. Comun. Jorn. Com. Esp. Deterg., 18, 231-245 (1987). (ii). Brunet, S., Germanaud, L., Perchec, P., and Sillion, B. Neutral phosphobetaines, their preparation, and their use in petroleum recovery. Fr. Demande, 2572078-A1 (1986).

229. Tucker. S. A, K. and Garrell, R. L. Simple Preparation and Application of TEMPOCoated $\mathrm{Fe}_{3} \mathrm{O}_{4}$ Superparamagnetic Nanoparticles for Selective Oxidation of Alcohols. Chem. A Eur. J., 16, 12718-12726 (2010).

230. Conibear, A. C., Lobb, K. A., and Kaye, P. T. ${ }^{31}$ P NMR kinetic study of the tandem cleavage of phosphonate esters by bromotrimethylsilane. Tetrahedron, 66, 8446-8449 (2010).

231. Martinelli, M. J., and Pollack, S. R. Encyclopedia of Reagent for Organic Synthesis. John Wiley and Sons Ltd, Bromotrimethylsilane, 1-21 (2001).

232. Paleos, C. M., Arkas, M. and Skoulios, A. Mesomorphic character of quaternary ammonium salts affected by secondary hydrogen bonding interactions. Molecular Crystals and Liquid Crystals Science and Technology, Section A: Molecular Crystals and Liquid Crystals, 309, 237-250 (1998).

233. Pinchuk, A. N., Rampy, M. A., Longino, M. A., Skinner, R. W. S., Gross, M. D., Weichert, J. P., and Counsell, R. E. Synthesis and Structure-Activity Relationship Effects on the Tumor Avidity of Radioiodinated Phospholipid Ether Analogues. J. Med. Chem., 49, 21552165 (2006).

234. Simoni, D. Gebbia, N., Invidiata, F. P., Eleopra, M., Marchetti, P., Rondanin, R., Baruchello, R., Provera, S. S., Marchioro, C., Tolomeo, M., Marinelli, L., Limongelli, V., Novellino, E., Kwaasi, A., Dunford, J., Buccheri, S., Caccamo, N., and Dieli, F. Design, Synthesis, and Biological Evaluation of Novel Aminobisphosphonates Possessing an in Vivo Antitumor Activity Through a gammadelta-T Lymphocytes-Mediated Activation Mechanism. $J$. Med. Chem., 51, 6800-6807 (2008). 
235. Gourves, J. P., Couthon, H., and Sturtz, G. A new efficient synthesis of $\omega$ aminoalkylidene-1,1-bisphosphonate tetraethyl esters. Phos. Sul. Silic. Rel. Elem., 132, 219-229 (1998).

236. Neidlein, R. E. T. Substituted methylphosphonates as synthons for alicyclic $\alpha$ functionalized phosphonates. Monatshefte fuer Chemie., 123, 1037-1043 (1992).

237. Tanaka, K. S., Houghton, T. J., Kang, T., Dietrich, E., Delorme, D., Ferreira, S. S., Caron, L., Viens, F., Arhin, F. F., Sarmiento, I., Lehoux, D., Fadhil, I., Laquerre, K., Liu, J., Ostiguy, V., Poirier, H., Moeck, G., Parr, TR. Jr., and Rafai Far, A. Bisphosphonated fluoroquinolone esters as osteotropic prodrugs for the prevention of osteomyelitis. Bioorg. Med. Chem., 16, 9217-9229 (2008).

238. Delorme, D., Houghton, T., Lafontaine, Y., Tanaka, K., Dietrick, E., Kang, T., and Rafai, F. A. Phosphonated oxazolidinones and uses thereof for the prevention and treatment of bone and joint infections and their preparation. WO 2007138381A2 (2007).

239. Lehn, J. M., Blacker, J., and Jazwinski, J. Preparation of 2,7-diazapyrenium dications for photochemical cleavage of nucleic acids. WO 1987-FR142, Compagnie Oris Industrie, Fr., (1987).

240. Polomoscanik, S. C., Holmes-Farley, S. R., Petersen, J. S., Sacchiero, R. J., and Dhal, P. K. Hydrophobically Modified Poly (Allylamine) Hydrogels Containing Internal Quaternary Ammonium Groups as Cholesterol Lowering Agents: Synthesis, Characterization, and Biological Studies . J. Macromol. Sci., Part A: Pure Appl. Chem., 49, 1011-1021 (2012).

241. Cavero, E., Zablocka, M., Caminade, A., and Majoral, J. P. Design of BisphosphonateTerminated Dendrimers. Eur. J. Org. Chem., 2759-2767 (2010).

242. Chougrani, K., Boutevin, B., David, G., Seabrook, S., and Loubat, C. Acrylate based anticorrosion films using novel bis-phosphonic methacrylates. J. Polym. Sci. Part A: Polym. Chem., 46, 7972-7984 (2008).

243. Chougrani, K., Boutevin, B., David, G., and Boutevin, G. New $N, N$-aminodiphosphonate-containing methacrylic derivatives, their syntheses and radical copolymerizations with MMA. Eur. Polym. J., 44, 1771-1781 (2008).

244. Gatlin, L. W. Alkylaminoalkyl substituted triazine sulfide scavenger. US 20030089641, Clearwater International (2003).

245. Kauffman, W. J. Synthesis and characterization of $N, N^{\prime}, N^{\prime \prime}$-tris(dimethylaminopropyl) hexahydro-s-triazine and isolable intermediates. J. Heterocylic Chem., 12, 409-11 (1975).

246. Jasiński, M., Mlostoń, G., Mucha, P., Linden, A., and Heimgartner, H. Synthesis of New Bis-Imidazole Derivatives. Helvetica Chimica Acta., 90, 1765-1780 (2007). 
247. Hu, N., Johnson, L. M., Pothayee, N., Pothayee, N., Lin, Y., Davis, R. M., and Riffle, J. S. Synthesis of ammonium bisphosphonate monomers and polymers. Polymer, 54, 3188-3197 (2013).

248. Bhattacharya, S., and Snehalatha, K. Synthesis and Esterolytic Chemistry of Some (Dialkylamino)pyridine-Functionalized Micellar Aggregates. Evidence of Catalytic Turnover. Langmuir, 11, 4653-4660 (1995).

249. Haldar, J., Venkateswarlu, Y., and Padma, A. Cationic antibacterial vancomycin derivatives and compositions. WO 2013072838 A1 (2013).

250. Romeo, R., Carnovale, C., Giofre, Salvatore V., Romeo, G., Macchi, B., Frezza, C., Marino-Merlo, F., Pistara, V., and Chiacchio, U. Truncated phosphonated C-1'-branched N,Onucleosides: A new class of antiviral agents. Bioorg. Med. Chem., 20, 3652-3657 (2012).

251. Hunter, C. A., Misuraca, M. C. and Turega, S. M. Dissection of Complex Molecular Recognition Interfaces. J. Amer. Chem. Soc., 133, 582-594 (2011).

252. Kaplanek, R., Briza, T., Havlik, M., Martasek, P., and Kral, V. Three-fold polyfluoro alkylated amines and isocyanates based on [tris(hydroxymethyl)amino]methane (TRIS). J. Fluor. Chem., 128, 179-183 (2007).

253. Rannard, S. P., and Davis, N. J. The Selective Reaction of Primary Amines with Carbonyl Imidazole Containing Compounds: Selective Amide and Carbamate Synthesis. Org. Lett., 2, 2117-2120 (2000).

254. Acton, A. L., and Fante, C. Janus PEG-Based Dendrimers for Use in Combination Therapy: Controlled Multi-Drug Loading and Sequential Release. Biomacromol., 14, 564-574 (2013).

255. Babu, D. A., Manohar, C. S., and Visweswaran, V. Synthesis, characterization and antimicrobial activity studies of 2-[\{2-(3,4-dimethoxyphenyl) ethyl $\}$ methylamino]sulphonylnaphthalenes. Asian J. Chem., 20, 1411-1419 (2008).

256. Shahane, S., Saurabh, S., Fadila, L., Moreau, J., Hurvois, J. P., Renaudde, J-L., and Thierry Roisnel, T. Synthesis of alkaloids of Galipea officinalis by alkylation of an $\alpha$-amino nitrile. Eur. J. Org. Chem., 27, 4622-4631 (2008).

257. Top, S., Kaloun, E. B., Vessières, A., Laïos, I., Leclercq, G., and Jaouen, G. The first titanocenyl dichloride moiety vectorised by a selective estrogen receptor modulator (SERM). Synthesis and preliminary biochemical behaviour. J. Organomet. Chem., 643-644, 350-356 (2002).

258. Saettone C., Alderigi, B., Giannaccini, C., Anslemi, M., Rossetti, M., and Scotton, R. Substantivity of sunscreens-prepatation and evaluation of some quaternary ammonium benzophenone derivatives. Int. J. Cosm. Sci., 10, 99-109 (1988). 
259. Wang, X. and Schneider, H. Binding of dansylamide derivatives to nucleotides and nucleic acids. J. Chem. Soc. Perkin Trans., 2, 1323-1328 (1998).

260. Jacobsen, D. W. Preparation of cryptofluorescent analogs of cobalamin coenzymes. Methods Enzymol., 67, 12-19 (1980).

261. Simonin, J., Vernekar, S. K. V., Thompson, A. T., Hothersall, J. D., Connolly, C. N., Lummis, S. R. L., and Lochner, M. High-affinity fluorescent ligands for the 5-HT 3 receptor. Bioorg. Med. Chem. Lett., 22, 1151-1155 (2012). 